\title{
ID: GA2 \\ Antibody-dye conjugate detects modest epidermal growth factor receptor expression in high-graded gliomas: a feasibility study for fluorescence-guided resection
}

\author{
Quan Zhou, Stanford University, qzh@stanford.edu
}

\section{Category: Neuroscience}

\begin{abstract}
Body : Background: The epidermal growth factor receptor (EGFR) is a biomarker heterogeneously expressed in high-grade gliomas (HGGs). Administered systemically, nearinfrared fluorescent dye-labeled antibody targeting EGFR may be able to detect HGGs expressing modest amount of EGFR and serve as an image-guided surgical probe. Objective: To assess the feasibility of imaging HGGs using panitumumab-IRDye800 (pan800) in mice bearing orthotopic HGG xenografts with modest EGFR expression, and characterize EGFR protein expression levels in human HGG tissues. Methods: Tumor mice were imaged in vivo after systemic pan800 injection to assess its tumor-specific uptake macroscopically over 14 days, and microscopically ex vivo. EGFR immunohistochemical staining of tumor specimens from 35 HGG patients during 2006-2019 was scored by pathologists and expression levels were compared to that of mouse xenografts and subsequently stratified to identify clinical characteristics that may be predictive of EGFR expression. Results: In HGG xenografts, intratumoral distribution of pan 800 correlated with the EGFR expression and the fluorescence signal. Fluorescence signal distinguished tumor cells with $90 \%$ specificity and $82.5 \%$ sensitivity. Target-to-background ratios maximized at 14 hours post pan800 infusion and reached 19.5 in vivo and 7.6 ex vivo, respectively. Equivalent or higher EGFR protein expression compared to the mouse xenografts was present in $77.1 \%$ HGG patients. Age, combined with IDH-wildtype cerebral tumor, was predictive of positive EGFR protein expression in patients. Conclusion: Tumor specific uptake of pan 800 provided remarkable contrast and a flexible imaging window for fluorescence-guided identification of HGGs despite modest EGFR expression. Targeted imaging and therapeutic attempts could benefit a significant HGG population.
\end{abstract}

Complete Status: Complete

First Name: Quan

Last Name: Zhou

Email: qzh@stanford.edu

Organization: Stanford University

Country: United States 


\title{
ID: GA3 \\ Kidney-specific nanoparticles for detection of Renal cell carcinoma by multimodality imaging
}

\author{
Li Liu, UT Southwestern Medical Center, li.liu@utsouthwestern.edu
}

Category: Systemic Diseases (Kidney, Liver and Pancreas)

\begin{abstract}
Body : Background Renal cell carcinoma (RCC) is known for a lack of early warning signs, accounting for $\sim 3 \%$ of adult malignancies and $90-95 \%$ of kidney neoplasms [1,2]. An effective contrast agent could assist in the early detection of kidney cancer. Recently, we developed that peptide-conjugated N-acetyl glucosamine-based polymer nanoparticles (NPs) have significant kidney accumulation without major toxicity. We hypothesize that these agents may provide an effective contrast to distinguish normal from the cancerous kidney. Our research objective is to characterize and evaluate kidney-targeted NPs using fluorescence imaging (FLI) and multispectral optoacoustic tomography (MSOT) imaging methods in RCC tumor xenografts including patient-derived xenograft (PDX) mouse models. Material and Methods The experimental group of nude mice( group $n>6$ ) was implanted in the right kidney with mouse and human RCC and Â XP490 PDX RCC tissue [3], with a control group composed of healthy nude mice. As tumors developed, we injected NPs intravenously and performed imaging at different time points over several days using in-vivo FLI and MSOT in both experimental and control groups. We additionally injected IRDye $800 \mathrm{CW}-2 \mathrm{DG}$ into tumor-bearing mice as the control contrast agent. After in vivo imaging, mice were sacrificed and kidney, liver, and spleen were excised to do ex-vivo organ FLI and histology studies. Results The NPs strongly enhanced MSOT signal in normal kidneys in both experimental and control groups, with much less enhancement in tumor-bearing kidneys, thereby distinguishing normal kidney from kidney orthotopic tumor (Figure lower imaging). Similarly, the Â in-vivo FLI signal of the right tumorbearing kidney was lower than the left normal kidney, while both kidneys in the control group showed higher signals (Figure upper imaging). In tumor-bearing mice, IRDye800CW-2DG selectively enhanced the FLI signal of the right kidney with a tumor ( Data did not show). Exvivo FLI and histology results validated in-vivo image observations. Conclusion The Kidneyspecific NPs clearly detected Â XP490 tumor-bearing kidney from the normal kidney (yellow arrow showed kidney tumor, red arrow showed the normal kidney). These NPs also could be a promising functional contrast agent for kidney and kidney disease. Further optimization of the specific experimental conditions is necessary to facilitate the clinical application of these NPs in kidney tumors.
\end{abstract}

References: 1. Sun, G. Lughezzani, et al. Treatment of metastatic renal cell carcinoma, Nature Review Urology, 7 (2010) 327-38. 2. J.J. Hsieh, M.P. Purdue, et al. Renal cell carcinoma, Nature Reviews Disease Primers, 3 (2017) 19. 3.W.F. Chen, H. Hill, et al. Targeting renal cell carcinoma with a HIF-2 antagonist, Nature, 539 (2016) 112-117.

\section{Image/Figure:}


Complete Status: Complete

First Name: Li

Last Name: Liu

Email: li.liu@utsouthwestern.edu

Organization: UT Southwestern Medical Center

Country: United States 


\title{
ID: GA6 \\ [Ga-68]-Pentixafor and [F-18]-NaF identify in vivo inflamed, micro-calcified atherosclerotic plaques in various mouse models
}

\author{
Alexandru Florea, Uniklinik RWTH Aachen, aflorea@ukaachen.de
}

\section{Category: Cardiovascular \& Pulmonary}

\begin{abstract}
Body : Introduction. Inflammation and micro-calcification are established hallmarks of the vulnerable plaque. [Ga-68]-Pentixafor and [F-18]-NaF have been proposed as molecular probes to specifically target the aforementioned processes, however they have mostly been assessed in separate retrospective clinical studies (1). Here, we used [Ga-68]-Pentixafor and [F$18]-\mathrm{NaF}$ to investigate the distinct mechanisms in vulnerable plaques in a unitary series of atherosclerotic mouse models using small animal PET/CT. Materials and methods. ApoEknockout mice were fed a western type diet for 12 weeks in order to develop de novo atherosclerosis. To establish a mouse model that develops intimal calcification in early stage plaques, one group (calcification group) was switched to a Warfarin + Vitamin K1 supplemented chow diet for an additional 12 weeks. Warfarin causes lethal bleedings in mice, a side-effect that can be avoided by adding vitamin $\mathrm{K} 1$ to the chow, still inducing vascular vitamin $\mathrm{K}$ deficiency (2). For the inflammation group, the western type diet was maintained up to 24 weeks. For the control, two types of groups were established: ApoE-knockout mice fed a western type diet for 12 weeks, then switched to a normal chow diet for additional 12 weeks, in order to assess the influence of Warfarin + Vitamin K1; and the second group of wild type mice on normal diet for 24 weeks. The entire feeding scheme is available in Figure 1. All four groups were injected with both [Ga-68]-Pentixafor and [F-18]-NaF and scanned using the small animal PET/CT in subsequent days. Plaque morphology was assessed via various ex vivo histological, immunohistological and micro-autoradiography techniques. Results. The calcification group consistently developed spotty calcifications (on $\hat{\mathrm{I}}_{4} / \mathrm{CT}$ ) in the aortic arch, which were confirmed by von Kossa stainings. Moreover, these calcifications had consistent [F-18]-NaF uptake. Tracer uptake correlated with plaques on haematoxylin-eosin and with inflammation markers positive areas as well as von Kossa for [Ga-68]-Pentixafor and [F-18]-NaF, respectively. Moreover, von Kossa staining and [F-18]-NaF micro-autoradiography showed that the inflammation group developed micro-calcifications in the aortic root and aortic arch regions. These micro-calcifications were not detected by CT, but could be visualised by increased [F-18]-NaF uptake. Conclusion. To our knowledge, this is the first mouse model that develops exclusively vascular spotty calcifications detectable by CT. Moreover, [Ga-68]-Pentixafor is able to specifically target inflamed, CXCR-4 positive areas, while [F-18]-NaF correctly targets micro-calcified atherosclerotic plaques.
\end{abstract}

References: (1) Florea, A. et al. (2020) â€ Locking and loading the bullet against microcalcificationâ€TM, Eur J Prev Cardiol; (2) KrÃ¹/4ger, T et al. (2013) â€ ${ }^{\sim}$ Warfarin Induces Cardiovascular Damage in Miceâ€ ${ }^{\mathbf{T M}}$ Arterioscler Thromb Vasc Biol.

\section{Image/Figure:}


https://www.xcdsystem.com/wmis/abstract/File6959/GA6_ImageFigure_0422023902.jpg

Full Name of Abstract's 1st Author : Alexandru Florea

Complete Status: Complete

First Name: Alexandru

Last Name: Florea

Email: aflorea@ukaachen.de

Organization: Uniklinik RWTH Aachen

Country: Germany 


\title{
ID: GA8 \\ Zr-89 oxine PET elucidates glucocorticoid-induced CXCR4-dependent migration of eosinophils from circulation to the bone marrow
}

\author{
Noriko Sato, National Cancer Institute, NIH, saton@mail.nih.gov
}

Category: Immunology: Inflammation \& Infection

\begin{abstract}
Body : Glucocorticoids (GCs) are first-line drugs in a variety of eosinophilic disorders. A transient and profound drop in circulating eosinophils occurs within hours of GC administration and the mechanism by which this phenomenon occurs has remained unexplained since it was first described in the 1940s 1 . Where eosinophils go after they disappear from the circulation also remains unknown. To investigate the migration of eosinophils in response to GC administration, we employed a 89Zr-oxine ex vivo cell labeling technique for real time cell tracking by positron emission tomography (PET)2. Examinations of the response of circulating eosinophils to $\mathrm{GC}$ administration in humans, rhesus macaques (RMs) and mice revealed that the response in RMs, but not in mice, closely resembles that in humans. We thus selected RM as the experimental model. Purified eosinophils were labeled with 89Zr-oxine, which did not alter viability, determined by annexin $\mathrm{V} /$ propidium iodide staining, activation status, examined by CD69 expression, nor in vitro chemotaxis toward CCL11. RMs received autologous transfer of $89 \mathrm{Zr}$-oxine labeled eosinophils intravenously $(0.37-2.7 \mathrm{MBq}, 10.5-97.3 \times 106$ cells, $35.3 \pm 8.0$ $\mathrm{kBq} / 106$ cells) and underwent serial PET/computed tomography (CT) imaging for a total of 3 days. GC (methylprednisolone, $4 \mathrm{mg} / \mathrm{kg}$ ) was administered intravenously immediately after the baseline imaging on day 1 and the subsequent eosinophil response was monitored hourly for 4 hours. The same imaging regimen was employed on day 1 for control RMs. Deferoxamine was infused throughout the imaging period to chelate and enhance renal clearance of free $89 \mathrm{Zr}$ released from dead/dying cells, preventing binding of $89 \mathrm{Zr}$ to the bone. In the control group $(n=3)$, eosinophils showed rapid migration kinetics after transfer; the cells quickly transited through the lungs and distributed to the bone marrow (BM), liver and spleen within 1 hour (approximately 25\%,19\% and 5\% injected dose, respectively), where they remained for the duration of the study. In the treatment group $(n=3), \mathrm{GC}$ administration increased 89Zr activity in the BM over the 4 hours ( $p<0.05)$, whereas $89 \mathrm{Zr}$ activity in other organs in GC treated animals or all four organs in the control group showed no significant change, indicating that GC induced BM homing of eosinophils. As GC administration in humans has been found to induce expression of the chemokine receptor in circulating eosinophils3, and that human and rhesus macaque genomes (but not the mouse genome4) have GC response elements near the CXCR4 transcription start site, we next examined the effects of GC administration on eosinophil CXCR4 expression in RMs. Administration of GC to RMs induced CXCR4 upregulation on eosinophils in blood with a peak at 4-hour, which coincided with disappearance of eosinophils from the circulation $(n=3)$. To assess whether the bone marrow migration of eosinophils after GC administration was CXCR4-dependent, we used the CXCR4 inhibitor plerixafor in combination with GC. GC-induced eosinopenia was significantly reduced or eliminated by CXCR4 blockade $(n=3)$, indicating that $\mathrm{GC}$-induced CXCR4 upregulation in the circulating eosinophils led to their BM migration, resulting in eosinopenia. In conclusion, live
\end{abstract}


tracking of 89Zr-oxine labeled eosinophils by PET revealed that GC administration induces rapid $\mathrm{BM}$ migration of eosinophils, which is mediated by GC-induced CXCR4 expression on eosinophils. These findings provide insight into the mechanism of action of GCs in eosinophilic disorders and may inform the development of more targeted therapeutic strategies and the study of glucocorticoid resistance.

References: 1. A.G. Hills, P.H. Forsham, C.A. Finch. Blood, 1948;3:755“768. 2. N. Sato, H. Wu, K.O. Asiedu, et al. Radiology, 2015;275:490-500. 3. P. Khoury, K. Stokes, M. Gadkari, et al. Allergy, 2018;73:2076“2079. 4. B. Guo B, X. Huang, S. Cooper, et al. Nat. Med., 2017;23:424“428.

Image/Figure:

https://www.xcdsystem.com/wmis/abstract/File6959/GA8_ImageFigure_0128112938.png

Complete Status: Complete

First Name: Noriko

Last Name: Sato

Email: saton@mail.nih.gov

Organization: National Cancer Institute, NIH

Country: United States 


\title{
ID: GA9 \\ Fluorescence-guided resection of tumors in mouse models of oral cancer.
}

\author{
Paula Demetrio de Souza Franca, MSKCC, demetrip@mskcc.org
}

\section{Category: Oncology}

\begin{abstract}
Body : Introduction: Complete removal and negative margins are the goal of any surgical resection of primary oral cavity carcinoma. Negative margins are strongly associated with a lower risk of local recurrence and higher survival rates [1]. On the other hand, over resection often leads to large surgical defects that may cause irreversible impairment of phonation, mastication, gustation, and swallowing [2]. Intraoperative optical imaging could represent a potential tool to address this unmet clinical need [3]. Here we explored the use of an intravenously injected fluorescent imaging agent, PARPi-FL, to delineate the tumor and to identify positive margins in real time. PARPi-FL targets the enzyme poly(ADPribose)polymerase 1 that is overexpressed in cancer $[3,4]$ editing a fluorescent signal. Methods: In this study, three different types of mouse models, two orthotopic xenograft models (using FaDu and Cal 27 cells) and one with chemically induced tumors, were used. Tumor bearing mice, along with controls, were intravenously injected with $0.043 \mathrm{mg} / \mathrm{kg}$ of PARPi-FL, sacrificed after 90 minutes and excised tongues were immediately imaged. Compromised margins were obtained by performing glossectomies and intentionally leaving small tumor tissue in the main tongue specimen. Tongues were imaged using both macroscopic (IVIS epifluorescence imaging system and Lumar stereoscope) and microscopic (hand held and a table top confocal microscope) tools. Results were further compared against histology. Results: PARP1 expression allowed histological tumor delineation ( $\mathrm{p}$ With epifluorescence imaging PARPi-FL was identified in areas where tumors were present, whereas very faint signal was observed in healthy tissue allowing precise tumor delineation. Control tongues had an average radiance efficiency signal significantly lower (P Further exploring the potential of PARPi-FL to guide surgical decision-making, we modeled partial glossectomies in mice. PARPi-FL was able to identify all compromised margins by means of its fluorescent emission. Free of disease margins had no visual signal in both IVIS and Lumar. PARPi-FL is also suitable for contemporaneously assessing margins at a cellular level. In exposed tongue lesions, it gave rise to a bright specific signal detected by confocal microscopy, whereas no specific signal could be seen in the normal muscle cells or dysplasia. Conclusions: In this study, PARPi-FL was able to achieve contemporaneous and specific visualization of tumors and compromised margins in oral cancer mice models. Intravenously injected PARPi-FL has great potential for clinical translation as it can improve the identification of tumor-positive resection margins during surgery, which are associated with lower survival rates [2]. It can also help clinicians reduce the amount of healthy tissue resected, leading to significant improvements in patientâ€ ${ }^{\mathrm{TM}_{\mathrm{S}}}$ quality of life [5].
\end{abstract}

References: 1. Zanoni, D.K., et al., A Proposal to Redefine Close Surgical Margins in Squamous Cell Carcinoma of the Oral Tongue. JAMA Otolaryngol Head Neck Surg, 2017. 143(6): p. 555560. 2. Rajapakshe, R.M., et al., A retrospective analysis to determine factors contributing to the survival of patients with oral squamous cell carcinoma. Cancer Epidemiol, 2015. 39(3): p. 360-6. 
3. Kossatz, S., et al., Detection and delineation of oral cancer with a PARP1 targeted optical imaging agent. Sci Rep, 2016. 6: p. 21371. 4. Kossatz, S., et al., PARP1 as a biomarker for early detection and intraoperative tumor delineation in epithelial cancers â $€$ " first-in-human results. bioRxiv, 2019: p. 663385. 5. Fedele, S., Diagnostic aids in the screening of oral cancer. Head Neck Oncol, 2009. 1: p. 5.

Full Name of Abstract's 1st Author : Paula Demetrio de Souza Franca

Complete Status: Complete

First Name: Paula

Last Name: Demetrio de Souza Franca

Email: demetrip@mskcc.org

Organization: MSKCC

Country: United States 


\title{
ID: GA10 \\ Redesigned LRP Reporter Improves CEST MRI Contrast in LRP-Expressing Mouse Tumor
}

\author{
Or Perlman, Massachusetts General Hospital and Harvard Medical School, \\ operlman@mgh.harvard.edu
}

Category: New Chemistry, Biology \& Bioengineering

\begin{abstract}
Body : Introduction: The genetically engineered lysine rich protein (LRP) reporter gene constitutes a means for expanding the capacity of MRI, allowing the direct detection and imaging of molecular and biological processes [1]. Built on the chemical exchange saturation transfer (CEST) mechanism, this reporter generates an on/off switchable contrast that can be controlled by a radio-frequency (RF) saturation pulse. LRP is biocompatible, biodegradable, and does not involve any metallic substance. It was demonstrated as potentially useful for several invivo applications, allowing the imaging of cells in tumors [2], oncolytic virus delivery and spread [3], and cardiac viral vector expression [4]. However, in its current form, the LRP reporter gene is unstable, as the repetitive DNA sequence encoding the reporter leads to DNA recombination events and hence the expression of a range of truncated protein fragments, resulting in decreased and limited CEST sensitivity [2]. Objective: The goal of this work was to redesign an improved and stable LRP-based CEST-MRI reporter gene (rdLRP) and to validate its CEST-MRI sensitivity in-vitro and in-vivo. Methods: The rdLRP was designed using an RHGP amino acid motif that consisted of arginine, histidine, glycine, and proline amino acids separated by different numbers of lysines. The rdLRP plasmid, under control of the cytomegalovirus (CMV) promoter, was transfected into HEK293T cells using lipofectamine. After 48 hours of incubation, the cells were lysed in pH 7.5 RIPA buffer and underwent 3 freeze/thaw/sonication cycles. The protein content of the supernatant was measured and protein concentrations of control and LRP transfected cell lysates were adjusted to be equivalent (typically 10 Â $\mu \mathrm{g} / \hat{A} \mu l$ protein). Control and rdLRP cell lysate samples were loaded into $5 \mathrm{~mm}$ od sample tubes and placed into $50 \mathrm{ml}$ Falcon tubes containing water. CEST Z-spectra were acquired on a 4.7T Bruker MRI scanner using a CEST-EPI sequence (saturation pulse power, duration, and frequency offset of $3.6 \hat{\mathrm{A}} \mu \mathrm{T}, 5000 \mathrm{~ms}$, and 7 to $-7 \mathrm{ppm}$ with $0.25 \mathrm{ppm}$ offsets, respectively). The average magnetization transfer ratio asymmetry (MTRasym) measure was calculated for each cell-lysate, following B0 field correction [5]. The rdLRP was then expressed in a murine glioma GL261N4 cell line and transplanted into mice brains ( $\mathrm{n}=10,50 \%$ served as control). In-vivo images were acquired at 11 and 18 days post implantation using a similar CEST-EPI sequence on a Bruker 7T scanner with a pre-saturation pulse power of $0.7 \hat{A} \mu \mathrm{T}$. Mapping of the CEST amide compound amplitude (3.5 ppm) was performed using a 5-pool Lorentzian fitting model, implemented in MATLAB. Results: A distinct increase in MTRasym was observed at the amine (1.8 ppm) and amide (3.5 ppm) exchangeable proton frequencies of cells transfected with the rdLRP relative to control and untransfected cells. The rdLRP contained no DNA repeats or GC rich regions resulting in a significantly more stable protein with $30 \%$ less positively charged amino acids. The improved stability was evident in RT-PCR cell lysates of cells transfected with the rdLRP, where only a single well-defined band is observed at the base
\end{abstract}


pair length expected for full-length rdLRP. In contrast, a ladder profile of different bands was observed previously in RT-PCR of cell lysates transfected with the original LRP reporter gene [1]. In-vivo imaging of brain tumor-bearing mice demonstrated a significant increase in tumor CEST contrast $(\mathrm{p}=0.0275, \mathrm{n}=10$ imaging data points) for LRP expressing tumors $(5.88 \hat{\mathrm{A}} \pm 1.44 \%)$ compared to control $(4.34 \hat{\mathrm{A}} \pm 1.28 \%)$. Conclusions: The redesigned LRP reporter demonstrates excellent stability with distinct CEST contrast observed for both amide and guanidyl amine exchangeable protons of the redesigned reporter. The redesigned reporter gene was detectable invivo in a mouse tumor model engineered to express the rdLRP.

References: [1] Gilad, A. A., et al., Nature biotechnology 25.2 (2007): 217-219. [2] Minn, I., et al., Magnetic resonance in medicine 74.2 (2015): 544-549. [3] Farrar, C. T., et al., Radiology 275.3 (2015): 746-754. [4] Meier, S., et al., Scientific reports 8.1 (2018): 1-10. [5] Kim, M., et al., Magnetic Resonance in Medicine 61.6 (2009): 1441-1450.

Full Name of Abstract's 1st Author : Or Perlman

Complete Status: Complete

First Name: Or

Last Name: Perlman

Email: operlman@mgh.harvard.edu

Organization: Massachusetts General Hospital and Harvard Medical School

Country: United States 


\title{
ID: GA11 \\ Optical Imaging of Radiofrequency Hyperthermia-Enhanced Oncolytic Therapy for Hepatic Cancer
}

\author{
Guanhui Zhou, University of Washington School of Medicine, zhouguanhui@126.com
}

\section{Category: Oncology}

\begin{abstract}
Body : Purpose: To investigate the feasibility of using indocyanine green (ICG)-based optical imaging to assess the efficacy of radiofrequency hyperthermia (RFH)-enhanced direct oncolytic therapy of hepatic cancer. Materials and Methods: This study included thee portions of (1) optimizing the ICG dose for intracellular uptake by rat hepatic cancer cells (MCA-RH7777); (2) optimizing ICG time-window for ICG-enhanced optical imaging; and (3) investigating the capability using ICG-based optical imaging to monitor the efficacy of RFH-enhanced oncolytic therapy using oncolytic 9â€'mer cationic peptide Â (LTX-315) for treatment of the same cancer cells. In optimizing ICG dose and time-window, tumor cells in each group were treated with various concentrations of ICG from 0 to $200 \hat{\mathrm{I}}^{1} / 4 \mathrm{~g} / \mathrm{mL}(\mathrm{n}=6 /$ concentration) for 24 hours, followed by different incubation times from 0 to 48 hours ( $n=6 /$ incubation time) at the optimized ICG concentration. The half maximal inhibitory concentration (IC50) of LTX-315 was quantified by MTS assay. To determine the sensitivity of ICG-based optical imaging in monitoring RFHenhanced LTX therapeutic effect, all tumor cells were labelled by ICG using the optimized protocol above, and then divided into four study groups (nâ€\% $=\hat{a} € \%$ o6/group) with different treatments, including $\hat{\mathrm{A}}$ (1) RFH alone at $42 \hat{\mathrm{a}} € \%{ }_{0} \hat{\mathrm{A}}^{\circ} \mathrm{C}$ for $30 \mathrm{a} € \%$ min; (2) oncolytic therapy with LTX-315 alone; (3) combination therapy of oncolytic therapy plus RFH; and (4) saline to serve as a control. MTS assay, fluorescence microscopy, optical/x-ray imaging and flow cytometry were used to evaluate and compare cell viabilities and apoptosis among different treatment groups. ï» ¿Fluorescent optical imaging of ICG/cells was performed using both Bruker optical/xray imaging system and an 18-gauge interventional optical imaging needle. The $\ddot{\imath} \neg$, uorescent signal intensities (SI) were statistically compared among various cell groups. Results: Â ICG was taken up by cancer cells at a linear concentration-dependent fashion from 0 to $100 \hat{\mathrm{I}} 1 / 4 \mathrm{~g} / \mathrm{mL}$ and then plateaued at $100 \hat{\mathrm{A}} \hat{\mathrm{I}} 1 / 4 \mathrm{~g} / \mathrm{mL}$. At the optimized ICG concentration of $100 \hat{\mathrm{I}} 1 / 4 \mathrm{~g} / \mathrm{mL}$, the fluorescent SI reached the peak at the incubation time of 24 hours. IC50 value of LTX-315 for MCA-RH7777 cells was $25.4 \hat{\mathrm{I}}^{1 / 4} \mathrm{M}$. MTS assay demonstrated the lowest cell viability in combination therapy group $(19.6 \hat{\mathrm{A}} \pm 2.8 \%)$ compared with LTX-315 alone $(50.2 \hat{\mathrm{A}} \pm 4.0 \%)$, RF alone $(93.6 \hat{\mathrm{A}} \pm 1.6 \%)$ and saline group $(99.0 \hat{\mathrm{A}} \pm 1.7 \%)(\mathrm{ppï} 1 / 4 œ 0.001)$, which was further confirmed by Bruker optical/X-ray imaging (Figure). Conclusion: This study demonstrates the feasibility of using ICG-based optical imaging to assess the treatment effect of RFH-enhanced oncolytic therapy for hepatic cancers, which may open a new avenue to further develop $\hat{A}$ a new intraoperative optical imaging technique for instantly guiding complete tumor kill at the ablated tumor margin, a current clinical problem of peripheral tumor persistence or recurrence post ablation of medium-to-large tumors.
\end{abstract}

References: 1. Haug BE, et al. Discovery of a 9-mer Cationic Peptide (LTX-315) as a Potential First in Class Oncolytic Peptide. J Med Chem 2016; 59: 2918. 2. Yamazaki T, et al. The 
oncolytic peptide LTX-315 overcomes resistance of cancers to immunotherapy with CTLA4 checkpoint blockade. Cell Death Differ 2016; 23(6): 1004-15. 3. Kepp O, et al. Oncolysis without viruses - inducing systemic anticancer immune responses with local therapies. Nat Rev Clin Oncol 2020; 17(1): 49-64. 4. Heo J, et al. Randomized dose-finding clinical trial of oncolytic immunotherapeutic vaccinia JX-594 in liver cancer. Nat Med 2013; 19(3): 329-36. 5. Harrington K, et al. Optimizing oncolytic virotherapy in cancer treatment. Nat Rev Drug Discov 2019; 18(9): 689-706. 6. Ji J, et al. Non-Small-Cell Lung Cancer: Feasibility of Intratumoral Radiofrequency Hyperthermia-enhanced Herpes Simplex Virus Thymidine Kinase Gene Therapy. Radiology 2018; 288(2): 612-20. 7. Shi Y, et al. Orthotopic Esophageal Cancers: Intraesophageal Hyperthermia-enhanced Direct Chemotherapy in Rats. Radiology 2017; 282(1): 103-12. 8. Hernot $\mathrm{S}$, et al. Latest developments in molecular tracers for fluorescence imageguided cancer surgery. Lancet Oncol 2019; 20(7): e354-e67. 9. Nishino H, et al. Real-time navigation for liver surgery using projection mapping with indocyanine green fluorescence development of the novel medical imaging projection system. Ann Surg 2018; 267(6): 1134-40. 10. Song J, et al. Orthotopic hepatocellular carcinoma: molecular imaging-monitored intratumoral hyperthermia-enhanced direct oncolytic virotherapy. Int J Hyperthermia 2019; 36(1): 344-50.

\section{Image/Figure:}

https://www.xcdsystem.com/wmis/abstract/File6959/GA11 ImageFigure 0218071626.jpg

Complete Status: Complete

First Name: Guanhui

Last Name: Zhou

Email: zhouguanhui@126.com

Organization: University of Washington School of Medicine

Country: United States 


\title{
ID: GA12 \\ Interventional Real-Time Optical Imaging-Guided Complete Tumor Ablation
}

\author{
Xuefeng Kan, Image-Guided Bio-Molecular Intervention Research, Department of \\ Radiology, University of Washington School of Medicine, Seattle, USA. , \\ xkliulang1314@163.com
}

\section{Category: Oncology}

\begin{abstract}
Body : Purpose:Â To develop the indocyanine green (ICG)-enhanced interventional real-time optical imaging $(\mathrm{OI})$ technique for instantly detecting peripheral residual tumors during

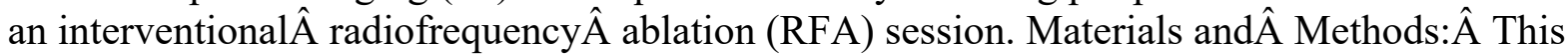
study included three portions of (1) optimizing the protocol for ICG -enhanced OI via serial invitro experiments using tumor cells; (2) building the interventional OI system; and (3) validating its technical feasibility via serial in-vivo experiments using animal models with orthotopic hepatic tumors. In optimizing ICG protocol, $1 \tilde{\mathrm{A}}-104 \mathrm{VX} 2$ tumor cells were treated with various concentrations of ICG from 0 to $125 \hat{\mathrm{A}} \hat{\mathrm{I}} 1 / 4 \mathrm{~g} / \mathrm{mL}$ for 24 hours, and then with the optimized ICG concentration for different incubation duration from $0 \mathrm{~min}$ to 48 hours. Compared to non-heattreated cell group, ICG-VX2 cells were treated by thermal ablation at $80 \hat{a},, f$ for 10 minutes. Fluorescence signal intensities (SI) of different cell groups $\hat{A}$ were measured by Bruker optical/xray imager, $\hat{A}$ and cell viability was evaluated by MTS assay and apoptosis analysis. For in-vivo validation, we first built the interventional real-time optical imaging system, constructed with a micro-OI needle (18G) with excitation wavelength at 760-nm and emission wavelength at 830$\mathrm{nm}$. Then, the created rabbit models with hepatic VX2 tumors were randomly divided into two groups with (1) incomplete RFA at $80 \hat{a},, f \hat{A}$ with partial opening prongs of the RFA electrode; and (2) complete RFA at 80â, $f$ with full opening all prongs $\hat{A}$ of the RFA electrode. Before and after RFA, under ultrasound imaging guidance, the micro-OI needle was positioned into the tumor $\hat{A}$ and its surrounding normal liver parenchyma, and fluorescence signals at six points of the tumor periphery acquired for statistical analysis. The SIs were measured and signal-tobackground ratio (SBR) was calculated using the equation: SItumor/SIparnchyma, which was correlated with ex-vivo OI and final pathology confirmation. Results: $\hat{A}$ OfÂ in-vitro experiments, the optimal concentration and time-window for ICG-enhanced OI of VX2 cells was $100 \hat{\mathrm{I}}^{1 / 4} \mathrm{~g} / \mathrm{mL}$ and 24 hours. $\hat{\mathrm{A}}$ The ICG fluorescent SI of dead tumor cells were significantly lower than untreated living cells $\hat{A}(63.7 \hat{A} \pm 5.7 \hat{A}$ au vs. $189.3 \hat{A} \pm 7.6$ au, P̈̈1/4œ0.001). $\hat{A}$ Of in-vivo experiments, ICG-enhanced OI clearly shows, at the first time in-vivo, the three zones of tumor. For tumors with incomplete RFA, SBR of residual tumor tissues was significantly higher than ablated tumor tissues $\left(2.30 \hat{\mathrm{A}} \pm 0.08\right.$ vs. $\left.0.58 \hat{\mathrm{A}} \pm 0.05, \mathrm{pi} \mathrm{i}^{1} / 4 œ 0.001\right)$, while no significant difference of SBR was found between the pre-treatment tumor and the residual tumor post-treatment $(2.29 \hat{\mathrm{A}} \hat{\mathrm{A}} \pm \hat{\mathrm{A}} 0.08$ vs. $2.30 \hat{\mathrm{A}} \pm 0.08, \mathrm{p} \hat{\mathrm{A}}=0.785)$. For tumors with complete RFA, SBR of ablated tumors was significantly decreased compared with that of pre-ablation tumors $\left(0.59 \hat{\mathrm{A}} \hat{\mathrm{A}} \pm \hat{\mathrm{A}} 0.03\right.$ vs. $\left.\hat{\mathrm{A}} 2.29 \hat{\mathrm{A}} \pm \hat{\mathrm{A}} 0.04, \mathrm{pi}{ }^{1} / 4 œ 0.001\right)$. These SBR changes of interventional OI were very well correlated the ICG-SIs measured by â€œstandardâ€• ex-vivo optical imaging and confirmed by pathology (Figure). Conclusion:Â We are developing a new interventional oncologic technique, â€œInterventional real-time optical imaging-guided complete tumor
\end{abstract}


ablation,â€• which may open new avenues for intraoperative real-time guidance, to ensure the complete removal of medium-to-large tumors during a percutaneous interventional ablation session.

References: 1.Kokudo N,Â et al. ClinicalÂ application of Â fluorescenceÂ imaging $\hat{A}$ of liverẦ cancer $\hat{A}$ using $\hat{A}$ indocyanine green. Liverâ€,Cancer, $\hat{A}$ 2012, 1:15. 2.Ishizawa T, et al.

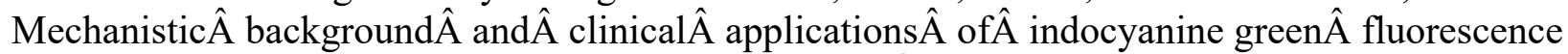
imaging of hepatocellular carcinoma. Ann Surg Oncol, $\hat{A}$ 2014, 21:440. 3.Huang SW, et al. The use of Ầ indocyanine green $\hat{A}$ imaging technique in patient with $\hat{A}$ hepatocellular carcinoma. Transl Gastroenterol Hepatol, 2018, 3: 95. 4.Azumi M,Â et al. Prognostic Impact of Â Indocyanine GreenÂ Plasma Disappearance Rate inÂ Hepatocellular CarcinomaÂ Patients after Radiofrequency Ablation: A Prognostic Nomogram Study. Intern Med,Â 2017, 56: 1001. 5.He JM,Â et al. Laparoscopic Anatomical Segment VII Resection forÂ Hepatocellular CarcinomaÂ Using the Glissonian Approach withÂ Indocyanine Green $\hat{A}$ Dye Fluorescence. J Gastrointest Surg.Ầ 2020; Jan 14. doi: 10.1007/s11605-019-04468-7. 6. Mei J,ÂA et al. A novel treatment strategy using $\hat{A}$ indocyanine green $\hat{A}$ for transarterial chemoembolization in BCLC stage CÂ hepatocellular carcinoma. Cancer Med,Â 2020 9: 62. 7.Ishizawa T,Â et al. Realtime $\hat{A}$ identification $\hat{A}$ of $\hat{A}$ liver cancers $\hat{A}$ by using $\hat{A}$ indocyanine green $\hat{A}$ fluorescent $\hat{A}$ imaging.

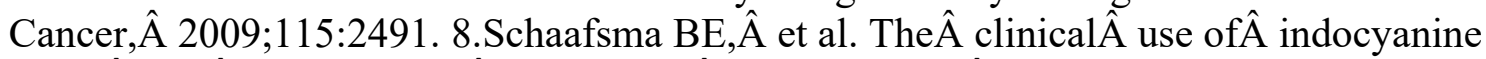
green $\hat{A}$ as a $\hat{A}$ near-infrared $\hat{A}$ fluorescent $\hat{A}$ contrast agent $\hat{A}$ for image-guided oncologic surgery. J Surg OncolÂ 2011, 104:323. Â 9.Carr JA, $\hat{A}$ et al. Shortwave infrared fluorescence imaging with the clinically approvedÂ near-infraredÂ dyeÂ indocyanine green. Proc Natl Acad Sci U S A.Â 2018, 115:4465. 10.Liu Y, $\hat{A}$ et al. First in-human intraoperative imaging of HCC using the fluorescence goggle system and transarterial delivery of $\hat{A}$ nearinfraredÂ fluorescent $\hat{A}$ imaging Â agent: a pilot study. Transl Res,ÂA 2013, 162:324.

\section{Image/Figure:}

https://www.xcdsystem.com/wmis/abstract/File6959/GA12_ImageFigure_0219044420.jpg

Full Name of Abstract's 1st Author : Xuefeng Kan

Complete Status: Complete

First Name: Xuefeng

Last Name: Kan

Email: xkliulang1314@163.com

Organization: Image-Guided Bio-Molecular Intervention Research, Department of Radiology, University of Washington School of Medicine, Seattle, USA.

Country: United States 


\title{
ID: GA13 \\ A novel CNS-homing peptide for targeting neuroinflammatory lesions in experimental autoimmune encephalomyelitis
}

\author{
Bodhraj Acharya, University of Maryland, School of Medicine/ Laboratory Alliance of \\ CNY, bodhach@gmail.com
}

Category: Immunology: Inflammation \& Infection

\begin{abstract}
Body : Using phage peptide library screening, we identified peptide-encoding phages that selectively home to the inflamed central nervous system (CNS) of mice with experimental autoimmune encephalomyelitis (EAE), a model of human multiple sclerosis (MS). A phage peptide display library encoding cyclic 9-amino-acid random peptides was first screened $\hat{A}$ exvivoÂ for binding to the CNS tissue of EAE mice, followed by $\hat{A}$ in vivoÂ screening in the diseased mice. Phage insert sequences that were present at a higher frequency in the CNS of EAE mice than in the normal (control) mice were identified by DNA sequencing. One of the phages selected in this manner, denoted as MS-1, was shown to selectively recognize CNS tissue in EAE mice. Individually cloned phages with this insert preferentially homed to EAE CNS after an intravenous injection. Similarly, systemically-administered fluorescence-labeled synthetic MS-1 peptide showed selective accumulation in the spinal cord of EAE mice. We suggest that peptide MS-1 might be useful for targeted drug delivery to CNS in EAE/MS.
\end{abstract}

Complete Status: Complete

First Name: Bodhraj

Last Name: Acharya

Email: bodhach@gmail.com

Organization: University of Maryland, School of Medicine/ Laboratory Alliance of CNY

Country: United States 


\title{
ID: GA15 \\ Imaging oxygen in tumors requires sub-millimeter spatial resolution to resolve of radiobiologically relevant hypoxia and predict treatment enhancement
}

\author{
Brian Pogue, Dartmouth College, brian.w.pogue@dartmouth.edu
}

\section{Category: Oncology}

\begin{abstract}
Body : Hypoxia in solid tumors is thought to be an important factor in resistance to therapy, but the extreme microscopic heterogeneity of the partial pressures of oxygen ( $\mathrm{pO} 2)$ between the capillaries makes it difficult to characterize the scope of this phenomenon without invasive sampling of oxygen distributions throughout the tissue. While several macroscopic tracers exist to image oxygen in tumors with PET, the spatial resolution achievable is near $3 \mathrm{~mm}$. While there does not exist a non-invasive way to image oxygen with sub-millimeter resolution throughout a tumor, here we developed a non-invasive method to track spatial oxygen distributions on the surface of tumors during fractionated radiotherapy. $\hat{A}$ The technique used oxygen-dependent quenching of phosphorescence, from oxygen probe Oxyphor PtG4 excited internally in the tissue by radiotherapy-induced Cherenkov light. Imaging of the phosphorescence lifetimes within the tissue was possible with a time-synchronized camera that captured the emission with a series of increasing time gates over the 22-44 microsecond lifetime. Mice bearing MDA-MB-231 breast cancer and FaDu head neck cancer xenografts were studied during 5 Gy per each of 5 daily fractions. Imaging of hypoxia was also achieved ex vivo through pimonidazole stains. $\hat{A}$ The tumor pO2 was spatially imaged daily, and the hypoxic fraction was also quantified from the spatial distribution. Imaging at different spatial resolutions illustrated that the hypoxic fraction could not be resolved at $3 \mathrm{~mm}$ accuracy, whereas below $1 \mathrm{~mm}$ the intercapillary pO2 values could be quantified to achieve a meaningful hypoxic fraction estimate. When animals were examined for response, only those with low hypoxic fractions could be found to have high response from radiotherapy, whereas those with high hypoxic fraction had less response the treatment course. $\hat{A}$ Imaging hypoxia in vivo has always been a challenge, and with several clinical imaging tools existing, it is important to realize the limitations in partial volume averaging that can suppress the molecular information desired, and this is especially important in the role of imaging oxygen, which is known to be heterogeneous on a distance scale near 100 microns or even less. $\hat{A}$
\end{abstract}

\section{Image/Figure:}

https://www.xcdsystem.com/wmis/abstract/File6959/GA15_ImageFigure 0228095615.png

Complete Status: Complete

First Name: Brian

Last Name: Pogue 
Email: brian.w.pogue@dartmouth.edu

Organization: Dartmouth College

Country: United States 


\title{
ID: GA16 \\ Standardization in Fluorescence Surgical Imaging Systems: 3D printed phantoms
}

\author{
Brian Pogue, Dartmouth College, brian.w.pogue@dartmouth.edu
}

\section{Category: Instrumentation}

\begin{abstract}
Body : Fluorescence guided surgery systems have increasingly been used for more procedures with indocyanine green, and the number of systems approved for human use has continued to grow, to nearly 10 to date. The variation in performance between systems has never been clearly defined nor has there been much study of intersystem stability and comparisons of data. While this may not be critical for the use case of ICG imaging, it can become more serious for the use case of molecular targeted imaging, where concentrations of agent in vivo can be significantly lower and the spectral optimization varies with the luminescent molecule. $\hat{A}$ Proposed standards have been outlined by the American Association of Physicists in Medicine Task Group on Fluorescence Guided Surgery Performance Assessment and will be outlined. A brief summary is that tissue-simulating phantoms should be used to validate and calibrate the systems for pertinent task-specific goals and will be specified with a suitable longevity. Some of the key recommendations for task-specific performance measures are fluorescent signal sensitivity, linearity with concentration, imaging dynamic range, depth of tissue sensitivity, and effects of scatter \& absorption upon signal from a fixed concentration. There can also be confounding issues of ambient light leakage and filtering efficiency that need to be assessed as they relate the task-specific performance. Anthropomorphic phantoms might be used for geometries that affect the interpretation or for physician training in proper use. $\hat{A} \hat{A} \hat{A} \hat{A} \hat{A} \hat{A} \hat{A} \hat{A} \hat{A} \hat{A}$ Â $\hat{A}$ These recommendations suggest that stable phantoms that mimic the ICG use case, or any molecular probe, should be developed, which are ideally stable and easily produced. It would be even more desirable to have them readily made for different geometries and different fluorophores. $\hat{A}$ This has led to our research project in developing 3D printed phantoms, with tailor produced fluorescence in the wavelengths of interest, and tailored absorption and scattering coefficients. $\hat{A}$ These are now produced from resin based low cost printers, and several have been distributed to users. $\hat{A}$ The incorporation of dye that directly matches ICG is possible with limits on use due to photobleaching. $\hat{\mathrm{A}} \hat{\mathrm{A}}$
\end{abstract}

\section{Image/Figure:}

https://www.xcdsystem.com/wmis/abstract/File6959/GA16_ImageFigure 0313105304.png

Complete Status: Complete

First Name: Brian

Last Name: Pogue 
Email: brian.w.pogue@dartmouth.edu

Organization: Dartmouth College

Country: United States 


\title{
ID: GA17 \\ Guidance for tumor radiotherapy response in vivo with Cherenkov-excited luminescence ink imaging
}

\author{
Brian Pogue, Dartmouth College, brian.w.pogue@dartmouth.edu
}

\section{Category: Instrumentation}

\begin{abstract}
Body : Radiation therapy is given in daily fractionated treatment to most patients for 30-40 days in a row, and in most cases the treatment is planned based upon the tumor volume as presenting on the CT scan. $\hat{A}$ However tumors respond differently to the radiotherapy, and so imaging or monitoring of in vivo tumor microstructural changes would help determine which tumors require a boost or which are responding to well. $\hat{A}$ By tracking the diffusive spread of injected intratumor UV excited tattoo ink using Cherenkov-excited luminescence imaging (CELI), the diffusion characteristics of the tumor being treated can be assessed during the daily fractions. Â Micro-liter quantities of luminescent tattoo ink with UV absorption and visible emission were injected at a depth of $2 \mathrm{~mm}$ into xenograft tumors, and they received 5 doses of $10 \mathrm{~Gy}$ radiation treatment. The therapeutic X-rays from a clinical linear accelerator were used for treatment, and for simultaneous excitation of the phosphorescent compounds within the tattoo ink through Cherenkov emission. In vivo phosphorescence was detected using a time-gated intensified camera.Â Diagnostic imaging was done immediately after injection, and then again at varying time points after the ink had broken down with the tumor as it was treated. Ex vivo tumors were imaged post-mortem using hyperspectral cryo-fluorescence imaging to quantify necrosis and compared to Cherenkov-excited light imaging of diffusive ink spread measured in vivo.Â Imaging of untreated control mice showed that ink distributions remained constant after four days with less than $3 \%$ diffusive spread measured using full width at $20 \%$ max. For all mice, in vivo CELI measurements matched the values estimated by the high-resolution ex vivo sliced luminescence imaging of the tumors. The tattoo ink spread in treated mice was found to correlate well with the nonperfusion necrotic core volume $(\mathrm{R} 2=0.92) . \hat{\mathrm{A}}$ Interestingly, the ink spread did not correlate all that well with total tumor volume changes $(\mathrm{R} 2=0.34)$, indicating that volume changes do not always reflect interstitial diffusion changes in the tumor microenvironment. In vivo and ex vivo findings indicate that the diffusive spread of the injected tattoo ink can be related to radiation-induced necrosis, independent of total tumor volume change.
\end{abstract}

References: Soter J, LaRochelle EPM, Byrd BK, Tendler II, Gunn JR, Meng B, Strawbridge RR, Wirth DJ, Davis SC, Gladstone DJ, Jarvis LA, Pogue BW."Tracking tumor radiotherapy response in vivo with Cherenkov-excited luminescence ink imaging." Phys Med Biol. 2020 Mar 5. doi: 10.1088/1361-6560/ab7d16. [Epub ahead of print] LaRochelle EPM, Soter J, Barrios L, GuzmÃ in M, Streeter SS, Gunn JR, Bejarano S, Pogue BW. "Imaging luminescent tattoo inks for direct visualization of linac and cobalt irradiation." Med Phys. 2020 Feb 13. doi: 10.1002/mp.14094. [Epub ahead of print]

\section{Image/Figure:}


Complete Status: Complete

First Name: Brian

Last Name: Pogue

Email: brian.w.pogue@dartmouth.edu

Organization: Dartmouth College

Country: United States 


\title{
ID: GA19 \\ Validation of A Noise Reduction Algorithm for Medium and High Energy Preclinical SPECT
}

\author{
Cesar Molinos Solsona, Bruker BioSpin , cesar.molinos@bruker.com
}

\section{Category: Instrumentation}

\begin{abstract}
Body : Background: SPECT imaging allows for the use of many radiotracers with Tc$99 \mathrm{~m}$ as the most common isotope. This means that frequently existing SPECT systems will be optimized to image the $140 \mathrm{keV}$ gamma emission from Tc99m and will typically require hardware investments in the form of new collimators able to shield isotopes giving off higher energy gammas. Examples of such isotopes are: I-111 with gamma emissions at $173 \mathrm{keV}(91 \%)$ and $247 \mathrm{keV}$ (94\%) common in antibody imaging, for instance In-111 capromab pendetide, Lu177 with main gamma emissions at $113 \mathrm{keV} \mathrm{(3 \% )} \mathrm{and} 208 \mathrm{keV}(11 \%)$ used in theranostic agents like 77Lu-DOTE-TATE for neuroendocrine tumor models. Many compounds based on the Iodine radioisotopes I-123 $159 \mathrm{keV}(83 \%)$ and I-131 $364 \mathrm{keV}(81 \%)$ gamma emissions also fall in this category of medium to high energy SPECT tracers. The motivation of the present study is to validate a method which minimizes background noise in SPECT imaging in those medium to high energy cases without the need of collimator upgrades. Method: The background noise depends on the collimator, the gamma spectrum, the activity distribution and the object. Multiple methods modelling background noise in high energy SPECT imaging have been documented, see [1-4]. In this particular case, a dual head and large FOV SPECT preclinical system featuring single pin-hole collimator, was employed [2][3] and based on the specific features of this system (detectors and collimators), a noise reduction algorithm developed. The noise estimation takes place during the reconstruction process by projecting the image obtained in the previous iteration through the collimator taking into account the isotope energy and the activity distribution to estimate the background noise distribution in all projections. Initial tests were carried out with phantoms and after verifying that the correction had the desired effect, an in vivo validation was carried out. Phantom studies involved a range of isotopes: In-111, I-123, Lu-177 and I-131. In vivo tests included In-111 and Lu-177 although only the former is presented here. A 7 weeks old $\mathrm{BALB} / \mathrm{c}$ nude female mouse was imaged 14 days after $3 \times 106$ SK-OV-3 ovarian cancer cells were grafted subcutaneously on the shoulder of the animal. Acquisitions were carried out 6 days after the injection of $18 \mathrm{MBq}$ of In-111 radiolabeled anti-HER2 antibody. The SPECT acquisition employed Pinhole collimators, a FOV of $80 \mathrm{~mm}$ and lasted $180 \mathrm{~min}$. The SPECT image reconstruction settings were: OSEM algorithm, 3 iterations and a $0.25 \mathrm{~mm}$ voxel size. Images were analyzed with the software tools PMOD 4.1 and Amide. Results: The results with phantoms showed a significant reduction in noise in the gamma energies tested. The results for the in vivo validation also showed a background noise reduction. In particular for In-111, the correction showed very good results imaging the $173 \mathrm{keV}$ gamma emission as the correction considerably reduces the induced noise from the higher $247 \mathrm{keV}$ energy emission, see figure. Conclusion: An algorithm for noise reduction in medium and high energy SPECT isotopes was implemented and successfully tested. This kind of correction allows for higher image quality in existing systems without the need to invest in hardware. The algorithm tested showed a
\end{abstract}


significant reduction in noise both in phantoms and in vivo tests. With In-111, the correction seems particularly effective at reducing the noise introduced by $247 \mathrm{keV}$ gammas when imaging $173 \mathrm{keV}$ gammas. Although it also has the potential of allowing to image the higher energy emission in isotopes like Lu177, further work to explore such an effectiveness in vivo will be required.

References: 1. Dewaraja, Y K et al. "Characterization of scatter and penetration using Monte Carlo simulation in 131I imaging." Journal of nuclear medicine: official publication, Society of Nuclear Medicine vol. 41,1 (2000): 123-30. 2. Laymon CM et al. "Nuclear Medicine Communications: November 2006 - Volume 27 - Issue 11 - p 901-909 3. Lewis DP et al, "Characterization of medium and high energy collimators using ray-tracing and Monte Carlo methods," 1998 IEEE Nuclear Science Symposium Conference Record. 1998 IEEE Nuclear Science Symposium and Medical Imaging Conference (Cat. No.98CH36255), Toronto, Ontario, Canada, 1998, pp. 2026-2030 vol.3. 4. Song X et al. "Fast modelling of the collimator-detector response in Monte Carlo simulation of SPECT imaging using the angular response function" Physics in Medicine \& Biology vol. 50,8 (2005). 5. Spinks TJ et al. "Quantitative PET and SPECT performance characteristics of the Albira Trimodal pre-clinical tomograph" 2014 Phys. Med. Biol. 59 715. 6. González AJ, et al. "A PET Design Based on SiPM and Monolithic LYSO Crystals: Performance Evaluation" IEEE Transactions on Nuclear Science, vol. 63, no. 5, pp. 2471-2477, Oct. 2016

\section{Image/Figure:}

https://www.xcdsystem.com/wmis/abstract/File6959/GA19_ImageFigure 0318054530.png

Complete Status: Complete

First Name: Cesar

Last Name: Molinos Solsona

Email: cesar.molinos@bruker.com

Organization: Bruker BioSpin

Country: United States 


\title{
ID: GA21 \\ Masking Effect of Enhanced MRI Relaxivity from Gadolinium Depositions in ECM bioploymers
}

\author{
Patrick Werner, Leibniz Research Institute for Molecular Pharmacology (FMP), \\ werner@fmp-berlin.de
}

Category: New Chemistry, Biology \& Bioengineering

\begin{abstract}
Body : Introduction The instability of certain gadolinium-based contrast agents (GBCAs) in molecular environments that provide endogenous ligands for released Gd3+ ions is of huge biochemical interest, . because they might play a crucial role in the long-term deposition of Gd3--ions in the body. Endogenous ions like $\mathrm{Zn}^{2+}$ can lead to a transmetallation of GBCAs and glycosaminoglycans (GAGs) are prime candidates for the binding of released Gd3 + [1]. Hyperintensities in T1-weighted images of patients after multiple GBCA administrations were repeatedly observed in recent years [2-4] and might be linked to this phenomenon. In this study, chondroitin-sulfate (CSA) and dextran-sulfate (DS) solutions as well as THP-1 cells [5] were used to investigate the transchelation of Gd3+-ions into GAG structures under in vivo-like conditions. Materials and methods CSA and DS were used as models for the transchelation of $\mathrm{Gd} 3+$-ions to polysaccharides. Glycan-rich THP-1 cell-suspensions were incubated with $\mathrm{GdCl} 3$ $(25 \mu \mathrm{M})$ or Magnevist ${ }^{\circledR} /$ Dotarem ${ }^{\circledR}(75 \mu \mathrm{M})$ for 20 min. All T1 measurements were performed on a $9.4 \mathrm{~T}$ preclinical MRI-system. T1 measurements were performed using a dephasing recovery sequence consisting of $50 \pi / 2$ pulses with subsequent gradient spoiling and gradient echo image acquisition. Results Figure 1 shows R1 values as functions of the concentration ratio between the different polysaccharides/ cells and Gd3+ in solution. R1 enhancements were observed upon interaction between polysaccharides and $\mathrm{Gd} 3+$, followed by an unexpected decrease of R1 (regime 2). R1 increases from $0.63 \mathrm{~s}-1$ (R1 of $25 \mu \mathrm{M} \mathrm{Gd} 3+$ in $\mathrm{H} 2 \mathrm{O}$ ) to $1.05 \mathrm{~s}-1$ in CSA (Fig. 1A) and to $0.84 \mathrm{~s}-1$ in DS (Fig. 1B). Subsequently, R1 decreases to about $0.35 \mathrm{~s}-1$ at a ratio of 300 for DS and to about $0.43 \mathrm{~s}-1$ at a ratio of 2000 for CSA. In both cases, the final value is almost identical with the R1 value of pure water (R1 of pure H2O: $0.35 \mathrm{~s}-1)$. The results of the cell supernatant experiments show no effect for intact GBCAs (Fig. 1C). Contrary, the cell-Gd3+ titration (Fig. 1D) shows decreasing R1 values with increasing numbers of cells similar to the regime 2 in CSA/DS. Discussion GAGs are an essential part of the extra-cellular matrix (ECM) of the human body. The observed increase of R1 caused by the binding of gadolinium to GAGs could underpin their important role for the explanation of clinically observed hyperintensities [1]. However, for very high $[\mathrm{GAG}] /[\mathrm{Gd} 3+]$ ratios we observed an unexpected signal loss. We assume that theincreasing number of available coordination sides from the sulfate groups of the polysaccharides causes a coordination of the $\mathrm{Gd} 3+$ ions in a way that excludes any water in the inner sphere. This lowers the relaxation effect of the gadolinium on the surrounding water and is masking the real amount of deposited Gd3+-ions detectable by MRI relaxometry. In experiments with THP-1 cells, similar decreasing R1-values could also be observed and correlated to the binding of Gd3+-ions to the glycocalyx. Conclusion Our results show that the binding of Gd3+ions to GAGs can cause an increase or decrease of R1, depending on the experimental conditions. This demonstrates the importance of more systematic studies with selected
\end{abstract}


endogenous components in various concentration regimes. Besides the well documented hyperintensities it might be possible for certain components of the glycocalyx to 'silence' the dissociated $\mathrm{Gd} 3+$ ions after chelation. This raises the question if the amount of deposited gadolinium in biological tissues was underestimated over the last years.

References: 1. Taupitz,M. (2013). Contrast media \& molecular imaging, 8(2),108-116 2. Kanda, T. (2013).Radiology, 270 (3), 834-841 3. Radbruch, A. (2015). Radiology, 275 (3), 783-791 4. Gianolio, E. (2019). European Journal of Inorganic Chemistry, 2019 (2), 137-151 5. Tsuchiya, S. (1980). International journal of cancer, 26 (2), 171-176.

\section{Image/Figure:}

https://www.xcdsystem.com/wmis/abstract/File6959/GA21_ImageFigure 0410012341.png

Full Name of Abstract's 1st Author : Patrick Werner

Complete Status: Complete

First Name: Patrick

Last Name: Werner

Email: werner@fmp-berlin.de

Organization: Leibniz Research Institute for Molecular Pharmacology (FMP)

Country: Germany 


\title{
ID: GA22 \\ 3D fluorescence tomography of chick embryos: a novel in vivo approach for testing anti-cancer therapeutics
}

\author{
Valerie Rouffiac, Gustave Roussy, Université Paris-Saclay, UMS AMMICa INSERM \\ US23-CNRS 3655., valerie.rouffiac@gustaveroussy.fr
}

\section{Category: Oncology}

\begin{abstract}
Body : Introduction In vivo experimental models are crucial for studying metastatic cancer progression in a physiological context and for the development and screening of efficient therapies before clinical transfer. Experimental setups bring focus on mice, as protocols could be established on large cohorts and various genotypes developed by knocking-out specific genes implicated in carcinogenesis are currently available. However, tumor engraftments in mice are sometimes hard to achieve, especially for patient derived xenografts, and may require months of follow-up. We propose here to overcome some limitations of the mouse model by using the chick embryo model as an alternative for in vivo preclinical studies. Different tumoral cell lines were successfully implanted in this model that has an immature immune system limiting tumor graft rejection and advanced 3D fluorescence tomography allowed to visualize tumoral growth over few days. Materials \& Methods Tumor cells of non-small cell lung cancer (NSCLC) and prostate cancer $(\mathrm{PCa})$ were implanted on chick chorioallantoic membrane (CAM) at incubation day 10 (ID) throughout a window created on the egg shell. 103 cells $/ 20 \mu \mathrm{L}$ of matrigel medium were implanted per egg. After closing the window, eggs were placed in an incubator. Tumor growth and embryo viability were examined onto a fluorescent macroscope AZ100M with emission/excitation GFP and mCherry filters depending on the cell line. At ID 17, complete analysis of tumor growth was performed post-mortem after removing embryo from the shell and placing it onto an IVIS spectrum imaging stage. 2D epifluorescence or 3D CT scan combined with transillumination fluorescence using adequate filters were acquired. 3D reconstruction was performed using the Living image software, also used to compute fluorescent signal intensities inside regions of interest drawn around whole embryo or detected tumoral lesions. Results \& Discussion Reproducible results were obtained by implantation of 103 cells per egg, resulting in up to $80 \%$ of successfully formed nodule in the case of lung cancer cell lines and $100 \%$ for prostate cancer cell lines. Sensitivity of whole body imaging system was sufficiently high to detect small metastatic foci in specific anatomical regions after 3D reconstruction. The presence of these tumor lesions was confirmed in different isolated organs of chick embryos evaluated separately on the IVIS Spectrum. Moreover, our data suggest that combined measurement of whole body fluorescence intensity and number of foci may be a sensitive tool to evaluate the metastatic seeding capacity of cancer cells. In conclusion, the chick embryo model, combined to sensitive whole body imaging technology could be a relevant and promising tool for future preclinical studies testing anti-cancer therapeutics.
\end{abstract}

Complete Status: Complete

First Name: Valerie 
Last Name: Rouffiac

Email: valerie.rouffiac@gustaveroussy.fr

Organization: Gustave Roussy, Université Paris-Saclay, UMS AMMICa INSERM US23-CNRS 3655.

Country: France 


\title{
ID: GA23 \\ Sensitive whole-body monitoring of spontaneous metastasis dynamics using a positive contrast MR reporter
}

\author{
Nivin Nystrom, Robarts Research Institute, nnasri@uwo.ca
}

\section{Category: New Chemistry, Biology \& Bioengineering}

Abstract Body : Introduction. Metastasis accounts for $>90 \%$ of cancer-related deaths. 1 However, $75 \%$ of preclinical cancer studies do not examine metastasis, focusing only on primary tumor growth, in part due to a lack of technologies for accurate quantification of metastatic spread over time.2 Bioluminescence imaging (BLI) is the tool of choice for assessing metastatic burden because of its high throughput and sensitivity, but assessing true burden with BLI, particularly in spontaneous metastasis models, remains challenging. Small superficial lesions cannot be readily differentiated from larger lesions in deep tissues, and light from large superficial tumors obfuscates detection of deeper lesions. To better understand the metastatic process and advance our ability to test therapies targeting metastatic disease, tools for accurate spatiotemporal quantification of metastasis are needed. Recently, a magnetic resonance imaging (MRI) reporter gene called Organic anion-transporting polypeptide $1 \mathrm{~b} 3$ (Oatp1b3) was developed, which encodes a transporter that takes up the positive contrast agent Gd-EOB$\mathrm{DTPA} \uparrow .3$ Our objective was to assess Oatp1b3 for whole-body in vivo tracking of cancer cells in a spontaneous metastasis model.4 Methods. Breast cancer cells (MDA-MB-231) were engineered with lentivirus to express luciferase for BLI. A subset of these cells was additionally engineered to co-express Oatp1b3 for MRI. $3 \times 105$ luciferase control (Luc-CTL) or luciferase/Oatp1b3 (Luc-1B3) cells were implanted into the 4th mammary fat pad of female nod scid gamma mice. BLI was performed after $150 \mathrm{mg} / \mathrm{kg}$ D-luciferin injection. T1-weighted 3TMRI was performed before and $5 \mathrm{~h}$ post $1-\mathrm{mmol} / \mathrm{kg}$ Gd-EOB-DTPA injection. Detectability of widespread metastases was first evaluated by imaging Luc-CTL and Luc-1B3 mice ( $\mathrm{n}=3$ each) 30 days after cell implantation. Next, a Luc-1B3 cohort $(n=7)$ was imaged over time until endpoint (up to Day 24) to assess the ability to monitor metastasis dynamically. Regions of interest were segmented and statistical significance was determined via analysis of variance. Results. For mice imaged at endpoint, macro-metastases $(>1 \mathrm{~mm} 3)$ located at axillary lymph nodes exhibited significantly increased MR signal intensity in Luc-1B3 mice on post-contrast images relative to pre-contrast images, and to Luc-CTL mice both pre- and post-contrast $(\mathrm{n}=3, \mathrm{p}$ $<0$.0001). Importantly, numerous micro-metastases $(<1 \mathrm{~mm} 3)$ were detected throughout lungs of Luc-1B3 mice on post-contrast MRI, which were not detected in corresponding pre-contrast MRI, with BLI, nor on post-contrast images of Luc-CTL mice (see Main Figure). Analyzing mean signal intensity and volume of individual foci ( $\mathrm{N}=60$ across 3 mice) demonstrated that lesions around $0.01 \mathrm{~mm} 3$, correlating to $\sim 104$ cells, could be detected. In the Luc-1B3 cohort imaged over time, metastasis was first detected at the ipsilateral axillary lymph node on average $11 \pm 1.3$ days after mammary fat pad implantation, with significantly increased MR signal intensity relative to surrounding muscle tissue $(n=7, p<0.0001)$. Five out of 7 mice displayed significant lymph node enhancement within 24 hours following first detection of BLI signal in that region. Impressively, the remaining two mice showed MR signal enhancement up to two 
days before BLI signal detection. Follow-up MRI at 20-days post-implantation revealed tumor growth at the initial ipsilateral axillary lymph node lesion, as well as subsequent metastases at other loci, including the contralateral axillary and ipsilateral brachial lymph nodes. These subsequent metastases were not at all detectable with BLI. Discussion. BLI provides whole-body information on locations of engineered cells, but smaller populations can go undetected. Oatp1b3-MRI mitigates these limitations for effective tracking of metastatic spread. We show that this reporter can dynamically track the metastatic process at its earliest stages, including small single lymph node lesions, even prior to BLI. At late stages, we demonstrate its superiority over BLI to track cancer spread to multiple lymph nodes and other organs such as the lungs. Oatplb3 fulfills a long-standing gap in our ability to accurately study metastatic disease over time, and offers a path towards deep-tissue tracking of any Oatp1b3-engineered cell type with combined high resolution, sensitivity, 3D spatial information, and surrounding anatomical context.

References: Abbreviations $\dagger$ gadolinium ethoxybenzyl diethylenetriaminepentaacetic acid Work Cited 1. Lambert et al. Cell 2017; 168(4): 670-91. 2. Gengenbacher et al. Nature Reviews Cancer 2017; 17(12): 751-65. 3. Nyström et al. Radiol Img Can 2019; 1(2): e190035. 4. Iorns et al. PLoS One. 2012; 7(10): e47995.

\section{Image/Figure:}

https://www.xcdsystem.com/wmis/abstract/File6959/GA23_ImageFigure_0421092820.png

Full Name of Abstract's 1st Author : Nivin N. Nyström

Complete Status: Complete

First Name: Nivin

Last Name: Nystrom

Email: nnasri@uwo.ca

Organization: Robarts Research Institute

Country: Canada 


\title{
ID: GA24 \\ Quantifying the detection limit of 89Zr-labelled cells for cellular tracking using clinical PET/CT and PET/MRI
}

\author{
Laura Lechermann, University of Cambridge, Imtl2@cam.ac.uk
}

\section{Category: New Chemistry, Biology \& Bioengineering}

\begin{abstract}
Body : Introduction: In vivo imaging and tracking of cells can be used to noninvasively probe the pharmakinetics, efficacy and safety of novel cell therapies, such as Chimeric Antigen Receptor (CAR) T-cells. Zirconium-89 has a long half-life (t1/2=78.4 h) and has therefore been used for labeling and imaging cells in vivo using Positron Emission Tomography (PET), in particular in the form of [89Zr]Zr(oxinate)4 [1-4]. This work presents an in vitro approach to quantify the detection limit for in vivo PET imaging of Jurkat T cells directly labeled with [89Zr]Zr(oxinate)4 utilizing clinical PET/CT and PET/MRI. Methods: [89Zr] Zr(oxinate)4 was synthesized according to previously published methods [4]. Jurkat Tcells were labeled with varying concentrations of [89Zr]Zr(oxinate) 4 to generate different cell specific activities $(0.43-31.91 \mathrm{kBq} / 106$ cells). Different concentrations of labeled cell suspensions (104, 105 and 106 cells) were seeded on 6 -well plates and into a $3 \times 3$ cubic-well plate with $1 \mathrm{~cm} 3$ cubic wells as a gel matrix. Plates were imaged on clinical PET/CT and PET/MRI scanners for $30 \mathrm{~min}$. The total activity in each well was determined by drawing volumes of interest over each well on PET images. The total cells associated activity was measured using a well counter and correlated with imaging data. Simulations for non-specific signal were performed to model the effect of non-specific radioactivity on detection. Results: [89Zr] $\mathrm{Zr}$ (oxinate) 4 was synthesized in an aqueous solution at a mean radiochemical yield of 93.5 $\% \pm 3.1( \pm \mathrm{SD}, \mathrm{n}=8)$, as indicated by thin layer chromatography. The total activity on cells per well ranged from $0.02-15.57 \mathrm{kBq}$ and $0.01-24.47 \mathrm{kBq}$, as measured by the gamma counter and PET imaging, respectively. The lowest cell number that could be visualized on 6-well plates images was $6.8 \times 104$, when the specific activity was $27.8 \mathrm{kBq} / 106$ cells in a volumne of $2 \mathrm{~mL}$. For the $3 \times 3$ cubic-well plate, of $3.3 \times 104$ cells could be detected on images with a specific activity of $15.4 \mathrm{kBq} / 106$ cells in a volumne of $1 \mathrm{~mL}$. The total activity in each well, as determined from PET/CT and PET/MR imaging, showed a strong correlation with the total cellassociated activity from cell suspensions as measured in the well counter (PET/CT: $r=0.84, p$ Conclusion: The results show the feasibility of detecting [89Zr]Zr(oxinate)4-labeled Jurkat Tcells on clinical PET systems. The results provide a best case scenario, as in vivo detection using $\mathrm{PET} / \mathrm{CT}$ or PET/MRI will be affected by cell number, specific activity per cell, the density of cells within the target volume, and non-specific signal. This work has important implications for cell labeling studies in patients, particularly when using radiosensitive cells (e.g. T-cells), which require detection of low cell numbers while minimizing radiation dose per cell.
\end{abstract}

References: [1] Ferris TJ, Charoenphun P, Meszaros LK, et al.Dalton Trans. 2014; 43(39):14851-14857 [2] Asiedu KO, Koyasu S, Szajek LP, Choyke PL, Sato N. Clin Cancer Res. 2017; 23(11):2759-2768 [3] Man F, Lim L, Volpe A, et al. Mol. Ther. 2019; 27(1):219-229 [4] Weist MR, Starr R, Aguilar B, et al. J Nucl Med. 2018; 59(10):1531-1537 


\section{Image/Figure:}

https://www.xcdsystem.com/wmis/abstract/File6959/GA24_ImageFigure_0418043426.png

Full Name of Abstract's 1st Author : Laura Melanie Tessa Lechermann

Complete Status: Complete

First Name: Laura

Last Name: Lechermann

Email:1mt12@cam.ac.uk

Organization: University of Cambridge

Country: United Kingdom 


\title{
ID: GA25 \\ Breast carcinoma detection in ex-vivo fresh human breast surgical specimens Using fast slide-free confocal microscopy scanner: the HIBISCUSS project
}

\author{
Muriel Abbaci, IR4M UMR8081 Equipe Gustave Roussy, muriel.abbaci@gustaveroussy.fr
}

\section{Category: Oncology}

\begin{abstract}
Body : Breast cancer is the commonest cancer in female worldwide and breastconserving surgery (BCS) became the most frequently performed surgical treatment for women with early-stage breast cancer. The ability to identify subclinical and deep-seated tumor during BCS is still difficult, and the surgeon must rely on nonspecific visual changes and manual palpation of subtle irregularities to guide cancer excision (Rosenthal, 2016). A meta-analysis suggested that the odds of local recurrence were 2.42 for positive versus negative margins in women undergoing BCS (Houssami, 2010). Although there is no consensus on negative surgical margins, at least $20 \%$ of patients undergo more than one procedure to achieve acceptable margins as part of breast-conserving strategy (Mac Neill, 2017). The development of reliable intraoperative assessment tools which can provide a timely indication of whether re-excision in tumor bed after BCS is indicated at the time of primary surgery would be an asset. The Histolog Scanner (HScan) is a recent confocal microscope designed for fresh tissue ex-vivo imaging with high resolution and speed $(1 \mathrm{~min}$ for $10 \mathrm{~cm} 2$ ) to guide intraoperative assessment and offer support in clinical decision making. Recently studies in dermatology and breast cancer provided first supportive data for this new imaging device (Elfgen, 2019; Krishnamurthy, 2020). Allowing the quickest and most effective intraoperative assessment of breast surgical specimen regardless of center clinical workflow and intraoperative availability of pathologist, is the aim of our international project HIBISCUSS. The main objective is to demonstrate that both pathologists and surgeons are able to detect breast cancer tissues in HScan images of breast surgical specimens. 105/195 patients with breast cancer undergoing breast conserving surgery and without previous neoadjuvant chemotherapy were already included at Gustave Roussy between June 2019 and March 2020. All fresh resected specimens are stained with Histolog dip-contrast agent. Two large-field images are acquired by lumpectomy with Histolog Scanner (SamanTree Medical, Lausanne, Switzerland). HScan images are then compared to corresponding histopathological diagnosis and hematoxylin eosin saffron digital slides. Two pathologists and eight surgeons are trained for the interpretation of a set of HScan images from 45 samples. Then all newly skilled physicians have to propose diagnosis for 150 samples in a blind study. Fifteen invasive ductal carcinoma, 15 invasive lobular carcinoma and 15 in situ ductal carcinoma have been imaged for the training part. Histological architecture and cellular morphology were captioned by dedicated pathologists to provide training sessions. Learning phase is underway for the ten physicians. For the second part of the study, 60 lumpectomies have already been imaged and are under preparation for the further blind interpretations. At the end of this study, if we can demonstrate that surgeons, as pathologists, are able to propose a quick diagnosis on HScan images then this new imaging tool could improve the intraoperative management of breast tumor resection.
\end{abstract}


References: Elfgen, C., Papassotiropoulos, B., Varga, Z., Moskovszky, L., Nap, M., Güth, U., Baege, A., Amann, E., Chiesa, F., \& Tausch, C. (2019). Comparative analysis of confocal microscopy on fresh breast core needle biopsies and conventional histology. Diagnostic Pathology, 14(1), 58. https://doi.org/10.1186/s13000-019-0835-z Houssami, N., Macaskill, P., Marinovich, M. L., Dixon, J. M., Irwig, L., Brennan, M. E., \& Solin, L. J. (2010). Meta-analysis of the impact of surgical margins on local recurrence in women with early-stage invasive breast cancer treated with breast-conserving therapy. European Journal of Cancer, 46(18), 3219-3232. https://doi.org/10.1016/j.ejca.2010.07.043 Krishnamurthy, S., Brown, J. Q., Iftimia, N., Levenson, R. M., \& Rajadhyaksha, M. (2019). Ex Vivo Microscopy: A Promising NextGeneration Digital Microscopy Tool for Surgical Pathology Practice. Archives of Pathology \& Laboratory Medicine, 143(9), 1058-1068. https://doi.org/10.5858/arpa.2019-0058-ra MacNeill, F., \& Karakatsanis, A. (2017). Over surgery in breast cancer. Breast (Edinburgh, Scotland), 31, 284-289. https://doi.org/10.1016/j.breast.2016.10.023 Rosenthal, E. L., Warram, J. M., de Boer, E., Basilion, J. P., Biel, M. A., Bogyo, M., Bouvet, M., Brigman, B. E., Colson, Y. L., DeMeester, S. R., Gurtner, G. C. G. C., Ishizawa, T., Jacobs, P. M., Keereweer, S., Liao, J. C., Nguyen, Q. T., Olson, J. M., Paulsen, K. D., Rieves, D., ... van Dam, G. M. (2016). Successful Translation of Fluorescence Navigation During Oncologic Surgery: A Consensus Report. Journal of Nuclear Medicine : Official Publication, Society of Nuclear Medicine, 57(1), 144-150. https://doi.org/10.2967/jnumed.115.158915

Complete Status: Complete

First Name: Muriel

Last Name: Abbaci

Email: muriel.abbaci@gustaveroussy.fr

Organization: IR4M UMR8081 Equipe Gustave Roussy

Country: France 


\title{
ID: GA28 \\ Design, Synthesis and Evaluation of a Novel PSMA Probe for NIR-II Imaging and Intraoperative Guidance in Mice
}

\author{
Longfei Zhang, Beijing Nornal University, Ifzhang13@lzu.edu.cn
}

\section{Category: Oncology}

\begin{abstract}
Body : Design, Synthesis and Evaluation of a Novel PSMA Probe for NIR-II Imaging and Intraoperative Guidance in Mice Longfei Zhang, Mengchao Cui* Key Laboratory of Radiopharmaceuticals, Ministry of Education, Beijing Normal University, Beijing 100875, China Objectives: Prostate cancer ( $\mathrm{PCa})$ is a prevalent cancer in adult males and one of the leading causes of tmour-related deaths[1]. In recent years, incident rates of PCa were dramatically increased and become a severe threat to men's health worldwide. Radical prostatectomy is the most effective way to treat prostate cancer (PCa). However, the abundant nerves and blood vessels in this region may be injured during surgery, leading to various postoperative complications such as frequent urination and urinary incontinence. Moreover, incomplete resection of a tumor could cause recurrence and metastasis of the tumor. Accordingly, the development of suitable optical imaging probes to distinguish tumor from normal tissue is an urgent issue. Prostate-specific member antigen (PSMA) is a type
\end{abstract} II transmembrane glycoprotein and its overexpression level is positively correlated with the stage of the disease and Gleason score. As a result, PSMA has attracted widespread attention as an effective biomarker for PCa. According to previous reports, imaging quality could be significantly improved in the near-infrared II (NIR-II) window (1000 nm - $1700 \mathrm{~nm})$ [2]. Inspired by these advances, we conjugated two Glu-urea-Lys moieties to the ethanol side chains of the benzo-bis(1,2,5-thiadiazole) fluorophore to form a NIR-II PSMA dimer probe for in vivo imaging and intraoperative guidance in living mice. Methods: The structure and optical properties of probe (NSN-BATP, Figure1A) were fully characterized by spectrum-based methods. The quantitive binding affinity of the probe was determined by inhibition assay using lysate of LnCAP cell. Results: The absorption and fluorescence emission maxima (Figure1B) of the probe in DMSO are $725 \mathrm{~nm}$ and $1095 \mathrm{~nm}$, respectively, with a large stokes shift $(370 \mathrm{~nm})$. NSN-BATP displayed high photostability during 30 minutes uninterrupted irradiation of excitation light. Competitive binding assay revealed that NSN-BATP displayed a high affinity toward PSMA $(\mathrm{Ki}=80 \mathrm{pM})$. The above excellent properties could ensure good performance obtained in further imaging studies. Conclusions: All these preliminary results indicated that NSN-BATP, as a new NIR-II probe, displayed excellent biological and optical properties towards PSMA. Further studies and applications of this probe are currently underway. Acknowledgements: Supported by the National Natural Science Foundation of China (No. U1967221). Reference: [1] Freddie Bray. et al. CA: A Cancer Journal for Clinicians, 2018, 68: 394-424 [2] Hong, G. et al. Nat Biomed Eng 1, 2017: 1 - 22. Corresponding Author: Mengchao Cui, Phone: +86-13811995064. E-mail: cmc@bnu.edu.cn

References: [1] Freddie Bray. et al. CA: A Cancer Journal for Clinicians, 2018, 68: 394-424 [2] Hong, G. et al. Nat Biomed Eng, 2017: 1 - 22. 


\section{Image/Figure:}

https://www.xcdsystem.com/wmis/abstract/File6959/GA28_ImageFigure_0422062104.jpg

Full Name of Abstract's 1st Author : Longfei Zhang

Complete Status: Complete

First Name: Longfei

Last Name: Zhang

Email: 1fzhang13@1zu.edu.cn

Organization: Beijing Nornal University

Country: China 


\title{
ID: GA32 \\ Multiplexed Molecular Ultrasound Imaging using Temporal Traces of Targeted Perfluorocarbon Nanodroplets
}

\author{
Austin Van Namen, Dartmouth College, avn.th@dartmouth.edu
}

\section{Category: Computational \& Data Science}

\begin{abstract}
Body : Background: There is a need in molecular ultrasound (US) imaging for differentiable contrast agents in order to target and visualize multiple biomarkers simultaneously. Ultrasound, due to its contrast mechanism, cannot easily distinguish two populations of contrast agents. Here we combine two phase-change nanodroplets with distinct kinetics and a novel method of differentiation to provide real-time, multiplex images. Phase-change perfluorocarbon nanodroplets (NDs) are emerging US contrast agents which when triggered with acoustic or optical energy can undergo a liquid-to-gas phase transition and generate strong US contrast. They are comprised of a lipid shell and liquid perfluorocarbon core. Similar to the microbubbles commonly used in US molecular imaging, the nanodroplet's lipid shell is readily functionalized with targeting ligands for molecular binding and identification (Tran et al., 2007). However, unlike microbubbles, the liquid nanodroplet core determines the functionality and properties of the contrast agents. In this project, we used both Perfluoropentane (boiling point $=28{ }^{\circ} \mathrm{C}$, denoted ND28) and Perfluorohexane nanodroplets $\left(56^{\circ} \mathrm{C}\right.$, denoted ND56). After vaporization, the low-boiling point ND28 form stable microbubbles, while high-boiling point ND56 recondense back to their liquid nanodroplet state in milliseconds(Hannah et al., 2016). Multiplex US imaging has previously been achieved with these nanodroplets using multiwavelength optical activation (Santiesteban et al., 2019), but this study will use high intensity focused ultrasound to simultaneously vaporize both nanodroplet contrast agents. Acoustic nanodroplet vaporization permits faster acquisition and increased activation depth. This study leverages the distinct temporal behaviors of ND28 and ND56 post-vaporization to determine spatially varying concentrations and form ratiometric images of the two contrast agents.

Results: First, nanodroplets were fabricated and molecularly targeted by attaching HER 2 and EGFR antibodies to the lipid shell using maleimide-thiol binding. Nanodroplets were then bound to HER2 and EGFR overexpressing cells and the binding was quantified by vaporizing the NDs and imaging the resulting microbubble providing statistically significant confirmation of selective binding. The nanodroplets were triggered using a short pulse of $1.1 \mathrm{MHz}$ high intensity focused ultrasound (HIFU) and imaged using a high-frequency Verasonics Vantage 256 system. An acquisition sequence consisting of 30 US frames was collected with 5 interleaved HIFU pulses. After confirmation of binding and targeting potential, the multiplexing potential of the NDs was explored. Polyacrylamide tissue-mimicking phantoms were made containing a homogeneous mix of nanodroplets with varying ratios from $100 \%$ ND28 to $100 \%$ ND56. The predicted temporal behavior of ND28 is a single vaporization event on the first HIFU pulse, while ND56 should vaporize and recondense with each HIFU pulse. Based on the predicted temporal behavior of ND28 and ND56 during the acquisition, a matrix of three ideal time signals was constructed as shown in Fig: a). By multiplying the pseudoinverse of this matrix and a pixel's US intensity over time, we calculate a map of the relative contribution of each ideal
\end{abstract}


signal. This results in images showing relative spatial concentration of ND28 and ND56 (Fig: cg). Averaging across the area of activation, we obtain ratios of ND56 activity to total activity. Fig: b) shows a linear relationship between the calculated and actual ratio of nanodroplets. Conclusion: The linear relationship shown in Fig: b) infers a way to image unknown concentrations of ND28 and ND56. To date, contrast-enhanced US can reliably visualize only a single population of contrast agents. Combined with demonstrated targeting potential of the nanodroplets, this research can be expanded to image multiple cell biomarker overexpressions simultaneously. These results demonstrate the ability to differentiate between two phase change nanodroplets based on their temporal responses with implications for improving molecular US imaging.

References: Hannah, A. S., Luke, G. P., \& Emelianov, S. Y. (2016). Blinking Phase-Change Nanocapsules Enable Background-Free Ultrasound Imaging. Theranostics, 6(11), 1866-1876. https://doi.org/10.7150/thno.14961 Santiesteban, D. Y., Hallam, K. A., Yarmoska, S. K., \& Emelianov, S. Y. (2019). Color-coded perfluorocarbon nanodroplets for multiplexed ultrasound and photoacoustic imaging. Nano Research, 12(4), 741-747. https://doi.org/10.1007/s12274019-2279-x Tran, T. D., Caruthers, S. D., Hughes, M., Marsh, J. N., Cyrus, T., Winter, P. M., Neubauer, A. M., Wickline, S. A., \& Lanza, G. M. (2007). Clinical applications of perfluorocarbon nanoparticles for molecular imaging and targeted therapeutics. In International Journal of Nanomedicine (Vol. 2, Issue 4, pp. 515-526). Dove Press.

\section{Image/Figure:}

https://www.xcdsystem.com/wmis/abstract/File6959/GA32_ImageFigure_0612034302.jpeg

Image/Figure Caption: FIGURE: a) Time signature matrix encodes the predicted temporal behavior of the nanodroplets over an activation sequence of $5 \mathrm{HIFU}$ pulses that vaporize the nanodroplets. Experimental data in b) shows a linear relationship between the calculated and true ND28:ND56 ratios. Images c)-g) are ratio images of mixed nanodroplets where each pixel has been fit to the ideal time signature matrix and matched to ND28 or ND56.

Complete Status: Complete

First Name: Austin

Last Name: Van Namen

Email: avn.th@dartmouth.edu

Organization: Dartmouth College

Country: USA 


\title{
ID: GA34 \\ Preclinical assessment of a targeted vs untargeted $\mathrm{pH}$-sensitive probe based on CypHer5E fluorophore in optical imaging procedures.
}

\author{
Federica Chianale, Bracco Imaging SpA, federica.chianale@bracco.com
}

\section{Category: Oncology}

\begin{abstract}
Body : Optical imaging (OI) offers several advantages in terms of sensitivity and safety for the non-invasive detection of human cancers. Fluorescent contrast agents in the nearinfrared spectrum of light $(650-900 \mathrm{~nm})$ carry ideal features due to the relatively high penetration in tissues and low background fluorescence. Tumor extracellular microenvironment is characterized by $\mathrm{pH}$ values in the range 6.4-7, lower than physiological, due to the metabolic shift toward anaerobic glycolysis in hypoxic conditions. This tumor-specific acidosis can be exploited using $\mathrm{pH}$-responsive fluorescent contrast agents which are "turned on" in an acidic environment. In addition, targeting of cancer-specific cell surface receptors, able to mediate internalization in endocytic compartments $(\mathrm{pH}$ 6.3-4.7), can induce intracellular accumulation of $\mathrm{pH}$-sensitive probes. Integrin $\alpha \mathrm{V} \beta 3$, overexpressed by several cancer cells and by endothelial cells of neoangiogenic vessels, is an attractive candidate due to an intrinsic rapid turnover of endocytosis and recycling. As a proof of concept study, the possibility to visualize the acidic tumor microenvironment by OI was assessed using the $\mathrm{pH}$-sensitive dye CypHer5E $(\mathrm{pKa}=7.3)$ in a subcutaneous hypoxic tumor model of human colorectal carcinoma HT-29 cells in nude mice. Then, to assess the possibility to visualize tumor masses upon cellular internalization, CypHer5E was conjugated to the integrin-targeting moiety $\mathrm{cRGDfK}$, giving rise to the $\mathrm{pH}$-sensitive probe cRGDfK-CypHer5E. Both CypHer5E and cRGDfK-CypHer5E were evaluated in $\alpha$ V $\beta 3$ overexpressing subcutaneous tumors of human glioma U-87 MG cells in nude mice. After intravenous administration of CypHer5E in HT-29 tumor-bearing mice, in vivo OI was performed up to $24 \mathrm{~h}$, time of sacrifice. A diffuse fluorescent signal throughout mice body was recorded at the earliest timepoint (30 min), rapidly decaying in all districts, reflecting the distribution of the dye through the blood flow and indicating an at least partial activation at physiological $\mathrm{pH}$. At all timepoints, no contrast could be appreciated, evaluated as tumor-tobackground ratio (TBR). No significant differences were found after ex vivo analysis of excised tumors, organs and tissues from either untreated or CypHer5E-treated mice, with tumor/muscle (T/M) fluorescence ratio close to 1 . Before mice sacrifice, pimonidazole was administered intravenously and tumor hypoxia was confirmed by ex vivo immunofluorescence staining of pimonidazole adducts in tumor sections. Prior to in vivo evaluation, the cellular uptake of both CypHer5E and cRGDfK-CypHer5E was assessed in vitro in $\alpha \mathrm{V} \beta 3$-overexpressing WM-266 human melanoma cells. Flow cytometry analysis showed that both CypHer5E and cRGDfKCypHer5E were internalized and turned on in acidic intracellular compartments. Upon $2 \mathrm{~h}$ incubation, cRGDfK-CypHer5E-treated cells displayed a 30-time greater fluorescence than CypHer5E-treated ones, indicating the efficient receptor-mediated internalization. Receptordependent endocytosis was estimated to account for $77.4 \pm 0.8 \%$ of total cRGDfK-CypHer5E cellular uptake. In vivo OI analysis of U-87 MG tumor-bearing mice after administration of CypHer5E showed no contrast of tumor over background at all timepoints, with a diffuse
\end{abstract}


fluorescent signal throughout mice body peaking $2 \mathrm{~h}$ after administration, then rapidly decaying. Otherwise, administration of cRGDfK-CypHer5E showed accumulation of the probe at tumor site, with TBR peaking after $30 \mathrm{~min}$ with a value of $1.9 \pm 0.4$. At $24 \mathrm{~h}$, time of sacrifice, ex vivo fluorescence analysis of excised tumors, organs and tissues showed T/M ratios of $1.2 \pm 0.1$ and $2.9 \pm 0.4$ for CypHer5E- and cRGDfK-CypHer5E-treated mice respectively. Of note, the time of sacrifice and ex-vivo analysis was far from the recorded TBR peak in vivo in cRGD-CypHer5Etreated mice, suggesting that optimization of observation time is required to completely appreciate the performance of this probe. In conclusion, integrin targeting of CypHer5E provided significant advantage in OI procedures compared to administration of the CypHer5E alone. These results support further investigation on molecular-targeted $\mathrm{pH}$-sensitive probes as a promising strategy to improve tumor detection by OI.

References: Boedtkjer and Pedersen, The Acidic Tumor Microenvironment as a Driver of Cancer. Rev. Physiol. 2020;82:103-126. Casey et al., Sensors and Regulators of Intracellular pH. Nat Rev Mol Cell Biol 2010;11(1):50-61. Moreno-Layseca et al., Integrin Trafficking in Cells and Tissues. Cell Biol 2019;21:122-132.

\section{Image/Figure:}

https://www.xcdsystem.com/wmis/abstract/File6959/GA34 ImageFigure 0608104103.png

Image/Figure Caption: The optical imaging (OI) efficacy of the $\mathrm{pH}$-responsive dye CypHer5E and the integrin-targeted cRGDfK-CypHer5E probe were compared in mice subcutaneous xenograft tumors of human glioma U-87 MG cells. While no differences between tumor and muscle OI signal could be recorded in CypHer5E-treated mice, integrin targeting of cRGDfKCypHer5E showed advantage in OI procedures in vivo.

Complete Status: Complete

First Name: Federica

Last Name: Chianale

Email: federica.chianale@bracco.com

Organization: Bracco Imaging SpA

Country: Italy 


\title{
ID: GA38 \\ Longitudinal imaging of activated $T$ cells and inflammatory demyelination using MRI and [18F]F-AraG PET in a novel multiple sclerosis model
}

\author{
Caroline Guglielmetti, University of California San Francisco, \\ caroline.guglielmetti@ucsf.edu
}

Category: Immunology: Inflammation \& Infection

\begin{abstract}
Body : Background Multiple sclerosis (MS) lesions are highly heterogeneous, as shown by ex vivo analysis of central nervous system (CNS) tissue from biopsies or post mortem1. Active MS lesions have been classified in different types (I-III) that are likely associated with distinctive disease progressions, and harbor singular macrophages and/or T-cells content1,2. While conventional magnetic resonance imaging (MRI) can detect active/inactive lesions 3,4, no clinical imaging method can presently provide specific information on lesion type, thus preventing proper stratification of patients, and impacting choice of therapeutic regimen. In this study, we used a newly developed positron emission tomography (PET) tracer, 2'-deoxy-2'$[18 \mathrm{~F}]$ fluoro-9- $\beta$-D-arabinofuranosylguanine $([18 \mathrm{~F}] \mathrm{F}-\mathrm{AraG})$ that showed relatively high specificity for activated T-cells in animal models and humans5-8. We combined this PET agent with high field MRI, and evaluated the potential of this multimodal approach to longitudinally image a newly characterized MS model9,10 that present two distinct brain lesion types: type I/IIresembling lesions with high levels of T-cells and macrophages/microglia, and type IIIresembling lesions with oligodendrocytes apoptosis and elevated microglia/macrophages. Objective/Hypothesis We investigated the potential of [18F]F-AraG PET imaging to noninvasively assess infiltrating activated T-cells and to provide, in combination with high field MRI, a unique way to assess lesion type. Methods Animals: C57/BL6J mice received cuprizone (CPZ) diet $(0.25 \%)$ for three weeks (W0-W3). At W5, experimental autoimmune encephalomyelitis (EAE) was induced9,10. Mice underwent MRI and PET imaging at W0 and W7 $(n=9)$ and a subset at W0, W3 and W7 $(n=5)$ (Suppl.Fig.1A). MRI: A 14.1T MR scanner was used to acquire T2-weighted images, and T1-weighted images post intravenous gadolinium injection (Suppl.Table1). PET: PET/CT was acquired one hour after [18F]F-AraG intravenous tail injection $(\sim 200 \mu \mathrm{Ci})$. Imaging analyses: For brain, we co-registrated PET/CT and MR data using VivoQuant software, delineated the corpus callosum, calculated PET and T2 signal intensity values, and T1 enhancing lesions' volume. Additionally, PET signal was quantified in the spinal cord, subiliac lymph nodes and muscle. Ex-vivo: Immunofluorescence analyses were performed at W0, W3 and W7 for T-cells (CD3) and microglia/macrophages (Iba1). Statistical analyses: Statistical significance was evaluated using a One-Way ANOVA with post-hoc Tukey (repeated measures) and paired t-test. Correlations analyses were performed using Pearson coefficient correlation. Results At W3, mice developed inflammatory lesions in the corpus callosum, characterized by elevated Iba1+ macrophages/microglia $(\mathrm{p}$ At W7, mice presented limb weakness and paralysis, demonstrating spinal cord damage. [18F]F-AraG was increased in the spinal cord $(\mathrm{p} \leq 0.0003)$ and correlated with T-cells levels $(\mathrm{r} 2=0.88, \mathrm{p}=0.045)$ (Suppl.Fig.1B). [18F]F-AraG signal was also increased in the lymph nodes $(\mathrm{p} \leq 0.0004)$ at $\mathrm{W} 7$, in agreement with elicited adaptive immune responses. Interestingly, no change was observed in leg
\end{abstract}


muscles where no inflammation is expected (Suppl.Fig.1C). Conclusions We demonstrated that, in a MS mouse model, high field MRI can detect brain lesions independently of the lesion type, and that [18F]F-AraG PET imaging provides a new way to non-invasively differentiate between lesion types by detecting activated T-cells. Upon clinical translation, these methods might provide new tools to enable patient stratification, monitor disease progression and response to therapies in MS.

References: 1 Reich, D. S., Lucchinetti, C. F. \& Calabresi, P. A. Multiple Sclerosis. N Engl J Med 378, 169-180, doi:10.1056/NEJMra1401483 (2018). 2 Lucchinetti, C. et al. Heterogeneity of multiple sclerosis lesions: implications for the pathogenesis of demyelination. Ann Neurol 47, 707-717 (2000). 3 Thompson, A. J. et al. Diagnosis of multiple sclerosis: 2017 revisions of the McDonald criteria. Lancet Neurol 17, 162-173, doi:10.1016/S1474-4422(17)30470-2 (2018). 4 Filippi, M. et al. Multiple sclerosis. Nat Rev Dis Primers 4, 43, doi:10.1038/s41572-018-0041-4 (2018). 5 Ronald, J. A. et al. A PET Imaging Strategy to Visualize Activated T Cells in Acute Graft-versus-Host Disease Elicited by Allogenic Hematopoietic Cell Transplant. Cancer Res 77, 2893-2902, doi:10.1158/0008-5472.CAN-16-2953 (2017). 6 Colevas, D., Bedi N, Chang S, Moreno Nieves U, Chatterjee S, Davidzon G, Srinivas S, Le Q, Gambhir A, Sunwoo J. A Study to evaluate immunological response to PD-1 inhibition in squamous cell carcinoma of the head and neck (SCCHN) using novel PET imaging with [18F]F-AraG [poster]. American Society of Clinical Oncology, Conference; 2018 June 1-5, Chicago (Ill): ASCO; 2018. Abstract nr 6050 (2018). 7 Franc, B. L. et al. In Vivo PET Imaging of the Activated Immune Environment in a Small Animal Model of Inflammatory Arthritis. Mol Imaging 16, 1536012117712638, doi:10.1177/1536012117712638 (2017). 8 Levi, J. et al. Imaging of Activated T Cells as an Early Predictor of Immune Response to Anti-PD-1 Therapy. Cancer Res 79, 3455-3465, doi:10.1158/0008-5472.CAN-19-0267 (2019). 9 Scheld, M. et al. Neurodegeneration Triggers Peripheral Immune Cell Recruitment into the Forebrain. J Neurosci 36, 1410-1415, doi:10.1523/JNEUROSCI.2456-15.2016 (2016). 10 Ruther, B. J. et al. Combination of cuprizone and experimental autoimmune encephalomyelitis to study inflammatory brain lesion formation and progression. Glia 65, 1900-1913, doi:10.1002/glia.23202 (2017).

\section{Image/Figure:}

https://www.xcdsystem.com/wmis/abstract/File6959/GA38_ImageFigure_0421112401.PNG

Full Name of Abstract's 1st Author : Caroline Guglielmetti

Complete Status: Complete

First Name: Caroline

Last Name: Guglielmetti

Email: caroline.guglielmetti@ucsf.edu

Organization: University of California San Francisco

Country: United States 


\title{
ID: GA42 \\ Bipyramid-like Mesoporours Pt Coated Au Nanoparticles with Superior Biological Behaviors and Catalase-like Activity in Pancreatic Cancer and Atherosclerosis Plaque
}

\author{
xiongfeng cao, Affiliated Hospital of Jiangsu University, 1353671692@qq.com
}

\section{Category: New Chemistry, Biology \& Bioengineering}

\begin{abstract}
Body : Mesoporous metal nanoparticles (MMNPs) have been widely developed for theranostic purposes. The synthesis of bipyramid-like MMNPs and its shape effect on biological behaviors are not investigated. Herein, bipyramid-like mesoporours Pt coated $\mathrm{Au}$ nanoparticles (Au-mesoPt bipyramid) were successfully prepared using gold nanoparticles as shape templates and Pluronic F127 as meso-structure directing agent. The three AumesoPt nanoparticles displayed uniform morphology and uniform size (length $\times$ width) $(\sim 85 \times 85 \mathrm{~nm}$ for Au-mesoPt sphere, $\sim 116 \times 38 \mathrm{~nm}$ for Au-mesoPt rod and $\sim 99 \times 38 \mathrm{~nm}$ for Au-mesoPt bipyramid, respectively). Apart from showing higher cellular uptake efficiency than that of Au-mesoPt sphere (3-fold) and Au-mesoPt rod (1.5-fold), Au-mesoPt bipyramid also exhibited greater multicellular spheroid permeability. In vivo Au-mesoPt bipyramid displayed longer blood circulation time than that that of Au-mesoPt sphere (9.6fold) and Au-mesoPt rod (4.8-fold), as well as more accumulation in tumor and atherosclerosis plaque. Inspired by the superior biological behaviors and instinct hydrogen peroxide catalase-like activity, Au-mesoPt bipyramid showed effective pancreatic cancer photothermal therapy and atherosclerosis plaque burden alleviation, both of which are notoriously for the excessive oxidative stress microenvironment. Overall, this work firstly synthesized bipyramid-like Au-mesoPt nanoplatform with greater biological behaviors than spherical and rod-like counterparts.
\end{abstract}

Full Name of Abstract's 1st Author : Xiongfeng Cao

Complete Status: Complete

First Name: xiongfeng

Last Name: cao

Email: 1353671692@qq.com

Organization: Affiliated Hospital of Jiangsu University

Country: China 


\title{
ID: GA43 \\ Heterogeneity of salivary gland cancers based on patient-derived salivary tumoroid models
}

YOONJIN ROH, Chung-Ang University, shdbswls@naver.com

\section{Category: Oncology}

\begin{abstract}
Body : Organoid culture is the 3D cultivation of source tissue in a support matrix and specialized media that closely resembles the physiological environment. The self-renewal ability of stem cells is dependent on extrinsic niche signals that have not been unraveled for the salivary gland. The ductal compartment in the salivary gland harbors stem cells. The specific requirements of the niche remain unclear for many cell types, including the proacinar cells that give rise to which generate secretory acinar epithelial cells that produce saliva. The addition of salivary glands to epithelial cells in vitro improves the ability of organoids to recapitulate the features of the tissue and enhance the viability of organoids. Tumor organoids are cultures of cancer cells that can be derived on an individual patient basis with a high success rate. This creates opportunities to build large biobanks with relevant patient material that can be used for drug screening and development. In this study, we have highlighted the current knowledge regarding tumor organoid culture, the advantages of the model system, and biobanks of patientderived tumor organoids that are used in drug development research.
\end{abstract}

References: Bartfeld, S., Clevers, H. (2017) Stem cell-derived organoids and their application for medical research and patient treatment. J Mol Med 95, 729-738. Michael A. Lensink et al. (2020) Responsible use of organoids in precision medicine: the need for active participant involvement. Development 147:7, dev177972. Li M, Izpisua Belmonte JC (2019) Organoids Preclinical Models of Human Disease. New England Journal of Medicine 380: 569-579. James T. et al., (2018) Organoid Modeling of the Tumor Immune Microenvironment, Cell, Volume 175, Issue 7. Kaushik, Garima et al. (2018) "Concise Review: Current Status of ThreeDimensional Organoids as Preclinical Models." Stem cells (Dayton, Ohio) vol. 36,9, 1329-1340. Fleur Weeber et al.,(2017) Tumor Organoids as a Pre-clinical Cancer Model for Drug Discovery, Cell Chemical Biology, Volume 24, Issue 9, 1092-1100,

\section{Image/Figure:}

https://www.xcdsystem.com/wmis/abstract/File6959/GA43 ImageFigure 0407011054.jpg

Full Name of Abstract's 1st Author : Yoon Jin Roh

Complete Status: Complete

First Name: YOONJIN

Last Name: $\mathrm{ROH}$ 
Email: shdbswls@naver.com

Organization: Chung-Ang University

Country: Korea, Republic of 


\title{
ID: GA44 \\ Varying Oleylamine to Dibenzyl Ether Ratio for Fine-Tuning Manganese Oxide Nanoparticle Diameter and MRI Signal Intensity
}

\author{
Celia Martinez de la Torre, West Virginia University, cemartinezdelatorre@mix.wvu.edu
}

\section{Category: Oncology}

\begin{abstract}
Body : The ultimate goal of this research project is to develop a contrast agent to reduce the number of false-positive and false-negative imaging results associated with current breast cancer diagnostic imaging techniques. Gadolinium chelates are the current clinically approved contrast agents for breast magnetic resonance imaging (MRI), but they are always "on" and highlight any vascularized structure. Due to their lack of targeting, both benign and malignant breast tumors are enhanced with gadolinium. By utilizing tumor-targeted $\mathrm{pH}$-sensitive manganese oxide $(\mathrm{MnO})$ nanoparticles, a contrast agent can be developed that will only turn "on" in the presence of the lower $\mathrm{pH}$ of endosomes/lysosomes inside cancer cells. Reducing the size of $\mathrm{MnO}$ nanoparticles should increase the dissolution of $\mathrm{MnO}$ to $\mathrm{Mn} 2+$ at low $\mathrm{pH}$ to enhance the MRI signal. MnO nanoparticles were synthesized through thermal decomposition of $\mathrm{Mn}(\mathrm{II})$ acetylacetonate in dibenzyl ether (DE) and oleylamine (OA) at a peak reaction temperature of $280 \mathrm{oC}$ for 30 minutes. The following ratios of OA:DE were used: 60:0, 50:10, 40:20, 30:30, 20:40, and 10:50 to investigate the effects of reactant conditions on nanoparticle size, $\mathrm{Mn} 2+$ release, and MRI signal. MnO nanoparticles were characterized physically and chemically using transmission electron microscopy (TEM), x-ray diffraction (XRD), and Fourier-transform infrared spectroscopy (FTIR). According to TEM, the 10:50 ratio created the smallest nanoparticle diameter of $19 \pm 6 \mathrm{~nm}$, while the 50:10 ratio created the largest nanoparticle diameter of $39 \pm 15 \mathrm{~nm}$. All nanoparticles were comprised of MnO (XRD) coated with oleylamine (FTIR). To further elucidate the effect of nanoparticle size and composition on the release of $\mathrm{Mn} 2+$ and resulting MRI signal, the smallest MnO nanoparticles (19 $\mathrm{nm}$ ) were compared with $\mathrm{MnO}$ nanoparticles of a larger diameter $(32 \mathrm{~nm})$ and $\mathrm{Mn} 3 \mathrm{O} 4$ nanoparticles of similar diameter $(17 \mathrm{~nm})$. Nanoparticles were incubated at 3 different $\mathrm{pHs}$ to measure $\mathrm{Mn} 2+$ release over time including $\mathrm{pH} 7.4$ (blood $\mathrm{pH}), 6.5$ ( $\mathrm{pH}$ of tumor extracellular space), and 5 (endosome/lysosome $\mathrm{pH}$ ) using inductively coupled plasma-optical emission spectroscopy (ICPOES) and 1T MRI. The smallest MnO nanoparticles were the most efficient MRI contrast agents by releasing the highest amount of $\mathrm{Mn} 2+$ at $\mathrm{pH} 5(\sim 50 \%$ at $24 \mathrm{hr})$ and $6.5(\sim 11 \%$ at $24 \mathrm{hr})$, which produced the lowest T1 value $(646 \mathrm{~ms}$ at $24 \mathrm{hr}, \mathrm{pH} 5)$ and maximum MRI signal at 1T; Mn3O4 nanoparticles generated the lowest MRI signal. Therefore, MnO nanoparticles instead of Mn3O4 should be utilized, and the nanoparticles should be targeted intracellularly to maximize MRI contrast generation. Future work includes fabricating hydrophilic MnO nanoparticles using phospholipid encapsulation and adding breast cancer-targeting peptides to enable specific detection of malignant versus benign breast tumors.
\end{abstract}

Full Name of Abstract's 1st Author : Celia Martinez de la Torre

Complete Status: Complete 
First Name: Celia

Last Name: Martinez de la Torre

Email: cemartinezdelatorre@mix.wvu.edu

Organization: West Virginia University

Country: United States 


\title{
ID: GA45 \\ Ultra-compact radiosynthesizer for clinical-scale production of PET tracers
}

\author{
Jia Wang, University of California, Los Angeles, jiajiawang@ucla.edu
}

\section{Category: Instrumentation}

\begin{abstract}
Body : Background Microfluidic-based radiochemistry, especially microdroplet reactors, could help increase the availability of various tracers for positron emission tomography (PET) by lowering the cost of production. Reduced reagent volumes lower the reagent cost, the simple apparatus can be built inexpensively, and the small footprint requires relatively little shielding or hot cell space. Previously, we developed a microdroplet reactor chip consisting of a hydrophilic trap patterned in a hydrophobic surface, built a compact apparatus to implement radiochemical reactions automatically[1], and showed the successful synthesis of [18F]fallypride and [18F]FDOPA in sufficient quantities for preclinical imaging[2]. Recently, we showed, by integrating a droplet reactor with a radionuclide concentrator, that we could produce up to 7.2 $\mathrm{GBq}$ of $[18 \mathrm{~F}]$ fallypride in droplet reactions and also studied the impact of upscaling on the reaction performance[3]. Here, we perform activity scaling for additional examples of clinicallyrelevant PET tracers $([18 \mathrm{~F}] \mathrm{FET}$ and $[18 \mathrm{~F}] \mathrm{FBB})$ on the droplet reactor and demonstrate the production of clinically-relevant amounts of these tracers and results of QC testing(Figure 1). Methods Fabrication of the disposable microfluidic chips and the compact system(Figure S1) were previously reported in detail[1]. The synthesis of several tracers was performed on this new platform, using protocols previously optimized using low starting activity $(\sim 19 \mathrm{MBq}[\sim 0.5 \mathrm{mCi}])$, for the synthesis of [18F] fallypride, [18F]FET, and [18F]FBB[3-4](Figure S2). The setup of reagents in designated dispensers for synthesizing the different tracers is shown in Figure S3. Activity scale was increased either using the radionuclide concentrator or by loading greater volume of the radioisotope solution. Synthesis performance was assessed via measurements of radioactivity (via dose calibrator) and radiochemical purity (via radio-TLC and Cerenkov luminescence imaging [5]). The final products were purified via analytical-scale radio-HPLC and reformulated in saline. Quality control tests were then performed to determine safety for eventual clinical use. Results The [18F]fluoride drying time in the syntheses of [18F]FET and [18F]FBB was increased from 1 to 7 min due to the larger volume of radioisotope solution when scaling from $19 \mathrm{MBq}[0.5 \mathrm{mCi}](10 \mu \mathrm{L})$ to $3.7 \mathrm{GBq}[100 \mathrm{mCi}](105 \mu \mathrm{L})$. As shown in Figure $\mathrm{S} 4$, with the increase of starting activity on the chip, crude radiochemical yield (crude RCY) of $[18 \mathrm{~F}]$ fallypride remained high until the starting activity exceeded $7.4 \mathrm{GBq}[200 \mathrm{mCi}]$ activity and then began to drop. For $[18 \mathrm{~F}] \mathrm{FET}$, the crude RCY also remained relatively constant at activities up to $3.7 \mathrm{GBq}[100 \mathrm{mCi}]$ and then began to drop. The crude RCY of [18F]FBB did not appear to be significantly impact by starting activity at the activity scales tested. For all tracers, it was possible to prepare at least one clinical dose amount of formulated product while only starting with $\sim 0.9 \mathrm{GBq}[25 \mathrm{mCi}]$ of activity. Compared to previously reported conventional synthesis methods[6]-[8](Table S1), the microdroplet reactor provides much higher molar activity even starting with 10-100x less activity, likely due to the small reagent amount and precursor amount. Example radio-HPLC chromatograms for $[18 \mathrm{~F}] \mathrm{FET}$ are shown in Figure S5, exhibiting excellent separation of product from impurities. The reformulated products of the tracers all passed the
\end{abstract}


clinical-relevant quality control tests. Conclusion In this work, we demonstrated that the microdroplet platform is not limited only to producing small batches of PET tracers for preclinical research but can also successfully produce multiple PET tracers at clinically-relevant scales. Compared to conventional synthesis methods, yields are comparable, reagent consumption is dramatically lower, and the resources needed to perform the synthesis are significantly reduced: potentially this platform could help to make these and other tracers more widely available. We are currently exploring the integration with other microfluidic elements including radioisotope concentration, purification and formulation[9] to further shrink the overall apparatus and shorten the overall synthesis time.

References: [1] Wang et al., Lab. Chip 2415-2424, 2019. [2] Wang et al., React. Chem. Eng. 2019. [3] Wang et al., RSC Adv. 10: 7828-7838, 2020. [4] Lisova et al., EJNMMI Radiopharm. Chem. 5: 1, 2019. [5] Wang et al., Nucl. Med. Biol. 82-83: 41-48, 2020. [6] Seok Moon et al., Appl. Radiat. Isot. 68: 2279-2284, 2010. [7] Siddiq et al., Appl. Radiat. Isot. 133: 38-44, 2018. [8] Wang et al., Appl. Radiat. Isot. 71: 41-46, 2013. [9] Chao et al., Lab. Chip 17: 1802-1816, 2017.

\section{Image/Figure:}

https://www.xcdsystem.com/wmis/abstract/File6959/GA45 ImageFigure 0408014347.png

Full Name of Abstract's 1st Author : Jia Wang

Complete Status: Complete

First Name: Jia

Last Name: Wang

Email: jiajiawang@ucla.edu

Organization: University of California, Los Angeles

Country: United States 


\title{
ID: GA47 \\ [89Zr]Zr(oxinate)4 allows radiolabelling and in vivo PET imaging of small extracellular vesicles
}

\author{
Azalea Khan, King's College London, azalea.khan@kcl.ac.uk
}

Category: New Chemistry, Biology \& Bioengineering

\begin{abstract}
Body : Introduction: Small extracellular vesicles (sEVs; diameter $\sim 30-150 \mathrm{~nm}$ ) are actively involved in cell-cell communication and disease progression 1 , and have the potential to be used as biomarkers and drug delivery systems 2 . It is important to understand the in vivo behaviour of exogenously administered sEVs, and PET imaging offers excellent properties to achieve this. Using PANC1 sEVs as a model system we report that [89Zr]Zr(oxinate)4, with proven efficiency of transporting $89 \mathrm{Zr}$ across the lipid bilayer of cells 3 and liposomes 4 , can be used to radiolabel sEVs and track them in vivo. Methods: [89Zr]Zr(oxinate)4 (89Zr-oxine) was synthesised by converting [89Zr]Zr(oxalate) 4 to [89Zr]ZrCl4, followed by addition of a buffered aqueous mixture containing oxine (Fig. 1A). PANC1 cells were cultured in CLAD1000 bioreactor flask and the supernatant collected weekly from which PANC1 sEVs were isolated by differential ultracentrifugation, and characterised for size using NanoSight and biomarker expression (CD63 and CD9) by dot blot. sEVs (1x1012 sEVs) were radiolabelled by incubating with [89Zr]Zr(oxinate) 4 at $37^{\circ} \mathrm{C}$ and purified by size-exclusion chromatography (Fig. 1B). Neutralised [89Zr]ZrCl4 was used as control. For the in vivo PET imaging study, healthy C57BL/6 mice were injected intravenously with 89Zr-PANC1 sEVs $(0.2-1 \mathrm{MBq}, \sim 1 \mathrm{x} 1010$ $\mathrm{sEVs}$ /mouse), as intact $(\mathrm{n}=3)$ or as heat-damaged $(\mathrm{n}=2)$, followed by whole-body PET-CT imaging and ex vivo biodistribution at 2 or $24 \mathrm{~h}$. Results: [89Zr]Zr(oxinate) 4 was synthesised with $95.1 \pm 2.6 \%$ purity, and radiolabelled PANC1 $\mathrm{sEVs}$ with a radiolabelling yield of $28.4 \pm$ 4.2\% (Fig. 1C). sEVs derived from other cell lines can also be radiolabelled (Supplementary data). Radiolabelling did not result in significant changes in the modal diameter or biomarker expression of the sEVs (Fig. 1D). In vivo imaging in C57BL/6 mice showed high uptake of intact sEVs in liver, spleen, and several lymph nodes (Fig. 1E), and brain (Fig. 1F). Spleen uptake reduced when sEVs were heat-damaged. At $24 \mathrm{~h}$ p.i., liver and spleen signal remained but fewer lymph nodes and no brain signal were visible. Bone uptake increased, particularly for heatdamaged sEVs. Ex vivo quantification showed significantly higher uptake of intact sEVs in spleen $(58 \pm 10 \% \mathrm{ID} / \mathrm{g})$ compared to heat-damaged sEVs $(19 \pm 10 \% \mathrm{ID} / \mathrm{g}), \mathrm{P}=0.03$ (Fig. $1 \mathrm{G})$. Both liver:bone and spleen:bone ratios were $>7$ for intact $\mathrm{sEVs}$, but Conclusion: [89Zr]Zr(oxinate)4 allows radiolabelling of PANC1 sEVs. PET imaging of 89Zr-PANC1 sEVs showed $89 \mathrm{Zr}$ uptake in liver, spleen, lymph nodes and brain, and demonstrated that heatdamaged sEVs show significant differences in spleen uptake and liver:bone and spleen:bone uptake ratios. Although these results are still preliminary and require further evaluation, particularly on the nature of the extensive lymph node and brain $89 \mathrm{Zr}$ uptake, our results indicate that $[89 \mathrm{Zr}] \mathrm{Zr}$ (oxinate) 4 is an efficient radiolabelling agent for sEVs and that liver:bone and spleen:bone ratios have potential as imaging biomarkers to assess the in vivo status of [89Zr]Zr(oxinate)4-labelled sEVs.
\end{abstract}


References: 1. C. Thery, et al. Nat Rev Immunol, 2002, 2, 569-579. 2. S. Walker, et al. Theranostics, 2019, 9, 8001-8017. 3. F. Man, et al. Molecular Therapy, 2019, 27, 219-229. 4. S. Edmonds, et al. ACS nano, 2016, 10, 10294-10307.

\section{Image/Figure:}

https://www.xcdsystem.com/wmis/abstract/File6959/GA47_ImageFigure 0614112132.jpg

Image/Figure Caption: Figure 1: A) Schematic of [89Zr]Zr(oxinate)4 synthesis. B) Schematic showing sEV radiolabelling strategy. C) Radiolabelling yield of PANC1 sEVs with [89Zr]Zr(oxinate)4 and control 89Zr. D) Characterisation of sEVs before and after radiolabelling by NanoSight for size (top) and by dot blot for CD63 and CD9 expression (bottom). E) PET maximum intensity projection of "Intact" and "Heat-damaged" 89Zr-PANC1 sEVs' biodistribution in healthy mice $1.5 \mathrm{~h}$ and $24 \mathrm{~h}$ p.i., showing uptake in liver, spleen and lymph nodes. F) Image slices of a mouse injected with intact 89Zr-PANC1 sEVs showing uptake in brain. G) Ex vivo biodistribution showing uptake of "intact" $(n=3)$ and "heat-damaged" $(n=2)$ PANC1 sEVs by liver, spleen and bone. $\mathrm{H}$ ) Ratio of liver:bone uptake and spleen:bone uptake highlighting reduced uptake by these organs, when sEVs are heat-damaged. Key: $*=\mathrm{P}<0.05$, ns $=$ non-significant (unpaired t-test); p.i. = post injection; $\mathrm{LN}=$ lymph node; $\mathrm{B}=$ bladder.

Complete Status: Complete

First Name: Azalea

Last Name: Khan

Email: azalea.khan@kcl.ac.uk

Organization: King's College London

Country: United Kingdom 


\title{
ID: GA49 \\ Ferrocene - cucurbit[7]uril : host-guest pretargeted PET imaging in a xenograft model
}

\author{
Vilma Jallinoja, Vanderbilt University, vilma.i.jallinoja@vanderbilt.edu
}

Category: New Chemistry, Biology \& Bioengineering

\begin{abstract}
Body : A variety of pretargeting approaches have been reported, sharing the common objective of decreasing the radiation doses to non-target tissue in antibody-based nuclear imaging and therapy procedures. [1] Previously developed systems rely on pretargeting agents that struggle with in vivo stability, immunogenicity, and/or modularity, which we believe are critical for achieving wide-spread clinical translation. [2-5] We are reporting for the first time a ferrocene (Fc) - cucurbit[7]uril (CB7) pretargeting platform based on host-guest complex formation between a CB7-modified antibody and a ferrocene-based small molecule radioligand. Design of this approach was based on literature reports that CB7, a macrocyclic host compound, encapsulates positively charged ferrocene-based guest compounds with high affinity $(\mathrm{K}=1012$ 1015 M-1). [6] We hypothesize that our approach will overcome the challenges of current popular platforms owing to the components' stability and the platform's modularity. [7,8] In these studies, the ferrocene - CB7 pretargeting agents consist of a host CB7-conjugated anticarcinoembryonic antigen (CEA) antibody called M5A and a 68Ga-radiolabeled ferrocene guest radioligand. We successfully conjugated CB7 to M5A (CB7-M5A) and determined via cellular binding assay (Lindmo) using CEA expressing BxPC3 cell line that the modified antibody's immunoreactivity remains $>95 \%$. Further each antibody was analyzed to have 1.14 CB7moieties on average. Additionally, a small molecule $68 \mathrm{Ga}$-radiolabeled ferrocene radioligand (68Ga-NOTA-PEG3-NMe2-Fc) was prepared (RCY\%: 78.3\% \pm 10.2 ; RCP\%: 96.7\% \pm 2.4 ). In preclinical studies performed in BxPC3 tumor bearing mice, we demonstrated that our novel platform can achieve tumor-specific uptake and rapid clearance of the radioligand. The Fcradioligand was pretargeted upon an administration of the CB7-anti-CEA-M5A $72 \mathrm{~h}$ prior to the radioligand injection. The pretargeted 68Ga-NOTA-PEG3-NMe2-Fc showed specific tumor uptake compared to the non-pretargeted 68Ga-NOTA-PEG3-NMe2-Fc $(p=0.0094)$. There was no significant decrease in tumor uptake observed during the radioligand's $4 \mathrm{~h}$ in vivo monitoring period. No significant bone uptake was observed $(0.32 \pm 0.050 \% \mathrm{ID} / \mathrm{g}$ at $4 \mathrm{~h})$, implying that the ligand's $68 \mathrm{Ga}-\mathrm{NOTA}$ complex was stable in vivo. The in vivo biodistribution studies also revealed that the $\mathrm{CB} 7$-unbound radioligand is excreted through the hepatobiliary and renal system. Our future studies will include optimizing the clearance time of the radioligand by adjusting its hydrophilicity and experimenting with the number of PEG-units as part of the ligand's structure. In order to maximize tumor-to-background ratio and minimize radiation to non-target tissue, we plan to study the ferrocene-CB7 pretargeting system's efficacy when longer interval times $(>72 \mathrm{~h})$ between the administration of the pretargeting components are applied.
\end{abstract}

References: [1] Bailly C, Bodet-Milin C, Rousseau C,Faivre-Chauvet A, Kraeber-Bodéré F, Barbet J. EJNMMI Radiopharm Chem. 2017;2(1). [2] Rossin R, van den Bosch SM, ten Hoeve Q, et al. Bioconjug Chem. 2013;24:1210-1207 [3] Rusckowski M, Fogarasi M, Fritz B, 
Hnatowich DJ. Nucl Med Biol. 1997;24:263-268. [4] Kalofonos HP, Rusckowski M, Siebecker DA, et al. J Nucl Med. 1990;31:1791-1796. [5] Weiden PL, Breitz HB. Crit Rev Oncol Hematol. 2001;40:37-51. [6] Barrow SJ, Kasera S, Rowland MJ, del Barrio J, Scherman OA. Chem.

Rev. 2015;115:12320-12406. [7] Masson E, Ling X, Joseph R, Kyeremeh-Mensah L, Lu X. RSC Adv. 2012; 2:1213-1247 [8] Das D, Assaf KI, Nau WM. Front Chem. 2019;7

\section{Image/Figure:}

https://www.xcdsystem.com/wmis/abstract/File6959/GA49 ImageFigure 0422120951.png

Full Name of Abstract's 1st Author : Vilma Jallinoja

Complete Status: Complete

First Name: Vilma

Last Name: Jallinoja

Email: vilma.i.jallinoja@vanderbilt.edu

Organization: Vanderbilt University

Country: United States 


\title{
ID: GA50 \\ The Fluorescent Probes First Visualize Tau fibrils in Astrocytic
}

\author{
Yuying Li, Beijing Normal University, liyuyingyyl@163.com
}

\section{Category: Neuroscience}

\begin{abstract}
Body : Introduction: Tauopathies are heterogeneous neurodegenerative diseases in clinical, biochemical and anatomical, which are defined by the depositing of hyperphosphorylated tau protein in nerve cells. Astrocytes as a kind of important nerve cells are involved in many metabolic activities in the central nervous system. Abundant researches demonstrated the astrocytes not only as reactive cells to neuronal damage but also as primary actors in the pathological process of many diseases, such as Alzheimer's disease (AD), corticobasal degeneration (CBD), progressive supranuclear palsy (PSP) and globular glial tauopathy (GGT). The histopathologic results stated tau aggregates in astrocytes give rise to different morphological types in diverse tauopathies. However, the reported noninvasive tau probes generally detected neurofibrillary tangles (NFTs, tau deposits in neurons) in AD, but not other types of tau inclusions, which could be only recognized by immunohistochemical analysis. Therefore, it is crucial to develop new imaging probes specific for astrocytic tau pathologies. With the help of sensitive probes, we could fulfill further biochemical and pathological studies, which may be useful to increase our understanding of these tauopathies related to disease and to develop novel therapeutic strategies for patients. Objective: To design and evaluate near-infrared (NIR) fluorescent probes with high-efficient detection of astrocytic tau pathologies.

Methods: Fluorescent properties of these compounds were measured by conventional methods. In vitro fluorescent neuropathological staining was used to recognize accumulated tau in astrocytes on plenty of human brain sections. In addition, in vitro immunofluorescence staining on the same brain sections clarified the affinities towards tau fibrils in astrocytic of these probes. Results: As shown in Figure 1, one of the NIR fluorescent probe, FTA-5 displayed high specific and high affinity to astrocyte tau pathologies in gray and white matters of human brain sections including $\mathrm{AD}$ patient and non-AD patient, and verified by immunofluorescence staining of GFAP (the specific antibody of astrocytes). In addition, none of the amyloid plaques and other non-target proteins were labeled by FTA-5. Conclusion: In this study, we screened out several new probes with high affinity and selectivity for astrocytic tau pathologies in human brain tissue. Further modification and application of these probes are currently underway to allow the direct imaging of astrocytic tau pathologies in animal models and exploring the relationships between this pathology and different neurodegenerative diseases. Funding: supported by the National Natural Science Foundation of China (No. U1967221).
\end{abstract}

References: [1] Isidro Ferrer, Neuroglia, 2018, 1: 126-150. [2] Shane A. Liddelow, Kevin A. Guttenplan, Laura E. Clarke, et. al., Nature, 2017. 541: 581-587.

\section{Image/Figure:}


Full Name of Abstract's 1st Author : Yuying Li

Complete Status: Complete

First Name: Yuying

Last Name: Li

Email: liyuyingyy1@163.com

Organization: Beijing Normal University

Country: China 


\title{
ID: GA51 \\ Rare earth luminescent probes for bioimaging, diagnosis, and navigation
}

\author{
Ruichan Lv, Xidian University, rclv@xidian.edu.cn
}

\section{Category: New Chemistry, Biology \& Bioengineering}

\begin{abstract}
Body : I focus on the bioimaging and early-diagnosis applications of luminescent materials, especially I have done lots of efforts on the rare earth luminescence probes. In order to "improve the sensitivity and accuracy of molecular imaging probes for detecting microscopic tumors", the following four aspects are carried out: (1) Heuristic algorithms are used to guide the optimization and synthesis of luminescent materials with higher intensity. Rare earth luminescent probes can respond to near-infrared excitation light by doping with different elements, ensuring the near infrared excitation has the largest penetration depth and negligible self-interference of the light source. (2) The luminescence properties of rare earth probes were adjusted by the introduction of gold nanocrystals. The lanthanide-gold composite probes with different shapes and sizes were simulated by discrete dipole approximation method, and verified by actual test results. The feedback from such an algorithm can guide the fabrication of the optimal composite nanocrystal structure required. (3) The multi-functional probes with photoactive synergistic anticancer therapy composites were designed with multi-modal imaging methods (photoacoustic, upconversion luminescence, down-conversion NIR II imaging, etc.). (4) The multi-modal molecular imaging platform was used for in vivo real-time luminescence-guided surgery navigation with high precision and sensitivity.
\end{abstract}

References: References: 1. Wang, Y.; Lü, W.-D.; Lin, B.; Yang, F.; Feng, M.; Lv, R.* , An optimized lanthanide-chlorophyll nanocomposite for dual-modal imaging-guided surgery navigation and anti-cancer theranostics. Biomaterials Science 2020, 8 (5), 1270-1278. 2. Lv, R. *; Yang, F.; Jiang, X.; Hu, B.; Zhang, X.; Chen, X.; Tian, J., Plasmonic modulated upconversion fluorescence by adjustable distributed gold nanoparticles. J. Lumin. 2020, 220, 116974. 3. Lv, R.; Yang, P.; He, F.; Gai, S.; Li, C.; Dai, Y.; Yang, G.; Lin, J. *, A Yolk-like Multifunctional Platform for Multimodal Imaging and Synergistic Therapy Triggered by a Single Near-Infrared Light. Acs Nano 2015, 9 (2), 1630-1647. 4. Lv, R.; Yang, P.; Hu, B.; Xu, J.; Shang, W.; Tian, J. *, In Situ Growth Strategy to Integrate Up-Conversion Nanoparticles with Ultrasmall CuS for Photothermal Theranostics. Acs Nano 2017, 11 (1), 1064-1072. 5. Lv, R.; Yang, P.; He, F.; Gai, S.; Yang, G.; Dai, Y.; Hou, Z.; Lin, J. *, An imaging-guided platform for synergistic photodynamic/photothermal/chemo-therapy with $\mathrm{pH} /$ temperature-responsive drug release. Biomaterials 2015, 63, 115-127. 6. Lv, R.; Yang, D.; Yang, P.; Xu, J.; He, F.; Gai, S.; Li, C.; Dai, Y.; Yang, G.; Lin, J. * Integration of Upconversion Nanoparticles and Ultrathin Black Phosphorus for Efficient Photodynamic Theranostics under $808 \mathrm{~nm}$ Near-Infrared Light Irradiation. Chem. Mater. 2016, 28 (13), 4724-4734. 7. Lv, R.; Zhong, C.; Li, R.; Yang, P.; He, F.; Gai, S.; Hou, Z.; Yang, G.; Lin, J. *, Multifunctional Anticancer Platform for Multimodal Imaging and Visible Light Driven Photodynamic/Photothermal Therapy. Chem. Mater. 2015, 27 (5), 1751-1763. 8. Lv, R. *; Feng, M.; Parak, W. J., Up-Conversion Luminescence Properties of Lanthanide-Gold Hybrid Nanoparticles as Analyzed with Discrete Dipole Approximation. 
Nanomaterials (Basel, Switzerland) 2018, 8 (12). 9. Lv, R.; Xiao, L.; Wang, Y.; Yang, F.; Tian, J.; Lin, J. *, Searching for the Optimized Luminescent Lanthanide Phosphor Using Heuristic Algorithms. Inorg. Chem. 2019, 58 (9), 6458-6466.

\section{Image/Figure:}

https://www.xcdsystem.com/wmis/abstract/File6959/GA51_ImageFigure_0410060301.jpg

\section{Complete Status: Complete}

First Name: Ruichan

Last Name: Lv

Email: rclv@xidian.edu.cn

Organization: Xidian University

Country: China 


\title{
ID: GA53 \\ Model corrected blood input function to compute rate of cerebral FDG uptake from dynamic total-body PET images of rats in vivo: comparison with arterial blood sampling
}

\author{
Bijoy Kundu, University of Virginia, bkk5a@virginia.edu
}

\section{Category: Computational \& Data Science}

\begin{abstract}
Body : Objectives: Recently, a 3-compartment dual output model with spill over (SP) and partial volume (PV) corrections was developed to simultaneously estimate the kinetic parameters and model corrected blood input function (MCIF) from dynamic 2-[18F] fluoro-2deoxy-D-glucose positron emission tomography (FDG PET) images of rodent heart in vivo $[1,2]$. In the current study this model was further optimized and the estimated MCIF was utilized to compute cerebral FDG uptake rate, Ki, from dynamic total-body FDG PET images of control Wistar-Kyoto (WKY) rats for the first time. The computed area under the MCIF (direct) and Ki (indirect) were compared to those derived from arterial blood sampling from the same rats in vivo. Materials and methods: Dynamic FDG PET scan of $n=6$ WKY rats, fasted for 6 hours, was performed using the Albira Si Trimodal Total Body PET/SPECT/CT imager [3] for 60 minutes. A 3-bed computed tomography (CT) scan was performed for attenuation correction right after the dynamic PET scan. Twelve arterial blood samples were collected following methods as described [1] for the entire dynamic PET imaging duration, more frequently at the early time points, and with lower frequency at the late time points. Briefly, for arterial blood sampling, an incision was made under anesthesia in the throat or groin area and the carotid or femoral artery catheterized using PE-50 tubing. Blood draws were followed by flushing the catheter with a small volume of heparinized saline. Blood loss volume was replaced with an equal volume of sterile saline at the end of the first 10 minute period. Each whole blood sample was weighed and FDG activity measured using a well counter. The 60-minute dynamic list mode PET data, corrected for attenuation, scatter and randoms, was reconstructed using maximum likelihood expectation maximization (MLEM) algorithm with 6 iterations and 0.75 $\mathrm{mm}$ isotropic voxel resolution into 23 time bins: (frames, time (seconds): 11,8; 1,12; 2,60;1,180; 8,400). A 15-parameter dual output model [1] with SP and PV corrections optimized the following objective functions: O1(p) $=\sum \mathrm{i}$ [(Model IDIF, $\mathrm{i}-$ PET IDIF,i)2 + (Model myo,i - PET myo,i)2], O2(p) = [(ModelPeak IDIF - PETPeak IDIF)2 + (ModelPeakmyo - PETPeak myo $) 2]$, where ModelIDIF, Modelmyo are model output equations and PETIDIF, PETmyo are image derived blood and myocardium time activity curves, respectively in objective function $\mathrm{O} 1(\mathrm{p})$ and $\mathrm{p}$ is the 15-parameter vector as described [1]. The second objective function, $\mathrm{O} 2(\mathrm{p})$, introduced for the first time, minimizes the square of the difference between the model and image-derived blood and myocardium peak values. Optimization of the objective functions resulted in estimation of MCIF, which was then used to compute cerebral $\mathrm{Ki}=\mathrm{K} 1 . \mathrm{k} 3 /(\mathrm{k} 2+\mathrm{k} 3)$ in a 4parameter compartment model [4]. To obtain the ground truth, the arterial blood sample data was first fitted to a 7-parameter function as described [1]. The peak of this blood model was forced through the maximum tissue activity value to compensate for artificially low blood sample values as a result of limitations in blood sample timing. This was then used to compute
\end{abstract}


experimental cerebral $\mathrm{Ki}$ as described above. The computed area under the curve (AUC under $\mathrm{MCIF}$, direct) and $\mathrm{Ki}$ (indirect) were compared to that derived from arterial blood samples. Results: Experimental and computed AUC were 1910.44 $\pm 392.97 \mathrm{kBq} \mathrm{min} / \mathrm{cc}$ and $1732.21 \pm 345.06 \mathrm{kBq} \mathrm{min} / \mathrm{cc}$, respectively $(\mathrm{p}=0.42)$. Experimental and computed cerebral FDG uptake rates were $0.036 \pm 0.003 \mathrm{~min}-1$ and $0.044 \pm 0.019 \mathrm{~min}-1$, respectively $(\mathrm{p}=0.31)$. Conclusions: Direct and indirect comparisons of model computations with arterial blood sampling were performed for the first time in control WKY rats. MCIF computed from the left ventricular blood pool compared well with arterial blood samples. Computed cerebral FDG uptakes rates agreed well with that obtained from arterial blood samples. Acknowledgements: This study was supported in part by a grant funding from the National Institutes of Health R01 HL123627.

References: [1] Zhong M, Kundu BK. Optimization of a Model Corrected Blood Input Function from Dynamic FDG-PET Images of Small Animal Heart in vivo. IEEE Trans Nucl Sci. 2013 October; 60(5):3417-3422. [2] Li J, Kemp BA, Howell NL, Massey J, Minczuk K, Huang Q, Chordia MD, Roy RJ, Patrie JT, Davogustto GE, Kramer CM, Epstein FH, Carey RM, Taegtmeyer H, Keller SR, Kundu BK. Metabolic Changes in Spontaneously Hypertensive Rat Hearts Precede Cardiac Dysfunction and Left Ventricular Hypertrophy. J Am Heart Assoc 2019;8:e010926. [3] González AJ, Aguilar A, Conde P, Hernández L, Moliner L, Vidal LF, Sánchez F, Sánchez S, Correcher C, Molinos C, Barberá J, Lankes K, Junge S, Bruckbauer T, Bruyndonckx P, Benlloch JM. A PET Design Based on SiPM and Monolithic LYSO Crystals: Performance Evaluation. IEEE Trans Nucl Sci. 2016;63:2471-2477. [4] Huang Q, Massey JC, Minczuk K, Li J, Kundu BK. Non-invasive determination of blood input function to compute rate of myocardial glucose uptake from dynamic FDG PET images of rat heart in vivo: comparative study between the inferior vena cava and the left ventricular blood pool with spill over and partial volume corrections. Phys Med Biol 2019;64:165010.

\section{Image/Figure:}

https://www.xcdsystem.com/wmis/abstract/File6959/GA53 ImageFigure 0411123723.jpg

Complete Status: Complete

First Name: Bijoy

Last Name: Kundu

Email: bkk5a@virginia.edu

Organization: University of Virginia

Country: United States 


\title{
ID: GA54 \\ Development and Preclinical Validation of a Naturalized Hepatitis B Core Particles Targeting Integrin avß3 for Near-infrared Fluorescent-guided Surgery in Breast Cancer
}

\author{
Rui-Qin Yang, School of Medicine, Xiamen University, yrqxm0822@163.com
}

Category: Oncology

\begin{abstract}
Body : Background Tumor-free surgical margins are critical in breast-conserving surgery (BCS), as high positive margins rates associate with high local recurrence rates. Nearinfrared (NIR) fluorescence imaging is a promising strategy for guiding the surgery, due to which can reliably communicate real-time feedback to the surgeon, thus potentially reduces the positive margins rates. Fluorescent probe is crucial for NIR fluorescence imaging technology. At present, the fluorescent dyes are delivered to the tumor mainly by specific antibodies and peptides. However, these methods are limited to dyes-delivery efficiency. With large surface to volume ratio, nanoparticles can achieve more surface modification, which can apparently improve the drug-delivery efficiency[1]. In addition, the protein-based nanoparticles, composed of natural self-assembling protein subunits, possess the advantages of biocompatibility, biodegradation, and low toxicity as compared to synthetic polymers. Therefore, we use a naturalized Hepatitis B Core nanoparticles remoldedwith RGD peptide, as the delivery carrier to develop a novel fluorescent nano-probe, aiming to achieve more effective intraoperative imaging. Methods The RGD-HBc fusion protein was expressed in Escherichia coli. Then the NIR fluorescent dye ICG was loaded into RGD-HBc virus-like particles (VLPs) through disassembly/reassembly method to obtain the RGD-HBc/ICG nano-probe. After physical and chemical properties were characterized, the applicability of the nano-probewas tested for cell and tissue binding characteristics using cell-based plate assays, subcutaneoustumor mice, and MMTV-PyVT mammary tumor transgenic mice. Subsequently, an invasive intramuscular model was utilized to evaluate the efficacy of the RGD-HBc/ICG-guided surgery. Finally, biosafety of nano-probe in vivo was evaluated after intravenous injection of the probe in mice. Results ICG was successfully loaded into RGD-HBc VLP and retained specific binding affinity to integrin $\alpha v \beta 3$ receptor in cell-based plate assays. In subcutaneous tumor models, a significantly higher tumor-to-background ratio (TBR) was seen in RGD-HBC/ICG group compared to $\mathrm{HBc} / \mathrm{ICG}$ and free ICG group $(6.0 \pm 0.6$ vs.3.1 $\pm 0.8 \mathrm{vs} .2 .2 \pm 0.2 ; \mathrm{P}(\mathrm{P}$. In MMTV-PyVT mammary tumor transgenic mice, receiver operating characteristic (ROC) curve showed the ability of NIR fluorescence imaging to differentiate between benign and malignant breast tissues with an AUC of 0.81 . No significant change was observed in hematology chemistry blood panels and vital organs pathology after intravenous injection of nano-probe. Conclusions In this study, the RGD-HBc/ICG shows specific targeting to integrin av $\beta 3$ receptor, and holds promise as an effective tool to delineate tumor boundary for fluorescence-guided BCS. These results may be translatable into clinical practice to improve surgical and patients' outcomes.
\end{abstract}


References: [1]Harmsen S, Teraphongphom N, Tweedle MF, Basilion JP, Rosenthal EL. Optical Surgical Navigation for Precision in Tumor Resections. Mol Imaging Biol. 2017;19(3):357-362.

\section{Image/Figure:}

https://www.xcdsystem.com/wmis/abstract/File6959/GA54_ImageFigure 0416074041.jpg

Complete Status: Complete

First Name: Rui-Qin

Last Name: Yang

Email: yrqxm0822@163.com

Organization: School of Medicine, Xiamen University

Country: China 


\title{
ID: GA55 \\ A new method of quantitative analysis of fluorescence image uniformity for intraoperatively evaluating graft blood supply at biliary anastomotic site on liver transplantation
}

\author{
Kunshan He, Beihang University, hekunshan@fingerpass.net.cn
}

\section{Category: Systemic Diseases (Kidney, Liver and Pancreas)}

\begin{abstract}
Body : Introduction: Biliary complications (BC) are one of the most frequent types of surgical complications in liver transplantation patients affecting from $10 \%$ to $45 \%$. The incidence of $\mathrm{BC}$ is associated with reduced graft survival and a mortality rate of $10 \%$ after liver transplantation. BC also affect the quality of life of patients because of the need for multiple hospitalizations and repeated interventions. Later complications, especially those that occur more than 12 months after liver transplantation, are more difficult to handle. Among them, the related complications of biliary anastomosis are one of the main causes of $\mathrm{BC}$. The main points for the prevention of complications related to biliary anastomosis are the protection of the blood supply of the bile duct at the anastomotic site and the delicate anastomosis technique. How to detect the blood supply of the biliary anastomosis in real time to ensure the smooth blood supply of the biliary anastomosis is the key problem we need to solve urgently. So we developed a new method of quantitative analysis of fluorescence image uniformity for evaluating blood supply at biliary anastomotic site after liver transplantation. Methods: In this study, we used a new fluorescent imaging system (HUIYAN, DPM, China). A total of three patients were recruited in the preliminary trial phase. Intraoperatively, after the biliary anastomosis is completed, patients were intravenously injected $0.5 \mathrm{mg} / \mathrm{kg}$ ICG. Video-rate color, fluorescence and merged images were displayed for evaluation of blood supply at biliary anastomotic site and recorded for data analysis. First, select the region of interest (ROI) at the biliary anastomosis. Then randomly select 9 sub-regions in the ROI, and respectively calculate their average fluorescence brightness value I. The maximum and minimum values are denoted by Imax and Imin, respectively. The uniformity of fluorescence signal intensity of ROI is defined as: $U=[1-($ Imax- Imin) $/($ Imax + Imin) $]^{*} 100 \%$ Results : The representative images collected during surgery were shown in Fig. 1. A few seconds after ICG injection, fluorescence signals began to appear in the area of biliary anastomosis. After about 2 minutes, fluorescence signals reached a plateau and it was appropriate to calculate U. Of the three patients, all objectives' uniformity of fluorescence signal intensity of ROI exceeded $72 \%$. After operation, none of them had obvious biliary complications. Conclusions: Using the method of quantitative analysis of fluorescence image uniformity for evaluating blood supply at biliary anastomotic site after liver transplantation is feasible. We will further expand the amount of data and explore the relationship between the uniformity of fluorescence signal intensity of ROI and biliary complications. FIG.1 The representative images collected during surgery. (A) Color image after biliary anastomosis, the rectangular area represents the area of interest. (B) Fluorescence and color fusion image after biliary anastomosis, the circular area represents a randomly selected sub-area.
\end{abstract}

Full Name of Abstract's 1st Author : Kunshan He 
Complete Status: Complete

First Name: Kunshan

Last Name: $\mathrm{He}$

Email: hekunshan@fingerpass.net.cn

Organization: Beihang University

Country: China 


\title{
ID: GA56 \\ PET imaging of human lung cancer xenograft model with $64 \mathrm{CuCl} 2$
}

\author{
Lei Jiang, Shanghai Pulmonary Hospital, leijiang1031@163.com
}

\section{Category: Oncology}

\begin{abstract}
Body : Purpose: Human copper transporter 1 (CTR1) has been proven to be overexpressed in many types of cancer cells, and copper (II)-64 chloride $(64 \mathrm{CuCl} 2)$ has been used as an effective tracer for positron emission tomography (PET) imaging in tumor-bearing animal models. Thus, this study aimed to investigate the potential application of $64 \mathrm{CuCl} 2$ in PET imaging of lung cancer through targeting CTR1. Methods: The expression of CTR1 in a series of lung cancer cell lines was identified by quantitative real-time polymerase chain reaction (QPCR), western blot, enzyme-linked immunosorbnent assay (ELISA), and immunofluorescent staining. Then in vitro cell uptake assay of $64 \mathrm{CuCl} 2$ was investigated in human lung cancer cell lines with different levels of CTR1 expression. Small animal PET imaging and quantitative analysis were performed in human lung cancer tumor-bearing mice after intravenous injection of $64 \mathrm{CuCl} 2$, respectively. Results: The CTR1 expression in multiple human lung cancer cells was identified and confirmed, and H1299 cell lines with high CTR1 expression, H460 with moderate CTR1, and H1703 with low CTR1 were selected for further experiments. In vitro cellular uptake assay displayed that the $64 \mathrm{CuCl} 2$ uptake by these three kinds of cells was positive correlated with their CTR1 expressed levels. And the blocking experiments testified the specificity of $64 \mathrm{CuCl} 2$ to target CTR1. Moreover, small animal PET imaging and quantitative results showed that $64 \mathrm{CuCl} 2$ accumulation in $\mathrm{H} 1299, \mathrm{H} 460$, and H1703 tumor-bearing mice was consistent with CTR1 levels and cell uptake experiments. Conclusions: The expression of CTR1 in human lung cancer xenograft model could be successfully visualized by $64 \mathrm{CuCl} 2 \mathrm{PET}$ examination. With the expected growth of PET/CT examination to be an essential strategy in clinical lung cancer management, $64 \mathrm{CuCl} 2$ has the great potential to be a promising PET imaging agent of lung cancer.
\end{abstract}

\section{Image/Figure:}

https://www.xcdsystem.com/wmis/abstract/File6959/GA56_ImageFigure 0412091443.jpg

Complete Status: Complete

First Name: Lei

Last Name: Jiang

Email: leijiang1031@163.com

Organization: Shanghai Pulmonary Hospital 
Country: China 


\title{
ID: GA58 \\ Developing a novel cell-based cancer therapy expressing potent TRAIL fusion proteins
}

\author{
Danish Mahmood, Robarts Research Institute, mahmood.danish@outlook.com
}

\section{Category: New Chemistry, Biology \& Bioengineering}

\begin{abstract}
Body : Introduction: Cell-based therapies represent a relatively new approach to treating cancer. Healthy cells can be genetically engineered ex vivo to produce powerful anticancer proteins prior to reinfusion into a patient. A major goal of this strategy is to specifically kill cancer cells. The tumour necrosis factor apoptosis-inducing ligand (TRAIL) is proapoptotic protein that has been shown to specifically kill cancer cells. However, systemically administered soluble TRAIL failed to show significant clinical benefit because the TRAIL complexes degraded into inactive subunits (de Miguel, et al., 2016; von Karstedt, et al., 2017). Previous studies show that cell-based therapies expressing soluble TRAIL require assistance of common chemotherapeutic drugs (e.g. doxycycline, mitoxantrone, or 5-fluorouracil), increasing risk for off-target effects (Loebinger, et al., 2009; Yu, et al., 2013). Recently, more stable and highly potent TRAIL fusion proteins have been developed (Hutt, et al., 2017; Naval, et al., 2019). Here we explored the ability to genetically engineer cells, including human mesenchymal stem cells (hMSCs), with these novel TRAIL variants as first steps in engineering a more potent cell-based cancer therapy. hMSCs are an attractive cell type for this trojan-horse strategy, as they have been shown to actively migrate into inflammatory lesions (D'souza, et al., 2012). Methods: We constructed lentiviral transfer plasmids encoding the fluorescence reporter tdTomato along with membrane-bound TRAIL (memTRAIL) or one of two covalently linked TRAIL fusion proteins (scTRAIL or fc-scTRAIL) (Fig 1.A). Lentiviruses were produced and we genetically engineered human mesenchymal stem cells (hMSCs) and embryonic kidney cells (HEK293T) to stably co-express tdTomato and either memTRAIL, scTRAIL, or fc-scTRAIL. MDA-MB-231 triple negative breast cancer, PC3MLN4 prostate cancer, and OVCAR-8 ovarian cancer cell lines were genetically engineered to stably express firefly luciferase for bioluminescence imaging (BLI). We co-cultured TRAIL-expressing or naïve HEK293T or hMSC cells with each luciferase-expressing cancer cell line and performed BLI at $24 \mathrm{~h}$ and $48 \mathrm{~h}$ to compare the cancer killing activity of the TRAIL variants. Results: Flow cytometry of the tdTomato reporter revealed expression levels within stably transduced HEK293T cell lines (Fig 1.B-E). BLI showed that HEK293T cells expressing memTRAIL induced a concentration dependent decrease of MDA-MB-231 (Fig 2.C-D), PC3MLN4 (Fig 2.G-H), and OVCAR-8 (Fig 2.K-L) cancer cell viability. HEK293T cells transiently expressing scTRAIL and fc-scTRAIL inhibited MDA-MB231 (Fig 2.E-F), PC3MLN4 (Fig 2.I-J), and OVCAR-8 (Fig 2.M-N) cancer cell viability. OVCAR-8 resisted TRAIL therapy the most, while PC3MLN4 were the most sensitive to it. The HEK293T cells stably expressing fc-scTRAIL inhibited prostate cancer cell viability more than HEK293T cells expressing memTRAIL before normalization ( $p$ Conclusion: Our work is the first to genetically engineer therapeutic cells to express recently described highly potent TRAIL fusion proteins. We used BLI to monitor the cancer killing activity of these therapeutic cells when co-cultured with breast, prostate, and ovarian cancer cells. Our initial data shows that
\end{abstract}


HEK293T cells producing fc-scTRAIL were superior at killing prostate cancer cells compared to cells expressing other TRAIL variants. Future work will explore hMSCs expressing fc-scTRAIL, as well as moving our work into preclinical models of cancer metastasis. Continued work may yield a new and more potent cell-based therapy for the treatment of metastatic disease.

References: de Miguel, D., et al. (2016, 5 4). Onto better TRAILs for cancer treatment. Cell Death \& Differentiation, 23(5), 733-747. D'souza, N., et al. (2012). MSC and Tumors: Homing, Differentiation, and Secretion Influence Therapeutic Potential. In B. Weyand, M. Dominici, R. Hass, R. Jacobs, \& C. Kasper, Mesenchymal Stem Cells: Basics and Clinical Application II (pp. 209-266). Berlin: Springer. Hutt, M., et al. (2017, 12 1). Superior Properties of Fc-comprising scTRAIL Fusion Proteins. Molecular Cancer Therapeutics, 16(12), 2792-2802. Loebinger, M., et al. Cancer Research, 69(10), 4134-4142. Naval, J., et al. (2019, 3 29). Importance of TRAIL Molecular Anatomy in Receptor Oligomerization and Signaling. Implications for Cancer Therapy. Cancers, 11(4). von Karstedt, S., et al. (2017, 6 24). Exploring the TRAILs less travelled: TRAIL in cancer biology and therapy. Nature Reviews Cancer, 17(6), 352-366. Yu, R., et al. (2013, 221). Delivery of sTRAIL variants by MSCs in combination with cytotoxic drug treatment leads to p53-independent enhanced antitumor effects. Cell Death and Disease, 4(2), e503-e503.

Complete Status: Complete

First Name: Danish

Last Name: Mahmood

Email:mahmood.danish@outlook.com

Organization: Robarts Research Institute

Country: Canada 


\title{
ID: GA62 \\ Epitope alteration by small molecules and its application for drug screening
}

\author{
Biyue Zhu, Massachusetts General Hospital, 413897689@qq.com
}

\section{Category: New Chemistry, Biology \& Bioengineering}

\begin{abstract}
Body : Antibody-epitope interactions are high-affinity bindings and daily utilized in various biological studies. Conventionally, small molecules are thought to lack the ability to alter this strong binding. In the present study, however, we discovered several small molecules that could alter the surface properties of amyloid beta $(\mathrm{A} \beta)$ epitopes. Consequently, the alteration could lead to inhibition or enhancement of the antibody-epitope recognition. Moreover, the alteration of $A \beta$ epitopes showed changes of $A \beta$ functions, including inhibited $A \beta$ aggregation, downregulated $A \beta$ oligomers-induced proinflammatory cytokine secretion from microglial cells and decreased in vivo microglial activation. In addition, several reported ligands of tau and PDL1 proteins could also alter the antibody recognitions to corresponding epitopes. Based on the above discovery, we proposed a screen platform based on epitope alteration for drug discovery (SPEED). With a small library of compounds for a proof-of-concept study, SPEED was successfully applied to seek new ligand leads for $\mathrm{A} \beta$ species. Lastly, this strategy was further validated by automated dot blotting, Meso Scale Discovery (MSD) assay and single molecule array (Simoa), a newly established digital ELISA. Our findings disclosed the largely overlooked phenomenon of epitope alteration with small molecules and we demonstrated its application to complement current label-free drug screening strategies.
\end{abstract}

References: 1. Zhou, M., Li, Q. \& Wang, R. Current Experimental Methods for Characterizing Protein-Protein Interactions. ChemMedChem 11, 738-56 (2016). 2. Wilson, A.J. Inhibition of protein-protein interactions using designed molecules. Chem Soc Rev 38, 3289-300 (2009). 3. Liang, Y., Guttman, M., Davenport, T.M., Hu, S.L. \& Lee, K.K. Probing the Impact of Local Structural Dynamics of Conformational Epitopes on Antibody Recognition. Biochemistry 55, 2197-213 (2016). 4. Rubinstein, N.D. et al. Computational characterization of B-cell epitopes. Mol Immunol 45, 3477-89 (2008). 5. Ward, E.S., Gussow, D., Griffiths, A.D., Jones, P.T. \& Winter, G. Binding activities of a repertoire of single immunoglobulin variable domains secreted from Escherichia coli. Nature 341, 544-6 (1989). 6. Quevedo, C.E. et al. Small molecule inhibitors of RAS-effector protein interactions derived using an intracellular antibody fragment. Nat Commun 9, 3169 (2018). 7. Peng, H.P., Lee, K.H., Jian, J.W. \& Yang, A.S. Origins of specificity and affinity in antibody-protein interactions. Proc Natl Acad Sci U S A 111, E265665 (2014). 8. Zhou, H.X. \& Pang, X. Electrostatic Interactions in Protein Structure, Folding, Binding, and Condensation. Chem Rev 118, 1691-1741 (2018). 9. Bakou, M. et al. Key aromatic/hydrophobic amino acids controlling a cross-amyloid peptide interaction versus amyloid self-assembly. J Biol Chem 292, 14587-14602 (2017). 10. Panza, F., Lozupone, M., Logroscino, G. \& Imbimbo, B.P. A critical appraisal of amyloid-beta-targeting therapies for Alzheimer disease. Nat Rev Neurol 15, 73-88 (2019). 11. Landau, M. et al. Towards a pharmacophore for amyloid. PLoS Biol 9, e1001080 (2011). 12. Heppner, F.L., Ransohoff, R.M. $\&$ Becher, B. Immune attack: the role of inflammation in Alzheimer disease. Nat Rev Neurosci 
16, 358-72 (2015). 13. Dodel, R. et al. Naturally occurring autoantibodies against beta-amyloid: investigating their role in transgenic animal and in vitro models of Alzheimer's disease. $\mathrm{J}$ Neurosci 31, 5847-54 (2011). 14. Ramakrishnan, M., Kandimalla, K.K., Wengenack, T.M., Howell, K.G. \& Poduslo, J.F. Surface plasmon resonance binding kinetics of Alzheimer's disease amyloid beta peptide-capturing and plaque-binding monoclonal antibodies. Biochemistry 48 , 10405-15 (2009).

\section{Image/Figure:}

https://www.xcdsystem.com/wmis/abstract/File6959/GA62 ImageFigure 0414112233.jpg

Full Name of Abstract's 1st Author : Biyue Zhu

Complete Status: Complete

First Name: Biyue

Last Name: Zhu

Email: 413897689@qq.com

Organization: Massachusetts General Hospital

Country: United States 


\title{
ID: GA63 \\ Visualizing tumour self-homing with magnetic particle imaging
}

\author{
Katie Parkins, Johns Hopkins School of Medicine, kparkin8@uwo.ca
}

\section{Category: Oncology}

\begin{abstract}
Body : Tumour self-homing describes a phenomenon where circulating tumour cells (CTCs) preferentially colonize established tumours rather than non-malignant tissues1. In recent years, self-homing CTCs have been repurposed as delivery vehicles for anti-cancer therapeutics. This strategy has shown exciting progress towards treating primary tumours, single organ metastases and most recently, multi-organ metastases2-4. However, further refinement is needed in order to optimize self-homing CTCs for potential clinical translation. Imaging tools that enable the fate of systemically-administered CTCs to be noninvasively monitored over time would provide valuable information about any unwanted off-tumour accumulation as well as the kinetics and efficiency of CTC infiltration, proliferation and survival within tumours to provide surrogate measures of therapeutic response in individual subjects. Magnetic particle imaging (MPI) is a highly sensitive modality that directly images iron oxide nanoparticles and clinical MPI scanners are being rapidly developed. Here we show for the first time that MPI can be used to track the fate of iron-loaded experimental CTCs in mice bearing pre-established breast cancer lesions. Methods: Primary mammary fat pad tumours were generated by injecting 1 x 106 MDAMB-231 breast cancer cells into the MFP of female NOD scid gamma (NSG) mice. After 41 days of tumour growth, mice received an intracardiac injection of 5 x 105 iron-labeled MDAMB-231BR-eGFP cells into the left ventricle. Full body MPI images were acquired 72 hours later using a 3D high sensitivity scan mode. Total MPI signal was plotted against iron content to derive calibration lines and quantify iron content in tumours and contralateral non-tumour bearing fat pads. Complementary MRI scans were performed on a 3T clinical scanner using a balanced Steady State Free Precession imaging sequence that was previously optimized for iron detection5. Results: MPI signal was visualized in the tumour bearing fat pad (Figure 1A). MPI signal was also detected in lungs, likely due to iron labeled cells that were trapped following intracardiac injection, as well as, in the abdomen, presumably due to iron content in the mouse feed. Iron content in tumour bearing fat pads $(\mathrm{M}=0.798 \pm 0.184 \mu \mathrm{g})$ was significantly higher than in contralateral non-tumour bearing fat pads $(\mathrm{M}=0.318 \pm 0.044 \mu \mathrm{g}$; $\mathrm{p}$ Conclusions: In this work, we employed high sensitivity MPI to noninvasively visualize the self-homing of systemicallyadministered CTCs into preestablished breast lesions. Iron-labeled CTCs migrated into and were readily visible and quantified at the established tumour site 72 hours following administration. Future work is focused on developing MPI as a valuable tool for visualizing tumour self-homing in mice bearing multi-organ metastases as well as co-engineering CTCs with therapeutic genes for tumour treatment.
\end{abstract}

References: 1. Norton \& Massague, 2006. Nature Medicine. 12:875-878. 2. Power, et al., 2007. Molecular Therapy. 15, 123-130. 3. Reinshagen, et al., 2018. Science Translational Medicine. 10(449). 4. Parkins, et al., bioRxiv 746685; doi: https://doi.org/10.1101/746685. 5. Ribot, et al., 2011. Magnetic Resonance Imaging 34, 231-238. 


\section{Image/Figure:}

https://www.xcdsystem.com/wmis/abstract/File6959/GA63_ImageFigure_0414113041.png

Full Name of Abstract's 1st Author : Katie Parkins

Complete Status: Complete

First Name: Katie

Last Name: Parkins

Email: kparkin8@uwo.ca

Organization: Johns Hopkins School of Medicine

Country: United States 


\title{
ID: GA64 \\ Image-Guided Drug Delivery and Cancer Therapy
}

\author{
Hak Soo Choi, Massachusetts General Hospital, Harvard Medical School, \\ HCHOI12@mgh.harvard.edu
}

Category: New Chemistry, Biology \& Bioengineering

\begin{abstract}
Body : Two fundamental and unsolved problems facing image-guided drug delivery and image-guided cancer therapy are nonspecific uptake of intravenously administered diagnostic and/or therapeutic agents by normal tissues and organs, and incomplete elimination of unbound targeted agents from the body. To solve these problems, we have synthesized a series of indocyanine near-infrared (NIR) fluorophores that varied systematically in net charge, conformational shape, hydrophilicity-lipophilicity, and charge distribution. Using 3D molecular modeling and machine learning, we have defined the relationship among the key independent variables that dictate biodistribution and tissue-specific targeting such as lung and sentinel lymph nodes, human prostate cancers, and human melanomas (1-4). Recently, we have developed a new pharmacophore design strategy "structure-inherent targeting," where tissue-specific targeting is engineered directly into the non-resonant structure of an NIR fluorophore, thus creating the most compact possible optical contrast agent for bioimaging and nanomedicine $(5,6)$. The biodistribution and targeting of these compounds vary with dependence on their unique physicochemical descriptors and cellular receptors, which permit 1) selective binding to the target tissue/organ, 2) visualization of the target specifically and selectively, and 3) provide curing options such as image-guided surgery or targeted therapy (7-10). Our study solves two fundamental problems associated with image-guided drug delivery and image-guided cancer surgery and lays the foundation for additional targeted agents with optimal optical and in vivo performance.
\end{abstract}

References: 1. Choi HS, Liu W, Misra P, Tanaka E, Zimmer JP, Itty Ipe B, Bawendi MG, Frangioni JV. Renal clearance of quantum dots. Nat Biotechnol. 2007; 25: 1165-70. 2. Choi HS, Liu W, Liu F, Nasr K, Misra P, Bawendi MG, Frangioni JV. Design considerations for tumourtargeted nanoparticles. Nat Nanotechnol. 2010; 5(1): 42-7. 3. Choi HS, Gibbs SL, Lee JH, Kim SH, Ashitate Y, Liu F, Hyun H, Park G, Xie Y, Bae S, Henary M, Frangioni JV, Targeted zwitterionic near-infrared fluorophores for improved optical imaging. Nat Biotechnol. 2013; 31: 148-53. 4. Choi HS. Nanoparticle assembly: building blocks for tumour delivery. Nat Nanotechnol. 2014; 9(2): 93-4. 5. Hyun H, Park MH, Owens EA, Wada H, Henary M, Frangioni JV, Choi HS. Structure-inherent targeting of near-infrared fluorophores for parathyroid and thyroid gland imaging. Nat Med. 2015; 21: 192-7. 6. Owens E, Henary M, El Fakhri G, Choi HS. Tissue-specific near-infrared fluorescence imaging. Acc Chem Res. 2016;49(9):1731-40. 7. Kang H, Gravier J, Bao K, Wada H, Lee JH, Baek Y, El Fakhri G, Gioux S, Rubin BP, Coll JL, Choi HS. Renal clearable organic nanocarriers for bioimaging and drug delivery. Adv Mater. 2016; 28(37):8162-8. 8. Kang H, Stiles WR, Baek Y, Nomura S, Bao K, Shuang H, Park GK, Jo MJ, I H, Coll JL, Rubin BP, Choi HS. Renal clearable theranostic nanoplatforms for gastrointestinal stromal tumors. Adv Mater. 2020;32(6):1905899. 9. Choi HS, Kim HK. 
Multispectral image-guided surgery in patients. Nat Biomed Eng. 2020;4:245-6. 10. Park GK, Lee JH, Soriano E, Choi M, Bao K, Kim DY, Paik JH, Yun SH, Frangioni JV, Clancy TE, Kashiwagi S, Henary M, Choi HS. Rapid and selective targeting of heterogeneous pancreatic neuroendocrine tumors for early diagnosis. iScience 2020;23(4):101006.

Complete Status: Complete

First Name: Hak Soo

Last Name: Choi

Email: HCHOI12@mgh.harvard.edu

Organization: Massachusetts General Hospital, Harvard Medical School

Country: United States 


\title{
ID: GA65 \\ PET/ CT guided radioactive seed implantation treatment for malignant tumor
}

\author{
Wei Chang, The First Affiliated Hospital of Zhengzhou, changweichang@163.com
}

\section{Category: Oncology}

\begin{abstract}
Body : 【Abstract】 Objective : To explore the value of PET-CT in 125I seeds permanent braehytherapy treatment for malignant tumor patients, provide the basis for the development of clinical programs. Methods : Collected from January 2011 to November 2017 in our hospital by cytology or pathology diagnosed malignant solid tumor and these cases both underwent CT and 18F-FDG PET / CT examination. Then the three-dimensional tumor imaging generated data was converted into DICOM format by the treatment planning system. Virtual implantation was also manipulated according to the three-dimensional position of the tumor. Biological target volume (BTV) and Gross tumor volume (GTV) and matched peripheral dose were compared in different imaging methods, PResults : patients the gross tumor volume , the mean number of seeds were $(22.74 \pm 15.57) \mathrm{cm} 3$ in PET-CT group and GTV $(27.33 \pm 16.31) \mathrm{cm} 3$ in CT group. BTV of PET-CT group reduced 22.16\% (P5). Conclusion : PET / CT imaging could process specify molecule target image on tumor, which might offer importance informations for treatment plan and treatment effects. It can be very good to distinguish tumor tissue and surrounding tissue inflammation or atelectasis, precise outline to guide radioactive seed implantation target range, and accurate clinical staging for next treatment planning provide a reliable basis. The disadvantages of traditional radioactive seeds implantation are less objectivity and poor individualization, which could be overcome by PET / CT guided radioactive seeds implantation and biological conformal radiotherapy can be implemented in the future.
\end{abstract}

References: 1.Zhonghua, Wai Ke, Za Zhi. The current status of diagnosis and treatment of pancreatic cystic neoplasm in China: a report of 2251 cases. 2018 Jan 1, 56(1) : 24-29. 2. Wu $\mathrm{VW}$, Leung WS, Wong KL, et a1.The impact of positron emission tomography on primary tumour delineation and dosimetric outcome in intensity modulated radiotherapy of early T-stage nasopharyngeal carcinoma[J]. Radiat Oncol, 2016, 11(1) : 109. 4. Matsuura T, Nishimura $\mathrm{Y}$, Nakamatsu K, et a1.Clinical outcomes of IMRT planned with or without PET/CT simulation for patienLs with pharyngeal eancers[J].Int J Clin Oncol，2017， 3. Spratt DE, Diaz R, Mc Elmurray J, et al. Impact of FDG PET/CT on delineation of the gross tumor volume for radiation planning in non-small-cell lung cancer. Clin Nucl Med, 2010, 35(4) : 237-243.

Complete Status: Complete

First Name: Wei 
Last Name: Chang

Email: changweichang@163.com

Organization: The First Affiliated Hospital of Zhengzhou

Country: China 


\title{
ID: GA66 \\ The immune checkpoint PD-L1 metabolic interactome in triple negative breast cancer cells
}

\author{
Jesus Pacheco Torres, Johns Hopkins University, jpachec6@jh.edu
}

\section{Category: Oncology}

\begin{abstract}
Body : Introduction Cancer immunotherapy is designed to activate the immune system against cancer cells. The field has been revitalized with the discovery of immune checkpoints such as programmed cell death protein-1 (PD-1) and its ligand (PD-L1, CD274)1. As the regulators of the immune system activation, these immune checkpoints are utilized by cancer cells to escape immune surveillance. PD-L1 is often overexpressed in cancers and it has been successfully exploited for immune therapy 2 in different tumor types, such as melanoma3,4. However, treatment outcomes have not been as effective in other cancers such as breast5 and pancreatic cancer6. The explanation for this is likely multifactorial, with metabolism playing an important role7-9. Aberrant choline metabolism, one of the most common tumor associated metabolic alteration, is characterized by increased phosphocholine (PC) and total cholinecontaining compounds. This alteration is mainly due to increased expression of choline kinase$\alpha($ Chk- $\alpha)$. Here, for the first time, we investigated the relationship between Chk- $\alpha$, PD-L1 and metabolism in triple negative MDA-MB-231 human breast cancer cells. Methods Experiments were performed using triple negative breast MDA-MB-231 and SUM-149 cells transiently transfected with small interfering RNA (siRNA) against either luciferase or scrambled siRNA (as controls), and Chk- $\alpha$ and PD-L1 given singly, or in combination, following standard protocols. Real-time PCR and immunoblotting were performed following established protocols 10. For high resolution $1 \mathrm{H}$ MRS, MDA-MB-231 cell extracts were obtained using a dual-phase extraction method10. Integrals of the metabolites of interest were determined and normalized to the TSP reference and the number of cells. Metabolites were estimated from at least three experimental samples. Statistical significance was evaluated using the Student t-test. Results and Discussion Silencing Chk- $\alpha$ resulted in a significant increase of PD-L1 expression and silencing PD-L1 resulted in a significant increase of Chk- $\alpha$ expression both at the mRNA and at the protein level (Persuasive data Figures 1 and 2 respectively). This inverse relationship was eliminated when both PD-L1 and Chk- $\alpha$ were silenced. Metabolic data obtained using high resolution 1H MRS of cell extracts showed a significant increase of PC in cells transfected with PD-L1 siRNA, in good agreement with the molecular changes (Figure 1a). Metabolic changes, however, extended well beyond the choline containing compounds, affecting amino acids, organic acids, and nucleotides. Chk- $\alpha$ downregulation also altered the metabolic profile, increasing the concentration of multiple metabolites as lactate, glutamate and glutathione. Furthermore, we found that PD-L1 downregulation profoundly altered the lipid profile of MDAMB-231 cells, increasing the total amount of lipids, the total amount of unsaturated lipids as well as different individual lipids (Figure 1b). Finally, to further assess whether these results were relevant in the clinic, we analysed expression data from The Cancer Genome Atlas, finding an inverse relationship between the mean expression of PD-L1 and Chk- $\alpha$ across different tumor types (Persuasive data Figure 3). These data suggest that treatments that decrease Chk- $\alpha$ may
\end{abstract}


also result in cancer cells escaping immune surveillance through increased expression of PDL1. Metabolic characterization showed that cells with downregulated Chk- $\alpha$ levels shifted towards a more immunosuppressive profile through metabolic reprogramming, increasing the production of metabolites linked to decreased effectiveness of T-cells11. Our data also suggest that low levels of PD-L1 could skew cancer cells towards a more immunosuppressive profile by significant changes in the lipid profile12. These results provide new insights in the role of PD-L1 in the cancer metabolome that may be exploited to improve the outcome of treatment with immune checkpoint inhibitors. Acknowledgement: This work was supported by NIH R35CA209960 and R01CA82337. JPT was founded by Fundación Alonso Martín Escudero and MSCA.

References: 1 Keir, M. E., Francisco, L. M. \& Sharpe, A. H. PD-1 and its ligands in T-cell immunity. Curr Opin Immunol 19, 309-314, doi:10.1016/j.coi.2007.04.012 (2007). 2 Mittendorf, E. A. et al. PD-L1 expression in triple-negative breast cancer. Cancer Immunol Res 2, 361-370, doi:10.1158/2326-6066.CIR-13-0127 (2014). 3 Tang, J. et al. Trial watch: The clinical trial landscape for PD1/PDL1 immune checkpoint inhibitors. Nat Rev Drug Discov 17, 854-855, doi:10.1038/nrd.2018.210 (2018). 4 Prat, A. et al. Immune-Related Gene Expression Profiling After PD-1 Blockade in Non-Small Cell Lung Carcinoma, Head and Neck Squamous Cell Carcinoma, and Melanoma. Cancer Res 77, 3540-3550, doi:10.1158/0008-5472.CAN-16-3556 (2017). 5 Tan, T. J., Chan, J. J., Kamis, S. \& Dent, R. A. What is the role of immunotherapy in breast cancer? Chin Clin Oncol 7, 13, doi:10.21037/cco.2018.04.01 (2018). 6 Aroldi, F. \& Zaniboni, A. Immunotherapy for pancreatic cancer: present and future. Immunotherapy 9, 607616, doi:10.2217/imt-2016-0142 (2017). $7 \mathrm{Li}$, X. et al. Navigating metabolic pathways to enhance antitumour immunity and immunotherapy. Nat Rev Clin Oncol, doi:10.1038/s41571019-0203-7 (2019). 8 Singer, K., Cheng, W. C., Kreutz, M., Ho, P. C. \& Siska, P. J. Immunometabolism in cancer at a glance. Dis Model Mech 11, doi:10.1242/dmm.034272 (2018). 9 Wang, X., Ping, F. F., Bakht, S., Ling, J. \& Hassan, W. Immunometabolism features of metabolic deregulation and cancer. J Cell Mol Med 23, 694-701, doi:10.1111/jcmm.13977 (2019). 10 Penet, M. F. et al. Metabolic imaging of pancreatic ductal adenocarcinoma detects altered choline metabolism. Clin Cancer Res 21, 386-395, doi:10.1158/1078-0432.CCR-14-0964 (2015). 11 Xia, X. et al. Glutaminolysis Mediated by MALT1 Protease Activity Facilitates PDL1 Expression on ABC-DLBCL Cells and Contributes to Their Immune Evasion. Frontiers in oncology 8, 632, doi:10.3389/fonc.2018.00632 (2018). 12 Herber, D. L. et al. Lipid accumulation and dendritic cell dysfunction in cancer. Nature medicine 16, 880-886, doi:10.1038/nm.2172 (2010).

\section{Image/Figure:}

https://www.xcdsystem.com/wmis/abstract/File6959/GA66 ImageFigure 0423054102.png

Image/Figure Caption: Figure 1. Metabolic heat map generated from quantitative analysis of high-resolution 1H MR spectral data of the (a) aqueous and (b) lipid phase, displaying differences in the metabolic profile of MDA-MB-231 cells. The heat map displays metabolites from untreated cells, cells transfected with $100 \mathrm{nM}$ luciferase siRNA for 48h, cells transfected with $100 \mathrm{nM} \mathrm{Chk-a}$ siRNA for 48h, cells transfected with $100 \mathrm{nM}$ PD-L1 siRNA for 48h, and cells transfected with a mixture of $50 \mathrm{nM}$ PD-L1 \#1 and $50 \mathrm{nM}$ Chk-a siRNA for 48h. Heat maps were created using MATLAB software (MATLAB R2012b, MathWorks) to visualize the 
metabolic patterns. Due to the high dynamic range of metabolites, we normalized the highest intensity of a metabolite in each of the four groups to $100 \%$. This normalization provides a dynamic range between $0-100 \%$, allowing a better presentation of heat maps. The heat map represents the average of 3-6 replicates per group. The integral area under the peak was normalized to the number of cells for each sample. TSP dissolved in D2O was used as a quantitative reference in the spectral analysis. ${ }^{*} \mathrm{p} \leq 0.05 * * \mathrm{p} \leq 0.01 * * * \mathrm{p} \leq 0.001$ compared to untreated + cells transfected with luciferase siRNA cells. GPC: glycerophosphocholine, PC: phosphocholine, Cho: choline, GSH: glutathione, GSSG: oxidized glutathione, MTA: S-methyl5 '-thioadenosine. Lipids (-CH3): methyl groups of fatty acids, Lipids (-CH2-): methylene groups of fatty acids (truncated), OOC-CH2: methylene groups at the a position of the carboxylic function, OOC-CH2-CH2: methylene groups at the b position of the carboxylic function, ARA: arachidonic acid, EPA: eicosapentaenoic acid, PtdEA: phosphatidylethanolamine, PtdCholine: phosphatidylcholine, $(\mathrm{CH}=\mathrm{CH}-\mathrm{CH} 2-\mathrm{CH}=\mathrm{CH})$ n: diallylic methylene protons, $\mathrm{CH}=\mathrm{CH}-\mathrm{CH} 2$ : methylene groups at the a position of a double bond, $\mathrm{CH}=\mathrm{CH}$ : fatty acid double bonds.

Full Name of Abstract's 1st Author : Jesus Pacheco-Torres

Complete Status: Complete

First Name: Jesus

Last Name: Pacheco Torres

Email: jpachec6@jh.edu

Organization: Johns Hopkins University

Country: United States 


\title{
ID: GA67 \\ Investigating innate immune activation in a mouse model of Parkinson's disease using highly specific PET tracers for TREM1 and TSPO.
}

\author{
Katherine Lucot, Stanford University, kllucot@stanford.edu
}

\section{Category: Neuroscience}

\begin{abstract}
Body : Objectives: Parkinson's disease (PD) is the most prevalent movement disorder, characterized by loss of dopaminergic neurons and accumulation of misfolded $\alpha$-synuclein protein. Maladaptive innate immune responses are another important hallmark of PD, including microglial activation and infiltration of peripheral myeloid cells into the central nervous system (CNS). While there are techniques to assess certain aspects of PD pathology, they are mostly postmortem and those for detecting innate immune activation in living subjects are suboptimal. For example, positron emission tomography (PET) imaging of translocator protein $18 \mathrm{kDa}$ (TSPO), a biomarker of microglial activation, suffers from poor specificity (i.e., it is also expressed in endothelial cells, astrocytes, and platelets).3,4 We recently identified TREM1 as a promising new, highly-specific biomarker of proinflammatory innate immune cells. Herein, we assess the utility of TREM1 compared to TSPO as an imaging biomarker for detecting innate immune responses in the CNS of a widely-used animal model of PD. Methods: C57/BL6J ( $\mathrm{n}=14)$ and TREM1-knockout mice $(n=3)$ were stereotaxically injected with 6-OHDA $(10 \mu \mathrm{g} / \mu \mathrm{L}$ in $1 \mu \mathrm{L}$ ) in their left striatum (coordinates: $\mathrm{A} / \mathrm{P}=0.5, \mathrm{~L}=1.8, \mathrm{D} / \mathrm{V}=-3.5$; Fig. 1.c), while control C57/BL6J mice $(n=6)$ were injected with saline at the same coordinates. At day 7 or 14 postsurgery, mice were administered intravenously with [64Cu]TREM1-mAb or [64Cu]Isotype control-mAb and imaged by PET/CT after 19 hours. Ex vivo autoradiography (ARG) was performed to obtain high resolution images of tracer binding within the striatum, and ratios of tracer signal in the ipsilateral versus contralateral striatum were quantified for both PET and ARG. An additional group of C57/BL6J mice stereotaxically injected with either 6-OHDA (n $=8)$ or saline $(\mathrm{n}=3)$ were administered with $[18 \mathrm{~F}] \mathrm{GE}-180$ (TSPO-tracer) at 7 and 14 days postsurgery, followed by ex vivo ARG. Statistically, all groups were compared to the saline group. Immunohistochemistry (IHC) was performed on a subgroup of ARG brain slices, to confirm myeloid cell activation (CD68 staining), and dopaminergic cell death (tyrosine hydroxylase, TH, staining). Results: Copper-64 radiolabeling of TREM1 mAb (chelator:mAb ratio =0.96) and isotype control (chelator:mAb ratio $=1.9$ ) resulted in $25 \%$ and $80 \%$ decay-corrected radiochemical yield, respectively (radiochemical purity $>99 \%$ ). PET/CT images revealed TREM1-positive myeloid cells in the injured brain hemisphere of 6-OHDA injected mice. PET image analysis verified significant tracer signal within the ipsilateral striatum of 6-OHDAinjected mice compared to the saline-injected mice at 7 days post-surgery ( $P$ Ex vivo ARG of mice injected with $[18 \mathrm{~F}] \mathrm{GE}-180$ showed significant tracer signal within the ipsilateral striatum of 6-OHDA- compared to saline-injected mice at 7 and 14 days (P Conclusion: TREM1-PET represents a highly specific approach for visualizing the presence of early innate immune activation, whereas [18F]GE180 shows promise for visualizing prolonged neuroinflammation in this PD rodent model. Further investigation of these imaging techniques is warranted in other PD rodent models and human postmortem tissue to assess their translational potential.
\end{abstract}


References: 1 Moore, D. J., West, A. B., Dawson, V. L. \& Dawson, T. M. Molecular pathophysiology of Parkinson's disease. Annu Rev Neurosci 28, 57-87,

doi:10.1146/annurev.neuro.28.061604.135718 (2005). 2 Holmqvist, S. et al. Direct evidence of Parkinson pathology spread from the gastrointestinal tract to the brain in rats. Acta Neuropathol 128, 805-820, doi:10.1007/s00401-014-1343-6 (2014). 3 James, M. L. et al. PET imaging of translocator protein $(18 \mathrm{kDa})$ in a mouse model of Alzheimer's disease using N-(2,5dimethoxybenzyl)-2-18F-fluoro-N-(2-phenoxyphenyl)acetamide. J Nucl Med 56, 311-316, doi:10.2967/jnumed.114.141648 (2015). 4 Chaney, A. et al. (11)C-DPA-713 Versus (18)F-GE180: A Preclinical Comparison of Translocator Protein $18 \mathrm{kDa}$ PET Tracers to Visualize Acute and Chronic Neuroinflammation in a Mouse Model of Ischemic Stroke. J Nucl Med 60, 122-128, doi:10.2967/jnumed.118.209155 (2019). 5 Wadsworth, H. et al. [(1)(8)F]GE-180: a novel fluorine-18 labelled PET tracer for imaging Translocator protein $18 \mathrm{kDa}$ (TSPO). Bioorg Med Chem Lett 22, 1308-1313, doi:10.1016/j.bmcl.2011.12.084 (2012).

Full Name of Abstract's 1st Author : Katherine L. Lucot

Complete Status: Complete

First Name: Katherine

Last Name: Lucot

Email: k1lucot@stanford.edu

Organization: Stanford University

Country: United States 


\title{
ID: GA68 \\ Development of a convolutional neural network to reduce contrast agent dose in collagen-targeted molecular MR imaging of liver fibrosis
}

\author{
Iris Zhou, Massachusetts General Hospital and Harvard Medical School, \\ iris.zhou@mgh.harvard.edu
}

\section{Category: Computational \& Data Science}

\begin{abstract}
Body : Introduction: Molecular imaging not only expands our ability to detect and manage diseases but also plays an increasingly important role in preclinical and clinical phases of drug development. Despite the great potential, the newly developed targeted contrast agents require extensive evaluations of their safety and efficacy while their synthesis needs to meet good manufacturing practice standards, both of which create a large cost barrier for first-inhuman evaluation. This cost can be substantially reduced by minimizing the dose, which is equally important to improve patient safety. Recent advances in machine learning aided PET showed promise in reducing tracer dose without sacrificing image contrast and quality [1,2]. Here we hypothesize that a convolutional neural network $(\mathrm{CNN})$ can predict the relaxation change induced by gadolinium-based type I collagen-targeted MRI agent EP-3533 at a full dose from that at a fractional dose in a rat model of nonalcoholic steatohepatitis (NASH). Methods: Male Wistar rats were fed a choline-deficient, L-amino acid-defined, high-fat diet (CDAHFD) $(\mathrm{N}=34)$ to induce NASH. CDAHFD rats underwent diet reversal or treated with elafibranor were included as a treatment group $(\mathrm{N}=35)$ and rats receiving normal chow were used as controls $(\mathrm{N}=16)$. Animals were anesthetized with isoflurane $(1-2 \%)$ and imaged with a $1.5 \mathrm{~T}$ Siemens clinical MRI scanner. To obtain longitudinal relaxation (R1) maps, respiratory-gated, $3 \mathrm{D}$ inversion recovery images were acquired prior to and 45 minutes after intravenous administration of EP-3533 $(10 \mu \mathrm{mol} / \mathrm{kg})$. The pre- and post-contrast R1 maps were co-registered with non-rigid image registration using the Medical Image Registration Toolbox (MIRT) in Matlab. After registration, the R1 maps at 50\% contrast dose were produced based on the R1 changes induced by a full dose. A total of 903 sets of pre-contrast, half-dose, and full-dose 2D images from the 85 animals were fed into the proposed CNN model with $85 \%$ of them for training/validation and $15 \%$ for testing. The CNN has a U-Net architecture [3] with a ResNet based encoder and decoder elements [4] to minimize image quality loss while maintaining the training efficiency. Results: The CNN was successfully trained for 100 epochs with a batch size of 32 and at a learning rate of 0.001 . The accuracy and loss plots for the training and validation data showed that the loss became less than 0.05 with the accuracy at above 0.8 after 100 epochs. Figure 1 shows the comparison between the CNN predicted full-dose post-contrast R1 maps with comparison to the ground-truth full-dose post-contrast data. Differences between the predicted images and the ground truth were only noticed in the stomach and major blood vessels. Discussion and Conclusion: Our preliminary results suggest that machine learning presents a promising solution to reduce contrast agent dose needed for molecular imaging, where it is challenging to synthesize targeted molecular probes in large quantities at a low cost. Further improvements including larger datasets, training with further reduced fractional doses and full-
\end{abstract}


dose, and more advanced models with rigorous optimization of training parameters will allow us to expand this approach to other targeted probes and even other molecular imaging modalities.

References: [1] Chen KT, Gong E, de Carvalho Macruz FB, Xu J, Boumis A, Khalighi M, et al. Ultra-Low-Dose (18)F-Florbetaben Amyloid PET Imaging Using Deep Learning with MultiContrast MRI Inputs. Radiology 2019;290:649-656. [2] Ouyang J, Chen KT, Gong E, Pauly J, Zaharchuk G. Ultra-low-dose PET reconstruction using generative adversarial network with feature matching and task-specific perceptual loss. Med Phys 2019;46:3555-3564. [3]

Ronneberger O, Fischer P, Brox T. U-Net: Convolutional Networks for Biomedical Image Segmentation. 2015; Cham: Springer International Publishing; 2015. p. 234-241. [4] Chen H, Zhang Y, Kalra MK, Lin F, Chen Y, Liao P, et al. Low-Dose CT With a Residual EncoderDecoder Convolutional Neural Network. IEEE Transactions on Medical Imaging 2017;36:25242535 .

\section{Image/Figure:}

https://www.xcdsystem.com/wmis/abstract/File6959/GA68_ImageFigure 0614042033.png

Image/Figure Caption: Figure 1. Convolutional neural network predicted full-dose postcontrast images from pre-contrast and simulated half-dose post-contrast images compared with the ground truth full-dose post-contrast MR images. Fibrosis imaging was performed using a collagen-binding molecular probe EP-3533 in a rat model of NASH.

Complete Status: Complete

First Name: Iris

Last Name: Zhou

Email: iris.zhou@mgh.harvard.edu

Organization: Massachusetts General Hospital and Harvard Medical School

Country: United States 


\title{
ID: GA69 \\ Non-invasive treatment decision support for non-small cell lung cancer with deep learning radiomics
}

\author{
Wei Mu, H. Lee Moffitt Cancer Center, wei_mu2016@163.com
}

\section{Category: Computational \& Data Science}

\begin{abstract}
Body : Purpose The emergence of two major treatment strategies, tyrosine kinase inhibitors (TKIs), and immune checkpoint inhibitors (ICIs), has revolutionized cancer treatment and improved long-term survival for patients with advanced non-small cell lung cancer (NSCLC) $[1,2]$ According to NCCN Guideline Version 2.2020 for NSCLC treatment [3], epidermal growth factor receptor (EGFR) and programmed death-ligand 1 (PD-L1) are two important biomarkers in treatment planning. However, these biomarkers are easily affected by the sampling bias and their dynamic nature $[4,5]$. Therefore, there is a compelling need to identify comprehensive biomarkers that can be used longitudinally and non-invasively to help guide therapy choice. Notably, patient responses to TKI and ICI are known to be reciprocal. Herein, we test the hypothesis that EGFR mutation status and PD-L1 expression status can be captured non-invasively using 18F-FDG-PET/CT images with artificial intelligence (AI) algorithms, and subsequently associated with clinical outcomes following treatment with TKIs or ICIs. Materials and methods Images and clinical data of 837 NSCLC patients were curated from Shanghai Pulmonary Hospital (SPH), the Fourth Hospital of Hebei Medical University (HBMU), the Fourth Hospital of Harbin Medical University (HMU) and Moffitt Cancer Center (HLM). To predict EGFR and PD-L1 mutation status, PET/CT images of 616 patients from SPH and HBMU were split into training $(\mathrm{N}=429)$ and validation $(\mathrm{N}=187)$ cohorts, and were used to train two residential-convolutional-network ( $\mathrm{ResCNN}$ ) models to obtain two deep-learning-based signatures (EGFR-DLS and PD-L1-DLS) to predict the EGFR mutation status and PD-L1 expression status, respectively. Patients from HMU with EGFR mutation status $(\mathrm{N}=65)$ and patients from HLM with PD-L1 expression status $(\mathrm{N}=85)$ were used as independent external test cohorts to test these two deep learning models. Further, these two DLS models were further associated progression-free survival (PFS) in HMU EGFR-TKI-treated patients $(\mathrm{N}=67)$ and HLM ICI-treated patients $(\mathrm{N}=149)$. Results The EGFR-DLS demonstrated high accuracy in EGFR mutation status prediction with AUCs of 0.86 (95\%CI: $0.83-0.90), 0.83$ (95\%:0.78-0.88) and 0.81(95\%CI: 0.72-0.91) in training, validation, and external HMU cohorts. Further, the PDL1-DLS also significantly discriminated PD-L1 positive and negative patients with AUCs of 0.89 (95\%CI: 0.84-0.94), 0.84 (95\%CI: 0.76-0.92) and 0.82 (95\%CI: 0.74-0.89) for training, validation and external HLM test cohorts. A high EGFR-DLS was significantly associated with longer PFS (Hazard ratio (HR): 0.24, pConclusion: The EGFR-DLS and PD-L1-DLS obtained from PET/CT images provide an innovative and non-invasive method for precise quantification of EGFR mutation status and PD-L1 expression status in NSCLC patients, which is a promising strategy to identify NSCLC patients sensitive to EGFR-TKI or ICI-treatments
\end{abstract}

References: 1. Kim, J. and D. Chen, Immune escape to PD-L1/PD-1 blockade: seven steps to success (or failure). Annals of Oncology, 2016. 27(8): p. 1492-1504. 2. Paez, J.G., et al., EGFR 
mutations in lung cancer: correlation with clinical response to gefitinib therapy. Science, 2004. 304(5676): p. 1497-1500. 3. (NCCN), N.C.C.N. NCCN Clinical Practice Guidelines in Oncology. Non-small Cell Lung Cancer Version 2.2020. [cited 2019 December 23, 2019]; Available from: https://www.nccn.org/professionals/physician_gls/pdf/nscl.pdf. 4. Taniguchi, K., et al., Intratumor heterogeneity of epidermal growth factor receptor mutations in lung cancer and its correlation to the response to gefitinib. Cancer science, 2008. 99(5): p. 929-935. 5. Yu, H., et al., PD-L1 expression in lung cancer. Journal of Thoracic Oncology, 2016. 11(7): p. 964-975.

\section{Image/Figure:}

https://www.xcdsystem.com/wmis/abstract/File6959/GA69 ImageFigure 0415035620.jpg

Full Name of Abstract's 1st Author : Wei Mu

Complete Status: Complete

First Name: Wei

Last Name: $\mathrm{Mu}$

Email: wei_mu2016@163.com

Organization: H. Lee Moffitt Cancer Center

Country: United States 


\title{
ID: GA70 \\ Anatomical atherosclerosis phantom for use in the MR imaging of intraplaque hemorrhage
}

\author{
Matteo Bomben, University of Toronto, matteo.bomben@mail.utoronto.ca
}

\section{Category: Cardiovascular \& Pulmonary}

\begin{abstract}
Body : Introduction Stroke is responsible for over 5.5 million annual deaths worldwide [1] with $15-20 \%$ being attributable to the rupture of atherosclerotic plaques in the carotid artery [2]. Rupture is strongly correlated to the presence of intraplaque hemorrhage (IPH) sites inside these plaques [3]. As such, researchers have developed a technique that uses a T1-weighted gradient echo MRI sequence (Siemens MPRAGE) to detect IPH and identify those at risk of stroke [4]. While promising, this method cannot be widely implemented in clinical settings unless there exists a method of standardizing images acquired across a variety of MR scanner models. With currently available atherosclerosis phantoms failing to include IPH $[5,6]$, the objective of this research was to synthesize two types of novel phantoms that replicate IPH and thus, by allowing for better image standardization, facilitate clinical IPH imaging. The first type of phantom was an IPH site size-assessment tool that modelled IPH sites of varying diameters, and the second consisted of anatomical replicas of IPH-positive plaques. Methods IPH sites
\end{abstract} appear as hyperintense regions on T1-weighted images as they possess short T1 relaxation times. As such, a material that mimics the imaging properties of IPH was created by adding $\mathrm{GdCl} 3$ to a $0.15 \%$-alginate, $1.5 \%$-agarose gel. Alginate (an anionic polymer) was added to the gel to prevent the diffusion of $\mathrm{Gd} 3+$ ions and thus preserve the signal intensity of IPH sites over time. To demonstrate the efficacy of this diffusion-prevention strategy, gels doped with different $\mathrm{GdCl} 3$ concentrations were created, embedded in an agar block, and their T1 relaxation times tracked over time. Once the IPH material was developed, the IPH site size-assessment phantom was fabricated. This was done by casting the IPH material into 1-8 mm diameter cylinders and embedding them in 2\% agar. Lastly, the anatomical atherosclerosis phantoms were created by casting multi-component plaques, layer-by-layer, using 3D printed moulds. Each replica plaque possessed an atheroma cap made of $2 \%$ agar, a lipid core consisting of a $50 \%$ canola oil-water emulsion solidified with $1.5 \%$ agarose, and an IPH site. Once fabricated, plaques were slid inside a mock, $8 \mathrm{~mm}$ diameter, blood vessel (made of $2 \%$ agar). All phantoms were imaged using the MPRAGE pulse sequence. Results Results showed that gels made from the IPH-mimicking material (with varying concentrations of $\mathrm{GdCl} 3$ ) maintained their $\mathrm{T} 1$ relaxation times over time. Over a 14-day period, the average T1 relaxation time of these sites was found to decrease by $2.32 \%$, a statistically insignificant margin. Interestingly, when alginate was not added to the IPH gel, a hypointense region appeared at the centre of the site, indicating diffusion of $\mathrm{Gd} 3+$ ions out of the site. The IPH-mimicking gel was then used to create a phantom that possessed IPH sites of varying diameters (Figure A). Each site mimicked the hyperintense signal of clinical IPH while also demonstrating statistically insignificant changes in signal intensity when stored for 7 days. Lastly, two anatomical atherosclerosis phantoms, each mimicking plaques with either a large or small IPH site (Figures B and C), were made. Similar to clinical plaques, these phantoms possessed hyperintense IPH sites, medium-intensity vessel walls, and a hypointense lumen. 
Conclusions A castable material that maintains its T1 relaxation time over time was created. Notably, by altering the concentration of $\mathrm{GdCl} 3$, a variety of $\mathrm{T} 1$ times can be achieved and thus this material can be used in a wide variety of MRI phantoms. Also produced were two novel IPH phantoms that model IPH sites of varying diameters and anatomical IPH-positive plaques. These phantoms can be used to standardize plaque images which will help facilitate the clinical imaging of IPH-positive atherosclerosis.

References: [1] Romain, G., A. Mariet, V. Jooste, G. Duloquin, Q. Thomas, J. Durier, M. Giroud, C. Quantin, and Y. Béjot. Long-Term Relative Survival after Stroke: The Dijon Stroke Registry. Neuroepidemiology 1-8, 2019.doi:10.1159/000505160 [2] Mughal MM, Khan MK, DeMarco JK, Majid A, Shamoun F, Abela GS. Symptomatic and asymptomatic carotid artery plaque. Expert Rev Cardiovasc Ther. 2011;9(10):1315-1330. doi:10.1586/erc.11.120 [3] Schindler A, Schinner R, Altaf N et al. Prediction of Stroke Risk by Detection of Hemorrhage in Carotid Plaques. JACC: Cardiovascular Imaging. 2019. doi:10.1016/j.jcmg.2019.03.028 [4] Singh N, Moody A, Rochon-Terry G et al. Identifying a High-risk Cardiovascular Phenotype by Carotid MRI-depicted Intraplaque Hemorrhage. Int J Cardiovasc Imaging. (2013) 29:1477-1483 DOI 10.1007/s10554-013-0229-3 [5] Chueh J, van der Marel K, Gounis M et al. Development of a high resolution MRI intracranial atherosclerosis imaging phantom. J Neurointerv Surg. 2017;10(2):143-149. doi:10.1136/neurintsurg-2016-012974 [6] Surry K, Austin H, Fenster A, Peters T. Poly(vinyl alcohol) cryogel phantoms for use in ultrasound and MR imaging. Phys Med Biol. 2004;49(24):5529-5546. doi:10.1088/0031-9155/49/24/009

\section{Image/Figure:}

https://www.xcdsystem.com/wmis/abstract/File6959/GA70_ImageFigure_0608124626.png

Image/Figure Caption: Figure A shows an image (acquired using the MPRAGE sequence) of a phantom that will allow for better measurement of IPH site size. It consists of mock-IPH sites, with diamters ranging from 1 to $8 \mathrm{~mm}$, embedded in a block of agar. Figures $\mathrm{B}$ and $\mathrm{C}$ show cross-sectional MPRAGE images of anatomical, IPH-positive, atherosclerosis phantoms. Figure $\mathrm{B}$ models an advanced plaque with a large IPH site, while Figure $\mathrm{C}$ models a plaque with a smaller IPH site and a distinguable lipid core. Note that the vessels displayed in Figures B and C had an $8 \mathrm{~mm}$ diameter.

Full Name of Abstract's 1st Author : Matteo Bomben

Complete Status: Complete

First Name: Matteo

Last Name: Bomben

Email: matteo.bomben@mail.utoronto.ca

Organization: University of Toronto

Country: Canada 


\title{
ID: GA72 \\ Near-infrared light-harvesting iron pyrite nanocrystals for phototheranostics of breast cancer
}

\author{
Jiang Yang, Sun Yat-sen University Cancer Center, terence0731@gmail.com
}

Category: New Chemistry, Biology \& Bioengineering

\begin{abstract}
Body : OBJECTIVE Phototheranostic nanomedicines have been continuously explored as a promising strategy for improved diagnosis and therapy with minimal exposure to radiation hazards, as exemplified by translational successes like AuroLase and Sebacia particles. Most other potential phototheranostic agents involve toxic shape-directing surfactants during synthesis and thus would encounter insurmountable translational hurdles. Pyrite iron disulfide (FeS2), also widely known as "fool's gold", is an earth-abundant, inexpensive and non-toxic semiconductor material proposed to be used in photovoltaic solar panels. It has a high optical absorption coefficient in the near-infrared (NIR) region and a narrow band gap of $0.95 \mathrm{eV}$ that is demonstrated to show NIR photoresponses. Therefore, we aimed to develop a covalently bound chalcogenide nanocrystal (NC) of FeS2 with scaled-up synthesis and to covalently functionalize the FeS2 NCs via a series of facile chemistry steps with 8-arm branched polyethylene glycol (PEG) polymers for aqueous dispersion and biocompatibility. The functionalized FeS2-PEG8 was investigated its suitability for multispectral optoacoustic tomography (MSOT) in phantoms and cells. We hypothesized that photothermal therapy can be accomplished in a human breast cancer xenograft model by focal hyperthermia and intended to verify if simultaneous guidance by thermographic and deep-tissue MSOT diagnostic imaging is attainable. METHODS FeS2 NCs were first synthesized from the $\mathrm{FeCl} 2$ precursor and we fabricated a photovoltaic module to harvest NIR light and test photoresponses. FeS2 NCs were then covalently functionalized on the surface by $10 \mathrm{kDa} 8$-arm-PEG-NH2 which has one amine group thiolated. We characterized the crystallinity, optical properties and cytotoxicity of FeS2-PEG8. In vitro optoacoustic features were surveyed in phantoms. Uptake in MDA-MB-231 breast cancer cells was validated by MSOT imaging and we explored the uptake mechanism using specific pharmacological inhibitors by Turnbull's Blue staining for Fe2+. Static 3D in vivo MSOT imaging was performed in NU/J xenograft mice bearing MDA-MB-231 tumors. We also systemically studied the photothermal effects of FeS2-PEG8. Lastly, photothermal therapy of breast cancer was carried out with visualization of thermographic imaging. RESULTS Despite the inherent insolubility of FeS2 NCs, superior aqueous-phase dispersity is realized with the covalent modification of 8-arm branched PEGs, accompanied by a solvent transition from the organic phase. Branched PEGylated FeS2 NCs well preserve the optical features in the NIR region with a spectrum that is distinctly different and thus can be unmixed from major significant intrinsic biological absorbers like oxygenated and deoxygenated hemoglobins for in vivo imaging. It presents an acceptable MSOT photostability upon continuous multispectral irradiation by a pulsed laser, retaining $78.9 \%$ of the original signal over two hours. FeS2-PEG8 can be detected by MSOT at a depth as deep as $20 \mathrm{~mm}$ in a tissue-mimicking phantom. Uptake in MDA-MB-231 human breast cancer cells was visualized by MSOT, which is predominantly mediated by PI3K-dependent macropinocytosis. In a xenograft of human breast cancer, FeS2-PEG8 was observed to be
\end{abstract}


delivered by tumor-feeding blood vessels and intra-tumoral accumulation. Primary organ distribution was found in the liver, spleen and lungs with low uptake in kidneys following tailvein injections, manifesting a hepatic-biliary clearance. FeS2-PEG8 displayed superior, stable and repetitive photothermal effects and photothermal therapy was successfully implemented in breast cancer xenograft models accordingly, resulting in statistically significant decreases of tumor burden. CONCLUSION Since breast tumors are most frequently located relatively superficially (esp. after breast compression during the imaging, which is performed routinely during mammography), our approach holds a realistic chance for clinical use. Its abundance, low toxicity and NIR light-harvesting capability have endowed FeS2 with translational opportunities after PEG modification, also because MSOT has been a readily-translatable imaging modality. Eradication of breast tumors without remnant cancer cells that may cause recurrence and metastatic disease may be achieved by post-surgical photothermal

\section{References: N/A}

Image/Figure Caption: Captions for Persuasive Data (A) Functionalization of FeS2 nanocrystals with 8-arm PEGs. Through strictly controlling the reaction molar ratio of EDC:NHS:DTBA at 1:1:1, one carboxyl side chain of 4,4'-dithiodibutyric acid (DTBA) is activated into NHS ester. N,N-Diisopropylethylamine (DIEA) as a non-nucleophilic organic base in the reaction effectively stabilizes 8-arm-PEG-NH3+Cl- (MW: 10k) (tripentaerythritol core) in the form of 8-arm-PEG-NH2 favoring formation of amide bonds with activated DTBA at a molar ratio of 1:1. Reactive thiol group is then activated by 1,4-dithiothreitol (DTT) through S-S bond cleavage, followed by precipitation with diethyl ether, and then reacts with FeS2 in chloroform by forming new S-S bonds to achieve covalent functionalization. (B) Schematic illustration of the photovoltaic module consisting of FeS2 coating on a fluorine-doped tin oxide (FTO) glass substrate, a $\mathrm{Ag} / \mathrm{AgCl}$ reference, and a Pt wire counter electrode in an alkaline electrolyte $(\mathrm{pH}=$ 13.5) with SO32-/S2O32- as the sacrificial agent. (C) Current-time characteristics of FeS2 nanocrystals on FTO under on/off cycles of a $808 \mathrm{~nm}$ NIR laser illumination at a constant bias of $0.1 \mathrm{~V}$. (D) The optoacoustic spectrum of FeS2-PEG8 shares similar features with its absorption spectrum. Inset shows single-wavelength optoacoustic images of FeS2-PEG8. (E) Multispectral optoacoustic stability. FeS2-PEG8 is subject to continuous spectral sweeping from 700-900 nm by a pulse laser of multispectral optoacoustic tomography (MSOT). $78.9 \%$ of the original MSOT signal is retained post exposure. Inset shows corresponding images at various time points. (F) Xray diffraction patterns. $2 \theta$ peaks of FeS2 nanocrystals closely match the indexed planes of a FeS2 pyrite reference standard (ICSD 53935), while covalent conjugation of 8-arm-PEGs well preserves the single-phase pyrite crystal structure. Additionally, two distinct peaks around $27^{\circ}$ corresponding to branched PEGs can be clearly observed only after PEGylation1. (G) FeS2PEG8 shows strong optical absorption in the NIR region. Characteristics of the absorption spectrum for FeS2-PEG8 are almost identical in chloroform and aqueous solution, also resembling to the pristine FeS2 nanocrystals. (H) Detection of different concentrations of FeS2PEG8 in an agarose phantom by MSOT. (I) Uptake of FeS2-PEG8 by MDA-MB-231 human breast cancer cells was confirmed by MOST imaging of cell phantoms. (J) Cellular uptake was further confirmed by Turnbull's Blue staining for intracellular Fe2+ species. FeS2-PEG8 is mainly localized both near the cell membrane and within the cytoplasm. Such endocytosis can be effectively blocked by pharmacological inhibitors of 5-(N-ethyl-N-isopropyl)-amiloride (EIPA) and wortmannin, indicating that PI3K-dependent macropinocytosis is one possible endocytic 
mechanism. (K) Specific MSOT signals of FeS2-PEG8 can be observed in the tumor and lungs post intravenous injeciton. Tumor feeding blood vessels also displayed increased signals after injection. (L) Ex vivo confirmation by histology. Turnbull's Blue staining with $\mathrm{H} \& \mathrm{E}$ as background contrast reveals distribution of FeS2-PEG8 in the tumor and the tumor-feeding blood vessels. H\&E staining is included as reference. (M) Single-wavelength optoacoustic transverse images of a NU/J mouse $1 \mathrm{~h}$ following intravenous injection. Enhanced optoacoustic contrast is observed primarily in the liver and spleen with minor distribution in the lungs. (N) In vivo optoacoustic spectra of FeS2-PEG8 remain identical in distributed organs, also bearing resemblance to the in vitro spectrum. $(\mathrm{O})$ MSOT imaging of a tissue mimicking phantom containing FeS2-PEG8 at different depths. The MSOT signals clearly decrease with increasing depths as expected, but positive signals can still be detected at a rather deep inclusion around 20 $\mathrm{mm}$. (P) Optical spectra of major intrinsic photoabsorbers in biological tissues, oxygenated $(\mathrm{HbO} 2)$ and deoxygenated $(\mathrm{Hb})$ hemoglobins. The distinctly different exogenous spectrum of FeS2-PEG8 in the NIR imaging window can therefore be spectrally unmixed. (Q) In vivo MSOT imaging discloses FeS2-PEG8 is primarily accumulated in the liver, spleen and lungs, but not the kidneys, following intravenous injeciton. Therefore, the predominant pathway is through the hepatic-biliary clearance. (R) Ex vivo histological confirmation. Turnbull's Blue staining with $\mathrm{H} \& \mathrm{E}$ as background contrast reveals distribution of FeS2-PEG8 primarily in the liver, spleen and lungs. (S) Change of tumor volume in different treatment groups over time $(n=5)$. (T) Photographic images of tumors from different treatment groups harvested at the endpoint of therapy. (U) Tumor weight in different treatment groups harvested at the endpoint of therapy. (V) Thermographic images of mice under different treatment at the baseline and different time points. (W) Averaged temperature of the tumor area during photothermal therapy for different treatment groups. An obvious elevated increase in tumors post injection of FeS2-PEG8 can be observed upon laser excitation. (X) Body weight changes of mice over time for different treatment. No significant changes were observed.

\title{
Complete Status: Complete
}

First Name: Jiang

\section{Last Name: Yang}

Email: terence0731@gmail.com

Organization: Sun Yat-sen University Cancer Center

\author{
Country: China
}




\title{
ID: GA73 \\ 111In-Radiolabeling of Mensenchymal Stem Cell-derived Extracellular Vesicles for Quantitative In Vivo Imaging Studies
}

\author{
Cheng-Hsiu Lu, National Yang-Ming University, rocket2350@yahoo.com.tw
}

\section{Category: New Chemistry, Biology \& Bioengineering}

\begin{abstract}
Body : Purpose: Mesenchymal stem cells (MSCs) are multipotent stromal cells, which have the great potential in tissue engineering, regenerative medicine and the treatment of various diseases. Extracellular vesicles (EVs) serve as an important mode of cell-to-cell communication, and these vesicles are capable of horizontal transfer of protein, nucleic acids and lipids from parental cells to recipient cells. Currently, EVs have been reported to be in charge of mediating the therapeutic and paracrine effects of MSCs. In order to assess the pharmacokinetics and biodistribution of EVs, the effective method to in vivo tracking EVs is needed. Here, we used 111 In-oxine to label Wharton's jelly MSC derived EVs (WJ-MSC-EVs) and studied their in vivo biodistribution with small animal SPECT/CT imaging. Procedures: WJ-MSC-EVs were isolated from conditioned medium when the cells reached $90 \%$ confluence, followed by characterized using western blot, transmission electron microscopy (TEM), nanoparticle tracking analysis (NTA). For in vivo tracking, WJ-MSC-EVs were labeled with 111In-oxine and imaged by SPECT/CT. Further, radioactivity was assessed for quantitative analysis of ex vivo biodistribution. Result: When the EVs were labeled with $111 \mathrm{In}$-oxine, the radiochemical purity of 111 In-oxine-EVs was generally over $90 \%$ and kept stable until 24 hours. Further, WJ-MSCEVs still remained characteristics after radiolabeling with 111In-oxine. The result showed that SPECT/CT images of the mice administrated with 111In-oxine-EVs accumulated majorly in liver and spleen, whereas mice administrated with 111In-oxine exhibited high lung, liver, and kidneys uptake until 24 hours. The ex vivo biodistribution of mice injected with 111In-oxineEVs and 111In-oxine showed the similar pattern to the result of in vivo imaging. Conclusions: Our noninvasive imaging of $111 \mathrm{In}$-oxine-EVs is able to reflect the in vivo behavior of WJ-MSCEVs. Further, the radiolabeling procedure did not damage the EVs and maintain the characteristics of EVs. In conclusion, we developed a simple and valid molecular probe to monitor the dynamic change of exogenously administered WJ-MSC-EVs.
\end{abstract}

References: 1. Di Rocco G, Baldari S, Toietta G (2016) Towards Therapeutic Delivery of Extracellular Vesicles: Strategies for In Vivo Tracking and Biodistribution Analysis. Stem Cells Int 2016:5029619. 2. Hwang DW, Choi H, Jang SC, et al. (2015) Noninvasive imaging of radiolabeled exosome-mimetic nanovesicle using (99m)Tc-HMPAO. Sci Rep 5:15636. 3. Rani S, Ryan AE, Griffin MD, Ritter T (2015) Mesenchymal Stem Cell-derived Extracellular Vesicles: Toward Cell-free Therapeutic Applications. Mol Ther 23:812-823. 4. Grange C, Tapparo M, Bruno S, et al. (2014) Biodistribution of mesenchymal stem cell-derived extracellular vesicles in a model of acute kidney injury monitored by optical imaging. Int J Mol Med 33:1055-1063. 5. Takahashi Y, Nishikawa M, Shinotsuka H, et al. (2013) Visualization and in vivo tracking of the exosomes of murine melanoma B16-BL6 cells in mice after intravenous injection. J Biotechnol 165:77-84. 6. Smyth T, Kullberg M, Malik N, Smith-Jones P, Graner 
MW, Anchordoquy TJ (2015) Biodistribution and delivery efficiency of unmodified tumorderived exosomes. J Control Release 199:145-155. 7. Malviya G, Nayak T, Gerdes C, Dierckx RA, Signore A, de Vries EF (2016) Isolation and (111)In-Oxine Labeling of Murine NK Cells for Assessment of Cell Trafficking in Orthotopic Lung Tumor Model. Mol Pharm 13:1329-1338. 8. Chow TH, Lin YY, Hwang JJ, et al. (2008) Diagnostic and therapeutic evaluation of 111 Invinorelbine-liposomes in a human colorectal carcinoma HT-29/luc-bearing animal model. Nucl Med Biol 35:623-634. 9. Batrakova EV, Kim MS (2015) Using exosomes, naturally-equipped nanocarriers, for drug delivery. J Control Release 219:396-405. 10. Haney MJ, Klyachko NL, Zhao Y, et al. (2015) Exosomes as drug delivery vehicles for Parkinson's disease therapy. J Control Release 207:18-30.

\section{Image/Figure:}

https://www.xcdsystem.com/wmis/abstract/File6959/GA73 ImageFigure_0418022639.png

Full Name of Abstract's 1st Author : Cheng-Hsiu Lu

Complete Status: Complete

First Name: Cheng-Hsiu

Last Name: $\mathrm{Lu}$

Email: rocket2350@yahoo.com.tw

Organization: National Yang-Ming University

Country: Taiwan 


\title{
ID: GA75 \\ Identification and Preclinical Validation of [18F]AS3504073-00, Novel PET Ligand for Fatty-Acid Oxidation, for Cardiac Imaging.
}

\author{
Hiroshi Fushiki, Astellas Pharma Inc., hiroshi.fushiki@astellas.com
}

\section{Category: Cardiovascular \& Pulmonary}

\begin{abstract}
Body : Objectives: Myocardial energy metabolism is mainly dependent on Fatty Acid Oxidation (FAO) in ATP production, however, FAO alteration in cardiac dysfunction is still unclear. Some SPECT and PET tracers have been developed to measure beta-oxidation. We have identified [18F]AS3504073-00 as a novel PET probe for FAO with good property on higher uptake in heart and no defluorination in vivo. To understand the character of [18F]AS350407300, PET imaging examination with [18F]AS3504073-00 in ischemic heart disease model, preclinical toxicity and dosimetry studies were conducted. Methods: Three secondary fluorinated analogs, 17-fluoro-4-thia-oleate (17-FTO), 15-fluoro-4-thia-oleate (15-FTO), and 7-fluoro-4thia-oleate, (7-FTO, AS3504073-00) were designed based on FTO as a lead compound and performed $18 \mathrm{~F}$-labeled synthesis. 18F-labeled tracers were administered to cynomolgus monkeys and PET measurements were demonstrated. Heart infraction model in monkey was established by occluding left anterior descending coronary artery (LAD). PET imaging with [18F]AS3504073-00 was conducted in ischemic heart disease monkey models. A 7-day rat toxicity study with AS3504073-00 was conducted. Dosimetry estimation by whole-body PET scans in 3 male cynomolgus monkeys after IV bolus injection of [18F]AS3504073-00 was performed. Results: All three compounds were successfully synthesized with enough quality and stability for animal experiment in a similar manner. In monkey PET biodistribution studies, 17[18F]FTO and 15-[18F]FTO were seen the bone accumulation, however, only [18F]AS350407300 showed much more uptake in the heart than that of $[18 \mathrm{~F}] \mathrm{FTO}$ and no significant accumulation of radioactivity was observed in the bone. In monkey LAD occlusion model, [18F]AS350407300 was visualized ischemic region. A 7-day rat toxicity study with AS3504073-00 did not show any test article-related changes. [18F]AS3504073-00 showed highest organ absorbed doses for the urinary bladder wall (119 $\mathrm{Gy} / \mathrm{MBq})$. The effective dose was $19.1 \mu \mathrm{Sv} / \mathrm{MBq}$ Conclusions: [18F]AS3504073-00 is shown to be a potential tool of FAO PET probe. The character of [18F]AS3504073-00 warrants the usefulness with good specificity and imaging efficacious window in heart disease. [18F]AS3504073-00 would be tolerable for clinical PET imaging studies.
\end{abstract}

Complete Status: Complete

First Name: Hiroshi

Last Name: Fushiki

Email: hiroshi.fushiki@astellas.com 
Organization: Astellas Pharma Inc.

Country: Japan 


\title{
ID: GA76 \\ Randomized Double-Blind Controlled Study to Evaluate Safety and Pharmacokinetics of Single Ascending Doses of ASP5354, an Investigational Imaging Product, in Healthy Adult Volunteers
}

\author{
Open Health, Open Health, ASP2215Publications@succinctchoice.com
}

\section{Category: New Chemistry, Biology \& Bioengineering}

\begin{abstract}
Body : Background: Iatrogenic ureteral injury is a complication that can occur during abdominal surgery. There is a medical need to better identify ureters during surgery in order to minimize the risk of injury. ASP5354 is a novel indocyanine green (ICG) derivative. The molecular size and hydrophilic nature of ASP5354 allow excretion into urine, enabling ureteralspecific imaging and visualization using existing near-infrared ICG detection devices. Preclinical results showed that ASP5354 $0.01 \mathrm{mg} / \mathrm{kg}$ allowed visualization of ureters for up to 3 hours postadministration and revealed that ASP5354 was generally well tolerated. Aim/Objective: The primary objective was to assess the safety/tolerability of a single ASP5354 dose in healthy subjects. Plasma and urine pharmacokinetic assessment of single-dose ASP5354 was a secondary objective. Methods: This first-in-human (FIH) double-blind study included five dose cohorts of healthy adults. Subjects were randomly assigned 2:1 to ASP5354 or placebo, and each received a single IV bolus dose of ASP5354 or placebo under fasting conditions, followed by a 24-hour blood/urine sampling period. Subjects were dosed sequentially with ASP5354 0.1, 0.5, 2, 8, and $24 \mathrm{mg}$. Safety/tolerability were assessed at each dose level to determine the maximum tolerated dose. Cumulative safety and pharmacokinetic data from the preceding cohort(s) were included in the dose-escalation assessment. Results: Patients $(\mathrm{N}=30)$ received either ASP5354 ( $\mathrm{n}=20$; each dose cohort, $n=4)$ or placebo $(n=10)$. In each cohort, $50 \%$ of subjects were female; the overall mean age was 43 (SD, 11.3) years, mean body mass index (BMI) was 27.3 (SD, 2.96) kg/m2, and $67 \%$ were white. Mean age and BMI were similar across cohorts. Overall, three subjects (15\%) who received ASP5354 experienced six adverse events (AEs) that were unrelated to treatment: pyelonephritis, urinary tract infection, oral herpes, headache, presyncope, and dysuria (each $n=1)$. Pyelonephritis was a serious AE caused by the urethral catheter. Infusions were well tolerated with no evidence of histamine release or hypersensitivity. No subjects discontinued due to an AE and no subjects died during the study. Exposure to ASP5354 was approximately dose proportional. The mean terminal half-life ranged from 2.1 to 3.6 hours, and total body clearance and terminal volume of distribution were consistent across the dose range. Urinary ASP5354 concentrations were quantifiable up to 6 hours postdose in the 0.1 and $0.5 \mathrm{mg}$ cohorts and up to 24 hours postdose in the 2, 8, and $24 \mathrm{mg}$ cohorts (Figure). Urinary excretion of ASP5354 was nearly complete by 24 hours across the dose range (unchanged excreted drug, 77-100\%). Green urine coloration was observed until 3 to 3.5 hours after the 8-mg dose in three of four subjects and until 8 to 12 hours and 12 to 24 hours after the 24-mg dose in two of four subjects in each time frame. Conclusions: Results of this FIH study showed that a single dose of ASP5354 was well tolerated across the tested doses. Plasma exposure of ASP5354 was approximately doseproportional and pharmacokinetics appeared linear across the dose range. These results support further evaluation of ASP5354.
\end{abstract}




\section{Image/Figure:}

https://www.xcdsystem.com/wmis/abstract/File6959/GA76_ImageFigure_0608083349.png

Image/Figure Caption: Figure. Arithmetic Mean (SD) Point Urine Concentration Profiles of ASP5354.

Complete Status: Complete

First Name: Open

Last Name: Health

Email: ASP2215Publications@succinctchoice.com

Organization: Open Health

Country: United States 


\title{
ID: GA77 \\ Novel Intra-Operative Fluorescent Imaging Dye, ASP5354, for Optical Surgical Navigation of Ureter Imaging, Preclinical Development, and Validation
}

\author{
Open Health, Open Health, ASP2215Publications@succinctchoice.com
}

Category: New Chemistry, Biology \& Bioengineering

\begin{abstract}
Body : Purpose: Iatrogenic ureteric injury (IUI) during abdominal surgery is a devastating surgical complication. The incidence rate of IUI with hysterectomy has been reported as $0.3-2.5 \%$, potentially resulting in serious adverse outcomes for patients, putting an extra burden on healthcare systems, and increasing the risk of litigation for clinicians.

Intraoperative ureteral visualization is thus critical to reduce the frequency of IUIs. Fortunately, some methodologies for ureter imaging have already been developed. Among these, nearinfrared fluorescent (NIRF) imaging is thought to represent an advantage by allowing real-time visualization through the retroperitoneum. Herein, we report on the novel indocyanine green derivative NIRF probe, ASP5354, with the inclusion of nonclinical imaging and development results. Methods and Results: ASP5354 emitted NIRF at a wavelength of $820 \mathrm{~nm}$ when irradiated with near-infrared excitation light at a wavelength of $780 \mathrm{~nm}$. Göttingen minipigs under anesthesia were laparotomized and then administered ASP5354 intravenously at doses of 0.001 and $0.01 \mathrm{mg} / \mathrm{kg}$. Videos of the abdominal cavity were taken using a NIRF camera and assessed visually by three independent clinicians. The number of animals whose ureter was recognized for up to 3 hours after administration of ASP5354 at 0.001 and $0.01 \mathrm{mg} / \mathrm{kg}$ was one out of three and three out of three, respectively. ASP5354 had no toxicologically significant changes in clinical signs when toxicity studies were performed in cynomolgus monkeys. Conclusion: Overall, these findings suggest that intravenous administration of ASP5354 at a dose of $0.01 \mathrm{mg} / \mathrm{kg}$ allows for sufficient intraoperative imaging of the ureter for up to 3 hours, serving as the basis for subsequent first-in-human investigations. At the present time, ASP5354 is set to commence phase 2 studies.
\end{abstract}

Complete Status: Complete

First Name: Open

Last Name: Health

Email: ASP2215Publications@succinctchoice.com

Organization: Open Health

Country: United States 


\title{
ID: GA78 \\ Endobronchial Ultrasound and Drug-Loaded Nanodroplets for Targeted Lung Cancer Therapy in an In Vivo Mouse Model
}

\author{
Sean McGrath, University of Toronto, seanfrancis.mcgrath@mail.utoronto.ca
}

\section{Category: Oncology}

\begin{abstract}
Body : Introduction Lung cancer is the leading cause of cancer-related death [1]. Endobronchial ultrasound (EBUS) is a clinical diagnostic tool developed for lung cancer staging [2], where a modified bronchoscope is introduced into the patient's airway and, using the integrated ultrasound probe, image-guided transbronchial biopsies of cancerous tissues deep within the chest are obtained [3]. This study explores the use of EBUS as a technique for targeted lung cancer therapy through the use of intravenously-injected, drug-loaded, acousticallyactivated, nanodroplet agents. Previous non-EBUS therapeutic ultrasound studies have shown that with sufficient acoustic pressure, drug-loaded nanodroplets can be cavitated to physically damage or weaken tumour vasculature [4] and deliver loaded drugs [5] for combined drug delivery and vascular disruption therapy. Although EBUS overcomes the limitations of standard clinical ultrasound systems that cannot access the inner lung due to limited sound wave penetration through alveolar air, clinical EBUS systems emit lower power than non-EBUS therapeutic ultrasound systems and cannot easily activate nanodroplets for therapy. Additionally, nanodroplets typically contain a hydrophobic perfluorocarbon core, limiting the loading yield of hydrophilic chemotherapeutic drugs. The aim of this study is to develop nanoparticles that can be loaded with high concentrations of drugs (such as the front-line chemotherapeutic cisplatin), that can then be incorporated into nanodroplets for successful activation by EBUS for therapy. This system will have the dual benefit of bringing targeted drug delivery and vascular disruptive therapy to lung cancer tumours. Methods Drug-loaded silica nanoparticles were synthesized [6]. To validate colocalization of the drug in the nanodroplets, a hydrophilic fluorescent dye, calcein (Fluka), was used as an optical surrogate for hydrophilic chemotherapeutics. Dynamic light scattering (DelsaMAX, Beckman Coulter, USA) was used to determine nanoparticle size. Nanoparticle-loaded nanodroplets, composed of a decafluorobutane core (C4F10, Synquest labs) and a phospholipid shell (DPPA, DPPC, DPPE-PEG5k, Avanti Polar Lipids), were synthesized in-house first as nanoparticle loaded lipid-stabilized bubbles, which were then condensed to form nanoparticle-loaded nanodroplets [7]. These were characterized for their stability and size distribution using a Coulter counter (Multisizer 4e, Beckman Coulter, USA). Calcein-loaded nanodroplets were imaged using fluorescence microscopy to verify nanoparticle incorporation. Nanodroplets were tested for their cavitation response to ultrasound (Verasonics, USA) by flowing dilute nanodroplets at $37^{\circ} \mathrm{C}$ through a $3 \%$ agar phantom with variable mechanical index (MI) ultrasound $(\mathrm{MI}=0.95-1.5)$. Murine AE17-OVA mesothelioma tumours were inoculated subcutaneously in the right flank of C57BL/6 mice (Taconic Bioscience, NY), as analogs for thoracic malignancy. A $150 \mu \mathrm{L}(\sim 4 \times 107$ nanodroplets $/ \mathrm{mL})$ injection of nanodroplets was followed by ultrasound ( $\mathrm{f}=5 \mathrm{MHz}, \mathrm{MI}=\sim 1.2$ ) using EBUS (EU-ME2 PREMIER ultrasound processor with BF-UC180F EBUS bronchoscope, Olympus, Japan). Contrast-enhanced ultrasound $(\mathrm{f}=3-4.2 \mathrm{MHz}, \mathrm{MI}=0.05)$ and microbubbles were used before and after the procedure,
\end{abstract}


to compare tumour blood perfusion and evaluate the effectiveness of vascular disruption (comparison through MATLAB, MathWorks). Results Drug-loaded nanoparticles with a mean size of $49.7 \pm 0.4 \mathrm{~nm}$ ( $\mathrm{PDI}=0.078 \pm 0.016$ ) were successfully synthesized. Calcein nanoparticleloaded droplets were fluorescent, indicating the nanoparticles were successfully incorporated. Nanodroplets ranged from $>0.3 \mu \mathrm{m}-2 \mu \mathrm{m}$, with a number peak at $0.4 \mu \mathrm{m}$. At 5 hours post-synthesis (at physiological temperature, $37^{\circ} \mathrm{C}$ ), Coulter counter measurements indicated $68 \%$ of nanodroplets remained in solution. Nanodroplets had a cavitation threshold of $\mathrm{MI}=1.0$, below the maximum output $(\sim \mathrm{MI}=1.2)$ of the EBUS system. Using murine mesothelioma tumours and nanodroplets in combination with EBUS system, nanodroplets caused a $73 \%$ reduction in peak intratumoral microbubble intensity after a single treatment, indicating a reduction in perfusion. Conclusion This study developed a procedure for synthesizing and successfully incorporating hydrophilic drug-loaded nanoparticles into nanodroplets. These nanodroplets can be cavitated by current EBUS technology, enabling combined targeted drug delivery and vascular disruptive therapy for the treatment of lung cancer.

References: [1] "Cancer." World Health Organization, www.who.int/news-room/factsheets/detail/cancer. [2] Kinsey, C., Arenberg, D., "Endobronchial ultrasound-guided transbronchial needle aspiration for non-small cell lung cancer staging" American journal of respiratory and critical care medicine, vol. 189, no. 6, pp. 640 - 649, 2014 [3] CzarneckaKujawa, K. and K. Yasufuku, "The role of endobronchial ultrasound versus mediastinoscopy for non-small cell lung cancer" Journal of Thoracic Disease, 9(Suppl 2), pp. S83 S97, 2017 [4] Zhou, Y., "Application of acoustic droplet vaporization in ultrasound therapy" Journal of Therapeutic Ultrasound, vol. 3, no. 20, 2015 [5] Ho, Y., Yeh, C., "Concurrent anti-vascular therapy and chemotherapy in solid tumors using drug-loaded acoustic nanodroplet vaporization" Acta Biomateriaia, vol. 49, pp. 472 - 485, 2017 [6] Kamegawa, R., Naito, M., Miyata, K., "Functionalization of silica nanoparticles for nucleic acid delivery" Nano Research, vol. 11, no. 10, pp. 5219 - 5239, 2018 [7] Seo, M., Williams, R., Matsuura, N., "Size reduction of cosolventinfused microbubbles to form acoustically responsive monodisperse perfluorocarbon nanodroplets" Lab Chip, vol. 15, no. 17, pp. 3581 - 3590, 2015

\section{Image/Figure:}

https://www.xcdsystem.com/wmis/abstract/File6959/GA78 ImageFigure 0614035208.JPG

Image/Figure Caption: Pre-treatment compared to post treatment tumor perfusion (outlined by pink ROI at peak enhancement under EBUS contrast): a) Before injection of microbubbles or NDs, b) after injection of microbubbles for imaging pre-treatment perfusion, and c) after injection of microbubbles for imaging post-treatment perfusion. d) Tumour microbubble perfusion profile pre- and post-nanodroplet treatment. e) Quantification of blood flow reduction with bolus injection peak intensity processing.

Complete Status: Complete

First Name: Sean

Last Name: McGrath 
Email: seanfrancis.mcgrath@mail.utoronto.ca

Organization: University of Toronto

Country: Canada 


\title{
ID: GA80 \\ Near IR fluorescence imaging guided photothermal therapy to enhance starvation therapy of breast tumor
}

\author{
Yuqiong Xia, Xidian University, yqxia@xidian.edu.cn
}

\section{Category: Oncology}

\begin{abstract}
Body : Tumor starvation therapy specifically blocks the nutrient supply to inhibit tumor growth. The most used reagent for starvation therapy is glucose oxide (GOx), which can not only exhaust glucose, blocking the nutrient supply of tumor, but also can produce $\mathrm{H} 2 \mathrm{O} 2$, leading to oxidative toxicity to tumor. However, in vivo application of GOx fails to inhibit tumor efficient in most studies. There are two reasons: one is that the delivery of GOx is poor and little GOx reaches tumor tissue; the other is that hypoxic tumor does not have enough oxygen for GOx to exhibit its maximum tumor inhibition. To overcome the problems, GOx was delivered by biocompatible liposomes (DiR@Lipo) to enhance tumor delivery. On the other hand, near-IR fluorophore DiR-containing liposomes DiR@Lipo were used to perform near-IR fluorescence image-guided photothermal therapy, which can increase the enhance the oxygen supply of tumor and kill tumor through heat effect precisely (Figure 1A). The two liposome formulations GOx@Lipo and DiR@Lipo were synthesized first with sizes of $145 \pm 6 \mathrm{~nm}$ and $140 \pm 4 \mathrm{~nm}$, respectively (Figure 1B). The similar size distributions will lead to similar pharmacokinetics, which facilitate combination therapy. Later, the catalytic activity of GOx@Lipo was characterized and was like that of free GOx (Figure 1C), which indicated that GOx encapsulation in liposomes did not affect the catalytic activity of GOx. Further, the photothermal effect of DiR@Lipo was carefully characterized and the result showed that DiR@Lipo reached 46 oC after $5 \mathrm{~min}$ of illumination $(808 \mathrm{~nm}, 2 \mathrm{~W} / \mathrm{cm} 2)$ at $10 \mu \mathrm{g} / \mathrm{mL}$ DiR (Figure 1D), which implied that DiR@Lipo with DiR at $10 \mu \mathrm{g} / \mathrm{mL}$ or above were efficient in inhibiting tumor. Finally, the combination of starvation therapy and photothermal therapy was performed on 4T1 cells. It was found that photothermal therapy by DiR@Lipo indeed increased cytotoxicity of GOx@Lipo against 4T1 cells. The study demonstrated that image-guided photothermal therapy was efficient in enhancing the efficacy of starvation therapy.
\end{abstract}

\section{Image/Figure:}

https://www.xcdsystem.com/wmis/abstract/File6959/GA80 ImageFigure 0417035127.jpg

Complete Status: Complete

First Name: Yuqiong

Last Name: Xia

Email: yqxia@xidian.edu.cn 
Organization: Xidian University

Country: China 


\title{
ID: GA81 \\ Integrating Radiomics into Manual Diagnosis for Increasing the Discovery Rate of Unresectable Tumors in Patients with Pancreatic Cancer
}

\author{
Wei Qin, Xidian University, wqin@xidian.edu.cn
}

Category: Systemic Diseases (Kidney, Liver and Pancreas)

\begin{abstract}
Body : Purpose Pancreatic tumors can be surgically removed. Some patients who are not suitable for surgery do not have obvious characteristics before surgery. During the surgery, ithe tumor was found to be unresectable and the surgery must be terminated. If it is possible to determine whether the patient's tumor is resectable by preoperative imaging, then the damage to the unresectable patients and treatment delay caused by the diagnosis errors can be avoided. Procedures One-hundred and twenty-three patients ( 82 in the training cohort and 41 in the validation cohort) with pancreatic cancer were enrolled. A total of 8676 radiomic features were extracted from dynamic enhanced T1-weighed images (arterial and portal venous phases). The 2sample t-test and the least absolute shrinkage and selection operator regression were used for feature selection, whereupon the Rad-score was calculated for each patient as a linear combination of selected features. Radiomic signatures were built using Rad-score. The doctors manually diagnoses through preoperative information to determine whether patients with resectable tumors. The performance of the manual diagnosis from the doctor was assessed by accuracy and true positive rate (TPR). The standard of the combination of manual diagnosis from the doctors and radiomic signature from the imaging is that if the common judgment is a resectable tumor, it is considered as a resectable tumor. The performance of the radiomic signature and the combination of manual diagnosis and radiomic signature were assessed by accuracy and TPR within the independent validation. Results The manual diagnosis by doctors showed good with an accuracy of $81.3 \%$ and a TPR of $52.1 \%$. The radiomics signature, which derived from T1-weighted imaging and comprised 8 selected features, showed better discrimination performance in both the training (accuracy: 82.9\%, TPR: $80.0 \%$ ) and validation cohorts (accuracy: 78.1\%, TPR: 66.7\%). The combination of manual diagnosis and radiomic signature showed the best discrimination performance in both the training (accuracy: 87.8\%, TPR: 93.3\%) and validation cohorts (accuracy: 85.4\%, TPR: 83.3\%). Conclusions The TPR of the model we constructed is higher, which means that the probability of correct judgment for unresectable cases is higher. When manual diagnosis and Magnetic resonance imaging are fused, the MRI could correct the judgment of doctors, so that patients with unresectable tumors are more likely to be found. The use of non-invasive images and the method of radiomics may help doctors diagnose, thereby increasing the discovery rate of unresectable tumors, which has important clinical significance.
\end{abstract}

Complete Status: Complete

First Name: Wei

Last Name: Qin 
Email:wqin@xidian.edu.cn

Organization: Xidian University

Country: China 


\title{
ID: GA83 \\ A kit formulation for [89Zr] $\mathrm{Zr}$ (oxinate)4: in vitro comparison with [111In]In(oxinate) 3 for white blood cell radiolabelling
}

\author{
Francis Man, King's College London, francis.man@kcl.ac.uk
}

\section{Category: New Chemistry, Biology \& Bioengineering}

\begin{abstract}
Body : Introduction: With new developments in immunology and cell-based therapies, there is an increased need to track the migration of individual cell types, such as immune cells (neutrophils, eosinophils, dendritic cells, CAR-T cells, etc.) and stem cells $(1,2)$. As the fate of administered therapeutic cells remains largely unknown, nuclear imaging techniques could shed light on the migration and survival of cells in patients. Further developments in cell-based therapies will require detection of small lesions and low numbers of cells, as well as better quantification, all of which could be achieved by positron emission tomography (PET).
\end{abstract} [89Zr]Zr(oxinate)4 (89Zr-oxine) is a PET radiotracer that has been evaluated in preclinical models of cell tracking (3-5) and could represent an advance on [111In]In(oxinate)3 (111Inoxine), the established gold standard radiotracer for cell tracking by scintigraphy and SPECT, because of the better sensitivity, spatial resolution and quantification of PET. However, a clinically usable formulation of $89 \mathrm{Zr}$-oxine is lacking. This study demonstrates a 1-step procedure for preparing $89 \mathrm{Zr}$-oxine and evaluates it against 111In-oxine in white blood cell (WBC) labelling. Methods: Commercial 89Zr-oxalate was added to a kit formulation containing oxine, a buffering agent, a base and a surfactant or organic solvent. The formation rate and stability of 89Zr-oxine in solution were evaluated by radio-thin-layer chromatography. Radiotracer recovery from the vial was evaluated by gamma-counting. WBC were isolated on two separate occasions from 10 human volunteers and radiolabelled with 89Zr-oxine and 111Inoxine for $15 \mathrm{~min}$ following a standard clinical protocol for radiolabelling WBC. An intraindividual comparison against 111In-oxine was performed in vitro, evaluating labelling efficiency, radionuclide retention and cell viability. Leukocyte function after radiolabelling was evaluated as fMLP-induced chemotaxis across a polycarbonate membrane. The impact of radiolabelling on DNA damage was assessed by measuring $\gamma \mathrm{H} 2 \mathrm{AX}$ foci (double-strand breaks) formation by immunofluorescent confocal microscopy. The uptake of $89 \mathrm{Zr}$ in different leukocyte populations was measured by flow-assisted cell sorting and gamma-counting. Results: An optimised formulation of $89 \mathrm{Zr}$-oxine was developed, containing $0.5 \mathrm{mg} / \mathrm{mL}$ oxine, $1 \mathrm{mg} / \mathrm{mL}$ polysorbate 80 and 1 M HEPES at $\mathrm{pH}$ 7.9-8.0 (Fig. 1A). The amount of surfactant was found to be critical for radiotracer recovery from the vial (Fig. 1B). This formulation enabled 1-step radiolabelling of oxine with commercial $89 \mathrm{Zr}(0.1-25 \mathrm{MBq})$ in $5 \mathrm{~min}$ and radiotracer stability for 1 week (Fig. 1C). The formulation also enabled the formation of 64Cu-oxine and 68Ga-oxine. Labelling efficiency of WBC with $89 \mathrm{Zr}$-oxine was $49 \pm 6 \%$, compared to $89 \pm 10 \%(\mathrm{P}<0.0001$, $\mathrm{n}=10$ ) for $111 \mathrm{In}$-oxine (Fig. 1D). The intracellular retention of $89 \mathrm{Zr}$ and cell viability $24 \mathrm{~h}$ after radiolabelling with $89 \mathrm{Zr}$ were $87 \pm 3 \%$ and $93 \pm 5 \%$, respectively, with no significant difference compared to 111In (Fig. 1D). The leukocyte chemotaxis assay revealed no significant differences between $89 \mathrm{Zr}$ - and 111In-labelled WBC (Fig. 1E). Similarly, there were no significant differences in DNA damage between cells labelled with 89Zr-oxine or 111In-oxine, although 
both show significantly more damage compared to unlabelled cells (Fig. 1F). When labelling mixed WBC, the uptake of $89 \mathrm{Zr}$ per cell was similar in most cell populations. Conclusion: Here we present a formulation that allows reproducible, rapid, stable, high-yield preparation of $89 \mathrm{Zr}$ oxine in a single step, without further processing of commercially available 89Zr. Furthermore, our results using a clinical protocol demonstrate that $89 \mathrm{Zr}$-oxine is a suitable PET alternative to 111In-oxine for WBC imaging as radiotracer retention, cell viability and functionality were similar. This will facilitate the clinical translation of cell tracking by PET using 89Zr-oxine.

References: (1) Ponomarev V. Molecular Imaging and Biology (2017) 19:379-384, doi:10.1007/s11307-017-1069-7 (2) Fruhwirth GO et al. Molecular Imaging and Biology (2018) 20:696-704, doi:10.1007/s11307-018-1254-3 (3) Charoenphun P et al. European Journal of Nuclear Medicine and Molecular Imaging (2015) 42:278-87, doi:10.1007/s00259-014-2945-x (4) Sato N et al. Radiology (2015) 275:490-500, doi:10.1148/radiol.15142849 (5) Man F et al. Molecular Therapy (2019) 27(1):219-29, doi:10.1016/j.ymthe.2018.10.006

\section{Image/Figure:}

https://www.xcdsystem.com/wmis/abstract/File6959/GA83 ImageFigure 0421062047.png

Full Name of Abstract's 1st Author : Francis Man

Complete Status: Complete

First Name: Francis

Last Name: Man

Email: francis.man@kcl.ac.uk

Organization: King's College London

Country: United Kingdom 


\title{
ID: GA85 \\ Dual modality imaging of human breast cancer cells derived from patient brain metastases
}

\author{
Natasha Knier, Western University, nknier@uwo.ca
}

\section{Category: Oncology}

Abstract Body : Introduction: Recently, there is momentum towards implementing patientderived xenograft (PDX) models in cancer research to better reflect the histopathology, tumor behavior, and metastatic properties observed in the original tumor1,2. Visualizing disease progression in these models could allow for evaluating therapies and identifying biomarkers in a clinically relevant way. Importantly, PDXs have shown to be predictive of clinical outcomes and are superior to cell lines for preclinical drug evaluation and personalized medicine strategies3-5. To study PDX cells preclinically, we have used both bioluminescence imaging (BLI) and Magnetic Particle Imaging (MPI). MPI is a new imaging technology that allows for direct detection and quantification of iron nanoparticles, which can be complemented by bioluminescence imaging (BLI), a commonly used modality that images viable cells. Here, we report the first successful iron labeling of breast cancer cells derived from patient brain metastases and dual modality imaging during tumor development. Objective: To utilize dual modality imaging to monitor PDX cells from breast cancer brain metastases in vivo Methods: Luciferase/GFP+ expressing human breast cancer PDX cells (F2-7, from the Cittely lab at UC Denver) were cultured and labeled with $54 \mathrm{uL}$ and $108 \mathrm{uL}$ of micron-sized paramagnetic ironoxide nanoparticles (MPIO). An additional trial using 54uL of MPIO was tested with a magnetic plate for magnetofection. Perl's Prussian Blue (PPB) staining was used to visualize cells with intracellular iron and labeling efficiency was determined using ImageJ. To evaluate the relationship between cell number and BLI signal, F2-7 cells were seeded at concentrations of $2 \times 105$ cells, 6x105 cells, and 1x106 cells. NOD/SCID/ILIIrg-/- $(\mathrm{n}=2)$ mice received injections of 1x106 iron-labeled F2-7 cells into the fourth mammary fat pad (MFP) from the 54uL labeling trial. BLI was performed on day $4,28,35,42$, and 49 and assessed for signal. MPI was performed on day 26, 27 and 42 and assessed for signal, and iron content was quantified. Ex vivo MPI of an iron-labeled and unlabeled MFP tumour was performed to further validate results. Results: PPB staining revealed iron labeling of F2-7 was successful for the 54uL of MPIO trial, showing $81.80 \pm 10.14 \%$ labeling efficiency with viability over $90 \%$. Labeling was deemed unsuccessful for the $108 \mathrm{uL}(27.51 \pm 1.19 \%)$ and $54 \mathrm{uL}$ with magnetic plate $(5.55 \pm 1.65 \%)$ trials (Fig. 1A). In vitro BLI revealed a significant positive correlation between the number of cells and BLI signal $(\mathrm{R} 2=0.9664)$ (Fig. $1 \mathrm{~B}, \mathrm{C})$. In vivo BLI revealed signal from the F2-7 cells injected into the MFP, and over time, signal increased with tumor development (Fig. 1D). MPI revealed signal from the PDX tumors as hot spots (Fig. 1E), with iron content increasing over time. Ex vivo MPI showed signal from an iron-labeled MFP tumor and no signal from an unlabeled MFP tumor. Conclusions: Here, we demonstrate the first application of high sensitivity MPI to monitor the growth of an iron-labeled PDX MFP tumour and the first successful labeling of PDX cells with iron-oxide nanoparticles. PDX cells are more sensitive to labeling with iron nanoparticles than other cell lines. We found that iron content increased over 
time, which was unexpected as iron content dilutes with cell proliferation6, however, death of iron-labeled cells and subsequent cell uptake by tumour-associated macrophages or circulating tumour cells returning to the primary tumour is a possible explanation for this finding. Using MPI in conjunction with BLI provided valuable information on both the iron content and the viability of the labeled MFP tumours and revealed that our cell populations were viable and proliferating. The ability to employ dual modality imaging of PDX cells provides a powerful system to study models that better predict metastatic behaviour for identification of biomarkers and personalized therapies targeting breast cancer brain metastasis.

References: 1. DeRose, Y. S., Wang, G., Lin, Y. C., Bernard, P. S., Buys, S. S., Ebbert, M. T., ... $\&$ Neumayer, L. (2011). Tumor grafts derived from women with breast cancer authentically reflect tumor pathology, growth, metastasis and disease outcomes. Nature medicine, 17(11), 1514. 2. Cassidy, J. W., Caldas, C., \& Bruna, A. (2015). Maintaining tumor heterogeneity in patient-derived tumor xenografts. Cancer research, 75(15), 2963-2968 3. Zhang, X., Claerhout, S., Pratt, A., Dobrolecki, L. E., Petrovic, I., Lai, Q., ... \& Wong, H. (2013). A renewable tissue resource of phenotypically stable, biologically and ethnically diverse, patient-derived human breast cancer xenograft models. Cancer research 4. Landis, M. D., Lehmann, B. D., Pietenpol, J. A., \& Chang, J. C. (2013). Patient-derived breast tumor xenografts facilitating personalized cancer therapy. Breast Cancer Research, 15(1), 1. 5. Reyal, F., Guyader, C., Decraene, C., Lucchesi, C., Auger, N., Assayag, F., ... \& De Cremoux, P. (2012). Molecular profiling of patient-derived breast cancer xenografts. Breast cancer research, 14(1), R11 6. Economopoulos, V., Chen, Y., McFadden, C., \& Foster, P. J. (2013). MRI detection of nonproliferative tumor cells in lymph node metastases using iron oxide particles in a mouse model of breast cancer. Translational oncology, 6(3), 347.

\section{Image/Figure:}

https://www.xcdsystem.com/wmis/abstract/File6959/GA85 ImageFigure_0609011929.jpg

Image/Figure Caption: Figure 1. A) Perl's Prussian Blue (PPB) stain identifying iron in blue of three iron-labeling trials of F2-7 cells with micron-sized paramagnetic iron-oxide nanoparticles (MPIO). B) F2-7-luc/GFP+ cells seeded at varying concentrations and imaged with BLI. C) A significant, positive correlation between the number of cells and BLI signal $(\mathrm{R} 2=0.9664)$. D) Bioluminescence imaging (BLI) of a mouse at day 4, 28, 35, 42, and 49 showing signal over time. E) Magnetic particle imaging (MPI) of an in vivo mammary fat pad tumour of a mouse at day 27 and 42, and an ex vivo mammary fat pad tumour from another mouse removed at day 26.

Full Name of Abstract's 1st Author : Natasha Knier

Complete Status: Complete

First Name: Natasha

Last Name: Knier

Email: nknier@uwo.ca 
Organization: Western University

Country: Canada 


\title{
ID: GA86 \\ In vitro assessment of biomarker encapsulation for liquid biopsies
}

\author{
Matthew Chen, University of Toronto, mattalex.chen@mail.utoronto.ca
}

\section{Category: Oncology}

\begin{abstract}
Body : Introduction Liquid biopsies is a technique that involves the use of molecular biomarkers to characterize tumours. 1 Focused ultrasound in conjunction with phospholipid microbubbles circulating in the bloodstream, can promote the release of molecular biomarkers from the brain into the bloodstream by locally disrupting the blood brain barrier in rodent models. 2 However, as the concentration of biomarkers that are released at safe acoustic pressures is low, it can be challenging to detect these biomarkers upon dilution in circulation. 3 The aim of this study was to develop a system for encapsulating biomarkers at their source, prior to dilution, for their subsequent isolation and identification using echogenic microbubbles. Our hypothesis is that after exposure to focused ultrasound, phospholipid microbubble debris can spontaneously form liposomes that encapsulate local biomarkers in situ. By co-injecting fluorescent molecules with the microbubbles, the newly formed liposomes will also contain fluorescent molecules enabling identification and isolation with optical techniques after blood sampling. We evaluate the capture of a fluorescent identifier and a surrogate biomarker (i.e., DsRed2) after phospholipids microbubble exposure to focused ultrasound as a function of acoustic pressure in a flow channel. Methods Perfluorobutane (C4F10, Synquest Laboratories) microbubbles were synthesized in-house using the phospholipids DPPA, DPPC, and DPPE-mPEG5k (Avanti Polar Lipids). U87-RFP cells (Anticancer) were grown in DMEM with 10\% fetal bovine serum (Wisent). To verify echogenicity, microbubbles (gas volume concentration $=\sim 1.4 \times 106$ $\mu \mathrm{m} 3 / \mathrm{mL}$ ) were flowed through a tissue-mimicking flow phantom (8mm channel, Model 523A, ATS Laboratories-Phantoms) and imaged using an iU22 clinical ultrasound system (Philips) with an L9-3 transducer $(\mathrm{MI}=0.1)$. Microbubbles under B-mode imaging were assessed in 20s segments every two minutes over 14-minute intervals and repeated at 0,30, 60, and 90 minutes after the initial microbubble activation. Analysis was conducted using MATLAB (MathWorks). For cavitation experiments, microbubbles with DsRed2 as a surrogate protein biomarker and fluorescein as a fluorescent identifier were flowed through a $1.5 \%$ agarose ultrasound phantom with a $1.4 \mathrm{~mm}$ channel. Focused ultrasound $(1.15 \mathrm{MHz}$, duty cycle of $1 \%$, 0-3.04 MPa) was applied with a transducer diameter of $75 \mathrm{~mm}$ and a focal length of $60 \mathrm{~mm}$ (FUS Instruments, Toronto, CAN). Microbubbles and cavitation debris were separated and washed with phosphate buffered saline ( $\mathrm{pH}$ 7.4) using centrifugation. Size distribution and concentration of isolated microbubbles and collected material post-cavitation were measured using Multisizer 4e (Beckman-Coulter) with 10 and $20 \mu \mathrm{m}$ apertures. Microscopy was conducted using an Axiovert 200M (Zeiss). Results Acoustically responsive perfluorobutane microbubbles were synthesized (concentration $8.2 \pm 0.7 \times 109$ microbubbles $/ \mathrm{mL}$, peak diameter of $1.1 \pm 0.1 \mu \mathrm{m})$. Synthesized microbubbles retained $31 \pm 4 \%$ of their starting population over $55 \mathrm{~min}$ in vitro and were verified to be echogenic through contrast ultrasound. Microbubble cavitation by focused ultrasound resulted in the formation of new particles, ranging in diameter from $\sim 1000 \mathrm{~nm}$ to below to limit of measurement (i.e., $\pm 3 \mathrm{~nm}$. The concentration of the particles
\end{abstract}


formed increased with focused ultrasound $(2.50 \pm 0.42 \times 107,4.26 \pm 0.02 \times 107,4.78 \pm 0.06 \times 107$, and 4.6 $\pm 0.22 \times 107$ particles $/ \mathrm{mL}$, from pressures of $0,1.06,2.07$, and $3.07 \mathrm{MPa}$, respectively). Fluorescence microscopy showed that the particles formed after cavitation in the presence of both DsRed2 and fluorescein were able to successfully capture both molecules in comparison to control microbubbles (not exposed to focused ultrasound). Conclusions Synthesized phospholipid-stabilized microbubbles were echogenic and stable, similar to commercial ultrasound contrast agents. After microbubble exposure to focused ultrasound, new particles were formed however more acoustic pressure did not result in significantly more particles. These particles encapsulated local molecules from the microenvironment at pressures upwards of 1.06 MPa. In the future, the potential of this system to encapsulate biomarkers in vivo with fluorescent identifiers as a tool for cancer diagnosis treatment planning will be evaluated.

References: 1. Gorgannezhad, Lena, Muhammad Umer, Md Nazmul Islam, Nam-Trung Nguyen, and Muhammad J. A. Shiddiky. 2018. "Circulating Tumor DNA and Liquid Biopsy: Opportunities, Challenges, and Recent Advances in Detection Technologies." Lab on a Chip 18 (8): 1174-96. 2. Bettegowda, Chetan, Mark Sausen, Rebecca J. Leary, Isaac Kinde, Yuxuan Wang, Nishant Agrawal, Bjarne R. Bartlett, et al. 2014. "Detection of Circulating Tumor DNA in Early- and Late-Stage Human Malignancies." Science Translational Medicine 6 (224): 224ra24-224ra24. 3. Pacia, Christopher Pham, Lifei Zhu, Yaoheng Yang, Yimei Yue, Arash Nazeri, H. Michael Gach, Michael R. Talcott, Eric C. Leuthardt, and Hong Chen. 2020. "Feasibility and Safety of Focused Ultrasound-Enabled Liquid Biopsy in the Brain of a Porcine Model." Scientific Reports 10 (1): 7449.

\section{Image/Figure:}

https://www.xcdsystem.com/wmis/abstract/File6959/GA86_ImageFigure_0614035256.png

Image/Figure Caption: Figure. (A) Microbubbles synthesized had a polydisperse population centered around $1.2 \mu \mathrm{m}$ as measured with a particle sizer. (B) Microbubbles reduced in population in vitro in static conditions (measured up to $55 \mathrm{~min}$ ). Microscope images of particles formed after cavitation of microbubbles at 3.07 MPa in the presence of fluorescent molecules. (C) Particles were presumed to be liposomes as they were characteristically difficult to observe under DIC conditions and were able to co-localize water-soluble fluorescent molecules as shown by (D) fluorescein inside the liposome aggregate was observed with fluorescence microscopy (E), along with the DsRed2 protein.

Complete Status: Complete

First Name: Matthew

Last Name: Chen

Email: mattalex.chen@mail.utoronto.ca

Organization: University of Toronto

Country: Canada 


\title{
ID: GA89 \\ TREM1-PET detects maladaptive innate immune responses associated with active disease in a mouse model of relapsing-remitting multiple sclerosis
}

\author{
Aisling Chaney, Stanford University, aislingc@stanford.edu
}

\section{Category: Neuroscience}

\begin{abstract}
Body : OBJECTIVES: Relapsing-remitting multiple sclerosis (RRMS) is a central nervous system (CNS) disorder characterized by unpredictable cycles of disease exacerbation, in the form of injurious inflammation and demyelination, followed by periods of significant remission. While several disease-modifying immunomodulatory therapies are available for RRMS1, disease manifestation and treatment response are highly variable and difficult to predict in patients. Thus, there is a critical need for non-invasive imaging strategies to monitor active disease and therapeutic response in vivo. Myeloid cells (e.g., macrophages, microglia, monocytes and neutrophils) are fundamental to the progression and remission of MS, with activated macrophages and microglia being the predominant immune cells associated with acute and chronic-active CNS lesions 2. Positron emission tomography (PET) imaging of triggering receptor expressed on myeloid cells 1 (TREM1) is a highly specific method for detecting pathogenic peripheral CNS-infiltrating myeloid cells, and has been shown to identify progressive disease in the experimental autoimmune encephalomyelitis (EAE) mouse model of MS. Here, we investigate the ability of TREM1-PET to identify active disease and distinguish recovery in the clinically relevant relapsing-remitting (RR-EAE) mouse model of MS. METHODS: Animals: SJL mice were induced with RR-EAE using PLP139-151 emulsified in immune adjuvant. Mice were scored daily and grouped by disease stage; 1 st wave of EAE disease (exhibiting paresis and/or paralysis, $n=3$ ), remission (exhibiting complete recovery following initial EAE symptoms, similar to what occurs in RR-MS patients, $n=5$ ) and relapse (exhibiting paresis after complete recovery, $n=4$ ) (Suppl. Fig1A). Results were compared with naïve mice ( $\mathrm{n}=13)$. Imaging: Anti-TREM1 monoclonal antibody (mAb) was DOTA-conjugated and radiolabeled with $64 \mathrm{Cu}$. PET/CT imaging was performed $20 \mathrm{~h}$ post-injection of [64Cu]TREM1-mAb $(95-120 \mu \mathrm{Ci},>99 \% \mathrm{RCP})$. Ex vivo: Following PET, mice were perfused with saline to remove any unbound intravascular tracer and spinal cords were analyzed via highresolution autoradiography. PET analysis: Thresholding of CT images was used to identify vertebrae and enable quantification of lumbar and thoracic/cervical spinal cord regions. A mouse brain atlas was used to quantify whole brain, medulla, pons and cerebellum regions.

RESULTS: TREM1-PET images demonstrated high signal in the spinal cords of RR-EAE mice during periods of active disease (i.e., EAE 1st wave and relapse), and markedly reduced signal during remission (Fig. 1Ai). Quantification of PET images revealed that tracer binding in the lumbar and cervical/thoracic spinal cord of EAE mice during 1st wave and relapse disease was significantly increased compared to naïve mice (p Suppl. Fig1Bi-iv). Specifically, there was increased tracer binding in the medulla of EAE 1st wave and relapse mice compared to EAEremission mice ( $\mathrm{p}$ CONCLUSION: TREM1-PET successfully identified CNS-infiltrating proinflammatory myeloid cells during active RR-EAE disease. Importantly during disease remission, TREM1-PET signal was significantly reduced. Therefore, PET imaging of TREM1
\end{abstract}


has high potential for enabling accurate and early detection of active diseases and prediction of therapeutic response for individual RR-MS patients. Future studies will investigate the specificity of [64Cu]TREM1-mAb in this mouse model using a control mAb.

References: 1. Vargas DL., Tyor WR. Update on disease-modifying therapies for multiple sclerosis. J Investig Med., 2017; 65:883-891. 2. Steinman, L., Immunology of Relapse and Remission in Multiple Sclerosis. Annu. Rev. Immunol., 2014; 32 57-81. 3. Robinson et al. The experimental autoimmune encephalomyelitis (EAE) model of MS: utility for understanding disease pathophysiology and treatment. Handb Clin Neurol. 2014; 173-189

\section{Image/Figure:}

https://www.xcdsystem.com/wmis/abstract/File6959/GA89_ImageFigure_0422011307.jpg

Full Name of Abstract's 1st Author : Aisling Chaney

Complete Status: Complete

First Name: Aisling

Last Name: Chaney

Email: aislingc@stanford.edu

Organization: Stanford University

Country: United States 


\title{
ID: GA91 \\ Cardiomyocyte-Targeting Nanocomposites with Multiparametric MR Theranostic Imaging in Myocardial Injury Animal Models
}

\author{
Chia-Hao Su, Institute for Translational Research in Biomedicine, Kaohsiung Chang Gung \\ Memorial Hospital, chiralsu@gmail.com
}

Category: New Chemistry, Biology \& Bioengineering

\begin{abstract}
Body : Congestive heart failure is the leading cause of morbidity and mortality in the United States and throughout the worldwide. There are an estimated 4.9 million cases of heart failure in the United States alone. Currently, $80 \%$ of men and $70 \%$ of women die within 8 years of diagnosis. In the area of myocardial diagnosis, despite echocardiography provides the advantages in cardiac imaging, cardiac MR imaging has emerged as non-invasive imaging modality in cardiovascular application, and not only could provide excellent spatial resolution, tissue contrast, flexibles imaging plan selection in 3-dimention, but also use for assessment of ventricular volume, mass and rejection in ventricles research. Nanocomposite-based MR molecular imaging set a unique platform for cellular tracking, targeted diagnostic studies, and image-monitored therapy. Herein, we have developed an iron oxide NPs@neuregulin-1 (NRG1) nanocarrier, and conjugated tagged with cardiac specific peptide (CSP) on the particle surface. These nanocarriers are able to target cardiomyocytes and to protect cells, both in vitro and in vivo. For in vitro studies, the nanocomposite could target on the H9C2 cells and showed higher targeting efficiency of the damage H9C2 cells than health one. In addition, we also evaluated the cardio protectivity of NRG1, the isoproterenol (ISO)-induced damaged cells were treated with NRG-1 and nanocarrier, we could find the differentiated $\mathrm{H} 9 \mathrm{C} 2$ cell were pretreated with NRG-1, and the cell viability has been shown to be cardio protective in ISO-induced cytotoxicity. Furthermore, the in vivo MR cardiac imaging also shown the therapeutic effects of the nanocarrier for in situ ISO-treated mice. The nanocarrier were administrated by intravenous injection at a Fe dosage of $5 \mathrm{mg} \mathrm{kg}-1$. Efficacy of the NPs as a contrast agent, measured as signal changes was monitored with pre- and post-injection MRI. Finally, we also applied diffusion weighted MR imaging to observe the changes in connectivity and map the density of axon and fiber tracts between key nuclei involved in brain stem cardiovascular regulation, and find the causal relationship between brain and heart during myocardial injury. In addition, we predict that the investigations on myocardial injury prognosis will benefit from the technical developments in imaging sensitivity and specificity, enhancing the accuracy of the non-invasive tools, or even be included in the standard health examination in the near future.
\end{abstract}

Full Name of Abstract's 1st Author : Chin-Lai Lee

Complete Status: Complete

First Name: Chia-Hao 
Last Name: $\mathrm{Su}$

Email: chiralsu@gmail.com

Organization: Institute for Translational Research in Biomedicine, Kaohsiung Chang Gung Memorial Hospital

Country: Taiwan 


\title{
ID: GA94 \\ Click-activated anti-ACQ NIR-cyanine with Z/E-isomerization for in vitro and in vivo tumor imaging
}

\author{
Xianghan Zhang, Xidian University, xhzhang@xidian.edu.cn
}

Category: New Chemistry, Biology \& Bioengineering

\begin{abstract}
Body : Cancer is a serious hazard to human life and health. Encouragingly, nearinfrared (NIR) fluorescent technology has employed in cancer diagnostics, image-guided surgery and therapeutic evaluation owing to its low autofluorescence and relative deep-penetration. Small organic fluorescence dyes, especially Cyanines have been enabled great progress in bioimaging area, but several hurdles have hindered its use for tumor molecular imaging. For example, the only FDA approved NIR fluorophore, indocyanine green (ICG), still suffers from small Stokes shifts, aggregation-caused quenching (ACQ), short retention time in vivo and so on. In this work, we have developed a new method to anti-ACQ and large stokes shift by clickactivated E/Z-isomerization with steric hindrance (Figure 1A). By simply introducing nitrogensubstituents with click-moiety on the meso-heptamethine chain, the cyanines firstly show large Stokes shifts, subsequently a transformation from E-isomer to Z-isomer and larger out-ofplanarity are activated once click-chemistry reacted. This method could inhibit stacking and enable significant imaging enhancement for traditional cyanines, that anti-ACQ probe generates fluorescence enhancement with increasing concentrations (Figure 1B). Furthermore, the imaging performance of anti-ACQ probe was investigated in vitro and in vivo. The obvious NIR fluorescence signals were observed for the CyP7T group, revealing the large Stokes shift; antiACQ group exhibited bright NIR signals in cytoplasmic regions, suggesting that aggregates were suppressed markedly by click-activated Z/E-isomerization (Figure 1C). As shown in Figure 1D, the anti-ACQ probe markedly achieves high target-to-background ratios for tumor imaging in vivo. The proposed method shows a new path for improving imaging sensitivity by clickactivated anti-ACQ not only for cyanines but also can be expanded for other organic fluorophores.
\end{abstract}

\section{Image/Figure:}

https://www.xcdsystem.com/wmis/abstract/File6959/GA94_ImageFigure_0610053211.pdf

Complete Status: Complete

First Name: Xianghan

Last Name: Zhang

Email: xhzhang@xidian.edu.cn

Organization: Xidian University 
Country: China 


\title{
ID: GA95 \\ A GSH-activated NIR-II fluorescent-MRI dual-modality probe for sensitive diagnosis of breast cancer
}

\author{
Zhongliang Wang, Xidian University, wangzl@xidian.edu.cn
}

\section{Category: Oncology}

\begin{abstract}
Body : Surgical resection is still the main treatment for malignant tumors, but clinicians usually rely on palpation and visual examination for surgery, which makes it difficult to accurately locate tumors and achieve complete clearance [1]. The visualization of malignant tumors can provide accurate guidance for surgical treatment. However, the traditional molecular imaging probe has the disadvantages of low sensitivity and poor signal-to-noise, and the singlemode imaging method is difficult to provide accurate imaging information in all aspects of the tumor. In recent years, multi-modality molecular imaging probes with the intelligent response has played a crucial role in promoting the development of clinical research on malignant tumors [2], especially the dual-mode imaging probe combined with magnetic resonance (MRI) and nearinfrared second region (NIR-II) fluorescence has a unique advantage in the diagnosis and treatment of malignant tumors [3]. Herein, we developed a GSH-activated NIR II/MRI dualmodality nanoprobe to sensitively detect the breast cancer and precisely resect the tumor by imaging guided surgery. The nanoprobe was synthesized by coating NIR-II dyes (IR820) with manganese dioxide. In this state, dye molecules in the nanoprobes aggregated and induced the fluorescence quenching. Once the probe reached the tumor site through the passive targeting effect, highly expressed GSH in the tumor cells can degrade manganese dioxide, so that the dyes can restore the molecular state and the fluorescence can be restored. Meanwhile, the probe disintegrates to release the divalent manganese ion and enhance the MRI signal. MRI can provide anatomic images with infinite penetration depth and high spatial resolution, which can promote the preoperative diagnosis of deep tumors. NIR-II fluorescence in turn produces highly sensitive images that help guide tumor surgery. The probe has the size with about $100 \mathrm{~nm}$ (Figure 1A) and showed 65-fold fluorescence enhancement once GSH activation, which resulted from high specificity and low background signal of the probe. After 6 hours of in vivo imaging, the MRI signal value at the tumor was enhanced by about 2 times (Figure 1C), and the signal-tonoise ratio of IR820@MnO2 was nearly 10 times higher than that of IR820@SiO2 (Figure 1D). The study indicates that IR820@MnO2 can be used as a powerful tool for accurate intraoperative imaging of tumors
\end{abstract}

References: [1] F. Bray, et al. Global cancer statistics. CA Cancer J Clin. 2018, 68, 394-424. [2] X. Chen, et al. Simultaneous Fenton-Like Ion Delivery and Glutathione Depletion by MnO2Based Nanoagent Enhances Chemodynamic Therapy. Angew. Chem.-Int. Edit. 2018, 57, 49024906. [3] D. Ye, et al. Activatable NIR Fluorescence/MRI Bimodal Probes for in Vivo Imaging by Enzyme-Mediated Fluorogenic Reaction and Self-Assembly. J. Am. Chem. Soc. 2019, 141, 10331-10341.

\section{Image/Figure:}


Complete Status: Complete

First Name: Zhongliang

Last Name: Wang

Email: wangzl@xidian.edu.cn

Organization: Xidian University

Country: China 


\title{
ID: GA96 \\ Biomolecular ultrasound imaging of in vivo phagolysosomal function
}

\author{
Bill Ling, California Institute of Technology, blling@caltech.edu
}

Category: Systemic Diseases (Kidney, Liver and Pancreas)

\begin{abstract}
Body : Phagocytosis and lysosomal degradation of pathogens and debris by the innate immune system is critical to organismal homeostasis, and malfunctions of this system underlie several major diseases [1]. However, evaluation of phagolysosomal function in vivo is challenging because most imaging agents compatible with non-invasive techniques comprise non-biodegradable synthetic materials. Unable to be metabolized, these agents are instead retained indefinitely within the cell [2]. Here, we overcome this challenge using an ultrasound contrast agent based on gas vesicles $(\mathrm{GVs})$, a unique class of air-filled nanostructures originating in aquatic microbes as a means to regulate buoyancy [3]. Since GVs are thermodynamically stable particles whose ability to scatter sound waves is dependent on the integrity of their allprotein shells, we hypothesized that their elimination kinetics may be tracked with ultrasound and used as a reporter of phagocytic and lysosomal function. We show that upon systemic administration into mice, GVs are filtered from the blood by liver macrophages, as demonstrated through a combination of vascular and liver ultrasound, pharmacological perturbation, and tissue immunohistology. After phagocytic uptake, GVs undergo lysosomal processing, resulting in a loss of ultrasound contrast, as shown through in vitro live cell optical and acoustic microscopy. The temporal dynamics of ultrasound signal in the blood and liver provide parameters for a twocompartment model describing the pharmacokinetics of GV clearance and degradation, enabling quantitation of reticuloendothelial function. When applied to two models of liver dysfunctionpharmacologic depletion of macrophages and diet-induced non-alcoholic steatohepatitis - this approach detected uptake and elimination rates that were significantly reduced compared to those of healthy mice. Thus, the combination of nanoscale, lysosomally-degradable contrast agents with quantitative ultrasound imaging enables non-invasive imaging and quantitation of phagolysosomal function.
\end{abstract}

References: 1. Chow, A. et al. Studying the mononuclear phagocyte system in the molecular age. Nat. Rev. Immunol. 11, 788-798 (2011). 2. Poon, W. et al. Elimination Pathways of Nanoparticles. ACS Nano 13, 5785-5798 (2019). 3. Shapiro, M. G. et al. Biogenic gas nanostructures as ultrasonic molecular reporters. Nat. Nanotechnol. 9, 311-316 (2014).

\section{Image/Figure:}

https://www.xcdsystem.com/wmis/abstract/File6959/GA96_ImageFigure_0419101628.jpg

Full Name of Abstract's 1st Author : Bill Ling

Complete Status: Complete 
First Name: Bill

Last Name: Ling

Email: blling@caltech.edu

Organization: California Institute of Technology

Country: United States 


\title{
ID: GA97 \\ H2S-activated NIR-II-Emitting nanoprobes for accurate diagnosis of colon cancer
}

\author{
Zhongliang Wang, Xidian University, wangzl@xidian.edu.cn
}

\section{Category: Oncology}

\begin{abstract}
Body : Colon cancer is one of the leading cancers with high morbidity and mortality, and early diagnosis of colon cancer still faces serious challenges to improve accuracy and specificity. At present, the most-common clinical diagnostic methods for colon cancer, including colonoscopy and biopsy, have non-negligible drawbacks and limitations, such as false positive or false negative results. In this work, we choose hydrogen sulfide (H2S) as a specific mark of colon tumor and develop a H2S-activated NIR-II-Emitting fluorescent probe, achieving a noninvasive diagnostic method with high sensitivity and specificity for colon cancer. Compared with current NIR fluorescent probes $(700-900 \mathrm{~nm})$, the second near-infrared wavelength window (NIR-II, 1,000-1,700 nm) fluorescence has low scattering and absorption in tissues, making it has higher resolution and sensitivity in vivo and more accurate for reflection of lesions. Among them, the lanthanide downconversion nanoparticals (DCNPs) have advantages of low toxicity, long stokes shift, the adjustable size and so on. As we know, Prussian blue (PB), a clinical drug approved by FDA, have great biosafety, compatibility and stable structure. More importantly, PB possesses strong NIR-II absorbance and can be decomposed by the redox reaction of Fe $3+$ and $\mathrm{H} 2 \mathrm{~S}$ in aqueous solution with a high sensitivity. Consequently, luminescence of PB-coated DCNPs can be quenched by Förster resonance energy transfer (FRET) and be recovered once $\mathrm{H} 2 \mathrm{~S}$ activation. Herein, we synthesized core-shell-structure lanthanide nanoparticles as luminescent materials, which can be excited by $808 \mathrm{~nm}$ laser to emit $1060 \mathrm{~nm}$ fluorescence. Then we developed a new method to grow PB on the surface of DCNP (Fig. 1a). DCNP @ PB has the size with about $120 \mathrm{~nm}$, and has a stable crystal structure. Further, the probe showed increased luminescence by 30 fold after $\mathrm{H} 2 \mathrm{~S}$ response (Figs. 1b and c), and excellent imaging with high signal-to-noise $(>10)$ in mice model of colon tumor (Fig. $\mathrm{d}$ and e). Thus, PB-coated DCNPs may be an ideal NIR-II imaging probe with high specificity and sensitivity for diagnosis and imaging-guided surgery in colon cancer.
\end{abstract}

References: [1] R. Labianca, et al. Colon Cancer. Crit. Rev. Oncol./Hematol. 2010, 74, 106-133. [2] C. Szabo, et al. Tumor-Derived Hydrogen Sulfide, Produced By Cystathionine- $\beta$-Synthase, Stimulates Bioenergetics, Cell Proliferation, And Angiogenesis In Colon Cancer. PNAS. 2013, 110, 12474-12479. [3] Y. Liu, et al. Rationally Designed Pure-Inorganic Upconversion Nanoprobes For Ultra-Highly Selective Hydrogen Sulfide Imaging And Elimination In Vivo. Chem. Sci. 2019, 10, 1193-1200. [4] Z. Deng, et al. Endogenous H2S-Triggered In Situ Synthesis of NIR-II-Emitting Nanoprobe for In Vivo Intelligently Lighting Up Colorectal Cancer. iScience. 2019, 17, 217-224.

\section{Image/Figure:}


Complete Status: Complete

First Name: Zhongliang

Last Name: Wang

Email: wangzl@xidian.edu.cn

Organization: Xidian University

Country: China 


\title{
ID: GA98 \\ Persistent regulation of tumor microenvironment by Dual drugs-loaded MOFs to enhance photodynamic therapy
}

\author{
Zhongliang Wang, Xidian University, wangzl@xidian.edu.cn
}

\section{Category: Oncology}

\begin{abstract}
Body : The therapeutic effect of reactive oxygen species (ROS)-involved cancer therapies, such as photodynamic therapy (PDT), is significantly limited by the shortage of oxygen-substrates concentration in tumor microenvironment and the intracellular adaptive redox homeostasis. The insufficient stock of oxygen (O2) and overexpressed antioxidant glutathione (GSH), greatly limit the efficiency of intracellular ROS accumulation during PDT. At present, there has been few reports about nanosystems which play both the role of hypoxia mitigation and GSH depletion. In view of the diploma of intracellular ROS accumulation, we propose a strategy to increase output while reduce loss. Broadening source and throttling expenditure of ROS, will bring the intracellular oxidative stress to an improved level, so as to enhance the efficacy of PDT. For adapting to the time window of PDT, the persistence of microenvironment improvement must be considered. It has been found that some small molecular inhibitors can effectively and persistently regulate the material metabolism and energy metabolism.[1] Such as, Metformin (MET) can reduce the oxygen consumption of malignant cells for a long time by regulating cell respiration,[2] and L-Buthionine-sulfoximine (BSO) can maintain the low intracellular GSH level by blocking its synthesis pathway.[3] In order to construct a drugdelivery nanosystem with capability of rapid uptake and responsive release, Metal-Organic Framework materials (MOFs) was selected as the vehicle to ensure the controllable structure and high drug-loading content. Herein, we report a sustained tumor microenvironment modulator and photodynamic therapeutic agent, (BSO-Met@PCN)@Lip-RGD, which consist of dual-drug (BSO and Met)-supported porphyrinic MOFs (PCN) with further surface modification of lipid bilayer and RGD Peptide. Once uptake by tumor cells, nano-agents rapidly release small molecule drugs at a low $\mathrm{pH}$ environment. Met made the mitochondrial respiratory chain suppressed, so as to reduce the metabolic oxygen consumption in advance, providing sufficient oxygen concentration to improve the ROS generation efficiency in photosensitive reaction. BSO, released at the same time, rapidly downregulate the level of intracellular GSH, thus weakening the ability of tumor cells to resist oxidative stress, so as to protect ROS from scavenging. Under laser irradiation, ROS erupted due to photosensitive reaction of PCN in the tumor cells regulated by microenvironment in advance, and broke the redox homeostasis to induce the rapid cell cytotoxicity. Experiments, both in in vitro and vivo, showed persistent microenvironment improvement according to PA imaging (Fig. 1A) and ROS effective accumulation (around sixfold improvement shown in Fig. 1B), as well as significant tumor inhibition in animal models (Fig. 1C). This ROS enhancement strategy shows the clinical potential of photodynamic therapy in tumor eradication, and is expected to provide benefits for various ROS-involved cancer therapies.
\end{abstract}


References: [1] Y. Fan, et al. Modulation of Intracellular Oxygen Pressure by Dual-Drug Nanoparticles to Enhance Photodynamic Therapy. Adv Funct Mater. 2019, 29, 1806708. [2] X. Song, et al. Liposomes co-loaded with metformin and chlorin e6 modulate tumor hypoxia during enhanced photodynamic therapy. Nano Res. 2017, 10, 1200-1212 [3] H. Min, et al. Biomimetic Metal-Organic Framework Nanoparticles for Cooperative Combination of Anti-angiogenesis and Photodynamic Therapy for Enhanced Efficacy. Adv Mater. 2019, 31, 1808200.

\section{Image/Figure:}

https://www.xcdsystem.com/wmis/abstract/File6959/GA98 ImageFigure 0418075811.png

Complete Status: Complete

First Name: Zhongliang

Last Name: Wang

Email: wangzl@xidian.edu.cn

Organization: Xidian University

Country: China 


\title{
ID: GA99
}

Noble metal nanoclusters as biocompatible probes for fluorescence imaging

\author{
Karolína Šišková, Faculty of Science, Palacký University in Olomouc, \\ karolina.siskova@upol.cz
}

Category: New Chemistry, Biology \& Bioengineering

\begin{abstract}
Body : Fluorescent gold nanoclusters (AuNCs) were synthesized using microwaveassisted procedure, $\mathrm{Au}(\mathrm{III})$ ions, and bovine serum albumin (BSA) of different purity and fatty acids (FAs) content [1]. Selected spectroscopic (circular dichroism, infrared absorption, fluorescence, dynamic light scattering) and other (zeta potential measurements) experimental techniques were employed for the characterization of our samples. Further addition of fatty acids positively affected fluorescence features of AuNCs as is demonstrated in Figure 1; namely, quantum yield increased from $5.7 \pm 0.2 \%$ to $7.2 \pm 0.3 \%$. The long-term stability followed for several months was investigated and a non-monotonic time behaviour of integral fluorescence intensity was revealed if the samples were not dialyzed. Simultaneously, AuNCs did not affect viability of HepG2 cell line in selected time interval, which as evidenced by Alamar Blue assay. Acknowledgement: This research was funded by Grant Agency of the Czech Republic, grant number 19-03207S, and Internal Grant Agency of Palacký University, grant number IGA_PrF_2019_030.

References: [1] P. Andrýsková et al., Nanomaterials 2020, 10, 343, doi: 10.3390/nano10020343

\section{Image/Figure:}

https://www.xcdsystem.com/wmis/abstract/File6959/GA99 ImageFigure 0418103635.jpg

Complete Status: Complete

First Name: Karolína

Last Name: Šišková

Email: karolina.siskova@upol.cz

Organization: Faculty of Science, Palacký University in Olomouc

Country: Czech Republic 


\title{
ID: GA100 \\ A sequentially activatable Optical/Photoacoustic ultrasensitive probe for imaging tumor acidity and GSH
}

\author{
Zhongliang Wang, Xidian University, wangzl@xidian.edu.cn
}

\section{Category: Oncology}

\begin{abstract}
Body : Early accurate diagnosis of tumor can greatly improve the effectiveness of cancer treatment. For cancer detection, the precisely imaging demands great sensitivity and specificity, and preferably multiple biomarkers. However, the sensitivity and the imaging contrast of currently available probes are substandard for diagnosis. Directly targeting multiple biomarkers with different imaging probes are limited by the intrinsic overexpression levels of the molecular targets. In order to improve the sensitivity, some optical imaging probes with highperformance have been developed such as quantum dots and rare-earth nanoprobes. Yet the specificity of these probes is typically restricted to passive targeting or single biomarker. In addition, near-infrared fluorescence imaging (NIR) only permits shallow visualization, despite it has high spatial and temporal resolution and low biological autofluorescence. This disadvantage can be overcome by photoacoustic (PA) imaging. Therefore, the development of a NIR/PA dualmode probe with high sensitivity and high specificity is essential for the accurate diagnosis of tumors. Herein, we report a NIR/PA dual-mode nanoprobe IR780SS@CaP that responds to two different targeted stimuli successively, to greatly increase the detection accuracy. We chose two established tumor microenvironment signals, namely low extracellular $\mathrm{pH}$ and high intracellular glutathione (GSH). As we know, solid tumor tissues accumulate acid metabolites due to their rapid proliferation, resulting in a weakly acidic microenvironment. In addition, different GSH content is an important distinction between tumor cells and normal cells. The fluorescence sensitivity of the probe was tested, and it was found that under continuous stimulation of acidity and GSH, the signal was nearly 100 times higher than that of the control group without stimulation (Figs. A and B). Fluorescent imaging of tumor-bearing mice was performed by intravenous injection of the probe (Fig. C). The tumor-to-normal tissue ratio $(\mathrm{T} / \mathrm{N})$ was over 10 after injection of the probe for 24 hours. At the same time, by photoacoustic imaging of tumorbearing mice, we can accurately obtain information on the deep tissues of tumors (Fig. D). IR780SS@CaP is continuously activated with acidity and GSH to further enhance imaging sensitivity and specificity and improve imaging accuracy through dual-mode imaging. We anticipate that the probe will inspire the design of other molecular imaging probes for the ultrasensitive and specific early diagnosis of cancer or for image-guided surgery.
\end{abstract}

References: [1] X. Zheng, et al. Successively Activatable Ultrasensitive Probe for Imaging Tumor Acidity and Hypoxia. Nat. Biomed. Eng. 2017, 1, 0057. [2] C. Yan, et al. A SequenceActivated and Logic Dual-channel Fluorescent Probe for Tracking Programmable Drug Release. Chem. Sci. 2018, 9, 6176-6182. [3] Y. Tang, et al. "Dual Lock-and-Key"-Controlled Nanoprobes for Ultrahigh Specific Fluorescence Imaging in the Second Near-Infrared Window. Adv. Mater. 2018, 30, 1801140. 


\section{Image/Figure:}

https://www.xcdsystem.com/wmis/abstract/File6959/GA100 ImageFigure 0419020811.jpg

\section{Complete Status: Complete}

First Name: Zhongliang

Last Name: Wang

Email: wangzl@xidian.edu.cn

Organization: Xidian University

Country: China 


\title{
ID: GA103 \\ Construction of biodegradable mesoporous silica nanocomposites for in vivo breast cancer chemotherapy combined with photothermal therapy
}

\author{
Hanrui Li, Xidian University, hrli330@163.com
}

\section{Category: Oncology}

\begin{abstract}
Body : Construction of biodegradable mesoporous silica nanocomposites for in vivo breast cancer chemotherapy combined with photothermal therapy Hanrui Li1, Yingying Guo1, Wenhua Zhan2, Qi Zeng1, Yun Zeng1, Ke Li3, Xueli Chen1 and Yonghua Zhan1,* 1Engineering Research Center of Molecular \& Neuro Imaging of the Ministry of Education, School of Life Science and Technology, Xidian University, Xi'an 710071, Shaanxi, China 2Department of Radiation oncology, General Hospital of Ningxia Medical University, Yinchuan 750004, Ningxia, China 3Shaanxi Key Laboratory of Brain Disorders, Shaanxi Key Laboratory of Ischemic Cardiovascular Disease, Institute of Basic and Translational Medicine, Xi'an Medical University, Xi' an 710021, Shaanxi, China *Corresponding E-mail: yhzhan@xidian.edu.cn, Tel.: +86 2981891070. Abstract Breast cancer is the highest incidence of cancer in Chinese women. Surgical resection is a main method for the treatment of early breast cancer, but surgical treatment has greatly reduced the quality of life of patients, and lacks better treatment for tumor metastases. Therefore, the development of new treatments for breast cancer is urgently needed. Photothermal therapy is a new method for tumor treatment with great development potential. Many advantages, such as non-invasive, less pain for patients, short treatment time and small side effects, which makes photothermal therapy an important means of tumor treatment. However, due to the limitation of the laser irradiation, the penetrating ability is weak, and the heat distribution is uneven, which greatly reduces the effect of photothermal therapy and even cause tumor metastasis. Chemotherapy is one of the most effective means of cancer treatment. The drugs can reach the metastatic sites and achieve a good therapeutic effect. But chemotherapy also has many disadvantages, such as drug toxicity and drug resistance. In view of the above, chemotherapy and photothermal therapy are combined as a treatment for breast cancer, which have better synergistic therapeutic effects. In this report, biodegradable mesoporous silica nanoparticles (bMSN NPs) were synthesized, which has a large specific surface area and a controlled pore size. IR780 can be loaded into mesopores by electrostatic adsorption (bMSN@IR780). Hyaluronic acid encapsulates doxorubicin (DOX) to form DOX@HA, and DOX@HA is loaded on the surface of bMSN@IR780 to form bMID NPs. In addition, bMSN NPs can be loaded with IR780 and DOX at the same time, which can realize the synergistic treatment of photothermal therapy and chemotherapy, while reduce the toxicity of DOX, prolong circulation time, and achieve continuous release of drugs. The TEM image showed that bMID NPs were about $74 \mathrm{~nm}$. The results of in vitro experiments proved that bMID NPs had lower toxicity, good targeting and stability, and good antitumor effects. In order to verify the in vivo distribution and antitumor effect of bMID NPs, a subcutaneous tumor model of breast cancer was established. The results showed that bMID NPs had good antitumor effect and provided a good platform for the treatment of breast cancer. Key words: Chemotherapy; Photothermal therapy; Doxorubicin; IR780; Biodegradable mesoporous silica nanoparticles;
\end{abstract}


Breast cancer. Figure. (A) Schematic illustration of bMID NPs for breast cancer chemotherapy and photothermal therapy; (B) Morphology, size and zeta potential of bMSN NPs and bMID NPs; (C) In vitro internalization of bMID NPs; (D) In vivo tumor inhibition of bMID NPs.

\section{Image/Figure:}

https://www.xcdsystem.com/wmis/abstract/File6959/GA103_ImageFigure 0419073626.jpg

Full Name of Abstract's 1st Author : Hanrui Li

Complete Status: Complete

First Name: Hanrui

Last Name: Li

Email: hrli330@163.com

Organization: Xidian University

Country: China 


\title{
ID: GA104 \\ Pifithrin- $\mu$ loaded gold@melanin nanoprobes for CT/MRI-guided photothermal therapy amplified by pro-apoptotic unfolded protein response cascades
}

\author{
Shouju Wang, 1st affiliated hospital of Nanjing Medical University, \\ shouju.wang@gmail.com
}

Category: Oncology

\begin{abstract}
Body : Background: The efficacy of photothermal therapy has been limited by the upregulated Binding immunoglobulin protein (Bip) in cancer cells, which maintains the homeostasis of cells and results in resistance. Object: To construct a theranostic nanoprobe that can deliver a Bip inhibitor (pifithrin- $\mu, P E S$ ) to cancer to enhance the therapeutic efficacy of photothermal therapy, as well as to locate the cancer by MRI and CT. Method: The gold@melanin nanoparticles were synthesized by spontaneous polymerization of dopamine in a mild alkaline condition over gold nanoparticles. The PES was attached to the surface of melanin nanoparticles through pi-pi stacking. Results: The nanoprobes (PES-Au@PDA, 63.3 $\pm 3.1 \mathrm{~nm}$ ) has a loading efficiency of PES approximately $40 \%$. The reported nanoprobes release PES upon laser irradiation. In vitro and in vivo studies demonstrate that PES-Au@ PDA can activate proapoptotic unfolded protein response (UPR) cascades, leading to remarkably improved photothermal therapy efficiency to glioma. Furthermore, the distribution of nanoprobes can be monitored by MRI and CT in vivo. Conclusion: A versatile theranostic nanoprobe was synthesized to guide photothermal therapy by MRI/CT and promote the therapeutic efficacy by selectively activating pro-apoptotic UPR cascade pathways.
\end{abstract}

References: C. Hetz, The unfolded protein response: controlling cell fate decisions under ER stress and beyond, Nat. Rev. Mol. Cell Biol. 13 (2012) 89-102, https://doi.org/10.

1038/nrm3270. Y. Liu, P. Bhattarai, Z. Dai, X. Chen, Photothermal therapy and photoacoustic imaging via nanotheranostics in fighting cancer, Chem. Soc. Rev. 48 (2019) 2053-2108, https://doi.org/10.1039/c8cs00618k.

\section{Image/Figure:}

https://www.xcdsystem.com/wmis/abstract/File6959/GA104_ImageFigure 0419032337.png

Complete Status: Complete

First Name: Shouju

Last Name: Wang

Email: shouju.wang@gmail.com

Organization: 1st affiliated hospital of Nanjing Medical University 
Country: China 


\title{
ID: GA106 \\ Simple Mn-based complex detects stimulated zinc secretion in the prostate by MRI
}

\author{
Manyu Jin, Massachusetts General Hospital, mjin2@mgh.harvard.edu
}

\section{Category: New Chemistry, Biology \& Bioengineering}

\begin{abstract}
Body : Zinc is one of the most essential and abundant microelements in the human body, required for hundreds of cellular processes and a cofactor for more than 300 enzymes (1). The prostate gland contains the highest levels of zinc in the body and it has been shown that these levels are markedly reduced in prostate cancer while maintaining high levels in other pathologically benign prostatic conditions. Recently, we demonstrated that the prostate secretes high levels of zinc when stimulated by a bolus of glucose in fasted animals (2). This mechanism allowed for extracellular molecular probes to detect secreted zinc in the prostate and thus detect malignant lesions by MRI. Here we designed a Mn-based zinc responsive MRI contrast agent to detect zinc secretion in the prostate by MRI. The mechanism of detection relies on the idea that a chelated paramagnetic ion, for example $\mathrm{Mn}(\mathrm{II})$, can undergo transmetallation by high concentrations of zinc. This zinc displacement would then result in an increase in transverse (r2) and longitudinal ( $\mathrm{r} 1$ ) relaxivity rendering it more efficient at shortening the neighboring water protons longitudinal (R1) and transverse (R2) relaxation rates. And this in turn translates into an increase in signal in a T1-weighted MRI image (Figure 1a). Here, we took advantage of this displacement by choosing the appropriate chelate in order to obtain an optimal displacement rate to image zinc secretion in the prostate. MnEDTA is commercially available, low cost, and easy to manufacture. Because EDTA has a higher affinity for zinc than manganese, $\mathrm{Mn}$ (II) can be displaced when exposed to high concentrations of zinc (3). Figure $1 \mathrm{~b}$ shows the $\Delta \mathrm{R} 2$ of MnEDTA and MnDOTA, which is a stable chelate unable to undergo transmetallation after zinc exposure and thus can be used as a negative control. As expected, $\triangle \mathrm{R} 2$ of MnEDTA increased slowly after adding zinc while MnDOTA exhibited almost no change in R2 indicating that MnEDTA is better suited for imaging zinc secretion. Figure 1c shows the protocol for the in vivo MRI studies on healthy male mice. Briefly, we infused MnEDTA, and MnDOTA i.v for 20 minutes until the concentration of probe in the prostate reached a steady state, then we administered a bolus of glucose or saline i.p while continuously infusing Mn-probe for another 20 minutes at which time we stopped the infusion. Images were collected serially throughout the duration of the infusion (40 mins) and during an additional 20 minutes without infusion to monitor probe wash out. Figure $1 \mathrm{~d}$ shows the change in prostate signal normalized to back muscle for both MnEDTA and MnDOTA after glucose or saline injections. It is evident that the prostate gland (more specifically the ventral lobe) is significantly more enhanced for the animals who received glucose and MnEDTA compared to MnDOTA. This indicates that the zinc secreted as a result of glucose stimulation displaces the Mn from EDTA but not from DOTA. Figure 1e shows the quantitative results where it is evident that in the ventral lobe only the MnEDTA plus glucose group shows significant increase in signal after $\sim 30$ minutes post glucose stimulation. In conclusion, these results suggest that by careful selection of a chelate, it is
\end{abstract}


possible to detect zinc secretion by displacing the $\mathrm{Mn}(\mathrm{II})$ ion from its ligand only after glucosestimulated zinc secretion in the prostate.

References: References: 1. Kelleher S L, et al. Adv. Nitr. 2011, 2(2), 101-111. 2. a) Jordan, M. Veronica Clavijo, et al, PNAS. Int. Ed. 2016, August 25, E5464-E5471; b) Jordan, M. Veronica Clavijo, et al, Inorg. Chem. 2019, 58, 13654-13660 3. Kálmán, Ferenc K, et al, Inorg. Chem. 2012, 51, 10065-10067

\section{Image/Figure:}

https://www.xcdsystem.com/wmis/abstract/File6959/GA106 ImageFigure 0609032423.jpg

Image/Figure Caption: Figure 1. a) Mechanism of signal enhancement. b) In vitro zinc competition showing $\Delta \mathrm{R} 2$ after adding 1eq of zinc. (Conditions: Final concentration of $\mathrm{Mn}=0.2$ $\mathrm{mM}$, Final concentration of $\mathrm{Zn}=0.2 \mathrm{mM}, 37^{\circ} \mathrm{C}, \mathrm{B} 0=1.5 \mathrm{~T}$, plasma). c) In vivo studies design, mice fasted overnight underwent serial T1-weighted MR imaging ( $\mathrm{N}=4$ for each group). Infusion conditions: $0.3 \mathrm{mmol} / \mathrm{kg} / \mathrm{hr}$ for MnEDTA and MnDOTA; Rate: $5 \mathrm{ul} / \mathrm{min}$, Bolus conditions: $2.2 \mathrm{mmol} / \mathrm{kg}$ D-Glucose. 2D T1-weighted gradient echo, TE/TR $=2.9 / 279.2 \mathrm{~ms}$, Flip angle $=60 \mathrm{deg}$, Averages $=4, \mathrm{FOV}=33 \times 33 \mathrm{~mm}$, Matrix $=140 \times 140$, Slice thickness $=1 \mathrm{~mm}$, B0 $=4.7$ T. d) Representative images for each group showed the difference between probes and saline or glucose. e) Change in normalized prostate signal relative to pre-glucose/saline administration.

Full Name of Abstract's 1st Author : Manyu Jin

Complete Status: Complete

First Name: Manyu

Last Name: Jin

Email:mjin2@mgh.harvard.edu

Organization: Massachusetts General Hospital

Country: United States 


\title{
ID: GA108 \\ Mesenchymal Stem Cell Derived Exosomes Reduce Amyloid- $\beta$ Burden, Downregulate HDAC4 and Ameliorate Alzheimer's Disease Deficits
}

\author{
Yi-An Chen, , yachen0414@gmail.com
}

\section{Category: New Chemistry, Biology \& Bioengineering}

\begin{abstract}
Body : Background: Accumulation of extracellular $\beta$-amyloid (A $\beta$ ) plaques within the brain is unique to Alzheimer's disease (AD) and thought to induce synaptic deficits, neuronal alterations and neurodegeneration. Optimal therapies should tackle core pathophysiology of AD as well as prevent the decline in memory and cognitive functions. This study aimed to evaluate the therapeutic performance of mesenchymal stem cell (MSC) derived exosomes, one of the secreted forms of extracellular vesicles encapsulating variety of MSC factors, on AD and investigate possible working mechanism. Methods: A 3D human neural cell culture model overexpressing human APP with double FAD mutations in human neuroblastoma cell line was established and co-cultured with purified MSC derived exosomes. AD transgenic mice were intravenously weekly injected with MSC derived exosomes for 4 weeks. 2-[18F]Fluoro-2-deoxyd-glucose ([18F]FDG) and novel object recognition (NOR) testing were performed before/after treatment. The AD-related pathology and the expression of neuronal memory/synaptic plasticityrelated genes were also evaluated. Results: MSC derived exosomes reduce A $\beta$ expression and restore the expression of neuronal memory/synaptic plasticity-related genes in a 3D human neural cell model of AD. [18F]FDG-PET imaging and cognitive assessment reveal a significant improvement in brain glucose metabolism and cognitive function in AD transgenic mice. Moreover, treatment of MSC derived exosomes regulated the phase of neurons and astrocytes in the brain of AD mice. Further, a potential biomarker-HDAC4 was identified for diagnosis and treatment for $\mathrm{AD}$. Conclusions: These results not only demonstrate the therapeutic mechanism but also provide evidence that MSC-derived exosomes can potentially serve as cell-free therapies for AD.
\end{abstract}

References: 1. Jia Q, Deng Y, Qing H. Potential therapeutic strategies for Alzheimer's disease targeting or beyond beta-amyloid: insights from clinical trials. BioMed research international. 2014; 2014: 837157. 2. Menting KW, Claassen JA. beta-secretase inhibitor; a promising novel therapeutic drug in Alzheimer's disease. Frontiers in aging neuroscience. 2014; 6: 165. 3. Duncan T, Valenzuela M. Alzheimer's disease, dementia, and stem cell therapy. Stem cell research \& therapy. 2017; 8: 111. 4. Teixeira FG, Carvalho MM, Neves-Carvalho A, Panchalingam KM, Behie LA, Pinto L, et al. Secretome of mesenchymal progenitors from the umbilical cord acts as modulator of neural/glial proliferation and differentiation. Stem cell reviews. 2015; 11: 288-97. 5. Zilka N, Zilkova M, Kazmerova Z, Sarissky M, Cigankova V, Novak M. Mesenchymal stem cells rescue the Alzheimer's disease cell model from cell death induced by misfolded truncated tau. Neuroscience. 2011; 193: 330-7. 6. Oh SH, Kim HN, Park HJ, Shin JY, Lee PH. Mesenchymal Stem Cells Increase Hippocampal Neurogenesis and Neuronal Differentiation by Enhancing the Wnt Signaling Pathway in an Alzheimer's Disease Model. Cell transplantation. 2015; 24: 1097-109. 7. Phinney DG, Pittenger MF. Concise Review: MSC-Derived Exosomes 
for Cell-Free Therapy. Stem cells. 2017; 35: 851-8. 8. Mendt M, Rezvani K, Shpall E. Mesenchymal stem cell-derived exosomes for clinical use. Bone marrow transplantation. 2019; 54: 789-92. 9. Pant S, Hilton H, Burczynski ME. The multifaceted exosome: biogenesis, role in normal and aberrant cellular function, and frontiers for pharmacological and biomarker opportunities. Biochemical pharmacology. 2012; 83: 1484-94. 10. Zhang Y, Liu Y, Liu H, Tang WH. Exosomes: biogenesis, biologic function and clinical potential. Cell \& bioscience. 2019; 9: 19.

\section{Image/Figure:}

https://www.xcdsystem.com/wmis/abstract/File6959/GA108 ImageFigure 0420010904.jpg

Full Name of Abstract's 1st Author : Yi-An Chen

Complete Status: Complete

First Name: Yi-An

Last Name: Chen

Email: yachen0414@gmail.com 


\title{
ID: GA112 \\ pO2-weighted Imaging In Vivo by Delayed Fluorescence of Intracellular Protoporphyrin IX
}

\author{
Marek Scholz, Dartmouth college, marasolc@centrum.cz
}

\section{Category: Instrumentation}

\begin{abstract}
Body : Hypoxic regions of tumors are known to be significantly less responsive to treatment by radiotherapy, chemotherapy, and particularly photodynamic therapy (PDT), which is an alternative treatment modality for cancer and various skin diseases. PDT consumes oxygen in treated tissues, which in turn lowers the treatment efficacy. Tools for online monitoring of intracellular pO2 for treatment planning and control are thus highly desirable. This work reports on imaging of tissue oxygen (pO2) in vivo by means of time-gated wide-field lifetime imaging of the microsecond-scale delayed fluorescence from Protoporphyrin IX (PpIX). PpIX is produced intracelullarly from a clinically used aminolevulinic acid (ALA) by most cells. Here, experiments were conducted in vivo in mouse skin, mouse subcutaneous tumors, and human skin after topical application of Ameluz(TM) cream or by intraperitoneal injection of $250 \mathrm{mg} / \mathrm{kg}$ ALA. Importantly, ALA is probably the most widely used drug for clinical PDT of skin diseases and cancer. Therefore, the need for monitoring of pO2 in PDT treatment could be met by detecting the long-lived luminescence of PpIX, without the necessity of using any additional luminescence probe. This approach could be used also beyond photodynamic therapy in diagnostic imaging, if lower non-therapeutic light doses are used. The lifetime of delayed fluorescence (DF) is strongly modulated by oxygen, which efficiently quenches the intermediate triplet state of PpIX.[1] Lower oxygen translates to longer lifetimes, up to $2 \mathrm{~ms}$ for hypoxic/anoxic regions. PpIX in the tissues was excited by a $635 \mathrm{~nm}$ pulsed laser and the DF emission was captured by an intensified time-gated camera. Collecting a series of gated exposures at specific time-delays after the excitation pulse enabled us to reconstruct pO2 images by fitting the decay kinetics in each pixel.[2,3] Macroscopic lifetime-based images were found to be related to the tissue oxygenation, and pO2-weighted images were obtained with a low light dose $(\sim 10 \mathrm{~mJ} / \mathrm{cm} 2)$, spatial resolution of $0.3 \mathrm{~mm}$, dynamic range from 1 to $50 \mathrm{mmHg}$, and capture times of several seconds, which allows for diagnostic imaging. Further, dose-rate effect on oxygen consumption during PDT was investigated, showing that fluence rate of $1.2 \mathrm{~mW} / \mathrm{cm} 2$ did not cause any appreciable oxygen depletion, whereas $6 \mathrm{~mW} / \mathrm{cm} 2$ caused severe oxygen depletion after radiant exposures of only $0.4-0.8 \mathrm{~J} / \mathrm{cm} 2$. Reoxygenation after PDT was studied too, showing that oxygen recovery can take many minutes, depending on the light dose delivered. pO2 distribution in general was spatially very non-uniform, which underlines the necessity of using imaging over point-detection. Further, the mitochondrial pO2 strongly correlated with breathing rate. In conclusion, time-gated imaging of PpIX DF seems to be a unique tool for direct on-line monitoring of $\mathrm{pO} 2$ changes during PDT with a promising potential not only for research purposes, but also for translation into clinics.
\end{abstract}

References: [1] Mik, E. G. et al. Mitochondrial PO2 measured by delayed fluorescence of endogenous protoporphyrin IX. Nat. Methods 3, 939-45 (2006). [2] Scholz, M., Cao, X., Gunn, 
J. R., Brůža, P. \& Pogue, B. pO2 -weighted imaging in vivo by delayed fluorescence of intracellular Protoporphyrin IX. Opt. Lett. 45, 284 (2020). [3] Scholz, M., Petusseau, A. F., Gunn, J. R., Chapman, M. S., \& Pogue, B.Imaging of hypoxia, oxygen consumption and recovery in vivo during ALA-photodynamic therapy using delayed fluorescence of

Protoporphyrin IX. Photodiagnosis and Photodynamic Therapy (2020), accepted

\section{Image/Figure:}

https://www.xcdsystem.com/wmis/abstract/File6959/GA112 ImageFigure 0421033719.png

Complete Status: Complete

First Name: Marek

Last Name: Scholz

Email: marasolc@centrum.cz

Organization: Dartmouth college

Country: United States 


\title{
ID: GA113 \\ Refined Analysis of [11C]L-Glutamine Metabolism in Triple Negative Breast Cancer Xenograft Model
}

\author{
Prashanth Padakanti, University of Pennsylvania, ppada@pennmedicine.upenn.edu
}

\section{Category: Oncology}

\begin{abstract}
Body : OBJECTIVE: Glutamine is the most abundant amino acid present in the human body. Aggressive cancer cells such as triple negative breast cancer (TNBC) utilize L-glutamine (L-Gln) as a nutrient for their energy production and biosynthesis, which is necessary for their cellular growth and proliferation. These tumors deploy glutaminolysis, a metabolic pathway, which starts with conversion of L-Gln to L-glutamate (L-Glu) by glutaminase (GLS). The L-Glu then enters the TCA cycle to produce various intermediates needed for the survival and growth of cancer cells. Here we sought to describe the glutamine metabolism in human TNBC
\end{abstract} (HCC1806) xenografted in mice using [11C]L-Gln PET. METHODS: Human TNBC cells exhibiting high GLS activity[1,2] were xenografted in athymic nu/nu mice. Mice were treated with GLS inhibitor (CB839, provided by Calithera Biosciences Inc., South San Francisco, CA) at $200 \mathrm{mg} / \mathrm{kg}$ or vehicle (VEH) by oral gavage twice daily for $2 \mathrm{~d}$ ( 4 doses). After completion of treatment, mice were injected with [11C]L-Glutamine $(\sim 70-420 \mu \mathrm{Ci}$ in $\sim 0.2 \mathrm{~mL})$, and euthanized at 10, 20 and 30min p.i. ( $\mathrm{n} \geq 3$ for each time point). Blood and tumor were collected upon euthanasia, and extracted (tumor tissue extracted after homogenization) for HPLC analyses of metabolites. The fraction of [11C]L-glutamine and [11C]L-glutamate in both blood and tumor samples were measured. The volatile metabolite $([11 \mathrm{C}] \mathrm{CO} 2)$ information was obtained by treating the blood and tumor tissue samples under acid/base conditions. The radioactive fractions from $\mathrm{HPLC}$, and the samples from the [11C]CO2 experiments were measured on gamma counter. Radioactivity information from these metabolite fractions will be used in the compartment model proposed to investigate [11C]L-Gln PET as a possible pharmacodynamic marker of GLS-targeted therapy. RESULTS: In VEH-treated mice, [11C]LGlutamine metabolite studies (Fig.1A-B) revealed that the majority of the radioactivity was in the form of [11C]L-glutamate in both blood and tumor. This increase in the ratio of [11C]Glu to [11C]Gln was observed in all time points (up to $30 \mathrm{~min}$ ). In contrast, in the CB839 treated group (Fig.1C-D), [11C]Gln was the major radioactive compound in the tumor over time, consistent with blockade of Gln to Glu conversion resulted from GLS inhibition. After $10 \mathrm{~min}$, [11C]Glu level in the blood was increased accompanied by a decrease in [11C]Gln, consistent with the fact that GLS activity in normal tissues (liver) was not inhibited by CB839[1]. The [11C]CO2 levels accounted for about $20-25 \%$ of blood radioactivity after $20 \mathrm{~min}$ (Fig.1E), and slightly lower levels were observed in the tumor, suggesting labelled $\mathrm{CO} 2$ is likely generated by organs outside the tumor. The data obtained from this metabolite studies will be compared with the \%ID values obtained from the microPET images. The combined information from metabolism studies, and the microPET images will allow us to estimate the input function of [11C]Gln for modeling of [11C]PET of the tumor. CONCLUSION: Mouse xenograft studies with [11C]L-glutamine demonstrated rapid metabolism of the radiotracer through the glutaminase enzyme to [11C]Lglutamate. Upon glutaminase inhibition, [11C]L-glutamine became the dominant species. 
$[11 \mathrm{C}] \mathrm{Glu}$ and $[11 \mathrm{C}] \mathrm{CO} 2$ in the blood in CB839 treated mice suggest peripheral metabolism of [11C]Gln by non-tumoral tissues. These metabolite studies will be utilized to develop an input function and compartmental model for [11C]Gln PET studies to advance this technology.

ACKNOWLEDGMENTS: We thank Calithera Biosciences Inc. for providing CB839 and Dr. Marina Gelman for insightful discussions. Supported by NIHR01233771, R21CA198563 \& R01CA211337.

References: 1. Gross MI. et al. Mol Cancer Ther 2014;13:890-901 2. Zhou R. et al. Cancer Res 2017;77:1476-84

\section{Image/Figure:}

https://www.xcdsystem.com/wmis/abstract/File6959/GA113 ImageFigure 0610044611.jpg

Image/Figure Caption: Figure 1. [11C]L-Glutamine metabolism in HCC1806 mice ( $\mathrm{n} \geq 3$ ): 1A) Vehicle treatment group, blood 1B) Vehicle treatment group, tumor 1C) CB839 treatment group, blood 1D) CB839 treatment group, tumor 1E) Volatile radioactivity ([11C]CO2) from whole blood and tumor

Complete Status: Complete

First Name: Prashanth

Last Name: Padakanti

Email: ppada@pennmedicine.upenn.edu

Organization: University of Pennsylvania

Country: United States 


\title{
ID: GA114 \\ In Vitro and In Vivo Bioluminescence Imaging of Mesenchymal Stromal Cells Expressing a Luciferase Analogue
}

\author{
Francesco Amadeo, NHS Blood and Transplant Liverpool, \\ francesco.amadeo@liverpool.ac.uk
}

Category: New Chemistry, Biology \& Bioengineering

\begin{abstract}
Body : Introduction. Bioluminescence imaging (BLI) is widely used in preclinical research to identify and track the biodistribution and fate of cells administered in small animals. The firefly Luciferase (FLuc) and D-luciferin system has been used for years for this purpose, but its light emission peak is at $560 \mathrm{~nm}$, resulting in poor light transmission in biological tissues. A novel BLI system, composed of a luciferin analogue, the Akalumine- $\mathrm{HCl}$, and a mutated form of the FLuc, the AkaLuc has been recently developed $[1,2]$. This BLI system, called AkaBLI, has an emission peak at around $650 \mathrm{~nm}$, implying an increase in the tissue penetration of the signal, and has been reported to give much stronger signal in BLI; with claims of single cell detection in small animals [1]. However, to date, this system has not been validated for applications in regenerative medicine. Aim. The present study was performed to (i) test whether the introduction of the FLuc and AkaLuc reporters has any impact on the properties of umbilical cord mesenchymal stromal cells (UC-MSCs) and (ii) directly compare the sensitivity of the FLuc and AkaLuc systems for imaging MSCs in vivo. Methods. Lentiviral vectors were used to generate two populations of UC-MSCs expressing either FLuc or AkaLuc under the same promoter. A bicistronic construct including a fluorescent reporter, ZsGreen, was used to sort the transduced cells and obtain pure populations. The FLuc and AkaLuc expressing cells were characterised and compared with untransduced cells in terms of doubling time, morphology, and expression of established MSC markers. An IVIS Spectrum system was used to characterise the two BLI systems in vitro and to assess their sensitivity in vivo, via the imaging of MSCs intravenously administrated to C57BL/6 albino mice. Results. FLuc and AkaLuc cells displayed similar properties to untransduced cells in terms of morphology, proliferation (doubling time of 50 hours circa), and expression of MSC markers (positive for CD44, CD73, CD90 and CD105). The in vitro BLI analysis revealed that in the AkaLuc system, light emission saturates at relatively low substrate concentrations $(80 \mu \mathrm{M}$ Akalumine-HCl). FLuc, on the other hand, requires higher substrate concentrations to saturate the signal (5.12 mM D-Luciferin). At saturation, the light output of the cells at $660 \mathrm{~nm}$ was similar, with 1346 photons/s/cell and 1139 photons/s/cell for AkaLuc and FLuc cells, respectively. In vivo, both systems were able to show that MSCs were delivered to the lungs after IV administration. However, the signal intensity of cells expressing AkaLuc was not stronger than FLuc when standard substrate doses were used (30 mM Akalumine- $\mathrm{HCl}$ and $47 \mathrm{mM}$ D-Luciferin). By increasing the D-luciferin dose to 144.5 $\mathrm{mM}$, we were able to increase the light output obtained with the FLuc system, which was then 4.5-fold stronger than AkaLuc. Conclusions. Despite the promising properties of the AkaBLI system, we saw no advantage in using this system for tracking UC-MSCs in vivo. Our data suggest that FLuc/D-Luciferin is still among the most sensitive BLI reporter systems that are available.
\end{abstract}


References: 1. Iwano, S., et al., Single-cell bioluminescence imaging of deep tissue in freely moving animals. Science, 2018. 359(6378): p. 935-939. 2. Kuchimaru, T., et al., A luciferin analogue generating near-infrared bioluminescence achieves highly sensitive deep-tissue imaging. Nat Commun, 2016. 7: p. 11856.

Complete Status: Complete

First Name: Francesco

Last Name: Amadeo

Email: francesco.amadeo@liverpool.ac.uk

Organization: NHS Blood and Transplant Liverpool

Country: United Kingdom 


\title{
ID: GA115 \\ Severity assessment: Influence of repeated CT measurements on animal welfare and study results
}

\author{
Jasmin Baier, University Clinic RWTH Aachen, jbaier@ukaachen.de
}

\section{Category: Oncology}

\begin{abstract}
Body : Introduction In preclinical research, non-invasive imaging is often used to observe physiological processes. For clinical applications most imaging devices are considered to be safe nowadays. However, in preclinical research necessary anesthesia, contrast agent application or the handling procedure can induce stress 1 and, therefore, influence animal welfare and study results. Low-dose radiation ( 2 and affect animal behavior3. So far, no systematic study has been published, examining the effect of repeated CT measurements on animal welfare and study results in mice. Thus, we investigated the influence of standard contrast-enhanced FMT/CT and CT protocols on welfare and physiological parameters. Methods For this purpose, healthy BALB/C mice were scanned with FMT/CT (4 mGy) or CT (50 mGy) three times per week over a four weeks period. Imaging was performed under isoflurane anesthesia for $30 \mathrm{~min}$. Since mice hair scatters light, animals that underwent optical imaging (FMT/CT) were repeatedly shaved before the measurements and ICG (120 nmol/ kg BW) was used as contrast agent. Regular CT scans were performed with Imeron 400 (2 mg/kg BW). A Rotarod test and blood pressure measurements were performed twice per week to investigate behavioral alterations. The general condition of the animals was evaluated daily using a score sheet. For the investigation of physiological parameters, blood was taken for hemograms and organs like liver, spleen, lung, heart and kidneys were removed for histological examinations. Results/ Discussion The investigations have shown that even after repeated contrast-enhanced FMT/CT and CT measurements neither the Rotarod tests nor the blood pressure measurements showed any alterations. However, according to the score sheets, the burden of the animals measured with FMT/CT (4 mGy) exceeded a medium range after first time shaving, which can be attributed to a reversible weight loss after the procedure. Furthermore, alterations of physiological parameters were observed. Mice imaged by CT ( $50 \mathrm{mGy})$ showed lower liver, spleen and kidney weights than control animals. Moreover, a reduction of the white blood cell count and an increase of leukocyte infiltration in liver and spleen were observed in FMT/CT and CT imaged mice (figure 1) which are in line with the literature4. Conclusion Based on the results the burden of the animals during CT imaging can be considered mild, whereas FMT/CT imaging led to a medium burden. The observed alterations in physiological parameters need to be further investigated. Therefore, DNA strand breakage and fecal corticosterone metabolites examinations are currently ongoing. Furthermore, healthy mice will be imaged without contrast agents to unravel the mechanisms behind the observations and distinguish between possible influences of the contrast agent and the radiation dosage. Moreover, the influence of CT measurements on study results will be investigated in 4T1-breast cancer bearing mice. Acknowledgement This research was funded by the Deutsche Forschungsgesellschaft (DFG) within the framework of the research group FOR2591 (project number 321137804) and the Research Training Group 2375 "Tumortargeted Drug Delivery".
\end{abstract}


References: (1) Hurst, J. L.; West, R. S. Taming Anxiety in Laboratory Mice. Nat.Methods 2010, 7(10), 825-826. (2) Tang, F. R.; Loke, W. K.; Khoo, B. C. Low-Dose or Low-Dose-Rate Ionizing Radiation-Induced Bioeffects in Animal Models. J.Radiat.Res. 2017, 58(2),165-182. (3) Raber, J.; Allen, A. R.; Weber, S.; Chakraborti, A.; Sharma, S.; Fike, J. R. Effect of Behavioral Testing on Spine Density of Basal Dendrites in the CA1 Region of the Hippocampus Modulated by (56)Fe Irradiation. Behav.BrainRes. 2016, 302, 263-268. (4) Maks, C. J.; Wan, X. S.; Ware, J. H.; Romero-Weaver, A. L.; Sanzari, J. K.; Wilson, J. M.; Rightnar, S.; Wroe, A. J.; Koss, P.; Gridley, D. S.; Slater, J. M.; Kennedy, A. R. Analysis of White Blood Cell Counts in Mice after Gamma- or Proton-Radiation Exposure. Radiat.Res. 2011, 176 (2), 170-176.

\section{Image/Figure:}

https://www.xcdsystem.com/wmis/abstract/File6959/GA115 ImageFigure_0609040923.png

Image/Figure Caption: Figure 1: Influence of contrast-enhanced FMT/CT and CT imaging on physiological parameters and animal welfare. A: Repeated CT measurements did not increase the overall burden of the animals, but first time shaving for FMT/CT measurements led to a reversible decrease of body weight. B: Liver weight significantly decreased in the Imeron 400 $\mathrm{CT}$ group in comparison to the other investigated groups. C: Mice showed a lower spleen weight after repeated CT measurements with Imeron 400 contrast enhancement. D: The white blood cell count was slightly decreased in CT investigated mice in comparison to the control and isoflurane group. E: Leukocyte infiltration (CD45) in mice livers was significantly increased in CT and $\mathrm{CT} / \mathrm{FMT}$ investigated mice in comparison to the control group.

Complete Status: Complete

First Name: Jasmin

Last Name: Baier

Email: jbaier@ukaachen.de

Organization: University Clinic RWTH Aachen

Country: Germany 


\title{
ID: GA116 \\ Highly sensitive imaging of miRNA-21 and in vivo synergetic cancer therapy based on a functionalized polydopamine theranostic nanoprobe
}

\author{
Fu Wang, Xidian Unniversity, fwang@xidian.edu.cn
}

\section{Category: Oncology}

\begin{abstract}
Body : MicroRNAs (miRNAs) are emerging as vital biomarkers since their abnormal expression is associated with various disease types including cancer. Therefore, it is essential to develop a sensitive and specific platform to monitor the dynamic expression of miRNAs for early clinical diagnosis and treatment. In this study, we designed a functionalized polydopamine (PDA)-based theranostic nanoprobe for efficient detection of miRNA-21 and in vivo synergistic cancer therapy. PDA was modified with polyethylene glycol (PEG) and the obtained PDA-PEG nanoparticles showed good stability in different solutions. PDA-PEG nanoparticles were loaded with FITC-labeled hairpin DNA (hpDNA) and an anticancer drug doxorubicin (DOX). In the absence of miRNA-21, PDA effectively quenched the fluorescence of FITC-labeled hpDNA. The presence of miRNA-21 specifically recognized hpDNA and induced the dissociation of hpDNA from PDA-PEG, and subsequent recovered the fluorescence signals. Upon cellular uptake of these nanoprobes, a dose-dependent fluorescence activation and synergetic cytotoxic effect were observed due to the release of DOX and inhibition of miRNA-21 function. Furthermore, PDA-PEG-DOX-hpDNA nanoparticles can afford long-term monitoring of miRNA-21 and combined therapeutic efficacy in the nude mice bearing 4T1 tumors. Our results demonstrate the capability of PDA-PEG-DOX-hpDNA as a theranostic nanoprobe for continuously tracking of miRNAs and synergetic cancer therapy.
\end{abstract}

\section{Image/Figure:}

https://www.xcdsystem.com/wmis/abstract/File6959/GA116 ImageFigure 0420084629.jpg

Full Name of Abstract's 1st Author : Wenjie Mao

Complete Status: Complete

First Name: $\mathrm{Fu}$

Last Name: Wang

Email: fwang@xidian.edu.cn

Organization: Xidian Unniversity

Country: China 


\title{
ID: GA117 \\ Ultra-small nano square for an enhanced nuclear imaging
}

\author{
Qize Zhang, Memorial Sloan Kettering Cancer Center, zhangq@mskcc.org
}

\section{Category: Oncology}

\begin{abstract}
Body : The most commonly used isotopes for positron emission tomography (PET) imaging, such as $18 \mathrm{~F}, 89 \mathrm{Zr}, 68 \mathrm{Ga}$, and the radiotherapy isotope $90 \mathrm{Y}$ all produce Cerenkov light 1,2. This optical emission could be utilized to further enhance in vivo imaging capabilities. Cerenkov radiation is predominantly in the UV/blue region of the spectrum and therefore is readily absorbed by body tissue. This endogenous attenuation limits the in vivo application of direct Cerenkov visualization, but an alternative approach is to position beacons that will capture the Cerenkov light, emitting at frequencies that escape the body tissue more easily, e.g. by shifting the spectrum to the infrared. Europium oxide displays a narrow emission at $620 \mathrm{~nm}$, large Stokes' shifts, and long lifetimes, which make such materials strong candidates for secondary Cerenkov emission fluorescence imaging (SCIFI)3. Among the various known probes, Europium oxide, exhibiting sensitized luminescence that can be excited with Cerenkov light, are advantageous over other lanthanide elements [WHY?]. However, so far no suitable preparation was available, commercial ones were either too large or not compatible with in vivo use. In this study, we developed a strategy to shift the blue Cherenkov light to a more penetrated red-light region through fluorescent Europium oxide nanoparticles. We synthesized a square shape ultrasmall Eu2O3 nanoparticle (uEuNP). By adding a mild oxidation agent during the synthesis, we achieved significantly increased the brightness of the particle without changing its size $(<1$ $0 \mathrm{~nm})$. We functionalized the particle with PEG to enhance dispersity in vitro and biocompatibility in vivo. These particles were further labeled with a radioisotope and intravenous injection into mice to visualize the lymph node metastasis and tumor. These features advance europium oxide nanoparticles as a preclinical cancer diagnosis platform.
\end{abstract}

References: 1)Cerenkov imaging-a new modality for molecular imaging DLJ Thorek, $\mathrm{R}$ Robertson, WA Bacchus... - ... molecular imaging, 2012 - ncbi.nlm.nih.gov 2)Optical imaging of Cerenkov light generation from positron-emitting radiotracers R Robertson, MS Germanos, C Li... - Physics in Medicine ..., 2009 - iopscience.iop.org 3)Luminescent europium (III) nanoparticles for sensing and imaging of temperature in the physiological range $\mathrm{H}$ Peng, MIJ Stich, J Yu, L Sun, LH Fischer... - Advanced ..., 2010 - Wiley Online Library

\section{Image/Figure:}

https://www.xcdsystem.com/wmis/abstract/File6959/GA117_ImageFigure_0608112550.png

Image/Figure Caption: Scheme showing the preparation UEuNP@PEG and the in vivo study on tumor mice model

Complete Status: Complete 
First Name: Qize

Last Name: Zhang

Email: zhangq@mskcc.org

Organization: Memorial Sloan Kettering Cancer Center

Country: United States 


\title{
ID: GA118 \\ A two-step transcriptional amplification reporter for dynamic mimicking and imaging of pre-mRNA splicing in living subjects
}

\author{
Fu Wang, Xidian Unniversity, fwang@xidian.edu.cn
}

Category: New Chemistry, Biology \& Bioengineering

\begin{abstract}
Body : Pre-mRNA splicing requires removing introns and connecting exons to generate mature transcripts in eukaryotes. However, because of the limited methods for noninvasive monitoring pre-mRNA splicing process, the dynamic changes for intron sequence with their loss and gain are poorly detected. Here we constructed an activatable two-step genetically-encoded transcriptional amplification reporter system, Ret-GV:U-Fluc, for real-time imaging of splicing process in living subjects. By taking advantage of the strong trans-activating properties of the GAL4-VP16 fusion protein, which can target to upstream activator sequence (UAS) elements to boost the subsequent firefly luciferase reporter gene expression, we successfully and consistently detected the pre-mRNA splicing process in response to exogenous splicing modulators in living cells and animals. Our study gives the advantageous properties to high-throughput screen of splicing modulators, which would speed up the development of new drugs in treatment of splicing disordered diseases.
\end{abstract}

\section{Image/Figure:}

https://www.xcdsystem.com/wmis/abstract/File6959/GA118_ImageFigure_0420104828.png

Full Name of Abstract's 1st Author : Si Chen

Complete Status: Complete

First Name: $\mathrm{Fu}$

Last Name: Wang

Email: fwang@xidian.edu.cn

Organization: Xidian Unniversity

Country: China 


\title{
ID: GA121 \\ Comparison of Iron Oxide Nanoparticles for Magnetic Particle Imaging
}

\author{
Julia Gevaert, University of Western Ontario, jgevaert@uwo.ca
}

\section{Category: Instrumentation}

\begin{abstract}
Body : Introduction: Magnetic particle imaging (MPI) is an emerging imaging modality capable of tracking cells in-vivo. Superparamagnetic iron oxide (SPIO) particles are used to generate MPI signal that varies linearly with iron content. This allows iron content to be directly quantified and used to calculate cell numbers. Along with this direct detection and quantification of iron, MPI is both sensitive and specific, overcoming limitations of other modalities such as MRI. Signal detection in MPI depends almost entirely on the type of tracer used, since the non-linear magnetization of the particle is directly detected by the system without interference from background noise or tissue. For improved resolution and sensitivity, tailored tracers with specific properties are therefore required. This study comparatively analyses four commercially available particles along with five novel variations of Synomag derivatives. Each tracer has varying core diameters, surface coatings, and iron concentrations, to determine optimal MPI performance. Materials and Methods: 5 existing commercial nanoparticles were compared: (i) Ferumoxytol (Feraheme ${ }^{\mathrm{TM}}$, AMAG Pharmaceuticals, Inc.), composed of a single iron oxide core and a carboxymethyl-dextran coat with a hydrodynamic diameter of $\sim 30 \mathrm{~nm}$, (ii) Vivotrax $^{\text {TM }}$ (Magnetic Insight Inc.), a multicore particle with a bimodal distribution consisting of $5 \mathrm{~nm}(70 \%)$ and $25 \mathrm{~nm}(30 \%)$ cores and a dextran coat with a hydrodynamic diameter of $\sim 60 \mathrm{~nm}$ (iii) Synomag-D ${ }^{\mathrm{TM}}$ (MicroMod $\mathrm{GmbH}$ ), a multicore 'nanoflower'-shaped particle with a dextran coat and a hydrodynamic diameter of $\sim 50 \mathrm{~nm}$, (iv) Synomag without dextran and with $\mathrm{COOH}$ groups on the surface, and (v) Bangs beads (Bangs laboratories), which have a hydrodynamic diameter of about 1 micron and multiple, small iron cores embedded in a polystyrene matrix. In addition, the Shuhendler lab modified the Synomag (iv) tracer with other surface coatings, including Pyridinium sulfobetaine (PSB), Carboxylic acid (COOH), L-Cysteine (CYS), D,LHomocysteine (HCYS), and Sulfobetaine (SB). Imaging was done on a Field Free Line projection MOMENTUM ${ }^{\mathrm{TM}}$ MPI scanner (Magnetic Insight Inc., Almeda, CA, USA). Relaxometer data was collected using 10uL (undiluted) of each tracer, producing a point spread function with FWHM and peak amplitude as a measure of MPI resolution and sensitivity. All relaxometer data was normalized to iron content. 231BR cancer cells were labeled with the Synomag tracers by coincubation for 4 and 24 hours to test labeling efficiency with these new agents. Results: Synomag-D ${ }^{\mathrm{TM}}$ and the non-dextran coated Synomag derivatives showed almost twice as much signal output, and therefore higher sensitivity than Vivotrax ${ }^{\mathrm{TM}}$, followed by Bangs beads, Vivotrax ${ }^{\mathrm{TM}}$, and Feraheme ${ }^{\mathrm{TM}}$. As a measure of resolution, FWHM was evaluated with Vivotrax ${ }^{\mathrm{TM}}$ having the best resolution, followed by the Synomag derivatives, Bang's beads, and Feraheme $^{\mathrm{TM}}$. Coincubation of cancer cells with Synomag showed greater uptake after 24 hours compared to 4 hours, although qualitatively the labeling efficiency was less than that for cells labeled with Vivotrax or Bangs beads by coincubation. Discussions and Conclusions: SPIO tracers were examined for MPI performance by comparing relative sensitivity and resolution. Comparing MPI performance of tracers will provide important information on which properties
\end{abstract}


to target in new tracers. A suitable tracer for MPI should produce a large signal for detection, with efficient cell uptake, and be used in-vivo. Future work will include testing labelling strategies for improved cell uptake accompanied by in-vivo imaging.

\section{Image/Figure:}

https://www.xcdsystem.com/wmis/abstract/File6959/GA121_ImageFigure 0614124548.jpg

Image/Figure Caption: Relaxometer data for Synomag-D, Vivotrax, Feraheme, and Bang's Beads (MPIO) showing (A) relative resolutions shown by peak FWHM and (B) relative sensitivities shown by peak amplitudes.

Full Name of Abstract's 1st Author : Julia Gevaert

Complete Status: Complete

First Name: Julia

Last Name: Gevaert

Email: jgevaert@uwo.ca

Organization: University of Western Ontario

Country: Canada 


\title{
ID: GA122 \\ Biodistribution of 89Zr-labeled prolactin receptor directed antibodies: PET studies in mouse tumor models and healthy NHPs
}

Martin Voorbach, AbbVie Inc., martin.j.voorbach@abbvie.com

\section{Category: Oncology}

\begin{abstract}
Body : ABBV-176 is a prolactin receptor (PRLR) directed antibody drug conjugate (h16f- Pyrrolobenzodiazepine, PBD) for the treatment of breast cancer. 89Zr-h16f was evaluated as a potential tool for PET imaging and assessing the delivery of ABBV-176. Pre-clinical studies including in vivo positron emission tomography (PET) imaging studies in a mouse xenograft model and in healthy non-human primates (NHP) were performed. In the mouse xenograft study 89Zr-h16f and 89Zr-ABBV-176 were tested for tumor uptake in both target positive and target negative MDA-MB-435-LM flank tumors. Tumor uptake for both 89Zr-ABBV-176 and 89Zrh16f was higher in a PRLR (+) tumor model compared to a PRLR (-) model. Tumor uptake and blood clearance of 89Zr-ABBV-176 and 89Zr-h16f were similar. In the PET study in healthy NHP, the biodistribution of 89Zr-ABBV-176 and 89Zr-h16f at low mass doses $(0.03 \mathrm{mg} / \mathrm{kg})$ and the biodistribution of $89 \mathrm{Zr}-\mathrm{h} 16 \mathrm{f}$ at low $(0.03 \mathrm{mg} / \mathrm{kg})$ and high mass doses $(3 \mathrm{mg} / \mathrm{kg})$ were compared. The biodistribution of $89 \mathrm{Zr}$-h16f and $89 \mathrm{Zr}-\mathrm{ABBV}-176$ were similar, with highest tissue concentrations observed in the adrenal glands. The high mass dose with 89Zr-h16f showed reduced adrenal uptake, consistent with PRLR binding specificity in this organ. For both the parent antibody and ADC, the liver uptake was consistent with hepatic clearance. Because the behavior of 89Zr-labeled h16f and 89Zr-labeled ABBV-176 appear similar, imaging with 89Zrlabeled h16f may give insight into the drug distribution of ABBV-176 in patients without exposing subjects to additional PBD.
\end{abstract}

Complete Status: Complete

First Name: Martin

Last Name: Voorbach

Email: martin.j.voorbach@abbvie.com

Organization: AbbVie Inc.

Country: United States 


\title{
ID: GA132 \\ Dual-Bioluminescence Imaging of a Novel Mouse Model of Synchronous Bilateral Breast Cancer
}

\author{
Shirley Liu, Western University, sliu826@uwo.ca
}

\section{Category: Oncology}

\begin{abstract}
Body : Introduction: Synchronous bilateral breast cancer (SBBC) is the detection of malignant lesions in both breasts at the time of diagnosis or within 3-12 months. It occurs in approximately $2 \%$ of breast cancer patients 1 , and compared to women with unilateral breast cancer, SSBC patients have higher rates of metastasis and lower overall survival2,3. Studies show that the worse prognosis is not due to increased tumor aggressiveness, but due to the combined effect of two tumors resulting in a higher chance of metastasis 3,4 . However, little is known about the metastatic properties of cells from each tumor and whether metastatic cells from one tumor contribute to the growth of metastases from the other tumor. In this study the first mouse model of SBBC was developed by orthotopically implanting human breast cancer cells into bilateral mammary fat pads. The objective was to characterize the distribution of metastatic cells from each tumour using non-invasive dual-bioluminescence imaging (dual-BLI)
\end{abstract} with the highly sensitive reporters Akaluc and Antares2, as well as endpoint fluorescence microscopy. Methods: MDA-MB-231 human breast cancer cells were engineered with lentivirus to co-express either Antares 2 and the fluorescence reporter zsGreen (zsG), or Akaluc and the fluorescence reporter tdTomato (tdT). Transduced cells were FACS sorted to obtain stable engineered cell populations. Female nod-scid-gamma (NSG) mice received orthotopic injections of $3 \times 105 \mathrm{zsG} /$ Antares 2 cells and $3 \times 105 \mathrm{tdT} /$ Akaluc cells into contralateral fourth mammary fat pads (day $0 ; n=10$ ). Antares2 and Akaluc dual-BLI was performed weekly for up to 6 weeks. Three mice were sacrificed on day 29 upon detection of both Antares2- and Akaluc-expressing lung metastasis. Dual-BLI was performed on the remaining seven mice until endpoint as determined by primary tumor necrosis (up to day 42). Mammary fat pad tumors and lungs were fixed, cryopreserved, and sectioned, and nuclei were stained with Hoechst. The cell composition of micrometastases in the lungs, identified as clusters of cells with a diameter greater than 200 $\mu \mathrm{m}$, was analyzed using fluorescence microscopy. Results: Metastases were first detected in the lungs in six of the 10 mice on day 15 using Akaluc-BLI, with all mice showing Akaluc signal in the lungs by day 29. Antares2-BLI detected lung metastases in four of the 10 mice by day 21 , and in six of seven mice by day 35 (Fig. 1A). Fluorescence microscopy of the lungs of mice sacrificed on day 42 showed both zsG- and tdT-expressing micrometastases, with the majority $(60 \%)$ of micrometastases composed of cells from both primary tumors $(n=2 ;$ Fig. $1 \mathrm{~B} / \mathrm{C})$. Discussion: This study was the first to characterize metastatic SBBC in an animal model. The high proportion of metastases composed of cells from both primary tumors in preliminary results may be explained by metastatic cross-seeding, a phenomenon where circulating tumor cells that have disseminated from a primary tumor preferentially seed onto an established metastatic lesion. This process may contribute to tumour heterogeneity in SBBC patients with inter-tumoral molecular subtype discordance and lead to treatment resistance. These unexpected findings may 
explain the worse outcome of SBBC patients and should provide future insight on patient treatment and management.

References: 1. Jobsen, J. J., J. van der Palen, F. Ong \& J. H. Meerwaldt (2003) Synchronous, bilateral breast cancer: prognostic value and incidence. Breast, 12, 83-8. 2. Ozturk, A., G. Alco, D. Sarsenov, S. Ilgun, C. Ordu, U. Koksal, K. Nur Pilanci, Z. Erdogan, F. Izci, F. Elbuken, F. Agacayak, F. Aktepe \& V. Ozmen (2018) Synchronous and metachronous bilateral breast cancer: A long-term experience. J BUON, 23, 1591-1600. 3. Jobsen, J. J., J. van der Palen, F. Ong, S. Riemersma \& H. Struikmans (2015) Bilateral breast cancer, synchronous and metachronous; differences and outcome. Breast Cancer Res Treat, 153, 277-83. 4. Mejdahl, M. K., J. Wohlfahrt, M. Holm, E. Balslev, A. S. Knoop, A. Tjønneland, M. Melbye \& N. Kroman (2019) Breast cancer mortality in synchronous bilateral breast cancer patients. Br J Cancer, 120, 761-767.

\section{Image/Figure:}

https://www.xcdsystem.com/wmis/abstract/File6959/GA132_ImageFigure_0608104546.png

Image/Figure Caption: Figure 1. Dual-BLI and fluorescence microscopy of lung metastases of contralateral tdT/Akaluc and zsG/Antares2-expressing mammary fat pad tumors. A)

Representative images of Akaluc BLI and Antares2 BLI of lung metastasis. B) Fluorescence microscopy of lungs showing metastasis of tdT- and zsG-expressing cells. White arrows locate micrometastases ( $>200 \mu \mathrm{m}$ diameter) composed of cells derived from both mammary fat pad tumors while green arrows locate micrometastases composed of only zsG-expressing cells. No tdT-expressing micrometastases were identified in this field of view. C) Pilot data of cell composition of lung micrometastases $(n=2)$. The number of micrometastases composed of only tdT-expressing cells, only zsG-expressing cells, or both tdT- and zsG-expressing cells were counted in 5 fields of view at 3 lung sections for each mouse.

Complete Status: Complete

First Name: Shirley

Last Name: Liu

Email: sliu826@uwo.ca

Organization: Western University

Country: Canada 


\title{
ID: GA133 \\ Near-infrared fluorescence imaging of carotid plaques with TLR4-conjugated zwitterionic fluorophore in atherosclerotic murine model
}

\author{
Xiaotian (Dennis) Wu, Gordon Center for Medical Imaging (MGH/HMS), \\ xwu23@mgh.harvard.edu
}

Category: Cardiovascular \& Pulmonary

\begin{abstract}
Body : Objectives: The early detection of plaques can have improved outcomes for patients suffering from coronary artery disease (CAD). Toll-like receptor 4 (TLR4) has been recognized as an important biomarker for atheroma development as it plays critical roles in macrophage differentiation into foam cells and inflammation[1]. We propose to use near-infrared fluorescence (NIRF) imaging with TLR4-conjugated zwitterionic NIR fluorophore (ZW8001C)[2] targeting macrophage TLR4 in this novel application to locate such inflamed plaques. Methods: A carotid atherosclerotic model was developed using 8-week-old male FVB/NJ mice ( $\mathrm{n}=4$, The Jackson Laboratory). Mice were kept on $0.2 \%$ high cholesterol Western Diet (HCD, TD.88137, Envigo) for a total of eight weeks after initial acclimation. After four weeks on HCD, mice were intraperitoneally injected with Streptozotocin $(40 \mathrm{mg} / \mathrm{kg}$, Sigma-Aldrich) for 8 consecutive days to induce diabetes. Two weeks after the diabetic induction, the left carotid artery (LCA) was surgically ligated to cause stenosis and the right carotid artery (RCA) was kept intact as a negative control. At the end of the eight weeks, TLR4-ZW800-1C (10 nmol, excitation/emission: $753 / 772 \mathrm{~nm}$ ) was injected retro-orbitally before imaging. The labeling ratio between ZW800-1C NHS ester and TLR4, calculated based on the Beer-Lambert law, was found to be 1.40. The mice were imaged in vivo with an in-house built multispectral NIRF imaging system (Ex: $760 \mathrm{~nm}$, Em: $785 \mathrm{LP}$ ) at various time points (baseline, $1 \mathrm{~h}$, and 5-6.5 h)[2]. Baseline images were acquired before the contrast agent injection. The experiment was done under anesthesia (Isoflurane) according to MGH IACUC-approved protocol (2019N000104). Animals were euthanized appropriately at the final imaging time point and organs (especially the LCA and RCA) were harvested for ex vivo NIRF imaging and further histology analysis. Image processing and analysis were performed using custom-written MATLAB code. Results: $50 \%$ of the mice exhibited prominent plaques in the LCA. Ex vivo imaging of the harvested heart, aortic arch, and ligated LCA showed significantly higher $(1.8 \times)$ fluorescent signal compared to the control RCA for the mice with plaques (Fig. 1 A-B). Imaging of various harvested organs showed highest uptake in lungs (signal-to-background ratio, $\mathrm{SBR}=13.6)$ and kidneys $(\mathrm{SBR}=$ 13.9) at the 5-6.5 h time point (Fig. $1 \mathrm{C}$ ). The high-uptake in the heart was considered to reflect physiological expression of TLR4 in cardiomyocytes[3]. Before harvest, in vivo imaging showed high fluorescent signal over the chest region, but specific signal from individual carotid arteries could not be detected, possibly due to low signal and scatter through the skin. Conclusion: NIRF imaging in conjunction with TLR4-conjugated ZW800-1C was able to detect atherosclerotic plaques with high sensitivity. Since TLR4 is involved in critical steps in plaque formation, this technology might be able to identify high-risk plaques likely to progress. Continued investigation will include larger sample sizes with histological analyses in mice models, and in large animals using intravascular NIRF catheters. Acknowledgements: The authors would like to thank the
\end{abstract}


MGH Center for Comparative Medicine animal facility staff for animal support. Funding support came from the National Heart, Lung, and Blood Institute and the National Institute of Biomedical Imaging and Bioengineering of the National Institutes of Health (R00HL127180 and T32EB013180).

References: [1] M. H. K. Roshan, A. Tambo, and N. P. Pace, "The Role of TLR2, TLR4, and TLR9 in the Pathogenesis of Atherosclerosis," International Journal of Inflammation, vol. 2016, 2016, doi: 10.1155/2016/1532832. [2] Y. Ji et al., "Targeted molecular imaging of TLR4 in hepatocellular carcinoma using zwitterionic near-infrared fluorophores," Quantitative Imaging in Medicine and Surgery, vol. 9, no. 9, pp. 1548-1555, 2019, doi: 10.21037/qims.2019.09.04. [3] S. Frantz et al., "Toll4 (TLR4) expression in cardiac myocytes in normal and failing myocardium," Journal of Clinical Investigation, vol. 104, no. 3, pp. 271-280, 1999, doi: 10.1172/JCI6709.

\section{Image/Figure:}

https://www.xcdsystem.com/wmis/abstract/File6959/GA133 ImageFigure 0422110915.png

Full Name of Abstract's 1st Author : Xiaotian (Dennis) Wu

Complete Status: Complete

First Name: Xiaotian (Dennis)

Last Name: $\mathrm{Wu}$

Email: xwu23@mgh.harvard.edu

Organization: Gordon Center for Medical Imaging (MGH/HMS)

Country: United States 


\title{
ID: GA134 \\ PET imaging of pulmonary Aspergillus fumigatus infection with 18F- fluorodeoxysorbitol
}

\author{
Jianhao Lai, National institute of health, jianhao.lai@nih.gov
}

Category: Immunology: Inflammation \& Infection

\begin{abstract}
Body : Background. Invasive pulmonary aspergillosis (IPA), mainly caused by Aspergillus fumigatus (A. fumigatus), affects over 200,000 patients worldwide, with mortality rates between $30 \%$ and $90 \%$ [1]. The diagnosis of IPA, however, remains challenging and new non-invasive biomarkers of IPA, especially in the early stages of disease, are urgently needed. Since some Aspergillus species are known to utilize sorbitol as part of the oxido-reductive Dgalactose pathway [2], we wanted to evaluate 2-[18F]-fluorodeoxysorbitol (FDS) [3] uptake in animal models of A. fumigatus infection. Methods. In vitro FDS uptake assays were performed in live and heat killed A. fumigatus. We used Escherichia coli (E. coli) as a gram negative control for comparison. PET/CT imaging was performed in a mouse model of pulmonary infection with $\mathrm{A}$. fumigatus as well as a mouse model of sterile lung inflammation (induced by poly (I:C) administration) and uninfected mice. Static PET images were obtained 120 minutes after injection of 18F-FDS. We also performed 18F-FDG PET/CT for comparison. Lung tissues from infected and healthy mice were GMS stained to confirm fungal presence/burden. To compare the uptake of FDS in fungi and bacteria, FDS uptake was also evaluated in the murine myositis models, infected with E. coli or Staphylococcus aureus (S. aureus). Results. In vitro uptake assays showed FDS uptake by live but not by heat killed A. fumigatus. The uptake in A. fumigatus, however, was much lower than that of E. coli, when normalized for weight. PET imaging of A. fumigatus pneumonia mice showed very low uptake in lung consolidations, slightly higher than healthy and sterile lung inflammation. 18F-FDG imaging, on the other hand, showed high uptake in both infection and sterile lung inflammation models compared to controls. In the murine myositis models, as expected, high FDS uptake was seen in live compared to heatkilled E.coli with no appreciable uptake corresponding to live or heat-killed S. aureus.

Conclusion. A. fumigatus is able to accumulate FDS in vitro, albeit to a much lower extent than gram negative bacteria. This corresponded to very low uptake of $18 \mathrm{~F}-\mathrm{FDS}$ in a fungal pneumonia animal model. We do not believe 18F-FDS PET imaging is a viable ligand for imaging Aspergillus infection in the lungs.
\end{abstract}

References: 1. Brown, G.D., et al., Hidden killers: human fungal infections. Sci Transl Med, 2012. 4(165): p. 165rv13. 2. Koivistoinen, O.M., et al., Sorbitol dehydrogenase of Aspergillus niger, SdhA, is part of the oxido-reductive D-galactose pathway and essential for D-sorbitol catabolism. FEBS Lett, 2012. 586(4): p. 378-83. 3. Weinstein, E.A., et al., Imaging Enterobacteriaceae infection in vivo with $18 \mathrm{~F}$-fluorodeoxysorbitol positron emission tomography. Sci Transl Med, 2014. 6(259): p. 259ra146.

\section{Image/Figure:}


Image/Figure Caption: (A) 18F-FDS uptake in E. coli and A. fumigatus cultures incubated with 18F-FDS for 120 minutes. (B) Quantified PET signal of 18F-FDG (30 minutes) and 18F-FDS (120 minutes) in the lungs of control, sterile inflammation, and A. fumigatus infected animals. (C) PET/CT imaging of Aspergillus infected and control animals using 18F-FDG (30 minutes post injection) or 18F-FDS (120 minutes post injection). Representative coronal images of lung $\mathrm{CT}$, PET and PET/CT fusion are shown.

Complete Status: Complete

First Name: Jianhao

Last Name: Lai

Email: jianhao.lai@nih.gov

Organization: National institute of health

Country: United States 


\title{
ID: GA135 \\ Reporting amyloid beta levels via bioluminescence imaging with amyloid reservoirs in Alzheimer's disease models
}

\author{
Biyue Zhu, Massachusetts General Hospital, 413897689@qq.com
}

\section{Category: Neuroscience}

\begin{abstract}
Body : Bioluminescence imaging has changed daily practice in preclinical research of cancers and other diseases in the last decades; however, it has been rarely applied in preclinical research of Alzheimer's disease (AD). In this report, we demonstrated that bioluminescence imaging could be used to report the levels of amyloid beta $(\mathrm{Ab})$ species in vivo. We hypothesized that AkaLumine, a newly discovered substrate for luciferase, could bind to $\mathrm{Ab}$ aggregates and plaques. We further speculated that the Ab species have the reservoir capacity to sequester and release AkaLumine to control the bioluminescence intensity, which could be used to report the levels of Abs. Our hypotheses have been validated via in vitro tests, mimic phantom imaging, and in vivo imaging using transgenic AD mice that were virally transduced with aka Luciferase (AkaLuc), a new luciferase that generates bioluminescence in the near infrared window. As expected, compared to the control group, we observed that the Ab group showed lower bioluminescence intensity due to AkaLumine sequestering at early time points, while higher intensity due to AkaLumine releasing at later time points. Our approach can be easily implanted into daily imaging experiments. We believe that our method has tremendous potential to change daily practice of preclinical AD research.
\end{abstract}

References: 1. Long, J.M. \& Holtzman, D.M. Alzheimer Disease: An Update on Pathobiology and Treatment Strategies. Cell 179, 312-339 (2019). 2. Tolar, M., Abushakra, S. \& Sabbagh, M. The path forward in Alzheimer's disease therapeutics: Reevaluating the amyloid cascade hypothesis. Alzheimers Dement (2019). 3. Sabbagh, M.N., Hendrix, S. \& Harrison, J.E. FDA position statement "Early Alzheimer's disease: Developing drugs for treatment, Guidance for Industry". Alzheimers Dement (N Y) 5, 13-19 (2019). 4. Jack, C.R., Jr., et al. NIA-AA Research Framework: Toward a biological definition of Alzheimer's disease. Alzheimers Dement 14, 535562 (2018). 5. Panza, F., Lozupone, M., Logroscino, G. \& Imbimbo, B.P. A critical appraisal of amyloid-beta-targeting therapies for Alzheimer disease. Nat Rev Neurol 15, 73-88 (2019). 6. Cummings, J., Lee, G., Ritter, A., Sabbagh, M. \& Zhong, K. Alzheimer's disease drug development pipeline: 2019. Alzheimers Dement (N Y) 5, 272-293 (2019). 7. Cummings, J.L., Morstorf, T. \& Zhong, K. Alzheimer's disease drug-development pipeline: few candidates, frequent failures. Alzheimers Res Ther 6, 37 (2014). 8. Cummings, J., Reiber, C. \& Kumar, P. The price of progress: Funding and financing Alzheimer's disease drug development. Alzheimers Dement (N Y) 4, 330-343 (2018). 9. Manni, I., de Latouliere, L., Gurtner, A. \& Piaggio, G. Transgenic Animal Models to Visualize Cancer-Related Cellular Processes by Bioluminescence Imaging. Front Pharmacol 10, 235 (2019). 10. Mezzanotte, L., van 't Root, M., Karatas, H., Goun, E.A. \& Lowik, C. In Vivo Molecular Bioluminescence Imaging: New Tools and Applications. Trends Biotechnol 35, 640-652 (2017). 11. Massoud, T.F. \& Gambhir, S.S. Molecular imaging in living subjects: seeing fundamental biological processes in a new light. 
Genes Dev 17, 545-580 (2003). 12. Hochgrafe, K. \& Mandelkow, E.M. Making the brain glow: in vivo bioluminescence imaging to study neurodegeneration. Mol Neurobiol 47, 868-882 (2013). 13. Dawson, T.M., Golde, T.E. \& Lagier-Tourenne, C. Animal models of neurodegenerative diseases. Nature neuroscience 21, 1370-1379 (2018). 14. King, A. The search for better animal models of Alzheimer's disease. Nature 559, S13-S15 (2018).

\section{Image/Figure:}

https://www.xcdsystem.com/wmis/abstract/File6959/GA135 ImageFigure 0420034843.jpg

Full Name of Abstract's 1st Author : biyue zhu

Complete Status: Complete

First Name: Biyue

Last Name: Zhu

Email: 413897689@qq.com

Organization: Massachusetts General Hospital

Country: United States 


\title{
ID: GA136 \\ Smart chemiluminescence probes and dual-amplification of signal for detection of amyloid beta species in Alzheimer's disease model
}

\author{
Biyue Zhu, Massachusetts General Hospital, 413897689@qq.com
}

\section{Category: Neuroscience}

\begin{abstract}
Body : Fluorescence and chemiluminescence imaging are the most widely applied optical emissive imaging methods in biomedical research. "Smart" (turn-on) fluorescence imaging has been routinely used for in vitro, cellular, and in vivo imaging; however, smart chemiluminescence imaging has been rarely explored. In this report, we designed chemiluminescence probe ADLumin-1 and validated that ADLumin-1 was a smart chemiluminescence probe for amyloid beta $(\mathrm{Ab})$ species, evidenced by a 216 -fold amplification of chemiluminescence intensity upon mixing with Abs in vitro. In vivo two photon imaging indicated that ADLumin-1 could efficiently cross blood-brain- barrier (BBB) and provided excellent contrast both for $\mathrm{Ab}$ plaques and cerebral amyloid angiopathy (CAA). In vivo whole brain imaging showed that the chemiluminescence signal of ADLumin-1 from 5-month-old transgenic $\mathrm{AD}(5 \mathrm{xFAD})$ mice was 1.80-fold higher than that from the age-matched wild-type mice. Moreover, we demonstrated that it was feasible to further dually-amplify signal via chemiluminescence resonance energy transfer (DAS-CRET) using two non-conjugated smart probes (ADLumin-1 and CRANAD-3) in solutions, brain homogenates, and in vivo whole brain imaging. Our results showed that DAS-CRET could provide a 2.25-fold margin between 5month-old 5xFAD mice and wild type mice. To our knowledge, this is the first report that a chemiluminescence probe could be used for detecting $\mathrm{Ab}$ species both in vitro and in vivo. Although ADLumin-1 was designed for Abs, we believe that our strategy could be potentially extended to a wide range of targets, including other aggregating-prone proteins. Notably, our results suggested that the strategies for turning-on fluorescence could be used for amplifying chemiluminescence, and we believe that our studies could inspire considerably more research on chemiluminescence imaging.
\end{abstract}

References: 1. Massoud, T. F.; Gambhir, S. S., Molecular imaging in living subjects: seeing fundamental biological processes in a new light. Genes Dev 2003, 17 (5), 545-80. 2. Rudin, M.; Weissleder, R., Molecular imaging in drug discovery and development. Nature reviews. Drug discovery 2003, 2 (2), 123-31. 3. Rao, J.; Dragulescu-Andrasi, A.; Yao, H., Fluorescence imaging in vivo: recent advances. Curr Opin Biotechnol 2007, 18 (1), 17-25. 4. Ntziachristos, V.; Bremer, C.; Weissleder, R., Fluorescence imaging with near-infrared light: new technological advances that enable in vivo molecular imaging. Eur Radiol 2003, 13 (1), 195-208. 5. Lakowicz, J., Principles of Fluorescence Spectroscopy. 2nd edition ed.; Plenum Publishing Corporation: 1999. 6. Rice, B. W.; Contag, C. H., The importance of being red. Nat Biotechnol 2009, 27 (7), 624-5. 7. Ueno, T.; Nagano, T., Fluorescent probes for sensing and imaging. Nat Methods 2011, 8 (8), 642-5. 8. Kobayashi, H.; Ogawa, M.; Alford, R.; Choyke, P. L.; Urano, Y., New strategies for fluorescent probe design in medical diagnostic imaging. Chem Rev 2010, 110 (5), 2620-40. 9. Frangioni, J. V., In vivo near-infrared fluorescence imaging. Current opinion in chemical 
biology 2003, 7 (5), 626-34. 10. Miao, Q.; Xie, C.; Zhen, X.; Lyu, Y.; Duan, H.; Liu, X.; Jokerst, J. V.; Pu, K., Molecular afterglow imaging with bright, biodegradable polymer nanoparticles. Nat Biotechnol 2017, 35 (11), 1102-1110. 11. Hananya, N.; Shabat, D., A Glowing Trajectory between Bio- and Chemiluminescence: From Luciferin-Based Probes to Triggerable Dioxetanes. Angew Chem Int Ed Engl 2017, 56 (52), 16454-16463. 12. Hananya, N.; Shabat, D., Recent Advances and Challenges in Luminescent Imaging: Bright Outlook for Chemiluminescence of Dioxetanes in Water. ACS Cent Sci 2019, 5 (6), 949-959. 13. Suzuki, K.; Nagai, T., Recent progress in expanding the chemiluminescent toolbox for bioimaging. Curr Opin Biotechnol 2017, 48, 135-141. 14. Roda, A.; Pasini, P.; Mirasoli, M.; Michelini, E.; Guardigli, M., Biotechnological applications of bioluminescence and chemiluminescence. Trends Biotechnol 2004, 22 (6), 295-303.

\section{Image/Figure:}

https://www.xcdsystem.com/wmis/abstract/File6959/GA136 ImageFigure 0420062440.jpg

Full Name of Abstract's 1st Author : biyue zhu

Complete Status: Complete

First Name: Biyue

Last Name: Zhu

Email: 413897689@qq.com

Organization: Massachusetts General Hospital

Country: United States 


\title{
ID: GA137 \\ Characterizing Prostate Cancer Organoids with Prostate Specific Membrane Antigen (PSMA)-Targeted and Metabolic PET Imaging Agents
}

\author{
Margaret White, National Institutes of Health, maggie.white@nih.gov
}

\section{Category: Oncology}

\begin{abstract}
Body : Objectives: PSMA has emerged as a relevant target for clinical prostate cancer (PC) imaging, although variability in its overexpression as it relates to response to androgen deprivation therapy (ADT), disease progression and genotypic signatures remain to be established. Therefore, assessing PC with additional metabolic imaging agents in the same patient would address the heterogeneity found in PC and may be informative in prognosis and treatment management. With the development of PC LuCaP patient derived xenograft (PDX) mouse models [1] and organoid cultures designed to preserve the genomic integrity of the PDX [2], the in vitro screening of multiple imaging agents as it relates to disease diversity is possible. Herein, in vitro protocols were established using the PSMA PET agent [18F]DCFPyL (FDC) and metabolic PET agents [18F]Fluorodeoxyglucose (FDG), [18F]Fluoroglutamine (Glu), and [18F]Fluoroarachidonic Acid (ArA) in LuCaP organoids to characterize PSMA and its relationship with metabolic markers. Methods: FDC, Glu and ArA were synthesized following published methods [3-5] with radiochemical yields of $15-43 \%$ and purities $>98 \%$. PC $3 /$ PSMA + [PSMA-transfected;+control) and LuCaP PDX models (4 adenocarcinomas [AD]; 1 neuroendocrine $[\mathrm{NE}]$ ) were evaluated for PSMA with FDC in vivo and in vitro (binding assays $[B \max ]$ ), in association with phenotype, metastatic location, androgen receptor (AR, immunohistochemistry [IHC]) [1], and ADT response (Fig.1). ADT response was determined by measuring tumor volumes (caliper) in PDX-bearing mice (control and ADT treated [Degarelix, $25 \mathrm{mg} / \mathrm{kg}]$ ) over a 3-week ADT paradigm (Fig.1). Organoids of the same PDX were cultured (7 days) and evaluated for PSMA (FDC; Bmax) and metabolic agents (FDG, Glu, and ArA uptake; 2h; Fig.1). Results: PSMA levels detected by FDC in PDXs and PDX-derived organoids were comparable and corresponded with in vivo PET images (Fig.1). Three ADs, LuCaP77, 141 and 136, exhibited high ADT sensitivity and intense AR IHC but varied in PSMA: LuCaP136 was 50-100 fold lower than LuCaP141 and 77. The ADT non-responsive AD LuCaP167 had high AR with the lowest PSMA, whereas the other ADT non-responder, NE LuCaP145.1, exhibited negligible AR but moderate PSMA levels. FDG uptakes of LuCaP77, 145.1, and 167 were 3-fold higher than LuCaP141 and 136, indicating non-correspondence to PSMA or ADT sensitivity. ArA uptakes were higher than FDG and Glu for all LuCaP PDXs, suggesting that PC organoids import and possibly metabolize more arachidonic acid compared to glucose and glutamine. Furthermore, the NE LuCaP145.1 exhibited higher FDG, Glu, and ArA uptake compared to AD LuCaP models, indicating that it may be more metabolically active. These results highlight the potential clinical value of combining FDC imaging with metabolic imaging as a means to characterize PC heterogeneity. Conclusions: The favorable comparison of PSMA levels between in vitro organoids and in vivo PDXs indicate that organoid models may be useful for the direct comparison of multiple PET agents towards the generation of a database to study PC biology.
\end{abstract}


The use of patient-derived genomically-heterogeneous models coupled with PET imaging is anticipated to provide insights into clinical PC.

References: Works Cited: 1. Nguyen, H.M., et al., LuCaP Prostate Cancer Patient-Derived Xenografts Reflect the Molecular Heterogeneity of Advanced Disease and Serve as Models for Evaluating Cancer Therapeutics. Prostate, 2017. 77(6): p. 654-671. 2. Beshiri, M.L., et al., A PDX/Organoid Biobank of Advanced Prostate Cancers Captures Genomic and Phenotypic Heterogeneity for Disease Modeling and Therapeutic Screening. Clin Cancer Res, 2018. 24(17): p. 4332-4345. 3. Basuli, F., et al., Fast indirect fluorine-18 labeling of protein/peptide using the useful 6-fluoronicotinic acid-2,3,5,6-tetrafluorophenyl prosthetic group: A method comparable to direct fluorination. J Labelled Comp Radiopharm, 2017. 60(3): p. 168-175. 4. Nagatsugi, F., et al., 20-[18F]fluoroarachidonic acid: tissue biodistribution and incorporation into phospholipids. Biol Pharm Bull, 1996. 19(10): p. 1316-21. 5. Zhang, X., et al., Automated synthesis of [(18)F](2S,4R)-4-fluoroglutamine on a GE TRACERlab FX-N Pro module. Appl Radiat Isot, 2016. 112: p. 110-4.

\section{Image/Figure:}

https://www.xcdsystem.com/wmis/abstract/File6959/GA137 ImageFigure 0505123410.png

Image/Figure Caption: Figure 1: PSMA imaging (coronal PET image at $1 \mathrm{~h}$ post FDC injection) in PC3/PSMA+ [PSMA-transfected;+control) and LuCaP PDX mouse models in vivo (A) reflect PSMA levels (Bmax) determined from in vitro FDC saturation binding assays using corresponding LuCaP PDX and organoid membrane preparations (fmoles PSMA/ $\mu$ g protein membrane prep) from either fresh/frozen excised whole mouse xenografts or PDX-derived organoids, respectively (B). An imaging agent grid was generated (B) detailing the PC model, original patient histology and metastatic location [1], in vivo response to ADT (Degarelix) and AR protein levels determined by immunohistochemistry (AR IHC) in vivo [1]; PSMA levels in PDXs and PDX-derived organoids were paired with metabolic PET agent uptake (\% uptake per 106 live cells $=[($ cpmuptake $) /($ cpmtotal added $) \times($ live cellstotal added $)] \times(106$ live cells $) \times 100)$ after 120 min of incubation in organoids. PC3/PSMA+ cells were maintained as a single cell suspension from $2 \mathrm{D}$ culturing for metabolic agent uptake assays.

Complete Status: Complete

First Name: Margaret

Last Name: White

Email: maggie.white@nih.gov

Organization: National Institutes of Health

Country: United States 


\title{
ID: GA138 \\ Non-invasive quantification of neuroinflammation tracer in non-human primates
}

\author{
Lucero Aceves-Serrano, University of British Columbia, aceves.lucero@alumni.ubc.ca
}

\section{Category: Neuroscience}

\begin{abstract}
Body : Introduction: Positron emission tomography tracers that target the translocator protein (TSPO) have become an essential tool in the assessment of neuroinflammation. However, tracer quantification can be difficult as it traditionally requires arterial cannulation. Thus, four alternative quantitative and semi-quantitative blood-free measurements of the TSPO tracer [11C]-PBR28 binding were evaluated and compared to the standard plasma input measurements. Methods: Thirteen [11C]-PBR28 scans from rhesus monkeys were acquired along with metabolite and plasma activity used to create arterial input functions (AIF). Total distribution volume (VT) and non-displaceable binding potential (BPND) were quantified with AIF (VTAIF, BPNDAIF)(1). For BPNDAIF, activity in white matter (WM) was used to estimate VND. Four additional metrics were computed: VT and BPND using a population-derived metabolitecorrected plasma input function (VTPBIF, BPNDPBIF), standard uptake value (SUV), SUV ratio to $\mathrm{WM}$ (SUVr), and BPND using WM time activity curve as tissue input function (BPNDWM)(2) using (i)the 20-50 min and the (ii)30-70 minutes post-injection time frames. 4 animals were scanned twice, providing test-retest reproducibility data. Results: $20-50$ min postinjection time frames; Parent and metabolite fractions were similar in arterial $(\mathrm{N}=9)$ and venous samples $(\mathrm{N}=3)$. There was good reproducibility between the test and retest scans for BPNDAIF (ICC $=0.72)$ BPNDPBIF (ICC $=0.69)$ SUVr $(\mathrm{ICC}=0.85)$ and BPNDWM $(\mathrm{ICC}=0.86)$ but not for SUV (ICC $=0.22)$. There was a good correlation between the VTAIF and VTPBIF within individual animals $(\mathrm{r}=0.98 \pm 0.01)$ but not across animals $(\mathrm{r}=0.52)$ (Fig. 1). Correlation between the BPNDAIF and BPNDPBIF was good both within $(\mathrm{r}=0.98 \pm 0.01)$ and across animals $(\mathrm{r}=0.97)$. Furthermore, the correlation was moderate for SUV, SUVr, and BPNDWM within animals $(\mathrm{r}=0.8 \pm 0.09)$, moderate for BPNDWM $(\mathrm{r}=0.71)$ across animals and poor for SUV and SUVr across animals ( $\mathrm{r}=0.21$ ). 30-70 min time frames; BPNDAIF, BPNDPBIF, and SUV showed increased variability compared to using the 20-50 min time frame suggesting that some [11C]PBR28 metabolite(s) can cross the blood-brain barrier, as their effect would be most impactful on data acquired later in the scan . Conclusion: In monkeys, the stability of peripheral metabolism permits the use of a PBIF to measure BPND. The high correlation between BPNDAIF and BPNDPBIF and the good test-retest value suggests that a population-derived curve may be a good alternative to individual sampling, as previously shown in humans(3). Furthermore, these results suggest that using earlier (20-50 min) time frames might yield more reliable measurements than using later (30-70 min) time frames. Acknowledgments: The authors would like to acknowledge TRIUMF for providing the radiotracer, and Brain Canada for funding this project.
\end{abstract}

References: 1. J. Logan et al., J. Cereb. Blood Flow Metab. 10, 740-747 (1990). 2. J. Logan et al., J. Cereb. Blood Flow Metab. 16, 834-840 (1996). 3. M. Schain, F. Zanderigo, J. J. Mann, R. T. Ogden, Neuroimage. 146, 121-131 (2017). 
Complete Status: Complete

First Name: Lucero

Last Name: Aceves-Serrano

Email: aceves.lucero@alumni.ubc.ca

Organization: University of British Columbia

Country: Canada 


\title{
ID: GA139 \\ Differentiating Aß40 and Aß42 with a small molecule fluorescence probe
}

\author{
Biyue Zhu, Massachusetts General Hospital, 413897689@qq.com
}

\section{Category: Neuroscience}

\begin{abstract}
Body : Differentiating amyloid beta (Ab) subspecies Ab40 and Ab42 has long been considered as an impossible mission with small-molecule probes. In this report, based on recently published structures of $\mathrm{Ab}$ fibrils, we designed iminocoumarin-thiazole (ICT) fluorescence probes to differentiate Ab40 and Ab42, among which Ab42 has much higher neurotoxicity. We demonstrated that ICTAD-1 robustly responds to Ab fibrils, evidenced by turn-on fluorescence intensity and red-shifting of emission peaks. Remarkably, ICTAD-1 showed different spectra towards Ab40 and Ab42 fibrils. In vitro results demonstrated that ICTAD- 1 could be used to differentiate Ab40/42 in solutions. Moreover, our data revealed that ICTAD- 1 could be used to separate Ab40/42 components in plaques of $\mathrm{AD}$ mouse brain slides. In addition, two-photon imaging suggested that ICTAD-1 was able to cross the BBB and label plaques in vivo. Interestingly, we observed that ICTAD-1 was specific toward plaques, but not cerebral amyloid angiopathy (CAA) on brain blood vessels. Given Ab40 and Ab42 species have significant differences of neurotoxicity, we believe that ICTAD-1 can be used as an important tool for basic studies and has the potential to provide a better diagnosis in the future.
\end{abstract}

References: 1. Hardy, J.; Selkoe, D. J., The amyloid hypothesis of Alzheimer's disease: progress and problems on the road to therapeutics. Science 2002, 297 (5580), 353-6. 2. Goedert, M.; Spillantini, M. G., A century of Alzheimer's disease. Science 2006, 314 (5800), 777-81. 3. McLean, C. A., Cherny, R.A., Fraser, F.W., Fuller, S.J., Smith, M.J., Beyreuther, K., Bush, A.I., Masters, C.L., Soluble pool of Abeta amyloid as a determinant of severity of neurodegeneration in Alzheimer's disease. Ann Neurol. 1999, 46 (6), 860-6. 4. Lue, L. F., Kuo, Y.M., Roher, A.E., Brachova, L., Shen, Y., Sue, L., Beach, T., Kurth, J.H., Rydel, R.E., Rogers, J., Soluble amyloid beta peptide concentration as a predictor of synaptic change in Alzheimer's disease. Am J Pathol. 1999, 155 (3), 853-62. 5. Terry, R., Masliah E, Salmon DP, Butters N, DeTeresa R, Hill R, Hansen LA, Katzman R., Physical basis of cognitive alterations in Alzheimer's disease: synapse loss is the major correlate of cognitive impairment. Ann Neurol. 1991, 30 (4), 572-80. 6. Bernstein, S. L.; Dupuis, N. F.; Lazo, N. D.; Wyttenbach, T.; Condron, M. M.; Bitan, G.; Teplow, D. B.; Shea, J. E.; Ruotolo, B. T.; Robinson, C. V.; Bowers, M. T., Amyloid-beta protein oligomerization and the importance of tetramers and dodecamers in the aetiology of Alzheimer's disease. Nat Chem 2009, 1 (4), 326-31. 7. Qiu, T.; Liu, Q.; Chen, Y. X.; Zhao, Y. F.; Li, Y. M., Abeta42 and Abeta40: similarities and differences. J Pept Sci 2015, 21 (7), 522-9. 8. Li, Y.; Yang, J.; Liu, H.; Yang, J.; Du, L.; Feng, H.; Tian, Y.; Cao, J.; Ran, C., Tuning the stereo-hindrance of a curcumin scaffold for the selective imaging of the soluble forms of amyloid beta species. Chem Sci 2017, 8, 7710-7717.

\section{Image/Figure:}


https://www.xcdsystem.com/wmis/abstract/File6959/GA139 ImageFigure 0420071112.jpg

Full Name of Abstract's 1st Author : biyue zhu

Complete Status: Complete

First Name: Biyue

Last Name: Zhu

Email: 413897689@qq.com

Organization: Massachusetts General Hospital

Country: United States 


\title{
ID: GA141 \\ MR guided radiotherapy imaging data quantification for early radiotherapy response measurement in pancreatic cancer patients
}

\author{
Michal Tomaszewski, Moffitt Cancer Center, michal.tomaszewski@moffitt.org
}

\section{Category: Computational \& Data Science}

\begin{abstract}
Body : Purpose/Objectives: Magnetic Resonance Image guided stereotactic body radiotherapy (MRgRT) is an emerging technology increasingly utilized in localized pancreatic adenocarcinoma (PDAC)[1]. Given the variable response rates and short progression times in PDAC, there is an unmet clinical need for a method to assess early RT response and ultimately predict survival that may allow us to better personalize therapy. We hypothesize that quantitative image feature analysis (radiomics) of the daily MR scans before and during MRgRT may be used to extract information related to early treatment response. Materials/Methods: 9 standard histogram [2] radiomic features were extracted from MRgRT images [3] (MRidian System, ViewRay, 0.35T, TRUFI sequence, $1.5 \mathrm{~mm} / 3 \mathrm{~mm}$ voxel size/slice thickness) from $\mathrm{n}=19$ locally advanced and borderline resectable patients, scanned at simulation (SIM) 14 days before SBRT start, and prior to each of 5 daily fractions (F1-F5) of 10Gy to Gross Tumor Volume (GTV). 9 patients had CA19-9 serum levels recorded pre/post induction chemotherapy (IC) pre RT (median days IC end to RT start:17). They were stratified for IC response by the CA19-9 [4] ratio post/pre IC (good if median). Normal tissue region (ROI_tis) was defined as whole abdomen minus GTV in 10 slices around the tumor center (Fig.1 $\bar{A}$ ). Spatial stability was quantified as Kendall's Concordance Coefficient (CC) between feature values when GTV Region of Interest (ROI) was moved $+/-1.5 \mathrm{~mm}$ in $\mathrm{x}$ and $\mathrm{y}$. Temporal image stability was quantified as coefficient of variation $(\mathrm{CoV})$ of mean ROI_tis signal values SIM-F5. Significance of changes SIM-F1 and F1-F5 were assessed using two-sided paired t-test. Results: All considered features apart from min, max and kurtosis showed very good spatial stability with $\mathrm{CC}>0.95$. Mean signal intensity ratio F1/SIM in GTV correlates strongly with corresponding changes in ROI_tis (Pearson r=0.87, p Suppl.Tab.1, Fig.1B). No trends were seen in ROI_tis $(\mathrm{IQR} p=0.50, \mathrm{SD} \mathrm{p}=0.48)$. Patients with good biochemical response to IC [5] (post/pre CA $19-9$ ratio Fig.1C), consistently with the observed heterogeneity drop after XRT. Conclusion: With MRgRT traditionally only used for spatial dose planning, these results present a novel framework for MRgRT image quantification using histogram analysis. Efficient normalization method was found to remove technical value drift and improve sensitivity. Significant changes in heterogeneity metrics were observed prior and after MRgRT, found to correlate with tumor response to induction chemotherapy, suggesting that our methods can be used for surrogate treatment response quantification. More work is required to understand the biological basis of the observed trends.
\end{abstract}

References: 1. Rudra S, Jiang N, Rosenberg SA, Olsen JR, Roach MC, Wan L, et al. Using adaptive magnetic resonance image-guided radiation therapy for treatment of inoperable pancreatic cancer. Cancer Med 2019;8:2123-32 2. Just N. Improving tumour heterogeneity MRI assessment with histograms. Br J Cancer. 2014;111(12):2205-13. 3. Mutic S, Dempsey JF. The 
ViewRay system: magnetic resonance-guided and controlled radiotherapy. Semin Radiat Oncol 2014;24:196-9 4. Hess V, Glimelius B, Grawe P, Dietrich D, Bodoky G, Ruhstaller T, et al. CA 19-9 tumour-marker response to chemotherapy in patients with advanced pancreatic cancer enrolled in a randomised controlled trial. Lancet Oncol. 2008;9(2):132-8.

\section{Image/Figure:}

https://www.xcdsystem.com/wmis/abstract/File6959/GA141 ImageFigure 0420071726.png

Full Name of Abstract's 1st Author : Michal Tomaszewski

Complete Status: Complete

First Name: Michal

Last Name: Tomaszewski

Email: michal.tomaszewski@moffitt.org

Organization: Moffitt Cancer Center

Country: United States 


\title{
ID: GA142 \\ T2 MRI heterogeneity provides a sensitive measure of early tumor response to radiotherapy
}

\author{
Michal Tomaszewski, Moffitt Cancer Center, michal.tomaszewski@moffitt.org
}

\section{Category: Oncology}

\begin{abstract}
Body : BACKGROUND: External beam radiotherapy (XRT) is a widely used cancer treatment, yet responses vary dramatically between patients. In this work, a novel Magnetic Resonance Imaging (MRI) approach is presented, utilizing the histogram analysis of a clinicallyused T2 relaxation measurement to provide a quantitative measure of tumor heterogeneity [1], indicative of early response to radiation, which can be used to adapt the treatment and maximize its effect. Relationship to treatment response, robustness, and biological underpinnings of the proposed biomarker candidate are shown. METHODS: MRI was used to measure the response to single dose 10Gy X-ray irradiation (XRAD 320, Precision X-Ray, CT) in two murine subcutaneous models of pancreatic ductal adenocarcinoma (Panc02, $n=11$ and BXPC3, $n=8)$. T2 relaxation mapping (MSME, $\mathrm{TR}=3427 \mathrm{~ms}, 32 \times \mathrm{TE}=7-224 \mathrm{~ms}, 128 \times 128$ points) was performed on a $7 \mathrm{~T}$ preclinical MRI (Bruker) every $72 \mathrm{~h}$ after treatment, and quantitative histogram analysis was used to identify most sensitive metrics of response. H\&E stained sections were processed in Tissue Studio (Definiens) and MATLAB to derive local cellular density maps at resolution matching MRI, approximated as the nuclear fraction, a pixel area fraction occupied by nuclei. RESULTS: 72h after XRT clear changes in tumor T2 maps were observed, apparent in histogram analysis (Fig.1). Interquartile range (IQR) of T2 showed excellent sensitivity for detection of changes due to early XRT response in both tumor types, compared to other histogram metrics (Tab.1). Non-irradiated tumors $(n=6$ Panc02) showed no change in the metrics. Histogram analysis of local cell density maps and T2 MRI maps showed matching IQR differences between the tumor types with elevated IQR in the heterogeneous BXPC3 tumors (Suppl.Fig.1). Additionally, histological co-registration showed higher IQR in areas of necrosis ( $7.4 \pm 1.1 \mathrm{~ms}$ vs. $4.0 \pm 0.4 \mathrm{~ms}, \mathrm{p}=0.025)$ suggesting that the changes are driven by radiation induced cell death. Importantly, a significant correlation was shown between early IQR change and tumor volume change ratio at the point of regrowth (Pearson $r=-0.67, p=0.035$ Panc02, $r=-0.70$, $\mathrm{p}=0.036$ BXPC3, Suppl.Fig.2), illustrating the utility of the metric for prediction of further tumor response. Furthermore, the IQR showed excellent test-retest agreement, superior to other metrics (Tab.1, last column). CONCLUSION: The study presents a novel, robust method and an associated imaging biomarker for measurement of early radiotherapy response in murine models of pancreatic cancer, based on readily translatable MRI T2 mapping. Histological comparison reveals the biological underpinnings of the metric, showing that T2 IQR is sensitive to cellular heterogeneity, strongly affected by necrosis as caused by irradiation [2]. Importantly, the predictive power of the IQR for tumor response was shown. This approach can be directly validated clinically thanks to the advent of MRI-guided radiotherapy [3,4]. Further, the method can be used for patient stratification, with escalation of radiation dose when a poor response to standard therapy protocol is predicted by the model.
\end{abstract}


References: 1. Just N. Improving tumour heterogeneity MRI assessment with histograms. Br J Cancer 2014;111:2205-13 2. Eriksson D, Stigbrand T. Radiation-induced cell death mechanisms. Tumour Biol 2010;31:363-72 3. Pollard JM, Wen Z, Sadagopan R, Wang J, Ibbott GS. The future of image-guided radiotherapy will be MR guided. Br J Radiol 2017;90:20160667 4. Rudra S, Jiang N, Rosenberg SA, Olsen JR, Roach MC, Wan L, et al. Using adaptive magnetic resonance image-guided radiation therapy for treatment of inoperable pancreatic cancer. Cancer Med 2019;8:2123-32

\section{Image/Figure:}

https://www.xcdsystem.com/wmis/abstract/File6959/GA142 ImageFigure 0421054540.png

Full Name of Abstract's 1st Author : Michal Tomaszewski

Complete Status: Complete

First Name: Michal

Last Name: Tomaszewski

Email:michal.tomaszewski@moffitt.org

Organization: Moffitt Cancer Center

Country: United States 


\title{
ID: GA146 \\ A high-throughput screen identifies compounds that regulate 18F-FDG consumption in cancer cell xenografts
}

\author{
Peter Clark, UCLA, pclark@mednet.ucla.edu
}

\section{Category: Oncology}

\begin{abstract}
Body : Background. One of the most exciting uses of 18F-FDG PET in cancer is as an early functional biomarker of drug efficacyl. However, identifying for which therapies and in which cancers 18 F-FDG PET can perform this function remains a significant challenge. We have an incomplete understanding of how cancers regulate glucose consumption and only lowthroughput methods for measuring this key pathway. High-throughput screens are powerful tools to identify new regulators of a pathway. Objective. To develop and validate a highthroughput assay for measuring glucose consumption, and to conduct a high-throughput screen to identify new compounds that regulate glucose consumption and for which 18F-FDG PET could function as a biomarker of efficacy in lung cancer. Methods. We adapted and optimized a luminescence assay for measuring glucose consumption to achieve the necessary properties to use in a high-throughput screen. We screened 3555 clinically relevant compounds against three genetically distinct lung cancer cell lines (H460, HCC827, and A549) and measured changes in glucose consumption using our high-throughput assay. We validated 15 of the compounds in cell culture by measuring IC50 values and further validated one compound, Milciclib, in vivo in H460 cells using 18F-FDG PET. We overexpressed GLUT1 in H460 cells to evaluate whether blocking glucose consumption was necessary for Milciclib to block cell growth. Results. We identified conditions that yielded a high-throughput assay for measuring glucose consumption with a Z-factor of $0.59 \pm 0.05$, indicating a large dynamic range and low variability. We discovered over 100 clinically relevant compounds across three genetically distinct lung cancer cell lines that blocked glucose consumption by $>50 \%$. Surprisingly, very few of the compounds overlapped between the different cell lines, suggesting that the genetic background of a cancer strongly determines the mechanisms used to regulate glucose consumption. The most potent inhibitors of glucose consumption include topoisomerase inhibitors such as camptothecin (IC50: 464 and $98 \mathrm{nM}$ in HCC827 and H460 cells, respectively) and the tyrosine kinase inhibitor dovitinib (IC50: 554 and $1511 \mathrm{nM}$ in HCC827 and H460 cells, respectively). Milciclib is a CDK and TRK inhibitor in clinical trials. Milciclib inhibits glucose consumption in H460 cells in culture with an IC50 of $1.5 \mathrm{mM}$ by decreasing GLUT1 levels and decreases 18F-FDG consumption in $\mathrm{H} 460$ xenografts by $30.4 \pm 2.5 \%$. GLUT1 overexpression blocked Milciclib from inhibiting glucose consumption and inhibiting cell growth, suggesting that blocking glucose consumption is a major mechanism through which Milciclib limits cell growth2. Innovation. We developed a new method to rapidly identify compounds that block cancer cell glucose consumption and for which 18F-FDG PET could be used an early functional biomarker. Impact. We anticipate that this method will enable studies that expand the use of 18 F-FDG PET and suggest new therapies for which 18F-FDG PET could function as a biomarker of drug efficacy in cancer.
\end{abstract}


References: 1. Pinker K, et al. Eur J Nucl Med Mol Imaging. 2017 44:55-66 2. Ghezzi C, et al. Nat Commun. 2019 10(1):5444

\section{Image/Figure:}

https://www.xcdsystem.com/wmis/abstract/File6959/GA146_ImageFigure_0421013142.png

Complete Status: Complete

First Name: Peter

Last Name: Clark

Email: pclark@mednet.ucla.edu

Organization: UCLA

Country: United States 


\title{
ID: GA147 \\ Evaluation of calcification in the heart of partially nephrectomized mice by computed tomography and $18 \mathrm{~F}-\mathrm{NaF}$ positron emission tomography
}

\author{
Rira Watanabe, Daiichi Sankyo, watanabe.rira.vi@daiichisankyo.co.jp
}

\section{Category: Cardiovascular \& Pulmonary}

\begin{abstract}
Body : Coronary artery calcification is routinely evaluated by an unenhanced low-dose CT scan, while sodium fluoride-18 (18F-NaF) positron emission tomography (PET), which can highlight active mineralization, are getting more popular and applied in the clinic1). In the course of drug discovery and development, preclinical CT/18F-NaF PET study is demanded before clinical implementation to ensure the usefulness of conducting the imaging studies in the clinic especially for evaluation of drug efficacy with consideration for the mechanism of action of the drug. In this study, we demonstrated usefulness of micro CT/18F-NaF PET for calcification in the heart in a nephrectomized animal model. Five/six nephrectomized DBA/2J mice (Nx mice) on high-phosphorus and calcium diet (acceleration diet) was used since they develop severe coronary artery calcification. Each $4 \mathrm{Nx}$ mice were on the acceleration diet for 1, 2, 3 or 14 weeks and each 2 sham-operation mice were fed standard-chow for 3 or 14 weeks. Micro-CT images of the chest were taken with cardiac synchronization and calcification volume of the heart was calculated by imaging analysis. The day after CT scanning, $18 \mathrm{~F}-\mathrm{NaF}$ was injected to all mice. $18 \mathrm{~F}-\mathrm{NaF}$ uptake was assessed by 10 -minute 2D-PET scanning followed by dissection of the heart for counting radioactivity accumulated. The dissected hearts were then dried completely and analyzed for calcium content by colorimetric analysis. Results showed calcium content increased over the course of the study. Micro-CT and 18F-NaF 2D-PET imaging were able to visualize heart calcification. Micro-CT demonstrated mild to severe calcification in the heart of Nx mice after 2, 3 or 14 weeks of the acceleration diet-loading, while calcification was not apparent in the heart of sham-operation mice and Nx mice after 1 week of the diet-loading. $18 \mathrm{~F}-\mathrm{NaF} 2 \mathrm{D}-\mathrm{PET}$ imaging also showed higher signals around the heart of $\mathrm{Nx}$ mice than shamoperation mice, however, delineation of the heart was difficult because of the limitation of 2DPET: overlay of the physiological accumulation of $18 \mathrm{~F}-\mathrm{NaF}$ on the bone and low resolution. Calcification volume measured by micro-CT and $18 \mathrm{~F}-\mathrm{NaF}$ accumulation in the heart correlated well with calcium content respectively $(\mathrm{R}=0.90$ and 0.95$)$. However, limitation of detection by CT were suggested for small calcification at early phase of this animal model whereas high sensitivity and good linearity of $18 \mathrm{~F}-\mathrm{NaF}$ accumulation was indicated. In this model mice, calcium content in the heart was considerably variable among individuals from early to late phase, so development of heart calcification should be ideally assessed by individual basis. These in vivo imaging methods are considered useful for serial monitoring of progression of lesions because micro-CT is minimally invasive and can be applied consecutively. This imaging modality could be useful for allocation of individual model mice into groups to evaluate anticalcification efficacy of drugs with small number of animals. In assessment of mineralization, combination of quantitative micro-CT and $18 \mathrm{~F}-\mathrm{NaF}$ accumulation would be useful to assess early and late phase calcification and allow us to evaluate anti-calcification effect of compounds
\end{abstract}


under development in preclinical, which eventually encourages clinical application of these imaging analyses.

References: 1. Joshi NV, Vesey AT, Williams MC, et al. Lancet. 2014;383(9918):705-13

Complete Status: Complete

First Name: Rira

Last Name: Watanabe

Email: watanabe.rira.vi@daiichisankyo.co.jp

Organization: Daiichi Sankyo

Country: Japan 


\title{
ID: GA149 \\ PD-L1 Aptamer-based Nanoscale Metal-Organic Framework Reduces Toxic and Side Effects in the Chemo-, Photodynamic Therapy Primed Cancer Immunotherapy
}

\author{
YANG DU, CAS Key Laboratory of Molecular Imaging, Institute of Automation, Chinese \\ Academy of Sciences, duyang@fingerpass.net.cn
}

Category: Oncology

\begin{abstract}
Body : In the U.S., colorectal cancer (CRC) is the third most common type of cancer, and the 5-year survival rate for patients with metastatic CRC is only $14 \%$. Although a substantial of methods have been invented to cure cancer, chemotherapy is still a golden standard in late CRC. Chemotherapy always use drugs to kill tumor cells, but in some cases the side effects and toxic are also nonnegligible. The anti-PD-1/PD-L1 antibodies, as the checkpoint inhibitors, are deemed as the other alternative way and the most promising immunotherapies for the advanced CRC patients. However, their durable response rate is low, and instances of severe treatmentrelated adverse events were also reported due to the systemic administration of immunostimulatory agents. We herein report the fabrication of near infrared dye Cy5.5 labeled immunogenic nanoparticles of PD-L1 aptamer-based, oxaliplatin-loading nanoscale metalorganic frameworks (Cy5.5-NMOFs@OXA@Apt) for monitoring its in vivo tumor targeting effect and therapeutic efficacy. The Cy5.5-NMOFs@OXA@Apt as immunogenic agents is used for the combined chemotherapy and photodynamic therapy (PDT), which can also synergize with the aptPD-L1 immunotherapy. The data showed that, first, Cy5.5-NMOFs@OXA@Apt showed a specific and targeted fluorescence imaging of MC38 CRC; Second, synergy between PDT and oxaliplatin effectively kills tumor cells and induces immune response, resulting in the exposure of calreticulin (CRT) on tumor cells and antitumor vaccination effect; Third, the Cy5.5NMOFs@OXA@Apt treatment induced the regression of both light-irradiated primary tumors and also non-irradiated distant tumors through inducing a strong tumor-specific immune response with less observable systemic toxicity. In conclusion, our work indicated that the Cy5.5-NMOFs@OXA@Apt not only showed a specific and targeted tumor therapy efficacy, but also decrease the risk during the therapeutic process. This simple and translatable approach of engineered aptPD-L1 targeted NMOFs may present a novel and safer approach in immune checkpoint blockade therapy.
\end{abstract}

Full Name of Abstract's 1st Author : YANG DU

Complete Status: Complete

First Name: YANG

Last Name: DU

Email: duyang@fingerpass.net.cn 
Organization: CAS Key Laboratory of Molecular Imaging, Institute of Automation, Chinese Academy of Sciences

\section{Country: China}




\title{
ID: GA151 \\ Preclinical tumor imaging using monoclonal antibody ramucirumab labeled with zirconium-89
}

\author{
Zbynek Novy, Palacky University Olomouc, novy.zbynek@gmail.com
}

\section{Category: Oncology}

\begin{abstract}
Body : Aim: The angiogenesis suppression has become one of the key anti-tumor strategies. This also includes blocking of VEGFR2 binding site by the monoclonal antibody ramucirumab (RAM). The aim of this study was to prepare RAM radiolabeled with positron emission tomography (PET) imaging radionuclide zirconium-89 and subsequently determine in vitro and in vivo biological properties of $89 \mathrm{Zr}$-labelled ramucirumab. Methods: The conjugation of RAM with the bifunctional chelator p-SCN-Bn-deferoxamine (DFO) was carried out at pH 9.0 in sodium bicarbonate. DFO-RAM was radiolabeled with $(89 \mathrm{Zr}) \mathrm{Zr}$-oxalate at $\mathrm{pH} 5.5$ in sodium acetate solution. The binding affinity of prepared 89Zr-DFO-RAM to VEGFR2 was tested in vitro on prostate adenocarcinoma (PC-3) and ovary adenocarcinoma (SK-OV-3) cell lines. The $\mathrm{PET} / \mathrm{CT}$ imaging and ex vivo biodistribution studies were performed in mice engrafted with PC3 and SK-OV-3 tumors. Results: The in vitro experiments showed preserved binding ability of 89Zr-DFO-RAM to VEGFR2 with the KD values $38.93 \pm 2.04$ and $36.59 \pm 8.36 \mathrm{nM}$ for PC-3 and SK-OV-3 cells respectively. The obtained ex vivo biodistribution data revealed the activity uptake in PC-3 and SK-OV-3 tumors at about $8.7 \pm 0.2$ and $12.1 \pm 1.6 \% \mathrm{ID} / \mathrm{g}$ respectively. The tumor-to-blood ratio for 1,3 and 6 days p.i. was $0.4,0.6$ and 0.8 for PC-3 and 0.5, 1.0 and 1.3 for SK-OV-3 tumors respectively. The PET/CT images showed high radioactivity accumulation in the tumors starting already at first day p.i. Conclusions: The labeling of DFO-RAM with Zr-89 provided anti-VEGFR2 radiopharmaceutical with promising binding to the target receptors both in vitro and in vivo. The results of in vivo experiments proved the potency of 89Zr-DFO-RAM to target and image VEGFR2-positive tumors. However, several non-target organs like e.g. heart, spleen and liver showed also increased accumulation of the radioactivity.
\end{abstract}

References: none

Complete Status: Complete

First Name: Zbynek

Last Name: Novy

Email: novy.zbynek@gmail.com

Organization: Palacky University Olomouc

Country: Czech Republic 


\title{
ID: GA152 \\ A machine learning reconstruction method for nuclear optical imaging
}

\author{
Zeyu Zhang, Beihang University, zhang.zey.doc@gmail.com
}

\section{Category: Computational \& Data Science}

\begin{abstract}
Body : Background: Cerenkov luminescence tomography (CLT) provides an effective way to harness the advantages of both nuclear imaging and optical imaging. The previous studies have shown that CLT is highly sensitive and timesaving for in vivo tumor detection. However, the accuracy of CLT is still limited, because the conventional mathematical functions are difficult to precisely describe the photon propagation in vivo. Therefore, the calculation error is inevitable. Herein, a machine learning method was developed to perform in vivo CLT. Using the ANN reconstruction method, the calculation error could be avoided and the reconstruction accuracy was able to be improved. Evaluation with Monte-Carlo simulation and glioma mouse models have shown that the ANN CLT approach owned significantly higher reconstruction accuracy compared with the conventional methods. Methods: As shown in Figure 1A, the developed ANN for CLT contained five different layers. From left to right, the first one was the input layer, and then there were three hidden layers followed by the output layer. For a welltrained ANN CLT model, the reconstructed Cerenkov luminescence sources could be generated when a surficial optical flux was given. Monte-Carlo simulation data were generated for the ANN to learn the relationship between detected signals and the actual photon sources.

Approximately 6,000 Monte-Carlo CLI data were simulated, which covered nearly 500 different positions in the mouse brain. To take the simulation randomness into account, three different source shapes (ellipsoid, cylinder and cube) were used, and five repetitive simulations were conducted in each source position. Molecular optical simulation environment (MOSE) version 2.3 and a standard digital mouse model was applied to perform the simulation. The ANN CLT reconstruction performance was evaluated through photon sources in different numbers, depth and shapes. Conventional propagation model based reconstruction method was also applied for contrast. Moreover, orthotropic glioma mouse models $(n=4)$ were constructed for in vivo validation. $4 \times 106$ U87-MG glioma cells mixed with PBS $(10 \mu \mathrm{L})$ were injected in the mouse brains. 11C-methionine (11C-MET, $600 \mu \mathrm{Ci}, 100 \mu \mathrm{L})$ was injected through the tail vein and performed in vivo CLI after $20 \mathrm{~min}$, followed by CT imaging. H\&E staining results cryo-slices of the mouse head were obtained as the "golden standard" to validate the reconstruction accuracy. Results: Figure 1B illustrates the CLT results using the ANN CLT method. Explicit consistency can be observed between the simulated tumor tissues and the reconstructed results. Compared with the conventional CLT method, the ANN CLT results provided more accurate tumor reconstruction results, both in tumor positions and tumor shapes. These observations demonstrated the proposed reconstruction strategy was effective and promising. The improvements of the ANN CLT strategy were also be seen from the in vivo imaging on mice. The reconstruction accuracy was verified by the cryo-slice $\mathrm{H} \& \mathrm{E}$ results. Higher reconstruction accuracy was shown in the ANN CLT, compared with the conventional method. This outcome indicated that the proposed ANN CLT method was feasible for in vivo tumor imaging and was able to enhance the tumor diagnostic precision. Conclusion: In this study, a novel CLT
\end{abstract}


reconstruction method based on ANN was developed, which could avoid the reconstruction errors generated from the calculation of photon propagation. The improvements of the ANN CLT were presented by both Monte-Carlo simulation and in vivo imaging on orthotropic glioma bearing mice. Compared with the conventional CLT method, the proposed ANN CLT reconstruct the inner photon sources with higher accuracy. The in vivo evaluation on mouse models further indicated that the ANN CLT effectively detected the inner tumor tissues. We believe this CLT method could promote the application of CLT, and boost the combination of artificial intelligence and molecular imaging.

References: [1] Zhu B, Liu JZ, Cauley SF, Rosen BR, Rosen MS. Image reconstruction by domain-transform manifold learning. Nature. 2018 Mar;555(7697):487-92. [2] Ouyang W, Aristov A, Lelek M, Hao X, Zimmer C. Deep learning massively accelerates super-resolution localization microscopy. Nature biotechnology. 2018 May;36(5):460-8. [3] Gao Y, Wang K, An Y, Jiang S, Meng H, Tian J. Nonmodel-based bioluminescence tomography using a machinelearning reconstruction strategy. Optica. 2018 Nov 20;5(11):1451-4. [4] Huang C, Meng H, Gao Y, Jiang S, Wang K, Tian J. Fast and robust reconstruction method for fluorescence molecular tomography based on deep neural network. InImaging, Manipulation, and Analysis of Biomolecules, Cells, and Tissues XVII 2019 Mar 4 (Vol. 10881, p. 108811K). International Society for Optics and Photonics.

\section{Image/Figure:}

https://www.xcdsystem.com/wmis/abstract/File6959/GA152_ImageFigure_0421040536.png

Complete Status: Complete

First Name: Zeyu

Last Name: Zhang

Email: zhang.zey.doc@gmail.com

Organization: Beihang University

Country: China 


\title{
ID: GA154 \\ The imaging of anti-EGFR specific fluorescent nanobody probe generated via bioorthogonal reaction in Esophageal Squamous Cell Carcinoma
}

\author{
Xueli Chen, Xidian University, xlchen@xidian.edu.cn
}

\section{Category: Oncology}

\begin{abstract}
Body : The overexpression of epidermal growth factor receptor (EGFR) is the key molecular marker for poor prognosis factor in esophageal squamous cell carcinoma. The antiEGFR nanobodies which are modified with imaging tracer can be used as specific molecular imaging probes. In view of this, $\mathrm{N}-\varepsilon$-acryllysine was genetically incorporated into a specific targeted EGFR nanobody 7D12. A photo-triggered cycloaddition reaction between the 7D12 and the tetrazole compounds has been used to generate a fluorescent anti-EGFR nanobody probe (7D12-AcrK-T3). Cellular experiments showed that the 7D12-AcrK-T3 fluorescent probe had specific uptake in the EGFR-overexpressing cells. In contrast, few fluorescence signal were found in the EGFR negative expression and control groups. The xenograft nude (nu/nu) mice of esophageal squamous cell were intravenously injected 7D12Acrk-T3. The imaging results showed that fluorescent values in the tumor area of regions of interest were significantly higher than that in the muscle tissues and in the blocking groups at $3 \mathrm{~h}$ post injection. The above results indicate the bioorthogonal reaction to achieve site-specific labeling of nanobodies, and this is a fast and versatile method for establishing fluorescent nanobodies probes, which can reduce the heterogeneity of probes caused by traditional chemical methods. This study will accelerate the development of the specific molecular imaging probes and application for the early tumor diagnosis of esophageal squamous cell carcinoma.
\end{abstract}

\section{Image/Figure:}

https://www.xcdsystem.com/wmis/abstract/File6959/GA154 ImageFigure 0421044558.jpg

Complete Status: Complete

First Name: Xueli

Last Name: Chen

Email: xlchen@xidian.edu.cn

Organization: Xidian University

Country: China 


\title{
ID: GA155 \\ An engineering secreted luciferase reporter for noninvasive visualization of gasdermin D-induced pyroptotic cell death
}

\author{
Fu Wang, Xidian Unniversity, fwang@xidian.edu.cn
}

\section{Category: New Chemistry, Biology \& Bioengineering}

\begin{abstract}
Body : Pyroptotic cell death is a phenomenon that runs through all life activities and plays an important role in the physiological and pathological processes of the body's metabolism. It is of big biological significance to understand the phenomenon and nature of cell pyroptosis, In the process of cell pyroptosis, the pore-forming effector GSDMD (Gasdermin D) is cleaved to form oligomers, which are inserted into the cell membrane, causing rapid cell death. However, the effective cell death induced by GSDMD complicates our ability to understand the biology of this protein. In this work, we performed mutagenesis and molecular modeling to develop a pyroptosis reporter, which strategically place secreted luciferase Gluc in the p30-p20 tolerated junction of GSDMD to support natural pyrophosphorylation and promote live imaging of cell pyroptosis. In addition, we demonstrated that this fused Gluc-GSDMD reporter executed inflammatory body-dependent cell death in response to multiple stimuli, and that the lysed p30GSDMD can be secreted out of the cell and can be detected in the culture medium and animal blood. Therefore, our study provides a valuable tool that not only non-invasive and real-time monitoring of cell pyroptosis, but also achieve a high-throughput functional screening of pyroptosis-targeted compounds in cell cultured cells and animal models.
\end{abstract}

\section{Image/Figure:}

https://www.xcdsystem.com/wmis/abstract/File6959/GA155 ImageFigure 0421050659.jpg

Full Name of Abstract's 1st Author : Moxuan Ji

Complete Status: Complete

First Name: Fu

Last Name: Wang

Email: fwang@xidian.edu.cn

Organization: Xidian Unniversity

Country: China 


\title{
ID: GA156 \\ Unsupervised clustering of magnetic resonance image features reveals mild cognitive impairment subtypes with distinct clinical prognoses
}

\author{
Liyu Huang, Xidian University, huangly@mail.xidian.edu.cn
}

\section{Category: Neuroscience}

\begin{abstract}
Body : Mild cognitive impairment (MCI) is a heterogeneous syndrome with the multiple underlying pathologies[1]. It is difficult to accurately characterize the MCI heterogeneity by using the current subtyping method[2]. Magnetic resonance imaging (MRI) combined with various machine learning algorithms provides tools to investigate alterations in brain structure and function of MCI patients[3]. Hence, this work aims to propose a new method for subtyping the MCI patients based on MRI image features. Figure 1 shows the overall design of the study. The data used in this study were approved by the ADNI database (http://adni.loni.usc.edu/). Standard T1-weighted imaging was obtained by 3D-MPRAGE with slightly different resolutions across patients. FreeSurfer was used to calculate seven kinds of features that reflect brain structure. Then, quantitative imaging features were clustered by using consensus clustering algorithm to identify and validated MCI subtypes in a $3 \mathrm{~T}$ discovery cohort $(n=295)$ and a heterogeneous $1.5 T$ validation cohort $(n=202)$. Four clusters were selected (cluster number $\mathrm{k}=4$ ), and each cluster represented a MCI subtype. As shown in Figure p1A (supplemental data), the four-cluster solution had the smallest incremental changes in the area (delta area) under the cumulative distribution function (CDF) curves. The consensus matrices showed that the four-cluster solution had the maximum consensus within clusters and the minimum rate of ambiguity across 10,000 bootstraps. This resulted in 30 patients in subtype 1 , 128 patients in subtype 2, 89 patients in subtype 3 and 48 patients in subtype 4 in the discovery cohort. In order to evaluate the stability and repeatability of the clustering model (Figure p1B), we independently validated the same clustering process in the $1.5 \mathrm{~T}$ validation cohort. As shown in Figure 2B, the optimal cluster size in this cohort was also four. To compare differences across the subtypes, we performed ANOVA and Kaplan-Meier survival analysis on the neuropsychological measures by pooling both cohorts together. As shown in Figure p2A in the ADAS11, ADAS13, FAQ and GDS, subtype 1 had the highest value, while subtype 3 had the lowest value. On the other hand, in the MMSE, ADNI MEN, ADNI_EF, RAVLT and Logical Memory II tests, subtype 3 had the highest value, and subtype 1 had the lowest value. These results indicate that patients in subtype 1 have the most severe impairments in cognition, memory, and executive function, while the patients in subtype 3 had the least impairment at baseline diagnosis. The value of subtypes 2 and 4 on cognition, memory and executive function was between subtypes 1 and 3, showing moderate impairment. ADNI EF can significantly distinguish the differences between subtypes in post hoc tests, suggesting that the differences between the subtypes may be more concentrated in executive functions. Furthermore, in line with previous results, conversion differences also existed across the subtypes (Figure p2B). Patients in subtype 1 had a higher probability of converting to $\mathrm{AD}$, and the rate of conversion was faster than in other subtypes. Most patients in subtype 3 were able to maintain stable MCI status. The other two subtypes had moderate conversion rates that did not significantly differ
\end{abstract}


from each other. We speculate that the difference between subtype 2 and subtype 4 may be reflected in more detailed aspects, such as a specific behavioral domain, and it may take longer to observe the differences. For example, the survival curve showed that the survival difference between subtype 2 and subtype 4 in MCI status became larger after 100 months. This result requires more future exploration in neuropsychological and imaging association studies.

References: 1. Korolev IO, L. SL, C. BA, One HKJP Predicting Progression from Mild Cognitive Impairment to Alzheimer's Dementia Using Clinical, MRI, and Plasma Biomarkers via Probabilistic Pattern Classification. 11 (2):e0138866- 2. Nettiksimmons J, DeCarli C, Landau S, Beckett L Biological heterogeneity in ADNI amnestic mild cognitive impairment. 3. Mcconathy J, Sheline YIJBP (2014) Imaging Biomarkers Associated With Cognitive Decline: A Review. 77 (8):685-692 4. Destrieux C, Fischl B, Dale A, Halgren E (2010) Automatic parcellation of human cortical gyri and sulci using standard anatomical nomenclature. Neuroimage 53 (1):1-15

\section{Image/Figure:}

https://www.xcdsystem.com/wmis/abstract/File6959/GA156 ImageFigure 0421051547.jpg

Complete Status: Complete

First Name: Liyu

Last Name: Huang

Email: huangly@mail.xidian.edu.cn

Organization: Xidian University

Country: China 


\title{
ID: GA157 \\ Imaging system xc- activity using the novel PET radiotracer [18F]FRPG
}

\author{
Hannah Greenwood, UCL, hannah.greenwood.16@ucl.ac.uk
}

\section{Category: Oncology}

\begin{abstract}
Body : Introduction The amino acid transporter system xc- is over-expressed in multiple cancer types, providing intracellular cysteine for glutathione biosynthesis. System xcactivity in tumours is altered in response to chemotherapy and in drug-resistant cells, which can be imaged by PET using the L-glutamate analogue [18F]FSPG (1-2). Little is known regarding transporter-recognition of non-natural amino acids or their utility for cancer imaging. Motivated by this, we compared the in vitro and preclinical characteristics of [18F]FSPG to its stereoisomer $[18 \mathrm{~F}] \mathrm{FRPG}$ in models of cancer and inflammation. Methods [18F]FRPG and [18F]FSPG uptake was assessed in H460 lung cancer cells ( $3 \times 104$ cells/well, 24-well plate; $0.25 \mathrm{MBq}$; 1h), with efflux measured $30 \mathrm{~min}$ after removal of exogenous activity. Specificity of [18F]FRPG for system xc- was further examined following transporter inhibition and blocking studies with system xc- substrates and inhibitor $(1 \mathrm{mM})$. Tissue uptake of [18F]FRPG and [18F]FSPG was quantified in mice bearing subcutaneous A549, H460, VCAP and PC3 tumours by gamma counting 60 min post-injection. Additionally, mice bearing A549 tumours were dynamically imaged by PET/CT over 60 min following injection of radiotracer (3 MBq). Radiometabolite analysis was performed on tissue and blood samples following imaging. Lung inflammation was induced in BALB/c mice through intratracheal administration of lipopolysaccharide (LPS; $1.5 \mathrm{mg} / \mathrm{kg})$. [18F]FRPG and [18F]FSPG uptake in LPS-treated lungs were compared to PBS controls by PET, $24 \mathrm{~h}$ post induction of inflammation (3 MBq; 40-60min post-injection). Finally, [18F]FRPG uptake was evaluated in an orthotopic H460 lung cancer model (3 MBq; 40-60min post-injection). Results and discussion [18F]FRPG uptake was specific for the glutamate/cystine antiporter system xc- (Fig 1A), matching that of [ $\left.{ }^{18} \mathrm{~F}\right] \mathrm{FSPG}$ (3). In H460 cells, [18F]FRPG uptake was lower than [18F]FSPG over $60 \mathrm{~min}(7.9 \pm 0.2 \%$ radioactivity/100,000 cells vs. $15.1 \pm$ $0.7 \%$ radioactivity $/ 100,000$ cells; $n=3 ; p=0.004$; Fig 1B). However, the percentage of radiotracer retained in cells 30 min after removal of exogenous activity was higher for [18F]FRPG than [18F]FSPG (91\% vs. 69\%; $n=3 ; p=0.002$; Fig 1C). In vivo biodistribution studies showed rapid clearance of [18F]FRPG from the blood, liver and pancreas. Tumour uptake of [18F]FRPG was lower compared to [18F]FSPG in prostate VCAP tumours and lung A549 tumours (Fig 2D\&F). However, [18F]FRPG tumour-to-blood was either elevated or equal to that of [18F]FSPG (Fig 2E). [18F]FRPG and [18F]FSPG showed in vivo metabolism to a single metabolite, with $[18 \mathrm{~F}]$ FRPG showing a higher percentage of parent tracer in tumours compared to [18F]FSPG (Sup data 1). Radiotracer uptake in inflammatory cells may complicate the interpretation of tumour uptake. However, [18F]FRPG uptake in orthotopic tumours was 2.5fold higher than inflamed tissue, allowing for excellent tumour visualisation (Sup data 2). Conclusion These results confirm the specificity of [18F]FRPG for system xc- and desirable properties for cancer imaging. High [18F]FRPG uptake was measured in a range of human tumours, with background tissue uptake reduced in comparison to [18F]FSPG. The uptake of [18F]FRPG in LPS-induced inflammation increased compared to healthy tissue, however,
\end{abstract}


tumour uptake exceeded these values significantly. Future work will determine the redox sensitivity of [18F]FRPG and its ability to image response and resistance to therapy.

References: 1. Greenwood H, McCormick P, Gendron T, et al. Measurement of tumor antioxidant capacity and prediction of chemotherapy resistance in preclinical models of ovarian cancer by positron emission tomography. Clinical Cancer Research. 2019;25:2471-2482. 2. McCormick P, Greenwood HE, Glaser M, et al. Assessment of tumor redox status through (S)-4(3-[18F]fluoropropyl)-L-glutamic acid PET imaging of system Xc- activity. Cancer Research. 2019;79:853-863. 3. Koglin N, Mueller A, Berndt M, et al. Specific PET Imaging of XcTransporter Activity Using a 18F-Labeled Glutamate Derivative Reveals a Dominant Pathway in Tumor Metabolism. Clinical Cancer Research. 2011;17:6000-6011.

\section{Image/Figure:}

https://www.xcdsystem.com/wmis/abstract/File6959/GA157_ImageFigure_0603060908.png

Image/Figure Caption: Figure 1. Characterisation of [18F]FRPG in in vitro and in vivo cancer models. A. [18F]FRPG uptake in H460 cells was blocked in the presence of FSPG, L-glutamate and the system xc- inhibitor p-carboxyphenylglycine (CPG) (1 mM; 30 min uptake; $\mathrm{n}=3)$. B. Time course of [18F]FRPG and [18F]FSPG uptake over 90 min in H460 cells $(n=3)$. C. $[18 \mathrm{~F}] \mathrm{FRPG}$ and $[18 \mathrm{~F}] \mathrm{FSPG}$ retention following $30 \mathrm{~min}$ efflux in media alone or with the addition of L-glutamate. D. Radiotracer uptake in a range of subcutaneous tumour models, 60 min post-injection. E. Tumour to blood ratios for [18F]FRPG and [18F]FSPG. F. PET/CT maximum intensity projection images representing the same mouse bearing an A549 s/c tumour imaged with [18F]FRPG and [18F]FSPG (40-60 min p.i.). Images were taken from the same animal $24 \mathrm{~h}$ apart. **, $\mathrm{p}<0.01 ; * * *, \mathrm{p}<0.001$. L-Glu, L-glutamate; SG, salivary glands; $\mathrm{P}$, pancreas; B, bladder.

Full Name of Abstract's 1st Author : Hannah Greenwood

Complete Status: Complete

First Name: Hannah

Last Name: Greenwood

Email: hannah.greenwood.16@ucl.ac.uk

Organization: UCL

Country: United Kingdom 


\title{
ID: GA158 \\ Next-Generation PARP Imaging with $\left[{ }^{18}\right.$ F]FPyPARP - A Comparison with the Benchmark Radiotracers $\left[{ }^{18}\right.$ F]PARPi and $\left[{ }^{18}\right.$ F $]$ FTT.
}

\author{
Sophie Stotz, University of Tuebingen/Werner Siemens Imaging Center, \\ sophie.stotz@med.uni-tuebingen.de
}

\section{Category: Oncology}

\begin{abstract}
Body : Ever since PARP inhibitors emerged as reliable drugs for cancer treatment, huge effort was taken to broaden their use and develop PET imaging agents for detection and characterization of malignant lesions. Up until now, two gold-standard PARP radiotracers are applied in clinical settings, [18F]PARPi [1] and [18F]FTT [2], while ongoing research focuses on optimized compounds and derivatives. Still, both tracers suffer from strict hepatobiliary clearance in rodents, limiting their use for abdominal lesions due to high background uptake. Herewith, we present an logP-optimized PARPi variant, [18F]FPyPARP (logP 2.49), with expected shift towards renal excretion, and the first side-by-side comparison of [18F]PARPi $(\log \mathrm{P} 3.36)$ and $[18 \mathrm{~F}] \mathrm{FTT}(\log \mathrm{P} 3.09)$ together with the novel [18F]FPyPARP in a standardized mouse study. [18F]FPyPARP was synthesized in a one-pot reaction using the synthon $[18 \mathrm{~F}] \mathrm{FPyTF}$, in a synthesis time of $71 \pm 4 \mathrm{~min}$ with a decay-corrected radiochemical yield of $12 \pm 7 \%$ and a molar radioactivity of $31 \pm 15 \mathrm{GBq} / \mu \mathrm{mol}(\mathrm{n}=6)$. Additionally, the rather complicated [18F]PARPi synthesis was automated using [18F]SFB, while the original one-step [18F]FTT synthesis was simply adopted. HCC1937 cells, chosen because of their decent PARP expression, were subcutaneously injected into female NOD.CB17-Prkdcscid/J mice ( $\mathrm{n}=5)$. After xenograft growth, the mice were injected with $13.0 \pm 0.5 \mathrm{MBq}$ of the respective radiotracer and subjected to dynamic $(n=2)$ or static $(n=3)$ PET scans with anatomical MR imaging. Subsequent ex vivo biodistribution studies and analysis of the time-activity-curves indeed revealed clearly improved renal clearance as indicated by visible bladder uptake and comparable but heterogeneous radiotracer uptake in the xenograft. Nevertheless, all radiotracers showed high abdominal uptake especially in liver, spleen, intestine and kidney, indicating that the shift to renal excretion was only partial. The tumor-to-muscle ratios of the [18F]FPyPARP cohort $(2.10 \pm 0.41)$ were significantly $(\mathrm{p}=0.0085)$ lower compared to [18F]PARPi $(5.30 \pm 1.57)$ due to high muscle uptake. In contrast, the tumor-to-blood ratios were increased $(2.33 \pm 1.47,3.39 \pm 0.47$ and 2.97 \pm 0.92 , respectively, for [18F]PARPi, [18F]FPyPARP and [18F]FTT), suggesting good blood clearance and possibly lower unspecific background. In conclusion, we developed an alternative PARP radiotracer with a facilitated synthesis and validated it in a side-by-side comparison to the current benchmark tracers. Our tracer exhibits similar results in detecting PARP expression and met the main goal of this work by demonstrating a shift to partial renal clearance. Taking into account that first clinical data for $[18 \mathrm{~F}]$ PARPi show $\sim 30 \%$ renal clearance in humans [3], an even higher rate for [18F]FPyPARP is likely. This provides great potential for clinical PARP imaging of abdominal lesions and thus might aid developing new strategies in personalized cancer therapy.
\end{abstract}


References: [1] Zhou D et al. Nucl Med Biol. 2018;66:26-31 [2] Carney B et al. Mol Imaging Biol. 2016;18:386-392 [3] Schoder H et al. Clin Cancer Res. 2020

Full Name of Abstract's 1st Author : Sophie Stotz

Complete Status: Complete

First Name: Sophie

Last Name: Stotz

Email: sophie.stotz@med.uni-tuebingen.de

Organization: University of Tuebingen/Werner Siemens Imaging Center

Country: Germany 


\title{
ID: GA159 \\ A deeply supervised convolutional neural network with attention for semantic segmentation of thymoma
}

\author{
Yi Zhang, Xidian University, zhangyiuf@gmail.com
}

\section{Category: Computational \& Data Science}

\begin{abstract}
Body : Objective: Thymoma is the most common primary anterior mediastinal tumor accounting for $20 \%$ to $40 \%$ of all mediastinal tumors in adults [1]. Accurate and automated thymoma segmentation is highly desired in an image-driven thymoma analysis, since it has potential to increase the efficiency and reproducibility of computing more complete tumor measurements and imaging biomarkers, compared to human measurements [2]. Moreover, the segmentation of thymomas for radiologists is a time-consuming and inefficient task in clinical practice. Thus, it is necessary to develop a method to accurately and efficiently realize automatic segmentation of thymomas. Methods: A total of 310 subjects who underwent contrast-enhanced CT scanning were included in this ethically-approved retrospective study. All of the CT slices were manually labeled by four experienced radiologists. The CT images of 247 patients $(80 \%)$ were randomly selected to constitute the training set, and the remaining 63 patients $(20 \%)$ constituted the testing set. A convolutional neural network for automatic segmentation of thymoma was proposed in this study, called deeply supervised attention network (DSA-Net). DSA-Net used the encoder-decoder first proposed in U-Net [3] as the basic framework. The encoder was composed of several convolution blocks that added maxpooling layers between convolution blocks for downsampling. The detials of convolution block were shown in Fig. 1.The decoder was symmetrical with the encoder, except that the deconvolution layers for the upsampling were used instead of the maxpooling layers. The skip connection method same with U-Net was applied to the network, and attention blocks [4] were added to the skip connections. Since the features extracted in the shallow layer of the network contain more low-level semantic information, such as edge information, an early supervision path [5] was added to the network. The features in the first two convolution blocks in the encoding path were upsampled to obtain the thymoma edge as early supervision. The structure of the network is shown in Fig.1. The network was trained for 100 epochs using the cross-entropy loss function and RMSProp optimizer. The performance of segmentation was evaluated by calculating the accuracy, intersection over union ( $\mathrm{IoU}$ ) and Boundary F1 contour matching score (BFScore) between the prediction and manual labels. Results: The proposed network was tested in the testing set and resulted in accuracy, IoU and BFScore of $95.64 \%, 0.9024$ and 0.916 respectively. The best values were produced by U-net were $89.04 \%, 83.92 \%$ and 0.8847 . Compared to the U-Net method, the DSA-Net model improved IoU by $6.23 \%$. At the patient-level, the variance of the IoU was 0.0083 , which showed the robustness in different patents. The speed of $0.16 \mathrm{~s}$ per slice of the CT image showed the effect of DSA-Net to reduce the radiologists' workload. Conclusions: Deeply supervised and attention mechanism of the network based on the inputoutput characteristics of the layers resulted in improvement of the overall segmentation accuracy in thymoma segmentation. The method showed the ability of consistent delineation to assist radiologists.
\end{abstract}


References: [1] Marom EM. Advances in Thymoma Imaging. Journal of Thoracic Imaging, 2013. [2] Benveniste MFK, Rosado-De-Christenson ML, Sabloff BS, et al. Role of Imaging in the Diagnosis, Staging, and Treatment of Thymoma. Radiographics A Review Publication of the Radiological Society of North America Inc, 2011. [3] Ronneberger O, Fischer P, Brox T. U-Net: Convolutional Networks for Biomedical Image Segmentation. Medical Image Computing \& Computer-assisted Intervention, 2015. [4] Lian S, Luo Z, Zhong Z, et al. Attention guided U-Net for accurate iris segmentation. Journal of Visual Communication \& Image Representation, 2018. [5] Zhang Z, Fu H, Dai H, et al. ET-Net: A Generic Edge-aTtention Guidance Network for Medical Image Segmentation. Computer Science - Computer Vision and Pattern Recognition, 2019.

\section{Image/Figure:}

https://www.xcdsystem.com/wmis/abstract/File6959/GA159 ImageFigure_0421075530.jpg

Full Name of Abstract's 1st Author : Jingyuan Li

Complete Status: Complete

First Name: Yi

Last Name: Zhang

Email: zhangyiuf@gmail.com

Organization: Xidian University

Country: China 


\title{
ID: GA160 \\ Synergistic Chemo/Radiotherapeutic Effects of Theranostic Cellulose \\ Nanocrystal-based Drug Delivery System with Enhanced Retention in Metastatic Melanoma in the Lung
}

\author{
Surachet Imlimthan, Inselspital, Bern University Hospital, \\ surachet.imlimthan@extern.insel.ch
}

Category: New Chemistry, Biology \& Bioengineering

\begin{abstract}
Body : Theranostic nanoparticle-based drug delivery systems (DDS) allows the diagnosis and treatment of cancer using a single pharmaceutical platform.1 Non-invasive nuclear molecular imaging techniques, such as PET and SPECT have great utility to visualize and quantitatively determine the biological events in many diseases in vivo. 2 Nanomaterials tagged with a suitable radioactive isotope can be traced after systemic administration to determine their biodistribution, specific uptake, elimination, and metabolism. Moreover, they can be used to detect and monitor pathological and molecular changes related to chemo- and radiotherapeutic delivery, providing the information as theranostic nanosystems in vivo. Nanocrystalline cellulose (CNC) is the most abundant organic polymer that has excellent physicochemical properties, including facile and cost-effective preparation, biodegradability, biocompatibility, and versatile chemical accessibility. CNC has a rod-like shape with the typical dimensions of 5-10 nm in width and 100-300 nm in length, which is suitable for nanoscale DDS applications.3 Previously, we reported the in vivo behavior of $111 \mathrm{In}$-labeled CNC in both healthy and tumor-bearing animal models. The results showed that $\mathrm{CNC}$ had a transient uptake and retention in the lung, suggesting that they could be used to target the highly vascularized metastases through the EPR effect. 4 If undetected early, melanoma can easily metastasize from the primary site to other organs within the body, such as lung, spleen, bone marrow, and lymph nodes. In melanoma, the activating BRAF V600E mutation is the most common oncogene expressed by the majority of the cells (>90\%).5 Recently, vemurafenib, a kinase inhibitor targeting BRAF V600E mutation, got FDA approval for metastatic melanoma treatment in humans. However, the therapeutic efficacy of vemurafenib chemotherapy is limited by drug pharmacokinetics in vivo and the development of resistance after repeated cycles of treatment. 6 In this work, we have further developed theranostic CNC-based DDS targeting lung metastatic melanoma for potential synergy of concomitant chemo- and radiotherapy. First, we radiolabeled $\mathrm{CNC}$ with $177 \mathrm{Lu},(\mathrm{t} 1 / 2$ $=6.73 \mathrm{~d}$ ) that emits both beta and gamma radiation, allowing both diagnosis (with SPECT) and radiotherapy. Then, vemurafenib was entrapped to $177 \mathrm{Lu}$-labeled $\mathrm{CNC}$ surface in the presence of cationic polymer poly-L-lysine in a one-pot reaction (177Lu-CNC-V). We then evaluated the in vitro drug release profiles in physiological media, cytotoxicity, cellular uptake, and cell survival as well as the biodistribution of 177Lu-CNC-V in BRAF V600E mutation-harboring YUMM1.G1 lung metastatic melanoma murine model. Vemurafenib exhibited sustained release behavior under physiological conditions, suggesting the prolongation of drug half-life in vivo. The in vitro cell viability was decreased in murine YUMM1.G1 and human A375 melanoma (BRAF V600E positive) cell lines while there was no effect observed in murine B16-F10 skin melanoma (wild-type BRAF), demonstrating the specificity of the construct. Moreover,
\end{abstract}


theranostic $177 \mathrm{Lu}-\mathrm{CNC}-\mathrm{V}$ demonstrated better cellular internalization compared to free $177 \mathrm{Lu}$ and $177 \mathrm{Lu}-\mathrm{CNC}$. The cell survival assay was carried out to confirm the synergistic therapeutic effects of vemurafenib chemotherapy and 177Lu radiotherapy in both YUMM1.G1 and A375 cell lines. The results revealed that cells treated with $177 \mathrm{Lu}-\mathrm{CNC}-\mathrm{V}$ had the lowest survival fraction (SF) after 24 hours of treatment, suggesting a superior therapeutic efficacy of $177 \mathrm{Lu}-$ $\mathrm{CNC}-\mathrm{V}$. Further, the ex vivo biodistribution results were in agreement with our previous studies where the radiolabeled $\mathrm{CNC}$ showed a transient accumulation in the lung before clearance through MPS organs. Radiation dosimetry estimation showed a high absorbed dose in the spleen after $72 \mathrm{~h}$ post-injection. Overall, the in vitro and in vivo results suggest the potential use of the developed theranostic $\mathrm{CNC}$ as single chemo- and radiotherapeutic DDS platform for enhanced targeting metastatic melanoma in the lung. A long-term survival study with the theranostic DDS in the YUMM1.G1 model is underway.

References: 1. Ahmed N., et al., Drug Discov. Today 2012, 17(17-18), 928-934. 2. Kircher M. and Lapa C., Curr. Cardiovasc. Imaging Rep. 2017, 10(2):6. 3. Sacui I. A., et al., ACS Appl. Mater. Int. 2014, 6(9), 6127-6138. 4. Imlimthan S., et al., Biomacromolecules 2019, 20(2), 674683. 5. Erdei E. and Torres S. M., Expert Rev. Anticancer Ther. 2010, 10(11), 1811-1823. 6. Johannessen C. M., et al., Nature 2010, 468, 968-972.

\section{Complete Status: Complete}

First Name: Surachet

Last Name: Imlimthan

Email: surachet.imlimthan@extern.insel.ch

Organization: Inselspital, Bern University Hospital

Country: Switzerland 


\title{
ID: GA162 \\ Potential physiological information in ultrasound images to muscular atrophy under simulated weightlessness
}

\author{
Zhang yue, XIDIAN, 983242574@qq.com
}

\section{Category: Computational \& Data Science}

\begin{abstract}
Body : The protection against muscular atrophy is an important guarantee for the successful completion of manned space missions, especially deep-space exploration missions. This study aims at exploring potential information from ultrasonic image related to physiological process of muscular atrophy. Sprague Dawley (SD) rats that had induced weightless muscle atrophy with tail hanging were selected as the experimental objects. After the Gastrocnemius (Gas) and Soleus (Sol) muscles in the ultrasonic images were segmented manually, 368 features were extracted from ultrasound images. Clustering image feature trajectories reveals nonlinear variation of the image with muscle atrophy. The fusion of classification models based on image features brought more accurate identifying ability of muscular atrophy. The results add to the evidence that ultrasonography is a powerful method for the evaluation of rodent muscle atrophy in vivo.
\end{abstract}

\section{Image/Figure:}

https://www.xcdsystem.com/wmis/abstract/File6959/GA162_ImageFigure_0524082226.png

Image/Figure Caption: Inspection of predictive of multivariable texture model constructed from 2 feature sets: (i) Gas, (ii) Sol. Estimation of prediction performance is shown for combinations of 1 to 25 features.

Complete Status: Complete

First Name: Zhang

Last Name: yue

Email: 983242574@qq.com

Organization: XIDIAN

Country: China 


\title{
ID: GA165 \\ Characterization of a Novel PET Imaging Agent (18F-LCE470) for Cardiac Growth Hormone Secretagogue Receptor in a Large Animal Model of Heart Failure
}

\author{
Rebecca Sullivan, Lawson Health Research Institute, rsulli4@uwo.ca
}

\section{Category: Cardiovascular \& Pulmonary}

\begin{abstract}
Body : Introduction Cardiac imaging technologies, including echocardiography and cardiac magnetic resonance imaging (cMRI), enable the non-invasive detection of changes in heart function that may indicate disease progression. These modalities detect changes in structure and anatomy, and thus there is a critical need for the detection of biochemical and molecular changes that precede gross structural changes. The development of imaging agents that target molecular changes, combined with hybrid imaging technology, will be a powerful means to address this need. There is evidence that the cardiac growth hormone secretagogue receptor (GHSR) could be a potential molecular imaging target $2-4$. An array of imaging agents are being characterized for detection of myocardial GHSR by positron emission tomography (PET) in vivo in preclinical studies. With one of these novel agents, termed 18F-LCE470, I will be using the emerging technology of hybrid PET and MRI (PET/MRI) to detect and quantify changes in regional distribution of myocardial GHSR in a canine model of heart failure. Methods Female hounds (11-12 months of age, $n=4)$ were used in this study. A myocardial infarct (MI) was induced by 2-hour occlusion of the left descending coronary artery, followed by reperfusion. Dogs were imaged at specific timepoints: 18F-LCE470 for GHSR (baseline, days 3, 21, months 4 and 11 post-MI) and 13N-NH3 for perfusion (day 21, 4 and 11 months post-MI). For PET/MRI, dogs were injected with 100-150 MBq of the tracer, immediately followed by a 1hour (18F-LCE470) or 30 min (13N-NH3) dynamic PET scan. Cine MRI maps were obtained to calculate heart function. The contractile function of the left and right ventricles was determined by CircleCVI software. The regional distribution of GHSR in the left ventricle, represented by the volume of distribution (DV) of 18F-LCE470, was determined by two-tissue compartmental modeling based on reversible binding of the tracer. Polar maps representing a 3D version of the entire left ventricle myocardium [Fig1] were segmented initially into 17 segments, which allowed for 3 regions of delineation: Infarct (apex and part of the left coronary artery [LAD] near apex); Remote (remaining LAD and right coronary artery); and territory served by the left circumflex artery (LCX). Remote and LCX regions were kept the same across all time points, but infarct regions were recalculated for each dog as infarct size was highly variable. Results PET/MR images and regional compartmental analysis showed differences in distribution patterns of 18F-LCE470 (DV, ml/ml) and 13N-NH3 (flow, $\mathrm{ml} /(\mathrm{ml} * \mathrm{~min})$ ) [Fig1]. There was relatively low uptake of both tracers in the infarct region, and 13N-NH3 was taken up uniformly in noninfarcted tissue. In contrast, 18F-LCE470 distributed largely to LCX region [Fig1]. Therefore, we can infer that 18F-LCE470 uptake represents GHSR density in each region of the left ventricle. In one dog with a large infarct ("Hope"), absolute GHSR density was significantly elevated in the LCX compared to the infarct and remote regions at all time points post-MI. In another dog with a small infarct ("Merry"), GHSR density was significantly elevated in LCX
\end{abstract}


from infarct at all time points post-MI, and from remote regions at day 3 (Fig 2B). These changes in tracer uptake were seen in the absence of changes in the left ventricular and right ventricular ejection fraction [Fig 3]. Conclusions Quantitative compartmental analysis of a novel small molecule PET tracer, 18F-LCE470, revealed changes in regional distribution of GHSR in the canine heart after a heart attack. These results illustrate regional dynamics of GHSR post-MI and may indicate changes in GHSR signaling in the LCX region. This PET molecular imaging tool may potentially detect subclinical biochemical changes in myocardium prior to structural changes detected by conventional imaging methods used for diagnosis of heart disease.

References: 1. Boogers MJ, Fukushima K, Bengel FM, Bax JJ. The role of nuclear imaging in the failing heart: Myocardial blood flow, sympathetic innervation, and future applications. Heart Fail Rev. 2011;16(4):411-423. doi:10.1007/s10741-010-9196-0 2. Douglas GAF, McGirr R, Charlton CL, et al. Characterization of a far-red analog of ghrelin for imaging GHS-R in P19derived cardiomyocytes. Regul Pept. 2014;54:81-88. doi:10.1016/j.peptides.2014.01.0113. Sullivan R, McGirr R, Hu S, et al. Changes in the Cardiac GHSR1a-Ghrelin System Correlate With Myocardial Dysfunction in Diabetic Cardiomyopathy in Mice. J Endocr Soc. 2018;2(2):178-189. doi:10.1210/js.2017-00433 4. Sullivan R, Randhawa VK, Stokes A, et al. Dynamics of the Ghrelin/Growth Hormone Secretagogue Receptor System in the Human Heart Before and After Cardiac Transplantation. J Endocr Soc. 2019;3(4):748-762.

doi:10.1210/js.2018-00393

\section{Image/Figure:}

https://www.xcdsystem.com/wmis/abstract/File6959/GA165 ImageFigure 0613042223.jpg

Image/Figure Caption: Figure 1. Polar map analysis of tracers (18F-LCE470 [Top] and 13N$\mathrm{NH} 3$ [Middle]) across acute and chronic time points. LCX - left circumflex artery; LAD - left coronary artery; RCA - tight coronary artery; Apex. PET/MR Images [Bottom] of 18F-LCE470 show uptake in the left ventricle. White arrow - infarct, blue arrows - remote tissues. Figure 2. Representative graphs of volume of distribution (receptor density) for a large infarct ("Hope") and small infarct ("Sunny") showing significant differences between LCX to both remote and infarct regions at all time points post-MI with bigger differences seen in the larger infarct area. Colour Timepoints: Black - Baseline; Red - Day 3; Green - Day 21; Blue - Month 11 (*p

Figure 3. Heart function data using cine MRI images where there is no significant difference in the left ventricular or right ventricular ejection fractions at any timepoint.

Full Name of Abstract's 1st Author : Rebecca Sullivan

Complete Status: Complete

First Name: Rebecca

Last Name: Sullivan

Email: rsulli4@uwo.ca

Organization: Lawson Health Research Institute 
Country: Canada 


\title{
ID: GA167 \\ Development of a naphthofluorescein-based probe for in vivo Cerenkov Imaging of tumor $\mathbf{p H}$
}

\author{
Andrea Rodriguez, University of Pennsylvania, andrea.estefania2493@gmail.com
}

Category: New Chemistry, Biology \& Bioengineering

\begin{abstract}
Body : The tumor microenvironment (TME) exhibits increased acidity due to cancer cells undergoing glycolysis at an elevated rate. This decrease in $\mathrm{pH}$ plays a role in invasion and metastasis, and is also related to chemotherapeutic resistance 1,2 . Cerenkov imaging provides a novel and non-invasive tool for $\mathrm{pH}$ imaging in vivo. Fluorescent $\mathrm{pH}$ sensors can be coupled to Cerenkov emitters to detect Selective Bandwidth Quenching (SBQ) and Cerenkov Radiation Energy Transfer (CRET) as a function of $\mathrm{pH} \mathrm{3,4,5.} \mathrm{We} \mathrm{hypothesize} \mathrm{that} \mathrm{selective} \mathrm{SBQ} \mathrm{and}$ CRET from a Cerenkov-active $\mathrm{pH}$ sensor will be detected by Cerenkov imaging, providing accurate $\mathrm{pH}$ measurements in vivo. For this project, 5,6-carboxynaphthofluorescein succinimidyl ester was conjugated to 4-aminobutyl DOTA in a 1:1 molar ratio, producing a naphthofluorescein-based molecular construct $(\mathrm{NFbD})$ suitable for chelation with Cerenkov emitters such as 68Ga. NFbD maintained its $\mathrm{pH}$ sensitivity $(\mathrm{pKa}=7.7, \lambda \mathrm{ex}=600 \mathrm{~nm}, \lambda \mathrm{em}=669$ $\mathrm{nm}$ at $\mathrm{pH} 9)$, showed increased photostability, compared to naphthofluorescein $(\mathrm{t} 1 / 2=1.95 \mathrm{X}$ $103 \mathrm{~min}, 10.1 \mu \mathrm{M}$ ) and was successfully chelated with $68 \mathrm{Ga}$, to produce NFbD-Ga. Cerenkov imaging was performed as a function of $\mathrm{pH}$ in vitro to construct $\mathrm{NFbD-Ga} \mathrm{pH}$ titration curves. NFbD-Ga showed strong pH-dependent SBQ and CRET, with a CRET pKa of 7.17 (SBQmax = $600 \mathrm{~nm}$, CRETmax $=700 \mathrm{~nm}$ ). In vivo Cerenkov imaging was performed following intratumoral NFbD-Ga injections in athymic nude mice bearing 4175-Luc + triple negative breast cancer tumor xenografts, to detect $\mathrm{pH}$-dependent SBQ and CRET. Cerenkov tumor $\mathrm{pH}$ values were obtained by interpolation, fitting CRET signals to the in vitro titration curves yielding a $\mathrm{pHCerenkov}=7.23 \pm 0.03, \mathrm{n}=5$. In vivo Cerenkov imaging was validated with 31P Magnetic Resonance Spectroscopy (MRS) on the same cohort of mice, using the chemical shift of 3aminopropylphosphonate (3-APP) to measure an external pHMRS $=6.92 \pm 0.04, n=5$. Higher experimental Cerenkov signals were observed, a 35\% overestimation, likely due to increased signal attenuation from tissue scattering and absorption. To account for these effects, an empirical correction factor was applied and a corrected Cerenkov $\mathrm{pH}$ value of $6.94 \pm 0.03, \mathrm{n}=5$ was obtained. The measurement of accurate $\mathrm{pH}$ values will benefit from our current studies on depth penetration and scattering effects on SBQ and CRET Cerenkov signals. Nonetheless, the development of this Cerenkov-active $\mathrm{pH}$-sensor, $\mathrm{NFbD}$, indicates a new and promising biomedical application for Cerenkov imaging.
\end{abstract}

References: 1. Estrella, V., Chen, T., Lloyd, M., et al. (2013). Acidity generated by the tumor microenvironment drives local invasion. Cancer Res. 73, 1524-1535. 2. Kato, Y., Ozawa, S., Miyamoto, C., et al. (2013). Acidic extracellular microenvironment and cancer. Cancer Cell International 13, 89-96. 3. Czupryna, J., Kachur, A.V., Blankemeyer, E., et al. (2015). Cerenkov specific contrast agents for detection of $\mathrm{pH}$ in vivo. J Nucl Med 56, 483-488. 4. Dothager, R.S., Goiffon, R.J., Jackson, E., et al. (2010). Cerenkov Radiation Energy Transfer (CRET) Imaging: 
A Novel Method for Optical Imaging of PET Isotopes in Biological Systems. PLoS One 5, e13300. 5. Arroyo, A.D., Guzmán, A.E., Kachur, A.V., et al. (2019). Development of fluorinated naphthofluoresceins for Cerenkov imaging. Journal of Fluorine Chemistry 225, 27-34.

Full Name of Abstract's 1st Author : Andrea E. Guzmán Ríos

Complete Status: Complete

First Name: Andrea

Last Name: Rodriguez

Email: andrea.estefania2493@gmail.com

Organization: University of Pennsylvania

Country: United States 


\title{
ID: GA168 \\ Complex Relationship Between Iron Oxide Nanoparticle Degradation and Signal Intensity in Magnetic Particle Imaging
}

\author{
Erik Shapiro, Michigan State University, shapir86@msu.edu
}

\section{Category: New Chemistry, Biology \& Bioengineering}

\begin{abstract}
Body : In principle, Magnetic Particle Imaging (MPI), using superparamagnetic nanoparticles as an imaging tracer, is touted as quantitative and MPI signal can be equated to tracer quantity, given proper signal calibration. Yet, MPI signal properties have never been characterized for magnetic nanoparticles undergoing biodegradation, and deviations from the original calibration would impact quantification. We performed serial in vitro and in vivo MPI on various magnetic nanoparticles and measured MPI signal and nanoparticle resolution as a function of nanoparticle degradation and iron oxide dissolution. The findings of this work are critical to interpreting MPI data, particularly where long term biological processes are being studied. First, in Experiment 1, we measured change in total signal intensity, peak signal intensity and nanoparticle resolution of four magnetic nanoparticles with varying degradation rates (Table 1), in solutions that mimicked extracellular conditions (PBS, pH 7.4), harsh endosomal/lysosomal conditions (50 mM citrate buffer, $\mathrm{pH} 5.5)$ or mild endosomal/lysosomal conditions $(10 \mathrm{mM}$ citrate buffer, $\mathrm{pH} 6.5)$, during incubation at $37^{\circ} \mathrm{C}$ for 4200 hours. The principle findings were: in $50 \mathrm{mM}$ citrate, $\mathrm{pH} 5.5$, particles exhibited varied reduction in total and peak MPI signal intensity and changes in nanoparticle resolution over 4200 hours. In $10 \mathrm{mM}$ citrate, $\mathrm{pH}$ 6.5, significantly less decrease in signal intensities and changes in nanoparticle resolution were measured. In PBS, MPI signal and nanoparticle resolution remained constant for the first 1500 hours for all particles. Unpredictably, Vivotrax exhibited 30\% increase in total signal intensity at 1500 hours, coincident with a drop in peak signal intensity of $\sim 30 \%$, and a doubling in nanoparticle resolution that was maintained through 4200 hours. Experiments where the dextran coating was enzymatically removed showed that this signal increase was resultant from nanocrystal aggregation. Enzymatically responsive MPI experiments are thus suggested. In general, for all four particle types, TEM confirms nanoparticle decomposition during the experiment, albeit to different degrees depending on the nanoparticle type and the incubation solution and the disappearance in signal intensity is well explained by nanoparticles decomposition and dissolution of the $\mathrm{Fe} 3 \mathrm{O} 4$ nanocrystals in citrate at $\mathrm{pH} 5.5$. To further probe MPI signal change during degradation, in Experiment 2, we measured MPI signal for PLGA encapsulated Fe3O4 nanoparticles (CLIPs) as a function of PLGA molecular weight and size of Fe3O4 nanocrystal during degradation. We show that signal can either increase, decrease or remain constant, depending on the MW of the PLGA polymer, the degradation rate encouraged by the buffer, and the size of the Fe3O4 nanocrystal. In general: 1) $8 \mathrm{~nm}$ Fe3O4 nanocrystals exhibited decrease in MPI signal when degrading in PBS and phosphate buffer, $\mathrm{pH}$ 6.5, and 25 $\mathrm{nm}$ Fe3O4 nanocrystals exhibited increase in MPI signal; 2) high MW PLGA slowed degradation rate versus $5 \mathrm{kDa}$ PLGA. These signal changes are in line with our current understanding of magnetic Relaxometry and the transition from Neel relaxation to Brownian relaxation. Lastly, we performed in vivo MPI on mice injected with the fast degrading Vivotrax
\end{abstract}


$(\mathrm{n}=2)$ and the slow degrading Magnefy $(\mathrm{n}=2)$ to determine how well the in vitro experiments predict in vivo biodegradation. Immediately after injection, mice had high total signal intensity at the location of the liver, which decreased over 3082 hours. Vivotrax exhibited slower MPI signal loss in vivo ( $31.7 \mathrm{~d}$ half-life) as compared to Vivotrax incubated in vitro in $50 \mathrm{mM}$ citrate, $\mathrm{pH}$ 5.5 (13.2 d half-life). For the Magnefy particles, the rate of MPI signal change in vitro in $50 \mathrm{mM}$ citrate, $\mathrm{pH} 5.5$ was slower ( $69.5 \mathrm{~d}$ half-life) than in vivo ( $36.2 \mathrm{~d}$ half-life). Indeed, we show that long-term in vitro MPI experiments only roughly approximate long-term in vivo MPI signal properties.

\section{Image/Figure:}

https://www.xcdsystem.com/wmis/abstract/File6959/GA168 ImageFigure 0421105234.jpg

Complete Status: Complete

First Name: Erik

Last Name: Shapiro

Email: shapir86@msu.edu

Organization: Michigan State University

Country: United States 


\title{
ID: GA171 \\ Dual-acting Micelles for Cocktail Targeted Therapy of Metastatic Breast Carcinoma
}

\author{
ZHUORAN GONG, PEKING UNIVERSITY, 1701111666@pku.edu.cn
}

\section{Category: Oncology}

\begin{abstract}
Body : Background, Motivation and Objective Stage IV breast cancer, which has a high risk of invasion, often develops into metastases in distant organs, especially in the lung, and this could threaten the lives of women.[1] Traditional chemotherapeutics is the standard clinic treatment of stage IV breast cancer, while suffers from low delivery efficiency to the tumor site with significant variation among different patients. [2] Microtubules are key components of the cytoskeleton and play a crucial role in mitotic cell division, inhibited by vinca alkaloids, such as hydrophobic vinorelbine (VIN). [3] Moreover, biosynthesis of DNA can be inhibited by doxorubicin (DOX), a routinely used chemotherapeutic agent. [4] However, currently, owing to low penetration and limited distribution of them in tumor, the chemotherapy efficacy in metastatic lesion is low. [5] Therefore, a kind of site-specific new platforms that can shrink the primary tumor, and target metastases while minimizing unwanted distribution in normal tissues is urgently needed. Fibronectin, a class of adhesive glycoproteins, plays a major role in extracellular matrix functions of cancer cells such as cell adhesion, proliferation, and migration, highly expressed in the invasive or metastatic tumor sites. Herein, a cocktail targeted dual-acting micelle encapsulating doxorubicin and vinorelbine functionalized with CREKA peptides served to target the fibronectin-expressing tumor area was rationally fabricated, termed CTM. Statement of Contribution/Methods CREKA peptide and DSPE-PEGMAL were mixed and stirred for $4 \mathrm{~h}$, formed DSPE-PEG-CREKA (DPC). Then the equal molar ratio of DOX and VIN were incorporated into DPC aqueous solution with acoustics, prepared CTMs respectively. Results/Discussion The as-prepared CTMs are ultra-small with diameters around $11.0 \mathrm{~nm}$ in uniformity shown in TEM image (Fig. 1a), demonstrated that the efficient assembled CTMs have potential for targeted drug delivery to tumors. The fluorescence microscopy showed significant DOX uptake in cells treated with CTMs at $6 \mathrm{~h}$, compared with low uptake in free chemotherapy agents group (FC, including free DOX and VIN in 1:1 molar ratio). (Fig. 1b) Due to high efficiency of CTMs uptake, the microtubule network of 4T1 cells was inhibited by CTMs treatment, while with moderate microtubule disruption in FC treatment. (Fig. 1c) It could be illustrated that CTMs promoted the hydrophobic VIN delivery efficiency and made the significant effect. The 4T1 metastatic tumor mice model was built and evaluated the CTMs biodistribution and cocktail targeted treatment efficacy in vivo. Twenty-four hours after intravenous drug administration, CTMs were enriched in lung metastatic tumor lesion, compared to the ineffectiveness enrichment in FC group. (Fig. 1d) suggested that the simple small CTMs opened a new avenue for drug delivery in metastasis. Importantly, CTMs showed a significant high efficacy in suppressing metastatic tumors in vivo, compared to FC group at the end of the treatment. (Fig. 1e) Meanwhile, the reduction in invasive metastatic foci was apparent in CTM-treated mice, which the CTM-treated residual lesion area was approximately one-third of that in FC group. (Fig. 1f) Moreover, the CTM-treated micrographs showed neither noticeable
\end{abstract}


damage nor inflammation in major organs, (Fig. 1g) suggesting CTMs had few pathological effects on normal tissues and biosafety in vivo. Conclusion In summary, the dual-acting CTM for cocktail targeted therapy was successfully prepared, which could overcome the shortcomings of traditional chemotherapy in metastasis. The aforementioned persuasive data shows that the CTM is a great promising further modality for high efficacy breast carcinoma metastasis therapeutics.

References: [1] Weigelt, B., Peterse, J. L. \& van 't Veer, L. J. Breast cancer metastasis: markers and models. Nat Rev Cancer 5, 591-602 (2005). [2] Szakacs, G., Paterson, J. K., Ludwig, J. A., Booth-Genthe, C. \& Gottesman, M. M. Targeting multidrug resistance in cancer. Nat Rev Drug Discov 5, 219-234 (2006). [3] Rossi, A. et al. Single agent vinorelbine as first-line chemotherapy in elderly patients with advanced breast cancer. Anticancer Res 23, 1657-1664 (2003). [4] Hameed, S. et al. Self-assembly of porphyrin-grafted lipid into nanoparticles encapsulating doxorubicin for synergistic chemo-photodynamic therapy and fluorescence imaging. Theranostics 8, 5501-5518 (2018). [5] Quail, D. F. \& Joyce, J. A. Microenvironmental regulation of tumor progression and metastasis. Nat Med 19, 1423-1437 (2013).

\section{Image/Figure:}

https:/www.xcdsystem.com/wmis/abstract/File6959/GA171 ImageFigure 0422013554.png

Full Name of Abstract's 1st Author : Zhuoran Gong

Complete Status: Complete

First Name: ZHUORAN

Last Name: GONG

Email: 1701111666@pku.edu.cn

Organization: PEKING UNIVERSITY

Country: China 


\title{
ID: GA172 \\ Novel antibody-drug conjugate shows promise for PSMA $(+)$ prostate cancer treatment
}

\author{
Sudath Hapuarachchige, Johns Hopkins School of Medicine, shapuar1@jh.edu
}

\section{Category: Oncology}

\begin{abstract}
Body : Introduction Prostate cancer (PC) is a devastating disease and 1 million men are diagnosed with PC each year in the world. Approximately, 30\% of PC patients die of the disease. Typical treatments for PC are surgery, radiation therapy, and hormone deprivation therapy (HDT). However, almost all PCs eventually become castrate-resistant prostate cancer (CRPC).1,2 In this stage PC progresses rapidly and metastasizes. Common chemotherapeutic drugs, such as docetaxel and cabazitaxel, are not efficient to control the disease because of low therapeutic activity and high systemic toxicities of these drugs. Therefore, novel rationally designed targeted therapeutics and therapeutic strategies are urgently needed for management of aggressive PC. 5D3 is a novel monoclonal antibody $(\mathrm{mAb})$ rose against PSMA antigen, which is a marker of aggressive PC.3 5D3 shows higher binding affinity and fast internalization characteristics in PSMA $(+) \mathrm{PC}$ cells. This $\mathrm{mAb}$ is robust and stable in chemical conjugation reactions, and doesn't change the binding affinities after conjugations.4,5 Hence, 5D3 is an ideal candidate for the development of antibody-drug conjugates (ADC) for PC therapy. In this study, we conjugated 5D3 mAb with anti-tubulin agent mertansine (DM1) and developed a novel ADC (Figure 1A). Novel 5D3-DM1 ADC was evaluated in PSMA(+) human PC subcutaneous mouse models. Methods Anti-PSMA mAb, 5D3, 5B1 and F(ab')2 of 5D3 were labeled with AlexaFluor-488. A comparative in vitro cellular study in PSMA(+) PC3-PIP cells was performed for understanding the internalization characteristics. For the internalization study, PSMA $(+)$ PC3-PIP cells in chamber slides were treated with 5D3(AF-488)2, 5B1(AF-488)2, and $\mathrm{F}(\mathrm{ab}) 2(\mathrm{AF}-488) 2$ and imaged using a confocal fluorescence microscope to identify the specifics for ADC development. The best candidate, $5 \mathrm{D} 3 \mathrm{mAb}$ was first functionalized with MCC groups by reacting with sulfo-SMCC-NHS and conjugated with DM1 drug molecules. 5D3-DM1 ADC was purified and characterized by MALDI-TOF and DLS. The degree of drug conjugation per mAb was 2.8 in the synthesized ADC. PSMA $(+)$ PC3-PIP PC mouse models were used for the evaluation of 5D3-DM1 ADC with PSMA(-) PC3-Flu mouse models as the control. Mice in both groups were administered with two doses of 5D3-DM1 ADC two week apart. Therapeutic response was examined based on the change of tumor volumes. Results and Discussion 5D3 $\mathrm{mAb}$ shows fastest internalization characteristics and higher cytoplasmic accumulation in PSAM(+) PC3-PIP cells compared to anti-PSMA 5B1 mAb and F(ab')2 fragments of 5D3
\end{abstract} (Figure 1B). After drug conjugation, molecular weight of ADC was measured by MALDI-TOF (Figure 1C) and the degree of drug conjugation per $\mathrm{mAb}$ was calculated based on the molecular weight change. In the treatment study, PSMA(+) PC3-PIP tumors showed significant growth reduction compared to PSMA(-) PC3-Flu tumors (Figure 1D). After intravenous administration, 5D3-DM1 internalizes fast in PSMA $(+)$ tumor cells, is processed, and releases DM1 drugs arresting the cancer cell mitosis. A Kaplan-Meier graph was plotted considering 4-flod tumor growth with respect to the initial tumor volume as the terminal point for euthanization unless 
animals die naturally (Figure 1E). The plot reveals significantly higher survival rate in PSMA(+) tumor bearing mice after the 5D3-DM1 treatment compared to the PSMA(-) tumor bearing control mice and untreated control mice. No adverse systemic effects of the treatment were observed in either animal group. Conclusions 5D3 has high binding affinities on PSMA(+) PCPIP cells and shows fast internalization properties. $5 \mathrm{D} 3 \mathrm{mAb}$ is stable and robust in conjugation reactions and can be conjugated with DM1 without changing its binding affinities with PSMA. 5D3-DM1 ADC has shown high therapeutic efficacy on PSMA(+) PC3-PIP prostate cancer subcutaneous mouse models exhibiting a reduction of tumor growth and increasing the survival.

References: References 1. Cho, S et al. Mol Cancer Ther 2018, 17, 2176-2186 2. Semenas, J et al. Curr Drug Targets 2012, 13, 1308-1323 3. Novakova, Z et al. Prostate 2017, 77, 749-764 4. Banerjee, S R et al. J Nucl Med 2019, 60, 400-406 5. Hapuarachchige, S et al. Mol Pharm 2020, $17,98-108$

\section{Image/Figure:}

https://www.xcdsystem.com/wmis/abstract/File6959/GA172 ImageFigure 0614034537.jpg

Image/Figure Caption: Figure 1. 5D3-DM1 ADC for PSMA-positive prostate cancer therapy. (A) Strategy of 5D3-DM1 ADC therapeutic activity. (B) Internalization study of anti-PSMA $\mathrm{mAb} 5 \mathrm{D} 3,5 \mathrm{~B} 1$, and $\mathrm{F}\left(\mathrm{ab} \mathrm{b}^{\prime}\right) 2$ of 5D3 in PSMA(+) PC3-PIP cells after $1 \mathrm{~h}$ treatment. (C) MALDITOF spectra of 5D3 mAb (blue) and 5D3-DM1 (red). (D) Change of tumor volumes in PSMA(+) PC3-PIP and PSMA(-) PC3-Flu human PC subcutaneous mouse models after 5D3DM1 ADC treatment. (E) Kaplan-Meier plot of survival. 4-fold increment of tumor volume with respect to initial tumor volume was considered as the euthanization set point of mice.

Full Name of Abstract's 1st Author : Sudath Hapuarachchige

Complete Status: Complete

First Name: Sudath

Last Name: Hapuarachchige

Email: shapuar1@jh.edu

Organization: Johns Hopkins School of Medicine

Country: United States 


\title{
ID: GA173 \\ Design and characterization of an angular domain optical projection tomography system
}

\author{
Veronica Torres, Dartmouth College, veronica.c.torres@dartmouth.edu
}

\section{Category: Instrumentation}

\begin{abstract}
Body : Evaluation of lymph nodes plays a key role in staging patients' cancer, and helps determine adjuvant therapy, which has been shown to affect overall survival. In breast cancer for example, sentinel lymph node biopsy is the standard of care where the sentinel or few of the closest draining lymph nodes are assessed. 1 In head and neck2, colorectal3, and pancreatic cancer4, however, it is common for dozens of lymph nodes to be removed and pathologically evaluated. The conventional method of interrogating a single lymph node is both time and labor intensive, and so tissues are often under-sampled and high rates of false negatives occur. Optical imaging offers a potential solution for whole node evaluation, but application is limited because of scatter in biological tissue. Various methods of scatter rejection exist to improve spatial resolution, often with an interplay between performance and technical complexity. Previously, we have demonstrated through Monte Carlo simulation5 and preliminary studies6 that a low-cost angular domain optical projection tomography imaging system (ADEPT) and simple filtered backprojection (FBP) is sufficient to detect and localize clinically relevant metastases in lymph nodes. Here, we evaluate performance of the ADEPT system to determine if and how the proof of concept study results can be improved, and to push the limits of sensitivity and specificity. Solid cylindrical resin phantoms with varying diameters $(6,8,10,12$ $\mathrm{mm}$ ) were created with scattering and absorbing agents added to match the optical properties and size range of lymph nodes (previously determined experimentally5). A single $\sim 1.5 \mathrm{~mm}$ diameter well was drilled at half the radius of each; these were filled with capillary tubes containing 10 $\mu \mathrm{M}$ of fluorescent IRDye-800CW to represent metastases. Each of the phantoms underwent tomographic transmission and fluorescence imaging on the ADEPT system for open aperture (conventional imaging) and closed aperture (angular domain imaging) configurations. Images were collected in $5^{\circ}$ intervals over $360^{\circ}$ and FBP reconstruction was employed. Results demonstrated that the fluorescent inclusions could easily be observed for all phantom sizes and both aperture setups [Fig. 1(a)]. Quantitatively, as shown in the bar plots below, angular domain imaging with a closed aperture provided higher contrast (with the exception of the $12 \mathrm{~mm}$ sample) and more accurate spatial resolution, where full width half maximum values of the detected inclusions were closer to the expected range of $1.1-1.2 \mathrm{~mm}$ based on the inner diameter of the capillary tubes (closed: $0.93 \pm 0.06 \mathrm{~mm}$, open: $2.61 \pm 0.09 \mathrm{~mm}$ ). While results of contrast-to-noise ratio (CNR) showed better performance for the open setup, it should be noted that exposure time was not scaled; as such, if noise characteristics were normalized, closed CNR values would be increased by a factor of about three. This however, demonstrates an important tradeoff of angular restriction - balancing the number of rejected and detected photons (improved spatial resolution vs increased noise) - that should be considered in system design. Overall, these findings along with demonstrations of accurate geometric and fluorescence
\end{abstract}


concentration reconstructions (see supplementary) support the potential of the ADEPT system for improving lymph node pathology.

References: 1. D. L. Weaver, Mod. Pathol. 23 Suppl 2, S26 (2010). 2. Chen et al. Radiation Oncology (2015) 10:181 3. S. Shen et al.,Arch Pathol Lab Med. 2009;133:781-786 4. W. S. Tummers et al., Ann Surg Oncol. 2018 July ; 25(7): 1880-1888 5. L. Sinha et al., Biomed Opt Exp. (2019), 10:2 6. V. C. Torres et al., J. Biomed. Opt. 24(11), 110501 (2019).

\section{Image/Figure:}

https://www.xcdsystem.com/wmis/abstract/File6959/GA173_ImageFigure_0611041704.jpg

Image/Figure Caption: Figure 1. ADEPT imaging of lymph node optical property matching phantoms with increasing diameter from left to right $(6,8,10,12 \mathrm{~mm})$. (a) Each phantom shows a schematic (green circle indicates the fluorescence inclusion), absorption reconstruction, and fluorescence sinogram and reconstruction using open and closed aperture configurations. (b) Contrast and (c) contrast-to-noise ratio of open versus closed aperture configurations for all samples. (d) Full width half maximum of a line profile across the center of the inclusion. Grey bar indicates the range of expected values.

Full Name of Abstract's 1st Author : Veronica Torres

Complete Status: Complete

First Name: Veronica

Last Name: Torres

Email: veronica.c.torres@dartmouth.edu

Organization: Dartmouth College

Country: United States 


\title{
ID: GA174 \\ Synthesis, Relaxivity and In Vivo Assessment of an Amphiphilic Gd-DTPA Multimer assembled on Icosahedral-B122- Core as MRI Blood Pool Contrast Agent
}

\author{
Shatadru Chakravarty, Michigan State University, shatadru@msu.edu
}

\section{Category: New Chemistry, Biology \& Bioengineering}

\begin{abstract}
Body : Objective: Noninvasive detection of vascular abnormalities that manifest in a variety of conditions such as atherosclerosis, tumor initiation and progression are of critical importance for disease prognosis and patient care.[1,2] To this end, magnetic resonance angiography (MRA) is a dynamic technique that can be used to acquire high resolution angiographic images as well as to evaluate the hemodynamic effects of various vascular lesions. [3, 4] Despite the fact that a number of contrast agents based on low molecular weight (LMW) Gd(III) polyamino polycarboxylic acid chelates (e.g. Gd-DTPA and Gd-DOTA) have received clinical approval for Magnetic Resonance Imaging (MRI), [5] these are not ideal for MRA due to their non-specific biodistribution and minimal vascular retention.[6] The design of contrast agents for MRA can be approached either by the attachment of multiple LMW Gd3+chelate to a macromolecular core[7] or by utilizing a receptor induced magnetization enhancement strategy where a LMW Gd3+-chelate can bind to plasma proteins (e.g. Human Serum Albumin or HSA) in a non-covalent fashion. [8] In this work, we propose to combine both these strategies in the following three steps; firstly, a novel DTPA based ligand with a lipophilic moiety is designed for non-covalent binding with HSA. Secondly, this ligand is appended onto the twelve vertices of a closo-B12(OH) 12 core in a radial fashion resulting in a multimeric medium sized configuration, termed by us as closomers.[9] Finally, 12-fold Gd-complexation yields the MRA closomer contrast agent CCA-I. In mice injected with CCA-I, significant contrast enhancement is observed in the vena cava, heart, liver, kidneys and the bladder with minimal Gd retention in tissues $24 \mathrm{~h}$ post-injection. Methods, Results and Discussion: A novel amphiphilic ligand, MRA Ligand-I is synthesized by incorporating a hydrophobic substituent comprised of a 3, 3-diphenylpropan-1-amine moiety off the DTPA backbone. Subsequent reaction with $\mathrm{GdCl} 3.6 \mathrm{H} 2 \mathrm{O}$ affords the corresponding Gd-MRA Ligand-I chelate, although the aqueous solubility of this final complex is not optimal. To impart better aqueous solubility, a short oligoethylene glycol (OEG) linker with a $-\mathrm{NH} 2$ group is introduced as a side chain resulting in the synthesis of MRA Ligand-II. To this end, Closomer-I, a B12-analogue with pendant meta-chloro phenylcarbonate arms, is reacted with MRA Ligand-II to obtain the 12-fold functionalized carbamate entity, Closomer-II in $80 \%$ yield. Next, removal of all tert-butyl ester groups from Closomer-II, followed by subsequent reaction with $\mathrm{GdCl} 3 \cdot 6 \mathrm{H} 2 \mathrm{O}$ leads to the formation of CCA-I in 79\% yield. Next, the percentage binding of the CCA-I with HSA is tested and found to be significantly high $(97 \%)$ as compared to its parent ligand Gd-MRA Ligand-I (44\%) and is also slightly higher compared to that of MS-325 (88\%), a blood pool MRA contrast agent approved for clinical use.[10] T1-weighted MRI phantom images of CCA-I at $7 \mathrm{~T}$ at various $\mathrm{Gd} 3+$ ion conc. in PBS and $\mathrm{HSA} / \mathrm{PBS}(4.5 \% \mathrm{w} / \mathrm{v}=0.67 \mathrm{mM})$ shows noticeable contrast enhancement for the solutions of CCA-I in presence of HSA. Finally, representative T1-weighted
\end{abstract}


MRA scans of mice injected with CCA-I at a Gd dose of $0.1 \mathrm{mmol} / \mathrm{kg}$ display significant contrast enhancement of blood vessels that persisted for about 10 min post-injection (p.i.) and diminish over a $1 \mathrm{~h}$ time period. Some contrast enhancement in the liver tissues at $3 \mathrm{~h} \mathrm{p}$.i. is indicative of the hepatic uptake of CCA-I that is common for compounds with a considerable amphiphilic component. Further, contrast observed in the bladder at $3 \mathrm{~h}$ p.i. indicates the renal system is the primary clearance route for CCA-I. Conclusion: In summary, we report the synthesis, relaxivity measurements and in vivo MRA imaging results of an amphiphilic variation of the DTPA ligand, appended on a multimeric closomer core.

References: 1. Rajendran P, Rengarajan T, Thangavel J, et al (2013) The Vascular Endothelium and Human Diseases. Int J Biol Sci 9:1057-1069. https://doi.org/10.7150/ijbs.7502. 2. Mulligan PR, Prajapati HJS, Martin LG, Patel TH (2014) Vascular anomalies: classification, imaging characteristics and implications for interventional radiology treatment approaches. Br J Radiol 87:20130392. https://doi.org/10.1259/bjr.20130392. 3. Young P, Brinjikji W (2015) Update on state of the art magnetic resonance angiography techniques. J Vasc Diagnostics 3:9-16. https://doi.org/10.2147/JVD.S73647. 4. Di Leo G, Fisci E, Secchi F, et al (2016) Diagnostic accuracy of magnetic resonance angiography for detection of coronary artery disease: a systematic review and meta-analysis. Eur Radiol 26:3706-3718. https://doi.org/10.1007/s00330015-4134-0. 5. Wahsner J, Gale EM, Rodríguez-Rodríguez A, Caravan P (2019) Chemistry of MRI Contrast Agents: Current Challenges and New Frontiers. Chem Rev 119:957-1057. https://doi.org/10.1021/acs.chemrev.8b00363. 6. Herborn CU (2012) Contrast Agents for MR Angiography. In: Carr JC, Carroll TJ (eds) Magnetic Resonance Angiography: Principles and Applications. Springer New York, New York, NY, pp 381-385. 7. Tang J, Sheng Y, Hu H, Shen Y (2013) Macromolecular MRI contrast agents: Structures, properties and applications. Prog Polym Sci 38:462-502. https://doi.org/https://doi.org/10.1016/j.progpolymsci.2012.07.001. 8. Caravan P (2009) Protein-Targeted Gadolinium-Based Magnetic Resonance Imaging (MRI) Contrast Agents: Design and Mechanism of Action. Acc Chem Res 42:851-862.

https://doi.org/10.1021/ar800220p. 9. Safronov A V, Jalisatgi SS, Hawthorne MF (2017) Closomers: Versatile Monodisperse Molecular Nanoparticles. Eur J Inorg Chem 2017:43784392. https://doi.org/10.1002/ejic.201700594. 10. Caravan P, Cloutier NJ, Greenfield MT, et al (2002) The Interaction of MS-325 with Human Serum Albumin and Its Effect on Proton Relaxation Rates. J Am Chem Soc 124:3152-3162. https://doi.org/10.1021/ja017168k.

\section{Image/Figure:}

https://www.xcdsystem.com/wmis/abstract/File6959/GA174_ImageFigure 0614094154.jpg

Image/Figure Caption: Figure 1. (a.) Synthesis of MRA Ligand-I and Gd-5; (b.) Synthesis of MRA Ligand-II; (c.) Synthesis of Closomer MRA Contrast Agent, CCA-I; (d.) Percentage binding of Gd-5 and CCA-I and MS-325 with HSA $(4.5 \% \mathrm{w} / \mathrm{v}=0.67 \mathrm{mM})$ in PBS at $\mathrm{pH} 7.4$ and $37^{\circ} \mathrm{C}$; (e.) Representative in vivo T1-weighted MRI scans of mice at various time points post injection of CCA-I $(\mathrm{Gd} 3+$ dose of $0.1 \mathrm{mmol} / \mathrm{kg})$.

Complete Status: Complete

First Name: Shatadru 
Last Name: Chakravarty

Email: shatadru@msu.edu

Organization: Michigan State University

Country: United States 


\title{
ID: GA175 \\ Painted Radioluminescence Imaging of tumor uptake with 18F-FDG
}

\author{
xu cao, xidian university, caoxu327@gmail.com
}

\section{Category: Oncology}

\begin{abstract}
Body : Purpose Radioluminescence imaging has been utilized for imaging 18F-FDG uptake in tumor by detecting the scintillation light converted from ionizing radiation sources with a scintillator. The modality suffers from low spatial resolution due to the air gap between the scintillator and the imaging animal. Here we present a painted radioluminescence imaging (PRLI) to enhance spatial resolution and optical conversion efficiency by directly painting a gadolinium oxysulfide: terbium $(\mathrm{Gd} 2 \mathrm{O} 2 \mathrm{~S}$ : $\mathrm{Tb})$ scintillator on the body surface of small animals. Procedures The spatial resolution, imaging depth and signal linearity of PRLI were first characterized with in vitro phantoms. In vivo experiments utilizing a nude mouse injected with the $18 \mathrm{~F}-\mathrm{FDG}$ in matrigel ball was then conducted to verify the proposed PRLI. Alternative modalities including flexible radioluminescence imaging (Flex-RLI) and Cerenkov luminescence imaging (CLI) were also performed to characterize PRLI. Results With respect to spatial resolution, full-width half maximum values across a $1 \mathrm{~mm}$ capillary tube were 2.1, 5.2 and 1.2 $\mathrm{mm}$ for PRLI, Flex-RLI and CLI respectively. The imaging depth of PRLI can reach to $9 \mathrm{~mm}$ in biological tissue medium. In vivo, the signal intensity of PRLI was 1555.6 and 21.6 times stronger than CLI and flex-RLI, respectively. The spatial resolution of PRLI was similar with CLI and higher than flex-RLI for the mouse imaging. Conclusions PRLI provides higher spatial resolution than flex-RLI, and has highest signal intensity comparing with CLI and flex-RLI for imaging 18F-FDG.
\end{abstract}

\section{Image/Figure:}

https://www.xcdsystem.com/wmis/abstract/File6959/GA175 ImageFigure 0421115844.png

Complete Status: Complete

First Name: $x u$

Last Name: cao

Email: caoxu327@gmail.com

Organization: xidian university

Country: China 


\title{
ID: GA176 \\ Systematic review and meta-analysis concerning near-infrared imaging with fluorescent agents to identify the sentinel lymph node in oncology patients
}

\author{
Bernadette Jeremiasse, Princess Maxima Center, b.jeremiasse- \\ 4@prinsesmaximacentrum.nl
}

Category: Oncology

\begin{abstract}
Body : Introduction Sentinel node procedures (SNP) are performed with the use of tracer-agents, mainly radio-colloid and/or blue dye, to identify and resect the sentinel lymph node (SLN) in oncology patients. Fluorescent agents have emerged as a new tracer-agent to identify the SLN intra-operatively with near-infrared imaging. Our aim is to compare the SLN detection rate and adverse events of fluorescent agents to current "golden standards" (blue dye and/or radio-colloid) for the SNP by means of a systematic review and meta-analysis without any restrictions based on tumor type. Methods A systematic search in PubMed, Embase and The Cochrane Library was performed. Articles that compared the detection rates of fluorescent agents with radio-colloid and/or blue dye were included. Risk of bias was assessed using the QUADAS-2 tool. Meta-analyses were performed for breast, gynecological and dermatological cancer using a random effects model. Results In total 6195 articles were screened which resulted in a final inclusion of 55 articles. These studies concerned patients with breast $(n=26)$, gynecological $(n=13)$, dermatological $(n=12)$, urological $(n=2)$ and miscellaneous $(n=2)$ cancer types. All studies used indocyanine green (ICG) as fluorescent agent. Meta-analyses comparing ICG with blue dye showed a significant and clinically relevant difference in detection rate ranging from $11 \%$ up to $36 \%$ in favor of ICG, for breast, dermatological, and gynecological cancer (see figure for breast cancer). Meta-analyses comparing ICG with radio-colloid did not show any significant differences, with the exception of ICG versus radio-colloid + blue dye for the bilateral SLN detection in gynecological cancer. The only reported side effect reported of ICG was temporary skin staining. For blue dye, a few cases of permanent tattooing were reported. Conclusion Near-infrared fluorescence imaging using ICG provides a higher SLN detection rate compared to blue dye for the SNP in a range of different tumor types. SLN detection rates of ICG are comparable to radio-colloid. Due to their complementary characteristics in terms of spatial resolution and transdermal sensitivity, we suggest to use a combination of both ICG and a radio-colloid. In facilities with limited or no access to radiocolloids, ICG appears to be a good alternative for blue dye, especially in patients with gynecological cancer and in patients with other cancers with fairly predictable locations of the SLN.
\end{abstract}

\section{Image/Figure:}

https://www.xcdsystem.com/wmis/abstract/File6959/GA176 ImageFigure 0603075657.png 
Image/Figure Caption: Figure: Forest plots depicting the risk differences in SLN detection rates for breast cancer 1. Comparison of the SLN detection rate using ICG versus blue dye 2 .

Comparison of the SLN detection rate using ICG versus radio-colloid (RC).

Full Name of Abstract's 1st Author : Bernadette Jeremiasse

Complete Status: Complete

First Name: Bernadette

Last Name: Jeremiasse

Email: b.jeremiasse-4@prinsesmaximacentrum.nl

Organization: Princess Maxima Center

Country: Netherlands 


\title{
ID: GA178 \\ In vivo PET-MR imaging relates hypoxia to increased PD-L1 expression in tumors
}

\author{
Katie Parkins, Johns Hopkins School of Medicine, kparkin8@uwo.ca
}

\section{Category: Oncology}

\begin{abstract}
Body : Immune checkpoint inhibitors have shown remarkable success in the treatment of some cancer types such as melanoma. However, for some patients, such as those with triple negative breast cancer (TNBC), the benefits of this therapy remain underwhelming1. This poor response has been largely attributed to tumor heterogeneity and limited immune cell infiltration2. An understanding of mechanisms that drive the heterogeneity of response to immune checkpoint inhibitors can lead to improved therapeutic outcomes. Hypoxia and acidic extracellular $\mathrm{pH}(\mathrm{pHe})$ are frequently encountered in tumors3. Here, for the first time, we have used combined PET-MR and optical imaging to understand the role of hypoxia and $\mathrm{pHe}$ in PDL1 expression using a syngeneic TNBC model in immune competent mice. Methods: Murine 4T1 breast cancer cells were engineered to express firefly luciferase (luc) under the control of hypoxia response elements (HRE). Tumors were generated by inoculating 2 x 106 cells into the upper mammary fat pad of female Balb/c mice $(n=5)$. Tumor growth was monitored over time and tumor hypoxia visualized and measured with in vivo bioluminescence imaging (BLI) using
\end{abstract} an IVIS Spectrum scanner. Once the presence of hypoxia was detected in tumors with BLI, mice were intravenously injected with $14 \pm 2 \mathrm{MBq}$ of [64Cu]Avelumab, a PD-L1 antibody, and imaged at $24 \mathrm{~h}$ and $48 \mathrm{~h}$ post injection using a simultaneous 7 T Bruker PET-MR scanner. Tumor pHe was assessed by acquiring chemical exchange saturation transfer (CEST) images following an $80 \mu \mathrm{L}$ intravenous injection of $100 \%$ iopamidol. A RARE pulse sequence with rectangular saturation transfer module of $2 \mathrm{sec}$ duration and $3 \mathrm{mT}$ power was used to acquire CEST images for $\sim 30$ min repeatedly at 2 saturation offsets $4.2,5.5 \mathrm{ppm}$ and last $5 \mathrm{M} 0$ images. Other imaging parameters were $\mathrm{TR} / \mathrm{TE}=10000 / 6.23 \mathrm{~ms}$; matrix size $=48 \times 32$; rare factor $=16$; slice thickness $=$ $2 \mathrm{~mm}$ and $\mathrm{FOV}=12.6 \times 8.6 \mathrm{~mm} 2$ respectively. Mice were subsequently sacrificed, tumors were sliced in the corresponding orientation of the PET-MR images, and ex vivo BLI of tumor slices was performed. BLI and PET/MR images were co-registered using MATLAB software. Masks were then generated from BLI images using ImageJ to define areas based on the level of tumor hypoxia. These optical masks were then overlaid onto the registered PET/MR slice and used as the region of interest (ROI) to calculate PET PD-L1 signal and CEST pH levels within those respective areas. Results: Tumor hypoxia was visualized with in vivo BLI as shown in Figure 1A. Representative in vivo PET-MR images of [64Cu]Avelumab distribution in mice are presented in Figure 1B. Representative ex vivo (BLI) and corresponding in vivo images of the tumor are displayed in Figure 1C. We observed significantly higher [64Cu]Avelumab uptake in tumor regions with the highest BLI intensity (75-100\% mask) compared to areas with the lowest intensity $(0-25 \%)(p<0.05)$ (Figure 1D). Furthermore, we found a significant positive correlation between the mean BLI signal and the mean PET PD-L1 signal in the tumors ( $p<0$ .05 ). Our preliminary data from corresponding CEST images suggests that hypoxic regions with high PD-L1 expression are also more acidic compared to normoxic regions (Figure 1D). 
Conclusions: These results identify, for the first time, the close association between areas of hypoxia and increased PD-L1 expression, suggesting that hypoxia increases PD-L1 expression. These results have direct relevance to treatment with PD-L1 or PD-1 immune checkpoint antibodies, since poorly perfused hypoxic tumor regions will have higher PD-L1 expression but lower delivery of the antibody, allowing cells in hypoxic tumor areas to escape immune surveillance. Future studies should investigate the role of hypoxia in regulating immune checkpoints, and the use of hypoxic cell targeting in improving the outcome of immune checkpoint inhibitor treatment.

References: 1. Voutsadakis IA. Immune Blockade Inhibition in Breast Cancer. Anticancer research. 2016;36(11):5607-22. PubMed PMID: 27793883. 2. Kleponis J, Skelton R, Zheng L. Fueling the engine and releasing the break: combinational therapy of cancer vaccines and immune checkpoint inhibitors. Cancer biology \& medicine. 2015;12(3):201-8. doi: 10.7497/j.issn.2095-3941.2015.0046. PubMed PMID: 26487965; PubMed Central PMCID: PMC4607816. 3. Penet MF, Glunde K, Jacobs MA, Pathak AP, Bhujwalla ZM. Molecular and functional MRI of the tumor microenvironment. Journal of Nuclear Medicine. 2008; 49(5), 687690. Acknowledgement: Supported by NIH R35 CA209960, R01 CA82337, RO1 CA236616, P30 CA006973 and P41 EB024495.

\section{Image/Figure:}

https://www.xcdsystem.com/wmis/abstract/File6959/GA178 ImageFigure 0610012322.png

Image/Figure Caption: Figure 1: Hypoxia detected in 4T1-HRE-luc tumors in five different mice (A). Representative PET-MR images showing PD-L1 imaging in mice (B). The highest PD-L1 levels were detected in the tumor, followed by lungs and spleen. Representative BLI images reporting hypoxia, PET-MR images showing PD-L1 localization and a CEST image showing $\mathrm{pH}(\mathrm{C})$. These images show the close association between hypoxia and PD-L1. BLI and PET/MR images were co-registered using MATLAB software. Masks generated from BLI images were used to define areas based on the level of tumor hypoxia. These optical masks were overlaid on the co-registered PET/MR slice and used to calculate the PET PD-L1 signal and CEST pHe (D).

Complete Status: Complete

First Name: Katie

Last Name: Parkins

Email: kparkin8@uwo.ca

Organization: Johns Hopkins School of Medicine

Country: United States 


\title{
ID: GA179 \\ Micron-sized iron particles for the detection of cancer cells by magnetic particle imaging
}

\author{
Kierstin Melo, Western University, kmelo4@uwo.ca
}

\section{Category: Instrumentation}

\begin{abstract}
Body : Introduction: Magnetic particle imaging (MPI ) is a new imaging modality that sensitively and specifically detects superparamagnetic iron oxide nanoparticles (SPIONs) within the body. Our lab has been developing cellular magnetic resonance imaging (MRI) tools for well over a decade using both SPIONs and fluorine-19 $(19 \mathrm{~F})$-based contrast agents for numerous important applications, including tracking of immune and stem cells used for cellular therapies. We have shown that SPION-based MRI cell tracking has very high sensitivity, but low specificity. Contrastingly, our work has demonstrated that 19 F-MRI cell tracking has high specificity, but low sensitivity. SPION-MPI cell tracking could overcome the challenges of MRIbased cell tracking allowing for both high sensitivity and high specificity cell detection. This study is the first to demonstrate that micron-sized iron oxide particles (MPIO) can be used for quantitative MPI of iron-labeled cancer cells in the mouse brain. Methods: Human breast cancer cells (231BR) were labeled with micron-sized iron oxide nanoparticles (MPIO), Immune compromised mice (NSG) were injected with either $2.5 \times 105$ or $5.0 \times 105$ cells. MPIO-labeled cells were administered intracardially using ultrasound guidance. MRI was performed in vivo the same day at 3T using a balanced steady-state free precession (bSSFP) sequence. After imaging, the mice were euthanized, and the brains were fixed and removed for MPI scans. MPI was performed on a Momentum TM scanner. MRI images were used to quantify the percentage of black pixels which represent the black voids created by iron labeled cells. MPI images were used to quantify the amount of MPI signal which is related linearly to the amount of iron. In a second experiment, NSG mice were also injected with 5 x 104 4T1BR cells, a murine breast cancer cell line, labeled with either MPIO or the SPION ferucarbotran (Vivotrax) which is currently the gold standard SPION for MPI. MRI and MPI was performed in vivo. Results: Figure 1 shows representative MRI and MPI images. Signal voids due to MPIO-labeled cells can be detected in the in vivo brain MRI of mice injected with either $2.5 \times 105$ or $5.0 \times 105$ cells (Figure 1A,B). Ex vivo MPI also showed signal in the brain from iron, which is visible as a hot spot (Figure 1C,D). More black pixels were measured in the brain MRI of mice receiving an injection of $5.0 \mathrm{x}$ 105 cells. This agreed with MPI data which showed that there is less iron in the brains of mice injected with $2.5 \times 105$ cells compared to those injected with $5.0 \times 105$ cells. In the second experiment, in vivo MRI was able to detect signal voids in the brains of mice injected with $5.0 \mathrm{x}$ 104 cells which were labeled with either MPIO or Vivotrax (Figure 2A,C), although voids were fainter in Vivotrax labeled cells. In vivo MPI signal was only detectable in mice injected with MPIO-labeled cells (Figure 2B). Conclusions: This is the first example of the use of MPIO for cell tracking with MPI. The major advantage of MPI is the ability to quantify iron content and estimate the number of iron labeled cells. With an intracardiac cell injection, approximately $15 \%$ of the injected cells are expected to arrest in the brain vasculature. For our lowest cell injection
\end{abstract}


of $5.0 \times 104$ cells, this is $\sim 10000$ cells. The next steps include increasing sensitivity for MPI by testing different MPI tailored iron nanoparticles.

\section{Image/Figure:}

https://www.xcdsystem.com/wmis/abstract/File6959/GA179_ImageFigure_0604122336.png

Image/Figure Caption: Figure 1: (A) MRI of mouse brains injected with $5.0 \times 105$ (B) and 2.5 x 10 5231BR cells labeled with MPIO. (C) MPI of mouse brain injected with $5.0 \times 10$ 5(D) and 2.5 x 105 231BR cells labeled with MPIO. Figure 2: (A) MRI of mouse brain injected with 5.0 x 104 4T1BR5 labeled with MPIO. (B) MPI scan (C) MRI of mouse brain injected with $5.0 \mathrm{x}$ 104 4T1BR5 labeled with Vivotrax TM.

Full Name of Abstract's 1st Author : Kierstin Melo

Complete Status: Complete

First Name: Kierstin

Last Name: Melo

Email: kmelo4@uwo.ca

Organization: Western University

Country: Canada 


\title{
ID: GA180 \\ Computational Methods Including B1-Correction to Improve Kinetic Modeling of Hyperpolarized 13C MR EPSI
}

\author{
Philip Lee, University of California, San Francisco, philip.lee2@ucsf.edu
}

\section{Category: Computational \& Data Science}

\begin{abstract}
Body : Background \& Motivation: Hyperpolarized (HP) 13C MR spectroscopic imaging (MRSI) enables quantitative monitoring of enzyme-catalyzed metabolism in human subjects. Using HP [1-13C]pyruvate, the rate of conversion of pyruvate to lactate (kPL) via lactate dehydrogenase (LDH) can be computed per voxel in patients. However, a high noise floor can obscure the peaks of downstream metabolites including lactate and alanine, limiting the achievable spatial resolution and rendering kPL estimations inaccurate in these cases. While surface transmit/receive (T/R) coils can be used to improve the sensitivity and spatial resolution in HP experiments, these coils produce an inhomogeneous B1+ profile, resulting in varying flip angles across the regions of interest. Hence, in computing the $\mathrm{kPL}$ for each voxel, it is critical to correct the nominal flip angle based off the coil's B1+ profile. The goal of this project was to develop a novel post-processing pipeline to improve spectral SNR using tensor rank truncation and to correct HP kPL images for B1 variations in human studies. Methods \& Results: In this case study, a patient with pancreatic neuroendocrine tumor liver metastases was imaged with HP [1-13C]pyruvate. An axial T1-weighted spoiled gradient-echo anatomical reference was acquired with the target lesions, approximately $5 \mathrm{~cm}$ deep, highlighted (Figure 1a). A 13C T/R surface coil was placed on the patient's abdomen on the right side closest to the target lesion to ensure coverage by the coil's T/R field. [1-13C]pyruvate and [1-13C]lactate signals were acquired with a single-slice 2D echoplanar spectroscopic imaging sequence over 1 minute with a temporal resolution of $3 \mathrm{~s}$ and a spatial resolution of $1.2 \mathrm{~cm}$ isotropic. Constant flip angles of $10^{\circ}$ for pyruvate and $20^{\circ}$ for lactate were applied. The $\mathrm{B} 1+$ profile of the $13 \mathrm{C} \mathrm{T} / \mathrm{R}$ coil was measured from a separate phantom scan using the double angle method.1 Vitamin E capsules within the coil, serving as fiducial markers, were visible in the T1-weighted anatomical images acquired during the patient scan (yellow dots in Figure 1b). Using these markers, the B1+ profile was rotated and translated in MATLAB to match the coil's position during the patient scan as shown in Figure 1b. The adjacent color bar indicates the scaling factor relative to the nominal flip angle. The EPSI post-processing pipeline consisted of a B0-correction, zero- and first-order phase corrections, and a tensor rank truncation method.2 In an HP tensor, the spectral, spatial, and temporal dynamics are inherently low-rank allowing for most ranks to be truncated, preserving selected principal components. The number of ranks preserved in each dimension was optimized using a bias-variance tradeoff algorithm. The before- and after-processing spectra are shown in Figure 1c-d. Pyruvate and lactate images summed over time after processing are shown in Figure 1e-f. The brightest voxels correspond with the tumors of interest. After correcting the flip angles based on the oriented B1+ profile, kPL values for each voxel were then computed using an inputless two-site model to generate kPL maps. 3 The kPL maps before and after this correction are shown in Figure 1g-h. For a healthy voxel over-flipped by 3\%, the kPL before and after correction were 0.025 and $0.027 \mathrm{~s}-1$, a $4.91 \%$ under-estimation without B1-correction. For a
\end{abstract}


tumor voxel under-flipped by $3 \%$, the kPL before and after correction were 0.044 and $0.042 \mathrm{~s}-1$, a 5.22\% over-estimation without B1-correction. Discussion: The HP 13C EPSI post-processing pipeline developed here recovers metabolite signals allowing for improved kPL estimations. Over-flipping led to an underestimation of kPL (a 4.91\% under-estimation without the coil-correction) whereas under-flipping led to overestimation (a 5.22\% overestimation without the coil-correction), which agree with simulations.3 Acknowledgements: This work was supported by NIH grants U01 EB026412, R01 CA183071, and P41 EB013598.

References: 1. Cunningham $\mathrm{CH}$ et al. Saturated double-angle method for rapid B1+ mapping. Magn Reson Med. 2006;55:1326 -JG-MO-HY33. 2. Chen H-Y, et al. Tensor Image Enhancement and Optimal Multichannel Receiver Combination Analyses for Human Hyperpolarized 13C MRSI. Magn Reson Med. 2020. 3. Larson PEZ et al. Investigation of analysis methods for hyperpolarized 13C-pyruvate metabolic MRI in prostate cancer patients. NMR in Biomedicine. 2018;31:e3997.

\section{Image/Figure:}

https://www.xcdsystem.com/wmis/abstract/File6959/GA180 ImageFigure 0610061103.png

Image/Figure Caption: Figure 1. (a) An axial T1-weighted spoiled gradient-echo image with a highlighted tumor voxel in red (whose spectrum is shown in (c-d)and other target lesions highlighted. (b) A contour map of the $13 \mathrm{C}$ T/R coil's excitation profile acquired using the double angle method. The color bar indicates the scaling factor relative to the nominal flip angle. In the immediate proximity to the coil, regions of tissue $200 \%$ of the prescribed flip angle. Fiducial markers embedded within the coil were visible and are highlighted by yellow dots. (c-d) Before and after B0-correction, phase correction, and tensor rank truncation of the highlighted tumor voxel's spectrum, respectively. (e-f) [1-13C]pyruvate and [1-13C]lactate images summed over time after processing, respectively, with low SNR voxels dropped (pyruvate SNR threshold: 10.5; lactate SNR threshold: 3.74). (g-h) kPL maps before and after the flip angle correction, respectively. For a healthy voxel under-flipped by $3 \%$, the $\mathrm{kPL}$ before and after correction were 0.025 and $0.027 \mathrm{~s}-1$, a $4.91 \%$ under-estimation without B1-correction. For a tumor voxel overflipped by $3 \%$, the kPL before and after correction were 0.044 and $0.042 \mathrm{~s}-1$, a $5.22 \%$ overestimation without B1-correction.

Full Name of Abstract's 1st Author : Philip Meng-en Lee

Complete Status: Complete

First Name: Philip

Last Name: Lee

Email: philip.lee2@ucsf.edu

Organization: University of California, San Francisco

Country: United States 


\title{
ID: GA183 \\ Intraoperative lymph node mapping using fluorescently-labeled mannose receptor-specific nanobodies
}

\author{
Lukasz Mateusiak, Vrije Universiteit Brussel, lukasz.mateusiak@vub.be
}

\section{Category: Oncology}

\begin{abstract}
Body : INTRODUCTION: Nanobodies (Nbs) are recombinant camelid-derived singledomain antibody fragments $(12-15 \mathrm{kDa})$, characterized by excellent kinetic parameters for molecular imaging due to their size and robustness. They offer considerable potential in diagnostic and therapeutic applications [1]. Lymphadenectomy, the surgical removal of lymph nodes (LNs), is performed in clinically node-negative patients who have a high chance of expressing nodal metastases. For quality assurance, a minimum number of LNs in the area of interest needs to be harvested. Samples of resected tissue are controlled for signs of cancer to assess its progress and guide further treatment. Intraoperative identification of LNs can be a complex and demanding procedure. We propose to use fluorescence molecular imaging to highlight all LNs within the surgical field to facilitate node picking during surgery. To realize LN staining we fluorescently labeled a previously developed [2][3] anti-mannose receptor (MR, CD206) $\mathrm{Nb}$ that yields significant uptake in LNs. MATERIALS AND METHODS: The Nb MMR3.49 with high affinity for MR as well as a non-targeting control $\mathrm{Nb}$ were fluorescentlylabeled with Cy5 or IRDye $800 \mathrm{CW}$. One hour after intravenous injection of $2 \mathrm{nmol}$ of the labeled compound in healthy mice ( $\mathrm{n}=3$ per group), axillary, inguinal and popliteal LNs were imaged in situ and ex vivo with the KIS (Kaer Labs, excitation 640nm, collection 820nm) (for detection of IRDye $800 \mathrm{CW}$ signals). The translational nature of the approach was studied by upscaling the experiment to a healthy porcine model $(\mathrm{n}=1,35 \mathrm{~kg})$. Here, $\mathrm{LN}$ mapping in the pelvic area, following intravenous injection of $73 \mathrm{nmol}$ of MMR3.49-Сy5, was evaluated 90 minutes after injection. LNs, muscle and fat tissue were procured for further ex vivo assessment. Fluorescent signals and target-to-background ratios (TBRs) were quantified using ImageJ software by superimposing them on white light images to anatomically localize the highlighted structures. RESULTS: Both Cy5 and IRDye800CW-labeled anti-MR Nbs enabled clear and specific in situ visualization of the LNs in mice, with ex vivo TBRs of $2.64 \pm 0.63$ and $4.62 \pm 0.50$
\end{abstract} respectively. No uptake in LNs was observed for the control Nb (TBR $1.40 \pm 0.18$ and $1.14 \pm 0.32$ ) (Figure 1A). Comparably, in the pig, MMR3.49-Cy5 helped to realize distinct in vivo fluorescent staining of pelvic LNs (Figure 1B). TBRs of the excised LNs were respectively 3.49 with muscle, and 2.98 with fat used as background. CONCLUSIONS: Intravenous injection of fluorescently-labeled anti-MR Nbs enables intraoperative localization of LNs within the whole surgical view. This could potentially increase the accuracy of extended nodal dissections. Early data suggests the imaging findings in mice are scalable to porcine models.

References: [1] S. Muyldermans, "Nanobodies: Natural Single-Domain Antibodies,” Annu. Rev. Biochem., vol. 82, no. 1, pp. 775-797, 2013. [2] A. Blykers et al., "PET imaging of macrophage mannose receptor-expressing macrophages in tumor stroma using 18F-radiolabeled camelid single-domain antibody fragments," J. Nucl. Med., vol. 56, no. 8, pp. 1265-1271, 2015. 
[3] C. Xavier et al., "Clinical Translation of [68Ga]Ga-NOTA-anti-MMR-sdAb for PET/CT Imaging of Protumorigenic Macrophages," Mol. Imaging Biol., vol. 21, no. 5, pp. 898-906, 2019.

\section{Image/Figure:}

https:/www.xcdsystem.com/wmis/abstract/File6959/GA183_ImageFigure_0602055727.png

Image/Figure Caption: Figure 1. (A) In situ fluorescence imaging of inguinal LNs in mice using MR-targeting fluorescent $\mathrm{Nb}$ or control $\mathrm{Nb}, 60$ minutes after intravenous injection. (B) In site highlighting of an $\mathrm{LN}$ in the pelvic region of a pig using Cy5-labeled MR-targeting $\mathrm{Nb}, 90$ minutes after intravenous injection.

Complete Status: Complete

First Name: Lukasz

Last Name: Mateusiak

Email: lukasz.mateusiak@vub.be

Organization: Vrije Universiteit Brussel

Country: Belgium 


\title{
ID: GA184 \\ Hyperpolarized 13C MR imaging of metastatic prostate cancer murine models
}

\author{
Shubhangi Agarwal, University of California, San Francisco, shubhangi.agarwal@ucsf.edu
}

\section{Category: Oncology}

\begin{abstract}
Body : Purpose: An organ's microenvironment plays a critical role in becoming a preferred site for metastasis and contributes towards tumor progression and response to therapy 1,2. Bone and liver are common sites for prostate cancer (PCa) metastasis3. Patients with liver metastases have poor prognosis 4 relative to patients with bone metastases alone and have shown variable response to treatments5. There is a current gap in knowledge of the mechanistic underpinnings, such as the tumor-microenvironment and metabolism, which will be critical in evaluating diagnosis and treatment response of metastatic PCa. Potent androgen pathway inhibitors have shown to induce neuroendocrine prostate cancer (NEPC), a lethal subtype of castration-resistant prostate cancer with poor survival and limited treatment options6,7. Hyperpolarized 13C (HP) MRI is a valuable technique for dynamic, real-time and non-invasive evaluation of metabolism in-vivo8 for metastases which are often not amenable to biopsy. In this study, we analyzed the differences in metabolism of NEPC tumor models grown in three different sites and their response to chemotherapy. Methods: NEPC tumor grafts were established in the renal capsule (tumor sections9), bone and liver (isolated tumor cells 10, 2×105 in $20 \mu \mathrm{L}$ ) using LuCaP9311, LTLs 352 and 61012 PDXs (patient-derived xenografts). Baseline and post-one-week (bone and liver mice treated with $60 \mathrm{mgs} / \mathrm{kg}$ carboplatin) imaging were conducted on a Bruker $3 \mathrm{~T}$ scanner with dual-tuned $1 \mathrm{H} / 13 \mathrm{C}$ volume coil. T2-weighted images and diffusion-weighted images were acquired for tumor delineation and generation of apparent diffusion coefficient (ADC) maps. Hyperpolarization was performed using a 3.35T dynamic nuclear polarizer. The copolarized $80 \mathrm{mmol} / \mathrm{L}$ [1-13C] pyruvate and $13 \mathrm{C}$ urea were rapidly dissolved with dissolution buffer and injected into mouse over 12s. Dynamic 13C spectra were acquired using a 2D-CSI sequence. Metabolite maps were obtained via SIVIC13 and analyzed using MATLAB. Statistical analysis was conducted via t-tests, pyruvate-to-lactate conversion rate, $\mathrm{kPL}$ was calculated as described previously 14 and normalized to ADCs to adjust for the differential cellularity of the tumors9. Tumors were harvested for immunohistochemical staining. Results: The T2-weighted images were used to visualize the tumor (Fig1) and calculate volumes (TableP1). All the PDXs implanted in kidney had similar kPL and ADCs (Fig1). The L93 kidney tumors had significantly lower kPL as compared to the bone and liver tumors. Bone tumors had the lowest ADCs when compared to kidney $(\mathrm{p}<0.05)$ and liver tumors (not significant). LTL610 bone tumors also had higher kPL and lower ADC than kidney, but not significant. LTL610 bone tumors had significantly lower ADC as compared to L93 bones (FigP2). Tumor volumes post-carboplatin increased for all the PDXs in bone and liver (FigP3C). LTL610 bone tumor kPL decreased by 41\% ( $<<0.05)$ from baseline (FigP3A). The ADCs for LTL610 and L93 bone post-carboplatin increased significantly by $15 \%$ and $24 \%$ respectively (FigP 3 B). Discussion: ADC maps show that bone tumors had higher cellularity as compared to kidney (L93 and LTL610) and liver tumors (L93). The L93 kPL indicates that bone and liver tumors were significantly more metabolic than kidney tumors. The kPL of bone tumors could partly be
\end{abstract}


attributed to increased cellularity or contamination of the muscle cells (due to the infiltrative tumor) which are naturally higher in LDHA. Significant decrease in kPL of LTL610 bone tumors post-carboplatin indicates response, while the liver tumors show a slight increase alluding to differential impact due to the tumor-microenvironment. These changes are currently being evaluated by protracted follow up on tumor volume. Ongoing biochemical and immunohistochemical assays will further inform on the mechanism behind the higher kPL of bone tumors. We will investigate the molecular and metabolic mechanisms responsible for the differential response to chemotherapy of the tumors as a result of the tumor site. HP-MRI has the potential to provide early metabolic readout of metastases and aid in monitoring therapy.

References: 1. Ganguly, S. S., Li, X. \& Miranti, C. K. Front. Oncol. 4, 364 (2014). 2. Mussawy, H. et al. BMC Cancer 18, 979 (2018). 3. Body, J.-J., Casimiro, S. \& Costa, L. Nat. Rev. Urol. 12, 340-356 (2015). 4. Singh, A. et al. World J. Oncol. 6, 265-269 (2015). 5. Drake, C. G. Oncology 28, 974-980 (2014). 6. Crona, D. J. \& Whang, Y. E. Cancers (Basel) 9, (2017). 7. Aggarwal, R., Zhang, T., Small, E. J. \& Armstrong, A. J. J Natl Compr Canc Netw 12, 719-726 (2014). 8. Kurhanewicz, J. et al. Neoplasia 13, 81-97 (2011). 9. Sriram, R. et al. Cancers (Basel) 10, (2018). 10. Valta, M. P. et al. Clin Exp Metastasis 31, 573-584 (2014). 11. Lam, H.-M., Nguyen, H. M. \& Corey, E. Methods Mol. Biol. 1786, 1-27 (2018). 12. Choi, S. Y. C. et al. Adv. Drug Deliv. Rev. 79-80, 222-237 (2014). 13. Crane, J. C., Olson, M. P. \& Nelson, S. J. Int J Biomed Imaging 2013, 169526 (2013). 14. Larson, P. E. Z. et al. NMR Biomed. 31, e3997 (2018).

\section{Image/Figure:}

https://www.xcdsystem.com/wmis/abstract/File6959/GA184_ImageFigure_0614073606.png

Image/Figure Caption: Fig 1: Representative Images and characteristics of PCa murine models. (A) The first column shows the T2-weighted proton image, pyruvate to lactate conversion rate, kPL (normalized to apparent diffusion coefficient (ADC)) and the ADC maps in the kidney of mice implanted with NEPC L93, LTL352 and LTL610 PDX tumors. (B) The pyruvate to lactate conversion rate, $\mathrm{kPL}$ and (C) ADC maps of the three PDXs in kidney. (D\&E) Comparison of kPL and ADC between different tumor implantation sites for L93 and LTL610 implanted mice, respectively.

Complete Status: Complete

First Name: Shubhangi

Last Name: Agarwal

Email: shubhangi.agarwal@ucsf.edu

Organization: University of California, San Francisco

Country: United States 


\title{
ID: GA185 \\ Molecularly targeted piezoelectric nanotransducers for minimally invasive stimulation of neurons
}

\author{
Tomas Jordan, Dartmouth College, tomas.jordan.th@dartmouth.edu
}

\section{Category: Neuroscience}

\begin{abstract}
Body : Minimally invasive stimulation of select neurons with great spatiotemporal precision remains a challenging feat in neuroscience. The field is a high priority research area since neural circuit manipulation is heavily utilized in neuroscience research. Further, neurostimulation shows promising results in treatment of a number of conditions, including Parkinson's disease, epilepsy, or depression. The technique has also been increasingly used in brain-machine interfacing applications and retinal or cochlear implants. However, established neurostimulation methods suffer from numerous limitations constraining the spatial resolution, penetration depth, or chronic use. Ultrasound (US) appears to be a modality with great potential for neuroscience applications as it offers important advantages over electrical or light-based methods. First, US has low attenuation in soft tissue, allowing up to $5-7 \mathrm{~cm}$ penetration and, thus, enabling non-invasive stimulus delivery into regions deep in the brain. Second, its spatial and temporal resolution are exquisite: the beam can be focused into a sub-mm spot at frequencies in the $\mathrm{MHz}$ range (which highly exceeds the maximum firing rates of neurons). A growing body of research suggests that ultrasound is capable of modulating neuronal activity by altering the neuron excitability. However, this method does not enable reliable activation of a small brain region due to the lack of US-sensitive ion channels. To enhance the sensitivity of neurons to ultrasound waves, we utilize piezoelectric nanoparticles molecularly targeted to the neuronal membranes. Through the direct piezoelectric effect, these nanoparticles transduce mechanical deformations into electric charges, activating voltage-gating ion channels. Here, we demonstrate the effects in cultured rat hippocampal neurons. We used 300-nm tetragonal barium titanate nanoparticles (BTNPs) and verified their crystal structure with X-ray powder diffraction as tetragonality is a necessity for the piezoelectric properties. To enhance the piezoelectricity of the particles, we electrically poled them in a $900 \mathrm{~V} / \mathrm{mm}$ electric field at $110^{\circ} \mathrm{C}$. This procedure led to aligning of the ferroelectric domains, resulting in a 50 -fold increase in the piezoelectric conversion. To prepare a stable nanoparticle dispersion, we coated the BTNPs with polyethylene glycol (PEG). Through the use of bifunctionalized PEG polymers, we were able to conjugate IgG antibodies to the BTNP surface to enable cell-specific binding. We utilized an anti-Neurofascin antibody which targets a cell adhesion molecule expressed in the axon initial segment. We incubated our BTNP-antibody conjugates with cultured hippocampal neurons and observed successful attachment to the membranes. In our studies of the ultrasound excitation of neuron bound BTNPs, we were interested in the effects on calcium signaling and glutamate release. For calcium studies, we utilized GCaMP6f, a genetic calcium indicator, expressed in the presynaptic bouton. To monitor the release of glutamate, we transfected the cells with a glutamate fluorescent reporter GluSnFr. We applied ultrasound stimulation with a focused ultrasound (FUS) transducer (Sonic Concepts H-151) while imaging the fluorescence of the genetically encoded reporters. We observed that FUS stimulation (5 x $1 \mathrm{~ms}$ pulses, $1.1 \mathrm{MHz}$ frequency, 4.63
\end{abstract}


MPa focal pressure) induced a calcium influx into the presynaptic boutons. The ultrasound excitation of BTNPs was also found to cause neurotransmitter vesicle fusion and release of glutamate (single FUS pulse of $45.5 \mu$ s duration, $4.2-4.6 \mathrm{MPa}$ focal pressure, $1.1 \mathrm{MHz}$ frequency). Ultrasound in the absence of the BTNPs was found not to be a reliable way of inducing calcium or glutamate response. These results indicate that FUS excitation of membrane bound BTNPs generates sufficient electric charges to activate primary hippocampal neurons. Combining the advantages of molecular targeting and the favorable properties of ultrasound, our technique has a strong potential to become widely utilized in minimally invasive neurostimulation applications.

References: Jordan, T.; O’Brien, M. A.; Spatarelu, C.-P.; Luke, G. P. Antibody-Conjugated Barium Titanate Nanoparticles for Cell-Specific Targeting. ACS Appl. Nano Mater. 2020, 3 (3), 2636-2646. https://doi.org/10.1021/acsanm.0c00019

\section{Image/Figure:}

https://www.xcdsystem.com/wmis/abstract/File6959/GA185 ImageFigure 0421123736.jpg

Complete Status: Complete

First Name: Tomas

Last Name: Jordan

Email: tomas.jordan.th@dartmouth.edu

Organization: Dartmouth College

Country: United States 


\title{
ID: GA186 \\ Cytokine Scavenging Nanoparticles based on Heparin for Cancer Therapy
}

\author{
Zhihang Chen, Johns Hopkins University, zchen19@jhu.edu
}

Category: New Chemistry, Biology \& Bioengineering

\begin{abstract}
Body : Cytokines, which are rich in the tumor microenvironment (TME), are a broad category of small proteins $(\sim 5-20 \mathrm{kD})$ secreted by cells to influence the cellular behavior of surrounding cells. Because cytokines play an important role in tumor growth, immune evasion, and promotion of invasion and metastasis, they are attractive targets in cancer treatment. Here, we have developed an entirely new class of nanoparticles (NPs) to act as scavengers (Sca) for cytokines to disrupt multiple functions of cancer cells and their interactions with cytokines. Many reports indicate that the glycosaminoglycan (GAG) molecules such as heparin, demonstrate specific binding with cytokines and chemokines. Hence, we incorporated biocompatible heparin to construct the Sca-NPs to scavenge cytokines in the tumor. The heparin molecules were conjugated to nano-sized cores that were magnetic iron oxide nanoparticles (MIONPs) or dextran in our investigations, to improve the efficiency of capture. To shield the interaction between the Sca-NPs and proteins during circulation and transport, inert polyethylene glycol (PEG) polymers were conjugated to Sca-NPs through acid sensitive acetal bonds. This PEG shell is stable under normal conditions, but is cleaved in weak acid condition, such as in cancerous or inflamed tissues, to expose the Sca-NP to capture the cytokines (Figure 1). Following degradation in the endosomal compartment, or following clearance by circulation, the Sca-NP bound cytokines and chemokines would be removed from the TME. These Sca-NPs were found to reduce the concentration of IL-6 in MB-MDA-231 cell medium. Following a 1 hour incubation, the Sca-NPs were removed from the medium, and the concentration of IL-6 decreased to $53 \%$ of control. In medium that was incubated with the PEGSca-NP, concentration of IL-6 changed slightly (reduced to $92 \%$ of control). When we treated the medium with cleaved PEG-Sca-NP (in which the PEG shield was removed), the IL-6 concentration was reduced to $56 \%$ of control. These Sca-NPs also were applied to scavenge IL-6 in mouse serum. Following treatment, the concentration of IL-6 was reduced to $70 \%$ of control. These Sca-NPs also can carry an imaging reporter to allow noninvasive detection of their localization in tumors. These scavenging NPs (Sca-NPs) may also have potential to reduce the cytokine storm to prevent organ failure.
\end{abstract}

\section{Image/Figure:}

https://www.xcdsystem.com/wmis/abstract/File6959/GA186_ImageFigure 0608092257.jpg

Image/Figure Caption: Figure 1. Schematic of Sca-NPs

Complete Status: Complete

First Name: Zhihang 
Last Name: Chen

Email: zchen19@jhu.edu

Organization: Johns Hopkins University

Country: United States 


\title{
ID: GA188 \\ Delta-like Ligand 3 (DLL3) is a novel target for molecular imaging of Neuroendocrine Prostate Cancer
}

\author{
Joshua Korsen, Weill Cornell Medicine, jak2058@med.cornell.edu
}

\section{Category: Oncology}

\begin{abstract}
Body : Objective: Transdifferentiation of prostate adenocarcinoma to Neuroendocrine Prostate Cancer (NEPC) has emerged as one of the leading causes of resistance to androgen deprivation therapy (ADT) [1]. The patients have aggressive disease with visceral metastasis and have short survival time (7-10 months) [2]. Genomic and proteomic characterization of biopsy samples of NEPC lesions indicates loss of androgen receptor (AR) signaling [3]. Therefore, current PET imaging agents such as 18F-FDHT and 68Ga-PSMA11 that rely on functional AR cannot be used. Our goal is to develop a PET based molecular imaging agent that can uniquely identify NEPC lesions. Taking advantage of highly specific expression of Delta-like ligand 3 (DLL3) in NEPC lesions, we have developed zirconium-89 labeled immunoPET agent that can specifically identify NEPC lesions. Methods: We have used well characterized NCI-H660 cell line as representative NEPC model and compared it with AR dependent LNCaP and AR independent PC3 and DU145 cell lines. qPCR was used to measure relative levels of ARregulated gene transcripts (AR, PSMA, PSA) and NEPC marker DLL3 and normalized to b-actin in the cell lines. Relative protein levels of AR regulated genes and DLL3 were measured by western blot analysis of cellular extracts using b-actin as our control. ImmunoPET agent - 89ZrSC16 - was developed through the conjugation of the DFO chelator to SC16 (DLL3 specific) $\mathrm{mAb}$ and radiolabeled with zirconium-89. Saturation binding assay was performed on the cell line NCI-H660 to determine Bmax and Kd values. For in vivo PET imaging and biodistribution studies, NCI-H660 (DLL3/+) or DU145 (DLL3/-) xenografts were established in 6-8 week old male athymic nude mice. The mice were administered with $89 \mathrm{Zr}-\mathrm{SC} 16$ and imaged at 24, 48, 72, 96, and $120 \mathrm{~h}$ post injection on a PET scanner and at chosen time points mice were euthanized and organs collected for biodistribution studies. Results: Saturation binding assay reveals that $\mathrm{Kd}$ $=0.35 \mathrm{nM}$ and $\mathrm{Bmax}=863 \mathrm{fm} / 106$ cells for $89 \mathrm{Zr}-\mathrm{SC} 16$. In vitro studies indicated that NCI-H660 cell line was positive for DLL3 and negative for AR, PSA, and PSMA both at transcriptional and translational level. As expected all other cells except PC3 were negative for DLL3. In vivo PET imaging with 89Zr-SC16 showed clear delineation of NCI-H660 (DLL3/+) tumor xenografts. Biodistribution studies showed tumor uptake of $18.4 \pm 3.8 \% \mathrm{ID} / \mathrm{g}$ in the NCI-H660 tumors compared to $5.5 \pm 0.5 \% \mathrm{ID} / \mathrm{g}$ in the DU145 tumors, demonstrating the selective accumulation of the radiotracer in the DLL3-expressing tumors. Conclusion: Our findings demonstrate that only NEPC cells selectively express DLL3 and using DLL3 targeting PET agent 89Zr-SC16 we can non-invasively and uniquely identify NEPC lesions in vivo. Acknowledgement: Partial funding for these studies was provided by 2019 Geoffrey Beene Cancer Research Center Grant.
\end{abstract}

References: 1. Watson, P.A., V.K. Arora, and C.L. Sawyers, Emerging mechanisms of resistance to androgen receptor inhibitors in prostate cancer. Nat Rev Cancer, 2015. 15(12): p. 701-11. 2. Apostolidis, L., Nientiedt, C., Winkler, E.C., et al., Clinical characteristics, treatment 
outcomes and potential novel therapeutic options for patients with neuroendocrine carcinoma of the prostate. Oncotarget, 2018. 10: p. 17-29. 3. Puca, L., Gavyert, K., Sailer, V., et al., Delta-like protein 3 expression and therapeutic targeting in neuroendocrine prostate cancer. Sci Transl Med, 2019. 11(484).

Full Name of Abstract's 1st Author : Joshua Korsen

Complete Status: Complete

First Name: Joshua

Last Name: Korsen

Email: jak2058@med.cornell.edu

Organization: Weill Cornell Medicine

Country: United States 


\title{
ID: GA189 \\ A pilot study of 18F-FSPG PET/CT in patients referred for exclusion of active cardiac sarcoidosis and negative, equivocal or non-diagnostic 18F-FDG PET/CT
}

\author{
Andrei Iagaru, Stanford University, aiagaru@stanford.edu
}

\section{Category: Cardiovascular \& Pulmonary}

\begin{abstract}
Body : OBJECTIVES: 18F-FDG PET is accepted for the assessment of active cardiac sarcoidosis; however, up to $1 / 3$ of patients fail to follow the required complex dietary restrictions, resulting in non-diagnostic scans. In addition, there are equivocal results. $18 \mathrm{~F}-$ labeled FSPG, an L-glutamate derivative, is a promising radiotracer for PET imaging of the aminoacid antiporter system $\mathrm{xC}$ - that is involved in detoxification processes and balancing oxidative stress, including in sarcoidosis. No dietary restrictions are required prior to $18 \mathrm{~F}-\mathrm{FSPG}$ PET. This prospective pilot study evaluates the utility of $18 \mathrm{~F}-\mathrm{FSPG}$ PET for assessment of cardiac sarcoidosis, in comparison to negative or non-diagnostic 18F-FDG PET. MATERIALS AND METHODS: Patients referred to Nuclear Medicine to rule out active cardiac sarcoidosis were prospectively enrolled after the $18 \mathrm{~F}-\mathrm{FDG}$ PET/CT results were negative, equivocal or nondiagnostic. 18F-FSPG whole-body imaging started 46-67 minutes (mean \pm SD: $53.8 \pm 6.0$ ), while dedicated cardiac imaging started 55-77 minutes (mean $\pm \mathrm{SD}: 65.9 \pm 5.9$ ) after injection of 7.1-8.9 $\mathrm{mCi}$ (mean \pm SD: $8.1 \pm 0.5$ ). The mean delay time between 18F-FDG PET and 18F-FSPG PET was 23.3 days. A state-of-the art SiPM-based PET/CT scanner (GE Discovery MI) was used for all scans. RESULTS: Data from 4 women and 11 men, 32-79 year-old (mean \pm SD: 58.2 \pm 12.9 ) were collected. Eight of the participants had no $18 \mathrm{~F}-\mathrm{FDG}$ uptake compatible with cardiac sarcoidosis; 2 of the 8 had 18F-FDG uptake in lung nodules and mediastinal lymph nodes, while 3 of the 8 had 18F-FDG uptake in mediastinal lymph nodes compatible with sarcoidosis. 18F-FSPG uptake was seen in the lung nodules and mediastinal lymph nodes in the same 5 participants with $18 \mathrm{~F}$ FDG uptake, while no 18F-FSPG uptake was identified in the myocardium. The remaining 7 patients had non-diagnostic cardiac 18F-FDG PET due to non-compliance with dietary instructions; 18F-FDG uptake in lung nodules and mediastinal lymph nodes was seen in 1 of these 7 patients. These lung nodules and mediastinal lymph nodes also had 18F-FSPG uptake. No 18F-FSPG myocardial uptake was identified in the 7 patients with non-diagnostic cardiac 18F-FDG PET. CONCUSIONS: 18F-FSPG may play a role in ruling out cardiac sarcoid involvement in a cohort with negative, equivocal or non-diagnostic 18F-FDG PET. Further evaluation in larger cohorts is needed to confirm our data. In addition, future studies should evaluate 18 F-FSPG usage in patients with confirmed active cardiac sarcoidosis.
\end{abstract}

\section{Image/Figure:}

https://www.xcdsystem.com/wmis/abstract/File6959/GA189 ImageFigure 0421011659.png

Complete Status: Complete

First Name: Andrei 
Last Name: Iagaru

Email: aiagaru@stanford.edu

Organization: Stanford University

Country: United States 


\title{
ID: GA191 \\ Preliminary Results of a Prospective Study of 68Ga-RM2 PET/MRI in Patients with Biochemically Recurrent Prostate Cancer and Negative Conventional Imaging
}

\author{
Andrei Iagaru, Stanford University, aiagaru@stanford.edu
}

\section{Category: Oncology}

\begin{abstract}
Body : Purpose: 68Ga-RM2 is a synthetic bombesin receptor antagonist targeting gastrin-releasing peptide receptors (GRPr) that are overexpressed in several human tumors, including prostate cancer (PC). Methods: We enrolled 119 men with BCR PC, 45-83-year-old $($ mean \pm SD: $68.2 \pm 6.9$ ). Imaging started at 40-89 minutes (mean \pm SD: $51.1 \pm 9.1$ after injection of 113.8-152.6 MBq (mean \pm SD: $140.6 \pm 6.3$ ) of $68 \mathrm{Ga}-\mathrm{RM} 2$ using a time-of-flight (TOF)-enabled simultaneous positron emission tomography (PET) / magnetic resonance imaging (MRI) scanner. Results: All patients had rising PSA and negative conventional imaging prior to enrollment. 68Ga-RM2 PET identified recurrent PC in 81 of the 119 participants. Positivity rate of 68GaRM2 PET was: $30.4 \%$ for PSA $5.0 \mathrm{ng} / \mathrm{dl}(\mathrm{n}=40)$. PSA velocity values were $1.9 \pm 2.7 \mathrm{ng} / \mathrm{ml} / \mathrm{year}$ (range: 0-9.1) in patients with negative PET scans and $5.8 \pm 9 \mathrm{ng} / \mathrm{ml} /$ year (range: $0.2-45.4$ ) in patients with positive PET scans (P: 0.01). Conclusions: 68Ga-RM2 PET identifies GRPr expression in BCR PC lesions despite negative conventional imaging, indicating it is a promising PET radiopharmaceutical in this clinical scenario. 68Ga-RM2 may identify higher risk patients given the highly statistically significant difference PSA velocity values between patients with negative and positive scans and may be a complementary radiopharmaceutical to the PSMAtargeting tracers to ultimately allow for personalized medicine.
\end{abstract}

\section{Image/Figure:}

https://www.xcdsystem.com/wmis/abstract/File6959/GA191 ImageFigure 0421013343.png

Complete Status: Complete

First Name: Andrei

Last Name: Iagaru

Email: aiagaru@stanford.edu

Organization: Stanford University

Country: United States 


\title{
ID: GA192 \\ Immunotherapeutic cell tracking using fluorine-19 MRI at 3 Tesla: Demonstrating longitudinal detection and quantification of human peripheral blood mononuclear cells and regulatory $T$ cells in immunocompromised mice
}

\author{
Olivia Sehl, Robarts Research Institute, osehl@uwo.ca
}

Category: Immunology: Inflammation \& Infection

\begin{abstract}
Body : Cell-based immunotherapy refers to the administration of immune cells for the treatment of an ever-increasing list of cancerous, autoimmune, degenerative, and infectious diseases; some of which are rare and/or difficult to treat. For example, administration of peripheral blood mononuclear cells (PBMC) has been FDA approved for prostate cancer $(1,2)$ and regulatory $\mathrm{T}$ cells (Treg) are under development to replace druginduced immunosuppression for patients with autoimmune disease, organ transplantation, or blood cancers (3). For immunotherapy, migration of administered cells to secondary lymphoid tissues is essential to elicit adaptive immune responses. It is crucial to know whether the cells have migrated to lymph nodes, and in what quantity, to determine the magnitude of this response $(1,2)$. Researchers are now being challenged to verify the presence and quantity of cells in vivo to gain regulatory approval of cell therapies (4). Fluorine-19 (19F) magnetic resonance imaging (MRI) has the potential to answer these fundamental questions. 19F images display distribution of perfluorocarbon- (PFC)-labeled cells and allow for measurements of cell number, providing information about cell delivery and engraftment $(4,5)$. Our objective is to conduct longitudinal cell tracking of human PBMC and Treg in immunocompromised mice using 19F MRI at 3 Tesla (T), to study cell migration, persistence, and quantity in vivo. Methods: Following preparation of human PBMC (2) and Treg (3), cells were cocultured overnight with PFC (Celsense Inc.). PFC+ PBMC were injected into $\mathrm{Nu} / \mathrm{Nu}$ mice in the right $(3 \times 106$ cells $)$ and left $(6 \times 106$ cells $)$ footpads $(n=4)$ or by intraperitoneal injection $(5 \times 106$ cells, $\mathrm{n}=2$ ). In another cohort, a mixture of 10x106 PFC+ Treg and 10x106 unlabeled PBMC were administered intravenously $(\mathrm{n}=4)$ or subcutaneously $(\mathrm{n}=4)$. Unlabeled PBMC was included to repopulate lymphoid organs and promote Treg migration and retention at lymph nodes. 24 and 48 hours later, $1 \mathrm{H}$ and $19 \mathrm{~F}$ images were acquired on a 3T clinical MRI using a $4.31 \mathrm{~cm}$ dual-tuned surface coil and a 3D balanced steady state free precession (bSSFP) sequence $(1,6)$. 19F signal in images was quantified by comparison to reference tubes and $19 \mathrm{~F}$ atoms/cell was measured by NMR. Results: Labeling was successful with 1.5x1012 19F spins/PBMC and 1.73x1012 19F spins/Treg, as determined by NMR. 19F images of mice receiving PFC+ PBMC show the accumulation of 19F signal in the popliteal and inguinal lymph nodes post-adoptive transfer. 19F images of mice receiving PFC+ Treg show the accumulation of $19 \mathrm{~F}$ signal in inguinal and axillary lymph nodes. The included figure shows representative 19F images from each cohort and tables summarizing the quantification of PFC+ cells detected at lymph nodes.

Discussion: We demonstrated detection and quantification of PBMC and Treg in vivo which accumulate at lymph nodes. This is the first study to conduct in vivo 19F imaging of Treg. This is also the first study to image 19F-positive PBMC in vivo under clinical conditions; and overall the third $19 \mathrm{~F}$ cell tracking mouse study at 3T. By developing this technology at 3T, we move
\end{abstract}


towards clinical translation. This work is central to understand the fate of cells after administration and to improve therapeutic benefit. For example, our images suggest that the route of cellular administration may impact the treatment efficacy (i.e. the number of cells reaching lymph nodes). $19 \mathrm{~F}$ imaging of cellular immunotherapy may also play a clinical role, to inform physicians whether a patient may need repeat dosing or other interventions.

References: (1) Fink C, Gaudet JM, Fox MS, Bhatt S, Viswanathan S, Smith M, Chin J, Foster P, Dekaban GA. 19F-perfluorocarbon-labeled human peripheral blood mononuclear cells can be detected in vivo using clinical MRI parameters in a therapeutic cell setting. Sci Rep.

2018;8(1):1-13. (2) Fink C, Smith M, Sehl OC, Gaudet JM, Meagher TC, Sheikh NA, Dikeakos JD, Rieder MJ, Foster PJ, Dekaban GA. Quantification and characterization of granulocyte macrophage colony-stimulating factor activated human peripheral blood mononuclear cells by fluorine-19 cellular MRI in an immunocompromised mouse model. Diagn Interv Imaging. 2020. (3) MacDonald KN, Piret JM, Levings MK. Methods to manufacture regulatory T cells for cell therapy. Clin Exp Immunol. 2019;197(1):52-63. (4) Ahrens ET, Bulte JWM. Tracking immune cells in vivo using magnetic resonance imaging. Nature Reviews Immunology. 2013. (5) Chapelin F, Capitini CM, Ahrens ET. Fluorine-19 MRI for detection and quantification of immune cell therapy for cancer. J Immunother Cancer. 2018;6(1):1-11. (6) Makela A V., Foster PJ. Preclinical 19 F MRI cell tracking at 3 Tesla. Magn Reson Mater Physics, Biol Med. 2019;32(1):123-32.

\section{Image/Figure:}

https://www.xcdsystem.com/wmis/abstract/File6959/GA192_ImageFigure_0603094011.png

Image/Figure Caption: Figure: 19F/1H MRI overlay of mice showing detection of PFC+ PBMC after intraperitoneal or footpad (red arrows) injections and PFC+ Treg after intravenous or subcutaneous injections. PFC+ cells accumulate in popliteal lymph nodes, inguinal lymph nodes, the spleen, and axillary lymph nodes (see legend). Images were aqcuired both 24 and 48 (not shown) hours post-adoptive transfer. The colour scale bar represents 19F spins. From each cohort of mice, the average measurement of cells from each lymph node is shown in tables. In the table, (-) indicates that the specified nodes were outside the imaging field of view.

Full Name of Abstract's 1st Author : Olivia C. Sehl

Complete Status: Complete

First Name: Olivia

Last Name: Sehl

Email: osehl@uwo.ca

Organization: Robarts Research Institute

Country: Canada 


\title{
ID: GA193 \\ [18F]F-AraG PET for CD8 profiling of tumors and assessment of immunomodulation by chemotherapy
}

\author{
Jelena Levi, CellSight Technology, Inc., jlevi@cellsighttech.com
}

\section{Category: Oncology}

\begin{abstract}
Body : Certain types of chemotherapy are shown to induce anticancer immune response and are actively being investigated for their synergy with immunotherapy $[1,2]$. Of the several hundred clinical trials exploring various combinations of chemo- and immunotherapy [3] very few utilize molecular imaging to evaluate immune response. Here, we assess the value of using [18F]F-AraG, an agent relatively specific for activated T cells [4-6], as a non-invasive tool that could profile tumors based on the key players in adaptive antitumor response, CD8+ cells, and evaluate immunomodulatory effects of chemotherapy. To mimic the variations in cancerimmune phenotypes observed in the clinic, we imaged six syngeneic tumor models with different immune contextures: MC38 (colon), CT26 (colon), LLC (lung), A9F1 (immunogenic Lewis lung clone), 4T1 (breast), and B16F10 (melanoma). [18F]F-AraG revealed strikingly different uptake patterns in these tumors: signal in the tumor core (MC38 and A9F1), signal encircling the tumor (CT26), signal at the tumor margin (LLC and B16F10) and absence of signal (4T1). To evaluate the ability of $[18 \mathrm{~F}] \mathrm{F}-\mathrm{AraG}$ to report on the presence of $\mathrm{CD} 8+$ cells within the tumor microenvironment, the signal intensity was correlated with the number of lymphocytes isolated from the tumors one day post imaging. While $[18 \mathrm{~F}] \mathrm{F}-\mathrm{AraG}$ intensity did not correlate with the number of total lymphocytes (CD45+ cells), statistically significant correlation was found with the number of PD-1 positive CD8+ cells ( $\mathrm{r} 2=0.528$, $\mathrm{p}$ To assess the ability of [18F]F-AraG to detect immunomodulatory effects of chemotherapy, we performed longitudinal imaging of tumor bearing mice (MC38, A9F1 and 4T1) undergoing two types of chemotherapy: oxaliplatin/cyclophosphamide, shown to induce immunogenic cell death and paclitaxel/carboplatin, reported to cause immunogenically silent tumor cell death. In MC38 model, paclitaxel/carboplatin did not result in appreciable change in signal post therapy, but oxaliplatin/cyclophosphamide treatment led to close to 2.4 fold higher [18F]F-AraG signal. The increase in signal post oxaliplatin/cyclophosphamide was also observed in A9F1, but not in 4T1 model. These imaging results corresponded to the differences in the tumor microenvironment that were detected between chemotherapies and tumor models. Compared to paclitaxel/carboplatin, oxaliplatin/cyclophosphamide therapy led to an increase in total lymphocytes in both MC38 and A9F1 tumors, but not in 4T1. In MC38 tumors that showed the most striking difference between the two chemotherapies, the oxaliplatin/cyclophosphamide group showed 27-fold higher ratio of effector CD8+ to regulatory CD4+FOXP3+ cells than the paclitaxel/carboplatin treated mice. Our data suggest $[18 \mathrm{~F}] \mathrm{F}-\mathrm{AraG}$ PET is a promising tool for CD8 profiling of the tumors and evaluation of immune induction strategies. The ability of $[18 \mathrm{~F}] \mathrm{F}-$ AraG PET to assess the location and function of CD8+ cells, as well immune activity within tumors post immune priming therapy warrants further investigation into its utility for patient selection, evaluation of optimal time to deliver immunotherapies, and assessment of combinatorial therapy approaches.
\end{abstract}


References: 1. Voorwerk, L., et al., Immune induction strategies in metastatic triple-negative breast cancer to enhance the sensitivity to PD-1 blockade: the TONIC trial. Nat Med, 2019. 25(6): p. 920-928. 2. Galluzzi, L., et al., Immunological Effects of Conventional Chemotherapy and Targeted Anticancer Agents. Cancer Cell, 2015. 28(6): p. 690-714. 3. Xin Yu, J., et al., Trends in clinical development for PD-1/PD-L1 inhibitors. Nat Rev Drug Discov, 2020. 19(3): p. 163-164. 4. Ronald, J.A., et al., A PET Imaging Strategy to Visualize Activated T Cells in Acute Graft-versus-Host Disease Elicited by Allogenic Hematopoietic Cell Transplant. Cancer Res, 2017. 77(11): p. 2893-2902. 5. Franc, B.L., et al., In Vivo PET Imaging of the Activated Immune Environment in a Small Animal Model of Inflammatory Arthritis. Mol Imaging, 2017. 16: p. 1536012117712638. 6. Levi, J., et al., Imaging of Activated T Cells as an Early Predictor of Immune Response to Anti-PD-1 Therapy. Cancer Research, 2019. 79(13): p. 3455-3465.

Complete Status: Complete

First Name: Jelena

Last Name: Levi

Email: jlevi@cellsighttech.com

Organization: CellSight Technology, Inc.

Country: United States 


\title{
ID: GA194 \\ Comparison of 52Mn-complexing bifunctional chelators for radioimmunoconjugation: labelling efficiency and in vivo stability
}

\author{
George Firth, King's College London, george.firth@kcl.ac.uk
}

\section{Category: New Chemistry, Biology \& Bioengineering}

\begin{abstract}
Body : Introduction Manganese is a versatile essential metal that has found an important role at the heart of medical imaging, in the form of paramagnetic complexes as contrast agents for MRI and, most recently, as radionuclides for PET. Manganese-52 has favourable imaging properties ( $\mathrm{t} 1 / 2=5.6$ days, $\beta+=30 \%)$ that make it a desirable option for imaging long-lived biological processes, notably cell and antibody tracking.1,2 In order to permit successful immunoPET studies, high radiolabelling efficiency and good stability of the radioimmunoconjugate are paramount. In this study several different bifunctional chelators were labelled with 52Mn. The radiolabelling efficiency was determined and successful lead candidates where selected for in vivo stability and PET biodistribution studies. Methods 52Mn was produced at the Hevesy lab following the irradiation of pressed chromium powder (GE PETtrace; $16 \mathrm{MeV}$ protons; $20 \mu \mathrm{A}$ for $4 \mathrm{~h}$ ). Several trastuzumab-like (Herzuma ${ }^{\circledR}$, trastuzumab biosimilar) immunoconjugates of bifunctional chelators (BFCs) (DOTA, oxo-DOTA, NOTA, PCTA, DTPA, CDTA) were synthesised and radiolabelled following incubation at room temperature with [52Mn] $\mathrm{MnCl} 2$ using range of antibody concentrations $(0.002-20 \mu \mathrm{M})$ and $\mathrm{pH}$ (5 and 7.2). Reactions were quenched after 45 minutes with EDTA (50 mM). Labelling efficiencies from each dilution assay were determined by iTLC (iTLC-SG; 0.1 M citrate buffer). Chelator to antibody ratios were calculated by radiolabelling the immunoconjugates with $111 \mathrm{In}$ and co-incubating with varying concentrations of $\mathrm{InCl3}$. The labelling efficiency at different indium to immunoconjugate ratios was calculated, and from this, the number of moles of indium specifically bound at each ratio was determined for each immunoconjugate. A known concentration of immunoconjugate thus allowed for the number of chelators to be determined. The biodistribution of [52Mn]DOTA-Her and [52Mn]PCTA-Her (1.5 MBq; $200 \mu \mathrm{L})$ selected as lead compounds on the basis of in vitro labelling efficiency were investigated in healthy BALB/c mice over 13 days by PET/CT. Ex vivo biodistribution at 13 days post injection was obtained following tissue dissection. [52Mn] $\mathrm{MnCl} 2 \mathrm{PET}$ images over 3 days and ex vivo biodistribution at 4 days are shown for comparison. Results/discussion The class of cyclic chelators tested demonstrated successful radiolabelling yields with quantitative labelling achieved at concentrations above $2 \mu \mathrm{M}$, compared to acyclic chelators which had considerably lower yields ( $90 \%$ at $200 \mathrm{~nm}$ immunoconjugate concentration; room temperature; $\mathrm{pH} \mathrm{5).} \mathrm{Conjugation} \mathrm{to} \mathrm{each}$ of the isothiocyanate-derived BFCs yielded approximately 5 chelators per antibody for DOTAHer and PCTA-Her, and 7 for oxo-DOTA-Her and NOTA-Her using identical conjugation conditions for each BFC (Supplementary data 1A). In vivo biodistribution of the lead candidate, PCTA-Her, was investigated using PET imaging (Fig. 1B). This was then compared to the gold standard chelator, DOTA, as a DOTA-Her BFC, which has previously demonstrated good in vivo stability.1 Both immunoconjugates demonstrated prolonged in vivo stability, with radioactivity present in the blood pool up to $166 \mathrm{~h}$ post injection. The subsequent time point at
\end{abstract}


$318 \mathrm{~h}$ exhibits metabolism of the immunoconjugates, and is likely due to the biological half-life of the antibody and not the stability of the BFC. Conclusion The results presented here suggest that PCTA could offer advantages for long-term PET imaging, not from an in vivo stability perspective, as DOTA immunoconjugates already permit good in vivo stability, but from a radiolabelling efficiency perspective. The ability to quantitatively radiolabel PCTA-Her with $52 \mathrm{Mn}$ using mild conditions (room temperature, neutral $\mathrm{pH}, 2 \mu \mathrm{M}$ BFC concentration) makes it a desirable choice for future applications in immunoPET. NOTA and oxo-DOTA are additional options with superior radiolabelling over DOTA, however their in vitro and in vivo stability are currently under investigation.

References: 1. S. A. Graves, R. Hernandez, J. Fonslet, C. G. England, H. F. Valdovinos, P. A. Ellison, T. E. Barnhart, D. R. Elema, C. P. Theuer, W. Cai, R. J. Nickles and G. W. Severin, Bioconjug. Chem., 2015, 26, 2118-2124. 2 P. Gawne, F. Man, J. Fonslet, R. Radia, J. Bordoloi, M. Cleveland, P. Jimenez-Royo, A. Gabizon, P. J. Blower, N. Long and R. T. M. De Rosales, Dalt. Trans., 2018, 47, 9283-9293.

\section{Image/Figure:}

https://www.xcdsystem.com/wmis/abstract/File6959/GA194 ImageFigure 0610082812.png

Image/Figure Caption: Fig. 1 Radiolabelling and in vivo biodistribution of various 52Mn immunoconjugates. (A) Panel of bifunctional chelators selected for the study. (B) Radiolabelling efficiency of immunoconjugates under increasingly dilute conditions expressed as \% labelling efficiency against effective concentration of bifunctional chelator. (B) Serial maximum intensity projection PET/CT images of BALB/c mice at 1, 24, 72, 166 and $318 \mathrm{~h}$ post injection with [52Mn]DOTA and PCTA-Herzuma ${ }^{\circledR}$-immunoconjugates $(\mathrm{n}=1)$. Radioimmunconjugates remain stable in vivo up to $166 \mathrm{~h}$ post injection showing characteristic blood pool biodistribution, including heart, descending aorta and carotid arteries. There is a notable lack of pancreas and salivary gland uptake which are typical features of [ $52 \mathrm{Mn}] \mathrm{MnCl} 2$ biodistribution. At $310 \mathrm{~h}$ post injection, these features are much more prominent confirming the metabolism of the radiotracer.

Full Name of Abstract's 1st Author : George Firth

Complete Status: Complete

First Name: George

Last Name: Firth

Email: george.firth@kcl.ac.uk

Organization: King's College London

Country: United Kingdom 


\title{
ID: GA195 \\ Imaging macrophage density and distribution in 4T1 mouse mammary tumours using fluorine-19 MRI at 3 Tesla
}

\author{
Olivia Sehl, Robarts Research Institute, osehl@uwo.ca
}

\section{Category: Oncology}

\begin{abstract}
Body : Introduction: Tumour-associated macrophages (TAMs) are the most abundant and influential cells infiltrating breast tumours. Accumulating evidence suggests that TAMs actively promote all aspects of tumour initiation, development, immunosuppression, metastasis, and resistance to therapies (1-7). A meta-analysis of clinical data has indicated a strong link between TAM burden and the prognosis of breast cancer patients (8). Considering the identification of TAMs as a biomarker and the emergence of TAM-targeted immunotherapies $(6,9)$, a critical step involves the development of diagnostic tools to detect and quantify TAMs in vivo. Fluorine-19 (19F) MRI is a promising technique to monitor TAM burden because it has the ability to visualize and quantify the number of TAMs (10). The aim of this study is (1) to determine the density and distribution of TAMs in murine mammary tumours over time using fluorine-19 (19F) MRI and (2) to deplete macrophages by systemic administration of clodronate liposomes, then determine how this effects tumour growth and TAM presence using $1 \mathrm{H} / 19 \mathrm{~F}$ MRI. Methods: 3x105 4T1 breast cancer cells were implanted to the mammary fat pad of BALB/c mice $(n=17)$. Phagocytic macrophages, including TAMs, were labeled through intravenous delivery of perfluorocarbon (V-Sense, CelSense Inc.). 24 hours later, 1H/19F images were acquired on a 3T clinical MRI using a dual-tuned surface coil and 3D balanced steady state free precession (bSSFP) sequence. Study 1: 1H/19F images were acquired $11(n=4), 17(n=5)$ and $20(\mathrm{n}=5)$ days after tumour implantation. Study 2: Clodronate liposomes $(1 \mathrm{mg})$ was delivered intravenously 5, 10, and 15 days after tumour implantation to induce apoptosis in phagocytic macrophages. 1H/19F images were acquired on day $17(\mathrm{n}=3)$. Regions of $19 \mathrm{~F}$ signal were assessed for changes in spatial distribution, then delineated manually and quantified relative to reference tubes of known 19F content. Results: Study 1: Average tumour size increased from day $11(162.13 \mathrm{~mm} 3)$ to day $17(468.50 \mathrm{~mm} 3)$ and day $20(788.62 \mathrm{~mm} 3)(\mathrm{p}$ Study 2: The average tumour volume in $1 \mathrm{H}$ images was $468.5 \mathrm{~mm} 3$ in control mice and 441.2 $\mathrm{mm} 3$ in mice administered clodronate (Fig $\mathrm{E}, \mathrm{p}=0.7133$ ). 19F signal was found in the liver, spleen, and tumours of control mice and mice that receive clodronate due to the accumulation of perfluorocarbon (Fig D).19F signal measured in control tumours (1.01 x1019 19F spins) was not significant from mice administered clodronate (1.01 x1019 19F spins) (Fig F, p=0.9941). 19F signal in the liver and spleen was $13.5 \times 1019$ 19F spins in control mice and $4.62 \times 101919 \mathrm{~F}$ spins in mice administered clodronate $(\mathrm{p}=0.2511)$. Discussion: $19 \mathrm{~F}$ MRI cell tracking can provide insights about the role of TAMs in the tumour microenvironment. We have shown that 19Flabeled TAMs are found predominately at the periphery of tumours and that TAM burden increases as tumours grow (between day 17 and 20). Future study will involve more timepoints to further understand the timing of TAM infiltration. 19F MRI can be used to provide a measure of TAM burden when testing novel therapies. In this study, we did not observe an effect of systemic administration of clodronate on TAMs, nor on macrophages in the liver and spleen.
\end{abstract}


More rigorous treatments, including combination treatments, may be required to deplete TAMs. With 19F MRI cell tracking, we can identify when TAMs persist following treatments to assess how TAMs contribute to tumour recurrence and resistance.

References: 1. Carron EC, Homra S, Rosenberg J, Coffelt SB, Kittrell F, Zhang Y, et al. Macrophages promote the progression of premalignant mammary lesions to invasive cancer. Oncotarget. 2017. 2. Yuan ZY, Luo RZ, Peng RJ, Wang S Sen, Xue C. High infiltration of tumor-associated macrophages in triple-negative breast cancer is associated with a higher risk of distant metastasis. Onco Targets Ther. 2014. 3. Gwak JM, Jang MH, Kim D Il, Seo AN, Park SY. Prognostic value of tumor-associated macrophages according to histologic locations and hormone receptor status in breast cancer. PLoS One. 2015. 4. Obeid E, Nanda R, Fu YX, Olopade OI. The role of tumor-associated macrophages in breast cancer progression (review). Int J Oncol. 2013;43(1):5-12. 5. Condeelis J, Pollard JW. Macrophages: Obligate partners for tumor cell migration, invasion, and metastasis. Cell. 2006;124(2):263-6. 6. De Palma M, Lewis CE. Macrophage regulation of tumor responses to anticancer therapies. Cancer Cell [Internet]. 2013;23(3):277-86. 7. Qian B, Deng Y, Im JH, Muschel RJ, Zou Y, Li J, et al. A distinct macrophage population mediates metastatic breast cancer cell extravasation, establishment and growth. PLoS One. 2009;4(8). 8. Bingle L, Brown NJ, Lewis CE. The role of tumour-associated macrophages in tumour progression: Implications for new anticancer therapies. Journal of Pathology. 2002. 9. Guerriero JL. Macrophages: The Road Less Traveled, Changing Anticancer Therapy. Trends Mol Med. 2018;24(5):472-89. 10. Makela A V., Gaudet JM, Foster PJ. Quantifying tumor associated macrophages in breast cancer: A comparison of iron and fluorinebased MRI cell tracking. Sci Rep. 2017;7(February):1-9.

\section{Image/Figure:}

https://www.xcdsystem.com/wmis/abstract/File6959/GA195 ImageFigure 0603085636.png

Image/Figure Caption: Figure: A) $1 \mathrm{H}$ and corresponding 19F images of mammary tumours on day 11,17 , and 20 of development. Tumours are outlined in $1 \mathrm{H}$ images. 19F signal from TAMs is found predominately at the periphery of tumours. Over time, B) tumour volume and C) $19 \mathrm{~F}$ signal (representing TAM burden) increase. D) 3-dimensional rendering of $19 \mathrm{~F} / 1 \mathrm{H}$ overlay showing perfluorocarbon uptake in the liver, spleen, and tumour. Clodronate treatment did not lead to differences in E) tumour volume or F) $19 \mathrm{~F}$ signal in tumours.

Full Name of Abstract's 1st Author : Olivia C. Sehl

Complete Status: Complete

First Name: Olivia

Last Name: Sehl

Email: osehl@uwo.ca

Organization: Robarts Research Institute 
Country: Canada 


\title{
ID: GA196 \\ Targeted photoimmunotheranostics of myeloid-derived suppressor cells in tumor-bearing mice
}

\author{
James Barnett, The Johns Hopkins University School of Medicine, jbarne55@jhmi.edu
}

\section{Category: Oncology}

\begin{abstract}
Body : Introduction: The heterogenous population of myeloid-derived suppressor cells (MDSCs) plays an important role in promoting an immunosuppressive tumor microenvironment (TME) by disrupting host immune recognition1. MDSCs inhibit immune surveillance through mechanisms that involve reactive oxygen species (ROS), nitric oxide synthase (NOS), arginase-1 and prostaglandin E2 (PGE2)-mediated suppression of immune cells. These cells are identified phenotypically as polymorphonuclear cell-like PMN-MDSCs and monocyte-like M-MDSCs that differentiate from immature myeloid cells during cancer, trauma and autoimmune disorders. MMDSCs are CD11b+ and Ly6C+, PMN-MDSCs are CD11b+ and Ly6G+. Antibody mediated MDSC depletion using anti-mouse granulocyte receptor-1 antigen (Gr1) Clone RB6-8C5 (Ly6GHi/Ly6CLo) has been used to effectively eliminate MDSCs in some tumor models, although further investigation must be carried out to validate its efficacy on both MDSC subtypes2. To achieve targeted near-infrared photoimmunotherapy (NIR-PIT)3, we synthesized an IR700-labeled Gr1 antibody-photosensitizer conjugate. This proof of principle study evaluates the specificity of the Gr1-IR700 conjugate to detect and eliminate splenic MDSCs in culture. Methods: Preparation of Gr1-IR700 and IgG-IR700 was carried out through the attachment of an NHS-activated NIR phthalocyanine dye, IR700, to the free amine residues on the RB6-8C5 monoclonal antibody and rat IgG2b, $\kappa$ isotype control. Orthotopic 4T1 tumor models were established through the inoculation of $2 \times 1064 \mathrm{~T} 1$ cells in the mammary fat pad of 4-6-week-old female, BALB/c mice. Mice bearing tumors with volumes ranging from $1.5-2.0$ $\mathrm{cm} 3$ were euthanized, and the spleens were harvested for MDSC isolation. After spleen dissociation and red blood cell lysis, immunomagnetic isolation of murine MDSCs was performed following the EasySep ${ }^{\mathrm{TM}}$ Mouse MDSC (CD11b+Gr1+) Isolation Kit protocol (77\% purity shown in Figure 1). To validate in vitro targeting of MDSCs, viability assays were conducted. MSDCs were incubated at a density of $1 \times 106$ cells $/ \mathrm{mL}$ with $1-10 \mathrm{ug} / \mathrm{mL}$ of Gr1IR700. Reagent specificity was also determined using PBS and IgG-IR700 as controls. The cells were exposed to $16 \mathrm{~J} / \mathrm{cm} 2$ of NIR irradiation. CCK-8 assays were used to measure cell viability. In ongoing in vivo PIT studies to demonstrate MDSC targeting, tumor-bearing mice will receive i.v. injection of $100 \mu \mathrm{g}$ of Gr1-IR700 followed by splenic and tumor irradiation at $200 \mathrm{~J} / \mathrm{cm} 2$. MDSC cell death will be characterized by fluorescence-activated cell sorting (FACS) analysis of spleens and tumors in PBS, IgG-IR700 and Gr1-IR700-treated groups. Results and Discussion: We confirmed that light-activated Gr1-IR700 caused Gr1-specific cell death in splenic MDSCs. Representative cell viability data for all groups $(\mathrm{n}=2)$ are shown in Figure 2. At the NIR light dose of $16 \mathrm{~J} / \mathrm{cm} 2$, Gr1-mediated cell killing was not dependent on the conjugate concentration, and phototoxicity was partially inhibited by 5-fold excess RB6-8C5. These findings confirm the ability of Gr1-IR700 PIT to eliminate MDSCs, illustrating its potential application for targeting MDSCs in the TME.
\end{abstract}


References: (1) Gabrilovich DI. Cancer Immunol Res. 2017 (2) Xing YF. et al. Ann Rheum Dis. 2016 (3) Jin, J. et al. Sci.Rep. 2016

\section{Image/Figure:}

https://www.xcdsystem.com/wmis/abstract/File6959/GA196_ImageFigure_0421023349.jpg

Full Name of Abstract's 1st Author : James Dion Barnett

Complete Status: Complete

First Name: James

Last Name: Barnett

Email: jbarne55@jhmi.edu

Organization: The Johns Hopkins University School of Medicine

Country: United States 


\title{
ID: GA198 \\ Development of a novel class of activatable near-infrared II fluorophores for imaging phospholipase $\mathrm{A} 2$ activity in lung cancer
}

\author{
Michael Hart, University of Pennsylvania, mihart@pennmedicine.upenn.edu
}

\section{Category: New Chemistry, Biology \& Bioengineering}

\begin{abstract}
Body : Overexpression of cytosolic phospholipase A2 (cPLA2) is implicated in a variety of different cancer types such as triple-negative breast (TNBC) and non-small cell lung (NSCLC) cancers.1-3 NSCLC is the leading cause of cancer related death in the United States and is primarily treated by surgical resection of the primary tumor.4 Currently, surgeons rely on tissue palpation and visual inspection to guide surgical resection and identify malignant tissues intraoperatively. Previously, a novel cPLA2-activable fluorophore, DDAO-arachidonate, was developed for tumor margin detection in TNBC models. DDAO-arachidonate fluorescence is caged by esterification of DDAO to an unsaturated fatty acid. Enzymatic cleavage by cPLA2 releases DDAO resulting in a measurable fluorescence at $660 \mathrm{~nm} .5$ The emission of DDAO in the visible spectrum of light is not ideal for in vivo imaging due to tissue absorption and scattering at these wavelengths. The purpose of the present study was twofold. First, we validated DDAO-arachidonate activation in human lung cancers in preparation for translation to imaging NSCLC. Second, new caged long wavelength fluorophores were synthesized and tested for NIR-II fluorescence to improve upon the in vivo optical properties of our probe. Liposomal formulations of DDAO-arachidonate were tested for size and zeta potential to demonstrate feasibility for in vivo applications. A solution of egg-phosphatidylcholine and DDAOarachidonate (95:5 mole \%) in chloroform was dried under nitrogen before reconstituting in $0.1 \%$ PBS. Membrane extrusion was performed using a $100 \mathrm{~nm}$ filter. The size and zeta potential for the formulation were determined to be $105.7 \mathrm{~nm}$ and $-15.13 \pm 2.4 \mathrm{mV}$ using a Malvern ZetaSizer Nano. To test feasibility of translation to NSCLC imaging, $20 \mathrm{nmol}$ of liposomal DDAOarachidonate were painted on normal and cancerous human lung tissue in triplicate immediately following tissue resection. The tissue was incubated at $37^{\circ} \mathrm{C}$ for 3 hours to before imaging using the PerkinElmer IVIS Spectrum with $640 \mathrm{~nm}$ excitation and $680 \mathrm{~nm}$ emission. Fluorescence was seen only in the tumor tissue with a tumor to background ratio (TBR) of approximately 5.2. Second, a new class of caged cyanine dyes has been synthesized with emission in the NIR-II region. An absorbance spectrum for one of these compounds, JAM317, was obtained using a PerkinElmer Lambda35 with peak absorbance at $950 \mathrm{~nm}$. Optical studies using the PhotonEtc. IR VIVO imaging system have demonstrated measurable fluorescence from $1050 \mathrm{~nm}$ to 1538 $\mathrm{nm}$, with peak emission observed using the $1050 \mathrm{~nm}$ filter. Continuous excitation by an $808 \mathrm{~nm}$ laser over 5 min demonstrated that JAM317 is resistant to photobleaching. The synthesized fluorophores are modifiable and can be targeted to specific disease states or physical properties such as hydrophilicity may be altered through the introduction of different moieties. This study has demonstrated that DDAO-arachidonate has a promising TBR for imaging NSCLC in human tissue and new NIR-II fluorophores have been developed that may further improve the imaging of NSCLC.
\end{abstract}


References: 1) Caiazza F, McCarthy NS, Young L, Hill AD, Harvey BJ, Thomas W. Cytosolic phospholipase A2-alpha expression in breast cancer is associated with EGFR expression and correlates with an adverse prognosis in luminal tumours. Br J Cancer 2011;104(2):338-44. 2) Murakami M, Taketomi Y, Miki Y, Sato H, Hirabayashi T, Yamamoto K. Recent progress in phospholipase A(2) research: from cells to animals to humans. Prog Lipid Res 2011;50(2):15292. 3) Laye JP, Gill JH. Phospholipase A2 expression in tumours: a target for therapeutic intervention? Drug Discov Today 2003;8(15):710-6. 4) Cancer Facts \& Figures. Atlanta, Georgia: American Cancer Society; 2020. 5) Chiorazzo MG, Tunset HM, Popov AV, Johansen B, Moestue SA, Delikatny EJ. Detection and differentiation of breast cancer sub-types using a cPLA2 $\alpha$ activatable fluorophore. Scientific Reports 2019;9. 6) Zhu S, Yung BC, Chandra S, Niu G, Antaris AL, Chen X. Near-infrared-II (NIR-II) bioimaging via off-peak NIR-I fluorescence emission. Theranostics 2018;8(15):4141-4151.

\section{Image/Figure:}

https://www.xcdsystem.com/wmis/abstract/File6959/GA198_ImageFigure_0421035129.PNG

Full Name of Abstract's 1st Author : Michael C. Hart

Complete Status: Complete

First Name: Michael

Last Name: Hart

Email:mihart@pennmedicine.upenn.edu

Organization: University of Pennsylvania

Country: United States 


\title{
ID: GA199 \\ Head and neck cancer margin assessment with ratiometric angular domain fluorescence imaging
}

\author{
Veronica Torres, Dartmouth College, veronica.c.torres@dartmouth.edu
}

\section{Category: Oncology}

\begin{abstract}
Body : Surgical margin assessment remains a critical step in the treatment of head and neck squamous cell carcinoma (HNSCC), where the extent of margin involvement determines postoperative treatment, and in turn influences patient prognosis. Cancer within $1 \mathrm{~mm}$ of the surface is defined as a "close" margin; within 1-5 mm, a "wide" margin. In either case, reoperation is carried out if possible. If not possible, patients with "close" margins require postoperative chemo-radiation, which substantially increases patient morbidity; whereas patients with only "wide" margins are given radiotherapy without the concomitant use of chemotherapy.1,2 Current margin assessment protocols require lengthy tissue processing in surgical pathology departments, where results often take more than $24 \mathrm{~h}$ to process, with only a small percentage of the margin being interrogated. Fluorescence molecular imaging has proven to be a useful tool for intra-operative assessment of tumor margins. For example, in a study of HNC patients, the injection of cetuximab-IRDye800CW enabled real-time evaluation of resected margin status with a sensitivity and specificity of $100 \%$ and $91 \%$, respectively. 3 Although fluorescence intensity can indicate positive/negative status of margins, the signal alone does not provide any information of cancer depth; i.e. is the positive margin "close" or "wide". To address this, a ratiometric approach using angular domain fluorescence imaging is proposed. It is hypothesized that differences in depth sensitivity profile shapes of photons detected with an open aperture (conventional imaging) versus a narrow aperture (angular domain imaging) can be used to distinguish the depth from which the fluorescence signal comes from. Monte Carlo simulations were conducted using the open-source MCmatlab4 where average soft biological tissue optical properties were assumed ( $\mu \mathrm{a}=0.4 \mathrm{~cm}-1, \mu \mathrm{s}=10 \mathrm{~cm}-1, \mathrm{~g}=0.9$ ). Fluorescence detection sensitivity profiles were modeled as an isotropically emitting point source focused at the tissue surface for open aperture detection, and as a pencil beam focused at half the depth for narrow aperture detection. Fluorescence uptake of tumors at varying depths was simulated for a $0.7 \mathrm{~cm}$ thick tissue, and open and narrow aperture wide-field reflectance images of the volume were generated using the sensitivity profiles [Fig.1(a-c)]. Results demonstrated the potential for ratiometric angular domain imaging to offer depth resolution and improved identification of both close and wide margins. While the inclusion closest to the surface was easily identified, neither open nor narrow aperture imaging alone was able to detect wide margins. However, after taking the ratio of the two images [Fig. 1(d)], the deeper tumor was delineated and relative signal intensity differences as a function of depth were maintained. With such information, a depth map [Fig. 1(e)] can be generated after thresholding to provide surgeons with rapid analysis of margin involvement to better guide postoperative treatment strategies. Although these simulations are simplified, the normalization of the ratiometric approach can account for common issues of fluorescence guided surgery such as heterogeneity
\end{abstract}


of tissue optical properties, fluorescence distribution, and surgical artifacts (e.g. blood on tissue surfaces or imaging probes).

References: 1. R. Haque, et al., BMC Ear, Nose and Throat Disorders 2006, 6:2. 2. M. D.

Williams, Curr Oncol Rep, 2016, 18:54. 3. F. J. Voskuil, et al., Theranostics. 2020; 10(9): 39944005. 4. D. Marti et al., J Biomed Opt. 2018 Dec;23(12):1-6.

\section{Image/Figure:}

https://www.xcdsystem.com/wmis/abstract/File6959/GA199_ImageFigure_0611043021.png

Image/Figure Caption: Figure 1. Simulated representations of (a) fluorescence inclusions as a function of depth; wide-field reflectance fluorescence images collected with a (b) open aperture and (c) narrow aperture. (d) Ratio image of (b) and (c). (e) Color-coded depth map.

Full Name of Abstract's 1st Author : Veronica Torres

Complete Status: Complete

First Name: Veronica

Last Name: Torres

Email: veronica.c.torres@dartmouth.edu

Organization: Dartmouth College

Country: United States 


\title{
ID: GA201 \\ Early Detection and Staging of Lung Fibrosis enabled pMRI by Novel Protein Contrast Agents
}

\author{
Jenny Yang, Georgia State University, jenny@gsu.edu
}

Category: Immunology: Inflammation \& Infection

\begin{abstract}
Body : Chronic lung diseases, such as chronic obstructive pulmonary disease (COPD) and idiopathic pulmonary fibrosis (IPF), are major leading causes of death worldwide. Recently the usage of E-cigarettes has grown rapidly in the US contributing to the sharp increase of chronic lung disease. There is a pressing unmet medical need to develop noninvasive imaging methodologies and contrast agents to detect early stages of lung fibrosis, and stage fibrosis severity for progression of IPF and COPD to facilitate treatment. Here, we first demonstrate that overexpressed collagen 1 is an ideal molecular biomarker for detection, progression and monitoring of lung fibrosis in both COPD and IPF resulted lung damage. We then report the development of a new class of collagen targeted human protein-based Gd 3+ -based contrast agent, hProCA32.collagen. hProCA32.collagen exhibits strong affinity to collagen 1, high relativities per particle ( $\mathrm{r} 1$ and $\mathrm{r} 2$ ) at both 1.4 and 7.0 T, and negligible immunogenicity. We have achieved the first detection of early stage lung fibrosis in both COPD and IPF animal models using hProCA32.collagen (pMRI) correlated with histological patterns and lung function analysis. In addition to early detection, hProCA32.collagen stages fibrosis progression in lung shown by receiver operating characteristic (ROC) curve. hProCA32.collagen mitigates metal toxicity due to its lower dosage, strong resistance to transmetalation and unprecedented metal selectivity for Gd 3+ over physiological metal ions. We anticipate hProCA32.collagen having strong translational potential in facilitating effective treatment to halt further chronic lung disease progression as well as reveal key factors that contribute to lung fibrosis including emerging public health issue associated with e-smoking.
\end{abstract}

References: 1. Salarian M, Turaga RC, Xue S, Nezafati M, Hekmatyar K, Qiao J, Zhang Y, Tan S, Ibhagui OY, Hai Y, Li J, Mukkavilli R, Sharma M, Mittal P, Min X, Keilholz S, Yu L, Qin G, Farris AB, Liu ZR, Yang JJ. Early detection and staging of chronic liver diseases with a protein MRI contrast agent. Nat Commun 2019;10(1):4777 PMC6820552. 2. Salarian M, Yang H, Turaga RC, Tan S, Qiao J, Xue S, Gui Z, Peng G, Han H, Mittal P, Grossniklaus HE, Yang JJ. Precision detection of liver metastasis by collagen-targeted protein MRI contrast agent. Biomaterials 2019;224:119478. 3. Tan S, Yang H, Xue S, Qiao J, Salarian M, Hekmatyar K, Meng Y, Mukkavilli R, Pu F, Odubade OY, Harris W, Hai Y, Yushak ML, Morales-Tirado VM, Mittal P, Sun PZ, Lawson D, Grossniklaus HE, Yang JJ. Chemokine receptor 4 targeted protein MRI contrast agent for early detection of liver metastases. Sci Adv 2020;6(6):eaav7504 PMC7007242. 4. Xue S, Yang H, Qiao J, Pu F, Jiang J, Hubbard K, Hekmatyar K, Langley J, Salarian M, Long RC, Bryant RG, Hu XP, Grossniklaus HE, Liu ZR, Yang JJ. Protein MRI contrast agent with unprecedented metal selectivity and sensitivity for liver cancer imaging. Proceedings of the National Academy of Sciences of the United States of America 2015. 


\section{Image/Figure:}

https://www.xcdsystem.com/wmis/abstract/File6959/GA201 ImageFigure 0421033509.jpg

\section{Complete Status: Complete}

First Name: Jenny

Last Name: Yang

Email: jenny@gsu.edu

Organization: Georgia State University

Country: United States 


\section{ID: GA202}

Cisplatin biofunctionalized magnetic Fe3O4@SiO2@Au nanoparticles for targeted chemo-phototherapy of cancer cells

Natalia Lopuszyńska, Institute of Nuclear Physics Polish Academy of Sciences, natalia.lopuszynska@ifj.edu.pl

Category: Oncology

Full Name of Abstract's 1st Author : Natalia Łopuszyńska

Complete Status: Complete

First Name: Natalia

Last Name: Łopuszyńska

Email: natalia.lopuszynska@ifj.edu.pl

Organization: Institute of Nuclear Physics Polish Academy of Sciences

Country: Poland 


\title{
ID: GA203 \\ Comparison of image reconstruction algorithms for RF-acoustic tomography molecular imaging
}

\author{
Brian Lee, Stanford University, dlwns87@stanford.edu
}

\section{Category: Instrumentation}

\begin{abstract}
Body : Introduction Molecular imaging (MI) techniques offer unique functional and molecular information non-invasively at the cellular level [1]. While a variety of imaging modalities have been developed, they come with trade-offs between imaging depth, sensitivity, and/or spatial resolution. Hence, there is a need to develop a highly sensitive MI device that provides images with high spatial resolution and deep imaging depths without relying on ionizing radiation. Radio-frequency (RF)-Acoustic Tomography Molecular Imaging (RATMI) is an imaging technique that generates ultrasound images by irradiating tissue with non-ionizing RF fields for deeper penetration [2-4]. Despite the technical feasibility, the limited choices of biocompatible RF absorbing agents have hindered the use of RATMI [5]. The development and characterization of cancer-targeted RF nanodroplets have been recently studied which highlighted the ability to image the accumulation of the agent in prostate cancer with high specificity; however, the image reconstruction methods showed limitations with poor image quality. In this study, various image reconstruction methods were performed in order to enhance image quality and sensitivity. Materials and Methods The image reconstruction was performed on raw data sets acquired by the RATMI prototype system (ENDRA Life Sciences Inc.). The prototype is comprised of a pair of customized horn RF antennas (433 MHz) and an ultrasound linear-array transducer (Acuson L382) for generating and receiving the signals. The stage is attached to a rotational motor for generating tomographic images (Figure 1). In this study, the sample stage was rotated 36 times with a 10-degree interval to cover the full tomographic angle. At each angle, 4,095 RF pulses were excited and the acquired acoustic signals were averaged for a better signal-to-noise ratio. Two saturated saline $(35 \%$ of $\mathrm{NaCl})$ phantoms were used for image analyses; (1) a needle (ID: $0.7 \mathrm{~mm}$ ) placed at the isocenter of the field-of-view for assessing the spatial resolution and (2) two tubes (ID: $3 \mathrm{~mm}$ ) placed $7.5 \mathrm{~mm}$ away from the center for evaluating the contrast. Four different image reconstructions were performed; (a) Default filtered back projection (FBP), (b) Delay-and-Sum (DAS), (c) Universal back-projection (UBP [6]), and (d) Model-based algorithm (MBA [7]). Algorithms (a), (b), and (c) are the most commonly used algorithms, while (d) accounts for the effects of acoustic absorption, detector element, impulse response, and directivity pattern. Quantitative image assessments were performed via a contrast-to-noise ratio (CNR) analysis and line profile assessment. The CNR was evaluated from the two-tube data set using Equation 1 (Persuasive data). Three regions-ofinterest (ROIs) with a diameter of $3 \mathrm{~mm}$ were selected; two ROIs for signal tubes (Figure 2) and one for the background. In addition, a line profile was evaluated from the needle phantom image by drawing horizontal/vertical lines through the needle and calculating the full-width at half maximum (FWHM) of a gaussian fit. Results Two quantitative metrics were used to evaluate the image reconstruction performance; CNR and line profile (Figure 2). The CNR for the MBA was $44.4 \pm 27.1 \%$ higher compared to other methods (Figure 3 ). For the line profile analysis, the MBA
\end{abstract}


showed significantly higher accuracy compared to other methods in the needle phantom image (Figure 4). The FWHM of MBA needle image, $0.6 \mathrm{~mm}$, showed significantly lower error, while other algorithms suffered from some blurring artifacts with FWHMs of $1.3 \mathrm{~mm}$. These parameters may serve as a quantitative and intuitive parameter to assess the performance and quality of clinical investigations. Conclusion The image reconstruction methods were evaluated for the RATMI system. The preliminary results produced by MBA were promising in CNR and line profile assessments; however, further evaluations through a resolution phantom and small animal studies are needed to fully assess and compare the imaging performance.

References: [1] James ML, Gambhir SS. A molecular imaging primer: modalities, imaging agents, and applications. Physiol Rev. 2012 Apr;92(2):897-965. [2] Bowen T. RadiationInduced Thermoacoustic Soft Tissue Imaging. In: 1981 Ultrasonics Symposium. 1981. p. 81722. [3] Olsen RG, Lin JC. Acoustical imaging of a model of a human hand using pulsed microwave irradiation. Bioelectromagnetics. 1983;4(4):397-400. [4] Kruger RA, Liu P, Fang YR, Appledorn CR. Photoacoustic ultrasound (PAUS)--reconstruction tomography. Med Phys. 1995 Oct;22(10):1605-9. [5] Ogunlade O, Beard P. Exogenous contrast agents for thermoacoustic imaging: an investigation into the underlying sources of contrast. Med Phys. 2015 Jan;42(1):170-80. [6] Xu M, Wang LV. Universal back-projection algorithm for photoacoustic computed tomography. Phys Rev E Stat Nonlin Soft Matter Phys. 2005 Jan;71(1 Pt 2):016706. [7] Rosenthal A, Razansky D, Ntziachristos V. Fast semi-analytical model-based acoustic inversion for quantitative optoacoustic tomography. IEEE Trans Med Imaging. 2010 Jun;29(6):1275-85.

Full Name of Abstract's 1st Author : Brian Jun Lee

Complete Status: Complete

First Name: Brian

Last Name: Lee

Email: dlwns87@stanford.edu

Organization: Stanford University

Country: United States 


\title{
ID: GA204 \\ Density-adapted 3D projection-reconstruction MRI for sodium imaging of rat glioblastoma at 3 Tesla
}

\author{
Xin Yue Wang, University of Western Ontario, xwan9@uwo.ca
}

\section{Category: Oncology}

\begin{abstract}
Body : Introduction: Tissue sodium concentration (TSC) is a sensitive indicator of disease. Changes in tissue sodium distribution are implicated in many diseases 1 . Sodium concentration increases in cancer cells as a result of aerobic glycolysis2. Longitudinal changes in TSC could potentially be a useful tool for understanding treatment response. Sodium has previously been imaged preclinically with magnetic resonance imaging (MRI) at high field strengths3. Since sodium has limited in vivo concentration, low gyromagnetic ratio, and quadrupolar relaxation $(\mathrm{T} 1=10-40 \mathrm{~ms}, \mathrm{~T} 2$, fast $=1-5 \mathrm{~ms}$, T2,slow $=15-30 \mathrm{~ms})$, adequate signal-tonoise ratio (SNR) is difficult to achieve1. The density-adapted three-dimensional projectionreconstruction (DA3DPR) sequence has been demonstrated to achieve improved sodium SNR compared to cartesian imaging sequences, and without exotic hardware requirements4. Improved sodium imaging adapted for preclinical studies at 3T lends significance to eventual translation of sodium imaging for clinical use. Hypothesis: Implementation of a DA3DPR sequence at 3T for preclinical sodium MRI will be able to detect changes in the TSC of rat glioma as a result of altered metabolism. Methods: DA3DPR imaging was optimized on a GE Discovery MR750 3.0T MRI. Performance was evaluated at $1-\mathrm{mm}$ and $3-\mathrm{mm}$ isotropic resolution $(\mathrm{TE}=0.5 \mathrm{~ms}, \mathrm{TR}=100$ $\mathrm{ms}$, Taq $=8 \mathrm{~ms}$, flip angle $(\mathrm{FA})=90^{\circ}$, gradient slew rate $=20 \mathrm{mT} / \mathrm{m}$, isotropic $\left.\mathrm{FOV}=80 \mathrm{~mm}\right)$. Sodium imaging was performed using a purpose-built, curved transmit/receive butterfly radiofrequency (RF) surface coil. A phantom of 50-mmol/L sodium (physiological sodium concentration) and $1 \%$ agarose was imaged to determine the coil sensitivity profile. Reference vials containing $2 \mathrm{~mL}$ of $30-, 50-, 70-$, and 100-mM concentrations of sodium were placed within the imaging area of the coil. Reproducible RF excitation of the imaging area was maintained using a reference vial containing 2-M sodium doped with $200 \mathrm{mM}$ of the shift reagent TmDOTP. DA3DPR imaging at 3-mm resolution was further evaluated in an animal model.

Stereotactic surgery was performed to implant 1'106 C6 glioma cells into the right hemisphere of a Wistar rat brain. Seven days following implantation, rats were imaged with DA3DPR sodium MRI $(\mathrm{NEX}=10$, total acquisition time $=12 \mathrm{mins})$ and 3D T2-weighted proton MRI (CUBE) every 3 days until experimental endpoint. Proton MRI was performed using a custom birdcage for small animal imaging (ID $=85.7 \mathrm{~mm}$ ). Regional intensity variations in the sodium imaging data due to RF inhomogeneity from the surface coil were corrected by normalization to the measured coil sensitivity profile. In vivo sodium images were manually co-registered to the 3D coil sensitivity data with 3D-slicer using fiducial markers visible in both imaging data sets. This yielded normalized in vivo maps of absolute TSC (in units of $\mathrm{mM}$ ). Manual segmentation of the tumour and healthy brain were performed using ITK-SNAP to determine differences in TSC. Conclusions: A DA3DPR sequence for sodium imaging was successfully implemented with a 3$\mathrm{mm}$ isotropic resolution at 3T for preclinical imaging research. Our implementation can visualize regional changes in sodium with enough SNR $(>30)$ for assessment of brain tumours. TSC
\end{abstract}


concentrations within the area of interest showed a standard deviation of $\pm 3.02-\mathrm{mM}$, demonstrating successful correction of surface coil related intensity variation. This correction was applied in vivo with longitudinal monitoring of TSC in rats implanted with C6 tumours showing increased sodium signal in areas of the tumour compared to the healthy brain. Future work will focus on increasing the study size as well as monitoring longitudinal changes of TSC in response to therapy.

References: References: [1] Madelin, G., et al., Prog Nucl Magn Reson Spectrosc. 2014, 79:1447 [2] Vander Heiden, M.G. et al., Science. 2009, 324(5930):1029-1033 [3] Schepkin, V.D., et al., Magn Reson Med. 2005. 53(1):85-92 [4] Nagel, A.M., et al., Magn Reson Med. 2009, 62(6): $1565-1573$

\section{Image/Figure:}

https://www.xcdsystem.com/wmis/abstract/File6959/GA204_ImageFigure 0613043153.jpg

Image/Figure Caption: Figure 1. A) Longitudinal sodium and proton overlay images of an axial view of a rat implanted with C6 glioma cells. B) Average tissue sodium concentration in tumour region and healthy brain region over time.

Full Name of Abstract's 1st Author : Xin Yue Wang

Complete Status: Complete

First Name: Xin Yue

Last Name: Wang

Email: xwan9@uwo.ca

Organization: University of Western Ontario

Country: Canada 


\title{
ID: GA206 \\ In vivo measurement of metabotropic glutamate receptor subtype 5 with positron emission tomography in humans with fragile $X$ syndrome
}

\author{
James Brasic, Johns Hopkins University, zauberfuchs@gmail.com
}

\section{Category: Neuroscience}

\begin{abstract}
Body : Introduction Fragile X syndrome (FXS), the most common genetic cause of intellectual disability (ID) and autism spectrum disorder (ASD), results from deficits of Fragile X Mental Retardation Protein (FMRP). Although animal models of FXS have demonstrated dysfunction of the metabotropic glutamate receptor subtype 5 (mGluR5) that is ameliorated by pharmacological interventions, clinical trials of glutamatergic agents have not demonstrated beneficial effects in adult or adolescent humans with FXS (1). Although studies with positron emission tomography (PET) have shown these drugs do target mGluR5 (1), mGluR5 expression has not been measured in FXS (2). The goal of this study was to demonstrate the feasibility of measurements of mGluR5 expression in the living brain of humans with FXS. Methods Positron emission tomography (PET) with a high resolution research tomograph (HRRT) was conducted at for 90 minutes after the intravenous bolus injection of 185 megabequerels (MBq) (5 millicuries [mCi]) 3-[18F]fluoro-5-(2-pyridinylethynyl)benzonitrile ([18F]FPEB), a potent, selective mGluR5 inhibitor (3) to two men with the full mutation for fragile $\mathrm{X}$ syndrome
\end{abstract} (FXS) aged 24-27 (25.5+2.12) years, six men with ASD aged 18-23 (20+2.10) years, and three individuals (one man and two women) with typical development (TD) aged 19 to $24(21+2.9)$ years (4). The nondisplaceable binding potential (BPND) (5) was calculated for relevant volumes of interest (VOIs) on PET images coregistered with magnetic resonance imagery (MRI)

(3). First, VOIs of cortical regions were obtained automatically by Freesurfer $6.0(6,7)$ and VOIs of subcortical regions were obtained automatically utilizing the subcortical segmentation tools of the software library of the Oxford Centre for fMRI of the Brain (8-10). We used cerebellar white matter $(\mathrm{CbWM})$ for the reference region since this structure demonstrated negligible densities of mGluR5s (11). Then, VOIs were transferred from MRI to PET space according to MRI-to-PET coregistration parameters obtained with the coregistration module (12) of Statistical Parametric Mapping (SPM) (13), and applied to PET frames to obtain regional time activity (radioactivity) curves (TACs). Regional BPNDs were obtained by reference tissue graphical analysis (RTGA) (14) and the multilinear reference tissue method with two parameters (MRTM2) (15). Results In contrast to both the ASD and TD groups, BPNDs for the men with FXS were reduced in all VOIs The nondisplaceable binding potentials (BPNDs) (5) of [18F]FPEB by both reference tissue graphical analysis (RTGA) (14) and the multilinear reference tissue method with two parameters (MRTM2) (15) in each VOI of two men with FXS and ASD are greater than a standard deviation below the means of six men with autism spectrum disorder and three healthy people with typical development $(3,4)$ (Figure). Reductions in the men with FXS were particularly marked in the limbic system and the striatum (Figure). Conclusion This study supports the viability of PET to measure mGluR5 density in FXS (16). The results are confirmed through performance of a similar protocol on six participants with FXS (17). The marked reductions of mGluR5 density in the cortices and the limbic system support the finding that two 
distinct but interrelated social behavior abnormalities, one linked to impaired cognitive processes (delayed socialization) and the second one to disturbance in limbic circuits (avoidance), play a role in the development of ASD in boys with FXS (18). The protocol suggests that PET may be used to quantify a mGluR5 binding to confrim target engagement for clinical trials of novel glutamatergic agents for FXS.

References: 1 Budimirovic, DB, et al. J Neurodevel Disord, 2017; 9: 14,doi: 10.1186/s1 1689017-9193-x.[PubMed: 28616097].[PubMedCentral: PMC5467057] [sêp] 2. Brašić JR, et al. Arch Neurosci. 2019; 6 (Brain mapping): e91831. doi: 10.5812/ans.91831.3. Wong, DF, et al. J Nucl Med. 2013; 54, 388-396, doi: 10.2967/jnumed.112.107995, PubMed PMID: 23404089.SE: 4. Fatemi, SH, et al. Cerebellum \& Ataxias, 2018; 5: 3 http://rdcu.be/GQb3 PubMed PMID: 29449954; PubMed Central PMCID: PMC5810020. 5. Innis RB, et al. J Cereb Blood Flow Metab. 2007; 27: 1533-1539. 6. Fischl B, et al. Cereb Cortex. 2004; 14: 11-22. 7. Hoopes A. https://surfer.nmr.mgh.harvard.edu/fswiki/DownloadAndInstall (2020). 8. Jenkinson M, et al. Neuroimage. 2012; 62: 782-790. 9. Patenaude B, et al. Neuroimage. 2011; 56: 907-922. 10. Woolrich MW, et al. Neuroimage. 2009; 45 (1 supplement 1): S173-S186. 11. Sullivan JM, et al. J Cereb Blood Flow Metab. 2013; 33: 532-541. 12. Ashburner J, Friston KJ. In: Frackowiak RSJ, Ashburner J, Penny WD, Zeki S, Friston KJ, Frith C, Dolan R, Price CJ, editors. Human Brain Function, 2nd edition. Waltham, Massachusetts: Academic; 2004. p. 635673. 13. Wellcome Centre for Human Neuroimaging. Statistical Parametric Mapping (SPM). 2020. http://www.fil.ion.ucl.ac.uk/spm/ 14. Logan J, et al. J Cereb Blood Flow Metab. 1996; 16: 834-840. 15 . Ichise M, et al. J Cereb Blood Flow Metab. 2002; 22: 1271-1281. 16. Brasic J, et al, J Nucl Med. 2020; 61 (supplement 1): 1552. [abstract] http://jnm.snmjournals.org/content/61/supplement_1/1552.abstract?sid=7a58a0b9-ec27-42f3b06e-7ded1 f2dc2c8 17. Russell D. A PET brain imaging study of mGluR5 in subjects with neuropsychiatric conditions (FPEB). http://clinicaltrials.gov/ct2/show/NCT00870974?term=A+PET+Brain+Imaging + Study + of + mGlu R5+in+Subjects+With+Neuropsychiatric+Conditions+\%28FPEB\%29\&rank=1 Accessed 25 Apr 2020. 18. Budimirovic, DB, et al.. Am J Med Genet. 2006; Part A, 140A, 1814-1826. 19. Brasic J, et al.. J Nucl Med 2018; 59 (supplement 1): 1774. Available at: http://jnm.snmjournals.org/content/59/supplement_1/1774.abstract

\section{Image/Figure:}

https://www.xcdsystem.com/wmis/abstract/File6959/GA206_ImageFigure 0606075805.jpg

Image/Figure Caption: Histogram of nondisplaceable binding potential (BPND) images (5) by the multilinear reference tissue method with two parameters (MRTM2) (Ichise, et al., 2003) of volumes of interest on positron emission tomography (PET) for 90 min after participants with FXS and ASD and ID $(\mathrm{N}=2)$, ASD without FXS or ID $(\mathrm{N}=6)$, and TD $(\mathrm{N}=3)$ received intravenous bolus injections of 185 megabecquerels (MBq) [5 mCi] [18F]FPEB (3, 4). Am: Amygdala; aFr: Anterior frontal lobe; ASD: Autism spectrum disorder; aTp: Anterior temporal lobe; BPND: Nondisplaceable binding potential; BS: Brainstem; cAC: caudal anterior cingulate; CB: Cerebellum; CN: Caudate nucleus: CC: Cingulate cortex; Cu: Cuneus; dpTp: Dorsal posterior temporal lobe; ER: Entorhinal area; FO: Frontal operculum; Fs: Fusiform gyrus; FXS: Fragile X syndrome; GP: Globus pallidus; Hp: Hippocampus; IC: Isthmus/cingulate; ID: Intellectual disability; In: Insula; iPa: Inferior parietal lobe; iTp : Inferior temporal lobe; LG: 
Lingual gyrus; 1Oc: Lateral occipital lobe; 1OG:Lateral orbital gyrus; mdOG: Medial orbital gyrus; $\mathrm{mFr}$ : Medial frontal lobe; $\mathrm{mTp}$ : Medial temporal lobe; OO: Orbital operculum; $\mathrm{paC}$ :

Paracentral; pC: Pericalcarine cortex; pCg: posterior cingulate; PH: Parahippocampus; Pr:

Precuneus; Prc: Precentral gyrus; PS: Postcentral gyrus; PT: Pars triangularis; Pu: Putamen: rAC:

Rostral anterior cingulate; rFr: Rostral frontal lobe; sFr: Superior frontal lobe; SM:

Supramarginal; sPa: Superior parietal lobe; sTp: Superior temporal lobe; TD: typical

development; Th: Thalamus; tT: Tranverse temporal; vS: Ventral striatum

Complete Status: Complete

First Name: James

Last Name: Brasic

Email: zauberfuchs@gmail.com

Organization: Johns Hopkins University

Country: United States 


\title{
ID: GA208 \\ Increased Metabolic Activity of Inflamed Organs in Convalescent Patients Post Severe COVID-19 Infection
}

\author{
Meiyun Wang, Henan Provincial People's Hospital \& the People's Hospital of Zhengzhou \\ University, mywang@ha.edu.cn
}

Category: Immunology: Inflammation \& Infection

\begin{abstract}
Body : The severe acute respiratory coronavirus 2 (SARS-CoV-2) has resulted in an ongoing outbreak of corona virus disease 2019 (COVID-19) worldwide [1]. According to a large sample study, the proportion of severe type COVID-19 patients is 15.7\% (173 of 1099) [2]. The extent of the effects, as well as the mechanisms of progress and outcome in these severe type patients are not clear but of great importance. Other than the lungs, COVID-19 was reported to induce dysfunction of other organs, such as liver and spleen [3]. 18F-Fluorodeoxyglucose (18FFDG) PET/CT allows in vivo mapping of cellular glycolytic rate directly, which can reflect the metabolic activities related to inflammation. In the retro-study of four highly suspected COVID19 patients, PET/CT revealed increased 18F-FDG uptake in pulmonary lesions and mediastinal lymph nodes [4]. However, metabolic information about other inflammation related organs of COVID-19 patients remains unknown. Herein, PET/CT findings in the lungs, mediastinal lymph nodes, spleen, and liver of seven severe COVID-19 patients during convalescent phase were reported. The patients underwent PET/CT scans after two negative SARS-CoV-2 nucleic acid RT-PCR testing. Compared to control group with matched age and sex, 18F-FDG Standard Uptake Value (SUV) in these convalescent patients was higher in the residual pulmonary lesions, mediastinal lymph nodes, spleen and liver (all $\mathrm{p}$ The relationships between 18F-FDG uptakes and inflammation features need to be further investigated in COVID-19 patients to evaluate the infectious potential of the disease post recovery.
\end{abstract}

References: 1. Fauci AS, et al. NEJM 2020; doi:10.1056/NEJMe2002387. 2. Guan WJ, et al. NEJM 2020; doi:10.1056/NEJMc2005203. 3. Zhang C, et al. Lancet Gastroenterol Hepatol 2020; doi: 10.1016/S2468-1253(20)30057-1. 4. Qin C, et al. Eur J Nucl Med Mol Imaging 2020;47:1281-6.

Complete Status: Complete

First Name: Meiyun

Last Name: Wang

Email: mywang@ha.edu.cn

Organization: Henan Provincial People's Hospital \& the People's Hospital of Zhengzhou University 
Country: China 


\title{
ID: GA209 \\ Neuroprotective effect of the microglia depletion by CSF-1R inhibition in the Parkinson's animal
}

\author{
Jae Yong Choi, Korea Institute Radiological and Medical Sciences, smhany@kirams.re.kr
}

\section{Category: Neuroscience}

\begin{abstract}
Body : Purpose Although colony-stimulating factor 1 receptor (CSF-1R) inhibition has been shown to reduce neuroinflammation levels in the animal brain, it is unclear whether this therapeutic approach leads to the improvement of PD symptoms. Therefore, the purpose of the present study is to address the anti-inflammatory influence on behavioral and neuronal circuits. Methods In PD and PLX3397 treatment in PD groups, the rats $(n=6)$ were pretreated with desipramine hydrochloride (12.5 mg/kg i.p.). After ketamine/xylazine anesthesia (40 $\mathrm{mg} / \mathrm{kg}$ and $5 \mathrm{mg} / \mathrm{kg}$, respectively), $6-\mathrm{OHDA}$ ( $8 \mathrm{ug}$ in $2 \mathrm{uL} / \mathrm{site}$ in $0.2 \%$ ascorbic acid) was injected into the four coordinate sites in the right striatum. The inserted needle was withdrawn after 5 min, and the wound was sutured. In the sham group, the procedure was exact the same for the PD $(n=6)$ except that the $2 \%$ ascorbic acid saline was injected into the striatum. PLX3397 treatment (Tx) in PD groups ( $n=6)$ was given to PLX3397 treatment for 21 days in the PD group. After the treatment of PLX3397, all groups underwent behavioral tests. Then, PET and MR images were acquired sequentially. Results Activated microglia depletion by the administration of CSF-1R inhibitor causes an improvement of motor function and depressive-like behavior. In addition, this therapeutic treatment generates partial recovery of dopaminergic and glutamatergic systems as well as neuroinflammation. In the immunoassay, we can observe the significant reduction of activated microglia in the drug treatment group. Conclusions In the present study, we demonstrated that microglia depletion suppressed the neuroinflammatory progress, and which has a beneficial effect on motor and non-motor symptoms of PD.
\end{abstract}

References: Oh SJ, Ahn H, Jung KH, Han SJ, Nam KR, Kang KJ, Park JA, Lee KC, Lee YJ, Choi JY. "Evaluation of the Neuroprotective Effect of Microglial Depletion by CSF-1R Inhibition in a Parkinson's Animal Model" Mol Imaging Biol. In press

Complete Status: Complete

First Name: Jae Yong

Last Name: Choi

Email: smhany@kirams.re.kr

Organization: Korea Institute Radiological and Medical Sciences

Country: Korea, Republic of 


\title{
ID: GA210 \\ Evaluation of therapeutic effect of taurine treatment in an Alzheimer's disease using glutamate PET
}

\author{
Jae Yong Choi, Korea Institute Radiological and Medical Sciences, smhany@kirams.re.kr
}

\section{Category: Neuroscience}

\begin{abstract}
Body : Purpose Alzheimer's disease (AD) is the most common cause of dementia in elderly individuals, which leads to problems including memory loss, cognitive deficits, and intellectual disabilities. The therapeutic drugs developed so far have various side effects such as dizziness, headache, and confusion. Therefore, it is necessary to develop alternative therapeutic treatments that are free from adverse effects. Taurine, 2-aminoethanesulfonic acid, is the second most abundant endogenous amino acid after glutamate, in the central nervous system. The chemical has nontoxic properties in the body, so taurine has been used in foods and drugs for the treatment of liver and heart diseases. In the present study, we investigate the effect of taurine supplementation in AD mice by glutamate PET. Materials Three groups of mice have used for these studies; the B6/SJL F1 hybrids (WT, $n=5), 5 x F A D$ mice $(A D, n=5)$, and 5xFAD mice that were orally administered the taurine as drinking water from 2 months to 9 months of age (ADTaurine, $\mathrm{n}=5$ ). Taurine was administered at a dose of $1,000 \mathrm{mg} / \mathrm{kg} / \mathrm{day}$. At the time at 9 months of age, all groups underwent the glutamate PET study. Mice were anesthetized with $2.0 \%$ isoflurane, and $18 \mathrm{~F}-\mathrm{FPEB}(9.4 \pm 1.2 \mathrm{MBq} / 200 \mathrm{uL})$ was injected through a tail vein. Simultaneously dynamic PET scan was performed for $60 \mathrm{~min}$ and images were reconstructed with the 3 -dimensional ordered subset expectation maximization algorithm with 4 iterations $(14 \times 30 \mathrm{~s}, 3 \times 60 \mathrm{~s}, 4 \times 300 \mathrm{~s}, 3 \times 600 \mathrm{~s}$, totally 24 frames $)$. The cortex, striatum, hippocampus, thalamus, and cerebellum were selected as volumes of interests (VOIs). The decay-corrected regional time-activity curves (TACs) were acquired from VOIs and normalized in units of standardized uptake value (SUV), which is calculated to normalize for differences in injected dose and body weight. Pharmacokinetic parameters (Cmax, Tmax, AUC) were estimated from TACs. In addition, the regional distribution volume ratio (DVR) was estimated using Logan graphical analysis. Results By visual inspection, the AD group showed significantly lower striatal and hippocampal 18F-FPEB uptake than those of the WT group. The ADtaurine group showed relatively higher uptakes than those of AD group. In AD group, all AUC values of the target regions were $27-38 \%$ decrease compared with the corresponding values in WT mice. The taurine group showed a 37-46\% higher AUC value than the AD group. Cmax values of the target regions for WT were between 1.95 and $2.48 \mathrm{~min}$, and the Cmax values for the AD were comparable (1.67-2.44). However, the Cmax values of ADtaurine increased relative to other groups (2.41-3.30). 18F-FPEP reached a maximum concentration at $3.25 \mathrm{~min}$ p.i in WT.

However, the peak arrival times of the AD and ADtaurine groups were relatively fast (AD: $1.55-$ $2.34 \mathrm{~min}$, ADtaurine: 1.35-1.45 min). The mean DVR values for AD group were $29-42 \%$ lower than those for WT. However, ADtaurine group showed 15-20\% higher DVR values compared to those for AD. Conclusions In summary, glutamate PET showed successfully differentiate the disease severity of AD mouse model. Moreover, the improvement of glutamate-related function in the brain by taurine was observed. From these perspectives, taurine caused glutamate related
\end{abstract}


therapeutic effect in AD. Moreover, mGluR5 PET could be a useful tool to reflect the therapeutic effect of taurine in AD rodents, which is expected to be the basis for future studies for early diagnosis of $\mathrm{AD}$.

Complete Status: Complete

First Name: Jae Yong

Last Name: Choi

Email: smhany@kirams.re.kr

Organization: Korea Institute Radiological and Medical Sciences

Country: Korea, Republic of 


\title{
ID: GA211 \\ High-resolution positron emission microscopy of patient-derived tumor organoids
}

\author{
Syamantak Khan, Stanford University, drskhan@stanford.edu
}

\section{Category: Oncology}

\begin{abstract}
Body : Tumor organoids are self-organizing three-dimensional micro-tumors that are grown from patient-derived tumor tissue. They closely recapitulate the structural and functional tumor microenvironment of the original tumor, and therefore, function as a superior personalized cancer model1. Although emerging evidence indicates that organoids can be used to accurately predict drug responses in a personalized treatment setting, clinically relevant methods to perform high-throughput drug and immune-therapy screens are still missing. Here we present a proof of concept for using positron emission microscopy (o-PEM) to screen drug response of tumor organoids derived from head and neck cancer patients. Using 18-Fluorodeoxyglucose (FDG) as a radiotracer, we show that high-resolution images of these organoids can be captured rapidly revealing metabolic maps which in combination with brightfield imaging functionally resembles the PET/CT images from the clinic. Tumor organoids were fed with FDG and mounted on a thin CdWO4 scintillator plate. Scintillator flashes were directly imaged by a single-photon-sensitivity electron-multiplying charge-coupled devices (EMCCD) camera as previously described by Kim et al in their radioluminescence imaging platform2. The EMCCD signal was calibrated with known radioactivity of FDG for dose calculation or accurate quantification of PEM images. A reference fluorescence imaging with live/dead cell marker revealed that PEM imaging recognizes the viable region of a patient-derived tumor explant with high signal to noise ratio. Cisplatin treatment of tumor organoids caused significantly lower signal or metabolic activity in the PEM images, while organoids derived from cisplatin-resistant patients showed higher metabolic activity. The highest resolution of PEM $(18 \mu \mathrm{m})$ for organoid imaging was found to be 200 fold better than the standard PET images $(5 \mathrm{~mm})$. Unlike a cell viability assay, this method can accurately identify the cell fate much early form its metabolic indication. This is the very same reason why FDG-PET has proved to be the most useful way to monitor therapeutic response in the patients. Microdosimetry calculation reveals the exact doses accumulated in each organoid. The quantification was performed with respect to a calibrated signal obtained from a known dose. The obtained time-dependent data falls very close to the radioactive decay curve of 18 -F even up to a very low activity and camera signal, confirming the reliability of microdosimetry conversions. In this technique, the photon budget is only dependent on the radioactivity of $18-\mathrm{F}$, which is an energy-independent autonomous long term phenomenon and warrant the excess amount of photon yield in expense of imaging time or speed. Finally, the influx rate constant $(\mathrm{Ki})$ of FDG into the tumor organoids was estimated with an approximation of the standard two-tissue compartmental model with irreversible uptake. The influx rate into the tumor organoids from multiple patients was found to be similar to the patient tumor from which the organoids were grown. Our data suggest that the metabolic heterogeneity of the patient tumor is significantly conserved in the organoid tumors in vitro and o-PEM can functionally recapitulate the original PET imaging of the patient performed in the clinic. In conclusion, due to
\end{abstract}


the advantages of improved resolution and rapid data acquisition ability in a quantitative manner, this in-vitro PEM imaging platform has the potential to complement PET imaging in the clinic and emerge as the next-generation high-throughput tool for cancer precision therapy.

References: 1. F. Weeber, S. N. Ooft, K. K. Dijkstra, E. E. Voest, Tumor organoids as a preclinical cancer model for drug discovery. Cell chemical biology 24, 1092-1100 (2017). 2. T. J. Kim, S. Türkcan, G. Pratx, Modular low-light microscope for imaging cellular bioluminescence and radioluminescence. Nature protocols 12, 1055 (2017).

Full Name of Abstract's 1st Author : Syamantak Khan

Complete Status: Complete

First Name: Syamantak

Last Name: Khan

Email: drskhan@stanford.edu

Organization: Stanford University

Country: United States 


\title{
ID: GA213 \\ PET/MR Findings of a Severe COVID-19 Patient
}

\section{Meiyun Wang, Henan Provincial People's Hospital \& the People's Hospital of Zhengzhou University, mywang@ha.edu.cn}

Category: Immunology: Inflammation \& Infection

\begin{abstract}
Body : The pandemic of corona virus disease 2019 (COVID-19) is evolving rapidly since December 2019 with possibility of repeated outbreak. Much remains unknown about the mechanism of action and the effects of the disease. Although the clinical manifestations are dominated by respiratory symptoms, other effects of the disease include dysfunction of other organs, such as cardiology [1,2], liver [3], head [4], and gonadal [5]. Previous reports mainly used different indicators to in-directly evaluate possible damages of different organs (such as blood pressure, CK-MB, and high-sensitivity cardiac troponin I for the cardiovascular system [1,2]; alanine aminotransferase and aspartate aminotransferase for liver [3]; and luteinizing hormone for gonadal effects [5]). Additional imaging techniques are needed to directly explore the details of these possible damages thus addressing the possible mechanisms. Herein, PET/MR hybrid scanner was used on a severe COVID-19 patient to investigate the effects of the virus infection on different organs (heart, spleen, head, liver, and lymph nodes). The patient underwent PET/MR scan using 18F-FDG as the PET tracer. T2WI and T1WI sequences were used for liver and spleen MR; T2-FLAIR and DTI(FA) were used for head MR; Cine, Black blood, and late gadolinium-enhanced sequences were used for cardiac MR. The detailed analyses of the MR, PET, and fusion images, were conducted. From the PET/MR images, no remarkable cardiovascular, spleen and brain damage was observed. While the uptake of 18F-FDG in liver and lymph nodes were increased. The incensement of 18F-FDG uptake in liver and lymph nodes could be originated from the inflammatory response. The absence of cardiovascular effects suggests that the distribution of ACE2 and the organs' damage are possibly not correlated in this case. Further studies about individual differences as well as the mechanisms of action are required. Continuous tracking of the patient is in progress to give more information.
\end{abstract}

References: 1. Huang C, et al. Lancet. 2020;395(10223):497-506. 2. Zheng Y-Y, et al. Nat Rev Cardiol 2020. Doi: 10.1038/s41569-020-0360-5. 3. Zhang C, et al. Lancet Gastroenterol Hepatol 2020. Doi: 10.1016/S2468-1253(20)30057-1. 4. Poyiadji N, et al. Radiology 2020. Doi:

10.1148/radiol.2020201187. 5. Ma L, et al. medRxiv 2020. Doi: 10.1101/2020.03.21.20037267.

Complete Status: Complete

First Name: Meiyun

Last Name: Wang

Email: mywang@ha.edu.cn 
Organization: Henan Provincial People's Hospital \& the People's Hospital of Zhengzhou University

Country: China 


\title{
ID: GA215 \\ In Vivo Tracking of Iron Labeled Extracellular Vesicles by Magnetic Particle Imaging
}

\author{
Victoria Toomajian, Michigan State University, toomaji2@msu.edu
}

\section{Category: Oncology}

\begin{abstract}
Body : Introduction: Breast cancer remains one of the most common cancers worldwide. When breast cancer results in brain metastases, this fate is typically fatal for patients [1]. In order to combat this, therapies need to be made to treat brain metastasis. While there are existing treatments such as surgery and radiation treatment, the life span of patients remains fairly low, with a median overall survival of 7 months [2]. Targeted therapeutics may not be able to cross the blood brain barrier (BBB), or have limited penetration, resulting in reduced efficacy in treating these metastases [3]. Therefore, it is important to examine potential therapeutic delivery vehicles that may cross the BBB, such as extracellular vesicles (EVs). EVs are small particles released from cells, and are a naturally occurring method of cell to cell communication that have been implicated in a number of biological processes, including metastasis [4]. EVs can be exploited to deliver a therapeutic agent to recipient cells, allowing them to be used as a potential treatment for various diseases including cancer [5]. They are also capable of crossing the $\mathrm{BBB}$, which would allow them to deliver a therapeutic to brain metastases [5][6]. However, before they can be used as such, it is important to understand the timing, localization, and accumulation of EVs during metastatic progression. Magnetic particle imaging (MPI) can aid in characterizing EVs in metastases after labeling them with superparamagnetic iron oxide nanoparticles (SPION). MPI is a new sensitive imaging method that is specific to superparamagnetic iron and provides quantitative data. Methods: Murine breast cancer (4T1) and macrophage (J774A.1) cells were labeled with SPIONs, using protamine-sulfate to enhance uptake. Following 24-hour labeling, the cells were washed to eliminate any extracellular iron and iron-labeled EVs (Fe-EVs) were collected by ultracentrifugation 24 hours later. Nanoparticle tracking analysis (NTA) provided information on size and a measure of concentration of Fe-EVs. Fe-EV pellets were imaged by MPI and iron content was quantified using a calibration curve. For in vivo experiments, Balb/C mice will receive an intracardiac injection to deliver 4T1-BR Fluc/GFP cells, a breast cancer cell line that forms brain metastases and stably expresses firefly luciferase and fluorescence; this allows for imaging of the metastatic sites using bioluminescence imaging (BLI). J774 or 4T1 Fe-EVs will then be injected into the mice at either early or late stages of metastatic growth, and subsequently imaged using MPI and BLI to determine timing and accumulation of EVs arriving to the metastatic site. Results: MPI was used to image J774 (Fig 1A) and 4T1 (Fig 1B) Fe-EV pellets. A calibration curve (Fig 1C) was generated and was used to quantify the total amount of iron within each Fe-EV pellet. NTA identified the average size $(107.3 \mathrm{~nm} \& 98.5 \mathrm{~nm})$ and concentration of the Fe-EVs (Fig 1D, E), allowing for a measure of 213 and 222 attograms Fe/EV, for J774 or 4T1 Fe-EVs, respectively. With this extent of ironloading, a lower detection limit of $2.5 \times 108$ Fe-EVs could be identified using MPI, although high sensitivity imaging could reduce that number further. Future experiments will use MPI and BLI to identify Fe-EVs within brain metastases, in vivo. Imaging will be validated by histological
\end{abstract}


examination of the brain. Conclusions: This multi-model imaging technique will demonstrate that EVs can cross the blood brain barrier and accumulate in metastatic sites. These findings will allow for further study of EVs for use as a therapeutic delivery vehicle to treat brain metastasis.

References: [1] Aversa C, Rossi V, Geuna E, Martinello R, Milani A, Redana S, et al. Metastatic breast cancer subtypes and central nervous system metastases. Breast. 2014;23:623-8. [2] Witzel I, Laakmann E, Weide R, Neunhöffer T, Park-Simon TJ, Schmidt M, et al. Treatment and outcomes of patients in the Brain Metastases in Breast Cancer Network Registry. Eur J Cancer. 2018;102:1-9. [3] Bendell JC, Domchek SM, Burstein HJ, Harris L, Younger J, Kuter I, et al. Central nervous system metastases in women who receive trastuzumab-based therapy for metastatic breast carcinoma. Cancer. 2003;97:2972-7. [4] Harris DA, Patel SH, Gucek M, Hendrix A, Westbroek W, Taraska JW. Exosomes released from breast cancer carcinomas stimulate cell movement. PLoS One. 2015;10:e0117495-e0117495. [5] Jia G, Han Y, An Y, Ding Y, He C, Wang X, et al. NRP-1 targeted and cargo-loaded exosomes facilitate simultaneous imaging and therapy of glioma in vitro and in vivo. Biomaterials. 2018;178:302-16. [6] Yang T, Martin P, Fogarty B, Brown A, Schurman K, Phipps R, et al. Exosome delivered anticancer drugs across the blood-brain barrier for brain cancer therapy in Danio rerio. Pharm Res. 2015;32:2003- 14 .

\section{Image/Figure:}

https:/www.xcdsystem.com/wmis/abstract/File6959/GA215_ImageFigure_0614071654.png

Image/Figure Caption: Figure 1. MPI signal produced by (A) J774 Fe-EV pellet (1.42E9 particles) and (B) 4T1 Fe-EV pellet (2.9E9 particles) collected after ultracentrifugation and resuspended in phosphate buffered saline. The white outline represents the axial view of the conical tube containing the pellet of Fe-EVs. A calibration curve was generated using the total MPI signal from a known amount of iron (D). Particle concentration was calculated using NTA, and the size of these particles for (C) J774 Fe-EVs and (D) 4T1 Fe-EVs were similar to the average size of EVs.

Complete Status: Complete

First Name: Victoria

Last Name: Toomajian

Email: toomaji2@msu.edu

Organization: Michigan State University

Country: United States 


\title{
ID: GA217 \\ Deep CEST MR Fingerprinting Reveals Tumor Apoptotic Response to Oncolytic Virotherapy In Vivo
}

\author{
Or Perlman, Massachusetts General Hospital and Harvard Medical School, \\ operlman@mgh.harvard.edu
}

\section{Category: Computational \& Data Science}

\begin{abstract}
Body : Introduction Oncolytic virotherapy (OV) is a promising treatment for high mortality cancers [1]. Non-invasive monitoring of OV is essential for optimizing the clinical outcome and providing an improved understanding of the interactions between the virus and its tumor-host. Chemical exchange saturation transfer (CEST) MRI is a molecular imaging technique that may shed new light on $\mathrm{OV}$, as it is capable of detecting protein concentration and $\mathrm{pH}$ changes. However, clinical translation of CEST methods has been hindered by the qualitative nature of the image contrast and the long image acquisition times. Objective The goal of this work was to develop a deep-learning-based CEST MR fingerprinting (MRF) method for quantitative and rapid multi-pool imaging of OV treatment response. The approach was evaluated in mice undergoing virotherapy treatment and translated to clinical scanners. Methods CEST-MRF Acquisition Two SE-EPI CEST-MRF acquisition protocols (105s each) were employed sequentially, designed for obtaining MT and amide proton exchange-rate and volumefraction maps. Both protocols varied the saturation power, but employed different saturation offsets (first: 6-14 ppm, second: $3.5 \mathrm{ppm}$ ). T1, T2, and B0 maps were acquired using variable repetition-time, multi-echo spin-echo, and water saturation shift referencing [2], respectively. CEST-MRF Dictionary Generation The CEST-MRF signal trajectories for 184,800 and 70,224,000 multi-pool parameter combinations were synthesized for the two protocols using a numerical solution of the Bloch-McConnell equations [3-4]. Deep Reconstruction Networks To avoid the exceedingly long dictionary matching-time required for conventional dot-product MRF [5] and to improve the multi-parameter reconstruction ability, image reconstruction was performed using a series of two Deep RecOnstruction NEtworks (DRONEs) [6], trained on the synthesized data. The pixel-wise signal trajectories from the MT specific MRF schedule were input to the first DRONE, together with the water T1, T2 and B0 values. The two MT exchange parameter outputs, together with the water pool and $\mathrm{B} 0$ parameters were then input into the second DRONE, together with the pixel-wise signal trajectories from the amide-pool MRF schedule. In Vivo Imaging U87 tumors were implanted in the brain of 16 mice $(25 \%$ used as control). The mice were imaged at 8-11 days after implantation, using a $7 \mathrm{~T}$ preclinical MRI (Bruker, Germany). Next, a herpes simplex-derived oncolytic virus, NG34, was inoculated, and the mice were imaged $48 \mathrm{hrs}$ and $72 \mathrm{hrs}$ later. For clinical translation, the continuous wave saturation pulse was replaced by a train of spin-lock saturation pulses and the read-out was done using GRE-EPI. A healthy volunteer was recruited and imaged at 3T (Siemens Healthineers, Germany). Results Before virus inoculation, the semi-solid and amide proton concentrations were both decreased in the tumor, consistent with increased edema decreasing the protein concentrations. The tumor amide proton exchange-rate was increased, indicative of increased intracellular $\mathrm{pH}$. Following $\mathrm{OV}$, the core of the tumor presented significantly lower amide proton
\end{abstract}


concentration and exchange-rate compared to the tumor rim and the contralateral region. Both effects are indicative of apoptosis as it is known to inhibit protein synthesis [7] and decrease cytosolic $\mathrm{pH}$ [8]. At baseline, control mice demonstrated similar MT and amide effects as OVtreated mice, however, no apoptotic process was evident. The preclinical MRI findings were in good agreement with the histology and immunohistochemistry findings (HSV antibodies, H\&E, Caspase-3, Coomassie). The normal human subject parameter maps yielded MT volume fraction and exchange-rate values in good agreement with the literature [9]. The amide proton exchangerate was in good agreement with previous water exchange spectroscopy-based measurements [10]. The total reconstruction time for the 4 molecular maps using the neural-network scheme was $94 \mathrm{~ms}$, compared to $2.3 \mathrm{hrs}$ for conventional dot-product reconstruction. Conclusions The deep CEST-MRF technique successfully and rapidly quantified $\mathrm{pH}$ and molecular concentration changes, potentially serving as important biomarkers for OV-induced apoptosis.

References: [1] Russell, S. J., et al., Nature biotechnology 2012;30(7):658. [2] Kim, M., et al., Magn Reson Med. 2009;61(6):1441-1450. [3] Cohen, O., et al., Magn Reson Med. 2018;80(6):2449-2463. [4] Perlman, O., et al., Magnetic Resonance in Medicine 2020;83:462478. [5] Ma, D., et al., Nature 2103;495(7440):187. [6] Cohen, O., et al., Magn Reson Med. 2018;80(3):885-894. [7] Jeffrey, I., et al., Cancer Research. 2002;62(8):2272-2280. [8] Nilsson, C., et al., Apoptosis. 2006;11(7):1149. [9] Stanisz, G. J., et al., Magn Reson Med.

2005;54(3):507-512. [10] Van Zijl, P. C., et al., Magn Reson Med. 2003;49(3):440-449.

Full Name of Abstract's 1st Author : Or Perlman

Complete Status: Complete

First Name: Or

Last Name: Perlman

Email: operlman@mgh.harvard.edu

Organization: Massachusetts General Hospital and Harvard Medical School

Country: United States 


\title{
ID: GA219 \\ Molecular imaging of prostate cancer targeting CD46 using immunoPET with 89Zr-DFO-YS5
}

\author{
Sinan Wang, ucsf, sinan.wang@ucsf.edu
}

\section{Category: Oncology}

\begin{abstract}
Body : Purpose: Molecular imaging and targeted theranostic agents are playing an increasing role in prostate cancer detection and therapy1. CD46 represents a novel prostate cancer (PCa) biomarker with high expression in aggressive and late stage PCa such as
\end{abstract} neuroendocrine prostate cancer2,3. ImmunoPET imaging is a noninvasive molecular imaging modality combined the excellent targeting specificity of antibodies or antibody fragments and the superior sensitivity and resolution of PET4,5. In this work, we developed a novel CD46-targeted positron emission tomography (PET) radiotracer, [89Zr]DFO-YS5, and evaluated its performance for immunoPET imaging in murine PCa models. Materials and methods: The radiolabeling of 89Zr oxalate to DFO-YS5 or control antibody was accomplished in a typical, two step procedure by first conjugating with desferoximine (DFO), and then subsequent chelation of the isotope. Mice bearing DU145 and 22Rv1 xenografts or LTL-331 patient derived xenografts were grown to at least $0.5 \mathrm{cc}$, and PET imaging was performed on a Siemens Inveon micro PET/CT system. Results: [89Zr]DFO-YS5 was isolated in $74 \pm 11 \%(n=6)$ yield based on starting $89 \mathrm{Zr}$ oxalate with molar activities ranging from $7.42 \mathrm{mCi} / \mathrm{mg}$ to $9.49 \mathrm{mCi} / \mathrm{mg}(274.2$ $\mathrm{MBq} / \mathrm{mg}-351.1 \mathrm{MBq} / \mathrm{mg}$ ). Purity was greater than 95\%. PET imaging and biodistribution analysis of [89Zr]DFO-YS5, [89Zr]DFO-IgG (control antibody) and 68Ga-PSMA-11 was performed in mice bearing DU145 (CD46+, PSMA-) and 22Rv1 (CD46+, PSMA+) xenografts. Both DU145 and 22Rv1 showed high uptake of [89Zr]DFO-YS5 (13.3 $\pm 3.9 \%$ ID/gram and $11.2 \pm 2.5 \% \mathrm{ID} /$ gram respectively, Figure C, E). As a comparison, control antibody [89Zr]DFOIgG showed a low uptake in DU145 and 22Rv1 xenografts (Figure D, E). In 68Ga-PSMA-11 imaging, the PSMA negative cell line DU145 showed very low uptake compared to the PSMA positive cell line 22Rv1, which demonstrated 4\% ID/g uptake. Taken together, these data demonstrate the feasibility of imaging CD46 positive, PMSA negative tumors with [89Zr]DFOYS5. PET/CT analysis of the LTL-331 patient derived xenograft model also demonstrated high tumoral uptake of [89Zr]DFO-YS5. Human equivalent dosimetry was performed in wild type male mice, demonstrating a radiation dose of $0.447 \pm 0.043 \mathrm{mSv} / \mathrm{MBq}$. Discussion: Taken together, the data presented herein demonstrate that [89Zr]DFO-YS5 is an effective radiopharmaceutical for imaging CD46 positive prostate cancer. CD46 is highly expressed in $\mathrm{PCa}$, particularly in de-differentiated forms of prostate cancer including treatment emergent small cell/neuroendocrine prostate cancer, where expression is generally higher than PSMA. Theranostic targeting with prostate specific membrane antigen (PSMA) is highly promising for both imaging and therapy for prostate cancer. However, some PCa cells express low level of PSMA, which could not be detected through PSMA based imaging7. Our imaging study in the dual DU145 and 22Rv1 model mimics the situation seen in the clinic, with heterogeneous target expression. Thus, CD46 targeted imaging and therapy could be a complementary option for PSMA negative PCa. Conclusion: Our data demonstrate that [89Zr]DFO-YS5 imaging provides 
excellent imaging contrast with high uptake specific for CD46-expressing prostate cancer cells, including in PSMA negative tumors and patient derived xenografts. Overall, the novel radiotracer [89Zr]DFO-YS5 represents a promising candidate for clinical translation as an immunoPET agent for the detection of CD46-positive primary and metastatic prostate cancers.

References: References 1. Fanti S, Minozzi S, Antoch G, et al. Consensus on molecular imaging and theranostics in prostate cancer. Lancet Oncol 2018;19:e696-e708. 2. He J, Wang Y, Feng J, et al. Targeting prostate cancer cells in vivo using a rapidly internalizing novel human singlechain antibody fragment. J Nucl Med 2010;51:427-32. 3. Su Y, Liu Y, Behrens CR, et al. Targeting CD46 for both adenocarcinoma and neuroendocrine prostate cancer. JCI Insight 2018;3. 4. Knowles SM, Wu AM. Advances in immuno-positron emission tomography: antibodies for molecular imaging in oncology. J Clin Oncol 2012;30:3884-92. 5. Wei W, Rosenkrans ZT, Liu J, Huang G, Luo QY, Cai W. ImmunoPET: concept, design, and applications. Chem Rev 2020;120:3787-851. 6. Sharma SK, Chow A, Monette S, et al. Fcmediated anomalous biodistribution of therapeutic antibodies in immunodeficient mouse models. Cancer Res 2018;78:1820-32. 7. Mannweiler S, Amersdorfer P. Heterogeneity of prostatespecific membrane antigen (PSMA) expression in prostate carcinoma with distant metastasis. Pathol Oncol Res 2009;15:167-72.

\section{Image/Figure:}

https://www.xcdsystem.com/wmis/abstract/File6959/GA219_ImageFigure_0611015850.png

Image/Figure Caption: [89Zr]DFO-YS5 preparation and PET imaging on CD46 positive malignancy in murine prostate cancer models

Full Name of Abstract's 1st Author : Sinan Wang

Complete Status: Complete

First Name: Sinan

Last Name: Wang

Email: sinan.wang@ucsf.edu

Organization: ucsf

Country: United States 


\title{
ID: GA220 \\ Development of CD46-targeting immunoPET probe for prostate cancer imaging
}

\author{
Sinan Wang, ucsf, sinan.wang@ucsf.edu
}

\section{Category: Oncology}

\begin{abstract}
Body : Prostate cancer $(\mathrm{PCa})$ is the most prevalent noncutaneous cancer in men1. Prostate-specific membrane antigen (PSMA) is a well-established biomarker for PCa, making it the target of a number of imaging and therapeutic approaches2,3. CD46 has recently been identified as a novel and underexplored therapeutic target in prostate cancer (PCa) with high expression in de-differentiated forms of prostate cancer including treatment emergent small cell/neuroendocrine prostate cancer4,5. Herein, we developed [89Zr]DFO-YS5 as a CD46targeting immunoPET probe, and demonstrated that [89Zr]DFO-YS5 could specifically target CD46 positive tumor cell lines with excellent imaging contrast. Our previous work demonstrate that [89Zr]DFO-YS5 could be synthesized in $74 \pm 11 \%(n=6)$ yield based on starting $89 \mathrm{Zr}$ oxalate with molar activities ranging from $7.42 \mathrm{mCi} / \mathrm{mg}$ to $9.49 \mathrm{mCi} / \mathrm{mg}(274.2 \mathrm{MBq} / \mathrm{mg}-351.1$ $\mathrm{MBq} / \mathrm{mg}$ ). The in vitro study has shown that [89Zr]DFO-YS5 had a high binding affinity for CD46 at $6.0 \pm 0.6 \mathrm{nM}$. With the radiotracer in hand, a multi-time point micro PET/CT and biodistribution study was performed. Mice with CD46 positive DU145 xenograft model was imaged and their organ were harvested and measured at different time point. It showed a specific uptake at tumor site as early at 24 hours post injection. Biodistribution data revealed that the tumor uptake as measured in increased over time, measuring up to $18.8 \pm 11.3 \% \mathrm{ID} / \mathrm{gram}$ at day 7. In contrast, the blood pool retention, which started at $10.8 \pm 1.2 \% \mathrm{ID} / \mathrm{gram}$ at 24 hours, decreased to $3.8 \pm 1.6 \% \mathrm{ID} / \mathrm{gram} \%$ by $168 \mathrm{~h}$. The uptake at other organs remained below $5 \%$ ID/gram all the time, providing an excellent imaging contrast. To further evaluate the imaging ability of [89Zr]DFO-YS5, and compare it to the PSMA based prostate cancer imaging agent 68Ga-PSMA-11, [89Zr]DFO-YS5 or 68Ga-PSMA-11 were administered to athymic mice xenografted with 22Rv1 cells subcutaneously. Mice administered with [89Zr]DFO-YS5 were imaged 4 days post i.v. injection and then sacrificed for a biodistribution study. The probe could specifically localize at tumor site and had a low accumulation at other organs. The biodistribution study showed that the tumor uptake was $14 \% \mathrm{ID} / \mathrm{gram}$ in average, the uptake at other organs was all below 5\%. Mice administered with 68Ga-PSMA-11 showed high tumor, spleen, and especially kidney uptake. The biodistribution study showed 4\% ID/gram uptake at tumor and over 120\% ID/gram uptake at kidney because of its high expression level of PSMA. These results demonstrate that [89Zr]DFO-YS5 can specifically image CD46 positive prostate cancer with favorable imaging characteristics 68Ga-PSMA-11 in the 22Rv1 model. To validate [89Zr]DFO-YS5 that specifically targets CD46 positive tumor, and compare the uptake difference between CD46 positive tumor and CD46 negative tumor, a PET imaging and biodistribution study was performed in mice bearing CD46 positive DU145 and CD46 negative MC38 xenografts (see persuasive data figure). Mice were administered [89Zr]DFO-YS5, imaged at day 4 followed by a biodistribution study. The uptake at DU145 xenograft was $15 \pm 5 \%$ ID/gram, almost three folds of the uptake at MC38. The DU145 tumor/muscle uptake ratio could reach 90, which is also almost 3 folds of MC38 tumor/muscle ratio, suggesting a good imaging
\end{abstract}


contrast and high specificity for CD46-expressiong xenograft. Overall, [89Zr]DFO-YS5 showed high binding affinity to CD46, excellent imaging contrast against CD46 positive xenograft DU145 and 22Rv1 in vivo. The imaging was also verified by a control experiment using CD46 negative xenograft MC38. Taken together, our data demonstrated that [89Zr]DFO-YS5 can be specifically taken up by CD46 positive PCa xenograft, including in PSMA negative tumors, demonstrating strong potential for clinical translation.

References: 1. Miyahira AK, Cheng HH, Abida W, et al. Beyond the androgen receptor II: New approaches to understanding and treating metastatic prostate cancer; Report from the 2017 Coffey-Holden Prostate Cancer Academy Meeting. Prostate. 2017;77:1478-88. 2. Holland JP, Divilov V, Bander NH, Smith-jones PM, Larson SM, Lewis JS. 89Zr-DFO-J591 for immunoPET of prostate-specific membrane antigen expression in vivo. J Nucl Med 2010; 51:1293-1300. 3. Kratochwil C, Bruchertseifer F, Giesel FL, et al. 225Ac-PSMA-617 for PSMA-targeted $\alpha$-radiation therapy of metastatic castration-resistant prostate cancer. J Nucl Med 2016; 57:1941-44. 4. He J, Wang Y, Feng J, et al. Targeting prostate cancer cells in vivo using a rapidly internalizing novel human single-chain antibody fragment. J Nucl Med 2010;51:427-32. 5. Su Y, Liu Y, Behrens CR, et al. Targeting CD46 for both adenocarcinoma and neuroendocrine prostate cancer. JCI Insight 2018;3.

\section{Image/Figure:}

https://www.xcdsystem.com/wmis/abstract/File6959/GA220_ImageFigure_0612113408.jpg

Image/Figure Caption: Comparison of [89Zr]DFO-YS5 PET imaging in mice bearing CD46 positive (DU145) and CD46 negative (MC38) tumors

Full Name of Abstract's 1st Author : Jun Li

Complete Status: Complete

First Name: Sinan

Last Name: Wang

Email: sinan.wang@ucsf.edu

Organization: ucsf

Country: United States 


\title{
ID: GA221 \\ Effectual labeling of NK cells with upconverting nanoparticles by electroporation for in vivo animal image-tracking and biodistribution assessment
}

\author{
Kwan Soo Hong, Korea Basic Science Institute, kshong@kbsi.re.kr
}

Category: New Chemistry, Biology \& Bioengineering

\begin{abstract}
Body : Natural killer (NK) cells, which are cytotoxic lymphocytes of the innate immune system and recognize cancer cells via various immune receptors, are promising agents in cell immunotherapy. To utilize NK cells as a therapeutic agent, their biodistribution and pharmacokinetics need to be evaluated following systemic administration. Therefore, in vivo imaging and tracking with efficient labeling and quantitative analysis of NK cells are required. However, the lack of the phagocytic capacity of NK cells makes it difficult to establish breakthroughs in cell labeling and subsequent in vivo studies. Herein, we propose an effective labeling method using upconverting nanoparticles (UCNPs) for NK cells using electroporation with high sensitivity and stability. The crucial parameters such as cell viability and cytotoxic function of the labeled NK cells were also studied at various electroporation conditions. The labeling of NK cells enabled tracking and biodistribution imaging analysis of NK cells injected intravenously, which showed that the labeled NK cells were initially distributed primarily in lungs and then spread to the liver and spleen. These advances will accelerate the application of NK cells as key components of immunotherapy against cancer. Figure. Schematics of in vivo image-tracking of NK cells labeled with UCNPs using electroporation technique.
\end{abstract}

\section{Image/Figure:}

https://www.xcdsystem.com/wmis/abstract/File6959/GA221_ImageFigure_0421112914.jpg

Complete Status: Complete

First Name: Kwan Soo

Last Name: Hong

Email: kshong@kbsi.re.kr

Organization: Korea Basic Science Institute

Country: Korea, Republic of 


\title{
ID: GA222 \\ Activatable fluorescent probe for in vivo hypoxia detection and animal imaging
}

\author{
Kwan Soo Hong, Korea Basic Science Institute, kshong@kbsi.re.kr
}

\section{Category: Oncology}

\begin{abstract}
Body : Hypoxia enlargement in tumor is closely related with its expanded aggressiveness and strong resistance to therapy, leading to poor prognosis in several cancer types. Herein, we synthesized a highly water soluble NIR fluorescent probe that contains folate as a tumor targeting moiety and nitro benzyl as sensing moiety. The molecule shows a highly accurate response on the diagnosis of hypoxia. Forming spectroscopic characterization, and biological evolution of folate conjugated hypoxia sensitive BODIPY forms, fol-BODIPY is presented as a diagnostic agent in cancer detection applications. The nitro-benzyl-modified folBODIPY probe serves as a rapid fluorescence enhancement factor for monitoring and bioimaging under hypoxia condition. Confocal fluorescence images and flow cytometry of hypoxic CT26 cells confirmed the nitro-reductase detection ability of fol-BODIPY more advanced at the cellular level compared with controls. Significantly fol-BODIPY can detect tumor hypoxia in xenograft mouse model, showing a target specific fluorescence bio-imaging without disrupting major organs. This advanced probe could provide a potential guideline for the construction of new cancer diagnosis and its monitoring system. Figure. (a) Schematics of cancer targeting and active fluorescence switching on under the hypoxic condition, and (b) fluorescence emission spectra of fol-BODIPY in various concentrations of nitro-reductase. (c) CLSM imaging of CT26 cells incubated with fol-BODIPY, (d) in vivo optical imaging of xenograft CT26 tumor mice following IV injection of fol-BODIPY probe, and (e) corresponding fluorescence intensity in the tumor region.
\end{abstract}

\section{Image/Figure:}

https://www.xcdsystem.com/wmis/abstract/File6959/GA222 ImageFigure 0421115322.jpg

Complete Status: Complete

First Name: Kwan Soo

Last Name: Hong

Email: kshong@kbsi.re.kr

Organization: Korea Basic Science Institute

Country: Korea, Republic of 


\title{
ID: GA223 \\ Novel 18F-radiolabeled anti-TNF $\alpha$ imaging agent: A mismatch between radioisotope half-life and biomolecule pharmacokinetics that works.
}

\author{
Denis Beckford Vera, University of California San Francisco, \\ denis.beckfordvera@ucsf.edu
}

Category: Immunology: Inflammation \& Infection

\begin{abstract}
Body : Objectives: Upregulation of tumor necrosis factor alpha (TNF $\alpha)$ in joints affected by rheumatoid arthritis (RA) drives inflammation and bone degradation. Varied response in RA patients to anti-TNF $\alpha$ biologics highlights the need to quantify TNF $\alpha$ in joints. We recently developed a 89Zr-labelled anti-TNFa certolizumab pegol (89Zr-CZP) that successfully assessed human TNF $\alpha$ expression in paws of transgenic mice. 1 Dosimetric estimation of 89Zr-CZP revealed high potential human radiation exposure due to slow CZP clearance matched with the long half-life of $89 \mathrm{Zr}$. However, observation that TNFa was visualized as early as $4 \mathrm{~h}$ post-injection with $89 \mathrm{Zr}$-CZP1 suggested that $89 \mathrm{Zr}$ could be substituted with a shorter-lived isotope such as $18 \mathrm{~F}$. This mismatch would significantly decrease radiation exposure while still providing suitable detection of TNF $\alpha$. Herein, we described the preparation of [18F]-fluorobenzoyl-CZP (18F-CZP) and its evaluation as a potential PET tracer for molecular imaging of TNFa in vivo. Methods: 18F-CZP synthesis was fully automated on an ELIXYS radiochemical synthesizer (SOFIE). The final product was characterized by sizeexclusion HPLC (SE-HPLC). The biological activity of 18F-CZP was assessed by quantifying the percentage of $18 \mathrm{~F}-\mathrm{CZP}$ that binds human recombinant TNF $\alpha$ using SE-HPLC. A longitudinal $\mu \mathrm{PET} / \mathrm{CT}$ imaging study of $18 \mathrm{~F}-\mathrm{CZP}$ in transgenic mice expressing human TNF $\alpha$ was performed over a period of 1 year. This animal model resembles the progression of RA in humans. $\mu \mathrm{PET} / \mathrm{CT}$ scans were acquired every 4-6 weeks to follow RA progression. TNF $\alpha$ transgenic mice (male, 8 weeks old, $\mathrm{n}=6$ ) were administered with $7.4 \mathrm{MBq}(10-40 \mu \mathrm{g}) 18 \mathrm{~F}-\mathrm{CZP}$ via tail vein. Images were acquired 1 and $4 \mathrm{~h}$ post-injection (pi). The specificity of $18 \mathrm{~F}-\mathrm{CZP}$ to TNF $\alpha$ in vivo was assessed by blocking TNF $\alpha$ with 1-2 $\mathrm{mg}$ of CZP administered 24h before administration of 18F-CZP. Additionally, another cohort of transgenic mice $(n=4)$ was intravenously injected with 7.4 MBq of 18F-FDG. Mice from these control cohorts were also scanned 1 and $4 \mathrm{~h}$ pi. Human dosimetry of $18 \mathrm{~F}-\mathrm{CZP}$ was estimated by extrapolation of the pharmacokinetics data obtained from the $\mu \mathrm{PET} / \mathrm{CT}$ images and OLINDA 2.0 (ICRP103). For the dosimetry study, mice were scanned $0.5,1,2$ and $4 \mathrm{~h}$ pi. Results: $18 \mathrm{~F}-\mathrm{CZP}$ was prepared in $2 \mathrm{~h}$ with an activity yield of $35 \pm 3 \%(n=7)$. After SE chromatography the radiochemical purity of $18 \mathrm{~F}-\mathrm{CZP}$ was $98 \%$. Protein aggregation was not observed. More than $90 \%$ of $18 \mathrm{~F}-\mathrm{CZP}$ bounded TNF $\alpha$ in vitro suggesting that radiolabeling of CZP with $18 \mathrm{~F}$ did not affect its biological activity. During the longitudinal $\mu$ PET/CT study, 18F-CZP accumulated in inflamed extremities joints of TNF $\alpha$ transgenic mice $1 \mathrm{~h}$ pi with excellent signal to noise ratio (Fig 1). No uptake was observed in extremity joints of TNF $\alpha$ transgenic mice after administration of $18 \mathrm{~F}$ FDG (Fig 1). PET/CT longitudinal study revealed 18F-CZP uptake correlated with disease severity. The blocking study showed $\sim 50 \%$ reduction in the uptake in inflamed extremity joints. The calculated effective dose of $18 \mathrm{~F}-\mathrm{CZP}$ was $0.0126 \pm 0.00028 \mathrm{mSv} / \mathrm{MBq}$ which is 30 -fold
\end{abstract}


lower than that from $89 \mathrm{Zr}$-CZP. If we assume $37 \mathrm{MBq}$ administration for $89 \mathrm{Zr}-\mathrm{CZP}$ and 74 $111 \mathrm{MBq}$ for $18 \mathrm{~F}-\mathrm{CZP}$, as we plan for clinical studies, RA patients could potentially receive a 14-20 -fold lower dose from a single injection of 18F-CZP compared to 89Zr-CZP.1

Conclusions: TNF $\alpha$ expression in joints of a RA animal model was assessed using 18F-CZP $1 \mathrm{~h}$ pi. Clinical translation of this approach may enable personalization of anti-TNF $\alpha$ drugs therapy in patients with RA while significantly reducing radiation exposure.

References: 1. Beckford Vera, D. R. et al. Mol Imaging Biol 36, $25-10$ (2019).

\section{Image/Figure:}

https://www.xcdsystem.com/wmis/abstract/File6959/GA223 ImageFigure 0609124251.png

Image/Figure Caption: Figure 1. Small animal PET/CT maximum intensity projections of 18F$\mathrm{CZP}$ and $18 \mathrm{~F}-\mathrm{FDG} 1 \mathrm{~h}$ post-injection in 12 weeks old transgenic mice that express human TNF $\alpha$.

Complete Status: Complete

First Name: Denis

Last Name: Beckford Vera

Email: denis.beckfordvera@ucsf.edu

Organization: University of California San Francisco

Country: United States 


\title{
ID: GA224 \\ Amphiphilic fluorescent probe: detecting pH-fluctuations within cancer cells and animal tumor tissues
}

\author{
Kwan Soo Hong, Korea Basic Science Institute, kshong@kbsi.re.kr
}

\section{Category: Oncology}

\begin{abstract}
Body : Abnormal anaerobic metabolism leads to a lowering of the $\mathrm{pH}$ of many tumors, both within specific intracellular organelles and in the surrounding extra-cellular regions. Information related to $\mathrm{pH}$-fluctuations in cells and tissues could aid in the identification of neoplastic lesions and in understanding the determinants of carcinogenesis. Here we report an amphiphilic fluorescent $\mathrm{pH}$ probe (CS-1) that provides $\mathrm{pH}$-related information in cancer cell membranes and selected intracellular organelles, such as lysosome and mitochondria. In contrast, CS-1 only labels normal cells marginally with a very low Pearson's correlation coefficient. Selfaggregation is seen for CS-1 in acidic microenvironments, a feature that is thought to improve its localization within live cells. Ex-vivo experiments provide support for the suggestion that CS-1 acts as a fluorescent labeling the periphery of tumors, presumably as the result of proton-induced aggregation, comparing to a much lower response is seen for muscle and liver. On the basis of the results presented here, we propose that sensors such as CS-1 may have a role to play in the pathological detection of tumor tissues as a surgical guidance. Figure. (a) Structure of the probe CS-1, and (b) schematic view of the proposed acid-promoted self-assembly of probe CS-1. (c) Colocalization CLSM images of CS-1 and LysoTracker in A549 cancer cells in a time dependent manner, and (d) corresponding Pearson's correlation coefficients (PC).
\end{abstract}

\section{Image/Figure:}

https://www.xcdsystem.com/wmis/abstract/File6959/GA224_ImageFigure 0422120953.jpg

Complete Status: Complete

First Name: Kwan Soo

Last Name: Hong

Email: kshong@kbsi.re.kr

Organization: Korea Basic Science Institute

Country: Korea, Republic of 


\title{
ID: GA225 \\ Intraoperative imaging technique to highlight peripheral nerves during cancer surgery using spectral reflectance
}

\author{
Ngai Nick Alex Wong, The Hong Kong Polytechnic University, \\ 18041854r@connect.polyu.hk
}

\section{Category: Oncology}

\begin{abstract}
Body : Nerve preservation is vital for surgical treatment to reduce postoperative adverse effect. Accidental nerve transection during cancer surgery might result in significant complications such as chronic pain, sensory loss, or even ability loss such as erectile dysfunction and urinary incontinence after prostate cancer surgery, and abnormal vocal cord function after thyroid cancer surgery. Unfortunately, there is lack of reliable technique to identify peripheral nerve in the current operating room. Surgeons typically rely on appearance, tactile information, and invasive electromyography to distinguish nerves. Real-time visualization of nerves with high specificity and safety during surgery is critical to guide surgeon to avoid nerve damage or transection. Here, we develop a label-free imaging strategy to provide specific and safe intraoperative imaging guidance for detecting peripheral nerves. Focusing on strong wavelengthspecific reflectance from myelin sheaths surrounding nerve fiber by thin film interference phenomenon, we demonstrate myelin induced reflectance imaging can be useful to specifically visualize peripheral nerves during surgery without any exogenous label or other invasive procedures. Using this label-free technique, we visualized the reflectance signal change of nerve and non-nerve tissues at different wavelengths and different depths under ex vivo and in vivo conditions with multiphoton microscope equipped with wavelength tunable laser and reflectance detection filters. The detected patterns highlight several peripheral nerves with myelin showing strong reflectance at 820-900 nm over other non-nerve tissues including muscle, fat, and vein. Excellent nerve-to-muscle contrast was shown using spectral reflectance technique (contrast value of 5.1 at $850 \mathrm{~nm}$ ) compared to conventional nerve staining dye, fluoromyelin (contrast value of 1.9 at $850 \mathrm{~nm}$ ). Notably, evaluation of intraoperative imaging performance with multiple murine cancer models demonstrated significantly higher nerve-to-muscle contrast in specific wavelength regions in cancer models compared to normal mice. Imaging in breast cancer model depicted contrast value of 3.2 at $900 \mathrm{~nm}$ and 4.3 at $960 \mathrm{~nm}$ whereas normal model imaging showed 2.0 at $900 \mathrm{~nm}$ and 2.3 at $960 \mathrm{~nm}$. This result implicates structural change of nerve by interaction with cancer cells resulting in enhanced myelin-induced reflectance signal which provides potential for clinical translation of our developed nerve identification technique in real cancer surgery. Intraoperative imaging in xenograft models of 4T1-lec2-RFP breast cancer and K562-GFP myelogenous leukemia showcased successful visualization of peripheral nerves under heterogenous cancer environment. Our novel technique provides high potential for a powerful navigation tool highlighting the exact location of peripheral nerves with high specificity and no toxicity to guide surgeons, which allows complete preservation of nerve structures. This enables minimally invasive surgery to improve overall patient outcome and to provide better quality of patient's life after cancer treatment.
\end{abstract}




\section{Image/Figure:}

https://www.xcdsystem.com/wmis/abstract/File6959/GA225_ImageFigure 0423014139.jpg

Full Name of Abstract's 1st Author : Ngai Nick Alex Wong

Complete Status: Complete

First Name: Ngai Nick Alex

Last Name: Wong

Email: 18041854r@connect.polyu.hk

Organization: The Hong Kong Polytechnic University

Country: Hong Kong 


\title{
ID: GA227 \\ 18F-FAC PET can image immune activation and reveals potential functional role of deoxyribonucleoside salvage pathway in EAE mouse model of MS.
}

\author{
Bao Ying Chen, University of California, Los Angeles, BaoYingChen@mednet.ucla.edu
}

\section{Category: Neuroscience}

\begin{abstract}
Body : Introduction: The immune system is complex, with different activities occurring at different anatomical locations. Autoreactive activated lymphocytes are responsible for pathology in diseases such as multiple sclerosis (MS) and promote the activation of additional leukocyte populations. Limiting the activity of these activated cells is the main goal of immunomodulatory therapies 1,2 . However, there is no strategy that specifically images immune cell activation as a whole. Positron emission tomography (PET) imaging can non-invasively image biochemical processes in vivo3. 18F-FAC is a deoxycytidine analogue PET radiotracer that measures deoxyribonucleoside salvage, a pathway that is enriched in activated immune cells4,5 and controlled by the rate-limiting enzyme deoxycytidine kinase (dCK). Objective: To characterize 18F-FAC PET across disease progression in experimental autoimmune encephalomyelitis (EAE), a mouse model of MS, to investigate whether 18F-FAC PET can function as a biomarker for an immunomodulatory therapy in EAE, and to determine whether the deoxyribonucleoside salvage pathway has functional relevance in immune activation. Methods: To induce EAE, 10-week-old female immunocompetent C57BL/6 were injected with myelin oligodendrocyte glycoprotein in Freund's complete adjuvant and pertussis toxin. To study 18FFAC PET across disease progression, EAE and control mice were imaged on Day 7 (presymptomatic state), Day 14 (peak disease), Day 21 (stabilized state), and Day 28 (late disease) post-induction. Ex vivo biodistribution studies on perfused EAE mice were conducted to validate the image quantification. To determine whether $18 \mathrm{~F}-\mathrm{FAC}$ can be a biomarker for immunomodulatory treatments, mice were treated with fingolimod $(0.5 \mathrm{mg} / \mathrm{kg})$ or vehicle and imaged on Day 14. TRE-515 is a dCK inhibitor. To start to study the deoxyribonucleoside salvage pathway in EAE, we evaluated the pharmacodynamics of TRE-515 in EAE. C57BL/6 female mice were treated with TRE-515 $(75 \mathrm{mg} / \mathrm{kg}) 16$ and 4 hours before 18F-FAC PET imaging. For all imaging, mice were injected with $\sim 40 \mu \mathrm{Ci}$ of $18 \mathrm{~F}-\mathrm{FAC}$ and imaged on the HiPET one-hour post-injection. Results: Immune activation differs in different organs across different time points in EAE and can be visualized using 18F-FAC PET. 18F-FAC accumulation was increased two-fold in the brain, spleen, and lymph nodes by day 7, even before clinical symptoms. This accumulation was maintained in the brain throughout day 14, and in the spleen and lymph nodes through day 28. Biodistribution studies confirmed the PET quantification results. Ex vivo studies of cells isolated from the brain showed that CD4 and CD11b cells accumulate the radiotracer at similar levels. 18F-FAC PET can be a biomarker to examine the efficacy of immunomodulatory treatments in EAE. Fingolimod is an immunomodulatory drug used in MS that sequesters leukocytes in the lymph nodes and spleen. Fingolimod significantly decreased brain 18F-FAC accumulation by two-fold in fingolimod compared to vehicle-treated EAE mice. Fingolimod had no effect on 18F-FAC accumulation in the spleen and lymph nodes. TRE-515, a small molecule inhibitor of dCK, blocks the deoxyribonucleoside salvage pathway in
\end{abstract}


vivo. Mice treated with TRE-515 accumulated two-fold less 18F-FAC in the spleen than control treated mice, suggesting that TRE-515 has efficacy in vivo. Future studies will evaluate how TRE-515 effects immune cells in EAE mice. Innovation/Impact: These results are the first to show that $18 \mathrm{~F}-\mathrm{FAC}$ PET can be used as a biomarker for immune activation and assessing immunomodulatory therapies. This study is also the first to assess the role of the deoxyribonucleoside salvage pathway in immune activation. Understanding the biology behind 18F-FAC PET can enhance our understanding of the PET images. If translated into the clinic, patients with autoimmune diseases could benefit from using PET imaging to guide immunomodulatory treatments.

References: 1. Compston A, Coles A. Multiple sclerosis. Lancet. 2008;372(9648):1502-1517. doi:10.1016/S0140-6736(08)61620-7 2. Wingerchuk DM, Carter JL. Multiple sclerosis: Current and emerging disease-modifying therapies and treatment strategies. Mayo Clin Proc. 2014;89(2):225-240. doi:10.1016/j.mayocp.2013.11.002 3. Phelps ME. Positron emission tomography provides molecular imaging of biological processes. Proc Natl Acad Sci U S A. 2000;97(16):9226-9233. doi:10.1073/pnas.97.16.9226 4. Radu CG, Shu CJ, Nair-Gill E, et al. Molecular imaging of lymphoid organs and immune activation using positron emission tomography with a new 18 F-labeled 2'-deoxycytidine analog HHS Public Access Author manuscript. Nat Med. 2008;14(7):783-788. doi:10.1038/nm1724 5. Chen BY, Ghezzi C, Villegas $\mathrm{B}$, et al. 18 F-FAC PET visualizes brain-infiltrating leukocytes in a mouse model of multiple sclerosis . J Nucl Med. October 2019:jnumed.119.229351. doi:10.2967/jnumed.119.229351

Full Name of Abstract's 1st Author : Bao Ying Chen

Complete Status: Complete

First Name: Bao Ying

Last Name: Chen

Email: BaoYingChen@mednet.ucla.edu

Organization: University of California, Los Angeles

Country: United States 


\title{
ID: GA228 \\ In vivo trafficking of the anti-cancer drug [Ga(oxinate) 3] (KP46) studied with gallium-68 PET imaging
}

\author{
afnan darwesh, Biomedical Engineering \& Imaging Sciences department, King's College \\ London, afnan.darwesh@kcl.ac.uk
}

\section{Category: Oncology}

\begin{abstract}
Body : Background: Tris (8-hydroxyquinolinato) gallium (III), (KP46, [Ga(oxinate)3]) has been investigated in recent years as an orally administered anti-cancer drug.1,2 Despite extensive preclinical and clinical evaluation of its efficacy, its trafficking to tumours and other tissues remains poorly understood. $68 \mathrm{Ga}$ is a readily available positron-emitting radionuclide used for positron emission tomography (PET), offering the opportunity to study the in vivo trafficking of KP46 by PET. Here we report findings of $68 \mathrm{Ga}$ trafficking in vitro and in vivo in mice after oral and intravenous administration of [68Ga]KP46. Methods: KP46 and [68Ga]KP46 were synthesised by literature methods3,4. Binding of [68Ga]KP46 to Apotransferrin and albumin was measured by size exclusion chromatography. In vivo: Mice in groups A, B and C (n =3) carried A375 human melanoma xenografts. Group A were given intravenous injections of [68Ga]KP46 subjected to PET imaging over $4 \mathrm{~h}$. Groups B and C were orally administered with [68Ga]KP46 or with [68Ga]KP46 combined with KP46 respectively. Mice were allowed to recover for 3 hours before being re-anaesthetised and scanned for $1 \mathrm{~h}$. All groups were sacrificed for organ harvesting at $4 \mathrm{~h}$. Octanol extraction and tissue digestion was performed on tissue samples from groups B and $\mathrm{C}$ to test the form of [68Ga]KP46 and measure gallium-69 by ICPMS. Results: [68Ga]KP46 showed no binding to Apotransferrin and minimal binding to human serum albumin (HSA) indicating the stability of [68Ga]KP46. In group A mice, [68Ga]KP46 did not deliver $68 \mathrm{Ga}$ to tumour $(2.6 \pm 0.9 \% \mathrm{ID} / \mathrm{g})$ and most activity concentrated in liver $(28.4 \pm 6.6 \%$ $\mathrm{ID} / \mathrm{g})$ and heart $(16.9 \pm 2.1 \% \mathrm{ID} / \mathrm{g}) .68 \mathrm{Ga}$ acetate, on the other hand, showed tumour accumulation $(5.8 \pm 2.4 \% \mathrm{ID} / \mathrm{g})$ probably by transferrin dependant mechanism. In group B, most activity remained in the large intestine $(61.8 \pm 21.5 \% \mathrm{ID} / \mathrm{g})$, with minimal trafficking of radioactivity to other tissues. The majority of radioactivity in tissue samples was in a hydrophilic form, i.e. no longer in the form of [68Ga]KP46. Adding therapeutic dose of KP46 to group $\mathrm{C}$ showed no trafficking change and most activity remained in the large intestines $(56.5 \pm 21.4 \% \mathrm{ID} / \mathrm{g})$. ICP-MS measurement of gallium-69 in tissue samples was consistent with $68 \mathrm{Ga}$ trafficking results. Conclusion: After oral administration of [68Ga]KP46 either as tracer or as bulk drug, gallium is not absorbed from the gut and is not delivered to tumour within the timeframe investigated. We suggest that in this short timescale, delivery of gallium to tumour is not a significant component of the mode of action of this drug. Delivery of 8-hydroxyquinoline could be investigated in the future as an alternative hypothesis for its mode of action. Longer half-life [67Ga]KP46 can be administered orally with KP46 to study the in vivo trafficking of [67Ga]KP46 in the presence of its therapeutic dose at longer time period.
\end{abstract}

References: 1. C. R. Chitambar., Expert Opin. Investig Drugs, 2004, 13, 531-541. 2. H. Yu, J. R. Lou and W. Q. Ding., Anticancer Res, 2010, 30, 2087-2092. 3. P. Collery, J. L. Domingo and B. 
K. Keppler., Anticancer Res, 1996, 16, 687-692. 4. Y. Yano, T. F. Budinger, S. N. Ebbe, C. A. Mathis, M. Singh, K. M. Brennan and B. R. Moyer., J. Nucl. Med., 1985, 26, 1429-1437.

\section{Image/Figure:}

https://www.xcdsystem.com/wmis/abstract/File6959/GA228_ImageFigure_0422055230.PNG

Complete Status: Complete

First Name: afnan

Last Name: darwesh

Email: afnan.darwesh@kcl.ac.uk

Organization: Biomedical Engineering \& Imaging Sciences department, King's College London

Country: United Kingdom 


\title{
ID: GA230 \\ A fast, robust method for the synthesis of radiomanganese (Mn-52) porphyrin complexes: applications in cell and liposome labelling.
}

\author{
Peter Gawne, King's College London, peter.gawne@kcl.ac.uk
}

\section{Category: New Chemistry, Biology \& Bioengineering}

\begin{abstract}
Body : Manganese porphyrins are known to have several therapeutic and imaging applications. Their superoxide dismutase activity makes them efficient tumour chemo/radiosensitisers - with several agents in clinical trials.[1] Additionally, their paramagnetism allows them to be used as MRI contrast agents.[2] Porphyrins also have a wellestablished affinity for lipid bilayers which also makes them candidates as cell and liposome labeling agents. It was hypothesised that metallation with the PET radionuclide $52 \mathrm{Mn}(\mathrm{t} 1 / 2=5.6$ d) would allow long-term in vivo biodistribution studies of Mn-porphyrins as well, as a method to label and track cells and liposomes. Despite this, methods for fast and efficient radiolabelling of radiomanganese porphyrins are lacking. In this work, a radiosynthesis method for $52 \mathrm{Mn}-$ porphyrins was designed, and the cell and liposome labelling ability of these agents were then tested. $52 \mathrm{MnCl} 2$ and the porphyrin ligands $1-4$ (Fig. 1A) were produced as previously
\end{abstract} reported.[3,4] Porphyrins $1-4$ were added to neutralised 52MnCl2 and heated at $165 \mathrm{oC}$ for $1 \mathrm{~h}$ in a microwave (MW) synthesizer (Fig. 1A) at a porphyrin concentration of $0.6-0.7 \mathrm{mM}$. These conditions were compared with heating at $70 \mathrm{oC}$ using a heating block. The radiochemical yields (RCYs) were calculated by iTLC/TLC and HPLC, and the resulting 52Mn-porphyrin complexes characterised by comparison with their non-radioactive 55Mn counterparts and $\log \mathrm{P}$ measurements. Following this, the porphyrin complexes 52Mn- $(1-4)$ were used to radiolabel empty DOXIL-like liposomes. 52Mn- $(1-4)$ were incubated with the liposomes at $50 \mathrm{oC}$ for 30 min and purified by size-exclusion chromatography. Additionally, the porphyrin complexes $52 \mathrm{Mn}-(1-2)$ were taken forward and used to radiolabel MDA-MB-231 cells in vitro which was directly compared with a previously reported direct cell labelling agent, 52Mn-oxine.[5] The MW radiosynthesis method allowed fast $(1 \mathrm{~h})$ and efficient radiolabelling with $>95 \%$ radiochemical yields with ligands $1,2 \& 4$, and $85 \pm 7 \%$ for 52Mn-(3). The RCY of 52Mn-(3) increased to $95 \pm 2 \%$ by using a ligand 3 concentration of $0.7 \mathrm{mM}$ (Fig. 1B). Conversely, nonMW heating at $70 \mathrm{oC}$ for $1 \mathrm{~h}$ resulted in ca. $25 \% \mathrm{RCY}$ for $52 \mathrm{Mn}-(2 \& 4)$, with no conversion for $52 \mathrm{Mn}-(1 \& 3)$ (Fig. 1B). Whilst the RCYs increased after $24 \mathrm{~h}$ at $70 \mathrm{oC}$, none of the reactions reached completion. Formation of the 52Mn-complexes were confirmed with radioHPLC (representative 52Mn-(1) trace in Fig 1C), iTLC (Fig. 1D) and log P. 52Mn- $(1-3)$ labelled empty DOXIL-like liposomes with high efficiencies of $78 \pm 3 \%, 86 \pm 2 \%$ and $74 \pm 7$ $\%$, respectively (Fig. 1E). 52Mn- $(1-2)$ radiolabelled MDA-MB-231 cells with moderate efficiencies of $10 \pm 7 \% \mathrm{LE}$ and $12 \pm 1 \% \mathrm{LE}$, respectively (Fig. $1 \mathrm{~F}$ ) whereas $52 \mathrm{Mn}$-oxine had a higher LE of $42 \pm 2 \%$ as previously reported.[5] However, the cellular retention of $52 \mathrm{Mn}$ after $24 \mathrm{~h}$ via $52 \mathrm{Mn}$-oxine radiolabelling was significantly lower $(14 \pm 1 \%)$, compared with 52Mn-(1) $(32 \pm 5 \%)$ and $52 \mathrm{Mn}-(2)(45 \pm 4 \%)$ (Fig. 1G). This suggests a distinct mechanism of trapping from the 52Mn-oxine methodology. In contrast to standard heating methods, MW heating allows the fast, efficient radiosynthesis of a variety of 52Mn-porphyrins with $>95 \%$ RCYs - avoiding 
the need for purification. This technique can be exploited for the radiolabeling and subsequent in vivo imaging of Mn-porphyrin therapeutics, as well as for the accurate in vivo quantification of Mn-porphyrin-based MRI agents. 52Mn-porphyrins also show promising cell and liposome labelling properties, with improved 52Mn cell retention over direct labelling methods using 52Mn-oxine.

References: [1] Batinic-Haberle I et al., Redox Biology. 2019, 25, 101139 [2] Calvete M. et al., Coord Chem Rev. 2017, 333, 82-107 [3] Fonslet J. et al., App Rad Isop. 2017, 121, 38-43 [4] Monteiro C. et al., Tetrahedron, 2008, 64, 5132-5138 [5] Gawne P. et al., Dalton Trans. 2018, 47, 9283-9293

\section{Image/Figure:}

https://www.xcdsystem.com/wmis/abstract/File6959/GA230_ImageFigure_0609084627.jpg

Image/Figure Caption: Fig. 1: A) Structures of the porphyrin ligands 1-4 used in this work along with the reaction scheme for the microwave synthesis method. B) Comparison of the RCYs achieved over $24 \mathrm{~h}$ for the microwave synthesis method compared to that achieved with labelling at $70 \mathrm{oC}$ for the 52Mn-porphyrins. C) Radio HPLC trace (top) of 52Mn-(1), UV trace of non-radioactive Mn-(1) (bottom). Note the presence of isomers for the porphyrin ligand. D) iTLC/TLC characterisation of the 52Mn-porphyrin complexes. E) Graph showing the $\%$ liposome labelling of the 52Mn-porphyins complexes. F) Graph showing the \% cell labelling of $52 \mathrm{Mn}-(1)$ and 52Mn-(2) with MDA MB-231 cells compared with 52Mn-oxine and unchelated $52 \mathrm{Mn}$. G) Graph showing the cellular retention of 52Mn in MDA MB-231 cells labelled with $52 \mathrm{Mn}$-oxine, 52Mn-(1) and 52Mn-(2) after $24 \mathrm{~h}$. Error bars represent mean $\pm \mathrm{SD}(\mathrm{n}=3)$ except for the $70 \mathrm{oC} \operatorname{RCY}(\mathrm{n}=1)$

Complete Status: Complete

First Name: Peter

Last Name: Gawne

Email: peter.gawne@kcl.ac.uk

Organization: King's College London

Country: United Kingdom 


\title{
ID: GA233 \\ Radial k-space sampling combined with KWIC-reconstruction enables motion robustness in DCE-MRI of genetically engineered mouse model of pancreatic cancer
}

\author{
Jianbo Cao, University of Pennsylvania, caojianboce@gmail.com
}

\section{Category: Systemic Diseases (Kidney, Liver and Pancreas)}

\begin{abstract}
Body : Introduction: Pancreatic ductal adenocarcinoma (PDA) is a highly lethal disease with a 5-year survival rate only $8 \%$. PDA is characterized with a dense stroma which underlies the chemoresistance and immune-suppressive microenvironment and pose the barrier for the treatment. DCE-MRI is a sensitive tool for the tumor microvascular perfusion/permeability assessment, has shown the potential for the PDA stroma-directed drug effect monitor1. The genetically engineered mouse (GEM) model of PDA recapitulates the human disease including the dense stroma, is a perfect model for therapeutic and biological investigation for PDA. However, the upper abdomen location of the pancreatic cancer makes it highly motion susceptible, Moreover, the fast respiration rate as well as the small blood vessel size, poses unique challenges for the DCE-MRI study in mice. In this study, we present a radial k-space sampled DCE-MRI protocol that achieves motion-robustness simultaneously with high temporal resolution for capturing the arterial input function (AIF) and spatial resolution of tumor DCE images . Methods: A genetically engineered mouse (GEM) that harbors Kras and p53 mutation in pancreas specific Cre allele, referred to as KPC model of PDA, was used in this study2. MRI was performed on 9.4T system 1. DCE series was acquired using multi-slice 2D cardiac gated saturation recovery GRE employing golden-angle $\left(111.246^{\circ}\right)$ radial k-space sampling $(\mathrm{TR} / \mathrm{TE}=2 \mathrm{x}$ cardiac period $\sim 200 / 0.91 \mathrm{msec}, \mathrm{FOV}=38$ x $38 \mathrm{~mm} 2)$. Radial data were regridded to $256 \times 256$ matrix with or without employing k-space-weighted image contrast (KWIC) filter3,4 (Fig 1A). For KWIC reconstruction, 25 views encoding the center and 200 views encoding the outer-most regions of k-space region were used. Non-KWIC reconstruction utilized 200 views (according to Nyquist criterion) throughout the entire k-space. The AIF was extracted from the blood signal in heart left ventricle. Pixel-wise DCE series combined with T10 map and individual-AIF was least squares fit to the "shutter-speed" pharmacokinetic model to extract parameter maps including Ktrans. Results: The AIF extracted from fully sampled images (Fig 1 B-C) exhibits a blunted profile (Fig 1 F-G) due to relatively low temporal resolution, whereas AIF extracted from KWIC-reconstructed images (Fig 1 D-E) provide sufficient temporal resolution to fully characterize the initial rise of the AIF. . In the absence of respiratory gating, KWIC reconstruction resulted in motion-free high-resolution abdominal images including the PDA tumor (Fig 2A-C). Selected DCE images reconstructed without KWIC (Fig 3C1C5)and with KWIC (Fig 3A1-A5) demonstrated minimal differences in image quality . The tumor ROI DCE signal time course resulted from the two reconstruction methods revealed that KWIC time course captures more time points during rapid wash-in phase of the contrast agent (Fig 3B, D). Tumor T1, Ktrans and kep values $(\mathrm{T} 1=2.5 \pm 0.15 \mathrm{~s}$, Ktrans $=0.09-0.33 / \mathrm{min}$, and $\mathrm{kep}=0.5-1.6 / \mathrm{min}$ ) obtained from parameter maps (Fig 3E, F, G) are comparable to previously reported values generated using Cartesian sampling methods at significantly lower spatial
\end{abstract}


resolution1. Conclusion: Radial k-space sampling yields motion-free DCE images of PDA tumor in mice. The view sharing technique (KWIC) is highly compatible with radial data which is flexible in degrees of under sampling during image reconstruction to obtain optimal temporal resolution of AIF and spatial resolution of tumor DCE images. Acknowledgements This study was supported by U24CA231858 (Penn Quantitative Imaging Resource for Pancreatic Cancer), R21CA198563, R01CA211337 and P30-CA-016520-42.

References: 1. Clinical Cancer Research; 2019;25(7):2314-2322 2. Cancer Cell 2003;4(6):437450. 3. Magn Reson Med; 2000;44(6):825-832. 4. Magn Reson Med 2004;52(4):815-824.

\section{Image/Figure Caption:}

Full Name of Abstract's 1st Author : Jianbo Cao

Complete Status: Complete

First Name: Jianbo

Last Name: Cao

Email: caojianbocc@gmail.com

Organization: University of Pennsylvania

Country: United States 


\title{
ID: GA234 \\ Effects of PK model and AIF selection for DCE-MRI detection of pancreatic cancer response to stroma-directed drug
}

\author{
Jianbo Cao, University of Pennsylvania, caojianbocc@gmail.com
}

\section{Category: Computational \& Data Science}

\begin{abstract}
Body : Introduction: The dense stroma poses a desmoplastic microenvironment in the pancreatic ductal adenocarcinoma (PDA)1,2, poses a barrier for the effective treatment. Overcoming this barrier has motivated the development of stroma-directed drugs to reduce or degrade the extracellular matrix components in PDA. The DCE-MRI has been proved to be an effective way to monitor the effect of this kind of drug3 while some questions need to be investigated more thoroughly to facilitate the clinical translation of this technique. In this study, we compared DCE-metric obtained from pharmacokinetic (PK) model in combination with individual- arterial input function (AIF) or a group-AIF for the ability to detect treatment effect. Corroborating imaging and immunostaining data, this study aims to provide rationale supporting choice of PK model and AIF approach which lead to quantitative imaging marker with optimal sensitivity and specificity for stroma-directed drug. Methods: The orthotopic PDA model was generated by injection $1.25 \times 1054662-\mathrm{KPC}$ cells into the pancreas of syngeneic mice by a surgical procedure4. On a 9.4 system (Agilent) with a $12-\mathrm{cm}$ gradient coil, DCE-MRI were performed at baseline and 24 hours after the PEGPH $20(1 \mathrm{mg} / \mathrm{kg}$ ) or vehicle (VEH) treatment. The imaging protocol include the T10 map of tumor and blood (left ventricle) and DCE series3. The individual AIFs were extracted from the DCE series and the group-AIF was the average of 20 individual AIFs in 10 mice, two from each mouse obtained at baseline and post treatment. Pixel wise DCE data were fitted for three different PK models including Tofts (T), ModifiedTofts (M-T) and Shutter-Speed (SS) model using the least-squares method combined with the group or individual AIF 5-8. Pixel-wise parametric maps of Ktrans, kep, Ve and Vp were obtained as output. Responses to PEGPH20 was confirmed by reduction hyaluronan level by IHC compared to VEH-treated mice as reported earlier3. Results: While all mice were from the same strain and similar in age, individual AIFs exhibit a great difference in amplitude, leading to the highest SD at the peak point of group-AIF (Fig 1A), the variation of AIF may result from physiological conditions at the time of study or occasionally imperfect bolus injection of contrast agent. Mean Ktrans of each tumor (Fig 1B) exhibit good correlation between individual versus group-AIF with Pearson coefficient $r=0.86$. For kep metric, individual-AIF allows both SS- and T-model to detect a significant increase of kep from baseline to post-PP, whereas using group-AIF, only SS model detects such increase (Fig 2A, D). In comparison, for Ktrans metric, group-AIF combined with SS-model detected a significant increase of this metric from pre and post-treatment but individual-AIF is not able to do when combining with Tofts or SS PK model (Fig 2B, E). For the metric of \%change of Ktrans, SS model combined with either individual- or group-AID can detect a significant difference between PP vs. VEH treatment (Fig 2C, F). Notably, using the group-AIF, the M-T model revealed a moderate but significant increase of Vp after PP treatment (Fig 3A). Correspondingly, the "vascular lumen area" index obtained from CD31 analysis exhibited an average of $48 \%$ increase after PP versus VEH treatment, although
\end{abstract}


statistical significance was not reached due to small sample size (Fig 3B-3C). Conclusion: SS model appears to be more sensitive than Tofts or M-T model for detection of treatment effect when applied with either individual- or group-AIF. Tofts model, however, is capable of detecting the treatment effect only when combined with Individual-AIF approach. Further validation of Vp metric is ongoing by IHC analysis. Acknowledgements: This study was supported by U24CA231858 (Penn Quantitative Imaging Resource for Pancreatic Cancer), R21CA198563, R01CA211337 and P30-CA-016520-42.

References: 1. Gut 2018;67(2):320-332. 2. J Clin Oncol 2018;36(4):359-366 3. Clinical cancer research; 2019;25(7):2314-2322 4. Cancer Res 2015;75(14):2800-2810 5. J Magn Reason Imaging 1997;7(1):91-101 6. Magn Reson Med 2004;52(2):248-257 7. Magn Reson Med 2005;54(6):1351-1359 8. Magnetic Resonance in Medicine 2012;67(1):226-236

Full Name of Abstract's 1st Author : Jianbo Cao

Complete Status: Complete

First Name: Jianbo

Last Name: Cao

Email: caojianbocc@gmail.com

Organization: University of Pennsylvania

Country: United States 


\title{
ID: GA235 \\ Breast cancer risk analysis based on the parenchyma region in mammographic images
}

\author{
Liyu Huang, Xidian University, huangly@mail.xidian.edu.cn
}

\section{Category: Computational \& Data Science}

\begin{abstract}
Body : Objectives: To propose a way to predict the risk of breast cancer as early as possible, especially before a visible tumor appears. Methods: The parenchyma region was framed near to but not overlapping with the malignant tumor region delineated by professional radiologists. This region of interest was then used for the extraction of quantitative features, which were filtered by two methods consecutively: statistical analysis and the least absolute shrinkage and selection operator. The selected features were integrated to construct a radiomics signature, which was then evaluated by statistical analysis and support vector machine (SVM). Subsequently, a radiomics nomogram was established with this signature and "age", which was assessed by $\mathrm{C}$-index, calibration curves and decision curves. Results: A radiomics signature, which consisted of 16 selected textural features, showed significantly differences (P 0.05 for the three cohorts, Hosmer-Lemeshow test) and clinically useful. Conclusion: The parenchyma region contains some lesion information before a visor tumor appears. Thus, it is possible to apply this information in breast cancer risk prediction.
\end{abstract}

References: [1] Kontos D, Winham SJ, Oustimov A, et al. Radiomic Phenotypes of Mammographic Parenchymal Complexity: Toward Augmenting Breast Density in Breast Cancer Risk Assessment. Radiology 2019;290(1):41-9. [2] Li H, Mendel KR, Lan L, Sheth D, Giger ML. Digital Mammography in Breast Cancer: Additive Value of Radiomics of Breast Parenchyma. Radiology 2019;291(1):15-20. [3] Marin Z, Batchelder KA, Toner BC, et al. Mammographic evidence of microenvironment changes in tumorous breasts. Med Phys 2017;44(4):1324-36. [4] Yang J, Wang T, Yang L, et al. Preoperative Prediction of Axillary Lymph Node Metastasis in Breast Cancer Using Mammography-Based Radiomics Method. Sci Rep 2019;9(1):4429. [5] Li H, Zhu Y, Burnside ES, et al. Quantitative MRI radiomics in the prediction of molecular classifications of breast cancer subtypes in the TCGA/TCIA data set. Npj Breast Cancer 2016;2:16012. [6] Guo Y, Hu Y, Qiao M, et al. Radiomics Analysis on Ultrasound for Prediction of Biologic Behavior in Breast Invasive Ductal Carcinoma. Clin Breast Cancer 2018;18(3):e335-e44. [7] Yang L, Yang J, Zhou X, et al. Development of a radiomics nomogram based on the 2D and 3D CT features to predict the survival of non-small cell lung cancer patients. Eur Radiol 2019;29(5):2196-206. [8] Zhao L, Gong J, Xi Y, et al. MRI-based radiomics nomogram may predict the response to induction chemotherapy and survival in locally advanced nasopharyngeal carcinoma. Eur Radiol 2020;30(1):537-46.

\section{Image/Figure:}


Complete Status: Complete

First Name: Liyu

Last Name: Huang

Email: huangly@mail.xidian.edu.cn

Organization: Xidian University

Country: China 


\title{
ID: GA236 \\ Comparison of Motion-Robustness and Reproducibility of Diffusion-Weighted Radial and Multishot EPI MRI of Orthotopic Pancreatic Cancer in Mice
}

\author{
Jianbo Cao, University of Pennsylvania, caojianbocc@gmail.com
}

\section{Category: Oncology}

\begin{abstract}
Body : Introduction Rapid respiration in mice and high magnetic fields of preclinical MRI scanners pose major challenges for quantitative diffusion weighted MRI (DWI) of the mouse abdomen. Two motion-resistant acquisition methods, radial spin-echo and EPI were evaluated for their effectiveness in mitigating motion and susceptibility artefacts in diffusionweighted images in a genetically engineered mouse (GEM) model of pancreatic ductal adenocarcinoma (PDA). The repeatability and accuracy in measuring diffusion coefficient were also assessed in a test-retest setting. Methods MRI was performed on a 4.7T horizontal bore system (Agilent) interfaced with a 12-cm gradient coil. Animals were anesthetized via 1.5-3\% isoflurane, the temperature and respiration were monitored and maintained at the physiological level during the scan. Two phantoms containing water and butanol, respectively, were placed alongside the mouse for reference. Prospective respiratory gating was applied to both radially sampled diffusion weighted spin echo sequence(DW-SE-RAD) $(\mathrm{TE}=30 \mathrm{msec}, \mathrm{TR}=2-3$ respiratory periods, slice thickness $=0.5 \mathrm{~mm}, \mathrm{FOV}=32 \times 32 \mathrm{~mm} 2$, matrix $=64$ read $\times 101$ views, averages $=1$, total acquisition time $=20-38 \mathrm{~min}$, depending on respiratory rate and number of slices) and adiffusion-weighted spin-echo multi-shot EPI (DW-SE-EPI) sequence (TE $=26 \mathrm{msec}, \mathrm{TR}=4$ respiration periods, slice thickness $=1.5 \mathrm{~mm}$, FOV $=32 \times 32 \mathrm{~mm} 2$, matrix $=$ $128 \times 128$, averages $=16$, segments $=4$, total acquisition time $=20-35 \mathrm{~min}$ ) that spans the same tumor volume. Both sequences employ the same diffusion preparation parameters 1: diffusion time $\Delta=14.4 \mathrm{msec}$, diffusion gradient duration, $\delta=9 \mathrm{msec}$ with five $\mathrm{b}$-values $(0.64,535,1071$, $1478,2141 \mathrm{~mm} 2 / \mathrm{s})$. All diffusion gradients were oriented along the diagonal direction; images were acquired with both positive and negative diffusion gradients. DW-SE-RAD and DW-SEEPI protocols were also applied to a pair of tumor-bearing mice with and without prospective respiratory gating to assess the need for such gating. For each diffusion protocol, a test-retest study was performed in ten mice with 2-4 hours interval when mice were fully recovered from anesthesia. Apparent diffusion coefficient (ADC) was estimated in phantoms, tumours, and spinal muscles. The correlation of ADC between the two protocols was evaluated by GEE model in the R software; the reproducibility metrics include within-subject SD and CV and repeability coefficient (RC). Results Images from the DW-SE-EPI protocol without prospective gating were highly motion corrupted and meaningful ADC maps could not be generated. A comparison between DW-SE-RAD images generated with and without respiratory gating revealed streaking artifacts in non-gated images, resulting in increased noise in the ADC maps (Figure 1). Therefore, respiratory gating was applied to both protocols in all studies reported below. The radial-DWI protocol produces artifact-free images over all b-values examined whereas ghosting is evident in the phase encoding direction (left to right) at all b-values and has comparable magnitude to the desired signal in the high b-value images. (Figure 2). ADC of water phantom estimated by Radial-DWI is consistent with well-known literature value 3 whereas it is
\end{abstract}


underestimated 12\% (P for Pancreatic Cancer), R21CA198563, R01CA211337 and P30-CA016520-42

References: 1. Nano Today 2015;10(1):11-21 2. J Magn Reson Imaging 2019;49(7):e101-e121 3. J Cereb Blood Flow Metab;1998;18(7):776-802.

Full Name of Abstract's 1st Author : Jianbo Cao

Complete Status: Complete

First Name: Jianbo

Last Name: Cao

Email: caojianbocc@gmail.com

Organization: University of Pennsylvania

Country: United States 


\title{
ID: GA237 \\ CX3CL1/CX3CR1-dependent accumulation of regulatory macrophages promotes recurrence of intrahepatic cholangiocarcinoma in clinical and animal studies
}

\author{
Kwan Man, The University of Hong Kong, kwanman@hku.hk
}

\section{Category: Oncology}

\begin{abstract}
Body : Background: Understanding of the mechanism of intrahepatic cholangiocarcinoma (iCCA) recurrence after curative surgery will be critical for development of prophylactic strategies. Regional immune-regulation in graft and tumor microenvironment may play a critical role of iCCA recurrence after curative surgery. Regulatory macrophages (Mregs) have potent immunosuppressive function. Currently, the existence and role of Mregs in iCCA remain unknown. Here, we aim to investigate the role and mechanism of Mregs on iCCA recurrence after curative surgical treatment. Methods: Gene expression data from our institute and TCGA were analysed to find out possible interaction between iCCA and monocytes. The serum CX3CL1 concentration and peripheral CX3CR1+ monocytes were assessed. A total of 75 patients with iCCA was included, and prospectively followed-up since 2001 . The clinical significance of CX3CL1 expression and Mregs was evaluated by Cox proportional hazards regression model. The phenotypic analyses of Mregs from fresh iCCA tissue were conducted by flow cytometry. Results: 1 . Higher serum CX3CL1 concentration and more peripheral CX3CR1+ monocytes were detected in post-transplantation patients (Fig.1). CX3CR1+ CD68+ macrophages could be observed in LT explant with iCCA. 2. Elevated CX3CL1 expression and more CX3CR1+ CD68+ macrophages in iCCA tissue were correlated with shorter disease-free survival (DFS) in iCCA patients (Fig.2); 3. In multivariate model, CX3CR1+ CD68+ macrophages were identified as independent prognostic factor for DFS (HR:3.400, 95\%CI: 1.900-6.084, P Conclusion: CX3CL1/CX3CR1axis promoted iCCA recurrence through recruiting regulatory macrophages. Background: Understanding of the mechanism of intrahepatic cholangiocarcinoma (iCCA) recurrence after curative surgery will be critical for development of prophylactic strategies. Regional immune-regulation in graft and tumor microenvironment may play a critical role of iCCA recurrence after curative surgery. Regulatory macrophages (Mregs) have potent immunosuppressive function. Currently, the existence and role of Mregs in iCCA remain unknown. Here, we aim to investigate the role and mechanism of Mregs on iCCA recurrence after curative surgical treatment. Methods: Gene expression data from our institute and TCGA were analysed to find out possible interaction between iCCA and monocytes. The serum CX3CL1 concentration and peripheral CX3CR1+ monocytes were assessed. A total of 75 patients with iCCA was included, and prospectively followed-up since 2001. The clinical significance of CX3CL1 expression and Mregs was evaluated by Cox proportional hazards regression model. The phenotypic analyses of Mregs from fresh iCCA tissue were conducted by flow cytometry. Results: 1. Higher serum CX3CL1 concentration and more peripheral CX3CR1+ monocytes were detected in post-transplantation patients (Fig.1). CX3CR1+ CD68+ macrophages could be observed in LT explant with iCCA. 2. Elevated CX3CL1 expression and more CX3CR1+ CD68+ macrophages in iCCA tissue were correlated
\end{abstract}


with shorter disease-free survival (DFS) in iCCA patients (Fig.2); 3. In multivariate model, CX3CR1+ CD68+ macrophages were identified as independent prognostic factor for DFS (HR:3.400, 95\%CI: 1.900-6.084, P Conclusion: CX3CL1/CX3CR1axis promoted iCCA recurrence through recruiting regulatory macrophages.

\section{Image/Figure:}

https://www.xcdsystem.com/wmis/abstract/File6959/GA237 ImageFigure 0422030917.jpg

Complete Status: Complete

First Name: Kwan

Last Name: Man

Email: kwanman@hku.hk

Organization: The University of Hong Kong

Country: China 


\title{
ID: GA243 \\ Specific $\alpha$-toxin radiotracer for the detection of $S$. aureus infections by immunoPET imaging
}

\author{
María Isabel González, Fundación para la Investigación Biomédica Hospital Gregorio \\ Marañón, migonzalez@hggm.es
}

Category: Immunology: Inflammation \& Infection

\begin{abstract}
Body : Introduction: Gram-positive Staphylococcus aureus (S. aureus) bacterium is one of the most frequent causes of infection in humans[1], which severity partially lies in evading defensive barriers through the release of virulent factors like $\alpha$-toxins. Current lack of specific and selective methods for its non-invasive diagnosis leads to false positives caused by erroneous detection of inflammatory processes. This work presents the synthesis and evaluation of a specific $\alpha$-toxin tracer for the detection of S. aureus infections by immunoPET/CT imaging. Methods: Anti S. aureus $\alpha$-toxin antibody ( $\alpha$-ToxAb, $1 \mathrm{mg} / \mathrm{ml}$ ) was conjugated with p-DFO-Bz$\mathrm{NCS}$ at $37^{\circ} \mathrm{C}$ and $\mathrm{pH} 9.0$ for 30 min, employing 1:5 molar excess of chelator. Conjugated product was mixed with $89 \mathrm{Zr}$-oxalic at $25^{\circ} \mathrm{C}$ and $\mathrm{pH} 5.5$ for $1 \mathrm{~h}$ and purified with centrifugal filters[2,3]. Radiochemical purity was measured by HPLC (PBS, $0.2 \mathrm{ml} / \mathrm{min}$ ). Two animal models of local infection were established in Sprague Dawley rats: active S. aureus was injected in left hind leg while right hind leg was inoculated with either heat-killed S. aureus or active E. coli. Bacteria suspensions $(0.4 \mathrm{ml}, 108 \mathrm{CFU} / \mathrm{ml})$ were inoculated $24 \mathrm{~h}$ before radiotracer administration. Ex vivo microbiological analysis and immunohistochemistry were carried out at endpoint. Pharmacokinetics and biodistribution of $89 \mathrm{Zr}-\alpha-\mathrm{ToxAb}$ were assessed by in vivo blood half-life, ex vivo evaluation and digital autoradiography ( $\mathrm{n}=3$ per animal model). Animals underwent PET/CT imaging ( $400 \mu 1,250-300 \mu \mathrm{Ci}, \mathrm{n}=2-5$ per time point) at 1, 24, 48 and 72 h. Results: Synthesized $89 \mathrm{Zr}-\alpha$-ToxAb reached a specific activity of $14.45 \mathrm{mCi} / \mathrm{mg}$ and 96.16 $\%$ purity (Fig1A). Blood half-life was 2.33 days (Fig1B). In vivo PET/CT confirmed the specificity of $89 \mathrm{Zr}-\alpha-\mathrm{ToxAb}$ for infections induced by $\mathrm{S}$. aureus, reaching an infected-toinflammed ratio of 5.33 and a S. aureus-to-E. coli ratio of 5.89 at $48 \mathrm{~h}$ (Fig1C). These results correlated with ex vivo autoradiography (Fig1D). Microbiological and immunohistochemical analysis confirmed the existence of infections or inflammation (Fig1E). Biodistribution results suggested hepatobiliary metabolism of the tracer $(1.92 \pm 0.59$ and $3.42 \pm 1.79 \% \mathrm{ID} / \mathrm{g}$ in liver and spleen at $48 \mathrm{~h})$, with partial urinary excretion $(3.50 \pm 0.66 \% \mathrm{ID} / \mathrm{g}$ in kidneys at $48 \mathrm{~h})$.

Conclusions: We synthesized a novel $89 \mathrm{Zr}-\alpha$-ToxAb immunoPET tracer for the selective and specific detection of S. aureus infections through targeting the a-toxin released by the bacterium. Our results support the ability of $89 \mathrm{Zr}-\alpha-$-ToxAb to avoid false positives caused by inflammatory processes and its capacity to specifically detect $\mathrm{S}$. aureus against gram negative bacteria.

Promising applications can be found in the diagnosis, treatment optimization and prognosis of staphylococcal infection. Acknowledgment: Authors thank Yolanda Sierra, Alexandra de Francisco and María de la Jara Felipe for their excellent work with animal preparation and imaging protocols. Fundings: This work has been supported by"Diagnosis and treatment followup of severe Staphylococcal Infections with Anti-Staphylococcal antibodies and Immune-PET Grant Fundación BBVA a Equipos de Investigación Científica 2018. This study has been also
\end{abstract}


supported by Ministerio de Ciencia, Innovación y Universidades, Instituto de Salud Carlos III, project "PI16/02037", co-funded by European Regional Development Fund (ERDF), "A way of making Europe" and by Comunidad de Madrid, project "Y2018/NMT-4949 (NanoLiver-CM)" and "S2017/BMD-3867 (RENIM-CM)", co-funded by European Structural and Investment Fund. The CNIC is supported by the ISCIII, the Ministerio de Ciencia e Innovación and the Pro CNIC Foundation, and is a Severo Ochoa Center of Excellence (SEV-2015-0505).

References: References: [1] Baddour, L.M. et al. (2007) Mayo Clin. Proc., 82(10), p.1163-1164; [2] Van Dongen, G. A. M. S. et al. (2010) Nature Protocols, 5(4), p.739-743; [3] Lewis, J.S. et al. (2015) J. Vis. Exp., 96, e52521

\section{Image/Figure:}

https://www.xcdsystem.com/wmis/abstract/File6959/GA243_ImageFigure_0422063220.JPG

Complete Status: Complete

First Name: María Isabel

Last Name: González

Email: migonzalez@hggm.es

Organization: Fundación para la Investigación Biomédica Hospital Gregorio Marañón

Country: Spain 


\title{
ID: GA246 \\ Hybrid PET-MRI evaluation of myelin biomarkers in a NHP model for MS
}

\author{
Marine BREUILLY, CNRS / CRNL, breuilly@cermep.fr
}

\section{Category: Neuroscience}

\begin{abstract}
Body : I. Introduction Conventional MRI measurements in Multiple Sclerosis patients remain poorly correlated with disability and lack long-term prognostic value. A subsequent challenge is to identify the most reliable measure of myelin content in the white matter. Advanced MRI approaches (magnetization transfer, relaxometry...) are investigated (1). PET with repurposed amyloid tracers proved promising in translational studies (2), and allowed the quantification of myelin changes in patients (3). Here, we used an EAE model in NHP to concurrently evaluate candidate MRI markers, and PET radiotracers, namely [11C]PiB and [18F]BF227. The MRI markers that are presented here are the T1w/T2w ratio (T1T2r), the Magnetization Transfer Ratio (MTR) and the inhomogeneous Magnetization Transfer Ratio (ihMTR). MT and ihMT are MRI techniques sensitive to the macromolecular content of biological tissues which has been proven to be strongly sensitive to myelin-rich content. II. Methods Experimental Autoimmune Encephalitis (EAE) was induced in one adult female primate (Macaca fascicularis) through intradermal immunizations with recombinant human myelin oligodendrocyte glycoprotein (rhMOG) and Freund's Incomplete Adjuvant (FIA) performed every 4 weeks. The animal underwent 3 simultaneous PET-MRI exams, under a Siemens Biograph mMR hybrid camera. PET consisted in a dynamic 60-min acquisition after [11C]PiB injection, immediately followed by 120-min acquisition after [18F]BF227 injection, while MRI included T1w, T2w, FLAIR, as well as MT_OFF, MT_ON and ihMT (7). For longitudinal analysis, T1w images were spatially normalized to a reference T1 template with corresponding atlas (4). For visual inspection, T2w, FLAIR, MTs, ihMT and PET images were co-registered to FLAIR. MRI-derived images are computed according to the literature: T1T2r, MTR (6), and ihMTR (7). WM lesion was manually segmented on FLAIR images. The contralateral region was defined as the symmetric of the segmented lesion on the reference $\mathrm{T} 1$ template images. Then, the contralateral region was co-registered back to the native space used for measurement. III. Results The animal developed motor symptoms (transient unilateral left paresis) twenty days after the second immunization, and was treated with dexamethasone for one week to prevent aggravation. Imaging was performed at D4, D11 and D39 after the appearance of symptoms. Paresis persisted until the final exam and sacrifice of the animal, though attenuated in frequency and intensity. Hyper-intense WM lesion was detected on the right hemisphere (see Figure). WM lesion volume was measured and decreased over time: 1170mm 3 (D4); 500mm3 (D11); 220mm3 (D39). Asymmetrical WM uptake was noticed on late PET images at D11 of both [11C]PiB and [18F]BF227. Left/Right asymmetry was also clearly visible on the MRderived images, T1T2r, MTR and ihMTR images. Lesion/contralateral ratio was measured in MR-derived images at D11: 0.67 (T1T2r), 0.56 (MTR), and 0.58 (ihMTR). These measured ratio are in accordance with the hyposignal observed in the WM lesion. IV. Conclusions This preliminary report on one animal suggests that the EAE NHP model is relevant for testing PETMRI biomarkers of myelin changes over the course of the disease. The study design will allow a
\end{abstract}


back-to-back comparison of the "candidate" [18F]BF227 (previously evaluated on a rat model (5)), against the "reference" radiotracer [11C]PiB. PET quantification will be performed as standardized uptake values (SUV) relatively to cerebellum after adequate definition of regions of interest (lesion core, border, and normal appearing white matter (NAWM). In parallel, a crossanalysis will be performed to identify the best myelin biomarkers combination to obtain a specific and sensitive PET-MRI biomarker to myelin content variation.

References: (1) O’Muircheartaigh J, Vavasour I, Ljungberg E, et al. Quantitative neuroimaging measures of myelin in the healthy brain and in multiple sclerosis. Hum Brain Mapp.

2019;40(7):2104-2116. doi:10.1002/hbm.24510

(2) Stankoff B, Freeman L, Aigrot MS, et al. Imaging central nervous system myelin by positron emission tomography in multiple sclerosis using [methyl-11C]-2-(4-methylaminophenyl)- 6hydroxybenzothiazole. Ann Neurol. 2011;69(4):673-680. doi:10.1002/ana.22320

(3) Bodini B, Stankoff B. Imaging Central Nervous System Demyelination and Remyelination

by Positron-Emission Tomography. Brain Plast. 2016;2(1):93-98. doi:10.3233/bpl-160042

(4) Ballanger B, Tremblay L, Sgambato-Faure V, et al. A multi-atlas based method for automated anatomical Macaca fascicularis brain MRI segmentation and PET kinetic extraction. Neuroimage. 2013;77:26-43. doi:10.1016/j.neuroimage.2013.03.029

(5) Zhang M, Chauveau F, Billard T, et al. Quantitative longitudinal imaging of demyelination and remyelination in the lysolecithin-induced rat model of multiple sclerosis using 18F -BF227 PET and MRI. J Nucl Med. 2017;58(supplement 1):1363-1363.

http://jnm.snmjournals.org/cgi/content/short/58/supplement_1/345. Accessed December 4, 2019.

(6) Henkelman RM, Stanisz GJ, Graham SJ. Magnetization transfer in MRI: A review. NMR Biomed. 2001;14(2):57-64. doi:10.1002/nbm.683

(7) Duhamel G, et al. Validating the sensitivity of inhomogeneous magnetization transfer (ihMT) MRI to myelin with fluorescence microscopy. Neuroimage. 2019;199(January):289-303. doi:10.1016/j.neuroimage.2019.05.061

\section{Image/Figure:}

https://www.xcdsystem.com/wmis/abstract/File6959/GA246 ImageFigure 0612102431.png

Image/Figure Caption: WM lesion in a EAE NHP model. Coronal view at D11 after first MS relapse. This figure exhibits the coronal view of both MRI-derived images and molecular images : a) T1w, b) T2w, c) FLAIR, d) MT0, e) MT_ON, f) summed [11C]PiB PET image (40$60 \mathrm{~min}$ ) overlaid on FLAIR, g) summed [18F]BF227PET image (40-60min) overlaid on FLAIR, h) T1T2r, i) MTR, j) ihMTR. Images are centred on the largest lesion (delineated by red outline), localised in the right hemisphere. The Left-Right asymmetry of signal is clearly visible in all types of images : Normal Appearing Whit Matter (NAWM) vs Lesional White Matter. Unit of PET colorbar scale is given in SUV.

Complete Status: Complete

First Name: Marine

Last Name: BREUILLY 
Email: breuilly@cermep.fr

Organization: CNRS / CRNL

Country: France 


\title{
ID: GA248 \\ Simple radiosynthesis of $68 \mathrm{Ga}$-desferrioxamine $\mathrm{B}(68 \mathrm{GaDFO})$ for infection imaging
}

\author{
afnan darwesh, Biomedical Engineering \& Imaging Sciences department, King's College \\ London, afnan.darwesh@kcl.ac.uk
}

Category: Immunology: Inflammation \& Infection

\begin{abstract}
Body : Background: Endovascular stent graft infections are difficult to diagnose.1A PET radiotracer that is taken up selectively by microorganisms could provide more specific detection than currently used [18F]FDG. The Fe3+ complex of DFO, a siderophore, is taken up by bacteria, making its isostructural $68 \mathrm{Ga}$ complex applicable for imaging infection. $2 \mathrm{DFO}$ is a clinically approved drug3, hence its $68 \mathrm{Ga}$ complex has a low regulatory barrier to clinical translation. Here, we report a GMP method for $68 \mathrm{GaDFO}$ preparation, its binding to Escherichia coli (E.coli) in vitro, and its in vivo pharmacokinetics in healthy mice. Methods: $68 \mathrm{GaDFO}$ was radiolabelled by mixing DFO dissolved in water $(20 \mu 1)$, aqueous solution of sodium acetate (3.6 M) $(20 / 30 \mu 1)$, and $68 \mathrm{Ga}$ chloride $(200 \mu \mathrm{l})$. The mixture was diluted with water to $1 \mathrm{ml}$ then incubated for $10 \mathrm{~min}$ at room temperature. The radiochemical purity and chemical identity were assessed by TLC and HPLC. natGaDFO was added to $68 \mathrm{GaDFO}$ and analysed by LC-MS to determine the radioactive and stable compound retention time. Stability in human serum was measured by RP-HPLC after 60 -min incubation at $37 \mathrm{o}$ C. $68 \mathrm{GaDFO}$ uptake in E. coli was studied in vitro and compared to $68 \mathrm{Ga}$ ferrichrome-C, enterobactin, and siderophore-free $68 \mathrm{Ga}$. Healthy Balb/C mice $(n=3)$ were injected intravenously with $68 \mathrm{GaDFO}$ and PET/CT scanned dynamically for $60 \mathrm{~min}$. Mice were sacrificed for organ harvesting at $60 \mathrm{~min}$, and organs were counted in a gamma-counter. Results: $68 \mathrm{GaDFO}$ was labelled with high radiochemical purity $(\geq$ $95 \%$ ) as measured by TLC and HPLC. natGaDFO and $68 \mathrm{GaDFO}$ showed similar retention time. $68 \mathrm{GaDFO}$ demonstrated no binding to serum proteins at any time point by RP-HPLC. All $68 \mathrm{Ga}$ labelled siderophores showed higher E. coli uptake than siderophore free $68 \mathrm{Ga}$, with $68 \mathrm{Ga}$ ferrichrome-C showing the highest uptake. $68 \mathrm{GaDFO}$ exhibited rapid renal excretion and low blood retention. Ex vivo biodistribution at $60 \mathrm{~min}$ showed most of the activity resided in urine. Conclusion: $68 \mathrm{GaDFO}$ radiolabelling with GMP graded reagents gave a product with high radiochemical purity identified as a 1:1 complex of Ga3 + with DFO. The complex showed specific uptake in E. coli. $68 \mathrm{GaDFO}$ did not release $68 \mathrm{Ga}$ to serum proteins, indicating high stability of the compound in serum. $68 \mathrm{GaDFO}$ is rapidly cleared with no significant retention in any organ except kidneys. In vivo biodistribution of $68 \mathrm{GaDFO}$ on infected animal models is now planned.
\end{abstract}

References: 1 J. C. Marc Elieson,T. Mixon, Case Reports, 2012, 39, 884-889. 2 W. Rabsch and G. Winkelmann, Biol. Met., 1991, 4, 244-250. 3 M. Naser, S. Mehrnoosh, E. Hassan, N. Hajar, S. Mehdi, S. Mohsen, G. Mehdi, P. Hoda and A. Mehdi, Int. J. Hematol. stem cell Res., 2016, $10,239-247$.

\section{Image/Figure:}


https://www.xcdsystem.com/wmis/abstract/File6959/GA248 ImageFigure_0422045123.PNG

Complete Status: Complete

First Name: afnan

Last Name: darwesh

Email: afnan.darwesh@kcl.ac.uk

Organization: Biomedical Engineering \& Imaging Sciences department, King's College London

Country: United Kingdom 


\title{
ID: GA249 \\ Collagen-coated superparamagnetic nanoparticles for in vivo MR imaging
}

\author{
Mario González-Arjona, Fundación para la Investigación Biomédica del Hospital Gregorio \\ Marañón,mgarjona@hggm.es
}

Category: Immunology: Inflammation \& Infection

\begin{abstract}
Body : Introduction: Due to their superparamagnetic properties, ultra-small superparamagnetic iron oxide nanoparticles (USPIO) represent the main contrast agent for Magnetic Resonance Imaging (MRI) in T2-weighted images [1-3]. One of the challenges for the in vivo application of these nanoparticles is the need of chemical approaches to increase their stability, biocompatibility and biospecificity towards particular diseases. We present the covalent biofunctionalization of USPIOs with collagen [4-6], due to its high biocompatibility, null toxicity and high affinity of this protein towards metalloproteinase MMP11, MMP8 and MMP13 (collagenases) [7], enzymes highly involved in inflammatory processes [8,9]. Methods: USPIOs were initially synthesized by thermal decomposition of iron precursors in organic phase (NPs-Ole) [10] and further stabilized in aqueous media by the oxidation of the oleic double bounds in presence of $\mathrm{KMnO} 4$ to generate a carboxylic acid (NPs-Aze) [11]. Covalent biofunctionalization of NPs-Aze was then performed with collagen I in presence of EDC and sulfo-NHS. A full characterization of the new nanoparticles, NPs-Col, included determination of morphology and size by TEM and DLS. Surfactant incorporation (collagen I) was assessed by Thermogravimetric analysis (TGA) and $\zeta$-potentiometry. Magnetic properties were determined with a Vibrating Sample Magnetometer (VSM) and phantom imaging by MRI. In vitro studies of cellular uptake and cytotoxicity were assessed on RAW 264.7 cells, analyzed by Prussian Blue staining and XTT assay, respectively. In vivo MRI evaluation was carried out in 11-week old, female, C57/BL6 mice (NPs-Aze n=1, NPs-Col n=2) using a 7T Agilent-Varian MRI scanner. Results/Discussion: NPs-Ole were successfully synthesized by thermal decomposition as previously reported [10]. Increase in hydrodynamic size (NPs-Ole: $13.5 \mathrm{~nm}$; NPs-Aze: $35.6 \mathrm{~nm}$; NPs-Col: $52.7 \mathrm{~nm}$ ) and decrease in surface charge potential (NPs-Aze: $-34.3 \mathrm{mV}$; NPs-Col: $15.4 \mathrm{mV}$ ) confirmed bioconjugation with collagen. TGA also confirmed the presence of collagen on the surface of NPs-Col, showing different decomposition points (NPs-Aze: $300^{\circ} \mathrm{C}$; NPs-Col: $300^{\circ} \mathrm{C}$ and $500^{\circ} \mathrm{C}$ ). TEM images confirmed a small core size of $<5 \mathrm{~nm}$, ideal for the use of these USPIOS as imaging agent, spherical shape and great homogeneity [12]. VSM showed absence of magnetic hysteresis, which confirmed superparamagnetic behavior of both NPs-Aze and NPs$\mathrm{Col}$, along with high magnetization values of $89.3 \mathrm{emu} / \mathrm{g}$ and $70.9 \mathrm{emu} / \mathrm{g}$, respectively. A better RAW 264.7 viability was observed with collagen coating at both 24 and $48 \mathrm{~h}$ in comparison to NPs-Aze. This value was slightly higher at $48 \mathrm{~h}$ and with higher concentration of collagen, in agreement with previous literature $[6,13]$. Prussian Blue staining confirmed cell uptake in a dosetime response model: an increase of NPs uptake was observed over time and depending on NPs concentration (from 0 (control) to $40 \mu \mathrm{g} / \mathrm{mL}$ ). MRI phantoms confirmed the magnetic behavior of NPs-Col as T2 contrast agent in vitro, showing a relaxation time of $115.06 \pm 3.2 \mathrm{~ms}$. This capability was confirmed in vivo, showing higher stability in blood of NPs-Col compared to nonfunctionalized NPs, with blood circulation times larger than $2 \mathrm{~h}$ (Fig 1). Higher contrast in liver
\end{abstract}


and spleen confirmed the typical hepatobiliary metabolism of nanoparticles of this size and shape in healthy animals. Conclusions: We have successfully synthetized USPIO covalently coated with collagen protein. The improvement in the biocompatibility and toxicity of the novel NPsCol was confirmed in vitro. In vivo MRI studies confirmed their high stability in blood due to the presence of the protein and their negative contrast in T2 images, supporting further use of these nanoparticles for in vivo detection of MMPs by MRI. Acknowledgments: Authors thank Izaskun Bilbao and Yeny Rojas for their excellent work with animal preparation and imaging protocols. This work has been supported by "Diagnosis and treatment follow-up of severe Staphylococcal Infections with Anti-Staphylococcal antibodies and Immune-PET - Grant Fundación BBVA a Equipos de Investigación Científica 2018. This study has been also supported by Comunidad de Madrid, project "Y2018/NMT-4949 (NanoLiver-CM)" and "S2017/BMD-3867 (RENIM-CM)", co-funded by European Structural and Investment FUND.

References: [1] Roca, A. G. et al. "Agents Based on High Quality Magnetite Nanoparticles". Interactions 7033-7039 (2009). [2] Baghi, M. et al. "The efficacy of MRI with ultrasmall uperparamagnetic iron oxide particles (USPIO) in head and neck cancers". Anticancer Res. 25, 3665-3670 (2005). [3] Tran, N. \& Webster, T. J. "Magnetic nanoparticles: Biomedical applications and challenges". J. Mater. Chem. 20, 8760-8767 (2010). [4] Lu, An-Hui, E. emsp14L Salabas, and Ferdi Schüth. "Magnetic nanoparticles: synthesis, protection, functionalization, and application." Angewandte Chemie International Edition 46.8 (2007): 1222-1244 [5] Gupta, Ajay Kumar, and Mona Gupta. "Synthesis and surface engineering of iron oxide nanoparticles for biomedical applications." biomaterials 26.18 (2005): 3995-4021. [6] Liu, $\mathrm{X}$. et al. "Human-like collagen protein-coated magnetic nanoparticles with high magnetic hyperthermia performance and improved biocompatibility". Nanoscale Res. Lett. 10, 28 (2015). [7] Nagase, H., Visse, R. \& Murphy, G. Structure and function of matrix metalloproteinases and TIMPs. Cardiovasc. Res. 69, 562-573 (2006). [8] Tang, M., Bai, X., Li, Y., Dai, X. \& Yang, F. MMP-1 Over-expression Promotes Malignancy and Stem-Like Properties of Human Osteosarcoma MG-63 Cells In Vitro. Curr. Med. Sci. 38, 809-817 (2018). [9] Chakrabarti, S. \& Patel, K. D. Matrix metalloproteinase-2 (MMP-2) and MMP-9 in pulmonary pathology. Experimental Lung Research (2005). [10] Roca, A. G., Morales, M. P. \& Serna, C. J. "Synthesis of Monodispersed Magnetite Particles From Different Organometallic Precursors". IEEE Trans. Magn. 42, 3025-3029 (2006). [11] Herranz, F., Morales, M. P., Roca, A. G., Desco, M. \& RuizCabello, J. "A new method for the rapid synthesis of water stable superparamagnetic nanoparticles". Chem. - A Eur. J. 14, 9126-9130 (2008). [12] Sun, Shouheng, and Hao Zeng. "Size-controlled synthesis of magnetite nanoparticles." Journal of the American Chemical Society 124.28 [13] Sinani, V. A. et al. Collagen Coating Promotes Biocompatibility of Semiconductor Nanoparticles in Stratified LBL Films. (2003). doi:10.1021/n10255045

\section{Image/Figure:}

https://www.xcdsystem.com/wmis/abstract/File6959/GA249 ImageFigure 0422055002.png

\section{Complete Status: Complete}

First Name: Mario

Last Name: González-Arjona 
Email: mgarjona@hggm.es

Organization: Fundación para la Investigación Biomédica del Hospital Gregorio Marañón Country: Spain 


\title{
ID: GA252 \\ In vitro and in vivo fluorescent detection of inflammatory processes using milk exosomes
}

\author{
Ana Santos-Coquillat, Hunter College/ Instituto Investigación Sanitaria Gregorio \\ Marañón, ascoquillat@hggm.es
}

Category: Immunology: Inflammation \& Infection

\begin{abstract}
Body : Introduction: Inflammatory processes underlie the evolution and pathogenesis of many diseases $(1,2)$. New nanoplatforms for the detection of these processes are emerging during the last decade (3). In the development of novel nanoparticles for diagnosis, milk-derivate exosomes stand out due to their liposome-like structure, non-toxicity, easy production, and their role in biological processes(4), including the immune system. The objective of this work is the evaluation of labeled exosomes as non-invasive optical imaging tools for the selective detection of inflammation processes. Materials and methods: Exosomes from goat milk were isolated by ultracentrifugation and size exclusion chromatography and covalently labeled with BDP or SCy5 fluorophores in PBS at $4^{\circ} \mathrm{C}$ and $90 \mathrm{~min}$. A complete evaluation of their physicochemical properties, purity and stability was obtained by TEM, DLS, NTA, HPLC, and flow cytometry. In vitro cellular uptake of Exo-BDP was assessed by confocal microscopy in RAW 264.7 murine macrophages at different time points. Cytotoxicity was evaluated by XTT and LDH assays at 24 and $48 \mathrm{~h}$. Flow cytometry and confocal imaging were used to evaluate the uptake of labelled exosomes after the stimuli of either IL-4 or LPS/IFN- $\gamma$ in RAW 264.7 cells. An in vivo experiment was performed in a thioglycolate-induced mice model of sterile peritonitis (5) to assess the ability of circulating myeloid cells (macrophages and neutrophils) to internalize exosomes and infiltrate inflammed tissues. Sulfo-Cyanine 5-conjugated exosomes (Exo-SCy5) were administrated intravenously (i.v. $20 \mu \mathrm{g}, \mathrm{n}=6$ ). Inflammation was followed up by in vivo optical imaging at 9 and 24 hours after peritoneal inflammation induction. At 24 hours animals were sacrificed, and peritoneal exudates evaluated by flow cytometry, and ex vivo and organ distribution imaging were performed. Comparisons between control and Exo-SCy5 groups were performed by using Student's $t$ test, after checking for normality. Results/discussion: Milk derived exosomes were successfully optically labeled, showing high yields of labeling ( $99 \%$ by flow cytometry) as well as high purity ( $>95 \%$ ) and stability (up to $72 \mathrm{~h}$ ), estimated by HPLC. Original morphology and size of the exosomes were no modified, according to TEM, DLS and NTA. In vitro evaluation confirmed the uptake of Exo-SCy5 from short time points $(1 \mathrm{~h})$ with the highest uptake after $24 \mathrm{~h}$, and no significant cytotoxicity was found. The different macrophage subpopulations after the stimulation showed an in vitro uptake increase in the M1 subpopulation versus non-activated condition. In vivo results showed a predominant uptake in the peritoneal area, consistent with the peritonitis model. Macrophage and neutrophil populations in peritoneal fluid were assessed by flow cytometry and showed a two-fold increase of median fluorescence intensity, with statistically significant differences compared to control condition. Moreover, $15 \%$ of the neutrophils and $25 \%$ of the macrophages of the total population showed probe uptake, thus confirming its ability to track circulating myeloid cells, and therefore the inflammatory process. Conclusions: We have synthesized a new probe based on natural
\end{abstract}


nanoparticles (milk exosomes) able to detect inflammatory processes in vitro and in vivo through the direct labeling of macrophages and/or neutrophils. Acknowledgments: This study has been supported by Ministerio de Ciencia, Innovación y Universidades, Instituto de Salud Carlos III, project "PI16/02037", co-funded by European Regional Development Fund (ERDF), "A way of making Europe" and by Comunidad de Madrid, project "Y2018/NMT-4949 (NanoLiver-CM)" and "S2017/BMD-3867 (RENIM-CM)", co-funded by European Structural and Investment Fund. This work has also been supported by "Diagnosis and treatment follow-up of severe Staphylococcal Infections with Anti-Staphylococcal antibodies and Immune-PET - Grant Fundación BBVA a Equipos de Investigación Científica 2018. The CNIC is supported by the ISCIII, the Ministerio de Ciencia e Innovación and the Pro CNIC Foundation, and is a Severo Ochoa Center of Excellence (SEV-2015-0505).

References: 1. Y. Kim, H. Mok, Citraconylated exosomes for improved internalization into macrophages. Applied Biological Chemistry 62, 26 (2019). 2. D. Okin, R. Medzhitov, Evolution of inflammatory diseases. Curr Biol 22, R733-R740 (2012). 3. H. Yan et al., Engineering Cell Membrane-Based Nanotherapeutics to Target Inflammation. Adv Sci (Weinh) 6, 19006051900605 (2019). 4. X. Li et al., Challenges and opportunities in exosome research-Perspectives from biology, engineering, and cancer therapy. APL Bioeng 3, 011503-011503 (2019). 5. J. Garcia-Prieto et al., Neutrophil stunning by metoprolol reduces infarct size. Nat Commun 8 , 14780 (2017).

\section{Image/Figure:}

https://www.xcdsystem.com/wmis/abstract/File6959/GA252_ImageFigure_0422100133.jpg

Complete Status: Complete

First Name: Ana

Last Name: Santos-Coquillat

Email: ascoquillat@hggm.es

Organization: Hunter College/ Instituto Investigación Sanitaria Gregorio Marañón

Country: United States 


\title{
ID: GA255 \\ Calculation of System Response Matrix based on the Analytical Derivations Method for the Dual-head PET System
}

\author{
Fanzhen Meng, Xidian University, mengfz0316@xidian.edu.cn
}

\section{Category: Instrumentation}

\begin{abstract}
Body : In PET reconstruction, the iterative algorithms often offer a better trade-off between noise and resolution in comparison with the filtered back projection (FBP), especially in the case where the acquired data has the incomplete information. While the imaging performance of the iterative methods has close relationship with the construction of a system response matrix (SRM). Previous efforts have been taken to model the SRM through analytical derivations, Monte Carlo (MC) simulation, and empirical measurement. The MC-based method and empirical measurement are accuracy but often associated with huge storage requirement and high computation cost. In this study, we have utilized the analytical derivations to construction the SRM. We have modeled respectively the photon propagation in the space and into crystals. In the space, the solid angle model is utilized to calculation the SRM. In the crystals, the crystals are divided into several subcrystals in the depth direction in order to model the depth of information (DOI) effect. In order to verify the performance of proposed method, we simulated one dual-head PET system with the software of Geant4 Application for Tomographic Emission (GATE). Each detector head of this PET system consists of $26 \times 52$ LYSO crystals with a size of $2.0 \times 2.0 \times 13 \mathrm{~mm} 3$. The reconstructed field of view $(\mathrm{FOV})$ is $52 \times 52 \times 104 \mathrm{~mm} 3$ with the voxel size $0.5 \times 0.5 \times 0.5 \mathrm{~mm} 3$. We simulated one planar source with $10 \times 40 \times 80 \mathrm{~mm} 3$. The reconstructed image with the MC-based SRM has been as the reference standards. The results show that when only the solid angle model is utilized, the image intensity in the center of field of view (FOV) is higher than the region in the edge. When the DOI information is model, the image is more uniform which is very close to that of the MC simulation..
\end{abstract}

Complete Status: Complete

First Name: Fanzhen

Last Name: Meng

Email: mengfz0316@xidian.edu.cn

Organization: Xidian University

Country: China 


\title{
ID: GA256 \\ Imaging of CD80 to monitor the immune-activating potential in cancer
}

\author{
Claudia Adriana Castro Jaramillo, ETHZ, claudia.castro@pharma.ethz.ch
}

\section{Category: Oncology}

\begin{abstract}
Body : Introduction Growing evidence shows that inflammation is highly involved in the regulation of carcinogenesis, including the disruption of the immune response and the regulation of the tumor microenvironment. For instance, therapies modulating the patients' immune system, such as immune checkpoint inhibitors, had a positive impact on the field of oncology, showing promising clinical results in a variety of tumors. Unfortunately, their efficacy is frequently limited by heterogeneity, complexity and diverse immunosuppressive mechanisms of tumors. And as additional drawback they can potentially generate severe immune-related adverse effects. Hence, the stratification of patients who would benefit from immunotherapy could help to design individualized therapy regimens and improve clinical management. Currently, patient stratification is based on the analysis of biopsied material being limited by tumor heterogeneity and lacking information about spread in the body. An alternative are molecular imaging techniques allowing the non-invasive visualization and quantification of molecular signatures in the whole body. Offering additional and complementary information to the in vitro/ex vivo analysis that rely on biopsies. A very attractive biomarker for the noninvasive visualization of the immune status of tumors is CD80. CD80 is a co-stimulatory molecule present on the cell surface of antigen-presenting cells (APCs) such as macrophages and dendritic cells and gets upregulated upon their activation. It binds CD28 and CTLA-4 on T cells and helps to orchestrate appropriate T cell responses. CD80 may, therefore, serve as an imaging marker for the assessment and monitoring of the inflammation status of tumors and the associated microenvironment, which could be particularly relevant for tumor staging and quantification of immunotherapeutic responses. Methods We conducted preclinical proof-ofconcept studies imaging CD80/CD86 with radiolabeled Abatacept and Belatacept. These therapeutic Fc-fusion proteins are based on the extracellular sequence of CTLA-4, the endogenous high-affinity ligand of CD80 and CD86. Target affinity was determined by surface plasmon resonance (SPR). Both proteins were conjugated with NCS-NODAGA and labeled with $64 \mathrm{Cu}$. Tracers were evaluated by in vitro autoradiography and in vivo by PET imaging, using CT-26 syngrafts in immune-competent mice (mCD80) and Raji/Daudi xenografts in immune suppressed mice (hCD80). Target expression and co-localization with relevant immune markers were analyzed by RT-qPCR, flow cytometry and confocal microscopy. Results and discussion We successfully developed two novel, fusion protein based, radio ligands for noninvasive imaging of murin and human CD80 with affinities in the low nanomolar range determined by SPR. The developed tracers allowed the specific localization of hCD80 and $\mathrm{mCD} 80$ rich tissues in vivo. We characterized the levels of expression and expression patterns of CD80 and other inflammation markers in the different types of tumors. In vitro autoradiography and in vivo studies revealed high accumulation of the tracers in tissues with high expression of CD80. Accumulation was reduced in the presence of an excess of unlabeled protein (Figure 1), confirming specific uptake. Imaging results were confirmed by ex vivo biodistribution analysis.
\end{abstract}


The radioligands are currently further optimized and will be evaluated towards the monitoring of therapy-related changes in CD80 expression in cancer and further application in other inflammatory diseases.

References: [1] Sounni N.E. and Noel A. Targeting the Tumor Microenvironment for Cancer Therapy. Clin Chem. 2013;59:85-93. [2] Taddio M.F., Mu L., Castro Jaramillo C.A., Bollmann T., Schmid D.M., Muskalla L.P., Gruene T., Chiotellis A., Ametamey S.M., Schibli R. and Krämer S.D. Synthesis and structure-affinity relationship of small molecules for imaging human CD80 by positron emission tomography. J Med Chem. 2019;62:8090-8100. [3] Meletta R, Muller Herde A, Dennler P, Fischer E, Schibli R, Kramer SD. Preclinical imaging of the costimulatory molecules CD80 and CD86 with indium-111-labeled belatacept in atherosclerosis. EJNMMI Res 2016; 6(1):1.

\section{Image/Figure:}

https://www.xcdsystem.com/wmis/abstract/File6959/GA256 ImageFigure 0610093210.JPG

Image/Figure Caption: Figure 1: Representative PET images of CT26 syngrafts in BALB/c mice, 48 hours after tail vein injection of $25 \mu \mathrm{g}$ 64Cu-NODAGA-abatacept $(0.74$ to $2.3 \mathrm{MBq}$, decay corrected to scan start), under baseline (tracer only) and blocking (tracer $+1 \mathrm{mg}$ uncongugated abatacept) conditions. Maximal intensity projection. CT, grey scale; PET, color scale. SUV, standardised uptake value. Yellow arrow indicates expected position of the syngrafts. One representative out of 4 mice each.

Complete Status: Complete

First Name: Claudia Adriana

Last Name: Castro Jaramillo

Email: claudia.castro@pharma.ethz.ch

Organization: ETHZ

Country: Switzerland 


\title{
ID: GA258 \\ Chemiluminescence resonance energy transfer in nano-scale for enhanced cancer phototheranostics
}

\author{
Jueun Jeon, Sungkyunkwan University, wndmsd13451@gmail.com
}

\section{Category: Oncology}

\begin{abstract}
Body : Chemiluminescence resonance energy transfer (CRET) refers to a non-radiative transfer mechanism between chemiluminescence (CL) donors and adjacent acceptors, usually in the nanoscale. Recently, reactive oxygen species (ROS) that are the representative hallmark of cancer were visualized by CRET-mediated molecular imaging [1]. In addition, there is no doubt about the resolution of CL imaging, responsible for high signal-to-noise ratio and superior specificity. Thus, CRET has received increasing for its applications to phototheranostics, particularly CRET-NPs have been extensively investigated for chemiluminescence imaging and photodynamic therapy (PDT). However, the short duration of their chemiluminescence signal and low therapeutic efficacy remain major limitations to translational application. Innovative strategies to achieve quantum yield-enhanced cancer phototheranostics should reflect the unique natures of CRET. Herein, we describe a new approach utilizing CRET-NPs capable of enhancing both photoacoustic (PA) and reactive oxygen species (ROS) quantum yield. CRET-NPs were prepared by physically encapsulating a CL donor, oxalate, into self-assembled NPs composed of biopolymer as the hydrophilic shell and chlorin e6 as the hydrophobic CL acceptor. Based on the hidden nature of oxalate and photosensitizer, we hypothesized that CRET-NPs could be longacting cancer phototheranostic agents, allowing effective PA imaging and PDT by the following feasible mechanisms: PA signal improvement via thermal expansion-induced vaporization (TEIV) and ROS quantum yield enhancement through both type I and II photochemical reactions, respectively. The CRET-NPs from this study facilitated the enhancement of PA quantum yield by TEIV, which is the most efficient mechanism of light-sound energy conversion, allowing for long-lasting PA imaging of $\mathrm{H} 2 \mathrm{O} 2$-abundant tumor tissues. As a result, when CRET-NPs were intravenously injected into tumor-bearing mice, a strong PA signal was detected at the tumor site for at least $12 \mathrm{~h}$. while CL signal lasted $10 \mathrm{~min}$. Interestingly, there was no PA signal from CRET-NPs in normal liver tissue with a low level of ROS. These results suggesting that CRET-NPs allow long-term ROS imaging in a real-time and non-invasive manner. In addition to PA imaging, the CRET phenomenon of the NPs enhanced the ROS quantum yield of photosensitizer through both oxygen-independent type I and oxygen-dependent type II photochemical reactions. As a result, CRET-NPs effectively inhibited the growth of malignant tumors with complete regression in $60 \%$ of cases with only a single treatment. To the best of our knowledge, this is the first demonstration that CRET-NPs can generate an enhanced ROS quantum yield through both electron transfer and self-illumination. In summary, we developed CRET-NPs that maintain a stable nanostructure with high PA and ROS quantum yield for cancer phototheranostics. Quantum yield-enhanced CRET-NPs, resulting from the hidden nature of the CRET phenomenon, offer a new phototheranostic modality. Beyond cancer treatment, the findings of this study have broad implications for diagnosis and treatment of intractable diseases.
\end{abstract}


References: [1] Lee, D. et al. In vivo imaging of hydrogen peroxide with chemiluminescent nanoparticles. Nat. Mater. 6, 765-769 (2007). [2] Lee, Y.-D. et al. Dye/peroxalate aggregated nanoparticles with enhanced and tunable chemiluminescence for biomedical imaging of hydrogen peroxide. ACS Nano 6, 6759-6766 (2012). [3] Kwon, S., et al. Nanomedicines for Reactive Oxygen Species Mediated Approach: An Emerging Paradigm for Cancer Treatment. Acc. Chem. Res. 52, 1771-1782 (2019).

\section{Image/Figure:}

https://www.xcdsystem.com/wmis/abstract/File6959/GA258 ImageFigure 0422074505.jpg

Image/Figure Caption: Schematic illustration of CRET-NPs for cancer phototheranostics. CRET-NPs with a high ROS quantum yield can generate an intense PA signal by thermal expansion-induced vaporization. Because of its ROS-responsive $\mathrm{CO} 2$ production, the strong PA signal of CRET-NPs can be generated at the tumor tissue for a long period of time. In addition, the CRET phenomenon of NPs enhances the ROS quantum yield through both electron transfer in the oxygen-independent type I photochemical reaction and self-illumination in the oxygendependent type II photochemical reaction

Full Name of Abstract's 1st Author : Jueun Jeon

Complete Status: Complete

First Name: Jueun

Last Name: Jeon

Email: wndmsd13451@gmail.com

Organization: Sungkyunkwan University

Country: South Korea 


\title{
ID: GA259 \\ L-cysteine-mediated modulation of copper trafficking in prostate tumour cells: an in vitro and in vivo investigation with $\mathrm{Cu}-64 \mathrm{PET}$
}

\author{
Joanna Bartnicka, King's College London, joanna.bartnicka@kcl.ac.uk
}

\section{Category: Oncology}

\begin{abstract}
Body : Copper plays a role in cancer biology1 and PET imaging with [64Cu]64CuCl2 has been used to visualise tumours in mice2, as well as in prostate cancer patients3. Copper is transported in the blood bound to proteins and amino-acids but their role in the delivery of $[64 \mathrm{Cu}] 64 \mathrm{CuCl} 2$ to cancer cells is unknown. We explored whether uptake of $64 \mathrm{Cu}$ into prostate cancer-derived cells PC3 and DU145 cells was influenced by the presence of biomolecules known to bind or transport copper. Since our previous work suggested that serum proteins did not enhance $64 \mathrm{Cu}$ uptake, we focused on the copper-binding amino acids: L-histidine, Lmethionine, L-cysteine and L-threonine. We investigated in vitro whether these amino acids can affect $64 \mathrm{Cu}$ delivery to cells, and if so, by what mechanism? We then explored whether the conclusions drawn were relevant to whole-body trafficking of ionic $64 \mathrm{Cu}$ to prostate tumours in vivo using Positron Emission Tomography (PET). In vitro cell uptake of [64Cu] $64 \mathrm{CuCl} 2$ was measured in Hank's Buffered Salt Solution (control) or with added amino-acids. Glutathione in DU145 cells was depleted with buthionine sulfoximine (1 mM, 24 hours). For the in vivo study, 11-12 weeks old male SCID/beige mice bearing Du145 subcutaneous xenografts were injected intravenously (i.v.) with $150 \mathrm{mg} / \mathrm{kg}$ dose of N-acetyl cysteine (NAC, a clinically approved prodrug of L-cysteine) or saline ( $n=3$ per group) and after 5 minutes with $4 \pm 2 \mathrm{MBq}$ of acetatebuffered $64 \mathrm{Cu}$. Animals were imaged by PET for $1 \mathrm{~h}$ post $64 \mathrm{Cu}$ administration (p.i.). Areas under the time-activity curves of $64 \mathrm{Cu}$ uptake over 1 hour were compared by an un-paired t-test. In vitro uptake of 64Cu in DU145 cells (Fig.1A) was unchanged with $100 \mu \mathrm{M}$ L-histidine, Lmethionine or L-threonine but with L-cysteine, the intracellular/extracellular ratio of $64 \mathrm{Cu}$ was increased by $5.15 \pm 2.98$ fold (p We conclude that the effective delivery of ionic $64 \mathrm{Cu}$ to cellular transporters on prostate cancer DU145 cells can occur in vitro without the involvement of amino acids intermediates. The intracellular retention of $64 \mathrm{Cu}$ in prostate cancer cells is increased with high intracellular levels of L-cysteine and NAC by a yet unreported mechanism. The latter effect was not demonstrably apparent in in vivo in tumour xenografts of these cells. Further investigation of the link between thiols and copper metabolism in cancer biology could help elucidate the mechanism of accumulation of ionic $64 \mathrm{Cu}$ tracers in tumours.
\end{abstract}

References: 1. D. Denoyer, S. Masaldan, S. La Fontaine and M. A. Cater, Targeting copper in cancer therapy: 'Copper That Cancer', Metallomics, 2015, 7, 1459-1476. 2. X. L. Fangyu Peng, James Janisse, Otto Muzik, Anthony F. Shields, PET of Human Prostate Cancer Xenografts in Mice with Increased Uptake of 64CuCl2, J Nucl Med, 2006, 47, 4. 3. A. Piccardo, F. Paparo, M. Puntoni, S. Righi, G. Bottoni, L. Bacigalupo, S. Zanardi, A. DeCensi, G. Ferrarazzo, M. Gambaro, F. G. Ruggieri, F. Campodonico, L. Tomasello, L. Timossi, S. Sola, E. Lopci and M. Cabria, (64)CuCl2 PET/CT in Prostate Cancer Relapse, J Nucl Med, 2018, 59, 444-451. 4. J. Lee, M. M. Pena, Y. Nose and D. J. Thiele, Biochemical characterization of the human copper 
transporter Ctr1, J Biol Chem, 2002, 277, 4380-4387. 5. T. Ishii, Y. Sugita and S. Bannai, Regulation of glutathione levels in mouse spleen lymphocytes by transport of cysteine, J Cell Physiol, 1987, 133, 330-336.

\section{Image/Figure:}

https://www.xcdsystem.com/wmis/abstract/File6959/GA259_ImageFigure_0614124823.png

Image/Figure Caption: Fig 1: Effect of thiols on the in vitro and in vivo accumulation of $64 \mathrm{Cu}$ in prostate cancer cells. A-D: 64Cu uptake (60-90 minutes) in vitro in DU145 cells incubated in HBSS with added amino acid. Graphs show means $\pm \mathrm{SD}, \mathrm{n}=3$. In $\mathrm{A}, \mathrm{B}$ and $\mathrm{C}$ dotted lines represent levels of $64 \mathrm{Cu}$ uptake in control samples incubated in HBSS alone. A. Effect of amino acids: L- histidine, L-methionine, L-cysteine and L-threonine $(100 \mu \mathrm{M})$. B. Effect of various reducing agents: L-cysteine and D-cysteine - $100 \mu \mathrm{M}$, glutathione $(\mathrm{GSH})-10 \mu \mathrm{M}$, ascorbate -1 $\mathrm{mM}$. C. Effect of the pre-incubation with increasing concentrations of L-cysteine, with or without amino-acids competing for L-cysteine uptake (1 mM L-serine and $1 \mathrm{mM} \mathrm{L}$-threonine) on the subsequent $64 \mathrm{Cu}$ uptake. D. Effect of glutathione depletion (by BSO treatment) on $64 \mathrm{Cu}$ accumulation with/without L-cysteine. E: SCID/beige mice bearing Du145 xenografts were i.v. injected with $\mathrm{N}$-acetyl cysteine (NAC, $150 \mathrm{mg} / \mathrm{kg}$ ) or saline (control) and 5 minutes later i.v. with $64 \mathrm{Cu}$-acetate $(4 \pm 2 \mathrm{MBq})$. Left panel shows maximum intensity projections of PET/CT images 50-60 minutes p.i. of 64Cu. Arrows point to the Du145 xenografts. Right panel shows uptake of $64 \mathrm{Cu}(\% \mathrm{ID} / \mathrm{g})$ in key organs between 1 and $60 \mathrm{~min}$ p.i. Areas under the curve were calculated and compared between groups with an un-paired t-test.

Complete Status: Complete

First Name: Joanna

Last Name: Bartnicka

Email: joanna.bartnicka@kcl.ac.uk

Organization: King's College London

Country: United Kingdom 


\title{
ID: GA260 \\ Changes in tumor pathophysiology and therapy outcome in triple negative breast cancer in mice induced by diagnostic contrast-enhanced ultrasound
}

\author{
Anne Rix, RWTH Aachen International University, arix@ukaachen.de
}

\section{Category: Oncology}

\begin{abstract}
Body : In preclinical imaging, contrast-enhanced ultrasound (CEUS) is an established technique to characterize the vascularization and expression of molecular markers in tumors. Next to that, US contrast agents can be used to intentionally permeabilize vessels during sonoporation 1 or induce a vascular shutdown after inertial cavitation2. So far, no data are available to exclude an influence of diagnostic CEUS on tumor pathophysiology. Therefore, the present study should discover possible influences of diagnostic contrast-enhanced US on tumor pathophysiology and therapy outcome after antiangiogenic and antitumoral treatment in orthotopic triple negative breast cancer in mice. To achieve this, $\mathrm{n}=100$ female orthotopic 4T1tumor bearing BALB/c mice were allocated randomly to the following groups: no imaging, isoflurane anesthesia alone, ultrasound including a destructive pulse (destructive US), contrastenhanced ultrasound (non-destructive CEUS) or contrast-enhanced ultrasound with destruction of the contrast agent (destructive CEUS). Animals of each group were subdivided to either receive $10 \mathrm{mg} / \mathrm{kg}$ regorafenib or vehicle solution daily by oral gavage. On day 7, 10 and 14 after tumor cell injection, US measurements were performed using phospholipid microbubbles (MB) for non-destructive US or phospholipid MB targeting the vascular endothelial growth factor receptor-2 for destructive US. Furthermore, the impact of anesthesia and US imaging on animal welfare was evaluated twice a week by assessing heartrate, motor coordination and fecal corticosterone metabolites. The tumor size was assessed daily by caliper measurements. Before euthanization blood samples were collected and a complete blood count was performed. Fluoresecently labelled lectin was injected intravenously to assess perfused tumor vessels. Tumors were histologically characterized regarding vascularization and immune cell infiltration. Welfare assessment showed no impact of longitudinal ultrasound examinations on the animals' heartrate, motor coordination or fecal corticosterone metabolite levels. Histological characterization showed a significantly higher tumor vascularization and angiogenesis in vehicle treated animals examined with non-destructive CEUS (Fig. 1 A-C.). Next to that, macrophage infiltration was significantly higher in tumors after non-destructive CEUS (Fig. 1 E-F.), but no influence of the different imaging protocols on the tumor size could be detected. Both, nondestructive and destructive CEUS induced a systemic immune response of 4T1-tumor bearing mice represented by a reduced leukocyte count and spleen size. In conclusion, longitudinal US imaging did not increase the burden of the animals during the experiment. The significantly increased 4T1-tumor vascularization, perfusion, angiogenesis and immune cell infiltration after diagnostic CEUS resulted in a different interpretation of therapy outcome after regorafenib treatment. Furthermore, CEUS influenced the systemic immune reaction of 4T1-tumor bearing mice. These results show the need to carefully choose the right control groups to prevent unwanted falsification of study results and to improve the reproducibility of results between studies. Further investigations are ongoing regarding alterations of the tumor composition (e.g.
\end{abstract}


collagen content, matrix metalloproteinases) and a second syngenic tumor model (CT26) will be included to explore if these changes are tumor type specific.

References: [1] Theek B, Baues M, Ojha T et. al. Sonoporation enhances liposome accumulation and penetration in tumors with low EPR J Control Release. 2016 Jun 10; 231: 7785. [2] Goertz DE, Karshafian R, Hynynen K. IEEE. Antivascular effects of pulsed low intensity ultrasound and microbubbles in mouse tumors. IEEE Ultrason Symp 2008;1-4:670-3.

\section{Image/Figure:}

https://www.xcdsystem.com/wmis/abstract/File6959/GA260_ImageFigure_0422081739.jpg

Full Name of Abstract's 1st Author : Anne Rix

Complete Status: Complete

First Name: Anne

Last Name: Rix

Email: arix@ukaachen.de

Organization: RWTH Aachen International University

Country: Germany 


\title{
ID: GA261 \\ Molecular Magnetic Resonance Imaging of Alpha-v-beta-3 Expression in Tumors using Ultrasound Microbubbles
}

\author{
Vertika Pathak, EXMI, vpathak@ukaachen.de
}

\section{Category: Oncology}

\begin{abstract}
Body : Angiogenesis is one of the most studied hallmarks of cancer. Here, molecular ultrasound (US) with targeted microbubbles (MB) has shown potential in the early detection of malignant tumors as well as in the assessment of anti-angiogenic therapy response (1). Magnetic Resonance Imaging (MRI) is another widely used imaging modality, which is highly desirable for molecular imaging of angiogenesis. However, MRI suffers from its inherently low sensitivity to probes. To circumvent the sensitivity issue of traditionally used contrast agents, nanoparticles loaded with greater payloads of gadolinium, or ultrasmall superparamagnetic iron oxide nanoparticles (USPIO) have been extensively developed. However, due to their small size, nanoparticles extravasate from the vessels and, their high unspecific uptake by the reticuloendothelial system makes it difficult to properly quantify the target-bound fraction. Therefore, to generate a potent intravascular MR contrast agent, USPIOs were loaded into the shell of micrometer-sized polymeric microbubbles. In this context, the location of the USPIOs at the air-liquid interface strongly enhances susceptibility effects, thus making the agent sensitively detectable in $\mathrm{T} 2 * \mathrm{~W}$ images (2). As a proof of concept, we in this study investigated the capability of USPIO-MB as dual-modality 'molecular MRI and ultrasound contrast agents' to assess $\alpha v \beta 3$-integrin expression at the neovasculature of murine breast carcinomas. USPIO-MB were synthesized as described previously (2), functionalized with c[RGDfK], and scrambled $\mathrm{c}[\mathrm{RADfK}]$ peptides. $\alpha v \beta 3$-integrin-expressing 4T1 cells were incubated with the USPIO-MB for 8 minutes (in presence/absence of excess free-RGD), washed and embedded in gelatin phantoms. T2 relaxation times of the phantoms were obtained using a 7.0 T MRI system (Bio-Spec 70/20 USR, Bruker). Finally, an in vivo proof-of-principle study was performed in Balb/c mice bearing subcutaneous 4T1 tumours. 1x109 USPIO-MB were intravenously injected as a bolus and dynamic susceptibility contrast (DSC) scans with a three-shot EPI readout (TE/TR: 8/333 ms; FOV: 35x35; image size: 112x112; FA: 450) were acquired for 5 minutes. Targeted-US imaging was performed in the same set of animals that underwent MRI using a VEVO 3100 US system equipped with an MX-250 transducer (21MHz central frequency). After USPIO-MB administration, cine loops at 20 frames per second and $4 \%$ power were acquired for about a minute. Eight minutes post-USPIO-MB injection, a destructive pulse was applied to destroy USPIO-MB within the frame and immediately another 1500 frames were recorded to assess the unbound USPIO-MB fraction. Statistical analysis was performed using student multiple t-test and ANOVA in GraphPad Prism 8. USPIO-MB were characterized by narrow size distribution and high USPIOs encapsulation (Figure 2A-F). Cells incubated with RGD-USPIO-MB showed higher R2 values than cells incubated with USPIO-MB. Adding free RGD significantly reduced the RGD-USPIO-MB binding (Figure 2G). The time-intensity curves obtained from DSC-MRI showed a prolonged signal loss in tumours due to RGD-USPIO-MB binding whereas both USPIO-MB and RAD-USPIO-MB only caused a transient signal loss due to MB passaging
\end{abstract}


in the blood (Figure 1). The normalized signal intensity change before and after MB injection was quantified from T2W MR images. (Figure $2 \mathrm{H}, \mathrm{n}=5$ ). Contrast-enhanced US results were in line with those from MRI showing a significantly higher binding of RGD-USPIO-MB than USPIO-MB or RAD-USPIO-MB to the tumor neovasculature (Figure 2I, $n=5$ ). In summary, we present the proof-of-principle for molecular MRI of tumor angiogenesis with targeted-USPIOMB. From a translational point of view, our dual imaging methodology may be suitable for interventional procedures where the lesion is initially detected by MRI and the subsequent ultrasonic real-time feedback may be used to guide tissue biopsy or excision.

References: 1. Gibson, Raymond E. "Molecular Imaging, Principles and Practices." (2011): 594. 2. Koczera, Patrick, Lia Appold, Yang Shi, Mengiiao Liu, Anshuman Dasgupta, Vertika Pathak, Tarun Ojha et al. "PBCA-based polymeric microbubbles for molecular imaging and drug delivery." Journal of controlled release 259 (2017): 128-135.

\section{Image/Figure:}

https://www.xcdsystem.com/wmis/abstract/File6959/GA261_ImageFigure 0609033354.png

Image/Figure Caption: Number-based size distribution of USPIO nanoparticles ( $25 \pm 4 \mathrm{~nm})$ and microbubbles $(1.8 \pm 2 \mu \mathrm{m})$ measured using DLS and Coulter counter (A, B). TEM images of USPIO-loaded MB with inlets showing USPIO nanoparticles in the shell (C,D) compared with unloaded controls (E,F). In vitro MRI results: T2 relaxation rates of cells in gelatin incubated with USPIO-MB, RGD-USPIO-MB, and RGD-USPIO-MB in presence of excess RGD (Comp.) $(\mathrm{G}, \mathrm{n}=3)$. In vivo MRI: Normalized signal intensities in $4 \mathrm{~T} 1$ tumours before and after the contrast injection calculated from $\mathrm{T} 2 \mathrm{~W}$ images $(\mathrm{H}, \mathrm{n}=5)$. In vivo US: Target binding of RGDUSPIO-MB was confirmed by using the destruction-replenishment method and subtracting the post- from pre-destructive mean acoustic intensity. (I, $\mathrm{n}=5$ ).

Full Name of Abstract's 1st Author : VERTIKA PATHAK

Complete Status: Complete

First Name: Vertika

Last Name: Pathak

Email: vpathak@ukaachen.de

Organization: EXMI

Country: Germany 


\title{
ID: GA262 \\ In vivo assessment of cirrhosis pathophysiology by PET imaging
}

\author{
Mario González-Arjona, Fundación para la Investigación Biomédica del Hospital Gregorio \\ Marañón, mgarjona@hggm.es
}

Category: Systemic Diseases (Kidney, Liver and Pancreas)

\begin{abstract}
Body : Introduction: Cirrhosis represents relevant social and public health diseases, with a progressively increasing incidence [1]. This scenario combines with the limited knowledge about the pathogenesis as well as with severe limitations in the diagnosis and treatment of the pathology. In this work we aimed to assess by non-invasive PET imaging some of the main pathophysiological processes involved in chronic liver disease, including angiogenesis, inflammation and metabolic disorders. For this purpose, three different radiotracers were employed: 68Ga-NOTAGA-RGD2 specific for angiogenesis [2], [18F]Fluoro6TiaHeptadecanoic acid (FTHA) selective of fatty acid metabolism [3], and 18FDG for the evaluation of glucose metabolism as surrogate of inflammation [4]. Methods: The tracers were tested in a control vs. cirrhotic rat model ( $\operatorname{control} n=2$; cirrhotic $n=3$ ). PET/CT scans were obtained from each rat employing the three different tracers, following the scheme: day $118 \mathrm{~F}$ FDG, day 2 18F-FTHA and day 3 68Ga-RGD2 plus ex vivo biodistribution. 18F-FTHA was synthetized based on previous bibliography [5] by azeotropic nucleophilic substitution of the tosylated precursor. The $68 \mathrm{Ga}$-labeling of commercial NODAGA-RGD2 was optimized from previous works [6] concerning temperature, reaction time and radio-isotope concentration (20 nmol of NODAGA-RGD2, $\mathrm{pH}=5.2,7 \mathrm{mCi} 68 \mathrm{GaCl} 3,15 \mathrm{~min}, \mathrm{RT}$ ). $68 \mathrm{Ga}$ was obtained from a $68 \mathrm{Ge} / 68 \mathrm{Ga}$-generator, based on nano-SnO2 and developed at CIEMAT [7]. 18FDG was commercially available from CuriumPharma. Cirrhosis was induced in Sprague-Dawley rats (male, 5 wk-o, 200g) by the administration of carbon tetrachloride (CCl4) by oral gavage twice a week for 12 weeks. Oral gavage with water $(\mathrm{H} 2 \mathrm{O})$ was given to control animals. Enzyme inducer for $\mathrm{CCl} 4$, Phenobarbital, was added to drinking water $(35 \mathrm{mg} / \mathrm{dL})$ from 2 weeks before CCl4 administration until the end of the experiments [8]. PET/CT imaging was carried out in non-fasting rats at different time points according to the radiotracer employed (18FDG: $30 \mathrm{~min}$ post-injection/one 30-min static frame; 18F-FTHA: 0 min post.inj/dynamic 3x10min frames; 68Ga-NODAGA-RGD2: 0 min post.inj/dynamic 4x10min frames). On the CT images, cylindrical regions of interest (ROIs) were delimited in the three liver lobes and afterwards applied to the coregistered PET images to obtain the SUVmean. Ex vivo 68Ga-NODAGARGD2 biodistribution study was performed at the study endpoint, 40 min post injection. Results/Discussion: Cirrhosis led to different findings about the three pathophysiological processes evaluated. Confirming previous histological studies [9], in vivo 18FDG-PET/CT imaging showed higher uptake in the cirrhotic model $(0.83 \pm 0.05)$, as compared to control $(0.60 \pm 0.04)$, probably reflecting an inflammatory response of the organ. Maximum uptake of 18F-FTHA was found at 10-20 min, but there were no apparent differences in fatty acid metabolism between control (4.48 \pm 1.29$)$ and cirrhotic rats (3.74 \pm 1.47$)$. Finally, 68GaNODAGA-RGD2 results showed an increase of tracer uptake in rats with cirrhosis (cirrhotic: $1.30 \pm 0.16$ vs. control: $0.68 \pm 0.13$ ), suggesting increased hepatic angiogenesis (Figure 1A and B).
\end{abstract}


These results were confirmed by ex vivo bioDi (control liver: $0.10 \pm 0.05$; cirrhotic liver: $0.34 \pm 0.12 \% \mathrm{ID} / \mathrm{g}$ ) (Figure $1 \mathrm{C}$ ). Conclusions: We have carried out a preliminary assessment of three of the main pathophysiological processes present in chronic liver disease. The increased 18FDG and 68Ga-NODAGA-RGD2 uptake in cirrhotic animals may indicate tissue inflammation together with angiogenesis, while the low difference in 18F-FTHA uptake suggests no significant changes in fatty acid metabolism. These non-invasive molecular imaging tools may provide a better insight into pathogenesis and may facilitate development of novel therapeutic strategies in cirrhosis. Acknowledgments: Authors thank Yolanda Sierra, Alexandra de Francisco and María de la Jara Felipe for their excellent work with animal preparation and imaging protocols. This work has been supported by "Diagnosis and treatment follow-up of severe Staphylococcal Infections with Anti-Staphylococcal antibodies and Immune-PET - Grant Fundación BBVA a Equipos de Investigación Científica 2018; Comunidad de Madrid, project "Y2018/NMT-4949 (NanoLiver-CM)" and "S2017/BMD-3867 (RENIM-CM)", co-funded by European Structural and Investment FUND.

References: [1] Abubakar, I. I., T. Tillmann, and A. Banerjee. "Global, regional, and national age-sex specific all-cause and cause-specific mortality for 240 causes of death, 1990-2013: a systematic analysis for the Global Burden of Disease Study 2013." Lancet 385.9963 (2015): 117171. [2] Knetsch, Peter A., et al. "[68 Ga] NODAGA-RGD for imaging $\alpha$ v $\beta 3$ integrin expression." European journal of nuclear medicine and molecular imaging 38.7 (2011): 13031312. [3] Iozzo, Patricia, et al. "Liver uptake of free fatty acids in vivo in humans as determined with $14(\mathrm{R}, \mathrm{S})-[18 \mathrm{~F}]$ fluoro-6-thia-heptadecanoic acid and PET." European journal of nuclear medicine and molecular imaging 30.8 (2003): 1160-1164. [4]Cussó, Lorena, et al. "Effects of a ketogenic diet on [18 F] FDG-PET imaging in a mouse model of lung cancer." Molecular Imaging and Biology 21.2 (2019): 279-285. [5] DEGRADO, Timothy R. Synthesis of 14 (R, S)[18F] fluoro-6-thia-heptadecanoic acid (FTHA). Journal of Labelled Compounds and Radiopharmaceuticals, 1991, vol. 29, no 9, p. 989-995. [6] Isal, Sibel, et al. "PET imaging of 68 Ga-NODAGA-RGD, as compared with 18 F-fluorodeoxyglucose, in experimental rodent models of engrafted glioblastoma." EJNMMI research 8.1 (2018): 51. [7] Romero, E., and M. A. Morcillo. "Inorganic oxides with potential application in the preparation of a $68 \mathrm{Ge} / 68 \mathrm{Ga}$ generator system." Applied Radiation and Isotopes 119 (2017): 28-35. [8] Fortea, José I., et al. "Comparison of two protocols of carbon tetrachloride-induced cirrhosis in rats-Improving yield and reproducibility." Scientific reports 8.1 (2018): 1-10. [9] Zhou, Tianhao, and Shannon S. Glaser. "Pleiotropic effects of CD5L in hepatic inflammation and fibrosis." EBioMedicine 44 (2019): 22-23.

\section{Image/Figure:}

https://www.xcdsystem.com/wmis/abstract/File6959/GA262 ImageFigure 0422084925.png

Complete Status: Complete

First Name: Mario

Last Name: González-Arjona

Email: mgarjona@hggm.es 
Organization: Fundación para la Investigación Biomédica del Hospital Gregorio Marañón Country: Spain 


\title{
ID: GA267 \\ Light-triggered and image-guided CO delivery with multimodal CORM-1- loaded PBCA microbubbles
}

\author{
Patrick Koczera, Uniklinik RWTH Aachen University, pkoczera@ukaachen.de
}

Category: New Chemistry, Biology \& Bioengineering

\begin{abstract}
Body : Gases like O2, NO, Xe or H2S show therapeutic potential for disease models of myocardial infarction and for cardio/neuro-protection. As signaling molecule carbon monoxide (CO) bears the chance to modulate inflammation, cell survival and vascular tone in cardiovascular disease. However, handling and delivery as compressed gas is difficult in vivo [1]. The CO releasing molecule-1 (CORM-1) releases $\mathrm{CO}$ as "active compound" upon lightirradiation. Furthermore, the manganese central atoms bear the potential for utilization as an MRI contrast agent. However, as CORM-1 is highly hydrophobic, the utilization of drug carriers or potentially harmful organic solvents is required. To avoid these solvents, microbubbles (MB) can be used as drug delivery systems. MB enable enhanced drug delivery to tumors, and contrast-enhanced ultrasound imaging [2]. To exploit the beneficial effects of $\mathrm{CO}$ for myocardial infarction, we developed CORM-1-loaded poly(butyl cyanoacrylate) (PBCA) MB (COMB), which release $\mathrm{CO}$ upon light irradiation, for US- and MR-imaging-guided triggered delivery of $\mathrm{CO}$ to coronary arteries. Synthesis of COMB was performed by emulsifying an aqueous solution of the monomer n-butyl cyanoacrylate (BCA) with $1 \%$ Triton-X at $\mathrm{pH} 2.5$ with CORM-1 in dimethylformamide at 10000 RPM for 1 hour [2]. Sized-distribution, drug-loading and -release, and US- and MR-imaging contrast enhancement were determined for characterization of the synthesized MB. Cell toxicity was tested for J774A.1-, A549- and NIH/3T3-cells. Physiological CO delivery upon light irradiation was spectrophotometrically evaluated by quantifying the saturation of hemoglobin with CO. Apoptosis of HL-1 cardiomyocytes under hypoxia was assessed by immunofluorescence of activated caspase-3. Statistical evaluation was performed by ANOVA and Tukey's test. Relevant CORM-1-related toxicity was not detected in vitro. Unloaded MB and COMB showed high yield per synthesis with a narrow-size distribution in the range of 1-4 micrometers. Successful loading of CORM-1 in microbubbles was confirmed at $0.37 \mathrm{mM}$ CORM-1 in 109 COMB per mL. No significant differences could be detected between unloaded and CORM-1-loaded MB by quantification of contrast enhancement in US imaging (B-Mode in Figure 1A). Upon white light irradiation, COMB released approximately one molecule of CO per molecule CORM-1, whereas no release was detected in the dark. Accordingly, light irradiation induced significant enhancement of T1 and $\mathrm{T} 2$ relaxation rates in MR imaging, as detectable in T1- and T2-weighted imaging (Fig. 1B). Incubation of whole human blood with $\mathrm{COMB}$ and subsequent light irradiation increased percentage of hemoglobin saturated with CO significantly (Fig. 1C). Apoptosis of hypoxic cardiomyocytes was significantly reduced upon light irradiation and incubation with COMB (Fig. 1D). We developed a one-pot synthesis protocol for preparation of CORM-1-loaded PBCA MB named COMB. COMB enabled white light-triggered local generation and delivery of CO and were suitable for US imaging. CO release could be monitored by MRI. The potential of COMB for adjuvant therapy in myocardial infarction was demonstrated by light-triggered
\end{abstract}


delivery of $\mathrm{CO}$ to human blood and by reducing apoptosis in hypoxic cardiomyocytes. The impact on inflammation in macrophages is evaluated in ongoing investigations. Further validation in vivo needs to be performed in order to explore the feasibility of COMB for CO delivery in myocardial infarction. For clinical applications COMB could improve recanalization therapy after myocardial infarction. As catheters for coronary angioplasty can easily be mounted with light probes, COMB could improve recanalization therapy by (I) displaying recanalization success by US imaging, by (II) light-triggered CO delivery and by (III) MRI monitoring of CO delivery to the heart in order to enhance vascular healing and myocardial recovery, and reduce the rate of in-stent restenosis.

References: [1] Fix SM, Borden MA, Dayton PA, Therapeutic gas delivery via microbubbles and liposomes, Journal of Controlled Release, 209: 139-149, 2015. [2] Koczera P, Appold L, Shi Y, Liu M, Dasgupta A, Pathak V, Ojha T, Fokong S, Wu Z, van Zandvoort M, Iranzo O, Kuehne A, Pich A, Kiessling F, Lammers T, PBCA-based polymeric microbubbles for molecular imaging and drug delivery, Journal of controlled release 259: 128-135, 2017.

\section{Image/Figure:}

https://www.xcdsystem.com/wmis/abstract/File6959/GA267 ImageFigure 0422092544.jpg

Complete Status: Complete

First Name: Patrick

Last Name: Koczera

Email: pkoczera@ukaachen.de

Organization: Uniklinik RWTH Aachen University

Country: Germany 


\title{
ID: GA269 \\ Development of Targeted Near-Infrared Imaging Agents for Detection of Junction Adhesion Molecule-A Protein
}

\author{
Ethan Walker, Case Western Reserve University, yvv@case.edu
}

\section{Category: Oncology}

\begin{abstract}
Body : Purpose: Prostate and breast cancer are the most prevalent primary malignant human tumors globally. Prostatectomy and breast conservative surgery remain the most common definitive treatment option for the $>500,000$ men and women newly diagnosed with localized prostate and breast cancer each year in the US [1,2]. Morphological examination is the mainstay of diagnosis but margin under-sampling of the excised cancer tissue may lead to local recurrence $[3,4]$. Therefore, there is an unmet clinical need for technologies that rapidly and globally visualize cancerous tissues in the surgical cavity and can be used to guide their surgical resections. Near-infrared (NIR) imaging probes, which employ monoclonal antibody (Ab)phototoxic phthalocyanine dye IR700 conjugates, opened a window of opportunities to identify and visualize new potential targets in oncology. Molecular imaging is a potential method of performing in vivo imaging that permits the specific, image-guided, live, and controlled visualization of targeted cancer proteins that may define a surgical margin. Recently, junctional adhesion molecule-A (JAM-A) has been reported to be highly expressed on many tumor types and functionally has been shown to necessary and sufficient for self-renewal and tumor growth, making it a putative therapeutic target [5,6]. Procedures: JAM-A Ab/IR700 conjugates were used as an imaging agent to visualize prostate and breast tumor mouse xenografts in vivo. Results: The intravenously injected JAM-A Ab/IR700 enabled the non-invasive detection of heterotopic human prostate PC3pip and breast MDA-MB-231 cancer cells by NIR fluorescence imaging. A single dose of JAM-A Ab/IR700 significantly reduced number of mitotic cancer cells in vivo, indicating theranostic ability of this imaging agent. Conclusions: The JAM-A Ab/IR700 conjugates allowed us to image a specific receptor expression in prostate and breast tumors without post-image processing.
\end{abstract}

References: [1] Siegel R, Naishadham D, Jemal A (2012) Cancer statistics, 2012. CA Cancer J Clin 62:10-29. [2] Giordano SB, Gradishar W (2017) Breast cancer: updates and advances in 2016. Curr Opin Obstet Gynecol 29:12-17. [3] van den Ouden D, Kranse R, Hop WC, van der Kwast TH, Schroder FH (1998) Microvascular invasion in prostate cancer: prognostic significance in patients treated by radical prostatectomy for clinically localized carcinoma. Urologia internationalis 60:17-24. [4] Leong AS, Zhuang Z (2011) The changing role of pathology in breast cancer diagnosis and treatment. Pathobiology 78:99-114. [5] Lathia JD, Li M, Sinyuk M, Alvarado AG et al (2014) High-throughput flow cytometry screening reveals a role for junctional adhesion molecule a as a cancer stem cell maintenance factor. Cell reports 6:117-129. [6] Alvarado AG, Turaga SM, Sathyan P et al (2016) Coordination of self-renewal in glioblastoma by integration of adhesion and microrna signaling. Neuro-oncology 18:656-666.

\section{Image/Figure:}


Image/Figure Caption: Figure 1. In vivo fluorescence imaging of PC3pip and MDAMB231 tumors. In vivo IR700 fluorescence real-time imaging of right flank PC3pip human prostate tumor (A) and left chest MDAMB231 human breast tumor xenografts (C) in nude mice after tail vein injection of Jam-a mAb labeled by IR700 (Jam-A/IR700=probe). The fluorescence intensity of the probe decreased gradually in tumor and it was lower when it competed with pure Jam-A $\mathrm{mAb}$ (probe competition). Controls: 1) mix of unlabeled Jam-a mAb and Jam-a mAb/R700 (2-to1 ratio) as probe competition, 2) non-specific mouse monoclonal IgG labeled with IR700 as Norm mAb, and 3) IR700 alone. Black arrows - tumor xenografts. Pseudo-color images of IR700 fluorescence inside of (A) and (C) are presented in the same scale. Quantitative analysis of IR700 intensities in PC3pip prostate tumors (B) and MDAMB231 breast tumors (D). The fluorescence intensities were significantly higher in both PC3pip and MDAMB231 tumor xenografts labeled with probe as compared with controls. After pre-image (before i.v. injection), images were taken at 1-, 3-, 6-, 12-, 18-, 24-, and 48-hr time points. Each time-point indicates an average of IR700 fluorescence per $\mathrm{mm} 2$ of tumor xenograft. Notes: Vertical bars - SD. Data were normalized to the levels of IR700 fluorescence of pre-images. $\mathrm{N}=5$ for each type of

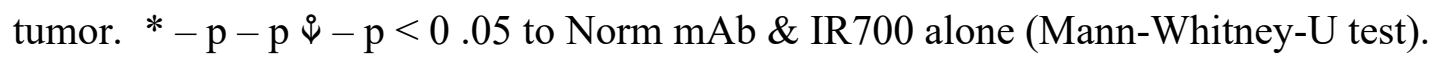

Complete Status: Complete

First Name: Ethan

Last Name: Walker

Email: yvv@case.edu

Organization: Case Western Reserve University

Country: United States 


\title{
ID: GA271 \\ pH-sensitive nanoparticle mediated TOPK inhibitor delivery to overcome drug- resistant triple negative breast cancer
}

\author{
Hoon Choi, University of Pennsylvania, hoonchoi@pennmedicine.upenn.edu
}

\section{Category: Oncology}

\begin{abstract}
Body : OBJECTICVE: PDZ-binding kinase/T-LAK cell-originated protein kinase (PBK/TOPK) encoded by a single gene has been identified as a promising target of breast cancer(1), especially for triple negative breast cancer (TNBC), which lacks subtype-specific treatment. While TNBC patients initially respond to chemotherapy, majority of them develop drug resistant shortly after, leading to cancer progression. Potent inhibitors of TOPK (TOPKi) have been developed however substantial hematological toxicity were noted, which can be mitigated by liposomal delivery(2). Meanwhile, TOPKi (e.g., OTS964) has been shown to sensitize drug-resistant glioma cells to temozolomide (3), however, their effect for chemoresistant TNBC has not been examined; furthermore, off-target effect and hematological toxicity induced by OTS964 require tumor-specific delivery platform (3). Here we formulate TOPKi into an ultra-pH sensitive nano delivery platform ( $\mathrm{pH}-\mathrm{NP})(4)$ and demonstrate a remarkable tumor growth delay in paclitaxel-resistant TNBC by TOPKi-pH-NP. METHODS: Succinimidyl PEGNHS (MW: 2000Da, NANOCS) in borate buffer solution at $\mathrm{pH} 8.5$ was conjugated to $\mathrm{pH}-$ sensitive peptide, KK-(GHFFH)3 reported earlier (4). The $\mathrm{pH}$-sensitive nanoparticles encapsulating indocyanine green (ICG-pH-NP) or OTS964 (OTS-pH-NP) were manufactured similarly as described earlier (4). A paclitaxel (Taxol)-resistant version was derived from human triple negative breast cancer line (HCC1806, ATCC) and 106 Taxol-resistant cells were inoculated into athymic nude mice. When xenografts reached $\sim 200 \mathrm{~mm} 3$, mice were assigned randomly into one of the four groups: (1) OTS-pH-NP (10mg OTS964/kg, i.v.)+Taxol (10mg Taxol/kg, i.p.); (2) Taxol only (10mg/kg, i.p.); (3) Free OTS (10mg/kg, i.v.)+Taxol (10mg/kg, i.p.); (4) OTS-pH-NP only (10mg OTS964/kg, i.v.). OTS-pH-NP or Free OTS were treated at day 5,7,9,12,14,16 while Taxol at day $0,2,5,7,9,12,14,16$. Tumor size was measured by caliper. In separate cohort, tumor-bearing mice were injected with ICG-pH-NP or free ICG (at 2 and 10 $\mathrm{mg} \mathrm{ICG/kg,} \mathrm{i.v.),} \mathrm{NIR} \mathrm{fluorescent} \mathrm{imaging} \mathrm{was} \mathrm{performed} \mathrm{(24} \mathrm{h} \mathrm{post} \mathrm{injection)} \mathrm{on} \mathrm{IVIS} \mathrm{Lumina}$ II (PerkinElmer) with ex/em $=745 / 820 \sim 880 \mathrm{~nm}$. The ICG was extracted from tumor tissues and was quantified by HPLC (JASCO). RESULTS: Figure 1A-B show that pH-NP enhanced ICG accumulation in the tumor, owing to its ability to dissolve itself along the $\mathrm{pH}$ gradient (highàlow) thus releasing the cargo favorably at mild acidic extracellular $\mathrm{pH}$ of the tumor. In contrast, despite a large dose, free ICG did not penetrate in the tumor well and tumor concentration is less than 1/4th of that achieved by pH-NP (Fig 1B). For this Taxol-resistant TNBC, it is not surprising that tumor growth was not delayed by Taxol only treatment (Figure 1C). In comparison, Taxol+free OTS moderately delayed tumor growth likely due to free OTS's unfavorable PK profile (data not shown) while OTS-pH-NP by itself also had moderate effect. OTS-pH-NP+Taxol treatment achieved a remarkable tumor regression $(\mathrm{P}=0.0004$ compared to free OTS+ Taxol), suggesting that OTS964, when achieving effective concentration in tumor mediated by $\mathrm{pH}-\mathrm{NP}$, can overcome the drug-resistant TNBC to Taxol. CONCLUSION:
\end{abstract}


OTS964 delivered by pH-NP was able to sensitize drug-resistant human TNBC to Taxol, leading to remarkable tumor regression. Such impact is mediated by $\mathrm{pH}-\mathrm{NP}$ 's ability to protect its cargos through the protein-rich plasma and release them into mildly acidic tumor extracellular space as it is confirmed by significantly higher tumor accumulation of ICG delivered by $\mathrm{pH}-\mathrm{NP}$.

References: 1. Park JH, Lin ML, Nishidate T, Nakamura Y, Katagiri T. PDZ-binding kinase/TLAK cell-originated protein kinase, a putative cancer/testis antigen with an oncogenic activity in breast cancer. Cancer Res 2006;66:9186-95 2. Matsuo Y, Park J-H, Miyamoto T, Yamamoto S, Hisada S, Alachkar H, et al. TOPK inhibitor induces complete tumor regression in xenograft models of human cancer through inhibition of cytokinesis. Science Translational Medicine 2014;6:259ra145-259ra145 3. Lu H, Xiao J, Ke C, Ni X, Xiu R, Tian Q, et al. TOPK inhibits autophagy by phosphorylating ULK1 and promotes glioma resistance to TMZ. Cell death \& disease 2019;10:583 4. Choi H, Liu T, Nath K, Zhou R, Chen IW. Peptide nanoparticle with pHsensing cargo solubility enhances cancer drug efficiency. Nano Today 2017;13:15-22

\section{Image/Figure:}

https://www.xcdsystem.com/wmis/abstract/File6959/GA271 ImageFigure 0422035450.jpg

Complete Status: Complete

First Name: Hoon

Last Name: Choi

Email: hoonchoi@pennmedicine.upenn.edu

Organization: University of Pennsylvania

Country: United States 


\title{
ID: GA274 \\ Glioblastoma staging with fluorescence molecular imaging of macrophages/microglia polarization
}

\author{
Arpan Mahanty, The Hong Kong Polytechnic University, arpan.mahanty1@gmail.com
}

\section{Category: Oncology}

Abstract Body : The glioblastoma multiforme (GBM), known as human brain cancer (Class IV), is very aggressive and lethal in nature. The overall $90 \%$ of patients survival period extended up to only 14 months even though its early diagnosis or treatment with cutting edge technologies. The GBM does not have any forestanding boundary, which makes difficult to properly identify it from the normal brain tissues. Surgery is a current gold standard for GBM treatment though complete surgical resection of GBM tissues is practically impossible ascribe to their diffusive characteristics into circumvent normal brain tissues, thus resulting in high recurrence rate. Temozolomide chemotherapy is an additional treatment option, however, its efficacy is not satisfactory and therapeutic resistance is high because of molecularly heterogeneous malignancy of GBM. To address this issue, there is an urgent need to develop a sensitive imaging tool for accurate staging and prognosis of GBM and for image-guided surgery to show a clear margin of GBM in the surgery room. Previous studies already reported that the number of immune responsive microglias and macrophages infiltrating in glioblastoma tumor is surprisingly high, which comprises $30 \%-50 \%$ of the tumor mass which can be classified into M1 (inflammatory) and M2 (non-inflammatory) subsets. The M1 subset exhibits tumor suppressive indication promoting immune vigilance alternatively M2 subset shows tumor supportive functions with tumor progression, angiogenesis. The identification of M2 macrophages (tumor associated macrophages/TAM) has taken current spotlight in onco-immunotherapy for better cancer diagnosis and prognosis. In past few years there are extensive research has been carried out on small molecules and has proven that small molecules can successfully apprach to thair target across the physiological barrier. Here, we effort to localize M1 and M2 macrophages together by fluorescene molecular imaging technique to illucidaete circumference of glioblastoma apart from normal brain tissues which leads a surgical guidance to surgeon for maximum tumor resecetion and improving patient outcome. Elicited from this avidity, we have synthesized/selected M1 and M2 specific molecular probes for M1/M2 macrophage/microglia identification. In vitro we demonstrated on differentiated mouse bone marrow derived macrophage (BMDM) and mouse microglia cell line (BV2) to target M1/M2 probe. Differentiated M1 and M2 macrophages/microglias were characterized with CD86+ and CD206+staining, flow cytometry and qRT-PCR analysis.In vivo fluorence imaging on orthotopic U87-MG-Red Fluc GBM model exhibited an excellent localization of M2

macrophages/microglias at tumor site. In vivo Histophathological staining analysis also elucidated the presence of M1 and M2 macrophage/microglial localization around the GBM tumor (co-localized with GFAP+). Ex- vivo organ distribution imaging also indicate specificity of molecular probe with negligible non specific organ distribution. The presented molecular probes allows to elucidate the condition of GBM tumors with prominent boundaries which may provide new purview of GBM treatment. 
Full Name of Abstract's 1st Author : Arpan MAHANTY

Complete Status: Complete

First Name: Arpan

Last Name: Mahanty

Email: arpan.mahanty1@gmail.com

Organization: The Hong Kong Polytechnic University

Country: Hong Kong 


\title{
ID: GA277 \\ Spectral photoacoustic and contrast-enhanced ultrasound imaging of placental function in response to preeclampsia therapy
}

\author{
Dylan Lawrence, Tulane University, dlawren@tulane.edu
}

Category: Systemic Diseases (Kidney, Liver and Pancreas)

\begin{abstract}
Body : Preeclampsia, a hypertensive disorder affecting 5-8\% of pregnancies, is associated with placental ischemia and hypoxia during early development which causes systemic endothelial dysfunction responsible for the later onset of maternal symptoms. While potential therapies targeting cardiovascular dysfunction reduces maternal symptoms in preclinical models, the impact on in vivo placental function has not been demonstrated. Here we present multimodal imaging of in vivo placental function in the reduced uterine perfusion pressure (RUPP) rat model of preeclampsia. Using spectral photoacoustic (SPA) and contrast-enhanced ultrasound (CEUS) imaging, we demonstrate longitudinal measures of placental oxygenation and perfusion in response to tempol, a superoxide dismutase mimetic previously shown to improve the maternal symptoms of preeclampsia [1]. Timed-pregnant Sprague Dawley rats were administered tempol via drinking water beginning on gestational day (GD) 12. Imaging was performed every other day from GD14 to 18. Post-imaging on GD14, the RUPP procedure was implemented following established techniques [2]. sPA images were acquired at the relative optical absorption peaks of oxyhemoglobin and deoxyhemoglobin and the resulting photoacoustic signal was fit using a linear spectral unmixing algorithm to estimate the concentration of both chromophores in the image. Using co-registered B-mode US images of anatomy, the placenta was then manually selected and average oxygen saturation was calculated. To assess longitudinal changes in placental perfusion during normal pregnancy, nonlinear CEUS images were acquired on a separate cohort of untreated animals over a 10-minute period following a bolus injection of microbubble contrast agent. Conventionally, perfusion is quantified using a time-intensity curve (TIC) fit using the average CEUS signal in a region of interest. We implemented a pixel-wise TIC analysis in order to capture the complex flow profile in the placenta and surrounding tissue that's typically lost with conventional methods. Hemodynamic parameters related to blood flow and blood volume in the placenta were then calculated. Two days after the RUPP procedure, average placental oxygen saturation significantly decreased from $55 \%$ to $48 \%$, while in normally developing placenta the oxygenation remains constant. Treatment of the pregnancy animal with tempol increased placental oxygen saturation in the RUPP by $6 \%$ - effectively restoring placental oxygenation to normal pregnant levels. This improved oxygenation was maintained through GD18 when tempol was also found to reduce mean arterial pressure in RUPP animals. A pixel-wise TIC analysis of CEUS images was used to assess longitudinal changes in placental perfusion during normal pregnancy. From GD14 to 18, relative blood flow and placental blood volume increased while mean transit time of microbubbles in the placenta decreased, demonstrating an increase in placental perfusion during normal pregnancy. We have demonstrated SPA and CEUS imaging of in vivo placental hypoxia and perfusion in response to therapeutic intervention for preeclampsia. Future work will adapt these multimodal imaging
\end{abstract}


methods to include measures of vascular growth and provide a more complete understanding of overall placental function.

References: [1] M. Sedeek et al., "Role of Reactive Oxygen Species in Hypertension Produced by Reduced Uterine Perfusion in Pregnant Rats," Am J Hypertens, vol. 21, no. 10, pp. 1152-6, Oct 2008, doi: 10.1038/ajh.2008.239. [2] J. Li, B. LaMarca, and J. F. Reckelhoff, "A model of preeclampsia in rats: the reduced uterine perfusion pressure (RUPP) model," Am J Physiol Heart Circ Physiol, vol. 303, no. 1, pp. H1-8, Jul 2012, doi: 10.1152/ajpheart.00117.2012.

\section{Image/Figure:}

https://www.xcdsystem.com/wmis/abstract/File6959/GA277_ImageFigure 0422102548.jpg

Full Name of Abstract's 1st Author : Dylan J. Lawrence

Complete Status: Complete

First Name: Dylan

Last Name: Lawrence

Email: dlawren@tulane.edu

Organization: Tulane University

Country: USA 


\title{
ID: GA279 \\ 1 H MRS Analysis of Muscle Metabolites altered by Cachexia
}

\author{
Raj Kumar Sharma, The Johns Hopkins University, School of Medicine, \\ rsharm40@jhmi.edu
}

Category: Oncology

\begin{abstract}
Body : Skeletal muscle wasting is one of the hallmarks of cachexia, a syndrome that affects almost $80 \%$ of pancreatic cancer patient [1]. The syndrome is characterized by unintentional weight loss, loss of appetite, reduced physical ability and poor quality of life [2]. In a recent study, the role of mitochondrial dysfunction was identified in the amino acid derangement observed in cachectic muscle from a mouse model [3]. $1 \mathrm{H}$ magnetic resonance spectroscopy (MRS) studies have identified metabolic changes in muscle tissues associated with glioma and gastric cancer induced cachexia $[4,5]$. Here we have investigated metabolic changes in muscle tissue occurring with pancreatic cancer-induced cachexia. Identification of metabolic changes in skeletal muscle may provide novel targets to treat this syndrome. Metabolic changes were quantified using high-resolution quantitative 1H MRS of muscle tissue obtained from normal, non-cachectic (Panc1) and cachectic (Pa04C) mice bearing pancreatic ductal adenocarcinoma (PDAC) xenografts. The Panc1 cell line was obtained from ATCC and the $\mathrm{Pa} 04 \mathrm{C}$ cell line was kindly provided by Dr. Maitra. Tumors were generated by inoculating cancer cells in the right flank of six to eight-week-old male severe combined immunodeficient mice. Muscle tissue from the hind limb of euthanized normal $(\mathrm{n}=10), \mathrm{Pa} 04 \mathrm{C}(\mathrm{n}=10)$ and Panc1 $(\mathrm{n}=9)$ tumor bearing mice, was excised once tumors were $\sim 300 \mathrm{~mm} 3$, snap frozen and stored at $80^{\circ} \mathrm{C}$ prior to dual phase extraction. $1 \mathrm{H}$ MRS was performed on the water and lipid soluble phases. All 1H MR spectra with water suppression were acquired on a $750 \mathrm{MHz}$ MR spectrometer using a single pulse sequence. All data analyses and quantification were performed with TOPSPIN 3.5 software. The presence of cachexia was confirmed by the significant weight loss observed in Pa04C tumor bearing mice, compared to Panc1 tumor bearing mice and normal mice. We identified a muscle metabolic signature in mice with and without cachexia inducing PDAC xenografts as summarized in Figure 1. A significant decrease of alanine, glutathione, aspartate, creatine, glycine, lactate, glucose and an increase in isoleucine, acetate, phenylalanine and formate was detected in cachectic muscle tissue compared to control muscle tissue (Figure 1A). Previous studies have reported a decrease of glucose in muscle tissue of glioma induced cachexia and gastric cancer induced cachexia that indicated higher utilization of glucose by the tumor during cachexia $[4,5]$. Reduced alanine in the cachectic group indicated an imbalance of systemic glucose hemostasis and amino acid metabolism [6]. Investigating the role of glycine could be important as supplementation of glycine may reduce muscle wasting [7]. We observed significant depletion of lipid $(\mathrm{CH} 3, \mathrm{CH}=\mathrm{CH})$ and triglycerides in cachectic muscle (Figure 1 B) that is consistent with a previous study [8]. Our results advance the understanding of changes in muscle metabolism with cachexia, and support investigating metabolic targets and biomarkers to reduce pancreatic cachexia-associated morbidity. Supported by NIH R01CA193365 and R35CA209960.
\end{abstract}


References: 1. Argiles et al. (2014) Nat. Rev. Cancer 14 (11), 754-62; 2. Siddiqui et al. (2020) Biochimica et Biophysica Acta 1873 (2), 188359; 3. Kunzke et al. (2020) J Cachexia Sarcopenia and Muscle 11 (1), 226-240; 4. Cui et al. (2019) Skeletal muscle 9 (1), 3-3; 5. Cui et al. (2019) J. of Proteome Res. 18 (4), 1880-1892; 6. Winnard et al. (2016) Cancer Res. 76 (6), 1441-50; 7. Ham et al. (2014) Clin. Nutr. 33 (3), 448-58; 8. Penet et al. (2011) Cancer Res. 71(22), 69486956.

\section{Image/Figure:}

https://www.xcdsystem.com/wmis/abstract/File6959/GA279 ImageFigure 0610034217.jpg

Image/Figure Caption: Figure 1: Bar plots summarizing metabolic differences in the muscle between control mice (Normal), and mice with Panc1 and Pa04C tumors. (A) water phase metabolites, (B) lipids. Values represent Mean + SEM. P X control vs Panc1, * Panc1 vs $\mathrm{Pa} 04 \mathrm{C})$.

Complete Status: Complete

First Name: Raj Kumar

Last Name: Sharma

Email: rsharm40@jhmi.edu

Organization: The Johns Hopkins University, School of Medicine

Country: United States 


\title{
ID: GA280 \\ Biodistribution and dosimetry of Gallium-68 hexa-lactoside for imaging asialoglycoprotein receptor
}

\author{
Mei-Hui Wang, INER, mhwang@iner.gov.tw
}

Category: Systemic Diseases (Kidney, Liver and Pancreas)

\begin{abstract}
Body : Introduction: Gallium-68 hexa-lactoside is an asialoglyco-peptide that specifically binds the asialoglycoprotein receptor on the membrane of mesenchymal hepatocyte. The primary purpose of this study was to measure the whole-body biokinetics and radiation dosimetry in healthy human volunteers. The study was conducted within a regulatory framework that required its pharmacological safety to be assessed simultaneously. Methods: The sample includes 7 men and 5 women ranging in age from 23-44 yr. Whole-body PET scans were acquired sequentially for 64 min after the intravenous administration of $129.5 \pm 9.25 \mathrm{MBq}$ $(3.5 \pm 0.25 \mathrm{mCi})$ [68Ga]hexa-lactoside. The fraction of the administered dose in major organs was quantified from the attenuation and background corrected geometric mean counts in conjugate views. Multiexponential fractions were iteratively fit to each time-activity curve using a nonlinear, least squares regression algorithm. These curves were numerically integrated to yield source organ residence time. The radiation dose estimates will be calculated using OLINDA/EXM program 1.1. Results: There were no pharmacological effects of the radiotracer on any of the subjects. The images showed rapidly increased activity in liver within $8 \mathrm{~min}$. PET images demonstrated that the radiopharmaceutical localized in liver that was consistent with asialoglycoprotein receptor binding. The normal reference range (\%ID) of liver reserve at $0-8$ min, 10-18 $\mathrm{min}, 20-28 \mathrm{~min}, 30-46 \mathrm{~min}$ and 48-64 $\mathrm{min}$ in the healthy volunteers were $42.18 \pm 5.87 \%, 54.08 \pm 3.97 \%, 56.55 \pm 4.84 \%, 57.34 \pm 5.06 \%$ and $55.83 \pm 4.52 \%$, respectively. A transient low level of $42 \%$ measured in $0-8$ min post-injection for gallium-68 hexa-lactoside was due to the initial biodistribution. The radiation doses in human were $0.0098 \mathrm{mSv} / \mathrm{MBq}$ in liver and $0.0268 \mathrm{mSv} / \mathrm{MBq}$ in whole-body (i.e. $0.9916 \mathrm{mSv} / \mathrm{mCi}$ ). For maximum injection dose 3.75 $\mathrm{mCi}$, the systemic exposure for [68Ga]hexa-lactoside is $3.7185 \mathrm{mSv}$ which is acceptable safe enough for human administration, compared to [18F]FDG in clinical use (7-14 mSv). Conclusion: [68Ga]hexa-lactoside may be a safe and effective radiotracer for imaging asialoglycoprotein receptor in the liver and the body.
\end{abstract}

Complete Status: Complete

First Name: Mei-Hui

Last Name: Wang

Email: mhwang@iner.gov.tw

Organization: INER 
Country: Taiwan 


\title{
ID: GA281 \\ PARP1 targeted radiotheranostics for brain tumor
}

\author{
Giacomo Pirovano, Memorial Sloan Kettering Cancer Center, pirovang@mskcc.org
}

\author{
Category: Oncology
}

\begin{abstract}
Body : Introduction Glioblastoma (GBM) has a median survival of 12 - 15 months and a 5 -years survival of $5 \%$ in the United States of America [1,2]. In order to meet this challenge, we developed and characterized a cancer-specific theranostic agent, 123I-MAPi, an Auger emitter. In an effort to pursue the clinical translation of this molecule for GBM treatment, we tested 18F-PARPi in a Phase I clinical trial. Methods 18F-PARPi synthesis has been previously described [3]. 123I-MAPi synthesis has been previously described [4]. In vivo experiments were performed in accordance with protocols approved by the Institutional Animal Care and Use Committee of MSK and followed National Institutes of Health (NIH) guidelines. Orthotopic DIPG and TS543 GBM model were used for in vivo experiments. ALZET® Osmotic Pumps were implanted subcutaneously for delivery flow of $1 \mu \mathrm{L} / \mathrm{h}$, over $3 \mathrm{~d}$. Dosimetry was calculate using PARaDIM [5]. Results DIPG mice were intravenously co-injected with FITC-Dextran and 18F-PARPi (Fig.1A) to test blood-brain barrier (BBB) permeability (Fig.1B). A higher BBB permeability to 18F-PARPi compared to FITC-Dextran was observed (Fig.1C). TS543 orthotopic GBM mouse model showed improved survival for 123I-MAPi treatment cohort $(\mathrm{n}=$ 10), with a median survival of 58 days as opposed to 40 days for the control cohort $(\mathrm{n}=12)$ (Fig.1D). Convection enhanced delivery (CED) showed therapeutic efficacy of 123I-MAPi: median survival of $72 \mathrm{~d}$ as opposed $48 \mathrm{~d}$ in the control (Fig.1E). Dosimetry (Fig.1F) confirmed tumor accumulation with minimal absorbed dose to healthy organs. Relative biological efficacy (RBE) of nearly 50, compared to standard external beam X-ray, allowed us to determine equivalent dose of the Auger radiation (Fig.1G). Conclusion We developed and characterized the first stable Auger-emitting PARP1 inhibitor capable to show cancer-specific cytotoxicity preclinically. This, and the promising results observed in patients using 18F-PARPi, could open the door for Auger-based theranostic clinical translation for GBM patients.
\end{abstract}

References: [1] Wen PY, Kesari S (2008). Malignant gliomas in adults. New England Journal of Medicine [2] Weissleder R, Pittet MJ (2008). Imaging in the era of molecular oncology. Nature [3] Carney, B et al. (2018) Target engagement imaging of PARP inhibitors in small-cell lung cancer. Nature communications [4] Pirovano et al. (2020) Targeted brain tumor radiotherapy using an Auger emitter. Clinical Cancer Research [5] Carter LM et al. (2019) PARaDIM - A PHITS-based Monte Carlo tool for internal dosimetry with tetrahedral mesh computational phantoms. Journal of Nuclear Medicine

\section{Image/Figure:}

https://www.xcdsystem.com/wmis/abstract/File6959/GA281_ImageFigure_0422121554.jpg

Complete Status: Complete 
First Name: Giacomo

Last Name: Pirovano

Email: pirovang@mskcc.org

Organization: Memorial Sloan Kettering Cancer Center

Country: United States 


\title{
ID: GA282 \\ 18F-DPA714 PET imaging in EBOV infected monkeys
}

\author{
Swati Shah, National Institutes of Health, swati.shah@nih.gov
}

\section{Category: Immunology: Inflammation \& Infection}

\begin{abstract}
Body : Background The Ebola Virus (EBOV) is the causative agent of the Ebola virus disease (EVD) that is known to have a very high mortality rate. The EBOV has been shown to possess broad cell tropism and once inside the host, it infects multiple cell types. Previous studies have shown that early infection of macrophages, dendritic cells and monocytes are largely responsible for the systemic dissemination of the virus. EVD is largely characterized by lymphopenia, breach in vascular integrity, cytokine storm and multi-organ failure. Here, we measured translocator protein $18 \mathrm{kDa}$ (TSPO) binding by $18 \mathrm{~F}-\mathrm{DPA} 714$ PET imaging to evaluate peripheral disease progression in EBOV infected monkeys since TSPO is expressed in various immune cells at baseline and is generally upregulated in inflammatory conditions. Thus, correlating the changes in organ TSPO expression with disease markers can shed light on the underlying pathophysiology of EBOV infection. Methods We used 18F-DPA714, a PET ligand, to longitudinally quantify the peripheral TSPO binding in Rhesus macaques $(n=10)$ at baseline and multiple time points after EBOV/Makona inoculation. We performed 40 min dynamic PET imaging of the chest and abdomen and used Logan plot (PMOD) with an image-derived blood input function (left ventricle) to measure lung, liver, spleen and bone marrow (BM) distribution volumes $(\mathrm{Vt})$. Whole blood samples were collected during each imaging time point to measure various biomarkers such as plasma viral load, cytokine expression, differential blood counts, kidney and liver function panels. Post-mortem splenic tissue sections were immunostained for various markers of macrophages (CD68), T cells (CD3), B cells (CD20), TSPO, apoptosis markers (cleaved caspase 3(CC3) and cleaved Poly (ADP-ribose) polymerase 1 (PARP1)) and EBOV (vp40) by multiplex fluorescence immunohistochemistry (MF-IHC). Results Assessment of DPA714 Vt values over time (post-infection), using a mixed effect linear regression model, demonstrated a significant negative correlation of time with spleen and lung $\mathrm{Vt}$ and positive correlation with BM Vt. Multiple plasma cytokines including IFN- $\gamma$, MIP-1a, IL-6, IL-10, IL1 ra, IL-15 and IL-18 correlated negatively with lung/spleen Vt and positively with BM Vt (p Conclusions Our findings suggest a monocytic and lymphocytic depletion in the spleen, rather than immune activation and inflammatory reaction as would be expected in inflammatory conditions. Similarly, decreased uptake in lungs may reflect the depletion of alveolar macrophages, which could explain predisposition of EBOV patients to superimposed infections including pneumonia. However, increased uptake in the BM suggests hematopoietic activation in response to thrombocytopenia, monocyte depletion and neutrophilia. This is supported by BM Vt showing significant negative correlation with platelet counts and positive correlations with neutrophil percentages.
\end{abstract}

\section{Image/Figure:}


Image/Figure Caption: (A) Spleen Vt decreased over time after EBOV inoculation while (B) bone marrow Vt was found to increase over time. (C) Coronal and sagittal PET imaging showing decreased uptake in the spleen (red circle) and increased uptake in the bone marrow (red arrows) on the terminal scan compared to baseline.

Complete Status: Complete

First Name: Swati

Last Name: Shah

Email: swati.shah@nih.gov

Organization: National Institutes of Health

Country: United States 


\title{
ID: GA283 \\ Acute statin treatment improves antibody accumulation in HER2-, EGFR-, and PSMA-expressing tumors
}

\author{
Patricia Ribeiro Pereira, WUSTL, ribeiropereirap@wustl.edu
}

\section{Category: Oncology}

\begin{abstract}
Body : Introduction:Statins are low-cost cholesterol-depleting drugs used to treat patients with hypercholesterolemia. Preclinically, statins modulate caveolae-mediated endocytosis. Membrane receptors defined as tumor biomarkers and therapeutic targets are often internalized by an endocytic pathway. Indeed, receptor endocytosis and recycling are dynamic mechanisms that can affect receptor density at the cell surface. In therapies using monoclonal antibodies, a downregulation in receptor density at the cell surface decreases antibody binding to the extracellular domain of the membrane receptor. Here, we used immunoPET to demonstrate that statins can temporally modulate human epidermal growth factor receptor 2 (HER2), epidermal growth factor receptor (EGFR), and prostate-specific membrane antigen (PSMA) receptor density at the tumor cell surface for binding therapeutic monoclonal antibodies. Methods:Small-animal PET was used to study the binding of 89Zr-labeled antibodies in ectopic xenografts and patient-derived xenografts (PDXs). Lovastatin, simvastatin, and rosuvastatin were used to modulate caveolae. To retrospectively compare the non-statin vs.statin Kaplan-Meier survival curves, retrospective data from patients treated with trastuzumab were obtained from pharmacy records. Therapeutic assays were performed in PDXs to determine tumor populations that benefit from the combination of statin/antibody and to determine the mechanisms by which statins synergize with therapeutic monoclonal antibodies. Results: Using data from The Cancer Genome Atlas and the Cancer Cell Line Encyclopedia we observed a negative correlation at the protein level between the cholesterol-binding protein caveolin-1 (CAV1) and HER2, EGFR, or PSMA. In xenografts and patient samples, wefound that tumors with high CAV1 expression localized less receptor at the cell membrane and these features decreased antibody uptake and efficacy. In cultured cells and tumor xenografts, statins temporally depleted CAV1 expression in ways than enhanced tumors' avidity for anti-HER2, anti-EGFR, and anti-PSMA antibodies (Fig. 1A,B). In HER2- and EGFR-expressing xenografts, treating mice with a statin accelerated and increased 89Zr-labeled antibody accumulation at the tumor site. The hydrophilic rosuvastatin demonstrated a lower ability to enhance antibody binding to tumors when compared with the lipophilic lovastatin and simvastatin. 89Zr-labeled huJ591 tumor accumulation was higher in PSMA-expressing tumors of statin-treated mice when compared with tumors of saline-treated mice at later time-points of antibody accumulation $(24 \mathrm{~h}$ and $48 \mathrm{~h})$, but the values were similar at $4 \mathrm{~h}$ and $8 \mathrm{~h}$. These results suggest that treating mice with statins increases, but does not accelerate, anti-PSMA antibody accumulation. Anti-DLL3 antibody accumulation was similar in saline versus statin treated tumors. Retrospective data demonstrated that patients with HER2+/CAV1HIGH(29.4\% of total HER2+gastric tumors) have a significantly worse overall survival than those expressing low CAV1 (Fig. 1C). Kaplan-Meier analyses of statin use and HER2+gastric cancer disease outcome in patients treated with trastuzumab suggested that patients without statin treatment have worse survival than patients treated with a statin (Fig. 1C).
\end{abstract}


Additionally, statins synergize with therapeutic antibodies to decrease oncogenic signaling pathways. Conclusions:Our data suggest that acute statin treatment with appropriate pharmacokinetics/pharmacodynamics are potential adjuvants for specific antibody-targeted therapies. Statins enhanced antibody accumulation while also enhancing target density at the cell membrane. In DLL3+tumors containing low levels of CAV1, statins did not alter antibody binding. Because lipophilic statins accumulate in cancer cells through passive diffusion, we observed higher antibody accumulation when compared with tumors from mice treated with rosuvastatin. Further investigations are necessary to define the statin doses necessary for tumoral CAV1 depletion, and the therapeutic value of combining cytotoxic antibodies with statins able to temporally stabilize receptor availability at the cell membrane. The translational significance of these findings lies in the potential of statins to modulate the heterogeneous staining of receptors at the cell membrane, a characteristic often associated with poor response in tumors to therapeutic antibodies.

\section{Image/Figure:}

https://www.xcdsystem.com/wmis/abstract/File6959/GA283 ImageFigure 0511085449.png

Image/Figure Caption: Figure 1. (A,B)Representative coronal images (48 h) and biodistribution of tumor uptake at 4, 8, 24, and $48 \mathrm{~h}$ p.i. of [89Zr]Zr-DFO-trastuzumab, [89Zr]Zr-DFOpanitumumab, [89Zr]Zr-DFO-huJ591, and [89Zr]Zr-DFO-SC16 in athymic nude mice bearing, respectively subcutaneous HER2+NCIN87, EGFR+A431, PSMA+PC3pip, and DLL3+H82 tumors. Lovastatin $(8.3 \mathrm{mg} / \mathrm{kg}$ of mice) was orally administrated $12 \mathrm{~h}$ prior and at the same time as the tail vein injection of $89 Z$ Zr-labeled antibodies. Bars, $n=5$ mice per group, mean \pm S.E.M. MIPs maximum intensity projection. \%ID g-1, percentage of injected dose per gram. ${ }^{* *} \mathrm{P}<0.01,{ }^{*} \mathrm{P}<0.05$ based on a Student's t-test and compared with tumor uptake of salinetreated mice at the respective time-point. (C, top) Kaplan-Meier analyses of CAV1 expression and gastric cancer disease outcome in patients treated with trastuzumab. Patients with HER2+/CAV1High(blue) phenotype have a worse survival than HER2+/CAV1Low(red) (n = 51 patients). Log rank; $\mathrm{p}<1 \mathrm{e}-4$. (C, bottom) Kaplan-Meier analysis of statin use and gastric cancer disease outcome in patients treated with trastuzumab. Patients without statin treatment (blue) have a worse survival than patients treated with statin (red) $(n=56$ patients). Log rank; $p=0.02$.

Full Name of Abstract's 1st Author : Patricia M. R. Pereira, $\mathrm{PhD}$

Complete Status: Complete

First Name: Patricia

Last Name: Ribeiro Pereira

Email: ribeiropereirap@wustl.edu

Organization: WUSTL

Country: United States 


\title{
ID: GA285 \\ A MUC16-targeted Antibody for immunoPET Imaging of Pancreatic \& Ovarian Cancer
}

Kyeara Mack, Memorial Sloan Kettering, mackk1@mskcc.org

\section{Category: Oncology}

\begin{abstract}
Body : Objectives: MUC16 ranks high among viable targets for the diagnosis and treatment of a class of ovarian and pancreatic adenocarcinomas. Aberrantly expressed O-linked glycans on mucins such as MUC16 are implicated in the biology that promotes the malignancy of tumors of epithelial origin. Such truncated MUC16 glycoforms are being investigated as potential targets for cancer treatment. We hypothesize that antibodies that bind to aberrantly expressed glycoforms of MUC16 can serve as a class of novel immunoPET tracers for noninvasive detection of ovarian and pancreatic tumors. Here, we utilized a humanized antibody, huAR9.6, that targets fully-glycosylated as well as hypoglycosylated isoforms of MUC16 to noninvasively image pancreatic and ovarian tumors via immunoPET. Methods: huAR9.6, was conjugated to desferrioxamine (DFO) and radiolabeled with zirconium-89 (89Zr) for immunoPET imaging. Radioligand binding assays were used to assess huAR9.6 binding to MUC16 on OVCAR3, SKOV3, Capan-2, and MiaPaCa-2 cell lines. OVCAR3 and Capan-2 cell lines were used as MUC16-positive ovarian and pancreatic cancer cell lines respectively, whereas SKOV3 and MiaPaCa-2 were used as MUC16-negative cell lines. Subcutaneous xenografts were developed by injecting the above-mentioned cell lines in the right flanks of athymic nude mice. Upon tumor development, mice were injected with [89Zr]Zr-DFO-huAR9.6 and serial PET imaging was performed at 24, 48, 72, 96, 120, and $144 \mathrm{~h}$. Ex vivo biodistribution analyses were conducted at $144 \mathrm{~h}$ after injection of [89Zr]Zr-DFO-huAR9.6. Results: [89Zr]ZrDFO-huAR9.6 was successfully synthesized with $>99 \%$ radiochemical purity and high specific activity $(\sim 10 \mu \mathrm{Ci} / \mu \mathrm{g})$. Radioligand binding assays demonstrated specific binding of [89Zr]ZrDFO-huAR9.6 to OVCAR3 and Capan-2 cell lines. The radiolabeled antibody showed $>95 \%$ stability in human serum over a 7-day period. Significant tracer uptake was observed in Capan-2 tumors $(\sim 25 \% \mathrm{ID} / \mathrm{g})$ whereas low uptake was shown in MiaPaCa-2 tumors $(\sim 5 \% \mathrm{ID} / \mathrm{g})$. In vivo tumor uptake of [89Zr]Zr-DFO-huAR9.6 in Capan-2 tumors could be blocked by a co-injection of a 25-fold excess of unlabeled huAR9.6 antibody. Conclusion: Here, we successfully synthesized a 89Zr-labeled variant of the huAR9.6 antibody. Data from in vitro binding assays and in vivo PET imaging shows that radiolabeled huAR9.6 specifically binds to pancreatic cancer cells that express MUC16. Radioligand binding data also shows specific binding to ovarian cancer cells that are MUC16-positive. These findings demonstrate potential for using [89Zr]Zr-DFO-huAR9.6 as a non-invasive imaging agent to detect cancer of the pancreas and ovaries. Acknowledgments: This work was supported by the Emerson Collective Cancer Research Fund, OncoQuest Inc., and NIH R35 CA232130.
\end{abstract}

\section{Image/Figure:}


Image/Figure Caption: Figure 1. In vivo data for 89 [Zr]Zr-DFO-huAR9.6 in subcutaneous mouse models. A. Serial PET/CT imaging of 89[Zr]Zr-DFO-huAR9.6 in MUC16+ capan-2 tumors shows clear uptake after $48 \mathrm{~h}$, with high tumor uptake enabling clear delineation of capan-2 tumors after $144 \mathrm{~h}$. Tumor uptake is successfully blocked at $72 \mathrm{~h}$. Corresponding biodistribution is shown in B, reaching $\sim 25 \% \mathrm{ID} / \mathrm{g}$ at $144 \mathrm{~h}$. C. Lower uptake is observed with serial PET/CT imaging in MUC16- MiaPaCa-2 tumors, with corresponding biodistribution (5\% $\mathrm{ID} / \mathrm{g}$ after $144 \mathrm{~h}$ ) is shown in panel D.

Complete Status: Complete

First Name: Kyeara

Last Name: Mack

Email: mackk1@mskcc.org

Organization: Memorial Sloan Kettering

Country: United States 


\title{
ID: GA286 \\ Facile synthesis and characterization of 11-acetate 21 tosylate budesonide and its fluoride radiolabelling for lung imaging application
}

\author{
Mei-Hui Wang, INER, mhwang@iner.gov.tw
}

\section{Category: Cardiovascular \& Pulmonary}

\begin{abstract}
Body : Introduction: Bronchopulmonary dysplasia is a common pulmonary complication in preterm infants and lung inflammation control by budesonide has been used to reduce the incidence and severity of bronchopulmonary dysplasia. For pharmacokinetic study of budesonide, 11-acetate 21 tosylate budesonide was prepared for F-18 radiolabelling and [18F]budesonide is used for imaging application. Methods: 11 -acetate 21 tosylate budesonide was prepared by protecting the two hydroxyl groups of commercial budesonide with tosyl and acetyl group, sequentially. The tosylation of budesonide is performed by tosyl chloride using DMAP as an activating reagent and triethylamine as an $\mathrm{HCl}$ scavenger. Then, the acetylation for another hydroxyl group is performed by acetic anhydride to have 11-acetate 21 tosylate budesonide. For radiolabeling, [18F]fluoride was trapped on an anion exchange cartridge and eluted using kryptofix-2.2.2. After the drying procedure, [18F]fluoride was reacted with 11acetate 21 tosylate budesonide at $110^{\circ} \mathrm{C}$ for $10 \mathrm{~min}$ in anhydrous dimethyl fluoride via an aromatic nucleophilic substitution, in which the tosyl leaving group is substituted with $[18 \mathrm{~F}]$ fluoride. After passing through a Sep-Pak C-18 to remove the free $[18 \mathrm{~F}]$ fluoride and followed by hydrolysis with $\mathrm{NaOMe} /$ methanol, [18F]budesonide was synthesized. The radiolabeled products were analyzed by radio-HPLC using LichroCART column $(5 \mathrm{~mm}$ x $25 \mathrm{cmx}$ $10 \mathrm{~mm}$ ) and eluted with $50 \%$ ethanol for $30 \mathrm{~min}$ at a flow rate of $0.5 \mathrm{~mL} / \mathrm{min}$. The intratracheal pharmacokinetic study in SD rats was measured by PET/CT. Results: The 11-acetate-21$[18 \mathrm{~F}]$ budesonide showed coupling peaks at retention times of approximately $21 \mathrm{~min}$ and $22 \mathrm{~min}$, which is because commercial budesonide has mixed budesonide $\mathrm{R}(+)$ and $\mathrm{S}(-)$ isomers. However, after de-acetylation, the epimers of [18F]budesonide were eluted at $25 \mathrm{~min}$ as a single peak. In animal study, [18F]budesonide was strongly detected in the trachea at 15 min after intratracheal injection, but rapidly metabolized to liver. With surfactant supplement in $[18 \mathrm{~F}]$ budesonide, $[18 \mathrm{~F}]$ budesonide can steadily stay in lung for more than $60 \mathrm{~min}$. Conclusion: 11 -acetate 21 tosylate budesonide and [18F]budesonide were successfully prepared and purified. PET/CT images showed that surfactant supplement is necessary for [18F]budesonide to have better distribution in lung for more than $60 \mathrm{~min}$.
\end{abstract}

Complete Status: Complete

First Name: Mei-Hui

Last Name: Wang

Email: mhwang@iner.gov.tw 
Organization: INER

Country: Taiwan 


\title{
ID: GA287 \\ Progress Towards Enzyme-Responsive Peptide Nanoparticles for Imaging of Metastatic Cancer
}

\author{
Douglas MacPherson, City University of New York, dmacpherson@gc.cuny.edu
}

Category: New Chemistry, Biology \& Bioengineering

\begin{abstract}
Body : Cancers metastasize, making the disease difficult to effectively detect and treat. Metastatic cancer cells uniquely overexpress a class of enzymes called matrix metalloproteinases (MMPs). MMP9 expression and activity is correlated with metastasis and tumor spread in breast and prostate cancer. Using enzyme-cleavable peptide sequences, we will be able to harness these MMPs for use against the disease. My central hypothesis is that radiolabeled enzyme-responsive peptides will provide a means to image metastatic cancer characterized by elevated MMP expression. Our laboratory has developed enzyme-responsive self-assembling peptide amphiphiles (ERSAPAs), that adopt a micelle structure, and switch conformation to a fiber when cleaved by enzymes. Short hydrophobic peptide fibers have been demonstrated to adhere to tumor tissue. We propose to tether a radioactive iodine molecule to an ERSAPA, permitting it to travel freely in the body, and when it arrives at a site of MMP9-expressing cancer tissue, the resulting cleavage and fiber formation will "anchor" the radio-label at the cancer site. Using established peptide design rules, we have synthesized peptides with three distinct regions: (i) a hydrophobic fiber-forming sequence, (ii) a hydrophilic sequence that adjusts the amphiphilic balance of the peptide to favor micelle formation, and (iii) an MMP9-cleavable sequence separating the hydrophilic and hydrophobic regions. Our negative control consists of a micelleforming peptide sequence in which the MMP-cleavable sequence is scrambled, preventing cleavage. Our positive control is a micelle-forming, cleavable peptide that does not have a radioligand attached. We have used atomic force microscopy (AFM) and fluorescence experiments to determine peptide assembly and nanostructure formation. Liquid chromatography - mass spectrometry (LCMS) has been used to determine cleavage of the peptide by the enzyme. Thus far, we have demonstrated micelle formation of our peptides, MMP-cleavage of our positive control peptide, and effective "cold" iodine coupling to the peptides. Remaining work involves demonstrating cleavage and micelle -to- fiber transition of the iodine-labeled peptide. Following this, we will assess the tumor-targeting effectiveness using radioactive "hot" iodine in murine models of MMP9 -expressing cancer. This technology will provide a nuclear imaging agent established from an enzyme-responsive nanoparticle, and will be the first imaging nanoparticle to undergo a programmed morphological switch. Funder Acknowledgement: This work is supported by the National Science Foundation CREST Center for Interface Design and Engineered Assembly of Low Dimensional systems (IDEALS), NSF grant number HRD1547830 .
\end{abstract}

References: References: Kalafatovic D, Nobis M, Javid N, Frederix PW, Anderson KI, Saunders BR, Ulijn RV. MMP-9 triggered micelle-to-fibre transitions for slow release of doxorubicin. Biomaterials science. 2015;3(2):246-9. Son J, Kalafatovic D, Kumar M, Yoo B, Cornejo MA, Contel M, Ulijn RV. (2019) Customizing Morphology, Size, and Response Kinetics of Matrix 
Metalloproteinase- Responsive Nanostructures by Systematic Peptide Design. ACS Nano. 13 (2), pp 1555- 1562 .

Full Name of Abstract's 1st Author : Douglas MacPherson

Complete Status: Complete

First Name: Douglas

Last Name: MacPherson

Email: dmacpherson@gc.cuny.edu

Organization: City University of New York

Country: United States 


\title{
ID: GA295 \\ Exploiting the MUC5AC Antigen for Noninvasive Identification of Pancreatic Cancer
}

\author{
Kelly Henry, Invicro, kelly.henry89@gmail.com
}

\section{Category: Oncology}

\begin{abstract}
Body : Objectives: Pancreatic cancer (PC) remains the 4th leading cause of cancer death; therefore, there is a clinically unmet need for novel therapeutics and diagnostic markers to treat this devastating disease. Physicians often rely on biopsy or CT for diagnosis, but more specific protein biomarkers are highly desired to assess the stage and severity of PC in a noninvasive manner. Serum biomarkers such as CA19.9 are of particular interest as they are commonly elevated in PC but have exhibited suboptimal performance in the clinic. MUC5AC has emerged as a useful serum biomarker that is specific for PC vs. inflammation. We developed RA96, an anti-MUC5AC antibody, to gauge its utility in PC diagnosis through immunohistochemical (IHC) analysis and whole-body PET/CT imaging in PC. Methods: We determined the utility of RA96 for MUC5AC IHC on clinical PC and pre-clinical PC by staining on tissue microarrays containing 300 cores of normal ducts and PanINs. These samples originated from 21 disease-free pancreata and 81 resected pancreata of different neoplasms, 30 alcoholic chronic pancreatitis and 10 autoimmune pancreatitis specimens, and 48 pancreatic ductal adenocarcinomas (PDACs). Immunohistochemical staining was performed with RA96 and the intensity of the reactions was scored as mild, moderate, or strong (score of 1, 2, or 3, respectively) by an experienced pathologist. Finally, we radiolabeled RA96 with zirconium-89 to assess its applications as a whole-body PET radiotracer for MUC5AC quantification in preclinical models of PC. RA96 and isotype-matched IgG were functionalized with pisothiocyanatobenzyl-desferrioxamine (DFO-Bn-NCS) and radiolabeled with zirconium-89 and characterized via instant thin-layer chromatography. Serial PET/CT imaging was performed in MUC5AC+ capan-2 xenografts. Results: Immunohistochemical staining with RA96 distinguished chronic pancreatitis (CP), PanIN, and varying grades of PDAC in clinical samples. The specific activity and molar activity for both [89Zr]Zr-DFO-RA96 and [89Zr]Zr-DFO-IgG was $0.3-0.4 \mathrm{MBq} / \mathrm{mg}$ and $45-55 \mathrm{GBq} / \mathrm{mmol}$, respectively. Serial PET imaging with [89Zr]ZrDFO-RA96 shows significant uptake in MUC5AC+ capan-2 tumors $(14.6 \pm 1.5 \% \mathrm{ID} / \mathrm{g})$ compared to the blocking group $(6.6 \pm 1.5 \% \mathrm{ID} / \mathrm{g})$ and $[89 \mathrm{Zr}] \mathrm{Zr}-\mathrm{DFO}-\mathrm{IgG}$ control $(8.8 \pm 0.3$ $\% \mathrm{ID} / \mathrm{g}$ ), with a P Conclusions: Our study demonstrates that RA96 can differentiate between inflammation and PC, improving the fidelity of PC diagnosis. Our immuno-PET tracer [89Zr]ZrDFO-RA96 shows specific detection of MUC5AC+ tumors in vivo, highlighting the utility of MUC5AC targeting for diagnosis of PC. We expect that immuno-PET targeting MUC5AC has high potential to be clinically useful for early detection of PC. Acknowledgments: We would like to acknowledge financial support from NIH grant R35 CA232130-01A1 and the MSK Center for Molecular Imaging and Nanotechnology Tow Fellowship.
\end{abstract}

References: 1. Kaur S, Smith LM, Patel A, Menning M, Watley DC, Malik SS, Krishn SR, Mallya K, Aithal A, Sasson AR, Johansson SL, Jain M, Singh S, Guha S, Are C, Raimondo M, 
Hollingsworth MA, Brand RE, Batra SK. A Combination of MUC5AC and CA19-9 Improves the Diagnosis of Pancreatic Cancer: A Multicenter Study. Am J Gastroenterol. 2017;112(1):17283.

\section{Image/Figure:}

https://www.xcdsystem.com/wmis/abstract/File6959/GA295_ImageFigure_0614051354.png

Image/Figure Caption: Figure: Serial PET/CT imaging of [89Zr]Zr-DFO-RA96 and [89Zr]ZrDFO-IgG with corresponding ex vivo biodistribution at $144 \mathrm{~h}$. Images are represented as coronal slices (A) and maximum intensity projections (B). Tumors are on the right flank and indicated by the dotted line. Significant differences are noted when comparing the [89Zr]Zr-DFO-RA96 to all control groups (P 89Zr]Zr-DFO-RA96. Block: full block with 30× amount of unlabeled antibody coinjected with [89Zr]Zr-DFO-RA96. IgG: injection of isotype-matched control [89Zr]Zr-DFO$\mathrm{IgG}$. Mice were retro-orbitally bled from the right eye one week prior, resulting in non-specific uptake of radiotracer in the eye region.

Full Name of Abstract's 1st Author : Kelly E. Henry

Complete Status: Complete

First Name: Kelly

Last Name: Henry

Email: kelly.henry89@gmail.com

Organization: Invicro

Country: United States 


\title{
ID: GA296 \\ Azido Conjugated Nitroimidazole (ACN): An Antibody-independent Alternative to Pimonidazole Immunohistochemistry for Mapping Cellular Hypoxia
}

\author{
Faisal Rashed, University of Alberta, Canada, frashed@ualberta.ca
}

\section{Category: Oncology}

\begin{abstract}
Body : Abnormal vasculature in solid tumours often results in poor oxygenation, a phenomenon that is known as tumour hypoxia. Hypoxic cells are resistant to chemotherapy, radiation therapy and immunotherapy, and thus contribute to treatment failure and cancer progression. Therefore, detection of tumour hypoxia is crucial for making appropriate therapy choices and monitoring treatment response. Nitroimidazoles (NI) have demonstrated a significant potential in hypoxia diagnosis because they get selectively activated in hypoxic cells and react with proteins to form drug-protein adducts. Pimonidazole, a commercially available 2NI derivative, is used in immunohistochemistry (IHC) to detect hypoxic regions in tissue biopsies. Here, we report the synthesis and characterization of a novel 2-NI-derived hypoxia mapping agent named Azido Conjugated Nitroimidazole (ACN). Instead of immunodetection, our method utilizes copper (I) catalyzed "click chemistry". Using a fluorophore conjugated alkyne, we have been able to detect the hypoxia-selective entrapment of ACN in cells and in mouse tumour models. By combining the hypoxic affinity of NIs with the ease and versatility of click chemistry, we have developed a histological tool that bypasses the necessity of antibodies to facilitate a rapid detection of hypoxia. Our approach offers an easier and less expensive alternative to Pimonidazole IHC, while still maintaining high fidelity and precision. A direct comparison of the two methods shows that ACN click chemistry is equally efficient at detecting histological hypoxia as Pimonidazole IHC. The modular nature of the reaction also allowed us to use ACN and a biotin labelled alkyne to pull down its potential protein targets, which were then characterized using liquid chromatography-coupled mass spectrometry (LC-MS/MS). A total of 62 candidate proteins were identified, and we analyzed them using various bioinformatic platforms (PANTHER, IPA) for biological significance. ACN target proteins appeared to be predominantly cytoplasmic $(\sim 71 \%)$ with only $\sim 21 \%$ being nuclear proteins. Canonical pathways analysis suggests that most of these proteins are involved in stress and glucose metabolism pathways. We studied the effects of ACN binding to one of its top protein targets, glyceraldehyde-3-phosphate dehydrogenase (GAPDH). While ACN, at IC50 concentration, does not affect GAPDH protein levels or cellular localization, it significantly reduces GAPDH enzymatic activity under hypoxia. This information is valuable because NIs have long been explored for hypoxia directed cancer therapy. Given the therapeutic potential of these compounds, our study provides insights into the molecular mechanism of NI drugs.
\end{abstract}

Full Name of Abstract's 1st Author : Faisal Bin Rashed

Complete Status: Complete

First Name: Faisal 
Last Name: Rashed

Email: frashed@ualberta.ca

Organization: University of Alberta, Canada

Country: Canada 


\title{
ID: GA300 \\ Evaluation of 89Zr-Oxine labeling of dendritic cells for PET monitoring.
}

\author{
Mikhail Doubrovin, Columbia University Medical Center, md2367@cumc.columbia.edu
}

\section{Category: Immunology: Inflammation \& Infection}

\begin{abstract}
Body : Background: 111In-Oxine has been successfully used for imaging of different types of white blood cells and immune cells using SPECT over decades. It was also previously used to monitor in vivo trafficking of dendritic cells [1]. Use of 89Zr-Oxine provides advantages of quantitative approach to the immune cell imaging using PET, as it was reported for lymphocytes. In the current study we demonstrate feasibility of use of 89Zr-Oxine for stable ex vivo labeling of dendritic cells for PET imaging. Materials/Methods: porcine dendritic cells were obtained by apheresis of monocytes from the pigs. $89 \mathrm{Zr}$ labeling of Oxine was performed, as previously described [2] with $98 \%$ radiochemical purity. In in vitro testing a range of $89 \mathrm{Zr}$ Oxine doses from 101 to $2 * 102 \mathrm{uCi} / 106$ cells were tested with incubation time from 30 to 60 minutes, at room temperature in PBS. Cell viability at $0,4,24$ - and 72 -hours following incubation was tested. The labeling efficiency and label stability over time was evaluated. The most optimal labeling condition was used to pre-label $1.2 * 108$ cells 1 hour prior to the administration to the animal (according to IACUC approved protocol). PET images were acquired at 4, 24 and 72 hours to evaluate the stability of 89Zr-Oxine labeling in vivo. Results: In vitro, the labeling efficiency for 30 - and 60-minute incubation did not differ significantly ( $54 \%$ and $62 \%$, respectively). In the range of tested radiotracer concentrations, no significant difference of labeling efficiency was observed ( $\mathrm{p}>0.05$ ). Loss of 89Zr-Oxine in medium was observed from $8.1+/-2.7 \%$ at 24 hours to $17.3+/-5.6 \%$ at 72 hours. No significant differences in cell viability was observed at 24 and 72 hours for all tested radiotracer concentrations and exposition times ranging from $74 \%$ to $88 \%(\mathrm{p}>0.1$ ). For improved sensitivity of in vivo visualization, the maximal dose and labeling time values were selected. At the time of in vivo experiment, $89 \mathrm{Zr}$-Oxine labeling efficiency was $58 \%$ with $77 \%$ viability. $3.1 \mathrm{mCi}$ total activity of 89Zr-Oxine was administered. PET imaging demonstrated expected biodistribution of the ex vivo labeled cells to the pulmonary vasculature as early as 4 hours following administration (SUVmax 43.3). Minimal radiotracer uptake was observed in the liver at 4, 24 and 72 hours, where excretion of free $89 \mathrm{Zr}$ released from the cells could have been expected (SUVmax 5.2, 3.3 , and 2.8, respectively). Conclusions: 89Zr-Oxine ex vivo labeling of porcine dendritic cells can be performed with reliable efficiency and stability in the dose range applicable for the in vivo PET studies. 89Zr-Oxine labeling appears stable over time and does not affect the viability of the labeled cells. In vivo PET imaging demonstrates localization of reinfused dendritic cells labeled ex vivo with 89Zr-Oxine without significant in vivo loss of the label. This approach appears feasible for quantitative PET imaging of dendritic cells in vivo in the future studies. Support: research is supported through NIH HLT32.
\end{abstract}

References: 1. Garrovillo M, Ali A, Depaz HA, Gopinathan R, Oluwole OO, Hardy MA, Oluwole SF., Induction of transplant tolerance with immunodominant allopeptide-pulsed host lymphoid and myeloid dendritic cells. Am J Transplant. 2001 Jul;1(2):129-37. PMID: 12099360. 
2. Charoenphun P, Meszaros LK, Chuamsaamarkkee K, Sharif-Paghaleh E, Ballinger JR, Ferris TJ, Went MJ, Mullen GE, Blower PJ. [(89)Zr]oxinate4 for long-term in vivo cell tracking by positron emission tomography. Eur J Nucl Med Mol Imaging. 2015 Feb;42(2):278-87. PMID: 25359636.

Complete Status: Complete

First Name: Mikhail

Last Name: Doubrovin

Email: md2367@cumc.columbia.edu

Organization: Columbia University Medical Center

Country: United States 


\title{
ID: GA301 \\ Ratio dependent fluorescence intensity of trimetallic nanoclusters serving as potential bimodal imaging probes
}

\author{
Petr Běčák, Faculty of Science, Palacký University in Olomouc, petr.becak01@upol.cz
}

Category: New Chemistry, Biology \& Bioengineering

\begin{abstract}
Body : New fluorescent nanoclusters (NCs) were created using ions of $\mathrm{Au}(\mathrm{III}), \mathrm{Ag}(\mathrm{I})$, $\mathrm{Fe}(\mathrm{II})$ and bovine serum albumin (BSA). Au(III) was selected for the formation of fluorescent AuNCs in the presence of BSA (1); $\mathrm{Ag}(\mathrm{I})$ was chosen in order to increase fluorescence intensity of NCs (2); and Fe ions served as precursors for the formation of a potential contrast agent being employed in magnetic resonance imaging (MRI) (3). Different ratios of these ions used during the synthesis of NCs substantially influenced NCs fluorescence intensity, as well as the position of the characteristic fluorescence peak. Decrease of the fluorescence intensity was observed depending on the ratios of metal ions as demonstrated in Figure 1. Moreover, it was revealed that NCs fluorescence intensity is a nonmonotonic function of time if the samples were not dialysed. It was tested that our NCs can be used for MRI if the concentration of incorporated $\mathrm{Fe}$ ions is high enough.
\end{abstract}

References: (1) Xie, J.; Zheng, Y.; Ying, J. Y.; Protein-directed Synthesis of Highly Fluorescent Gold Nanoclusters. J. Am. Chem. Soc. 2009, 131,888-889 (2) Ganguly M.; Jana J.; Pal A.; Pal T.; Synergism of gold and silver invites enhanced fluorescence for practical applications.; RSC Adv., 2016, 6, 17683-17703 (3) An L.; Yan Ch.; Mu X.; Tao Ch.; Tian Q.; Lin J.; Yang S.; Paclitaxel-Induced Ultrasmall Gallic Acid-Fe@BSA Self-Assembly with Enhanced MRI Performance and Tumor Accumulation for Cancer Theranostics.; ACS Appl. Mater. Interfaces $2018,10,28483-28493$

\section{Image/Figure:}

https://www.xcdsystem.com/wmis/abstract/File6959/GA301_ImageFigure_0602024629.jpg

Image/Figure Caption: Fig. 1: Dependence of integral fluorescence intensity on different $\mathrm{Au}: \mathrm{Ag}: \mathrm{Fe}$ molar ratios in days.

Full Name of Abstract's 1st Author : Petr Běčák

Complete Status: Complete

First Name: Petr

Last Name: Běčák

Email: petr.becak01@upol.cz 
Organization: Faculty of Science, Palacký University in Olomouc

Country: Czech Republic 


\title{
ID: GA302 \\ PET kinetics of the myocardial norepinephrine transporter tracer [11C]mHED in mice
}

\author{
Stefanie Kramer, ETH Zurich, skraemer@pharma.ethz.ch
}

\section{Category: Cardiovascular \& Pulmonary}

\begin{abstract}
Body : Purpose The PET tracer [11C]meta-hydroxyephedrine ([11C]mHED) is a substrate for the norepinephrine transporter (NET), which takes up its substrates from the synaptic cleft into the presynaptic nerve terminals. Clinical [11C]mHED PET provides information on sympathetic innervation in brain and heart. PET studies with [11C]mHED in mice are scarce (ref. 1) and its kinetics have not been studied in detail in mice. To further evaluate whether [11C]mHED PET may be useful in basic research with mouse models of heart diseases, we investigated by PET the specificity of [11C]mHED for NET in the mouse heart and characterized the kinetics of [11C]mHED uptake by compartmental modelling. Methods Female FVB/N mice (20-24 g) were assigned to 3 groups, (i) baseline scan ( $\mathrm{n}=5$ ), (ii) scan with NET blocking by desipramine $(20 \mathrm{mg} / \mathrm{kg}$ intraperitoneally $10 \mathrm{~min}$ before [11C]mHED administration; $\mathrm{n}=6$ ), and (iii) baseline scan with simultaneous recording of the blood coincidences by a femoral arterio-venous shunt guided through a blood coincidence counter $(n=3)$. [11C]mHED was synthesized by direct $\mathrm{N}$-methylation of metaraminol with [11C]methyl triflate and injected intravenously (3-14 MBq; 1.5-62 nmol/kg combined mHED and metaraminol), $1 \mathrm{~min}$ before (baseline, desipramine blocking) or $1 \mathrm{~min}$ after (kinetics) scan start. PET was acquired for 60 min. Standardized uptake values (SUV) of left ventricle wall averaged from 1 to 30 or 1 to 60 min were plotted in a dose-response curve against the logarithmic injected dose as nmol mHED plus residual metaraminol plus desipramine (if applicable) to determine the dose at half maximal NET saturation and the maximally tolerated dose for linear (dose-independent) kinetics. PET data with blood coincidences were fit to one- and two-tissue compartment models. PET data from the blocking group were analysed using one of the blood coincidence curves from the kinetic modelling group. Results Desipramine reduced the SUV(1-30min) of left ventricle wall from 4.4 (upper plateau of dose response curve; SE 0.18) to 1.0 (lower plateau, SE 0.13; Figure). Half-saturation of NET uptake was fit to $88.3 \mathrm{nmol} / \mathrm{kg}$ combined $\mathrm{mHED}$ and metaraminol (SE 30.3; Figure), in agreement with earlier findings in mice (ref. 1) and rats (ref. 2). This suggests linear kinetics up to a dose of $10 \mathrm{nmol} / \mathrm{kg}$ combined $\mathrm{mHED}$ and metaraminol. PET data of the kinetic modelling group (1.5-2.0 nmol/kg tracer and metaraminol) followed two-tissue compartment models. Blocking by desipramine reduced $\mathrm{K} 1$, the transfer clearance parameter from blood to the region of interest and had no effect on the other fit parameters in serial twotissue compartment models. This indicated that NET transport is represented by K1 in our models. K1 determined from the 3 scans with blood coincidence data was $0.947 \pm 0.146$ $\mathrm{mL} / \mathrm{cm} 3 / \mathrm{min}$ (input function corresponding to parent tracer in plasma). The fit partial volume of blood in the region of interest was $0.46 \pm 0.035$. This high number is in agreement with the high fraction of blood in the region of interest due to myocardial motion and partial volume effects. According to the model, this partial volume in addition includes tracer in extracellular space with rapid exchange with blood. SUV(1-30min) correlated with K1 ( $\mathrm{p} \mathrm{n}=14)$, indicating that
\end{abstract}


[11C]mHED SUV may be used as a surrogate parameter for NET function in mouse. Conclusion [11C]mHED is NET-specific in mouse heart and follows a two-tissue compartment model. NET function is represented by $\mathrm{K} 1$ in serial two-tissue compartment models and SUV averaged from 1-30 or 1-60 min correlates well with K1, suggesting SUV as a parameter for estimating NET function in the mouse heart. We suggest [11C]mHED PET as a useful method to address sympathetic innervation in the mouse heart in basic research. The combined dose of tracer and precursor should be kept below $10 \mathrm{nmol} / \mathrm{kg}$.

References: ref. 1) Law MP, Schafers K, Kopka K, Wagner S, Schober O, Schafers M. Molecular imaging of cardiac sympathetic innervation by 11C-mHED and PET: from man to mouse? J. Nucl. Med. 2010;51:1269-76. ref. 2) Law MP, Osman S, Davenport RJ, Cunningham VJ, Pike VW, Camici PG. Biodistribution and metabolism of [N-methyl-11C]mhydroxyephedrine in the rat. Nucl. Med. Biol. 1997;24:417-24.

\section{Image/Figure:}

https://www.xcdsystem.com/wmis/abstract/File6959/GA302 ImageFigure 0609025702.jpg

Image/Figure Caption: Dose-response curve for [11C]mHED PET in the mouse heart. The dose in $\mathrm{nmol} / \mathrm{kg}$ includes the tracer and residual precursor (metaraminol). The highest dose corresponds to $20 \mathrm{mg} / \mathrm{kg}$ desipramine $\mathrm{HCl}$. Insert, SUV(1-30min) of mouse hearts at the indicated doses or with desipramine.

Complete Status: Complete

First Name: Stefanie

Last Name: Kramer

Email: skraemer@pharma.ethz.ch

Organization: ETH Zurich

Country: Switzerland 


\title{
ID: GA307 \\ Evaluation of 3,2-HOPO versus DFO-based chelation of zirconium-89 mesothelin antibody as a diagnostic imaging agent for [227Th]Th-3,2-HOPO-MSLN-mAb
}

\author{
Jyoti Roy, National Institutes of Health, jyotiroy14@gmail.com
}

\section{Category: Oncology}

\begin{abstract}
Body : BACKGROUND Mesothelin (MSLN) targeted monoclonal antibody (MSLN$\mathrm{mAb}$ ) based thorium-227 conjugate [227Th]Th-3,2-HOPO-MSLN-mAb) has demonstrated in vivo efficacy in mesothelin positive tumors 1,2 and is currently being evaluated in Phase I clinical trial. Due to the low gamma emission with low abundance of measurable photons in the decay chain of thorium-227, tumor imaging using thorium-227 based conjugate is technically challenging3,4. Besides, forming a complex with thorium-227, the 3,2-HOPO chelator can also
\end{abstract} form complex with zirconium-89, one of the commonly used PET radionuclides5,6. This approach thus would allow for radiolabeling the 3,2-HOPO-MSLN-mAb either with therapeutic or imaging radionuclide. Thus, the 3,2-HOPOH-MSLN-mAb was radiolabeled with zirconium89 , resulting in [89Zr]Zr-3,2-HOPO-MSLN-mAb. In parallel, the MSLN-mAb was conjugated to deferoxamine (DFO; [89Zr]Zr-DFO-MSLN-mAb), one of the widely used chelators of zirconium-89. Both the zirconium-89 conjugates were compared in vitro and in vivo using MSLN positive tumor models. METHOD 3,2-HOPO-MSLN-mAb and DFO-MSLN-mAb conjugates were radiolabeled with zirconium-89 to synthesize [89Zr]Zr-3,2-HOPO-MSLN-mAb and [89Zr]Zr-DFO-MSLN-mAb respectively. Radiochemical purity (RCP) of both the conjugates were determined by HPLC using size exclusion column. Both the conjugates were evaluated in vitro (binding affinity) and in vivo for biodistribution and PET imaging of HT29MSLN (transfected) cells and two patient-derived (PDXs, NCI-Meso21 and NCI-Meso16) tumor xenografts. After injecting $(50 \mu \mathrm{Ci}, \mathrm{i} . \mathrm{v})$ tumor bearing mice (Athymic, nu/nu, female) with the radioactive conjugates, biodistribution and PET imaging were performed on days 1, 3, and 6 for HT29-MSLN xenografts and on day 3 for PDXs . Radioactivity associated with the tissues/organs were determined by gamma counter and used to calculate $\%$ injected dose $/ \mathrm{g}$ (\%ID/g), tissue: blood (T: B) and tissue: muscle (T: M) ratios. RESULT The RCP of [89Zr]Zr3,2-HOPO-MSLN-mAb and [89Zr]Zr-DFO-MSLN-mAb was 52-76\% $(\mathrm{n}=20)$ and 90-92\% $(\mathrm{n}=8)$ respectively. Both [89Zr]Zr-3,2-HOPO-MSLN-mAb and [89Zr]Zr-DFO-MSLN-mAb exhibited a low nanomolar binding affinity for MSLN. [89Zr]Zr-DFO-MSLN-mAb showed higher HT29MSLN tumor uptake (28-33\%ID/g) ) at all time-points compared to [89Zr]Zr-3,2-HOPOMSLN-mAb (7-11\%ID/g). Similarly, at day 3 PDX tumor accumulation of [89Zr]Zr-DFOMSLN-mAb (NCI-Meso 16:15.88 \%ID/g, NCI-Meso21:19.49\%ID/g) were greater than [89Zr]Zr-3,2-HOPO-MSLN-mAb (NCI-Meso 16:7.95 \%ID/g, NCI-Meso21:13.07 \%ID/g). T: B and T: $\mathrm{M}$ ratios were also lower for [89Zr]Zr-3,2-HOPO-MSLN-mAb than the DFO conjugate. However, femur uptake of [89Zr]Zr-3,2-HOPO-MSLN-mAb (6.74\%) was 2-fold higher compared to [89Zr]Zr-DFO-MSLN-mAb (3.57\%ID/g) at day 1 and then increased to $\sim 3-4$-fold over 6 days. At all times, PET imaging results paralleled the biodistribution pattern of both the conjugates. CONCLUSION Although, in vitro, both the conjugates exhibited a high binding affinity for MSLN, in vivo, [89Zr]Zr-DFO-MSLN-mAb showed higher tumor uptake and lower 
femur uptake than [89Zr]Zr-3,2-HOPO-MSLN-mAb. Higher femur uptake observed with [89Zr]Zr-3,2-HOPO-MSLN-mAb may interfere with the uptake of this agent in MSLN positive tumor lesions in the bone. As [89Zr]Zr-3,2-HOPO-MSLN-mAb uses the same chelator as [227Th]Th-3,2-HOPO-MSLN-mAb, the same 3,2-HOPO-MSLN-mAb conjugate could be used to study organ distribution and lesion uptake and to guide clinical development.

References: 1. Hagemann UB, Ellingsen C, Schuhmacher J, et al. Mesothelin-Targeted Thorium-227 Conjugate (MSLN-TTC): Preclinical Evaluation of a New Targeted Alpha Therapy for Mesothelin-Positive Cancers. Clin Cancer Res. 2019. 2. Hagemann UB, Mihaylova D, Uran SR, et al. Targeted alpha therapy using a novel CD70 targeted thorium-227 conjugate in in vitro and in vivo models of renal cell carcinoma. Oncotarget. 2017;8(34):56311-56326. 3. Dahle J, Borrebaek J, Jonasdottir TJ, et al. Targeted cancer therapy with a novel low-dose rate alphaemitting radioimmunoconjugate. Blood. 2007;110(6):2049-2056. 4. Dahle J, Larsen RH. Targeted Alpha-Particle Therapy with Th-227-Labeled Antibodies. Curr Radiopharm. 2008;1(3):209-214. 5. J NT, Pandya DN, Pailloux SL, et al. Evaluation of a 3-hydroxypyridin-2one (2,3-HOPO) Based Macrocyclic Chelator for (89)Zr(4+) and Its Use for ImmunoPET Imaging of HER2 Positive Model of Ovarian Carcinoma in Mice. Theranostics. 2016;6(4):511521. 6. Deri MA, Ponnala S, Zeglis BM, et al. Alternative Chelator for Zr-89

Radiopharmaceuticals: Radiolabeling and Evaluation of 3,4,3-(LI-1,2-HOPO). J Med Chem. 2014;57(11):4849-4860. b

Full Name of Abstract's 1st Author : Jyoti Roy

Complete Status: Complete

First Name: Jyoti

Last Name: Roy

Email: jyotiroy14@gmail.com

Organization: National Institutes of Health

Country: United States 


\title{
ID: GA312 \\ Imaging early stage metastases using an 18F labeled VEGFR-1 specific single chain VEGF mutant
}

\author{
Christian Mason, Sylvestor Comprehensive Cancer Center, cam32302@med.miami.edu
}

\section{Category: Oncology}

\begin{abstract}
Body : Metastatic breast cancer is a leading cause of death among women in the
\end{abstract} United States, and approximately $30 \%$ of initially node-negative breast cancer patients will develop metastatic lesions. The 5-year survival rate for metastatic breast cancer remains near 27 $\%$ due to the complexity and challenges associated with treating this stage of the disease. 1 Consequently, significant research efforts have been trained at interrogating the underlying biology of metastasis formation and growth in an effort to develop therapeutic strategies that can more effectively combat metastatic cancers.2-5 While the specific processes involved in metastatic dissemination are not fully understood, the recruitment of hematopoietic stem cells with high expression of the vascular endothelial growth factor receptor 1 (VEGFR-1) has been implicated in early stages of metastasis formation. 6 Herein, we propose the use of an 18F sitespecifically labeled, single-chain version of VEGF121 re-engineered to selectively bind to VEGFR-1 (scVR1), as a positron emission tomography (PET) imaging agent to non-invasively image early stage metastases. The scVR 1 was radiolabeled with $18 \mathrm{~F}$ via a biorthogonal click reaction between site-specifically trans-cyclooctene functionalized scVR1 and an Al18F labeled tetrazine-NODA (1,4,7-triazacyclononane-1,4-diiacetic acid). The [18F]AlF-NODA-scVR1 was purified using a PD10 column and subsequently analyzed on HPLC to determine radiochemical purity. Animal experiments were performed in 6-8 week old female BALB/c mice, bearing orthotopic primary 4T1 breast tumors or 4T1 metastatic lesions. The [18F]AlF-NODA-scVR1 tracer was administered via tail vein injection, PET imaging and ex vivo analysis was performed 2 hours post-injection. The [18F]AlF-NODA-scVR1 was prepared with a $98.2 \pm 1.5 \%$ radiochemical purity and specific activity of $7.5 \pm 1.2 \mathrm{GBq} / \mu \mathrm{mol}$. The specific binding of scVR1 to VEGFR-1 was confirmed via bead-based assay. The ex vivo biodistribution showed tumor uptake of $2.75 \pm 0.30 \% \mathrm{ID} / \mathrm{g}$ and was readily observable in PET images. Metastasis formation was detected with [18F]AlF-NODA-scVR1 tracer showing colocalization with bioluminescent imaging as well as ex vivo autoradiography and immunofluorescent staining of VEGFR-1. In addition, the $[18 \mathrm{~F}] \mathrm{AlF}-\mathrm{NODA}-\mathrm{scVR} 1$ tracer uptake in metastatic lesions correlated with VEGFR-1 expression. Systemic treatment strategies such as chemotherapy and endocrine therapy have improved outcomes when performed in patients early in progression and metastatic dissemination. However, the ability to image the initial stages of metastatic development and progression remains elusive. A PET tracer that could provide clinicians a means of imaging and diagnosing metastatic disease could further improve survival rates for metastatic breast cancers. The [18F]AlF-NODA-scVR1 PET tracer was developed and its selectivity for the VEGFR-1 receptor was established. In addition, the in vivo PET/CT and ex vivo analyses support the potential of VEGFR-1 specific tracers to image early stages of metastatic tumor formation and growth. While further development of [18F]AlF-NODA-scVR1 is necessary to improve its performance, the results presented herein provide support for its prospective implementation in 
clinical applications. The [18F]AlF-NODA-scVR1 PET tracer offers a promising tool that could help physicians make informed decisions when determining if a patient requires adjuvant or neoadjuvant therapies.

References: 1. Waks, A. G. \& Winer, E. P. Breast Cancer Treatment: A Review. JAMA 321, 288-300 (2019). 2. Psaila, B. \& Lyden, D. The Metastatic Niche: Adapting the Foreign Soil. Nat. Rev. Cancer 9, 285-293 (2009). 3. Pachmayr, E., Treese, C. \& Stein, U. Underlying Mechanisms for Distant Metastasis - Molecular Biology. Visc. Med. 33, 11-20 (2017). 4. Hunter, K. W., Crawford, N. P. \& Alsarraj, J. Mechanisms of metastasis. Breast Cancer Res. BCR 10, S2 (2008). 5. Valastyan, S. \& Weinberg, R. A. Tumor Metastasis: Molecular Insights and Evolving Paradigms. Cell 147, 275-292 (2011). 6. Meng, D. et al. Effects of VEGFR1+ hematopoietic progenitor cells on pre-metastatic niche formation and in vivo metastasis of breast cancer cells. J. Cancer Res. Clin. Oncol. 145, 411-427 (2019).

\section{Image/Figure:}

https://www.xcdsystem.com/wmis/abstract/File6959/GA312_ImageFigure_0505025118.jpeg

Image/Figure Caption: Figure 1: Imaging and ex vivo analysis of [18F]AlF-NODA-scVR1 in lung metastases. A) PET/CT images of [18F]AlF-NODA-scVR1. B) Bioluminescent imaging of 4T1luc cells in chest cavity associated with lung metastases. C) Ex vivo analysis of lung tissue including autoradiography, hematoxylin and eosin staining, and immunofluorescence staining, VEGFR-1 (red), CD-31 (green), and 4',6-diamidino-2-phenylindole (DAPI) (blue). D) Correlation between relative autoradiography signal intensity and VEGFR-1+ cells in lung metastases $(\mathrm{P}<0.05)$. E) Correlation between relative autoradiography signal intensity and VEGFR-1+ cells in surrounding lung tissue of metastatic mice $(\mathrm{P}<0.05)$. F) Percentage of $\mathrm{CD} 31+$ cells in metastatic tumors, surrounding lung tissue in metastatic mice, and healthy lung tissue. G) Percentage of VEGFR-1+ cells in metastatic tumors, surrounding lung tissue in metastatic mice, and healthy lung tissue.

Complete Status: Complete

First Name: Christian

Last Name: Mason

Email: cam32302@med.miami.edu

Organization: Sylvestor Comprehensive Cancer Center

Country: United States 


\title{
ID: GA315 \\ Developing fluorescent small molecule ChoKa inhibitors for delineating tumor margins in an intraoperative setting
}

\author{
Sofya Osharovich, University of Pennsylvania, sofyaosharo@gmail.com
}

\section{Category: Oncology}

\begin{abstract}
Body : In this study, we evaluate a novel small molecule choline kinase inhibitor, JAS239, for its toxicity in mice, ability to delineate tumor margins in vivo, and measure ChoK $\alpha$ expression in spontaneous canine lung adenocarcinomas. Our goal is to translate JAS239 to the veterinary clinic for intraoperative tumor imaging of spontaneous canine patient lung adenocarcinomas. Choline Kinase $\alpha(\mathrm{ChoK} \alpha)$ is an enzyme overexpressed in $39 \%$ of human breast and $60 \%$ of human lung tumors. It is associated with an aggressive phenotype, high histological tumor grade, and poor clinical outcome in many human cancers 1-3. ChoK $\alpha$ catalyzes the phosphorylation of choline to generate phosphocholine, a precursor of the cell membrane phospholipid, phosphatidylcholine (PtdCho)4. A novel small-molecule near infrared (NIR) fluorescent ChoK $\alpha$ inhibitor, JAS239, has been developed in our lab (excitation: $742 \mathrm{~nm}$, emission: $775 \mathrm{~nm})$. In the NIR window $(700-900 \mathrm{~nm})$, light absorption by water, hemoglobin, and tissue is reduced, decreasing light scattering and allowing for better depth penetration and tumor margin differentiation5-7. JAS239 binds to the active site of $\mathrm{ChoK} \alpha$, competitively inhibiting phosphocholine production, and thus can be used as a targeted imaging agent for intraoperative tumor resection guidance in ChoK $\alpha$-overexpressing tumors. Tumor resection continues to be the most effective approach to cure patients with local disease. Surgeons typically use visual inspection and finger palpation to define solid tumor margins8. However, this approach is often insufficient for the detection of residual disease surrounding the wound bed, thus leading to local recurrence in up to $40 \%$ of patients and a significantly reduced 5-year surviva19,10. Clinically-approved intraoperative tumor imaging fluorophores, such as indocyanine green (ICG), have shown promise in detecting tumor margins and residual tumor during surgery, however, they are limited by their lack of specificity between tumorigenic cells and inflammation, relying predominantly on leaky vasculature to localize to the tumor. Toxicity studies showed that there were no hematological, biochemical, or histopathological differences when mice were treated with 50x the imaging dose of JAS239 (five times the imaging dose of $40 \mathrm{nmol}$ for 10 days). This study provides the data necessary for a clinical toxicology study in non-patient canines. In mice, detection of tumor margins was complicated byvariable background fluorescence in different models of cancer, while JAS239 tumor localization and fluorescence remains relatively similar. We hypothesize that this could be due to variable ChoK $\alpha$ expression in the muscle tissue of different mouse strains. Nude mice exhibited the least amount of fluorescent background signal, while DBA/2 mice had large fluorescent background signal when compared to tumor signal. In preparation for translation to canines, we are accumulating a panel of patient-derived canine lung tumors and histologically staining them for ChoK $\alpha$ expression. Spontaneous lung cancers from canine patients express high levels of ChoK and are suitable candidates for JAS239 fluorescence-guided surgery.
\end{abstract}


References: 1. Cuadrado, A., Carnero, A., Dolfi, F., Jimenez, B. and Lacal, JC. "Phosphorylcholine: a novel second messenger essential for mitogenic activity of growth factors.” Oncogene 8.11 (1993): 2959-2968. PMID: 8414498. 2. Molina, A., SarmenteroEstrada, J., Belda-Iniesta, C., Taron, M., Ramirez De Molina, V., Cejas, P., Skrzypski, M., Gallego-Ortega, D., De Castro, J., Casado, R., Garcia-Cabezas, M., Sanchez, J., Nistal, M., Rosell, R., Gonzalez-Baron, M., and Lacal, JC. "Expression of Choline Kinase Alpha to Predict Outcome in Patients with Early-stage Non-small-cell Lung Cancer: A Retrospective Study." The Lancet Oncology 8.10 (2007): 889-97. PMID: 17851129. 3. Molina, A., Rodriguez-Gonzalez, A., Gutierrez, R., Martinez-Pineiro, L., Sanchez, J., Bonilla, F., Rosell, R., and Lacal, JC. "Overexpression of Choline Kinase Is a Frequent Feature in Human Tumor-derived Cell Lines and in Lung, Prostate, and Colorectal Human Cancers." Biochemical and Biophysical Research Communications 296.3 (2002): 580-83. PMID: 12176020. 4. Molina, A., Báñez-Coronel, M., Gutiérrez, R., Rodríguez-González, A., Olmeda, D., Megías, D., Lacal, JC. "Choline Kinase Activation Is a Critical Requirement for the Proliferation of Primary Human Mammary Epithelial Cells and Breast Tumor Progression." Cancer Research 64.18 (2004): 6732-739. PMID: 15374991. 5. Bokobza, L. "Near Infrared Spectroscopy." Journal of Near Infrared Spectroscopy 6.1 (1998): 3. SLỆ. 6. Hong, G., Antaris, A., and Dai, H. "Near-infrared Fluorophores

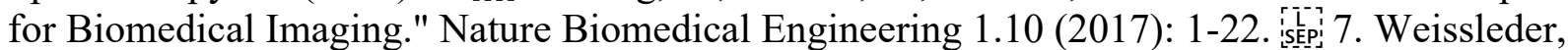
R. "A Clearer Vision for in Vivo Imaging." Nature Biotechnology 19.4 (2001): 316-17. PMID: 11283581. 8. Tipirneni, K., Warram, J., Moore, L., Prince, A., De Boer, E., Jani, A., Wapnir, I., Liao, J., Bouvet, M., Behnke, N., Hawn, M., Poultsides, G., Vahrmeijer, A., Carroll, W., Zinn, K., and Rosenthal, E. "Oncologic Procedures Amenable to Fluorescence-guided Surgery." Annals of Surgery 20.20 (2016): 1-12. PMID: 28045715. 9. V. S. Klimberg, S. Harms, and S. Korourian, “Assessing margin status.” Surg. Oncol. 8.2 (1999): 77-84. PMID: 10732959. 10. Sienko, A., Allen, T., Zander, D., and Cagle, P. "Frozen

Full Name of Abstract's 1st Author : Sofya Osharovich

Complete Status: Complete

First Name: Sofya

Last Name: Osharovich

Email: sofyaosharo@gmail.com

Organization: University of Pennsylvania

Country: United States 


\title{
ID: GA317 \\ Development and imaging of 124I-Trametinib as a novel radiotracer for MEK in-vivo
}

\author{
Edwin Pratt, Memorial Sloan Kettering Cancer Center, pratte@mskcc.org
}

\section{Category: Oncology}

\begin{abstract}
Body : Trametinib (MEKINIST ${ }^{\mathrm{TM}}$ ) is an extremely potent allosteric inhibitor of MEK1/2 that has been approved for treatment of metastatic melanoma and anaplastic thyroid cancer in patients with confirmed BRAFV600E/K mutations. Though highly efficacious, adverse side effects including skin, gastrointestinal and hepatic toxicity, are dose limiting and can lead to treatment termination. Development of a non-invasive tool to visualize and quantify the delivery and distribution of trametinib (either as single agent or in combination with other therapeutics) to tumors and organs would be very helpful in assessing therapeutic index, personalizing individual dose and potentially predict resistance to therapy. To address these issues, we have developed a radiolabeled trametinib and evaluated the in vitro and in vivo properties. 123I-, 124I- and 131Itrametinib, pure tracer analogs to trametinib, were synthesized in $>95 \%$ purity from a boronatotrametinib precursor with average yields of $69.7 \%$ and $>100 \mathrm{GBq} / \mu \mathrm{mol}$ specific activity. Overall, 124I-trametinib uptake in a panel of cancer cell lines can be blocked with cold trametinib along with other Type-III MEK inhibitors confirming specificity of the radiotracer in vitro and in vivo. 124I-Trametinib was taken up at higher rates in KRAS and BRAF mutant cell lines compared to wild type KRAS cancer cell lines. In vivo, biodistribution revealed high uptake in the liver 2 hours post injection followed by clearance through the gastrointestinal tract over 4 days. Importantly, higher than expected uptake was observed in the lung and heart for up to 24 hours. Peak uptake in the skin and gastrointestinal tract was observed between 6 and 24 hours in c57bl/6j mice while in B16F10 melanoma bearing mice peak tumor concentrations were achieved between 24 to 48 hours. Tumor uptake at 48 hours peaked at $4 \%$ injected dose per gram while tumor to muscle or skin ratios were relatively low, peaking at 3.4- to 8.1-fold by 72 hours, respectively. Biodistribution of 124I-trametinib was significantly reduced in mice on trametinib therapy providing a quantitative method to observe MEK inhibition or expression in vivo. 124I-trametinib serves as a tool for dose personalization in vivo instead of the current fixed dosage scheme and when combined with radiomic data monitor emergence of therapy resistance. In addition, the production of iodinated trametinib affords researchers the ability to measure changes in MEK during therapy as well as drug distribution for improved drug delivery studies.
\end{abstract}

References: EC, Pratt et al, J Nucl Med May 22, 2020 jnumed.120.241901

\section{Image/Figure:}

https://www.xcdsystem.com/wmis/abstract/File6959/GA317_ImageFigure_0424124903.jpg

Image/Figure Caption: Synthesis and cell line specificity of radioiodinated trametinib. A) Synthesis scheme started from trametinib into a boronato-pinacol precursor readily available for 
iodination. Radiosynthesis and purification was complete within 1.5 hours with a radiochemical yield (RCY) of $70 \%$ for 124 I-trametinib with a specific activity $>100 \mathrm{GBq} / \mu \mathrm{mol}$. B) HPLC purification shows majority of radioiodinated product is distinct from boronato-precursor and identical to cold trametinib. C) Trametinib is a type-III MEK allosteric inhibitor designed to reversibly inhibit ATP from phosphorylating MEK1/2. 124I-trametinib addition to cell lines expressing reveal a nearly ten-fold range of uptake after $2 \mathrm{~h}$, all of which are blocked with $1 \mu \mathrm{g}$ cold trametinib. Human KRAS mutant (G12V, G12C or G12D) or BRAFV600E cell lines retained a higher percentage of 124I-trametinib with the double KRAS BRAF mutant having the highest uptake differentiating KRAS and BRAF mutant tissue from normal. Murine cancer cell lines were all similarly avid for 124I-trametinib except Raw264.7 representing normal KRAS and BRAF and lower uptake.

Full Name of Abstract's 1st Author : Edwin Charles Pratt

Complete Status: Complete

First Name: Edwin

Last Name: Pratt

Email: pratte@mskcc.org

Organization: Memorial Sloan Kettering Cancer Center

Country: United States 


\title{
ID: GA319 \\ A Pharmacokinetic Comparison of Two Albumin Binders on an Integrin alphavbeta6 Binding Peptide by Positron Emission Tomography and Biodistribution
}

\author{
Ryan Davis, University of California Davis, rydavis@ucdavis.edu
}

\section{Category: Oncology}

\begin{abstract}
Body : Introduction: The integrin $\alpha v \beta 6$ is a cell surface receptor that is undetectable in healthy adult tissue but is over expressed in many cancers and has been proven a good target for positron emission tomography (PET) imaging of metastatic disease with a fluorine-18 radiolabeled peptide [18F]avb6-BP.1-3 The integrin avb6 binding peptide (avb6-BP)3 however is rapidly cleared from the blood, thus, the use of a serum albumin binding moiety $(A B M)$ to increase blood half-life and tumor accumulation4,5 was explored. We compared two ABMs, Evans blue (EB) and p-iodophenylbutyryl (IP) for their ability to enhance the pharmacokinetic profile of avb6-BP. The ABM-peptides were synthesized with the DOTA chelator for radiolabeling with copper-64 to yield $[64 \mathrm{Cu}] \mathrm{Cu}$ DOTA-EB-avb6-BP $([64 \mathrm{Cu}] 1)$ and $[64 \mathrm{Cu}] \mathrm{Cu}$ DOTA-IP-avb6-BP ([64Cu $] 2$; Fig. A) and were evaluated in vitro and in vivo. Methods: The peptides were synthesized using Fmoc-chemistry and radiolabeled with copper-64 to give $[64 \mathrm{Cu}] 1$ and $[64 \mathrm{Cu}] 2$. Integrin avb6 affinity of $[64 \mathrm{Cu}] 1$ and $[64 \mathrm{Cu}] 2$ was determined by competitive ELISA against biotinylated latency associated protein (LAP) and for selectivity and internalization by cell binding assay with engineered DX3puro $\beta 6(\alpha v \beta 6+)$ cells and DX3puro $(\alpha \mathrm{v} \beta 6-)$ cells, and pancreatic BxPC-3 $(\alpha \mathrm{v} \beta 6+)$ cells. Albumin binding and serum stability assays (mouse $\&$ human at $37^{\circ} \mathrm{C}$ ) were performed, followed by PET/CT imaging and biodistribution in mice bearing BxPC-3 xenograft tumors $(4 \mathrm{~h}, 24 \mathrm{~h}, 48 \mathrm{~h}, 72 \mathrm{~h}$ post-injection [p.i.]). Results: [64Cu] 1 and $[64 \mathrm{Cu}] 2$ were obtained in $>97 \%$ radiochemical purity; the addition of the ABMs did not affect avb6-affinity (IC50 $=14 \pm 2$ and $19 \pm 5 \mathrm{nM}$, respectively, vs. DOTAavb6-BP: $28 \pm 3 \mathrm{nM}$ ). Cell binding was avb6-selective (DX3puro $\beta 6:$ [64Cu]1 55.8 $\pm 3.0 \%$,

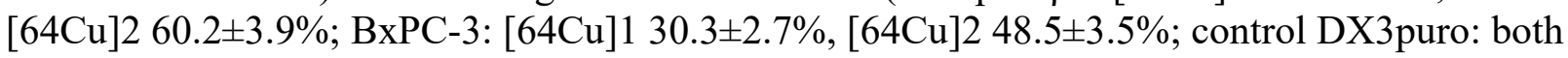
Conclusions: The p-iodophenylbutyryl (IP) ABM modified avb6-BP [64Cu]2 had more favorable pharmacokinetics with higher tumor uptake, less kidney retention, lower liver background, and better serum stability. These favorable properties resulted in the IP-ABM modified avb6-BP as the lead candidate for future integrin avb6 targeted studies.
\end{abstract}

References: References: 1Tumor Biol,2016,37,5117; 2Curr Drug Targets,2009,10,645; 3Clin Cancer Res,2019,25,1206; 4Chem Soc Rev, 2016,45,1432; 5Nucl Med Biol,2019,70,46

\section{Image/Figure:}

https://www.xcdsystem.com/wmis/abstract/File6959/GA319 ImageFigure_0613035455.png

Image/Figure Caption: Figure. A. Chemical structures of $[64 \mathrm{Cu}] 1$ and $[64 \mathrm{Cu}] 2 \mathrm{~B}$. Timeactivity graphs for tumor, liver, and kidney obtained in BxPC-3 (avb6 (+)) xenograft tumor nude 
athymic mouse model (bars: standard deviation). C. Maximum intensity projection PET/CT images of animals from the same mouse model obtained at $72 \mathrm{~h}$ (Arrow indicates tumor, PET data are shown in color scale, CT data in gray).

Complete Status: Complete

First Name: Ryan

Last Name: Davis

Email: rydavis@ucdavis.edu

Organization: University of California Davis

Country: United States 


\title{
ID: GA323 \\ Evaluation of the impact of an albumin-binding fragment on the pharmacokinetics of a copper-64 labeled peptide targeting the integrin $\alpha v \beta 6$ for PET imaging
}

\author{
Tanushree (Nina) Ganguly, University of California Davis, tganguly@ucdavis.edu
}

\section{Category: Oncology}

\begin{abstract}
Body : Objectives: The integrin $\alpha v \beta 6$ is a cell surface receptor that is undetectable in healthy adult tissue but is overexpressed in several carcinomas and an indicator of poor prognosis [1-4]. Although 64Cu-labeled peptide-based positron emission tomography (PET) imaging agents targeting $\alpha v \beta 6$ have been reported, their high kidney accumulation and limited circulation half-life remain a challenge $[5,6]$. The incorporation of non-covalent albumin-binding motifs has shown to increase the circulation time of radiopharmaceuticals, resulting in higher uptake in target tissue, and, in some cases, reduced kidney uptake [7-9]. Here we evaluated the effect of incorporating an albumin-binding motif (ABM) into the $\alpha \mathrm{v} \beta 6$-BP (binding peptide). Methods: Peptides were synthesized without (1) and with (2) an iodophenyl-based ABM using Fmoc solid-phase chemistry and radiolabeled with copper-64 to yield [64Cu]1 and $[64 \mathrm{Cu}] 2$ respectively. The affinity of natCu1 and natCu 2 for $\alpha v \beta 6$ was assessed by ELISA. $[64 \mathrm{Cu}] 1$ and $[64 \mathrm{Cu}] 2$ were evaluated in vitro (cell binding, internalization) in the DX3puro 36 $(\alpha v \beta 6+)$ and DX3puro ( $\alpha v \beta 6-)$, and the pancreatic BxPC-3 $(\alpha v \beta 6+)$ cells, in an albumin binding assay, and for serum stability. In vivo (PET/CT imaging) and biodistribution studies were done in mice bearing either the paired DX3 puro $\beta 6 / \mathrm{DX} 3$ puro or BxPC-3 xenograft tumors. Results: $[64 \mathrm{Cu}] 1$ and $[64 \mathrm{Cu}] 2$ were synthesized in $\geq 97 \%$ radiochemical purity. In vitro, natCu1 $(28 \pm 3 \mathrm{nM})$ and natCu2 $(19 \pm 5 \mathrm{nM})$ maintained nanomolar affinity for $\alpha \mathrm{v} \beta 6$ in ELISA, [64Cu]1 and $[64 \mathrm{Cu}] 2$ show comparable binding to $\alpha \mathrm{v} \beta 6+$ cells (DX3puro $\beta 6: \geq 70 \%, \geq 42 \%$ internalized; BxPC-3: $\geq 19 \%, \geq 12 \%$ internalized), $[64 \mathrm{Cu}] 2$ was $\geq 98 \%$ stable in human serum at $24 \mathrm{~h}$ and showed 6 -fold higher binding than $[64 \mathrm{Cu}] 1$ to human serum protein by albumin-binding assay. In vivo, clear and selective uptake in the $\alpha v \beta 6+$ tumors was seen, with prolonged imaging for up to $72 \mathrm{~h}$ for $[64 \mathrm{Cu}] 2$ (Figure). $3-5$ fold higher $\alpha v \beta 6(+)$ tumor uptake for $[64 \mathrm{Cu}] 2 \mathrm{vs}$. [64Cu]1 was observed at all time points (\% ID/g at 4 and $48 \mathrm{~h}-\mathrm{DX} 3$ puroß6: [64Cu]1: $2.94 \pm 0.32,0.62 \pm 0.31$; $[64 \mathrm{Cu}] 2: 11.65 \pm 3.11,1.80 \pm 0.47$. BxPC-3: $[64 \mathrm{Cu}] 1: 1.61 \pm 0.7,1.18 \pm 0.39 ;[64 \mathrm{Cu}] 2: 5.93 \pm 0.6$ 4.9 \pm 0.57$)$, and at least 3-fold improved tumor/off target ratios at $48 \mathrm{~h}$ (BxPC-3/kidney: [64Cu]1: $0.18 \pm 0.09,[64 \mathrm{Cu}] 2: 0.48 \pm 0.09$; BxPC-3/blood: $[64 \mathrm{Cu}] 1: 7.01 \pm 2.35,[64 \mathrm{Cu}] 2: 22.61 \pm 1.54)$. Conclusions: Incorporation of an ABM in the $\alpha v \beta 6$-binding peptide $([64 \mathrm{Cu}] 2)$ resulted in improved pharmacokinetics, including increased uptake in $\alpha v \beta 6+$ tumors, increased circulation time and improved tumor/off target contrast. Acknowledgements: This work was funded by NIH NCI RO1CA199725 Figure. Reconstructed PET/CT maximum intensity projections (MIPs) of mice bearing DX3puro $\beta 6(\alpha \mathrm{v} \beta 6+$, green arrow) and DX3puro ( $\alpha \mathrm{v} \beta 6$-, red arrow) or BxPC-3 $(\alpha v \beta 6+$, magenta arrow) tumors using $[64 \mathrm{Cu}] 1$ (without $\mathrm{AB}$ group) and $[64 \mathrm{Cu}] 2$ (with $\mathrm{AB}$ group). Mice were anesthetized using $2-3 \%$ isoflurane in medical grade oxygen and received tail vein injections of the radiotracer $(200-250 \mu \mathrm{Ci})$ formulated in saline. Static 15-minute scans
\end{abstract}


were acquired at 4 and $24 \mathrm{~h}$ p.i.; 30-minute and 60-minute scans were acquired at $48 \mathrm{~h}$ and $72 \mathrm{~h}$, respectively. PET data are shown in color scale, CT data in gray.

References: [1] Carcinogenesis 2002, 23, 237. [2] Current Drug Targets 2009, 10, 645. [3] J Clin Invest 2005, 115, 339. [4] Cancer letters 2017, 403, 128. [5]. J Nuc Med 2010, 51, 251. [6] Mol Imaging Biol 2014, 16, 567 [7] Mol Imaging Biol 2013, 15, 649. [8] J Nucl Med 2017, 58, 590. [9] Theranostics 2017, $7,1928$.

\section{Image/Figure:}

https://www.xcdsystem.com/wmis/abstract/File6959/GA323 ImageFigure_0422114955.jpg

Complete Status: Complete

First Name: Tanushree (Nina)

Last Name: Ganguly

Email: tganguly@ucdavis.edu

Organization: University of California Davis

Country: United States 


\title{
ID: GA325 \\ Hypoxia modifies normal prostate fibroblast metabolism towards a prostate cancer associated fibroblast phenotype
}

\author{
Jesus Pacheco Torres, Johns Hopkins University, jpachec6@jh.edu
}

\section{Category: Oncology}

\begin{abstract}
Body : Introduction: Fibroblasts are versatile cells that produce several extracellular matrix (ECM) proteins such as collagen 1 and extradomain A containing fibronectin, as well as degradative enzymes such as matrix metalloproteinases1. Fibroblasts play an important role in wound healing, and in different pathological conditions such as the extensive fibrosis observed in chronic conditions, and in cancer progression. We recently identified increased cancer associated fibroblasts (CAFs) in more metastatic prostate cancers2. To understand the influence of hypoxia that is frequently observed in prostate cancer in modifying fibroblast metabolism, here we characterized human prostate normal and cancer associated fibroblast metabolites under normoxic and hypoxic conditions. Methods: Experiments were performed using human prostate fibroblasts (WPMY-1, ATCC, Manassas, VA) and human prostate cancer associated fibroblasts (PCAFs, Asterand Bioscience, Detroit, MI). WPMY-1 were derived from stromal cells from the peripheral zone of the histologically normal adult prostate3. PCAFs were obtained from an adenocarcinoma of the prostate gland. In order to induce hypoxia, WPMY-1 and PCAF cells were incubated for $48 \mathrm{~h}$ under hypoxic conditions $(0 \% \mathrm{O} 2)$ using an induction chamber where the oxygen was displaced by $100 \% \mathrm{~N} 2$ at the start of incubation. For high resolution $1 \mathrm{H}$ MRS, cell extracts were obtained using a dual-phase extraction method4. High-resolution $1 \mathrm{H}$ MR spectra were recorded on a Bruker Biospin Avance-III $750 \mathrm{MHz}$ NMR spectrometer. 1H MR spectra were manually phased and automated baseline corrected using TOPSPIN 3.2 software. Integrals of the metabolites of interest were determined and normalized to the TSP (trimethylsilylpropanoic acid) reference and the number of cells. Metabolites were estimated from at least four experimental samples. Statistical significance was evaluated using the Student $t$ test. Results: Metabolic profiles were significantly different between WPMY-1 and PCAFs under normoxic conditions, as shown in the heat map in Figure 1a, with significant differences observed in several water-soluble metabolites. On the other hand, other than a slight elevation in the levels of sphingomyelin and the total lipid content, lipid profiles were similar in WPMY-1 and PCAFs under normoxia. WMPY-1s responded to hypoxia by significantly increasing several metabolites although the lipids remained unaffected, as indicated in Figure $1 \mathrm{~b}$ (Persuasive data Figure 1). PCAFs, on the other hand, responded to hypoxia by significantly increasing the lipid content, whereas only a few metabolites showed changes, such as an increase in phosphocholine and a decrease in glutamine, as shown in Figure 1c (Persuasive data Figure 2). Discussion: These results are especially relevant in the view of recent studies reporting that, in the context of cancer metastasis, cancer cells do not behave differently but the fibroblasts respond differently to cancer cells5. Here we found that the metabolic response to hypoxia of normal (WMPY-1) and cancer associated (PCAF) fibroblasts was very different. This is important, as these two cell populations are spatially localized in different regions. Normal fibroblasts are usually found at the interface of the tumor and adjacent normal tissue and are
\end{abstract}


associated with HIF expression6. We previously reported that hypoxia triggered significantly faster degradation of the extracellular matrix by fibroblasts suggesting that under hypoxic conditions these fibroblast may be assisting invasion and facilitating metastasis. PCAFs are located within the tumor. The metabolic changes observed in PCAFs in response to hypoxia suggest that they may be playing an important role in immune suppression. Different studies reported that lipid accumulation was found to skew tumor infiltrating myeloid cells towards immunosuppressive and anti-inflammatory phenotypes7,8. These results support targeting fibroblasts within and at the periphery of prostate cancers to improve survival. Metabolic or lipid inhibitors can provide treatment strategies to target normal fibroblasts and PCAFs. Acknowledgements: This work was supported by NIH R35CA209960 and R01CA82337. JPT was funded by Fundación Alonso Martín Escudero y MSCA.

References: 1. Zent, J.; Guo, L. W., Signaling Mechanisms of Myofibroblastic Activation: Outside-in and Inside-Out. Cell Physiol Biochem 2018, 49 (3), 848-868. 2. Penet, M. F.; Kakkad, S.; Pathak, A. P.; Krishnamachary, B.; Mironchik, Y.; Raman, V.; Solaiyappan, M.; Bhujwalla, Z. M., Structure and Function of a Prostate Cancer Dissemination-Permissive Extracellular Matrix. Clin Cancer Res 2017, 23 (9), 2245-2254. 3. Webber, M. M.; Trakul, N.; Thraves, P. S.; Bello-DeOcampo, D.; Chu, W. W.; Storto, P. D.; Huard, T. K.; Rhim, J. S.; Williams, D. E., A human prostatic stromal myofibroblast cell line WPMY-1: a model for stromal-epithelial interactions in prostatic neoplasia. Carcinogenesis 1999, 20 (7), 1185-92. 4. Penet, M. F.; Shah, T.; Bharti, S.; Krishnamachary, B.; Artemov, D.; Mironchik, Y.; Wildes, F.; Maitra, A.; Bhujwalla, Z. M., Metabolic imaging of pancreatic ductal adenocarcinoma detects altered choline metabolism. Clin Cancer Res 2015, 21 (2), 386-95. 5. Kshitiz; Afzal, J.; Maziarz, J. D.; Hamidzadeh, A.; Liang, C.; Erkenbrack, E. M.; Kim, H. N.; Haeger, J. D.; Pfarrer, C.; Hoang, T.; Ott, T.; Spencer, T.; Pavlicev, M.; Antczak, D. F.; Levchenko, A.; Wagner, G. P., Evolution of placental invasion and cancer metastasis are causally linked. Nat Ecol Evol 2019, 3 (12), 1743-1753. 6. Sivridis, E.; Giatromanolaki, A.; Koukourakis, M. I., Proliferating fibroblasts at the invading tumour edge of colorectal adenocarcinomas are associated with endogenous markers of hypoxia, acidity, and oxidative stress. J Clin Pathol 2005, 58 (10), 1033-8. 7. AlKhami, A. A.; Zheng, L.; Del Valle, L.; Hossain, F.; Wyczechowska, D.; Zabaleta, J.; Sanchez, M. D.; Dean, M. J.; Rodriguez, P. C.; Ochoa, A. C., Exogenous lipid uptake induces metabolic and functional reprogramming of tumor-associated myeloid-derived suppressor cells. Oncoimmunology 2017, 6 (10), e1344804. 8. Niu, Z.; Shi, Q.; Zhang, W.; Shu, Y.; Yang, N.; Chen, B.; Wang, Q.; Zhao, X.; Chen, J.; Cheng, N.; Feng, X.; Hua, Z.; Ji, J.; Shen, P., Caspase-1 cleaves PPARgamma for potentiating the pro-tumor

\section{Image/Figure:}

https://www.xcdsystem.com/wmis/abstract/File6959/GA325 ImageFigure 0601092429.jpg

Image/Figure Caption: Figure 1. Heat map showing altered metabolites in the cell extracts of (a) PCAFs compared to WMPY-1 under normoxic conditions; (b) WMPY-1 incubated under normoxic and hypoxic conditions; and (c) PCAFs incubated under normoxic and hypoxic conditions. Each column represent a biological replicate. Two-tail unpaired t-test; ${ }^{*} \mathrm{p} \leq 0.05, * *$ p unsaturation; EPA: eicosapentaenoic acid; ARA: arachidonic acid.

Full Name of Abstract's 1st Author : Jesus Pacheco-Torres 
Complete Status: Complete

First Name: Jesus

Last Name: Pacheco Torres

Email: jpachec6@jh.edu

Organization: Johns Hopkins University

Country: United States 


\title{
ID: GA326 \\ BOLD mechanism for patients with ArterioVenous Malformations: effect of distance of the lesion to the BOLD responses
}

\author{
Bob Hou, WVU, bhou@hsc.wvu.edu
}

\section{Category: Computational \& Data Science}

\begin{abstract}
Body : Many physiologic and pathologic factors (1-4) impact BOLD fMRI signal intensity (i.e., the BOLD mechanism) of a human subject. We hypnotized that for a patient with Arteriovenous Malformations (AVM) the distance of the lesion to the BOLD signal is one of the most important parameters on the BOLD mechanism. Hence in this investigation, we applied
\end{abstract} BOLD task fMRI and resting state fMRI modalities to AVM patients and healthy human subjects for understanding the impact from the distance on the BOLD mechanisms. Retrospectively we studied brain MRI data of 8 AVM patients with their lesions in or adjacent to left frontal or temporal lobes, and 7 age and gender matched healthy human subjects. The MRI data were from T1-weighted images, task BOLD fMRI from the right hand only finger tapping task and resting state BOLD fMRI (rsfMRI). The task and resting state fMRI data's group and individual analyses were performed by using FSL (6) and AFNI (7). We also did a t-test for determining the statistically significant difference between AVM and normal subjects in motor function. A 2 tails pair T-Test was applied, and a $\mathrm{P}$ value is less than 0.05 to be considered statistically significant. Results: In Figure 1, we showed the task fMRI BOLD, and the resting state BOLD co-registered on the T1 weighted image for an AVM case (the top row) and a normal subject (the bottom row). The averages and standard deviations (STDs) of the BOLD signals (i.e., r values) in the AVM and non-AVM (NAVM) sides for these 8 patients and the signals in two brain hemispheres for the 7 normal (Nor) subjects were measured and listed in the Table 1, and the P values for the signals of the AVM to NAVM sides for the patients and for the left to right brain hemispheres of the NOR were also calculated by the T-Test and listed in the table. Also the distances between AVMs to the PMC fMRI signals for the patients were included in the table. The correlation plot and linear fitting for the fMRI BOLD ratios (AVM/NAVM) VS. the distance $(\mathrm{mm})$ based on the data in the Table 1 was plotted in the Figure 2. The images from the group analyses for the task BOLD (the top raw) and resting state (the bottom row) BOLD images of the AVM and NOR subjects groups were shown in the Figure 3, in which the time course and the power spectrum for each image were also demonstrated. In Table 2 we listed the Z (i.e., the signal) values from the group analyses for the AVMs' task BOLD (i.e., fMRI) and resting state BOLD (i.e., rsfMRI) and also for the Normals' task BOLD and resting state BOLD. Figure 2 demonstrated that with the lesion distance increasing the BOLD fMRI signal ratio (AVM/NAVM) increased, and the linear correlation was significant ( $r>95 \%)$. This suggested that there was dependency between the lesion distance and the task BOLD functional signal of the AVM patients. The BOLD fMRI signal in the AVM side seems to be reduced linearly with the distance due to AVM's stealing effect on CBF to the functional areas. From the results of the group analyses, the differences between the fMRI and rsfMRI BOLD for the patients were demonstrated from the BOLD ratios, and corresponding timing courses and power spectra. The 
group analyses results implied that the AVM has great impact on the BOLD responses resulting likely from its disruption of the blood supply to the functional areas.

References: References: [1] Buxton, RB, et. al., Neuroimage, 2004, 23 Suppl 1:S220-33. [2] RW, COX, Computers and Biomedical Research, 1996, 162-173. [3] Smith, SM., et. al., NeuroImage, 2004, 208-219. [4] Hou,BL, et. al., Neuroimage, 2006, 489-497. [5] The AVM Study Group, N Engl J Med, 1999, 1812-1818. [6]Smith, SM., et. al., NeuroImage, 2004, 208219. [7] COX, RW, Computers and Biomedical Research, 1996, 162-173.

\section{Image/Figure:}

https://www.xcdsystem.com/wmis/abstract/File6959/GA326_ImageFigure_0614070401.JPG

Image/Figure Caption: Figure 1 : The task fMRI BOLD, and the resting state BOLD coregistered on the T1 weighted image for an AVM case (the top row) and a normal subject (the bottom row). Table 1 :The averages and standard deviations (STDs) of the BOLD signals (i.e., $r$ values) in the AVM and non-AVM (NAVM) sides for these 8 patients with the distances between AVMs to the PMC fMRI signals, and the signals in two brain hemispheres for the 7 normal (Nor) subjects. Figure 2: The correlation plot and linear fitting for the fMRI BOLD ratios (AVM/NAVM) VS. the distance $(\mathrm{mm})$. Figure 3: The images from the group analyses for the task BOLD (the top raw) and resting state (the bottom row) BOLD images of the AVM and NOR subjects groups. Table 2: The $Z$ (i.e., the signal) values from the group analyses for the AVMs' task BOLD (i.e., fMRI) and resting state BOLD (i.e., rsfMRI) and also for the Normals' task BOLD and resting state BOLD.

Complete Status: Complete

First Name: Bob

Last Name: Hou

Email: bhou@hsc.wvu.edu

Organization: WVU

Country: United States 


\title{
ID: GA327 \\ First PET tracer for imaging human CD19-positive B lymphocytes: initial evaluation in a humanized mouse model of multiple sclerosis
}

\author{
Carmen Azevedo, Stanford University, ecarmenazevedo@gmail.com
}

\section{Category: Neuroscience}

\begin{abstract}
Body : Objectives: Multiple sclerosis (MS) affects over two million people globally1 and costs millions of dollars in lost income and healthcare. Several disease modifying therapies (DMTs) exist for certain types of MS but not all patients respond due to disease heterogeneity. One such DMT that has shown dramatic benefits for some MS patients targets B lymphocytes, but there is currently no way to predict response. Positron emission tomography (PET) imaging of B cells could aid in selecting patients for anti-B cell therapy and monitor their response in real time. The aim of this study was to create the first anti-human CD19 antibody (mAb) PET tracer and assess its utility for detecting B cell infiltration into the CNS of humanized CD19 transgenic mice (hCD19-tg) induced with experimental autoimmune encephalomyelitis (EAE). Methods: A mutated variant of inebilizumab (B cell DMT in clinical trials) was radiolabeled; this mAb lacks the ability to deplete B cells due to mutations at its FcR $\gamma$ binding site while maintaining high affinity for CD19.2 Female hCD19-tg C57/BL6J mice age 9 - 13 weeks were induced with EAE through inoculation of myelin oligodendrocyte glycoprotein (MOG1-125) and observed daily for signs of paralysis from day 8 onward. On day 12 post inoculation, $3.0 \pm 0.5 \mathrm{MBq}[64 \mathrm{Cu}] \mathrm{hCD} 19-$ $\mathrm{mAb}$ was injected intravenously into 12 mice $(\mathrm{n}=8 \mathrm{EAE}, \mathrm{n}=4$ naïve). PET/CT imaging was performed on all mice $19-20$ hours post injection. Percent of injected dose per gram $(\% \mathrm{ID} / \mathrm{g})$ was determined by gamma counting of perfused organs of interest and PET signal was normalized to heart \% $\mathrm{ID} / \mathrm{g}$. Autoradiography (ARG) was performed to obtain high resolution images of tracer distribution in brain regions, expressed as mean pixel intensity/decay corrected dose. Results: Radiolabeling of DOTA-conjugated mAb (2 - 3 DOTA/mAb, MALDI-MS) was achieved by incubating $20 \mu \mathrm{g} \mathrm{mAb}$ with $37 \mathrm{MBq}[64 \mathrm{Cu}] \mathrm{CuCl} 3$ at $38^{\circ} \mathrm{C}$ for 30 minutes, producing a radiotracer with high radiochemical purity ( $>99 \%$, ITLC) and molar activity of 2 $\mathrm{MBq} / \mu \mathrm{g}$. The findings from PET, ARG, and biodistribution provide evidence that [64Cu]hCD19 can detect B cell infiltration into the CNS of EAE mice. Specifically, EAE mice had significantly higher PET signal in whole brain, medulla, and cerebellum, (Fig.1a, d; WB p Conclusion: The first human CD19-mAb was successfully radiolabeled and demonstrated its ability to detect B cells in humanized EAE mice. This new tracer represents a rapidly translatable tool for predicting and monitoring response to MS therapy.
\end{abstract}

References: 1. GBD 2016 Multiple Sclerosis Collaborators. Global, regional, and national burden of multiple sclerosis 1990-2016: a systematic analysis for the Global Burden of Disease Study 2016. 2019;18(3):269-285. 2. Oganesyan V, et al. Structural characterization of a human $\mathrm{Fc}$ fragment engineered for lack of effector functions. Acta Crystallogr D Biol Crystallogr. 2008;64(Pt 6):700-704.

\section{Image/Figure:}


Image/Figure Caption: Figure 1. [64Cu]hCD19 PET of hCD19-tg mice reveals increased tracer binding in the brain of EAE vs naïve mice, shown by representative sagittal PET/CT brain images taken $19 \mathrm{~h}$ post injection (a). Tracer signal in whole brain was calculated by coregistering a mouse brain atlas to PET/CT images $(\mathrm{d}, 0.160 \pm 0.0156$ vs $0.1265 \pm 0.01603 ; \mathrm{p}$ nissl stained brain tissue (b, overlay). Increased accumulation of tracer in brain was verified by ex vivo gamma counting and quantification of autoradiography images (e, brain stem $25.35 \pm$ 6.308 vs $17.64 \pm 2.457 \mathrm{MPI} /$ dose, p p p p ex vivo gamma counting (f, TSC $1.104 \pm 0.333 \mathrm{vs}$ $0.2715 \pm 0.0343 \% \mathrm{ID} / \mathrm{g}, \mathrm{p} \mathrm{p} * * * \mathrm{p}$-value $\mathrm{p}$-value $\mathrm{p}$-value $<0.05$

Full Name of Abstract's 1st Author : E Carmen Azevedo

Complete Status: Complete

First Name: Carmen

Last Name: Azevedo

Email: ecarmenazevedo@gmail.com

Organization: Stanford University

Country: United States 


\title{
ID: GA330 \\ Non-invasive Imaging of Human CD8+ Tumor Infiltrating Lymphocytes in Glioblastoma
}

\author{
Veronica Nagle, Memorial Sloan Kettering Cancer Center, naglev@mskcc.org
}

\section{Category: Oncology}

\begin{abstract}
Body : Objectives: Glioblastoma Multiforme (GBM) is the most common malignant brain tumor in adults. Patients with GBM suffer from inevitable relapse and a median survival of less than two years1. Immunotherapeutic agents have shown antitumor activity against a variety of human cancers, including melanoma, non-small cell lung cancer, as well as brain metastases from these tumor types. However, GBMs have failed all immunotherapeutic phase 3 clinical trials, likely due to the inability to stratify and monitor patients in an immunologically informed manner2. Cytotoxic CD8+ T cells mitigate the therapeutic response to the majority of FDA approved immunotherapeutics and are currently indiscernible from tumor tissue in brain tumors with the current imaging modalities. We assessed a human-CD8+ T cell-targeted minibody positron emission tomography (PET) tracer (IAB22M2C) to monitor systemic and brain parenchymal cytotoxic T cell infiltrate in GBM-bearing humanized immune system (HIS) mice. Methods: Anti-Human-CD8a minibody was conjugated to 2-S-(4-Isothiocyanatobenzyl)-1,4,7triazacyclononane-1,4,7-triacetic acid (p-SCN-NOTA) and radiolabeled with 64-Copper $(64 \mathrm{Cu})$ for positron emission tomography (PET). To generate HIS mice, 107 HLA-typed PBMCs from a single donor (Stem Cell Technologies) were injected into the tail vein of 5-8 week old female NOG CIEA (Taconic). To generate GBM PDX bearing HIS mice, 5-8 week old female NOG CIEA (Taconic) were IC implanted with PDX cells (provided by the MSK Brain Tumor Center). Three weeks following tumor implantation, mice were humanized with PBMCs with HLA partially matched to the major loci. Radiotracer was injected by tail vein and 15 min static $\mathrm{PET} / \mathrm{CT}$ images were acquired at $1,4,12$, and $24 \mathrm{~h}$ using a dual microPET/CT scanner (Inveon, Siemens). T cells were quantified by multi-channel flow cytometry (spleen) and immunohistochemistry (brain). High resolution autoradiography was performed on PBS perfused frozen brains. Results: Here, we show [64Cu]Cu-NOTA-anti-CD8 is able to quantitatively detect systemic human CD8+ T cell burden as well as CD8+ tumor-infiltrating T cells in an orthotopic GBM PDX HIS mouse model. Further, we demonstrate the variability of donor-dependent human T cell infiltrate in the peripheral blood mononuclear cell (PBMC) HIS mouse model and show that $[64 \mathrm{Cu}] \mathrm{Cu}-\mathrm{NOTA}$-anti-CD8 uptake correlates with donor-dependent $\mathrm{T}$ cell infiltrate in a orthotopic GBM patient-derived xenograft (PDX). Conclusion: These data indicate the capacity of $[64 \mathrm{Cu}] \mathrm{Cu}-\mathrm{NOTA}$-anti-CD8 to non-invasively monitor peripheral and brain tumor human $\mathrm{CD} 8+\mathrm{T}$ cell burden with remarkable specificity. The anti-CD8 tracer IAB22M2C has passed phase 1 clinical trials in peripheral solid tumors 3 and with the data herein, we propose the rapid clinical translation of this radiotracer for utility in human GBMs as well as patients suffering other brain tumors. The validation of this tracer as a tool to monitor GBM CD8+ tumor infiltrating lymphocytes will enable clinicians to non-invasively evaluate $\mathrm{T}$ cell responses in brain tumors, leading to both better designed and immunologically informed clinical trials.
\end{abstract}


References: 1. Ostrom, Q.T. et al. CBTRUS Statistical Report: Primary Brain and Other Central Nervous System Tumors Diagnosed in the United States in 2012-2016. Neuro Oncol 21, v1-v100 (2019). 2. Aldape, K. et al. Challenges to curing primary brain tumours. Nat Rev Clin Oncol (2019). 3. Pandit-Taskar, N. et al. First-in-Humans Imaging with (89)Zr-Df-IAB22M2C AntiCD8 Minibody in Patients with Solid Malignancies: Preliminary Pharmacokinetics, Biodistribution, and Lesion Targeting. J Nucl Med 61, 512-519 (2020).

Full Name of Abstract's 1st Author : Veronica L. Nagle

Complete Status: Complete

First Name: Veronica

Last Name: Nagle

Email:naglev@mskcc.org

Organization: Memorial Sloan Kettering Cancer Center

Country: United States 


\title{
ID: GA331 \\ Economical production of pre-clinical PET probes: microscale synthesis of [18F]Florbetaben
}

\author{
Ksenia Lisova, Crump Institute for Molecular Imaging,UCLA, ksenlisan@gmail.com
}

Category: New Chemistry, Biology \& Bioengineering

\begin{abstract}
Body : Introduction: Conventional automated radiosynthesizers are generally optimized for producing large batches of PET tracers, while small portions needed for a typical preclinical imaging cannot be economically produced with such techniques. In this work we describe the first reported microvolume procedure for production of injectable [18F]Florbetaben [2] ([18F]FBB) for use in imaging of $\beta$-amyloid - a hallmark of Alzheimer's disease. Methods: Typically, production of $[18 \mathrm{~F}] \mathrm{FBB}$ starts with a 2-step synthesis using an N-Boc-protected precursor (Fig. 1A), followed by semi-preparative HPLC purification and reformulation by solidphase extraction (SPE) [1], [3]. Reagent amounts in the conventional method were scaled down 90-fold to implement the synthesis in droplet format on Teflon-coated silicon chips [4]. In addition, semi-prep HPLC was replaced with an analytical scale method, and an automated SPE system based on a miniature cartridge was developed to formulate into small volumes (Fig. 1B). Several parameters of the fluorination step were optimized (temperature, base amount, precursor amount), resulting in the following procedure. Aqueous $[18 \mathrm{~F}]$ fluoride was azeotropically dried with $\mathrm{K} 2 \mathrm{CO} 3 / \mathrm{K} 222(275 / 383 \mathrm{nmol})$ and then reacted with the Boc-protected precursor $(80 \mathrm{nmol})$ in $10 \mu \mathrm{L} \mathrm{DMSO}$ at $130^{\circ} \mathrm{C}$ for $5 \mathrm{~min}$. The resulting intermediate was deprotected with $\mathrm{HCl}$ at $90^{\circ} \mathrm{C}$ for $3 \mathrm{~min}$ and recovered from the chip in aqueous acetonitrile solution $(80 \mu \mathrm{L}$ of $1: 1 \mathrm{v} / \mathrm{v}$ $\mathrm{H} 2 \mathrm{O}: \mathrm{MeCN}$ ). The crude product was purified via analytical scale HPLC (Kinetex $5 \mu \mathrm{m} \mathrm{C18}$ column, 250 x $4.6 \mathrm{~mm} ; 1: 1 \mathrm{v} / \mathrm{v}$ MeCN:20 mM phosphate buffer (pH 7.2), $1.5 \mathrm{~mL} / \mathrm{min} ; 254 \mathrm{~nm}$ ). Formulation was performed using a miniature cartridge (fabricated by packing frits and $\mathrm{C} 18$ resin into a short length of tubing) installed into a custom automated formulation system driven by a LabView program to carry out the following procedure. The HPLC purified fraction was diluted with water and trapped on the cartridge. The cartridge was next washed with $10 \mathrm{~mL}$ DI water, after which the product was eluted with $150 \mu \mathrm{L}$ of EtOH into amber vial with $180 \mu \mathrm{L}$ PEG 400 and $670 \mu \mathrm{L}$ DI water with $33 \mathrm{mg}$ of sodium ascorbate. Results: Starting with $270 \pm 100$ $\mathrm{MBq}(\mathrm{n}=3)$ of [18F]Fluoride, the method affords formulated [18F]FBB with RCY of $49 \pm 3 \%$ $(\mathrm{n}=3)$ and $>98 \%$ radiochemical purity and molar activity of $170 \pm 50 \mathrm{GBq} / \mu \mathrm{mol}(\mathrm{n}=3)$ after 55 min synthesis time. The miniature $\mathrm{C} 18$ cartridge enables efficient low-volume elution with EtOH and a small final volume $(1.0 \mathrm{~mL})$, which would not be feasible using standard cartridges, but ensures sufficient probe concentration for preclinical imaging even for small batches. Conclusion: This work describes an novel economic approach to produce large or small quantities of $[18 \mathrm{~F}] \mathrm{FBB}$ for preclinical applications. $1 \mathrm{~mL}$ containing $77 \pm 35 \mathrm{MBq}(\mathrm{n}=3)$ of formulated tracer was obtained in $55 \mathrm{~min}$ with very high molar activity. This method provides very high yields and molar activities compared to reported literature methods, and requires less space and shielding and significantly reduced amount of reagents.
\end{abstract}


References: [1] Rominger et al., J. Nucl. Med. 54: 1127-1134, 2013. [2] Zhang et al., Nucl.

Med. Biol. 32: 799-809, 2005. [3] Collins et al., Proc. Natl. Acad. Sci. 114: 11309-11314, 2017. [4] Wang et al., Lab. Chip 2415-2424, 2019.

\section{Image/Figure:}

https://www.xcdsystem.com/wmis/abstract/File6959/GA331_ImageFigure_0422071637.png

Full Name of Abstract's 1st Author : Ksenia Lisova

Complete Status: Complete

First Name: Ksenia

Last Name: Lisova

Email: ksenlisan@gmail.com

Organization: Crump Institute for Molecular Imaging,UCLA

Country: United States 


\title{
ID: GA336 \\ Targeted Delivery of Chemotherapy Agent SN38 to Intracranial Glioblastoma Using Sub-5 nm Ultrafine Iron Oxide Nanoparticles
}

\author{
Yuancheng Li, Emory University, yli41@emory.edu
}

\section{Category: Oncology}

\begin{abstract}
Body : Glioblastoma (GBM) is the most common and aggressive malignant primary brain tumor with extremely poor prognosis1. Treatment options for GBM remain limited, as systemically delivering effective dosage of therapeutics to GBM is challenging due to the physiological barriers, such as the blood-brain barrier (BBB) or blood-tumor barrier (BTB), blocking the delivery of most chemotherapy agents2. While theranostic nanoparticles offer advantages in drug delivery, including long blood circulation time, tumor targeted specificity and carrying water insoluble drugs, most nanoparticles, typically with core sizes over $10 \mathrm{~nm}$, have not been successfully applied to intracranial brain tumors3. In this abstract, we present the initial investigation of using sub-5 $\mathrm{nm}$ ultrafine iron oxide nanoparticles (uIONPs) with the core diameter of $3 \mathrm{~nm}$ to deliver a water insoluble chemotherapy agent SN38 to intracranial U87MG glioblastomas in mice. SN38 is the highly potent yet hydrophobic active metabolite of topoisomerase I inhibitor Irinotecan, and can be uploaded into the hydrophobic blocks of (polyethylene glycol)-block-(allyl glycidyl ether) (PEG-b-AGE) coating polymer of uIONPs in tetrahydrofuran (THF) upon mixing. Transferring the mixture of SN38 loaded PEG-b-AGE and UIONP to the aqueous phase promotes the hydrophobic moiety of PEG-b-AGE polymer to collapse onto the surface of uIONP, thus encapsulating SN38 molecules (uIONP/SN38). The loading efficiency of SN38 on the reported uIONP was determined to be $48.7 \pm 6.4 \%$ (SN38/Fe $\mathrm{wt} \%$ ) by measuring the UV absorbance of SN38. Cytotoxicity of uIONP/SN38 towards U87MG glioblastoma cells was observed in the in vitro assay with IC50 of $\sim 25 \mathrm{nM}$ (SN38 dosage) comparing to $\sim 2 \mu \mathrm{M}$ obtained from free SN38. uIONP/SN38 was then functionalized with cyclo(Arg-Gly-Asp-d-Phe-Cys) (RDG) peptide as the ligand to actively target tumor integrins $\alpha v \beta 3$ for delivering SN38 to the intracranial tumor of mouse model. RGD-conjugated uIONP/SN38 was labeled with near infrared dye NIR830 (NIR830-RGD-uIONP/SN38) for both MRI and optical imaging to track the delivery and intratumoral distribution of $\mathrm{uIONP/SN38}$. The results showed accumulation of NIR830-RGD-uIONP in the intracranial tumors as strong NIR signals were observed from ex vivo imaging of collected tumors at 12 hours after intravenous (i.v.) injection. The immunofluorescence staining of collected brain tissue sections using antibody for $\alpha v \beta 3$ integrins revealed the co-localized uIONPs with $\alpha v \beta 3$ integrins antibody, indicating targeting of NIR830-RGD-uIONP/SN38 to the $\alpha v \beta 3$ integrin over expressed U87MG glioblastoma. To study the efficacy of RGD-uIONP/SN38 in treating GBM, 24 mice bearing intracranial U87MG glioblastomas were randomly divided into four groups ( $n=6 /$ group) to receive different treatments, i.e., RGD-uIONP/SN38 as the treatment group (group 1), RGDuIONP vehicle only (group 2), SN38 formulated with Cremophor EL (group 3) as a free drug control, and no treatment (group 4). RGD-uIONP/SN38 and SN38/Cremophor EL were given to mice every 3 days at the SN38 dosage of $5 \mathrm{mg} / \mathrm{kg}$ body weight 10 days after tumor implantation with tumor growth monitored by Gd contrast enhanced MRI. RGD-uIONP was given to mice at
\end{abstract}


the Fe dosage equivalent to RGD-uIONP/SN38 in group 1. Longitudinal monitoring of tumor growth was performed after each administration of treatment. The results showed that mice receiving $\alpha \mathrm{v} \beta 3$ tumor integrin targeted RGD-uIONP/SN38 exhibited longer survival $(12.7 \pm 2.3$ days) than those treated with SN38/Cremophor EL, which did not demonstrate significant improvement in survival ( $7.5 \pm 3.8$ days, $\mathrm{P}>0.05)$ over the non-treated control ( $7.3 \pm 0.5$ days). In summary, sub-5 nm uIONP with PEG-b-AGE coating demonstrated a high loading efficiency for highly potent yet hydrophobic small molecule drug SN38, and $\sim 80$-fold higher cytotoxicity towards glioblastoma cells than free SN38 as shown in in vitro assay. In an intracranial U87MG glioblastoma mouse model, tumor-integrin-targeted uIONP/SN38 conjugated with RGD can be delivered to the intracranial tumors to achieve efficacious treatment.

References: References: [1] Weller M. et al., Nat. Rev. Dis. Primers 2015, 1, 15017. [2] Arvannitis CD. et al., Nat. Rev. Cancer 2020, 30, 26. [3] Fisusi, FA. et al., Nanomedicine (Loud) $2018,13,579$.

\section{Image/Figure:}

https://www.xcdsystem.com/wmis/abstract/File6959/GA336 ImageFigure 0610073651.jpg

Image/Figure Caption: Figure 1. (A) A TEM image of PEG-b-AGE polymer coated uIONPs in water indicates their mono-dispersed state. (B) Zeta potentials and hydrodynamic diameters of PEG-b-AGE polymer coated uIONPs measured before and after SN38 encapsulation, RGD conjugation and NIR830 labeling validate the successful modifications and retained monodispersion. (C) Near infrared imaging at 2 and 12 hours after i.v. injection of NIR830-RGDuIONPs shows the accumulation of uIONP in the brain tumor at 12 hours and other organs, noticing renal clearance of uIONP at $12 \mathrm{~h}$ after injection. (D) H\&E and immunofluorescence stained tumor tissue section reveals the accumulation of NIR830-RGD-uIONPs in the $\alpha v \beta 3$ overexpressing U87MG tumor. (E) Gd enhanced T1 weighted MRI shows the growth of intracranial tumors in mice receiving RGD-uIONP/SN38, RGD-uIONP and free SN38. (F) The survival curve of tumor bearing mice treated with RGD-uIONP/SN38, RGD-uIONP and free SN38.

Full Name of Abstract's 1st Author : Yuancheng Li

Complete Status: Complete

First Name: Yuancheng

Last Name: $\mathrm{Li}$

Email: yli41@emory.edu

Organization: Emory University

Country: United States 


\title{
ID: GA337 \\ Artificial intelligence analysis of magnetic particle imaging for islet transplantation in a mouse model
}

\author{
Hasaan Hayat, Michigan State University, hayathas@msu.edu
}

\section{Category: Systemic Diseases (Kidney, Liver and Pancreas)}

\begin{abstract}
Body : Current approaches to biomedical image quantification and analysis of Magnetic Particle Imaging (MPI) are thwarted by the lack of reliable, standardized methods of segmentation of a region of interest (ROI) due to inability of various human raters to determine threshold regarding the integrity of nanoparticle signal from background in images and the high degree of inter-rater variability and resulting selection bias from human error1,2,3,4.

Furthermore, there are minimal methods for rapid and adequate prediction of Total Iron Value (TIV), which can be indicative of cellular nanoparticle uptake/content, from an ROI within an MPI Image scan5,6. This calls for the development of artificial intelligence (AI) systems for analysis of MPI. Here, we utilize a canonical algorithm in the domain of unsupervised machine learning, known as K-means++7,8,9,10, in order to precisely segment the regions of interest (ROI) of raw MPI image data and preform subsequent analysis of TIV. We have developed the algorithm to preform iron prediction tasks through a novel AI generated standard curve method, in which several fiducial markers within a scan are segmented by the algorithm, and their total pixel intensity sum and corresponding total iron amounts are used to generate a standard curve through linear regression, from which the total iron amount of an unknown ROI is predicted. For initial parameter optimization of the algorithm, 3D-printed phantoms of several sizes were generated in two shapes - Circle and a letter "S", and were injected with varying amounts of VivoTrax superparamagnetic iron oxide nanoparticles (SPIONs) and imaged with the Momentum MPI scanner (Magnetic Insight) to generate data on which the algorithm can be calibrated. From this, we observed a linear correlation between MPI signal intensity and iron amount within the phantoms, so the standard curve (SC) model was elected as a reliable model for total iron amount prediction and was used as the algorithm for quantification and predictive analysis of the segmented ROI throughout the rest of the study. In order to provide a robust domain of data to the algorithm and evaluate its performance in multiple MPI scenarios, we generated in vitro, in vivo, and ex vivo models of human pancreatic islets and applied the AI algorithm to these datasets in order to gain insight into segmentation and iron prediction on these images. In vitro models included labeling islets with VivoTrax SPIONs and imaging the cells in varying amounts. Immunofluorescence staining and fluorescence microscopy of labeled cells showed insulin expression and uptake of dextran coated VivoTrax nanoparticles by the islet cell clusters (Fig 1. A-D). In vivo mouse models were generated through transplantation of increasing numbers of the labeled human islets under the kidney capsule of NOD/scid mice and mice were scanned in 2D using the Momentum MPI scanner (Magnetic Insight). A 3D MPI scan was co-registered with CT to determine correct anatomical location of islet cell transplant under kidney capsule of mice (Fig 1. E-H). Ex Vivo data was collected from excised mouse kidneys which were imaged with the MPI scanner. The proposed K-means++ algorithm and SC model were then applied to the MPI data for segmentation, quantification and TIV analysis of the
\end{abstract}


transplanted labeled islets (Fig 1. I \& J). Through statistical evaluation of the algorithm performance via intraclass correlation coefficient validation on these datasets, we observed the ability of the K-means++ based machine learning model employed in this study to provide a novel, standardized method of segmentation, quantification, and analysis of MPI scans of transplanted human islets across a wide domain of data. This will provide a powerful tool for MPI segmentation and ROI analysis for applications in cell-based therapy including islet transplantation, stem cell transplantation, CAR T cell therapy, et al. in vivo.

References: 1. Lin, S. H., \& Ikram, M. A. (2020). On the relationship of machine learning with causal inference. Eur J Epidemiol, 35(2), 183-185. doi:10.1007/s10654-019-00564-9 2. GarciaLorenzo, D., Francis, S., Narayanan, S., Arnold, D. L., \& Collins, D. L. (2013). Review of automatic segmentation methods of multiple sclerosis white matter lesions on conventional magnetic resonance imaging. Med Image Anal, 17(1), 1-18. doi:10.1016/j.media.2012.09.004 3. Yamane, T., Ishii, K., Sakata, M., Ikari, Y., Nishio, T., Ishii, K., . . Group, J. A. S. (2017). Interrater variability of visual interpretation and comparison with quantitative evaluation of (11)CPiB PET amyloid images of the Japanese Alzheimer's Disease Neuroimaging Initiative (J-ADNI) multicenter study. Eur J Nucl Med Mol Imaging, 44(5), 850-857. doi:10.1007/s00259-016-35912 4. Paysen, H., Loewa, N., Stach, A., Wells, J., Kosch, O., Twamley, S., . . Wiekhorst, F. (2020). Cellular uptake of magnetic nanoparticles imaged and quantified by magnetic particle imaging. Sci Rep, 10(1), 1922. doi:10.1038/s41598-020-58853-3 5. Rawat, W., \& Wang, Z. (2017). Deep Convolutional Neural Networks for Image Classification: A Comprehensive Review. Neural Comput, 29(9), 2352-2449. doi:10.1162/NECO_a_00990 6. Thrall JH, Li X, Li Q, Cruz C, Do S, et al. (2018) Artificial Intelligence and Machine Learning in Radiology: Opportunities, Challenges, Pitfalls, and Criteria for Success. J Am Coll Radiol 15(3 Pt B): 504508. 7. Chandhok C, Chaturvedi S, Khurshid AA (2012) An approach to image segmentation using K-means clustering algorithm. International Journal of Information Technology 1(1): 11 17 8. Arthur D, Vassilvitskii S (2006) k-means++: The advantages of careful seeding. Stanford Infolab. 9. Agarwal S, Yadav S, Singh K (2012) Notice of violation of IEEE publication principles K-means versus k-means++ clustering technique. 2012 Students Conference on Engineering and Systems, IEEE. 10. Yuan, C., \& Yang, H. (2019). Research on K-value selection method of K-means clustering algorithm. J-Multidisciplinary Scientific Journal, 2(2), 226-235

\section{Image/Figure:}

https://www.xcdsystem.com/wmis/abstract/File6959/GA337_ImageFigure_0423031836.png

Image/Figure Caption: Figure 1. Artificial Intelligence Analysis of Magnetic Particle Imaging of Transplanted Human Islets in a Mouse Model. (Top left) Fluorescence immunostaining of human islets labeled with VivoTrax. A. Dextran staining of nanoparticles via anti-dextran antibody; B. Insulin staining via rabbit polyclonal anti-insulin antibody; C. DAPI staining of cell nucleus; D. Merged images (Top Right) 3D MPI Scan and CT Overlay human islet transplanted under left kidney capsule of mouse model. E. 3D MPI/CT merged image; F. Sagittal view of MPT/ CT image; G. Coronal view of MPI/CT image; H. Axial view of MPI/CT image. (Bottom) K-means++ segmentation and SC model TIV prediction of MPI of in vivo human islet transplanted mouse model. (Left) I. Segmentation results of mouse with 50 IEQ; 100 IEQ; 200 
IEQ; 400 IEQ; 800 IEQ (IEQ = Islet Equivalent) ; (Right) J. TIV prediction from extracted ROIs using Standard Curve (SC) model

Full Name of Abstract's 1st Author : Hasaan Muhammad Hayat

Complete Status: Complete

First Name: Hasaan

Last Name: Hayat

Email: hayathas@msu.edu

Organization: Michigan State University

Country: United States 


\title{
ID: GA340 \\ Characterization of Ovarian Lesions with KDR-targeted Ultrasound Molecular Imaging: Early Experiences on Correlation between In-Vivo Imaging Findings and Ex-Vivo Immunohistochemistry Outcomes in a Clinical Trial
}

\author{
Neha Antil, Stanford University, drantil7@stanford.edu
}

\section{Category: Oncology}

\begin{abstract}
Body : Purpose: Ovarian cancer (OC) is the fifth leading cause of cancer-related deaths in women and the most lethal gynecologic cancer. Ovarian cancer usually remains asymptomatic until advanced stages, but is curable when discovered early. Thus it is crucial to diagnose the disease at early stage for proper patient management. Conventional transvaginal ultrasound (US) imaging suffers from limited sensitivity and specificity in the detection of ovarian cancer. Adding molecularly targeted ligands to contrast microbubbles enables US to detect, characterize and monitor ovarian cancer at a molecular level. Using kinase insert domain receptor (KDR)targeted ultrasound molecular imaging (USMI) in this clinical study, we sought to correlate USMI signal of a variety of ovarian lesions with histology and immunohistochemistry (IHC) staining for KDR. Material and Methods: Ten women (mean age 55.0 \pm 14.6 years, range 28 to 76 years) with complex ovarian lesions were prospectively recruited in this HIPAA-compliant IRB-approved study after meeting eligibility criteria and obtaining informed consent. USMI was performed on clinical EPIQ 7 US scanner using endovaginal broadband curved array transducer (C10-3v). The complex ovarian lesion was first identified on B-Mode US and a plane showing a solid component (target lesion) was then selected for further evaluation. While imaging in dual mode with B-mode and contrast mode side-by-side, clinical grade KDR-targeted microbubbles (MBKDR, BR55, Bracco, $0.03 \mathrm{~mL} / \mathrm{kg}$ of body weight) were manually injected over 10 seconds followed by $10 \mathrm{~mL}$ saline flush. Imaging was obtained starting with initial 45 seconds acquisition to capture the wash-in phase of MBKDR, followed by 10 second acquisition every 2 mins until 30 minutes post injection. Blood pressure, ECG, oxygen levels, heart rate, $\mathrm{CBC}$, and metabolic panel were obtained before and after MBKDR administration. Surgery for these complex ovarian lesions was performed within 2-week interval following imaging (5.6 \pm 5.2 days, range 1-14 days) after USMI. Tumor regions overlying the imaged target lesions were marked by the surgeon for accurate correlation on histology. For USMI analysis, USMI stationary signal was categorized into 1: no, 2: weak and 3: strong signal, which was then correlated with ex-vivo KDR signal expression along with other marker expression like CD31, B7H3 and prostatespecific membrane antigen (PSMA). Results: All patients tolerated MBKDR without adverse events. 7 patients were included in the data analysis, surgery was not performed in one patient; ex vivo analysis was not yet completed in one patient; one lesion was intraoperatively found outside a severely atrophic ovary in one patient. In all 7 patients, successful intravenous administration of MBKDR was confirmed with enhancement of the solid component of lesions during wash-in phase of MBKDR within the first 45 seconds. 3 out of 7 lesions were benign $(43 \%)$, and 4 lesions were borderline (low malignant potential) tumors $(57 \%)$ on histology. The enhancement observed in the 7 lesions ranged from none $(n=2)$ over weak $(n=3)$ to strong $(n=2)$, respectively. In all 7 patients the imaged target lesions could be identified on gross specimens
\end{abstract}


and processed for IHC. In-vivo USMI signal showed correlations with ex-vivo IHC for KDR in $86 \%$ (6/7 lesions), whereas B7H3 and PSMA expression was observed in 57\% (4/7) 43\% (3/7) lesions, respectively. Conclusion: KDR-targeted USMI is feasible and safe, and enables noninvasive detection of KDR expression in patients with ovarian lesions. All imaged target lesions could be identified on gross specimens for IHC work-up and in-vivo USMI signals matched with ex-vivo KDR expression in the majority of cases and warrants further exploration. USMI has potential to be used with other types of US MBs targeted at novel biomarkers such as B7H3 and PSMA.

References: 1. Willmann JK, Bonomo L, Testa AC, Rinaldi P, Rindi G, Valluru KS, et al. Ultrasound molecular imaging with BR55 in patients with breast \& ovarian lesions: First-inhuman results. J Clin Oncol. 2017;35(19):2133-40. 2. Willmann JK, Lutz AM, Paulmurugan R, Patel MR, Chu P, Rosenberg J, et al. Dual-targeted contrast agent for US assessment of tumor angiogenesis in vivo. Radiology. 2008;248(3):936-44. 3. Lutz AM, Willmann JK, Drescher CW, Ray P, Cochran F V., Urban N, et al. Early diagnosis of ovarian carcinoma: Is a solution in sight. Radiology. 2011;259(2):329-45. 4. Lutz AM, Willmann JK, Cochran F V., Ray P, Gambhir SS. Cancer screening: A mathematical model relating secreted blood biomarker levels to tumor sizes. PLoS Med. 2008;5(8):1287-97. 5. Willmann JK, Cheng Z, Davis C, Lutz AM, Schipper ML, Nielsen $\mathrm{CH}$, et al. Targeted microbubbles for imaging tumor angiogenesis: assessment of wholebody biodistribution with dynamic micro-pet in Mice. Radiology. 2008;249(1):212-9. 6. Lutz AM, Bachawal S V., Drescher CW, Pysz MA, Willmann JK, Gambhir SS. Ultrasound molecular imaging in a human CD276 expression-modulated murine ovarian cancer model. Clin Cancer Res. 2014;20(5):1313-22.

\section{Image/Figure:}

https://www.xcdsystem.com/wmis/abstract/File6959/GA340_ImageFigure 0423012010.jpg

Full Name of Abstract's 1st Author : Dr Neha Antil

Complete Status: Complete

First Name: Neha

Last Name: Antil

Email: dranti17@stanford.edu

Organization: Stanford University

Country: United States 


\title{
ID: GA341 \\ Innovation of NIR luciferin analogues using firefly bioluminescence for in vivo imaging
}

\author{
Nobuo Kitada, The University of Electro-Communications, kitada@uec.ac.jp
}

Category: New Chemistry, Biology \& Bioengineering

\begin{abstract}
Body : We have developed near-infrared (NIR) luciferin analogues that exhibit firefly bioluminescence, including compounds dubbed "TokeOni" and "seMpai," which are now commercially available. In this conference, I will present the characteristics of NIR luciferin analogues. Firefly bioluminescence, a process whereby light is emitted with high efficiency, has found widespread use in life science applications. Notably, NIR light is suitable for use in in vivo imaging because deep tissues are highly permeable to light in the relevant wavelength range $(650-900 \mathrm{~nm})$. However, the wavelength of light emitted via firefly bioluminescence is usually yellow-green $(560 \mathrm{~nm})$. We performed studies on the structure-activity relationship of compounds producing firefly bioluminescence in order to synthesize luciferin analogues emitting NIR light. Thus, AkaLumine, a luciferin analogue that produces light at $675 \mathrm{~nm}$ wavelength, was developed. This compound was shown to yield useful results when applied to the in vivo imaging of mice. We have improved the characteristics of AkaLumine to optimize this compound for use in in vivo imaging, thus obtaining the compounds dubbed TokeOni and seMpai. Recently, Akaluc, an artificial enzyme that specifically catalyzes the light-producing oxidation of TokeOni, has been developed (Iwano S., et al., Science, 2018, 359, 935-939). As a result, it became possible to in vivo imaging for larger animals than mice like micro pig and marmoset. In this way, bioluminescence imaging technology is undergoing constant innovation, and we continue to develop NIR luciferin analogues that can be used as imaging tools. In this conference, I will present the characteristics of recently developed NIR luciferin analogues.
\end{abstract}

References: Iwano S., et al., Science, 2018, 359, 935-939 Kuchimaru, T. et al. Nat. Commun. 2016, 7, 11856. Saito, R. et al. BCSJ, 2019, 92, 608-618

Full Name of Abstract's 1st Author : Nobuo Kitada

Complete Status: Complete

First Name: Nobuo

Last Name: Kitada

Email: kitada@uec.ac.jp

Organization: The University of Electro-Communications

Country: Japan 


\title{
ID: GA344 \\ Surface protein targeted white blood cell tracking of inflammation using Magnetic Particle Imaging (WBC-MPI)
}

\author{
K. L. Barry Fung, University of California, Berkeley, barry.kl.fung@berkeley.edu
}

Category: Immunology: Inflammation \& Infection

\begin{abstract}
Body : Magnetic particle imaging (MPI) is a tracer imaging modality that detects superparamagnetic iron oxide nanoparticles (SPIOs). MPI has shown promise for sensitive ( $\sim 200$ cell), long term tracking of stem cells $[1,2]$. White blood cell (WBC) tracking using scintigraphy has traditionally allowed for the clinical diagnosis of inflammation and is ordered in cases of fever-of-unknown-origin $[3,4]$. Recently, antibody based immunotherapeutic approaches have been developed to selectively modulate immune cells, e.g. for cancer treatment [5].

Tracking WBCs in the tumor microenvironment, especially specific phenotypes, has proved crucial to monitoring and predicting outcomes of immunotherapy [6]. Objective: We developed the first in vivo approach for the in situ labelling and tracking of neutrophils with MPI for a radiation-free alternative to traditional WBC scans. The Ly6 family of glycosylphosphatidylinositol-linked proteins has been implicated in neutrophil migration and recruitment. Anti-Ly6 antibodies have been used at high concentration in mice to deplete neutrophils from circulation [5]. Fluorescent conjugated Anti-Ly6 antibodies have also been used to track neutrophils [7]. We utilized Anti-Ly6G antibodies conjugated to SPIOs for in situ labelling of WBCs in mice for MPI-based WBC tracking. Methods: Inflammation was induced in a C57BL6 mouse (7-8 wks) by injecting lipopolysaccharide (LPS) (O111:B4, $50 \mathrm{ug}$ ) in the right thigh. The biodistribution was imaged after i.v. tail vein administration of Anti-Ly6G antibody (IgG1, REA526 clone, Miltenyi Biotec, GmBH) SPIOs (6-6.5 mg Fe/kg, 40 ug of protein/mouse) using a $6.3 \mathrm{~T} / \mathrm{m}$ field-free line MPI scanner (field-of-view $=10.1 \times 4.7 \mathrm{~cm} 2$, tscan $=15 \mathrm{~min})$. The SPIOs were also injected in another healthy C57BL6 mouse and scanned. The inflamed mouse had neutrophil activity validated with a bioluminescence scan using an i.p. injection of luminol (XenoLight RediJect Inflammation Probe, Perkin Elmer) [8], imaged in an IVIS Lumina (Xenogen, Perkin Elmer). A high boost-filter was applied to the MPI scans to improve conspicuity. Separately, the Anti-Ly6G-Ab-SPIOs were physically and magnetically characterized using a vibrating sample magnetometer (Lakeshore Cryotronics), transmission electron microscope (JEOL, $80 \mathrm{KeV}$ ) and arbitrary wave relaxometer [9], and compared to Vivotrax (Magnetic Insight), a commercial formulation of ferucarbotran used as a common MPI tracer, to assess magnetic properties and imaging performance. Results: SPIO characterization is shown in Fig 1A-F. The Anti-Ly6G-Ab-SPIOs were larger than than VivoTrax (Fig 1A-B) and showed improved performance in both resolution and signal per $\mathrm{g}$ Fe as compared to VivoTrax (Fig 1C-E). Notably, the resolution of the Anti-Ly6G-Ab-SPIOs was better than Vivotrax (FWHMAnti-Ly6G = $8.8 \mathrm{mT}$, FWHMVivoTrax = $10.4 \mathrm{mT}$ ) despite the Anti-Ly6G-Ab-SPIO's saturation magnetization, an oft-cited metric for high-performing MPI tracers [10], being lower (Msat,Anti-Ly6G = $44 \mathrm{emu} / \mathrm{g}$, Msat,VivoTrax = $131 \mathrm{emu} / \mathrm{g}$ ), Moreover, the Ab-SPIOs show minimal change in sensitivity in whole blood versus in saline as compared to Vivotrax's variation in sensitivity (Fig 1F). In the in vivo experiments, MPI images taken 24-hr post i.v.
\end{abstract}


injection of SPIOs (Fig 1G) showed Anti-Ly6G Ab-SPIO distribution in organs of the reticuloendothelial system (liver, spleen, as well as cranial, lower limb, and pelvic bone marrow) in healthy mice. These results concur with the delayed distribution of labelled WBCs in In111 scans shown in Fig 1J [11]. Fig 1H is an MPI image in which the mouse's right flank is inflamed, and tracer accumulation at the inflamed site appears with high contrast. This image and inflammation were validated with our luminol-based images, showing the myeloperoxidase activity in the inflamed part (Fig 1K). Conclusion: We performed the first WBC tracking MPI study targeting a surface specific ligand on neutrophils and observed homing of labelled neutrophils to inflamed sites, and demonstrated antibody conjugated SPIOs' superior MPI behaviour as compared to VivoTrax. This zero-radiation approach can be invaluable for in situ labelled WBC-MPI scans for inflammation and infection and for optimizing antibody-based cancer immunotherapies.

References: [1] Zheng B et al., Sci Rep 2015; 5: 14055 [2] Zheng B et al., Theranostics 2016; 6(3): 291-301 [3] Lewis SS et al, Open Forum Infect Dis 2014; 1(2): ofu089. [4] Becker W, Eur J Nucl Med 1995; 22(10):1195-211. [5] Bucher KF et al., J Leukoc Biol 2015; 98(3): 365-372 [6] Hegde PS et al, Clin Cancer Res; 22(8); 1865-74 [7] Yip BG and Kubes P, Blood 2013; 121(1); 241-242 [8] Tseng JC and Kung AL, J Vis Exp 2013; (78): e50690 [9] Tay et al., Sci Rep 2016; 6: 34180 [10] Khandar AP et al., Biomaterials 2013; 34(15): 3837-3845 [11] Love C et al., J Nucl Med Technol 32(2): 47-57

\section{Image/Figure:}

https://www.xcdsystem.com/wmis/abstract/File6959/GA344_ImageFigure_0613074836.png

Image/Figure Caption: Figure 1: SPIO Characterization Representative transmission electron microscopy $($ TEM) images (scale bar $=50 \mathrm{~nm}$ ) were taken of both $(\mathrm{A})$ VivoTrax (VT) with core diameter $=5.4 \pm 1.4 \mathrm{~nm}$ and (B) Anti-Ly6 SPIOs with core diameter $=14.0 \pm 2.6 \mathrm{~nm}$. (C) The magnetization curve of VT and the Anti-Ly6G SPIOs as measured in a vibrating sample magnetometer was fit to a log-normal diameter distribution (Anti-Ly6G SPIO diameter mean $12.6 \mathrm{~nm}$, with log-normal standard deviation of 1.1, and VT diameter mean 8.6, log-normal standard deviation of 14) Notably, VT has a saturation magnetization that is 3 times that of the Anti-Ly6G particles. We used a homebuilt relaxometer to measure the MPI point spread function (PSF) of (D) VT and (E) Anti-Ly6G SPIOs in saline and in blood, normalized to the maximum MPI signal per $\mathrm{g} F$ fe from both particles. Note the solvent-dependent blur in the PSF of VT in comparison unchanging behaviour of the Anti-Ly6G SPIOs, as well the Anti-Ly6G SPIO's superior full width half max and signal. (F) A standard curve of MPI signal was taken versus iron concentration for all particles in mouse blood and in saline. Both particles show linearity with concentration (R2 > 0.99). VT showed significant ( $p 0.5)$ MPI images of in situ labeled neutrophils using anti-Ly6G iron oxide tracers and reference images from other modalities. $(\mathrm{G})$ The tracer signal reveals the distribution of neutrophils in the myeloid cells of the bone marrow, and the RES organs of liver and spleen. (H) MPI images of a C57BL6 mouse with inflamed right flank. Images 24-hours post anti-Ly6G tracer administration revealed the site of inflammation with high contrast - note the asymmetric signal in the flank of the mouse. (J) A healthy human WBC scan for comparison with $(\mathrm{G})$ - note the uptake in the organs of the reticuloendothelial system (bone marrow, liver, spleen) (adapted from Love and Palestro J. Nucl. Med. Tech. 2004) (K) A bioluminescence image of an inflamed mouse with an i.p injection of luminol, a marker of 
neutrophil myeloperoxidase activity, co-registered to a representative x-ray. Note the strong luminescence in the inflamed right flank, similar to $(\mathrm{H})$.

Full Name of Abstract's 1st Author : King Long Barry Fung

Complete Status: Complete

First Name: K. L. Barry

Last Name: Fung

Email: barry.kl.fung@berkeley.edu

Organization: University of California, Berkeley

Country: United States 


\title{
ID: GA345 \\ Brain pharmacokinetics of bispecific antibody-based radioligands is size- dependent
}

\author{
Rebecca Faresjö, Uppsala University, rebecca.faresjo@gmail.com
}

\section{Category: Neuroscience}

\begin{abstract}
Body : Introduction: If engineered to enter the brain, antibodies with high specificity to intra-brain proteins, for example amyloid-beta $(\mathrm{A} \beta)$, can be used in positron emission tomography (PET) to study diseases of the brain, such as Alzheimer's disease. Previously we have developed bispecific antibodies, utilising the mouse transferrin receptor as a transport shuttle, with high brain uptake1. These bispecific antibodies are large constructs with long residence time in blood, which is not optimal for PET. Here we have developed a smaller bispecific antibody-construct with improved faster blood clearance. However, knowledge of brain clearance time is lacking. The aim was to study brain pharmacokinetics of bispecific antibody-based ligands. Methods: Bispecific antibody ligands mAb3D6-scFv8D3 (210 kDA) and di-scFv3D6-8D3 (58 kDa) were expressed in Expi293-cells and purified by affinity chromatography. The ligands were 125I-labelled and each of them administered i.v. to wild-type mice (C57/B16). Brains were isolated 2h-24h post-injection, ligand uptake was measured with a gamma counter, then frozen. Brains were sectioned sagittaly and exposed to a phosphor screen for 7 days. Results: [125I]mAb3D6-scFv8D3 displayed slow kinetics, brain concentrations were high and stable for $8 \mathrm{~h}$ before elimination became apparent, while the smaller [125I]di-scFv3D6$8 \mathrm{D} 3$ started to be cleared from the brain earlier, at $4 \mathrm{~h}$ post administration. Retention and elimination phases of [125I]mAb3D6-scFv8D3 were confirmed by autoradiography (Fig. 1). Brain-to-blood ratio was 1.5-fold higher 12h after administration of [125I]di-scFv3D6-8D3 than for [125I]mAb3D6-scFv8D3 and the difference increased with time. Conclusions: Nonspecifically bound small non-IgG constructs (e.g. di-scFv3D6-8D3) clear faster from brain than larger conventional IgG-like constructs. This is likely to improve the specific-to-nonspecific signal, potentially allowing for fluorine-18 radiolabeling.
\end{abstract}

References: Sehlin D, Fang XT, Cato L, Antoni G, Lannfelt L, Syvänen S. Nat Commun. 2016;7:10759. doi:10.1038/ncomms 10759

\section{Image/Figure:}

https://www.xcdsystem.com/wmis/abstract/File6959/GA345 ImageFigure 0613013508.jpg

Image/Figure Caption: Fig.1: Sagittal brain sections of wild-type mice, euthanized at $2 \mathrm{~h}, 4 \mathrm{~h}$, $6 \mathrm{~h}, 8 \mathrm{~h}, 10 \mathrm{~h}, 12 \mathrm{~h}, 16 \mathrm{~h}$ or $24 \mathrm{~h}$ after administration of A) [125I]mAb3D6-scFv8D3 and B) [125I]di-scFv3D6-8D3.

Full Name of Abstract's 1st Author : Rebecca Faresjö 
Complete Status: Complete

First Name: Rebecca

Last Name: Faresjö

Email: rebecca.faresjo@gmail.com

Organization: Uppsala University

Country: Sweden 


\title{
ID: GA346 \\ Evaluation of fluorescently-labeled HN17 as a bacterial biofilm specific imaging agent
}

\author{
Landon Locke, The Ohio State University, Landon25@gmail.com
}

Category: Immunology: Inflammation \& Infection

\begin{abstract}
Body : Introduction: The clinical management of bacterial biofilm infections represents an enormous challenge in today's healthcare setting. The NIH estimates that at least two-thirds of bacterial infections are biofilm related (1). Early treatment can mitigate biofilmassociated recalcitrance to antibiotics (2); however, this relies on accurate detection, and there is no imaging technique to detect biofilms in vivo. Through optical-based screens, we identified a small, lipophile-enhanced peptide, (4Iph)(f)LPNSNHIKQGL (HN17), that targets the biofilm matrix that makes up $\sim 80 \%$ of biofilm volume (3). We previously established that HN17-Cy5 rapidly penetrated $\mathrm{P}$. aeruginosa biofilms and maintained biofilm selectively in epithelial cell infection models. Here, we expanded in vitro testing of HN17-Cy5 using a human primary neutrophil infection model and evaluated HN17-Cy5 in vivo in a biofilm-infected wound model. Materials and Methods: HN17 was synthesized by solid state methods at a purity of $>90 \%$ and labeled with Cy5. Imaging flow cytometry was used to examine HN17-Cy5 interaction with human primary neutrophils infected or not with GFP-expressing P. aeruginosa $(\mathrm{Pa}$; strain
\end{abstract} PAO1). Neutrophils were infected with Pa at an MOI of 5:1, washed, and incubated with HN17Cy5 $(2 \mu \mathrm{M})$ or PBS as a control for $20 \mathrm{~min}$ at $37^{\circ} \mathrm{C}$ with gently rocking. Following 2 additional washes, neutrophils were fixed and analyzed on an ImageStreamX Mark II (Amnis Corporation). Data for 10,000 cells were recorded for each sample with live/dead stain, GFP, and HN17-Cy5 fluorescence analyzed for each cell along with 60x brightfield images. Two replicate experiments were conducted using neutrophils from two different donors. Data were analyzed using IDEAS v6.0 software. In vivo biofilm targeting of HN17-Cy5 was evaluated using a biofilm-infected wound model. Briefly, wounds of 4 mice were infected with an inoculum of 107 cells consisting of a 50:50 mixture of lux-tagged and TdTomato-expressing Pa. $24 \mathrm{~h}$ after wound inoculation, mice were administered 60 nmoles of HN17-Cy5 via the tail vein. Control groups include $\mathrm{n}=3$ mice that were mock infected in both wounds and injected with 60 nmoles of HN17$\mathrm{Cy} 5$ and $\mathrm{n}=2$ mice that were infected with Pa but injected with PBS. At 6 and $18 \mathrm{~h}$ after probe injection, live whole-animal bioluminescence and fluorescence imaging was performed on an IVIS Lumina II optical imaging system to assess bacterial burden and HN17-Cy5 distribution. Region of interest analysis was performed using Living Image 4.4 software (Caliper Life Sciences, Hopkinton, MA). Following imaging, mice were euthanized and tissues harvested, fixed, and sectioned. Confocal laser scanning microscopy (CLSM) of the sectioned tissue was performed on the Olympus Fluoview 3000 confocal microscope using a 60x oil objective (N.A. 1.4). Images were processed using the Olympus Fluoview software. Results: HN17-Cy5 targeting, as measured by mean fluorescence intensity, was significantly higher in the Painfected compared to uninfected neutrophils $(\mathrm{p}=0.008)$. Discrete punctate spots of HN17-Cy5 fluorescence were apparent in the vast majority of infected neutrophils, though intracellular colocalization between HN17-Cy5 and GFP was lo $<(<0.2)$. In vivo, wound ROI analysis 
showed that HN17-Cy5 localized to biofilm-infected wounds 3.2 times greater than surrounding tissue and showed significantly higher uptake in biofilm-infected wounds compared to uninfected wounds at $18 \mathrm{~h}<(\mathrm{p}<0.001)$. Ex vivo CLSM imaging of wound tissue revealed punctate HN17-Cy5 signals that co-localized with P. aeruginosa. Minimal Cy5 fluorescence was observed in uninfected wound tissue. Conclusion: Bacteria encased within a self-produced matrix known as biofilms cause devastating, chronic infections. Through optical screens, we identified a small peptide (HN17) that specifically targeted P. aeruginosa biofilms in mammalian cell infection models and showed enhanced accumulation in biofilm-infected tissues in vivo. HN17 represents a novel approach to imaging biofilms and additional studies are warranted to determine pathogen range and targeting mechanism.

References: 1. Boisvert AA, Cheng MP, Sheppard DC, Nguyen D. Microbial Biofilms in Pulmonary and Critical Care Diseases. Ann Am Thorac Soc 2016;13:1615-23. 2. Ciofu O, RojoMolinero E, Macia MD, Oliver A. Antibiotic treatment of biofilm infections. APMIS 2017;125:304-19. 3. Flemming HC, Wingender J. The biofilm matrix. Nat Rev Microbiol 2010;8:623-33.

\section{Image/Figure:}

https://www.xcdsystem.com/wmis/abstract/File6959/GA346 ImageFigure 0614092223.jpg

Image/Figure Caption: HN17-Cy5 localizes to biofilm-infected wounds in vivo. Equally scaled fluorescence images overlaid on photographs showing increased HN17-Cy5 localization to biofilm-infected (A) vs. uninfected wounds (B). ROI analysis showed that HN17-Cy5 localized to biofilm-infected wounds 3.2 times greater than surrounding tissue and showed significantly higher uptake in biofilm-infected wounds compared to uninfected wounds $(C ; p<0.001)$. Ex vivo confocal microscopy imaging showing HN17-Cy5 (D3) co-localized with TdTomatoexpressing P. aeruginosa biofilms (D2) while not tracking with host cells (D1; stained with DAPI). Merged channels are shown in D4.

Full Name of Abstract's 1st Author : Landon Locke

Complete Status: Complete

First Name: Landon

Last Name: Locke

Email: Landon25@gmail.com

Organization: The Ohio State University

Country: United States 


\title{
ID: GA348 \\ 68Ga-SiO2 radiotracers for Positron Emission Particle Tracking (PEPT)
}

\author{
Juan Pellico, King's College London, juan.pellico@kcl.ac.uk
}

\section{Category: New Chemistry, Biology \& Bioengineering}

\begin{abstract}
Body : Introduction: Positron Emission Particle Tracking (PEPT) is based on the accurate localisation of a single labelled particle using a pair of back-to-back $\gamma$-rays generated by the annihilation of a positron.1 Our aim is to explore the use of PEPT for biomedical applications, however, there is a lack of research in particulates that allow the high specific activities $(0.5-2 \mathrm{MBq} /$ particle $)$ in the required size range $(<50 \mu \mathrm{m}) .2$ Whilst the radiolabelling of macroscopic materials provide isolated particles with high activity, the decrease in particle size brings associated a significant reduction in the activity per particle. Most importantly, there are no optimised protocols to isolate a single radiolabelled microparticle below $50 \mu \mathrm{m}$. In this work, a series of $\mathrm{SiO} 2$ particles ranging from $3 \mu \mathrm{m}$ to $3 \mathrm{~mm}$ were synthesised, radiolabelled with $68 \mathrm{Ga}$, and evaluated as potential PEPT radiotracers. Moreover, flow cytometry was evaluated for particle isolation. Hypothesis: Considering that the radiolabelling of $\mathrm{SiO} 2$ particles with 68Ga is a straightforward and fast protocol with high radiolabelling yields, 3 it seems a suitable candidate to render tracers with the radioactivity per particle required for PEPT. Flow cytometry has potential as the isolation/purification technique of PEPT tracers due to its ability to isolate single cells of the same range of size. Methods: $\mathrm{SiO} 2$ microparticles $(3 \mu \mathrm{m})$ were synthesised using a brief modification of the Stöber method.4 SiO2 particles of 150 - $250 \mu \mathrm{m}$ (Sigma-Aldrich) and 1 - $3 \mathrm{~mm}$ (VWR chemicals) were also used for 68Ga labelling. Radiolabelling: Aliquots of $68 \mathrm{GaCl} 3$ were adjusted to $\mathrm{pH}=5.2$ with HEPES buffer. The particles suspended at different concentrations were added over the $68 \mathrm{Ga}$ solution. The mixture was heated at $90^{\circ} \mathrm{C}$ for $30 \mathrm{~min}$. Isolation: $3 \mu \mathrm{m}$ particles at $0.25 \mathrm{mg} / \mathrm{mL}$ were isolated using a FACS MelodyTM. Sorting was carried out using a 6-well plate and a microcentrifuge tube holder independently. Results: Radiolabelling yields of $3 \mu \mathrm{m}$ particles were very high $(96 \%)$ even at a low concentration such as $2 \mu \mathrm{g} / \mathrm{mL}$ (Figure 1a). 1 - $3 \mathrm{~mm}$ and 150 - $250 \mu \mathrm{m}$ particles demonstrated high radiolabelling yields, 84 - 86\% (Figure 1b). Strating from approximately $90 \mathrm{MBq}$, remarkable specific activities of $8.2 \pm 1.0 \mathrm{MBq} /$ particle was obtained for $1-3 \mathrm{~mm} \mathrm{SiO} 2$ and 1.4 $\pm 0.3 \mathrm{MBq} /$ particle for $150-250 \mu \mathrm{m} \mathrm{SiO} 2$ (Figure 1c). 1 - $3 \mathrm{~mm}$ particles showed a high radiochemical stability in highly ionic aqueous solutions of $99.0 \pm 0.5 \%$ at $5 \mathrm{~min}$ postincubation, remaining almost identical after 2 hours $(99.2 \pm 0.5 \%)$. Moreover, $150-250 \mu \mathrm{m}$ particles demonstrated a radiochemical stability of $95.1 \pm 0.4 \%$ after 5 min of incubation increasing to $99.3 \pm 0.6 \%$ for 2 hours (Figure 1d). Finally, FACS sorting was used to isolate $3 \mu \mathrm{m} \mathrm{SiO} 2$ particles. The images showed good isolation capabilities for single particles, whilst aggregation was evident for 20 and 50 particles after sorting in a 6-well plate (Figure 1e). To solve this issue, sorting was carried out in a microcentrifuge tube with $20 \mu \mathrm{L}$ of $\mathrm{H} 2 \mathrm{O}$. After setting up a 10 particles sorting, images showed no aggregation with 8 spherical particles of homogeneous shape and size (Figure 1f). Conclusions: 68Ga-SiO2 particles have been evaluated as potential PEPT tracers. The larger $\mathrm{SiO} 2$ particles showed unprecedent specific activities suitable for PEPT applications. For the small particles, specific activities are close to those
\end{abstract}


required and optimisation of the radiochemistry protocol should allow for improvements. Noteworthy, a fresh generator could render required activities. Preliminary results show the potential of FACS for the isolation of single particles. Acknowledgements: This work was funded by the UK's Engineering and Physical Sciences Research Council (EPSRC) under the programme grant EP/R045046/1 "Probing Multiscale Complex Multiphase Flows with Positrons for Engineering and Biomedical Applications"

References: 1. D. J. Parker, C. J. Broadbent, P. Fowles, M. R. Hawkesworth and P. McNeil, Nucl. Instruments Methods Phys. Res. Sect. A Accel. Spectrometers, Detect. Assoc. Equip., 1993, 326, 592-607. 2. D. J. Parker and X. Fan, Particuology, 2008, 6, 16-23. 3. T. M. Shaffer, M. A. Wall, S. Harmsen, V. A. Longo, C. M. Drain, M. F. Kircher and J. Grimm, Nano Lett., 2015, 15, 864-868. 4. X. Lei, B. Yu, H.-L. Cong, C. Tian, Y.-Z. Wang, Q.-B. Wang and C.-K. Liu, Integr. Ferroelectr., 2014, 154, 142-146.

\section{Image/Figure:}

https://www.xcdsystem.com/wmis/abstract/File6959/GA348 ImageFigure 0605093534.png

Image/Figure Caption: Figure 1. a) Radiolabelling yields of $3 \mu \mathrm{m} 68 \mathrm{Ga}-\mathrm{SiO} 2$ particles at different particle concentrations ( $\mathrm{mg} \mathrm{SiO} 2 / \mathrm{mL}, \mathrm{b}$ ) Radiolabelling yield of $1-3 \mathrm{~mm} 68 \mathrm{Ga}-\mathrm{SiO} 2$ and $150-250 \mu \mathrm{m} 68 \mathrm{Ga}-\mathrm{SiO} 2$ particles after three different repetitions, c) Specific activity (MBq/particle) for 1-3 mm 68Ga-SiO2 and 150-250 $\mu \mathrm{m} 68 \mathrm{Ga}-\mathrm{SiO} 2$ particles after three different repetitions, d) Radiochemical stability of 1-3 mm 68Ga-SiO2 and 150-250 $\mu \mathrm{m} 68 \mathrm{Ga}-\mathrm{SiO} 2$ particles from $5 \mathrm{~min}$ to $120 \mathrm{~min}$ after heavy stirring in $5 \% \mathrm{NaCl}$, e) Optical microscope images taken after the sorting of 1, 20 and $50 \mathrm{SiO} 2(3 \mu \mathrm{m})$ particles in a 6-well plate, f) Optical microscope image of the Neubauer chamber after the sorting of $10 \mathrm{SiO} 2(3 \mu \mathrm{m})$ particles.

Complete Status: Complete

First Name: Juan

Last Name: Pellico

Email: juan.pellico@kcl.ac.uk

Organization: King's College London

Country: United Kingdom 


\section{ID: GA351}

Deep learning Radiomics for ultrasound images: interpret spatio-temporal signatures for quantitative decision-making

Kun Wang, Chinese Academy of Sciences, kunwang@nlpr.ia.ac.cn

Complete Status: Complete

First Name: Kun

Last Name: Wang

Email: kunwang@nlpr.ia.ac.cn

Organization: Chinese Academy of Sciences 


\section{ID: GA352}

Optically Modulatable Contrast Agents for Background-Free Photoacoustic and Ultrasound Imaging

Stanislav Emelianov, Georgia Tech, stas@gatech.edu

Complete Status: Complete

First Name: Stanislav

Last Name: Emelianov

Email: stas@gatech.edu

Organization: Georgia Tech

Country: United States 
ID: GA353

Shedding Light on Tumour Oxygenation Using Photoacoustic Imaging

Sarah Bohndiek, University of Cambridge, seb53@cam.ac.uk

Complete Status: Complete

First Name: Sarah

Last Name: Bohndiek

Email: seb53@cam.ac.uk

Organization: University of Cambridge

Country: United Kingdom 


\section{ID: GA354}

Role of Imaging in Response Assessment in Immune-oncology

Elisabeth GE de Vries, University Medical Center Groningen (UMCG), the Netherlands, e.g.e.de.vries@umcg.nl

Complete Status: Complete

First Name: Elisabeth GE

Last Name: de Vries

Email: e.g.e.de.vries@umcg.nl

Organization: University Medical Center Groningen (UMCG), the Netherlands

Country: Netherlands 
ID: GA355

Noninvasive monitoring of brain tumors using serum, CSF, and imaging biomarkers.

Elizabeth Gerstner, Massachusetts General Hostpial, EGERSTNER@mgh.harvard.edu

Complete Status: Complete

First Name: Elizabeth

Last Name: Gerstner

Email: EGERSTNER@mgh.harvard.edu

Organization: Massachusetts General Hostpial

Country: United States 


\section{ID: GA356}

CRISPR Genome Imaging in Human Cells

John Ronald, Robarts Research Institute, jronald@robarts.ca

Complete Status: Complete

First Name: John

Last Name: Ronald

Email: jronald@robarts.ca

Organization: Robarts Research Institute

Country: Canada 


\section{ID: GA357}

Imaging in the development of cell therapy

Ajay Verma, Codiak Biosciences, ajay.verma@codiakbio.com

Complete Status: Complete

First Name: Ajay

Last Name: Verma

Email: ajay.verma@codiakbio.com

Organization: Codiak Biosciences

Country: United States 


\section{ID: GA358}

PET Imaging at the Academic and Industry Interface. What's working well, and where can we improve?

Peter Scott, University of Michigan, pjhscott@umich.edu

Complete Status: Complete

First Name: Peter

Last Name: Scott

Email: pjhscott@umich.edu

Organization: University of Michigan

Country: United States 


\title{
ID: GA361 \\ A bridging phenyloxadiazolyl methyl sulfone reagent for the site-specific radiolabeling of antibody fragments
}

\author{
GUILLAUME DEWAELE-LE ROI, Memorial Sloan Kettering Cancer Center, \\ dewaeleg@mskcc.org
}

Category: New Chemistry, Biology \& Bioengineering

\begin{abstract}
Body : Over the last twenty years, immunoconjugates have become essential therapeutic and diagnostic tools. However, many antibody-drug conjugates (ADCs) and radioimmunoconjugates are still synthesized via the indiscriminate attachment of cargos (e.g. chelators, fluorophores, or toxins) to lysine residues, a remarkably imprecise approach that limits their overall efficacy. Indeed, these non-site-specific synthetic methods not only produce heterogeneous mixtures of immunoconjugates but also yield constructs with suboptimal immunoreactivity and in vivo performance. To overcome these problems, several "site-specific" bioconjugation strategies have been developed. The most established of these is the use of maleimide-based bifunctional probes for conjugations to cysteine residues. However, the resultant thioether linkages are unstable in vivo both spontaneously (via the retro-Michael reaction) as well as in the presence of competing thiols. In the context of radioimmunoconjugates, this can result in the detachment of radionuclides from the immunoglobulin vector, thereby reducing target-to-background activity concentration ratios and increasing radiation doses to healthy tissues. Herein, we present the synthesis, characterization, and evaluation of DiPODS, a novel bioconjugation reagent containing two oxadiazolyl methyl sulfone moieties. DiPODS irreversibly forms covalent bonds with two thiols, thus facilitating the re-bridging of reduced disulfide linkages in antibodies, antibody fragments, and proteins. DiPODS was synthesized from commercially available starting materials in 8 steps. Subsequently, a fluorescein-labeled derivative of the reagent - DiPODS-FITC - was created to interrogate the performance of DiPODS for the bioconjugation of both isotype-control and HER2-targeting Fab fragments. Ultimately, the modification of a HER2-targeting Fab with DiPODS-FITC produced an immunoconjugate with in vitro performance superior to an analogous construct created using a traditional, lysine-based approach to bioconjugation.
\end{abstract}

References: [1] Radiopharmaceutical Chemistry, 1 ed., Springer International Publishing, Springer Nature Switzerland AG, 2019. [2] a) N. Toda, S. Asano, C. F. Barbas, 3rd, Angew Chem Int Ed Engl 2013, 52, 12592-12596; b) J. T. Patterson, S. Asano, X. Li, C. Rader, C. F. Barbas, 3rd, Bioconjug Chem 2014, 25, 1402-1407. [3] a) P. Adumeau, M. Davydova, B. M. Zeglis, Bioconjug Chem 2018, 29, 1364-1372; b) M. Davydova, L. R. Dewaele, P. Adumeau, B. M. Zeglis, J. Vis. Exp. 2019, 145, e59063 [4] Khozeimeh, E. S.; Dewaele-Le Roi, G.; Shannon, W. E.; Zeglis, B. M. and Price E. W. 'DiPODS: A Reagent for Site-Specific Bioconjugation via the Irreversible Re-bridging of Disulfide Linkages' submitted manuscript, 2020.

\section{Image/Figure:}


Image/Figure Caption: A) Schematic site-specific bioconjugation of a bifunctional DiPODSbearing reagent to proteins via the re-bridging of disulfide linkages. B) Flow cytometry analysis of HER2-positive BT474 human breast cancer cells stained with (left) a site-specifically modified FabHER2-DiPODS-FITC) synthesized using DiPODS and (right) a non-sitespecifically modified HER2-targeting immunoconjugate (FabHER2-Lys-FITC) synthesized using a traditional lysine-based conjugation method.

Complete Status: Complete

First Name: GUILLAUME

Last Name: DEWAELE-LE ROI

Email: dewaeleg@mskcc.org

Organization: Memorial Sloan Kettering Cancer Center

Country: United States 


\title{
ID: GA362 \\ Dependence of Magnet Field Gradient on Spatial Resolution in MPI with Field- Free line
}

\author{
Kota Nomura, Mitsubishi Electric Corp., Nomura.Kota@cw.MitsubishiElectric.co.jp
}

Category: Instrumentation

\begin{abstract}
Body : Introduction: Magnetic Particle Imaging (MPI) is an imaging modality that directly detects the nonlinear response of magnetic nanoparticles (MNPs). Spatial encoding is realized by saturating MNPs almost everywhere except in the vicinity of a special point called the field-free point (FFP) using a static magnetic field[1]. Recently, It has been shown that the sensitivity of MPI can be significantly improved using a simultaneous encoding scheme by scanning the FOV with a field-free line (FFL) instead of FFP [2][3]. We have developed the MPI with FFL device that can change magnetic field gradient using a neodymium magnet and an iron yoke(Fig.1a). We study of the influence magnetic field gradient of FFL on imaging quality. Methods: The magnet for FFL was designed using magnetic field analysis so that magnetic field gradient changed from 1.0 to $3.0 \mathrm{~T} / \mathrm{m}$ while the distance of the pair of magnets(Fig. $1 \mathrm{~b}$ ).An oscillating magnetic field was generated using an excitation coil (pair of solenoid coils). The frequency and peak-to-peak strength of the oscillating magnetic field were taken as $500 \mathrm{~Hz}$ and $30 \mathrm{mT}$, respectively. The signal generated by MNPs was received by a gradiometer coil and the third harmonic signal was amplified by a lock-in amplifier. We used Resovist ${ }^{\circledR}$ (Fujifilm RI Pharma) as MNPs. Resovist ${ }^{\circledR}$ is an organ-specific contrast agent for magnetic resonance imaging and consists of superparamagnetic iron oxide (magnetite, $\gamma$-Fe2O3) coated with carboxydextran. The spatial resolution by the gradient magnetic field strength was evaluated using a cylindrical sample of $\varphi 5 \mathrm{~mm}$ and a volume of $0.15 \mathrm{~mL}$ (Fig. 1c). The spatial resolution was quantified by the full-width-at-half-maximum (FWHM) of the signal intensity. Results: Fig.1d shows the measurement results of the signal intensity distribution with various magnetic field gradient. It is shown that the higher magnetic field gradient, the better the spatial resolution. It is also shown that the signal intensity decreases as magnetic field gradient increases (Fig.1e). This indicates that the region of magnetically saturated MNPs increases with increasing magnetic field gradient. We will discuss the relationship between magnetic field gradient distribution of FFL and MPI signal. This research was supported by AMED under Grant Number JP19hm0102073h0001
\end{abstract}

References: [1] B.Gleich and J.Weizenecker (2005). "Tomographic imaging using the nonlinear response of magnetic particles". Nature, 435(7046):1214-1217. [2] J. Weizenecker, B.Gleich and J.Borgert(2009). "Magnetic particle imaging using a field free line". J.Phys. D41:105009. [3] K. Murase, S.Hiratsuka, R.Song, and Y.Takeuchi(2014). ” Development of a system for magnetic particle imaging using neodymium magnets and gradiometer". JJAP.53.067001

\section{Image/Figure:}


Image/Figure Caption: Figure 1. (a) Magnetic field analysis results of FFL and magnets to make FFL. (b) Magnetic field gradient at the center relative to the distance between the magnets (c) Resovist ${ }^{\circledR}$ encapsulated cylinder sample. (d) Spatial distribution of magnetic signals from MNPs measured by scanning FFL.FWHM of detected signal intensity distribution for magnetic field gradient. (e) Signal intensity distribution measured by scanning FFL.

Complete Status: Complete

First Name: Kota

Last Name: Nomura

Email: Nomura.Kota@cw.MitsubishiElectric.co.jp

Organization: Mitsubishi Electric Corp.

Country: Japan 


\title{
ID: GA365 \\ Dual Tracers of 18F-FES and 18F-FDG for Prediction of Progression-Free Survival After Fulvestrant Therapy in Patients with HR+/HER2- Metastatic Breast Cancer
}

\author{
Cheng Liu, Fudan University Shanghai Cancer Center, liucheng1226@126.com
}

\section{Category: Oncology}

\begin{abstract}
Body : Purpose: Heterogeneity of estrogen receptor (ER) expression may be an essential predictor of endocrine therapy failure and drug resistance in ER-positive metastatic breast cancer (MBC). The purpose of this study was to use dual tracers 18F-fluoroestradiol (18FFES) and 18F-fluorodeoxyglucose (18F-FDG) as imaging biomarkers in predicting progressionfree survival (PFS) in ER-positive MBC patients receiving fulvestrant therapy. Methods: We retrospectively analyzed thirty-five HR+HER2- metastatic breast cancer patients who underwent $18 \mathrm{~F}-\mathrm{FES}$ PET/CT and 18F-FDG PET/CT scans prior to $500 \mathrm{mg}$ fulvestrant therapy in our centre. The maximum of standard uptake value (SUVmax) across all metastatic lesions on the baseline PET/CT were assessed. The heterogeneity of ER expression was assigned by presence of $18 \mathrm{~F}-$ FES negative lesions, for patients with 100\% 18F-FES positive lesions were categorized into two groups by the median ratio of FES/FDG SUVmax, the FES/FDG and high FES/FDG. PFS were estimated by the Kaplan-Meier method and compared by the log-rank test. Univariate and multivariate analyses were performed using the Cox proportional hazard model. Results: In total, 235 metastatic lesions were identified in 35 patients. 12 of 35 patients' presence of $18 \mathrm{~F}-\mathrm{FES}$ negative lesion(s), suggesting that heterogeneity of ER expression in metastatic lesions. These patients had a low median PFS of 5.5 months $(95 \%$ CI $2.3-8.7)$. Of patients with $100 \% 18 \mathrm{~F}-\mathrm{FES}$ positive lesions, 11 had a low ratio of FES/FDG and 12 had a high ratio of FES/FDG. These groups had a median PFS of 29.4 months (95\%CI 2.3-56.5) and 14.7 months (95\%CI 10.9-18.5), respectively. The patients were stratified in three categories based on incorporating both $18 \mathrm{~F}-$ FES and 18F-FDG imaging results that were significantly correlated with PFS by univariate analysis $(\mathrm{P} 0.001)$ and multivariate analysis $(\mathrm{P}=0.006)$. Conclusions: 18F-FES and 18F-FDG PET could serve as prognostic imaging biomarkers for ER-positive MBC patients treated with fulvestrant therapy.
\end{abstract}

Complete Status: Complete

First Name: Cheng

Last Name: Liu

Email: liucheng1226@126.com

Organization: Fudan University Shanghai Cancer Center

Country: China 


\section{ID: GA368}

Multi modal/multi resolution tracking oncology targets

Adam Shuhendler, University of Ottawa, Adam.Shuhendler@uottawa.ca

Complete Status: Complete

First Name: Adam

Last Name: Shuhendler

Email: Adam.Shuhendler@uottawa.ca

Organization: University of Ottawa

Country: Canada 


\title{
ID: GA377 \\ PSMA-targeted photodynamic therapy in surgical prostate tumour samples
}

\author{
Melline Schilham, Radboud University Medical Center, melline.schilham@gmail.com
}

\section{Category: Oncology}

\begin{abstract}
Body : Introduction Complete surgical resection of the primary tumour is one of the most important aspects of curative therapy in prostate cancer (PCa) patients. Incomplete resection is associated with recurrent disease and worse prognosis(1). However, too extensive resection may cause damage to vital structures potentially causing post-surgical complications such as incontinence and impotence $(2,3)$. These surgical challenges emphasize the significance of improved intraoperative visualisation of tumour margins and adjuvant ablative therapies. Photodynamic agents have the potential of combining both fluorescence imaging for imageguided surgery as well as ablation of cancer cells induced by photodynamic therapy (PDT). Labelling prostate specific membrane antigen (PSMA) ligands with a photosensitiser may thus enable intraoperative tumour detection, delineation and tumour-targeted PDT (tPDT)(4). Previously, the PSMA-targeting tPDT ligand PSMA-N064 was developed and evaluated. To make the next translational step before clinical implementation, the present study aims to evaluate the potential for TPDT in an ex vivo incubation study on surgically obtained human PCa samples. Methods The PSMA-N064 peptide consists of a PSMA binding motif, a DOTA chelator and the photosensitizer IRDye700DX. In this study, PSMA-N064 is used to investigate the therapeutic effect of tPDT on human tumour and normal prostate tissue. We aim to include $25 \mathrm{PCa}$ patients scheduled for radical prostatectomy for obtaining prostate tissue samples. The samples of the first ten patients will be used for protocol optimisation. Immediately after resection of the prostate, four samples are taken from the tumour and one from contralateral normal prostate tissue. The samples are incubated with $0.05 \mathrm{nmol} / \mathrm{ml}$ of the ligand in binding buffer (or binding buffer only, for the control) for 2-4 hours at 37 degrees Celsius. After washing, fluorescence flatbed scanning is used to evaluate tissue distribution of the ligand. Three out of five samples are illuminated with $690 \mathrm{~nm}$ light $(50-150 \mathrm{~J} / \mathrm{cm} 2$ at a fluence rate of 450 $\mathrm{mW} / \mathrm{cm} 2$ ) using a light emitting diode. $4-24$ hours later, the samples are fixated in $4 \%$ formalin, embedded in paraffin, sectioned ( $4 \mu \mathrm{m}$ slices) and stained (HE and PSMA expression). The therapeutic effect of tPDT on cells is evaluated by immunohistochemistry for cleaved-caspase-3 and $\gamma$-H2AX to assess apoptosis and double-strand DNA breaks (DSB), respectively. Viability of the fully treated tumour sample (tPDT-tumour) is compared to the fully treated normal prostate sample (tPDT-normal) and control tumour samples that receive either NIR-light irradiation only (control-1), ligand incubation only (control-2) or no treatment at all (control-3). Results Thus far, we obtained and treated samples from two patients. Macroscopic fluorescence imaging showed favourable accumulation of the ligand in tumour tissue samples (mean fluorescence intensity (MFI) $65296 \pm 51284$ ) compared to normal prostate tissue samples (MFI $5391 \pm 2860$ ), indicating a specific uptake of the ligand in tumour tissue. Preliminary results of cleavedcaspase- 3 and $\gamma-\mathrm{H} 2 \mathrm{AX}$ stainings suggested more profound apoptosis and DSB in the treated tumour samples compared to controls. However, NIR-light alone also seemed to increase apoptosis and DSB compared with the non-treated tumour sample (Figure 1). Additional
\end{abstract}


apoptosis assays (e.g. TUNEL) will be performed and these results will be used to further optimise the protocol, i.e. duration of ligand incubation, washing steps, fluence and fluence rate of light delivery. After the optimal protocol has been determined, we will include and analyse samples from 15 additional patients. The results of this evaluation will be presented on the WMIC-2020 upon acceptation. Conclusion Although further evaluation of the results has to be completed, preliminary results of our study suggest an effect of tumour-targeted PDT using IRDye700DX-conjugated PSMA-N064 on human PCa-samples. In the future, this ligand may be of value in intraoperative tumour detection and tPDT by eradication of unresectable tumour rests and thus improving surgical outcomes.

References: 1. Wright JL, Dalkin BL, True LD, Ellis WJ, Stanford JL, Lange PH, et al. Positive surgical margins at radical prostatectomy predict prostate cancer specific mortality. The Journal of urology. 2010;183(6):2213-8. 2. Bellangino M, Verrill C, Leslie T, Bell RW, Hamdy FC, Lamb AD. Systematic Review of Studies Reporting Positive Surgical Margins After Bladder Neck Sparing Radical Prostatectomy. Curr Urol Rep. 2017;18(12):99. 3. Shikanov S, Woo J, AlAhmadie H, Katz MH, Zagaja GP, Shalhav AL, et al. Extrafascial versus interfascial nervesparing technique for robotic-assisted laparoscopic prostatectomy: comparison of functional outcomes and positive surgical margins characteristics. Urology. 2009;74(3):611-6. 4. Lutje S, Heskamp S, Franssen GM, Frielink C, Kip A, Hekman M, et al. Development and characterization of a theranostic multimodal anti-PSMA targeting agent for imaging, surgical guidance, and targeted photodynamic therapy of PSMA-expressing tumors. Theranostics. 2019;9(10):2924-38.

\section{Image/Figure:}

https://www.xcdsystem.com/wmis/abstract/File6959/GA377 ImageFigure 0430044547.jpg

Image/Figure Caption: Figure 1. Increased staining of apoptotic and DSB markers as a result of tPDT after incubation with PSMA-N064 on human prostate cancer samples. (A) Microscopic images of $4 \mu \mathrm{m}$ slice-sections in different samples (tPDT-tumour, tPDT-normal and control-1) stained with HE, PSMA, cleaved caspase- 3 and $\gamma$-H2AX from patient 2 are shown. tPDT-tumour and control-1 were taken from prostate tumour during surgery. tPDT-normal was taken from the contralateral presumed healthy prostate lobe. tPDT-tumour and tPDT-normal were incubated with the PSMA-N064 ligand, which contains the IRDye700DX photosensitiser. Control-1 received irradiation only. Both tumour samples (tPDT-tumour and control-1) show increased PSMA-expression compared to tPDT-normal. All depicted samples received NIR-light irradiation. The apoptotic effect of tPDT on cells is demonstrated by cleaved-caspase-3 and DNA damaging effect (DSB) by $\gamma$-H2AX staining. Increased staining in tPDT-tumour indicates favourable therapeutic effects induced by PSMA-N064. (B) Macroscopic fluorescence imaging of biopsy cores from patient 2 demonstrate the tissue distribution of the ligand in PSMAoverexpressing tissue (tPDT-tumour) compared to healthy prostate tissue (tPDT-normal).

\section{Complete Status: Complete}

First Name: Melline

Last Name: Schilham 
Email: melline.schilham@gmail.com

Organization: Radboud University Medical Center

Country: Netherlands 


\section{ID: GA378}

Single-cell analysis of the tissue microenvironment: the impact of machine learning on cell segmentation

Andrew Quong, Fluidigm, andrew.quong@fluidigm.com

Complete Status: Complete

First Name: Andrew

Last Name: Quong

Email: andrew.quong@fluidigm.com

Organization: Fluidigm

Country: United States 
ID: GA379

Bridging data across spatial and temporal scale in discovery research imaging trials

Matthew Silva, Invicro, LLC, msilva@invicro.com

Complete Status: Complete

First Name: Matthew

Last Name: Silva

Email:msilva@invicro.com

Organization: Invicro, LLC

Country: United States 


\section{ID: GA380}

How Can Molecular Imaging Interrogate Cancer Biology? Illuminating Metabolic Heterogeneity with PET and Big Data

David Lewis, Cancer Research UK Beatson Institute, David.Lewis@glasgow.ac.uk

Complete Status: Complete

First Name: David

Last Name: Lewis

Email: David.Lewis@glasgow.ac.uk

Organization: Cancer Research UK Beatson Institute

Country: United Kingdom 


\section{ID: GA388}

Antibody-based PET imaging in neurodegenerative disorders

Stina Syvänen, Uppsala University, stina.syvanen@pubcare.uu.se

Complete Status: Complete

First Name: Stina

Last Name: Syvänen

Email: stina.syvanen@pubcare.uu.se

Organization: Uppsala University

Country: Sweden 
ID: GA389

Imaging synaptic changes in neurodegenerative diseases using SV2A PET

Richard Carson, , richard.e.carson@yale.edu

Complete Status: Complete

First Name: Richard

Last Name: Carson

Email: richard.e.carson@yale.edu 


\section{ID: GA390}

Target Engagement of Macromolecules

Sridhar Nimmagadda, Johns Hopkins University, snimmag1@jhmi.edu

Complete Status: Complete

First Name: Sridhar

Last Name: Nimmagadda

Email: snimmag1@jhmi.edu

Organization: Johns Hopkins University

Country: United States 


\section{ID: GA393}

New Detection Methods for Simultanous PET, SPECT, CT, and XRF In Vivo Imaging

Ludek Sefc, First Faculty of Medicine, Charles University, sefc@cesnet.cz

Complete Status: Complete

First Name: Ludek

Last Name: Sefc

Email: sefc@cesnet.cz

Organization: First Faculty of Medicine, Charles University

Country: Czech Republic 


\section{ID: GA394}

PETRUS : Positron Emission Tomography Registered Ultrasound

Bertrand Tavitian, University of Paris, bertrand.tavitian@inserm.fr

Complete Status: Complete

First Name: Bertrand

Last Name: Tavitian

Email: bertrand.tavitian@inserm.fr

Organization: University of Paris

Country: France 


\title{
ID: GA395 \\ Subcellular Context in Multi-mm Samples of Functionally-Imaged Mammalian Brain - New Roads to Correlation using X-Rays
}

\author{
Carles Bosch, Francis Crick Institute, Carles.Bosch@crick.ac.uk
}

\section{Category: Neuroscience}

\begin{abstract}
Body : Understanding the mechanism of action of neural circuits in the mammalian brain poses a multi-faceted challenge: entire circuits must be recorded and imaged, so as to simultaneously interrogate their function and their structure. I will present a correlative multimodal imaging pipeline that combines in vivo $\mathrm{Ca} 2+$ imaging of brain function at a cellular scale with synchrotron X-ray microscopy of multi-millimetre samples providing subcellular context with serial block-face electron microscopy of volumes of nearly $1 \mathrm{~mm} 3$. In this approach, $\mathrm{X}$-ray imaging takes a central position and plays a dual role: as a bridge between imaging modalities, and as a source of subcellular context for vast regions of tissue that can inform subsequent detailed EM studies. Ongoing world-wide synchrotron upgrades are expected to further improve the performance of X-ray imaging, and it might reinforce its central position in correlative multimodal imaging pipelines for systems neuroscience.
\end{abstract}

References: Previous published work from myself related to the topic: - Schwarz, D., Kollo, M., Bosch, C., Feinauer, C., Whiteley, I., Margrie, T.W., Cutforth, T., and Schaefer, A.T. (2018). Architecture of a mammalian glomerular domain revealed by novel volume electroporation using nanoengineered microelectrodes. Nature communications 9, 183 - Bosch, C., Masachs, N., Exposito-Alonso, D., Martinez, A., Teixeira, C.M., Fernaud, I., Pujadas, L., Ulloa, F., Comella, J.X., DeFelipe, J., et al. (2016). Reelin Regulates the Maturation of Dendritic Spines, Synaptogenesis and Glial Ensheathment of Newborn Granule Cells. Cereb Cortex 26, 42824298. - Bosch, C., Martinez, A., Masachs, N., Teixeira, C.M., Fernaud, I., Ulloa, F., PerezMartinez, E., Lois, C., Comella, J.X., DeFelipe, J., et al. (2015). FIB/SEM technology and highthroughput 3D reconstruction of dendritic spines and synapses in GFP-labeled adult-generated neurons. Frontiers in neuroanatomy 9, 60. Previous published work from others related to the topic: - Khimchenko, A., Bikis, C., Pacureanu, A., Hieber, S.E., Thalmann, P., Deyhle, H., Schweighauser, G., Hench, J., Frank, S., Muller-Gerbl, M., et al. (2018). Hard X-Ray Nanoholotomography: Large-Scale, Label-Free, 3D Neuroimaging beyond Optical Limit. Adv Sci (Weinh) 5, 1700694. - Briggman, K.L., Helmstaedter, M., and Denk, W. (2011). Wiring specificity in the direction-selectivity circuit of the retina. Nature 471, 183-188. - Denk, W., and Horstmann, H. (2004). Serial block-face scanning electron microscopy to reconstruct threedimensional tissue nanostructure. PLoS biology 2, e329.

Complete Status: Complete

First Name: Carles

Last Name: Bosch 
Email: Carles.Bosch@crick.ac.uk

Organization: Francis Crick Institute

Country: United Kingdom 
ID: GA396

Correlative Imaging using Cryo Super-Resolution Fluorescence Microscopy and Cryo X-ray Tomography

Maria Harkiolaki, Diamond Light Source, maria.harkiolaki@diamond.ac.uk

Complete Status: Complete

First Name: Maria

Last Name: Harkiolaki

Email: maria.harkiolaki@diamond.ac.uk

Organization: Diamond Light Source

Country: United Kingdom 
ID: GA397

Introduction and Opening Remarks

Janet Eary, National Cancer Institute, janet.eary@nih.gov

Complete Status: Complete

First Name: Janet

Last Name: Eary

Email: janet.eary@nih.gov

Organization: National Cancer Institute

Country: United States 
ID: GA398

Quantification of immune cell infiltration in the tumor environment

Mangala Srinivas, Cenya Imaging, Mangala.Srinivas@Radboudumc.nl

Complete Status: Complete

First Name: Mangala

Last Name: Srinivas

Email: Mangala.Srinivas@Radboudumc.nl

Organization: Cenya Imaging

Country: Netherlands 
ID: GA399

Assessment of early responses to immunotherapy using molecular imaging

Anna Wu, City of Hope, awu@coh.org

Complete Status: Complete

First Name: Anna

Last Name: $\mathrm{Wu}$

Email:awu@coh.org

Organization: City of Hope

Country: United States 


\title{
ID: GA400 \\ Imaging the niche: tumour immune interaction in premetastatic tissue priming
}

\author{
Michel Eisenblatter, University Medical Center Freiburg, michel.eisenblaetter@uniklinik- \\ freiburg.de
}

Category: Oncology

\begin{abstract}
Body : The individual prognosis of a cancer patient is, much more than by characteristics of the primary tumour, determined by the fact whether of not metastases have already manifested. Treatment of patients with metastatic disease is considerably more challenging than local tumour therapy. Metastatic spread is not a random process. The distribution of metastases cannot be explained by mechanical properties such as vessel size or integrity alone but is specific for each tumour type. Tumours, by release of cytokines and other factors including exosomes, induce a premetastatic niche in the target tissue of metastasis. The premetastatic niche constitutes an envionment that can ideally host a circulating tumour cell. Protects the cell from anti-tumour immunity and provides it with growth factors. The major players in the niche are immune cells of various sort. And while tumour cells differ wildly, these immune cells are virtually the same across all different cancer types. The key to universal visualisation of the niche in order to unravel the process of metastatic spread for interference at a time, tumour cell seeding has not even happened is thus to address the immune cell components in the niche. Monocyte-specific, antibody-based imaging agents enabled for imaging of tumourinduced immune cell activity and accumulation in different models of metastatic cancer. The effects of the different tumours on target-tissue of metastasis reflected the individual malignant potential and allowed for prediction of the subsequent pattern of metastatic spread. Using this model system, different tumour-host communication media could be assessed for their respective effect. In this context, the distribution and biological effect of exosomes during premetastatic niche establishment could be shown. Imaging premetastatic tissue priming bears the potential to understand how tumours pave the way for systemic disease. Ultimately, these methods can help to make cancer controllable on a long term basis.
\end{abstract}

Complete Status: Complete

First Name: Michel

Last Name: Eisenblatter

Email: michel.eisenblaetter@uniklinik-freiburg.de

Organization: University Medical Center Freiburg

Country: Germany 


\title{
ID: GA402 \\ COX-2 overexpression reduces extracellular matrix (ECM) stiffness in a xenograft model of triple negative breast cancer (TNBC)
}

\author{
Mc Millan Ching, The Johns Hopkins University School of Medicine, mching5@jh.edu
}

\section{Category: Oncology}

\begin{abstract}
Body : Background:Cyclooxygenase-2 (COX-2) is an inducible enzyme that catalyzes the production of prostaglandin E2 (PGE2) as a cellular response to inflammation. COX-2 overexpression has a pleiotropic and multifaceted role in inflammation and carcinogenesis. Upregulation of COX-2/PGE2 is associated with poor prognosis in breast cancer and other malignancies [1]. We previously found that COX-2 downregulation resulted in a significant decrease of collagen 1 (Coll fibers), numbers of cancer associated fibroblasts (CAFs), and metastasis, whereas COX-2 overexpression resulted in a significant increase of Coll fibers, CAFs, and metastasis [2]. Our purpose in this study was to determine if COX-2 driven changes in the ECM, including changes in Coll fibers and their patterns, altered ECM stiffness. Altered ECM stiffness can provide mechanical cues to cancer cells through mechanotransduction pathways to modify migration and invasion. We used our established model of SUM-149 TNBC cells lentivirally transduced to overexpress COX-2 to characterize, for the first time, the effects of COX-2 overexpression on ECM stiffness using a novel microindentation assay. In parallel we are evaluating molecular changes in ECM proteins and proteases - such as fibronectin, kalinin, ADAM, ADAMTS1 - that actively play a dynamic role in the formation and degradation of ECM using immunohistochemistry (IHC), western blot analysis, and ELISA. Methods: Establishment of triple negative SUM-149 human breast cancer cells that stably overexpress COX-2 (SUM-149-COX-2) or with an empty vector (SUM-149-EV) was achieved through cloning and construction of a lentivirus vector expressing the COX-2 gene [2]. These cells were inoculated in the mammary fat pad of severe combined immunodeficient (SCID) mice. Nine weeks post-inoculation, excised xenograft tumors were measured using iNano nanoindenter (Nanomechanics Inc.) to measure tissue elastic modulus (i.e. stiffness). Downstream analyses of collected biological specimens including immunoblotting, immunohistochemistry, serum ELISA assay, and microscopy is ongoing. Indentation results were processed into resulting photomaps and heatmaps of indented regions and tissue stiffness, respectively. Results: Representative photomap and heatmap of tissue stiffness measured in a SUM-149-COX-2 tumor are shown in Figure 1A. Values for multiple indentation points measured per individual tumor and data summarized from elastic modulus-probed tumor tissues of SUM149-EV Control $(n=5)$ vs. SUM149-COX-2 ( $\mathrm{n}=5)$ are displayed in Figure 1B. Discussion: Our indentation data identified, for the first time, a reduction in tumor stiffness with COX-2 overexpression. We are currently evaluating the relationship between differences in tumor tissue stiffness with COX-2 overexpression to molecular changes in ECM proteins such as fibronectin, collagen, kalinin, and ECM proteases such as ADAM and ADAMTS to further understand the role of COX-2 in altering the ECM and tissue stiffness. These insights may lead to an expanded understanding of the role of COX-2 and inflammation in cancer aggression. Acknowledgements: This work was supported by Supported by NIH R35CA209960 and R01CA82337.
\end{abstract}


References: 1. Hoellen F et al., Anticancer research. 2011;31(12):4359-67. 2. Krishnamachary B et al., Oncotarget. 2017;8(11):17981-94.

\section{Image/Figure:}

https://www.xcdsystem.com/wmis/abstract/File6959/GA402_ImageFigure_0614053702.jpg

Image/Figure Caption: Figure 1A: Representative photomap (left) and heatmap (right) of microindentation measurements of a SUM-149-COX-2 tumor Figure 1B: Number of indentation points per individual tumor section (left); data summarized from stiffness-probed tissues of SUM-149-EV Control (n=5) vs. SUM-149-COX-2 (n=5) tumors (right).

Full Name of Abstract's 1st Author : Mc Millan Ching

Complete Status: Complete

First Name: Mc Millan

Last Name: Ching

Email: mching5@jh.edu

Organization: The Johns Hopkins University School of Medicine

Country: United States 


\title{
ID: GA406 \\ Varying CT reconstruction filtering parameters impact on Hounsfield Units
}

\author{
Wendy McDougald, University of Edinburgh, wmedoug2@ed.ac.uk
}

\section{Category: Computational \& Data Science}

\begin{abstract}
Body : Background/objective: Preclinical computed tomography (CT) is a widely used in vivo imaging technique providing anatomical information which is expanding its usefulness as a quantitative imaging tool. The expansion of utilizing CT as a dedicated system and with PET, SPECT or optical will aid with the interpretion and quantitative analysis of multi-modality imaging data sets. For example, cardiovascular research evaluating calcium deposits in vessels use CT Hounsfield Units (HU) to assist in quantifying the level of atherosclerotic plaque burden and vulnerability. Reconstructing CT images using different filters and parameters to improve spatial resolution and signal-to noise ratio is standard procedure. This study focuses on the potential impact reconstruction filters have on measured HU values (high or low pass frequency filters). Materials and Methods: A CT image was acquired of the air/water quality control and the tissue equivalent material (TEM) phantoms on the Mediso nanoPET/CT using a low dose protocol (tube voltage of $25 \mathrm{kVp}$, exposure of $170 \mathrm{~ms}$ and 360 projections, dose Results: As expected, data revealed slight variations across the different applied filters when other parameters remained constant. The greatest HU percent difference measured was between the high pass and low pass filters (Blackman vs Ram-Lak) of 15\% in muscle HU. Otherwise the biases remained Conclusion: Measured HU results for muscle (soft tissue) were the most impacted by varying the reconstruction parameters. Choosing suitable CT reconstruction parameters requires going beyond spatial resolution and noise trade-offs to include considering potential quantitative biases in measured HU. Thus, the choices of reconstruction filtering warrants revisiting not only for improved signal-to-noise ratio but also for HU quantification.
\end{abstract}

\section{Image/Figure:}

https://www.xcdsystem.com/wmis/abstract/File6959/GA406 ImageFigure 0515030711.png

Image/Figure Caption: Figure 1:Tukey box and whisker plots show each material (air, water, lung, muscle and cortical bone) HU measurements. Results represent bias generated by the different parameter variations (slice thickness, pixel size and filter cut-off value) within each filter. Densities for lung, muscle and cortical bone are $0.21,1.06$ and $1.57 \mathrm{~g} / \mathrm{mL}$, respectively. TEM phantom rods are $4 \mathrm{~mm}$ as reported by the manufacturer. A set template volume of interest was used on each CT image for HU extraction in PMOD.

Full Name of Abstract's 1st Author : Wendy McDougald

Complete Status: Complete

First Name: Wendy 
Last Name: McDougald

Email: wmcdoug2@ed.ac.uk

Organization: University of Edinburgh

Country: United Kingdom 


\title{
ID: GA407 \\ In vivo mapping of immune cell infiltration into extracellular matrix hydrogel in a rat model of stroke using $19 \mathrm{~F}$ magnetic resonance imaging.
}

\author{
Michel Modo, University of Pittsburgh, mmm154@pitt.edu
}

\section{Category: Neuroscience}

\begin{abstract}
Body : INTRODUCTION: Magnetic resonance imaging (MRI)-based guidance of extracellular matrix (ECM) hydrogel affords its implantation into a tissue cavity caused by a stroke (1). A major immune response occurs that leads to the biodegradation of the implanted hydrogel and a reconstitution of brain tissue ensues (2). To improve our understanding of the spatio-temporal dynamics of the immune cell infiltration into ECM hydrogel, we used 19F MR imaging to trace immune cells that were systemically labeled using perfluorcarbon (PFC) nanoemulsions. METHODS: Rats underwent middle cerebral artery occlusion to induce a stroke that lead to tissue cavitation. To tag circulating immune cells, PFC nanoemulsions (CelSense) were injected through the tail vein 12 days following stroke. On day 13 post-MCAo, a preimplantation 19F and T2-weighted MRI scans (9.4T) were acquired to visualize a baseline distribution of immune cells, as well as to verify the injection of PFC (i.e. blood vessels containing $19 \mathrm{~F}$ signal). On day $14, \mathrm{ECM}(4 \mathrm{mg} / \mathrm{mL})$ was implanted into the stroke cavity. On day 15,24 hours post-implantation $19 \mathrm{~F}$ and $\mathrm{T} 2$-weighted images were acquired to investigate the invasion and distribution of immune cells in vivo. Straight after MRI, animals were perfusionfixed for histological analyses. Fluorescently activated cell sorting (FACS) was performed to determine the phenotype of immune cells labeled in the blood. RESULTS: FACS indicated that of all PFC-labeled cells, $48 \%$ were macrophages, $40 \%$ neutrophils and $5 \%$

lymphocytes. Baseline $19 \mathrm{~F}$ images revealed no infiltration of labeled immune cells into the stroke-damaged brain, but verified that PFC was circulating with a strong 19F signal present within major blood vessels (Figure 1). 24 hours post-implantation, a strong 19F signal was evident within the lesion cavity implanted with ECM hydrogel, as well as the peri-infarct parenchyma. Moreover, PFC-labeled cells also produced a strong signal in the surgical wound on top of the head, providing a positive control for the host immune response to tissue damage. Histology further confirmed these in vivo results with $97 \%$ of macrophages in the ECM hydrogel containing PFC and $80 \%$ of neutrophils. No lymphocytes were present in the ECM hydrogel 24 hours post-implantation These results further indicate that the main immune response to ECM hydrogel implantation in the acute phase is mediated by invasion of peripheral immune cells into the CNS, rather than through a recruitment of local microglia. DISCUSSION \& CONCLUSIONS: The spatio-temporal dynamics of immune cell invasion into ECM hydrogel after implantation into stroke are difficult to capture using histological methods. However, we here demonstrated that $19 \mathrm{~F}$ MR imaging can potentially provide this type of information to improve our understanding of the contribution of immune response to hydrogel biodegradation and tissue restoration.
\end{abstract}

\section{Image/Figure:}


Image/Figure Caption: 19F MR imaging of PFC-labeled immune cells reveal no signal in the lesion cavity prior to implantation of ECM hydrogel, but visualize in vivo the distribution of immune cells 24 hours after ECM implantation

Complete Status: Complete

First Name: Michel

Last Name: Modo

Email: mmm154@pitt.edu

Organization: University of Pittsburgh

Country: United States 


\title{
ID: GA409 \\ Development of new luciferin analogues that emit near-infrared light through firefly bioluminescence
}

\author{
Genta Kamiya, The University of Electro-Communications, kamiya0801@uec.ac.jp
}

Category: New Chemistry, Biology \& Bioengineering

\begin{abstract}
Body : Firefly bioluminescence produce highly efficient light caused by the reaction of the firefly luciferin catalyzed with firefly luciferase (Fluc). Because of its high efficiency, firefly bioluminescence is utilized in life science applications as in vivo animal imaging. However, the yellow-green light emitted through natural firefly bioluminescence is not suitable for in vivo imaging, because this range of wavelength of light is absorbed and scattered by hemoglobin and oxidized hemoglobin in blood. Conversely, near-infrared (NIR) light (650 -900 nm) is less affected from absorption and scattering by blood, so it is more suitable for in vivo imaging. Our laboratory conducted studies on the structure-bioluminescence activity relationship and succeeded in synthesizing luciferin analogues that produce light in the visible region. In particular, a luciferin analogue emitting NIR light $(675 \mathrm{~nm})$ named AkaLumine was developed. In 2018, Iwano et al. developed Akaluc, the mutated enzyme of Fluc that specifically catalyzes bioluminescence reaction using Akalumine as a substrate. By using this Akaluc-Akalumine system, real-time imaging in large animals were succeeded. many efforts of researchers worldwide, higher than $700 \mathrm{~nm}$ wavelength bioluminescence imaging system has not yet been used in practical applications. In the present study, we aimed to develop bioluminescent systems emitting light at wavelengths $700 \mathrm{~nm}$; we thus synthesized luciferin analogues and evaluated their bioluminescence light using the natural enzyme Fluc and the artificial enzyme Akaluc. The synthesized luciferin analog GeKiaka showed 760-nm emission of light with both Fluc and Akaluc.
\end{abstract}

References: Iwano S., et al., Science, 2018, 359, 935-939 Kuchimaru, T. et al. Nat. Commun. 2016, 7, 11856.

Complete Status: Complete

First Name: Genta

Last Name: Kamiya

Email: kamiya0801@uec.ac.jp

Organization: The University of Electro-Communications

Country: Japan 


\title{
ID: GA410 \\ Dual-agent fluorescent labelling of soft-tissue sarcomas improves the contrast based upon targeting both necrotic and active components of the tumor milieu
}

\author{
Hira Shahzad Sardar, Dartmouth College, hira.shahzad.sardar.th@dartmouth.edu
}

\section{Category: Oncology}

\begin{abstract}
Body : Fluorescence guided surgery (FGS) is an imminent and promising solution to improving detection and surgical resection of solid tumors, such as soft-tissue sarcomas. Current practice utilizes a single fluorescent agent that accumulates with higher concentration in tumors. However, homogeneous distribution throughout the tumor is difficult to achieve. Molecular targeted fluorophores usually bind viable tumor cells, while perfusion-based fluorophores aggregate in tumor interstitia. It is known that sarcomas overexpress epidermal growth factor receptor (EGFR) in $43 \%$ to $78 \%$ of the cases (2-6). At Dartmouth, an anti-epidermal growth factor receptor (EGFR) Affibody molecule (Afibody, AB) labeled with IRDye 800CW (LI-COR Biosciences, Inc.), ABY-029, is currently in an on-going first-in-human Pzhase 0 trial (NCT0315411) for soft-tissue sarcomas (9). One of the main advantages of ABY-029 is its short plasma half-life (1) and rapid normal tissue clearance (7), which makes surgery possible only a few hours after administration. Our pre-clinical studies in xenograft sarcoma murine models demonstrate that maximal accumulation of ABY-029 within the tumor occurs between 4-8 hours after administration and significant tumor-to-background ratios (TBR) are observed starting at 4 hours (8). Here, we show in a xenograft model of human synovial sarcoma that the epidermal growth factor receptor (EGFR) targeted agent ABY-029 has a moderate positive correlation with viable tumor regions $(\mathrm{r}=0.22 \pm 0.4)$, while indocyanine green (ICG), a perfusion dye, demonstrates a strong negative correlation with viable tumor $(\mathrm{r}=-0.63 \pm 0.1)$. We hypothesized that administering these two agents at their optimal administration-to-imaging interval in the same animal, would improve whole-tumor contrast. Both agents can be imaged in the same channel of surgical system due to the significant spectral overlap, thus it is possible to assess the improvement in contrast without image manipulation. Image contrast - measured by contrast-tovariance ratio (CVR) — was highest in tumors that received both ABY-029 and ICG (2.45 \pm 0.8$)$, compared to animals that received ABY-029 (2.27 \pm 0.8$)$ or ICG $(2.01 \pm 0.5)$ alone. In softtissue sarcoma, where fluorescence is highly heterogeneous, measures of contrast that account for variability in the signal is essential. The combination of ABY-029 + ICG had the most improvement on the mean of CVR, which considers both the mean of the fluorescence signal and the standard deviation of the signal in both the tumor and normal tissues. Moreover, the combination of ABY-029 + ICG minimizes the correlation between viable tumor and fluorescence intensity $(r=-0.1 \pm 0.2)$ indicating that the fluorescence signal is more evenly distributed throughout the tumor milieu. "Dual-agent imaging" using region-specific fluorophores is a promising method to increase tumor contrast in a clinical setting.
\end{abstract}

References: 1. Samkoe KS, Bates BD, Tselepidakis NN, et al. Development and evaluation of a connective tissue phantom model for subsurface visualization of cancers requiring wide local excision. J Biomed Opt. 2017;22(12):1-12. doi:10.1117/1.JBO.22.12.121613 2. Keizman D, 
Issakov J, Meller I, et al. Expression and significance of EGFR in malignant peripheral nerve sheath tumor [published correction appears in J Neurooncol. 2009 Sep;94(3):389. Meimon, Natalie [corrected to Maimon, Natalie]]. J Neurooncol. 2009;94(3):383-388.

doi:10.1007/s11060-009-9862-z 3. Teng HW, Wang HW, Chen WM, et al. Prevalence and prognostic influence of genomic changes of EGFR pathway markers in synovial sarcoma. J Surg Oncol. 2011;103(8):773-781. doi:10.1002/jso.21852 4. Yang JL, Gupta RD, Goldstein D, Crowe PJ. Significance of Phosphorylated Epidermal Growth Factor Receptor and Its Signal Transducers in Human Soft Tissue Sarcoma. Int J Mol Sci. 2017;18(6):1159. Published 2017 May 30. doi:10.3390/ijms18061159 5. Yang JL, Hannan MT, Russell PJ, Crowe PJ. Expression of HER1/EGFR protein in human soft tissue sarcomas. Eur J Surg Oncol. 2006;32(4):466-468. doi:10.1016/j.ejso.2006.01.012 6. Sato O, Wada T, Kawai A, et al. Expression of epidermal growth factor receptor, ERBB2 and KIT in adult soft tissue sarcomas: a clinicopathologic study of 281 cases. Cancer. 2005;103(9):1881-1890. doi:10.1002/cncr.20986 7. Samkoe KS, Sardar HS, Bates BD, et al. Preclinical imaging of epidermal growth factor receptor with ABY-029 in soft-tissue sarcoma for fluorescence-guided surgery and tumor detection. J Surg Oncol. 2019;119(8):1077-1086. doi:10.1002/jso.25468 8. DSouza AV, Lin H, Henderson ER, Samkoe KS, Pogue BW. Review of fluorescence guided surgery systems: identification of key performance capabilities beyond indocyanine green imaging. J Biomed Opt. 2016;21(8):80901. doi:10.1117/1.JBO.21.8.080901 9. Samkoe KS, Gunn JR, Marra K, et al. Toxicity and Pharmacokinetic Profile for Single-Dose Injection of ABY-029: a Fluorescent Anti-EGFR Synthetic Affibody Molecule for Human Use. Mol Imaging Biol. 2017;19(4):512-521. doi:10.1007/s11307-016-1033-y

Full Name of Abstract's 1st Author : Hira Shahzad Sardar

Complete Status: Complete

First Name: Hira Shahzad

Last Name: Sardar

Email: hira.shahzad.sardar.th@dartmouth.edu

Organization: Dartmouth College

Country: United States 


\title{
ID: GA411 \\ In vivo Tracking of Stem Cell-derived Islet Organoids Using Magnetic Particle Imaging
}

\author{
Aixia Sun, Michigan state university, sunaixia@msu.edu
}

Category: Systemic Diseases (Kidney, Liver and Pancreas)

\begin{abstract}
Body : INTRODUCTION Transplantation of stem cell-derived islet organoids is a promising approach for Type 1 Diabetes (T1D) treatment. However, there is an absence of appropriate imaging techniques available to determine graft outcome after transplantation [1-4]. Magnetic particle imaging (MPI) is a novel imaging modality that directly detects the superparamagnetic properties of iron oxide nanoparticles (SPIONs), and is a specific, sensitive, and linearly quantitative technique. Here, we demonstrate the feasibility of in vivo tracking of transplanted stem cell-derived islet organoids using MPI [5-7]. METHODS Human iPSCs were differentiated to islet organoids according to the established protocol [8]. Islet organoids were generated after 21 days of differentiation and verified using immunofluorescence staining of islet cell markers followed by labeling with dextran coated SPIONs (VivoTrax, Magnetic Insight Inc., Alameda, CA, USA, $560 \mu \mathrm{g} / \mathrm{mL}$ ) for 48 hours at $37 \mathrm{oC}$. Cell labeling efficiency was examined by anti-dextran staining. Islet organoids phantoms, comprising of different numbers of labeled islet organoids $(25,50,100,200$ or 400, $\mathrm{n}=2)$, were imaged using a MOMENTUM imager (Magnetic Insight Inc., Alameda, CA). The iron content of samples was calculated from the quantification of MPI image and verified using the inductively coupled plasma (ICP) mass spectrometry analysis. Next, 800 SPIONs labeled organoids were transplanted into the left kidney capsule of NOD/scid mice ( $\mathrm{n}=3$, Jackson Laboratory, Bar Harbor, ME). Mice were examined by 3D MPI image at 1, 7, 14, 28, and 42 days post transplantation. Quantitative assessment of the islet organoids was performed using MPI image intensity calibrated against a fiducial marker of known iron content. Before euthanasia serum, the left kidney was collected from the mice and processed for human insulin ELISA and immunofluorescence staining of dextran in labeled islets organoids. RESULTS Immunofluorescence staining of islet organoids expressed islet cell markers including insulin and glucagon after 21 days of differentiation (Fig. $1 \mathrm{~A} \& B$ ). Ninety nine percent of the cells in islet organoids were labeled with SPIONs as confirmed by anti-dextran antibody staining (Fig. 1C). Image analysis of labeled islet organoid phantoms revealed a direct linear correlation between the iron content and the number of islet organoids ( $\mathrm{R} 2=0.997, \mathrm{P}<0.0001)$, and this was consistent with the result of the ICP analysis (Fig. 1D). Strong MPI signals were detected under the kidney capsule on the first day of imaging in all recipients; this represented the labeled islet organoids (Fig. 1E). During the course of the study the signal from 3D images gradually decreased, as expected. Immunofluorescence staining of the kidney sections showed the presence of SPIONs was under the left kidney capsule (Fig. 1F). CONCLUSION MPI can longitudinally monitor islet organoids labeled with SPIONs post transplantation and provide quantitative information of their presence in vivo.
\end{abstract}

References: 1. Bulte JWM, Daldrup-Link HE. Clinical Tracking of Cell Transfer and Cell Transplantation: Trials and Tribulations. Radiology 2018; 289: 604-615. 2. Crabbe A, 
Vandeputte C, Dresselaers T et al. Effects of MRI contrast agents on the stem cell phenotype. Cell Transplant 2010; 19: 919-936. 3. Janowski M, Walczak P, Kropiwnicki T et al. Long-term MRI cell tracking after intraventricular delivery in a patient with global cerebral ischemia and prospects for magnetic navigation of stem cells within the CSF. PLoS One 2014; 9: e97631. 4. Magnitsky S, Zhang J, Idiyatullin D et al. Positive contrast from cells labeled with iron oxide nanoparticles: Quantitation of imaging data. Magn Reson Med 2017; 78: 1900-1910. 5. Zheng B, Vazin T, Goodwill PW et al. Magnetic Particle Imaging tracks the long-term fate of in vivo neural cell implants with high image contrast. Sci Rep 2015; 5: 14055. 6. Zheng B, von See MP, $\mathrm{Yu}$ E et al. Quantitative Magnetic Particle Imaging Monitors the Transplantation, Biodistribution, and Clearance of Stem Cells In Vivo. Theranostics 2016; 6: 291-301. 7. Bulte JW, Walczak P, Gleich B et al. MPI Cell Tracking: What Can We Learn from MRI? Proc SPIE Int Soc Opt Eng 2011; 7965: 79650z. 8. Russ HA, Parent AV, Ringler JJ et al. Controlled induction of human pancreatic progenitors produces functional beta-like cells in vitro. EMBO J 2015; 34: 1759-1772.

\section{Image/Figure:}

https://www.xcdsystem.com/wmis/abstract/File6959/GA411 ImageFigure 0513101752.jpg

Image/Figure Caption: Figure 1 A. 3D differentiation of human pluripotent stem cells produce islet organoids. B. Immunofluorescence staining islet organoids for insulin and glucagon, both of them were expressed in the islet organoid (green, insulin; red, glucagon; blue, DAPI; bar $=20 \mu \mathrm{m}$ ). C. Since SPIONs coated with dextran, we stained labeled islet organoids for dextran. Labeled islet organoids showed an effective accumulation of SPIONs (green, dextran; blue, DAPI; bar $=10 \mu \mathrm{m}$ ). D. MPI image indicated the signal intensity was increased as the increasing number of islet organoids. The quantification of MPI signal manifested a direct linear correlation with the number of islet organoids $(\mathrm{R} 2=0.997, \mathrm{P}<0.0001)$. E. 3D MPI longitudinal monitoring of transplanted islet organoids in vivo. Signal was detected under the left kidney capsule and MPI image intensity of transplanted organoids gradually decreased over a period of 42 days post transplantation. F. Immunofluorescence staining the kidney section for dextran, the green florescence represents SPIONs were present under the left kidney capsule indicated transplanted the organoids (green, dextran; blue, DAPI; bar $=20 \mu \mathrm{m}$ ).

Full Name of Abstract's 1st Author : Aixia Sun

Complete Status: Complete

First Name: Aixia

Last Name: Sun

Email: sunaixia@msu.edu

Organization: Michigan state university

Country: United States 


\title{
ID: GA412 \\ T2 and UTE (Ultra-short TE) Molecular MR Imaging for Gastric Cancer Diagnosis using Polymeric Nanoparticles
}

\author{
Hwunjae Lee, Yonsei University College of Medicine, yohan0410@gmail.com
}

Category: New Chemistry, Biology \& Bioengineering

\begin{abstract}
Body : Several recently developed technologies for molecular imaging have been applied to magnetic resonance (MR) imaging for cancer. In particular, various MR sequences with biocompatible polymer-based magnetic nanoparticles (pMNPs) have been applied for the MR imaging of cancer. However, there are several limitations to this approach, and passive contrast agents are not yet sufficiently targeted. This is a particular challenge for gastric cancer owing to the interference from stomach contents. Therefore, in this study we developed an intelligent targeting contrast agent and assessed its feasibility for early gastric cancer diagnosis using a mouse model. Specifically, we synthesized pMNPs, which enable both T2-weighted (T2) and ultra-short TE (UTE) MR imaging using hyaluronic acid as the polymer, which binds to the receptor CD44, a recently identified biomarker of gastric cancer. Both MR sequences (T2, UTE) were analyzed with respect to imaging effects and targeting to the pMNPs. In vitro assessments showed no significant cytotoxicity of the pMNPs to MKN-45 and MKN-28 cells and confirmed the cellular uptake of the pMNPs. MR signal enhancement was identified after pMNPs.
\end{abstract}

Complete Status: Complete

First Name: Hwunjae

Last Name: Lee

Email: yohan0410@gmail.com

Organization: Yonsei University College of Medicine

Country: Korea, Republic of 


\title{
ID: GA413
}

PDPN Targetable Opto-MR Dual mode Nanocomposites for Glioma Multiforme In Mouse

Hwunjae Lee, Yonsei University College of Medicine, yohan0410@gmail.com

Category: New Chemistry, Biology \& Bioengineering

\begin{abstract}
Body : It is very desirable to design dual-mode nanocomposites for diagnostic applications via flexible strategies. Herein, we proposed to synthesize one type of multifunctional magnetic resonance (MR)/optic nanocomposites based on manganese ferrite magnetic nanoparticles (MFNPs) modified by fluorescent podoplanin (PDPN) antibodies. MFNPs were synthesized using a thermal decomposition method and were modified by the fluorescent PDPN antibodies on their surfaces. The fluorescent PDPN antibody-conjugated MFNPs enabled strong MR imaging and fluorescence imaging due to the antibodies that targeted brain tumors. These results demonstrate that MR/optic nanocomposites have potential as precision diagnostic systems for brain cancer.
\end{abstract}

Complete Status: Complete

First Name: Hwunjae

Last Name: Lee

Email: yohan0410@gmail.com

Organization: Yonsei University College of Medicine

Country: Korea, Republic of 


\title{
ID: GA416 \\ Multimodal in vivo imaging of $B$. pertussis colonization in the baboon respiratory tract: from body scale to cellular interactions
}

\author{
Thibaut Naninck, CEA, thibaut.naninck@cea.fr
}

Category: Immunology: Inflammation \& Infection

\begin{abstract}
Body : Introduction : Whooping cough, due to Bordetella pertussis infection, is today re-emerging worldwide despite high vaccination coverage 1,2 . The baboon model of $\mathrm{B}$. pertussis infection have risen the hypothesis of asymptomatic carriage and transmission3. It is then crucial to develop tools to assess bacterial colonization in primates. Here we developed in vivo multimodal imaging modalities combining [18F]-FDG PET-CT with Fibered Confocal Fluorescence Microscopy (FCFM) 4 to explore the respiratory tract and assess colonization and interactions between B. pertussis and antigen presenting cells (APCs) in baboons. Methods : In order to assess bacterial colonization and its inflammation counterparts, classical whole-body [18F]-FDG PET-CT was performed $45 \mathrm{~min}$ after injecting intravenously $5 \mathrm{MBq} / \mathrm{kg}$ $(0.135 \mathrm{mCi} / \mathrm{kg})$ of $[18 \mathrm{~F}]-\mathrm{FDG}$ at baseline, 8 days and 30 days post baboon exposure to $\mathrm{B}$. pertussis clinical strain by intranasal and intra-tracheal routes. For optical in vivo studies, GFPexpressing B. pertussis (derived from the previous clinical strain) was inoculated in young baboons. Besides, monoclonal anti-HLA-DR-AF647 antibody was administered by topical application in the trachea to specifically target and label APCs. FCFM, coupled with bronchoscopy, was performed in the lower respiratory tract weekly post-infection. Bacterial colonization was quantified using GFP positive signal area detected by FCFM.

Results/Discussion: Animals exposed with clinical B. pertussis strain harbor significant lung lesions and FDG uptake at early stage of the clinical disease (D8), especially in the right lung, with remission observed by day 30 (Figure 1). Animals infected with a GFP-expressing B. pertussis strain developed the classical clinical symptoms for whooping cough as previously described in baboons infected with wild-type strains5,6. Furthermore, in vivo FCFM coupled with bronchoscopy allowed us to detect and quantify bacterial colonization kinetics and APC interactions in the lower respiratory tract of young baboons after B. pertussis-GFP infection in baboons. Ex vivo co-culture analyses also confirmed the interactions between B. pertussis and APCs in lungs explants. We were also able to detect by FCFM bacterial tracheal colonization in animals challenged by intranasal route only providing new insights concerning bacterial localization after natural infection. Conclusion: This approach combining PET-CT and fluorescence imaging will then be a useful tool to describe the mechanisms of action of the bacteria both at macroscopic and cellular resolution during infection to develop more effective vaccines against pertussis. However, to better characterize B. pertussis colonization at wholebody scale, more specific PET radiotracer than FDG (eg. Antibodies, bacterial substrate analog) must be used in the future.
\end{abstract}

References: 1. WHO | Diphtheria-tetanus-pertussis (DTP3) immunization coverage. WHO http://www.who.int/gho/immunization/dtp3/en/ (2018). 2. WHO | WHO-recommended surveillance standard of pertussis. WHO 
http://www.who.int/immunization/monitoring_surveillance/burden/vpd/surveillance_type/passiv e/pertussis_standards/en/ (2018). 3. Warfel, J. M., Zimmerman, L. I. \& Merkel, T. J. Acellular pertussis vaccines protect against disease but fail to prevent infection and transmission in a nonhuman primate model. Proc. Natl. Acad. Sci. U. S. A. 111, 787-792 (2014). 4. Thiberville, L. et al. In Vivo Imaging of the Bronchial Wall Microstructure Using Fibered Confocal Fluorescence Microscopy. Am. J. Respir. Crit. Care Med. 175, 22-31 (2007). 5. Warfel, J. M., Beren, J., Kelly, V. K., Lee, G. \& Merkel, T. J. Nonhuman Primate Model of Pertussis. Infect. Immun. 80, 1530-1536 (2012). 6. Naninck, T. et al. In vivo imaging of bacterial colonization of the lower respiratory tract in a baboon model of Bordetella pertussis infection and transmission. Sci. Rep. 8, 12297 (2018).

\section{Image/Figure:}

https://www.xcdsystem.com/wmis/abstract/File6959/GA416_ImageFigure 0518041309.jpg

Image/Figure Caption: Figure $1:$ [18F]-FDG PET-CT imaging of baboon airways following B. pertussis exposure. Representative PET \& CT fusion slices (frontal: A-C, axial: D-F) at baseline (A, D), day $8(\mathrm{~B}, \mathrm{E})$ and day $30(\mathrm{C}, \mathrm{F})$ post challenge.

Complete Status: Complete

First Name: Thibaut

Last Name: Naninck

Email: thibaut.naninck@cea.fr

Organization: CEA

Country: France 


\title{
ID: GA417 \\ In vivo imaging of microglial activation by positron emission tomography with 11C-ER176 in a mouse model for post-infectious autoimmune encephalitis
}

\author{
Zeljko Tomljanovic, Columbia University, tomljanovic@gmail.com
}

\section{Category: Neuroscience}

\begin{abstract}
Body : Introduction Antibodies against neuronal receptors and synaptic proteins are associated with a group of ill-defined CNS autoimmune diseases named autoimmune encephalitis (AE), characterized by an abrupt onset of seizures, movement disorders, and psychiatric symptoms. Basal ganglia encephalitis (BGE), representing a subset of AE syndromes, can be triggered in children by repeated Group A Streptococcus (GAS) infections that lead to neuropsychiatric symptoms termed pediatric autoimmune neuropsychiatric disorders associated with streptococcus (PANDAS). Antibodies that are produced as a result of GAS infection crossreact with host neuronal proteins and cause a neuroinflammatory response. We have previously shown that multiple GAS infections of mice induce migration of Th17 lymphocytes from the nose into the brain, producing blood-brain barrier (BBB) breakdown, microglia activation, extravasation of autoantibodies into the CNS and loss of excitatory synapses of the olfactory bulb (OB). Prior PET studies in humans have also implicated microglia activation in the basal ganglia in PANDAS. 11C-ER176 is a third generation PET radio ligand for $18 \mathrm{kDa}$ translocator protein (TSPO) - a marker of activated microglia, and so far has not been used in an animal model of post-infectious BGE. We sought to evaluate the ability of 11C-ER176 to detect differences in TSPO binding in our animal model and correlate it to microglia activation by immunofluorescence. Methods Fifteen four-week-old C57BL/6 mice were intranasally infected weekly with either PBS (control group) or GAS-2W streptococci (treated group) for five weeks. Twenty-four hours after the last inoculation, mice were injected intravenously with 11C-ER176 $(103.12 \pm 20.97 \mathrm{uCi})$ and PET images were acquired 0-60 minutes post-injection. Images were co-registered and normalized to standard T2 MRI using PMOD 3.6. Standardized uptake values ratios (SUVR) were calculated using the rostral thalamus (rThal) as a "pseudo-reference" region. Statistical analysis was performed using frames from 30-60 min post-injection. Microglia activation was examined by immunofluorescence for Ibal and CD68 in serial sections from the olfactory bulb in treated and untreated mice of the same strain who did not undergo PET. Results GAS treated mice $(n=8,55.25 \pm 5.0$ days, weight $16.12 \pm 2.42 \mathrm{~g}, 7$ female) showed greater binding of 11C-ER176 than the PBS group $(n=11,58.81 \pm 5.59$ days, weight $19 \pm 2.14 \mathrm{~g}, 4$ female) in the olfactory bulb $(22.4 \%, p=0.004)$, and in the amygdala $(12.9 \%, p=0.023)$. Sexcorrected data showed similar results. The data were in agreement with immunofluorescence results in which there was a two-fold increase in CD68+ Iba1+ activated microglia in the olfactory bulbs of GAS treated mice compared to control mice. Conclusion GAS-treated C57BL/6 mice show increased binding of $11 \mathrm{C}$-ER 176 to TSPO in olfactory bulb and amygdala, suggesting an active neuroinflammatory process. Our findings agree with histological evidence of a leaky blood-brain barrier, serum IgG deposition in the olfactory bulb and amygdala and microglial activation in the olfactory bulb in the same mouse model for post-infectious BGE.
\end{abstract}


References: 1. Dileepan T, Smith ED, Knowland D, et al. Group A Streptococcus intranasal infection promotes CNS infiltration by streptococcal-specific Th17 cells. J Clin Invest. 2016;126(1):303-317. doi:10.1172/JCI80792 2. James ML, Belichenko NP, Shuhendler AJ, et al. [18F]GE-180 PET Detects Reduced Microglia Activation After LM11A-31 Therapy in a Mouse Model of Alzheimer's Disease. Theranostics. 2017;7(6):1422-1436. Published 2017 Mar 24. doi:10.7150/thno.17666

\section{Image/Figure:}

https://www.xcdsystem.com/wmis/abstract/File6959/GA417 ImageFigure 0527105237.png

Image/Figure Caption: Figure 1. Standardized uptake values ratios (SUVR) maps of 11CER176 superimposed over standardized T2 weighted mouse brain MRI. Highlighted regions show higher uptake of the radio tracer in the olfactory bulb (OB) and the amygdala (AM) in the GAS treated group compared to the control group of animals.

Complete Status: Complete

First Name: Zeljko

Last Name: Tomljanovic

Email: tomljanovic@gmail.com

Organization: Columbia University

Country: United States 


\title{
ID: GA418 \\ Changes of bladder a1-adrenoceptors in the urothelium of rat bladder following partial bladder outlet obstruction and subsequent relief
}

\author{
Juhyun Shin, Chungnam national university hospital, synthia2000@naver.com
}

Category: Systemic Diseases (Kidney, Liver and Pancreas)

\begin{abstract}
Body : Introduction and Objective: $\alpha 1$-adrenoceptor antagonists relieve voiding and storage symptoms in patients with bladder outlet obstruction. However, many patients with benign prostatic hyperplasia need treatment for remaining storage symptoms after surgery, even if the obstruction was successfully relieved. Because bladder $\alpha 1 \mathrm{D}$-adrenoceptors are upregulated in some rats with bladder outlet obstruction, we investigated the expression of bladder a1-adrenoceptor subtype after relief of partial bladder outlet obstruction in a rat model. Material and Methods: A total of 60 female Sprague-Dawley rats (body weight 180-210 g) were randomly divided into three groups: the sham-operated group (Sham, $\mathrm{n}=20$ ), the partial $\mathrm{BOO}$ group $(\mathrm{BOO}, \mathrm{n}=20)$ and the partial $\mathrm{BOO}$ relief group $(\mathrm{BOO}+\mathrm{R}, \mathrm{n}=20)$. $\mathrm{PBOO}$ rats were induced for 2 weeks, the obstruction has relived by removal of ligation after 2 weeks. The bladder tissue was carefully separated into urothelium and detrusor muscle layer under a dissecting microscope by cutting in through the lamina propria. The expression of $\alpha 1$-adrenoceptor subtype in the urothelium $(\mathrm{n}=5)$ and smooth muscle $(\mathrm{n}=5)$ tissues was examined by Western blot assay. Results: In the urothelium of the partial BOO group and the partial BOO relief group, the expression of $\alpha 1 \mathrm{D}$-adrenoceptor was significantly higher than the sham operated group ( $\mathrm{p}$ a1D-adrenoceptor was higher in the partial BOO group and the partial BOO relief group in the muscle (p In addition, $\alpha 1 \mathrm{~A}$-adrenoceptor was predominant in the sham operated group, but was significantly decreased in the urothelium in the partial BOO group, and there was no statistical difference in muscle and total bladder. The expression of $\alpha 1 \mathrm{~B}$-adrenoceptor was not statistically significant among the three groups. Conclusions: Our findings indicate a remarkable increase in bladder a1D-adrenoceptor expression after relief of partial bladder outlet obstruction. This finding suggest that urothelial $\alpha 1 \mathrm{D}$-adrenoceptor plays an important role in the persistence of storage symptoms in the partial BOO relief group. These results may also imply that selective $\alpha 1 \mathrm{D}-$ adrenoceptor antagonists such as naftopidil may be effective in persistent post-operative storage symptoms of BPH patients.
\end{abstract}

References: Martin C. Michel \& Wim Vrydag. a1-, a2- and b-adrenoceptors in the urinary bladder, urethra and prostate. Br J Pharmacol 2006;147:S88-S119. Barendrecht MM, Frazier EP, Vrydag W, Alewijnse AE, Peters SL, Michel MC. The effect of bladder outlet obstruction on alpha1- and beta-adrenoceptor expression and function. Neurourol Urodyn 2009;28:349-55.

Complete Status: Complete

First Name: Juhyun

Last Name: Shin 
Email: synthia2000@naver.com

Organization: Chungnam national university hospital

Country: Korea, Republic of 


\title{
ID: GA420 \\ Pretargeting radionuclide imaging of xenografted hepatocellular carcinoma with biotinylated aptamer JHIT2 and iodine-131 labled streptavidin
}

\author{
Min Yang, Department of Nuclear Medicine, the Third Affiliated Hospital of Sun Yat-sen \\ University, sarahyang124@163.com
}

\section{Category: Oncology}

\begin{abstract}
Body : Background: Previously, we discovered that aptamer JHIT2 could be used as a probe for radionuclide imaging of hepatocellular carcinoma (HCC), like radioimmunoimaging, but with an unsatisfying tumor to background ratio,. Moreover, biotin(strept)avidin system, one of the most specific and stable noncovalent interactions, has been used in radioimmunoimaging to improve imaging effect. Purpose: To figure out if biotin-streptavidin system can improve the efficiency of aptamer JHIT2 mediated HCC radionuclide-targeted imaging. Procedures: The biotinylated aptamer JHIT2 was synthesized by Biotech Company. Streptavidin (SA) was labeled with iodine-131 by Iodogen method. The biotinylated aptamer and 131I-SA was administrated to HepG2 cells in vitro sequentially for optimal binding time of aptamer to HCC and SA to biotinylated aptamer for establishing imaging protocol. The biodistribution of 131I-SA in tumor-bearing nude mice pretreated with biotinylated JHIT2 was observed. Finally, radionuclide-targeted imaging combining aptamer with biotin-streptavidin system was evaluated in HCC xenograft models. Results: The labeling rate of 131I-SA was $(92.35 \pm 0.23) \%$. Radiochemical purity of $131 \mathrm{I}-\mathrm{SA}$ was $(87.27 \pm 2.07) \%,(91.46 \pm 0.35) \%$ and $(82.27 \pm 1.93) \%$ in Phosphate Buffer Saline, saline and Dulbecco's Modified Eagle's Medium (including 10\% Fetal Bovine Serum) respectively for $24 \mathrm{~h}$ at room temperature. In vitro cell uptake experiment showed that the highest cell uptake ratio was obtained after 30min incubation with 131I-SA, when cells had been pre-incubated with biotinylated JHIT2 for 30min. The tumor uptake value and tumor/muscle ratio were $(5.61 \pm 1.23) \% \mathrm{ID} / \mathrm{g}$ and $3.21 \pm 0.56$ at $30 \mathrm{~min}$, and $(2.54 \pm 1.36) \% \mathrm{ID} / \mathrm{g}$ and $0.81 \pm 0.40$ at $2 \mathrm{~h}$ in vivo, respectively. SPECT/CT imaging showed significant radioactivity concentration on the tumor site at $30 \mathrm{~min}$ with a target/background ratio of 4.97 and 2.35 at $2 \mathrm{~h}$. Conclusions: The protocal of targeting radionuclide imaging mediated by aptamer JHIT2 and biotin-streptavidin system was preliminarily determined, indicated that this combination could obtain better targeting imaging effect of $\mathrm{HCC}$ in vivo.
\end{abstract}

References: 1.Bray F, Ferlay J, Soerjomataram I, Siegel RL, Torre LA, Jemal A (2018) Global cancer statistics 2018: GLOBOCAN estimates of incidence and mortality worldwide for 36 cancers in 185 countries. CA Cancer J Clin 68:394-424. 2.Rimmele M (2003) Nucleic acid aptamers as tools and drugs: recent developments. Chembiochem 4:963-971. 3.Ireson CR, Kelland LR (2006) Discovery and development of anticancer aptamers. Mol Cancer Ther 5:2957-2962. 4.Xiang D, Shigdar S, Qiao G, et al. (2015) Nucleic acid aptamer-guided cancer therapeutics and diagnostics: the next generation of cancer medicine. Theranostics 5:23-42. 5.Zhao M, Dong L, Liu Z, Yang S, Wu W, Lin J (2018) In vivo fluorescence imaging of hepatocellular carcinoma using a novel GPC3-specific aptamer probe. Quant Imaging Med Surg 8:151-160. 6.Xu J, Teng IT, Zhang L, et al. (2015) Molecular Recognition of Human Liver 
Cancer Cells Using DNA Aptamers Generated via Cell-SELEX. PLoS One 10:e0125863. 7.Min Y, Wenshan H, Yueming Z, Guixiong Z, Jiehua X (2019) Biodistribution and imaging of 131Ilabelled hepatoma aptamer in tumor-bearing nude mice. Chin J Hepat Surg(Electronic Edition) 8:260-264. 8.Hnatowich DJ, Virzi F, Rusckowski M (1987) Investigations of avidin and biotin for imaging applications. J Nucl Med 28:1294-1302. 9.Paganelli G, Malcovati M, Fazio F (1991) Monoclonal antibody pretargetting techniques for tumour localization: the avidin-biotin system. International Workshop on Techniques for Amplification of Tumour Targetting. Nucl Med Commun 12:211-234. 10.Li GP, Zhang H, Zhu CM, Zhang J, Jiang XF (2005) Avidin-biotin system pretargeting radioimmunoimaging and radioimmunotherapy and its application in mouse model of human colon carcinoma. World J Gastroenterol 11:6288-6294. 11.Sharkey RM, Karacay H, Cardillo TM, et al. (2005) Improving the delivery of radionuclides for imaging and therapy of cancer using pretargeting methods. Clin Cancer Res 11:7109s-7121s. 12.Yao Z, Zhang M, Kobayashi H, et al. (1995) Improved targeting of radiolabeled streptavidin in tumors pretargeted with biotinylated monoclonal antibodies through an avidin chase. J Nucl Med 36:837-841.

\section{Image/Figure:}

https://www.xcdsystem.com/wmis/abstract/File6959/GA420 ImageFigure 0607010139.png

Image/Figure Caption: SPECT/CT of biotin-streptavidin and aptamer mediated 131I imaging in vivo

Full Name of Abstract's 1st Author : Min Yang

Complete Status: Complete

First Name: Min

Last Name: Yang

Email: sarahyang124@163.com

Organization: Department of Nuclear Medicine, the Third Affiliated Hospital of Sun Yat-sen University

Country: China 


\title{
ID: GA421 \\ Combined value of PSMA-based 18F-DCFPyL, 18F-FDG and 18F-NaF PET/CT imaging in metastatic prostate cancer patients
}

\author{
Esther Mena, NCI. NIH, esther.menagonzalez@nih.gov
}

\section{Category: Oncology}

\begin{abstract}
Body : Objective: To investigate the combined performance of 18F-DCFPyL, a Prostate-Specific Membrane Antigen (PSMA) targeted PET agent, 18F-FDG and 18F-NaF $\mathrm{PET} / \mathrm{CT}$ imaging in a prospective cohort of patients with documented metastatic prostate cancer. Methods: This is a prospective IRB-approved, single-institution study, including 30 patients with known metastatic prostate cancer by conventional imaging (average PSA of $285.94 \mathrm{ng} / \mathrm{mL}$, range 0.15-2910 ng/mL). Eight patients were castrate-sensitive, eleven castrate-resistant prostate cancer and eleven treatment-naive. All patients underwent whole-body 18F-DCFPyL-PET/CT at $2 \mathrm{~h}$ p.i $(299.9 \pm 15.5 \mathrm{MBq}), 18 \mathrm{~F}-\mathrm{FDG}-\mathrm{PET} / \mathrm{CT}$ and 18F-NaF PET/CT imaging were performed about 2 weeks apart. PSMA-PET lesion detection rate and scan concordance were correlated with patient castrate status and treatment status at the time of the scan. Results: All patients showed a positive 18F-DCFPyL-PET scan consistent with metastatic disease. 18F-DCFPyL and 18F-FDG PET scans had overall concordant positive findings in $23.3 \%$ (7/30) of patients, who were mostly castrate-resistant, whereas $76.7 \%$ of patients had incongruent findings with $18 \mathrm{~F}-$ DCFPyL detecting more lesions than 18F-FDG, except for one patient with multiple liver lesions on FDG, not seen on DCFPyL. For bone lesions, 18F-DCFPyL and 18F-NaF showed good imaging concordance in $45 \%$ of patients with osseous lesions $(n=20)$, with 18F-DCFPyL-PET detecting more bone lesions in 5 patients, and 18F-NaF-PET detecting more lesions in 6 patients, 1 of them with the bone disease detected by $18 \mathrm{~F}-\mathrm{NaF}-\mathrm{PET}$ only. The congruence DCFPyL/NaF did not depend on patient's castrate status or prior treatment exposure. Pathologic confirmation of metastatic disease was performed for at least one positive-DCFPyL lesion in 48\% (12/25) of the patients (biopsy sites: 6 bones, 4 prostate beds, 1 nodal and 1 lung lesion); three patients are pending biopsy, and the remaining patients either had lesions not amenable for biopsy or refused biopsy. Patient accrual is ongoing for this trial and more results will be available at the time of presentation. Conclusions: 18F-DCFPyL-PET imaging was able to identify suspicious metastatic lesions in patients with castrate-sensitive, castrate-resistant, and treatment-naive metastatic prostate cancer. 18F-DCFPyL-PET detected significantly more lesions than 18F-FDG-PET, with good DCFPyL/FDG PET imaging concordance in about $23 \%$ of the cases, particularly in the setting of metastatic castrate-resistant prostate cancer. DCFPyL/NaF showed good imaging concordance in $45 \%$ of the patients with bone lesions, independent of the patient's castration status or treatment status.
\end{abstract}

References: 1. (18)F-DCFPyL PET/CT Imaging in Patients with Biochemical Recurrence Prostate Cancer after Primary Local Therapy. Mena E, Lindenberg ML, Turkbey IB, Shih JH, Harmon SA, Lim I, Lin FI, Adler S, Eclarinal P, McKinney Y, Citrin D, Dahut W, Wood B, Krishnasamy V, Chang R, Levy E, Merino M, Pinto P, Eary JF, Choyke PL. J Nucl Med. Nov. 1: Epub ahead of print, 2019. 2. A Prospective Comparison of 18F-Sodium Fluoride PET/CT and 
PSMA-Targeted 18F-DCFBC PET/CT in Metastatic Prostate Cancer. Harmon SA, Bergvall E, Mena E, Shih JH, Adler S, McKinney Y, Mehralivand S, Citrin DE, Couvillon A, Madan RA, Gulley JL, Mease RC, Jacobs PM, Pomper MG, Turkbey B, Choyke PL, Lindenberg ML. J Nucl Med. 2018 Nov;59(11):1665-1671.

Complete Status: Complete

First Name: Esther

Last Name: Mena

Email: esther.menagonzalez@nih.gov

Organization: NCI. NIH

Country: United States 


\title{
ID: GA424 \\ Disulfiram derivatives as inhalable nanotherapeutics for non-small cell lung cancer
}

\author{
Jiadong Pang, Sun Yat-sen University Cancer Center, pangjd@sysucc.org.cn
}

\section{Category: Oncology}

\begin{abstract}
Body : OBJECTIVE Lung cancer is among the most prevalent and the deadliest cancers worldwide, over $80 \%$ of which belongs to non-small cell lung cancer (NSCLC) with poor prognoses. NSCLC would usually respond to ALK inhibitors, but unfortunately resistance almost definitely develops during the treatment process. In addition, efficient delivery of targeted therapeutics to tumor lesions remains challenging. In order to fight against both ALK wild type and resistance in the treatment, we aim to synthesize a series of disulfiram derivatives that allow in situ self-assembly of nanoparticles (NPs) effectively encapsulating ceritinib, as screened from a panel of ALK inhibitors. Non-invasive inhalation enables rapid distribution and high enrichment of NPs in the tumor lesions to achieve targeted treatment. Meanwhile, the formation of ditiocarb-copper complex $(\mathrm{GnD}-\mathrm{Cu} 2+)$ from the disulfiram derivatives via glutathione cleavage in tumor provides a viable alternative ALK-independent tumor-suppressing pathway and thus tackles ALK resistance and wild type. Intriguingly, the residues are primarily excreted by renal clearance, which minimizes systemic toxicity. The blood circulation half-life by intravenous injection and inhalation is also investigated and compared. In summary, this new class of disulfiram derivatives is a possible solution to treat NSCLC. METHODS A click chemistry-based synthesis of disulfiram with glucosamine (GnDs) was proposed. Chemical structures of the final products were confirmed using fourier transform infra-red and $1 \mathrm{H}$ nuclear magnetic resonance. Thermal stability, thermal decomposition and critical micelle concentration of GnDs were measured. Sizes of GnD NPs encapsulated with ceritinib were studied using dynamic light scattering and TEM. The robustness of the optimal $\mathrm{GnD}$ was verified by different drying and storage methods. The cytotoxicity of $\mathrm{GnDs}$, $\mathrm{GnDs}-\mathrm{Cu} 2+, \mathrm{GnDs} /$ ceritinib was accessed in ALK mutant H3122 and wild-type A549 cells using MTT. The mechanism of GnD degradation forming $\mathrm{GnD}-\mathrm{Cu} 2+$ and its effect in $\mathrm{H} 3122$ cells were also studied. Biodistribution and renal clearance following inhalation were confirmed by imaging in two types of lung cancer models. H\&E staining and ALK immunofluorescence staining were performed to scrutinize distribution of $\mathrm{GnD}$ in tumors. The blood half-life of GnD complex was tracked and calculated from periodical imaging. RESUTLS GnDs can effectively encapsulate ceritinib into NPs. The NPs retains a high stability over 10 days in PBS, after several cycles of lyophilization, and by other standard drying including rotary evaporation and vacuum drying. Sustained drug release can be maintained for days. GnDs in the presence of $\mathrm{Cu} 2+$ and ceritinib have shown synergistic anti-tumor effect in H3122 cells, while only the former exhibits an appreciable inhibition in A549 cells. $\mathrm{GnD}$ bond-breaking is induced by excessive glutathione in tumor cells, leading to formation of disulfiram residues complexing excessive $\mathrm{Cu} 2+$ in tumors from blood, which reduces the level of P-glycoprotein and sensitize $\mathrm{H} 3122$ cells. $\mathrm{GnD} / \mathrm{Cy} 5.5-\mathrm{COOH}$ demonstrates renal excretion and long blood circulation with primary RES distribution. Interestingly, GnD from inhalation with only a proportion entering systemic absorption presents a different half-life
\end{abstract}


in both phases of distribution and elimination in contrast to intravenous injection.

CONCLUSION We demonstrate the chemical synthesis of a series of disulfiram derivatives that actively deliver ALK inhibitors for targeted therapy against NSCLC, while serving as a secondary ALK-indepedent therapeutic option by itself via forming cytotoxic ditiocarb-copper complex. The monosaccharide functionality mediates its efficient uptake in cancer cells. Its long blood circulation, extended pulmonary residence, and rapid renal clearance potentiate $\mathrm{GnDs}$ as promising nanotherapeutic agents. GnDs-Cu2+ formed in situ in cells can rescue ALK resistance. Furthermore, the inhalable nature endows major advantages in terms of noninvasiveness, repetitive administration, and expeditious accessibility to multiple lung cancer lesions and lobes at the same time. Its low toxicity and minimal off-target accumulation can potentially meet the unmet needs in clinic practice.

References: [1] Peters S, Camidge DR, Shaw AT, et al. Alectinib versus Crizotinib in Untreated ALK-Positive Non-Small-Cell Lung Cancer. New England Journal of Medicine, 2017, 377:829838. [2] Uchibori K, et al. Brigatinib combined with anti-EGFR antibody overcomes osimertinib resistance in EGFR-mutated non-small-cell lung cancer. Nature Communications. 2017, 8: 14768. [3] Anita K, Rolf W.S, Els W, Jos H.B, Alfred H.S. Brain accumulation of the EML4ALK inhibitor ceritinib is restricted by P-glycoprotein (P-GP/ABCB1) and breast cancer resistance protein (BCRP/ABCG2). Pharmacological Research, 2015, 102: 200-207. [4] Katayama R, Sakashita T, Yanagitani N. P-glycoprotein Mediates Ceritinib Resistance in Anaplastic Lymphoma Kinase-rearranged Non-small Cell Lung Cancer. EBioMedicine, 2016, 3 : 54-66. [5] Khozin S, Blumenthal, Gideon M, Zhang LJ, et al. FDA Approval: Ceritinib for the Treatment of Metastatic Anaplastic Lymphoma Kinase-Positive Non-Small Cell Lung Cancer. Clinical Cancer Research, 2015, 21(11): 2436-2439. [6] Skrott Z, Mistrik M, Andersen KK, et al. Targeting multidrug resistance in cancer. Nature Reviews Drug Discovery, 2002, 2: 48-58. [7] Liu P, Brown S, Channathodiyil P, et al. Reply: Cytotoxic effect of disulfiram/copper on human glioblastoma cell lines and ALDH-positive cancer-stem-like cells. British Journal of Cancer, 2012 9: 1488-1497. [8] Skrott, Z., et al. Alcohol-abuse drug disulfiram targets cancer via p97 segregase adaptor NPL4. Nature, 2017, 552, 194-199. [9] LI Y, Wang L H, Zhang H T, et al. Disulfiram combined with copper inhibits metastasis and epithelial-mesenchymal transition in hepatocellular carcinoma through the NF-kB and TGF- $\beta$ pathways. Journal of Cellular And Molecular Medicine, 2018, 22(1): 439-451. [10] Zhang C, Peng F, Liu W, et al. Preparation and optimization of triptolide-loaded solid lipid nanoparticles for oral delivery with reduced gastric irritation. Molecules, 2013, 18(11): 13340-13356. [11] Liu SL, Liu HG, Gang Ren1, et al. PET Imaging of Integrin Positive Tumors Using 18F Labeled Knottin Peptides. Theranostics, 2011, 1: 403-412.

Image/Figure Caption: a Schematic of the mode of action of the inhalable GnDs/ceritinib for damaging tumor tissue. Inhalation of $\mathrm{GnDs} /$ ceritinib enables targeted delivery of the drug in lung cancer and protects the drug from being metabolized. $b$ Structure of GnDs. c Critical micelle concentration (CMC) of G11D by pyrene fluorescence. Inset shows G11D could self assemble into nanostructures. $\mathrm{d}$ Images of G6D, G11D and G18D loading Cy5.5-COOH from $\mathrm{CHCl} 3$ after shaking $1 \mathrm{~h}$. Inset shows dye was encased in a hydrophobic cavity. e Cumulative release of Cy5.5-COOH from GnD NPs under $\mathrm{pH} 7.4$ at 1, 2, 3, 4, 5 and 6 d. Sustained drug release can be maintained for days. f Size distribution, PDI, and surface charge of G11D, and G11D/ceritinib by dynamic light scattering (DLS) and TEM images of G11D, and G11D/ceritinib. g Size 
distribution, PDI, and surface charge of G11D after different drying methods or different freezedrying times. The nanotherapeutic formulation retains a high stability with reproducible hydrodynamic sizes after a number of cycles of lyophilization, and by other standard pharmaceutical drying including rotary evaporation and vacuum drying. $\mathrm{h}$ Diameter change of G11D at $\mathrm{pH} 7.4$ in $10 \%$ PBS $\left(4^{\circ} \mathrm{C}\right)$ over time. i TEM images of G11D at pH 7.4 in $10 \%$ FBS (37 $\left.{ }^{\circ} \mathrm{C}\right)$. j Cell survival rate of G11D, G11D-Cu, G11D/ceritinib and G11D/ceritinib-Cu in H3122 cells and A549 cells by MTT. GnDs in the presence of $\mathrm{Cu} 2+$ and ceritinib have jointly shown synergistic anti-cancer effect in $\mathrm{H} 3122$ cells, while only the former exhibits an appreciable inhibition of proliferation in A549 cells. k Cu content of A549 and H3122 cells in tumor tissue or dish and $\mathrm{Cu}$ content of A549 and $\mathrm{H} 3122$ cells at 1, 2, 3, and $4 \mathrm{~d}$ after the addition of whole blood. Inset shows $\mathrm{Cu}$ in blood accumulated in tumor tissue. 1 Images of GnDs and $\mathrm{GnDs}-\mathrm{Cu} 2+$ degradating by $1 \mathrm{uM} \mathrm{GSH}$ after shaking $1 \mathrm{~h}$. GnD bond cleavage is induced in situ by GSH, leading to formation of disulfiram residues complexing excessive $\mathrm{Cu} 2+$ in tumors $\mathrm{m} \mathrm{GSH}$ content of $\mathrm{H} 3122$ cells $48 \mathrm{~h}$ after the addition of GnDs-Cu2+ and $\mathrm{GnDs} /$ ceritinib. GnDs-Cu2+ reduce the content of GSH in tumor cells. $\mathrm{n}$ Western blot detection of P-gp expression level in H3122 after treatment for $24 \mathrm{~h}$. o Cell survival rate of $24 \mathrm{~h}$ treated $\mathrm{H} 3122$ in $0.5 \mathrm{uM}$ ceritinib 48

h. GnDs-Cu2+ reduces the expression level of P-glycoprotein and sensitize ALK-resistant H3122 cells. p H3122 cells were pre-treated with EIPA, genistein or chlorpromazine for $1 \mathrm{~h}$ and exposed to $20 \mathrm{uM} \mathrm{GnDs} / \mathrm{Cy} 5.5-\mathrm{COOH}$ for $1 \mathrm{~h}$. q Confocal images of $\mathrm{H} 3122$ cells after the addition of G11D/Cy5.5-COOH for $1 \mathrm{~h}$ and $2 \mathrm{~h}$. $\mathrm{r} \mathrm{G11D}$ delivers the drug into tumor tissue and be excreted mainly through kidney. The H3122 lung tumor model was established by intravenously (i.v. injecting $1 \times 106 \mathrm{H} 3122$ cells) or in situ (injecting $5 \times 105 \mathrm{H} 3122$ cells) into $\mathrm{Nu} / \mathrm{Nu}$ mice. On day 7, after confirming development of multifocal metastases in both lungs, the mice were inhalation of G11D/Cy5.5-COOH. Cy5.5-COOH served as a control of G11D/Cy5.5$\mathrm{COOH}$. Live imaging photos of nude mice inhaled with G11D/Cy5.5-COOH or Cy5.5-COOH. s Luminescence images and fluorescent images of nude mice organs with G11D/Cy5.5-COOH. $\mathrm{t}$ Confocal images of lung tissue section from sacrificed mice treated with HE staining and ALK immunofluorescence staining. u Ffluorescent images of blood from C57 mice at different time. v Fluorescent intensity and calculation of half-life by fitting curve. G11D from inhalation with only a proportion entering systemic absorption presents a different half-life in both phases of distribution and elimination in contrast to intravenous injection.

Full Name of Abstract's 1st Author : Jiadong Pang

Complete Status: Complete

First Name: Jiadong

Last Name: Pang

Email: pangjd@sysucc.org.cn

Organization: Sun Yat-sen University Cancer Center

Country: China 


\title{
ID: GA425 \\ Theranostic Fe3O4-ZIF-8 Core-Shell Nanoparticles for Delivery of Arsenic Trioxide
}

\author{
Natalia Ziółkowska, First Faculty of Medicine, Charles University, \\ n.l.ziolkowska@gmail.com
}

Category: New Chemistry, Biology \& Bioengineering

\begin{abstract}
Body : Introduction Fe3O4-ZIF-8 core-shell nanoparticles, consists of the metalorganic framework (MOF) ZIF-8 (Zeolitic Imidazolate Framework-8) [1] and Fe3O4 cores, were developed and studied as promising candidates for future theranostic applications [2]. MOF is representing the therapeutic tool since it possesses a high drug loading capacity and a convenient $\mathrm{pH}$-dependent material stability. The $\mathrm{Fe} 3 \mathrm{O} 4$ moiety is included to the design to enable the material visualization with magnetic resonance imaging (MRI). As a model of a drug, arsenic trioxide was selected, which is a very promising anticancer agent. The aim of this study was to characterize the chemical stability, magnetic properties and in vitro cytotoxicity of prepared materials. Methods ZIF-8 was selected due to its non-toxic components and $\mathrm{pH}$-dependent material stability and arsenic trioxide was used as a model drug. The arsenic drug was introduced to the framework of the porous ZIF-8 shell via post-synthetic ligand exchange. To improve the material biocompatibility, the nanoparticles were coated with polyethylene glycol (PEG). Three samples were studied: Fe3O4, drug-free PEG-NH2@Fe3O4-ZIF-8 and drug-loaded PEGNH2@As@Fe3O4-ZIF-8. To mimic physiological conditions in the body, in vitro drug release studies were performed in a phosphate buffered saline at two different $\mathrm{pH}$ values $(\mathrm{pH} 7.4$, reflecting the healthy tissue and $\mathrm{pH} 6.0$, reflecting some tumours tissues). In addition, cytotoxicity studies of the drug free and arsenic-loaded core-shell nanocarrier were performed on two cell lines (fibroblast cell line - $\mathrm{pH} 7.4$; cancer cell lines - $\mathrm{pH}$ 6). MR experiments on phantoms verified nanoparticle superparamagnetic behavior and its performance as a contrast agent. Firstly, all probes were prepared in water solutions in five iron concentrations $(0.1 \mathrm{mM}-$ $1.5 \mathrm{mM}$ ) and T1/T2 relaxation times were measured using Inversion recovery (T1) and CarrPurcell-Meiboom-Gill (CPMG) (T2) sequences on 1.5 T Bruker relaxometer. Then, T1/T2 weighted MR images of nanoparticles were acquired on a $4.7 \mathrm{~T}$ Bruker Biospec scanner using a resonator coil with rapid acquisition with relaxation enhancement (RARE) multi-spin echo MR sequence. MR image processing and quantification were performed using ImageJ software. The signal-to-noise ratio (SNR), contrast-to-noise ratio (CNR), as the difference in SNR between phantom and water, and the fractional signal loss (FSL), representing percentage signal drop in phantoms related to the water signal intensity, were calculated. Results and discussion Drugloaded probe testing revealed that at $\mathrm{pH} 6$, the porous $\mathrm{ZIF}-8$ shell decomposed entirely rapidly and induced a complete arsenic release. Whereas at $\mathrm{pH} 7.4$, the arsenic-loaded core-shell nanoparticles released only half of the loaded arsenic after $168 \mathrm{~h}$. In vitro cytotoxicity showed that no toxic effect was found in both tested cell lines, what confirms that probes can possibly proceed to further in vivo testing. Results obtained in MR experiments supports that nanoparticles possess adequate magnetic properties to serve as a contrast agent and MR method is sufficiently sensitive for their visualization. Relaxivity $\mathrm{r} 2 / \mathrm{r} 1$ ratio of the $\mathrm{Fe} 3 \mathrm{O} 4$ nanocluster
\end{abstract}


(48.51) can be assigned as a "negative" contrast agent. Both drug free and arsenic-loaded coreshell nanocarriers exhibited $\mathrm{r} 2 / \mathrm{r} 1$ ratio of 10.67 and 12.39 , respectively, also provided very good MR contrast. This was verified by $\mathrm{T} 1$ and $\mathrm{T} 2$ weighted MR images, on which phantoms were visible as hypointense regions in all Fe-concentrations. The MR quantitative image analysis (SNR, CNR, FSL) confirmed that signal drop was in line with the Fe concentration. Results are summarized in Figure 1. Conclusion Overall, the Fe3O4-ZIF-8 core-shell nanoparticles fulfilled essential requirements for theranostics: they can be loaded with a high amount of the selected arsenic drug and possessing superparamagnetic probes can be used as contrast agents, as shown in vitro MRI studies. Additionally, it was confirmed that the nanocarrier could be used for $\mathrm{pH}$ triggered drug release and cytotoxicity tests revealed no toxic effect.

References: [1] K. S. Park, Z. Ni, A. P. Côté, J. Y. Choi, R. Huang, F. J. Uribe-Romo, H. K. Chae, M. O'Keeffe, O. M. Yaghi, Proc. Natl. Acad. Sci. USA 2006, 103, 10186. [2] R. Ettlinger, N. Moreno, N. Ziółkowska, A. Ullrich, H.-A. Krug von Nidda, D. Jirák, K. Kerl, H. Bunzen, submitted.

\section{Image/Figure:}

https://www.xcdsystem.com/wmis/abstract/File6959/GA425 ImageFigure 0608110829.jpg

Image/Figure Caption: Figure 1. T1 (left) and T2 (right) weighted MR images of (a) Fe3O4 nanoclusters, (b) drug-free PEG-NH2@Fe3O4-ZIF-8 and (c) drug-loaded PEGNH2@As@Fe3O4-ZIF-8 nanoparticles with different Fe concentrations $(0.1 \mathrm{mM}-1.5 \mathrm{mM})$. Corresponding SNR, CNR and FSL values are shown.

Full Name of Abstract's 1st Author : Natalia Ziółkowska

Complete Status: Complete

First Name: Natalia

Last Name: Ziółkowska

Email: n.1.ziolkowska@gmail.com

Organization: First Faculty of Medicine, Charles University

Country: Czech Republic 


\title{
ID: GA426 \\ Accurate Reconstruction of Cerenkov Luminescence Tomography Using Spike and Slab Prior for Breast Tumor Detection
}

\author{
Meishan Cai, Institute of Automation, Chinese Academy of Sciences, \\ caimeishan2016@ia.ac.cn
}

Category: Oncology

\begin{abstract}
Body : Introduction: Cerenkov luminescence tomography (CLT) is a novel biomedical imaging modality to display the three-dimensional (3-D) distribution of radioactive probes in vivo using the Cerenkov photons emitted from fast-moving charged particles in nuclear decay. CLT combines the superiority of nuclear imaging and optical imaging and thus facilitates diversities of preclinical and clinical researches such as tumor detection and lymph node visualization. However, the reconstruction performance is significantly limited owing to the severe scattering of Cerenkov photons in biological systems. Methods: We developed a novel reconstruction algorithm using spike and slab prior (SSP) to enhance the reconstruction accuracy of CLT. Compared with traditional algorithms based on Gaussian prior (GP) or Laplacian prior (LP), SSP encourages the sparsity of Cerenkov source better and thus could obtain the reasonably sparse reconstruction source, which improves the reconstruction accuracy greatly. To validate the reconstruction performance of the proposed algorithm, we conducted in vivo CLT imaging of breast tumor-bearing mouse model. A female Balb/c nude mouse (4-6 weeks, 15$20 \mathrm{~g}$ ) was injected with $\sim 1074 \mathrm{~T} 1$ breast tumor cells to establish the breast cancer xenograft. The xenograft was allowed to grow for one week. Then, $800 \pm 50 \mathrm{mCi}$ [18F]-Fluoro-2-deoxy-dglucose (18F-FDG) was injected into the tumor-bearing mouse via tail vein. 40 min later, the Cerenkov luminescence image was acquired by the electron-multiplying charge-coupled device (EMCCD) and the anatomical image was achieved by the micro-CT system. These images were employed to accomplish the reconstruction of CLT using proposed SSP algorithm and comparative algorithms including GP and LP. In addition, the positron emission tomography (PET) images were obtained to verify the real tumor area. Results: The CLT images of the breast tumor reconstructed by SSP algorithm show good image quality with low artifacts. The reconstructed results by SSP algorithm are more similar to the PET results, which enables CLT to be a strong complement for PET. In comparison, SSP algorithm improves the location accuracy of CLT for breast tumor detection over GP and LP algorithms. Conclusions: This study demonstrated the superiority of the proposed SSP algorithm accurate reconstruction for breast tumor detection. The results show that the location accuracy of CLT is greatly improved using SSP algorithm. This study could facilitate the development and biomedical application of CLT in the future.
\end{abstract}

\section{Image/Figure:}

https://www.xcdsystem.com/wmis/abstract/File6959/GA426 ImageFigure 0602043034.png 
Image/Figure Caption: Figure 1. The CLT reconstruction results of the bladder and the breast tumor using different reconstruction algorithms. Compared with the GP and LP algorithms, the CLT images reconstructed by SSP algorithm show good image quality with low artifacts and more similarity with the PET results.

Complete Status: Complete

First Name: Meishan

Last Name: Cai

Email: caimeishan2016@ia.ac.cn

Organization: Institute of Automation, Chinese Academy of Sciences

Country: China 


\title{
ID: GA427 \\ HA-Ce6-MnO2@SWNHs combine NIR-II PTT with Enhanced PDT by Modulating Tumor Hypoxia and Priming Tumor-Associated Macrophages toward M1-like Phenotype for cancer therapy
}

\author{
Pengli Zhang, Shanghai Jiao Tong University, plzhang2018@sjtu.edu.cn
}

\section{Category: Oncology}

\begin{abstract}
Body : Introduction: Tumor hypoxia is a general feature of most locally advanced solid tumors, which have negative effect on anticancer therapies, e.g. radio- and chemotherapy and photodynamic therapy (PDT). Manifold nanoparticles (NPs), like MnO2 NPs, have been designed to alleviate hypoxia. Hypoxia areas of tumors were frequently clustered abundant macrophages and most of them existed as M2-skewed TAMs. A large amount of reports indicated that low molecular weight HA (LMW HA) could bind CD44 receptors overexpressed on 4T1 cells and may activate pro-tumoral M2 TAMs to anti-tumor M1 macropthages. Herein, we synthesized $\mathrm{MnO} 2$ and Chlorine e6 (Ce6)-loaded, HA-modified single-walled Carbon nanohorns, designated as HA-Ce6-MnO2@SWNHs, for MRI/FI/PAI triple-modal imagingguided NIR-II PTT and enhanced PDT. Materials \& Methods: A high temperature acid-oxydic rection has been applied to synthesize $\mathrm{MnO} 2$ on SWNHs in situ. Briefly, MnO2@SWNHs were prepared by mixing the aqueous solutions of $\mathrm{SWNHs}$ and $\mathrm{KMnO} 4$ at $80 \mathrm{oC}, \mathrm{pH}$ was adjusted at 2.5 with $\mathrm{HCl}$ and stirred for $24 \mathrm{~h}$. The $\mathrm{pH}$ was adjusted to 7.4 when the reaction was finished. Then $\mathrm{MnO} 2-\mathrm{SWNHs}$ were loaded with $\mathrm{Ce} 6$ and modified with HA at room temperature. The ability of $\mathrm{MnO} 2$ generating oxygen by triggering $\mathrm{H} 2 \mathrm{O} 2$ and then enhancing PDT in vitro were monitored by dissolved oxygen tester and a SO sensor green (SOSG) probe. Flow cytometry analysis in vitro was performed to examine the function of HA to skew M2 macrophages to M1. The effect of PDT/PTT in vitro were examined by cell count kit-8 (CCK-8) assay. Tumor accumulation and biodistribution of HA-Ce6-MnO2@SWNHs in vivo were monitored by magnetic resonance imaging (MRI), photoacoustic imaging (PAI) and fluorescence imaging (FI). Tumor hypoxia relief and polarization of macrophages were evaluated by immunofluorescent staining and flow cytometry analysis. The therapeutic effect of PDT/PTT were also been examined with mices bearing 4T1 tumors. Results \& Discussion: Multifunctional HA-Ce6MnO2@SWNHs were stable in water, PBS and $10 \%$ FBS, and have a strong absortion at NIR-II $(\sim 1064 \mathrm{~nm})$ promising for both photothermal therapy and PAI in NIR II area. The content of dissolved O2 and SO increased with HA-Ce6-MnO2@SWNHs concentration in H2O2. In vitro flow cytometry showed prominent expression of iNOS on macrophages treated with HA-Ce6MnO2@SWNHs and HA, the former is more obvious than the latter, but not on Ce6MnO2@SWNHs group, which suggested that HA induced the polarization of macrophages to M1. Cell viability studies showed HA-Ce6-MnO2@SWNHs had no obvious cytotoxicity, but PDT and NIR-II PTT on 4T1 cells treated with HA-Ce6-MnO2@SWNHs induced cell death dramatically. FI/PAI revealed that HA-Ce6-MnO2-SWNHs had high tumor accumulation and relatively lower liver untake. After accumulated into tuomrs, HA-Ce6-MnO2@SWNHs consumed $\mathrm{H} 2 \mathrm{O} 2$ in the acidic tumor microenviroment by $\mathrm{MnO} 2$ producing $\mathrm{O} 2$ and $\mathrm{Mn} 2+$ ions that alleviate hypoxia for enhanced PDT and improved T1-weighted MRI signal intensity
\end{abstract}


simultaneously. Immunostaining and flow cytometry analysis of tumors showed the percentage of M1 macrophages increased and hypoxia relief. To compensate the insufficient peneatration depth of short wavelength light for PDT, a NIR-II laser (1064 nm) was utilized for PTT. PDT and NIR-II PTT combination therapy showed a splendid therapeutic effect on the primary tumors and simultaneously inhibted metastases in lungs and livers. Conclusions: HA-Ce6MnO2@SWNHs can numerously accumulate in tumors, and successfully relieve tumor hypoxia, enhance PDT and induce macrophage from M2 to M1 phenotype. Combined NIR-II PTT and enhanced PDT have more remarkable curative effect than single treatment, significantly prolong the survival of mices bearing $4 \mathrm{~T} 1$ tumors.

References: Manli Song, Ting Liu, et al. Bioconjugated Manganese Dioxide Nanoparticles Enhance Chemotherapy Response by Priming Tumor-Associated Macrophages toward M1-like Phenotype and Attenuating Tumor Hypoxia [J]. ACS Nano, 2016, 10, 633-647.

Complete Status: Complete

First Name: Pengli

Last Name: Zhang

Email: plzhang2018@sjtu.edu.cn

Organization: Shanghai Jiao Tong University

Country: China 


\title{
ID: GA428 \\ A quadrature 31P MRI surface coil for small animal 1H/31P MR imaging.
}

\author{
Martin Vit, Technical university of Liberec, vit.martin2@seznam.cz
}

\section{Category: Instrumentation}

\begin{abstract}
Body : Introduction New responsive phosphorus probes for magnetic resonance imaging (MRI) are currently being tested due to progress in phosphorus chemistry. For the animal testing phase, it is necessary to create appropriate MR equipment - especially radiofrequency coils designed for detection of phosphorus MR signal with higher efficiency. The design and construction of radiofrequency coil for small animal imaging at high magnetic field strength 4.7T are presented. The design of coil is dedicated for MR signals at $1 \mathrm{H}(200.4 \mathrm{MHz})$ and $31 \mathrm{P}(81.1 \mathrm{MHz})$ and optimized for in vivo rat experiments. Methods A coil is a combination of two galvanic separated radiofrequency parts $1 \mathrm{H}(200.4 \mathrm{MHz})$ and $31 \mathrm{P}(81.1 \mathrm{MHz})$. It consists of $1 \mathrm{H}$ coil generated linearly polarized B1 field and 31P coil generated circularly polarized B1 field for better sensitivity by $40 \%$ (1). 31P coil consist of a second order LCC trap (2) for stronger decoupling of the neighboring frequencies. This trap works at own frequency as parallel resonance circuit and suppressed the current of neighboring coil f31PTrap $=200.4 \mathrm{MHz}$ (3). A coil is incorporated in PLA rat holder with incorporated anaesthesia pipe and a stump for fixation of animal. In the body holder are inserted hot water pipes for stabilizing an animal temperature. A coil is intended for 4.7T system (Bruker Biospec 47/20, Ettlingen, Germany). Discussion A pilot experiment with the coil shows a suitable construction of mechanical parts and robust design which is needed for in vivo experimental instrumentation. Field of view of presented coil is consistent with edge of loops $40 \mathrm{~mm}$. A coils show good sensitivity for both protons and phosphorus MR experiments. Tuning range of phosphorus coils is $81 \pm 2 \mathrm{MHz}$ - actual capacity of tuning capacitor depends on inserted payload. Tuning range of $1 \mathrm{H}$ loop is slightly wider $200 \pm 5 \mathrm{MHz}$. Vertical (perpendicular) reach of coils is $42 \mathrm{~mm}$ for $-3 \mathrm{~dB}$ signal-to-noise attenuation. Electric quality of unloaded resonance for $31 \mathrm{P}$ coil was measured as $\mathrm{S} 11=279$, respectively $\mathrm{S} 11$ $=311$ for $1 \mathrm{H}$ coil. Coupling between coils was measured as S21 $=-12.3 \mathrm{~dB}(200.4 \mathrm{MHz}$, proton $>$ phosphorus $)$ and $\mathrm{S} 21=-9.8 \mathrm{~dB}(81.1 \mathrm{MHz}$, phosphorus $->$ proton $)$. A coil was compared with standard commercially available coil $(1 \mathrm{H} / 31 \mathrm{P} 50 \mathrm{~mm}$ coil $)$. Presented coil provides $+42 \%$ higher signal compared to commercial one. Conclusion The goal of this work was to construct a 1H/31P coil for animal experiments for bimodal imaging (Fig. 1). The coil setup enables $1 \mathrm{H}$ anatomical imaging as well as $31 \mathrm{P}$ imaging or spectroscopy at the same animal position. The pilot data indicates that coil could effectively acquire signal from both $1 \mathrm{H}$ and $31 \mathrm{P} \mathrm{MR}$ experiments.
\end{abstract}

References: 1. Mispelter Joel at al.(2015): NMR Probeheads: Volume Coils Constructions, s 257-259., Imperial College press, 2015. 2. Hosseinnezhadian S et al. A flexible 12-channel transceiver array of transmission line resonators for 7 T MRI. J Magn Reson 2018;296:47-59. 3. Meyerspeer M et al. An Improved Trap Design for Decoupling Multinuclear RF Coils, Magnetic Resonance in Medicine, 72:584-590 (2014) 


\section{Image/Figure:}

https://www.xcdsystem.com/wmis/abstract/File6959/GA428_ImageFigure_0602054201.png

Image/Figure Caption: MRI scan: Images acquired by newly constructed coil $200 \mathrm{ml}$ phantom containing distilled water and 9g K2HPO4 (0.23M). 1H - Spin echo localizer TE=36ms, $\mathrm{TR}=3300 \mathrm{~ms}, \mathrm{TA}=1: 20 \mathrm{~min}, 31 \mathrm{P}$ FID-CSI TR=500ms, $\mathrm{TA}=8 \mathrm{~min}$. 1H\&31P overlapped images $1 \mathrm{H}$ localizer and 31P image Design of the coil: a) View on completed coil with tuning sticks, b) complete electric design of coil, c) CAD model of central part of coil

Full Name of Abstract's 1st Author : Martin Vit

Complete Status: Complete

First Name: Martin

Last Name: Vit

Email: vit.martin2@seznam.cz

Organization: Technical university of Liberec

Country: Czech Republic 


\title{
ID: GA429 \\ Novel probe based on fluorinated 2-oxazoline polymer for $19 \mathrm{~F}$ magnetic resonance imaging
}

\author{
Martin Vit, Technical university of Liberec, vit.martin2@seznam.cz
}

Category: New Chemistry, Biology \& Bioengineering

\begin{abstract}
Body : Introduction Fluorine 19F magnetic resonance imaging (MRI) has emerged especially in experimental preclinical and clinical studies. The limited commercial supply of probes leads to further research on new substances including smart contrast agents (thermoresponsive, $\mathrm{pH}$-responsive and more (1)).Comparable sensitivity to proton and no background in the mammal physiological environment enabling absolute quantification of 19F MR signal class fluorine based probes as excellent ones. Here we present new MRI fluorine probe based on utilization of 2-oxazolines. Methods Probe is based on poly-2-oxazolines with 2-(3,3,3trifluoroprophyl) 2-(3,3,3-trifluoropropyl)-2-oxazoline as fluorine containing part.(2). For use in biological, hydrophilic behavior was done by 2-methyl-2-oxazoline (MeOx) and 2-ethyl-2oxazoline (EtOx). 2-n-octyl-2-oxazoline is hydrophobic modification and 2-n-propyl-2-oxazoline is thermoresponsive modification (Fig.1a). Probe was tested in vitro in tubes with $0.2 \mathrm{ml}$ of substance. Experiment was performed at 4.7T system (Bruker Biospec 47/20, Ettlingen, Germany) We analyzed 8 samples HF1, HTF7, HTF3, HTF4 HF3, HF4, HF5 and HLF4 (Fig.1). $19 \mathrm{~F}$ imaging procedure is combination of $1 \mathrm{H}$ navigator and $19 \mathrm{~F}$ imaging sequence; results are on figure 2. Fluorine T1 times were measured by spectroscopic method. Fluorine T2 times were measured as CPMG Results/Discussion T1 relaxation times were calculated in the range 40-251 ms. T2 assessment was successful only for HF5 due to low signal intensity; T2 relaxation for HF5 is $122 \mathrm{~ms}$. Results of HF5 are summarized on figure 1b. Images with high signal-to-noise ratio (SNR) were obtain for HF1 (12.8), HF3 (28.4), HF4 (53.6) and HF5 (63.1). These 4 polymers are nicely imaged by MRI. Thermoresponsivity of HF5 probe was measured with results on fig. 1b. SNR changes, or relaxation time changes are negligible. Low signal intensity for other polymers could be caused by extremely short relaxation times. In our case echo time of imaging sequence is $13.7 \mathrm{~ms}$. Shorter relaxation times are not clinical relevant. It suggests using sequences allowing shorter TE. Another reason of low signal intensity could be intended by low hydrophilicity of substance. Conclusion We tested different fluorine contrast probes intended as thermoresponsive agents. The best imaging properties for our experimental set up was achieved for HF5, the probes HF4, HF3 and HF 1 are still visible. HLF4, HTF3, HTF4 and HTF7 were not visualized. Next step will be imaging with shorter echo times and biological in vitro experiments to determine biocompatibility of the probes.
\end{abstract}

References: 1 Jirak D, Galisova A, Kolouchova K, Babuka D, Hruby M. Fluorine polymer probes for magnetic resonance imaging: quo vadis? MAGMA. 2019 Feb;32(1):173-185 2 Kaberov, L. I.; Verbraeken, B.; Riabtseva, A.; Brus, J.; Talmon, Y.; Stepanek, P.; Hoogenboom, R.; Filippov, S. K. ACS Macro Lett. 2018, 7 (1), 7-10.

\section{Image/Figure:}


Image/Figure Caption: a)Chemical structure and properties of the substances, b) Thermal dependence of MRI parameters, c) Vizualization of T1 realxation curve HF5 for 26 and $43^{\circ} \mathrm{C}$

Full Name of Abstract's 1st Author : Martin Vit

Complete Status: Complete

First Name: Martin

Last Name: Vit

Email: vit.martin2@seznam.cz

Organization: Technical university of Liberec

Country: Czech Republic 


\title{
ID: GA430 \\ Quantification of $19 \mathrm{~F}$ polymer implant biodegradation - long term in vivo MR study
}

\author{
Natalia Ziólkowska, First Faculty of Medicine, Charles University, \\ n.l.ziolkowska@gmail.com
}

Category: New Chemistry, Biology \& Bioengineering

\begin{abstract}
Body : Introduction In this long-term study, we monitored a fluorinated polymer containing thermo- [1] and $\mathrm{pH}$-responsive copolymers forming a slowly dissolving implant [2] in physiological conditions. Rats were injected with polymer subcutaneously and intramuscularly and measured with dual homemade $1 \mathrm{H} / 19 \mathrm{~F}$ MR coil on $4.7 \mathrm{~T}$ scanner to determine localization and dissolution time of the implant. 19F magnetic resonance imaging (19F MRI) quantification confirmed in vivo presence of implants up to 14 (intramuscular injection) and 20 (subcutaneous injection) months with sufficient MR sensitivity. The MRI was complemented with more sensitive 19F magnetic resonance spectroscopy (19F MRS) with signal detectability throughout the experiment (21 months). The aims of this study were to assess biodegradability of the implant, its long-term influence on animals welfare and chemical stability in vivo. Methods Healthy male Lewis rats were administrated with polymer (200 $\mu$ l) intramuscularly and subcutaneously to the left and right hind leg, respectively. Long-term in vivo MR experiment was performed using a dual surface $1 \mathrm{H} / 19 \mathrm{~F}$ coil optimized for small animal scanning on a $4.7 \mathrm{~T}$ scanner. Firstly, 19F MRS signal from the implant was measured with a single-pulse sequence, secondly a 1H/19F MRI experiment was conducted using multi-spin echo sequence to provide anatomical details and implant localization. 19F MRS signal was quantified using MATLAB (R2007b) script for signal-to-noise ratio (SNR) calculation. Data obtained from 19F MRI served for implant dissolution quantification: volume, signal intensity, SNR and contrast-to-noise ratio (CNR) calculation. To present fluorine signal changes in time, reflecting the implant degradation in vivo, implant volume was multiplied by SNR creating a course of dissolution (Dynamic changes, DCH). To confirm the injected implant biocompatibility, standard parameters, such as weight and blood biomarkers were measured, also ex vivo histological examination was proceeded. Results and discussion Signal from 19F MRI was detected for 20 months (14 months from intramuscular implant and 20 on subcutaneous injection site) with sufficient SNR and CNR (Fig. 1). DCH of implant dissolution shows a decrease of MR fluorine signal in time. Even though some incontinences can be observed due to in vivo variability, the overall trend is visible with small signal changes observed from 6 months post-injection. At the full dissolution time (14 months) on intramuscular injection site the DCH reaches $11.7 \%$ of its initial value and only $6.0 \%$ on the subcutaneous site with longer decomposition observed (20 months). 19F MRS enables to extract more subtle signal changes and was detected for 21 months from injection onset. No toxic effect of the implant was observed during its in vivo dissolution, as no abnormalities were found in blood markers results, weight and histological examination. Conclusion This experiment affirm that tested probe is an example of a great future clinical tracer with good chemical and MR properties. No negative influence was observed during chronic in vivo degradation,
\end{abstract}


measurement with all the major biochemistry indices maintained at levels comparable to those of the control.

References: [1] K. Kolouchova, O. Sedlacek, D. Jirak, D. Babuka, J. Blahut, J. Kotek, M. Vit, J. Trousil, R. Konefał, O. Janouskova, Self-Assembled Thermoresponsive Polymeric Nanogels for 19F MR Imaging, Biomacromolecules, 19 (2018) 3515-3524. [2] O. Sedlacek, D. Jirak, A. Galisova, E. Jager, J.E. Laaser, T.P. Lodge, P. Stepanek, M. Hruby, 19F Magnetic Resonance Imaging of Injectable Polymeric Implants with Multiresponsive Behavior, Chemistry of Materials, 30 (2018) 4892-4896.

\section{Image/Figure:}

https://www.xcdsystem.com/wmis/abstract/File6959/GA430_ImageFigure_0608095637.jpg

Image/Figure Caption: Figure 1. 1H/19F MR images measured in vivo on 4.7 T scanner and overlapped from coronal plane (red - 19F signal). Images represent rat injected with polymer forming implants (left site - intramuscular injection. right site - subcutaneous injection) and were acquired at different time points ( 1 month -21 months). In post-processing sine square was applied with the same weight filter on all images. Beneath the images 19F signal-to-noise ratio (SNR) and contrast-to-noise ratio (CNR) values are given at the corresponding site.

Full Name of Abstract's 1st Author : Natalia Ziółkowska

Complete Status: Complete

First Name: Natalia

Last Name: Ziółkowska

Email: n.1.ziolkowska@gmail.com

Organization: First Faculty of Medicine, Charles University

Country: Czech Republic 


\title{
ID: GA432 \\ Zwitterionic Porous Conjugated Polymers as a Versatile Platform for Antibiofouling Implantable Bioelectronics
}

\author{
JINJIA XU, University of Illinois at Urbana-Champaign, jinjiax@illionis.edu
}

\section{Category: New Chemistry, Biology \& Bioengineering}

\begin{abstract}
Body : $\pi$-Conjugated polymers (CPs) have attached considerable attentions for significant biomedical purposes, including bioelectronics and biosensing, tissue engineering, would healing, due to their great structural flexibility, tunable conductivity, compatible mechanical properties, and ease of fabrication over inorganic conducting materials. However, in vivo studies have shown that the improved electrochemical performance of devices by CPs coatings could not be sustained after implantation due to the non-specific adsorption of biomacromolecules on electrochemical device surfaces that reduces the sensitivity and performance of the device and triggers foreign body response that eventually leads to the failure of implanted devices. Here an integrated zwitterionic conjugated porous polymer-based biomaterial platform was designed and studied, which consists of conjugated polymer backbones, multifunctional zwitterionic side chains, and distorted unit in core components. Conjugated polythiophene was selected as the backbone due to its good electrical conductivity, chemical stability and interesting optical properties. The zwitterionic carboxybetaine side chain was introduced as the side chain because of its excellent antifouling property, high water solubility and biocompatibility. 9,9'-Bifluoreneylidene (BF) is a well-known distorted unit, where two fluorene units are connected through a $\mathrm{C}=\mathrm{C}$ double bond with $30^{\circ}$ twisting angle because of the steric hindrance of peripheral $1-\mathrm{H}$ and $8-\mathrm{H}$. We envision such twisted conformation will provide enough free volume inside zwitterionic CPs network to construct the porous skeleton. To prove our hypothesis, thiophene-based conjugated linear (PTh) and porous (PThBF) polymers were sucessfully synthesized via Yamamoto and Suzuki polycondensation. The photophysical properties of polymers were investigated by UV-vis and photoluminescence spectroscopies in solution and film states. The porous morphology of PThBF was visualized by SEM and TEM observations. The results suggested that porosity, optical and electronic properties of polymers are readily tuned by the insertion of distorted BF units. The conductivity and anti-biofouling properties against protein, cells and bacterial of polymers surface will be systematically investigated during this work. This platform can potentially be adapted to a wide range of applications (e.g. bioelectronics, organic electrochemical transistors and biofuel cell), which require high performance conducting materials with excellent antifouling/biocompatibility at biointerfaces.
\end{abstract}

References: J. Xu, J. Xu, H. Moon, H. O. Sintim, and H. Lee, ACS Appl. Polym. Mater., 2020, 2, 528-536.

Full Name of Abstract's 1st Author : Jinjia XU

Complete Status: Complete 
First Name: JINJIA

Last Name: XU

Email: jinjiax@illionis.edu

Organization: University of Illinois at Urbana-Champaign

Country: United States 


\title{
ID: GA433 \\ High Temporal Resolution Dynamic Contrast Enhanced MRI (DCE-MRI) Using SE-WMRI (Single Excitation-Wideband MRI)
}

\author{
Wei-Hao Huang, National Taiwan University, mark51614@gmail.com
}

\section{Category: New Chemistry, Biology \& Bioengineering}

\begin{abstract}
Body : Introduction: $\quad$ DCE MRI is a powerful tool to assess functional microcirculation and tumor angiogenesis [1]. By a series of repeated MR scans, we can get the time-signal curve to observe the kinetic characteristics of organ. In order to capture the subtle dynamic changes, temporal resolution is highly demanded. SE-WMRI is an acceleration technique which can improve temporal resolution but does not sacrifice image quality [2]. In this study, we aim to combine DCE MRI and Wideband technique and use this accelerated sequence to assess the renal function of mice. With temporal resolution improvement, we can get more information in same scan time, helping to perform more accurate analysis. Materials and Methods: $\quad$ MR experiments were performed on a Bruker (Ettlingen, Germany) 7T Biospec $70 / 30$ scanner. Two mice (Non-scid, weight $20 \mathrm{~g}$ ) were injected with $0.01 \mathrm{mmol} / \mathrm{mL}$ gadolinium (Gd) contrast agent $150 \mu \mathrm{L}$ and preformed conventional gradient echo (GRE) and SE-WMRI respectively. A series of conventional T1-weighted GRE and $\mathrm{W}=2, \mathrm{~S}=5$ SE-WMRI scans were acquired by the following parameters: TR/TE:90ms/3.1ms, FA: $40 \mathrm{deg}$, MTX: $112 \times 112$, FOV: $30 \times 30 \mathrm{~mm} 2$, spatial resolution $=0.268 \mathrm{~mm} / \mathrm{px}$, slice thickness: $0.7 \mathrm{~mm}, 10$ slices for each scan. The temporal resolution of conventinal GRE and SE-WMRI are $7.5 \mathrm{~s}$ and $3.75 \mathrm{~s}$ respectively. Results and Discussion: The conventional GRE and SE-WMRI images are demonstrated as Fig.(a)(b) and they also present the image intensity change between preinjection and post-injection. According to the Fig.(c), the physiological state of two mice causes slight differences to the perfusion curves but the kinetic characteristics in both sequence are similar. The image intensity of kidney rapidly elecated after Gd-injection. Then, image intensity start to decrease because the contrast agent was washed out by kidney. Additionally, the perfusion curves of cortex and medulla region show that there is around 40s time delay of intensity peak between two regions. The reason is contrast agent arrives renal vasculature in cortex region first and enters to renal tubules in medulla region [3]. Conclusion: In this study, we performed the high temporal resolution DCE MRI using Wideband technique and the perfusion curve also showed the same kinetic characteristics. Hence, the idea of combing SEWMRI with DCE MRI is feasible and it has potential to extend to various application where high-speed observation is needed. Currently, it is just a preliminary study so we only have two cases. In order to validate the stability of SE-WMRI, we will increase the case number in the future. Furthermore, we will perform the k-trans analysis of kidney to obtain more physiological information which can assist precision medical diagnosis.
\end{abstract}

References: [1] Padhani AR., J Magn Reson Imaging. (2002); [2] Wu, Edzer et al., Medical physics. (2015). [3] Bokacheva, Louisa et al., Magn Reson Imaging Clin N Am. (2008)

\section{Image/Figure:}


Image/Figure Caption: Fig.(a) demonstrates the image result of conventional GRE and the image enhancement caused by Gd contrast agent can be observed between Fig.(a)-1 and Fig.(a)2. Same as Fig.(a), the Fig.(b) show the image result of SE-WMRI. Fig.(c) is the perfusion curve of two sequences and the dynamic range of image intensity is normalized to $0-1$. The grey blocks in the Fig.(c) describe the pre-injection period. The blue line and red line represent the cortex and medulla region respectively. Green blocks are the time delay of intensity peak between two regions. The average time delay is $41.25 \mathrm{~s}$ (Conventional: $45 \mathrm{~s}$, SE-WMRI: $37.5 \mathrm{~s}$ ).

\section{Complete Status: Complete}

First Name: Wei-Hao

Last Name: Huang

Email: mark51614@gmail.com

Organization: National Taiwan University

Country: Taiwan 


\title{
ID: GA434 \\ Fast Image Processing Method for Different Clinical Modalities to Facilitate Diagnosis of Breast Cancer Progression at the Early Stages
}

\author{
Nikolai Slavine, UT Southwestern Medical Center at Dallas, Texas, \\ nikolai.slavine@utsouthwestern.edu
}

Category: Instrumentation

\begin{abstract}
Body : Purpose: To demonstrate an application of rapidly converging, efficient iterative deconvolution breast image enhancement method with a novel resolution subsets-based approach RSEMD that operates on patient DICOM images from different imaging modalities. The proposed method can be applied to clinical images to further improve image quality in order to aid in the diagnosis of breast cancer at the earliest stages. Materials and Methods: The method was tested on phantoms and clinical breast images. The method was applied to imaging data previously processed with commercial Naviscan Flex Solo II positron emission mammography scanner (PEM), Hologic Selenia Dimensions breast tomosynthesis system (DBT) and Naviscan Gamma Medica Molecular Breast Imaging scanner (MBI) to determine improvements in image resolution, SNR and CNR. The blurred clinical image is iterated against different resolution kernels to maximize SNR. The RSEMD as an efficient extension of the blind deblurring algorithm iterates the blurred image with different resolution parameters and a corresponding number of iterations for each subset are taken in turn. In this case, RSEMD method look similar to an extended Richardson-Lucy algorithm with multiple resolution levels (resolution subsets). During the RSEMD iteration procedure the SNR is checked in each iterative step and this process can be repeated until the deblurring restoration reaches the highest SNR with parameter can be set as a small fraction of the initial SNR. The second parameter is an initial deburring parameter (width). Results: The quality of breast images was evaluated in terms of image resolution, contrast and level of noise. In all of the phantom and patients' breast studies the improved images proved to have higher resolution, contrast and lower noise as compared with images reconstructed by conventional methods. Application of the RSEMD algorithm reduced the noise in the PEM, DBT and MBI images and preserved important image features. For example, values of both SNR and CNR reached a plateau after approximately 6 iterations (3 resolution subsets) with an improvement factor of about 1.7 in PEM images. The images generated after applying RSEMD showed both qualitative and quantitative improvements in breast lesion conspicuity for different imaging modalities compared to the image produced using conventional method of reconstruction. The processing time per iteration with RSEMD was improved by a factor of 4-5 as compared to other iterative algorithms with image post-filtering. Conclusions: An iterative deconvolution algorithm with a novel resolution subsets-based approach RSEMD that operates on patient images has been used for quantitative improvement in breast imaging. As shown, the RSEMD algorithm performs well and can be employed retrospectively on clinical DICOM images. The proposed rapidly converging, iterative deconvolution method RSEMD can be applied to PEM, DBT and MBI clinical images to further improve image quality in order to aid in the diagnosis of breast cancer at the earliest stages. The RSEMD method can be employed with already binned pixel/voxel intensities, using RSEMD
\end{abstract}


software supported by off-the-shelf operating system hardware (e.g. Intel NUC $®$ processor) that can be implemented during the breast image processing cycle.

References: [1] Valk PE, Bailey DL et al Positron emission tomography: basic science and clinical practice: Springer London; 2003. [2] Fletcher JW, Djulbegovic B et al.

Recommendations on the use of F-18 FDG PET in oncology. J Nuc Med. 2008;49:480-508. [3]

Tagliabue L, Del Sole A. Appropriate use of positron emission tomography with F-18

fluorodeoxyglucose for staging of oncology patients. Euro J Internal Med. 2014;25:6-11. [4]

Jelercic S, Rajer M. The rote of PET-CT in radiotherapy planning of solid tumours. Radiology

and Oncology. 2015;49:1-9. [5] Kramer-Marek G, Capala J. Can PET Imaging Facilitate

Optimization of Cancer Therapies? Current Pharmaceutical Design. 2012;18:2657-69. [6] Lewis

DY, Soloviev D, Brindle KM. Imaging Tumor Metabolism Using Positron Emission

Tomography. Cancer Journal. 2015;21:129-36. [7] Hooley RJ, Durand MA, Philpotts LE,

Advances in Digital Breast Tomosyntesis. Amer J Roent. 2017; 208:256-66. [8] Karellas A and

Vedantham S. Breast cancer imaging: A perspective for the next decade. Med Phys. 2008;

35(11):4878-97. [9] Friedewald SM et al. Breast Cancer Screening Using Tomosynthesis in Combination with Digital Mammography. JAMA, June 25 2014. [10] Haas BM et al.

Comparison of Tomosynthesis Plus Digital Mammography and Digital Mammography alone for Breast cancer screening. Radiology. 2013; 269:694-700. [11] O'Connor M, Rhodes D, Hruska C, Molecular Breast Imaging. Expert Rev Anticancer Ther; 2009: 9(8):1073-1080. [12] Shermis RB, Redfern RE, Burns J, Kudrolli JH. Molecular Breast Imaging in Breast Cancer Screening and Problem Solving. Radio Graphics. 2017; 37: 1309-1606. [13] Richardson W. BayesianBased Iterative Method of Image Restoration. JOSA.1972; 62:59. [14] Lucy LB, An iterative technique for the rectification of observed distributions. The Astronomical Journal.1974; 79:745750. [15] Slavine, NV "Rapidly converging 3D Iterative Deconvolution Method for Quality Image Improvement from Different Clinical Modalities", Patent disclosure UT 3794 , UT Southwestern Medical Center at Dallas, TX, 2020

\section{Image/Figure:}

https://www.xcdsystem.com/wmis/abstract/File6959/GA434 ImageFigure 0604110312.png

Image/Figure Caption: Figure proposed rapidly converging RSEMD method that operates on patient DICOM images can be applied to PEM, DBT and MBI clinical images to further improve image quality in order to aid in the diagnosis of cancer at the earliest stages.

Complete Status: Complete

First Name: Nikolai

Last Name: Slavine

Email: nikolai.slavine@utsouthwestern.edu

Organization: UT Southwestern Medical Center at Dallas, Texas

Country: United States 


\title{
ID: GA435 \\ Furin-Mediated Self-Assembly of Olsalazine Nanoparticles: Raman Spectroscopy Studies
}

\author{
Jeff Bulte, Russell H. Morgan Department of Radiology and Radiological Science, Division \\ of MR Research and Cellular Imaging Section, Institute for Cell Engineering, Johns \\ Hopkins University School of Medicine, Baltimore, MD, USA, jwmbulte@mri.jhu.edu
}

\section{Category: Oncology}

\begin{abstract}
Body : Introduction: Raman spectroscopy has great potential for in vivo molecular and cellular imaging due to its non-invasiveness, allowing the detection of specific targets without altering the internal structure or function of cells [1]. Examples of nanoparticles that have been introduced as exogenous labels for surface-enhanced Raman scattering (SERS) include metal nanoparticles and carbon wall nanotubes, but their toxicity may be of concern. [2]. It was recently reported that Olsalazine-RVRR nanoparticles (Olsa-RVRR NPs) can be self-assembled in cancer cells by the tumor-associated enzyme furin following a biocompatible condensation reaction [3]. The reduced effusion effect and prolonged retention time of Olsa-NPs enabled their use as an efficient chemical exchange saturation transfer (CEST) MRI theranostic contrast agent. In this study, we assessed the inherent differential Raman signal associated with intracellular self-assembled Olsa-NPs and whether they can be detected by Raman spectroscopy. Methods: For imaging of single fixed cells, 1x105 HCT116 (high furin-expressing) cells were cultured in six-well plates with quartz slides for $24 \mathrm{~h}$. Cells were incubated with $100 \mu \mathrm{M}$ Olsa-RVRR, Olsa, CTR1 (a control isomer of Olsa-RVRR with the scrambled peptide sequence RKRCRV in lieu of the furin substrate RVRR), and CTR2 (a control Olsa-conjugated KCRVRR sequence short of the CBT motif) for $3 \mathrm{~h}$ at $37{ }^{\circ} \mathrm{C}$. Cells were washed three times with PBS and fixed with $4 \%$ paraformaldehyde for $10 \mathrm{~min}$, and washed again before imaging. Raman spectra were recorded using an XploRA PLUS Raman microscope (HORIBA Instruments Inc., Edison, NJ, USA) with a motorized stage. A $785 \mathrm{~nm}$ laser and a 60x water immersion objective were used for sample excitation. For each spectrum, two acquisitions were obtained for 10 seconds each. Spectra were acquired with LabSpec6 (Horiba) software and processed using custom-written scripts in MATLAB. A fifth order least-squares polynomial curve fitting was applied with a moving window of 100. After area normalization, a spike correction was done after thresholding of zvalues. For imaging of cell pellets, $5 \times 106$ HCT116 cells were incubated as described above, and after washing at $200 \mathrm{~g}$ for 5 min cell pellets were collected and dried on an aluminum plate. For Raman spectroscopy, a $638 \mathrm{~nm}$ laser was used with 10x objective lens. For each spectrum, five acquisitions were obtained with for 100 seconds each. Results: For the the HCT116 slide-seeded cells, a clear Raman peak was detected at $1168 \mathrm{~cm}-1$ after incubation with Olsa-RVRR. (Fig. 1a). In contrast to Olsa-RVRR, the Raman images for the three control compounds (Olsa alone, CTR1, and CTR2) lacked peaks at $1168 \mathrm{~cm}-1$ due to the lack of aggregation of Olsa-NPs inside cells (Fig. 1b). To quantify the intracellular accumulation inside cells, Raman analysis was performed on cell pellets (Fig. 1c). Again, cell pellets showed a significantly higher Raman intensity for Olsa-RVRR compared to the other three control (P. Conclusions: A clear Raman signal could be detected at $1168 \mathrm{~cm}-1$ following furin-induced condensation and self-assembly
\end{abstract}


of Olsa nanoaggregates. Cell pellet experiments further validated an increased intracellular concentration of Olsa NPs. The inherent Raman scattering properties of Olsa-NPs may encourage future in vivo Raman imaging, with or without combining CEST MRI [3].

References: 1. Butler H. J., et al. Nat. Protoc. 2016, 11, 664-687. 2. Keren S., et al. Proc. Natl. Acad. Sci. USA 2008, 105, 5844-5849. 3. Yuan, Y., et al. Nat. Mater. 2019, 18, 1376-1383.

\section{Image/Figure:}

https://www.xcdsystem.com/wmis/abstract/File6959/GA435_ImageFigure_0604112507.png

Image/Figure Caption: Figure 1. a) Bright field image and Raman images of Olsa-RVRRincubated HCT116 cells. Olsa-NPs were detected at $1168 \mathrm{~cm}-1$ (red), the entire cytoplasm (peak at $1440 \mathrm{~cm}-1$ ) is shown in green. Scale bar $=10 \mu \mathrm{m}$. b) Raman images of HCT116 cells without/with incubation of Olsa, CTR1, or CTR2. Scale bar $=5 \mu \mathrm{m}$. c) Intensity values for each treatment group (total area under curve at $1168 \mathrm{~cm}-1, \mathrm{n}=17$ for each group) after peak normalization. $* \mathrm{P}<0.0001$ for Olsa-RVRR compared to the other groups.

Complete Status: Complete

First Name: Jeff

Last Name: Bulte

Email: jwmbulte@mri.jhu.edu

Organization: Russell H. Morgan Department of Radiology and Radiological Science, Division of MR Research and Cellular Imaging Section, Institute for Cell Engineering, Johns Hopkins University School of Medicine, Baltimore, MD, USA

Country: United States 


\title{
ID: GA436 \\ Large-scale preparation of gold nanoclusters and simultaneous labeling with Gallium-68
}

\author{
Benchao Zheng, Shanghai jiaotong university, 729721158@qq.com
}

\section{Category: Oncology}

\begin{abstract}
Body : Introduction Ultra-small gold nanoclusters (AuNCs) have gained extensive attentions for theranostics of tumors because of its renal clearance and high tumor accumulation efficiency through the enhanced permeability and retention (EPR) effect. AuNCs is also a good carrier for radioisotopes for preparation of novel nuclear medicine. However, for clinical translation, AuNCs has to be made in large scale. Moreover, the conventional radiolabeling methods involve chelating agents (DOTA or NOTA), which may alter the biological distribution of AuNCs in vivo [1]. Herein, a novel core-doping radiolabeling method was proposed, through which AuNCs could be produced in a large-scale and labeled by Gallium-68 simultaneously. Methods $68 \mathrm{Ga}$-labeled AuNCs was synthesized by co-reduction of $\mathrm{HAuCl} 4$ and $68 \mathrm{GaCl} 3$ using glutathione $(\mathrm{GSH})$. The product was purified by centrifugation. The radiolabeling and nanocluster stability of $68 \mathrm{Ga}$-labeled AuNCs was examined by incubating them with $10 \%$ rat serum in PBS. For large scale production and high dose labeling, the amount of reagent and the dose of $68 \mathrm{Ga}$ were multiplied according to the reaction volume. High dose labeling was performed by radioactive tracer method [2]. Results The size of the as-synthesized gold nanoclusters was around $1 \mathrm{~nm}$ (Figure 1a). The size did not change obviously when AuNCs were dispersed in DMEM and PBS for $48 \mathrm{~h}$ (Figure 1b). The labeling efficiency of $1 \mathrm{mCi}$ $68 \mathrm{Ga}$ in small batch of products $(10 \mathrm{~mL}, 5 \mathrm{mg} \mathrm{Au})$ was $86.67 \%$. For large scale production, one batch around one gram of AuNCs in $500 \mathrm{~mL}$ reaction solution could be produced. For radio labeling, it was found that the radiolabeling efficiency was not affected by the volume of reactants (Figure 2a). In the $100 \mathrm{~mL}$ reaction system (around $50 \mathrm{mg}$ AuNCs), when the radioactive activity increased from $100 \mathrm{mCi}$ to $1000 \mathrm{mCi}$, the labeling efficiency could still reach $75 \%$, which proved that this labeling method could be used to label high dose of radionuclides and meet the clinical requirements (Figure $2 \mathrm{~b}$ ). The labeling stability was examined by incubating $68 \mathrm{Ga}-\mathrm{AuNCs}$ in $10 \%$ normal rat serum in PBS for different time at $37^{\circ} \mathrm{C}$. As shown in Figure 2c, around 5\% of radioactivity was detached after the incubation for 90 min, indicating the excellent radiolabeling stability. Conclusions The novel radiolabeling method we report can realize the rapid, ligand-free labeling of radionuclides into gold nanoclusters with high labeling efficiency, overcoming the disadvantages of traditional ligand radiolabeling methods. In particular, the method can also achieve high dose of radionuclide labeling, which could promote the clinical translation of nuclear medicine based on gold nanoclusters.
\end{abstract}

References: [1] Irene F, María M, Juan P, Jesús R and Fernando H. (2018)Appl.Sci, 8, 1098. [2] Yongfeng Z, Lisa D, Deborah S, Matthew L C, Meng Y, et al. (2016)ACS Nano, 10, 5959-5970.

\section{Image/Figure:}


Image/Figure Caption: Figure 1. a) TEM image of AuNCs. Scale bar $20 \mathrm{~nm}$. b) Stability of the AuNCs dispersed in DMEM (red line) and PBS (black line). c) Photographs of AuNCs produced in $10 \mathrm{~mL}$ (left) and $100 \mathrm{~mL}$ volume (right). Figure 2. a) Labeling efficiency of 68Ga under various reaction volume. b) Labeling efficiency of $68 \mathrm{Ga}$ with various radioactivity in $100 \mathrm{~mL}$ reaction volume. c) Radioactive stability of $68 \mathrm{Ga}-\mathrm{AuNCs}$.

Complete Status: Complete

First Name: Benchao

Last Name: Zheng

Email: 729721158@qq.com

Organization: Shanghai jiaotong university

Country: China 


\title{
ID: GA437 \\ Gas-filled Protein Nanostructures as Cavitation Nuclei for Molecule-Specific Sonodynamic Therapy
}

\author{
Lin Song, The Hong Kong Polytechnic University, lin.song@connect.polyu.hk
}

Category: New Chemistry, Biology \& Bioengineering

\begin{abstract}
Body : Sonodynamic therapy (SDT) is a promising non-invasive therapeutic modality for cancer, based on the combination of a sonosensitizer and low-intensity ultrasound to generate cytotoxicity. It has the advantage of deep tissue penetration and high spatial resolution. The initiation of inertial cavitation is critical for ROS generation and subsequent cellular toxicity in SDT. Thus, precise control of the occurrence of cavitation is a key factor in increasing SDT's therapeutic efficacy. In this paper, we used recently reported gas-filled protein nanostructures (Bourdeau, R. W., Nature 2018; Shapiro, M. G., Nat Nanotechnol 2014) as nano-scaled acoustic cavitation nuclei to induce controlled cavitation. These gas vesicles (GVs) are nanobubbles derived from buoyant cyanobacteria, and have exceptional physical stability and EPR capability (Guohao, W., Biomaterials 2020). We first confirmed that cavitation could be acoustically induced at places where GVs were present and found the cavitation intensity depended on the ultrasound peak negative pressure. In addition, the production of ROS was dependent upon both the concentration of GVs as well as the duration of treatment, showing that cavitation and resulting ROS are controllable in our scheme. GVs were also functionalized with hyaluronic acid to target CD44+ cells, and SDT in vitro with these GVs led to increased ROS production and subsequently-enhanced cellular toxicity. In vivo, GVs showed an ability to specifically accumulate in the tumor site up to 12 hours after administration. The combined GVs-SDT treatment was found to significantly inhibit tumor growth and produce greater tumor damage and apoptosis than SDT or ultrasound alone without greater systemic toxicity. Thus, our results show that GV-mediated SDT treatment enabled targeted disruption of specific cells expressing a known surface marker within the area of insonation, providing a spatially-specific and targeted SDT treatment.
\end{abstract}

References: 1. R.W. Bourdeau, A. Lee-Gosselin, A. Lakshmanan, A. Farhadi, S.R. Kumar, S.P. Nety, M.G. Shapiro, Acoustic reporter genes for noninvasive imaging of microorganisms in mammalian hosts, Nature 553(7686) (2018) 86-90. 2. M.G. Shapiro, P.W. Goodwill, A. Neogy, M. Yin, F.S. Foster, D.V. Schaffer, S.M. Conolly, Biogenic gas nanostructures as ultrasonic molecular reporters, Nat Nanotechnol 9(4) (2014) 311-6. 3. G. Wang, L. Song, X. Hou, S. Kala, K.F. Wong, L. Tang, Y. Dai, L. Sun, Surface-modified GVs as nanosized contrast agents for molecular ultrasound imaging of tumor, Biomaterials 236 (2020) 119803.

\section{Image/Figure:}

https://www.xcdsystem.com/wmis/abstract/File6959/GA437_ImageFigure 0605051132.jpg 
Image/Figure Caption: Enhanced SDT mediated by functionalized GVs in vivo. (a) Photographic and fluorescent images of ICG-labeled GVs (Ex. 780 nm, Em. 845 nm). (b) Absorbance spectra of GVs, ICG, ICG-labeled GVs in phosphate-buffered saline (PBS). (c) In vivo NIR fluorescent imaging of tumor-bearing mice, taken at different times post-administration of of free ICG and ICG-labeled GVs. (d) Quantitative analysis of tumor intensity at different time-points. Bars represent the mean \pm SD from 4 independent experiments. (e) Tumor growth curves of SCC-7 tumor-bearing mice with different treatments. $\mathrm{n}=5$ mice per group, ${ }^{*} \mathrm{p}<0.05$. (f) Body weights of SCC-7 tumor-bearing mice after various treatments at different time-points. (g) Representative histological images of H\&E stained tumor slices collected from different groups. Scale bar represents $200 \mu \mathrm{m}$. (h) Representative images of a TUNEL assay on tumor slices collected from different groups. Scale bar represents $200 \mu \mathrm{m}$.

Full Name of Abstract's 1st Author : Lin Song

Complete Status: Complete

First Name: Lin

Last Name: Song

Email: lin.song@connect.polyu.hk

Organization: The Hong Kong Polytechnic University

Country: China 


\title{
ID: GA438 \\ A nano 'immune-guide' recruiting $T$ cells and modulating original macrophages ratio to enhance cancer Immunotherapy
}

\author{
Wenshe Sun, Shanghai Jiao Tong University, weihaihahaha@163.com
}

\section{Category: Oncology}

\begin{abstract}
Body : Introduction Tumor immunosuppressive microenvironment is a great obstacle for tumor immune therapy $[1,2]$. Artificially modulating the type, density and location of immune cells within the tumor microenvironment could restrain tumor growth and promote current immunotherapy efficiency. Herein, an iron oxide nanoparticles based 'immune-guide' are fabricated by functionalization of the nanoparticles with hyaluronic acid (HA), an extracellular matrix components that can target various CD44-overexpressed tumors and mediate adhesion and migration of multiple types of immune cells[3]. Its capability to recruit immune cells (T cell and $\mathrm{B}$ cell) to tumor sites and regulate the proportion of different original tumor-related macrophages (TAMs), and thus to inhibit tumor growth and facilitate cancer immunotherapy were carefully evaluated. Materials and methods The nano guide was prepared by first modification of $\mathrm{Fe} 3 \mathrm{O} 4$ with poly dopamine and then functionalization with hyaluronic acid (HAPDA@Fe3O4). Its specificity for CD44 overexpressed tumor cells and capability of recruiting immune cells and reprograming macrophage were examined in vitro. Its tumor targeting efficiency was evaluated by magnetic resonance imaging (MRI) and photoacoustic imaging (PAI). The immune cell recruitment and TAM conversion efficiency of HA-PDA@Fe3O4 were examined by flow cytometry after the tumor bearing mice were i.v. injected with the particles. Inhibition of the primary tumors and its metastases were also examined by treating the mice with HA-PDA@Fe3O4 alone or in combination with immune checkpoint inhibitors. Results and discussion HA-PDA@Fe3O4 assembled into cluster with hydrodynamic diameter about $200 \mathrm{~nm}$. $\mathrm{T} 2$ relaxivity $(\mathrm{r} 2)$ of the clusters was $34 \mathrm{~s}-1 \mathrm{mM}-1$. PDA coating improved the photothermal conversion performance of Fe3O4. HA-PDA@Fe3O4 could specifically target CD44overexpressed breast cancer, recruit immune cells and tilt M2 macrophage to M1 phenotype. After intravenous injection, HA-PDA@Fe3O4 could effectively accumulated in tumor, and compared with the control group, the proportion of total immune cells increased from $9 \%$ to $14 \%$. Among which, the most obviously change was T cells, which increased from $7 \%$ to $21 \%$, followed by B cells, which increased from $1 \%$ to $4 \%$, whereas, the proportion of tissue-resident macrophage decreased dramatically, from $23 \%$ to $4 \%$. As a result, tumor growth and metastases in lung were inhibited after the tumor-bearing mice were treated with HA-PDA@Fe3O4 alone, and completely eliminated in combination with immune checkpoint inhibitor PD-1and CD47. Conclusion: HA-PDA@Fe3O4 could penetrate and accumulate in the depth of tumor, and increase tumor inflammation, turning tumor into immune hot phenotype though recruit large amounts of T cells. Moreover, HA-PDA@Fe3O4 could also regulate the proportion of different original TAMs. Consequently, the biocompatible HA-PDA@Fe3O4 nanoparticles could synchronize facilitate $\mathrm{T}$ cell and macrophage based immunotherapy to efficiently inhibit tumors. More importantly, HA-PDA@Fe3O4 could also disturb pre-metastatic niches to prevent lung metastasis. Our study implicated HA-PDA@Fe3O4-based immunotherapy has tremendous
\end{abstract}


potential for clinical diagnostic and therapeutic applications. Acknowledgements: This study was supported by the National Natural Science Foundation of China (81571729), Innovation Research Plan supported by Shanghai Municipal Education Commission (ZXWF082101) and Med-Engineering Crossing Foundation from Shanghai Jiao Tong University (YG2017ZD05).

References: 1. Binnewies, M.; Roberts, E. W.; Kersten, K. et al. Understanding the tumor immune microenvironment (TIME) for effective therapy. Nature Medicine 2018, 24 (5), 541-550 2. Mantovani, F; Marchesi, A; Malesci et al. Tumour-associated macrophages as treatment targets in oncology. Nat Rev Clin Oncol. 2017 14(7):399-416. 3. McCarthy, J. B.; El-Ashry, D.; Turley, E. A., Hyaluronan, Cancer-Associated Fibroblasts and the Tumor Microenvironment in Malignant Progression (vol 6, 48, 2018). Frontiers in Cell and Developmental Biology 2018, 6.

\section{Image/Figure:}

https://www.xcdsystem.com/wmis/abstract/File6959/GA438_ImageFigure_0605095336.png

Image/Figure Caption: Figure 1: (a) MR imaging and Photoacoustic imaging of tumors after intravenous injection of HA-PDA@Fe3O4. (b) The percentage change of total immune cells and $\mathrm{T}$ cells in tumor after different treatments. (c) Different original macrophage proportion and expression of polarization markers after treatments implementation. (d) Tumor growth curve after different treatments.

Complete Status: Complete

First Name: Wenshe

Last Name: Sun

Email:weihaihahaha@163.com

Organization: Shanghai Jiao Tong University

Country: China 


\title{
ID: GA439 \\ In Vivo Photoacoustic Imaging of Gold Nanostars Distribution and Photothermal Effect Efficacy in Triple-Negative Breast Cancer
}

\author{
Wing Sum Cherry Lau, The Hong Kong Polytechnic University, cherrylauws@yahoo.com
}

\section{Category: Oncology}

\begin{abstract}
Body : BACKGROUND. Potentials of gold nanoparticles mediating photothermal therapy have expanded options for treating triple-negative breast cancer (TNBC) with enhanced curative efficacy. Gold nanostars demonstrate superior photothermal conversion efficiency for ablating tumor cells such as ovarian cancer and breast cancer, yet the non-invasive monitoring of gold nanostars in tumor at therapeutic dose remain unclear. AIM. This research aims at assessing the temporal uptake and distribution of PEGylated gold nanostars (pAuNSs) in TNBC tumour by photoacoustic imaging (PAI), and thus optimizing the pAuNSs-mediated photothermal effect. METHODS. In vivo photoacoustic imaging of 4T1 orthotopic mouse model was conducted using the Multispectral Optoacoustic Tomography (MSOT) system, at a series of time intervals upon intravenous administration of pAuNSs $(1,2,4,24,48 \mathrm{~h})$. Laser exposure of the tumor was carried out subsequent to the injection of pAuNSs, using a $808 \mathrm{~nm}$ laser $(1 \mathrm{~W} / \mathrm{cm} 2)$ irradiating the tumor for 3 min twice and a 2 min rest in between, while the surface temperature of tumor was recorded by thermal imaging camera. RESULTS. Post-injection signals of pAuNSs in tumor elevated significantly and remained similarly prominent during $2-24 \mathrm{~h}$. Signal intensity in tumor reached a four-fold increment at $24 \mathrm{~h}$ comparing with the pre-injection value. The tumor-tomuscle ratio obtained from MSOT signal quantification was analysed and displayed a close correlation with biodistribution data. The photothermal conversion effect was compared between laser irradiation at $2 \mathrm{~h}$ or $24 \mathrm{~h}$ post-injection. The tumor temperature reached up to $60^{\circ} \mathrm{C}$ and $55^{\circ} \mathrm{C}$ respectively, whereas the laser-only group increased from $31^{\circ} \mathrm{C}$ to $37^{\circ} \mathrm{C}$. The pAuNSs accumulated at these two timepoints effectively converted laser energy into heat generation in the tumor, at temperature that is sufficient for thermally ablating tumor cells by inducing cell death. CONCLUSION. PAI successfully tracked the pAuNS uptake in TNBC tumor both quantitatively and qualitatively, which helped identifying the optimal photothermal therapy window and predicting the anti-tumor effect. Not only does this theranostic nanoplatform achieve image-guided cancer therapy, but it may also facilitate the prognosis of TNBC patients.
\end{abstract}

\section{Image/Figure:}

https://www.xcdsystem.com/wmis/abstract/File6959/GA439 ImageFigure_0614121644.png

Image/Figure Caption: Fig. 1. MSOT maximum intensity projection images of the pAuNSs (green) distribution in orthotopic 4T1 tumor at different time intervals upon i.v. injection. Fig. 2. Thermal images of orthotopic 4T1 tumor-bearing mice receiving laser irradiation only or mediated by the administration of pAuNSs ( $2 \mathrm{~h}$ p.i., 24 p.i.).

Full Name of Abstract's 1st Author : Lau Wing Sum 
Complete Status: Complete

First Name: Wing Sum Cherry

Last Name: Lau

Email: cherrylauws@yahoo.com

Organization: The Hong Kong Polytechnic University

Country: Hong Kong SAR 


\title{
ID: GA440 \\ Non-Invasive Assessment of Treatment Response in Drug-resistant Colorectal Cancer by MRMI of Extradomain-B Fibronectin
}

\author{
Amita Vaidya, Case Western Reserve University, amv74@case.edu
}

\section{Category: Oncology}

\begin{abstract}
Body : Multidrug resistance represents a complex challenge for therapy and diagnostics of colorectal cancer (CRC). While contrast-enhanced MRI is routinely used for management of CRC, clinically used non-targeted gadolinium-based contrast agents (GBCAs) are limited in their ability to identify molecular changes in heterogenous drug-resistant tumors, causing delayed detection of negative response to therapy and eventual relapse1. To address this challenge, we evaluated the feasibility of monitoring therapeutic response of drug-resistant CRC tumors with MR molecular imaging (MRMI) using a targeted GBCA specific to the abundantly expressed extracellular matrix oncoprotein, extradomain-B fibronectin (EDB-FN)2. Although EDB-FN is known to be significantly upregulated in a plethora of neoplasms including CRC3,4, its expression profile in drug-resistant $\mathrm{CRC}$ remains unexplored. We discovered significant upregulation of EDB-FN at the mRNA and protein levels with concomitant increase in invasion of drug-resistant DLD1-DR cells generated from parent DLD-1 cells by long-term treatment with 5'-Fluorouracil (Fig. 1A). The 2 cell lines were then subcutaneously implanted in the flanks of athymic nude mice ( $n=5$ per group) to generate tumor xenografts. MRMI of EDB-FN was performed using macrocyclic ZD2-targeted contrast agent MT218 [ZD2-N3-Gd(HP-DO3A)]5 on 3T MRS 3000 scanner (MR Solutions). T1-weighted MR images were obtained before and 20 min post-injection of $40 \mu \mathrm{mol} / \mathrm{kg}$ dose of MT218 using an axial fast spin-echo sequence $(\mathrm{TR}=$ $305 \mathrm{~ms}, \mathrm{TE}=11 \mathrm{~ms}, \mathrm{FA}=90^{\circ}, \mathrm{FOV}=40 \mathrm{~mm} \times 40 \mathrm{~mm}$, slice thickness $=1 \mathrm{~mm}$, slice number $=15$, $\mathrm{Nav}=2$, matrix $=256 \times 256$ ) with respiratory gating and a mouse short quad coil. MRMI with MT218 facilitated accurate non-invasive assessment of drug resistance, with robust signal enhancement (Fig. 1B) and over 2.2-fold increase in contrast-noise ratio (CNR) observed in the EDB-FN-rich DLD1-DR tumors over the non-resistant DLD1 counterparts (Fig. 1C-D). The feasibility of non-invasive therapeutic monitoring using MRMI of EDB-FN was then evaluated in DLD1-DR tumor-bearing mice treated with the pan-AKT inhibitor MK2206-HC16 or vehicle DMSO ( $\mathrm{n}=5$ per group). The treated drug-resistant tumors failed to respond to therapy, demonstrating significantly increased tumor volumes $(109.7 \pm 12.21$ to $1250 \pm 199.3 \mathrm{~mm} 3)$, higher signal enhancement (Fig. 1E) and 1.4-fold elevated CNRs (Fig. 1F) in the 4-week followup MRMI scans, over the pre-treatment scans, which was further validated by strong EDB-FN staining observed in post-mortem histology. To date, this is the first and only report providing compelling in vivo evidence for exploiting drug resistance-mediated upregulation of EDB-FN as a molecular marker for imaging and therapeutic monitoring of CRC. Our research paves the way for development of novel molecular imaging technologies that can detect not only anatomical but also molecular changes in tumors and their microenvironment, early on in the treatment regimen, for improving the clinical outcomes of oncotherapies.
\end{abstract}


References: 1. Roberto G, et al. Advanced imaging of colorectal cancer: From anatomy to molecular imaging. Insights Imaging. 2016. 285-309. 2. White E. et al. New insights into form and function of fibronectin splice variants. J Pathol. 2008. 216:1-14. 3. Han Z \& Lu ZR. Targeting Fibronectin for Cancer Imaging and Therapy. J Mater Chem B. 2017. 639-54. 4. Santimaria, et al. Immunoscintigraphic detection of the ED-B domain of fibronectin, a marker of angiogenesis, in patients with cancer. Clin Cancer Res. 2003. 9:571-9 5. Ayat N, et al. Optimization of ZD2 Peptide Targeted Gd(HP-DO3A) for Detection and Risk-Stratification of Prostate Cancer with MRI. ACS Med Chem Lett. 2018. 9:730-35. 6. Agarwal E, et al. Akt inhibitor MK-2206 promotes anti-tumor activity and cell death by modulation of AIF and Ezrin in colorectal cancer. BMC Cancer. 2014. 145.

\section{Image/Figure:}

https://www.xcdsystem.com/wmis/abstract/File6959/GA440_ImageFigure 0605042407.png

Image/Figure Caption: Drug-resistant DLD1-DR cells generated by 5'-FU treatment in DLD-1 cells show A. increased invasion and EDB-FN upregulation, measured by EDB-FN-specific peptide probe ZD2-Cy5.5 binding. T1-weighted 2D Spin echo images were obtained before and $20 \mathrm{~min}$ post-injection of $40 \mu \mathrm{mol} / \mathrm{kg}$ dose of MT2 18 in athymic nu/nu mice show B. higher signal enhancement, C. increased CNR, and D. strong EDB-FN immunohistochemical staining in DLD1-DR tumors, over the non-resistant DLD-1 tumors. MRMI of DLD1-DR tumors treated with MK2206-HCl showed failed response to therapy, demonstrated by E. increased signal enhancement in representative T1-weighted axial image, and F. significantly higher average and individual CNRs at post-treatment, compared to the pre-treatment scans (M\#1-M\#5: mouse IDs).

Complete Status: Complete

First Name: Amita

Last Name: Vaidya

Email: amv74@case.edu

Organization: Case Western Reserve University

Country: United States 


\title{
ID: GA441 \\ Local aromatase expression in the breast is linked to breast density
}

\author{
Anat Biegon, Stony Brook University, anat.biegon@stonybrookmedicine.edu
}

\section{Category: Oncology}

\begin{abstract}
Body : Background: High breast density is an established risk factor for breast cancer, though the underlying mechanism it is not entirely clear. Estrogens are known to increase breast density, as evidenced by increased breast density during the luteal phase of the menstrual cycle, when estrogen levels are high, and decreased density after menopause, when estrogen levels are low. Aromatase is the enzyme catalyzing the last and obligatory step in estrogen biosynthesis in all organs; and a substantial proportion (approximately two thirds) of breast tumors in postmenopausal women overexpress this enzyme relative to non-malignant breast tissue. Recently, we found that aromatase expression in the unaffected breast of women with breast cancer was significantly higher than values in breasts of healthy women (1). Hypothesis: High expression of aromatase in the breasts of postmenopausal women and the resultant locally produced estrogen drive increased breast density and contribute to the risk of malignancy. Methods: Ten postmenopausal women with newly diagnosed, biopsy-confirmed breast cancer were recruited for a breast imaging PET-CT study with [11C]vorozole, a validated aromatase tracer $(2,3)$. PET-CT images were interpreted by a nuclear medicine physician. Quantitative assessments of radiotracer uptake was perfomred using MIM software, including size of lesion and mean and maximum standardized uptake vaous (SUV). Regions of interest were created for the unaffected (contralateral) breasts, lesions and the breast around the tumor. Breast density by area (percent death, PD) was assessed with Cumulus software applied to mediolateral oblique (MLO) mammographic view for both breasts, obtained during clinical workup. The SUVs from both breasts (contralateral whole breast and unaffected area around tumor) were split into two groups, classified as high aromatase (median or above) and low aromatase (below median) and the PD of the two groups was compared (Student's t-test, 2 tailed). In addition, we examined the relationship between individual breast SUVs and PD using linear regression. Results: The mean PD in the high aromatase group was two-fold higher than the PD in the low aromatase group $(\mathrm{p}=0.006)$. There was also a statistically significant, positive correlation between SUV and PD $(\mathrm{R}=0.58, \mathrm{p}=0.015)$. Discussion and conclusions: Our results show that high expression of aromatase in the breasts of postmenopausal women with breast cancer explains a significant proportion of the variance in breast density in this population, and the two measures are positively and significantly correlated. These findings suggest that high breast density in postmenopausal women reflects high levels and activity of aromatase, which provide for high levels local estrogen, capable of accelerating the growth of estrogen receptor positive tumors via an intracrine/paracrine mechanism. If this finding is replicated in a larger cohort, it may mean that high breast density could be used in the future to inform patient selection for preventive or adjuvant treatment with aromatase inhibitors.
\end{abstract}

References: 1. Biegon A, Shroyer KR, Franceschi D, Dhawan J, Tahmi M, Pareto D, Bonilla P, Airola K, Cohen J. Initial studies with [11C]vorozole positron emission tomography detect over- 
expression of intra-tumoral aromatase in breast cancer. J Nucl Med. 2019 Nov 22. pii: jnumed.119.231589. doi: 10.2967/jnumed.119.231589. (2019) 2. Kim SW, Biegon A, Katsamanis ZE, Ehrlich CW, Hooker JM, Shea C, Muench L, XuY, King P, Carter P, Alexoff DL, Fowler JS. Reinvestigation of the synthesis and evaluation of [N-methyl-(11)C]vorozole, a radiotracer targeting cytochrome P450 aromatase. Nucl Med Biol.;36(3):323-34. (2009) 3. Biegon A, Alexoff DL, Kim S-W, Logan J, Pareto D, Schlyer D, Wang G-J and Fowler J.S. Aromatase Imaging with [N-methyl-C-11]vorozole PET in Healthy Men and Women. J. Nuc Med. 56:580-585(2015)

\section{Image/Figure:}

https://www.xcdsystem.com/wmis/abstract/File6959/GA441_ImageFigure 0607075507.jpg

Image/Figure Caption: Relationship between aromatase availability and breast density in postmenopausal women with breast cancer A. PET image showing both breasts, arrow indicates tumor location on mammography. Pseudocolored using he rainbow spectrum, SUV scale on right. B. Mammpgram of unaffected breast $(\mathrm{B} \& \mathrm{~W})$ thresholded and binarized with cumulus software (red). Resultant percent dense breast (PD) shown on right. C. Scattergram of PD values in the high aromatase (h) and low aromatase (l) groups. D. Relationship between aromatase SUV in the breast (ASUVB) and PD. Linear fit (red line) : $\mathrm{PD}=-4.7+20 \mathrm{X}$ ASUV

\section{Complete Status: Complete}

First Name: Anat

Last Name: Biegon

Email: anat.biegon@stonybrookmedicine.edu

Organization: Stony Brook University

Country: United States 


\title{
ID: GA442 \\ Dual-drug phase-changing nanodroplets for ultrasound-guided cancer combination therapy
}

\author{
Catalina Spatarelu, Dartmouth College, catalina-paula.spatarelu.th@dartmouth.edu
}

\section{Category: Oncology}

\begin{abstract}
Body : Phase-changing nanodroplets are nanosized constructs with perfluorocarbon liquid cores that can be remotely vaporized to form gaseous microbubbles and expel their contents. This process can generate shock waves, shear forces, and microstreams in the interstitial tumor space, enhancing intratumoral drug delivery1. Their nanometric size brings several advantages over microbubbles, namely the ability to extravasate within the tumor tissue, and longer in vivo circulation times. After vaporization, the presence of microbubbles within the interstitial tumor space allows for ultrasound images of the agents to be obtained. Therefore, linking the ultrasound contrast and the delivery of therapeutics enables image-guided therapy with high temporal and spatial resolution and good sensitivity. This study focuses on the development and functionality of double-drug loaded nanodroplets towards ultrasound-guided co-delivery of doxorubicin and paclitaxel for combination therapy. As combination therapy was shown to be more effective for various types of cancer chemotherapy regimens $2-4$, dual-loaded nanodroplets were developed for this project. The benefits of co-encapsulation include the normalization of the pharmacokinetics of the two agents, paclitaxel and doxorubicin, that have different solubility and hydrophilicity properties. Moreover, the ratio between the two can be controlled from the design stage. Phase-changing nanodroplets (DDDs) were obtained by a double emulsion technique. The core emulsion consisted of aqueous droplets of doxorubicin dispersed in liquid perfluoropentane, while the shell was made up from a lipid layer intertwined with paclitaxel molecules and a NIR dye to allow for optical droplet vaporization (ODV). In vitro testing focused on determining the droplets' size and stability by DLS and optimizing their composition. The ultrasound contrast capabilities of the droplets were followed by imaging with a L22-8 Verasonics ultrasound transducer (18 MHz, 128 elements) before and after triggering events. For ODV a pulsed Phocus Opotek Mobile HE tunable laser system, operating at $1064 \mathrm{~nm}$ and $0.38 \mathrm{~J} / \mathrm{cm} 2$ was used. The activation depth of the droplets was investigated by using fresh chicken breast tissue of increasing thickness on top of droplets-containing polyacrylamide phantoms. For estimating the biological effects of DDDs, MCF 7 breast cancer cells and $\mathrm{FaDu}$ head and neck cancer cells were incubated with different formulations: free drugs, empty droplets and loaded droplets and results for all the groups were compared. Dual-drug droplets, containing paclitaxel (encapsulation efficiency 78\%) and doxorubicin (encapsulation efficiency $60 \%$ ), that can release their load and turn into microbubbles upon exposure to pulsed NIR light (Fig 1A) were synthesized. Their mean size was measured at $321 \pm 10 \mathrm{~nm}$. Phantom studies showed the localized triggering of droplets' vaporization, proven by ultrasound signal from microbubbles (Fig 1B), as well as a linear relation between the activation and the relative concentration of droplets in phantoms $(\mathrm{R} 2=0.97)$. The highest depth at which these nanodroplets can be vaporized by NIR laser was shown to be in the range of $18-19 \mathrm{~mm}$. Cell studies indicated that activated droplets have the highest cytotoxicity compared to empty
\end{abstract}


droplets, free drug formulations, and inactivated droplets with statistical differences between all the groups, while laser exposure alone did not account for a significant viability decrease (Fig 1C). The study investigating the timing of activation with respect to the incubation time revealed that for 30 minutes or more of incubation, the activation of droplets had a much higher effect on cell viability than the 10 minutes timeframe. Overall, the results indicate a promising and versatile platform, that could be used for combination therapy of several types of solid tumors. In vivo validation studies are ongoing.

References: 1. Xing, L. et al. Ultrasound-Mediated Microbubble Destruction (UMMD) Facilitates the Delivery of CA19-9 Targeted and Paclitaxel Loaded mPEG-PLGA-PLL Nanoparticles in Pancreatic Cancer. Theranostics 6, 1573-1587 (2016). 2. Gianni, L. et al. Phase III trial evaluating the addition of paclitaxel to doxorubicin followed by cyclophosphamide, methotrexate, and fluorouracil, as adjuvant or primary systemic therapy: European Cooperative Trial in Operable Breast Cancer. J. Clin. Oncol. 27, 2474-81 (2009). 3. Hu, C.-M. J. \& Zhang, L. Nanoparticle-based combination therapy toward overcoming drug resistance in cancer. Biochem. Pharmacol. 83, 1104-1111 (2012). 4. Xu, X., Ho, W., Zhang, X., Bertrand, N. \& Farokhzad, O. Cancer nanomedicine: from targeted delivery to combination therapy. Trends Mol. Med. 21, 223-232 (2015).

\section{Image/Figure:}

https://www.xcdsystem.com/wmis/abstract/File6959/GA442_ImageFigure_0608074003.png

Image/Figure Caption: Fig 1. A. Schematic of nanodroplets' ODV; B. Differential ultrasound image of phantoms with DDDs activated by pulsed NIR light within the laser spot (marked with the white circle); C. MTT results of cells exposed to different formulations of loaded droplets or free drugs.

Complete Status: Complete

First Name: Catalina

Last Name: Spatarelu

Email: catalina-paula.spatarelu.th@dartmouth.edu

Organization: Dartmouth College

Country: United States 


\title{
ID: GA443 \\ Synthesis, stability and binding studies of 89Zr-complexes of DFO/HOPO-CC49 antibody constructs for PET imaging applications
}

\author{
Michael Phipps, City University of New York, mphipps@gradcenter.cuny.edu
}

\section{Category: Oncology}

\begin{abstract}
Body : Background/Objectives: The membrane-bound glycoprotein complex known as tumor associated glycoprotein 72 (TAG-72) is overexpressed in many adenocarcinomas including colorectal carcinoma [1]. The CC49 monoclonal antibody (also known as minretumomab) binds TAG-72 and was one of the first radiolabeled antibodies to reach clinical trials [2,3]. Deglycosylated TAG-72 does not bind to CC49, suggesting that the epitope recognized by CC49 is likely a glucan. Desferrioxamine B (DFO) is an acyclic hexadentate siderophore ligand that has been used in various work to create $89 \mathrm{Zr}$ based imaging agents. 3,4,3-(LI-1,2-HOPO) (HOPO) is an acyclic octadentate hydroxypyridonate based chelator that has been demonstrated as an effective chelator for hard ions and has garnered interest for use in imaging and therapy, and in the treatment of heavy metal toxicity by chelation therapy $[4,5]$. HOPO has demonstrated superior in vivo stability with $89 \mathrm{Zr}$ over DFO, where some demetallation of the osteophilic $89 \mathrm{Zr}$ from DFO resulted in bone uptake several times greater than [89Zr]-HOPO [4]. Positron emission tomography (PET) images taken using [89Zr]-HOPOtrastuzumab in breast cancer models using BT474 xenografted mice, and it exhibited high in vivo stability [4]. Therefore, $89 \mathrm{Zr}$-CC49 constructs may be effective imaging agents for cancers overexpressing TAG-72. Our investigation created such constructs and evaluated their stability and their binding to TAG-72 in vitro. Methods: Bifunctional DFO-NCS and HOPO-NCS were used to synthesize DFO-CC49 and HOPO-CC49 by bioconjugation to lysine residues on CC49. Each ligand-antibody construct was radiolabeled with $\geq 100 \mathrm{uCi} 89 \mathrm{Zr}$. The radiolabeling reaction was purified by elution from a G-25 column with phosphate buffered saline (PBS) solution. Site specific binding of CC49 was demonstrated by a blocking assay in vitro using TAG-72 expressing LS174T cells. Stability studies in PBS, serum, and whole blood will be performed to evaluate and compare [89Zr]-DFO-CC49 and [89Zr]-HOPO-CC49. Results: Both DFO-CC49 and HOPO-CC49 exhibited comparable labeling with $89 \mathrm{Zr}$ at $37 \mathrm{oC}$ with $>90 \%$ radiochemical yield after 1 hour. Purification resulted $>98 \%$ radiochemical purity and a specific activity of 3-4 mCi/mg mAb. The cell block assay verified site specific binding of CC49. A preliminary stability study with [89Zr]-HOPO-CC49 in PBS at 37 oC demonstrated $89 \%$ stability after $7 \mathrm{~d}$. Conclusions/Outlook: We have synthesized HOPO and DFO conjugated CC49 constructs and are evaluating the stability of their $89 \mathrm{Zr}$-complexes in vitro.
\end{abstract}

References: [1] Scott, A.M., et al. Nat. Rev. Cancer, 2012, 12, 278-287 [2] Sheer, D.G., et al. Cancer Res., 1988, 48, 6811-6818 [3] Forero, A., et al. Cancer Biother. Radio., 2005, 20, 467478 [4] Deri, M.A., et al. Bioconjugate Chem., 2015, 26, 2579-2591 [5] Deblonde, G.J.-P., et al. New J. Chem., 2018, 42, 7649-7658

\section{Image/Figure:}


https://www.xcdsystem.com/wmis/abstract/File6959/GA443_ImageFigure_0614032418.png

Image/Figure Caption: Table 1: Radiolabeling conditions for CC49 conjugates with 89Zr

Complete Status: Complete

First Name: Michael

Last Name: Phipps

Email: mphipps@gradcenter.cuny.edu

Organization: City University of New York

Country: United States 


\title{
ID: GA444 \\ Electron paramagnetic resonance measurement of changes in tumor microenvironment after metastatic potential enhancement
}

\author{
Agnieszka Drzal, Jagiellonian University, agnieszka.drzal@doctoral.uj.edu.pl
}

\section{Category: Oncology}

\begin{abstract}
Body : Introduction Cancer cells are able to form metastases in a multi-step process where transforming growth factor $\beta$ (TGF- $\beta$ ) and cell migration play an important role. The aim of this study was to investigate two approaches to enhance tumor metastasis in orthotopic murine breast cancer model and the changes they introduce in the tumor microenvironment in vivo. Material and Methods C57bl mice $(\mathrm{N}=30)$ were inoculated into the mammary fat pad with E0771 murine breast cancer cells. Three variants of cells were used. The first one was cultured in standard conditions (control, $N=10)$, the second one was treated with TGF- $\beta(1 \mathrm{ng} / \mathrm{ml})$ for 4 weeks $(\mathrm{N}=10)$, and the last one was clonally selected by double transmigration through microporous membranes $(\mathrm{N}=10)$. In vivo experiments involved tumor vasculature assessment with 3D ultrasound Power Doppler imaging, 2D tumor redox state imaging with electron paramagnetic resonance (EPR) technique and two spin probes (one extra- and one intracellular), tumor pO2 measurement with EPR oximetry and Oxychip as an oxygen sensor and EPR measurement of $\mathrm{pH}$ and glutathione concentration using RSG and RSSR probes respectively. Additionally, ex vivo epithelial-mesenchymal transition (EMT), angiogenesis and oxidative stress biomarkers were assessed. Results and Discussions The highest number of metastases was found in mice inoculated with cells after clonal selection. However, mice injected with cells after the TGF- $\beta$ treatment were also characterized by elevated metastatic potential compared to the control group. Results from EPR measurements and Doppler ultrasonography show significant differences between metastatic and non-metastatic tumors. These findings are in agreement with immunohistochemistry and Western blot data showing enhanced oxidative stress, microvascularization and EMT markers in more invasive tumors. Conclusion Both clonally selected invasive cells and the cells treated with TGF- $\beta$ gave rise to more metastatic tumors compared to the control. More aggressive tumors were characterized by a slower growth rate, higher vascularization, and indications of oxidative stress.
\end{abstract}

Complete Status: Complete

First Name: Agnieszka

Last Name: Drzal

Email: agnieszka.drzal@doctoral.uj.edu.pl

Organization: Jagiellonian University

Country: Poland 


\title{
ID: GA445 \\ Peptide-based 68Ga PET radiotracer for noninvasive imaging of PD-L1 expression in non-small cell lung cancer patients
}

\author{
Xin Zhou, Peking university cancer hospital, zhouxinbjmu@163.com
}

\section{Category: Oncology}

\begin{abstract}
Body : Background: Immune checkpoint inhibitors (ICIs) are now a mainstay of advanced lung cancer treatment1, 2. Responses occur in a substantial fraction of patients and are frequently durable and even curative. Currently, the expression of PD-L1 tested by means of immunohistochemistry represents the most validated predictor of an ICI response, which is invasive and does not represent the heterogeneity of the whole body3. Radiolabeled antibodies and adnectin-derived small proteins have been investigated as imaging agents in preclinical and clinical studies4-9. Here, we report a peptide-based positron emission tomography (PET) imaging agent, 68Ga-NOTA-WL12, and an assessment of the safety, radiation dosimetry, and imaging characteristics of this radiopharmaceutical, a correlation of the tumor 68Ga-NOTAWL12 uptake with the PD-L1 immunohistochemistry results, and a comparison of 68Ga-NOTAWL12 with 18F-FDG in advanced non-small cell lung cancer (NSCLC) patients. Methods: Nine patients (mean age, 65.4 years; 8 males, 1 female) diagnosed with NSCLC were enrolled in this study (ethical approval No. 2019 KT62). Primary tumor PD-L1 expression was measured by immunohistochemistry (antibody clone, 22C3). WL12 was radiolabeled with 68Ga coupled with NOTA. The activity was $1.9-3.7 \mathrm{MBq} / \mathrm{kg}$ when administered by intravenous injection, corresponding to 10-20 $\mu \mathrm{g}$ of radiolabeled NOTA-WL12 and 0-120 $\mu \mathrm{g}$ of WL12 peptide to reduce liver uptake. PET/CT imaging was performed at 1 and $2 \mathrm{~h}$ after injection (two patients underwent imaging at only $1 \mathrm{~h}$ for physical reasons). The radiation dosimetry calculations were evaluated, and all patients were monitored for adverse events. 18F-FDG PET/CT scans were obtained within a week, and 3 of 9 patients underwent reexamination after immunotherapy.
\end{abstract} Results: No drug-related adverse events occurred in this study. The mean effective dose was 1.77 $\times 10-2 \pm 3.89 \times 10-3 \mathrm{mSv} / \mathrm{MBq}$. Tracer uptake was mainly physically distributed in the liver, spleen and kidneys. The liver uptake gradually declined with increasing cold precursor. The ratio of tumor to background (blood pool, lungs, and muscle) did not differ between $1 \mathrm{~h}$ and $2 \mathrm{~h}$. The one-hour primary SUVpeak correlated with the PD-L1 immunohistochemistry results $(\mathrm{r}=$ $0.9349, \mathrm{P}=0.002)$. The best parameter for FDG, i.e., SUVmean, showed a mild relationship $(\mathrm{r}=$ $0.6937, \mathrm{P}=0.0382$ ). Based on 3 cases, two patients treated with immunotherapy were evaluated as having achieved a "partial metabolic response", with mild to moderate WL12 uptake in the lesions, while one patient was rated as having "progressive disease", with nearly no WL12 uptake. Conclusion: This first-in-human study demonstrates that 68Ga-labeled anti-PD-L1peptide PET/CT imaging is safe and associated with acceptable dosimetry. The cold precursor benefits tumor visualization, and a delay of $1 \mathrm{~h}$ is sufficient for imaging. The uptake of $68 \mathrm{Ga}-$ NOTA-WL12 shows a good relationship with the PD-L1 immunohistochemistry results, while FDG shows a slight relationship(Fig. 1). The uptake of WL12 might indicate the efficacy of immunotherapy. 
References: 1. Gandhi L, Rodríguez-Abreu D, Gadgeel S, et al. Pembrolizumab plus Chemotherapy in Metastatic Non-Small-Cell Lung Cancer. The New England journal of medicine. 2018;378:2078-2092. 2. Socinski MA, Jotte RM, Cappuzzo F, et al. Atezolizumab for First-Line Treatment of Metastatic Nonsquamous NSCLC. The New England journal of medicine. 2018;378:2288-2301. 3. Hofman P. PD-L1 immunohistochemistry for non-small cell lung carcinoma: which strategy should be adopted? Expert Rev Mol Diagn. 2017;17:1097-1108. 4. Niemeijer AN, Leung D, Huisman MC, et al. Whole body PD-1 and PD-L1 positron emission tomography in patients with non-small-cell lung cancer. Nat Commun. 2018;9:4664. 5. Xing Y, Chand G, Liu C, et al. Early Phase I Study of a (99m)Tc-Labeled Anti-Programmed Death Ligand-1 (PD-L1) Single-Domain Antibody in SPECT/CT Assessment of PD-L1 Expression in Non-Small Cell Lung Cancer. J Nucl Med. 2019;60:1213-1220. 6. Donnelly DJ, Smith RA, Morin P, et al. Synthesis and Biologic Evaluation of a Novel (18)F-Labeled Adnectin as a PET Radioligand for Imaging PD-L1 Expression. J Nucl Med. 2018;59:529-535. 7. Bensch F, van der Veen EL, Lub-de Hooge MN, et al. (89)Zr-atezolizumab imaging as a non-invasive approach to assess clinical response to PD-L1 blockade in cancer. Nat Med. 2018;24:1852-1858. 8. Heskamp S, Hobo W, Molkenboer-Kuenen JD, et al. Noninvasive Imaging of Tumor PD-L1 Expression Using Radiolabeled Anti-PD-L1 Antibodies. Cancer Res. 2015;75:2928-2936. 9. Huisman M, Niemeijer A-L, Windhorst B, et al. Quantification of PD-L1 expression with [18F]BMS-986192 $\mathrm{PET} / \mathrm{CT}$ in patients with advanced stage non-small-cell lung cancer. Journal of Nuclear Medicine. 2020.

\section{Image/Figure:}

https://www.xcdsystem.com/wmis/abstract/File6959/GA445_ImageFigure_0607091826.png

Image/Figure Caption: The better linear relationship of 68Ga-NOTA-WL12 than 18F-FDG with PD-L1 TPS on IHC: In a sixty-eight-year-old male with advanced NSCLC, the PD-L1 TPS on IHC was $8 \%$. On 68Ga-NOTA-WL12 imaging, the tumor showed low uptake of tracer (a, b), while on $18 \mathrm{~F}-\mathrm{FDG}$ imaging, the tumor showed intense radioactive concentration (c). In an eight-year-old female with advanced NSCLC, the PD-L1 TPS on IHC was $80 \%$. On $68 \mathrm{Ga}$ NOTA-WL12 imaging, the tumor showed moderate uptake of tracer (d, e), while on 18F-FDG imaging, the tumor showed intense radioactive concentration (f).

Full Name of Abstract's 1st Author : Xin Zhou

Complete Status: Complete

First Name: Xin

Last Name: Zhou

Email: zhouxinbjmu@163.com

Organization: Peking university cancer hospital

Country: China 


\title{
ID: GA446 \\ MRSI-based preclinical Glioblastoma therapy follow-up: is the oscillatory pattern of response an immune system biomarker?
}

\author{
Ana Paula Candiota, Centro de Investigación Biomédica en red, \\ AnaPaula.Candiota@uab.cat
}

\section{Category: Oncology}

\begin{abstract}
Body : Introduction: Glioblastomas (GB) are malignant brain tumours with poor prognosis even after surgical resection and aggressive therapy. The participation of the immune system is key for sustained response [1]. We have used MRSI-based nosological images [2,3] for GB therapy response assessment through tumour responding index (TRI) calculation. An oscillatory TRI pattern (6-7 days) was shown in longitudinal studies. Glioma-associated microglia/macrophages (GAMs) constitute the most abundant non-tumour cell type in the GB microenvironment and can be activated and polarized into an anti-tumour (M1) or pro-tumour (M2) phenotype [4]. The purpose of our present work was to gain further insight into the contribution of immune cell populations to the MRSI spectral pattern changes recorded from
\end{abstract} Temozolomide (TMZ) - treated preclinical GL261 GB. Methods: C57BL/6 mice bearing GL261 GB tumours ( $\mathrm{n}=39$ ) were treated with TMZ in an immune-enhanced metronomic schedule (IMS) every 6 days, at $60 \mathrm{mg} / \mathrm{kg}(\mathrm{n}=23)$ [3], while the remaining $(\mathrm{n}=16)$ were administered with vehicle and used as controls. T2 $\mathrm{w}$ MRI and consecutive $14 \mathrm{~ms}$ volumetric TE MRSI were acquired every 2 days [1] and nosologic maps calculated [5]. Twenty mice ( $\mathrm{n}=10$ controls, $\mathrm{n}=10$ treated) were euthanized at TRI-guided time points for in vitro evaluation, while the remaining ones had longitudinal follow-up until endpoint or curation. Quantitative polymerase chain reaction (qPCR) was performed with genes associated to microglia/macrophage population (F4/80) and its polarization (NOS2 for M1 phenotype and CD206 for M2 phenotype). Results were normalized to two reference genes (HPRT and TPB). Immunostainings for CD3 (lymphocytes) and Iba-1 (microglia/macrophages) were performed in $\mathrm{n}=6$ additional mice [2]. Cured mice $(\mathrm{n}=8)$ were followed-up by T2w MRI and in case of non-tumour mass detection within one month of cure, a "re-challenge" experiment with GL261 was carried out. Results-Discussion: IMS-TMZ increased GL261 GB bearing mice survival, from $21 \pm 1$ days in untreated mice up to $295 \pm 279$ days, improving previous results [4]. TRI oscillations ( $6.2 \pm 1.5$ days, $n=11$, Fig. 1A) were in agreement with immune cycle length (Fig 1B). MRSI spectral changes could reflect immune system action involving lymphocytes and especially microglia/macrophages (GAMs), since they can represent up to $30 \%$ of GB mass [6]. Namely, immunohistochemistry shows CD3 and Iba-1 content significantly higher in responding zones. Significant differences were observed for F4/80 $(0.71 \pm 0.32$ vs $0.18 \pm 0.08$ relative expression; Fig. $1 \mathrm{C}$ and Nos $2 / \mathrm{CD} 206$ ratio $(0.26 \pm 0.16$ vs $0.11 \pm 0.09$ ratio; Fig. 1D) between responding and control tumours, respectively. Global GAMs population and M1/M2 ratio were higher in responding compared to control tumours. Since M1 and M2 have different metabolic profiles [7], this population difference could be one of the key causes for the differential MRSI-sampled pattern with time. This agrees with an increase in antitumour immune cells content at TRI-high peak times (Fig $1 \mathrm{~A}$ ). Regarding the re-challenged mice, only $1 / 8$ tumour grew after 10 days, which vanished again after being treated with only one 
additional IMS-TMZ dose. This oscillatory pattern may constitute an immune system activity biomarker for use in future work. Conclusions: Our results indicate that IMS-TMZ treated GL261 GB showed significantly more microglia/macrophages than untreated mice, with predominance of the M1 anti-tumour phenotype, which can be imaged non-invasively by MRSI. This can be of interest in pathologies/therapies in which immune system participation is foreseen. IMS-TMZ induced immune memory in GB cured mice, although the ongoing mechanism needs further clarification.

References: 1. Cloughesy TF et al. Nat Med (2019) 25:477-86 2. Arias-Ramos N et al. Metabolites (2017) 7:E20. 3. Wu S et al. NMR Biomed (2020) e4229. 4. Lisi L et al. Neurosci Lett (2017) 645:106-12. 5. Delgado-Goñi T et al. NMR Biomed (2016) 29:732-43. 6. Glass R \& Synowitz M. Acta Neuropathol (2014) 128:347-62. 7. Van den Bossche J et al. Trends Immunol (2017) 38:395-406

\section{Image/Figure:}

https://www.xcdsystem.com/wmis/abstract/File6959/GA446 ImageFigure 0607124551.jpg

Image/Figure Caption: Figure 1: A) Bottom: tumour volume (mm3, black line) and $\%$ of responding pixels (\% TRI, green line) for case C1264. Green columns indicate TMZ administration day. Top: nosological images superimposed to MRI. Color-coding: Blue, normal; Red, non-responding tumour; Green, responding tumour. Black arrows indicate TMZ administration days. B) Scheme of cancer immunity cycle, starting with cellular damage during treatment, release of immunogenic signals, antigen presentation, lymphocyte amplification followed by tumour infiltration, interaction with macrophages and cell killing. C) Nos2 to CD206 (M1/M2) ratio average in tumour from responding $(0.26 \pm 0.16, \mathrm{n}=10)$ and control $(0.11$ $\pm 0.09, \mathrm{n}=10)$ mice. Significant differences were observed with unpaired $\mathrm{t}$-test $(\mathrm{p}=0.025)$. Data are the mean \pm S.D.

\section{Complete Status: Complete}

First Name: Ana Paula

Last Name: Candiota

Email: AnaPaula.Candiota@uab.cat

Organization: Centro de Investigación Biomédica en red

Country: Spain 


\title{
ID: GA447 \\ 68Ga-NOTA- ZHER2:2891 PET/CT in the Evaluation of Breast Cancer: A Pilot Prospective Study
}

\author{
Jie Zang, Peking Union Medical College (PUMC) Hospital, Chinese Academy of Medical \\ Science and PUMC, 15901495106@163.com
}

\section{Category: Oncology}

\begin{abstract}
Body : Purpose: This prospective pilot study investigated the use of 68Ga-NOTAZHER2:2891, an affibody molecule targeting human epidermal growth factor receptor 2 (HER2), for evaluating breast cancer using PET/CT. Methods: With institutional review board approval and informed consent, 19 female patients suspected of breast cancer based on mammography or ultrasonography were recruited. Within 1 week before surgery, the patients underwent whole-body PET/CT scans at 60 minutes after intravenous injection of approximately $1.85 \mathrm{MBq}(0.05 \mathrm{mCi})$ per kilogram body weight of 68Ga-NOTA- ZHER2:2891 and 5.6MBq $(0.15 \mathrm{mCi})$ per kilogram body weight of $18 \mathrm{~F}-\mathrm{FDG}$, respectively. All patients were pathologically diagnosed with breast cancer and 12 cases had ipsilateral lymph node metastasis. Results: No radiopharmaceutical-related adverse event was observed in the patients. 68Ga-NOTAZHER2:2891 PET/CT detected the primary tumor well in 18/19 (94.7\%) patients, and the lymph node metastasis in 8/12 (66.7\%) of the patients. The SUVmax were1.78 \pm 1.14 in the breast cancer, and $1.12 \pm 1.67$ in the lymph node metastasis. The SUVmax of HER2(3+) tumors $(2.96 \pm 1.12)$ were significantly higher than those in HER2(-) or HER2 $(1+)$ tumors $(1.16 \pm 0.40$, $\mathrm{P}=0.004)$. There was a significant positive correlation between the SUVmax of 68Ga-NOTAZHER2:2891 and the HER2 expression level $(\mathrm{r}=0.637, \mathrm{P}=0.006)$. The normal breast tissue showed low 68Ga-NOTA- ZHER2:2891 binding, with a SUVmax of $0.45 \pm 0.14$ and a SUVmean of $0.25 \pm 0.07$. 18F-FDG PET/CT detected the primary tumor well in $19 / 19(100.0 \%)$ patients, and the lymph node metastasis in 8/12 (66.7\%) of the patients. There was no significant correlation between the SUVmax of 68Ga-NOTA- ZHER2:2891 and 18F-FDG $(\mathrm{P}=0.286)$. Conclusion: This pilot study indicated that the uptake of 68Ga-NOTA-ZHER2:2891 correlate with HER2 expression in breast cancer. 68Ga-NOTA- ZHER2:2891 PET/CT can provide specific, noninvasive evaluation of HER2 expression in breast cancer.
\end{abstract}

Complete Status: Complete

First Name: Jie

Last Name: Zang

Email: 15901495106@163.com

Organization: Peking Union Medical College (PUMC) Hospital, Chinese Academy of Medical Science and PUMC 
Country: China 


\title{
ID: GA448 \\ Terahertz wave irradiation promotes skin regeneration and new hair growths
}

\author{
Jung Sun Yoo, The Hong Kong Polytechnic University, jungsun.yoo1@gmail.com
}

\section{Category: New Chemistry, Biology \& Bioengineering}

\begin{abstract}
Body : Terahertz waves $(0.1 \mathrm{THz} \sim 10 \mathrm{THz})$ have attracted great interest with recent technological advances and are now the subject of interdisciplinary research. A variety of applications have shown initial promise for their biomedical use, particularly in broadband Terahertz based imaging including noninvasive or intraoperative cancer imaging and skin and cornea hydration sensing. However, efforts to reveal the Terahertz-induced effects at cellular and molecular levels, especially how biological objects interact with Terahertz wave having specific frequency and intensity, is scarce and remains challenging. Recent progress in Terahertz spectroscopy have shown that many important biomolecules have intrinsic vibrational resonances in the Terahertz range, implicating that appropriate Terahertz irradiation may direct biochemical reactions and biological energy transport $[1,2,3]$. In addition, mild inflammation response in mouse skin under Terahertz irradiation was reported recently, suggesting possibility of immune response mediated skin regeneration [4]. Herein, biological effects of Terahertz irradiation for skin regeneration and hair growth were investigated to develop a novel therapeutic technique for dermatological diseases. Hair loss, or alopecia, is a markedly increasing dermatological disease in aging society, involving an annual global market revenue of US\$85 billion and a growth rate of 5\%. Unfortunately, currently available therapeutic drugs such as Minoxidil and Finasteride have either low efficacy or significant adverse effects. Focusing on capability of Terahertz wave for nonionizing local irradiation and deep penetration to cover a whole hair follicle, skin exposure to Terahertz $(0.2 \mathrm{THz})$ was identified as an effective tool to promote cell proliferation and to activate hair stem cells. T-ray induced cell proliferation using Ki-67 marker was observed in the different aged mice (4- and 6-week-old balb/c male mice, each 3-4 mice per experimental group). Immunofluorescence staining and statistical analysis showed significantly increased expression of proliferation marker, Ki-67 over hair follicles and neighbouring skin regions in the T-ray illuminated group $(0.2 \mathrm{THz}$ wave for $10 \mathrm{~min})$ compared to the room-light illuminated control group. Interestingly, elevated enhancement was reported in 6-week old mice (more telogen and categen phases) compared to 4-week old mice (more anagen phases). This result indicates T-ray can promote proliferation of hair follicle cell components especially in the resting or regression phases of hair cycle. Hair stem cell regeneration was also measured with various hair stem cell markers, CD34 (bulge stem cell marker), $\beta$-catenin (Wnt/ $\beta$ catenin activity), P-cadherin (hair germ stem cell marker) in 6-week old balb/c mice. All three markers were upregulated within hair follicles, but not in the extra-follicular dermal macroenvironment. Particularly increased expression of $\beta$-catenin proves T-ray's regeneration capability because it is well reported that cyclic activation of hair stem cell involves periodic $\beta$ catenin activity and the eventual anagen initiation requires activation of Wnt/ $\beta$-catenin [5]. Altogether, we provide, for the first time, a powerful strategy to treat hair loss with high efficacy and no side effect using a new promising frequency regime, i.e., Terahertz wave. Such Terahertz
\end{abstract}


medicine will eventually revolutionize current clinical practice in dermatological treatment to improve overall patient outcome and reduce health care cost.

References: [1] X. Yang et al. Trends in Biotechnology 34(10) (2016) 810-824. [2] D.A. Turton et al. Nat Commun. 5:3999.(doi) (2014) 10.1038/ncomms4999. [3] G. Acbas et al. Nat Commun 5:3076.(doi) (2014) 10.1038/ncomms4076. [4] Y. Hwang et al. Opt Express. 22(10) (2014) 11465-75. doi: 10.1364/OE.22.011465. [5] E.R. Deschene et al. Science. 343(6177) (2014) 1353-6. doi: 10.1126/science. 1248373.

Image/Figure Caption: (A-F) Immunofluorescence analysis using markers for bulge stem cells (CD34, green in A and B), progency cells (P-cadherin, red in $\mathrm{E}$ and $\mathrm{F}$ ) and $\mathrm{Wnt} / \beta$-catenin activity ( $\beta$-catenin, red in $\mathrm{C}$ and $\mathrm{D})$. Active fluorescence signals (arrows) highlighting hair regeneration is found in the hair follicle inner layers of the THz-irradiated mice $(0.2 \mathrm{THz}, 10$ min). Scale bars, $100 \mu \mathrm{m}$. (G) Quantification of regeneration marker expression. All data were averaged from independent experiments ( $n=3$ to 4 mice per group). Statistical analysis was performed by unpaired t-test. $(* \mathrm{p}<0.05, * * \mathrm{p}<0.01, * * * \mathrm{p}<0.005)$. Error bars, mean \pm s.d.

Complete Status: Complete

First Name: Jung Sun

Last Name: Yoo

Email: jungsun.yoo1@gmail.com

Organization: The Hong Kong Polytechnic University

Country: Hong Kong SAR 


\title{
ID: GA449 \\ Assessment of Gender Differences in Thoracolumbar Bone Metabolism using [18F] Sodium Fluoride PET/CT
}

\author{
Helene Chesnais, Penn Medicine, chesnais@sas.upenn.edu
}

\section{Category: Computational \& Data Science}

\begin{abstract}
Body : Background: Structural imaging modalities such as DXA are currently used to assess bone quality in osteoporosis but do not provide any metabolic information about the status of the bone. The use of [18F]NaF PET/CT is increasing as a marker of bone metabolism. The purpose of this study was to analyze gender differences in the lumbar and thoracic spines of healthy males and females as measured by $[18 \mathrm{~F}] \mathrm{NaF}$ PET/CT SUVmean and SUVmax values. Methods: This retrospective study included 136 participants (68 females and 68 males, ages $21-75$ ) that had undergone $[18 \mathrm{~F}] \mathrm{NaF} \mathrm{PET} / \mathrm{CT}$. The operator guided computer software PMOD (PMOD Technologies LLC, Switzerland) was used to segment individual vertebra and calculate SUV. SUVmean and SUVmax of the thoracolumbar spine, lumbar spine, and thoracic spine were compared using linear regressions with age and BMI in all subjects, then stratified by gender, and by Young ( Results: In females but not males, SUVmean was negatively correlated with age ( $\mathrm{r}=-0.51$, PDiscussion: SUVmean demonstrated more consistent trends with age and BMI than did SUVmax in females, whereas SUVmax was a better predictor of age-related changes in males than in females. This can inform the analysis of future healthy, mixed-age and mixed-BMI cohorts for $[18 \mathrm{~F}] \mathrm{NaF} \mathrm{PET} / \mathrm{CT}$.
\end{abstract}

\section{Image/Figure:}

https://www.xcdsystem.com/wmis/abstract/File6959/GA449 ImageFigure 0614122120.png

Image/Figure Caption: Coronal maximum intensity projections of 18F-NaF PET/CT showing decreased vertebral $\mathrm{NaF}$ tracer uptake in an older female $(\mathrm{BMI}=23 \mathrm{~kg} / \mathrm{m} 2)$ compared to a younger female subject with similar BMI $(\mathrm{BMI}=25 \mathrm{~kg} / \mathrm{m} 2)$.

Complete Status: Complete

First Name: Helene

Last Name: Chesnais

Email: chesnais@sas.upenn.edu

Organization: Penn Medicine

Country: United States 


\title{
ID: GA450 \\ Long-Term Evaluation of Apoptosis Effect after Sevoflurane-Exposure in Neonatal Rat Brain
}

\author{
Xuan Zhang, NCTR, xuan.zhang@fda.hhs.gov
}

\section{Category: Neuroscience}

\begin{abstract}
Body : Numerous behavioral studies have disclosed that a long sevoflurane exposure in developing animals can lead to subsequent impairments in cognition, memory and learning ability. The aim of this study is to evaluate apoptotic effects in the developing brain after 8 hours of sevoflurane exposure at multiple timepoints for up to five weeks. Immunohistochemical analyses and molecular imaging approaches were employed to detect apoptotic cells in the developing brain. Postnatal day (PND) 7 rats were exposed to sevoflurane delivered in medical grade air at a clinically-relevant concentration of $2.5 \%$ and control animals were exposed to room air only for 8 hours. On PNDs 21, 28 and 35, caspase 3 (a biomarker for apoptosis) staining and Micro PET/CT scans with an apoptotic radiotracer, [18F]-ML10, were conducted. Radiolabeled tracer accumulation in regions of interest (ROIs) in the frontal cortex was converted into Standard Uptake Values (SUVs). In PND 21 and 28 rats, the uptake of [18F]ML10 was significantly increased. Preliminary analyses also show there were significant increases in caspase-3 positive cells in frontal cortical areas on PNDs $21(p=0.0109)$ and $28(p$ $=0.0357$ ). The neuroprotective agent acetyl-L-carnitine (ALC, $100 \mathrm{mg} / \mathrm{kg}$ ) administered before exposure was able to prevent the apoptotic effect of sevoflurane-induced neurotoxicity seen on PND 28. Thus, 8 hours of sevoflurane treatment can significantly increase neuroapoptosis in frontal cortical areas of the developing brain. This effect is likely to be related to the sevofluraneinduced behavioral dysfunctions noted in later life.
\end{abstract}

Complete Status: Complete

First Name: Xuan

Last Name: Zhang

Email: xuan.zhang@fda.hhs.gov

Organization: NCTR

Country: United States 


\title{
ID: GA451 \\ Exploring In Vivo Neural Imaging as a Readout for Histone Deacetylase 6 Inhibitor Rescue of Axonal Transport after Oxaliplatin-Induced Peripheral Neuropathy
}

\author{
Lucia Le Roux, MD Anderson Cancer Center, lucia.leroux@mdanderson.org
}

\section{Category: Neuroscience}

\begin{abstract}
Body : Background: Prospective studies in cancer patients indicate that $68 \%$ of patients suffer from chemotherapy-induced peripheral neuropathy (CIPN) [1]. This debilitating condition is a leading cause of dose reduction and discontinuation and can cause long-term disability. The exact molecular mechanism for chemotherapy induced axonal dysfunction is not known, but there is a growing body of evidence that altered microtubule function leading to axonal transport defects could be one of the causes for the development of neuropathic disease [2]. Axonal degeneration after administration of chemotherapeutic agents has been shown in several human and animal studies [3]. Oxaliplatin also causes axonal degeneration and causes CIPN in about $70 \%$ of patients exceeding accumulative doses of $540 \mathrm{mg} / \mathrm{m} 2$ [4]. We hypothesized that loss of axonal transport is an early event in the development of neuropathy. Histone deacetylase 6 (HDAC6) is a cytoplasmic class II HDAC with preference towards non-histone proteins, as alpha-tubulin. Selective HDAC6 inhibition and HDAC6 genetic deletion both prevent CIPN [5, 6]. Inhibition of HDAC6 increases tubulin acetylation, which has been linked to improved axonal transport using in vitro model systems. However, direct evidence that axonal transport is improved by HDAC6 inhibition following chemotherapy is lacking. The goal of this study is to use an in vivo neural imaging probe as a readout for axonal transport, to assess the effect of HDAC6 inhibition by the selective HDAC6 inhibitor ACY-1083 on axonal transport upon oxaliplatin induced neuropathy. Methods: We used a 2x2 factorial design: oxaliplatin (30mg/kg IP cumulative dose over 2 weeks) versus vehicle x ACY$1083(30 \mathrm{mg} / \mathrm{kg}$ IP daily for 2 weeks after completion of the oxaliplatin treatment) versus vehicle on 20 female SKH1 mice with 4 treatment groups for 5 mice per group. A fluorescently labeled neural imaging probe based on non-toxic, Tetanus Toxin C (TTc-790) was injected in the calf muscle of each animal followed by in vivo imaging after injection. In vivo whole animal imaging of the spine was done at baseline, after Oxaliplatin treatment, after ACY-1083 treatment and at the conclusion of the study, after 2 weeks of no treatment. Quantitative assessment of axonal transport was done by measuring TTc-790 uptake with a Region of Interest (ROI) over the spine at the thoracic-lumbar junction, 60 minutes after injection. Results: Qualitative in vivo neural imaging results show a reduction in the amount of TTc-790 transport over the spine for the oxaliplatin animals compared to baseline, with preservation of transport in the other groups, including the ACY-1083-oxaliplatin co-treatment group. There was no significant difference in TTc-790 transport between groups at baseline. At the conclusion of the study, after 2 weeks of no treatment, a main effect of oxaliplatin was observed, such that oxaliplatin significantly reduced TTc-790 transport $\mathrm{F}(1,15)=6.48$, after 2 weeks of no treatment ( $\mathrm{p}$ Conclusion: TTc790 transport in vivo can be successfully used to track the effect of neuroprotective agents on
\end{abstract}


chemotherapy-induced deficits in axonal transport. In this study the HDAC6 inhibitor ACY1083 showed imaging evidence of efficacy as a neural protectant for oxaliplatin-induced CIPN.

References: 1. Seretny, M., et al., Incidence, prevalence, and predictors of chemotherapyinduced peripheral neuropathy: A systematic review and meta-analysis. Pain, 2014. 155(12): p. 2461-70. 2. De Vos, K.J. and M. Hafezparast, Neurobiology of axonal transport defects in motor neuron diseases: Opportunities for translational research? Neurobiol Dis, 2017. 105: p. 283-299. 3. Starobova, H. and I. Vetter, Pathophysiology of Chemotherapy-Induced Peripheral Neuropathy. Front Mol Neurosci, 2017. 10: p. 174. 4. Argyriou, A.A., et al., Voltage-gated sodium channel polymorphisms play a pivotal role in the development of oxaliplatin-induced peripheral neurotoxicity: results from a prospective multicenter study. Cancer, 2013. 119(19): p. 3570-7. 5. Krukowski, K., et al., HDAC6 inhibition effectively reverses chemotherapy-induced peripheral neuropathy. Pain, 2017. 158(6): p. 1126-1137. 6. Van Helleputte, L., et al., Inhibition of histone deacetylase 6 (HDAC6) protects against vincristine-induced peripheral neuropathies and inhibits tumor growth. Neurobiol Dis, 2018. 111: p. 59-69.

\section{Image/Figure:}

https://www.xcdsystem.com/wmis/abstract/File6959/GA451 ImageFigure 0610101812.jpg

Image/Figure Caption: TTc-790 tracks axonal transport in vivo and can be used to measure the effect of neuroprotective agents in CIPN models. A) Study design timeline for oxaliplatin administration, ACY-1083 treatment and imaging time points. In this study a $2 \times 2$ factorial design was used with oxaliplatin ( $30 \mathrm{mg} / \mathrm{kg}$ IP cumulative dose over 2 weeks) versus vehicle $\mathrm{x}$ ACY $-1083(30 \mathrm{mg} / \mathrm{kg}$ IP daily for 2 weeks after completion of the oxaliplatin treatment) versus vehicle on 20 female SKH1 mice with 4 treatment groups for 5 mice per group. B) Qualitative in vivo neural imaging results show a reduction in the amount of TTc-790 transport over the spine for the oxaliplatin animals compared to baseline, with preservation of transport in the cotreatment control, ACY-1083, and the Oxaliplatin + ACY-1083 groups. C) Quantitative assessment of axonal transport expressed as average radiance $(\mathrm{p} / \mathrm{s} / \mathrm{cm} 2 / \mathrm{sr})$ was done by measuring TTc-790 uptake with a region of interest (ROI) over the spine at the thoracic-lumbar junction at 60 minutes after injection. Oxaliplatin treatment significantly reduced TTc-790 transport compared to controls and compared to the oxaliplatin + neuroprotection group $\mathrm{F}(1,15)$ $=6.48$, at the conclusion of the study after 2 weeks of no treatment $(\mathrm{p}<0.05)$. The ACY-1083 treated animals had significantly more TTc-790 axonal transport than the oxaliplatin treated group $(\mathrm{P}=0.03)$ and showed no significant difference from the control or ACY-1083 alone group. Image analysis was used to quantitatively compare control and treated groups, followed by 2way ANOVA and Tukey's corrected multiple comparisons in GraphPad Prism.

Complete Status: Complete

First Name: Lucia

Last Name: Le Roux

Email: lucia.leroux@mdanderson.org 
Organization: MD Anderson Cancer Center

Country: United States 


\title{
ID: GA452 \\ RELAXOMETRY OF CANCER: ROLE OF INTRACELLULAR WATER LIFETIME AS A TUMOUR BIOMARKER BY IN VIVO FAST FIELD CYCLING
}

\author{
Simonetta Geninatti Crich, University of Torino, simonetta.geninatti@unito.it
}

\section{Category: Oncology}

\begin{abstract}
Body : Conventional diagnostic magnetic resonance imaging (MRI) techniques have focused on the improvement of the spatial resolution by using high magnetic fields (1-7T). High field allows the visualization of small tumour mass but lacks to give a precise evaluation of tumour grading and metastatic potential. Recently, $[1,2]$ we showed that the intracellular water lifetime represents a hallmark of tumour tissue cells status that can be easily monitored by measuring T1 at different and relatively low magnetic field strengths, ranging from 0.2 to 200 $\mathrm{mT}$. A fast exchange through cell membranes indicates a high metabolic rate and thus a high activity of the tumor cells as also reported by Springer C Jr in many studies[3]. Thus it is possible to measure the high metabolic pressure by an enhance water exchange with the exterior of the cell. Therefore, intracellular water lifetime can be considered an important tumour biomarker directly depending on the rate of cell proliferation, cell migration and in responding to external stimuli as hypoxia or extracellular acidosis. 1/T1 relaxometric profiles are acquired on Fast Field Cycling relaxometers able to switch the magnetic field between different field strengths, during the measurement procedure. A field cycle overcomes the problem of the low sensitivity at low magnetic fields and allows rapid acquisition. The currently available relaxometers are designed for liquid samples measurements. Therefore, in order to host a mouse, the commercially available relaxometer was modified with the implementation of a $40 \mathrm{~mm} 0.5 \mathrm{~T}$ Field Cycling magnet and a dedicated $11 \mathrm{~mm}$ solenoid detection coil placed around the mouse's leg where is located the tumour xenograft prepared with mouse mammary adenocarcinoma cells, namely TSA, 4T1, 168Farn, injected in the leg muscle. These cell display different characteristics in terms of aggressiveness and metastatic potential (i.e. 168FarnAnother interesting application in which the low field relaxation can give fundamental information is the therapeutic outcome. Currently tumour responses to therapy are monitored primarily by imaging evaluating essentially the decrease of tumor size. This approach, however, lacks sensitivity and can only give a delayed indication of a positive response to treatment. In our study, we propose the use of FFC-NMR to provide relevant information about response to treatment by monitoring changes of water exchange rates through cell membranes that are directly dependent on the metabolism alterations caused by the chemo- or radio-therapy. Even though FFC-NMR instrumentationis not endowed with spatial resolution, the fundamental knowledge obtained in this study can enable new diagnostic opportunities in oncology that were previously unrecognized and are potentially transferable to the two prototype humanwhole-body-sized FFCMRI scanners recently built at Aberdeen University by Lurie and co-workers. Pilot studies performed on these FFC-MRI scanners have already demonstrated the potential use of FFC-MRI in a range of several pathologies such us musculoskeletal and cardiovascular diseases.
\end{abstract}


References: [1] Ruggiero et al Angew Chem Int Ed Engl 57, 7468-7472 (2018). [2] Ruggiero et al Molecular physics 117, 968-974 (2019). [3] Xin Li, Silvia Mangia, Jing-Huei Lee, Ruiliang Bai, and Charles S. Springer, J Magn Reson Med. 2019 Jul; 82(1): 411-424. [4] C. J. Springer, Jr., X. Li, L. A. Tudorica, K. Y. Oh, N. Roy, S. Y.-C. Chui, A. M. Naik, M. L. Holtorf, A. Afzal, W. D. Rooney, W. Huang, NMR Biomed. 2014, 27, $760-773$;

\section{Image/Figure:}

https://www.xcdsystem.com/wmis/abstract/File6959/GA452 ImageFigure 0609083339.jpg

Image/Figure Caption: NMRD profiles of the tumour tissues grown on hind limbs: 4T1 (triangles), TS/A, (squares) and 168FARN (circles) acquired 11, 13, and 25 days after intramuscular injection, respectively. R1tum is the averaged relaxation rate normalized to the tumour mass fraction compared to the whole hind limb. Error bars report the standard deviation (SD).

Complete Status: Complete

First Name: Simonetta

Last Name: Geninatti Crich

Email: simonetta.geninatti@unito.it

Organization: University of Torino

Country: Italy 


\title{
ID: GA453 \\ Molecular Imaging through Endogenous Tissue Metabolomics - from metabolomic marker discovery to imaging
}

\author{
Leo Cheng, MGH/Harvard Medical School, lcheng@mgh.harvard.edu
}

\section{Category: Oncology}

\begin{abstract}
Body : Molecular imaging can be performed through introductions of probing agents for specific molecular targets, it can also be achieved through untargeted measurements of endogenous disease metabolomic markers. Metabolomic imaging produces a disease distribution map and can be measured with localized magnetic resonance spectroscopy (MRS) or mass spectrometry imaging (MSI).. 7T MRS-based Metabolomic Imaging of Human Prostates. Twenty-two prostatectomy-removed whole prostates with biopsy-proven PCa were analyzed with a 7T human MRI scanner. Prostates were immersed in 70\% D2O to reduce magnetic susceptibility effects during measurements of T2-weighted (T2w)MRI and multi-voxel (mv)MRS of the middle transverse cross-sectional plane, which consists 16x16 voxels of a thickness of $3 \mathrm{~mm}$. Following MR measurements, prostates were sectioned, fixed, wholemounted, H\&E stained, and evaluated with traditional histopathology. The mvMRS data were processed using an in-house developed MATLAB-based program. After correction of the phase and baseline, the program performed an automatic curve-fitting with Lorentzian-Gaussian lineshapes. These 22 analyzed prostates were randomly divided into training and testing cohorts with 11 cases in each cohort. Through co-registrations of T2wMRI with the histological wholemounted images, and based on the results of histological identification of PCa lesions, metabolomic profiles for identification of PCa lesions were generated from the mvMRS results of the training cohort. Coefficients associated with these metabolomic profiles were applied onto the mvMRS results of the testing cohort and resulted in observations of statistically significant differentiations of PCa lesions for the latter, as shown in Fig. 1A, for means and standard errors calculated based on individual mvMRS voxels. Examples from training (1B) and testing (1C) cohorts are also illustrated in the figure. Mapping Human Lung Cancer Metabolomics with MSI. Our laboratory has engaged in the discovery and interrogation of LuCa metabolomic markers with intact tissue MRS analyses on paired LuCa tissue and serum samples obtained from LuCa patients. The initial feasibility study group of tissue MSI from our MRS studied tissueserum pairs included six LuCa patients of both squamous cell carcinoma and adenocarcinoma, as well as sera from three controls. Samples were embedded, frozen sectioned into 12 um thickness, mounted on indium tin oxide-coated microscope slides, and measured on a MALDI-TOF/TOF mass spectrometer. Our previous MRS metabolomic analyses of LuCa tissue-serum pairs identified a group of cellular metabolites as potential markers, as shown in Table 1. Here, we interrogate these markers further with tissue MSI and serum MS. Examples of our resulting MALDI MSI are shown in Fig. 2. Data in this figure illustrate that LuCa and necrosis identified by histology in Case A (M, 72yo, SCC, Stage I) presented increased metabolic intensities for both glutamine (Gln) and phosphocholine (PChol), which were not seen with necrosis alone (B, F, 72yo, Adeno, Stage I). Of note, the interpretation of PChol increase requires caution, as an increase in collagen can also result in the PChol increase as seen in (C, F, 60yo, SCC, Stage I).
\end{abstract}


However, in that case, the increase in Gln is almost absent. This observation further emphasizes the importance of conducting metabolomic evaluations, rather than merely observing individual metabolites. In conclusion, our results presented here demonstrate the potential of utilizing endogenous disease metabolomic markers for molecular imaging through MRS and MSI. While the former can be explored for in vivo non-invasive metabolomic disease evaluations, the latter can provide metabolomic characterizations at close to single cell level for molecular pathology examinations.

\section{Image/Figure:}

https://www.xcdsystem.com/wmis/abstract/File6959/GA453 ImageFigure 0608115845.jpg

Image/Figure Caption: Explained in Abs test.

Complete Status: Complete

First Name: Leo

Last Name: Cheng

Email: lcheng@mgh.harvard.edu

Organization: MGH/Harvard Medical School

Country: United States 


\title{
ID: GA454 \\ Comparing diffusion-weighted MRI and 18F-FDG PET/MRI for staging and restaging of Langerhans Cell Histiocytosis
}

\author{
Ramyashree Nyalakonda, Stanford University, nramya.rao@gmail.com
}

\section{Category: Oncology}

\begin{abstract}
Body : Abstract: PURPOSE: To compare the detection of lesions between DW-MRI and 18F-FDG PET/MR for staging and restaging of Langerhans Cell Histiocytosis (LCH), using all clinical outcomes and imaging data as the reference standard. Also, this study will compare the differences between LCH chemotherapy responders and non-responders. METHODS: In a prospective clinical trial, we enrolled 11 children (2-16 years old, 4 female and 7 male) with LCH, who underwent an 18F-FDG PET and DW-MRI scan on an integrated 3T PET/MR scanner at baseline $(\mathrm{n}=11)$ and after chemotherapy $(\mathrm{n}=7)$. We determined the presence or absence of tumor lesions in 5 anatomical areas per patient on 18F-FDG PET and DW-MRI. We calculated sensitivities, specificities and diagnostic accuracies of the two imaging modalities, with biopsy results and follow up imaging as the reference standard. In addition, we measured the SUVratio as the maximum standardized uptake value (SUVmax) of each lesion, normalized to the SUVmean of the liver and the apparent diffusion coefficients (ADCmean) of all lesions. Quantitative data before and after chemotherapy were compared with a Mann-Whitney U test. SUVratio and ADCmean were correlated with a linear regression analysis. RESULTS: On baseline scans, our patients had $37 \mathrm{LCH}$ lesions according to the reference standard. 18F-FDG PET detected 37 of 37 lesions and DW-MRI detected 35 of 37 lesions. The sensitivity and specificity for baseline scans were $100 \%$ and $100 \%$, respectively for $18 \mathrm{~F}$-FDG PET and $94.59 \%$ and $100 \%$ for DW-MRI. DW-MRI missed two lesions in the head and thorax as there was no restricted diffusion of the lesion in the head and the thorax had motion artifacts. Chemotherapy responders demonstrated significant decline in SUVratio $(\mathrm{p}=0.002)$ and significant increase in ADCmean ( $p$ that resolution of a lesion leads to a decrease in SUVratio and an increase in ADCmean. CONCLUSION: Our data provides preliminary evidence that DW-MRI can be used for staging and treatment monitoring of LCH. Since patients with multifocal LCH need multiple follow-up scans throughout their lifetime, using DW-MRI could substantially decrease the radiation exposure of these young patients.
\end{abstract}

\section{Image/Figure:}

https://www.xcdsystem.com/wmis/abstract/File6959/GA454_ImageFigure_0608020624.png

Image/Figure Caption: Figure 1: Concordant treatment response to chemotherapy seen on both 18F-FDG PET and DW-MRI in a $12 \mathrm{y} / \mathrm{o} \mathrm{M}$. A-H, Examples of pelvis images: A, axial fluorodeoxyglucose PET image at baseline; B, axial fused fluorodeoxyglucose PET and LAVA image at baseline; $\mathrm{C}$, axial diffusion weighted image at baseline; $\mathrm{D}$, fused diffusion weighted and LAVA image at baseline; E, axial fluorodeoxyglucose PET image at follow-up; F, axial fused fluorodeoxyglucose PET and LAVA image at follow-up; G, axial diffusion weighted image at 
follow-up; and $\mathrm{H}$, fused diffusion weighted and LAVA image at follow-up. Patient who had a $\mathrm{LCH}$ lesion in the pelvis showed a concordant response to chemotherapy on both 18F-FDG PET and DW-MRI.

Full Name of Abstract's 1st Author : Ramyashree Rao Nyalakonda

Complete Status: Complete

First Name: Ramyashree

Last Name: Nyalakonda

Email: nramya.rao@gmail.com

Organization: Stanford University

Country: United States 


\title{
ID: GA455 \\ Multi-parametric MRI-CEST imaging of tumor metabolism and acidosis to investigate cancer aggressiveness
}

\author{
Annasofia Anemone, University of Turin, annasofiaantonia.anemone@unito.it
}

\section{Category: Oncology}

\begin{abstract}
Body : Introduction In clinical practice, FDG-PET uptake is a well-established index for quantifying glucose utilization for the diagnosis and characterization of tumours, but it is limited by radiation exposure issues and reduced spatial resolution that limits heterogeneity assessments. The aim of this study is to combine two MRI-CEST (Chemical Exchange Saturation Transfer) methods for investigating different aspects of tumour aggressiveness, including dysregulated glucose uptake and tumor acidosis following the sequential injection of natural D-glucose [1] and iopamidol [3] in two murine models exhibiting distinct metabolic properties. In addition, we compared glucose and the non-metabolizable 3OMG derivative in assessing the altered glucose metabolism. Materials and Methods 4T1 (mouse mammary carcinoma) and PC3 (human prostate cancer) cells were subcutaneously implanted in both flanks of female BALB/c and male Athymic NudeFoxn1nu mice, respectively. CEST MRI protocol was performed on a Bruker 7T MRI scanner. Each mouse underwent i.v. injection of glucose (dose $5 \mathrm{~g} / \mathrm{kg}$ ) or of $3 \mathrm{OMG}$ (dose $3 \mathrm{~g} / \mathrm{kg}$ ) followed by Iopamidol injection (dose $4 \mathrm{gI} / \mathrm{Kg}$ ) $30 \mathrm{~min}$ later. Z-spectra before and after contrast media injections were acquired and CEST contrast was calculated between POST and PRE images. Contrast enhanced maps were calculated for glucose or $3 \mathrm{OMG}$ and tumor $\mathrm{pHe}$ maps for iopamidol. Two days after the MRI acquisition mice were kept fasted overnight and injected with 18FFDG for PET imaging. GLUT1 and NHE1 expression were quantified by Western Blot. Results The two tumour models showed a marked and different FDG uptake: higher FDG uptake in 4T1 tumours than in PC3 tumours (Fig.1e and j). Consistently with the higher glucose uptake, 4T1 tumour model presented a more acidic extracellular $\mathrm{pH}$ than the PC3 model (Fig. 1d and i), reflecting an increased tumour acidosis. GlucoCEST studies using glucose revealed a slightly lower CEST contrast (ST effects) in the PC3 tumor in comparison to the 4T1 ones (Fig. 1b), whereas the non-metabolizable 3OMG derivative showed a significant lower CEST contrast for the PC3 tumours (Fig. 1g) in accordance with the FDG-PET measurements. Increased expression of GLUT1 transporter in 4T1 cell line compared to PC3 validated the increased glucose metabolism for the 4T1 breast tumor model displayed in vivo (persuasive data). Conclusion These findings highlight i) multiparametric MRI-CEST approaches can be combined to characterize several aspects of tumor metabolism (glucose uptake and acidosis) in alternative to the FDG-PET technique and ii) the non-metabolizable 3OMG is more sensitive than glucose to distinguish glucose utilization in murine tumour models with different aggressiveness. This work was supported by the European Union's Horizon 2020 research and innovation programme (Grant Agreement No. 667510) and by the Associazione Italiana Ricerca Cancro (AIRC MFAG 2017 - ID. 20153 project).
\end{abstract}

References: [1] van Zijl PC, et al. Proc Natl Acad Sci U S A. 2007,4359 [2] Navon G, et al. Magn Res Med 2018 79,1061 [3] Longo DL, et al.; Cancer Research 2016, 76, 6463 


\section{Image/Figure:}

https://www.xcdsystem.com/wmis/abstract/File6959/GA455_ImageFigure_0610104953.jpg

Image/Figure Caption: Figure 1: Representative T2w images (a,f), GlucoCEST map after Dglucose (b) or 3OMG injection (g), CEST contrast (c,h) and $\mathrm{pH}$ maps (d,i) and fused FDG-PET/ CT images for 4T1 and PC3 tumor bearing mice $(e, j)$.

Full Name of Abstract's 1st Author : Annasofia Anemone

Complete Status: Complete

First Name: Annasofia

Last Name: Anemone

Email: annasofiaantonia.anemone@unito.it

Organization: University of Turin

Country: Italy 


\title{
ID: GA456 \\ Chemical Biology Tools for Aldehyde Dehydrogenases in Cancer Stem Cells
}

\author{
Michael Lee, University of Illinois at Urbana-Champaign, mcl4@illinois.edu
}

Category: New Chemistry, Biology \& Bioengineering

\begin{abstract}
Body : Cancer stem cells (CSCs) are a subpopulation of cancer cells that are suspected to drive metastasis, relapse, and resistance to therapeutics due to their ability for tumorigenesis, self-renewal, and differentiation. High aldehyde dehydrogenase (ALDH) activity has been correlated to CSC properties. Due to the limitations of current techniques and the large number of ALDH isoforms, it has been difficult to pinpoint specific isoform(s) responsible for CSC properties. Our lab has previously developed activity-based turn-on fluorescence reporters for the ALDH1A1 isoform. To build upon this, we have developed chemical biology approaches to study the role of ALDHs in CSCs. First, we have engineered a system to spatiotemporally knock-in ALDH1A1 via unnatural amino acid incorporation. Second, we have developed a mechanism-based probe for activity-based protein profiling of ALDHs. These new methods allow for the modulation and quantification of specific ALDH isoform activity via molecular imaging to further elucidate its roles in CSCs as well as explore potential new drug targets.
\end{abstract}

Full Name of Abstract's 1st Author : Michael C. Lee

Complete Status: Complete

First Name: Michael

Last Name: Lee

Email: mc14@illinois.edu

Organization: University of Illinois at Urbana-Champaign

Country: United States 


\title{
ID: GA457 \\ PET imaging for in vivo evaluation of brain myelination in a Down syndrome animal model
}

\author{
Daniele de Paula Faria, University of Sao Paulo Medical School, danielefaria1@gmail.com
}

\section{Category: Neuroscience}

\begin{abstract}
Body : Introduction: Down Syndrome (DS) or trisomy 21 is the most common genetic cause of mild to moderate intellectual disability in the world. Defects in myelination process can be associated to developmental delays and intellectual disability(1,2). Molecular neuroimaging allows in vivo and noninvasive studies of cellular and molecular processes, and therefore, it is an important tool to understand the cells changing over time. Positron Emission Tomography (PET) imaging using Pittsburgh Compound B labelled with carbon-11 (11C-PIB) is traditionally used for $\beta$-amyloid plaque quantification, however, in the last decade has also been used for myelin quantification(3-5). Objective: The aim of this study was to evaluate myelination progression in Ts65Dn young mice, model of Down syndrome, by 11C-PIB PET imaging Methods: Transgenic mice (TS65Dn) and its wild type (WT), were evaluated with 11C-PIB PET imaging in 2 different ages: 2 -month-old (WT $=31$ animals and Ts65Dn $=16$ animals) and 5-month-old (WT $=7$ animals and Ts65Dn $=6$ animals $) .11 \mathrm{C}$-PIB $( \pm 15 \mathrm{MBq})$ was injected intravenously and $30 \mathrm{~min}$ after tracer injection a static PET image of 20 min was acquired in a PET scanner for small animals ( $\beta$-cube, Molecubes, Belgium) with the animal anesthetized with isoflurane $2-3 \%$ in oxygen. Images were quantified by PMOD ${ }^{\mathrm{TM}}$ software (Switzerland) using T1 MRI as brain template and the data was expressed in SUV (Standardized Uptake Value) mean \pm standard deviation in different brain regions: striatum, cortex, hippocampus, thalamus, cerebellum, brainstem and midbrain. Group comparisons were analyzed by 2-way ANOVA (GraphPad Prism version 6). Statistical differences were considered significant when $\mathrm{P} \leq 0.05$. Results: All analyzed brain regions, in the WT group, showed 11C-PIB uptake increase between overtime . In the Ts65Dn animals there was an increase in tracer uptake, however this increase was not statistically significant between 2 and 5-month-old data. The 11C-PIB uptake (SUV) for the WT 2 month-old group was $0.065 \pm 0.027$ for the striatum, $0.072 \pm 0.029$ for the cortex, $0.068 \pm 0.028$ for the hippocampus, $0.064 \pm 0.026$ for the thalamus, $0.072 \pm 0.033$ for the cerebellum, $0.086 \pm 0.037$ for the brainstem and $0.075 \pm 0.031$ for the midbrain. Comparing with WT 5 monthold, the uptake was $0.108 \pm 0.039(\mathrm{p}=0.0057), 0.120 \pm 0.040(\mathrm{p}=0.0031), 0.113 \pm 0.037(\mathrm{p}=0.0045)$, $0.108 \pm 0.044(\mathrm{p}=0.0055), 0.121 \pm 0.040(\mathrm{p}=0.0057), 0.133 \pm 0.052(\mathrm{p}=0.0322)$ and $0.131 \pm 0.062$ $(\mathrm{p}=0.0039)$ in the respective brain regions. For the Down syndrome model with 2 -month-old, the 11C-PIB SUV was $0.064 \pm 0.034$ for the striatum, $0.067 \pm 0.035$ for the cortex, $0.066 \pm 0.034$ for the hippocampus, $0.061 \pm 0.035$ for the thalamus, $0.069 \pm 0.039$ for the cerebellum, $0.081 \pm 0.045$ for the brainstem and $0.071 \pm 0.040$ for the midbrain and in the 5-month-age was $0.091 \pm 0.020$ $(\mathrm{p}=0.2714), 0.094 \pm 0.015(\mathrm{p}=0.2900), 0.096 \pm 0.020(\mathrm{p}=0.1777), 0.082 \pm 0.019(\mathrm{p}=0.4962)$ $0.094 \pm 0.010(\mathrm{p}=0.4518), 0.103 \pm 0.020(\mathrm{p}=0.6739)$ and $0.094 \pm 0.023(\mathrm{p}=0.5785)$ in the respective brain regions. Conclusion: 11C-PIB PET imaging was able to detect differences in myelin content of different brain regions of WT and Ts65Dn animals. In the WT group was possible to detect increase in tracer uptake, meaning increase of myelin content between 2-month of age to
\end{abstract}


5-month of age. The increase in the Ts65Dn was not statically significant, meaning a slower development in myelin content in this animal model of Down syndrome what can be related to brain development.

References: 1. Aziz NM, Klein JA, Brady MR, Olmos-Serrano JL, Gallo V, Haydar TF. Spatiotemporal development of spinal neuronal and glial populations in the Ts65Dn mouse model of Down syndrome. Journal of Neurodevelopmental Disorders. 2019;11(1). 2. OlmosSerrano JL, Kang HJ, Tyler WA, Silbereis JC, Cheng F, Zhu Y, et al. Down Syndrome Developmental Brain Transcriptome Reveals Defective Oligodendrocyte Differentiation and Myelination. Neuron. 2016;89(6):1208-22. 3. Stankoff B, Freeman L, Aigrot MS, Chardain A, Dolle F, Williams A, et al. Imaging central nervous system myelin by positron emission tomography in multiple sclerosis using [methyl-(1)(1)C]-2-(4'-methylaminophenyl)- 6hydroxybenzothiazole. Ann Neurol. 2011;69(4):673-80. 4. Faria Dde P, Copray S, Sijbesma JW, Willemsen AT, Buchpiguel CA, Dierckx RA, et al. PET imaging of focal demyelination and remyelination in a rat model of multiple sclerosis: comparison of [11C]MeDAS, [11C]CIC and [11C]PIB. Eur J Nucl Med Mol Imaging. 2014;41(5):995-1003. 5. Carvalho RHF, Real CC, Cinini S, Garcez AT, Duran FLS, Marques FLN, et al. C-11 PIB PET imaging can detect white and grey matter demyelination in a non-human primate model of progressive multiple sclerosis. Multiple Sclerosis and Related Disorders. 2019;35:108-15.

Complete Status: Complete

First Name: Daniele

Last Name: de Paula Faria

Email: danielefaria1@gmail.com

Organization: University of Sao Paulo Medical School

Country: Brazil 


\title{
ID: GA458 \\ IMAGE GUIDED ROBOTIC PROSTATECTOMY USING THE PH- ACTIVATED MICELLAR IMAGING AGENT ONM-100 - A PHASE 2 STUDY OF TUMOR AND CAMERA AGNOSTIC DETECTION OF SOLID CANCERS
}

\author{
Brian Madajewski, OncoNano Medicine Inc, bmadajewski@onconanomed.com
}

\section{Category: Oncology}

\begin{abstract}
Body : Background Background ONM-100, a polymeric micelle covalently conjugated to indocyanine green (ICG), is being developed for intraoperative detection of solid cancers. ONM-100 micelles dissociate in the acidic environment of solid tumors via an ultra $\mathrm{pH}$-sensitive binary activation mechanism, resulting in ICG fluorescence. As low $\mathrm{pH}$ is a hallmark of all solid tumors, ONM-100 has the potential to be a broadly indicated, tumor-agnostic imaging agent. A 30 patient Phase 1 study was completed in breast (BC), head and neck (HNSCC), colorectal (CRC) and esophageal (EC) cancers, where a single intravenous (IV) dose $(0.1-1.2 \mathrm{mg} / \mathrm{kg})$ of ONM-100 was administered 24 hours before surgery. ONM-100 was well tolerated with no drug related safety issues, fluoresced in all four tumor types, and detected histology-confirmed positive margins and occult disease missed by SOC surgery in 30\% of the patients (WMIC 2019, oral presentation). This ongoing Phase 2a study (NCT03735680) optimizes imaging performance of ONM-100 over a range of doses and imaging schedules and explores potential for ONM-100 guided surgery in new tumor types and using additional FDA-approved imaging devices. Methods This is a non-randomized, open-label, multi-center 2 part study. The study is designed to be adaptive in terms of selection of dose, imaging schedule and tumor type based on continuous review and analysis of imaging and safety data. All patients receive a single IV dose $(0.5-7 \mathrm{mg} / \mathrm{kg})$ of ONM-100 the same day as, or previous to the day of surgery. Part 1 comprises up to 6 dose Cohorts of 3 patients each with confirmed biopsy of BC or HNSCC. In Part 2, up to 3 dose/schedule Groups are verified in multiple tumor types (prostate $(\operatorname{PrC})$, ovarian $(\mathrm{OC})$, nonsmall cell lung (NSCLC), urothelial (UC) cancers, CRC, HNSCC, BC). ONM-100 fluorescence is imaged intraoperatively before and after tumor excision, in the specimens on the operating room back table, and during pathology workflow. ONM-100 fluorescence is evaluated in terms of tumor visualization, tumor to background ratio, diagnostic performance vis-à-vis standard pathology and detection of positive margins or occult disease missed by SOC surgery. Results In this ongoing study, 16 patients have been enrolled in 3 Cohorts in Part 1 (4 BC, 5 HNSCC) and 2 Groups in Part 2 (6 PrC, 1 OC) at two US sites. Here, we show for the first time, ONM-100 imaging feasibility in PrC during robotic prostatectomy using the da Vinci Xi Firefly ${ }^{\mathrm{TM}}$. ONM100 fluorescence was detected through the prostatic capsule, and tissue slice fluorescence corresponded to pathologically confirmed tumor regions (Figure 1). ONM-100 imaging intraoperatively detected a clinically suspicious bladder neck margin in the wound bed of a prostatectomy patient. The excised margin was histologically confirmed to have tumor present. These data also demonstrated feasibility to image another new tumor type - ovarian cancer. All 4 enrolled tumor types were successfully imaged using the Visionsense ${ }^{\mathrm{TM}}$ camera. Pharmacokinetic data demonstrated dose proportionality across the dose ranges studied in Phase
\end{abstract}


1 and Phase $2(0.1-3 \mathrm{mg} / \mathrm{kg})$. ONM-100 was well tolerated at all dose levels. Strong fluorescence signals were observed even within few hours after dosing, which combined with a half life $>55$ hours increases the potential for a wide imaging window. Conclusion ONM-100 continues to exhibit favorable safety and PK profiles even at doses $3 \mathrm{X}$ higher than in Phase 1. The study extends the feasibility of detecting tumor positive margins in real-time to prostatectomies. The tumor and camera agnostic imaging is further confirmed in 2 additional tumor types (total 6) and 2 additional imaging devices (total 4) attesting to the robustness of the imaging agent and the mechanism of action, independent of the metabolic phenotype of the tumor.

\section{Image/Figure:}

https://www.xcdsystem.com/wmis/abstract/File6959/GA458_ImageFigure_0608075327.png

Image/Figure Caption: Figure 1: ONM-100 fluorescence imaging in prostate cancer. Patients received a single intravenous $3 \mathrm{mg} / \mathrm{kg}$ dose of ONM-100 approximately 6 hours prior to surgery. White light (A) and near-infrared (B) intraoperative in vivo imaging of the prostate in a patient undergoing robotic prostatectomy using the da Vinci Xi Firefly system. Ex vivo tissue slabs are processed to formalin fixed paraffin embedded (FFPE) blocks, cut, stained with hemtoxylin and eosin (H\&E) and analyzed for tumor (C). Annotations of tumor areas within the H\&E (C, blue outlines) are superimposed on fresh tissue slab NIR images (D) previously captured using the LiCOR PearlÒ Trilogy imager. Areas of prominent fluorescence are found to highly correlate with histologically confirmed areas of tumor

Complete Status: Complete

First Name: Brian

Last Name: Madajewski

Email: bmadajewski@onconanomed.com

Organization: OncoNano Medicine Inc

Country: United States 


\title{
ID: GA459 \\ Volumetric glutamate-weighted MR imaging enables in vivo detection of metabolic differences between human hippocampal subfields
}

\author{
Abigail Cember, University of Pennsylvania, cember@pennmedicine.upenn.edu
}

\section{Category: Neuroscience}

\begin{abstract}
Body : Introduction. The medial temporal lobe has been identified by modern neuroscience to be the locus of learning and memory formation, and many neurodegenerative conditions wreak havoc on cognitive ability because of their damage to these structures. Thus, this region of the brain has been the focus of much investigation; however, there remain many unanswered questions about its molecular physiology even in healthy individuals. Glutamate is the primary excitatory neurotransmitter in the mammalian central nervous system and implicated in almost all neurophysiological processes; accordingly, dysregulation of its metabolism has been observed in many disease states. Like many small molecules of interest, glutamate remains challenging to measure non-invasively in vivo. While 1HMRS, biochemical and histological measurements have endeavored to quantify the amounts of glutamate present in human and animal brain tissue, spatially-resolved detection of any differences in glutamate concentration between tissue types or anatomic regions of living brain has remained elusive. Glutamateweighted Chemical Exchange Saturation Transfer (gluCEST) magnetic resonance imaging (MRI) is nearly unique in its ability to provide non-invasive, spatially resolved measurements of glutamate in vivo. We recently developed and improved correction for B1 inhomogeneity of gluCEST images of the human brain which enabled truly volumetric (3D k-space) imaging, even in regions of the brain where B1 inhomogeneity is problematic. In this work, using 3D gluCEST imaging [1,2] at 7T with this improved post-processing strategy [3], glutamate-weighted MR images of a slab which includes the medial temporal lobe were generated and then analyzed by registration with a high-resolution hippocampal subfield segmentation. Hypothesis. Based on the general awareness of finely specific physiology and function in the medial temporal lobe [e.g. 4,5] and published findings on the presence of glutamate in rodent brains $[6,7,8]$, we hypothesized that there may be subtle, as of yet unmeasured physiological differences in glutamate concentration between various subfields of the hippocampus and neighboring structures of the human medial temporal lobe. Methods. Images of 13 healthy subjects ages 2169 were obtained on a Siemens 7.0T Terra outfitted with a single volume transmit/32 channel receive phased array head coil. Post-processing was performed using in-house code written in MATLAB and MEX (Matlab executable). Segmentation of the medial temporal lobe was performed using the Magdeburg 7T Atlas available in the Distributed Segmentation Service of ITK-SNAP [9,10,11], with T1 and T2-weighted structural images as input. Registration and calculation of ROI averages was then performed using the c3d package accompanying ITKSNAP. Results. Our results suggest that we are indeed able to detect gluCEST differences in tissue types in vivo; namely, an elevated amount of glutamate in the dentate gyrus in comparison with the other medial temporal lobe structures (which are all considered gray matter). This corroborates the findings in the preclinical works referenced; such a result has not previously been demonstrated in living human beings, for lack of technology capable of measuring it.
\end{abstract}


Although these preliminary results demonstrate the potential of 3D gluCEST MRI in measuring substructural glutamate differences in human medial temporal lobe, the method is equally applicable to measuring glutamate changes in the other parts of the brain. There are several points of validation needed, specific to the gluCEST acquisition and in particular the method for correcting for B1 inhomogeneity, which is an essential step in the processing of gluCEST raw data to generate CEST maps. Namely, we sought to demonstrate that the findings to date are not an artefact of extreme values or user-set thresholds, of the saturation B1 distribution of the head coil, nor of our method for correcting for it. Please see "Persuasive Data" for figures addressing these points.

References: 1] Cai K et al. Magnetic resonance imaging of glutamate. Nat Med. 2012;18(2):302-6. 2] Nanga RPR et al. Feasibility of Partial 3D GluCEST in Healthy Human Adults at 7.0T. Proc. Intl. Soc. Mag. Reson. Med. 27 (2019) 3996. 3] Cember A et al. Improved method for post-processing correction of B1 inhomogeneity in glutamate weighted CEST images of the human brain. Under Review. 4] Berron D et al. Age-related functional changes in domainspecific medial temporal lobe pathways. Neurobiol. Aging. 2018; 65: 86-97. 5] Baker S et al. Human dentate gyrus plays a necessary role in discriminating new memories. Curr. Biol. 2016; 26 (19); 2629-2634. 6] Pepin J et al. Imaging of neuronal compartment using gluCEST method. Proc. Intl. Soc. Mag. Reson. Med. 25 (2017) 3741 7] Bagga P et al. Mapping the alterations in glutamate with GluCEST MRI in a mouse model of dopamine deficiency. J Neurochem. 2016;139(3):432-439. 8] Crescenzi R et al. Longitudinal imaging reveals subhippocampal dynamics in glutamate levels associated with histopathologic events in a mouse model of tauopathy and healthy mice. Hippocampus. 2017;27(3):285-302. 9] Yushkevich et al. Automated volumetry and regional thickness analysis of hippocampal subfields and medial temporal cortical structures in mild cognitive impairment. Hum Brain Mapp. 2015; 36:258-287 10] Berron D et al. A protocol for manual segmentation of medial temporal lobe subregions in 7 Tesla MRI. Neuroimage Clin. 2017; 15:466-482. 11] Berron D et at. Higher CSF Tau Levels Are Related to Hippocampal Hyperactivity and Object Mnemonic Discrimination in Older Adults. J. Neurosci.: $2019 ; 39$ (44) 8788-8797.

\section{Image/Figure:}

https://www.xcdsystem.com/wmis/abstract/File6959/GA459_ImageFigure_0608093519.png

Image/Figure Caption: Left: Zoomed-in view of the medial temporal lobe of a 22-year-old male, shown with the Magdeburg Atlas 7T segmentation overlaid onto the structural image, featuring coronal and saggital views. Right: Box plot showing the ROI-averaged gluCEST values for all 13 subjects, by subfield according to the colorcode in the image. The scale of gluCEST values shown on this axis is $7-13 \%$, calculated using B0 and B1 corrected negativenormalized CEST asymmetry; the center line of the boxplot represents the subject with the median value for that segment, with edges of the colored bars being first and third quartile (standard boxplot structure). The dentate gyrus has a distribution that is clearly shifted higher than the other nearby segments. Both the median and the mean (not shown) ROI-averaged values for the dentate gyrus (bilateral) are 11\%, while the next highest gluCEST segments are CA1 and the hippocampal tail, at only $10.5 \%$. It should be noted that the apparently lower values in the more inferior subfields are likely to be an artefact of the B1 distribution, whereas we do not 
believe this to be the case for the relative values of the dentate gyrus and other immediately nearby structures (see Persuasive Data for B1 maps).

Complete Status: Complete

First Name: Abigail

Last Name: Cember

Email: cember@pennmedicine.upenn.edu

Organization: University of Pennsylvania

Country: United States 


\title{
ID: GA460 \\ Detection of Extracellular Matrix Remodeling in Atherosclerotic Lesions using a Selective PET Radiotracer for Matrix Metalloproteinase-13
}

\author{
Ariel Buchler, University of Ottawa Heart Institute, abuch052@uottawa.ca
}

\section{Category: Cardiovascular \& Pulmonary}

\begin{abstract}
Body : Introduction: Dysregulation of extracellular matrix remodeling by specific matrix metalloproteinases (MMPs) in vasculature is associated with atherosclerotic plaque vulnerability.1,2 Given that thrombosis underlies most adverse clinical outcomes including myocardial infarction and stroke, MMPs represent compelling biomarkers for imaging by positron emission tomography (PET).1,3 To date, differentiating stable atherosclerotic plaques from those susceptible to rupture using broad-spectrum MMP radiotracers has been unsuccessful potentially due to engagement with non-pathologic MMPs, possessing distinct and opposing functions in disease progression.4,5 Contrarily, MMP-13 has been shown to predominantly exhibit collagenolysis in atheromatous plaques and reduce smooth muscle cell accumulation, contributing directly to plaque destabilization by degradation of the fibrous cap.6-9 As such, a comparative analysis between non-selective [18F]BR-351 and a selective MMP-13 radiotracer, herein entitled [18F]FMBP, was performed to elucidate whether targeting of specific MMP isozymes augments imaging sensitivity, and improves correlation with markers of vulnerable atherosclerotic lesions. Methods: Preliminary screenings of [18F]FMBP and [18F]BR-351 were completed by in vitro autoradiography of en face aortae from atherogenic ApoE-/- mice. Ex vivo biodistribution and autoradiography were analogously performed in C57B1/6 and ApoE-/- mice under baseline or homologous blocking conditions to assess radiotracer localization, specific binding, and sensitivity. En face aortae were subsequently stained with Oil Red O, sectioned, and subject to immunofluorescence staining with Mac-2 and MMP-13 to correlate radiotracer uptake with histological biomarkers of plaque vulnerability. Results: Automated radiosyntheses afforded [18F]FMBP and [18F]BR-351 in high decay-corrected radiochemical yields, purities, and molar activities. During preliminary assessments, while both radiotracers localized within atherosclerotic lesions and demonstrated measurable specific binding, [18F]FMBP overall lesion uptake was 1.6-fold higher. Ex vivo biodistributions revealed that [18F]FMBP and [18F]BR-351 predominantly undergo renal clearance and hepatobiliary excretion with low accumulation of radioactivity in non-excretory organs $(\mathrm{t}=30 \mathrm{~min})$. Ex vivo autoradiography and histology of en face aortae revealed that [18F]FMBP, in comparison to [18F]BR-351, exhibited 2.2-fold greater lesion uptake, observable specific binding (63\%), improved disease sensitivity (2.7-fold vs 2.5 fold) and superior correlation to extent of lipid accumulation by Oil Red O (R2: 0.80 vs 0.72 ). As detected by Mac-2 and MMP-13 immunofluorescence staining, extent of inflammation and ECM remodeling were distinctly increased within the core and fibrous cap of atherosclerotic lesions and further correlated to $[18 \mathrm{~F}] \mathrm{FMBP}$ autoradiographic uptake ( $\mathrm{R} 2=0.71$ and 0.56 , respectively). MMP-13 additionally correlated with Mac-2 $(\mathrm{R} 2=0.85)$. Conclusion: The feasibility of imaging extracellular matrix remodeling in mouse models of atherosclerosis with MMP-targeted PET radiotracers has been established. Selective imaging of MMP-13 with [18F]FMBP showed improved sensitivity, specificity, and correlation to markers of plaque vulnerability relative to
\end{abstract}


pan-selective [18F]BR-351. Altogether, [18F]FMBP has proven useful for the detection of extracellular matrix remodeling in inflamed atherosclerotic lesions, suggesting that selective isozyme imaging represents a promising approach towards the characterization of high-risk atherosclerosis.

References: 1. Curr Opin Lipidol. 2014;25(5):358-66. 2. Physiol Rev. 2005;85(1):1-31 3. Eur Heart J. 2004;25:1077-1082. 4. Eur Respir J. 2011;38:191-208. 5. Vascul Pharmacol. 2012;56:232-244. 6. Circulation. 1999;99:2503-2509. 7. Arterioscler Thromb Vasc Biol. 2014;34:1179-1186. 8. Arterioscler Thromb Vasc Biol. 2011;31:2464-2472. 9.

Circulation.2005;112:2708-2715.

\section{Image/Figure:}

https://www.xcdsystem.com/wmis/abstract/File6959/GA460_ImageFigure 0609032046.jpg

Image/Figure Caption: (A) Chemical structure of [18F]FMBP (left) and [18F]BR-351 (right). (B) Representative aortic en face ex vivo autoradiographs following administration of [18F]FMBP or [18F]BR-351 in C57Bl/6 and ApoE-/- mice. Corresponding Oil Red O brightfield images are shown. (C) Quantification of autoradiographic lesion uptake. (D) Correlation of autoradiographic uptake with Oil Red O.

Full Name of Abstract's 1st Author : Ariel Buchler

Complete Status: Complete

First Name: Ariel

Last Name: Buchler

Email: abuch052@uottawa.ca

Organization: University of Ottawa Heart Institute

Country: Canada 


\title{
ID: GA461 \\ In Vivo Glutathione-Responsive Photoacoustic Imaging for Targeted Drug Delivery and Lung Cancer Imaging
}

\author{
Melissa Lucero, University of Illinois at Urbana-Champaign, MLucero3@illinois.edu
}

Category: New Chemistry, Biology \& Bioengineering

\begin{abstract}
Body : Personalized medicine promises to improve treatment outcomes by matching patients with therapy. A recent approach towards this goal involves employing companion diagnostics (CDx), which are tests that can identify the presence of a biomarker critical to the activation a drug. However, all FDA-approved CDx are limited to the in vitro setting which does not account for factors that may influence efficacy such as the influence of the tissue microenvironment. Technologies such as PA imaging can overcome this limitation by providing information that can account for changes in the body during disease progression. Here, we have developed the first PA imaging-based companion diagnostic (PACDx) specifically for identifying elevated concentrations of glutathione (GSH) in cancer. GSH plays essential biological roles, however it has also been associated with a variety of pathologies such as cancer. For instance, GSH was reported to be 4-fold higher in non-small cell lung carcinoma (NSCLC) compared to healthy lung tissue in humans. With this information in mind, we chose to develop a CDx assay for lung cancer based on GSH detection. Since GSH is the most abundant thiol in the body it is typically not considered an ideal target for cancer detection or treatment. Current sensing approaches that use thiol-disulfide exchange, Michael additions, or nucleophilic aromatic substitution reaction (SNAr) are too reactive to distinguish levels between cancer and healthy tissue. To address this issue, we took a physical organic approach to home into a reactivity that provided a dose-dependent response in the $1-10 \mathrm{mM}$ range. Importantly, it is widely accepted that cancer cells contain GSH closer to the $10 \mathrm{mM}$ range, while healthy cells are closer to $1 \mathrm{mM}$. By generating Hammett, Eyring, and Brønsted plots, we were able to tune the reactivity of the most commonly used 2,4-dinitrobenzenesulfonyl sensor (fully reacts with $100 \mu \mathrm{M} \mathrm{GSH}$ ) to a sensor that can accurately distinguish GSH concentrations. Further in vitro studies revealed that PACDx had good selectivity over various biologically relevant analytes including metals, amino acids, reactive oxygen and nitrogen species, and other thiols. Following cellular evaluation studies with PACDx, we ported this chemistry into a prodrug (PARx) which will essentially use PA imaging to track the delivery of a chemotherapeutic into lung cancer tissue. Via both local and systemic routes, PARx produced an enhanced PA signal in the tumor compared to a control and successfully inhibited tumor growth without causing toxicity in vital organs. In a blinded study, we further demonstrate the utility of PACDx to stratify groups of cancer types with varying GSH concentrations. Moreover, we demonstrated the high selectivity of PARx to lung cancer as it did not inhibit tumor growth in mice carrying tumor types (i.e., brain) with low GSH concentrations. Overall, we envision that this work will have potential applications in personalized therapy and drug development.
\end{abstract}

Full Name of Abstract's 1st Author : Melissa Lucero 
Complete Status: Complete

First Name: Melissa

Last Name: Lucero

Email: MLucero3@illinois.edu

Organization: University of Illinois at Urbana-Champaign

Country: United States 


\title{
ID: GA462 \\ Polypyrrole Nanoparticles for Photoacoustic Imaging: Their Size Matters
}

\author{
Vit Herynek, First Faculty of Medicine, Charles University, vit.herynek@If1.cuni.cz
}

\section{Category: New Chemistry, Biology \& Bioengineering}

\begin{abstract}
Body : Introduction Photoacoustic (PA) imaging is an emerging modality, usually combined with standard ultrasound imaging. The method is based on the photoacoustic effect. When light interacts with a material, part of the energy is absorbed and partially transformed into heat, which leads to thermal expansion and formation of acoustic waves detectable by an ultrasound transducer. Although PA imaging can be used natively, substantially higher number of applications uses contrast agents. Among them, polypyrrole nanoparticles represent an interesting option [1]. The aim of the study was to reveal, how the nanoparticle size affects PA signal. Materials and Methods We prepared uniform polypyrrole nanoparticles (PPy) with narrow size distributions via water-based redox precipitation polymerization technique of pyrrole monomer in the presence of iron(III) chloride (nPy:nOxi, 1:2.3) as an oxidant. To stabilize growing nanoparticles in reaction mixtures, non-ionic poly(vinylpyrrolide) (PVP, Mn $=40000$ ) was used as a stabilizer. The size, morphology and dispersity were directly evaluated from microphotographs obtained by a transmission electron microscope (FEI-TEM, Tecnai G2 Spirit, Oregon, USA) and analyzed with ImageJ software [2]. Suspensions of PPy samples were diluted to $0.2,0.51,2,3,4,5 \mathrm{mg} / \mathrm{mL}$, inserted into silicon tubes submerged in water and tested as a photoacoustic contrast agent in vitro by using an imaging platform Vevo 3100/LAZR-X (Fujifilm VisualSonics, Amsterdam, Netherlands) combining ultrasound and photoacoustic imaging (excitation wavelength range 680 - $970 \mathrm{~nm}$ and 1200 - $2000 \mathrm{~nm}$ ). Results and Discussion Various reaction conditions in the presence of PVP led to formation of almost uniform nanoparticles with size range from 48 to $130 \mathrm{~nm}$. The TEM images showed regularly spherical nanoparticles with narrow size distribution in all prepared samples. A strong dependence of the signal on particle size was observed. A relative PA signal recalculated per one particle is shown in the Figure. Both dependences on the particle diameter (A) and particle crosssection area (B) are presented. Particle size is important from two perspectives. First, the size may affect cytotoxicity, safety in vivo, NP distribution in the organism, and also elimination routes. The reasonable size ranges between tens to few hundreds of nanometers, which was fulfilled with the investigated set of nanoparticle suspensions. Secondly, particle size strongly influences signal intensity. If the thickness of the nanoparticle layer penetrated by the excitation light is substantially smaller than the core diameter (which may be fulfilled in the diameter range we used in our experiment), heat absorption is proportional rather to particle cross section [3] (or irradiated particle surface). Our data (Fig. B) indicated linear dependence of the signal on particle cross section, which was anticipated. The measured dependence also proved, that suspensions containing smaller particles (with the same solid content in the suspension) were more efficient than suspensions with bigger nanoparticles. Conclusion Polypyrrole nanoparticles represent a promising substance for photoacoustic imaging. Their physical properties, which determine photoacoustic performance, can be tuned by varying of polymerization conditions during NP preparation. Knowledge of the chemical structure and its relation to their physical
\end{abstract}


properties may help to optimize nanoparticle preparation for in vivo imaging in terms of biocompatibility and high photoacoustic efficiency. Acknowledgement The work was supported by Czech Science Foundation - (grant No. 18-05200S), Ministry of Education, Youth and Sports of the Czech Republic (Czech-BioImaging LM2015062), National Sustainability Program II (Project BIOCEV-FAR LQ1604). The facility infrastructure was supported by European Regional Development Fund No. CZ.02.01./0.0./0.0./16_013/0001775 (OPVVV project).

References: [1] Zha Z. et al. Biocompatible polypyrrole nanoparticles as a novel organic photoacoustic contrast agent for deep tissue imaging, Nanoscale 5(10) (2013) 4462-7. [2] Schneider C.A. et al. NIH Image to ImageJ: 25 years of image analysis, Nat Methods 9(7) (2012) 671-675. [3] Shahbazi K. et al. Photoacoustics of core-shell nanospheres using comprehensive modeling and analytical solution approach, Communications Physics 2(1) (2019) 119.

\section{Image/Figure:}

https://www.xcdsystem.com/wmis/abstract/File6959/GA462 ImageFigure 0609041309.png

Image/Figure Caption: Dependence of the relative photoacoustic signal intensity on the nanoparticle diameter (A) or cross-sectional area (B) recalculated per one particle. Measurements were performed at the same concentration $(3 \mathrm{mg} / \mathrm{mL})$ at selected wavelengths $(705,800,915$ $\mathrm{nm})$.

Complete Status: Complete

First Name: Vit

Last Name: Herynek

Email: vit.herynek@1f1.cuni.cz

Organization: First Faculty of Medicine, Charles University

Country: Czech Republic 


\title{
ID: GA463 \\ Site-specific dual-labeling of anti-EGFR VHH 7D12 with a chelator for nuclear imaging and a photosensitizer for targeted photodynamic therapy
}

\author{
Sanne van Lith, Radboudumc, sanne.vanlith@radboudumc.nl
}

Category: New Chemistry, Biology \& Bioengineering

\begin{abstract}
Body : Variable domains of heavy chain only antibodies (VHHs) are interesting molecules for tumor imaging and therapy, mainly because of their small size and fast clearance, leading to efficient tissue penetration, homogeneous tissue distribution and good tumor-tobackground ratios at early time points after injection. VHHs can be conjugated to both a diagnostic probe and a therapeutic compound, turning them into valuable theranostic agents. The most commonly used method is conjugation to naturally occurring lysines, however this random conjugation approach can affect the binding affinity of the VHH (1). Furthermore there is no control over the stoichiometry. In the current study, we employed an unpaired cysteine for sitespecific conjugation using a dichlorotetrazine platform. We site-specifically equipped anti-EGFR VHH 7D12 with both the chelator DTPA and the photosensitizer IRDye700DX, and characterized the properties of this conjugate preclinically. 7D12 with a C-terminal unpaired cysteine was reduced with tris-(2-carboxyethyl)phosphine and conjugated to BCN-PEG2maleimide. The bimodal probe DTPA-tetrazine-IRDye700DX was synthesized according to methods described in (2) and conjugated to 7D12-BCN, yielding DTPA-IRDye700DX-7D12 (Figure 1A). The conjugate was characterized with RP-HPLC-HRMS and UV-visible spectroscopy. Fluorescence quantum yields and generation of molecular singlet oxygen upon illumination were determined. DTPA-IRDye700DX-7D12 was labeled with indium-111 and stability of labeling in PBS and human serum was verified with instant thin layer chromatography. Subsequently, binding and internalization and the half maximal inhibitory concentration (IC50) of the parent 7D12 and DTPA-IRDye700DX-7D12 were determined using EGFR overexpressing A431 cells. Furthermore, A431 cells were incubated with various concentrations of DTPA-IRDye700DX-7D12 (0-100 nM) and illuminated with various doses and dose rates of $690 \mathrm{~nm}$ light using a light emitting diode $(30-90 \mathrm{~J} / \mathrm{cm} 2$ and $50-200 \mathrm{~mW} / \mathrm{cm} 2$, respectively). Cell viability was determined using a luminescence-based assay (CellTiterGloß). The bimodal DTPA-Tz-IRDye700DX probe was obtained with an overall yield of $18 \%$. 7D12- BCN and DTPA-IRDye700DX-7D12 were retrieved with a protein recovery of 74\% and $43 \%$, and a degree of labeling of 1 and 0.56 , respectively. Spectral properties of the IRDye700DX were retained upon conjugation (excmax $689 \mathrm{~nm}$, emmax $698 \mathrm{~nm}$ ). Fluorescence quantum yield of the conjugate was slightly lower compared to that of free IRDye700DX $(0.12$ vs 0.29 , respectively), as was the singlet oxygen generation upon illumination. The conjugate could be labeled with indium-111 and labeling was stable in both PBS and human serum for up to 24 hours. [111In]In-DTPA-IRDye700DX-7D12 bound to A431 cells, and the absence of binding in presence of an excess of unlabeled 7D12 confirmed EGFR specificity. The tracer was efficiently internalized, with $8.8 \pm 0.7 \%$ of added activity found in the intracellular compartment after 4 hours of incubation at $37^{\circ} \mathrm{C}$. IC50 concentrations of native $7 \mathrm{D} 12$ and conjugate were similar (22.8 and $18.1 \mathrm{nM}$, respectively; Figure 1B), indicating that conjugation of the bimodal
\end{abstract}


probe does not interfere with target binding. A431 cells were effectively killed upon illumination after incubation with DTPA-IRDye700DX-7D12 (Figure 1C). In conclusion, the dichlorotetrazine platform offers a feasible method for site-specific dual-labeling of VHH 7D12, without altering binding affinity. Currently, the compound is characterized further in vivo, by performing biodistribution, SPECT/CT imaging and photodynamic therapy studies in a murine subcutaneous A431 model. In the future, the compound could be used for both EGFR-targeted nuclear imaging and photodynamic therapy, in a theranostic approach. The described conjugation method can be applied to other VHHs and small proteins with introduced unpaired cysteines (e.g. DARPins, affibodies). Furthermore, the flexibility of the approach makes it easy to vary the nature of the probes if necessary.

References: 1. Debie P, Van Quathem J, Hansen I, Bala G, Massa S, Devoogdt N, et al. Effect of Dye and Conjugation Chemistry on the Biodistribution Profile of Near-Infrared-Labeled Nanobodies as Tracers for Image-Guided Surgery. Mol Pharm. 2017;14(4):1145-53. 2. Canovas C, Moreau M, Vrigneaud JM, Bellaye PS, Collin B, Denat F, et al. Modular Assembly of Multimodal Imaging Agents through an Inverse Electron Demand Diels-Alder Reaction. Bioconjugate Chem. 2019;30(3):888-97.

\section{Image/Figure:}

https://www.xcdsystem.com/wmis/abstract/File6959/GA463 ImageFigure 0609070202.jpg

Image/Figure Caption: Figure $1 \mathrm{~A}$ ) chemical structure of the bimodal DTPA-IRDye700DX7D12 B) competition of cell associated [111In]In-DTPA-IRDye700DX-7D12 by increasing concentrations of native 7D12 or unlabeled DTPA-IRDye700DX-7D12 showing similar half maximal inhibitory concentrations (IC50) of both compounds C) viability of A431 cells upon incubation with a concentration range of DTPA-IRDye700DX-7D12 and subsequent illumination with $90 \mathrm{~J} / \mathrm{cm} 2690 \mathrm{~nm}$ light at $200 \mathrm{~mW} / \mathrm{cm} 2$.

Complete Status: Complete

First Name: Sanne

Last Name: van Lith

Email: sanne.vanlith@radboudumc.nl

Organization: Radboudumc

Country: Netherlands 


\title{
ID: GA464
}

Machine-learning prediction of response to docetaxel/carboplatin therapy in TNBC Patient-Derived Tumor Xenografts (PDX) using reproducible FDG-PET radiomic features

\author{
Sudipta Roy, Washington University School of Medicine, St. Louis, MO, USA, \\ sudiptaroy@wustl.edu
}

Category: Computational \& Data Science

\begin{abstract}
Body : Introduction: We implemented a co-clinical trial to assess the utility of PET radiomic features in predicting response to docetaxel/carboplatin therapy in triple negative breast cancer (TNBC) PDX. The objective of this work was two-fold: 1) assess the reproducibility of FDG-PET radiomics features in preclinical imaging of TNBC PDX; and 2) implement a machine-learning algorithm (MLA) to predict response to docetaxel/carboplatin therapy. Methods: TNBC PDX were implanted into breast fat pad of NGS mice. In test-retest studies, mice were imaged under identical conditions on consecutive days (Day 1 and Day 2). In the therapy studies, mice were imaged at baseline (BL) and four days (4D) following docetaxel/carboplatin therapy. Therapeutic studies last for 4 weeks. Mice were fasted for four hours prior to imaging. FDG $(200 \mu \mathrm{Ci} / 100 \mu \mathrm{L})$ was injected via tail vein catheter for a $60 \mathrm{~min}$ dynamic PET-CT acquisition. One hundred twenty-three radiomics features were extracted as per ISBI guideline[1] from FDG-PET images of manually segmented 3D tumor: 37 first order, and 86 higher order features extracted after quantization into 64, (GLCM (23), GLRLM (13), GLSZM (13), NGTDM (5), GLDZM (16), and NGLDM (16)). The workflow for the analysis in shown in Figure 1. Lin correlation coefficient (LCC) and Bland-Altman(BA) plots were used to assess the reproducibility of the features using test retest protocol. Regression was used to determine if a radiomic feature was correlated to tumor-volume. A rank importance of predictors using ReliefF algorithm [2] was applied on radiomics to select predictive features as inputs to supervised machine learning. MLA [3-5] with $\mathrm{K}=10$ fold cross validation were applied on the selected features to predict the three category of treatment response (response /no response /partial response) from baseline alone, and from difference between 4D and BL features. The MLA utilized include binary decision tree, support vector machine (SVM), naive Bayes, and ensemble of learners. Response was categorized by volume change after start of treatment: response ( $\geq 20 \%$ volume reduction), partial response ( Results: Eighty eight out of 123 radiomics were identified as reproducible based on LCC on 40 PET images (See Figure 1 (B) for two radiomics). Twenty radiomics out of 88 were identified as volume dependent and those were removed from analysis (See Figure 1 (C) for two radiomics). Based on treatment response, a ReliefF algorithm identified 12 radiomic features (positive rank $\geq 0.05$ ) out of 68 features to use as input to the MLA (see Figure 1(D)). Multiclass binary decision tree(regression) and naïve Bayes performed better in predicting outcome at low error $\leq 20 \%$ (accuracy $>80 \%$ ) using only BL features. Conversely, SVM and naïve Bayes gave performed better at error $\leq 20 \%$ (accuracy $>80 \%$ ), using the differences between $4 \mathrm{D}$ and BL radiomics features. Conclusion: We identified unique, volume-independent, and reproducible radiomic feature to assess response to therapy. A MLA was implemented to predict response to therapy
\end{abstract}


with high-accuracy of $>80 \%$ using only BL radiomic features. Additional biomarkers can be used to further enhance prediction of response to therapy.

References: 1. Zwanenburg A, Vallières M, Abdalah MA, Aerts HJWL, Andrearczyk V, Apte A, et al. "The Image Biomarker Standardization Initiative: Standardized Quantitative Radiomics for High-Throughput Image-based Phenotyping," Radiology (2020);(5):191145. 2. RobnikSikonja, M., and I. Kononenko. (2003). "Theoretical and empirical analysis of ReliefF and RReliefF," Machine Learning, 53, 23-69. 3. Cheng, Zhi et al. "Evaluation of classification and regression tree (CART) model in weight loss prediction following head and neck cancer radiation therapy," Advances in radiation oncology vol. 3,3 346-355. 7 Dec. 2017, doi:10.1016/j.adro.2017.11.006 4. Gao, H., Zeng, X. \& Yao, C. “Application of improved distributed naive Bayesian algorithms in text classification," J Supercomput 75, 5831-5847 (2019). https://doi.org/10.1007/s11227-019-02862-1 5. Gopi Battineni, Nalini Chintalapudi, Francesco Amenta, "Machine learning in medicine: Performance calculation of dementia prediction by support vector machines (SVM)," Informatics in Medicine Unlocked, Volume 16, 2019, 100200, ISSN 2352-9148, https://doi.org/10.1016/j.imu.2019.100200.

\section{Image/Figure:}

https://www.xcdsystem.com/wmis/abstract/File6959/GA464 ImageFigure 0609082242.PNG

Image/Figure Caption: Figure 1: (A) Overall flows of the proposed method from PDX generation to radiomics extraction, (B) concordance plots of two radiomics for repeatability study(top: one repeatable, bottom: one non-repeatable), (C) two volume-dependent features and their relationship with volume, (D) depicts the features selection using ReliefF rank importance of predictors, and $(\mathrm{E})$ is the prediction error of machine learning methods using radiomics and SUV25.

Complete Status: Complete

First Name: Sudipta

Last Name: Roy

Email: sudiptaroy@wustl.edu

Organization: Washington University School of Medicine, St. Louis, MO, USA

Country: United States 


\title{
ID: GA465 \\ Impact of near-infrared-fluorescence labeling of drug carriers on biodistribution and tumor accumulation in vivo
}

\author{
Stefanie Rosenhain, Institute for Experimental Molecular Imaging, \\ srosenhain@ukaachen.de
}

Category: Oncology

\begin{abstract}
Body : Introduction: Fluorescence-mediated computed tomography (FLT-CT) enables to non-invasively assess the biodistribution and tumor accumulation of near-infrared fluorescently (NIRF) labeled drug carriers and thus, plays an important role in preclinical cancer research. However, NIRF dyes are actively taken up by transporting proteins and can affect the biodistribution pattern of the drugs. In this context, organic anion transporting proteins (OATP) recently gained attention, because they are involved in the uptake of fluorescence dyes. It is proven that not only hepatocytes but also most cancer cells overexpress different OATPs. Since this can cause false-positive results, especially in tumor and liver tissue, it is important to understand OATP-mediated transport of fluorescence dyes and their influence on biodistribution and tumor accumulation. Material/Methods: OATP-mediated uptake of the free dyes AlexaFluor750 (AF750), Cy7, DY-730, DY-736, DY-750, ICG, IR-783, IRDye 750, and 800 $\mathrm{CW}$ was investigated by FLT-CT phantom and absorption measurements. OATP-mediated uptake of the dyes in A431, HepG2, and HUVECs (control) were investigated at $4{ }^{\circ} \mathrm{C}$ and $37^{\circ} \mathrm{C}$ ( $20 \mu \mathrm{M} ; 2 \mathrm{~h}$ ) using fluorescence microscopy and flow cytometry. Inhibition of the OATPs was performed using Rifampicin $(20 \mu \mathrm{M})$. OATP expression (SLC01A2, SLC01B1, SLC01B3, and SLC02B1) was determined by qPCR. Effects on biodistribution and tumor accumulation of free AF750, Cy7, DY750, ICG (analysis is pending) and IR-783 were analyzed in five A431 tumorbearing athymic nude mice. After injection of the dyes $(2 \mathrm{nmol} / \mathrm{animal})$, mice were measured by FLT-CT at $0 \mathrm{~h}, 3 \mathrm{~h}, 6 \mathrm{~h}, 24 \mathrm{~h}, 48 \mathrm{~h}, 72 \mathrm{~h}$, and $96 \mathrm{~h}$. After $96 \mathrm{~h}$, excised organs were imaged by $2 \mathrm{D}$ fluorescence mode. Further in vitro and in vivo investigation of poly(N-(2hydroxypropyl)methacrylamide) (pHPMA) labeled with the above-mentioned NIRF dyes are ongoing. Results: qPCR demonstrated a higher expression of OATP1A2 and OATP1B1 in both A431 and HepG2. Microscopic analysis revealed no uptake of AF750 and Cy7 into A431, in contrast to the uptake of DY-750 and IR-783. These finding were also confirmed in vivo. After injection of AF750, no tumor signal but a strong bladder signal along with a fast clearance from the whole body until 6h post-injection can be seen. Fluorescence intensities of $\mathrm{Cy} 7$ were strong both in liver and gut at early points in time. Most of the dye is cleared not later than $48 \mathrm{~h}$ post injection via the hepatobiliary elimination route, although some signal remained in the kidneys. IR-783 was showing a strong tumor accumulation even at $96 \mathrm{~h}$. These findings were supported by the ex vivo results. The preliminary results indicate a possible impact of NIRF dyes on biodistribution assessment of fluorescence-tagged molecules. Especially IR-783 shows a strong tumor signal, possibly influencing tumor accumulation of molecules labeled with IR-783. Conclusion: A comprehensive knowledge of the transport processes of NIRF dyes which are mainly OATP-mediated but not exclusively will increase proper fluorescence labeling strategies without distorting the pharmacokinetic properties and can optimize the specificity of favorable
\end{abstract}


targeting. Further studies are needed to investigate the biodistribution of fluorescence-tagged molecules and the role of OATP-mediated transport in vitro and in vivo.

References: • Becker, A, et al. 2000. "Macromolecular Contrast Agents for Optical Imaging of Tumors: Comparison of Indotricarbocyanine-Labeled Human Serum Albumin and Transferrin." Photochemistry and Photobiology 72 (2): 234-41. • Buxhofer-Ausch, V., et al. 2013. "TumorSpecific Expression of Organic Anion-Transporting Polypeptides: Transporters as Novel Targets for Cancer Therapy." Journal of Drug Delivery 2013: 1-12. • Debie, P. 2017. "Effect of Dye and Conjugation Chemistry on the Biodistribution Profile of Near-Infrared-Labeled Nanobodies as Tracers for Image-Guided Surgery." Molecular Pharmaceutics 14 (4): 1145-53. - Lee, H., et al. 2015. "Contribution of Hepatic Organic Anion Transporting Polypeptides (OATPs) to Docetaxel Uptake and Clearance." Molecular Cancer Therapeutics 14 (4): 994-1003. • Snipstad, S., et al. 2016. "Labeling Nanoparticles: Dye Leakage and Altered Cellular Uptake." Cytometry Part A, April, n/a-n/a. https://doi.org/10.1002/cyto.a.22853. • Schulte, R. and Ho, R. 2019. "Organic Anion Transporting Polypeptides: Emerging Roles in Cancer Pharmacology.” Molecular Pharmacology • Thakkar, N., et al. 2015. "Role of Organic Anion-Transporting Polypeptides (OATPs) in Cancer Therapy.” The AAPS Journal 17 (3): 535-45. • Vasquez, K., et al. 2011. "Quantitative Whole Body Biodistribution of Fluorescent-Labeled Agents by Non-Invasive Tomographic Imaging." PLoS ONE 6 (6): e20594. • Yi, X., et al. 2015. "IR-780 Dye for NearInfrared Fluorescence Imaging in Prostate Cancer.” Medical Science Monitor : International Medical Journal of Experimental

\section{Image/Figure:}

https://www.xcdsystem.com/wmis/abstract/File6959/GA465_ImageFigure_0609101912.jpg

Image/Figure Caption: In vivo and ex vivo biodistribution analysis of intravenously injected free dyes: AF750, Cy7, DY750, and IR-783. After injection of IR-783, there is a strong tumor accumulation that last until $96 \mathrm{~h}$ p.i. AF750 exhibit a fast clearance from the whole body which is evidenced by the strong bladder signals. Cy7 depicted strong fluorescence intensities both in liver and gut at early points in time. Although some signal remains in the kidneys, most of Cy7 is hepatobiliary cleared not later than $48 \mathrm{~h}$ p.i. DY750 shows indistinct signals in the liver, but is completely cleared after $24 \mathrm{~h}$ with no remaining signal in the organs $96 \mathrm{~h}$ post injection. Further fluorescence intensity quantification needs to be performed. After injection of AF750, no tumor signal but a strong bladder signal along with. IR-783 was showing a strong tumor accumulation even at $96 \mathrm{~h}$. These findings were supported by the in vitro and ex vivo results.

Complete Status: Complete

First Name: Stefanie

Last Name: Rosenhain

Email: srosenhain@ukaachen.de

Organization: Institute for Experimental Molecular Imaging 
Country: Germany 


\title{
ID: GA466 \\ Molecular MRI enables high resolution mapping of post-reperfusion vascular inflammation in ischemic stroke
}

\author{
Thomas Bonnard, GIP Cyceron, UMR-S Inserm U1237 - PhIND, \\ thomasbonnard@gmail.com
}

\section{Category: Neuroscience}

\begin{abstract}
Body : Ischemic stroke is a sudden neurological disease caused by the obstruction of cerebral blood flow. It represents the third cause of death and the first cause of acquired handicap among adults in industrialized countries. The use of advanced medical imaging techniques in acute setting, such as computed tomography (CT), and perfusion- and diffusion-weighted magnetic resonance imaging (MRI), has recently significantly improved treatment decisionmaking for acute ischemic stroke.1 Smart optimization of clinical imaging protocols, in combination with novel treatment strategies, has enabled extension of the treatment window up to $24 \mathrm{~h}$ for some patients, while it was for decades limited to $4.5 \mathrm{~h}$ post stroke onset. 2 However, the information provided by perfusion and/or diffusion imaging in stroke patients has some limits. It does not directly inform on the status of vascular inflammation for example. Yet, inflammation is known to play an important role in ischemic stroke damage, suspected to remain once treatment restored brain vessel patency. 3 This preclinical research investigated the efficacy of a molecular MRI approach to reveal vascular inflammation in ischemic stroke. The dynamics of vessel flow, tissue perfusion, and vascular inflammation and their correlation to brain lesion growth were studied in a transient middle cerebral artery occlusion (tMCAO) rat model with a preclinical 9.4 T MRI scanner (Varian Inc.). MRI was performed at $1 \mathrm{~h}, 6 \mathrm{~h}, 24 \mathrm{~h}$ and $96 \mathrm{~h}$ post reperfusion. Microparticles of iron oxide targeted to vascular cell adhesion molecule 1 (MPIO$\alpha \mathrm{VCAM}-1)$ were injected intravenously to reveal endothelial cell activation on susceptibilityweighted images (SWI). Bolus injections of Gadolinium-based contrast agent (Gadovist ${ }^{\circledR}$ ) were used for perfusion-weighted imaging (PWI). Time-of-flight weighted sequences were used for magnetic resonance angiography (MRA). Brain lesions were measured on apparent diffusion coefficient (ADC) maps for early time points ( $1 \mathrm{~h}$ and $6 \mathrm{~h}$ post reperfusion) and on T2 weighted images for later time points (24h and $96 \mathrm{~h}$ post reperfusion). Twenty-four hours post reperfusion, the intravenous injection of microparticles of iron oxide functionalized with anti-VCAM-1 antibody (MPIO- $\alpha$ VCAM-1) induced localized negative signals in the susceptibility-weighted images (SWI) (cf. figure). The method enabled high resolution mapping of VCAM-1 expression. It revealed an area of vascular inflammation that appeared around the core of the lesion. We detected the strongest VCAM- 1 expression $6 \mathrm{~h}$ post reperfusion, whereas no signal was measured $1 \mathrm{~h}$ post reperfusion ( $\mathrm{p}$ The strategy of molecular MRI with VCAM-1-specific iron oxide contrast agent enabled in vivo detection of the dynamic vascular inflammation process involved after abrupt reperfusion in ischemic stroke. Implementation of this technique in clinical settings may provide complementary information on stroke patient status and could help to guide therapeutic decision-making for emergency radiologist.
\end{abstract}


References: 1. Rudkin, S., Cerejo, R., Tayal, A. \& Goldberg, M. F. Imaging of acute ischemic stroke. Emerg. Radiol. 25, 659-672 (2018). 2. Nogueira, R. G. et al. Thrombectomy 6 to 24 Hours after Stroke with a Mismatch between Deficit and Infarct. N. Engl. J. Med. 378, 11-21 (2018). 3. Kehrel, B. E. \& Fender, A. C. Resolving Thromboinflammation in the Brain After Ischemic Stroke? Circulation 133, 2128-2131 (2016).

\section{Image/Figure:}

https://www.xcdsystem.com/wmis/abstract/File6959/GA466 ImageFigure 0609102612.JPG

Image/Figure Caption: Molecular MRI at $24 \mathrm{~h}$ post reperfusion. A. coronal sections of susceptibility weighted images (SWI) before and after the intravenous injection of VCAM-1 specific MPIO contrast agent (MPIO- $\alpha$ VCAM-1). B. Coronal sections treated with brain extracted tool of corresponding T2 map revealing lesion area and SWI after MPIO injection. MPIO signal uptake is highlighted in yellow color. C. 3 Dimensions reconstruction of SWI acquisition treated with brain extraction tool. Lesion core and MPIO signal areas are highlighted in yellow and red color, respectively.

Full Name of Abstract's 1st Author : Thomas Bonnard

Complete Status: Complete

First Name: Thomas

Last Name: Bonnard

Email: thomasbonnard@gmail.com

Organization: GIP Cyceron, UMR-S Inserm U1237 - PhIND

Country: France 


\title{
ID: GA467 \\ Quantification of Trastuzumab-HER2 engagement in human breast cancer models using fluorescence lifetime FRET imaging
}

\author{
Alena Rudkouskaya, Albany Medical College, rudkoua@amc.edu
}

\section{Category: Oncology}

\begin{abstract}
Body : Overexpression of Human EGF Receptor 2 (HER2) in cancer tumors is a marker of aggressive metastatic disease and poor prognosis. At the same time, it presents a target for precision therapy such as trastuzumab (TZM). Anti-HER2 humanized monoclonal antibody trastuzumab (TZM) has been successfully used clinically over the last two decades. However, not all eligible patients benefit from this therapy due to either primary or acquired TZM resistance1,2. Further investigation of TZM-HER2 binding, internalization and trafficking/degradation in cancer cells in vitro and in vivo may provide better understanding of this chemoresistance. In particular, fluorescence lifetime Forster resonance energy transfer imaging (FL-FRET) offers a unique approach to monitor TZM-HER2 binding followed by their uptake into target cells via the reduction of donor fluorophore lifetime3,4. Since this approach does not rely on intensity of contrast agent, it provides an efficient method to quantify ligandreceptor binding and internalization in cancer cells with an additional benefit of sensing intracellular or intra-tumoral microenvironment heterogeneity. We validated quantification of target engagement both in vitro and in vivo using FL-FRET in transferrin-transferrin receptor system5,6 In this study, we report a multiscale quantification of TZM-HER2 engagement in AU565 breast cancer cells and tumor xenografts using FL FRET imaging and phasor analysis approach. First, we characterized TZM-AF700 uptake and its relation to HER2 expression in AU565 cells using confocal microscopy. Further, we have quantified the dimerization of HER2 via NIR TZM FLIM FRET in vitro microscopy. Extensive analysis confirmed high specificity and efficiency of TZM FRET signal. Interestingly, we observed a significant heterogeneity of FRET within the cells: the highest TZM FRET levels occurred at the plasma membrane, whereas a decreasing gradient of donor lifetime reduction was registered as the TZM-HER2 complexes undergo internalization and intracellular endocytic trafficking. Phasor analysis 7 confirmed these observations qualitatively and quantitatively. These results suggest that TZM-HER2 complexes undergo significant conformational changes, including possible dissociation or degradation upon TZM-HER2 binding and trafficking. In addition, we performed FL-FRET imaging of TZM AF700/AF750 internalization in AU565 tumor xenografts in live intact animals. We successfully quantified the fraction of dimerized HER2 receptors bound by TZM and internalized in the tumors, livers and bladders. Importantly, immunohistological analysis of tumors showed significant upregulation of HER2 and HER3 expression in the tumors of mice injected with TZM compared to the untreated ones, indicating that TZM modulates their heterodimerization and signaling. Altogether, our results demonstrate that FL-FRET imaging robustly and directly reports on TZM-HER2 engagement in multiscale cancer models.
\end{abstract}

References: References (1) Rye, I. H.; Trinh, A.; Sætersdal, A. B.; Nebdal, D.; Lingjærde, O. C.; Almendro, V.; Polyak, K.; Børresen-Dale, A. L.; Helland, Å.; Markowetz, F.; et al. Intratumor 
Heterogeneity Defines Treatment-Resistant HER2+ Breast Tumors. Mol. Oncol. 2018, 12 (11), 1838-1855. https://doi.org/10.1002/1878-0261.12375. (2) Vu, T.; Claret, F. X. Trastuzumab: Updated Mechanisms of Action and Resistance in Breast Cancer. Frontiers in Oncology. 2012. https://doi.org/10.3389/fonc.2012.00062. (3) Sun, Y.; Day, R. N.; Periasamy, A. Investigating Protein-Protein Interactions in Living Cells Using Fluorescence Lifetime Imaging Microscopy. Nat. Protoc. 2011. https://doi.org/10.1038/nprot.2011.364. (4) Rajoria, S.; Zhao, L.; Intes, X.; Barroso, M. FLIM-FRET for Cancer Applications. Curr. Mol. Imaging 2015, 3 (2), 144-161. https://doi.org/10.2174/2211555203666141117221111. (5) Abe, K.; Zhao, L.; Periasamy, A.; Intes, X.; Barroso, M. Non-Invasive in Vivo Imaging of near Infrared-Labeled Transferrin in Breast Cancer Cells and Tumors Using Fluorescence Lifetime FRET. PLoS One 2013, 8 (11). https://doi.org/10.1371/journal.pone.0080269. (6) Rudkouskaya, A.; Sinsuebphon, N.; Ward, J.; Tubbesing, K.; Intes, X.; Barroso, M. Quantitative Imaging of Receptor-Ligand Engagement in Intact Live Animals. J. Control. Release 2018, 286, 451-459.

https://doi.org/10.1016/j.jconrel.2018.07.032. (7) S-J Chen, N Sinsuebphon, A Rudkouskaya, M Barroso, X Intes and X Michalet, "In vitro and in vivo phasor analysis of stoichiometry and pharmacokinetics using near-infrared dyes," Journal of Biophotonics 12(3):e201800185

Complete Status: Complete

First Name: Alena

Last Name: Rudkouskaya

Email: rudkoua@amc.edu

Organization: Albany Medical College

Country: United States 


\title{
ID: GA468 \\ Engineered Exosomes as Cell Specific Imaging Probe
}

\author{
Ali Arbab, Augusta University, aarbab@augusta.edu
}

\section{Category: New Chemistry, Biology \& Bioengineering}

\begin{abstract}
Body : Exosomes are nano-sized (30-140nm) spherical vesicles, derived from the endosomal system for intercellular communications. Exosomes have clear advantages over synthetic nanoparticles like liposomes or any other metallic or chemically synthesized nanoparticles as a vehicle/probe because of their more biocompatibility, low toxicity and immunogenicity, enhanced permeability (even through blood-brain barrier) and stability in biological fluids, and ability to accumulate in the tumor or lesions with higher specificity. Different imaging modalities and techniques are being utilized to detect specific cells in vivo. Most of the published reports showed the use of peptide or antibody to target specific cells. The use of hydrophobic peptide/s make the imaging agent less attractive due to poor target-tobackground ratio, therefore, investigators have used nanoparticle-based contrast agent to image specific cells. Making of these Nanoparticles (synthetic) or peptide-based imaging agents needs the involvement of extensive chemistry or radiochemistry. Here we propose to utilize cellular and molecular biology (recombinant DNA technology) technology to make biocompatible, biodegradable, and non-immunogenic nanovesicles (exosome) to carry neutrophil or alternately activated macrophage targeting peptide/s to image neutrophils or macrophages in vivo. These exosomes can be tagged with single-photon emission computed tomography (SPECT) compatible radioisotope/s to be used as SPECT imaging probes. Recently our laboratory has achieved a few milestones in exosome technology; (1) we developed a platform (patent pending) to make engineered exosomes using non-tumorous HEK293 cells that carry and express specific cell targeting peptide to detect specific cells when administered intravenously, (2) we used these engineered exosomes as a therapeutic probe to deplete specific cells in the body, (3) we optimized the methods to collect exosomes in shortest possible time, (4) we showed in vivo biodistribution of exosomes collected from different cells by clinically relevant SPECT imaging. Both in vitro and in vivo studies showed the specificity of these engineered exosomes to target specific cells. In vivo SPECT studies showed targeting and delineating the specific cells in the body.
\end{abstract}

References: 1. Rashid MH, Borin TF, Ara R, Alptekin A, Liu Y, Arbab AS. Generation of novel diagnostic and therapeutic exosomes to detect and deplete pro-tumorigenic M2-macrophages. Advanced Therapeutics. 2020 May; doi: 10.1002.adtp.201900209. 2. Rashid MH, Borin TF, Ara R, Angara K, Cai J, Achyut BR, Liu Y, Arbab AS. Differential in vivo biodistribution of 131Ilabeled exosomes from diverse cellular origins and its implication for theranostic application. Nanomedicine. 2019 Oct;21:102072. doi: 10.1016/j.nano.2019.102072. Epub 2019 Aug 1. PubMed PMID: 31376572; PubMed Central PMCID: PMC6814553.

\section{Image/Figure:}


Image/Figure Caption: Figure 1: In vivo specificity of targeting engineered exosomes. (A) In vivo SPECT/CT images (coronal view) after $3 \mathrm{hrs}$ of intravenous injection showed significant accumulation of M2-targeting exo in tumor, lung, spleen, lymph node, and bones. 111In-oxinelabeled non-targeting exosomes (HEK293 exo) and CD206-positive M2-macrophage targeting exosomes (M2-targeting exo) were injected into the 4T1 tumor-bearing mice. One group was treated with Clophosome ${ }^{\circledR}$ to deplete macrophages. Yellow and green arrows denote lymph node and bone metastasis, respectively. (B) 3D surface images showing M2-targeting exo are profoundly distributed in both lung and tumor areas compared to the group injected with HEK293 exo and pre-treated with Clophosome ${ }^{\circledR}$. The yellow arrow indicates the tumor center.

Complete Status: Complete

First Name: Ali

Last Name: Arbab

Email: aarbab@augusta.edu

Organization: Augusta University

Country: United States 


\title{
ID: GA469 \\ Imaging biotin trafficking in mice with positron emission tomography
}

\author{
Salvatore Bongarzone, King's College London, salvatore.bongarzone@kcl.ac.uk
}

\section{Category: New Chemistry, Biology \& Bioengineering}

\begin{abstract}
Body : Water-soluble vitamin H (biotin) is essential for cellular growth, development and well-being. Biotin (vitamin B7) is a cofactor for five carboxylases involved in gluconeogenesis and catabolism of amino acids and fatty acid biosynthesis [1]. Biotin is taken up by cells via the sodium-dependent vitamin transporter (SMVT), expressed in the cytoplasm and mitochondrial membranes. SMVT is present in the gastrointestinal tract, liver, kidneys, retina, heart, brain and skin. Biotin conjugates have been radiolabelled using PET and single-photon emission computed tomography (SPECT) radionuclides such as 64Cu, 18F, 111 In, 125I, 131I, $68 \mathrm{Ga}$ and $99 \mathrm{mTc}$ [2-4]. These "biotin-inspired" imaging probes have been used in clinical and preclinical settings for infection and tumour imaging. However, there is no report of studies of radiolabeled isotopologue of biotin itself using non-invasive PET imaging modality in vivo to date. In this work, we have used a new radiochemical reaction [5] to achieve the first synthesis of carbon-11 radiolabelled biotin ([11C]biotin, Figure 1A) as an in vivo PET imaging agent for probing biotin trafficking. The urea moiety of biotin was radiolabelled using cyclotron-produced carbon dioxide $([11 \mathrm{C}] \mathrm{CO} 2)$ and diamino biotin via a Mitsunobu reaction. Total synthesis time including HPLC purification was $32 \pm 1$ minutes from end of [11C]CO2 delivery. The amount of [11C]biotin obtained was $352 \pm 38 \mathrm{MBq}$ in $4-5 \mathrm{~mL}$ PBS with $2.5 \%$ ethanol in injectable solution starting from $5.9 \pm 0.3 \mathrm{GBq}$ of cyclotron produced ([11C]CO2 with an isolated radiochemical yield of $19 \pm 2 \%$, radiochemical purity $>99 \%$, molar activity of $7 \pm 1 \mathrm{GBq} / \mu \mathrm{mol}$ at end of $[11 \mathrm{C}] \mathrm{CO} 2$ delivery. To examine [11C]biotin trafficking in vivo, the radiotracer was administered intravenously (IV) in healthy anaesthetised mice placed in a high-resolution microPET scanner. Sixteen mice were divided into three groups: group A1 (female, vehicle IV injection 10 minutes before [11C]biotin IV injection, Figure 1B), group A2 (male, vehicle IV injection 10 minutes before [11C]biotin IV injection, Figure 1B) and group A3 (female, biotin $5 \mathrm{mg} / \mathrm{Kg}$ IV injection 10 minutes before [11C]biotin IV injection, Figure 1B). Dynamic PET image data were acquired for 60 minutes. Preclinical experiments showed that [11C]biotin administered intravenously is distributed in the liver, kidneys, retina, heart, brain and brown adipose tissue (BAT) consistent with known in vivo biotin transporter expression (Figure 1C). A significant decrease of [11C]biotin uptake in the biotin-challenged group (group A3) compared to the control group A1 was observed in liver, brain and BAT with a remarkable increase in [11C]biotin excretion (Figure 1D). In conclusion, by incorporating a positron emission tomography (PET) radioisotope (carbon-11) in the structure of biotin ([11C]biotin), we have provided a qualitative and quantitative method for non-invasive evaluation of real-time and whole-body pharmacokinetic of $[11 \mathrm{C}]$ biotin in living organism (animals) using PET imaging. Potential applications of [11C]biotin include the diagnosis and localisation of bacterial infection and cancer via a directlabelling or pre-targeting strategy. Firstly, the uptake of biotin by E. Coli is highly efficient. [11C]Biotin might have prospects for imaging bacterial infection compared with the "biotininspired" radiotracers in which the carboxylic group is modified [6]. Secondly, various
\end{abstract}


aggressive cancer cell lines such as ovarian, leukemia, mastocytoma, colon, breast renal, and lung cancer cell lines overexpress SMVT. The use of [11C]biotin in cancer might be useful for cancer diagnosis/management and understanding the role of SMVT in tumorigenesis. Alongside the potential of [11C]biotin as an imaging biomarker in pathological conditions, its clinical translation will allow the study of whole-body biotin trafficking in healthy humans.

References: [1] Said, H. M. Biotin: biochemical, physiological and clinical aspects. Subcell. Biochem. 56, 1-19, doi:10.1007/978-94-007-2199-9_1 (2012). [2] Ren, W. X. et al. Recent development of biotin conjugation in biological imaging, sensing, and target delivery. Chem. Commun. (Camb) 51, 10403-10418, doi:10.1039/c5cc03075g (2015). [3] Lewis, M. R. et al. In vivo evaluation of pretargeted $64 \mathrm{Cu}$ for tumor imaging and therapy. J. Nucl. Med. 44, 1284-1292 (2003). [4] Shoup, T. M. et al. Synthesis of fluorine-18-labeled biotin derivatives: biodistribution and infection localization. J. Nucl. Med. 35, 1685-1690 (1994). [5] Dheere, A. K. H., Bongarzone, S., Taddei, C., Yan, R. \& Gee, A. D. Synthesis of C-11-Labelled Symmetrical Ureas via the Rapid Incorporation of [C-11]CO2 into Aliphatic and Aromatic Amines. Synlett 26, 2257-2260, doi:10.1055/s-0034-1381055 (2015). [6] Lazzeri, E. et al. Scintigraphic imaging of vertebral osteomyelitis with 111In-biotin. Spine 33, E198-204, doi:10.1097/BRS.0b013e31816960c9 (2008).

\section{Image/Figure:}

https://www.xcdsystem.com/wmis/abstract/File6959/GA469 ImageFigure_0609032306.png

Image/Figure Caption: Figure 1: Structure of [11C]biotin (A). Schematic illustration of the PET/CT image acquisition of intravenous (IV) administration of formulation A and [11C]biotin (B). PET images of group A1 and A3 mice at 5 minutes post-radiotracer injection (C and D, respectively).

Full Name of Abstract's 1st Author : Salvatore Bongarzone

Complete Status: Complete

First Name: Salvatore

Last Name: Bongarzone

Email: salvatore.bongarzone@kcl.ac.uk

Organization: King's College London

Country: United Kingdom 


\title{
ID: GA470 \\ Characterisation of myocardial fibrosis in a rat model of hypertensive heart failure for PET imaging with 18F-Fluoroprolines
}

\author{
Viktoria Balogh, University of Edinburgh, s1243323@sms.ed.ac.uk
}

\section{Category: Cardiovascular \& Pulmonary}

\begin{abstract}
Body : Background: Fibrosis is a fundamental process involved in healing and remodelling during heart failure (HF). Positron emission tomography (PET) with fluoroprolines 1 is a potential tool for investigating active collagen biosynthesis in the myocardium. In order to test the utility of $18 \mathrm{~F}$-fluoroprolines as markers of collagen biosynthesis, animal models of cardiovascular fibrosis need to be developed and characterised. It was hypothesised that rats treated with angiotensin II (AngII) would develop myocardial fibrosis and could be used for testing PET radiotracers targeting collagen biosynthesis. This study aims to investigate the development of myocardial fibrosis in AngII treated rats using ex vivo imaging and biochemistry techniques. Methods: Adult male Sprague-Dawley rats (13-15 weeks, $n=6-9)$ were administered AngII (250 or $500 \mathrm{ng} / \mathrm{kg} / \mathrm{min}$, s.c.) or saline (control) for 4 weeks (using osmotic mini-pumps) to induce left ventricular hypertrophy and diffuse fibrosis. Blood pressure was measured during this period by tail cuff plethysmography. Ex vivo assessment of myocardial collagen content (Picrosirius red (PSR) stain; hydroxyproline (HP) assay) were undertaken in tissues isolated at the end of the treatment period. Furthermore, immunofluorescence was performed to investigate the presence of myocardial inflammation using macrophage and endothelial cell markers (CD68 and isolectin, respectively). Results: Systolic blood pressure was increased after treatment with 250 or $500 \mathrm{ng} / \mathrm{kg} / \mathrm{min}$ AngII, for 2 weeks (p Conclusions: Infusion of AngII for 4 weeks results in the development of a promising diffuse fibrosis model able to mimic hypertensive HF. Results of the HP assay suggest potential for success using fluoroproline PET radiotracers in detecting active collagen biosynthesis in this model. This potential will be assessed by exposing the high dose (4 weeks of $500 \mathrm{ng} / \mathrm{kg} / \mathrm{min}$ AngII) group to PET/MRI using 18F-fluoroprolines.
\end{abstract}

References: 1 Geisler, S. et al. Isomers of 4-[18F]fluoro-proline: radiosynthesis, biological evaluation and results in humans using PET. Curr Radiopharm 7, 123-132 (2014).

\section{Image/Figure:}

https://www.xcdsystem.com/wmis/abstract/File6959/GA470 ImageFigure 0609112435.jpg

Image/Figure Caption: Figure A. Systolic blood pressure measurements measured at baseline and during angiotensin II treatment in rats with tail cuff plethysmography, showing increased values in both treatment groups compared to baseline. Figure B. Hydroxyproline (HP) assay results showing increased HP content in high dose angiotensin II treated rats compared to controls. Figure C. Example images of Picrosirius red staining of a heart section from all treatment groups, red areas labelling collagen deposition. Comparison of all three groups showed increased fibrosis in the high dose group only. Figure D. Example images of the same hearts 
(adjacent sections to PSR) stained for DAPI (blue- nuclei) and CD68 (red- total macrophages), global analysis of CD68 \% area showed no differences between groups. Figure E. The same sections as in D showing DAPI and isolectin-B4 staining (white- endothelial and macrophage marker), global analysis showed no differences between groups. Figure F. Graph shows the comparison of PSR \% area measurements in perivascular areas of the myocardium, suggesting increased collagen deposition with high dose treatment. Figure G. Graph shows CD68 \% area values of vascular areas in the myocardium compared between the three groups, suggesting an increase in macrophages with low dose treatment.

Complete Status: Complete

First Name: Viktoria

Last Name: Balogh

Email: s1243323@sms.ed.ac.uk

Organization: University of Edinburgh

Country: United Kingdom 


\title{
ID: GA471 \\ NOVEL CONTRAST AGENTS BASED ON QUADRUPOLAR PEAKS FOR FFC-MRI
}

\author{
Simona Baroni, University of Turin, simona.baroni@unito.it
}

Category: New Chemistry, Biology \& Bioengineering

\begin{abstract}
Body : The present study aims at developing an innovative class of MRI contrast agents for Fast Field Cycling-MRI applications. They represent a completely new class of contrast agents, able to generate $14 \mathrm{~N}-\mathrm{Quadrupolar}$ Peaks (QPs), i.e. a relaxation enhancement at magnetic fields where the proton NMR frequency and the $14 \mathrm{~N}$ nuclear quadrupole resonance frequency coincide [1]. Their detection requires the acquisition of images at variable magnetic field strength as provided by the innovative Fast Field Cycling MRI (FFC-MRI) scanners (Fig. 1). The QP from the contrast agents has to fall at frequencies well distinguishable from those associated with the amidic peptide bonds from endogenous proteins, which occur at 0.7, 2.1 and 2.8 MHz. The first generation of QP contrast agents contain a polymeric form of histidine, which imidazole groups produce a characteristic enhancement at about 1.3-1.4 MHz [2]. The QPs are detectable only when the contrast agent is in a gel- or solid-like form. Since the maintenance of the immobilized state depends on the $\mathrm{pH}$, the contrast agents may report on tissue $\mathrm{pH}$ changes, that can be associated to the occurrence of a pathological condition, such as cancer, or to cellular apoptosis/necrosis. These innovative contrast agents are suitable for the design of nano- and micro- devices, such as scaffolds for tissue engineering in the field of regenerative medicine. They represent a possible answer to the current medical needs, such as an almost complete lack of methods for the rapid, non-invasive and repeated monitoring of tissue implants. Furthermore, new methods are needed to monitor cell status and polymer degradation under physiological conditions (temperature, saline medium, $\mathrm{pH}$, enzymes etc.) thus allowing the physician to control, in real time, the status of the implanted scaffold. Herein, we present the preparation, relaxometric characterization, preliminary in vitro and in vivo data of porous poly-histidinePLGA scaffolds suitable as QPs contrast agent for FFC-MRI, a new technique that introduces the strength of the applied magnetic field as an entirely new dimension into MRI, which opens new avenues for non-invasive imaging technologies with human applications.
\end{abstract}

References: [1] C. Gösweiner et al. (2018) Tuning Nuclear Quadrupole Resonance: A Novel Approach for the Design of Frequency-Selective MRI Contrast Agents. Phys. Rev. X 8, 10.1103/PhysRevX.8.021076. [2] S. Geninatti Crich, S. Aime, R. Stefania, S. Baroni, M.R. Ruggiero, L. Broche, D. Lurie "Nuovi agenti di contrasto per risonanza magnetica per immagini", Patent number 102019000007647 (2019).

\section{Image/Figure:}


Image/Figure Caption: Figure 1. Graphic presentation of the new class of QP-based contrast agents.

Complete Status: Complete

First Name: Simona

Last Name: Baroni

Email: simona.baroni@unito.it

Organization: University of Turin

Country: Italy 


\title{
ID: GA472
}

\section{[18F]LW223, a TSPO PET radiotracer with binding to human tissue independent of the rs6971 genetic polymorphism, has low non-specific binding in vivo}

\author{
Agne Stadulyte, University of Edinburgh, a.stadulyte@sms.ed.ac.uk
}

\section{Category: Immunology: Inflammation \& Infection}

\begin{abstract}
Body : Introduction: One of the most widely studied biomarkers of inflammation in the field of molecular imaging is the $18 \mathrm{kDa}$ translocator protein (TSPO). Various TSPO Positron Emission Tomography (PET) radiotracers have been developed for preclinical and clinical use. Unfortunately, these have suboptimal properties, including the binding to human tissue dependent on the rs6971 polymorphism [1] and high non-specific and non-displaceable binding in vivo [2-4]. Recently, the first fluorine-18 labelled PET TSPO radiotracer, [18F]LW223, with binding to human tissue independent of the rs6971 polymorphism [5], has been developed, representing the resolution of a major limitation to clinical adoption of TSPO PET imaging. We hypothesise that $[18 \mathrm{~F}] \mathrm{LW} 223$ will also have low non-specific and non-displaceable binding in vivo, which would resolve the second bottle neck in the field of TSPO PET imaging. This study aims to investigate the specific binding and equilibrium dissociation constant $(\mathrm{Kd})$ of $[18 \mathrm{~F}] \mathrm{LW} 223$. Furthermore it aims to report the mass dose limits (5\% occupancy D5 and 10\% occupancy D10) of [18F]LW223 in mice. Methods: [18F]LW223 was injected to male $\mathrm{CD} 57 \mathrm{Bl} / 6 \mathrm{~J}$ mice $(\mathrm{age}=14.1 \pm 4.7$ weeks, weight $=28.4 \pm 3.1 \mathrm{~g}$; mean $\pm \mathrm{SD}, \mathrm{n}=16$ ) via femoral or tail vein $(8.1 \pm 3.1 \mathrm{MBq}, 100-200 \mu \mathrm{L})$ either on its own (baseline molar activity of $84.8 \pm 47$, mean $\pm \mathrm{SD}, \mathrm{n}=3)$ or with varying doses of non-radioactive LW223 $(0.0028 \pm 0.0008 \mathrm{mg} / \mathrm{kg}$ - 'Dose 1', $\mathrm{n}=6 ; 0.016 \mathrm{mg} / \mathrm{kg}$ - 'Dose 2', $\mathrm{n}=1 ; 0.22 \pm 0.04 \mathrm{mg} / \mathrm{kg}$ - 'Dose 3', $\mathrm{n}=3$; or $0.55 \pm 0.09 \mathrm{mg} / \mathrm{kg}$ 'Dose 4', $\mathrm{n}=3$ ). Dynamic PET scans were performed over $2 \mathrm{~h}$. Standard uptake values (SUVs) of brain, heart, lungs, liver, spleen, marrow, gall bladder, urinary bladder intestines, skeletal muscle and blood (vena cava) were measured. Averaged SUV at 60-120 minutes post-[18F]LW223 injection were compared for each organ across groups and used to calculate $\%$ specific binding $(\% \mathrm{SB})$ for plotting of saturation curves and subsequent derivation of constants using one site specific binding equation. Results/Discussion: In vivo dynamic PET imaging showed rapid uptake of [18F]LW223 in the mouse organs with known TSPO expression. The results also suggested that [18F]LW223 was mainly excreted via hepatobiliary system, with only negligible levels excreted via renal system. Significant homologous blocking with doses 3 and 4 was measured in the brain, heart and lungs (p Conclusion: [18F]LW223 has nanomolar affinity for TSPO in vivo in the mouse and low non-specific binding across all target organs, therefore holds huge potential for TSPO PET imaging of sites throughout the body. Mass dose limits demonstrate feasible use of radiotracer in small animals and easy translation to humans.
\end{abstract}

References: [1] D.R. Owen, A.J. Yeo, R.N. Gunn, K. Song, G. Wadsworth, A. Lewis, C. Rhodes, D.J. Pulford, I. Bennacef, C.A. Parker, P.L. Stjean, L.R. Cardon, V.E. Mooser, P.M. Matthews, E.A. Rabiner, J.P. Rubio, An 18-kDa Translocator Protein (TSPO) polymorphism explains differences in binding affinity of the PET radioligand PBR28, J. Cereb. Blood Flow 
Metab. 32 (2012) 1-5. doi:10.1038/jcbfm.2011.147. [2] D.R. Owen, Q. Guo, N.J. Kalk, A. Colasanti, D. Kalogiannopoulou, R. Dimber, Y.L. Lewis, V. Libri, J. Barletta, J. RamadaMagalhaes, A. Kamalakaran, D.J. Nutt, J. Passchier, P.M. Matthews, R.N. Gunn, E.A. Rabiner, Determination of [11C]PBR28 binding potential in vivo: A first human TSPO blocking study, J. Cereb. Blood Flow Metab. 34 (2014) 989-994. doi:10.1038/jcbfm.2014.46. [3] A. Jučaite, Z. Cselényi, A. Arvidsson, G. Åhlberg, P. Julin, K. Varnäs, P. Stenkrona, J. Andersson, C. Halldin, L. Farde, Kinetic analysis and test-retest variability of the radioligand [11C](R)-PK11195 binding to TSPO in the human brain - a PET study in control subjects, EJNMMI Res. 2 (2012) 1-13. doi:10.1186/2191-219X-2-15. [4] S. Lavisse, M. Guillermier, A.-S. Herard, F. Petit, M. Delahaye, N. Van Camp, L. Ben Haim, V. Lebon, P. Remy, F. Dolle, T. Delzescaux, G. Bonvento, P. Hantraye, C. Escartin, Reactive Astrocytes Overexpress TSPO and Are Detected by TSPO Positron Emission Tomography Imaging, J. Neurosci. 32 (2012) 10809-10818. doi:10.1523/JNEUROSCI.1487-12.2012. [5] M.G. MacAskill, T. Walton, L. Williams, T. Morgan, C.J. Alcaide-Corral, A. Stadulyte, C.A. McKenzie, A. Moss , R. BouHaidar, R. AlShahi Salman, M.R. Dweck, G.A. Gray, D.E. Newby, C.Lucatelli , A. Sutherland, S.L. Pimlott, A.A.S. Tavares, Poster Communication: Preclinical assessment of 18F-LW223; a novel TSPO radiotracer for the detection of inflammation using PET, SINAPSE 2018 (Edinburgh, UK).

\section{Image/Figure:}

https://www.xcdsystem.com/wmis/abstract/File6959/GA472 ImageFigure 0609124951.jpg

Image/Figure Caption: Figure 1. A) Chemical structure of [18F]LW223; B) Representative [18F]LW223 maximal intensity projection (MIP) image at baseline (average from 60-120 minutes post-radiotracer injection); C) LW223 in vivo saturation plots for various tissues measured using total-body PET imaging and average standard uptake values (SUVs, 60-120 minutes post-radiotracer injection). Legend: $\mathrm{B}=$ brain; $\mathrm{L}=$ lung; $\mathrm{H}=$ heart; $\mathrm{L}=$ liver; $\mathrm{GB}=$ gallbladder; $\mathrm{A}=$ adrenal; $\mathrm{K}=$ kidney; $\mathrm{I}=$ intestine.

Full Name of Abstract's 1st Author : Agne Stadulyte

Complete Status: Complete

First Name: Agne

Last Name: Stadulyte

Email: a.stadulyte@sms.ed.ac.uk

Organization: University of Edinburgh

Country: United Kingdom 


\title{
ID: GA473 \\ A single metal multifunctional nanotexaphyrin as a radiotheranostics agent for cancer imaging and therapy
}

\author{
Miffy Cheng, Princess Margaret Cancer Research Centre, hok-yan.cheng@uhnresearch.ca
}

\section{Category: New Chemistry, Biology \& Bioengineering}

\begin{abstract}
Body : Oligometastatic prostate cancer affects over 260,000 people worldwide and is potentially curable with surgery and external beam radiation therapy if diagnosed and treated appropriately. However, ablative therapies are currently hindered by the lack of precision imaging and low treatment efficacy, leading to poor prognosis for patients and cancer recurrence. To overcome these challenges, we propose a multifunctional nanoparticle as a non-invasive tool for radionuclide imaging and focal photodynamic therapy. Multifunctional nanoparticles can add both diagnostic and therapeutic value in the management of oligometastatic cancer. The "onefor-all" approach was developed, in which a nanoparticle is composed of a single building block that when self-assembled, offers a multitude of intrinsic functions while minimizing formulation complexity.[2] We developed a theranostics liposomal nanoparticle known as Lunanotexaphyrin. Lu-nanotexaphyrin is formed through the self-assembly of a texaphyrinphospholipid building block that has been chelated with a Lu metal ion. We utilized a postinsertion method to quantitively chelate the 'cold' $175 \mathrm{Lu}$ and the 'hot' $177 \mathrm{Lu}$ with high chelation efficiency (98\%). The Lu-nanotexaphyrin formulation was optimised for both structural and optical stability in different serum conditions. Lu-nanotexaphyrin showed excellent photostability in its intact form and as it disrupts, high levels of singlet oxygen can be produced under light irradiation. Fluorescence microscopy and ICP-MS also revealed excellent cellular uptake of Lu-nanotexaphyrin. In vitro PDT with PC3-luc-6 cells showed the combination of nanoparticles with laser treatment resulted in potent PDT effects. In vivo fluorescence imaging of a subcutaneous prostate tumour model demonstrated tumour accumulations and activation of Lu-nanotexaphyrin at $24 \mathrm{~h}$ post-i.v. injection, in vivo PDT was subsequently conducted to show PDT effect. Further studies investigated the radiolabelling of $177 \mathrm{Lu}$ and its in vivo disposition and pharmacokinetic profiles. In addition, we evaluated SPECT imaging sensitivity and specificity of Lu-nanotexaphyrins as an image-guided PDT agent. These results highlight the utility of metallo-nanotexaphyrins as a radiotheranostics agent with good stability and therapeutic capability for prostate cancers. This work aims to demonstrate the metallo-nanotexaphyrins as a customizable and multifunctional nanomedicine platform for non-invasive SPECT imaging and image-guided photodynamic therapy.
\end{abstract}

References: [1] F. Bray, J. Ferlay, I. Soerjomataram, RL. Siegel, LA. Torre, A Jemal, CA Cancer J Clin. 2018; 68:394-42 [2] J. F. Lovell, C. S. Jin, E. Huynh, H. Jin, C. Kim, J. L. Rubinstein, W. C. Chan, W. Cao, L. V. Wang, G. Zheng, Nat. Mater. 2011, 10, 324-332332

\section{Image/Figure:}


Image/Figure Caption: Schematic representation of the preparation

of multimodal $177 \mathrm{Lu} / 175 \mathrm{Lu}$-nanotexaphyrin for SPECT imaging and PDT treatment.

Full Name of Abstract's 1st Author : Miffy Hok Yan Cheng

Complete Status: Complete

First Name: Miffy

Last Name: Cheng

Email: hok-yan.cheng@uhnresearch.ca

Organization: Princess Margaret Cancer Research Centre

Country: Canada 


\title{
ID: GA474 \\ Developing Tools to Monitor and Modulate ALDH1A1 Activity in Cancer Stem Cells
}

\author{
Sarah Gardner, University of Illinois Urbana-Champaign, shgardn2@illinois.edu
}

\section{Category: Oncology}

\begin{abstract}
Body : Cancer stem cells (CSCs) are a population of cancer cells that are known for their chemoresistance and tumor-initiating capacity. Due to these features, CSCs are believed to survive chemotherapy and lead to recurrence.1 CSCs are often identified for study through a combination of cell surface markers, depending on cancer type. 2 However, an increase in aldehyde dehydrogenase 1A1 (ALDH1A1) has been poised as a universal intracellular marker.3 In order to monitor ALDH1A1 activity in CSCs, AlDeSense, an ALDH1A1-selective fluorescent probe, was developed. Using AlDeSense, an increase in ALDH1A1 activity was confirmed in CSCs of several cancer origins and culture methods. Additionally, CSCs were shown to maintain high ALDH1A1 activity upon implantation into mice. Similarly, metastases, originating from CSCs, were found to maintain high ALDH1A1 activity.4 To expand CSC imaging capabilities and answer deeper biological questions, a covalent inhibitor, AlDeStain, was developed from AlDeSense. AlDeStain utilizes an electrophilic group instead of an aldehyde substrate moiety to yield a fluorescent, covalent ALDH1A1 inhibitor. This compound can be applied in vivo to better visualize CSCs in tumors. As well, AlDeStain application can be used to determine whether ALDH1A1 activity is a product or contributor to the stem cell phenotype.
\end{abstract}

References: 1. Zhao, J. (2016). Cancer stem cells and chemoresistance: The smartest survives the raid. Pharmacol. Ther. 160, 145-158. 2. Jaggupilli, A., and Elkord, E. (2012). Significance of CD44 and CD24 as cancer stem cell markers: an enduring ambiguity. Clin Dev Immunol 2012, 708036. 3. Tomita, H., Tanaka, K., Tanaka, T., and Hara, A. (2016). Aldehyde dehydrogenase 1A1 in stem cells and cancer. Oncotarget 7, 11018-11032. 4. Anorma, C., Hedhli, J., Bearrood, T.E., Pino, N.W., Gardner, S.H., Inaba, H., Zhang, P., Li, Y., Feng, D., Dibrell, S.E., et al. (2018). Surveillance of Cancer Stem Cell Plasticity Using an Isoform-Selective Fluorescent Probe for Aldehyde Dehydrogenase 1A1. ACS Cent. Sci. 4, 1045-1055.

Full Name of Abstract's 1st Author : Sarah H. Gardner

Complete Status: Complete

First Name: Sarah

Last Name: Gardner

Email: shgardn2@illinois.edu

Organization: University of Illinois Urbana-Champaign 
Country: United States 


\title{
ID: GA475 \\ Imaging Cancer Immunology: Magnetic Particle Imaging of CD47 Immunotherapy
}

\author{
James Mansfield, Visiopharm, jim@visiopharm.com
}

\section{Category: Oncology}

\begin{abstract}
Body : The rapid growth of immuno-oncology research has fueled a need to evaluate the location, quantity, and phenotype of immune cells. Tumour-associated macrophages (TAMs) play a pivotal role in cancer development, metastatic spread, and response to therapy. However, current methods for qualitative and quantitative biodistribution measurements of TAM accumulations are insufficient. Magnetic Particle Imaging (MPI) is a novel tomographic molecular imaging technique used to non-invasively track cells labeled with magnetic nanoparticle tracers. 1 MPI is a tracer imaging technique that produces tomographic images of magnetic nanoparticle biodistribution, and does not see tissue. The technology is currently offered preclinically, and multiple groups are working on clinical translation. These images can be acquired longitudinally, have high sensitivity (20-300 picomolar of nanoparticle detection limit2), signal independent of depth, and linear quantitation across a large signal range ( $>3$ orders of magnitude). The superparamagnetic iron-oxide (SPIO) nanoparticles, which are the same as or similar to those used as T2* contrast agents in MRI, can be functionalized and targeted in many ways. 3 Magnetic nanoparticles can be used to image the immune system. Phagocytotic cells, such as macrophages, become labeled with the nanoparticles in situ following intravenous injection. These cells then accumulate in regions of inflammation, enabling them to be imaged and quantitated in vivo by MPI.4 CD47 mAb immunotherapy modulates phagocytosis of macrophages in cancer. Administration of the antibody should therefore increase phagocytosis and increase the resulting MPI signal.5 A murine breast tumour model was established by injecting $3 \times 1054 \mathrm{~T} 1$ cells into the 5 th mammary fat pad of 8-10-week-old female BALB/c mice. Immunotherapy treatment was with a CD47 mAb (200 ug for 5 days via tail vein), begun once the tumors were palpable. Two groups were used: a CD47-treated group $(\mathrm{N}=3)$ and an untreated control group $(\mathrm{N}=3)$. All mice were injected with a long-circulating SPIO MPI tracer (Synomag PEG 50, Micromod GmbH, $6 \mathrm{mg} / \mathrm{kg}$ dose) and then 3D images were acquired on MPI and MRI (T2-weighted multi-slice, multi-echo sequences). Images were acquired at 1, 3, and 7 days after tracer injection. MPI and MRI images were co-registered and quantitated. Tumour, liver, spleen, and draining lymph nodes were harvested, imaged, fixed, and stained with Perls Prussian blue for analysis of iron content. This preliminary study suggests that nanoparticle accumulation increases following CD47 mAb treatment. In Figure 1, we see that all mice showed an accumulation of tracer in the tumor and liver following injection. There was an increased tracer accumulation at the tumour site for the treated group compared to the control on days 1 and 7 following injection, but not on day 4 (Table 1). In the tumour, the signal was predominately detected in the expanding margins. In this work, we showed that MPI can return quantitative and spatial distribution information on how inflammation in a cancer model modulates in response to an immunotherapy. In situ tagging of phagocytic cells with magnetic tracers and MPI could significantly improve the specificity of inflammation detection,
\end{abstract}


particularly in combination with existing FDG-PET techniques that highlight increased glucose metabolism. Beyond measuring inflammation, MPI can image other types of immunotherapies notably, labeled T cells in an adoptive transfer model6. MPI enables researchers to monitor the efficacy of immunotherapies with the sensitivity, specificity, and linear quantitation.

References: 1 . Wu XLC, Zhang XY, Steinberg XG, et al. A review of magnetic particle imaging and perspectives on neuroimaging. Am J Neuroradiol. 2019;40(2):206-212.

doi:10.3174/ajnr.A5896 2. Arami H, Teeman E, Troksa A, et al. Tomographic magnetic particle imaging of cancer targeted nanoparticles. Nanoscale. 2017;9(47):18723-18730.

doi:10.1039/c7nr05502a 3. Savliwala S, Chiu-Lam A, Unni M, et al. Magnetic nanoparticles. In: Nanoparticles for Biomedical Applications. Elsevier; 2020:195-221. doi:10.1016/B978-0-12816662-8.00013-8 4. Makela A V., Gaudet JM, Schott MA, Sehl OC, Contag CH, Foster PJ. Magnetic Particle Imaging of Macrophages Associated with Cancer: Filling the Voids Left by Iron-Based Magnetic Resonance Imaging. Mol Imaging Biol. Published online 2020. doi:10.1007/s11307-020-01473-0 5. Logtenberg MEW, Scheeren FA, Schumacher TN. The CD47-SIRP $\alpha$ Immune Checkpoint. Immunity. 2020;52(5):742-752. doi:10.1016/j.immuni.2020.04.011 6. Rivera-rodriguez A, Hoang-minh LB, Chiu-lam A, Sarna N. Tracking Adoptive T Cell Therapy Using Magnetic Particle Imaging. Published online 2020:1-17.

Image/Figure Caption: MPI and MRI images of nanoparticle uptake in TAMs. (A) Representative untreated 4T1 tumor with overlaid micro-CT for reference. (B) MPI images from D7 for all animals. (C) MRI images from one animal in experimental and control groups as a comparison. Arrow indicates nanoparticle uptake.

Complete Status: Complete

First Name: James

Last Name: Mansfield

Email: jim@visiopharm.com

Organization: Visiopharm

Country: United States 


\title{
ID: GA476 \\ Multimodality Molecular Imaging of the Lung Disease using PET, CT and Optical Imaging
}

\author{
Andrei Molotkov, Columbia University PET center, am3355@cumc.columbia.edu
}

Category: Cardiovascular \& Pulmonary

\begin{abstract}
Body : Our objective in this work is to develop a translational quantifiable imaging scan that can be used to inform our COVID-19 drug discovery efforts in the preclinical setting and can be translated in the future to the clinical setting. Inflammatory changes caused by viruses, bacteria, exposure to toxins, commonly used drugs and even surgical intervention have the potential of causing abnormal epithelial permeability which is manifest as infiltrative processes on CT, including the widespread infiltrates seen in COVID-19 pneumonia and acute respiratory distress syndrome (ARDS). However, anatomic changes do not quantitate the underlying disease, prohibiting real-time diagnosis of sub-anatomic disease, response to therapy, or personalization of treatment regimens. We utilized a previously published mouse model of ARDS, intranasal delivery of LPS, to induce the alveolar-capillary barrier permeability seen in lung disease. Methods. We intravenously injected mice with $\mathrm{Cy} 7$ or 68 -Gallium (68Ga) labeled mouse albumin and imaged using optical imaging (OI)/CT and PET, respectively. Results. We observed significantly increased lung activity of Cy7-albumin on 3D OI/CT, which matched the abnormal appearance on microCT. Furthermore, this uptake correlated with fluorescence seen on sectioned lungs. In order to examine the translational potential of these findings, we radiolabeled albumin with $68 \mathrm{Ga}$, a short half-life positron emitting radioisotope that is used in clinical PET scans. We found that in mice with LPS-induced lung injury, 68Ga-albumin PET correlated with our optical imaging findings and demonstrated abnormal activity in the lung fields, indicative of abnormal epithelial permeability. Conclusions. These findings indicate $68 \mathrm{Ga}$-albumin can be utilized as a sensitive translational radiotracer for quantifying the abnormal epithelial permeability that is seen in various lung pathologies, including COVID-19 induced pneumonia and ARDS. Furthermore, the ability to use Cy7-albumin 3D OI/CT imaging as a preclinical translational surrogate for $68 \mathrm{Ga}$-albumin offers an accessible high throughput means of rapidly screening potential therapeutics against lung diseases that clinically manifest with endothelial permeability.
\end{abstract}

Complete Status: Complete

First Name: Andrei

Last Name: Molotkov

Email:am3355@cumc.columbia.edu

Organization: Columbia University PET center 
Country: United States 


\title{
ID: GA477 \\ Fluorogenic AggTag method based on Halo and SNAP-tags to simultaneously detect aggregation of two proteins in live cells
}

\author{
Kwan Ho Jung, The Pennsylvania State University, kxj80@psu.edu
}

Category: New Chemistry, Biology \& Bioengineering

\begin{abstract}
Body : Despite the considerable interest in protein aggregation for neurodegenerative diseases, research progress in this field has been hampered by the lack of an efficient tool to directly visualize protein aggregation in live cells. To address this, we recently developed an imaging method named the AggTag to detect an aggregation of a protein of interest (POI) in live cells with fluorescence microscopy. This method relies on a genetic fusion of a POI to a selflabelling protein tag to chemically label the POI with an AggTag probe, which bears a fluorophore that turns on its fluorescence upon aggregation of the POI. The previously developed AggTag probe P1, a Halo-tag substrate containing a red fluorescent molecular rotor, was shown to detect soluble oligomers and insoluble aggregates of a POI in live cells.[1] In this work, a new library of AggTag probes was further developed in order to expand the applications of the AggTag method.[2] The innovation of this work lies in the use of an orthogonal protein tag system to simultaneously detect aggregation of two different POIs in the same cells with dualcolor fluorescence. To this end, the AggTag probe P2, a SNAP-tag substrate bearing green solvatochromic fluorophore, was newly synthesized and shown to detect misfolded oligomers and insoluble aggregates of SNAPf-tagged superoxide dismutase 1 (SOD1) mutants, and insoluble aggregates of huntingtin exon 1 mutant (Htt-Q97). Because $\mathrm{P} 1$ and $\mathrm{P} 2$ possess orthogonal labelling specificities and excitation, we envisioned that P1 and P2 together could be used to simultaneously detect the aggregation of two different POIs with dual-color emission. By co-expressing Halo-tagged Htt-Q110 and SNAPf-tagged misfolding-prone SOD1 mutants and labelling with $\mathrm{P} 1$ and $\mathrm{P} 2$, we demonstrated that $\mathrm{P} 1$ and $\mathrm{P} 2$ reported on the co-aggregation between Htt-Q110 and SOD1 mutants, supporting the hypothesis that the Htt aggregates induces the aggregation of SOD1 mutants. Furthermore, by swapping each fluorophore on P1 and P2 with one another, additional AggTag probes, $\mathrm{P} 3$ and P4, were created and shown to retain similar performance in detecting the protein aggregation as the pair of P1 and P2. Given the increasing attention to protein aggregation in neurodegenerative diseases, the presented imaging probes are expected to offer an invaluable tool to unravel co-pathology of protein aggregation in live cells.
\end{abstract}

References: [1] Y. Liu, C. H. Wolstenholme, G. C. Carter, H. Liu, H. Hu, L. S. Grainger, K. Miao, M. Fares, C. A. Hoelzel, H. P. Yennawar, G. Ning, M. Du, L. Bai, X. Li, X. Zhang, J. Am. Chem. Soc. 2018, 140, 7381 -7384. [2] K. H. Jung, S. F. Kim, Y. Liu, X. Zhang, ChemBioChem 2019, 20, 1078-1087.

Complete Status: Complete

First Name: Kwan Ho 
Last Name: Jung

Email: kxj80@psu.edu

Organization: The Pennsylvania State University

Country: United States 


\title{
ID: GA478 \\ Combining Magnetic Particle Imaging (MPI) and Localized Hyperthermia for image guided therapy
}

\author{
Jeff Gaudet, Magnetic Insight, gaudet@magneticinsight.com
}

\section{Category: New Chemistry, Biology \& Bioengineering}

\begin{abstract}
Body : Introduction: Magnetic Particle Imaging (MPI) is an emerging molecular imaging technique that directly detects superparamagnetic iron oxide nanoparticles (SPIONs). MPI does not see tissue, and is often paired with a second anatomic imaging modality such as MRI or X-ray/CT. SPIONs in MRI and MPI have found uses in numerous applications, such as: tracking therapeutic cells, detection of localized inflammation, and visualization of blood flow and perfusion.1,2,3 SPIONs have also been utilized for Magnetic Fluid Hyperthermia (MFH). When subjected to high frequency $(>150 \mathrm{kHz})$ alternating magnetic fields, SPIONs dissipate magnetic energy as heat. This heat can be used for applications such as thermotherapy, activating or releasing nanotherapeutic drugs, and as an adjuvant to immunotherapy. However, MFH faces challenges associated with off-target accumulation and heating of particles, which can lead to unintentional damage to otherwise healthy tissue. Recent work has shown that the same magnetic fields used to produce images in MPI can be used to spatially control MFH.4,5 MPI produces images using a strong magnetic field gradient, or selection field, which contains a Field Free Region (FFR). SPIONS in the FFR are free to rotate, while SPIONs elsewhere are magnetically locked and cannot rotate. This same concept can be used to restrict magnetic heating to a localized region.4,5 In this study, we investigated the use of both MPI and localized MFH for image-guided hyperthermia. Methods: A targeted hyperthermia instrument was used for MFH (Figure 1A - HYPER, Magnetic Insight, 0.5-2.6 T/m gradient field, $15 \mathrm{mT}$ at $340 \mathrm{kHz}$ ). Permanent magnets control the size and position of the FFR, which can be moved across the sample to define a heating region (Figure 1B). Three SPIONs were evaluated for MPI and hyperthermia properties, VivoTrax (Magnetic Insight), SynomagD-70 (MicroMod), and Ferumoxytol (AMAG). In vitro hyperthermia localization was demonstrated with vials of VivoTrax $(2.75 \mu \mathrm{g} \mathrm{Fe} / \mu \mathrm{L})$ and vials of water. A MOMENTUM MPI (Magnetic Insight, $5.7 \mathrm{~T} / \mathrm{m}$ gradient field, $20 \mathrm{mT}$ at $45 \mathrm{kHz}$ excitation field) system was used to image and quantitate the iron present in SPION samples. For in vivo tests, BALB/c mice received an intramuscular injection of SPIONs in the left and right hindlimb. MPI was performed prior to MFH localized to the right hindlimb. Tissue was excised for histological examination. Results: All SPIONs tested produced both MPI signal and magnet-induced heating, with both VivoTrax and SynomagD-70 producing more signal and heating than ferumoxytol at the same concentration. During in vitro MFH localization test, MPI with a co-registered photograph shows SPIONs in three sample vials, and no MPI signal produced in a water vial (Figure 1C). By adjusting the selection field strength, the effective heating area can be varied (Figure 1D). At the highest field gradient, heating is only seen in the target (Vial \#1), and not seen in the other vials. As the gradient is weakened, heating is observed in the next vial (Vial \#2,12 $\mathrm{mm}$ away). For all gradient strengths, heating remained constant in Vial \#1 and no heating was observed in the vial furthest from the target (Vial \#3, $21 \mathrm{~mm}$ away) or in the water vial. In vivo, MPI signal was observed in both
\end{abstract}


hindlimb muscles with MPI. H\&E and TUNEL staining are currently in progress on excised tissue to examine tissue damage in heated versus untreated limbs. Conclusions: Here a theranostic workflow was demonstrated by combining MPI with magnetically localized hyperthermia. MPI allowed for the identification of target heating regions, and off-target nanoparticles to avoid. The combination of MPI and localized hyperthermia is promising as a clinically translatable technology for applications in targeted thermotherapy, nanotherapeutics, and in immune stimulation.

References: 1. Makela, Ashley V., et al. "Cellular imaging with MRI." Topics in Magnetic Resonance Imaging 25.5 (2016): 177-186. 2. Daldrup-Link, Heike E. "Ten things you might not know about iron oxide nanoparticles." Radiology 284.3 (2017): 616-629. 3. Bulte, Jeff WM. "Superparamagnetic iron oxides as MPI tracers: A primer and review of early applications." Advanced drug delivery reviews 138 (2019): 293-301. 4. D. Hensley et al., "Combining magnetic particle imaging and magnetic fluid hyperthermia in a theranostic platform," Phys. Med. Biol., vol. 62, no. 9, pp. 3483-3500, May 2017. 5. Tay, Zhi Wei, et al. "Magnetic particle imaging-guided heating in vivo using gradient fields for arbitrary localization of magnetic hyperthermia therapy." ACS nano 12.4 (2018): 3699-3713.

\section{Image/Figure:}

https://www.xcdsystem.com/wmis/abstract/File6959/GA478_ImageFigure_0612022920.png

Complete Status: Complete

First Name: Jeff

Last Name: Gaudet

Email: gaudet@magneticinsight.com

Organization: Magnetic Insight

Country: United States 


\title{
ID: GA479 \\ 18F-FPPRGD2 PET for noninvasive assessment of integrin $\alpha v \beta 3$ target engagement by a potent anti-fibrotic integrin antagonist in a mouse model of non-alcoholic steatohepatitis
}

\author{
Caiyuan Zhang, Massachusetts General Hospital , czhang34@mgh.harvard.edu
}

\section{Category: Systemic Diseases (Kidney, Liver and Pancreas)}

\begin{abstract}
Body : Introduction: Nonalcoholic steatohepatitis (NASH), a severe form of nonalcoholic fatty liver disease (NAFLD), is characterized on liver histology by lobular inflammation, hepatocyte ballooning and more progressive liver fibrosis1. The presence and severity of liver fibrosis in NASH patients determines the long-term prognosis2. Activated hepatic stellate cells (HSCs) are the major cellular source of myofibroblasts, which are responsible for producing excess extracellular matrix and the central driver of liver fibrosis3. Integrin avb3 is emerging as a potential antifibrotic target and an imaging marker in liver fibrosis because of its upregulated expression in activated HSCs4. Our aim was to test whether 18FFPPRGD2, a PET molecular imaging tracer for targeting integrin avb3 expressed on activated hepatic stellate cells, could noninvasively assess the target engagement by the novel avb1, avb3, avb6 integrin antagonist IDL-2965 in a choline-deficient, L-amino aciddefined, high-fat diet (CDAHFD) NASH mouse model. Method: C57B1/6 male mice were fed with CDAHFD for either 2, 6, 10, or 14 weeks to induce different stages of fibrosis $(n=6 /$ time point). Age-matched mice ( $n=6 /$ time point) fed on normal chow served as control. All mice were imaged by 4.7 Tesla MRI scanner (Bruker, Billerica MA) with a Bruker PET insert on the first day of each time point for 90 minutes after intravenously injection of $100-150 \mathrm{~mL}$ of $18 \mathrm{~F}$ FPPRGD2 tracer solution (100-200 mCi); PET/MRI was repeated in CDAHFD mice on the following day one hour post oral gavage of IDL-2965 (10 mg/Kg). Tissues were harvested for qRT-PCR, hydroxyproline assay, hematoxylin and eosin (H\&E), and Sirius red staining at the end of the study. PET signal was quantified by volume of interest $(1 \mathrm{~cm} 3)$ in liver avoiding large hepatic vessels. Statistical analysis was tested for all measurements among groups using one-way ANOVA with two-tailed distribution by GraphPad Prism 7 software. Results: PET quantification showed tracer uptake in the liver increased with the duration of CDAHFD up to 10 weeks (Figure 1). At each time point, the uptake of tracer in NASH liver was statistically higher than that in age-matched control mice (week 2: $0.55 \pm 0.12 \%$ ID vs. $0.26 \pm 0.05 \% \mathrm{ID}, \mathrm{P}$ a-SMA (marker of activated HSCs), Colla1, integrin $\alpha \mathrm{v}$ and $\beta 3$ showed increasing upregulation as the disease progressed and reached statistical significance at the advanced stage of NASH at week 14 (Figure 4). Conclusion: 18F-FPPRGD2 PET/MRI noninvasively detects increased expression of integrin $\alpha v \beta 3$ with progression of liver fibrosis in the CDAHFD-induced NASH model and shows potential to assess target engagement of a novel therapeutic. Acknowledgements: This work was supported by funding from the National Institutes of Health (DK121789, DK104956, OD023503, OD010650), the Shanghai Municipal Planning Commission of Science and Research Fund (201640143), and Indalo Therapeutics.
\end{abstract}


References: 1. Wong VW, Adams LA, de Ledinghen V, et al. Noninvasive biomarkers in NAFLD and NASH - current progress and future promise. Nat Rev Gastroenterol Hepatol 2018;15:461-478. 2. Angulo P, Kleiner DE, Dam-Larsen S, et al. Liver Fibrosis, but No Other Histologic Features, Is Associated With Long-term Outcomes of Patients With Nonalcoholic Fatty Liver Disease. Gastroenterology 2015;149:389-97. 3. Tsuchida T, Friedman SL. Mechanisms of hepatic stellate cell activation. Nat Rev Gastroenterol Hepatol 2017;14:397-411. 4. Patsenker E, Popov Y, Wiesner M, et al. Pharmacological inhibition of the vitronectin receptor abrogates PDGF-BB-induced hepatic stellate cell migration and activation in vitro. J Hepatol 2007;46:878-87.

\section{Image/Figure:}

https://www.xcdsystem.com/wmis/abstract/File6959/GA479_ImageFigure_0609033122.jpg

Complete Status: Complete

First Name: Caiyuan

Last Name: Zhang

Email: czhang34@mgh.harvard.edu

Organization: Massachusetts General Hospital

Country: United States 


\title{
ID: GA480 \\ Reducing the Dose of Whole-body PET Scans of Pediatric Cancer Patients to that of a Chest X-ray Using Neural Networks with Attention-weighted Loss Function
}

\author{
Yan-Ran Wang, stanford, joycewyr@stanford.edu
}

\section{Category: Computational \& Data Science}

\begin{abstract}
Body : Background: 18F-FDG PET/MRI can detect pediatric tumors with 80\% reduced radiation exposure compared to $18 \mathrm{~F}-\mathrm{FDG} \mathrm{PET} / \mathrm{CT}$ by replacing $\mathrm{CT}$ with $\mathrm{MRI}$ for anatomical co-registration of radiotracer data1. However, serial scans, as obtained in cancer patients, could still amount to considerable sum doses. Approaches to reduce the dose of the injected radiotracer have received little attention thus far. Under normal circumstances, reduced radiotracer doses lead to an increased image noise5 and resultant decreased diagnostic accuracy. We hypothesized that this problem could be solved by training a deep convolutional neural network $(\mathrm{CNN})$ in the augmentation of low-dose input images to standard-dose images. The purpose of our study was to generate a convolutional neural network $(\mathrm{CNN})$, which could generate PET images of the whole body with the radiation dose of a chest x-ray (0.1-0.2 mSv). Method: Studies were approved by the institutional review board at our institution. We enrolled baseline 18F-FDG-PET/MR scans of 24 patients with malignant lymphoma, including 15 female and 9 male patients with an age of 6-31 years. 18F-FDG PET data ( $3 \mathrm{MBq} / \mathrm{kg})$ were unlisted to simulate $6.25 \%$ reduced dose scans. We developed a reconstruction neural network with an attention-weighted loss function, which takes an ultra-low-dose PET along with a LAVA MRI image as inputs in order to predict a standard-dose PET image. The main reconstruction net shares the same architecture as EDSR7, which is the state-of-the-art reconstruction network. Multi-slice inputs were used to reduce noise and introduce vertical consistency by providing the network with 2.5D information. Prior knowledge was encoded into attention-weighted loss function where the significant regions - tumor and liver - contribute more to the loss function for training as opposed to relatively low-diagnostic-value regions. Such mechanism helped the model pay more attention to the significant regions and protected the training from overwhelming by relatively less-critical pixels in whole-body scans. Aa a result, less training data was required which is critical in the medical image domain where large-scale acquisition of medical data is challenging due to ethical and financial concerns. Leave-one-out cross validation was applied to provide a comprehensive evaluation given the limited number of subjects. The effectiveness of the proposed neural network model with an attention-weighted loss function on PET reconstruction was demonstrated in qualitative visual results (Figure 1). The peak signal-tonoise ratio (PSNR), structural similarity (SSIM), and normalized root mean square error (NRMSE) were computed between original standard-dose PET and AI-augmented PET images to measure image similarity. Then, the similarity metrics were compared between the pair of standard-dose and reduced dose PETs and the pair of standard-dose and AI-augmented PETs using Wilcoxon signed rank test with continuity correction. In addition, two nuclear medicine physicians, blinded to examination details, determined the presence or absence of tumor lesions in eight anatomical regions per patients according to a Likert scale. Results: PSNR, SSIM, and
\end{abstract}


NRMSE were $33.34 \pm 1.13,0.95 \pm 0.01$ and $0.29 \pm 0.08$, respectively, for standard-dose PET and AI-augmented PET image pairs, $30.07 \pm 1.14,0.93 \pm 0.01$, and $0.42 \pm 0.06$, respectively, for standard-dose PET and reduced dose PET image pairs. Compared to the standard-dose PET and reduced dose PET image pairs, the standard-dose PET and AI-augmented PET image pairs had significantly higher PSNR ( $p$ the AI-augmented PET image scan. Conclusion: We generated a CNN which could generate PET images of the whole body with the radiation dose of a chest $\mathrm{X}$ ray.

References: [1] Muehe, Anne M., Ashok J. Theruvath, Lillian Lai, Maryam Aghighi, Andrew Quon, Samantha J. Holdsworth, Jia Wang et al. "How to provide gadolinium-free PET/MR cancer staging of children and young adults in less than $1 \mathrm{~h}$ : the Stanford approach." Molecular Imaging and Biology 20, no. 2 (2018): 324-335. [2] Daldrup-Link, Heike E. "How PET/MR can add value for children with cancer." Current radiology reports 5, no. 3 (2017): 15. [3] Huang, Bingsheng, Martin Wai-Ming Law, and Pek-Lan Khong. "Whole-body PET/CT scanning: estimation of radiation dose and cancer risk." Radiology 251, no. 1 (2009): 166-174. [4] Frush, Donald P., Michael Callahan, Marilyn Goske, Sue Kaste, and Marta Hernanz-Schulman. "CT and radiation safety: content for community radiologists." Image Gently website www. pedrad. org (2009). [5] Karakatsanis, Nicolas A., Eleni Fokou, and Charalampos Tsoumpas. "Dosage optimization in positron emission tomography: state-of-the-art methods and future prospects." American journal of nuclear medicine and molecular imaging 5, no. 5 (2015): 527. [6] Whiteley, William, Wing K. Luk, and Jens Gregor. "DirectPET: full-size neural network PET reconstruction from sinogram data." Journal of Medical Imaging 7, no. 3 (2020): 032503. [7] Lim, Bee, Sanghyun Son, Heewon Kim, Seungjun Nah, and Kyoung Mu Lee. "Enhanced deep residual networks for single image super-resolution." In Proceedings of the IEEE conference on computer vision and pattern recognition workshops, pp. 136-144. 2017.

\section{Image/Figure:}

https://www.xcdsystem.com/wmis/abstract/File6959/GA480_ImageFigure 0609033909.png

Image/Figure Caption: Figure 1: a PET/MRI scan of a 16-year old female patient with hodgkin lymphoma. a). a coronal view of MRI LAVA; b). a coronal view of $6.25 \%$ ultra-low-dose PET scan; c). a coronal view of the AI-reconstructed PET image; d). a coronal view of standard-dose PET scan. The red arrows point to the tumors at mediastinum.

Full Name of Abstract's 1st Author : Yan-Ran (Joyce) Wang

Complete Status: Complete

First Name: Yan-Ran

Last Name: Wang

Email: joycewyr@stanford.edu

Organization: stanford 
Country: United States 


\title{
ID: GA481 \\ In vivo evaluation of Tantalum Oxide (TaOx) Nanoparticles for X-ray CT Imaging and Therapy
}

\author{
Shatadru Chakravarty, Michigan State University, shatadru@msu.edu
}

\section{Category: Oncology}

\begin{abstract}
Body : Objective: X-ray Computed Tomography $(\mathrm{CT})$ can potentially transform into a robust molecular imaging platform fueled by recent developments in contrast agent (CA) research.[1-3] We have previously reported TaOx nanocrystals (NCs) with the highest Ta content reported till date (78\%) as CAs for CT imaging.[4] In this work, the in vivo performance of this unique $\mathrm{NC}$ construct has been evaluated for contrast enhanced micro-CT imaging of the breast ductal tree in rodents. Briefly, we investigated an improved formulation of the TaOx NCs in ethanol as a high-resolution contrast agent to monitor in vivo filling of breast ductal trees and ablative effects of ethanol (EtOH) by CT imaging. Our hypothesis is that the local killing of mammary epithelial cells will be as effective as prophylactic mastectomy in preventing breast cancer, but with minimal side effects. In addition, we have used ethyl-cellulose (EC) as a gelling agent to further minimize collateral tissue damage. These TaOx NCs will also extensively evaluated for their in vivo toxicity in rats. Methods, Results and Discussion: We first synthesized the highly hydrophilic, homogenously sized $(9-12 \mathrm{~nm}) \mathrm{TaOx}$ NCs using a previously reported procedure.[4] In a recent study, we have demonstrated the ability of TaOx NCs to be administered locally and reveal in exquisite detail the continuous non-anastomosed branched structure of a murine ductal tree. We have also shown that intraductal (ID) injection of a $70 \%$ EtOH solution is effective at locally ablating mammary epithelial cells with limited collateral tissue damage and at preventing tumor formation in an aggressive and multifocal mouse model of breast cancer.[5] To advance the efficacy and prognosis of this treatment regimen, we designed an improved formulation of TaOx NCs with higher solubility in EtOH. Interestingly, $\mathrm{EC}$ has been used clinically to improve delivery of $\mathrm{EtOH}$ and to limit EtOH diffusion from the intended target area in the treatment of venous malformations. [6, 7] Thus, to improve the tolerability of the TaOx formulation, $\mathrm{EC}$ was added to it as a gelling agent. ID injection of $70 \%$ $\mathrm{EtOH} / 3 \% \mathrm{EC}$ in nontransgenic mice and rats achieved the same epithelial ablation rate as $70 \%$ $\mathrm{EtOH}$ alone, while significantly minimizing collateral tissue damage. The TaOx NCs within this novel formulation served to evaluate any local or systemic toxicity of this new imaging agent. These data demonstrate the compatibility, safety, and stability of this refined EtOH formulation and support its further investigation in cancer-prone rodent and larger animal models as an innovative primary intervention strategy for $\mathrm{BC}$ prevention. Conclusion: We report the in vivo performance of the highly hydrophilic TaOx NCs formulations in saline and $\mathrm{EtOH} / \mathrm{EC}$ via two exploratory experiments. Taken in unison, our work demonstrates the CT imaging application, theranostic potential and safety of TaOx NCs.
\end{abstract}

References: 1. Lusic H, Grinstaff MW (2013) X-ray-computed tomography contrast agents. Chem. Rev. 113:1641-1666. 2. Lee N, Choi SH, Hyeon T (2013) Nano-sized CT contrast agents. Adv Mater 25:2641-60. https://doi.org/10.1002/adma.201300081. 3. Rawson SD, Maksimcuka 
J, Withers PJ, Cartmell SH (2020) X-ray computed tomography in life sciences. BMC Biol 18:21. https://doi.org/10.1186/s12915-020-0753-2. 4. Chakravarty S, Hix JML, Wiewiora KA, et al (2020) Tantalum oxide nanoparticles as versatile contrast agents for X-ray computed tomography. Nanoscale 12:7720-7734. https://doi.org/10.1039/D0NR01234C. 5. Kenyon E, Westerhuis JJ, Volk M, et al (2019) Ductal tree ablation by local delivery of ethanol prevents tumor formation in an aggressive mouse model of breast cancer. Breast Cancer Res 21:129. https://doi.org/10.1186/s13058-019-1217-x. 6. Sannier K, Dompmartin A, Théron J, et al (2004) A New Sclerosing Agent in the Treatment of Venous Malformations: Study on 23 Cases. Interv Neuroradiol 10:113-127. https://doi.org/10.1177/159101990401000203. 7. Dompmartin A, Blaizot X, Théron J, et al (2011) Radio-opaque ethylcellulose-ethanol is a safe and efficient sclerosing agent for venous malformations. Eur Radiol 21:2647-2656. https://doi.org/10.1007/s00330-011-2213-4.

\section{Image/Figure:}

https://www.xcdsystem.com/wmis/abstract/File6959/GA481 ImageFigure 0609050551.jpg

Image/Figure Caption: Figure 1. (a) In vivo X-ray micro-CT imaging; (a) In vivo ductal tree imaging and longitudinal assessment of ductal tree changes. Abdominal mammary glands of mice and rats were intraductally (ID) injected with $70 \% \mathrm{EtOH} / 100 \mathrm{mM} \mathrm{TaOx}$ NCs. Top, images were captured immediately after ID injection in microCT instrument. Bottom, images from separate experiments were sequentially captured from the same animal at indicated times after ID injection. Scale bars are $5 \mathrm{~mm}$; (b) Ethyl cellulose minimizes collateral tissue damage of EtOH. H\&E staining of mouse (left) and rat (right) glands $7 \mathrm{~d}$ after ID injection with different solutions containing $100 \mathrm{mM} \mathrm{TaOx}$ nanocrystals and non-injected control. Intact (black arrow) and ablated ducts (orange arrow) are indicated. Scale bar is $200 \mathrm{~nm}$.

Complete Status: Complete

First Name: Shatadru

Last Name: Chakravarty

Email: shatadru@msu.edu

Organization: Michigan State University

Country: United States 


\title{
ID: GA482 \\ Performance evaluation of a novel SiPM-based preclinical SPECT scanner using experimental and theoretical data according to the NEMA NU 1-2018 standard
}

\author{
Matthew Strugari, Biomedical Translational Imaging Centre (BIOTIC), \\ matthew.strugari@dal.ca
}

Category: Instrumentation

\begin{abstract}
Body : Purpose: The SPARKTM (Cubresa Inc.) is a novel benchtop preclinical SPECT scanner for in vivo imaging. It is the first gamma camera of its kind to employ a monolithic $\mathrm{CsI}(\mathrm{Na})$ crystal coupled to silicon photomultipliers (SiPMs) which offers high-resolution images well-suited for multi-radionuclide and pharmacokinetic studies. This provides a unique opportunity for non-invasively studying disease progression or treatment response, in a quantitative manner, at the molecular level. Typical SPECT scanners utilize NaI crystals coupled to photomultiplier tubes which offer inferior photon detection and spatial resolution. In order to compare gamma cameras, the National Electrical Manufacturers Association (NEMA) provides well-established methodologies for measuring medical imaging equipment performance. In this study, we evaluated the intrinsic and tomographic performance of the SPARK with Tc-99m by physical experiment and Monte Carlo simulation according to the NEMA NU 1-2018 Standard for Performance Measurements of Gamma Cameras. Methods: Intrinsic spatial resolution and linearity were measured axially from a projection image produced with a $2.5 \mathrm{~mm}$-thick tungsten mask having $0.8 \mathrm{~mm}$-wide parallel slits. Conversely, intrinsic energy resolution and flood field uniformity were measured without a mask. An electronic mask was used to define the useful and central fields of view (UFOV $=84.5 \mathrm{~mm}$; CFOV $=63.375 \mathrm{~mm}$ ) as necessary. Reconstructed spatial resolution without scatter was measured with three point sources spaced apart $5 \mathrm{~mm}$ axially and $7.5 \mathrm{~mm}$ transaxially using a 25-pinhole coded-aperture collimator. The detector radius of rotation is fixed at $27.429 \mathrm{~mm}$ and the image was reconstructed with $0.25 \mathrm{~mm}$ isotropic voxels using the OSEM algorithm (iterations: 9; subsets: 4). Intrinsic measurements were repeated using the GATE v9.0 Monte Carlo toolkit to validate the modeled detector. Results: Experimental and theoretical intrinsic spatial resolution and linearity were measured from the parallel lines shown in Figure 1. In terms of the full width at half maximum (FWHM), the experimental/theoretical intrinsic spatial resolution for the UFOV and CFOV was 0.91/0.92 mm and $0.95 / 0.92 \mathrm{~mm}$, respectively. Projection images possess little distortion as the differential linearity was found to be $0.024 / 0.001 \mathrm{~mm}$ for both the UFOV and CFOV while absolute linearity was $0.102 / 0.003 \mathrm{~mm}$ and $0.055 / 0.003 \mathrm{~mm}$, respectively. The intrinsic energy resolution was $16.56 / 16.35 \%$ and the detector was found to respond uniformly to a flood field of radiation based on the integral uniformity for $1 \mathrm{~mm}$ pixels which was 5.72/2.01\% and 5.78/1.96\% for the UFOV and CFOV. Lastly, the reconstructed spatial resolution without scatter was $1.22 \mathrm{~mm}$ when averaged over all three points in all image directions. Tomographic data could not be simulated for direct comparison due to proprietary restrictions concerning the coded-aperture collimator. Conclusions: Our experimental and theoretical results demonstrate good agreement for intrinsic measurements made according to the NEMA NU 1-2018 standard, and where standard deviations could be calculated, all experimental and theoretical results are the same within error.
\end{abstract}


This aids in validating our GATE model of the SPARK. NEMA measurements of commercial clinical SPECT scanners are stated in [1] with an intrinsic resolution of 2.7-4.2 $\mathrm{mm}$ whereas commercial preclinical scanners, such as the U-SPECT and A-SPECT, state an intrinsic resolution of $3.2 \mathrm{~mm}$ and $2.0 \mathrm{~mm}$, respectively [2,3]. To our knowledge, the SPARK presents the highest intrinsic spatial resolution measurements reported to date for any gamma camera assessed with the NEMA standard. This is primarily due to the small form factor of SiPM microcells in combination with a $3 \mathrm{~mm}$-thick $\mathrm{CsI}(\mathrm{Na})$ crystal. Later work will assess the remainder of intrinsic, system, and tomographic measurements. A thorough understanding of the experimental and theoretical imaging performance will assist in improving data quality by adapting the complex SPECT system matrix based on, for example, photon scatter or septal penetration of collimators.

References: [1] Bushberg, J. T. (Ed.). (2012). The essential physics of medical imaging (3rd ed). Wolters Kluwer Health/Lippincott Williams \& Wilkins. [2] Beekman, F. J., van der Have, F., Vastenhouw, B., van der Linden, A. J. A., van Rijk, P. P., Burbach, J. P. H., \& Smidt, M. P. (2005). U-SPECT-I: A novel system for submillimeter-resolution tomography with radiolabeled molecules in mice. Journal of Nuclear Medicine: Official Publication, Society of Nuclear Medicine, 46(7), 1194-1200. [3] McElroy, D. P., MacDonald, L. R., Beekman, F. J., Yuchuan Wang, Patt, B. E., Iwanczyk, J. S., Tsui, B. M. W., \& Hoffman, E. J. (2002). Performance evaluation of A-SPECT: A high resolution desktop pinhole SPECT system for imaging small animals. IEEE Transactions on Nuclear Science, 49(5), 2139-2147. https://doi.org/10.1109/TNS.2002.803801

\section{Image/Figure:}

https://www.xcdsystem.com/wmis/abstract/File6959/GA482_ImageFigure_0609061856.png

Image/Figure Caption: Figure 1: Masked projection images generated via (a) experiment and (b) simulation for the measurement of intrinsic spatial resolution and linearity. Images were acquired with $50 \mathrm{MBq}$ of Tc-99m for 40 minutes in order to achieve the desired statistics outlined in the NEMA NU 1-2018 standard. Note that both images are generated with $0.1 \mathrm{~mm}$ pixels having dimensions of $956 \times 956$ and are presented using the same intensity scale. Furthermore, only the line profiles are used for analysis while the Derenzo pattern, with hole diameters of $\{0.7,0.8,0.9,1.0,1.2$, and 1.4$\} \mathrm{mm}$, highlights the performance capabilities of the detector. See text for further information.

Complete Status: Complete

First Name: Matthew

Last Name: Strugari

Email: matthew.strugari@dal.ca

Organization: Biomedical Translational Imaging Centre (BIOTIC)

Country: Canada 


\title{
ID: GA483 \\ Combining 1H MRS and deuterium labeled glucose for mapping of neural metabolism in humans
}

\author{
Abigail Cember, University of Pennsylvania, cember@pennmedicine.upenn.edu
}

\section{Category: Neuroscience}

\begin{abstract}
Body : Introduction Quantitative exchange label turnover MRS (qMRS) is a recently developed strategy which increases the range of applications for magnetic resonance based metabolic mapping without the requirement for specialized hardware [1]. Similar to recently developed deuterium metabolic imaging (DMI) [2], this technique relies on the administration of deuterium labeled glucose, resulting in accumulation of downstream $2 \mathrm{H}$ labeled metabolites. Since the $2 \mathrm{H}$ label is invisible in $1 \mathrm{H}$ MRS, accumulation of labeled metabolites leads to an overall reduction in the 1H MRS signal of corresponding resonances. To date, qMRS has yet to be applied to the measurement of neural metabolism in human beings; the first such data is presented here. Hypothesis Based on successful preliminary studies in animals [1], we hypothesized that upon ingestion of deuterated glucose by humans, a change in the $1 \mathrm{H}$ MRS spectrum could be detected in downstream neurometabolites. Methods This protocol was approved by the Institutional Review Board at the University of Pennsylvania, with informed consent obtained prior to the initial scan. Eight subjects participated in this study, four male and four female, of average age 29.5 years. MR experiments were performed on a $7 \mathrm{~T}$ scanner (MAGNETOM Terra, Siemens Healthcare, Erlangen, Germany) equipped with a 1Tx/32Rx head coil (Nova Medical, Wilmington, MA, USA). The volunteers fasted overnight before undergoing studies in the morning. Subjects were scanned for approximately two hours, beginning directly after oral ingestion of $0.8 \mathrm{~g} / \mathrm{kg}$ of body weight of [6,6'-2H2] glucose dissolved in water. Axial T1weighted FLASH images were obtained to enable localization of the cortex. Following localization, spectroscopy data were acquired using custom sequences for CSI with sLASER localization (MRSI) [3] and SVS with PRESS localization [4]. Specifically, SVS and the initial MRSI measurement were performed directly upon positioning the subject in the scanner $(\mathrm{t}=20$ 30 mins post-ingestion), with six subsequent MRSI acquisitions ( $t=50,60,70,80,90,100$ mins) and one additional SVS acquisition $(t=120$ mins $)$ at the end of the experiment. Voxel sizes of MRSI and SVS were 10mm3 and 10x30x10 mm, respectively. Analysis of the spectra was performed in LCModel and MATLAB. Results To assess the feasibility of qMRS-based metabolic mapping, we acquired consecutive MRSI datasets (baseline and 6 time points) in human volunteers upon oral ingestion of $\left[6,6^{\prime}-2 \mathrm{H} 2\right]$ glucose in water. The Figure shows plots derived from Glu/NAA metabolite ratio maps in all subjects upon ingestion of the $\left[6,6^{\prime}-\right.$ $2 \mathrm{H} 2$ ]glucose solution. The black rectangles in subfigure A show example ROIs designated as gray and white matter regions, which were chosen manually for each subject. As expected, gray matter regions had a higher baseline Glu/NAA ratio compared to white matter regions. Upon ingestion of [6,6'-2H2]glucose, a decrease was observed in the Glu/NAA ratio in both gray and white matter, with this decrease being more pronounced in gray matter (subfigure B). Analysis of SVS data, which was acquired only at two timepoints -- baseline and two hours post-glucose drink-- confirmed this finding (see Persuasive Data). In SVS, on average, a 1.0 mM reduction in
\end{abstract}


gray matter [Glu] and a $0.5 \mathrm{mM}$ reduction in white matter [Glu] was measured. However, NAA levels changed $0.15 \mathrm{mM}$ and $0.07 \mathrm{mM}$ in the same regions. Conclusions These findings demonstrate the feasibility of performing $1 \mathrm{H}$ MRSI in conjunction with deuterium labeling to perform spatially and temporally resolved measurements of neural metabolism. Compared to DMRI with a typical voxel size of $\sim 8 \mathrm{~mL}$ (and an acquisition time of $\sim 35 \mathrm{~min}$ ), the qMRSI voxel size of $\sim 1 \mathrm{~mL}$ (with an acquisition time of $\sim 10 \mathrm{~min}$ ) gives over eight-fold higher volumetric resolution. In addition to glutamate, qMRS enables tracking of other important metabolites including glutamine, GABA, and lactate.

References: References [1] Rich LJ, Bagga P, Wilson NE, Schnall MD, Detre JA, Haris M, Reddy, R. (2020) $1 \mathrm{H}$ magnetic resonance spectroscopy of $2 \mathrm{H}$-to-1H exchange quantifies the dynamics of cellular metabolism in vivo. Nature Biomedical Engineering 4, 335-342. [2] De Feyter HM, Behar KL, Corbin ZA, Fulbright RK, Brown PB, McIntyre S, Nixon TW, Rothman DL, de Graaf RA. (2018) Deuterium metabolic imaging (DMI) for MRI-based 3D mapping of metabolism in vivo. Science advances. 4(8), eaat7314 [3] Scheenen TWJ, Klomp DWJ, Wijnen JP, Heerschap A. (2008) Short echo time 1H-MRSI of the human brain at 3T with minimal chemical shift displacement errors using adiabatic refocusing pulses. Magnetic Resonance in Medicine. 59 (1), 1-6. [4] Bottomley, PA. (1984). Spatial Localization in NMR Spectroscopy in Vivo. Annals of the New York Academy of Sciences 508 (1), 333-348.

\section{Image/Figure:}

https://www.xcdsystem.com/wmis/abstract/File6959/GA483 ImageFigure_0609062019.png

Image/Figure Caption: Figure caption: A) Glu/NAA ratio map (MRSI) illustrating ROIs used for calculation of the plotted data designated as gray and white matter regions. B) Bar graphs showing the timecourse measurements of Glu and NAA by MRSI in gray and white matter, with individual subjects plotted as block dots and the mean value indicated by bar height. In these plots, the decreasing signal of the gray matter glutamate resonance is quite noticeable. The decrease in this resonance in the white matter is less salient, reflecting less uptake of glucose from the bloodstream by these cell types. The measured concentration of NAA, which does not become labeled, remains nearly constant. A sample SVS measurement used for confirmation of this trend is provided as Persuasive Data.

Complete Status: Complete

First Name: Abigail

Last Name: Cember

Email: cember@pennmedicine.upenn.edu

Organization: University of Pennsylvania

Country: United States 


\title{
ID: GA484 \\ Construction of Pediatric 18F-FDG PET Brain Template by Iterative Nonlinear Registration
}

\author{
Teng Zhang, Second Affiliated Hospital Of Zhejiang University School Of Medicine, \\ zhangteng630@zju.edu.cn
}

\section{Category: Computational \& Data Science}

Abstract Body : Purpose: The brain template that provides a priori knowledge about the human
brain facilitates image processing and defines a common stereotaxic space for analysis. Due to
the age-related anatomical and metabolic variability, the commonly-used templates created from
adult images are not appropriate for pediatric data analysis. This study aims to build a pediatric
18F-FDG PET brain template by iterative nonlinear registration approach. Method: A total of 36 pediatric patients ( 11 girls and 25 boys, age $12.4 \pm 2.9$ years) with lymphoma or bone neoplasm were retrospectively included in this study. All patients had no history of neurologic disorders, psychiatric illness, chemotherapy, or radiotherapy. The patients fasted for at least 6 hours before the 18F-FDG PET examination. After injection with 3.8 MBq/kg $18 \mathrm{~F}-\mathrm{FDG}$ and a 40 -minutes uptake period, a 5-minute three-dimensional acquisition was carried out using a PET/CT scanner (Biograph mCT, Siemens Medical Solution). The pediatric 18F-FDG PET template was built by an iterative-registration optimization approach. In each iteration, all individual PET images were aligned to a temporary template of the current iteration and then averaged. The average PET image was then warped by residual inverse transform and was used as the temporary template for the next iteration. The iterative optimization stopped when the mean of squared differences between two consecutive templates was less than a predefined threshold, i.e. 1e-6 in this study. The construction was initialized from Statistical Parametric Mapping (SPM) PET template to position the proposed pediatric template in the commonly-used International Consortium of Brain Mapping (ICBM) coordinate system. Iterative affine registration was first carried out using average inverse scaling-shearing transform as residual, and then iterative symmetric diffeomorphic registration using average inverse displacement field as residual. We compared the proposed pediatric 18F-FDG PET template with a conventional PET template using a linear approach. Voxelwise intensity standard deviation was computed to indicate metabolic variations of the two templates. All PET images were transformed into the space of both templates, and the similarities between warped individual images and templates were calculated using neighborhood correlation. A paired student's t-test was performed to compare image similarities. The logarithm Jacobian determinant of the displacement field was estimated to evaluate local tissue deformation. Voxelwise Student's t-test was performed on the absolute difference of average logarithm Jacobian determinant from zero. False discovery rate (FDR) of $5 \%$ was used to correct multiple comparisons. Result: The proposed pediatric 18F-FGD PET template was demonstrated in Figure (A). Figure (B) and (C) showed created brain templates, standard deviations, and logarithm Jacobian determinant t-maps using the conventional linear approach and the proposed method. Results indicated that the proposed pediatric template had better anatomical details, lower standard deviation ( 0.038 vs. 0.067$)$, and lower t-values ( 0.96 vs. 1.86) than the template by linear approach. No significant differences in the average logarithm 
Jacobian determinant from zero were found using the proposed method. In contrast, widespread significant regions were found using a template created by a linear approach, as shown in Figure (D). The paired Student's t-test showed that similarities of warped images to the proposed template $(0.662 \pm 0.027)$ were significantly higher than those to the conventional template $(0.469$ \pm 0.043 , $\mathrm{p}$ In conclusion, the proposed pediatric $18 \mathrm{~F}-\mathrm{FDG}$ PET template provides a priori knowledge about brain metabolism of pediatrics and therefore can facilitate subsequent image registration and statistical analysis.

\section{Image/Figure:}

https://www.xcdsystem.com/wmis/abstract/File6959/GA484 ImageFigure 0609103205.png

Image/Figure Caption: (A) Proposed pediatric 18F-FDG PET template; (B/C) Template, standard deviation, and logarithm Jacobian determinant t-map using conventional linear/proposed nonlinear approach; (D) Significant regions using the template by linear approach; (E) Similarities between warped individual PET images and templates.

Full Name of Abstract's 1st Author : Teng Zhang

Complete Status: Complete

First Name: Teng

Last Name: Zhang

Email: zhangteng630@zju.edu.cn

Organization: Second Affiliated Hospital Of Zhejiang University School Of Medicine

Country: China 


\title{
ID: GA485 \\ CRISPR/Cas9-edited triple-fusion reporter gene imaging of dynamics and metabolic changes after transplantation of human induced pluripotent stem cell- derived cardiomyocytes
}

\author{
Yuanxue Gao, ZheJiang University, 11818370@zju.edu.cn
}

\section{Category: Cardiovascular \& Pulmonary}

\begin{abstract}
Body : Objective: Human induced pluripotent stem cell-derived cardiomyocytes (hiPSC-CMs) are a promising cell source for regenerating injured heart tissue. Tracking the fate of implanted hiPSC-CMs allows us to understand the mechanisms underlying the functional improvement in the infarcted heart and accelerate the clinical translation of hiPSC-CM therapies. This study aims to investigate the posttransplantation behaviour and metabolic changes after transplantation of hiPSC-CMs following acute myocardial infarction (AMI). Methods: Genome editing with CRISPR/Cas 9 technology was used to infuse a triple-fusion (TF) reporter gene into the AAVS1 locus of human urinary induced pluripotent stem cell (TF-hUiPSCs) which stably express monomeric red fluorescent protein for fluorescence, firefly luciferase for bioluminescence imaging (BLI), and herpes simplex virus thymidine kinase for positron emission tomography (PET) imaging. TF-hUiPSCs were induced to differentiate into cardiomyocytes (TF-hUiCMs) and then were processed using a pro-survival cocktail protocol to enhance engraftment post transplantation. AMI was surgically induced in rats by ligation of the left anterior descending artery, TF-hUiCMs $(1.0 \times 107$ per rat $)$ in a volume of $100 \mu \mathrm{l}$ or an equal volume of PBS was injected directly into the border zone of the infarcted myocardium immediately after ligation. Transplanted cells were detected by BLI at day 4 and day 35 . The dynamic metabolic change was monitored by [18F]-FDG PET imaging. Results: In the rat model of acute myocardial infarction, bioluminescence imaging tracked the TF-hUiCMs noninvasively and the signal showed an increasing trend from day 4 to 35 post injection. [18F]-FDG microPET imaging showed an enhancement in myocardial glucose uptake in the peri-infarct zone at day 35 after TF-hUiCMs transplantation. Conclusion: Our study demonstrated that TF-hUiCMs engrafted and proliferated in the infarcted heart and enhanced cardiomyocytes glucose metabolism under ischaemic conditions. BLI and PET imaging could provide biological information of transplanted stem cells in myocardial infarction.
\end{abstract}

\section{Image/Figure:}

https://www.xcdsystem.com/wmis/abstract/File6959/GA485 ImageFigure 0610123524.jpg

Image/Figure Caption: Dynamics and metabolic changes after TF-hUiCMs transplantation. (A)Bioluminescence signals were obtained at day 4 and day 35 after injection of TF-hUiCMs into the heart of rat with myocardial infarction. (B) Quantitative analysis of BLI signals in TF-hUiCMs group. Signal activity is expressed as $\mathrm{p} / \mathrm{sec} / \mathrm{cm} 2 / \mathrm{sr}(\mathrm{n}=5)$.(C)Schematic diagram of 17-segment model for analysis of regional [18F]-FDG uptake differences in left ventricle. (D) Representative [18F]-FDG PET images (HLA, horizontal long axis; SA, short 
axis; VLA, vertical long axis) and corresponding polar maps (PMs) of the left ventricle at Day 4 and Day 35.

Full Name of Abstract's 1st Author : Yuanxue Gao

Complete Status: Complete

First Name: Yuanxue

Last Name: Gao

Email: 11818370@zju.edu.cn

Organization: ZheJiang University

Country: China 


\title{
ID: GA486 \\ Human cortical progenitors integrate into the stroke-injured cortex and prevent cortical seizures after stroke
}

\author{
Xiao He, Zhejiang University, hexiao310@163.com
}

\section{Category: Neuroscience}

\begin{abstract}
Body : Background: Loss of synapses and damage of neural circuits in the cerebral cortex are the fundamental causes of stroke-induced neurological deficits in adult humans. Stem cell-based therapies for synapse replacement and circuit repair after stroke require generation of specific neuronal subtypes and maintainence of excitatory and inhibitory balance closely resembling neuronal networks in vivo. In order to restore injured synapses and circuits more effectively, we used the human cortical progenitors (hCPs) derived from human induced pluripotent stem cells (iPSCs) to generate both excitatory and inhibitory neurons resembling the composition of the human cortex. Methods: Focal cortical infarct in rats was induced by the photothrombotic occlusion. Magnetic resonance imaging (MRI) was used to identify the exact location and size of the infarct, which provided a precise guidance for transplantation of hCPs to peri-stroke cortical area. Then, positron emission tomography (PET) imaging was utilized to monitor the metabolic changes of infarcted cortex after hCPs transplantation. Meanwhile, in vivo bioluminescence imaging (BLI) was used to evaluate the survival, proliferation and distribution of hCPs and their progeny cells. Moreover, we used immunofluorescence, immunoelectron microscopy and patch-clamp to evaluate the differentiation and maturation of hCPs after transplantation. Virus-based anterograde monosynaptic tracing technique was used to confirm the establishment of direct synaptic connections between progeny cells of hCPs and ventral thalamus of rat brain. To investigate the long-term effects of stem cell transplantation on brain function, we used EEG to monitor the epileptic discharge activity in the peri-infarct areas for up to 12 months after stem cell transplantation. Results: 18F-FDG PET imaging showed that the glucose metabolism began to improve at the 1st week after hCPs transplantation $(\mathrm{L} / \mathrm{H}=0.80)$, and the maximum improvement was achieved by the 4 th week $(\mathrm{L} / \mathrm{H}=0.90)$. BLI results showed that the number of hCPs increased gradually with the time of transplantation within 5 weeks after transplantation. At 2 months after transplantation, immunofluorescence, immunoelectron microscopy and patch-clamp results confirmed that hCPs successfully differentiated into all six layers of cortical neurons, and the progeny neurons formed direct synaptic connections with host neurons and obtained mature electrophysiological characteristics. Moreover, the virus-based anterograde monosynaptic tracing result demonstrated that the grafted neurons receive direct synaptic inputs from the ventral thalamus of the rat brain within 3 months after transplantation. The EEG data showed that the probability of epilepsy in the hCPs transplantation group was $1 / 8$ $(12.5 \%)$, while that in the control group was $3 / 5(60 \%)$, which indicated that hCPs transplantation has the potential to prevent epilepsy after focal cortical stroke. At 12 months after stroke, there was no tumor formation in immunocompromised rats. Conclusions: Our findings show, for the first time, that human iPSCs-derived hCPs could largely prevent chronic cortical seizures after stroke. Transplanted hCPs could differentiate into mature neuronal cells,
\end{abstract}


regenerate new synapses and restore circuitry, and thereby contribute to functional repair at later time points after intracortical transplantation in a rat stroke model.

\section{Image/Figure:}

https://www.xcdsystem.com/wmis/abstract/File6959/GA486_ImageFigure_0614124241.png

Image/Figure Caption: Figure 1. (A) Serial representative 18F-FDG PET images demonstrate metabolic recovery after hCPs transplantation in a rat model of stroke. (B) Semiquantitative analysis of glucose metabolic change in vivo in different time points. Data in various groups are shown as change of $\mathrm{L} / \mathrm{H}$ ratio. Statistical analysis was performed using a one-way (ANOVA) test, with $* * *$ indicating $\mathrm{p}$ The above three graphs represent the typical epileptic discharge patterns of three rats with focal cortical infarction in vehicle control group $(n=5)$. The lower one represents the typical epileptogram of a rat with focal cortical infarction in the hCPs transplantation group $(\mathrm{n}=8)$.

Full Name of Abstract's 1st Author : Xiao He

Complete Status: Complete

First Name: Xiao

Last Name: He

Email: hexiao310@163.com

Organization: Zhejiang University

Country: China 


\title{
ID: GA487 \\ Imaging diabetic foot ulcers (DFU) in Asian population using Spatial Frequency Domain Imaging (SFDI)
}

\author{
Kapil Dev, Singapore Bioimaging Consortium (SBIC), kapil_dev@sbic.a-star.edu.sg
}

Category: Systemic Diseases (Kidney, Liver and Pancreas)

\begin{abstract}
Body : Among first world nations, Singapore has one of the highest rates of diabetes related lower extremity amputation [1]. According to the national health survey 2010 , the prevalence of diabetes in Singapore has increased from $8.2 \%$ in the year 2004 to $11.3 \%$ in the year 2010 and is likely to increase to $15.9 \%$ by the year $2050[2,3]$. This has caused a huge burden including medical expenses and productivity loss on Singapore's economy. Chronic diseases like diabetes or peripheral vascular disease cause compromised lower limb vascularization impacting perfusion and tissue oxygenation. Current assessment tools for tissue oxygenation like monofilament test, ankle brachial index (ABI), toe brachial index (TBI) are subjective, time-consuming, and allow for local tissue quantification. Also, it has been studied that in the presence of other artery complications, these methods are inaccurate for diabetic foot ulcer screening [4]. Herein, we report the screening of fifteen (15) patients with diabetes in a currently ongoing clinical study using Reflect RSTM, a spatial frequency domain imaging (SFDI) based system to evaluate tissue health status in terms of perfusion and oxygenation. SFDI is a non-contact, non-invasive optical imaging technique that allows for large area $(20 \mathrm{~cm} \mathrm{x}$ $15 \mathrm{~cm}$ ) measurement of tissue chromophores and physiological parameters such as melanin, oxyhemoglobin ( $\mathrm{HbO} 2)$, deoxy-hemoglobin ( $\mathrm{HbR})$, oxygen saturation ( $\mathrm{StO} 2)$ and total haemoglobin ( $\mathrm{HbT}$ ) up to $\sim 3 \mathrm{~mm}$ below the skin surface. With the help of depth-resolved model analysis, SFDI evaluates tissue haemoglobin in the papillary (micro-circulation, $\mathrm{HbT} 1$ ) and reticular dermis (macro-circulation, HbT2) respectively [5]. From the analysis of acquired data, we observed that patients with diabetes show poor microcirculation in the papillary dermis ( $\mathrm{HbT} 1)$ of plantar foot as diabetes is known to affect the microcirculation due to diabetic neuropathy. One of the diabetic patients with foot ulcer (DFU) on the right hallux indicated lower microcirculation values than other diabetic subjects with no ulcer and elevated $\mathrm{StO} 2$ values all over both plantar feet. The lower values of micro and macrocirculation may be due to the arteriopathic condition supplying less blood throughout the feet. The reduced $\mathrm{HbT} 1$ and elevated $\mathrm{StO} 2$ could be attributed to arteriovenous shunting [5] resulting in under-extraction of oxygen and may help identify ulceration risk that other tools cannot identify. With the help of these imaging metrics, we envision that these objective measures augment the poorly reproducible and variable clinical parameters that are currently used to risk stratify patients with diabetes ensuring timely interventions and treatments delivered to those at most risk.
\end{abstract}

References: [1] A. Yee et al., Proceedings of Singapore Healthcare 2017, 26 (2): 76. [2] National Health Survey 2004, 2010, Ministry of Health, Singapore. [3] T. P. Phan et al., BMJ Open Diabetes Res Care. 2014, 2 (1): e000012. [4] L. Potier et al., Eur J Vasc Endovasc Surg. 2011, 41 (1):110-6. [5] Craig Weinkauf et al., J Vasc Surg. 2019, 69 (2):555. 
Complete Status: Complete

First Name: Kapil

Last Name: Dev

Email: kapil_dev@sbic.a-star.edu.sg

Organization: Singapore Bioimaging Consortium (SBIC)

Country: Singapore 


\title{
ID: GA488 \\ Imaging ketone metabolism in the mouse brain with hyperpolarized $13 \mathrm{C}$ magnetic resonance spectroscopy
}

\author{
Lydia Le Page, UCSF, lydia.lepage@ucsf.edu
}

\section{Category: Neuroscience}

\begin{abstract}
Body : Introduction After a ketogenic diet (KD), some cognitive improvements have been seen in Alzheimer's disease (AD) patients1-3. Monitoring ketone administration in humans involves measuring the highest concentration serum ketone - beta-hydroxybutyrate (BHB)4 - but this is not always coupled to brain ketone metabolism5. In vivo measurement of brain BHB metabolism is critically needed to properly assess patient response. Here, we evaluate a new imaging method, 13C magnetic resonance spectroscopy (MRS) of hyperpolarized (HP) [113C]BHB, for in vivo assessment of ketone metabolism. Methods [1-13C]BHB (6.4M in ddH2O) was hyperpolarized, dissolved in buffer (Tris-HCl and EDTA in dH2O) and injected both with BDH+NAD at $11.7 \mathrm{~T}$ (dynamic 13C MRS) and intravenously in a C57BL/6J mouse at $3 \mathrm{~T}$ (dynamic, non-localized data acquired, $1 \mathrm{H} / 13 \mathrm{C}$ surface coil, $\mathrm{TR}=3.2 \mathrm{~s}$; 30 repetitions; $\mathrm{FA}=15^{\circ} ; \mathrm{RG}=203$ ). Solution T1 was measured at $1.5 \mathrm{~T}$ (benchtop Pulsar), $3 \mathrm{~T}$ (Bruker BioSpec) and 11.7T (Agilent vertical bore). Further, non-HP [1-13C]BHB was injected in a C57BL/6J mouse and the brain snap frozen $\sim 1$ minute later. Brain tissue metabolites were extracted using chloroform/methanol, and 13C data acquired (11.7T, 1D decoupled zgpr with f1 presaturation, $\left.\mathrm{FA}=30^{\circ}\right) . \mathrm{C} 57 \mathrm{BL} / 6 \mathrm{~J}(\mathrm{n}=6-8)$ or AD hAPP-J20 mice $(\mathrm{n}=3)$ received either a KD (TD.160153, Envigo) or control diet (TD.150345, Envigo). Serum BHB levels (Precision Xtra) and brain BDH activity (BMR) were measured. T1 and enzyme data were analyzed using Mestrenova; in vivo data in jMRUI. Results $\mathrm{T} 1$ was $41 \mathrm{~s}$ at $1.5 \mathrm{~T} ; 37 \mathrm{~s}$ at $3 \mathrm{~T} ; 28 \mathrm{~s}$ at $11.7 \mathrm{~T}$, and polarization level was $21 \%$ (Figure A), comparable to other HP probes6. Metabolism of HP [1-13C] BHB (181ppm) to HP 13C AcAc (175ppm) by BDH could be detected (Figure B-C). Both [113C]BHB and 13C AcAc resonances were seen in brain tissue extracts, showing brain metabolism of the injected probe during the timeframe of the HP experiment (Figure D). Serum $\mathrm{BHB}$ and $\mathrm{BDH}$ activity were significantly elevated in healthy mice fed a KD compared to control diet (Figure E,F). However, importantly, there was no correlation between serum BHB and brain BDH activity (Figure G). In the AD mouse model, serum BHB and brain BDH activity were also significantly elevated in KD-fed mice compared to control-diet-fed (Figure H, I). As for healthy mice, there was no correlation between serum BHB and brain BDH activity (Figure J). After injection, HP [1-13C]BHB was observed in the brain at 3T over multiple timepoints (Figure K); on summing the data, HP 13C AcAc was detected. Discussion and conclusion Enzymatic conversion of HP [1-13C]BHB to AcAc by BDH occurs within the lifetime of our HP probe. While KD increased BHB serum levels and brain BDH activity in both healthy and AD mice, our results demonstrate that these two values are not correlated, confirming that serum BHB is an unsuitable marker of ketone metabolism following a KD. An in vivo method for assessing BDH activity would provide valuable information on the in vivo metabolic status of the AD brain. We observed HP [1-13C]BHB to AcAc conversion in vivo at 3T, demonstrating
\end{abstract}


the feasibility of our approach. Future imaging studies will assess control and KD-fed hAPP-J20 mice, compared to healthy animals.

References: 1. Kashiwaya, Y. et al. Neurobiol. Aging 34, 1530-1539 (2013). 2. Taylor, M.K., Sullivan, D.K., Mahnken, J.D., Burns, J.M. \& Swerdlow, R.H. Alzheimer's Dement. Transl. Res. Clin. Interv. 4, 28-36 (2018). 3. Croteau, E. et al. J. Alzheimer's Dis. 64, 551-561 (2018). 4. Laffel, L. Diabetes. Metab. Res. Rev. 15, 412-426 (1999). 5. Grabacka, M., Pierzchalska, M., Dean, M. \& Reiss, K. Int. J. Mol. Sci. 17, 2093 (2016). 6. Najac, C. et al. Sci. Rep. 9, 3402 (2019).

\section{Image/Figure:}

https://www.xcdsystem.com/wmis/abstract/File6959/GA488_ImageFigure_0609115754.jpg

Image/Figure Caption: Figure (A) Thermal (left) and hyperpolarized (right) spectra of [1-13C] $\mathrm{BHB}$, demonstrating high polarization. (B) Following injection of HP [1-13C] BHB to a mixture of $\mathrm{BDH}$ enzyme and NAD, production of $\mathrm{HP}[1-13 \mathrm{C}]$ acetoacetate was observed at $11.7 \mathrm{~T}(\mathrm{C})$ Time course of substrate and product signal from acquisition in B (D) 13C thermal NMR spectrum of brain tissue snap-frozen immediately after injection of $13 \mathrm{C} \mathrm{BHB}$, showing the resonances of [1-13C] BHB and [1-13C] AcAc. (E-J) Characterization of serum BHB and brain BDH activity in healthy and hAPP-J20 mice. In healthy mice, (E) serum BHB and (F) cerebral BDH activity were both significantly elevated following a ketogenic diet (Student's t-test). (G) No correlation was observed between these two parameters (Pearson correlation). Similarly in hAPP-J20 mice, (H) serum BHB and (I) cerebral BDH activity were both significantly elevated following a ketogenic diet. $(\mathrm{J})$ No correlation was observed between these two parameters. ${ }^{*} \mathrm{p} \leq 0.05, * * * \mathrm{p}(\mathrm{K})$ Following injection of HP [1-13C] BHB into an anesthetized control mouse, non-localized dynamic $13 \mathrm{C}$ spectra were acquired using a $3 \mathrm{~T}$ Bruker system, showing the presence of [1-13C] BHB (181 ppm) in the brain (T2 image inset). On summing the spectra, HP acetoacetate was observed (175.5ppm).

Complete Status: Complete

First Name: Lydia

Last Name: Le Page

Email: lydia.lepage@ucsf.edu

Organization: UCSF

Country: United States 


\title{
ID: GA490 \\ A novel framework for real-time margin assessment for solid cancers using a tumor acidosis sensitive optical imaging agent
}

\author{
Max Witjes, UMCG, m.j.h.witjes@umcg.nl
}

\section{Category: Oncology}

\begin{abstract}
Body : Introduction: Intra-operative management of surgical margins in patients diagnosed with solid tumors remains challenging as surgeons continue to rely primarily on visual and tactile information. Currently, no reliable, clinically-approved method supporting real-time intra-operative decision making exists. Fluorescence-guided surgery using tumor-specific compounds is an interesting clinical tool for aiding in real-time evaluation of the margin status. ONM-100 is a novel, $\mathrm{pH}$-activatable micelle encapsulating the fluorescent imaging agent, Indocyanine Green (ICG). ONM-100 exhibits a binary off/on mechanism that allows for specific fluorescence activation in the acidotic tumor microenvironment. This study investigates a standardized, specimen-driven, fluorescence imaging framework using ONM-100 as a clinical decision-making tool during the surgical treatment of head and neck squamous cell carcinoma (HNSCC) and breast cancer (BC). Material and Methods: 13 patients with histologically confirmed head and neck squamous cell carcinoma $(n=9)$ or breast cancer $(n=4)$ were included in this phase I study (Netherlands National Trial Register 7085). ONM-100 was administered intravenously $24 \pm 8$ hours before surgery. Fluorescence images of the excised surgical specimen and the surgical cavity were analyzed. Fluorescent lesions with a Tumor-toBackground Ratio $(\mathrm{TBR})>1.5$, either on the surgical specimen or in the surgical cavity, were considered to be fluorescence-positive. In vivo imaging was performed with the NovaDaq Spy Elite and the ex-vivo imaging with the Pearl-trilogy. Fluorescence positive specimens were collected and submitted for standard of care histopathological evaluation. Results: In total, nine histologically confirmed tumor-positive surgical margins were discovered immediately following primary tumor excision using ONM-100 fluorescence-guided intra-operative imaging, six in
\end{abstract} HNSCC patients and three in BC patients. Postoperative analysis showed a median $( \pm \mathrm{IQR}) \mathrm{TBR}$ of the fluorescent lesions at the margin of resection to be $3.36 \pm 1.62$ and $3.8 \pm 1.8$ for HNSCC and $\mathrm{BC}$, respectively. Three additional fluorescent lesions in the surgical cavity (2 HNSCC, 1 BC) were biopsied, and showed occult carcinoma, severe dysplasia and false-positive detection of muscle tissue. Conclusion: Our fluorescence framework using a novel, $\mathrm{pH}$-activatable, fluorescent imaging agent enables reliable and adequate surgical decision making in real-time. Using a fluorescence TBR cutoff value of 1.5, we demonstrate a strong correlation of fluorescence to tumor-positive resection margins across 13 patients and two tumor types. The binary mechanism of ONM-100 allows for sharp tumor delineation in HNSSC and BC, and therefore provides the surgeon with a highly sensitive clinical tool for real-time margin assessment. In current standard of care, intra-operative surgical margin analysis is performed on frozen sections harvested from the specimen and not from the woundbed, because this is considered the most reliable approach. The fluorescent framework allows detailed specimen driven analysis as well as surgical woundbed analysis, which in this study revealed additional lesions in the woundbed. There are three possible scenarios identified in this study following the 
detection of a fluorescent spot in the ex vivo specimen or the surgical woundbed. Fluorescence spots 1) can be resected with adequate margin without compromising vital structures, 2) an identified fluorescent spot cannot be resected or 3) new spots outside the identified tumor field are identified in the mucosa or deep margin (in transit metastasis). Situations 1 and 3 occurred during this study. In this fluorescence guided decision making, 8 out of 9 spots would have been re-resected in real time, demonstrating the potential clinical benefit of this fluorescent framework.

Complete Status: Complete

First Name: Max

Last Name: Witjes

Email: m.j.h.witjes@umcg.nl

Organization: UMCG

Country: Netherlands 


\title{
ID: GA491 \\ Accurate MR Diffusion Studies Using Multi-frequency Excitation Wideband EPI (MEWB-EPI) on 7T MRI
}

\author{
Po-Wei Cheng, National Taiwan University, zopm100@hotmail.com
}

\section{Category: New Chemistry, Biology \& Bioengineering}

\begin{abstract}
Body : Goal: $\quad$ In this study, we will first show that by using Multi-frequency Excitation Wideband EPI (MEWB-EPI), this technique can improve temporal resolution by two folds on the current MR imager without upgrading its hardware. This advantage can instead tradeoff to have higher signal to ratio image by averaging 2 fasten MR acquisitions while remain the same acquisition time. In this paper, we employ this higher SNR images to improve the accuracy of diffusion study analysis to achieve more consistent FA and accurate fiber tracking. Introduction: The selection of parameter is a tradeoff between time and image quality. Recently, simultaneous multiband studies have opened a new horizon to shorten the imaging time; and among them, ME-wideband MRI and SE-wideband MRI are two extraordinary examples. [1] [2] In this study, we apply this novel acquisition method, "Multi-frequency Excitation Wideband EPI (MEWB-EPI) on the 7T Bruker imager, and show that this technique can easily accelerate 2fold temporal resolution. We can average these ultrafast images to achieve better SNR in the same imaging time.

Material and Method The MEWB-EPI
\end{abstract} sequence was implemented in both EPI and EPI DTI sequence to assess the technique for the anatomy and diffusion weight imaging of rat brain. All acquisitions were obtained from 7T MRI (Bruker, Ettlingen, Germany) system with MEWB-EPI (W, wideband acceleration factor, =2) 2X Temporal Resolution Spin-Echo EPI Image The Spin-Echo EPI images are the following parameters: FOV $=2.5 \times 2.5 \mathrm{~cm} 2,1 \mathrm{~mm}$ thickness, Matrix Size $=96 \times 96, \mathrm{TR} / \mathrm{TE}=5000 / 70$ $\mathrm{ms}, \mathrm{NEX}=40$. The two set images are the same spatial resolution: $260 \mathrm{um} 2$. Scan time for standard EPI and WB-EPI are $43 \mathrm{~m} 20 \mathrm{~s} \& 21 \mathrm{~m} 40 \mathrm{~s}$, half of the original time respectively. $2 \mathrm{X}$ Average Number Diffusion Application The spin echo diffusion imaging is combined with 1 null-image and 12-diretion diffusion gradients. The parameters of the diffusion study are as follows: $\mathrm{FOV}=2.5 \times 2.5 \mathrm{~cm} 2,1 \mathrm{~mm}$ thickness, TR/TE $=2500 / 21 \mathrm{~ms}, \Delta / \delta=8 / 3 \mathrm{~ms}, \mathrm{~b}$-value $=$ $1500 \mathrm{~s} / \mathrm{mm} 2$, Average Number $=40 / 80$ respectively. Since $2 X$ ME-Wideband technique is 2 times faster, we can take this advantage to imaging an 80 average number instead of the original 40 average number imaging, while maintaining the same scan time in 43min 20s. FA analysis and fiber track MR images and analysis were implemented in MATLAB (MathWorks, Natick, U.S.A.). The diffusion tracking are reconstructed by DSI Studio (http://dsistudio.labsolver.org). To evaluate the reproducibility of FA performance, the same DTI sequence are repeated twice in this study. This Statistics of this difference of FA value can provide as a DTI performance information. Result and Discussion: In the MEWB-EPI 2x temporal resolution experiment, the acquisition time of EPI sequence is reduced from $43 \mathrm{~min} 20 \mathrm{~s}$ to $21 \mathrm{~min}$ 40 s. The signal to noise, contrast and details of this $2 \mathrm{X}$ fast image kept well at the equal quality of the original one (Fig.1a, 1b). In the $2 \mathrm{X}$ average number diffusion experiment, SNR of DTI null images can be increased from $76.2 \pm 8.2$ to $105.2 \pm 5.8$ (Fig.1c, 1d), which match the 1.414 theoretical average gain. Its associated calculated FA images accordingly have better tissue 
delineation (Fig.1e, 1f) and its error of FA values is evidently reduced in Fig.1g. And this enhanced reconstructed Corpus callosum (Fig. 1h, 1i) also clearly shows a smooth and stable fiber tracking. Conclusions: The purpose of this study is to show the feasibility of accurate diffusion studies by using the MEWB-EPI technique. Our results show, this novel technique could easily accelerate the temporal resolution and also can take the scan speed as a tradeoff into its associated 1.414 SNR gain for a $2 \mathrm{X}$ faster sequence. In general, user can use the speed gain to either benefit on either speed gain or on anatomical details gain.

References: [1] E. L. Wu et al., Med. Phys. (2014) [1] E. L. Wu et al., Med. Phys. (2015)

\section{Image/Figure:}

https://www.xcdsystem.com/wmis/abstract/File6959/GA491_ImageFigure_0610110235.png

Image/Figure Caption: In the MEWB-EPI 2x temporal resolution experiment, the acquisition time of EPI sequence is reduced from $43 \mathrm{~min} 20 \mathrm{~s}$ to $21 \mathrm{~min} 40 \mathrm{~s}$. The signal to. noise, contrast and details of this $2 \mathrm{X}$ fast image kept well at the equal quality of the original one (Fig.1a, 1b). In the $2 \mathrm{X}$ average number diffusion experiment, SNR of DTI null images can be increased from $76.2 \pm 8.2$ to $105.2 \pm 5.8$ (Fig.1c, 1d), which match the 1.414 theoretical average gain. Its associated calculated FA images accordingly have better tissue delineation (Fig.1e, 1f) and its error of FA values is evidently reduced in Fig. 1g. And this enhanced reconstructed Corpus callosum (Fig.1h, 1i) also clearly shows a smooth and stable fiber tracking to reveal fine fiber for better 3D brain mapping in the future.

Complete Status: Complete

First Name: Po-Wei

Last Name: Cheng

Email: zopm100@hotmail.com

Organization: National Taiwan University

Country: Taiwan 


\title{
ID: GA492
}

\section{Combining Imaging of 68Ga-DOTATATE and 18F-FDG Positron Emission Tomography/Computed Tomography in Localizing Ectopic Adrenocorticotropic Hormone-Secreting Tumors}

\author{
Qingxing Liu, Peking Union Medical Colleage Hospital, smu_lqx@163.com
}

\section{Category: Oncology}

\begin{abstract}
Body : Purpose: Localizing ectopic adrenocorticotropic hormone-secreting (ACTH) tumor in patients with ectopic ACTH syndrome (EAS) is a challenge. This study aimed to evaluate the diagnostic value of $68 \mathrm{Ga}-\mathrm{DOTATATE}$ and $18 \mathrm{~F}-\mathrm{FDG}$ PET/CT in ectopic ACTH tumors. Methods: Thirty patients (Female: Male=16:14, $43 \pm 18 \mathrm{y}$ ) with suspected ectopic ACTH tumor were recruited. All the patients underwent 18F-FDG PET/CT and 68GaDOTATATE PET/CT within 4 weeks for comparison. A final diagnosis was based on the histopathology of surgical resection or biopsy. Results: Ectopic ACTH tumors were located in 18 patients (Bronchial NET: thymic NET: mediastinal NET: pancreatic NET: rectal NET: intramyocardial NET: pheochromocytoma $=10: 2: 2: 1: 1: 1: 1)$ and were occult in 12 patients. The SUVmax of 18F-FDG and 68Ga-DOTATATE PET are higher in primary tumor diameter $>$ $1 \mathrm{~cm}$ than those $\leq 1 \mathrm{~cm}$ (SUVmax of 18F-FDG: $0.51 \pm 0.86$ vs $4.45 \pm 1.02, \mathrm{P}=0.009$; SUVmax of 68Ga-DOTATATE: $2.54 \pm 0.77$ vs $7.62 \pm 2.38, \mathrm{P}=0.059)$. Compared with cases without metastases, cases with metastases had higher primary tumor diameter, Ki 67 index, SSTR2 expression, SUVmax of 18F-FDG and SUVmax of 68Ga-DOTATATE (P $>0.05)$. Overall sensitivity of 68Ga-DOTATATE, 18F-FDG, combined 68Ga-DOTATATE and 18F-FDG was $46.4 \%, 39.3 \%, 64.3 \%$, respectively; accuracy was $50.0 \%, 36.7 \%, 60.0 \%$, respectively. While in histologically confirmed cases $(n=20)$, the sensitivity and accuracy of 68Ga-DOTATATE, 18FFDG, combined 68Ga-DOTATATE and 18F-FDG were higher (sensitivity, 72.2\%, 61.1\%, $100.0 \%$, respectively; accuracy, $75.0 \%, 55.0 \%, 90.0 \%$, respectively). In primary tumor and metastasis, the SUVmax of the 68Ga-DOTATATE correlated well with SSTR2 expression $(\mathrm{r}=$ $0.72, \mathrm{P}=0.002)$, but not with ACTH expression $(\mathrm{r}=-0.08, \mathrm{P}=0.778) . \mathrm{Ki} 67$ index has a negative correlation with SSTR2 expression $(\mathrm{r}=-0.57, \mathrm{P}=0.028)$, and correlated well with the SUVmax of 18F-FDG $(\mathrm{r}=0.74, \mathrm{P}=0.001)$. The primary tumor diameter correlated well with the SUVmax of 18F-FDG PET $(\mathrm{r}=0.67, \mathrm{P}=0.003)$, and the SUVmax of 68Ga-DOTATATE PET $(\mathrm{r}$ $=0.56, \mathrm{P}=0.015)$. Conclusion: Combined 68Ga-DOTATATE and 18F-FDG PET/CT had higher sensitivity and accuracy in detecting ectopic ACTH tumor than 68Ga-DOTATATE or 18 F-FDG PET/CT.
\end{abstract}

\section{Image/Figure:}

https://www.xcdsystem.com/wmis/abstract/File6959/GA492 ImageFigure 0610033126.jpg

Complete Status: Complete

First Name: Qingxing 
Last Name: Liu

Email: smu_lqx@163.com

Organization: Peking Union Medical Colleage Hospital

Country: China 


\title{
ID: GA493 \\ Microscopic Imaging as a Quantitative Tool to Assess the in Skin Malaria Parasite Deposition: Mosquito Inoculation vs Syringe Injection
}

\author{
Clarize de Korne, Leiden University Medical Center, c.m.de_korne@lumc.nl
}

Category: Immunology: Inflammation \& Infection

\begin{abstract}
Body : INTRODUCTION: In 2018, an estimated 228 million cases of malaria occurred worldwide leading to nearly half a million deaths. An highly efficacious vaccine is needed to break malaria transmission and reduce its burden. Vaccines comprising live attenuated malaria parasites (sporozoites) are currently considered most promising. After deposition in the skin, sporozoites aim to invade a blood vessel enabling them to get transported to the liver, where they can replicate inside liver cells. Previous studies showed that mosquito-inoculated sporozoites (msq-spz) migrate to the liver much more efficiently as compared to intradermally syringe-injected parasites (srg-spz)[1-2]. As a consequence, dermally injected live attenuated sporozoite vaccines are less efficacious as compared to mosquito-delivered attenuated sporozoites. In order to replicate the success of mosquito-based administration, we set out to unravel the differences between msq-spz and srg-spz in the skin with molecular imaging tools. We used microscopic imaging and custom image analysis software to compare 1) the appearance of the inoculation and injection site and 2) the migratory capacity of msq-spz and srg-spz. METHODS: Similar numbers of Plasmodium berghei sporozoites expressing mCherry and luciferase were administered to mice by 30 mosquito bites or by intradermal syringe injection of 3000 sporozoites suspended in $10 \mu \mathrm{l}$ of medium. The parasite burden in the liver of mice as a result of the different administration routes was assessed by bioluminescence imaging 44h post injection. The inoculation and injection site were visualized using a spinning disk confocal microscope showing both the morphology of the skin tissue and the distribution of the msq-spz and srg-spz throughout the skin. Movies were obtained of migrating msq-spz ( $\mathrm{n}=566)$ and srg$\operatorname{spz}(n=1251)$ and quantitatively analyzed with our in-house written analysis tool SMOOTmouse skin. The migration capacity of msq-spz and srg-spz was compared based on key motility parameters: movement pattern, track tortuosity and velocity. RESULTS: The parasite burden in the liver after sporozoite delivery by syringe injection was 3-fold lower compared to sporozoite delivery by mosquito inoculation. Mapping the inoculation site revealed that msq-spz were spread widely as individual sporozoites or clusters (median nearest neighbor distance (NND): 55 $\mu \mathrm{m}, \mathrm{IQR}: 18-132)$ in an environment of densely packed, polygonal shaped cells (mean cell roundness: 0.75 , SD: 0.11$)$ and occasional hematomas (23\% of hematomas contained sporozoites). In contrast, mapping the injection site revealed that srg-spz formed one hotspot (NND: $23 \mu \mathrm{m}$ IQR: 13-43) in tissue which had suffered from fluid expansion (mean cell roundness: 0.87 , SD: $0.07 ;>15 \mu \mathrm{m}$ gaps between cells). The analysis of sporozoite migration capacity using SMOOTmouse skin showed that $88 \%$ of the sporozoites tracked in mouse skin were motile. Msq-spz migrated with a constant turn angle (angular dispersion: 0.73, SD: 0.10) at relatively high speed (mean velocity: $2.41 \mu \mathrm{m} / \mathrm{s}, \mathrm{SD}: 0.31$ ). Compared to the msq-spz, srg-spz migrated slower (mean: $1.84 \mu \mathrm{m} / \mathrm{s}$, SD: 0.24 ) with a more random turn angle (angular dispersion: 0.57 , SD: 0.14$)$. CONCLUSION: In conclusion, microscopic imaging of the inoculation site and
\end{abstract}


the injection site combined with quantitative image analysis revealed differences regarding tissue morphology, sporozoite distribution and sporozoite migration behavior. These differences might explain the low efficacy of sporozoite administration by intradermal syringe injection. Given the practical need for intradermal vaccine delivery, these results indicated that engineering solutions (e.g. microneedle devices) should be explored that can better mimic mosquito inoculation and thereby potentially replicate the success of mosquito-based administration.

References: 1) Hoffman et al. Protection of Humans against Malaria by Immunization with Radiation-Attenuated Plasmodium falciparum Sporozoites. The Journal of Infectious Diseases. 2002; 185(8):1155-1164. 2) Epstein et al. Live Attenuated Malaria Vaccine Designed to Protect Through Hepatic CD8+ T Cell Immunity. Science. 2011; 334(6055):475-480.

\section{Complete Status: Complete}

First Name: Clarize

Last Name: de Korne

Email: c.m.de_korne@lumc.nl

Organization: Leiden University Medical Center

Country: Netherlands 


\title{
ID: GA494 \\ C-Met receptor-mediated fluorescence-guided surgery in penile cancer patients
}

\author{
Hielke de Vries, Netherlands Cancer Institute (NCI), hm.d.vries@nki.nl
}

\section{Category: Oncology}

\begin{abstract}
Body : Aim/Introduction: Surgery is still the mainstay of treatment in penile squamous cell carcinoma (pSCC). During surgery, the goal is to find the optimal balance between an oncologically safe surgical margin and minimal unnecessary mutilation. Unfortunately, positive margins currently occur in 7-36\% of patients. As overexpression of the c-Met receptor is reported for $87 \%$ of $\mathrm{pSCC}$, c-Met has the potential to serve as imaging target in receptormediated imaging of pSCC. In this study the feasibility of c-Met mediated imaging to support image-guided surgery applications was clinically investigated. Materials and Methods: A total of fifteen patients ( $\geq 18$ years of age with $\geq \mathrm{T} 1 \mathrm{pSCC}$ and scheduled for primary tumour surgery) were included in a prospective pilot study. For initial ex vivo assessment of tracer performance $(\mathrm{N}=10)$ surgically excised penile tumour samples were incubated in a solution containing the Cy5 labelled C-Met targeting tracer EMI137 [1]. In vivo assessment of EMI137 was performed in five patients after intravenous tracer administration $(0.13 \mathrm{mg} / \mathrm{kg}$; imaging at $3 \mathrm{~h}$ p.i.). White light and fluorescence imaging were conducted using a Cy5-dedicated prototype STORZ fluorescence laparoscope complemented with image-processing software that allowed colour coding and scaling of the fluorescence signal [1]. The location and intensity of signal enhancement in the fluorescence images was correlated to standard pathological assessment including c-Met immunohistochemistry (\% of cells with membranous c-Met expression and combined c-Met score (mean of multiple regions per tumour)) and H\&E staining. Results: CMet mediated fluorescence imaging combined with image-processing allowed tumour identification in all tumour specimens (diameter $5-55 \mathrm{~mm}$ and infiltration depth $2-24 \mathrm{~mm}$ ). Tumour-to-background ratios did not significantly differ between ex vivo (range: $2.0 \pm 0.6-3.4$ \pm 0.3 ) and in vivo (range: $2.0 \pm 0.4-4.2 \pm 0.9$ ) sample analyses. In line with previous in vivo studies in colon cancer [2], no tracer-related adverse effects occurred. Microscopic immunohistochemistry showed a visual (non-significant) correlation of macroscopic tumour-tobackground ratios with the percentage of c-Met expressing cells in the surgical specimens. This suggests that this application could potentially be used for tumour margin visualization. Interpatient differences were observed in the percentage of c-Met positive cells (30-100\%) and the combined c-Met score (0.3-2.3). Conclusion: C-Met receptor-mediated fluorescence imaging of primary pSCC tumours using EMI137 was shown to be feasible. This pilot study is the first to report in vivo application of a targeted fluorescent tracer in pSCC and paves the way for further development of this surgical guidance approach in order to visualize surgical margins and (potentially) assess tumour spread using c-MET specific tracers.
\end{abstract}

References: [1] van Willigen DM, van den Berg NS, Buckle T, KleinJan GH, Hardwick JC, van der Poel HG, et al. Multispectral fluorescence guided surgery; a feasibility study in a phantom using a clinical-grade laparoscopic camera system. Am J Nucl Med Mol Imaging 2017;7:13847. [2] Burggraaf J, Kamerling IMC, Gordon PB, Schrier L, de Kam ML, Kales AJ, et al. 
Detection of colorectal polyps in humans using an intravenously administered fluorescent peptide targeted against c-Met. Nat Med 2015;21:955-61. doi:10.1038/nm.3641.

Complete Status: Complete

First Name: Hielke

Last Name: de Vries

Email: hm.d.vries@nki.nl

Organization: Netherlands Cancer Institute (NCI)

Country: Netherlands 


\title{
ID: GA495 \\ Hybrid ICG-99mTc-nanocolloid, on the road towards becoming the new standard for sentinel node biopsy in penile cancer?
}

\author{
Hielke de Vries, Netherlands Cancer Institute (NCI), hm.d.vries@nki.nl
}

\section{Category: Oncology}

\begin{abstract}
Body : Aim/introduction: Sentinel node (SN) biopsy in penile cancer (PeCa) is typically performed using $99 \mathrm{mTc}$-nanocolloid and blue dye. Recent reports suggest that the hybrid tracer indocyanine green (ICG)-99mTc-nanocolloid may improve intraoperative SN identification through combined radioactive and fluorescence guidance. However, further investigation will be required before this technique could be widely adopted for treatment of PeCa. The aims of the current study were to confirm the reliability of the use of ICG-99mTcnanocolloid and to investigate the added value of intraoperative fluorescence imaging over visual detection of blue dye in the largest series of PeCa SN biopsies with ICG- 99mTc-nanocolloid to date. Materials and Methods: $400{ }^{3} \mathrm{~T} 1 \mathrm{G} 2 \mathrm{~N} 0 \mathrm{PeCa}$ patients scheduled for SN biopsy and treatment of the primary tumour at a single European centre were included in this study. SNs were preoperatively identified based on lymphoscintigraphy and SPECT/CT after peritumoural injection of ICG-99mTc-nanocolloid. Blue dye was administered intraoperatively in 266 patients . During surgery, SNs were pursued via gamma tracing, visual identification (blue dye) and nearinfrared fluorescence imaging. Intraoperative SN identification rates using radio- and fluorescence guidance were individually assessed in all patients. In those patients who received ICG-99mTc-nanocolloid and blue dye, fluorescence SN identification rates were compared with blue dye using a chi-square test. Results: 740 groins were assessed and no tracer-related adverse events were reported. All preoperatively defined SNs $(n=1163)$ were intraoperatively localized. $98 \%$ of all excised SNs could be detected using gamma tracing and in $96 \%$ with fluorescence imaging. $95 \mathrm{SN}$ tumour-positive SNs were found, which all proved to contain both a radioactive and fluorescence signal. A comparison of the optical identification methods showed that fluorescence imaging yielded a 39.2\% higher SN detection-rate relative to blue dye (95.3 vs. $56.1 \%$ ). $100 \%$ of the SNs that were tumour-positive at pathology could be intraoperatively visualized by fluorescence imaging, whereas merely $84 \%$ were stained blue. Conclusions: ICG$99 \mathrm{mTc}$-nanocolloid was shown to be a reliable $\mathrm{SN}$ tracer for $\mathrm{PeCa}$ in a large patient cohort of $400{ }^{3} \mathrm{~T} 1 \mathrm{G} 2 \mathrm{~N} 0 \mathrm{PeCa}$ patients. Fluorescence imaging was shown to significantly outperform optical SN detection based on blue dye.
\end{abstract}

Complete Status: Complete

First Name: Hielke

Last Name: de Vries

Email: hm.d.vries@nki.nl 
Organization: Netherlands Cancer Institute (NCI)

Country: Netherlands 


\title{
ID: GA496 \\ Feasibility of PSMA-mediated fluorescence-guided robot-assisted prostatectomy at tracer dose in a porcine model
}

\author{
Tessa Buckle, Leiden University Medical Center, t.buckle@lumc.nl
}

\section{Category: Oncology}

\begin{abstract}
Body : Introduction: Fluorescence-guidance is an upcoming methodology for improvement of surgical accuracy. However, the ability to provide intraoperative fluorescenceguidance is both dependent on the amount/concentration of the fluorescent dye accumulated in the lesion(s) of interest and hampered by tissue-dependent signal attenuation. This generally results in application of relatively high doses of a fluorescent tracer, which can have negative impact on toxicity as well as costs. While tracer dose application $(100 \mathrm{ug})$ is the mainstay in nuclear medicine, to date there is still controversy about the feasibility of receptor-mediated fluorescence imaging in vivo at tracer dose levels. Following validation of the performance of new bimodal tracer designs for PSMA in mouse tumor models we have set-out to validate the performance of fluorescence guidance in a large animal (porcine) model with increased translational character. Reasoning that basal PSMA levels in the prostate should already allow evaluation of the feasibility of PSMA-mediated fluorescence imaging in vivo, we evaluated the ability to identify the prostate in vivo after administration of a tracer dose of a PSMA-targeted tracer. Materials and Methods: Tracer kinetics and in vivo imaging of the prostate were assessed in a porcine model $(\mathrm{N}=15,35-40 \mathrm{~kg} /$ animal) after intravenous administration of a high affinity Cy5-functionalized PSMA targeting tracer (EuK-(SO3)Cy5-MAS3; 100 ug, KD: $19.2 \pm 5.8$ $\mathrm{nM}$ [1]). Blood and urine samples were obtained over the course of 240 minutes. In vivo imaging and robot assisted prostatectomy were performed at 4-6 hours post injection, in combination with Cy5 imaging using a clinical Cy5-modified laparoscopic system. Following in vivo imaging, tracer uptake in excised tissues (prostate, kidney, ureter, liver, abdominal fat, muscle, splenic tissue and salivary gland) was evaluated ex vivo using fluorescence confocal microscopy. Results: Presence of fluorescence in the blood was detected at early time points after tracer administration ( $\mathrm{t}=5-20$ minutes) with decreasing intensity over time. Fast urinary clearance was observed, with low levels of fluorescence still present in the urine at the time of imaging and subsequent prostatectomy $(\mathrm{t} 1 / 2=105 \mathrm{~min})$. In vivo fluorescence imaging allowed visualization of the prostate and permitted robot-assisted prostatectomy based on tracer uptake in the prostate. Confocal microscopy of excised tissues confirmed tracer uptake in the kidney and prostate and revealed a faint tracer-related fluorescence signal in the ureter. No fluorescence was detected in the liver, abdominal fat, muscle, splenic tissue or the salivary gland. Conclusion: Our findings indicate that intraoperative PSMA-mediated fluorescence imaging using a tracer dose regime is feasible. The applied approach in (non-tumor bearing) pigs widens translation opportunities from molecule to man and decreases the chance of applying non-successful imageguidance approaches in patients.
\end{abstract}

References: 1. Hensbergen AW, Buckle T, van Willigen DM, Schottelius M, Welling MM, van der Wijk FA, et al. Hybrid Tracers Based on Cyanine Backbones Targeting Prostate-Specific 
Membrane Antigen: Tuning Pharmacokinetic Properties and Exploring Dye-Protein Interaction. J Nucl Med. 2020; 61: 234-41.

Complete Status: Complete

First Name: Tessa

Last Name: Buckle

Email: t.buckle@lumc.nl

Organization: Leiden University Medical Center

Country: Netherlands 


\title{
ID: GA497 \\ Fluorescence background quenching as a means to increase signal to background ratio's - a proof of concept during nerve imaging
}

\author{
Tessa Buckle, Leiden University Medical Center, t.buckle@lumc.nl
}

\section{Category: Neuroscience}

\begin{abstract}
Body : Introduction: Signal to background ratios are critical for the implementation of fluorescence -guided surgery technologies. While local tracer administrations help to reduce the chance of systemic side effects, reduced spatial migration and non-specific tracer distribution can impair the discrimination between the tissue of interest and the background. In an attempt to reduce background signals in imaging procedures that make use of local tracer administration, we explored a pre-targeting concept aimed at quenching non-specific fluorescent signals. The efficacy of this concept was evaluated in an in vivo neuronal tracing set-up. Methods: Pretargeting of the nerves was achieved using a wheat germ agglutinin (WGA) lectin. functionalized with an azide containing Cy5 dye (N3-Cy5-WGA). The Cy7 quencher dye (Cy7-DBCO) can subsequently be used to yield Cy7-Cy5-WGA; a compound wherein the Cy5 emission is quenched by Förster resonance energy transfer to Cy7. The photophysical properties of N3-Cy5WGA and Cy7-Cy5-WGA were evaluated and de-activation kinetics were evaluated in situ, in vitro (Schwannoma cell culture), ex vivo (muscle tissue from mice; used for dose optimization) and in vivo (Nervus Ischiadicus in THY-1 YFP mice; 24h). Results: In situ, conjugation of Cy7DBCO to N3-Cy5-WGA resulted in $>90 \%$ reduction of the Cy5 fluorescence signal intensity after 30 minutes. In cells, pre-targeting with the N3-Cy5-WGA lectin resulted in membranous staining, which could efficiently be deactivated by Cy7-DBCO over the course of $30 \mathrm{~min}(91 \%$ signal decrease). In ex vivo muscle tissue, administration of Cy7-DBCO at the site of injection of N3-Cy5-WGA resulted in effective quenching of the Cy5-related signal (80-90\% after 10-20 minutes), while the Cy7-related signal remained stable over time. Moreover, the use of the different volumes of Cy7-DBCO resulted in different levels of distribution of the Cy7 signal over the muscle tissue specimens; $45.5 \pm 8.1 \%, 77.7 \pm 8.1 \%$ and $78.1 \pm 13.2 \%$ for respectively $5 \mu \mathrm{L}$, $10 \mu \mathrm{L}$ and $20 \mu \mathrm{L}$. In vivo, administration of Cy7-DBCO at the location of the injection site effectively quenched the non-specific background signal up to $73 \%$ after five minutes, resulting in a 50\% increase in the signal-to-background ratio between the nerve and injection site $(\mathrm{p}=$ $0.03)$. Conclusion: The described pre-targeted fluorescence-quenching technology allowed fast and effective reduction of the background signal at the injection site, while preserving in vivo nerve visualization. This proof-of-principle study was focused on imaging of nerves using a fluorescent WGA-lectin, but the same concept could in the future also apply to applications such as sentinel node imaging.
\end{abstract}

Complete Status: Complete

First Name: Tessa

Last Name: Buckle 
Email: t.buckle@lumc.nl

Organization: Leiden University Medical Center

Country: Netherlands 


\title{
ID: GA498 \\ Head-to-head comparison of PSMA-PET/CT and ferumoxtran-10-enhanced MRI for the diagnosis of lymph node metastases in prostate cancer patients
}

\author{
Melline Schilham, Radboud University Medical Center, melline.schilham@gmail.com
}

\section{Category: Oncology}

\begin{abstract}
Body : Introduction Assessing lymph node (LN) involvement in prostate cancer (PCa) patients is critical for prognosis and patient management. The current gold standard for LNstaging, pelvic lymph node dissection (PLND), is an invasive procedure and its curative and diagnostic performance are questioned. In the pursuit for less invasive and more accurate staging methods several imaging modalities including ferumoxtran-10 enhanced MRI (nano-MRI, $\mathrm{nMRI}$ ) and prostate-specific membrane antigen (PSMA)-PET/CT, have been evaluated for their performance and accuracy. Recently, the first large prospective study evaluating the accuracy of PSMA-PET/CT in patients with primary PCa reported a sensitivity and specificity of $85 \%$ and $98 \%$, respectively for nodal and distant metastases[1]. In the past nMRI demonstrated a comparable sensitivity (82\%) and specificity (93\%) [2]. Since both modalities rely on different biology of LN metastases, the aim of this study was to evaluate their consecutive findings in terms of size, number and anatomical localisation of suspicious LNs in order to understand the diagnostic potential of these techniques in the detection of LN metastases in PCa patients. Methods This study was approved by the local ethical review board and need for informed consent was waived. We retrospectively analysed the medical files of patients with either
\end{abstract} primary PCa or recurrent disease who were referred for nMRI at our centre and also underwent a PSMA-PET/CT between October 2015 and July 2017. Both scans were performed at the same institution according to local protocols: PSMA-PET/CT with 68Ga-PSMA-HBED-CC at 2 $\mathrm{MBq} / \mathrm{kg}$ bodyweight, imaging after 60 minutes with whole body low-dose-CT for attenuation correction and anatomical referencing; isotropic high-resolution 3-dimensional nMRI, 24 hours after slow-drip intravenous infusion of ferumoxtran-10 (weight adapted dosage of $2.6 \mathrm{mg} \mathrm{Fe} / \mathrm{kg}$ body weight) diluted in $100 \mathrm{~mL}$ sodium chloride. All scans were analysed independently by experienced nuclear physicians/radiologists. Of all suspicious lesions, size, anatomical location and Level of Suspicion (LoS) on a likelihood scale ranging from 1 (no metastasis) to 5 (very high probability of metastasis) were determined. Subsequently, findings from PSMA-PET/CT and nMRI were compared. Results A total of 45 patients were included; 8 primary PCa patients (mean PSA $28.9 \mathrm{ng} / \mathrm{ml}, \pm 49.3$ ) and 33 patients with recurrent PCa (mean PSA $5.0 \mathrm{ng} / \mathrm{ml}, \pm 9.6$ ). For one patient no historical data was available. Both scans were performed within a mean of 2 days (range 1-18 days) apart. A cumulative total of 179 suspicious LNs was identified, of which 160 were identified by nMRI in 33 patients, and 71 were identified by PSMA-PET/CT in 25 patients. Significantly more suspicious LNs were detected by nMRI ( $p$ Discussion and conclusion The present study evaluated and compared the findings of PSMA-PET/CT and nMRI and demonstrated that the differences between their findings were mostly related to the number of suspicious LNs per patient and the average size of the identified LNs. Although a prospective study with a standard of reference is warranted for confirmation, the results of this study provide 
important insight in the complementary role of both imaging modalities and may be considered in clinical diagnostic decisions.

References: 1. Hofman MS, Lawrentschuk N, Francis RJ, Tang C, Vela I, Thomas P, et al. Prostate-specific membrane antigen PET-CT in patients with high-risk prostate cancer before curative-intent surgery or radiotherapy (proPSMA): a prospective, randomised, multicentre study. Lancet (London, England). 2020;395(10231):1208-16. 2. Heesakkers RA, Hovels AM, Jager GJ, van den Bosch HC, Witjes JA, Raat HP, et al. MRI with a lymph-node-specific contrast agent as an alternative to CT scan and lymph-node dissection in patients with prostate cancer: a prospective multicohort study. The Lancet Oncology. 2008;9(9):850-6.

\section{Image/Figure:}

https://www.xcdsystem.com/wmis/abstract/File6959/GA498_ImageFigure_0610102206.jpg

Image/Figure Caption: FIGURE 1. Size distribution of suspicious LNs as detected by nMRI and PSMA-PET/CT. LN = lymph node; $\mathrm{nMRI}=$ nanoMRI; PSMA = prostate specific membrane antigen.

Complete Status: Complete

First Name: Melline

Last Name: Schilham

Email: melline.schilham@gmail.com

Organization: Radboud University Medical Center

Country: Netherlands 


\title{
ID: GA499 \\ Manganese-based blood pool $\mathrm{T} 1$ contrast agent from a single pot template reaction
}

\author{
Graeme Stasiuk, King's College LOndon, graeme.stasiuk@kcl.ac.uk
}

\section{Category: New Chemistry, Biology \& Bioengineering}

\begin{abstract}
Body : Magnetic resonance imaging (MRI) is a powerful non-invasive medical imaging technique that produces high spatial and temporal resolution functional 3D images anatomy and physiological functions. 1 Working on the principles of NMR, MRI offers clear images by monitoring the differences between longitudinal (T1) and transverse (T2) relaxation times of water protons in the body. Different tissues in the body present different T1 and T2 values due to the differential water content. MRI can detect these intrinsic differences and produce a unique visual distinction between normal and abnormal tissues. To enhance the MR signal contrast agents (CAs) are employed. Gadolinium-based MRI T1 contrast agents (GdCAs) are the current gold standard for contrast enhanced medical applications in radiology.2 However, due to the low kinetic stability of acyclic GdCAs in vivo, a red flag has been raised for most of the clinical GdCAs due to agravated nephrogenic systemic fibrosis (NSF)3,4 and detection of $\mathrm{Gd} 3+$ deposition in the brain upon recurrent injection of GdCAs.5 Due to these concerns, the European Medicines Agency (EMA) had suspended the marketing authorizations of four acyclic GdCAs such as gadodiamide, (Omniscan), gadoversetamide (OptiMARK), gadopentetate dimeglumine (Magnevist) and gadobenic acid (Multihance- specifically restricted "to be used in liver scans due to they are taken up in the liver and meet an important diagnostic need").6,7 The US - Food and Drug Administration (FDA) also advised against the use of linear acyclic GdCAs in renal compromised patients.6,8 To overcome these hurdles, the development of Gd3+-free MR contrast agents are critical in radiology. To tackle this, manganese-based contrast agents (MnCAs) are proposed as safer alternatives9 to GdCAs. Mn2+ has five unpaired d electrons, long electronic relaxation time and rapid water exchange. These properties are ideal for CAs capable of ennhancing T1.10 Manganese is a biogenic and vital metal that is found in all tissues (mean serum concentration is $0.5-1.2 \mu \mathrm{g} / \mathrm{L}$ ) and is required for bone development, neuronal health and several physiological processes including cellular (mitochondrial) functions.11 Despite the increased interest in MnCAs development, only, Manganese(II) chloride (LumenHanceTM), is currently used for animal MRI studies.12 Therefore, there is a significant interest in the development in next-generation MnCAs, with high thermodynamic stability, kinetic inertness, and fast clearance from the body. In this work, we have employed for the first time a single pot template synthesis strategy to develop a manganese-based MRI contrast agent MnLMe. MnLMe has been fully chemically characterized via, X-ray and NMR, determination of the hydrated states and stability and relaxometry has been performed. MnLMe displays relaxivity, $\mathrm{r} 1=4.2 \pm 0.2 \mathrm{mM}-1 \mathrm{~s}-1(400 \mathrm{MHz}, 298 \mathrm{~K})$ and $4.9 \pm 0.1 \mathrm{mM}-1 \mathrm{~s}-1(64 \mathrm{MHz}, 298 \mathrm{~K})$ which is superior to the several reported acyclic MnCAs. The stability of the MnLMe has been investigated by zinc transmetallation, via time-dependent relaxivity and absorption spectral titration methods. The MnLMe displays remarkable kinetic inertness even in the presence of $\mathrm{Zn} 2+$ ions. MnLMe shows binding to BSA $(\mathrm{Ka}=4.16 \times 103 \mathrm{M}-1)$ with an enhanced $\mathrm{r} 1=21.5$
\end{abstract}


mM-1 s-1 (20 MHz, $298 \mathrm{~K}, \mathrm{pH}$ 7.4). In vivo MRI studies in healthy mice show MnLMe ( $\mathrm{n}=5)$ has a very different biodistribution to the $\operatorname{GdCA}$ Magnevist $(\mathrm{n}=5)$, with greater excretion form the liver and prolonged blood half-life, as observed by the continuous high $\Delta \mathrm{SI}$ in the left ventricle of the heart. These data indicate that MnLMe interacts with albumin and other proteins throughout the imaging window, confirming the binding to serum albumin and potential for use as a blood pool agent.

References: (1) Strijkers, G. J.; Mulder, W. J. M.; van Tilborg, G. A. F.; Nicolay, K., Med. Chem. 2007, 7, 291-305. (2) Clough, T. J.; Jiang, L.; Wong, K.-L.; Long, N. J. Nature Commun., 2019, 10, 1420. (3) Dekkers, I. A.; Roos, R.; Van Der Molen, A. J. Eur. Radiol. 2018, 28, 1579-1584. (4) Thomsen, H. S.; Morcos, S. K.; Dawson, P., Clin. Radiol. 2006, 61(11), $905-$ 906.. (5) Gulani, V.; Calamante, F.; Shellock, F. G.; Kanal, E.; Reeder, S. B. Lancet Neurol. 2017, 16, 564-570. (6) V. M. Runge, Invest. Radiol. 2017, 52(6), 317-323. (7) Sieber, M. A.; Lengsfeld, P.; Frenzel, T.; Golfier, S.; Schmitt-Willich, H.; Siegmund, F.; Walter, J.; Weinmann, H. J.; Pietsch, H. Eur Radiol. 2008, 18(10), 2164-2173. (8) https://www.fda.gov/media/116492/download. (9) Gale, E. M.; Wey, H. -Y.; Ramsay, I.; Yen, Y. -F.; Sosnovik, D. E.; Caravan, P. Radiology 2018, 286, 865-872. (10) Morrow J. R.; Tóth, É. Inorg. Chem. 2019, 56 (11), 6029-6034. (11) Wedler, F. C. Prog. Med. Chem. 1993, 30, 89-133. (12) Pálmai, M.; Pethő, A.; Nagya, L. N.; Klébert, S.; May, Z.; Mihály, J.; Wacha, A.; Jemnitz, K.; Veres, Z.; Horváth, I.; Szigeti, K.; Máthé, D.; Varg, Z. J. Colloid Interface Sci. 2017, 498, 298-305.

\section{Image/Figure:}

https://www.xcdsystem.com/wmis/abstract/File6959/GA499_ImageFigure_0610102450.png

Image/Figure Caption: Figure 1. MnLMe application at the clinical dose enhances MRI contrast in healthy mice. (A) Representative contrast-enhanced T1-weighted image in the kidneys, liver, heart, and bladder after application of MnLMe at a clinical dose (second panel) in comparison to pre-contrast imaging (first panel). Difference in T1- signal intensity, heat map (third panel), Merged heat map and T1-weighted image (fourth panel).

Complete Status: Complete

First Name: Graeme

Last Name: Stasiuk

Email: graeme.stasiuk@kcl.ac.uk

Organization: King's College LOndon

Country: United Kingdom 


\title{
ID: GA500 \\ Imaging glucose-stimulated zinc secretion from the prostate and pancreas using a Mn(II)-based sensor
}

\author{
Andre Martins, Werner Siemens Imaging Center, andre.martins@med.uni-tuebingen.de
}

Category: Systemic Diseases (Kidney, Liver and Pancreas)

\begin{abstract}
Body : The divalent zinc ion (Zn2+) plays an essential structural and catalytic role in many metalloenzymes and zinc finger proteins but also plays a key role in the secretory function of many tissues including the brain, pancreas, mammary glands, and prostate.1 Dysregulation in tissue $\mathrm{Zn} 2+$ has been associated with diseases such as Alzheimer's disease, diabetes, transient neonatal zinc deficiency, and prostate cancer. For instance, the healthy human prostate has been reported to have the highest levels of zinc while malignant prostate cells undergo a metabolic transformation that results in reduced accumulation of $\mathrm{Zn}(\mathrm{II}) .2$ Pancreatic b-cells also store insulin in granules in crystalline form containing molecules of pro-insulin with $\mathrm{Zn} 2+$ ions. Upon release of zinc by glucose stimulation, the local concentration of $\mathrm{Zn} 2+$ ions around $\beta$-cells (pancreas) and epithelial cells (prostate acinar glands), transiently rises to $\sim 0.5 \mathrm{mM} .1$ This process is known as glucose-stimulated zinc secretion (GSZS). Imaging GSZS from secretory tissues has proven useful at assessing organ function and health. So far, the only successful probes to detect zinc secretion by MRI in vivo are gadolinium-based sensors.3 In this work we introduce a manganese-based zinc sensor and show that pancreatic and prostatic zinc detection is not compromised when using Mn instead of Gd for imaging GSZS in vivo. Given the current concerns associated with Gd deposition after repeated usage of some Gd-based MRI contrast agents, an effective manganese alternative for the MRI detection of GSZS has been developed herein (Figure 1). In the absence of Zn2+, the newly synthesized MnL zinc sensor has little to no affinity for human serum albumin (HSA) but after binding to 1 equivalent of $\mathrm{Zn} 2+$, the resulting complexes then bind to HSA with strong zinc binding $(\mathrm{KD}(\mathrm{Zn})=90 \mathrm{nM})$. This in turn results in a remarkable increase in $\mathrm{r} 1$ relaxivity $(\sim 430 \%)$. MnL showed also superior kinetic inertness toward transmetallation by $\mathrm{Zn} 2+$ and transchelation by HSA. This sensor is excreted via hepatobiliary pathways and as an intact complex via renal filtration; as observed by LC-MS of urine. Control experiments showed marginal enhancement in the prostate and pancreas when no D-glucose was co-injected with MnL. In turn, when glucose was co-injected with MnL both the pancreas and the prostate showed superior T1 contrast when compared to the respective controls. Data suggested that $\mathrm{MnL}$ is uniquely sensitive to zinc. Tissue measurements of Mn content by ICP-AES together with imaging shows that after 15 minutes post-injection, $\mathrm{MnL}$ is found primarily in the kidneys, liver, pancreas, and prostate. After 90 minutes, the Mn content was greatly diminished in the kidneys and liver tissues but accumulated in the spleen and in the prostate. The later observation may reflect $\mathrm{Mn}$ in the prostatic urethra during excretion of the agent. In summary, a zinc-responsive Mn-based MRI contrast agent has been used to image GSZS in mice, similar to that reported previously using Gd-based agents. 3-6 The tissue Mn content showed that the $\mathrm{MnL}$ chelate is excreted through hepatobiliary pathways and intact through renal filtration. These data suggest that a Mn-based zinc sensor is a reliable alternative to Gd agents for detection of GSZS by MRI.7
\end{abstract}


References: 1. Kelleher, S. L., McCormick, N. H., Velasquez, V. \& Lopez, V. Zinc in Specialized Secretory Tissues: Roles in the Pancreas, Prostate, and Mammary Gland. Adv. Nutr. Int. Rev. J. 2, 101-111 (2011). 2. Lo, S.-T., Martins, A. F., Jordan, V. C. \& Sherry, A. D. Zinc as an Imaging Biomarker of Prostate Cancer. Isr. J. Chem. 57, 854-861 (2017). 3. Martins, A. F. et al. Imaging Insulin Secretion from Mouse Pancreas by MRI Is Improved by Use of a ZincResponsive MRI Sensor with Lower Affinity for Zn2+ Ions. J. Am. Chem. Soc. 140, 1745617464 (2018). 4. Esqueda, A. C. et al. A New Gadolinium-Based MRI Zinc Sensor. J. Am. Chem. Soc. 131, 11387-11391 (2009). 5. Yu, J. et al. Amplifying the Sensitivity of Zinc(II) Responsive MRI Contrast Agents by Altering Water Exchange Rates. J. Am. Chem. Soc. (2015) doi:10.1021/jacs.5b09158. 6. Jordan, M. V. C. et al. Zinc-sensitive MRI contrast agent detects differential release of Zn(II) ions from the healthy vs. malignant mouse prostate. Proc. Natl. Acad. Sci. 113, E5464-E5471 (2016). 7. Chirayil, S. et al. A manganese(II)-Based Responsive Contrast Agent Detects Glucose- Stimulated Zinc Secretion from the Mouse Pancreas and Prostate by MRI. (2020) doi:10.26434/chemrxiv.12430082.v1.

\section{Image/Figure:}

https://www.xcdsystem.com/wmis/abstract/File6959/GA500 ImageFigure 0610102501.png

Image/Figure Caption: Figure 1. Zinc detection mechanism with Mn-based contrast agent. Schematic for the detection of glucose stimulated zinc secretion from the mouse pancreas and prostate in vivo by MRI.

Complete Status: Complete

First Name: Andre

Last Name: Martins

Email: andre.martins@med.uni-tuebingen.de

Organization: Werner Siemens Imaging Center

Country: Germany 


\title{
ID: GA501 \\ Intraoperative tissue characterisation during robotic surgery using fluorescence- based smart forceps - development and evaluation in large animal models
}

\author{
Matthias van Oosterom, Leiden University Medical Center, m.n.van_oosterom@lumc.nl
}

\section{Category: Oncology}

\begin{abstract}
Body : Aim: Robot-assisted surgery has become an established technique for many minimal invasive laparoscopic procedures. With the increase in surgical precision, there is a great potential to specifically integrate molecular image-guided surgery concepts within this robotic workflow, further improving treatment precision: radical excision of diseased tissues with minimal damage to healthy tissues. An example of this is the recently introduction DROPIN gamma probe, a small and tethered detection modality that integrates radioguided surgery in the robotic workflow, optimally exploiting the enhanced manoeuvrability in the robotic setup $[1,2,3]$. Next to the integration of radioguidance, fluorescence guidance is also increasingly being used during robotic surgery (e.g. lymphatic mapping and angiography using indocyanine green (ICG)). In addition to the limitations in manoeuvrability of the current fluorescence laparoscopes, frequent use of fluorescence imaging may restrict the surgical workflow: within laparoscopic surgery the overall visualization of the patient anatomy (e.g. bleedings) during fluorescence imaging has proven inferior to white light imaging. Therefore, implementation of fluorescence imaging often requires the operating surgeon to pause his/her resection, interrupting the surgical workflow. Furthermore, this means that, during the actual resection under white light, fluorescent tracers are not detected. For that reason, we developed and investigated small click-on fluorescence detectors that transform standard robotic surgical instruments into 'smart' fluorescence imaging devices (SmartForceps), monitoring the presence of fluorescent tracers in every tissue grasped during the surgical procedure. Materials and Methods: A click-on fibrebased fluorescence detector was engineered for the ProGrasp forceps of a da Vinci surgical robotic system, capable of detecting ICG. Translational investigation of this concept was evaluated in: 1) phantoms; 2) robotic surgery in porcine models $(n=5)$, investigating lymph node localisation and angiography settings; and 3) ex vivo human specimens from the prostate cancer sentinel node procedure ( $\mathrm{n}=2$; using the hybrid tracer ICG-99mTc-nanocolloid). Results: The smart robotic instrument, with the detectors clicked-on, was small enough to be introduced through the standard $12 \mathrm{~mm}$ trocars. After introduction in the porcine abdomen, the fluorescence detectors did not hinder the forceps manoeuvrability or grasping ability. As confirmed with Firefly fluorescence imaging, fluorescent counts were only detected in the tracer containing tissues. The porcine surgery demonstrated that grasping with the SmartForceps of the different tissues allowed for the identification of vascularization (kidney, bowel and bladder) and lymphatic nodes. The ex vivo human tissue evaluation indicated that the SmartForceps could distinguish sentinel lymph nodes from non-sentinel lymph nodes (confirmed with Firefly fluorescence and gamma probe investigation). Conclusion: This study describes the successful introduction of using click-on fluorescence-based sensing modules within the surgical robotic workflow, converting regular forceps into so-called SmartForceps. This concept allows for fluorescence detection during white light resections. This might open the way for various
\end{abstract}


biosensing applications, where the surgical instruments themselves are used to characterise the molecular aspects of tissues handled during surgery.

References: [1] M.N. van Oosterom et al., AJNMMI, 2016; [2] P. Meershoek et al., EJNMMI, 2019; [3] F.W.B. van Leeuwen et al., Clin Nucl Med, 2019

\section{Complete Status: Complete}

First Name: Matthias

Last Name: van Oosterom

Email: m.n.van_oosterom@lumc.nl

Organization: Leiden University Medical Center

Country: Netherlands 


\title{
ID: GA502 \\ Tracking of the DROP-IN gamma probe using fluorescence-based computer vision during robotic surgery - validation in porcine models
}

\author{
Matthias van Oosterom, Leiden University Medical Center, m.n.van_oosterom@lumc.nl
}

\section{Category: Oncology}

\begin{abstract}
Body : Aim: Robot-assisted laparoscopic surgery is claiming an increasing share of the minimal invasive interventions performed. With the successful in-human introduction of the DROP-IN gamma probe, radioguided surgery has become compatible with the surgical robotic setting (e.g. sentinel lymph node biopsy or even PSMA-targeted dissections for prostate cancer) $[1,2,3]$. As a next step in (molecular-based) precision surgery, 'GPS-like' navigation of surgical laparoscopic detection modalities using preoperative patient scans has been suggested [4]. However, the tool-tracking needed for such a surgical navigation setup is complex in a dynamic laparoscopic workflow. In an attempt to integrate surgical navigation with the DROP-IN modality, a fluorescence-based video-tracking setup was developed and evaluated in the robotic surgical setting. Materials and Methods: The Firefly fluorescence laparoscope, integrated in the da Vinci surgical platform and capable of multispectral fluorescence imaging of fluorescein (lex $=488 \mathrm{~nm}$, lem $=515 \mathrm{~nm}$ ) and indocyanine green $($ ICG; lex $=800 \mathrm{~nm}$, lem $=820 \mathrm{~nm}$ ), was used [5]. Computer vision video-processing algorithms were engineered to recognize and segment fluorescent tracking-markers on the DROP-IN probe housing. Based on this information, the dedicated algorithms were capable to calculate in real-time the relative position and orientation (pose) of the probe with respect to the robotic fluorescence laparoscope. These tracking algorithms were incorporated in a SPECT-based surgical navigation device, capable to also track the pose of the laparoscope itself and the patient, using near-infrared optical tracking. Using a torso phantom for robotic surgery, this enabled navigation of the DROP-IN probe towards radioactive lesions (marked with $99 \mathrm{mTc}$ as identified on preoperative SPECT/CT imaging). Functioning of the navigated DROP-IN probe concept was further investigated with robotic surgery in porcine models $(n=4)$. Results: Exploiting the multispectral fluorescence imaging capabilities, the fluorescein containing DROP-IN markers could be separated from ICG-positive lesions based on the differing emission wavelengths. Even under surgical conditions, preserving a direct line-of-sight with respect to these fluorescent markers allowed for intraoperative pose tracking of the DROP-IN modality with respect to tissue lesions. This enabled the augmented reality visualization of the real-time distance between the lesion targets and the DROP-IN probe, as visualized in the robotic console. Once integrated with SPECT/CT navigation, accurate guidance of the DROP-IN probe relative to preoperatively marked lesions became possible. In this setting, the real-time acoustic and numeric feedback, as delivered by the DROP-IN gamma probe, confirmed that the lesion localization was performed successfully. Conclusion: Using (multi-spectral) fluorescence imaging, we have demonstrated that future usage of the DROP-IN probe modality might be enriched with surgical navigation towards target-tissues as marked in preoperative images. With that a next step has been taken towards (molecular-based) imageguided surgery in the robotic setting.
\end{abstract}


References: [1] M.N. van Oosterom et al., AJNMMI, 2016; [2] P. Meershoek et al., EJNMMI, 2019; [3] F.W.B. van Leeuwen et al., Clin Nucl Med, 2019; [4] M.N. van Oosterom et al., J Urol, 2018; [5] P. Meershoek et al., J Nucl Med, 2018.

Complete Status: Complete

First Name: Matthias

Last Name: van Oosterom

Email: m.n.van_oosterom@lumc.nl

Organization: Leiden University Medical Center

Country: Netherlands 


\title{
ID: GA503 \\ Imaging tumor hypersialylation using metabolic labelling
}

\author{
Flaviu Bulat, Cancer Research UK, flaviu.bulat@cruk.cam.ac.uk
}

\section{Category: Oncology}

\begin{abstract}
Body : Introduction: Aberrant cell surface glycosylation has been described as one of the key hallmarks of cancer, with hypersialylation the most commonly observed alteration in cancer. 1 Levels of sialylation can be monitored by incorporating unnatural sugars bearing bioorthogonal chemical reporters. Monitoring hypersialylation could provide an insight into tumor progression, proliferation and ultimately could potentially be used for monitoring treatment response. 2,3 We describe here in vivo imaging of hypersialylation using a novel triacetylated cyclopropene mannosamine (Ac3ManNCyoc) derivative. Methods: Mannosamine was functionalised using a carbamate-linked methyl cyclopropene to yield an unnatural sugar (ManNCyoc) that can be metabolised by cancer cells. A panel of sugars was synthesised with different degrees of acetylation (1 to 4), which we expected to show different diffusion rates across the cell membrane and different solubilities. A 'click' chemistry fluorescent probe, tetrazine-PEG11-DyLight800 was synthesised and used to label the methyl cyclopropene moiety and sugar incorporation levels were assessed in vitro using flow cytometry. One candidate was selected for in vivo imaging as this gave the highest signal-to-baseline ratio (SBR) on flow cytometry. In vivo fluorescence imaging was performed in Colo205 xenografts. Results: Varying the levels of sugar acetylation resulted in significant differences in cell labelling indicating that there was an optimum level of acetylation that balanced sugar solubility and membrane penetration. Flow cytometry (Fig 1B) showed that of the 4-sugar panel, the triacetylated sugar Ac3ManNCyoc gave the highest SBR (8.7). The fluorescently labelled tetrazine-PEG11DyLight800 probe showed a favourable biodistribution profile, with quick renal clearance and minimal organ retention in the xenograft-bearing mice, at $24 \mathrm{~h}$ following administration. Mice treated with Ac3ManNCyoc and the tetrazine probe (+/+); Fig 1A) showed a 1.6-fold increase in fluorescence, compared to vehicle treated animals $(-/+)$; Fig $1 \mathrm{~A} \& 1 \mathrm{C}, \mathrm{n}=2)$. Ex vivo fluorescence imaging of tumors showed an average 2.9-fold increase in fluorescence between control and sugar treated lesions $(n=2$, Fig 1D). Conclusions: Ac3ManNCyoc is a novel candidate for probing hypersialylation by metabolic labelling and subsequent imaging. The triacetylated sugar represents the optimal compromise between sugar solubility and membrane penetration.
\end{abstract}

References: 1. Pinho S.S., Reis C.A. Glycosylation in cancer: mechanisms and clinical implications. Nat. Rev. Cancer. 15, 540-555 (2015). 2. Neves, A. A., Stoeckmann, H., Wainman, Y. A., Kuo, J. C., Fawcett, S., Leeper, F. J. \& Brindle, K. M. Imaging cell surface glycosylation in vivo using 'double click' chemistry. Bioconjug. Chem. 24, 934-941 (2013). 3. Neves, A. A., Wainman, Y. A., Wright, A., Kettunen, M. I., Rodrigues, T. B., McGuire, S., Hu, D. E., Bulat, F., Geninatti Crich, S., Stoeckmann, H., Leeper, F. J. \& Brindle, K. M. Imaging Glycosylation in Vivo by Metabolic Labeling and Magnetic Resonance Imaging. Angew. Chemie - Int. Ed. 55, 1286-1290 (2016). 


\section{Image/Figure:}

https://www.xcdsystem.com/wmis/abstract/File6959/GA503_ImageFigure 0610105447.jpg

Image/Figure Caption: Figure 1. Fluorescence imaging of tumor hypersialylation. (A) Animals treated with sugar and tetrazine-PEG11-DyLight800 (+/+) and control mice (-/+) treated with fluorescent probe only. (B) Average mean fluorescence intensity (MFI; $n=3$ ) generated in Colo205 cells by different levels of sugar acetylation. (C) Tumor fluorescence intensity (radiance levels) obtained by in vivo fluorescence imaging. (D) Tumor radiance levels obtained by ex vivo fluorescence imaging of tumors. All data were acquired $24 \mathrm{~h}$ post tetrazine administration.

Full Name of Abstract's 1st Author : Flaviu Bulat

Complete Status: Complete

First Name: Flaviu

Last Name: Bulat

Email: flaviu.bulat@cruk.cam.ac.uk

Organization: Cancer Research UK

Country: United Kingdom 


\title{
ID: GA504 \\ In-human evaluation of a DROP-IN gamma probe for robot-assisted radioguided surgery of prostate cancer related lymph nodes
}

\author{
Matthias van Oosterom, Leiden University Medical Center, m.n.van_oosterom@lumc.nl
}

\section{Category: Oncology}

\begin{abstract}
Body : Aim: Within the surgical management of prostate cancer, minimal invasive and robot-assisted surgery has become an established procedure. While the robot offers many advantages during laparoscopic surgery, specific challenges can arise when it is combined with traditional surgical instruments: for example, when radioguided surgery is applied using the traditional rigid laparoscopic gamma probe. The restricted maneuverability of this rigid probe limits in vivo lesion localization when low-activity lesions (i.e. low tracer uptake) are positioned near a high-activity background (i.e. high tracer uptake). In addition, the robotic setup forces the surgeon to rely on the scrub nurse for probe positioning, since the surgical console is located outside the sterile field. To promote maneuverability and regain autonomy during robot-assisted radioguidance, we created a small, tethered and highly maneuverable DROP-IN gamma probe in 2014 and translated it into humans in 2018 [1-4]. Using these first proof-of-concept studies, in vivo use of this technology was successfully demonstrated during the sentinel lymph node procedure and PSMA-targeted lymphatic dissections in prostate cancer. In this study, we investigate this concept in a larger sentinel lymph node patient cohort and investigate DROP-IN radioguidance with respect to the traditional laparoscopic radioguidance and fluorescence guidance. Materials and methods: The included patients $(n=25)$ underwent a sentinel lymph node dissection, extended pelvic lymph node dissection and prostatectomy. Following intraprostatic injection of the sentinel node tracer [(indocyanine green-)99mTechnetiumnanocolloid], preoperative mapping was achieved with lymphoscintigraphy and SPECT/CT. During surgical localization and excision of the sentinel nodes, a combination of DROP-IN radioguidance, traditional laparoscopic radioguidance and Firefly fluorescence guidance was used. Phantom experiments were performed to substantiate the in vivo radioguidance findings. Results: 47 sentinel nodes were pursued with DROP-IN gamma probe guidance in vivo, of which $100 \%$ was identified. Of these nodes, $91 \%$ could also be detected in vivo with fluorescence imaging. In vivo evaluation of these nodes with the laparoscopic gamma probe only identified $76 \%$. Similar as in the phantom evaluation, the anatomical location of the missed sentinel nodes appeared to be linked to the location of the assistant portal. Conclusion: This inhuman study further strengthens the potential that the DROP-IN gamma probe concept delivers, integrating radioguided surgery with the robot-assisted surgical platform. With its greatly improved maneuverability, even an increased detection rate was found with the DROP-IN probe with respect to the traditional laparoscopic gamma probe.
\end{abstract}

References: [1] M.N. van Oosterom et al., Am_J_Nucl_Med_Mol_Imaging, 2016 [2] B. Fuerst et al., IEEE Trans Med Imaging, 2016 [3] P. Meershoek et al., Eur_J_Nucl_Med_Mol_Imaging, 2019 [4] F.W.B. van Leeuwen et al., Clin_Nucl_Med, 2019 
Complete Status: Complete

First Name: Matthias

Last Name: van Oosterom

Email: m.n.van_oosterom@lumc.nl

Organization: Leiden University Medical Center

Country: Netherlands 


\title{
ID: GA505 \\ Rational design of a red-fluorescent probe for ALDH1A1 and applications in vitro
}

\author{
Thomas Bearrood, University of Illinois at Urbana-Champaign, bearroo2@illinois.edu
}

\section{Category: Oncology}

\begin{abstract}
Body : Tumors, both solid and liquid, are composed a complex mosaic of cells of varying degrees of specialization. One of the most important subpopulations in this mosaic is that of cancer stem cells (CSCs).[1] CSCs are believed to be the root cause of major problems associated with cancer such as recurrence and metastases. Through various pathways, CSCs demonstrate pro-survival mechanisms that enable them to survive therapeutic regimens as the rest of the tumor is debulked. Like healthy stem cells, the CSCs can then begin to divide symmetrically and asymmetrically to regenerate a complex tumor. Additionally, CSCs show increased tumor initiating capacities, strongly implicating their role in tumor metastasis. Our knowledge of CSCs continues to progress through cell ablation and lineage tracking technologies. We also continue to identify CSC-selective cell surface markers through cellular assays, but these struggle to identify and track these cells in vivo without perturbing the system of interest. Aldehyde dehydrogenase (ALDH) 1A1 is an enzyme which is upregulated in many CSC populations.[2] Since it is an intracellular enzyme, activity-based sensors can be developed which transiently interact with ALDH1A1 to provide an imaging readout without perturbing the tumor microenvironment. We previously reported AlDeSense, the first turn-on fluorescent probe that is selective for ALDH1A1.[3] We later discovered that the AM ether used to make AlDeSense cell permeable may perturb the system through release of formaldehyde. We report here the rational design and cellular applications of red-AlDeSense, a red-fluorescent probe for ALDH1A1. We hypothesized the TokyoMagenta dye platform should afford improved cell permeability and a desirable $100 \mathrm{~nm}$ bathochromic shift compared to the Pennsylvania Green dye platform used for AlDeSense. The dye platform shift produced those desired effects but destroyed the fluorescence turn on and isoform selectivity. Through rational design, a series of 12 new probes were synthesized to improve the fluorescence turn on and restore the isoform selectivity, culminating in red-AlDeSense. Red-AlDeSense was used to identify cells with high ALDH1A1 activity both via flow cytometry and confocal microscopy. Additionally, the redfluorescence profile enabled co-staining with a green fluorescent anti-CD44 antibody for the concurrent detection of ALDH1A1 and another CSC marker. Our results using red-AlDeSense suggest ALDH1A1 and CD44 are independent CSC markers in the A549 cell line. The rational design of red-AlDeSense provides a guide for the design of future ALDH1A1-selective probes, both fluorescent and of other molecular imaging modalities. This systematic approach may also be used as a template for other isoform selective probes such as probes for ALDH1A3.
\end{abstract}

References: [1] Annu. Rev. Pathol. Mech. Dis. 2016. 11. 47. [2] Cell Cycle. 2011. 10. 1378. [3] ACS Cent. Sci. 2018. 4. 1045.

\section{Image/Figure:}


Image/Figure Caption: The rational pursuit of a red-fluorescent probe for ALDH1A1 culminated in red-AlDeSense. Red-AlDeSense features improved cell permeability, faster reactivity, and spectroscopic properties that enable costaining with green fluorescent enzymes and antibodies.

Complete Status: Complete

First Name: Thomas

Last Name: Bearrood

Email: bearroo2@illinois.edu

Organization: University of Illinois at Urbana-Champaign

Country: United States 


\title{
ID: GA506 \\ Imaging of tumor cell death in vivo using [18F]FPenM-C2Am: a phosphatidylserine targeting agent.
}

\author{
Flaviu Bulat, Cancer Research UK, flaviu.bulat@cruk.cam.ac.uk
}

\section{Category: Oncology}

\begin{abstract}
Body : Introduction Cell death is an important target for imaging tumor response to treatment. Recently, derivatives of a phosphatidylserine-binding protein $(\mathrm{C} 2 \mathrm{Am})$ for imaging tumour cell death in vivo using SPECT have been implemented [1]. We describe here the development and validation in vivo of a 18F-labelled derivative for PET imaging of tumour cell death following therapy. Methods A one-pot, two-step synthesis of $\mathrm{N}-(5-$ $[18 \mathrm{~F}]$ fluoropentyl)maleimide has been implemented using an automated module (GE TracerLab FxFN, 60 min, $>98 \%$ radiochemical purity and $12 \pm 3 \%$ decay corrected yield). This method was used to label the single cysteine in C2Am within $30 \mathrm{~min}(\mathrm{Am}=212 \pm 30 \mathrm{GBq} / \mu \mathrm{mol}(\mathrm{n}=3))$. Tumor cell death was induced in xenograft models of breast and colorectal cancer, using a TRAIL-R2 agonist. The binding of [18F]FPenM-C2Am was assessed using cell death assays in vitro, and by dynamic PET imaging and biodistribution studies in vivo. Conventional histological cell death assays (CC3 and TUNEL) were used to validate C2Am-based cell death imaging. Results/Discussion [18F]FPenM-C2Am showed predominantly renal clearance and minimal retention in liver $(1.18 \pm 0.13 \% \mathrm{ID} / \mathrm{g})$, small intestine $(0.97 \pm 0.25 \% \mathrm{ID} / \mathrm{g})$, spleen $(0.79 \pm 0.05 \% \mathrm{ID} / \mathrm{g})$ and kidney $(6.90 \pm 0.56 \% \mathrm{ID} / \mathrm{g})$ at $2 \mathrm{~h}$ after probe administration. $[18 \mathrm{~F}] \mathrm{FPenM}-\mathrm{C} 2 \mathrm{Am}$ generated tumour-to-muscle $(\mathrm{T} / \mathrm{m})$ and tumour-to-blood $(\mathrm{T} / \mathrm{b})$ ratios following treatment of $[6.7 \pm 0.8 ; 1.89 \pm 0.23]$ and $[9.3 \pm 2.3 ; 3.1 \pm 0.4]$, respectively, in colorectal and breast tumour models, $2 \mathrm{~h}$ following administration. There was a significant positive correlation between [18F]FPenM-C2Am PET signal in tumors post treatment $(\% \mathrm{ID} / \mathrm{g}, 2 \mathrm{~h}$ post injection) and the corresponding histologic markers of cell death (CC3: Pearson $r=0.73$, $\mathrm{P}=0.0005$; TUNEL: Pearson $\mathrm{r}=0.53, \mathrm{P}=0.03, \mathrm{n}=9$ breast $+\mathrm{n}=9$ colorectal tumours). A statistically significant pairwise difference was also obtained between the tumor-to-blood contrast prior to and following therapy, 2 hours after the injection of the imaging agent, for both the breast $(\mathrm{P}=0.0053)$ and colorectal $(\mathrm{P}=0.0056)$ models (unpaired two-tailed t-test). Conclusions Given the favourable biodistribution profile of [18F]FPenM-C2Am, and its ability to produce rapid and cell death-specific image contrast, this agent has potential for clinical translation.
\end{abstract}

References: [1] Neves AA, Xie B, Fawcett S, Alam IS, Witney TH, de Backer MM, Summers J, Hughes W, McGuire S, Soloviev D, Miller J, Howat WJ, Hu DE, Rodrigues TB, Lewis DY, Brindle KM (2017) Rapid Imaging of Tumor Cell Death In Vivo Using the C2A Domain of Synaptotagmin-I. J Nucl Med. 58:881-887.

\section{Image/Figure:}


Image/Figure Caption: $\mathrm{PET} / \mathrm{CT}$ imaging of tumor cell death in a triple-negative breast cancer xenograft model, using [18F]FPenM-C2Am. Images are maximum intensity projection (M.I.P) images of PET signal of a representative tumour-bearing mouse, before (left) and 24 hours after (right) drug treatment $(0.4 \mathrm{mg} / \mathrm{kg}, \mathrm{MEDI} 3039, \mathrm{i} . \mathrm{v}$.$) . Images were acquired 2$ hours after the administration of [18F]FPenM-C2Am (0.25 mg/g, 5 MBq, i.v.). Tumour (T), liver (L), kidneys $(\mathrm{K})$ and bladder $(\mathrm{B})$ are shown.

Complete Status: Complete

First Name: Flaviu

Last Name: Bulat

Email: flaviu.bulat@cruk.cam.ac.uk

Organization: Cancer Research UK

Country: United Kingdom 


\title{
ID: GA507 \\ Noninvasive molecular magnetic resonance imaging of renal fibrogenesis in a mouse model of Alport Syndrome with the molecular probe GdOA.
}

\author{
Yinching Chen, Athinoula A. Martinos Center for Biomedical Imaging, Massachusetts \\ General Hospital and Harvard Medical School, ychen16@mgh.harvard.edu
}

Category: Systemic Diseases (Kidney, Liver and Pancreas)

\begin{abstract}
Body : Renal fibrosis is the final common injury pathway for nearly all chronic kidney diseases (CKD) 1 including diabetes, hypertension and chronic glomerulonephritis. Clinical studies have demonstrated a strong correlation between end-stage renal disease (ESRD) and the extent of renal fibrosis2. Fibrosis results in a loss of renal function and eventually leads to kidney failure. However, there is currently no reliable means to assess renal fibrosis quantitatively in vivo and non-invasively. Here, we demonstrate a novel MR probe designed to detect active fibrogenesis can image and stage renal fibrosis in a mouse model. Renal fibrosis involves excessive accumulation of extracellular matrix proteins such as collagen types I and III along with lysyl oxidase enzymes which crosslink the collagen fibrils through the formation of allysine residues3. GdOA is an oxyamine derivative of Gd-DOTA, designed to bind to allysine for MRI assessment of renal fibrosis in vivo and non-invasively via changes in tissue R14. GdDOTA which has no specific binding to allysine was used as a control. Renal Fibrosis Model We used a mice model of Alport syndrome (AS) for renal fibrosis in our study. AS is a progressive hereditary disorder of basement membranes caused by genetic mutations resulting in altered type IV collagen. As a result, AS carriers are usually marked with abnormalities of the kidney glomerulus and tubulointerstitium, and formation of renal fibrosis. In mice with collagen Col4a3 knocked out, there is a progressive glomerulonephritis with microhematuria and proteinuria, consistent with the human disease. Imaging these mice at different times allows for testing the ability of GdOA to detect and stage renal fibrosis. Method Col4a3-deficient (Col4a3KO) mice were initially obtained from Jackson Laboratory and maintained as a heterozygous colony. Col4a3 KO mice (KO) and heterozygous littermates (WT) were imaged at $6-9$ weeks of age. Kidney function was assessed by urinary levels of serum albumin and creatinine as well as plasma creatinine. MRI was carried out using a home-made 4-channel transmission/receive coil in a Bruker 9.4T scanner. Animals were under isoflurane anesthesia (1-1.5\% isoflurane), kept warm by a stream of warm air, and the respiratory rate was maintained at 40-60 breaths per minute (SAII, Stony Brook, NY). On the imaging day, basal T1 weighted (T1W) and R1map images (inversion recovery) were obtained before the injection of either GdOA or GdDOTA (100 nmol/g, iv), T1W and R1map were acquired again 4 hours later. $\Delta \mathrm{R} 1$ was calculated as R1(post GdOA)-R1(pre). Following MRI, kidneys were collected, sectioned into cortex and medulla, assessed ex vivo for hydroxyproline (Hyp), Gd, lysyl oxidase gene expression, and stained with Sirius Red (collagen/fibrosis) and hematoxylin/eosin (H\&E). Fibrosis extent was assessed by quantifying the collagen proportional area (CPA) of Sirius Red stained slides. Results Col4a3KO mice exhibited a wide spectrum of renal damage, including diffuse tubular injury, glomerulosclerosis, and fibrosis. Both serum albumin to creatinine ratio, Hyp, and CPA were significantly increased in $\mathrm{KO}$ mice compared to heterozygous controls. In
\end{abstract}


vivo imaging with GdOA showed significant $(\mathrm{P})$ increases in $\triangle \mathrm{R} 1$ in the cortex and medulla in KO mice $(n=14)$ when compared to controls $(n=12)$. However, the non-specific binding probe Gd-DOTA showed no significant differences in $\triangle \mathrm{R} 1$ between $\mathrm{KO}$ and control groups. The GdOA induced $\Delta \mathrm{R} 1$ changes were highly correlated to ex vivo measures of $\mathrm{Gd}$ and hydroxyproline in the renal cortex and medulla, indicating the specificity of GdOA to detect and stage fibrosis. Conclusion We demonstrated that GdOA enhanced molecular MR detects and quantifies renal fibrosis in a mouse model of AS. GdOA MRI may enable monitoring of disease progression and the effectiveness of potential new treatments for chronic kidney disease. NIH U01DK104302

References: 1. Nath KA. Tubulointerstitial changes as a major determinant in the progression of renal damage. American Journal of Kidney Diseases. 1992;20:1-17 2. Cohen E. Fibrosis causes progressive kidney failure. Medical hypotheses. 1995;45:459-462 3. Kagan HM, Trackman PC. Properties and function of lysyl oxidase. Am. J. Respir. Cell Mol. Biol. 1991;5:206-210 4. Waghorn PA, Jones CM, Rotile NJ, Koerner SK, Ferreira DS, Chen HH, et al. Molecular magnetic resonance imaging of lung fibrogenesis with an oxyamine-based probe. Angew Chem Int Ed Engl. 2017;56:9825-9828

\section{Image/Figure:}

https://www.xcdsystem.com/wmis/abstract/File6959/GA507_ImageFigure 0610122251.jpg

Image/Figure Caption: We demonstrated in 3 representative fibrosis stages that GdOA led to greater enhancement in $\mathrm{T} 1 \mathrm{~W}$ signal intensity and R1 (see R1 histogram) as fibrosis became severer. The severity of renal fibrosis was assessed ex vivo by CPA (Sirius Red staining) and hydroxyproline level. Both hydroxyproline level and GdOA-induced $\Delta \mathrm{R} 1$ changes are significantly higher in AS KO group than in the control group (WT). There was a good correlation between hydroxyproline level and $\Delta \mathrm{R} 1 .{ }^{*} \mathrm{p}<0.001$

Complete Status: Complete

First Name: Yinching

Last Name: Chen

Email: ychen16@mgh.harvard.edu

Organization: Athinoula A. Martinos Center for Biomedical Imaging, Massachusetts General Hospital and Harvard Medical School

Country: United States 


\title{
ID: GA508 \\ A detailed in vitro and in vivo comparison of D-Glucose and 3-O-Methyl-D- glucose as MRI-CEST contrast agents
}

\author{
Annasofia Anemone, University of Turin, annasofiaantonia.anemone@unito.it
}

\section{Category: Oncology}

\begin{abstract}
Body : Introduction Native glucose and the non-metabolizable derivative 3OMG have been proposed as MRI-CEST contrast agents for tumor detection and as likely alternatives to FDG-PET [1,2]. Although several studies have investigated their contrast efficiency at several magnetic fields in preclinical and clinical MRI studies, both molecules have never been compared in a strict and robust way to assess their CEST contrast efficiency and $\mathrm{pH}$ dependence for elucidating their contrast capabilities at two clinical magnetics fields (3T and 7T). This study investigated the effect of $\mathrm{pH}$, saturation power level (B1) and magnetic field strength (B0) on the MRI-CEST contrast generated by D-glucose [3] and 3-O-Methyl-D-glucose (3OMG) [4] with the aim of determining the in vivo detection limits of the two agents in the glucoCEST procedure in a murine melanoma tumor model after single bolus injection. Methods The experiments were conducted by comparing D-Glucose or 3OMG $(20 \mathrm{mM})$ in phosphate buffered solutions at different $\mathrm{pH}$ values on two preclinical MRI scanners working at $7 \mathrm{~T}$ and and at 3T. In vivo GlucoCEST images were obtained after injection of Glucose or 3OMG (at two different doses of 1.5 and $3 \mathrm{~g} / \mathrm{Kg}$ ) and with different administration routes (intravenous or oral) on subcutaneous B16-F10 melanoma bearing mice. The CEST signal was recorder over a period of 30 min after the injection; CEST contrast effects and percentage of tumor detection (fraction pixel) were compared between the two molecules. Results Glucose showed a marked $\mathrm{pH}$ dependent CEST contrast with higher $\mathrm{ST} \%$ values at more acidic $\mathrm{pH}$ values (Figure $1 \mathrm{a} ; 7 \mathrm{~T}, 37^{\circ} \mathrm{C}$ ). Conversely, 3OMG CEST contrast reached higher ST\% effect at neutral $\mathrm{pH}$ values (ca. 35\% between 7.2 and $6.8 \mathrm{pH}$, Figure $1 \mathrm{~b})$ with a constant reduction towards acidic $\mathrm{pH}$ values $(25 \%$ of ST effect at $\mathrm{pH}$ 6.0). At the lower magnetic field (Figure $1 \mathrm{~b}$ and $\mathrm{c}$ ), glucose showed a slightly higher CEST detectability in comparison to 3OMG. The opposite $\mathrm{pH}$ dependence of the CEST response that was observed for D-Glucose and 3OMG are maintained independently of the applied B1 saturation power level. Surprisingly, the glucoCEST contrast enhancement in the tumour regions following intravenous administration (at the of dose $3 \mathrm{~g} / \mathrm{kg} \mathrm{b.w}$ ) showed similar contrast between glucose and the non-metabolizable 3OMG (around 1.5-2\% at 3T and 2-4\% at 7T) (Figure1). Conclusion Despite a marked different $\mathrm{pH}$ dependence of the CEST contrast observed in vitro for glucose and $3 \mathrm{OMG}$, when injected at the same dose by i.v. in tumor bearing mice a comparable contrast was observed. No clear advantages were observed for the non-metabolizable glucose derivative 3OMG at both ultra-high field (7T) and high-field (3T). Both molecules showed a prolonged CEST enhancement up to the first $30 \mathrm{~min}$ following the administration. This work was supported by the European Union's Horizon 2020 research and innovation programme (Grant Agreement No. 667510) and by the Associazione Italiana Ricerca Cancro (AIRC MFAG 2017 - ID. 20153 project).
\end{abstract}


References: [1] van Zijl PC, et al. Magn Res Med 2020 Jul;84(1):247-262 [2]Golay X, et al. Nat Med 2013 Aug;19(8):1067-72 [3] van Zijl PC, et al. Proc Natl Acad Sci U S A. 2007,4359 [4] Navon G, et al. Magn Res Med 2018 79,1061

\section{Image/Figure:}

https://www.xcdsystem.com/wmis/abstract/File6959/GA508 ImageFigure 0610123819.png

Image/Figure Caption: Figure 1: ST effect plot of 20mM D-Glucose (a) and 3OMG (b) solution containing $10 \mathrm{mM}$ of phosphate buffer as a function of $\mathrm{pH}$ values, $(\mathrm{B} 1=3 \mu \mathrm{T})$ acquired at $37^{\circ} \mathrm{C}$ using a $7 \mathrm{~T}$ scanner and a $3 \mathrm{~T}$ scanner $(\mathrm{c}, \mathrm{d})$. GlucoCEST $\Delta \mathrm{ST} \%$ map obtained at $7 \mathrm{~T}$ injecting glucose solutions at $1.5 \mathrm{~g} / \mathrm{Kg}(\mathrm{e})$ and $3 \mathrm{~g} / \mathrm{Kg}$ (f) dose or $30 \mathrm{MG}$ solution at at $1.5 \mathrm{~g} / \mathrm{Kg}(\mathrm{g})$ and $3 \mathrm{~g} / \mathrm{Kg}(\mathrm{h})$ dose via intravenous route. Data are reported as the difference $(\Delta \mathrm{ST} \%)$ between the ST effect before and after the intravenous injection.

Full Name of Abstract's 1st Author : Annasofia Anemone

Complete Status: Complete

First Name: Annasofia

Last Name: Anemone

Email: annasofiaantonia.anemone@unito.it

Organization: University of Turin

Country: Italy 


\title{
ID: GA509 \\ Glutamine Imaging for the Detection of Renal Cell Carcinoma Lung Metastases
}

\author{
Federica Pisaneschi, UT MD Anderson Cancer Center, fpisaneschi@mdanderson.org
}

\section{Category: Oncology}

\begin{abstract}
Body : Approximately $90 \%$ of malignancies that occur in the kidney are renal cell carcinoma (RCC). 1 Clear cell renal cell carcinoma (ccRCC) is the most common RCC subtype $(\approx 70 \%)$. In ccRCC, even after nephrectomy, distant metastases are frequent with lungs being the most common site.1,2 This study aims to image ccRCC-derived lung tumors in mice using 4[18F]-(2S,4R)-fluoroglutamine ([18F]FGln), to understand glutamine transport and glutamine utilization in ccRCC. [18F]FGln is a reporter for the alanine-serine-cysteine transporter 2
\end{abstract} (ASCT2), a major glutamine transporter, overexpressed in a number of cancers, including RCC.3 In ccRCC, high ASCT2 expression was associated with lower overall survival and identified as an independent prognostic factor.4 Therefore, understanding the correlation of [18F]FGln and ASCT2 can provide the opportunity to use $[18 \mathrm{~F}] \mathrm{FGln}$ and PET imaging as a predictive biomarker.5 [18F]FGln was reliably produced with $8.5 \pm 2.9 \%(\mathrm{n}=7)$ radioactivity yield.6 Its uptake was investigated in vitro, in ccRCC cell lines SN12C, UMRC3, and its metastatic subline LUNG150, generated from lung metastasis tissue from an orthotopic UMRC3 kidney mouse model. In vivo, subcutaneous and lung-orthotopic models of ccRCC were imaged. UMRC3 and LUNG150 cell lines express luciferase and GFP, facilitating bioluminescence imaging (BLI) of tumor growth. [18F]FGln uptake cell experiments were carried out to observe initial influx of the tracer due to the transporter $(2 \mathrm{~min})$ and the net effect of tracer influx and efficiency of [18F]FGln trapping in the intracellular compartment (1 hour). The 2 min influx of [18F]FGln in UMRC 3 cells was blocked by saturation of the transporter with $20 \mathrm{mM}$ of glutamine in the media (Fig 1A). The same reduction of [18F]FGln was achieved when ASCT2 expression was reduced by siRNA (Fig 1A). Expression of ASCT2 in UMCR3 cells and siRNA knockdown was confirmed by Western blots. At 1 hour, [18F]FGln uptake was reduced in a dose-dependent manner, by addition of glutamine (Fig 1B). A similar effect was achieved upon treatment with the ASCT2 selective inhibitor V-9302 (Fig 1B). In LUNG150 cells, at 2 min, uptake of [18F]FGln is 15-fold higher than in UMRC3 cells (Fig 1C), despite lower ASCT2 expression. It is possible that the initial influx of glutamine in these cells is mediated by secondary mechanisms that are worth investigation. This is consistent with ASCT2 inhibition with V-9302, which has less effect than shown in UMRC3 cells (Fig 1D). Moreover, knockdown of ASCT2 using siRNA in LUNG150 does not lead to a reduction in [18F]FGln uptake (Fig 1C). In vivo, lung-orthotopic ccRCC mouse models were generated by injection of UMRC3 or LUNG150 cells into the left lung of nude mice. PET/MRI proved superior to PET/CT to image this orthotopic lung tumor model. Tumor tissue was easily identified using 3D MRI (Fig 1E). Tracer uptake was determined in the heart, contralateral lung, tumor, and muscle and data reported as the $\% \mathrm{ID} / \mathrm{cc}$ ratio of tumor versus various organs (Fig 1F). Tumor-to-muscle was about 1.5, whereas tumor-to-lung and tumor-to-heart were around 1. It is possible, however, that tumor-to-contralateral lung values could be biased as, after necropsy, bioluminescence/tumor growth was observed in both lungs. In summary, [18F]FGln uptake in ccRCC cell lines is tied to the expression of ASCT2. ccRCC 
orthotopic lung tumors were generated and [18F]FGln PET/MRI experiments performed. UMRC3 lung orthotopic tumors did not show significant contrast over contralateral lung tissue, however, contrast is observed over muscle. PET/MRI imaging of LUNG150 orthotopic tumors and control non-tumor bearing mice are underway. Based on cell studies, the metastatic line LUNG150 shows promise to give statistical contrast over healthy lung tissues.

References: 1. Hsieh, J.J., Purdue, M.P., Signoretti, S., Swanton, C.et al. Renal cell carcinoma. Nat Rev Dis Primers 2017, 3, 17009. 2. Bianchi, M., Sun, M., Jeldres, C., Shariat, S.F.et al. Distribution of metastatic sites in renal cell carcinoma: A population-based analysis. Ann Oncol 2012, 23, 973-980. 3. Schulte, M.L., Fu, A., Zhao, P., Li, J.et al. Pharmacological blockade of ASCT2-dependent glutamine transport leads to antitumor efficacy in preclinical models. Nat Med 2018, 24, 194-202. 4. Liu, Y., Yang, L., An, H., Chang, Y.et al. High expression of solute carrier family 1, member 5 (SLCA1A5) is associated with poor prognosis in clear-cell renal cell carcinoma. Sci Rep 2015, 5, 16954. 5. Hassanein, M., Hight, M.R., Buck, J.R., Tantawy, M.N.et al. Preclinical evaluation of 4-[18F]fluoroglutamine PET to assess ASCT2 expression in lung cancer. Mol Imaging Biol 2016, 18, 18-23. 6. Zhang, X., Basuli, F. Shi, Z-.D., Xu, B., Blackman, B., Choyke, P.L., Swenson, R.E. Automated synthesis of [18F](2S,4R)-4-fluoroglutamine on a GE TRACERlab ${ }^{\text {TM }}$ FX-N Pro module Applied Rad Isot, 2016, 112, 110-114

\section{Image/Figure:}

https://www.xcdsystem.com/wmis/abstract/File6959/GA509_ImageFigure_0610124802.png

Image/Figure Caption: [18F]FGln uptake experiments in UMRC3 cell line at 2 min (A) and 1 hour (B) and in LUNG150 cell line at $2 \mathrm{~min}(\mathrm{C})$ and 1 hour (D). PET/MR image of UMRC3 lung tumors, using 7T Bruker MR scanner equipped with a Cubresa NuPET insert. ROIs: lung tumor $(\mathrm{T})$ in yellow, contralateral lung $(\mathrm{CL})$ in blue, heart $(\mathrm{H})$ in red and muscle $(\mathrm{M})$ in green $(\mathrm{E})$. Ratio of $\% \mathrm{ID} / \mathrm{cc}$ in lung tumor tissue $(\mathrm{T})$ to contralateral lung $(\mathrm{CL})$, muscle $(\mathrm{M})$, or heart $(\mathrm{H}) ; \mathrm{n}=3$ mice $(\mathrm{F})$.

Complete Status: Complete

First Name: Federica

Last Name: Pisaneschi

Email: fpisaneschi@mdanderson.org

Organization: UT MD Anderson Cancer Center

Country: United States 


\title{
ID: GA510 \\ Efficacy of 225Ac-trastuzumab in breast cancer models with medium-low HER2 expression
}

\author{
Behlol Khan, University of Saskatchewan, khan.behlol@usask.ca
}

\section{Category: Oncology}

\begin{abstract}
Body : Background: About 25-30\% of breast cancer (BC) overexpress epidermal growth factor II (HER2) and this is associated with aggressive disease and poor prognosis. Trastuzumab a humanized monoclonal antibody targeting HER2 is approved against HER2positive BC. However, $20-50 \%$ of patients selected for treatment show no response, while up to $70 \%$ of initial responders subsequently acquire resistance. The high linear energy transfer and decay characteristics of alpha particles such as actinium-225 (225Ac) make it ideal for treating small lesions. In this study we hypothesize that targeting 225Ac-trastuzumab can enhance the therapeutic efficiency of trastuzumab. Materials and Methods: Trastuzumab was conjugated with an eighteen-membered macrocyclic chelator p-SCN-Bz-macropa and radiolabeled with 225Ac for alpha particle therapy. The radiochemical yield of $225 \mathrm{Ac}$-trastuzumab was $>90 \%$. The (radio)immunoconjugate was characterized by flow cytometry, radioligand binding assays, HPLC and internalization rate (live-cell imaging). HER2 positive JIMT-1 cells, a trastuzumab/pertuzumab resistant model with medium to low HER2 expression were used to study the in vitro cytotoxicity. In vivo radioimmunotherapy using 225Ac-trastuzumab was studied using JIMT-1 xenograft following treatment with three doses of $350 \mathrm{nCi} /$ dose administered 10 days apart. In vivo study endpoint was tumor volume $>=1500 \mathrm{~mm} 3$. Control treatment groups included PBS, trastuzumab and 225Ac-labeled isotype control IgG. Results: Flow cytometry showed $>90 \%$ binding to the cells. In vitro studies in JIMT-1 showed enhanced cytotoxicity (IC50) of 225Ac-trastuzumab $(5.5 \pm 1.14 \mathrm{nM})$ which was 62 folds less than unlabeled trastuzumab $(340 \pm 1.77 \mathrm{nM}) .225 \mathrm{Ac}$-trastuzumab was effective at inhibiting the growth of JIMT-1 tumors. 6/7 mice treated with 225 Ac-trastuzmab had tumor decreased to $<=40 \mathrm{~mm} 3$, with one complete remission. Median survival 225Ac-trastuzumab (not yet reached); PBStreated (51 days). Conclusion: 225Ac-trastuzumab showed promising therapeutic efficiency towards HER2 positive BC JIMT-1 with medium-low HER2 expression. Efficiency of this radioimmunoconjugate in 3D spheroids and other HER2 positive xenografts is ongoing.
\end{abstract}

Full Name of Abstract's 1st Author : Khan Behlol Ayaz Ahmed

Complete Status: Complete

First Name: Behlol

Last Name: Khan

Email: khan.behlol@usask.ca 
Organization: University of Saskatchewan

Country: Canada 


\title{
ID: GA511 \\ Evaluation of 18F-rhamnose PET imaging as a potential diagnostic probe for invasive aspergillosis
}

\author{
Swati Shah, National Institutes of Health, swati.shah@nih.gov
}

Category: Immunology: Inflammation \& Infection

\begin{abstract}
Body : Background Invasive aspergillosis (IA) is one of the most severe forms of fungal infection in patients with primary or secondary immunodeficiencies. The lungs are involved in the majority of cases of IA and Aspergillus fumigatus (A. fumigatus) is one of the most common causative agents. In view of high mortality and morbidity associated with IA, the development of non-invasive imaging biomarkers becomes necessary to provide a rapid and specific diagnosis. In this study, we aimed to evaluate L-rhamnose, a naturally occurring sugar known to be metabolized by different Aspergillus species, as a potential PET ligand for the diagnosis of pulmonary infection caused by A. fumigatus. Methods In vitro uptake of $3 \mathrm{H}-$ rhamnose was tested in live and heat killed A. fumigatus cultures, and was compared to two representative gram negative and gram positive bacteria (Escherichia coli ATCC \#25922 and Staphylococcus aureus ATCC \#29213) as well as mouse macrophages (Mus musculus J774.1) to assess the specificity of uptake, in comparison to 3H-2-deoxyglucose (3H-2-DG). 2-deoxy-2$18 \mathrm{~F}$-fluoro-L-rhamnose (18F-rhamnose) was then synthesized and evaluated for in vitro uptake by $\mathrm{A}$. fumigatus and E. coli. In vivo uptake was then assessed by autoradiography (3Hrhamnose) and PET/CT (18F-rhamnose) in murine models of pulmonary aspergillosis (2 days following post-pharyngeal inoculation). Standardized uptake values (SUVs) of 18F-rhamnose in infected mice were then measured, and compared to animals with sterile lung inflammation (24 hours following post-pharyngeal poly (I:C) administration) and healthy controls. Results In vitro uptake assays showed selective uptake of $3 \mathrm{H}$-rhamnose by live A. fumigatus cultures when compared to heat killed fungi, macrophages, and selected representative of Gram negative and Gram positive bacterial strains. The uptake of $3 \mathrm{H}$-rhamnose was also significantly higher than that of 3H-2-DG in A. fumigatus. Similarly, increased uptake of the tritium labeled ligand was seen in the lungs of the infected mice by autoradiography while there was no appreciable uptake in the healthy mice and minimal uptake in poly (I:C) treated animals (30 minutes uptake period). $18 \mathrm{~F}$-rhamnose was specifically internalized by live A.fumigatus cultures when compared to heatkilled cultures and E.coli. In vivo PET/CT imaging with a 60-minute dynamic 18F-rhamnose PET/CT imaging of a pulmonary IA model showed a slight increase of uptake in lung lesions compared to controls and poly (I:C) treated mice (Figure 1A and 1B). Attempted modification of the molecule with $18 \mathrm{~F}$ labeling of $\mathrm{C} 6$, to improve uptake, led to rapid defluorination. Conclusions Our study showed that A. fumigatus was able to selectively accumulate $18 \mathrm{~F}$ rhamnose in vitro but to a lesser extent in vivo, likely due to short serum half-life. Even though the uptake of $18 \mathrm{~F}$-rhamnose was significantly higher in lungs of infected mice compared to healthy controls and poly (I:C) treated mice, there was only mild delineation of the lung lesions on PET imaging. Additional studies are underway with different labeling approaches to improve the retention of $18 \mathrm{~F}$-labeled rhamnose and increase the uptake within infected lung tissues.
\end{abstract}




\section{Image/Figure:}

https://www.xcdsystem.com/wmis/abstract/File6959/GA511_ImageFigure_0610090155.jpg

Image/Figure Caption: (A) Representative dynamic average PET images, averaged from 5203520 seconds post injection, of 18F-rhamnose uptake in the lungs of control, sterile lung (poly I:C) and post-pharyngeally infected pulmonary IA (AF IPA) murine models. The first 520 seconds were removed from analysis to reduce potential effects of increased vascularity after injection. (B) Time activity curve of mean 18F-rhamnose uptake in control, Poly (I:C), and AF IPA models from 0-3370 seconds post $18 \mathrm{~F}$-rhamnose injection.

Complete Status: Complete

First Name: Swati

Last Name: Shah

Email: swati.shah@nih.gov

Organization: National Institutes of Health

Country: United States 


\title{
ID: GA512 \\ Improving evaluations of radiation therapy with dynamic contrast enhanced multispectral optoacoustic tomography (DCE MSOT)
}

\author{
Shreya Goel, The University of Texas MD Anderson Cancer Center, \\ shreya.goel.shreya@gmail.com
}

\section{Category: Oncology}

\begin{abstract}
Body : Introduction Tumor vascular perfusion and oxygenation dictate outcomes in cancer treatment, including radiation therapy (RT)1. However, precise measurements of these biomarkers are limited by drawbacks of current dynamic contrast enhanced (DCE) imaging methods2. Here, a new analysis method for DCE multispectral optoacoustic tomography (MSOT)3 is presented that can quantitatively measure vascular perfusion with high temporal and spatial resolution, while endogenous MSOT is employed to evaluate changes in $\mathrm{O} 2$ saturation (dsO2) as measure of $\mathrm{O} 2$ delivery. The method is tested in two xenograft tumor models with different vascular phenotypes and different response to RT. Methods Subcutaneous xenograft models, MIA PaCa-2 (slow growing, less aggressive) and Colo357 (fast growing, highly aggressive) were prepared by injecting female athymic nude mice with 1 x106 cells in lower right flank. Tumors were irradiated with two isocentric beams to deliver a single dose of $10 \mathrm{~Gy}$ (XRAD 320, Precision X-ray Inc.). MSOT measurements were acquired 2 days before and 1 and 4 days after RT, using an MSOT inVision 256-TF scanner (iThera Medical). DsO2 was evaluated with endogenous MSOT by switching inhalation gas from $21 \% \mathrm{O} 2$ to $100 \% \mathrm{O} 2$. DCE MSOT was performed over $15 \mathrm{~min}$ after intravenously injecting $2.5 \mathrm{mmol} / \mathrm{kg}$ ICG. Data analysis was implemented in MATLAB. Correlative histopathology confirmed in vivo imaging outcomes. Results/Discussion We used noninvasive MSOT to examine native differences in vascular perfusion and DmsO2 between two molecularly distinct tumor models (Fig. 1) and evaluate early response to RT (Fig. 2). Baseline DCE MSOT measurements of ICG kinetics indicated > 3-fold lower perfusion in Colo357 tumors compared to MIA PaCa-2 (Fig. 1c). Endogenous MSOT of hypovascular Colo357 tumors showed poor response to $\mathrm{O} 2$ challenge (DsO2) compared to vascular rich MIA PaCa-2 tumors (Fig.1d). Endogenous and DCE MSOT could sensitively and precisely detect early changes in the biomarkers in response to RT. A single 10 Gy dose caused a dramatic drop in vascular perfusion in MIA PaCa-2 tumors, 1 and 4 days post-RT (Fig. 2a). In contrast, resistant Colo357 tumors showed no change in ICG perfusion at day 1 followed by a 2.5-fold decrease at day 4 (Fig.2b). DsO2 emerged as a significant biomarker of heterogeneous $\mathrm{RT}$ response in the two tumor models (Fig.2c). Conclusions We demonstrate improved precision and application of endogenous MSOT and DCE MSOT in noninvasive assessment of molecularly different tumor models. Quantitative and synergistic measurements of tumor vascular perfusion and $\mathrm{DsO} 2$ enabled early and precise evaluations of response to RT. MSOT derived imaging biomarkers can potentially allow rapid and accurate cancer staging, treatment planning and monitoring, in preclinical and clinical settings.
\end{abstract}

References: [1] Laking GR, West C, Buckley DL, Matthews J, Price PM. Imaging vascular physiology to monitor cancer treatment. Crit Ref Oncology/Hematology 2006;58:95-113, 
Elsevier. [2] O'Connor JP, Tofts PS, Miles KA, Parkes LM, Thompson G, Jackson A. Dynamic contrastenhanced imaging techniques: CT and MRI. Br J Radiol. 2011 Dec;84 :S112-20, British Institute of Radiology Publications. [3] Hupple CW, Morscher S, Burton NC, Pagel MD, McNally LR, Cardenas-Rodriguez J. A lightfluence- independent method for the quantitative analysis of dynamic contrast-enhanced multispectral optoacoustic tomography (DCE MSOT). Photoacoustics. 2018;10:54-64, Elsevier.

\section{Image/Figure Caption:}

Full Name of Abstract's 1st Author : Shreya Goel

Complete Status: Complete

First Name: Shreya

Last Name: Goel

Email: shreya.goel.shreya@gmail.com

Organization: The University of Texas MD Anderson Cancer Center

Country: United States 


\title{
ID: GA513 \\ A "smart" paraCEST agent for imaging pH

\author{
James Soundrarajan, UT Southwestern Medical Center, \\ james.ratnakar@utsouthwestern.edu
}

Category: New Chemistry, Biology \& Bioengineering

\begin{abstract}
Body : Paramagnetic lanthanide-based DOTA-tetraamide complexes provide contrast in MRI by chemical exchange saturation transfer (paraCEST).1 The CEST signal is often quite sensitive to the $\mathrm{pH}$ which makes them well suited for the measuring $\mathrm{pH}$ in vivo. The in vivo sensitivity reduces due to the T2 through a T2ex mechanism caused by the presence of a Ln3+bound inner sphere water. 2 This effect is eliminated by having no Ln3+- bound inner sphere water, which is achieved by having three bulky coordinating methylphosphonate pendant arms (1), and a Ln3+-bound-OH group for the paraCEST signal (Figure1a). The protonation of the non-coordinating phosphonate oxygen atoms shows $\mathrm{pH}$ sensitivity to the chemical shift. Yb3+ was chosen because it induces large paramagnetic shifts while minimizing line-broadening effects. The $1 \mathrm{H}$ NMR spectrum of $\mathrm{Yb}(1)$ shows 27 sharp resonances consistent with a ligand structure having $\mathrm{C} 1$ symmetry and a single twisted square antiprism (TSAP) isomer. The broad highly-shifted resonance at $133.6 \mathrm{ppm}$ is assigned to the hydroxypropyl $(-\mathrm{OH})$ proton on the fourth pendant arm which is well outside the typical tissue magnetization transfer (MT) window. The 31P NMR spectrum of $\mathrm{Yb}(1)$ shows three resonances, the chemical shift of each was highly $\mathrm{pH}$ dependent. A simultaneous fit of those data to a three-step equilibrium model gave three pKa values: $\log \mathrm{K} 1=7.0, \log \mathrm{K} 2=5.8$ and $\log \mathrm{K} 3=4.2$. The CEST spectrum of the $\mathrm{Yb}(1)$ complex in water at $25^{\circ} \mathrm{C}$, displays a single highly shifted CEST peak reflecting proton exchange between the $\mathrm{Yb} 3+$-coordinated $-\mathrm{OH}$ group and bulk water protons at different $\mathrm{pH}$, and also shows the complex exists as a single coordination isomer in solution (Figure 1b). CEST images of phantoms at different $\mathrm{pH}$ were collected and a false color $\mathrm{pH}$ map of each phantom was constructed (Figure 1c). The $\mathrm{pH}$ value estimated from the frequency of the CEST peak showed good agreement with the solution $\mathrm{pH}$ measured using a glass electrode (Figure 1d). The strong, sharp CEST signal of $\mathrm{Yb}(1)$ was not observed in kidney images during infusion of the agent but the characteristic CEST signal of $\mathrm{Yb}(1)$ appeared in images of the bladder only late during the imaging experiment. These preliminary studies show that $\mathrm{Yb}(1)$ complex is cleared by renal filtration but the process is much slower than expected for a molecule of this size. To examine this unexpected observation in more detail, the $\mathrm{Gd}(1)$ complex was prepared and dynamic T1weighted images verified that the agent clears only slowly from blood. Further investigations of the origin of this effect are in progress.
\end{abstract}

References: References 1) S. Viswanathan, Z. Kovacs, K. N. Green, S. J. Ratnakar, A. D. Sherry, Chem. Rev., 2010, 110, 2960 - 3018. 2) T. C. Soesbe, M. E. Merritt, K. N. Green, F. A. Rojas-Quijano, A. D. Sherry Magn. Reson. Med. 2011, 66, 1697-703

\section{Image/Figure:}


Image/Figure Caption: Figure 1. a) Structure of the ligand b) The CEST spectra of $\mathrm{Yb}(1)$ at different $\mathrm{pH}$ values at $25^{\circ} \mathrm{C}$; c) The false colored CEST images of $25 \mathrm{mM} \mathrm{Yb}(1)$ at different $\mathrm{pH}$ values; d) Linear plot showing the agreement between the CEST measured pH compared to electrode measurements.

Complete Status: Complete

First Name: James

Last Name: Soundrarajan

Email: james.ratnakar@utsouthwestern.edu

Organization: UT Southwestern Medical Center

Country: United States 


\title{
ID: GA514 \\ Tunable Nonamethine Cyanines Enable Targeted Multicolor in vivo Imaging in the Shortwave-Infrared Region
}

\author{
Michael Luciano, National Cancer Institute, lucianomp@nih.gov
}

\section{Category: New Chemistry, Biology \& Bioengineering}

\begin{abstract}
Body : In vivo fluorescence imaging has the potential to probe biological processes in their native environments and inform surgical decision making. 1 While multiplexed imaging is routine in microscopy, such experiments are challenging in body-wide imaging due to the competitive autofluorescence and scattering encountered with visible ( 2 Recent progress has demonstrated the benefits of optical imaging using longer wavelengths (1000 to $2000 \mathrm{~nm}$ ), referred to as the Shortwave-Infrared (SWIR) or NIR-II range. These methods enable high resolution imaging in bulk tissue, with dramatic improvements in both resolution and depth relative to imaging in the NIR region. 3 As InGaAs detector-based imaging systems have become more accessible, a critical bottleneck in this field is access to biologically compatible fluorescent probes that operate in this range Indocyanine dyes are broadly used in fluorescence-based experiments. In the visible range, trimethine indocyanines (e.g. Cy3) and pentamethine indocyanines (e.g. Cy5, AF-647), find extensive use in applications spanning routine quantification to advanced microscopy. Derivatives of heptamethine- indocyanines (e.g. Cy7, IRDye $800 \mathrm{CW}$ and indocyanine green, ICG) are the most broadly applied molecules for in vivo near-infrared (NIR) imaging, including for clinical use. 4 The defining chemical feature of these molecules - two-carbon homologation - leads to $\sim 100 \mathrm{~nm}$ increases in absorbance maxima, making these scaffolds nearly ideal for simultaneous use in multiplexed experiments. Another feature of the heptamethine variants is their utility in SWIR imaging, due to significant emission extending beyond $1000 \mathrm{~nm} .5$ The use of heptamethine indocyanine "tail imaging" in the SWIR has been investigated in the clinic using the FDA-approved NIR fluorophore, ICG.6 This study extends the biological utility of the indocyanine scaffold to longer wavelengths. Guided by computational design, we report two classes of novel dyes with significant SWIR emission based on the nonamethine indocyanine scaffold. The first is a true per-sulfonated "Cy9" type derivative (FNIR-872, $\lambda \mathrm{abs}=872 \mathrm{~nm}$ ), which has been optimized for monoclonal antibody labeling. We also describe a novel chemical strategy to modify the nonamethine cyanine scaffold with a fused aryl ring, thereby extending the absorbance maximum beyond $1000 \mathrm{~nm}$ (FSWIR-1072, $\lambda$ abs $=$ $1072 \mathrm{~nm}$ ). These dyes exhibit cyanine-like exceptional absorption cross sections, high solubility, and excellent photo/chemical stability. Targeted tumor imaging was successfully performed using FNIR-872-mAb, revealing comparable TBR and tumor signal to clinically used IR$800 \mathrm{CW}-\mathrm{mAb}$. In addition, we also describe how using red-shifted (892 nm) excitation wavelengths exhibit reduced endogenous emission (autofluorescence) in vivo from multiple peritoneal organs. Lastly, we demonstrate the utility of mAb and dextran bioconjugates of these novel small molecule dyes in combination with the FDA-approved dye ICG for multicolor tumor imaging experiments in the SWIR region. These efforts demonstrate the high potential of substituted nonamethine cyanines for targeted SWIR imaging, and extend the utility of indocyanine dyes to multiplexed imaging in the SWIR region for the first time.
\end{abstract}


References: 1. Garland, M.; Yim, J. J.; Bogyo, M., A Bright Future for Precision Medicine: Advances in Fluorescent Chemical Probe Design and Their Clinical Application. Cell. Chem. Biol. 2016, 23 (1), 122-136. 2. Carr, J. A.; Aellen, M.; Franke, D.; So, P. T. C.; Bruns, O. T.; Bawendi, M. G., Absorption by water increases fluorescence image contrast of biological tissue in the shortwave infrared. Proc. Natl. Acad. Sci. U S A 2018, 115 (37), 9080-9085. 3. Zhu, B.; Kwon, S.; Rasmussen, J. C.; Litorja, M.; Sevick-Muraca, E. M., Comparison of NIR Versus SWIR Fluorescence Image Device Performance Using Working Standards Calibrated With SI Units. IEEE Trans. Med. Imaging. 2020, 39 (4), 944-951. 4. Barth, C. W.; Gibbs, S. L., Fluorescence Image-Guided Surgery - a Perspective on Contrast Agent Development. Proc. SPIE Int. Soc. Opt. Eng. 2020, 11222. 5. Carr, J. A.; Franke, D.; Caram, J. R.; Perkinson, C. F.; Saif, M.; Askoxylakis, V.; Datta, M.; Fukumura, D.; Jain, R. K.; Bawendi, M. G.; Bruns, O. T., Shortwave infrared fluorescence imaging with the clinically approved near-infrared dye indocyanine green. Proc. Natl. Acad. Sci. U S A 2018, 115 (17), 4465-4470. 6. Hu, Z.; Fang, C.; Li, B.; Zhang, Z.; Cao, C.; Cai, M.; Su, S.; Sun, X.; Shi, X.; Li, C.; Zhou, T.; Zhang, Y.; Chi, C.; He, P.; Xia, X.; Chen, Y.; Gambhir, S. S.; Cheng, Z.; Tian, J., First-in-human liver-tumour surgery guided by multispectral fluorescence imaging in the visible and near-infrared-I/II windows. Nat. Biomed. Eng. 2020, 4 (3), 259-271.

\section{Image/Figure:}

https://www.xcdsystem.com/wmis/abstract/File6959/GA514 ImageFigure 0611061934.png

Image/Figure Caption: Left: Prior biocompatible Indocyanine dyes used in visible/NIR optical imaging and novel NIR and SWIR-emissive nonamethine cyanine dyes reported in this work. Right: 3-Color live imaging of an athymic nude mouse bearing an MDA-MB-468 orthotopic tumor implanted in the mammary fat pad. The mouse was injected with ICG $(\lambda$ excitation $=785$ $\mathrm{nm}$, pseudo-colored green) and FSWIR-1072-Dex-10 kDa ( $\lambda$ excitation $=1064 \mathrm{~nm}$, pseudocolored red) 24 hours after administering FNIR-872-Pan ( $\lambda$ excitation $=892 \mathrm{~nm}$, pseudo-colored cyan) by intraveneous injection and images in all three channels were recorded using long pass filters.

Complete Status: Complete

First Name: Michael

Last Name: Luciano

Email: lucianomp@nih.gov

Organization: National Cancer Institute

Country: United States 


\title{
ID: GA515 \\ Anaesthesia effects in PET studies: Type of anaesthesia influences [11C]MDL100,907 binding to 5HT2A receptors in porcine brain
}

\author{
Aage Alstrup, Aarhus University Hospital, aagols@rm.dk
}

\section{Category: Neuroscience}

\begin{abstract}
Body : Anaesthesized pigs and other laboratory animals are widely used in positron emission tomography (PET) neuroimaging studies. However, you can hardly anaesthetize the brain without affecting its physiology, and the used anaesthetics may therefore modify tracer binding profiles at receptor targets. We evaluated effects of isoflurane and propofol on the binding of [11C]MDL100,907 on the serotonin 5HT2A receptors in pigs. Two groups of anaesthetised Göttingen minipigs were imaged with [11C]MDL100,907 PET and analysed using regions of interest and statistical non-parametric mapping. The [11C]MDL100,907 binding potentials in striatum under isoflurane anaesthesia significantly exceeded those obtained under propofol anaesthesia, probably due to higher cerebral blood flow in brain during isoflurane anaesthesia. In conclusion, the interactions between the used anaesthetics and brain tracers must be carefully evaluated prior to animal experimentation and in the interpretation of results.
\end{abstract}

Complete Status: Complete

First Name: Aage

Last Name: Alstrup

Email: aagols@rm.dk

Organization: Aarhus University Hospital

Country: Denmark 


\title{
ID: GA516 \\ Translocator Protein-targeted Photodynamic Therapy Induces Effective Immunogenic Cell Death in Colorectal Cancer
}

\author{
Qing Xie, Vanderbilt University Medical Ceter, qing.xie@vumc.org
}

\section{Category: Oncology}

\begin{abstract}
Body : Colorectal cancer (CRC) is the leading cause of death in all of the digestive system cancers and the third in all types of cancer, with a 5-year overall survival rate of only $58 \%-65 \% 1$. Many patients advance to late stages when metastasis has occurred in remote sites and standard treatments are ineffective2. This study aimed to develop a more effective therapeutic approach for CRC based on immunogenic cell death (ICD) and photodynamic therapy (PDT). Here we chose the $18 \mathrm{kDa}$ translocator protein (TSPO), a protein overexpressed in CRC, as the target for Photodynamic therapy (PDT) of CRC. Our TSPOtargeted photosensitizer (IR700DX-6T) 3 was found to be located in the mitochondria and specifically bound to TSPO in MC38 CRC cells (Figure A, B). PDT with IR700DX-

6T caused effective CRC cell death in a concentration- and light dose-dependent manner (Figure C). The mechanisms behind the killing effects were further investigated. The cleaved caspase-3 substrate and morphological changes with typical apoptotic characteristics suggest that our TSPO-PDT treatment induced apoptotic cell death. Remarkably, TSPO-PDT also induced Immunogenic cell death (ICD)4 as evidenced by cell membrane translocation/release of two key DAMPs (damage-associated molecular patterns), including calreticulin (CRT) and heat shock protein 70 (HSP70) after TSPO-PDT treatment (Figure D, E). Furthermore, our in vivo study using a syngeneic CRC mouse model showed that TSPO-PDT was not only effective on directly treating TSPO+ tumors, but also able to trigger host anti-tumor immune response resembling abscopal effect. Such favorable antitumor immunity was shown to be associated with elevated dendritic cell maturation and CD8+ T cell infiltration, as well as decreased regulatory $\mathrm{T}$ (Treg) cells. In conclusion, TSPO-PDT using IR700DX-6T displayed high therapeutic efficacy against $\mathrm{CRC}$ cells and tumors. This new therapeutic approach could efficiently treat CRC tumors and cause host anti-tumor immune response, suggesting that TSPO-PDT has great potential in the treatment of CRC.
\end{abstract}

References: 1 Siegel, R. L., Miller, K. D. \& Jemal, A. Cancer statistics, 2020. CA Cancer J Clin 70, 7-30, doi:10.3322/caac.21590 (2020). 2 Dekker, E., Tanis, P. J., Vleugels, J. L. A., Kasi, P. M. \& Wallace, M. B. Colorectal cancer. Lancet 394, 1467-1480, doi:10.1016/S01406736(19)32319-0 (2019). 3 Zhang, S. et al. Tumor mitochondria-targeted photodynamic therapy with a translocator protein (TSPO)-specific photosensitizer. Acta Biomater 28, 160-170, doi:10.1016/j.actbio.2015.09.033 (2015). 4 Garg, A. D., Dudek-Peric, A. M., Romano, E. \& Agostinis, P. Immunogenic cell death. Int J Dev Biol 59, 131-140, doi:10.1387/ijdb.150061pa (2015).

\section{Image/Figure:}


Image/Figure Caption: (A, B) IR700DX-6T specifically bound to TSPO. (A) IR700DX-6T was effectively uptaken by TSPO (+) MC38 cells and the fluorescence signal overlaid with mitotracker green (mitochondrial marker). TSPO (-) MCF7 cells showed negligible uptake of IR700DX-6T. (B) DAA1106, a small molecule ligand with a high binding affinity to TSPO, markedly decreased the uptake of IR700DX-6T in MC38 cells. MC38 cells treated with nontargeted IR700DX showed unconspicuous fluorescence signal. (C) Cell viabilities were measured after IR700DX-6T-PDT at various concentrations $(0.5-5 \mu \mathrm{M})$ and light doses (7.2-27.0 $\mathrm{J} / \mathrm{cm} 2$ ). (D, E) TSPO induced ICD was confirmed by using fluorescence microscope imaging. MC38 cells were treated with PDT using $0.5 \mu \mathrm{M}$ of IR700DX-6T and $18 \mathrm{~J} / \mathrm{cm} 2 \mathrm{of}$ light irradiation at $690 \mathrm{~nm}$. Three hours after the PDT treatment, cells were fixed, permeabilized, and subjected to immunofluorescence imaging (red) of calreticulin (D) and heat shock protein 70 (E).

Complete Status: Complete

First Name: Qing

Last Name: Xie

Email: qing.xie@vumc.org

Organization: Vanderbilt University Medical Ceter

Country: United States 


\title{
ID: GA517 \\ PET Imaging of Respiratory Burst
}

\section{Seth Gammon, UT MD Anderson Cancer Center, stgammon@mdanderson.org}

\section{Category: Immunology: Inflammation \& Infection}

\begin{abstract}
Body : The innate immune system integrates multiple pro- and anti-inflammatory stimuli, organize key effector functions, and in the proper context, culminate in the respiratory burst, or ROS burst. These systems are deeply conserved across species, although flux through these pathways is most robust in humans. The respiratory burst has long been studied in neutrophils, but is known to occur (with less intensity) in macrophages, microglia, and "immunostimulated" endothelial cells. In neutrophils and macrophages, high energy ROS are produced by NADPH oxidase 2 (NOX2) and myeloperoxidase (MPO). When functioning properly, these cells and ROS bursts provide front line defense to a variety of pathogens and degrade difficult to process foreign bodies, such as carbon nanotubes. When pathogenic, ROS degrade and inhibit the normal function of tissues from cartilage in arthritis to T-cells in tumors.1-5 Contrast MRI, [18F]FDG-PET, or 68/67Ga-Citrate-PET/SPECT lack the sensitivity and/or specificity to detect changes in the innate immunity system in many of these diseases, and novel approaches to detect inflammation are sought.7-11 Herein, we develop and test 4[18F]fluoronaphthol ([18F]4FN) as a novel radiopharmaceutical to detect ROS burst by PET. A robust and automated synthesis of [18F]4FN using copper-mediated radiofluorination was developed and validated on a commercial synthesizer (GE TracerLab-FX). 12 To date, [18F]4FN has been successfully synthesized with an average activity yield of $6.8 \pm 2.5 \%(\mathrm{n}>=22)$, with $>99 \%$ radiochemical purity, and up to $140 \mathrm{GBq} / \mu \mathrm{mol}$ molar activity. The precursor has now been validated for cGMP production by a third party, ABX. Mechanism-based testing was conducted in vitro. Physiologically-relevant concentrations of MPO and $\mathrm{H} 2 \mathrm{O} 2$ could readily oxidize $>95 \%$ of the reporter probe, significantly more than incubating in $\mathrm{H} 2 \mathrm{O} 2$ or vehicle alone ( $\mathrm{p} 95 \%$ of cell-associated retention ( $\mathrm{n}>=3$ experiments, $\mathrm{n}=3-4$ replicates per experiment $\mathrm{p}<0$ .0001), demonstrating selectivity for the reporter vs mitochondrial ROS and PMA-induced macropinocytosis or non-specific binding. A PMA model of mild contact dermatitis (earlobe and base of ear) was utilized to conduct pilot in vivo experiments. PET imaging of [18F]4FN at $1 \mathrm{hr}$ post injection of the radiotracer in vivo yielded good contrast-to-noise ratios in two independent strains of adult female mice $(\mathrm{Balb} / \mathrm{c}$ and $\mathrm{C} 57 \mathrm{Bl} / 6 \mathrm{~N})$. The reporter was sufficiently robust that both IP and IV injections yielded images with good contrast ratios and large effect sizes (Cohen's coefficient » 0.7 ). Furthermore, in this model, [18F]4FN yields superior contrast to $[18 \mathrm{~F}] \mathrm{FDG}(\mathrm{p}=0.004)$. In a murine model of whole body toxic shock, there was increased retention in many organs, including the kidneys, and retention was significantly greater than 68Ga-citrate, another small molecule PET imaging agent for inflammation. Finally, in an LPS model of arthritis, [18F]4FN correlated well with L-012, a validated bioluminescence reporter of ROS and activation of the innate immune system. Focal inflammation, swelling and immune infiltrate was cross-validated by immunohistochemistry. Broadly, [18F]4FN demonstrated mixed renal and hepatobiliary excretion similar to other clinically translatable PET agents. In summary, $[18 \mathrm{~F}] 4 \mathrm{FN}$ could be readily synthesized with high molar activity, good yields, was
\end{abstract}


stable in both buffer and mouse plasma and appeared to be a suitable PET agent for monitoring ROS produced by activation of the innate immune system in deep tissues.

References: (1) Stone, T.; McPherson, M.; Darlington, L. EBioMedicine 2018, 30, 14. (2) Hansson, G. N Engl J Med 2005, 352, 1685. (3) Li, Q.; Wu, H.; Liao, W.; Zhao, M.; Chan, V.; Li, L.; Zheng, M.; Chen, G.; Zhang, J.; Lau, C.-S.; Lu, Q. J. Autoimmun. 2018. (4) SkopeljaGardner, S.; Jones, J. D.; Rigby, W. F. C. J. Autoimmun. 2018, 88, 1. (5) Morell, M.; Varela, N.; Marañón, C. Clin Rev Allergy Immunol. 2017, 53, 198. (6) Bilusic, M.; Gulley, J. J Nat'l Cancer Inst 2017, 109, djx132. (7) Jiemy, W. F.; Heeringa, P.; Kamps, J. A. A. M.; van der Laken, C. J.; Slart, R. H. J. A.; Brouwer, E. Autoimmun. Rev. 2018, 17, 715. (8) Zinnhardt B, W. M., Honold L, Barca C, Schäfers M, Kiliaan AJ, Jacobs AH Theranostics 2018, 8, 2603. (9) Signore, A.; Glaudemans, A. W. J. M.; Gheysens, O.; Lauri, C.; Catalano, O. A. Semin Nucl Med. 2017, 47, 286. (10) Wang, C.; Keliher, E.; Zeller, M. W. G.; Wojtkiewicz, G. R.; Aguirre, A. D.;

Buckbinder, L.; Kim, H.-Y.; Chen, J.; Maresca, K.; Ahmed, M. S.; Motlagh, N. J.; Nahrendorf, M.; Chen, J. W. Proceedings of the National Academy of Sciences 2019, 116, 11966. (11) Hou, C.; Hsieh, C.-J.; Li, S.; Lee, H.; Graham, T. J.; Xu, K.; Weng, C.-C.; Doot, R. K.; Chu, W.; Chakraborty, S. K.; Dugan, L. L.; Mintun, M. A.; Mach, R. H. ACS Chemical Neuroscience 2018, 9, 578. (12) Tredwell, M.; Preshlock, S. M.; Taylor, N. J.; Gruber, S.; Huiban, M.; Passchier, J.; Mercier, J.; Génicot, C.; Gouverneur, V. Angewandte Chem. Int. Edit. 2014, 53, 7751. (13) Eastmond, D. A.; French, R. C.; Ross, D.; Smith, M. T. Chem.-Biol. Interact. 1987, $63,47$.

\section{Image/Figure:}

https://www.xcdsystem.com/wmis/abstract/File6959/GA517_ImageFigure_0610045846.jpg

Image/Figure Caption: A) Diagram of radiopharmaceutical [18F]4FN. B) Imaging of ROS burst in arthritis by PET/CT and BLI/Photograph.

Complete Status: Complete

First Name: Seth

Last Name: Gammon

Email: stgammon@mdanderson.org

Organization: UT MD Anderson Cancer Center

Country: United States 


\title{
ID: GA518 \\ Nanoscale Metal-Organic Frameworks Potentiate Radiotherapy for Cancer Immunotherapy
}

\author{
Kaiyuan Ni, Massachusetts Institute of Technology, kaiyuann@mit.edu
}

Category: New Chemistry, Biology \& Bioengineering

\begin{abstract}
Body : Checkpoint blockade immunotherapy (CBI) affords systemic and durable antitumor immunity by targeting $\mathrm{T}$ cell regulatory pathway, yet fails on immunosuppressive tumors with inadequate $\mathrm{T}$ cell infiltration. Radiotherapy as local immunomodulatory effects kills tumor cells in an immunogenic mode and alters tumor microenvironment to synergize with CBI. To maximize anti-tumor efficacy as well as immunoadjuvant effect of radiotherapy, intratumorally enriched high- $Z$ radiosensitizers are clinically investigated to enlarge therapeutic index. We developed nanoscale metal-organic frameworks (nMOFs) as next-generation radiosensitizers. Compared to nanoparticle-based radiosensitizer such as $\mathrm{HfO} 2$, Hf-based nMOF effectively amplifies energy deposition with ordered structure and facilitates diffusion of reactive species with pores. By crystal engineering, we systemically compared nMOFs with different Hfoxo clusters to find Hf12-oxo a better X-ray absorber. By molecular engineering, we employed photosensitizing porphyrin-based linker to elicit a new therapeutic modality, radiotherapyradiodynamic therapy (RT-RDT). Upon low-dose X-ray, Hf12-oxo clusters not only absorb incident energy to generate hydroxyl radical via radiolysis but also scatter secondary energy to proximate linker to produce singlet oxygen. Combination of nMOF-mediated RT or RT-RDT with CBI not only eradicated primary tumor, but also extended local therapeutic efficacy to distant tumor via abscopal effect, suggesting a strong anti-tumor immunity induced by nMOF technology.
\end{abstract}

References: 1. Nanoscale metal-organic frameworks enhance radiotherapy to potentiate checkpoint blockade immunotherapy. Nature Communications 2018, 9 (1), 1-12. 2. Ultrathin metal-organic layer-mediated radiotherapy-radiodynamic therapy enhances immunotherapy of metastatic cancers. Matter 2019, 1 (5), 1331-1353.

\section{Image/Figure:}

https://www.xcdsystem.com/wmis/abstract/File6959/GA518 ImageFigure 0610060510.jpg

Image/Figure Caption: Design of radiosensitizers and schematic showing nMOF engineering. Compared to solid NPs, lattice assembled with ultrasmall metal clusters amplifies secondary energy deposition and facilitates ROS generation and diffusion for better radiosensitization effect. By tuning metal-oxo clusters via crystal engineering, functionalizing organic bridging linkers via molecular engineering, and incorporating immunotherapeutics via immunoengineering, nMOF-mediated immunoadjuvant treatment amplifies local inflammation to potentiate systemic anti-tumor immunity when combined with immune checkpoint blockade. 
Full Name of Abstract's 1st Author : Kaiyuan Ni

Complete Status: Complete

First Name: Kaiyuan

Last Name: $\mathrm{Ni}$

Email: kaiyuann@mit.edu

Organization: Massachusetts Institute of Technology

Country: United States 


\title{
ID: GA519 \\ Characterization of Arterial and Portal Venous Contributions to Metabolic Imaging of the Human Liver Using Hyperpolarized 13C-pyruvate MRI
}

\author{
Philip Lee, University of California, San Francisco, philip.lee2@ucsf.edu
}

\section{Category: Systemic Diseases (Kidney, Liver and Pancreas)}

\begin{abstract}
Body : Background \& Motivation: Hyperpolarized (HP) 13C MRI enables in vivo quantitative dynamic imaging of enzyme-catalyzed cellular metabolism, with clinical applications in prostate, brain, heart, and kidney.1-4 However, the unique dual blood supply of the liver (via the hepatic artery and portal vein) complicates quantitative analyses of both the delivery of injected HP substrates and the timing of subsequent observed metabolism. Decomposing the pyruvate delivery into the contributions of each blood vessel would allow for improved real-time acquisition triggering and kinetic modeling accuracy by providing perfusion information. Here, we investigated the distinct vascular contributions in the human liver and assessed the effects on observed HP 13C signals in the liver. Methods \& Results: Two healthy subjects (Healthy \#1 and \#2), one subject with metastatic pancreatic cancer (Cancer \#1), and one subject with metastatic breast cancer (Cancer \#2) underwent HP 13C MRI on a clinical 3 T MR scanner with a 1-, 8-, or 16-channel surface coil/clamshell transmitter combination with an injection of $0.43 \mathrm{~mL} / \mathrm{kg}$ of $250 \mathrm{mM} \mathrm{HP}$ [1-13C]pyruvate. Axial T1-weighted spoiled gradientecho anatomical scans were acquired for blood vessel registration. [1-13C]pyruvate, and downstream metabolites [1-13C]lactate, [1-13C]alanine, and [13C]bicarbonate signals were acquired every $3 \mathrm{~s}$ using a specialized multi-slice $13 \mathrm{C}$ echo planar imaging acquisition with constant flip angles (10-20 for pyruvate, $20-30^{\circ}$ for lactate). 2 Total scan time was 1 minute with in-plane voxels of $1.0 \times 1.0$ to $2.0 \times 2.0 \mathrm{~cm}$, slice thicknesses of $2.0-3.0 \mathrm{~cm}$, and $3-5$

slices. Dynamic curves were summed, interpolated, and smoothed using a moving average to obtain a total $13 \mathrm{C}$ signal dynamic curve for selected voxels in the aorta, the inferior vena cava (IVC), the portal vein (PV), healthy liver (or normal appearing in cancer subjects), and liver metastasis. The total $13 \mathrm{C}$ dynamic curves for subject Healthy \#2 are shown in Figure 1a. The peak arrival times from the start of acquisition for each anatomical structure and subject are shown in Figure 1b. The cancer voxel for subject Cancer \#2 is not displayed as the voxel was within an unintended saturation band due to the single channel surface coil's inhomogeneous excitation profile. Overall, we observed a differential arrival of [1-13C]pyruvate signal, first in the aorta followed by the IVC, PV, and lastly the healthy liver and liver metastasis, matching physiologic expectations as the IVC rapidly receives venous return from the kidneys while the PV is delayed as it receives blood that passes first through the GI tract. The healthy subjects showed a consistent peak arrival time relative to the aorta in the PV (10-12 s) and healthy liver (14-17 s). A different pattern was observed in both cancer subjects; a shorter peak arrival time relative to the aorta was observed in the PV (3.4-4.5 s) and normal appearing liver (4.5-6 s). These consistencies within subject groups suggest that real-time triggering would be advantageous for future scans. Figure 1c shows the lactate/pyruvate ratios for each anatomical structure and subject except for subject Cancer \#2 because of poor lactate SNR across all voxels. On the time scale of our experiment, lactate in the IVC predominantly reflects that produced by
\end{abstract}


venous outflow from the kidneys while lactate in the PV predominantly reflects that produced from pyruvate in the small bowel and spleen. Discussion: Distinct vascular contributions can be observed from the liver's dual blood supply, despite the limited lifetime of the HP signal. For optimal HP 13C-pyruvate MRI, the timing of image acquisition is important. These results can help tailor real-time acquisition strategies, such as bolus tracking, to optimize HP 13C imaging of the liver.5 Acknowledgements: This work was supported by NIH grants NIDDK 5R01DK115987 and P41 EB013598.

References: 1. Chen HY et al. Hyperpolarized 13C-pyruvate MRI detects real-time metabolic flux in prostate cancer metastases to bone and liver: a clinical feasibility study. Prostate Cancer Prostatic Dis. 2020;23(2):269-76. 2. Gordon JW et al. Translation of Carbon-13 EPI for hyperpolarized MR molecular imaging of prostate and brain cancer patients. Magn Reson Med. 2019;81(4):2702-09. 3. Cunningham CH et al. Hyperpolarized 13C Metabolic MRI of the Human Heart: Initial Experience. Cancer Res. 2018;78(14):3755-60. 4. Tang S et al. A metabolite-specific 3D stack-of-spiral bSSFP sequence for improved lactate imaging in hyperpolarized [1-13C]pyruvate studies on a 3T clinical scanner. Magn Reson Med. 2020;Epub. 5. Tang S et al. A regional bolus tracking and real-time B1 calibration method for hyperpolarized 13C MRI. Magn Reson Med. 2018;81(2):839-51.

\section{Image/Figure:}

https://www.xcdsystem.com/wmis/abstract/File6959/GA519_ImageFigure_0610062334.png

Image/Figure Caption: Figure 1. (a) Total 13C signals plotted over time for selected tissue voxels in subject Healthy \#2 with ROIs labeled in the adjacent anatomical image. (b) Peak arrival times of total $13 \mathrm{C}$ signal for each subject $(\mathrm{t}=0$ coincides with the start of the acquisition). (c)Lactate/pyruvate ratios for each anatomical structure and subject except for subject Cancer \#2 due to poor lactate SNR across all voxels. A diamond indicates that the value was not computed due to poor SNR.

Full Name of Abstract's 1st Author : Philip Meng-en Lee

Complete Status: Complete

First Name: Philip

Last Name: Lee

Email: philip.lee2@ucsf.edu

Organization: University of California, San Francisco

Country: United States 


\title{
ID: GA520 \\ Precise and non-invasive detection of liver metastases via chemokine receptor 4 molecular MR imaging
}

\author{
Shanshan Tan, Johns Hopkins University, tanshanshan0425@gmail.com
}

\section{Category: Oncology}

\begin{abstract}
Body : Liver is the most predominant site for metastases. In most cases, these liver metastases lead to poor prognosis. Therefore, non-invasive detection of liver metastases at early stages is critically important for many types of cancers. However, the sensitivity of imaging detection for liver micro metastases is limited due to lack of molecular biomarker and specific imaging methodology. We first report our finding that chemokine receptor 4 (CXCR4) serves as a biomarker for liver metastases from uveal melanoma (UM). This finding is confirmed by elevated CXCR4 expression in the liver metastases from UM patients. High CXCR4 expression level is further confirmed in liver metastases of UM mouse models, regardless of the expression level in the primary site. We then report the development and optimization of a protein-based MRI contrast agent with significantly improved relaxivities $(\mathrm{r} 1=30.9 \mathrm{mM}-1 \mathrm{~s}-1, \mathrm{r} 2=43.2$ $\mathrm{mM}-1 \mathrm{~s}-1)$ and strong CXCR4 targeting capability. Taking advantage of molecular MR imaging and of this newly developed CXCR4 targeting contrast agent, we demonstrate that visualization of UM micro-metastases in the liver (of the mouse model), as small as $0.1 \mathrm{~mm} 3$, is now possible. The elevated CXCR4 expression in the liver metastases is associated with the specific liver microenvironment. Therefore, molecular MR imaging of CXCR4 is expected to have wide applications in studying liver metastases from different cancer types. We show detection of liver metastases from ovarian adenocarcinoma as an example to prove this concept. The imaging methodology fulfilled by CXCR4 targeted contrast agent is expected to fill the major gap in noninvasive early detection of liver metastases.
\end{abstract}

References: Tan S, Yang H, Xue S, Qiao J, Salarian M, Hekmatyar K, et al. Chemokine receptor 4 targeted protein MRI contrast agent for early detection of liver metastases. Sci Adv 2020;6(6):eaav7504 doi 10.1126/sciadv.aav7504.

\section{Image/Figure:}

https://www.xcdsystem.com/wmis/abstract/File6959/GA520 ImageFigure 0610074419.png

Image/Figure Caption: CXCR4 is associated with uveal melanoma (UM) liver metastases, and detection of micro metastases in the liver by CXCR4 molecular MR imaging. A. High CXCR4 expression was observed in liver metastases in different UM murine models. UM cell lines with high, medium, and low in vitro expression of CXCR4 were used to generate metastatic UM mice models. The CXCR4 expression level in hepatic metastases are high in all three mice models regardless of the expression levels in vitro and in primary site. B. Detection of liver micro metastases by CXCR4 molecular MR imaging. After the administration of CXCR4 targeted contrast agent, the early stage micro metastases in the liver were enhanced and identified in MR 
image. The metastatic lesions in the H\&E staining are well-correlated with the ones that recognized in the MR image. IHC staining confirmed the lesions are UM metastases (HMB45 positive) and with CXCR4 expression (CXCR4 positive).

Full Name of Abstract's 1st Author : Shanshan Tan

Complete Status: Complete

First Name: Shanshan

Last Name: Tan

Email: tanshanshan0425@gmail.com

Organization: Johns Hopkins University

Country: United States 


\title{
ID: GA521 \\ STEM CELLS IN TREATMENT OF CORONARY HEART DISEASE AND \\ ITS MONITORING AT 900 MHz MAGNETIC RESONANCE IMAGER
}

\author{
RAKESH SHARMA, Florida State University Research Foundation, rksz2009@yahoo.com
}

Category: New Chemistry, Biology \& Bioengineering

\begin{abstract}
Body : Background: Stem cell therapy is new strategy for patients with ischemic heart disease. Rat heart micro-level visualization model of cardiac functions, offers the solution in clinical use. Hypothesis: Success of stem cell therapy depends on cell delivery and monitoring the stem cell rehabilitation and functioning in heart by non-invasive ultra-sensitive imaging method. Results: The stem cell repaired cardiovascular territories and reduced infarct related mortality. Non-invasive cardiovascular imaging monitors real-time status of cadiovascular remodeling or differentiated stem cell autografting, possibly, after myocardial dysfunction and recovery. Clear visualization of ventricular muscle fiber orientation, coronary arteries, apex functioning, ejection fraction facilitated evaluation of rejuvenation perspectives. However, the stem cell long-time retention and poor survival rates were major limitations to achieve significant therapeutic effects. Discussion: Right choice of pluripotent cells, stem cell delivery route, real-time monitoring of stem cell trafficking at transplantation sites, are emerging as promising tools. The stem cell molecular event monitoring during delivery, setting and functioning as myocyte cells, may be possible to dog watch the rejuvenation in clinical success. Conclusion: Non-invasive monitoring of stem cell trafficking by ultrahigh $900 \mathrm{MHz}$ MR microscopy provides micro-level details of stem cell therapeutic efficacy in cardiac enlargement and differentiation.
\end{abstract}

References: 1. R. Sharma .Stem cells in clinical practice and Tissue Engineering. Ed.Rakesh Sharma. IntechOpen Publishers, London. 2018.. 2. R. Sharma. 1. R.Sharma. Stem Cells and Tissue Engineering in Medical Practice: Ethical and Regulatory Policies. Current Drug Targets. 20(4): 388 - 398. DOI: 10.2174/1389450119666180831095830

\section{Image/Figure:}

https://www.xcdsystem.com/wmis/abstract/File6959/GA521_ImageFigure 0610090047.jpg

Image/Figure Caption: Microstructures of cardiac tributaries

Full Name of Abstract's 1st Author : RAKESH SHARMA

Complete Status: Complete

First Name: RAKESH

Last Name: SHARMA 
Email: rksz2009@yahoo.com

Organization: Florida State University Research Foundation

Country: India 


\title{
ID: GA522 \\ Decrease in fibronectin level reduces mechanical stiffness in HIF -1 $\alpha$ silenced triple negative human breast cancer xenograft
}

\author{
Desmond Jacob, Johns Hopkins University, djacob4@jhmi.edu
}

\section{Category: Oncology}

Abstract Body : Introduction: Cancer cells are known to remodel other extracellular matrix (ECM) components in the tumor and modify the mechanical properties of the tumor. These mechanical properties provide cues that influence critical cell activities, such as migration, proliferation and stem cell differentiation, which are part of the metastatic cascade in primary tumors [1-4]. Mechanical properties of tumors studied previously, focused on bulk indicators using bulk tissue compression testing, or cellular mechanics at sub cellular level using atomic force microscopy. However these do not sufficiently address the heterogeneous nature of the tumor. We have therefore, for the first time, developed a micro-indentation method that bridges the gap between the bulk and sub-cellular scale techniques to understand the role of hypoxia in tumor stiffness, by characterizing changes in hypoxia inducible factor (HIF)-1 $\alpha$ silenced tumors. Methods: Generation of MDA-MB-231 cells expressing shRNA against HIF-1 $\alpha$ (231-HIF-1 $\alpha)$ or an empty vector control (231-EV) using lentiviral transduction were performed using previously established methods [5]. 231-EV $(n=8)$ or $231-H I F-1 \alpha(n=8)$ tumors derived from these cells were excised, sectioned and tested using a iNano nanoindenter (Nanomechanics, Inc.) equipped with a $500 \mu \mathrm{m}$ diameter flat cylindrical probe. A grid based on the size of each section was mapped to cover the maximum area, and the indentation tests were carried out at each of the grid points. Elastic modulus maps of tumor tissue were generated from the test results and the indentation sites were classified as viable, necrotic or interface based on visual observation. Subsequently, tumor sections were fixed in formalin and paraffin embedded. $5 \mu \mathrm{m}$-thick slices were obtained and immunostained for major ECM components and crosslinking enzymes such as fibronectin, laminin, COL1A1, lysyl oxidase (LOX) and alpha smooth muscle actin ( $\alpha$-SMA). H\&E stained sections were used to annotate viable and necrotic regions. Fractional immunostained areas for each component and enzyme were quantified by computing the fraction of strongly stained pixels identified using Aperio software. Results \& Discussion: Microindentation was used to investigate the effects of silencing HIF-1 $\alpha$ on tumor tissue stiffness. The elastic modulus in viable tumor regions decreased significantly in 231-HIF-1 $\alpha$ tumors compared to 231-EV tumors (Fig 1A-B) suggesting that structural changes occurred in the tumor ECM with HIF-1 $\alpha$ silencing. The fractional fibronectin immunostained area decreased significantly in 231-HIF-1 $\alpha$ tumor sections compared to 231-EV tumors (Fig 1C) suggesting that decreased fibronectin density observed with HIF-1 $\alpha$ silencing may contribute to the decreased tumor stiffness. The fractional $\alpha-$ SMA immunostained area also decreased in 231-HIF-1 $\alpha$ tumor sections compared to 231-EV tumors but not significantly. An increase in the immunostained area for collagen-1, LOX and laminin within 231-HIF-1 $\alpha$ tumors indicate a lesser role of these components and enzymes in determining tissue stiffness. Conclusion: HIF-1 $\alpha$ silencing is known to inhibit proliferation, impair migration and reduce metastatic abilities of breast cancer cells [67]. Our data suggest that HIF-1 $\alpha$ also regulates mechanical cues in the tumor microenvironment. 
Characterization of major ECM components and cross-linking enzymes suggest that fibronectin levels may be a major factor in contributing to tissue stiffness. Acknowledgement: Supported by NIH R35CA209960 and R01CA82337.

References: References: [1] Lo CM., et al., Biophysical Journal 2000, [2] Bangasser BL., et al., Nature communications 2017, [3] Engler AJ., et al., Cell 2006, [4] Huebsch N., et al., Nature materials 2010, [5] Krishnamachary B., et al., Plos One 2012, [6] Gilkes DM., et al., Future Oncology 2013, [7] Shah T., et al., Oncotarget 2015.

\section{Image/Figure:}

https://www.xcdsystem.com/wmis/abstract/File6959/GA522 ImageFigure 0610091402.jpg

Image/Figure Caption: Figure 1: (A) Representative images of tumor sections and corresponding elastic modulus maps for sections obtained from 231-EV (top) and 231-sh-HIF-1 $\alpha$ (bottom) tumors. (B) Quantification of elastic modulus for different regions within 231-EV and 231 -sh-HIF-1 $\alpha$ tumors. (C) Quantification of strongly positive pixels normalized to the area of the tumor section. Values represent Mean \pm SEM. $* * \mathrm{P} \leq 0.005, * \mathrm{P} \leq 0.05$.

Complete Status: Complete

First Name: Desmond

Last Name: Jacob

Email: djacob4@jhmi.edu

Organization: Johns Hopkins University

Country: United States 


\title{
ID: GA523 \\ Positron emission tomography of systemically-administered adeno-associated viruses
}

\author{
Jai Woong Seo, Stanford University, jaiwseo@stanford.edu
}

Category: New Chemistry, Biology \& Bioengineering

\begin{abstract}
Body : Adeno-associated viruses (AAVs), single-stranded deoxyribonucleic acid (ssDNA) encapsulated within a 25-nm capsid, are attractive gene delivery vectors. Recently, peptide insertion within key capsid regions has been shown to alter the AAV pharmacokinetics (PK) and result in enhanced brain-specific transduction and reduced off-target accumulation after systemic administration $[1,2]$. In the past, AAV PK has been determined via fluorescent, luminescent, or positron emission tomography (PET) reporter gene imaging or classical qPCR. PET imaging of systemically-administered AAV capsids can uniquely offer non-invasive whole-body monitoring and facilitates the estimation of receptor binding kinetics. Here, we set out to develop a viral tracking method using inverse electron demand Diels-Alder reactions
\end{abstract} (IEDDA). Due to the small volume of AAVs injected (pmol), a multichelator was developed and applied to evaluate the PK and brain accumulation of AAV capsids which display brain tropism [3]. In particular, AAV9-PHP.eB (PHP.eB) and a variant with reduced peripheral accumulation (AAV9-ReB10 (ReB10)) were studied and compared with AAV9. AAV labeling chemistry with IEDDA reaction. Surface lysines were modified with tetrazine-NHS ester (TzNHS). The purified Tz-AAVs were reacted with $64 \mathrm{Cu}$ pre-labeled multichelators composed of 8 chelator conjugates coupled with transcyclooctene ((NOTA)8-TCO). A 30 min IEDDA reaction afforded $64 \mathrm{Cu}-\mathrm{AAV}$ at a yield of $2 \sim 10 \%$ and radiochemical purity of $>98 \%$ on instant thinlayer chromatography (ITLC) (Figure 1A). K557 and K567 were the predominant locations of the labeled lysines as determined by proteomic analysis. These two residues have not been reported to be directly involved in host receptor binding. The reaction of Tz-AAV with the fluorescent multichelator ((NOTA)8-AF555-TCO)) demonstrated that 3 5 multichelators were coupled with each capsid, and cryo-EM confirmed a similar number of labels on the surface of the capsid. PET/CT imaging and biodistribution of AAVs in C57BL/6 mice. For PK and biodistribution of the three AAVs, PET/CT images of C57BL/6 mice were acquired at 0,4 , and 21 hours after IV administration of $64 \mathrm{Cu}-\mathrm{AAV} 9(421 \pm 25 \mathrm{KBq}), 64 \mathrm{Cu}-\mathrm{PHP} . \mathrm{eB}(628 \pm 581 \mathrm{KBq})$, and $64 \mathrm{Cu}-\mathrm{ReB} 10(738 \pm 64 \mathrm{KBq})(\mathrm{n}=3 /$ group). Brain uptake of $64 \mathrm{Cu}-\mathrm{PHP} . \mathrm{BB}(17.0 \pm 4.1 \% \mathrm{ID} / \mathrm{g})$, and $64 \mathrm{Cu}-\mathrm{ReB} 10(22.1 \pm 1.9 \% \mathrm{ID} / \mathrm{g})$ at 21 hours was significantly greater than that of $64 \mathrm{Cu}-$ AAV9 $(0.16 \pm 0.02 \% \mathrm{ID} / \mathrm{g})$ (Figure 1B). While the liver uptake of PHP.eB $(35.1 \pm 10.4$ $\% \mathrm{ID} / \mathrm{g})$ was significantly increased at 21 hours as compared to AAV9 $(18.3 \pm 1.2 \% \mathrm{ID} / \mathrm{g}$, $\mathrm{P}=0.0409)$, liver accumulation of $\mathrm{ReB} 10(22.2 \pm 3.6 \% \mathrm{ID} / \mathrm{g})$ was comparable (Figure $1 \mathrm{C})$. ROIand Logan analysis of AAVs. Blood circulation half-life of 64Cu-AAV9, 64Cu-PHP.eB, and $64 \mathrm{Cu}-\mathrm{ReB} 10$ was 5.0, 4.1, and 3.1 hours, respectively. A reversible accumulation model (Logan plot) demonstrated that the $30 \mathrm{~min}$ accumulation in the brain after administration was greater for PHP.eB $>$ ReB10 $>$ AAV9, with a distribution volume of $0.2,0.1$, and 0.01 , respectively (Figure 1D). Thus, the affinity of PHP.eB and ReB10 to brain endothelium was estimated to be 20- and 10-fold higher than for AAV9, respectively. In conclusion. PET imaging of AAVs with a 
multichelator mapped early receptor-mediated binding and longitudinal accumulation over 21 hours. PET identified differences in target and off-target accumulation of peripherally detargeted AAVs.

References: 1. Deverman, B.E. et al. Cre-dependent selection yields AAV variants for widespread gene transfer to the adult brain. Nature Biotechnology, 2016, 34, 204-209 2. Kumar, S.R. et al. Multiplexed Cre-dependent selection yields systemic AAVs for targeting distinct brain cell types. Nature Methods 2020, 17, 541-550 3. Seo, J.W. et al. Positron emission tomography imaging of novel AAV capsids maps rapid brain accumulation. Nature Communications 2020, 11, 2102

\section{Image/Figure:}

https://www.xcdsystem.com/wmis/abstract/File6959/GA523_ImageFigure_0610091629.png

Image/Figure Caption: Figure 1. A.64Cu-labeling scheme of AAVs, B. Brain accumulation was greater in PHP.eB and ReB10 than AAV9 at 21 h. C. Liver accumulation was less in ReB10 and AAV9 than PHP.eB at 21 h. D. Logan plot of brain uptake rate for 30 min after AAV administration.

Complete Status: Complete

First Name: Jai Woong

Last Name: Seo

Email: jaiwseo@stanford.edu

Organization: Stanford University

Country: United States 


\title{
ID: GA524 \\ TRIPODD: A novel fluorescence imaging platform for in situ measurement of drug distribution and proteomic therapeutic response
}

\author{
Nathan McMahon, Oregon Health and Science University, mcmahonn@ohsu.edu
}

\section{Category: Oncology}

\begin{abstract}
Body : Personalized cancer medicine strives to prolong treatment efficacy with a variety of molecularly targeted therapies aimed at genetic vulnerabilities in malignant cells. Importantly, deregulation of kinase function in cell signaling pathways has been implicated in numerous cancers leading to the development of tyrosine kinase inhibitors (TKIs) to interact with these kinases for highly specific treatment. Though nearly 50 TKIs have been FDAapproved, TKI monotherapy is seldom curative, largely owing to tumor heterogeneity and acquired resistance. Unraveling the complexities of acquired resistance is challenging because it is a complex interplay of drug distribution and target engagement as well as multifaceted and dynamic interactions between the tumor and its microenvironment. Additionally, intra-tumoral heterogeneity ensures treatment of sensitive subpopulations, while simultaneously promoting the outgrowth of resistant "persister cells." Efficacious therapeutics capable of treating heterogenous tumors requires robust drug target engagement (DTE) in the complex setting of the diseased tissue, where outcomes are dictated by duration, completeness and cellular heterogeneity of drug target engagement. Major contributing factors to therapeutic failure are insufficient DTE, offtarget activity and cell signaling pathway reprogramming as a mechanism of acquired resistance. However, DTE and off-target activity are typically assessed by bulk sampling of plasma or tissue, yielding a heterogenous average, and thus lacking single cell resolution of drug distribution and target binding. There also remains a need for methods of measuring mechanisms of resistance via cell signaling pathway reprogramming. It is necessary to characterize cell signaling pathway reprogramming on the same cell-by-cell basis as DTE for an accurate measure of therapeutic response. Typically cell signaling is measured in cell lysates, which lack spatial context and conventional in situ immunostaining techniques lack the dimensionality to fully characterize therapeutic response. Therefore, no established technology exists to quantify TKI DTE, concomitant with local protein expression, while assessing tumor response heterogeneity. With the ultimate goal of improving TKI therapeutic efficacy, our group has developed a novel fluorescence imaging platform we call TRIPODD (Therapeutic Response Imaging through Proteomics and Optical Drug Distribution and binding). TRIPODD combines innovations made by our group to (1) develop protocols to fluorescently label TKIs that mimic the native drug, (2) advance a novel intracellular paired agent imaging (iPAI) platform to quantify DTE with these fluorescent TKIs, and (3) establish a highly multiplexed immunostaining strategy utilizing DNA barcoded antibodies, enabling in situ oligonucleotide conjugated antibody cyclic immunofluorescence (Ab-oligo cyCIF) imaging. iPAI uses the spectrally distinct, fluorescently labeled targeted and untargeted drug derivatives synthesized by our group to correct for untargeted uptake and facilitate quantitative assessment of DTE. Ab-oligo cyCIF is capable of generating extreme multi-parametric images for quantifying dephosphorylated and phosphorylated protein expression to quantify protein activation, expression, and spatial
\end{abstract}


distribution to unravel mechanisms of therapeutic resistance. Crucially, iPAI and Ab-oligo cyCIF can be performed on the same tissue sample enabling spatially aligned in situ quantification of TKI target engagement and local protein expression. Herein, we demonstrate the complementarity of iPAI and Ab-oligo cyCIF imaging technologies to produce a high dimensional image dataset to quantify spatially resolved tissue DTE, epidermal growth factor receptor (EGFR) cell signaling and cell state, all with single cell resolution. The result is the only fluorescence imaging platform to simultaneously quantify and correlate therapeutic efficacy to the complex interactions of drug binding (iPAI) and proteomics (Ab-oligo cyCIF) at the single cell level. Fully realized personalized therapy will include curated drug selection based on interand intra-tumoral heterogeneity and how these dictate therapeutic response and efficacy for which TRIPODD meets a critically unmet analytical need.

References: 1. Nathan P. McMahon, Jocelyn A. Jones, Sunjong Kwon, Koei Chin, Michel A. Nederlof, Joe W. Gray, Summer L. Gibbs, "Oligonucleotide conjugated antibodies permit highly multiplexed immunofluorescence for future use in clinical histopathology," J. Biomed. Opt. 25(5), 056004 (2020), doi: 10.1117/1.JBO.25.5.056004 2. 2. McMahon NP, Solanki A, Jones J, Kwon S, Chang YH, Chin K, Nederlof MA, Gray JW, Gibbs SL. Fluorescent Imaging for In Situ Measurement of Drug Target Engagement and Cell Signaling Pathways. Proc SPIE Int Soc Opt Eng. 2020 Feb;11219:112190O. Epub 2020 Feb 19. PMID: 32296256 3. 3. Solanki A, Wang L, Korber J, McMahon N, Tichauer K, Samkoe KS, Gibbs SL. Intracellular paired agent imaging enables improved evaluation of tyrosine kinase inhibitor target engagement. Proc SPIE Int Soc Opt Eng. 2020 Feb;11219:112190F. Epub 2020 Feb 19. PMID: 32292225

Complete Status: Complete

First Name: Nathan

Last Name: McMahon

Email:mcmahonn@ohsu.edu

Organization: Oregon Health and Science University

Country: United States 


\title{
ID: GA525 \\ Early Detection and Staging of Liver and Lung Fibrosis by Novel human Protein Contrast Agent
}

\author{
Oluwatosin Ibhagui, Georgia State Uuniversity, oodubade1@student.gsu.edu
}

Category: Immunology: Inflammation \& Infection

\begin{abstract}
Body : Fibrosis; the scarring and thickening of tissue resulting from organ injury is characterized by overexpression of deposition of Extracellular matrix proteins such as collagen and accounts for an uprising burden of mortality and morbidity worldwide. Liver (liver fibrosis) and lung (idiopathic pulmonary fibrosis (IPF) and chronic obstructive pulmonary disease (COPD)) are among the more common site for fibrotic diseases with tendency to progress to liver and lung failure thereby necessitating the need for transplant. Despite major advancement in clinical research, Early diagnosis and precise noninvasive detection of these diseases remain a critical unmet medical need. In this study, we report the development and optimization of our novel collagen-targeted human protein-based magnetic resonance imaging contrast agent (hProCA32.collagen) in early diagnosis, progression and monitoring of fibrosis in liver and lung. hProCA32.collagen exhibits strong affinity to collagen 1 of $\mathrm{Kd}$ of $1 \mathrm{uM}$. It has high relativities per particle ( $\mathrm{r} 1$ and $\mathrm{r} 2$ ) at both 1.4 and $7.0 \mathrm{~T}$ that enable us to perform dual imaging for diffusive fibrosis detection. It also possesses strong metal stability, serum stability and unprecedented metal selectivity for Gd3+ over physiological metal ions. We then report the detection of liver fibrosis induced by non-alcoholic fatty liver disease and more aggressive model utilizing human Comparative Gene Identification-58 knock out (CGI-58 KO) mouse liver fibrosis mouse model for both early and late stage (Ishak 3 and 5 of 6). In addition, we further report the quantitative detection of early fibrosis in a diabetic mouse model by ProCA32.collagen and pMRI. We report distinct vascular architecture changes associated with portal hypertension and intrahepatic angiogenesis during late-stage fibrosis and molecular signatures of different liver fibrosis. We further report the detection of lung fibrosis in our developed mouse model by ProCA32.collagen 1 and pMRI. Pathological analysis verified our noninvasive detection and correlation of histopathological and radiological pattern of usual interstitial pneumonia (UIP) characterized by honeycombing and heterogeneous architecture and collagen accumulation in the lungs. Furthermore, we have achieved the first MRI detection of lung fibrosis of Scnn1b-Tg+ mouse upon exposure to E-cigarettes. We show that the pattern observed for IPF both in histology and MR imaging is markedly different from e-cigarette induced lung fibrosing for COP-disease. We anticipate hProCA32.collagen having strong clinical translational potential in facilitating effective treatment to prevent disease progression as well as reveal key factors that contribute to organ fibrosis including emerging public health issue associated acute viral infection such as COVID-19.
\end{abstract}

References: Yang JJ, Yang J, Wei L, Zurkiya O, Yang W, Li S, Zou J, Zhou Y, Maniccia AL, Mao H, Zhao F, Malchow R, Zhao S, Johnson J, Hu X, Krogstad E, Liu ZR. Rational design of protein-based MRI contrast agents. Journal of the American Chemical Society. 2008, PMID: 18576649. Xue S, Yang H, Qiao J, Pu F, Jiang J, Hubbard K, Hekmatyar K, Langley J, Salarian 
M, Long RC, Bryant RG, Hu XP, Grossniklaus HE, Liu ZR, Yang JJ. Protein MRI contrast agent with unprecedented metal selectivity and sensitivity for liver cancer imaging. Proceedings of the National Academy of Sciences of the United States of America. 2015, PMID: 25971726 Salarian M, Turaga RC, Xue S, Nezafati M, Hekmatyar K, Qiao J, Zhang Y, Tan S, Ibhagui OY, Hai Y, Li J, Mukkavilli R, Sharma M, Mittal P, Min X, Keilholz S, Yu L, Qin G, Farris AB, Liu ZR, Yang JJ. Early detection and staging of chronic liver diseases with a protein MRI contrast agent. Nat Commun. 2019, PMID: 31664017. Salarian M, Ibhagui, O., Yang, J. Molecular Imaging of Extracellular Matrix Proteins with Targeted Probes using MRI. WIREs Nanomedicine \& Nanobiotechnology. 2019.

Full Name of Abstract's 1st Author : Oluwatosin Ibhagui

Complete Status: Complete

First Name: Oluwatosin

Last Name: Ibhagui

Email: oodubade1@student.gsu.edu

Organization: Georgia State Uuniversity

Country: United States 


\title{
ID: GA527 \\ An Activity-Based Sensing Approach for the Detection of Cyclooxygenase-2 in Live Cells
}

\author{
Anuj Yadav, University of Illinois Urbana-Champaign, anuj@illinois.edu
}

Category: New Chemistry, Biology \& Bioengineering

\begin{abstract}
Body : Cyclooxygenase (COX) initiates the production of prostaglandins by catalyzing the rate limiting step through its cyclooxygenase and peroxidase active sites. 1 The prostaglandins are implicated in regulation of physiological processes and immune responses in health and diseases. There are two isoforms 2 of COX: COX-1 is constitutively present in most cell types whereas COX-2 overexpression is induced by inflammatory and proliferative conditions associated with inflammatory diseases, 3 neurodegenerative disorders 4 and cancer. 5 However, COX-2 levels are indirectly measured by mRNA quantification, western blot analysis, or downstream product quantification (e.g., ELISA assays) these methods are not suitable for determining the effect of local environment on COX-2 activity. COX inhibitors are commonly used in clinics as expansive class of NSAIDs. But it has been difficult to determine the activity of isoform selective COX towards inhibitor development and furthering our understanding of its biological roles. In recent years isoform selective activity-based sensing approaches are emerging as a frontier area in deciphering enzymatic activities at the molecular level.6 This strategy was exploited to develop isoform selective COX-2 probe - CoxFluor to detect its activity in live cells by confocal imaging and flow cytometry. 7 It was hypothesized that larger pocket size of cyclooxygenase portion of COX-2 (vs COX-1) can easily accommodate bulkier groups.8,9 So, Arachidonic acid (natural substrate of COX-2) was strategically appended to a reporter dye (Resorufin) through an amide coupling such that the CoxFluor is fluorescently inactive but upon reaction with cyclooxygenase part it forms CoxFluor-PGG2 intermediate which upon subsequent processing by peroxidase portion of the enzyme releases the active dye resorufin (fluorescent). Furthermore, CoxFluor's selectivity was demonstrated in presence of 10fold excess functionally similar enzymes (lipoxygenase, peroxidase, catalase, etc.) and biologically relevant reactive oxygen, reactive nitrogen or other oxidant species. Additionally, by using molecular dynamics calculations it was shown that the presence of large cyclooxygenase binding pocket in COX-2 allows for the ease in CoxFluor's binding compared to COX-1 thus accounting for isoform selectivity. Moreover, CoxFluor enabled interrogating COX-2 activity dependencies on local oxygen concentration. It was hypothesized that since oxygen is a substrate in the reaction its concentration may influence the activity of COX-2 either through direct binding to the natural substrate in the reaction or attaching to the prosthetic heme subunit.10 Thus, on incubating LPS stimulated RAW 264.7 macrophage cell lines under normoxic and hypoxic conditions with CoxFluor it was clearly demonstrated that COX-2 activities changed without any change in enzyme level by flow cytometry and ELISA, respectively.
\end{abstract}

References: References: 1. Marnett, et al. J. Lipid Res. 2009, 50, S29-S34. 2. Hla, et al. Biochem. J. 1994, 302, 723-727. 3. Woolf, et al. Nature 2001, 410, 471-475. 4. Przedborski, et 
al. Proc. Natl. Acad. Sci. USA 2003, 100, 5473-5478. 5. Rao, et al. Cancer Res. 2000, 60, $293-$ 297. 6. Lybrand, et al. Biochemistry 2006, 45, 3189-3205. 7. Chan et al. Angew. Chem. Int. Ed. 2020, 59, 3307-3314. 8. Marnett, et al. J. Med. Chem. 2007, 50, 1425-1441. 9. Ramesha, et al.

Biochemistry 1997, 272, 21181-21186. 10. Vrzheshch, et al. Biochemistry 2010, 75, 1368-1373.

Full Name of Abstract's 1st Author : Anuj K Yadav

Complete Status: Complete

First Name: Anuj

Last Name: Yadav

Email:anuj@illinois.edu

Organization: University of Illinois Urbana-Champaign

Country: United States 


\title{
ID: GA528 \\ Plasma Biomarkers of Pancreatic Cancer Induced Cachexia
}

\author{
Santosh K Bharti, Johns Hopkins University, sbharti1@jhmi.edu
}

Category: Systemic Diseases (Kidney, Liver and Pancreas)

\begin{abstract}
Body : Introduction: Pancreatic ductal adenocarcinoma (PDAC) is often accompanied by weight loss caused by cachexia. In pancreatic cancer, the syndrome affects nearly $80 \%$ of patients and is a major cause of morbidity and mortality 1. A major characteristic of cachexia is accelerated skeletal muscle and fat storage wasting causing nutrient mobilization both directly as lipids and amino acids, and indirectly as glucose derived from the exploitation of liver gluconeogenesis that reaches the tumor through the bloodstream 2 causing significant metabolic dysregulation. Early and reliable detection of the onset of cachexia are important to initiate early interventions. Here, we analyzed metabolites in human plasma from PDAC patients with weight loss and with no weight loss using high resolution MR spectroscopy to identify plasma derived metabolic biomarkers of cachexia. These metabolic biomarkers can also play a role in designing and monitoring response to nutritional interventions. Methods: Plasma from 49 patients with PDAC were included in this study under an approved IRB protocol. For 1H MRS analysis, plasma samples were thawed and homogenized using a vortex mixer. Then $350 \mu \mathrm{L}$ of D2O phosphate buffer saline $(\mathrm{NaCl} 0.9 \%$ in $90 \% \mathrm{D} 2 \mathrm{O})$ was added to $250 \mu \mathrm{L}$ of plasma and mixed. After centrifugation (12000 rpm, $5 \mathrm{~min}$ ), each sample was transferred to $5 \mathrm{~mm} \mathrm{NMR}$ tubes and high-resolution 1H MRS was acquired on an Avance III $750 \mathrm{MHz}$ Bruker MR spectrometer using a single pulse and a Carr-Purcell-Meiboom-Gill (CPMG) pulse sequence with water suppression. To investigate the effect of cachexia induced weight loss on plasma metabolic patterns, PDAC patients were divided into four groups (I) PDAC patients with no weight loss history ( $\mathrm{n}=17)$ (II) with weight loss of 5-15lb $(\mathrm{n}=13)$, (III) with weight loss between 16-30lb ( $\mathrm{n}=11)$, and (IV) with weight loss of more than $31 \mathrm{lb}(\mathrm{n}=8)$. Results and Discussion: We found that PDAC patients with higher weight loss showed significant plasma metabolic changes compared to PDAC patients with no weight loss. PDAC patients with more $30 \mathrm{lb}$ weight loss showed dramatic metabolic differences when compared with PDAC patients with no weight loss (Figure 1A). Total lipids, branch chain amino acids, polyunsaturated fatty acids, alanine, glutamine, glucose, lactate, acetate, pyruvate, beta-hydroxy butyrate were significantly altered in PDAC patients with the most weight loss (Figure 1A). The other two groups with a lower weight loss also showed similar trends. We investigated the correlation between mean weight loss of each group with plasma metabolite levels. Lipid $(\mathrm{r} 2=0.88)$, glucose $(\mathrm{r} 2=0.7)$, creatine $(\mathrm{r} 2=0.87)$, lactate $(\mathrm{r} 2=0.75)$ and glutamate $(\mathrm{r} 2=0.55)$ showed a strong negative correlation with weight loss (Figure 1B). These results provide the groundwork for plasma metabolite analysis to detect the onset of cachexia and changes with nutritional intervention, and to identify metabolic targets to arrest the progression of PDAC induced cachexia. Acknowledgement: Supported by NIH R01CA193365 and R35 CA209960.
\end{abstract}

References: References: 1. (a) Fearon, K. C.; Baracos, V. E., Cachexia in pancreatic cancer: new treatment options and measures of success. HPB (Oxford) 2010, 12 (5), 323-4; (b) Ozola Zalite, 
I.; Zykus, R.; Francisco Gonzalez, M.; Saygili, F.; Pukitis, A.; Gaujoux, S.; Charnley, R. M.; Lyadov, V., Influence of cachexia and sarcopenia on survival in pancreatic ductal adenocarcinoma: A systematic review. Pancreatology : official journal of the International Association of Pancreatology 2015, 15 (1), 19-24. 2. Porporato, P. E., Understanding cachexia as a cancer metabolism syndrome. Oncogenesis 2016, 5, e200.

\section{Image/Figure:}

https://www.xcdsystem.com/wmis/abstract/File6959/GA528 ImageFigure 0610112104.jpg

Image/Figure Caption: Figure 1A: Metabolic heat map showing differences in the metabolic profile of plasma from PDAC patients with no weight loss ( $\mathrm{n}=17$ ), weight loss between 1-15 lb $(\mathrm{n}=13), 16-30 \mathrm{lb}(\mathrm{n}=11)$ and $31-70 \mathrm{lb}(\mathrm{n}=8)$. The maximum level of a metabolite present in a group was set to $100 \%$ to which the corresponding levels in the other groups were normalized. Student t-test was performed and a P-value less than 0.05 was considered significant. (*P-value Figure 1B: Linear regression was performed to investigate the correlation between metabolite levels in plasma of PDAC patients and weight loss. Mean weight loss and mean metabolites concentration were used to determine the correlation. PDAC patients with no weight loss ( $\mathrm{n}=17)$, weight loss between 1-15 lb ( $\mathrm{n}=13), 16-30 \mathrm{lb}(\mathrm{n}=11)$ and 31-70 lb $(\mathrm{n}=8)$.

Complete Status: Complete

First Name: Santosh K

Last Name: Bharti

Email: sbharti1@jhmi.edu

Organization: Johns Hopkins University

Country: United States 


\title{
ID: GA529 \\ Lipid nanoparticles functionalized by KAT ligation with peptide, Gd-chelate, and sulforhodamine $B$, enhanced atheroplaques in apoE-l- mice by in vivo MRI and ex vivo cryo fluorescent imaging
}

\author{
Yoko Yamakoshi, ETH Zurich, yamakoshi@org.chem.ethz.ch
}

\section{Category: New Chemistry, Biology \& Bioengineering}

\begin{abstract}
Body : A synthetic lipid derivative with potassium acyltrifluoroborate (KAT) moiety was mixed with commercial lipids to form a lipid nanoparticle with KAT functional group on their surface. This chemically active KAT moiety was used for the reaction with hydroxylamine (HA) derivatives of biomolecules and imaging probes viainstant amide-forming ligation reaction (KAT ligation)[1] worked under physiological conditions with diluted concentration of reactants. In this study, we synthesized a lipid nanoparticle functionalized with an apoB100-mimetic peptide, Gd-chelate, and sulforhodamine B and subjected it to in vivo MRI and ex vivo cryo fluorescent imaging on atherosclerotic apoE-/-mice Lipid nanoparticle (LNP) was initially prepared by self-assembly of lipid materials (phosphatidyl chorine, cholesteryl oleate, triglyceride, and a synthetic oleic acid KAT derivative (OA-KAT)) by sonication and extrusion in buffer [2]. Prepared LNP (LNP-KAT) was stably-dispersed with a diameter of ca. $50 \mathrm{~nm}$, and shown to be a mixture of particles with lipid monolayer and bilayer as observed by cryoTEM (Figure 1a). HA derivatives of functional moieties such as (1) LDL receptor-binding peptide motif in apoB100 (apolipoprotein of LDL) (P), (2) Gd(DO3A-monoamide) (Gd), (3) fluorescein $(\mathrm{F})$, and (4) sulforhodamine B (R) were synthetized. By mixing the LNP-KAT with these HA derivatives (1.2 equiv in a concentration of $0.12 \mathrm{mM}$ in total) in $\mathrm{pH} 5.3$ buffer, functionalized LNPs were synthesized in high yield of surface functionalization. No aggregation or degradation of NP was observed. Initial in vitro cell assay of an LNP carrying peptide (P) and fluorescein (F) (PF-LNP) was carried out on monocyte-derived cell line (THP-1, known to express the LDL receptors) in comparison to F-LNP (without peptide) indicating the effect of peptide to enhance the accumulation of LNP into cells presumably viaLDL receptor-mediated mechanism (Figure $1 b)$. The LNP (PGd-LNP) carrying peptide (P) and Gd-chelate $(\mathrm{Gd})$ was tested by in vivo MRI on atherosclerotic apoE-/-mice. All of three tested mice, which were received PGd-LNP viatail vain, clearly shown the enhancement in aortic arch especially in the area of brachiocephalic artery (BA) in 48 hours post-injection and corresponding Gd accumulation by ex vivo ICP-MS analysis. To investigate more detailed biodistribution of LNP, which correlated to the in vivo enhancement, the triply labeled LNP (PGdR-LNP), carrying peptide (P), Gd-chelate (Gd), and sulforhodamine B (R) was synthesized. By MRI in 24 hours post-injection, two atherosclerotic apoE-/-mice, which were injected with PGdR-LNP, showed significant enhancement in BA together with nearby thymus, where macrophage cells are largely existing (Figure 1c). Mice were subjected to the ex vivocryo fluorescence imaging analysis. As results (Figure 1d), clear correlation of MRI enhancement and fluorescence emission was observed in both mice, indicating that the enhancement observed in MRI was directly due to the high distribution of PGdR-LNP efficiently accumulated in atheroplaque in BA and thymus. $\quad$ From these in vivo andex vivo results, it was suggested that PGd-
\end{abstract}


LNP and PGdR-LNP were accumulated into the atheroplaque presumably by the help of LDL receptor overexpressed in atheromas in a similar to our previous results with natural LDL-based MRI-CA on the enhancement of atheroma by MRI in an atherosclerotic mouse model[3]. Furthermore, the enhancement of thymus in current study may be due to the accumulation of LNP through non-LDL receptor-dependent mechanism and presumably by the help of scavenger receptor. Further in vivo studies of LNPs with oxidized peptide tag and without peptide are currently going on to investigate these mechanisms.

References: [1] Dummas, A. M.; Molander, G. A.; Bode, J. W.*: Amide-forming Ligation of Acyltrifluoroborates and Hydroxylamines in Water, Angew. Chem. Int. Ed. 2012, 51, 5683. [2] Oriana, S.; Fracassi, A.; Archer, C.; Yamakoshi, Y.*: Covalent Surface Modification of Lipid Nanoparticles by Rapid Potassium Acyltrifluoroborate Amide Ligation, Langmuir 2018, 34, 13244. [3] Lowell, A. N.; Qiao, H.; Liu, T.; Ishikawa, T.; Zhang, H.; Oriana, S.; Wang, M.; Ricciotti, E.; Fitzgerald, G. A.; Zhou, R.*; Yamakoshi, Y.* Functionalized Low-density Lipoprotein Nanoparticles for in vivo Enhancement of Atherosclerosis on Magnetic Resonance Images, Bioconjugate Chem. 2012, 23, 2313.

\section{Image/Figure:}

https://www.xcdsystem.com/wmis/abstract/File6959/GA529 ImageFigure 0611035846.jpg

Image/Figure Caption: Figure 1.(a) Cryo TEM images of LNP-KAT with monolayer and bilayer morphologies. (b) Fluorescent microscopy images of THP-1 cell with internalization of PF-LNPand F-LNP particles (co-incubation: 5h). (c) MRI image of atherosclerotic apoE-/mice (A and B) injected with PGdR-LNP(24 h post-injection). (c) Bright field and fluorescence cryo images of atherosclerotic apoE-/-mice (A and B) injected with PGdR-LNP (24 h postinjection).

Complete Status: Complete

First Name: Yoko

Last Name: Yamakoshi

Email: yamakoshi@org.chem.ethz.ch

Organization: ETH Zurich

Country: Switzerland 


\title{
ID: GA530 \\ Anti-PD-1-based 68Ga-PET tracer for imaging cancer
}

\author{
Silvia Migliari, Nuclear Medicine and Molecular Imaging Department, University Hospital \\ of Parma, smigliari@ao.pr.it
}

\section{Category: Oncology}

\begin{abstract}
Body : Background In recent years, the blockade of immune checkpoint molecules with monoclonal antibodies, like those targeting the PD-1/PD-L1 pathway, has enabled the development of breakthrough therapies in oncology, leading to delayed tumor growth and increased survival. PD-1/PD-L1 expression determined by immunohistochemistry however, correlates moderately with patient survival and response to immunotherapy. PET imaging with radiolabeled anti-PD-1 antibody may allow whole-body detection of PD-1 at high sensitivity and resolution and reveal expression heterogeneity within tumors. Aim Aim of this study was to develop an immunoPET tracer for imaging PD-1 expression using a checkpoint-blocking antibody with proven antitumoral activity. Methods The novel PET tracer was developed by conjugation of anti-PD-1 checkpoint-blocking antibody with the chelator NOTA and labeling with the radioisotope Ga-68. We conjugated the 1,4,7-triazacyclononane-N,N',N"-triacetic acid (NOTA) chelator to Nivolumab (BMS-936558, ONO-4538, or MDX1106, trade name Opdivo; Bristol-Myers Squibb, Princeton, NJ, USA), the first-in-human immunoglobulin G4 (IgG4) PD-1 immune checkpoint inhibitor antibody. The buffer of Nivolumab $(10 \mathrm{mg} / \mathrm{ml})$ protein solution was readily exchanged by loading the protein onto a Biorad micro Bio-Spin 6 column, preequlibrated in Sodium Phosphate 0.1M, pH 8.5; p-SCN-Bn-NOTA powder was dissolved in the same buffer at a concentration of $10 \mathrm{mg} / \mathrm{ml}$. The mixture of $\mathrm{p}-\mathrm{SCN}-\mathrm{Bn}-\mathrm{NOTA}$ and Nivolumab was incubated overnight at $4^{\circ} \mathrm{C}$. Then the complex was radiolabelled with $30-50 \mathrm{MBq}$ of Ga-68 in $500 \mu \mathrm{l}$ of sodium acetate buffer $(\mathrm{pH} 5.1)$ at room temperature. The resulting [68Ga]NOTANivolumab was purified using a Biorad micro Bio-Spin 6 column and a sample of the radiopharmaceutical product was tested for quality controls to determine Radiochemical Purity using instant thin layer chromatography (ITLC) and high pressure liquid chromatography (HPLC). For ITLC, TLC-SG strips are used as stationary phase and ammonium acetate (sol. aq. $10 \%): \mathrm{MeOH}$ as mobile phase to separate free Gallium-68, which remains at the bottom, while the radiolabelled (Mo)Abs moved to the top. For HPLC, a MAbPacTM SEC - 1 column ( $5 \mu \mathrm{m}$, $300 \AA, 7.8 * 300 \mathrm{~mm})$ was used as stationary phase and water $(0.1 \%$ TFA $)$ as mobile phase. Binding affinity of NOTA-Nivolumab to T-cell blasts expressing PD-1 receptor was assessed by its capacity to inhibit fluorescently-labeled PD-1 binding, measured by flow cytometry. Results The Radiochemical Purity of [68Ga]DOTA-Nivolumab, assessed by both ITLC and HPLC methods, resulted approximately 75\%. Unconjugated DOTA showed no binding, while a titration curve of Nivolumab and NOTA-Nivolumab showed comparable binding affinity for PD-1 receptor. Conclusions The promising labeling results show an efficient and rapid procedure to label (Mo)Abs with Gallium-68 allowing the promising use of [68Ga]NOTA-Nivolumab as immunoPET tracer for imaging PD-1 expressing tumors.
\end{abstract}

Complete Status: Complete 
First Name: Silvia

Last Name: Migliari

Email: smigliari@ao.pr.it

Organization: Nuclear Medicine and Molecular Imaging Department, University Hospital of Parma

Country: Italy 


\title{
ID: GA531 \\ Design, construction and testing of a scale down automated 68Gallium-labeling NODAGA-Exendin-4 synthesis for clinical use
}

\author{
Silvia Migliari, Nuclear Medicine and Molecular Imaging Department, University Hospital \\ of Parma, smigliari@ao.pr.it
}

Category: New Chemistry, Biology \& Bioengineering

\begin{abstract}
Body : Background Glucagon-like peptide 1 receptor (GLP-1R) is preferentially expressed in pancreatic islets, especially in $\beta$-cells, but it's highly expressed in human insulinomas and gastrinomas. In recent years several GLP-1 receptor-avid radioligands have been developed to image insulin-secreting tumors or to provide a tentative quantitative in vivo biomarker of pancreatic $\beta$-cell mass. Exendin-4 is a 39-amino acid high affinity ligand of the GLP1-R with full agonist activity. It may be labeled with PET isotopes for imaging purposes. Here, we report results of a semi manual procedure to label [Lys40,Nle14(AhxNODAGA)NH2] exendin-4, as a kit, with Ga-68 (68Ga-NODAGA-Exendin-4). Aim To develop and validate a labeling procedure to obtain $68 \mathrm{Ga}-\mathrm{NODAGA}-\mathrm{Exendin}-4$ in order to make it available for our clinical community. Methods An automated synthesis module (Scintomics GRP ${ }^{2}$ ) was used to elute a $68 \mathrm{Ge} / 68 \mathrm{Ga}$ generator (GalliaPharma ${ }^{\circledR}$, Eckert and Ziegler) with $0.1 \mathrm{M} \mathrm{HCl}$ and to obtain the purified eluate $68 \mathrm{GaCl} 3$ through a cationic-exchange cartridge (SCX). The peptide contained in the kit vial (prepared by the Institute of Atomic Energy, Radioisotope Center POLATOM) in different amounts (10 - 20 - $30 \mathrm{ug}$ ) was reconstituted with $3.2 \mathrm{~mL}$ of HEPES solution. $68 \mathrm{GaCl} 3(300-1850 \mathrm{MBq}$ in $1.5 \mathrm{~mL})$ was added manually to the dissolved peptide, followed by $15 \mathrm{~min}$ incubation at $100^{\circ} \mathrm{C}$ in a thermoblock. The reaction solution was then purified through an HLB cartridge and the resulted product was diluted with PBS buffer and passed through a $0.22 \mu \mathrm{m}$ sterile filter into a sterile $25 \mathrm{~mL}$ capped glass for the final formulation. A sample of the radiopharmaceutical product was tested for quality controls (e.g. radionuclidic purity, radiochemical and chemical purity, $\mathrm{pH}$, endotoxins and sterility).

Results The synthesis of 68Ga-NODAGA-Exendin-4 starting from $10 \mathrm{ug}$ of peptide gets the best radiochemical yield of reaction $(6.47 \pm 2.4 \%)$, specific radioactivity $(5.04 \mathrm{MBq} / \mu \mathrm{g})$ and radiochemical purity $(91.69 \%)$, also representing the ideal amount of peptide for clinical application. All the quality control parameters tested were in accordance with the European Pharmacopoeia. Conclusions The complex NODAGA-Exendin-4 was successfully labeled with Ga-68 with a semi manual procedure, fitting all the required QCs for release and clinical use.
\end{abstract}

Complete Status: Complete

First Name: Silvia

Last Name: Migliari

Email: smigliari@ao.pr.it 
Organization: Nuclear Medicine and Molecular Imaging Department, University Hospital of Parma

Country: Italy 


\title{
ID: GA532 \\ Development and validation of a HPLC method to determine chemical purity and radiochemical purity of [68Ga]-DOTA-Pentixafor PET tracer
}

\author{
Antonino Sammartano, University Hospital , ansammartano@ao.pr.it
}

\section{Category: New Chemistry, Biology \& Bioengineering}

\begin{abstract}
Body : INTRODUCTION Due to its overexpression in a variety of tumor types, the chemokine receptor 4 (CXCR4) represents a highly relevant diagnostic and therapeutic target in nuclear oncology. Recently, new imaging probes targeting CXCR4 have been developed for PET imaging of several different hematologic and other neoplasms including leukemia, lymphoma, multiple myeloma, adrenocortical carcinoma or small cell lung cancer and also in other solid tumors and disease conditions, such as splenosis, stroke, atherosclerosis, and myocardial infarction in humans and in animals. Among CXCR4-directed imaging agents, Pentixafor labeled with Gallium-68 (Ga-68) has shown a unique position, because of its elevate affinity and selectivity to CXCR4, its low unspecific binding and adequate distribution profile accompanied by quick renal excretion. Preparation conditions may influence the quality and in vivo behaviour of tracers, especially considering clinical grade compounds, and no standard procedure for the quality controls (QCs) of [68Ga]-DOTA-Pentixafor is available, neither a specific monograph in the Pharmacopoeia does exist. So that, high pressure analytical purity (HPLC) method has become crucial for identification/characterization of the final product, demanding higher resolution than standard thin layer chromatography (TLC) method. Before their use in clinical and research settings, analytical methods must be validated. AIM Aim of this study was to develop a new rapid and simple HPLC method of analysis for the routine QCs of [68Ga]-DOTAPentixafor to guarantee the high quality of the finished product before release. MATERIALS AND METHODS At first, we have validated the synthesis process of [68Ga]-DOTA-Pentixafor assessing on three consecutive batches tthe radiochemical purity (HPLC), radiochemical impurities as $68 \mathrm{Ga}+3$ (HPLC and TLC), chemical purity (HPLC and gas chromatography, GC), $\mathrm{pH}$ (pH-strips), radionuclidic purity (principal $\gamma$-photon), 68Ge-content, Ga-68 half-life ( $\gamma$-ray spectrometry/counting) and sterility/endotoxin assay (sterility test and LAL test). Validation of analytical HPLC method to determine the chemical and radiochemical purity of [68Ga]-DOTAPentixafor was carried out according to ICH Q2 (R1) and EDQM guidelines. HPLC analysis was performed on a Dionex Ultimate 3000 HPLC system (Thermo Fisher Scientific) equipped with a AcclaimTM $120 \mathrm{C} 18$ column $3 \mu \mathrm{m} 120 \AA(3.0 \mathrm{~mm} \times 150 \mathrm{~mm})$, usingwater $(0.1 \% \mathrm{TFA})$ and acetonitrile $(0.1 \%$ TFA) as mobile phases. The parameters assessed for the HPLC method validation were: specificity, linearity, precision (repeatability), accuracy and limit of quantification, assessed using a set of standard samples, prepared by serial dilution starting from a "mother" solution with the highest concentration of the analyte of interest (Pentixafor) $(4,3$, $2.25,1.25,0.8,0.50 \mu \mathrm{g} / \mathrm{mL})$. RESULTS The mean radiochemical purity of the three consecutive synthesis runs was $99.96 \%$ (98.5\% measured by ITLC). For the validation of HPLC method, excellent linearity was found between 0.5 and $4 \mu \mathrm{g} / \mathrm{ml}$, with a correlation coefficient (R2) for calibration curves equal to 0.9995 . The average coefficient of variation (CV\%) resulted CONCLUSIONS The developed HPLC method to assess the radiochemical and
\end{abstract}


chemical purity of [68Ga]-DOTA-Pentixafor resulted rapid, accurate and reproducible allowing routinely use of this PET tracer as diagnostic tool for imaging CXCR4 expression in vivo, also assuring simple and safe implementation of the produced radiopharmaceutical in the diagnostic activity.

Complete Status: Complete

First Name: Antonino

Last Name: Sammartano

Email: ansammartano@ao.pr.it

Organization: University Hospital

Country: Italy 


\title{
ID: GA533 \\ Multimodal image-guided surgery of colorectal peritoneal carcinomatosis: A phase I clinical trial
}

\author{
Jan Marie de Gooyer, Radboud University Medical Center, jan- \\ marie.gooyer@radboudumc.nl
}

\section{Category: Oncology}

\begin{abstract}
Body : Aim Successful treatment of patients with colorectal peritoneal carcinomatosis highly depends on complete surgical resection of all tumor tissue. Surgical outcomes can be improved by intraoperative imaging using a tumor-targeting antibody conjugated to a fluorophore and a radiotracer, enabling preoperative radionuclide imaging, real-time intraoperative fluorescence guidance and gamma detection. Carcino Embryonic Antigen (CEA) is a suitable biomarker for in vivo targeting of colorectal cancer, and CEA-targeted intraoperative fluorescence imaging has already been investigated in a clinical study in colorectal cancer patients $[1,2]$. A CEA-targeted multimodal approach combining fluorescence imaging and radiodetection has been extensively investigated in a preclinical setting, but not in clinical trials $[3,4]$. Here we report results of the first clinical study investigating the feasibility, accuracy and safety of CEA-targeted preoperative SPECT/CT and intraoperative fluorescence imaging and radioguidance in patients with colorectal peritoneal carcinomatosis. Methods This phase I single arm intervention study consists of a dose escalation with 3 dose levels $(n=5$ per dose level). Patients receive an intravenous injection of the CEA-targeting tracer [111 In]In-DOTAlabetuzumab-IRDye $800 \mathrm{CW}$. Four days after injection a thoracoabdominal SPECT/CT is acquired to determine intra-abdominal tumor load and to scan for extra-abdominal metastases. One or two days after imaging, surgical resection extended with fluorescence imaging and radioguidance is performed. After standard of care cytoreductive surgery, the peritoneal cavity is examined for residual disease with fluorescence imaging. Blood samples are drawn for safety and pharmacokinetic analysis and vital signs are recorded at every hospital visit. Resected tissue specimens are analyzed microscopically, immunohistochemically (H\&E and CEA), and by gamma counting. Results/Discussion No study-related adverse events were seen in the first 10 patients after intravenous injection of 2 or $10 \mathrm{mg}$ of the tracer. Fluorescence imaging was feasible for real-time visualization of peritoneal tumor deposits. Mean fluorescent tumor to background ratios of $1.7 \pm 0.6$ and $4.1 \pm 1.5$ were observed in the 2 and $10 \mathrm{mg}$ cohorts, respectively. Preoperative SPEC/CT imaging revealed extra-abdominal lymph node metastasis not seen on conventional imaging modalities in two patients, altering the clinical management strategy. Ex vivo analysis of resected specimens revealed clear correspondence of NIRfluorescence and localization of CEA-expressing tumor cells. These preliminary data show that CEA-targeted multimodal imaging in patients with colorectal peritoneal carcinomatosis is feasible and safe.
\end{abstract}

References: 1. Tiernan, J.P., et al., Carcinoembryonic antigen is the preferred biomarker for in vivo colorectal cancer targeting. Br J Cancer, 2013. 108(3): p. 662-7. 2. Boogerd, L.S.F., et al., Safety and effectiveness of SGM-101, a fluorescent antibody targeting carcinoembryonic 
antigen, for intraoperative detection of colorectal cancer: a dose-escalation pilot study. Lancet Gastroenterol Hepatol, 2018. 3. Rijpkema, M., et al., SPECT- and fluorescence image-guided surgery using a dual-labeled carcinoembryonic antigen-targeting antibody. J Nucl Med, 2014. 55(9): p. 1519-24. 4. Hekman, M.C.H., et al., Detection of Micrometastases Using SPECT/Fluorescence Dual-Modality Imaging in a CEA-Expressing Tumor Model. J Nucl Med, 2017. 58(5): p. 706-710.

\section{Image/Figure:}

https://www.xcdsystem.com/wmis/abstract/File6959/GA533 ImageFigure 0611114757.jpg

Image/Figure Caption: A) Preoperative SPECT/CT revealed extra abdominal lymph node metastases not seen on conventional CT (blue arrows). B) White light image of corresponding retroperitoneal lymph node location in the same patient. C) Fluorescence imaging reveals tracer uptake in corresponding lymph nodes (Fluorescence in green). Presence of CEA-expressing tumor cells confirmed by pathological analysis.

Complete Status: Complete

First Name: Jan Marie

Last Name: de Gooyer

Email: jan-marie.gooyer@radboudumc.nl

Organization: Radboud University Medical Center

Country: Netherlands 


\title{
ID: GA534 \\ Tuning MRI Contrast Effects of Sub-5 nm Magnetic Iron Oxide Nanoparticles Through Varying Oligosaccharide Coating
}

\author{
Manman Xie, Emory University Shool of Medicine, mxie30@emory.edu
}

\section{Category: New Chemistry, Biology \& Bioengineering}

\begin{abstract}
Body : Sub-5 nm ultrafine iron oxide nanoparticles (uIONPs) have been shown to exhibit dual T1 (bright) and T2 (dark) contrast for magnetic resonance imaging (MRI) duo to their lower coupled magnetic moment that leads to the increased ratio of longitudinal relaxivity r1 and transverse relaxivity $\mathrm{r} 2$. While the composition [1], size [2], and aggregation [3] of this class of iron oxide nanoparticles have been generally investigated for improving the dual contrast effect and its applications, there is limited research done in understanding the controlling factors of coating materials on $\mathrm{r} 1$ relaxivity. We have previously developed oligosaccharide coated uIONPs by in situ polymerization of glucose on the particle surface [4], and found that oligosaccharide coating can be modulated for the thickness and hydrophilicity, which in turn may affect the MRI contrast effects. In this work, we report the investigation on further improving the $\mathrm{T} 1$ contrast effect by altering oligosaccharide coating applied to uIONPs to adjust the $\mathrm{r} 2 / \mathrm{r} 1$ ratio with the objective to better understand the molecular exchange process between the bulky water and hydration of the surface that contributes MRI contrast behaviors of uIONPs. uIONPs with different oligosaccharide coating were prepared with in situ polymerization by altering the ratio of glucose and uIONPs. The results of transmission electron microscopy (TEM), dynamic light scattering (DLS) and zeta potential measurements show that the prepared water-soluble uIONPs with different oligosaccharide coatings have good uniformity and monodispersity (Fig. 1a). With the increase of the ratio of glucoses to Fe in the surface polymerization, the hydrodynamic sizes of water-soluble uIONPs increased (Fig. 1b). The hydrophobicity of the oligosaccharide surface coating was determined by using the hydrophobic molecule pyrene as a reporting probe [5]. After mixing pyrene with different water-soluble uIONPs, it was found that the intensity ratio of the first emission band (at $374 \mathrm{~nm}$ ) to the third band (at $385 \mathrm{~nm}$ ) of the pyrene molecules, which is the indication of the hydrophobic properties of coating ligands on the surface, first gradually increased and then decreased as the function of the amount of glucose polymerized on the surface. Thus, the hydrophobicity of the surface gradually decreased and reached the lowest value when the ratio of glucose to Fe increased from 20 to 60. The relaxivities of different UIONPs were measured at the field strength of $3 \mathrm{~T}$ using a multi-TE spin echo sequence to measure $\mathrm{T} 2$ relaxation times and an inversion recovery sequence varying the inversion times to measure $\mathrm{T} 1$ relaxation times. The $\mathrm{T} 1$ and $\mathrm{T} 2$ values at different concentrations of uIONPs were obtained by fitting the signal intensities to the inversion times or echo time using the equation of $\mathrm{S} 1=\mathrm{S} 0 \times 1-2 \times \mathrm{e}(-\mathrm{Ti} / \mathrm{T} 1)$ or $\mathrm{S} 1=\mathrm{S} 0 \times \mathrm{e}-\mathrm{TE} / \mathrm{T} 2$ (Fig. 1c). Then, fitting the obtained $\mathrm{T} 1$ and $\mathrm{T} 2$ relaxation times with the iron concentration to obtain $\mathrm{r} 1$ and $\mathrm{r} 2$. The $\mathrm{r} 1$ and r2 measurements of the water-soluble uIONPs with different coating showed a dependence of the proton relaxivities on the ratio of glucose to Fe (Fig. 1d). uIONPs coated with the glucose $/ \mathrm{Fe}$ ratio of 60 (uIONPs-60) exhibited consistently higher relaxation rates than those coated at a lower glucose/Fe ratio. The increase of the glucose/Fe ratio (from 20 to 60) may play the role in
\end{abstract}


the probability of replacing organic oleic acids on the surface of nanoparticles, and the exchange of water molecules between bulk aqueous phase and magnetic cores. Consequently, the highest r1 relaxivity (4.11 mM-1s-1), comparable to that of Gd-DTPA (Magnevist, $4.22 \mathrm{mM}-1 \mathrm{~s}-1$ ), is achieved with uIONPs-60, suggesting the replacement of oleic acids on the surface of the nanoparticles is at the highest level with the reported coating materials and coating method. Whereas, the further increase of glucose/Fe ratio to 200 can only overload oligosaccharide

References: [1] N. Xiao, W. Gu, H. Wang, Y. Deng, X. Shi, L. Ye, T1-T2 dual-modal MRI of brain gliomas using PEGylated Gd-doped iron oxide nanoparticles, Journal of colloid and interface science, 417 (2014) 159-165. [2] H. Jung, B. Park, C. Lee, J. Cho, J. Suh, J. Park, Y. Kim, J. Kim, G. Cho, H. Cho, Dual MRI T1 and T2(*) contrast with size-controlled iron oxide nanoparticles, Nanomedicine: Nanotechnology, Biology and Medicine, 10 (2014) 1679-1689. [3] L. Wang, J. Huang, H. Chen, H. Wu, Y. Xu, Y. Li, H. Yi, Y.A. Wang, L. Yang, H. Mao, Exerting enhanced permeability and retention effect driven delivery by ultrafine iron oxide nanoparticles with T1-T2 switchable magnetic resonance imaging contrast, Acs Nano, 11 (2017) 4582-4592. [4] J. Huang, L. Wang, X. Zhong, Y. Li, L. Yang, H. Mao, Facile non-hydrothermal synthesis of oligosaccharide coated sub-5 nm magnetic iron oxide nanoparticles with dual MRI contrast enhancement effects, Journal of Materials Chemistry B, 2 (2014) 5344-5351. [5] K. Nakashima, M.A. Winnik, K.H. Dai, E.J. Kramer, J. Washiyama, Fluorescent probe studies on the microstructure of polystyrene-poly (vinylpyridine) diblock copolymer film, Macromolecules, 25 (1992) 6866-6870.

\section{Image/Figure:}

https://www.xcdsystem.com/wmis/abstract/File6959/GA534 ImageFigure 0611020945.jpg

Image/Figure Caption: Figure 1. (a) A TEM image of oligosaccharide coated uIONPs with core size of 3nm; (b) Change in hydrodynamic size of the same sized uIONPs with different oligosaccharide coating; (c) Signal intensity changes of uIONPs with different coating as a functional of the inversion time for deriving longitudinal relaxation time (T1); (d) The longitudinal relaxivities of the same sized uIONPs with different oligosaccharide coatings; (e) Fat-suppressed T1-weighted MR images of a mouse bearing the subcutaneous PDX tumor of pancreatic cancer before and after administration of uIONPs-60 at a dosage of $10 \mathrm{mg} \mathrm{kg}-1$. Positive T1 contrast enhancement was observed in the blood vessels (red dotted circles), tumor (yellow dotted circles) and kidney (green dotted circles); (f) Time dependent change of T1 MRI signal intensities in different organs.

Full Name of Abstract's 1st Author : Manman Xie

Complete Status: Complete

First Name: Manman

Last Name: Xie

Email: mxie30@emory.edu 
Organization: Emory University Shool of Medicine

Country: United States 


\title{
ID: GA535 \\ Mapping Pyridoxal Kinase Activity With 2-Hydrazinonicotinic Acid By CEST- MRI
}

\author{
Adam Shuhendler, University of Ottawa, Adam.Shuhendler@uottawa.ca
}

Category: New Chemistry, Biology \& Bioengineering

\begin{abstract}
Body : Background: Vitamin B6 or pyridoxine, in its phosphorylated aldehyde form (pyridoxal 5'-phosphate or PLP) is critical for intermediary metabolism [1], with pyridoxal being the most predominant of the few aromatic aldehydes normally present in our bodies. An intracellular pyridoxal kinase (PDXK)-pyridoxal phosphatase (PDXP) system controls the intracellular pool of PLP available for metabolism [2]. It was found that low PDXK and high PDXP activity conferred cancer cell resistance to therapy[3]. Importantly, non-small cell lung cancer patients with low PDXK expression (PDXK Methods: The CEST-MRI contrast agent, 2hydrazinonicotinic acid (2-HYNIC), was synthesized from 2-chloronicotinic acid and hydrazine in moderate yield $(49 \%)$ and sufficient quantity $(2-3 \mathrm{~g})$ [5]. A previously reported CEST-MRI contrast agent preferentially mapping aliphatic aldehydes (MeONA3) was synthesized as previously reported [6]. Hydrazones of 2-HYNIC and MeONA3 were prepared with acetaldehyde, 2-sulfobenzaldehyde, pyridoxal, and pyridoxal phosphate and purified by precipitation. Solution phase Z-spectra were recorded on a $300 \mathrm{MHz}$ Bruker Avance II NMR. CEST-MRI maps were acquired on an MRS 3000 3T MR Solutions system using an EPI protocol, and maps were generated as previously published [7]. PDXK and PDXP expression was confirmed in human small cell lung cancer cell lines (A549 and H460) by Western blot. CEST-MRI was performed on cells treated with $20 \mathrm{mM} 2$-HYNIC or MeONA3 $\pm 5 \mathrm{mM}$ pyridoxine. Results: By exchanging the core of our first-generation anthranilic acid-based aldehyde-conditional CEST-MRI contrast agents for nicotinic acid, we have successfully tuned the preference from aliphatic aldehydes to aromatic aldehydes (Fig. 1A). Proton NMR experiments and molecular modeling calculations suggest that 2-HYNIC favours a planar hydrazone conformation for aromatic aldehydes, while anthranilic acid-derived compound (MeONA3) favours a planar hydrazone conformation for aliphatic aldehydes (Fig. 1B). Critically, the planar hydrazone conformation is associated with greater CEST-MRI signal [6], putatively underlying the difference in aldehyde selectivity of these two contrast agents. When applied to pyridoxal vs. PLP sensing, both MeONA3 and 2-HYNIC produced low CEST-MRI contrast (\%MTRasym Conclusions:2-HYNIC is a novel, aromatic aldehyde-reactive CEST-MRI contrast agents capable of reporting on the PDXK-PDXP balance that is emerging as a biomarker of lung cancer chemoresistance.
\end{abstract}

References: [1] R. Percudani, A. Peracchi. EMBO Rep., 2003, 4, 850-854. [2]J. P. Richard, et al. Curr. Opin. Chem. Biol., 2009, 13, 475-483. [3]L. Galluzzi, I. Vitale, L. Senovilla, et al., Cell Reports, 2012, 2, 257-269. [4] Y.W. Jun, M. Hebenbrook, E.T. Kool. Chem. Commun., 2020, 56, 317-320. [5] L. K. Meszaros, et al., Dalton Trans, 2011, 40, 6260-6267. [6] T. Dang, et al. Chem. Eur. J., 2018, 24, 9148-9156. [7] G. Liu, et al. Contrast Med. Mol. Imaging, 2010, 5, $162-$ 170. 


\section{Image/Figure:}

https://www.xcdsystem.com/wmis/abstract/File6959/ImageFigure_GA535_0616124316.jpg

\section{Complete Status: Complete}

First Name: Adam

Last Name: Shuhendler

Email: Adam.Shuhendler@uottawa.ca

Organization: University of Ottawa

Country: Canada 


\title{
ID: GA536 \\ Lead Optimization of Nerve-Specific Fluorophores for Image-Guided Nerve Sparing Surgical Procedures
}

\author{
Connor Barth, Oregon Health \& Science University, connorwbarth@gmail.com
}

\section{Category: Oncology}

\begin{abstract}
Body : Nerve damage plagues surgical outcomes, significantly affecting post-surgical quality of life. Intraoperative nerve detection is completed using anatomical knowledge and conventional white light visualization when possible. However, neuroanatomy is varilable between patients especially in injured and diseased states that would be present during surgery. Additionally, nerves are typically protected deep within the tissue, so that white light visualization is largely impossible. Fluorescence image-guided surgery offers a potential means for enhanced intraoperative nerve identification and preservation. To date, a variety of nerve specific fluorophores have been tested in preclinical models, however a clinically approved nerve-specific contrast agent does not yet exist. Synthesis and functional validation of a nervespecific library has resulted in the development of near infrared (NIR) nerve-specific fluorophores for translation to first-in-human studies using the clinical infrastructure of NIR fluorescence guided surgery (FGS) systems.1 Further synthetic tuning of the most promising fluorophores has generated improved brightness, solubility, and safety. Increased brightness levels have resulted in 2-3x increase in nerve signal-to-background ratio (SBR). Improved solubility has enable water-soluble nerve-specific fluorophores with dose-limiting toxicity (DLT) values improved 5-10x. Single-dose toxicity testing including blood marker analysis and 14-day monitoring has facilitated definition of the no observable adverse effect level (NOAEL) and informed plans for upcoming investigational new drug (IND) enabling studies. Pharmacodynamics studies have been completed to identify the optimal imaging time point and dose, where nerve contrast is generated within 15 minutes and remains present for up to 4 hours following systemic administration. Clinically relevant, minimally invasive surgical procedures guided by NIR nerve-specific fluorescence have been completed in large animal models using the da Vinci surgical robot, demonstrating efficacy in surgically relevant models using existing clinical FGS system intrastructure. Given the interplay between recent synthetic tuning efforts and safety and pharmacology testing, we anticipate translation to the clinic within the next two years.
\end{abstract}

References: 1. Wang, L.G. et al. Near-infrared nerve-binding fluorophores for buried nerve tissue imaging. Sci Transl Med 12 (2020).

Complete Status: Complete

First Name: Connor

Last Name: Barth 
Email: connorwbarth@gmail.com

Organization: Oregon Health \& Science University

Country: United States 


\title{
ID: GA537 \\ Near-infrared Nerve-Specific Fluorophores for Image-Guided Nerve Sparing Surgical Procedures
}

\author{
Connor Barth, Oregon Health \& Science University, connorwbarth@gmail.com
}

Category: New Chemistry, Biology \& Bioengineering

\begin{abstract}
Body : Intraoperative nerve injury is one of the most feared complications of surgery affecting all surgical specialties and often causing irreparable damage. Nerve damage occurs in $\sim 17 \%$ of all surgeries and intraoperative nerve injuries affect 50 million patients annually worldwide, incurring undue pain, loss of function, and high costs to the healthcare system. Currently, no clinically approved technology exists to enhance intraoperative nerve recognition surgeons rely solely on knowledge of nerve anatomy and naked eye visualization when sparing vital nerves. We have developed first-in-kind nerve targeted near-infrared (NIR) fluorophores that highlight nerve tissue with high affinity and can be imaged in real-time for direct nerve visualization using clinical imaging systems. Recent work has allowed modification of the base structures of our fluorophores to significantly improve brightness, solubility, and toxicity while maintaining high nerve specificity. Clinically relevant formulation strategies have been explored to enable solubilization of these promising fluorophores for systemic administration. Micelle and nanoparticle based formulations have been identified that provide equivalent performance to the previously utilized co-solvent formulation while possessing clinically relevant safety and stability profiles. Additional work is underway to identify the biomolecular target of these structure-inherent targeting small molecule fluorophores to enable a mechanistic understanding for nerve-specificity. Pharmacokinetic, pharmacodynamic, dose response, and biodistribution studies have quantified each agent's in vivo performance and identified the optimal administration dose and imaging time windows. Further validation has been performed in swine minimally invasive surgical models using the da Vinci Surgical Robot and its integrated Firefly fluorescence imaging channel, demonstrating successful nerve identification and imaging using a clinical imaging system. We believe that the continued development use of these fluorophores in humans will improve nerve identification during surgery and reduce nerve injury, improving outcomes across all surgical specialties.
\end{abstract}

Complete Status: Complete

First Name: Connor

Last Name: Barth

Email: connorwbarth@gmail.com

Organization: Oregon Health \& Science University

Country: United States 


\title{
ID: GA538 \\ The combination of photodynamic and bacterial therapy suppresses tumor growth in an orthotopic mouse colon tumor model
}

\author{
Su Woong Yoo, Chonnam National University Hwasun Hospital, yoosw.md@gmail.com
}

\section{Category: Oncology}

\begin{abstract}
Body : Background \& Purpose: Photodynamic therapy (PDT) leads to hypoxic conditions of tumors by damaging the tumor-associated vasculature. Meanwhile, therapeutic bacteria specifically accumulate in tumors and migrate to distal regions far from the vasculature, which is typically a hypoxic area. Therefore, the synergistic therapeutic outcome may enable while we inject bacteria to the hypoxic-conditioned tumor by PDT. In this study, we combined PDT and bacterial therapy in orthotopic mouse colon tumor model in vivo. Materials \& Methods: The orthotopic colon mouse tumor model was made in C57BL/ 6 mice with MC38 cancer cell line. The tumor formation in the mouse colonic wall was identified by a lab-built endoscopic system. The mice were categorized into three groups; Group 1: control (no treatment), Group 2: only PDT therapy, Group 3: bacterial therapy after PDT (PDT + Bac). For the PDT, $525 \mathrm{~nm}$ green LED was irradiated $(2.3 \mathrm{~J} / \mathrm{cm} 2)$ right after intratumoral injection of rose bengal $(10 \mathrm{mg} / \mathrm{ml}, 30 \mu \mathrm{l})$ into the colonic tumor. Attenuated bacterial strain (Salmonella $\Delta$ ppGpp, $1 \times 107 \mathrm{CFU} / 100 \mu \mathrm{l})$ were injected to the mouse via a tail vein 24 hours after PDT in group 3. The tumor size was followed up by the endoscopic system and the visual score was evaluated. The follow-up was terminated when the tumor size reaches more than half of the colonic circumference. Results: The initial tumor size of each group was no significant difference. The overall follow-up periods of group 1 (control) were $4.0 \pm 1.4$ days. Group 2 (only PDT) followed-up for $8.3 \pm 2.5$ days. The group 3 (PDT + Bac) combination therapy groups showed $10.5 \pm 3.0$ days. Conclusion: Combination therapy of PDT and bacteria tends to suppress tumor growth in an orthotopic mouse colon tumor model. Further study with an optimized therapeutic protocol will be needed for a better outcome.
\end{abstract}

References: Dolmans DE, et al., Photodynamic therapy for cancer. Nat Rev Cancer. 2003 Becker C, et al., High resolution colonoscopy in live mice. Nat Protoc. 2006 Forbes NS. Engineering the perfect (bacterial) cancer therapy. Nat Rev Cancer. 2010

Complete Status: Complete

First Name: Su Woong

Last Name: Yoo

Email: yoosw.md@gmail.com

Organization: Chonnam National University Hwasun Hospital 
Country: South Korea 


\title{
ID: GA539 \\ Characterising the tumour vascular microenvironment of breast cancer patient- derived xenografts using optoacoustic imaging
}

\author{
Emma Brown, University of Cambridge, emma.brown@cruk.cam.ac.uk
}

\section{Category: Oncology}

\begin{abstract}
Body : Introduction: Vascular phenotypes in breast cancer are highly diverse and influence tumour biology and therapy response1. Multispectral Optoacoustic Tomography (MSOT) is an emerging modality that allows non-invasive visualisation of tumour vascular function through the endogenous contrast of deoxy- and oxy-haemoglobin ( $\mathrm{Hb}$ and $\mathrm{HbO} 2) 2$. We hypothesised that MSOT could monitor vascular phenotypes in clinically relevant breast cancer patient-derived xenografts (PDXs). Materials and Methods: Two luminal B PDXs (AB580, STG143) and two basal PDXs (STG139, STG321) were implanted in vivo (nAB580(LumB) $=$ 13, nSTG143 $($ LumB $)=6$; nSTG139 $($ Basal $)=19$, nSTG321 $($ Basal $)=8)$. MSOT was conducted when tumours reached $\sim 0.4 \mathrm{~cm} 3$, including a gas challenge (between air and $100 \% \mathrm{O} 2$ ) 3 . Total haemoglobin $(\mathrm{THbMSOT}=\mathrm{Hb}+\mathrm{HbO} 2)$ and blood oxygen saturation images $(\mathrm{SO} 2 \mathrm{MSOT}=$ $\mathrm{HbO} 2 / \mathrm{THb}$ ) were captured under air [SO2MSOT(Air)] prior to gas challenge, and 5 minutes after, under $100 \% \mathrm{O} 2$ [SO2MSOT(O2)], with both SO2MSOT values taken as averages of 10 consecutive measurements every $\sim 30$ s. Average $\Delta$ SO2MSOT (SO2MSOT(O2) -

SO2MSOT(Air), Figure 1) and 'Responding Fraction' [RF, ratio of responding ( $\triangle \mathrm{SO} 2 \mathrm{MSOT}>$ $2 \%$ ) to total pixels] were calculated in the tumour region of interest (ROI). Rim-core segmentation was performed by shrinking the tumour ROI radially by $1 \mathrm{~mm}$, taking the outer region as the 'Rim' and the rest as 'Core'. Once excised, tumours underwent IHC to stain CD31 (endothelial cells), CAIX (hypoxia), VEGF (angiogenic factor) and ASMA (\%ASMA coverage of CD31+ vessels, indicating vessel maturity). Results: THbMSOT, measured from in vivo MSOT images, was significantly higher in basal PDXs (2958 \pm 195.8 a.u. vs. 1391 \pm 254.8 a.u., p Corresponding with SO2MSOT(O2) levels, vessel maturity is highest in the basal STG321 model (\%ASMA covered vessels $=36.75 \pm 1.27 \%$ vs. STG139 20.08 $\pm 1.15 \%$, AB580 $28.76 \pm 1.35 \%$, STG143 25.64 $\pm 0.75 \%$, p Discussion: We have examined breast cancer PDXs from the basal and luminal B subtypes, finding markedly different vascular phenotypes between individual models and also between subtypes. Our results suggest that the luminal B model AB580 contains fewer blood vessels, but they are relatively mature, perfusing the whole tumour and leading to a less pronounced rim-core response to the gas challenge. AB580 also shows high $\mathrm{SO} 2 \mathrm{MSOT}(\mathrm{O} 2)$ and low hypoxia. While there is currently a lack of gas challenge data for the luminal B STG143 model, the IHC data suggests relatively few vessels and low hypoxia, so it may respond similarly to the AB580 luminal B PDX. Both basal PDXs (STG139 and STG321) have high blood vessel content and high rim response to the gas challenge, however, differences in vessel maturity appear to govern the perfusion, oxygenation and ultimately lead to discordance in their overall levels of hypoxia. Conclusion: MSOT is sensitive to different vascular phenotypes in breast PDXs, underscored by the vascular phenotypes measured using IHC. Future work will aim to understand the origin of different vascular phenotypes.
\end{abstract}


References: 1. Junttila, M. R. and de Sauvage, F. J. (2013). Influence of tumour microenvironment heterogeneity on therapeutic response. Nature 501, 346. 2. Wang, L. V and Hu, S. (2012). Photoacoustic Tomography: In Vivo Imaging from Organelles to Organs. Science (80-. ). 335, 1458-1462. 3. Tomaszewski, M. R., Gonzalez, I. Q., O’Connor, J. P., Abeyakoon, O., Parker, G. J., Williams, K. J., Gilbert, F. J. and Bohndiek, S. E. (2017). Oxygen Enhanced Optoacoustic Tomography (OE-OT) Reveals Vascular Dynamics in Murine Models of Prostate Cancer. Theranostics 7, 2900-2913.

\section{Image/Figure:}

https://www.xcdsystem.com/wmis/abstract/File6959/GA539 ImageFigure 0612064711.png

Image/Figure Caption: Figure 1. Representative MSOT image of a mouse body in greyscale with the spatial distribution of $\triangle \mathrm{SO}$ MSOT overlaid in the tumour region. $\triangle \mathrm{SO} 2 \mathrm{MSOT}$ defined as the amplitude of signal change during a gas challenge (between air and $100 \% \mathrm{O} 2$ ). Scale bar $=$ $5 \mathrm{~mm}$.

Complete Status: Complete

First Name: Emma

Last Name: Brown

Email: emma.brown@cruk.cam.ac.uk

Organization: University of Cambridge

Country: United Kingdom 


\title{
ID: GA540 \\ Imaging CRISPR/Cas9 Gene Editing in the Adult Rat Brain
}

\author{
Sabina Marciano, Werner Siemens Imaging Center, sabina.marciano@med.uni- \\ tuebingen.de
}

\section{Category: Neuroscience}

\begin{abstract}
Body : Introduction Gene editing using the CRISPR/Cas9 technology has been shown to be a powerful tool to interrogate the mammalian DNA. However, its application in postmitotic neurons has been challenging due to the size of the CRISPR-associated endonuclease SpCas9 and the low in vivo efficiency. We report a highly efficient knockdown (KD) of the Slc18a2 gene, encoding the vesicular monoamine transporter 2 (VMAT2) in vitro and in vivo using Cas9 from Staphylococcus aureus (SaCas9). Methods The KD efficiency of the sgRNA targeting the Slc18a2 gene was evaluated in vitro in rat primary neurons using surveyor assay and immunofluorescence. Next, AAV vectors for SaCas9 and sgRNA targeting Slc18a2 ( $\mathrm{n}=14)$ or LacZ (control, $n=10$ ) were injected into the right substantia nigra of adult rats. Starting 8 weeks post-injection, VMAT2 expression, DAT availability, postsynaptic changes and inflammatory responses were quantified using [11C]DTBZ, [11C]methylphenidate (MP), [11C]raclopride (RAC) and [18F]GE-180 PET. The CRISPR/Cas9-induced VMAT2 KD was characterized with behavioral readouts of locomotor activity, gait, spontaneous and apomorphine-induced rotations. Ex vivo histology and HPLC were carried to validate the in vivo data. Results We observed a [11C]DTBZ decrease, indicative for VMAT2 KD, in the right striatum of KD rats $(-30 \% \pm 20$, pSimultaneous PET/fMRI experiments are ongoing to inspect the impact of the induced molecular changes on the whole brain functional connectivity. Conclusions Our study pioneers the combinatorial use of CRISPR/Cas9 gene editing and multimodal imaging to disclose in vivo the direct link between specific genes and molecular changes observed in disease, and their impact on the whole brain functional connectivity. Using CRISPR/Cas9, we targeted the Slc18a2 gene, engineering the adult rat brain, and reproducing several motor symptoms of Parkinson's disease.
\end{abstract}

Full Name of Abstract's 1st Author : Sabina Marciano

Complete Status: Complete

First Name: Sabina

Last Name: Marciano

Email: sabina.marciano@med.uni-tuebingen.de

Organization: Werner Siemens Imaging Center

Country: Germany 


\title{
ID: GA541 \\ Oxygen-enhanced optoacoustic tomography evaluates vascular oxygenation in lung tumor xenograft models
}

\author{
Yihang Guo, UT Southwestern, guo.yihang.ash@gmail.com
}

\section{Category: Oncology}

\begin{abstract}
Body : Purpose: Multispectral optoacoustic tomography (MSOT) is an emerging noninvasive imaging modality that can detect real-time dynamic information about the tumor microenvironment (TME) in animals and increasingly humans. Particularly, oxygen enhanced (OE)-MSOT can monitor tumor vasculature and oxygenation during disease development or in response to therapeutic treatment. It is a non-ionizing imaging technique exploiting the spectral sensitivity of optical imaging and the high spatial resolution and penetration depth of ultrasound to provide in vivo images with high temporal resolution. In particular, the well characterized endogenous absorption spectra of oxy- and deoxyhemoglobin ( $\mathrm{HbO} 2$ and $\mathrm{Hb}$, respectively) can be used to evaluate hemodynamics in blood-filled vasculature using a linear regression technique for multispectral unmixing of photoacoustic data. We used OE-MSOT to assess the dynamic changes of oxy- and deoxyhemoglobin following the administration of heme-sequestering peptide 2(HSP2) and cyclopamine tartrate (CycT) using a subcutaneous tumor xenograft model in mice. Methods: Human H1299-luc lung cancer cells were implanted subcutaneously in the flank region of NOD/SCID mice. Mice were randomized into treatment groups that received saline (for control), HSP2 ( $25 \mathrm{mg} / \mathrm{kg}$ every 3 days, in about $50 \mu \mathrm{l}$ by retro orbital I.V injection), and CycT (by retro orbital I.V $7.5 \mathrm{mg} / \mathrm{kg}$ every 3 days) respectively for 3 weeks. For MSOT measurements, depilated anesthetized mice breathed air $(21 \% \mathrm{O} 2)$ for 2 minutes followed by $100 \%$ oxygen for 5 minutes. In order to examine $\mathrm{HbO} 2$ and $\mathrm{Hb}$ mouse images were acquired in transaxial sections through the tumor region using seven wavelengths: $680,715,730,768,800$, 850 and $900 \mathrm{~nm}$ with an MSOT InVision 256-TF device (iThera Medical, Munich, Germany). Illumination used 7 excitation fiber bundles and detection was based on 270 detectors in toroidal geometry around the mouse. A model-based reconstruction was used prior to multispectral processing. Following the final imaging, mice were sacrificed and tumor tissues were prepared for pimonidazole staining and CD31 staining, separately. Results and Discussion: MSOT reveals the spatial distribution of baseline $\mathrm{Hb}$ (blue), $\mathrm{HbO} 2$ (red), and total hemoglobin (THb) (Fig.1 A). OE-MSOT measurements were applied with an oxygen gas breathing challenge to further elucidate the mechanism of action. Oxygen challenge significantly increased the $\mathrm{sO} 2$ levels and the amplitude of $\Delta \mathrm{sO} 2$ response in HSP2- or CycT-treated tumors compared to control tumors (Fig.1 B\&C). Segmenting the different depth-based concentric regions (Fig.2 B) reveals both $\mathrm{THb}$ and kinetic dynamic sO2 levels decrease monotonically with depth in Control tumors (Fig.2 A).Confirming the OE-MSOT results, the exogenous hypoxia marker pimonidazole was significantly reduced in HSP2- and CycT-treated tumors (Fig.3 A\&B). Meanwhile, the levels of CD31 were significantly increased (Fig.3 C\&D). Conclusions: MSOT revealed heterogonous perfusion in the tumor. Decreased perfusion was evident in response to HSP2 and CycT indicating efficiency as a heme targeting drugs. MSOT measures the changes of perfusion and
\end{abstract}


proportion of oxy-and deoxy-hemoglobin revealing real-time, dynamic information about tumor vasculature and oxygenation.

\section{Image/Figure:}

https://www.xcdsystem.com/wmis/abstract/File6959/GA541_ImageFigure_0612085717.jpg

Complete Status: Complete

First Name: Yihang

Last Name: Guo

Email: guo.yihang.ash@gmail.com

Organization: UT Southwestern

Country: United States 


\title{
ID: GA542 \\ A novel in vivo imaging approach for evaluation of immuno-oncology therapies in mice via robotic ultrasound in combination with enhanced dual color bioluminescence
}

\author{
Juan Rojas, SonoVol, Inc., jrojas@sonovol.com
}

\section{Category: Instrumentation}

\begin{abstract}
Body : Objective: The ability to see multiple components of cell therapy longitudinally in a preclinical cancer model is key to understanding complex mechanisms of action and therapeutic efficacy. This study illustrates visualization of intratumorally injected cells in live animals via the unique combination of automated, hands-free ultrasound (US) and enhanced dual-reporter bioluminescence imaging (BLI). The objectives were to demonstrate that integrated US and dual-reporter BLI can quantitatively assess a) tumor volume, viability, and growth and b) track cell therapy biodistribution and migration in an anatomical context. As in vivo models are advancing in sophistication, the need for in vivo multiplexing is on the rise. Dual-reporter imaging is attractive because it allows you to derive two signals from one model. However, luciferases other than Firefly luciferase (Fluc) and their respective substrates are less optimal in vivo, largely due to emission wavelength, solubility issues, and fast signal decay. Therefore, in addition to multimodal imaging, orthogonal luciferase/luciferin systems that perform well in vivo are needed to expand the number of channels available. To meet this need, we used NanoLuc luciferase and the newly developed hydrofurimazine ( $\mathrm{HFz})$ as our complementary bioluminescent system to Fluc/luciferin. Methods: Five C57B16/J mice were subcutaneously inoculated in their flank with 1M Fluc expressing KPC 4662 cells. After 15 days post inoculation, mice were imaged using a novel combined US/BLI instrument (Strata, SonoVol Inc.). First, animals were positioned in the lateral recumbent pose and US volumes were scanned robotically. Subsequently, mice were intraperitoneally (IP) injected with D-luciferin (Promega Inc.) at $150 \mathrm{mg} / \mathrm{kg}$ and imaged with BLI after a $17 \mathrm{~min}$ post-injection waiting period $(30 \mathrm{~s}$ exposure time). D-luciferin was then allowed to wash out for 2h. Next, 50,000 HEK 293 cells expressing NanoLuc (Promega Inc.) were loaded in a well plate and assayed with hydrofurimazine, $\mathrm{HFz}$ (Promega Inc.). Following in vitro confirmation of BLI signal, cells were injected intratumorally in each of the flank tumors of the mice to simulate cell trafficking. Finally, mice were IP injected with $480 \mathrm{uL}$ PBS solution of $\mathrm{HFz}$ formulated with poloxymer P407. BLI images were captured every $5 \mathrm{~min}$ for $70 \mathrm{~min}$ using a $1 \mathrm{sec}$ exposure to illustrate the spatiotemporal localization of the injected cells. For quantification, US volumes were segmented with SonoEQTM software (SonoVol Inc.) in 3D to derive tumor volume, and BLI images were rendered with pseudocolor overlay in units of counts/sec. Results: US imaging demonstrated excellent 3D tumor boundary delineation; 15-day tumor sizes ranged from 52 to $248 \mathrm{~mm} 3$. BLI showed that both Fluc tumor cells and intratumorally injected Nanoluc cells can be detected with high sensitivity. Overlay visualization confirmed spatial co-localization, and when normalized per second, the signal of the NanoLuc cells was 2.5x stronger than the signal of the Fluc tumor. NanoLuc signal was graphed for 70 min to evaluate the kinetic curve of signal intensity over time. Time to peak was 35 +/- 5 min, except for mouse \#1394 with the smallest tumor (52 mm3).
\end{abstract}


In this animal, the NanoLuc cells were observed to be migrating away from the tumor region of interest (ROI) towards a lymph node as identified by US (see figure). This drainage caused a premature decrease in overall NanoLuc signal intensity in the tumor ROI. Conclusion: Integrated US/BLI imaging combined with dual orthogonal luciferases utilizing substrates (luciferins) with good aqueous solubility and bioavailability offer a novel approach for the quantification of cell and immune therapy. Future studies will include additional measurement channels for fluorescence imaging and microbubble-enhanced US.

\section{Image/Figure:}

https://www.xcdsystem.com/wmis/abstract/File6959/GA542 ImageFigure 0612091520.jpg

\section{Complete Status: Complete}

First Name: Juan

Last Name: Rojas

Email: jrojas@sonovol.com

Organization: SonoVol, Inc.

Country: United States 


\title{
ID: GA543 \\ Deep Learning based Segmentation of Tumors from TNBC PDX MR Images and Sensitivity of Radiomics Features to Segmentation Probability Boundaries
}

\author{
Kaushik Dutta, Washington University in St. Louis, kaushik.dutta@wustl.edu
}

\section{Category: Computational \& Data Science}

\begin{abstract}
Body : Objective: Manual delineation by experts is considered a gold standard in segmentation of tumors. However, manual method is time intensive and has high interobserver variability along with low reproducibility. This work aims to develop and evaluate the performance of a convolutional neural network $(\mathrm{CNN})$ algorithm for automatic localization and segmentation of triple negative breast cancer (TNBC) patient-derived tumor xenografts (PDX) tumors in preclinical T2 weighted MR images, and to evaluate the sensitivity of radiomic
\end{abstract} features to tumor segmentation based on observer segmentation probability maps. Method: MR imaging was performed on the MR Solutions simultaneous 7T MR/PET scanner (MR Solutions, Guildford, UK). T2-weighted MR images (2D FSE multi slice, echo train length 7, echo spacing $15 \mathrm{~ms}$, effective TE $=45 \mathrm{~ms}$, respiratory gated with $\mathrm{TR}=5 \mathrm{~s}$, respiration kept around 60 breaths $/ \mathrm{min}$, spatial resolution $0.25 \mathrm{~mm} \times 0.25 \mathrm{~mm} \times 1.0 \mathrm{~mm}$ ) were obtained for 37 mice with PDX tumors implanted in the mammary fat pad. Three expert readers manually segmented the tumor boundaries for all 37 PDX mice. Among them 29 PDX were used for training and validation of the network and the remaining were used for testing the performance of algorithm. Data Augmentation was implemented on each training image to make it more robust to positional variations along with intensity normalization of the image. A Deep-Dense U-Net1 based CNN with skip-connections was implemented to perform automated tumor segmentation. The radiomics parameters2,3 were extracted by integrating the Radiomics Image Processing Toolbox 4 in $\mathrm{R}$ along with in-house developed Radiomics pipeline written in Python. First order features were directly extracted from the intensity histogram while second order (GLCM) and the higher order (GLRLM, GLSZM) features were extracted using 32 quantization level. The segmentation performance was evaluated based on Dice Similarity Coefficient (DSC) and Jaccard Index. Sensitivity of radiomics parameters to segmentation boundaries was evaluated based on the mean Pearson Correlation Coefficient (r) value of the parameters with respect to delineation by experts. Results: The DSC between the automated algorithm and manual segmentation was $0.941 \pm 0.017,0.942 \pm 0.008$ and $0.938 \pm 0.13$ for each of the three observers respectively. The sensitivity of radiomic features to segmentation boundaries was evaluated based on differences in delineation by manual segmentation. Forty-five extracted radiomics features were found statistically significant (i.e., $p \leq 0.05$ ) in correlating to all experts and were used to evaluate the correlation between the features extracted from the automatic segmentation to the manual marking. Out of the 45, 28 features showed significantly high correlation i.e. $r \geq$ 0.8 . The first-order radiomics features correlated significantly to radiomic features derived by experts with $r$ ranging from 0.76-0.99. Higher order features exhibited greater variability in correlation with experts with values of $r$ ranging for 0.314-0.98. Conclusion: Deep learningbased segmentation performed accurate localization and segmentation of tumors in preclinicalMR imaging. However, despite the high agreement in segmentation, there is wide variability in 
radiomic features suggesting that the sensitivity of the feature to segmentation in a consideration in utility. The automated model mitigates inter-observer variability in radiomic parameters and make their analysis more robust and reproducible.

References: References: 1. Ronneberger, O., Fischer, P., \& Brox, T. (2015, October). U-net: Convolutional networks for biomedical image segmentation. In International Conference on Medical image computing and computer-assisted intervention (pp. 234-241). Springer, Cham. 2. Lambin, P., Rios-Velazquez, E., Leijenaar, R., Carvalho, S., Van Stiphout, R. G., Granton, P., ... \& Aerts, H. J. (2012). Radiomics: extracting more information from medical images using advanced feature analysis. European journal of cancer, 48(4), 441-446. 3. Gillies, R. J., Kinahan, P. E., \& Hricak, H. (2016). Radiomics: images are more than pictures, they are data. Radiology, 278(2), 563-577. 4. Carlson, Joel, (2018). Radiomics Image Processing Toolbox. Link https://cran.r-project.org/package=radiomics

\section{Image/Figure:}

https://www.xcdsystem.com/wmis/abstract/File6959/GA543 ImageFigure_0612095316.PNG

Image/Figure Caption: Figure 1. T2 weighted MR of TNBC PDX and their segmentations. PDX segmentation from two different mices are shown in each row. First Column: Original Image, Second Column: Predicted segmentation mask by neural network and Third Column: Markings of experts with respect to the segmentation (cyan, yellow, magenta - expert marking, red - algorithm predicted marking) Figure 2: Mean Pearson Correlation Coefficient of T2 weighted radiomic parameters of predicted tumor region with respect to manual delineation by three experts. A total of 45 radiomic features $(\mathrm{p} \leq 0.05)$ were evaluated and correlation values greater than 0.8 was graded as stable parameters

Complete Status: Complete

First Name: Kaushik

Last Name: Dutta

Email: kaushik.dutta@wustl.edu

Organization: Washington University in St. Louis

Country: United States 


\title{
ID: GA544 \\ Lymph node micrometastases detection using paired-agent fluorescent imaging
}

\author{
Chengyue Li, Illinois Institute of Technology, cli55@hawk.iit.edu
}

\section{Category: Oncology}

\begin{abstract}
Body : The identification of lymph node status is recognized as a critical prognostic factor in many solid cancer treatment and is essential to guide future adjuvant treatment[1]. Currently, surgical removal of lymph node followed by pathology examination is commonly performed as a standard-of-care to detect node metastases. However, the conventional pathology protocols are time-consuming and examine less than $1 \%$ of lymph node volumes by Hematoxylin and Eosin (H\&E) staining, 5- $\mu \mathrm{m}$-thick slices of lymph node taken at 2-mm intervals by pathologists. Using these routines, it is estimated that $20-60 \%$ of micrometastases $(0.2-2 \mathrm{~mm}$ diameter)[2] were undetected. In response, we developed a paired-agent imaging approach by employing a control imaging agent to allow rapid, quantitative mapping of microscopic populations of tumor cells in lymph nodes to guide pathology sectioning to reduce the false negative rate. By employing paired-agent imaging strategy, a recent study demonstrated the ability to detect fewer than 200 cancer-cells using a wide-field non-invasive imaging of breast cancer spread to axillary lymph nodes in a human breast cancer xenograft model[3]. To test the suitability of the staining/rinsing protocol for the pair-agent imaging approach, a targeted and control imaging combination was used to evaluate the potential for the agents to diffuse into and out of swine lymph nodes. IRDye800-Aby029, an anti-EGFR affibody labeled with IRDye800-CW (LICOR) as targeted imaging agent and IRDye700-DX (LICOR) was hydrolyzed as control agent. A green fluorescence protein transfected human cancer cells, MDA-MB-231-GFP, known to overexpress epidermal growth factor receptor (EGFR), were cultured as tumor spheroids with $250 \mu \mathrm{m}$ in diameter and implanted in swine lymph nodes to mimic the clinical condition of cancer spread to tumor draining lymph nodes. Micrometastases and tumor-free control nodes were compared to evaluate the diffusion of both agents into and out of intact nodes. Lymph nodes were immersed in saline solution to remain moisture and infused via two needles by co-administrating both targeted and control imaging agent at a rate of $0.3 \mathrm{~mL} / \mathrm{min}$ controlled by a syringe pump for $1 \mathrm{~min}$, followed by 5 min rinsing with PBS at the same infusion rate. Subsequently, lymph nodes were frozen-sectioned as $200 \mathrm{um}$ thick slice and imaged under an 80-um resolution fluorescence imaging system (Pearl, LICOR) to evaluate the spatial distribution of both agents in the entire lymph nodes. Fig.1 demonstrates cross-sectional fluorescence signal of both control and targeted imaging agents in the pig lymph node. Neither the targeted nor the control agent fluorescent maps could be used to identify the absence or presence of tumor. However, ratioing the targeted/control images to estimate binding potential[4] (unitless measure of receptor concentration) allowed tumor-bearing from control nodes to be separating perfectly: measured binding potential of targeted agent between micrometastases and control nodes were $1.33 \pm 0.47$ and $0.05 \pm 0.64$ respectively. These finding demonstrates the promise of using paired-agent approach by two needle infusion staining and rinsing protocol to significantly improve the sensitivity of cancer detection for breast cancer sentinel lymph node biopsy.
\end{abstract}


References: . Swartz, M.A., et al., Microsc Res Tech, 2001 2. Tew, K., et al., Br J Surg, 2005. 3. Tichauer, K.M., et al., Nat Med, 2014. 4. Tichauer, K.M., et al., J Biomed Opt, 2013.

\section{Image/Figure:}

https://www.xcdsystem.com/wmis/abstract/File6959/GA544_ImageFigure_0612122702.png

Full Name of Abstract's 1st Author : Chengyue Li

Complete Status: Complete

First Name: Chengyue

Last Name: Li

Email: cli55@hawk.iit.edu

Organization: Illinois Institute of Technology

Country: United States 


\title{
ID: GA545 \\ Dynamic bioluminescence and multispectral optoacoustic tomography reveal efficacy of microtubule Inhibitor BPR0L075 as a vascular disrupting agent in $4 \mathrm{T1}$ breast tumors
}

\author{
Honghong Wang, UT Southwestern medical center, honghong.wang@utsouthwestern.edu
}

\section{Category: Oncology}

\begin{abstract}
Body : Purpose: Our research objective was to assess tumor vascular disruption in breast tumors in response to microtubule inhibitor BPR0L075 using dynamic bioluminescence imaging (BLI) and multispectral optoacoustic tomography (MSOT) imaging. Methods: Mouse breast cancer cell line, 4T1-luc-GFP, was implanted orthotopically in the lower mammary fat pad of syngeneic female BALB/c mice. When the tumors reached 5-8 $\mathrm{mm}$ in diameter, five mice were injected IP with BPR0L075 $(60 \mathrm{mg} / \mathrm{kg})$ and three with prototypic vascular disrupting agent (VDA) CA4P $(120 \mathrm{mg} / \mathrm{kg})$. Three additional tumor bearing mice were injected with the same volume of saline as control. All mice were evaluated at baseline, 2 and 24 hours after injection using BLI and MSOT systems. For BLI, D-luciferin $(40 \mathrm{mg} / \mathrm{ml}$ in $80 \mu \mathrm{L})$ was administered subcutaneously in the foreback neck region prior to each BLI session. A BLI time course was acquired over 30 minutes, and maximum signal was recorded and normalized. When performing MSOT imaging, the mice were imaged in transaxial sections through the tumor region using five wavelengths - $680730768800850 \mathrm{~nm}$. Initially the mice breathed air, which was switched to $100 \%$ oxygen. Images were reconstructed and processed using manufacturer's software. Percentage of hemoglobin saturation (\%SO2MSOT) was calculated as $\% \mathrm{SO} 2=[\mathrm{HbO} 2 /$ $(\mathrm{HbO} 2+\mathrm{Hb})]^{*} 100$. The change of $\% \mathrm{SO} 2$ was calculated as delta $\% \mathrm{SO} 2=\% \mathrm{SO} 2$ oxygen \%SO2air. Results: Dynamic BLI and MSOT imaging showed that both BPR0L075 and CA4P caused vascular impairment of $4 \mathrm{~T} 1$ breast tumors. Bioluminescence emission was significantly decreased, with reductions of $96.9 \pm 2.7 \%, 91.3 \pm 2.4 \%$ and $98.9 \pm 0.6 \%, 92.5 \pm 0.9 \% 2$ and 24 hours after administration of BPR0L075 and CA4P, respectively. These responses were significant compared with baseline (pThere were significant statistical differences at 2 and 24 hours post BPR0L075 or CA4P administration, compared to each baseline (2hours BPR0L075 : p Conclusions: BPR0L075 caused rapid shut down of tumor vasculature causing hypoxia in 4T1 breast tumors. Both dynamic BLI and label-free MSOT imaging revealed the efficacy of BPR0L075, indicating that BPR0L075 provides a new alternative to the first-generation vascular disrupting agent CA4P and setting a basis for future therapeutic investigations.
\end{abstract}

\section{Image/Figure:}

https://www.xcdsystem.com/wmis/abstract/File6959/GA545 ImageFigure 0612022309.jpg

Image/Figure Caption: Representative images of spatial distribution maps of oxygen saturation in air and oxygen regions, and changes of \%SO2 (delta \%SO2) at baseline, 2 hours and 24hours after BPR0L075 administration. The maximum signal BLI images at baseline, 2hours, 24hours 
post BPR0L075 treatment and the corresponding dynamic BLI time courses in representative mouse.

Full Name of Abstract's 1st Author : Honghong Wang

Complete Status: Complete

First Name: Honghong

Last Name: Wang

Email: honghong.wang@utsouthwestern.edu

Organization: UT Southwestern medical center

Country: United States 


\title{
ID: GA546 \\ Characterization of PEGylated TCP1 probes for molecular imaging of colorectal cancer
}

\author{
Zhonglin Liu, University of Arizona, zliu@email.arizona.edu
}

\section{Category: Oncology}

\begin{abstract}
Body : Objectives: c[Cys-Thr-Pro-Ser-Pro-Phe-Ser-His-Cys]OH (TCP1) is a small cyclic peptide that was originally identified in orthotopic mouse colorectal cancer (CRC) using phage display selection. The results of our previous studies indicate that 99mTc-labeled 6hydrazinopyridine-3-carboxylic acid (HYNIC) conjugated-TCP1 (99mTc- TCP1-HYNIC) can detect malignant lesions in the colon. To establish optimal probes for translatable imaging studies, we have synthesized a series of TCP1 analogues in which reporter groups $(99 \mathrm{mTc}$ chelators) and linking groups between reporter groups and TCP1 are varied. The objective of this study was to characterize a PEGylated analog of TCP1 for CRC imaging by in vitro and in vivo studies. Methods: Cyanine 7 (Cy7)-labeled TCP1 (Cy7-TCP1) was prepared by conjugation of Cy7-N-hydroxysuccinimide (NHS) ester with TCP1 at a molar ratio of 1:1. Cy7-TCP1-PEG4 was prepared by coupling amino-dPEG4-t-butyl ester with the $\mathrm{Cy} 7$ carboxylic salt to provide an intermediate for subsequent conjugation of TCP1 in the presence of N,N,N',N'-tetramethyl-O(1H-benzotriazol-1-yl)uranium hexafluorophosphate (HBTU) as coupling reagent. TCP1-PEG4HYNIC was prepared by reacting the N-hydroxysuccinimide ester of HYNIC first with aminodPEG4-acid to provide intermediate which was subsequently coupled with TCP1 using HBTU. Conjugates of TCP1-HYNIC and TCP1-PEG4-HYNIC were radiolabeled with 99mTc by incubation at $80^{\circ} \mathrm{C}$ for $30 \mathrm{~min}$ with tricine as co-ligand. In vitro cell uptake of each Cy7-labeled TCP-1 probe was visualized with fluorescence microscope in HCT116 colon cancer cells seeded on glass cover slips and incubated with culture media containing TCP1 fluorescent agents for 1 h. The cells were fixed and stained with 4',6-diamidino-2-phenylindole (DAPI) to allow counterstaining of nuclei for fluorescence microscopy. In vivo biodistributions of $99 \mathrm{mTc}-$ TCP1-HYNIC and 99mTc-TCP1-PEG4-HYNIC were characterized in nude mice carrying HCT116 CRC xenografts using a custom-built ionizing-radiation Quantum Imaging Detector (iQID) camera within 30-240 minutes post i.v. radiotracer injection. Results: Incorporation of the PEG4 spacer enhanced hydrophilicity of the TCP1-HYNIC conjugate as seen by a shorter retention time of TCP1-PEG4-HYNIC chromatography. Fluorescence microscopy images of Cy7-TCP1 and Cy7-TCP1-PEG4 demonstrated rapid internalization of TCP1 by HCT116 cells. When the cells were treated with unlabeled TCP1 peptide prior to the cell incubation with TCP1 probes, the fluorescent signals of HCT116 cells were significantly reduced relative to the cells without blockade. Co-registered Cy 7 and DAPI images revealed nuclear localization of the TPC1 fluorescent-probes in cancer cells. iQID imaging studies of two TCP-1 radioprobes showed that the xenografted HCT116 tumors could be readily visualized with good contrast. Relative to 99mTc-TCP1-HYNIC, 99mTc-TCP1-HYNIC-PEG4 was shown to be capable of better localization of xenografted tumors as evidenced by significantly higher radioactive tumorto-nontumor ratios (tumor/muscle) determined by postmortem analysis (16.1 \pm 2.7 vs. 33.1 $\pm 4.7, \mathrm{P}$ \pm 0.3 vs. $1.4 \pm 0.2, \mathrm{P}>0.05)$ compared to $99 \mathrm{mTc}-\mathrm{TCP} 1-\mathrm{HYNIC}$. Conclusions: The TCP1-based
\end{abstract}


probes undergoing endocytosis and localizing mainly in the nucleus are promising for CRC detection. For the first time, we have successfully delineated the biodistributions of $99 \mathrm{mTc}-$ labeled TCP1 probes by iQID whole-body mouse imaging. Tumor detectability of the newly designed cyclic TCP1 peptide with a PEG4 linker was improved probably because of enhanced tumor binding affinity and nontumor clearance kinetics.

Complete Status: Complete

First Name: Zhonglin

Last Name: Liu

Email: zliu@email.arizona.edu

Organization: University of Arizona

Country: United States 


\title{
ID: GA547 \\ PROSPECTIVE EVALUATION OF PERFORMANCE OF 68Ga-PSMA-11 PET/CT IN PROSTATE CANCER PATIENTS WITH BIOCHEMICAL RECURRENCE
}

\author{
Ayman Gaber, Sinai clinic hospital, ayman.maher@hotmail.com
}

\section{Category: Oncology}

\begin{abstract}
Body : OBJECTIVE: The primary objective of this prospective study was to determine the sensitivity of 68Ga-PSMA-11 PET/CT in detection of prostate cancer in a biochemical recurrence $(\mathrm{BCR})$ setting on a per-patient basis confirmed by histopathology/biopsy, conventional imaging, and/or follow up. METHODS : A total of 91 subjects with confirmed prostatic adenocarcinoma based on histopathology and biochemical recurrence following treatment were enrolled in this prospective study. The Gleason score ranged from $6(3+3)$ to $10(5+5) .7$ subjects had a Gleason score 3+3 (7.7\%), 20 subjects had a Gleason score 3+4 (22\%), 22 subjects had a Gleason score 4+3 (24.2\%), 17 subjects had a Gleason score 4+4 (18.7\%), 16 subjects had a Gleason score 4+5 (17.6\%), 6 subjects had a Gleason score 5+4 (6.6\%), 2 subjects had a Gleason score 5+5 (2.2\%) and 1 subject with an undefined Gleason score (1\%). The median age of all enrolled subjects was 66 years (range: 52 to 88 years). All subjects received 3 to $7 \mathrm{mCi}$ of $68 \mathrm{Ga}-\mathrm{PSMA}-11$ (average $4.7 \mathrm{mCi}$ ). PET/ CT imaging was performed $60 \pm 15$ minutes after injection. For subjects with a PSA, less than $2 \mathrm{ng} / \mathrm{ml}$ an additional late scan at 180 minutes post-injection was also done. PET/CT images were read by an experienced nuclear medicine physician aware of clinical data. Evaluation of the results of the 68Ga-PSMA-11 PET/CT scan was compared to other available conventional anatomical and functional imaging modalities. This study received FDA authorization and approval from Biomedical Research Alliance of New York Institutional Review Board (BRANY IRB). RESULTS : All the images were of high quality. For subjects with a PSA less than $0.2 \mathrm{ng} / \mathrm{ml}$ the detection rate was $43 \%$, with a PSA between $0.2 \mathrm{ng} / \mathrm{ml}$ to $0.5 \mathrm{ng} / \mathrm{ml}$ was $53.3 \%$, with a PSA between $0.5 \mathrm{ng} / \mathrm{ml}$ to 1.0 $\mathrm{ng} / \mathrm{ml}$ was $60.8 \%$, with a PSA between $1.0 \mathrm{ng} / \mathrm{ml}$ to $2.0 \mathrm{ng} / \mathrm{ml}$ was $72 \%$, with a PSA between 2.0 to $5.0 \mathrm{ng} / \mathrm{ml}$ was $100 \%$ and with a PSA of more than $5.0 \mathrm{ng} / \mathrm{ml}$, the detection rate was also $100 \%$. The average detection rate of $68 \mathrm{Ga}-\mathrm{PSMA}-11 \mathrm{PET} / \mathrm{CT}$ scan was $78.5 \%$ for the 91 patients. In comparison, 37/82 (45.1\%) patients had lesions detected by pelvic MRI; 34/89 (38.2\%) patients had lesions detected by (Chest /Abdomen/Pelvis) CT and 21/85 (24.7\%) patients had lesions detected on Bone Scintigraphy. For all the subjects enrolled in the study, there were no SAE /AEs observed. CONCLUSION : 68Ga-PSMA-11 PET/CT is a safe imaging technique with a considerably higher detection rate in comparison with different conventional imaging modalities in patients with prostate cancer. There is a direct correlation between PSA level and 68Ga-PSMA-11 positivity with $100 \%$ detection rate for PSA levels above $2.0 \mathrm{ng} / \mathrm{ml}$.
\end{abstract}

Complete Status: Complete

First Name: Ayman 
Last Name: Gaber

Email: ayman.maher@hotmail.com

Organization: Sinai clinic hospital

Country: Egypt 


\title{
ID: GA548 \\ Development of implantable NMR microcoils for biomedical applications
}

\author{
Justine Deborne, Institut des Sciences Moléculaires, Université de Bordeaux, Bordeaux, \\ France, justine.deborne@u-bordeaux.fr
}

Category: Instrumentation

\begin{abstract}
Body : Introduction This study evaluates the performance of implantable NMR microcoils used to acquire in vivo NMR spectra of diagnostic quality from very small volumes of interest $(\sim 500 \mathrm{~nL})$. For minimum tissue damage during microprobe implantation, the microcoils were designed with needle-shaped geometry and outer diameters limited to a few hundred micrometers. The RF magnetic field generated by the microcoils were simulated using the finite element method and the microcoils were validated in vitro and in vivo in preclinical MRI scanner. Methods Two examples of implantable NMR microprobes are shown in Figures 1a)-b). This filar-type architecture is based on the use of copper microwires (diameter of 150 $\mu \mathrm{m})$. In Figure 1a), the copper wire is bent to form an elliptical-shape loop with typical dimensions of $3 \mathrm{~mm}$ for the long axis and 200 micrometers for the short axis. In Figure 1b), the twist microcoil consists of two strands twisted over a length of $3 \mathrm{~mm}$. A constant pitch of 500 micrometers was used to twist the two strands resulting in 6 identical windings. The microcoils were matched and tuned to the proton resonance frequency using extra-corporeal variable capacitors. NMR experiments were performed at 7 and 17.2 T. NMR spectra and MR images were acquired using external coils for RF excitation. For in vivo experiments, cannulae were stereotaxically positioned the day before the insertion of the NMR microcoils in the cortex of male wistar rats. Results/Discussion The quality factor of the loaded coils was larger than 100. The detection volume of the microcoils, derived from RF simulations and from the MR images were measured to be $500 \mathrm{~nL}$ for the twist coil and $250 \mathrm{~nL}$ for the loop coil. For in vitro results, the linewidth of the water peak (full width at half maximum) was $6 \mathrm{~Hz}$ at $7 \mathrm{~T}$. Figure 1c) shows a typical 1H MRS spectrum (PRESS sequence) obtained in vivo from the rat brain using a loop microcoil. In this particular example of water-suppressed acquisition (512 averages, 17-minutes acquisition) at 7 Tesla, main resonances of brain metabolites (NAA, Glu, Gln, Cr, Cho, etc) can be easily identified and quantified. Compared to an acquisition with a conventional external RF coil, the gain in sensitivity was found to be on order of 1500 . Conclusions The MRS/MRI results obtained in vitro and in vivo illustrate the relevance of the microcoil design with respect to spectral resolution, detection sensitivity, spatial selectivity and limited invasiveness. Foreseen applications include the investigation of metabolism in submicroliter volumes in physiological and pathological conditions with metabolic dysfunctions (tumoral environment, neurodegenerative pathologies, etc). Acknowledgment The study received financial support from the «Laboratory of Excellence » TRAIL ANR-10-LABX-57 (research program Insight) and from France Life Imaging (FLI).
\end{abstract}

\section{Image/Figure:}


Image/Figure Caption: Figure 1. a) implantable loop microcoil. b) implantable twist microcoil. c) NMR spectrum acquired at 7 Tesla in the rat brain using a PRESS sequence with water suppression. Total acquisition time : 17 minutes. Acquisition volume : $250 \mathrm{~nL}$.

Complete Status: Complete

First Name: Justine

Last Name: Deborne

Email: justine.deborne@u-bordeaux.fr

Organization: Institut des Sciences Moléculaires, Université de Bordeaux, Bordeaux, France

Country: France 


\title{
ID: GA549 \\ Evaluation of a multi-slice single-shot MRI-CEST sequence for volumetric pH imaging
}

\author{
Daisy Villano, University of Turin, daisy.villano@unito.it
}

\section{Category: Oncology}

\begin{abstract}
Body : Introduction: Chemical Exchange Saturation Transfer (CEST)-MRI allows to investigates seeral aspects of tumor microenvironemt, including metabolite, proteins and acidosis. CEST-based tumor $\mathrm{pH}$ imaging is hampered by long acquisition times (due to long saturation pulses and multiple offsets sampling) that reduce both the volume coverage and spatial resolution capability, hence the information on tumor $\mathrm{pH}$ heterogeneity that can be obtained. At the preclinical level only few multislice CEST sequences have been implemented to solve this issue, but not yet optimal in terms of SNR [1], geometrical distortions [2] and image artifacts [3, 4]. This work aims to improve both volume coverage and spatial resolution by implementing an uneven irradiation scheme, as in [1], with a centric-reordered RARE readout, for a whole tumor $\mathrm{pH}$ imaging for assessing tumor acidosis heterogeneity. Methods: The proposed sequence is composed of a first long saturation period that induces the steady state CEST contrast and a second short saturation pulse repeated after each image acquisition to compensate signal losses. Since the slices are acquired just after the second short saturation pulse rather than following each long first saturation period, the total scan time for the whole volume is dramatically reduced. Different acquisition parameters (k-space acceleration factor, matrix size, slice scan order, saturation times and power, voxel size, slice thickness) were simulated and optimized. CEST images were acquired on a $7 \mathrm{~T}$ scanner at $310 \mathrm{~K}$. Other MRI parameters were
\end{abstract} TR/TE:3.49/ 12000 ms, Matrix size:64×64 and axial slice with a thickness of $1.5 \mathrm{~mm}$. Multislice sequence accuracy and homogeneity to measure $\mathrm{pH}$ along the $3 \mathrm{rd}$ dimension was tested in vitro on a $\mathrm{pH}$-varying iopamidol phantom. In vivo validation was performed by acquiring multislice $\mathrm{pH}$ images covering the whole tumors following iopamidol i.v. injection. Results: Figure 1 shows the results of the in vitro and in vivo validation experiments. 2D multislice $\mathrm{pH}$ maps are shown in Figures $1 \mathrm{a}$ and the corresponding average calculated $\mathrm{pH}$ values for each vial are shown in Figures $1 \mathrm{~b}$. The calculated $\mathrm{pH}$ maps are very homogenous along the slice direction within the same vial. The observed calculated $\mathrm{pH}$ values were close to the experimental ( $\mathrm{pH}$-meter) ones along all the slices, suggesting that the $\mathrm{pH}$ measurement is not affected by the slice position. Moreover, mean calculated $\mathrm{pH}$ values were highly correlated to the experimental ones with low standard deviations, confirming the accuracy along the third dimension. The optimized fast multislice sequence was applied in vivo to obtain multislice 2D $\mathrm{pH}$ images (Figure 1d). The 3D data set was exploited for 3D $\mathrm{pH}$ rendering of the whole tumors and to evaluate the $\mathrm{pH}$ gradients in the tumoral region (Figure 1e). Conclusions: The proposed fast multislice CEST-MRI protocol was tested and validated in vivo allowing high volume coverage and $\mathrm{pH}$ sensitivity. The optimized multislice sequence can cover a whole tissue in less than 10 minutes, providing a new tool for $3 \mathrm{D} \mathrm{pH}$ heterogeneity evaluation of whole tumors or organs both in physiological and pathological conditions. Acknowledgments: Funding: This work was supported by the European Union's Horizon 2020 research and innovation programme 
(Grant Agreement No. 667510) and by the Associazione Italiana Ricerca Cancro (AIRC MFAG 2017 - ID. 20153 project).

References: [1] Sun PZ et al; Magn Reson Med 2011, 65, 588 [2] Sun PZ et al; Magn Reson Med 2008, 59, 1175 [3] Randtke EA et al; Magn Reson Med. 2017, 78, 97 [4] Dixon WT et al; Magn Reson Med. 2010, 63, 253

\section{Image/Figure:}

https://www.xcdsystem.com/wmis/abstract/File6959/GA549 ImageFigure_0613051435.JPG

Image/Figure Caption: Figure 1: Multislice sequence validation. 2D multislice $\mathrm{pH}$ map of the $\mathrm{pH}$ varying iopamidol phantom (a). Mean compartmental $\mathrm{pH}$ as a function of the slice (points) compared to real $\mathrm{pH}$ (lines) (b). Mean correlation between calculated $\mathrm{pH}$ and real $\mathrm{pH}$.

(c). Multislice $2 \mathrm{D} \mathrm{pH}$ maps in the tumor region superimposed onto the anatomical image and $\mathrm{pH}$ map 3D rendering (d). $\mathrm{pH}$ variation in the tumoral region (e).

Full Name of Abstract's 1st Author : Daisy Villano

Complete Status: Complete

First Name: Daisy

Last Name: Villano

Email: daisy.villano@unito.it

Organization: University of Turin

Country: Italy 


\title{
ID: GA550 \\ Analysis of influencing factors on the efficacy of patients with high-risk differentiated thyroid cancer after initial treatment
}

\author{
Qian Zhou, The First Affiliated Hospital of Zhengzhou University, zqqz0705@163.com
}

\section{Category: Oncology}

\begin{abstract}
Body : Objective: Patients with differentiated thyroid cancer of the same risk stratification have very different initial treatment effects, especially for high-risk patients. This study aims to explore the influencing factors of high-risk DTC patients after initial treatment, in order to better guide clinical, Provide a basis for formulating individualized treatment and follow-up strategies. Methods: A retrospective analysis of DTC patients who were present in our hospital from January 2015 to January 2018 was performed to exclude patients with positive $\mathrm{TgAb}$ and incomplete medical records. A total of 217 high-risk patients were included in this study, including 61 males and 156 females. Cases; average age (44.81 \pm 12.13$)$ years old. According to the criteria of the 2015ATA guidelines for the classification of efficacy, the patients were divided into excellent response group (ER group), uncertain response group (IDR group), and biochemical insufficiency response group (BIR group) according to the data measured under the stimulation state of the patient half a year after surgery. The structural incomplete response group (SIR group) used the $\mathrm{c} 2$ test or Fisher's exact probability method to compare the differences between the four treatment groups of gender, tumor multifocality, TNM stage, and N stage; the Kruskal-Wallis rank sum test was used to compare the age and the largest tumor The difference in diameter, stimulating Tg (ps-Tg) level before iodine treatment and postoperative iodine treatment dose among the 4 therapeutic groups. The receiver operating characteristic curve (ROC curve) of the relationship between ps-Tg and tumor diameter and ER was established. Use ordered logistics regression to analyze the independent influencing factors of patients' therapeutic efficacy. Results: There were 92 patients $(42.4 \%)$ in the ER group, 61 patients $(28.1 \%)$ in the IDR group, 38 patients $(17.5 \%)$ in the BIR group, and 26 patients $(12.0 \%)$ in the SIR group. Univariate analysis showed: gender $(\mathrm{c} 2=13.426, \mathrm{P}=0.005)$, tumor diameter $(\mathrm{H}=21.368, \mathrm{P}=0.000)$, tumor multifocality $(\mathrm{c} 2=15.510, \mathrm{P}<0.001), \mathrm{N}$ stage $(\mathrm{c} 2=$ 46.819, $\mathrm{P}<0.001)$, Ps-Tg level $(\mathrm{H}=3597.206, \mathrm{P}<0.001)$ significantly affects the classification of patients' efficacy after initial treatment. The difference between ps-Tg and tumor diameter cutoff values obtained by ROC curve for ER and non-ER are $9.92 \mathrm{ng} / \mathrm{ml}$ and $1.45 \mathrm{~cm}$, the area under the curve (AUC) is 0.876 and 0.654 , and the sensitivities are $69.5 \%$ and $61.1 \%$, respectively. The specificity was $95.1 \%$ and $66.7 \%$, the negative predictive value was $70.2 \%$ and $60.1 \%$, and the positive predictive value was $91.5 \%$ and $60.1 \%$, respectively. Taking the excellent response group as a reference, ordered multivariate regression analysis showed that N1b stage, ps-Tg level, and tumor diameter are independent factors that affect the therapeutic effect. Patients with ps-Tg $>9.92 \mathrm{ng} / \mathrm{ml}$ had a 36.307 times worse degree of efficacy than patients with ps$\mathrm{Tg} \leq 9.92 \mathrm{ng} / \mathrm{ml}$; patients with N1b stage had a poorer degree of efficacy 2.210 times that of patients with N0 stage; tumors with diameters $>1.45 \mathrm{~cm}$ The degree of poor efficacy of the patient was 1.978 times that of patients with tumor diameter $\leq 1.45 \mathrm{~cm}$. Conclusion: High-risk patients still have nearly half of the patients who can achieve excellent response after six months
\end{abstract}


of initial treatment. Patients with N0 staging, ps-Tg $<9.92 \mathrm{ng} / \mathrm{ml}$, and tumor diameter $<1.45 \mathrm{~cm}$ have better curative effect after initial treatment. For this part of patients, 131I treatment dose and follow-up intensity can be appropriately reduced. Patients with $\geq 9.92 \mathrm{ng} / \mathrm{ml}$ and cancer lesion diameter $\geq 1.45 \mathrm{~cm}$ should appropriately increase the treatment dose of 131I and the intensity of TSH inhibition and increase the intensity of follow-up to detect metastatic lesions as early as possible.

\section{Image/Figure:}

https://www.xcdsystem.com/wmis/abstract/File6959/GA550 ImageFigure 0613093426.png

Image/Figure Caption: Figure 1: Distribution of different clinicopathological factors in different therapeutic groups. 1A: Gender distribution of patients in different therapeutic groups; 1B: Multifocal distribution of cancer in patients in different therapeutic groups; 1C: Distribution of $\mathrm{N}$ stages in patients in different therapeutic groups; 1D: Distribution of TNM stages in patients in different therapeutic groups ; 1E: The distribution of the largest diameter of tumors in patients in different treatment groups; Figure 1F: The distribution of ps-Tg levels in patients in different treatment groups

Complete Status: Complete

First Name: Qian

Last Name: Zhou

Email: zqqz0705@163.com

Organization: The First Affiliated Hospital of Zhengzhou University

Country: China 


\title{
ID: GA551 \\ 18F-FDG PET/CT Identified Residual Lesions for Recovered COVID-19 Subjects
}

\author{
Jijin Yao, the Fifth Affiliated Hospital of Sun Yat-sen University, \\ yaojj23@mail.sysu.edu.cn
}

Category: Cardiovascular \& Pulmonary

\begin{abstract}
Body : Objectives: In light of the widely documented multiple organ injuries related with coronavirus disease 2019 (COVID-19), concerns have been raised regarding the assessment of these injuries in discharged patients. We aim to identify residual lesions throughout the whole body by 18 F-FDG PET/CT in these subjects. Methods: Patients who were discharged for COVID-19 at our hospital over a period of one week from 5th Feb to 12th Feb 2020, were recruited. Diagnosis and treatment protocol for COVID-19 were followed according to the WHO interim guidance[1] and the guidance from China[2]. The lung function tests were performed one month after discharge, and PET/CT scans were carried out two to three months after discharge. Patients had negative nucleic acid test for SARS-CoV-2 within three days before PET/CT scans. A total of twenty-five organs, including digestive system, urinary system, reproductive system, and cardiovascular system, were included in our study. To reflect the SUVmax of each organ more objectively, we randomly obtained five SUVmaxe values from each organ, and the mean of the biggest three SUVmaxe was recorded for this organ. In addition, if a definite or suspicious lesion can be seen in an organ, SUVmax values were mainly collected from there. The definitions of SUVmax thresholds in normal organs were followed the criteria reported by Tan et al.[3] and Engel et al.[4], above which the organ was considered abnormal uptake. Results:

Seven patients were recruited, which included four cases of severe and three of mild pneumonia. The baseline clinical characteristics between severe and mild groups were well balanced. The mean time from discharge to the date of lung function test was 36.4 days, and the mean time from discharge to the date of PET/CT scan was 59.0 days. In the follow-up examinations, only one patient suffered an increased 18F-FDG uptake of lower lobe of bilateral lungs (SUVmax, $2.37 \pm 0.06[\mathrm{~L}]$ and $2.41 \pm 0.07[\mathrm{R}]$; Figure 1) and a slightly impaired of pulmonary function (i.e. FVC [75.7\%], DLCO [68\%], and DLCO/VA [76\%]), which recovered normally three months after discharge. In the other six patients, both the lung function tests and the 18F-FDG uptake of bilateral lungs were normal (Figure 1). Further analysis revealed that no significant difference was observed in 18F-FDG uptake and lung function between severe and mild groups $(\mathrm{p}>0.05$, Table 1). Moreover, no increase of 18F-FDG uptake was observed in any organs tested, such as stomach, colorectum, myocardium, liver, kidney, spleen, pancreas, testis, prostate, bladder, and so on, for all patients. Overall, there were no significant difference in 18F-FDG uptake among the lungs and extrapulmonary organs between severe and mild group $(\mathrm{p}>0.05$, Table 1$)$. Conclusion: Lung function and 18F-FDG uptake can be quickly recovered two to three months after discharge among COVID-19 survivors regardless of pneumonia severity. Our findings will provide new insights into the recovery of recent functional injury of various organs in the whole body among COVID-19 survivors, which may not only help to relieve patients' panic of
\end{abstract}


pneumonia-related organs injury, but also could help physicians to formulate an optimized follow-up healthcare strategy.

References: 1 . Clinical management of severe acute respiratory infection when COVID-19 is suspected. Released by World Health Organization on 13 March 2020.

https:/www.who.int/publications-detail/clinical-management-of-severe-acute-respiratoryinfection-when-novel-coronavirus-(ncov)-infection-is- suspected. 2. World Health Organization. Diagnosis and Treatment Protocol for Novel Coronavirus Pneumonia (Trial Version. https:/www.who.int/docs/default-source/wpro---documents/countries/china/covid-19briefingnhc/1-clinical-protocols-for-the-diagnosis-and-treatment-of-covid-19v7.pdf?sfvrsn=c6cbfba4_2. Released by National Health Commission \& State Administration of Traditional Chinese Medicine on March 3, 2020 3. Tan LT, Ong KL. Semi-quantitative measurements of normal organs with variable metabolic activity on FDG PET imaging. Ann Acad Med Singapore. 2004;33(2): 183-185. 4. Engel H, Steinert H, Buck A, Berthold T, Huch Böni RA, von Schulthess GK. Whole-body PET: physiological and artifactual fluorodeoxyglucose accumulations. J Nucl Med. 1996;37(3):441-446.

\section{Image/Figure:}

https://www.xcdsystem.com/wmis/abstract/File6959/GA551 ImageFigure 0613082907.png

Image/Figure Caption: Figure 1: CT imaging manifestation during illness (vertical: A1-G1), follow-up CT (vertical: A2-G2) and PET/CT (vertical: A3-G3) among seven COVID-19 survivors. Of the seven patients, the first four patients (Case 1-4) suffered severe pneumonia and the last three patients (Case 5-7) suffered mild pneumonia. Case 1 showed multiple patchy and light consolidation with patchy shadows in both lungs during illness (A1; red arrows). Follow-up CT showed obvious absorption of lesions in both lungs (A2; green arrows). PET/CT showed slightly increased 18F-FDG in some lesions of lower lobes (SUVmax, $2.37 \pm 0.06$ [L] and 2.41土 0.07 [R]; A3; green arrows). Other three severe pneumonia (Case 2-4) showed patchy groundglass opacities in both lungs with patchy consolidation lesions in it (B1-D1; red arrows) during illness. The interstitial pneumonia of both lungs has receded greatly in the follow-up CT, and only a small amount of grid-like thickening of interlobular septa can be seen (B2-D2; green arrows). Moreover, no increase of 18F-FDG-uptake was observed (C2-D2; green arrow) among Case 2 to 4 . The last three patients with mild pneumonia (Case 5-7) showed scattered groundglass opacities during illness (E1-G1; red arrows). Only scattered interlobular septum thickenings in the follow-up CT (E2-G2; green arrow), and increased 18F-FDG-uptake was not observed among the mild pneumonia (E3-G3; green arrows). Red arrow: Residual lesion; Green arrow: Dissipated lesion.

Full Name of Abstract's 1st Author : Jijin Yao

Complete Status: Complete

First Name: Jijin

Last Name: Yao 
Email: yaojj23@mail.sysu.edu.cn

Organization: the Fifth Affiliated Hospital of Sun Yat-sen University

Country: China 


\title{
ID: GA552 \\ Probing Nucleus Protein DDX24 in Live Animal
}

\author{
Jiebing Gao, Sun Yat-Sen University, gaojb@mail.sysu.edu.cn
}

\section{Category: New Chemistry, Biology \& Bioengineering}

\begin{abstract}
Body : Purpose: DDX24, an important ATP-dependent RNA helicase which mainly functions in nucleus in living cells. It has shown that DDX24 overexpressed in human cancer cells and have close relationship with multiple organs, vein and lymphatic vessel malformations.[1,2] To precisely study the function of DDX24, a specific probe of DDX24 is needed. We provide a polypeptide probe with a highly specific binding to DDX24 and demonstrate its potential value in DDX24 related disease precision medicine. Procedures: We designed a polypeptide, LT(p)FEHYWAQLTS(p)-RRRRRRRR, a modified polypeptide targeting to the binding complex of DDX24/MDM2/p53 [3-5]. CHO cells transfected with the DDX24 or vector control were incubated with the fluorescent probe along with the scrambling control peptide. Tumor subcutaneous xenograft models of DDX24 expressing CHO or vector control cells were generated in balb/c nude mice. In vivo fluorescence imaging was carried out to assess the binding specificity for fluorescent probe. For radiolabeling, the NOTA-modified peptide probe was dissolved in NaOAc buffer and incubated with $[64 \mathrm{Cu}] \mathrm{CuCl} 2$. A C-18 Sep-Pak Plus cartridge was used to trap the product. The collected product was evaporated under gentle nitrogen flow in ice bath and dissolved in normal saline for other experiments. The radiolabeled product was tested on Radio-TLC. MicroPET/CT were performed in balbc-nu mice engrafted subcutaneously DDX24 (+) and DDX24 (-) CHO tumors xenografts. Results: When the designed FITC-probe was incubated with CHO cells transfected with DDX24-mcherry showed substantial fluorescence compared to control CHO cell. The peptide probe was located in the nucleolus area, while the control probe was scattered in CHO cells transfected with DDX24. Fluorescence imaging showed intense uptake of Cy5-peptide probe in xenografts at 8-12 hours post-injection and DDX24-mcherry transfected CHO cell xenografts showed substantial fluorescence compared to control $\mathrm{CHO}$ xenografts. For radiolabeling, the $\mathrm{Rf}$ value of the product after purification is 0.0 . The peak of the $64 \mathrm{Cu} 2+$ was not found, the RCP (radiochemical purity) of product was more than 95\%. The RCY (radiochemical yield) was 62\%. DDX24+ tumor xenografts were clearly imaged by microPET/CT at 6 or $12 \mathrm{~h}$ p.i. of $64 \mathrm{Cu}-\mathrm{NOTA}-$ LT(p)FEHYWAQLTS(p)-RRRRRRRR. The SUV of DDX24 (+) tumors at $24 \mathrm{~h}$ was 2.8-fold higher than DDX24 (-) tumors. Conclusions: Our polypeptide probe targeting to nucleus DDX24 expressing cells or live animals resulted in substantial fluorescence, or 64Cu labeled microPET imaging compared to control xenografts in animal models suggesting this peptide probe can specifically target DDX24 in live animals.. Acknowledgments Funding This work was funded by the National Key R\&D Program of China (2018YFC0910601), the National Natural Science Foundation of China (No.81871382), and Starting Fund (310103050303-220904094238) from the Fifth Affiliated Hospital, Sun Yat-sen University.
\end{abstract}

References: 1. Pang P, Hu X, Zhou B, Mao J, Liang Y, Jiang Z, Huang M, Liu R, Zhang Y, Qian J et al: DDX24 Mutations Associated With Malformations of Major Vessels to the Viscera. 
Hepatology 2019, 69(2):803-816. 2. Oliver D, Ji H, Liu P, Gasparian A, Gardiner E, Lee S, Zenteno A, Perinskaya LO, Chen M, Buckhaults P et al: Identification of novel cancer therapeutic targets using a designed and pooled shRNA library screen. Sci Rep 2017, 7:43023. 3. Shi D, Dai C, Qin J, Gu W: Negative regulation of the p300-p53 interplay by DDX24 ( Supplementary Figures）. Oncogene 2016. 4. Kussie PH, Gorina S, Marechal V, Elenbaas B, Moreau J, Levine AJ, Pavletich NP: Structure of the MDM2 oncoprotein bound to the p53 tumor suppressor transactivation domain. Science 1996, 274(5289):948-953. 5. Hu B, Gilkes DM, Chen J: Efficient p53 activation and apoptosis by simultaneous disruption of binding to MDM2 and MDMX. Cancer Res 2007, 67(18):8810-8817.

\section{Image/Figure:}

https://www.xcdsystem.com/wmis/abstract/File6959/GA552 ImageFigure 0614061107.jpg

Image/Figure Caption: Figure 1: The polypeptide probe targeting to nucleus DDX24 expressing cells or live animals resulted in substantial fluorescence, or 64Cu labeled microPET imaging compared to control xenografts in animal. (A). The designed FITC-probe was incubated with CHO cells transfected with DDX24-mcherry showed the peptide probe was located in the nucleolus area and co-located with DDX24-mcherry (white arrow) (upper line). Control CHO cells transfected with vector had minor fluorescence (second line). The control probe and FITCcpp was scattered in CHO cells transfected with DDX24 (third line and lower line). (B). Fluorescence imaging showed intense uptake of Cy5-peptide probe in xenografts post-injection and DDX24-mcherry transfected CHO cell xenografts showed substantial fluorescence compared to control $\mathrm{CHO}$ xenografts. The probe could be blocked by unlabeled peptide. (C-E). $64 \mathrm{Cu}$ labeled microPET imaging showed the uptake of the polypeptide probe was substantial in DDX24 (+) tumor than DDX24 (-) tumor. The SUV of DDX24 (+) tumors reach the peak at 12 hours and was 2.8-fold higher than DDX24 (-) tumors.

Full Name of Abstract's 1st Author : Jiebing Gao

Complete Status: Complete

First Name: Jiebing

Last Name: Gao

Email: gaojb@mail.sysu.edu.cn

Organization: Sun Yat-Sen University

Country: China 


\title{
ID: GA553 \\ Micron scale direct conversion X-ray detector for phase contrast X-ray imaging in biomedical applications
}

\author{
Karim Karim, KA Imaging, kkarim@kaimaging.com
}

\section{Category: Instrumentation}

\begin{abstract}
Body : When conventional x-ray radiography presents inadequate absorption-contrast, higher sensitivity can be achieved using phase-contrast methods. The implementation of phasecontrast $\mathrm{x}$-ray imaging using propagation-based techniques requires stringent spatial resolution requirements that necessitate lengthy propagation distances and inefficient scintillator-based detectors. Thus, imaging throughput is limited, and the absorbed dose by the sample can be unacceptable for radiation sensitive life science and biomedical applications. This work develops a hybrid direct X-ray conversion amorphous selenium and complementary metal-oxidesemiconductor detector technology that offers a unique combination of high spatial resolution and quantum efficiency for hard x-rays. A semiconductor fabrication process was developed for large area compatible vertical detector integration by back-end processing. Characterization of signal and noise performance using Fourier-based methods was performed by modulation transfer function, noise power spectrum, and detective quantum efficiency experiments using radiography and microfocus x-ray sources. The measured spatial resolution at each stage of detector development was one of the highest, if not the highest reported for hard $\mathrm{x}$-rays. In fact, charge carrier spreading from $x$-ray interactions with amorphous selenium was shown physically larger than the pixel pitch for the first time. A simultaneous factor of three improvement in quantum efficiency was achieved compared to scintillator-based detectors, despite the detector being a relatively unoptimized prototype. Lastly, fast propagation-based phase-contrast $\mathrm{x}$-ray imaging in compact geometries is demonstrated using a conventional low power microfocus source and the phase-contrast technique was applied to a number of biological samples including mouse organs, seeds, and insects. The imaging results from this research suggest that hybrid semiconductor technology offers the potential to fill the large performance deficit in high spatial resolution scintillator-based detectors for phase-contrast X-ray imaging and to even enable high speed dynamic phase contrast X-ray imaging for in situ characterization of biological samples.
\end{abstract}

References: C.C. Scott, A. Parsafar, A. El-Falou, P. M. Levine, K.S. Karim, "High Dose Efficiency, Ultra-high Resolution Amorphous Selenium/CMOS Hybrid Digital X-ray Imager," IEEE International Electron Devices Meeting (IEDM) Technical Digest, December 2015.

\section{Image/Figure:}

https://www.xcdsystem.com/wmis/abstract/File6959/GA553_ImageFigure_0613115413.jpg

Image/Figure Caption: Propogation based phase contrast X-ray image of a 50-100 micron articular cartilage in a mouse knee (larger view shown in inset) captured using the micron scale 
novel direct conversion X-ray detector and a commercially available $5 \mathrm{um}$ focal spot microfocus $\mathrm{X}$-ray tube in a compact $18 \mathrm{~cm} / 8 \mathrm{~cm}$ geometry.

Complete Status: Complete

First Name: Karim

Last Name: Karim

Email: kkarim@kaimaging.com

Organization: KA Imaging

Country: Canada 


\title{
ID: GA554 \\ Fluorofurimazine: A Bright, Soluble Bioluminescent Substrate for NanoLuc based Antares
}

Thomas Kirkland, Promega, thomas.kirkland@promega.com

Category: New Chemistry, Biology \& Bioengineering

\begin{abstract}
Body : Sensitive detection of two biological events in vivo has long been a goal in bioluminescence imaging. Antares, a fusion of the luciferase NanoLuc to the orange fluorescent protein CyOFP, has emerged as a bright bioluminescent reporter with orthogonal substrate specificity to firefly luciferase (FLuc) and its derivatives such as AkaLuc. However, the brightness of Antares in mice is limited by the poor solubility and bioavailability of the NanoLuc substrate furimazine. Here, we report a new substrate, fluorofurimazine (FFz), with even higher brightness and better pharmacokinetics than furimazine in vivo. After establishing the superior performance of $\mathrm{FFz}$, we demonstrated the potential of multiplexing of FFz with orthogonal ATP dependent beetle luciferase systems. We show that we can use Antares with fluorofurimazine to track tumor size and AkaLuc with AkaLumine to visualize CAR-T cells within the same mice, demonstrating the ability to perform two-population imaging with these two luciferase systems.
\end{abstract}

References: Chu, J., et al. (2016). "A bright cyan-excitable orange fluorescent protein facilitates dual-emission microscopy and enhances bioluminescence imaging in vivo." Nat Biotech 34: 760-767. Su, Y. et al. (2020) "Novel NanoLuc substrates enable bright bioluminescence imaging in animals." Nat Meth Accepted for Submission

Complete Status: Complete

First Name: Thomas

Last Name: Kirkland

Email: thomas.kirkland@promega.com

Organization: Promega

Country: United States 


\title{
ID: GA555 \\ Hyperpolarized 15N-labeled,deutrated Tris(2-pyridylmethyl)amine as an MRI sensor of freely available $\mathrm{Zn} 2+$ in tissue
}

\author{
Eul hyun Suh, University of Texas Southwestern Medical Center, \\ eul.suh@utsouthwestern.edu
}

Category: New Chemistry, Biology \& Bioengineering

\begin{abstract}
Body : Dynamic nuclear polarization (DNP) coupled with $15 \mathrm{~N}$ magnetic resonance imaging (MRI) provides an opportunity to image quantitative levels of biologically important metal ions such as $\mathrm{Zn} 2+, \mathrm{Mg} 2+$ or $\mathrm{Ca} 2+$ using appropriately designed $15 \mathrm{~N}$ enriched probes. A $\mathrm{Zn}$-specific probe could prove particularly valuable for imaging the tissue distribution of freely available $\mathrm{Zn} 2+$ ions, an important known metal ion biomarker in the pancreas, in prostate cancer, and in several neurodegenerative diseases. 1 Specially, the amount of total $\mathrm{Zn} 2+$ in prostate tissue decreases significantly during the early stages of malignancy.2 Therefore, noninvasive, real time in vivo detection of $\mathrm{Zn} 2+$ homeostasis would be an important diagnostic biomarker for prostate cancer and perhaps a variety of other diseases. Nitrogen-15 (0.37\% natural abundance) has a favorable nuclear spin of $1 / 2$ and a wide chemical shift range $(900 \mathrm{ppm})$, yet $15 \mathrm{~N}$ NMR is much less widely used as a tool in biological systems compared to $13 \mathrm{C}$ and $1 \mathrm{H}$ because of its poor sensitivity.3 However, 15N NMR in combination with hyperpolarization techniques such as dissolution dynamic nuclear polarization (d-DNP) or parahydrogen induced polarization (PHIP) is beginning to find its way into the biological realm. Hyperpolarization refers to technologies that enhance the NMR signal-to-noise ratio by amplifying nuclear spin polarization. 4 Here we report a HP-15N based probe, $15 \mathrm{~N}$-enriched, d6-deuterated tris(2pyridylmethyl)amine (TPA) (Fig. 1) for the detection and quantification of total freely available $\mathrm{Zn} 2+$ in tissues. TPA is a tripodal ligand that has excellent selectivity for $\mathrm{Zn} 2+$ over other common biological cations. Most optical and Gd-based $\mathrm{Zn} 2+$ responsive agents contain a sensing moiety structurally derived from TPA.5-7 This compound is known to distribute across cell membranes6 so in principle should detect freely available tissue $\mathrm{Zn} 2+$ in all compartments and not just $\mathrm{Zn} 2+$ ions released from cells in response to a biological stimulus.7 It was hyperpolarized by microwave driven dynamic nuclear polarization (DNP) 4 and demonstrated that the polarized ligand had favorable T1 and linewidth characteristics for $15 \mathrm{~N}$ MRI (T1=70.9 $\mathrm{s}$, at 9.4T). Chelation experiments with hyperpolarized 15N-TPA-d6 and $\mathrm{Zn} 2+$ ions revealed that the $15 \mathrm{~N}$ NMR signal of $15 \mathrm{~N}-\mathrm{TPA}-\mathrm{d} 6$ shifted $20 \mathrm{ppm}$ upfield upon complexation. The $15 \mathrm{~N}$ spectra also indicated that $\mathrm{Zn} 2+$ exchange between free ligand and 15N-TPA-d6-Zn2 + complex is slow on the experimental NMR timescale (Figure 1c). The $\mathrm{Zn} 2+$ detection limit of hyperpolarized 15N-TPA-d6 was found to be around $5 \mu \mathrm{M} \mathrm{Zn2+.} \mathrm{Ligand} \mathrm{protonation} \mathrm{and}$ $\mathrm{Ca} 2+$ binding did not interfere with $\mathrm{Zn} 2+$ sensing. $15 \mathrm{~N}$ chemical shift images of phantoms containing HP $15 \mathrm{~N}-\mathrm{TPA}-\mathrm{d} 6$ in the absence and presence of $\mathrm{Zn} 2+$ were collected to illustrate the feasibility of in vivo imaging of Zn-distribution (Figure 1d). We also demonstrate how HP-TPA can be used to quantify freely available $\mathrm{Zn} 2+$ in human benign prostatic hyperplasia (BPH) tissue.
\end{abstract}


References: 1. De Leon-Rodriguez, L.; Lubag Jr, A. J. M.; Dean Sherry, A., Imaging free zinc levels in vivo - What can be learned? Inorganica Chimica Acta 2012, 393, 12-23. 2. Costello, L. C.; Franklin, R. B., Zinc is decreased in prostate cancer: an established relationship of prostate cancer! Journal of biological inorganic chemistry : JBIC : a publication of the Society of Biological Inorganic Chemistry 2011, 16 (1), 3-8. 3. Witanowski, M., Nitrogen n.m.r. spectroscopy. 1974, 37 (1-2), 225. 4. Ardenkjær-Larsen, J. H.; Fridlund, B.; Gram, A.; Hansson, G.; Hansson, L.; Lerche, M. H.; Servin, R.; Thaning, M.; Golman, K., Increase in signal-to-noise ratio of $>10,000$ times in liquid-state NMR. Proceedings of the National Academy of Sciences 2003, 100 (18), 10158-10163. 5. Dai, Z.; Canary, J. W., Tailoring tripodal ligands for zinc sensing. New Journal of Chemistry 2007, 31 (10), 1708-1718. 6. Huang, Z.; Zhang, X.-a.; Bosch, M.; Smith, S.; Lippard, S. J., Tris(2-pyridylmethyl)amine (TPA) as a membrane-permeable chelator for interception of biological mobile zinc(). Metallomics : integrated biometal science 2013, 5 (6), 648-655. 7. Clavijo Jordan, M. V.; Lo, S.-T.; Chen, S.; Preihs, C.; Chirayil, S.; Zhang, S.; Kapur, P.; Li, W.-H.; De Leon-Rodriguez, L. M.; Lubag, A. J. M.; Rofsky, N. M.; Sherry, A. D., Zinc-sensitive MRI contrast agent detects differential release of $\mathrm{Zn}$ (II) ions from the healthy vs. malignant mouse prostate. Proceedings of the National Academy of Sciences 2016, 113 (37), E5464-E5471.

\section{Image/Figure:}

https://www.xcdsystem.com/wmis/abstract/File6959/GA555 ImageFigure 0613040919.jpg

Image/Figure Caption: Figure 1. DNP-15N MR experiments of 15N-TPA-d6. (a) 15N NMR spectrum of hyperpolarized and thermal equilibrium 15N-TPA-d6 at 9.4 T, 298K (b) Sequential $[15 \mathrm{~N}]$ spectrum decay (c) $15 \mathrm{~N}$ NMR chemical shift of 15N-TPA-d6 in the absence and presence of $\mathrm{Zn} 2+(0.25 \mathrm{eq})(\mathrm{d}) 15 \mathrm{~N}$ CSI of HP [15N]-TPA-d6 in HEPES buffered phantom solutions containing different ratios of $\mathrm{Zn} 2+/[15 \mathrm{~N}] \mathrm{TPA}-\mathrm{d} 6$. (i) A diagram of the phantom arrangement; (ii) $15 \mathrm{~N}$ spectra at different $\mathrm{Zn} 2+$ to $[15 \mathrm{~N}] \mathrm{TPA}-\mathrm{d} 6$ ratios; (iii) $15 \mathrm{~N}$ images of free, uncomplexed [15N]TPA-d6 (40 ppm); (iv) images of the Zn2+-[15N]TPA-d6 complex (20 ppm). (v) and (vi) show the ratiometric images of each species, normalized by the total $15 \mathrm{~N}$ signal $(\mathrm{tN})$

Full Name of Abstract's 1st Author : Eul Hyun Suh

Complete Status: Complete

First Name: Eul hyun

Last Name: Suh

Email: eul.suh@utsouthwestern.edu

Organization: University of Texas Southwestern Medical Center

Country: United States 


\title{
ID: GA556 \\ In vitro intracellular paired agent imaging validation for small molecule drug- target engagement quantification
}

\author{
Allison Solanki, OHSU, solanki@ohsu.edu
}

\section{Category: Oncology}

\begin{abstract}
Body : In the era of targeted cancer therapy, genetic screening typically motivates treatment recommendations; however, gene profiling doesn't guarantee initial, or lasting, therapeutic efficacy. To ensure a drug is effective, it is important to consider that a therapeutic must first engage with its target(s) in the complex disease setting, where efficacious therapy is dictated by the duration, completeness and heterogeneity of drug target engagement (DTE)1, 2 . Indeed, quantification of local cellular drug distribution should play a key role in assessing and predicting patients' treatment success; yet despite being an integral piece of the therapeutic puzzle, such analysis is not routine. Instead, standard pharmacokinetic or biochemical drug screening tools are typically bulk in nature (e.g., plasma analysis, western blot) 3 and inherently limited in their utility for understanding heterogeneous diseases and mechanisms of initial or acquired therapy resistance. In response to this challenge, we have developed a novel imaging platform to quantify drug target engagement termed intracellular paired agent imaging (iPAI)4, 5. PAI utilizes untargeted imaging agents to correct for nonspecific uptake of spectrally distinct targeted agents, providing quantitative assessment of receptor density. PAI has recently been extended to small molecule therapeutics using fluorescently-labeled targeted and untargeted drug derivatives, including kinase inhibitors (KIs) in the epidermal growth factor receptor (EGFR) signaling pathway. Notably, although this technique relies on fluorescently-labeled drugs for quantification, all treatment is completed with the parent drug, classifying iPAI as a label-free method permitting assessment of the interaction between the parent drug and its native target. In preliminary work, we have designed, synthesized and performed in vitro validation on an iPAI reagent toolbox containing the EGFR KI erlotinib (Erl), in addition to downstream EGFR pathway-targeted drugs: buparlisib (PI3K), selumetinib (MEK1/2, ERK1/2), MK2206
\end{abstract} (AKT1/2/3), and LY3214996 (ERK1/2). Evaluation of this suite of drugs is particularly valuable, since it is largely understood that monotherapy will not provide a durable, long-term response, but instead could create an actionable window to exploit collateral sensitivities to other drugs generated by therapeutically-induced selective pressure within the existing or parallel pathways. Targeted and untargeted behavior of each iPAI pair was quantified and evaluated using a suite of four functional assays, including (1) competitive binding with the parent drug, (2) fluorescence imaging vs. the parent fluorophore and vs. target-specific antibodies, which were compared to (3) quantitative flow cytometry to enumerate protein expression per cell and (4) tandem liquid chromatography mass spectrometry (LCMS) measured uptake and stability. Initial screening on positively and negatively protein expressing cell lines showed substantial non-specific accumulation of the targeted iPAI agent that was recapitulated by the untargeted iPAI agent. Calculated ratiometric DTE images (where targeted signal is subtracted from the untargeted signal, and divided by the untargeted signal) revealed an expression pattern that was spatially matched to antibody staining, representing the number of drug targets available for binding. 
Cells were then administered therapy and validation studies were repeated to verify that the iPAI reagents were indeed able to predict cellular response, determined via simultaneous viability analysis. These in vitro studies provide a conduit to iPAI implementation in future in vivo work, where non-small cell lung cancer (NSCLC) models will be utilized to study both monotherapy and combination therapy that could provide a durable, long-term treatment response.

References: 1. Dubach, J.M. et al. Quantitating drug-target engagement in single cells in vitro and in vivo. Nat Chem Biol 13, 168-173 (2017). 2. Bunnage, M.E., Chekler, E.L. \& Jones, L.H. Target validation using chemical probes. Nat Chem Biol 9, 195-199 (2013). 3. Simon, G.M., Niphakis, M.J. \& Cravatt, B.F. Determining target engagement in living systems. Nat Chem Biol 9, 200-205 (2013). 4. Samkoe, K. et al. Simultaneous extracellular and intracellular quantification of EGFR using paired-agent imaging in an in ovo tumor model, SPIE Int Soc Opt Eng 10859 (2019). 5. Solanki, A. et al. Intracellular paired agent imaging enables improved evaluation of tyrosine kinase inhibitor target engagement. Proc SPIE Int Soc Opt Eng 11219 (2020).

Complete Status: Complete

First Name: Allison

Last Name: Solanki

Email: solanki@ohsu.edu

Organization: OHSU

Country: United States 


\title{
ID: GA557 \\ Minocycline treatment during adolescence prevents structural and oxidative stress brain abnormalities at adulthood: a functional and structural imaging study in the Poly I:C rat model of schizophrenia
}

\author{
Diego Romero-Miguel, Fundación para la Investigación Biomédica del Hospital Gregorio \\ Marañón Biomedical Imaging and Instrumentation Group (BiiG), Idromero@hggm.es
}

Category: Neuroscience

\begin{abstract}
Body : Introduction: Minocycline (MIN) is a tetracycline with antioxidant, antiinflamatory and neuroprotective properties. Given the likely involvement of inflammation and oxidative stress (IOS) in schizophrenia, MIN has been proposed as a potential adjuvant treatment in this pathology 1,2 . Thus, we aim to evaluate whether MIN treatment during adolescence in the PolyI:C maternal immune stimulation (MIS) animal model of schizophrenia could prevent the brain physiological and behavioral deficits described at adulthood. To our knowledge, this is the first work to tackle this topic using positron emission tomography (PET) with 2-deoxy-2-[18F]fluoro- D-glucose (FDG), together with MRI voxel-based morphometry (VBM), adapted to rodents. We also evaluated improvements in IOS and behavior deficits as outcomes. Methods: In gestational day 15, PolyI:C (4 mg/kg) or saline (VH) were injected to pregnant Wistar rats. 93 male offspring received MIN (30 mg/kg) or saline (Sal) daily from postnatal day (PND) 35-49. Four groups were evaluated attending to the study factors: MIS condition (Saline, MIS) and treatment (VH, MIN). At PND70, rats were submitted to prepulse inhibition test (PPI). FDG-PET and T2-weighted MRI brain studies were performed at adulthood (PND120) and analyzed by means of SPM12. IOS markers (iNOS, COX2, GPx, CAT, SOD, MDA, NRF2, KEAP1, HO1, NQO1, GSH free , GSH total and GSSG) were evaluated by western blot, enzymatic activity and concentration assays in frozen tissue of prefrontal cortex, hippocampus, caudate- putamen, and amygdala. Results: MIS-offspring showed PPI deficits compared with VH-offspring but MIN treatment did not fully prevent this behavioral deficit. In addition, MIN prevented the volumetric abnormalities in the third ventricle but not in the hippocampus in MIS-offspring. Also, MIN reduced brain metabolism in the cerebellum and increased FDG uptake in the nucleus accumbens. Besides, MIN reduced the expression of iNOS in PFC and caudate putamen and increased the activity/concentration of KEAP1 in PFC, HO1 and NQO1 in amygdala, HO1 in caudate-putamen and HO1 and NQO1 in hippocampus. Conclusions: Our study demonstrates that a MIN treatment during adolescence partially counteracts volumetric abnormalities and IOS deficits via iNOS and Nrf2-ARE pathways in the MIS model, increasing the expression of cytoprotective enzymes. However, MIN treatment during this peripubertal stage does not prevent sensorimotor gating deficit. Therefore, despite not preventing all the pathological levels assessed, MIN effectivity highlights the usefulness of antiIOS compounds to halt the disease course at early stages. Acknowledgements: This study was supported by the Ministerio de Ciencia, Innovación y Universidades, ISCIII (PI14/00860, PI18/01691, PI17/01766, CPII14/00005) and Consejería de Educación e Investigación, Comunidad de Madrid (grants PEJD-2018-PRE/BMD-7899, PEJ- 2017-TL/BMD-7385) cofinanced by European Regional Development Fund (ERDF), “A way of making Europe”,
\end{abstract}


Delegación del Gobierno para el Plan Nacional sobre Drogas (2017/085, 2019/041), Fundación Mapfre, Fundación Alicia Koplowitz, Fundación Tatiana Pérez de Guzmán el Bueno, Pro-CNIC Foundation, Spanish Ministry of Economy, Industry and Competitiveness (MINECO-EUFEDER) SAF2016-75500-R, CIBERSAM, Ministerio de Economía y Competitividad (RTI2018099778-B-I00), Programa Operativo de Andalucía FEDER, Iniciativa Territorial Integrada ITI 2014-2020 Consejería Salud, Junta de Andalucía” (PI-0080-2017 and PI-0009-2017), Consejería de Salud de la Junta de Andalucía" (PI-0134- 2018), University of Cádiz (PR2019-046), Instituto de Investigación e Innovación en Ciencias Biomédicas de Cádiz-INiBICA (IN-C22; LI19/06INCO22), and Consejería de Economía, Innovación, Ciencia y Empleo de la Junta de Andalucía" (CTS-510).

References: 1 . Chaudhry, I.B., et al., Minocycline benefits negative symptoms in early schizophrenia: a randomised double-blind placebo-controlled clinical trial in patients on standard treatment. J Psychopharmacol, 2012. 26(9): p. 1185-93. 2. Kelly, D.L., et al., Adjunctive Minocycline in Clozapine-Treated Schizophrenia Patients With Persistent Symptoms. J Clin Psychopharmacol, 2015. 35(4): p. 374-81.

\section{Image/Figure:}

https://www.xcdsystem.com/wmis/abstract/File6959/GA557 ImageFigure 0613041752.jpg

Full Name of Abstract's 1st Author : Diego Romero-Miguel

Complete Status: Complete

First Name: Diego

Last Name: Romero-Miguel

Email: 1dromero@hggm.es

Organization: Fundación para la Investigación Biomédica del Hospital Gregorio Marañón Biomedical Imaging and Instrumentation Group (BiiG)

Country: Spain 


\title{
ID: GA558 \\ Sterically Shielded Heptamethine Cyanine Dyes for Bioconjugation and High Performance Near-Infrared Fluorescence Imaging
}

\author{
Cynthia Schreiber, University of Notre Dame, cschrei2@nd.edu
}

Category: New Chemistry, Biology \& Bioengineering

\begin{abstract}
Body : The near-infrared window of fluorescent heptamethine cyanine dyes greatly facilitates biological imaging because there is deep penetration of the light and negligible background fluorescence. But dye instability, aggregation, and poor pharmacokinetics are current drawbacks that limit performance and the scope of possible applications. All these limitations are simultaneously overcome with a new molecular design strategy that produces a charge balanced and sterically shielded fluorochrome. The key design feature is a meso-Aryl group that simultaneously projects two shielding arms directly over each face of a linear heptamethine polyene. Cell and mouse imaging experiments compared a shielded heptamethine cyanine dye (and several peptide and antibody bioconjugates) to benchmark heptamethine dyes and found that the shielded systems possess an unsurpassed combination of photophysical, physiochemical and biodistribution properties that greatly enhance bioimaging performance.
\end{abstract}

\section{Image/Figure:}

https://www.xcdsystem.com/wmis/abstract/File6959/GA558_ImageFigure_0613060613.png

Image/Figure Caption: Just like a superhero, an ultrastable shielded heptamethine cyanine dye uses its two strong arms to ward off self-aggregation and non-specific biological interactions. Yet the arms are short enough to allow dye-labeled bioconjugates to selectively target cell receptors for high-contrast and photon-intense microscopy or tumor imaging in living subjects.

Complete Status: Complete

First Name: Cynthia

Last Name: Schreiber

Email: cschrei2@nd.edu

Organization: University of Notre Dame

Country: United States 


\title{
ID: GA559 \\ Removal of noise in MRI-CEST images for improving tumor pH imaging
}

\author{
Feriel Romdhane, University of Turin, feriel.ramdhane@unito.it
}

\section{Category: Computational \& Data Science}

\begin{abstract}
Body : Introduction MRI-CEST pH imaging has shown a great utility in characterizing tumor acidosis and in assessing tumor $\mathrm{pH}$ heterogeneity [1]. However, when pushing the spatial or temporal resolution of the CEST-MRI images, the acquired low signal-to-noise ratio (SNR) images may significantly affect the accuracy of the tumor $\mathrm{pH}$ quantification. This work aims to investigate a new denoising approach for MRI-CEST images in simulated and real situations, to compare with different denoising methods and to validate in vivo its robustness to improve CEST contrast quantification and $\mathrm{pH}$ measurements. Methods Synthetic CEST data for the Iopamidol contrast agent were contaminated with two different levels of Rician noise. In vitro data were imaged with a Bruker 7T MRI scanner for Iopamidol solution titrated at different concentrations and $\mathrm{pH}$ values. Moreover, in vivo data were acquired upon iopamidol injection in a prostate orthotopic tumor murine model and the CEST contrast was measured as the difference between pre- and post-injection images and exploited for $\mathrm{pH}$ calculation. A novel denoising technique based on the combination between Non-Local Mean and Anisotropic diffusion Tensor (NLmCED) [2] was evaluated on simulated data corrupted by noise and validated by comparing with the ground truth. Further evaluation was performed with both in vitro and in vivo data in order to improve the CEST contrast quantification and $\mathrm{pH}$ measurements and the robustness and accuracy of the proposed approach was compared with conventional denoising methods: Gaussian filter and Cubic Smoothing Splines [3]. Results/Discussion As quantitative measures, both PSNR and the similarity between the reference and the denoised image (SSIM index) were calculated on the denoised synthetic data at two different level of noise (Fig.1 a-b). The NLmCED method exceed the other techniques by providing highest quantitative measures for the two pools of iopamidol. For in vitro data, comparable performance of the NLmCED was obtained at different iopamidol concentrations and $\mathrm{pH}$ values (Fig. $1 \mathrm{c}-\mathrm{d}$ ). The obtained results for denoising in vivo data validated the performance of the NLmCED algorithm which succeeded to increase the fraction of pixels in the region of interest (ROI) (Fig.1 i-k) by surpassing both the Gaussian filter and the Cubic Smoothing Spline method, improving both the contrast detection and $\mathrm{pH}$ measurement (Fig.1 e-h). Conclusion The proposed denoising method is expected to have a great utility for robust and accurate tumor $\mathrm{pH}$ imaging and quantification with the MRI-CEST technique following Iopamidol injection. Acknowledgement This work was supported by grants from the Associazione italiana Ricerca Cancro (AIRC MFAG \#20153).
\end{abstract}

References: References [1] P. C. Zijl van and N. N. Yadav, "Chemical exchange saturation transfer (CEST): what is in a name and what isn't?", Magn Reson Med, vol. 65, pp. 927-48, Apr 2011. [2] F. Romdhane, F. Benzarti and H. Amiri, "A new method for three-dimensional magnetic resonance images denoising", Int. J. Computational Vision and Robotics, Vol. 8, No. 1, 2018. [3] J. Stancanello, E. Terreno, DD. Castelli, C. Cabella, F. Uggeri and S. Aime, " 
Development and validation of a smoothing-splines-based correction method for improving the analysis of CEST-MR images.", Contrast Media Mol Imaging. Jul-Aug 2008.

\section{Image/Figure:}

https://www.xcdsystem.com/wmis/abstract/File6959/GA559 ImageFigure 0614124518.jpg

Image/Figure Caption: Reduction noise for simulated and in vitro data and improvement of the CEST contrast and $\mathrm{pH}$ quantificationsfor ROI in vivo animal : (a) PSNR index, (b) SSIM index for ST $4.2 \mathrm{ppm}$, (c) ST for $4.2 \mathrm{ppm}$ results for different $\mathrm{pH}$, (d) ST for $4.2 \mathrm{ppm}$ results for different concentration for in vitro data, (e-h) $\mathrm{pH}$ map for different denoising methods for ROI in vivo animal data, and (i-k) Fraction pixel for $\Delta$ ST $4.2 \mathrm{ppm}, \Delta \mathrm{ST} 5.5 \mathrm{ppm}$ and pH respectively for ROI in vivo animal data.

Full Name of Abstract's 1st Author : Feriel Romdhane

Complete Status: Complete

First Name: Feriel

Last Name: Romdhane

Email: feriel.ramdhane@unito.it

Organization: University of Turin

Country: Italy 


\title{
ID: GA560 \\ Elucidating the Role of Hypoxia and CXCR4 on Acute Myeloid Leukemia Pathogenicity Using Intravital Imaging
}

\author{
Helen Jin, University of Toronto, helenjingshu.jin@uhnresearch.ca
}

\section{Category: Oncology}

\begin{abstract}
Body : Introduction: Acute myeloid leukemia (AML) is a disease of the bone marrow (BM) initiated by acquired mutations of resident hematopoietic stem cells (HSC). Interactions between AML and the supporting BM microenvironment play important protective and promotive functions for cancer development1. A hallmark of the BM is its low oxygen partial pressure, which is required for long-term maintenance of HSCs2. While in solid tumours hypoxia has been associated with poor prognosis3, its role in leukemias is not yet fully understood due to the inaccessibility of the BM space for in vivo study. Recently, an intravital imaging mouse model was developed that makes it possible to view and monitor leukemia cells orthotopically within the femoral BM for a few hours a session, repeatedly, over the course of two months4. Here we applied the novel femur window chamber (FWC) technique to study the role of hypoxia on AML proliferation, maintenance, and therapy resistance, and how these effects may be mediated by the chemokine receptor CXCR4. Methods: To monitor cellular hypoxia, the murine myeloid cell line 32DKit was transfected with a fluorescent hypoxia reporter (GFP driven by five hypoxia response elements5). 32DKit cells were also modified to express varying levels of CXCR4 to study the relationship between the chemokine, hypoxia, and AML development. 1x106cells were intravenously injected into each group of 8 week old $\mathrm{C} 3 \mathrm{H} / \mathrm{HeJ}$ mice, which were then transplanted with the FWC two weeks post-inoculation. FWC installation involved shaving the femoral corticalis to reveal the BM cavity, then securing a cover glass on top4 (Fig. A). Fluorescent cellular activity within the BM was monitored two times a week for four weeks via confocal microscopy (Zeiss LSM710). Leukemic burden was quantified as the percent total fluorescent signal within the BM space while levels of hypoxia were quantified as the ratio of GFP to mCherry signal above threshold intensities. Nonfluorescent leukemic and healthy mice were also imaged as controls. AML response to hypoxia-targeted treatment was evaluated using mouse groups administered with $10 \mu \mathrm{g} / \mathrm{kg}$ of the antibiotic echinomycin6, intraperitoneally injected for five consecutive days one week post-inoculation.
\end{abstract}

Results: Preliminary results validate the utility of our AML hypoxia reporter cell line in detecting varying hypoxic levels both in vitro and in vivo (Fig. B-C). Interestingly, we observed that AML cells tended to engraft as (and then proliferate within) distinct cell clusters surrounding sinusoids within the vascular niche of the BM, with cells localized at the edges of the clusters being more hypoxic than those towards the center. Longitudinal monitoring of the BM further revealed leukemia-associated changes in the overall proportion of hypoxic cells within the microenvironment. Treatment with echinomycin induced a noticeable transformation in AML cellular conformation, altering the large cell clusters seen prior to treatment to a more individualized and diffuse cellular arrangement three weeks post-treatment. We further examined the influence of CXCR4 on leukemia progression given its role in normal hematopoietic cell homing, growth, and proliferation, which may in part be mediated by BM hypoxia. AML cells 
induced to overexpress CXCR4 were observed to result in higher leukemic burdens when compared to unmodified cells (Fig. D-F). Discussion: Current efforts are now underway to clarify the role of hypoxia in activating AML CXCR4 by examining the direct spatiotemporal relationship between the two in vivo. Future studies will focus on targeting these factors using novel therapeutic agents, characterizing drug effect in real-time using the $\mathrm{FWC}$, and examining the potential of anti-CXCR4 and hypoxia combinatorial strategies. Integration of molecular imaging into therapy development can provide invaluable biological readouts regarding treatment action in vivo. Our FWC model provides an innovative means by which to quantitatively study AML cell response to new therapies within the living BM for preclinical trials.

References: 1. Zhou, H.-S., Carter, B. Z. \& Andreeff, M. Bone marrow niche-mediated survival of leukemia stem cells in acute myeloid leukemia: Yin and Yang. Cancer Biol. Med. 13, 248259 (2016). 2. Mimeault, M. \& Batra, S. K. Hypoxia-inducing factors as master regulators of stemness properties and altered metabolism of cancer- and metastasis-initiating cells. J. Cell. Mol. Med. 17, 30-54 (2013). 3. Vaupel, P. \& Mayer, A. Hypoxia in cancer: significance and impact on clinical outcome. Cancer Metastasis Rev. 26, $225-239$ (2007). 4. Chen, Y., Maeda, A., $\mathrm{Bu}, \mathrm{J}$. \& DaCosta, R. Femur Window Chamber Model for In Vivo Cell Tracking in the Murine Bone Marrow. J. Vis. Exp. JoVE (2016) doi:10.3791/54205. 5. Raman, V. et al. Characterizing Vascular Parameters in Hypoxic Regions: A Combined Magnetic Resonance and Optical Imaging Study of a Human Prostate Cancer Model. Cancer Res. 66, 9929-9936 (2006). 6. Wang, Y., Liu, Y., Malek, S. N., Zheng, P. \& Liu, Y. Targeting HIF $1 \alpha$ Eliminates Cancer Stem Cells in Hematological Malignancies. Cell Stem Cell 8, 399-411 (2011).

\section{Image/Figure:}

https://www.xcdsystem.com/wmis/abstract/File6959/GA560_ImageFigure_0613064930.png

Image/Figure Caption: Figure: a) Established protocol for FWC surgery: the femur is exposed by blunt dissection and a U-shaped bar is inserted underneath the bone to secure the FWC in place. b) Quantitative comparison of hypoxia reporter cell fluorescent signal under normoxia and hypoxia in vitro. c) Intravital image of the hypoxia reporter cell line within an AML cell cluster. d) Intravital images of BM AML burden with cells expressing basal vs. elevated levels CXCR4. Magnified images demonstrate ability to resolve individual cells; e) quantification of fluorescent signal and f) ex vivo flow cytometry confirms imaging results. Scale bars: $50 \mu \mathrm{m}$; Error bars $=$ mean $+\mathrm{SD}, * * * \mathrm{p}<0.01$ (two-tailed t test).

Full Name of Abstract's 1st Author : Helen Jingshu Jin

Complete Status: Complete

First Name: Helen

Last Name: Jin

Email: helenjingshu.jin@uhnresearch.ca 
Organization: University of Toronto

Country: Canada 


\title{
ID: GA561
}

NCI perspective: Imaging Cancer inflammation

\author{
Janet Eary, National Cancer Institute, janet.eary@nih.gov
}

Category: Immunology: Inflammation \& Infection

\begin{abstract}
Body : Inflammation is a normal process in our body; acute inflammation acts to suppress infections and support wound healing, while chronic inflammation can lead to cancer. Chronic inflammation often does not exhibit the classic signs and symptoms of inflammation and is thus often neglected until a disease like cancer is clinically apparent. The biological understanding of the transition from acute to chronic inflammation is important as it can provide an opportunity for clinical intervention and in monitoring disease progression. Current clinical evaluation for inflammatory status in an individual patient relies on immunological tests of biological fluids and pathological analyses of biopsied tissue samples. Molecular imaging techniques that can track the changes of molecular signatures, immune cell trafficking, metabolic, and functional parameters are essential for obtaining a better understanding of the role that inflammation plays in the development and progression of cancer. It can also help us understand the biology of cancer development and progression, while providing insights into new targets for cancer treatment. NCI is interested in providing a pathway for adoption of such molecular imaging techniques for the understanding of cancer inflammation.
\end{abstract}

Complete Status: Complete

First Name: Janet

Last Name: Eary

Email: janet.eary@nih.gov

Organization: National Cancer Institute

Country: United States 


\title{
ID: GA562 \\ Development of Novel 64Cu-Labeled PET Radiotracers for SARS-Cov-2
}

\author{
Jianzhong Xian, Fifth Affiliated Hospital of Sun Yat-sen University, \\ xianjzh@mail.sysu.edu.cn
}

Category: Immunology: Inflammation \& Infection

\begin{abstract}
Body : Objective: Coronavirus Disease 2019（COVID-19） which is caused by SARS-CoV-2 has become a pandemic all over the world. To specifically detect SARS-CoV-2 in live subject, PET tracers specifically recognize proteins of SARS-CoV-2 are urgently needed. Human angiotensin-converting enzyme 2 (hACE2) or the A-peptide of hACE2 from the binding interface, have shown specifically binding to $\mathrm{S} 1$ subunit in spike (S) protein of SARS-CoV-2[1]. After binding of receptor-binding domain (RBD) in S1 subunit of S protein, the heptad repeat 1 (HR1) and 2 (HR2) domains in S2 subunit of S protein interact with each other to form a sixhelix bundle (6-HB) fusion core, bringing viral and cellular membrane into close proximity for fusion and infection [2]. Herein, we reported our effort on developing potent recombinant ACE2, A-peptide, or HR peptide as PET radiotracers for SARS-CoV-2. Method: The recombined protein ACE2-PD (binding to S1), A-peptide (binding to S1) and HR-peptide (binding to S2) were modified by biotin, fluorescent dye $\mathrm{Cy} 5$ and NOTA to facilitate in vitro study, fluorescence cell sorting and in vivo PET imaging respectively. The biotinylated peptides were carried out in vitro assays to detect if they can specifically bind to S protein. The Cy5 labeled peptides were tested by FACS and cell immunofluorescence with S protein positive HEK-293 cells. S protein positive and negative HEK-293 cells were seeded in athymic nude mice to test the binding specificity of NOTA-modified peptides for S-protein of SARS-CoV-2 in vivo. The $64 \mathrm{Cu}-$ Labeled PET radiotracers including [64Cu]-NOTA-hACE2, [64Cu]-NOTA-A-peptide and [64Cu]-NOTA-HR-peptide were performed in vivo experiments. Results: The saturation binding revealed that biotin-ACE2 recombinant protein, biotin A-peptide and biotin HR-peptide specifically bound to the $\mathrm{S}$ protein with high affinity $(\mathrm{Kd}=0.046 \mathrm{nM}, 3.88 \mathrm{nM}, 3.56 \mathrm{nM}, \mathrm{Bmax}$ $=2.0,2.6,3.0$ (A450), respectively) (Figure 1). The competition assay determined binding potency for S1-RBD recombined protein (ACE2-PD, A-peptide) or S protein full-length recombined HR-peptide, IC50 value of $3.09 \mathrm{nM}, 10.3 \mathrm{nM}, 15.7 \mathrm{nM}$ respectively (Figure 1). FACS revealed that these peptides specifically bind to the cells expressing $\mathrm{S}$ protein. Immunofluorescence showed that the A-peptide was stained around the cellular membrane of S protein positive HEK-293 cells. The PET imaging revealed that the 64Cu-A-peptide-NOTA can specifically bind to S-protein positive tumor in live animals (Figure 1). Conclusion: Our data demonstrated that newly designed 64Cu-labled NOTA-peptides radiotracer specifically binds to $\mathrm{S}$ protein in vitro and in vivo. These peptide radiotracers represent a promising technique for imaging and quantification COVID-19 in clinic.
\end{abstract}

References: 1. Zhou, P. et al. A pneumonia outbreak associated with a new coronavirus of probable bat origin. Nature 579, 270-273 (2020). 2. Bosch, B. J. et al. Severe acute respiratory syndrome coronavirus (SARS-CoV) infection inhibition using spike protein heptad repeatderived peptides. Proc. Natl. Acad. Sci. USA 101, 8455-8460 (2004). 


\section{Image/Figure:}

https://www.xcdsystem.com/wmis/abstract/File6959/GA562_ImageFigure_0614124617.png

Image/Figure Caption: Figure 1: Characterization of rhACE2, A-peptide and HR-peptide binding to the $\mathrm{S}$ protein of SARS-coV-2. Enzyme-linked immunosorbent assay (ELISA) was performed to test recombined protein biotin-ACE2 binding (A), A-peptide (C), and HR-peptide (E) binding to $\mathrm{S}$ protein receptor binding district (S-RBD). B Cell immunofluorescence of rhACE2 or A-peptide for S-protein positive HEK-293 cells, the binding of S-protein to ACE2 receptor was recognized by the Virus-specific A-peptide signal of S-protein. The Cy5-labeled Apolypeptide (red) was used to accurately co-localize S-protein and the membrane-expressed ACE2 receptor (green). D. Fluorescent-activated cell sorting (FACS) was used to test the binding ability with S protein in cellular level of ACE2, A-peptide and HR-peptide. F. The microPET/CT images were captured from [64Cu]-NOTA-rhACE2 for in vivo studies indicated that the PET tracer showing high radioactive uptake only in S-protein positive tumor in live subjects.

Full Name of Abstract's 1st Author : Jianzhong Xian

Complete Status: Complete

First Name: Jianzhong

Last Name: Xian

Email: xianjzh@mail.sysu.edu.cn

Organization: Fifth Affiliated Hospital of Sun Yat-sen University

Country: China 


\title{
ID: GA563 \\ Imaging predictors of response to radioligand therapy of metastatic castrate- resistant prostate cancer
}

\author{
Boon Lee, GenesisCare, boon.quanlee@genesiscare.com
}

\section{Category: Oncology}

\begin{abstract}
Body : Aims: Mean and maximum standardized uptake values (SUV) of pre-therapy 68Ga-PSMA images and total tumour volume (TTV) were retrospectively evaluated in predicting treatment response and disease progression after 177Lu-PSMA therapy. Methods: Whole-body SUVmax, SUVmean and TTV on baseline PSMA PET scans were determined in 34 mCRPC patients. Tumour segmentation was performed by automatic SUV $>3$ thresholding using MIM Encore 6.9.4. Prostate-specific antigen (PSA) level measurements were determined every two weeks before and after therapy. Patients received between 1-5 cycles of 177Lu-PSMA. Patients with a PSA decline ( $>50 \%)$ were grouped as responders and assessed by Overall Survival (OS) and Progression-Free Survival (PFS) as endpoints. Results: Patient ages ranged from 54.45 to 92.47 years (mean 71.65 \pm 7.95 ). Baseline PSA levels ranged from 0.11-930 ng/ml (mean $76.62 \pm 174.20$ ). Nineteen $(56 \%)$ of the 34 men had a PSA reduction $>50 \%$ (mean reduction $82 \%$ ). Mann-Whitney analysis showed no statistically significant differences between SUVmax and SUVmean values when comparing responders ( $>50 \%$ decrease in PSA level) to non-responders ( Conclusion: PET/CT plays an important role in the staging and prediction of treatment response. In this heterogenous mCRPC group receiving 177Lu-PSMA therapy, SUVmean and SUVmax of PSMA PET were not predictive of treatment response but a small TTV could be predictive of response.
\end{abstract}

Full Name of Abstract's 1st Author : Boon Quan Lee

Complete Status: Complete

First Name: Boon

Last Name: Lee

Email: boon.quanlee@genesiscare.com

Organization: GenesisCare

Country: Australia 


\title{
ID: GA564 \\ In vivo Comparison of [18F]FBZA, [18F]P3BZA and [18F]DMPYs as PET imaging agents for malignant melanoma detection
}

\author{
Ayoung Pyo, Chonnam National University, payjal@naver.com
}

\section{Category: Oncology}

\begin{abstract}
Body : Objectives: Malignant melanoma has one of the highest mortality rates of any cancer because of its aggressive nature and high metastatic potential. Clinical staging of the disease at the time of diagnosis is very important for the prognosis and outcome of melanoma treatment. In this study, we designed and synthesized the $18 \mathrm{~F}$-labeled pyridinebased benzamide derivatives N-(2-(dimethylamino)ethyl)-5-[18F]fluoropicolinamide ([18F]DMPY2) and N-(2(dimethylamino)ethyl)-6-[18F]fluoronicotinamide ([18F]DMPY3) and compared with conventional positron emission tomography (PET) imaging agents in early stage primary and metastatic melanoma animal models. Methods: [18F]DMPY2 and [18F]DMPY3 were synthesized by direct radiofluorination of the bromo precursor, and radiochemical yields were $\sim 15-20 \%$. B16F10 (mouse melanoma) cells $(5 \times 104)$ were plated into a 24-well plate and were incubated at $37^{\circ} \mathrm{C}$ for 10,30 , and $60 \mathrm{~min}$ with $0.74 \mathrm{MBq}$ of [18F]DMPY2 and [18F]DMPY3. B16F10 cells $(1 \times 106)$ were subcutaneously implanted into the right shoulder of nude mice. After about 10 days, mice were used for experiments when the tumor size reached approximately 100 to $150 \mathrm{~mm} 3$. For the SK-MEL-3 (human melanoma) subcutaneous tumor model, $3 \times 106$ SK-Mel-3 cells in $100 \mu \mathrm{l}$ PBS were implanted into the right shoulder of nude mouse that were imaged after 21 days. The mircoPET images were obtained at 60 min after i.v. injection of N-[2(diethylamino)-ethyl]-4-[18F]fluorobenzamide ([18F]FBZA), N-[2-(diethylamino)-ethyl]-5[18F]fluoropicolinamide ([18F]P3BZA) and [18F]DMPY2 (7.4MBq) in B16F10 or SK-MEL-3 subcutaneous tumor bearing mouse model. The static images at $60 \mathrm{~min}$ were acquired for 10 min. Results: Cell uptakes of [18F]DMPY2 and [18F]DMPY3 were $>103$ fold and 18-fold higher, respectively, in B16F10 cells than in negative control cells. Biodistribution studies revealed strong tumor uptake and retention of [18F]DMPY2 $(24.8 \%$ injected dose per gram of tissue $[\mathrm{ID} / \mathrm{g}]$ at $60 \mathrm{~min})$ and [18F]DMPY3 $(11.7 \% \mathrm{ID} / \mathrm{g}$ at $60 \mathrm{~min})$ in B16F10 xenografts. MicroPET imaging of [18F]DMPY2 demonstrated strong tumoral uptake/retention and rapid washout, resulting in excellent tumor-to-background contrast in B16F 10 xenografts.

Furthermore, The tumor-to-liver ratio of [18F]DMPY2 was over 11.0-fold and 14.7-fold higher than that of [18F]P3BZA and [18F]FBZA, respectively ([18F]DMPY2: $36.29 \pm 3.55$; [18F]P3BZA: $3.28 \pm 1.34$; [18F]FBZA: $2.47 \pm 0.24 ; * \mathrm{P}<0.05$; Fig. 1C). This uptake pattern was reproduced in an SK-MEL-3 human melanoma xenograft Foxn1nu mouse model. The tumor-to-liver ratio of [18F]DMPY2 was more than 4.5- and 7.6-fold higher than that of [18F]P3BZA and [18F]FBZA, respectively ([18F]DMPY2: 10.30 \pm 2.22 ; [18F]P3BZA: $2.25 \pm$ 0.36; [18F]FBZA: $1.35 \pm 0.30 ;{ }^{*} \mathrm{P}<0.05$; Fig. 1F). Conclusion: [18F]DMPY2 might have strong potential for the diagnosis of early stage primary and metastatic melanoma using PET.
\end{abstract}


References: 1. H. Liu et al., Development of 18F-labeled picolinamide probes for PET imaging of malignant melanoma. J. Med. Chem. 56, 895-901 (2013) 2. G. Ren et al., Melanin-targeted preclinical PET imaging of melanoma metastasis. J. Nucl. Med. 50, 1692-1699 (2009).

\section{Image/Figure:}

https://www.xcdsystem.com/wmis/abstract/File6959/GA564_ImageFigure 0614031322.jpg

Image/Figure Caption: Figure 1. (A) MicroPET images of [18F]DMPY2, [18F]P3BZA and $[18 \mathrm{~F}] \mathrm{FBZA}$ at 60 min postinjection in B16F10 subcutaneous tumor model (blue arrow) (B) Tumor uptake values and $(\mathrm{C})$ tumor-to-liver ratios of [18F]DMPY2, [18F]P3BZA and [18F]FBZA at 60 min in B16F10 xenografts $(* \mathrm{P}<0.05)$. (D) MicroPET images of [18F]DMPY2, [18F]P3BZA and [18F]FBZA at 60 min postinjection in SK-MEL-3 subcutaneous tumor model (green arrow). (E) Tumor uptake values and (F) tumor-to-liver ratios of [18F]DMPY2, [18F]P3BZA and [18F]FBZA at 60 min in SK-MEL-3 xenografts $(* \mathrm{P}<0.05)$.

Full Name of Abstract's 1st Author : Ayoung Pyo

Complete Status: Complete

First Name: Ayoung

Last Name: Pyo

Email: payjal@naver.com

Organization: Chonnam National University

Country: Korea, Republic of 


\title{
ID: GA565 \\ ELECTRO-CARDIAC MAPPING AS CARDIAC ANATOMICAL DYNAMIC IMAGING IN STEM CELL THERAPY
}

\author{
RAKESH SHARMA, Florida State University, rksz2009@gmail.com
}

\section{Category: Cardiovascular \& Pulmonary}

\begin{abstract}
Body : Electro-cardiac mapping is a smart technique to visualize the cardiac muscle functionality and status of heart function. In case of incurable or unmanageable conditions of ischemic heart disease or heart failure, stem cell therapy is good option as optimal delivery of stem cells to revive the cardiac viability. Stem cell delivery is given as intramyocardial, epicardial and intracoronary injections. In present time, intramyocardial NOGA system is popular choice. The NOGA system delineates the cardiac target sites for stem cell therapy and NOGA system navigates the cardiac recovery and its rate of deployment. The system visualizes 3D heart endocardial surface using spatial, mechanical and electrophysilogical information of major left ventricle apex region undergoing necrosis, ischemia spreading over normal surface from base to apex, inferior to anterior, septal to lateral border zone regions (see the Figure). The NOGA system distinguishes infarct regions (low linear local shortening and low unipolar voltage) from ischemic regions(low linear local shortening and preserved unipolar voltage). The approaches of catherization in NOGA system are transfemoral and transradial guidewires. Still investigations and clinical trials are in progress. In future, clinical success will decide the safe application of NOGA system.
\end{abstract}

References: Rakesh Sharma(2018) Stem Cells in Clinical Practice and Tissue Engineering. InTech Publishers Pvt Co.London. https://www.intechopen.com/books/stem-cells-in-clinicalpractice-and-tissue-engineering

\section{Image/Figure:}

https://www.xcdsystem.com/wmis/abstract/File6959/GA565_ImageFigure_0614035226.jpg

Image/Figure Caption: Clear border zones at the interection of infarct and normal cardiac apex tissue are shown.

Full Name of Abstract's 1st Author : Rakesh Sharma

Complete Status: Complete

First Name: RAKESH

Last Name: SHARMA

Email: rksz2009@gmail.com 
Organization: Florida State University

Country: India 


\title{
ID: GA566 \\ Silica Coated Super-paramagnetic Iron Oxide Nanoparticles: A New Generation in Vivo T1 MRI Contrast Agent
}

\author{
M. Zubair Iqbal, Zhejiang Sci-Tech University, zubair@zstu.edu.cn
}

\section{Category: Oncology}

\begin{abstract}
Body : Abstract Magnetic resonance imaging (MRI), a sophisticated promising threedimensional tomographic noninvasive diagnostic technique, has intrinsic advantage in safety when compared with radiotracer and optical imaging modalities. However, MRI contrast agents are less sensitive than complexes used in other imaging techniques and toxicity issue still endures in nanoparticle-based MRI-T1 contrast agents. Therefore, demand for nontoxic novel (T1\&T2) T1-weighted MRI potential candidate with ultrasensitive imaging and advanced functionality is very high. In this research, silica coated ultra small monodispersed superparamagnetic iron oxide nanoparticles were synthesized via thermal decomposition which demonstrated high performance T1-weighted MRI contrast agent for heart, liver, kidney and bladder. Advanced characterization techniques were used to investigate the crystal structure, morphological evaluation, concentration of iron, size distribution, active modes and magnetization of as-synthesized nanoparticles. Transmission electron microscopy (TEM) results have illustrated that the diameter of SPIONPs was in the range of $4 \mathrm{~nm}$ and the average size of Fe3O4@SiO2 was about 30nm 40nm. X-ray diffraction (XRD) and Raman spectroscopy analyses revealed the purity in phase of the prepared SPIONPs. These magnetite nanoparticles exhibited weak magnetic moment at room temperature because of spin-canting effect which escorted high positive signal enhancement ability. MCF-7 and HeLa cell viabilities experiments demonstrated good biocompatibility of the SPIONPs. In addition, the first ever study has been made on T1 MRI contrast agent of silica coated ultra small (4nm-sized) magnetite nanoparticles exhibited a good $\mathrm{r} 1$ relaxivity of 1.2 and low $\mathrm{r} 2 / \mathrm{r} 1$ ratio of 6.5 , attributed to low magnetization, large surface area and 5 unpaired valence electrons on the surface of Fe3+. In vivo T1-weighted MR imaging of heart, liver, kidney and bladder after intravenous injection of nanoparticles further verified the high sensitivity and biocompatibility of as-synthesized magnetite nanoparticles. These results reveal silica coated SPIONPs as a promising candidate for T1 contrast agent with extraordinary capability to enhance MR images.
\end{abstract}

References: 9. M. Zubair Iqbal, Tianxiang Chen, Xuehua Ma, Ling'e Zhang, Wenzhi Ren et al, Silica Coated Super-paramagnetic Iron Oxide Nanoparticles (SPIONPs): A New Type Contrast Agent of T1 Magnetic Resonance Imaging (MRI), J. Materials Chemistry B, 2015, 3, 5172-5181. (Featured in Back Cover)

\section{Image/Figure:}

https://www.xcdsystem.com/wmis/abstract/File6959/GA566_ImageFigure 0614035801.jpg

Complete Status: Complete 
First Name: M. Zubair

Last Name: Iqbal

Email: zubair@zstu.edu.cn

Organization: Zhejiang Sci-Tech University

Country: China 


\title{
ID: GA567 \\ In vivo MRI-CEST tumor $\mathrm{pH}$ imaging can detect early resistance to proton pump inhibitors in human prostate cancer murine models
}

\author{
Pietro Irrera, Moffitt Cancer Center, pietro.irrera@moffitt.org
}

\section{Category: Oncology}

\begin{abstract}
Body : INTRODUCTION Tumor lesions have common characteristics among different type of cancer like genetic mutations, altered metabolism, hypoxia and extracellular acidosis. Because survival in the tumor microenvironment depends on $\mathrm{pH}$ homeostasis, interference with $\mathrm{pH}$ regulating systems is considered a new therapeutic strategy. Therefore, novel imaging approaches are urgently needed for non-invasively assessing the efficacy of novel anticancer therapies. Recent pre-clinical investigations reported promising results with proton pump inhibitors (PPIs) treatments in tumor therapy, both individually and combined with a chemotherapeutic drug [1-3]. Aim of this work was to evaluate MRI-CEST (chemical exchange saturation transfer) tumor $\mathrm{pH}$ imaging to assess treatment response to esomeprazole, a proton pump inhibitor (PPI) and to investigate the efficacy of this new therapeutic strategy in vivo. MATERIALS AND METHOD Cell viability and $\mathrm{pH}$ measurements were performed in vitro for the following drugs: lansoprazole, esomeprazole (V-ATPases), cariporide and amiloride (NHE1). Expression of V-ATP and NHE1 was quantified by RT-PCR and WB in vitro. For in vivo experiments, athymic nude mice were inoculated subcutaneously with human prostate PC3 or DU145 cells and treated with esomeprazole (dose: $2.5 \mathrm{mg} / \mathrm{kg}$ ) for two weeks and tumor growth was monitored. A fast-multi-slice CEST sequence was used for $\mathrm{pH}$ imaging following iopamidol injection. MRI-CEST tumor $\mathrm{pH}$ imaging and FDG-PET were perfomed one week after treatment for assessing early treatment response by measuring tumor acidosis and glucose uptake. RESULTS AND DISCUSSION Only esomeprazole and amiloride treatments reported a dosedependent and marked alkalinisation of the extracellular environment together with a dosedependent cell death in in vitro studies. Despite a potent effect of esomeprazole in vitro, in vivo studies showed no reduction in tumor growth for both the two prostate tumor murine models after two weeks of treatment (Fig.1 a,c). MRI pH imaging detected similar baseline tumor $\mathrm{pH}$ acidosis between PC3 and DU145 models. Interestingly, as early as one week after treatment, tumor $\mathrm{pH}$ imaging demonstrated no differences in tumor acidosis between treated and control mice (Fig.1 b,d). FDG-PET imaging confirmed the observed findings showing no differences in the glucose uptake levels for PC3 mice between treated and untreated groups (Fig.1 e). CONCLUSION Esomeprazole provided a marked $\mathrm{pH}$ alteration in vitro in both human prostate cell lines but it was not effective in reducing tumor growth rate in vivo. MRI-CEST tumor $\mathrm{pH}$ imaging showed, as early as one week after the treatment, no effects in tumor $\mathrm{pH}$ values for both the prostate murine models. Therefore, this approach can be used to detect upfront resistance to PPIs for validating novel therapeutic strategies that are effective only in in vitro conditions but not in vivo. Acknowledgements: This work was supported by the Compagnia San Paolo (Regione Piemonte, project grant \#CSTO165925) and by Associazione Italiana Ricerca Cancro (AIRC MFAG \#20153)
\end{abstract}


References: [1] Azzarito T et al; Cancer Lett. 2015;356(2 Pt B):697-703 [2] De Milito A et al; Cancer Res 2007;67(11):5408-5417 [3] Luciani F et al; J Natl Cancer Inst 2004;96(22):17021713

\section{Image/Figure:}

https://www.xcdsystem.com/wmis/abstract/File6959/GA567_ImageFigure 0614050351.jpg

Image/Figure Caption: Figure 1. Volume variations of tumor mass during esomeprazole treatment for DU145 and PC3 cells $(\mathrm{a}, \mathrm{c})$. Extracellular $\mathrm{pH}$ values after one week of esomeprazole treatment $(\mathrm{b}, \mathrm{d})$. Glucose consumption in PC3 tumor model after esomeprazole treatment (e).

Complete Status: Complete

First Name: Pietro

Last Name: Irrera

Email: pietro.irrera@moffitt.org

Organization: Moffitt Cancer Center

Country: United States 


\title{
ID: GA568 \\ MONITORING CARDIAC STEM CELL THERAPY BY NANOPARTICLES AT 900 MHz MAGNETIC RESONANCE MICROSCOPY
}

\author{
RAKESH SHARMA, Florida State University, rksz2009@gmail.com
}

\section{Category: New Chemistry, Biology \& Bioengineering}

\begin{abstract}
Body : Background:Cardiovascular and coronary heart diseases involve molecular and tissue level damage of blood vessels and heart. Coronary Heart Disease and heart failure are the leading cause of mortality worldwide. Stem cell transplantation is emerging as a new treatment option. Hypothesis:Stem cells are capable to reach and settle down at damaged cardiac tissue. This stem cell option also repairs the myocardial infarction area in heart or vascular territories and ultimately reduces the infarct-related mortality. Materials and Methods: Non-invasive cardiovascular imaging monitors the real-time status of cardiovascular remodeling or differentiated stem cell autografting. Cardiac magnetic resonance imaging (MRI) and bioluminescence are robust non-invasive monitoring techniques to visualize cardiovascular structure changes due to myocardial dysfunction or restorative myocardial recovery. Results: The present study highlights the sources, types, delivery methods of stem cells in cardiovascular treatment, advantages and current limitations of stem cell monitoring, scopes of ultra-high field cardiac $900 \mathrm{MHz}$ Bruker Biospin MRI and bioluminescence methods applied in stem cell transplantation, to translate stem cell molecular events into clinical success and evaluation of rejuvenation rate with future perspectives. At $900 \mathrm{MHz}$ magnetic field, microscopy offers the images up to 15 micron spatial resolution of cardiac tributaries and myocyte behavior. The imaging method is capable of recording physiological functionality of beating heart of animal placed in magnet. Possibly, pluripotent cells are MRI visible. Discussion: The in vivo imaging techniques are useful in dynamic monitoring of cardiac stem cell therapy following myocardial infarction. Choice of stem cells and mode of delivery are very crucial in getting successful stem cell therapy positive outcome. Cardiovascular remodeling evaluation by MRI has merits because it is safe, sensitive, lacks radiation, provides good resolution, generates a real-time events' blueprint or first-hand information of myocardial viability with functional information of cardiac territories and their physiochemical changes in cardiac functions during stem cell rejuvenating process and after myocardial repair. Present time, ultra-high magnetic field CMR possibly has preclinical prospects as in vivo noninvasive molecular imaging or restorative monitoring reporter of rejuvenating stem cell genes to evaluate success of transplantation and cardiac repair. On the other side of coin, researchers are continuously developing new real-time physiological cum functional MRSI options to explore new stem cell molecular probes and smart MRS imaging sequences with improved MRI sensitive specific stem cell differentiation and rejuvenating detection by targeting energy metabolites, myocardial viability, and vital physiochemical molecules. Noninvasive monitoring is necessary and bioluminescence or other radionuclide methods may be alarming because the potential biological damage caused by radionuclide exposed reporter genes and bioluminescence induced immune responses is concern in differentiating stem cells. Seriously, all these issues need research to minimize artifacts within safe limits. With the help of stem cell imaging and monitoring, transplantation of stem cells
\end{abstract}


sooner or later will be optimized for the effective long-lasting therapy of myocardial infarction and heart failure on some day. Conclusion: The right choice of stem cells, pluripotent stem cell delivery, transplantation and real-time monitoring of stem cell trafficking enhances the stem cell therapeutic efficacy in cardiac engraftment and differentiation.

References: Sharma, R. (2012). Antibodies in Nanomedicine and Microimaging Methods. In Antibody-Mediated Drug Delivery Systems (eds Y. Pathak and S. Benita).

doi:10.1002/9781118229019.ch15

\section{Image/Figure:}

https://www.xcdsystem.com/wmis/abstract/File6959/GA568 ImageFigure 0614051617.jpg

Image/Figure Caption: Superparamagnetic Iron Oxide bound antibody imaging at $900 \mathrm{MH}$ MRI microimager to visualize acrdiac muscle fibres orientation distorsion due to ischemic heart. The 15 micron level dissection is possible by imager.

Full Name of Abstract's 1st Author : Rakesh Sharma

Complete Status: Complete

First Name: RAKESH

Last Name: SHARMA

Email: rksz2009@gmail.com

Organization: Florida State University

Country: India 


\title{
ID: GA569 \\ A Case Of Meckel Diverticulum
}

Binh Duong, Vietnamese National Cancer Hospital (K hospital), ddbinhtm@gmail.com

\section{Category: Instrumentation}

\begin{abstract}
Body : Aim/Introduction: Gastrointestinal bleeding is quite common in children, often self-limiting and is a benign situation. However, hemorrhage could be dangerous without proper treatment and it is important to discover the causes. Meckel's diverticulum is one of the most reasons causing the lower gastrointestinal bleeding in healthy children, with more than $50 \%$ of patients in 2-year-old stage. In this article, a 22 months infant with recurrent lower gastrointestinal bleeding was reported, Meckel's diverticulum was diagnosed with 99mTcpertechnetate SPECT/CT. Patient had endoscopic resection and Meckel's diverticulum was confirmed with pathology. Materials and Methods: After injection of 2,3 mCi 99mTcpertechnetate at left hand dorsal vein, planar acquisition in chest, abdominal and pelvis areas with dynamic phase ( 3 second / frame in one minute), static phase ( 5 minutes / frame in 30 minutes; static images at 45 and 60 minute) and abdominal SPECT/CT with SPECT/CT Discovery NM/CT 670 Pro. Results: An abnormal, focus increased uptake in the right lower quadrant of abdomen with intensity was equivalent to the stomach and accumulated through time. It was considered as Meckel's diverticulum. Conclusion: Meckel's diverticulum is one of the most common gastrointestinal congenital diseases. Concisely pre-surgery diagnosis is difficult due to discrete clinical manifestations and limitation of conventional diagnostic radiology. SPECT/CT scintigraphy with $99 \mathrm{mTc}$-pertechnetate is an useful examination.
\end{abstract}

References: 1. Spottswood SE, Pfluger T, Bartold SP, et al. SNMMI and EANM Practice Guideline for Meckel Diverticulum Scintigraphy 2.0. J Nucl Med Technol. 2014;42(3):163-169. doi:10.2967/jnmt.113.136242. 2. Wong CS, Dupley L, Varia HN, Golka D, Linn T. Meckel's diverticulitis: a rare entity of Meckel's diverticulum. J Surg Case Reports. 2017;2017(1):rjw225. doi:10.1093/jscr/rjw225. 3. Society THER. Jayesh Sagar 1 Vikas Kumar 2 D K Shah 3. 2006:501-505. 4. Kotha VK, Khandelwal A, Saboo SS, et al. Radiologist's perspective for the Meckel's diverticulum and its complications. Br J Radiol. 2014;87(1037). doi:10.1259/bjr.20130743. 5. Elsayes KM, Menias CO, Harvin HJ, Francis IR. Imaging manifestations of Meckel's diverticulum. Am J Roentgenol. 2007;189(1):81-88. doi:10.2214/AJR.06.1257. 6. Levy AD, Hobbs CM. From the archives of the AFIP. Meckel diverticulum: radiologic features with pathologic Correlation. Radiographics. 2004;24:565-587. doi:10.1148/rg.242035187. 7. Tayeb M, Mohammad Khan F, Rauf F, Khan MM. Phytobezoar in a jejunal diverticulum as a cause of small bowel obstruction: A case report. J Med Case Rep. 2011;5(1):66-67. doi:10.1186/1752-1947-5-482. 8. Gelfand MJ, Parisi MT, Treves ST. Pediatric Radiopharmaceutical Administered Doses: 2010 North American Consensus Guidelines. J Nucl Med. 2011;52(2):318-322. doi:10.2967/jnumed.110.084327. 9. Activity B. Dosage Card. Eanm. 2008;43(0):1-2. doi:10.1111/j.1750-3841.2009.01080.x.

\section{Image/Figure:}


Complete Status: Complete

First Name: Binh

Last Name: Duong

Email: ddbinhtm@gmail.com

Organization: Vietnamese National Cancer Hospital (K hospital)

Country: Vietnam 


\title{
ID: GA570 \\ F18-Fluorodeoxy Glucose PET-Computed Tomography with High Magnetic Field Sodium MRI Hybrid Imaging To Test Chemosensitivity
}

\author{
RAKESH SHARMA, Florida State University, rksz2009@gmail.com
}

\section{Category: Oncology}

\begin{abstract}
Body : Background: Increased CT-PET and Sodium MRI image intensities of soft tissue prostate tumors were correlated with histostaining excised tumor characteristics. Hypothesis: Increased intracellular sodium $\mu \mathrm{MRI}$ signal intensities and flouro-2-deoxy-glucose utilization by $\mu \mathrm{PET}$ in apoptosis rich regions appearing dark-gray $\mathrm{CT}$ visible tumors were positively correlated as chemosensitivity assay of Taxotere. Validation: The PC-3 cancer cell line induced prostate tumor MRI and CT-PET images and histology slices were digitally captured and compared in pre- and post-Taxotere treated tumors. Clinical Correlation: The optimization of inversion recovery sodium MRI parameters was done to generate sodium images of prostate patients $(n=10)$. The 18FDG biotransformation was optimized to measure CT-PET image houncefield-SUV intensities. A criterion was developed to evaluate pre-malignancy by histology and correlate with hybrid CT-PET-MRI. Materials: For correlation, regression analysis was done using imaging, histology, and immunostaining data from soft tissue prostate tumor after 24 and 48 hours post-Taxotere treatment. Results: Apoptosis indices were calculated by histostaining and ss-DNA antibody assay. Sodium MRI and CT-PET signal intensity distributions were comparable at specific locations relatively and measured in tumor tissue data trained color coded set regions. In tumors, Taxotere induced an increase in intracellular sodium MRI signal 30\% (p 6\%). Apoptosis rich regions showed characteristic nuclei with S phase DNA histogram, appearing brighter on IC-Na images and mild active on CT-PET images (sensitivity $=65 \%$; specificity $=70 \%$ ). Conclusion: MRI and CT-PET multimodal imaging may be rapid non-invasive chemosensitivity assay to monitor the drug anticancer effect in soft tissue prostate tumors. Hybrid approach may validate the locations of different tumor stages as distinct by using immunosensitive, histology characterization.
\end{abstract}

References: Sharma, R., \& Katz, J. K. (2008). Taxotere Chemosensitivity Evaluation in Mice Prostate Tumor: Validation and Diagnostic Accuracy of Quantitative Measurement of Tumor Characteristics by MRI, PET, and Histology of Mice Tumor. Technology in Cancer Research \& Treatment, 175-185. https://doi.org/10.1177/153303460800700303

\section{Image/Figure:}

https://www.xcdsystem.com/wmis/abstract/File6959/GA570 ImageFigure 0614070252.jpg

Image/Figure Caption: The figure represents delineated tumor areas on sodium MRI (column 1), CT-PET images (column 2), fused MRI-CT-PET images (column 3) of soft tissue prostate tumors in top row and 24 hours post-Taxotere treated prostate tumors at bottom row. For clarity of trimmed MRI image A match with histology digital image (column 4) is shown with different 
tumor regions of 24 hours post-Taxotere treated tumor. These images show comparable tumor regions in MRI with CT-PET fused and histology images with distinct signal intensities with tumor area measurement to test diagnostic accuracy. The qualitative criterion suggested different tumor or pre-malignancy stages while quantitative criterion suggested severity of cell death during tumor progress and the post-Taxotere induced chemosensitive response.

Full Name of Abstract's 1st Author : Rakesh Sharma

Complete Status: Complete

First Name: RAKESH

Last Name: SHARMA

Email: rksz2009@gmail.com

Organization: Florida State University

Country: India 


\title{
ID: GA573 \\ Synthesizing and functionalizing of $\mathrm{X}$-ray luminescence nanoparticles and studying x-ray stimulated behavior of Caenorhabditis elegans for optogenetic studies
}

\author{
Meenakshi Ranasinghe, Clemson University, mranasi@g.clemson.edu
}

\section{Category: Neuroscience}

\begin{abstract}
Body : Rare-earth doped phosphors have interesting optical properties depending on their size, crystal structure and type of dopant. They are used in bioimaging, chemical sensing and radiology imaging screens. We are interested in synthesizing $\mathrm{x}$-ray excited nanophosphors for a non-invasive, in vivo optogenetic neuron simulation method. However, it is challenging to synthesize monodisperse nano-size particles that emit bright visible light photons when irradiated with X-ray. We are synthesizing Eu-doped NaGdF4 (NaGdF4: Eu) nanoparticles using a citrate method which involves lanthanides-citrate complex formation followed by nucleation and growth upon the addition of $\mathrm{NaF}$. Then, the nanoparticles are annealed at high temperature (up to $1000^{\circ} \mathrm{C}$ ) to increase the emission intensity by removing defect sites that can quench luminescence. To prevent nanoparticle sintering during annealing we encapsulated NaGdF4: Eu nanoparticle in a silica shell. The synthesized NaGdF4: Eu nanoparticles are characterized using dynamic light scattering, powder $\mathrm{x}$-ray diffraction, transmission electron microscopy and x-ray luminescence spectroscopy. The TEM images confirm that the size of the nanoparticles is around $100 \mathrm{~nm}$. According to the x-ray luminescence spectroscopy measurements, at low and high dopant levels, they show low emission intensity, likely due to lack of luminescent centers and self-quenching, respectively. Hydrothermal treatment increased the emission intensity by a factor of 2-3 and annealing without a silica shell increased the emission intensity by another factor of 5 which resulted in sintered particles, negating their bio-application. However, this may have potential to form thin films which use in solar cells and fluorescence screens. Formation of silica shell resulted in a decrease in emission intensity due to boundary defects and small molecules (H2O and CO2) trap in the shell. After annealing silica-coated NaGdF4: Eu, there is no large increase in intensity compared to pristine nanoparticles. Annealing at $350-450{ }^{\circ} \mathrm{C}$ resulted in porous NaGdF4: Eu coated with silica which may have potential drug delivery applications. At high temperatures, silica-coated NaGdF4: Eu change its structure to Eu doped sodium gadolinium silicate. We functionalized NaGdF4: Eu@SiO2 nanoparticles with Biotin and confirmed attaching to streptavidin by performing in vitro study using streptavidin-coated microbeads. The second part of the project is to study radiation-induced behavior of $\mathrm{C}$. elegans. They are widely used as unique model-organism in neuronal networks, cancer studies, aging studying, etc. Here, we are interested in the radiation-induced locomotory behavior of C. elegans. Our study shows that they show x-ray induced locomotory movements and their LITE-1 photoreceptor is responsible for the observation. In the future, we plan to optimize the synthesis, annealing, and surface passivation protocols to obtain bright monodisperse nanophosphors. We will also functionalize them and test their application as in situ light sources for sensing and actuating in vivo using $\mathrm{C}$. elegans is an animal model. Further, we will study the mechanism of $\mathrm{x}$-ray induced behavior of $\mathrm{C}$. elegans.
\end{abstract}


References: LITE-1 Dependent X-Ray Sensitivity in C. elegans Kelli E. Cannon, Meenakshi Ranasinghe, Anhkhoa Do, H. Mariah Pierce, Paul W. Millhouse, Ayona Roychowdhury, Stephen Foulger, Lynn Dobrunz, Jeffrey N. Anker, Mark Bolding bioRxiv 766568; doi: https://doi.org/10.1101/766568

Complete Status: Complete

First Name: Meenakshi

Last Name: Ranasinghe

Email:mranasi@g.clemson.edu

Organization: Clemson University

Country: United States 


\title{
ID: GA574 \\ Anti-PD-L1 immunoPET unravels receptor changes post-oncolytic virotherapy
}

\author{
Julia Hoebart, The Institute of Cancer Research, Julia.Hoebart@icr.ac.uk
}

\section{Category: Oncology}

\begin{abstract}
Body : Immune checkpoint inhibitors (ICIs) harness the body's immune system to fight cancer.[1] Despite promising clinical outcomes in some malignancies, the vast majority of cancer patients show poor to moderate responses owing to insufficient immune activation of tumours, as observed in head and neck cancer.[2] This pitfall can be addressed by combining ICIs with oncolytic virotherapy (OV), an approach to immunologically pre-condition tumours for the onset of ICI treatment.[3,4] However, the combination of ICIs and OV is still in the early stages of development and further research is required to better understand and fully exploit its potential. Hence, we investigate systemic expression of the immune checkpoint programmed death receptor ligand 1 (PD-L1) post-OV using immunoPET. An IgG2b-based anti-PD-L1 positron emission tomography (PET) radioconjugate (89Zr-DFO- $\alpha$ PD-L110F.9G2) was used to study spatiotemporal dynamics of PD-L1 expression after OV in a syngeneic mouse model of oral carcinoma. For OV, a single dose of a genetically engineered herpes simplex type 1 virus (RP1) was injected intratumourally. 89Zr-DFO- $\alpha$ PD-L110F.9G2 (2 MBq) was administered intravenously $(\sim 10 \mu \mathrm{g}$ of radiolabelled co-injected with $\sim 100 \mu \mathrm{g}$ of unlabelled anti-PD-L1 antibody) and static PET/CT scans with concomitant biodistribution studies were performed on days 3 and 7 post-OV. PD-L1 immunohistochemistry (IHC) was used to confirm antigen expression levels in tumour and spleen samples. Multiparametric flow cytometry (FC) was used to assess expression of PD-L1 and closely related immune checkpoint receptors on CD45+ cells residing in tumours and spleens. Using PET, we found that RP1 injection significantly increased 89Zr-DFO- $\alpha$ PD-L110F.9G2 uptake in spleens and tumour draining lymph nodes 3 days after treatment. By day 7, 89Zr-DFO- $\alpha$ PD-L110F.9G2 uptake in these organs had returned to pretreatment levels. In contrast, tumours of treated mice showed no significant change in radioconjugate uptake. Levels of intratumoural and intrasplenic 89Zr-DFO- $\alpha$ PD-L110F.9G2 uptake were in line with PD-L1 IHC performed on representative sections of both organs. FC analysis showed a trend toward an increase in PD-L1+CD45+ tumour-infiltrating cells on day 3 but not day 7 after treatment, while PD-L2+CD45+ cells followed an inverse trend. Interestingly, PD-1 expressing CD45+ cells were elevated on day 7 after OV in tumours and spleens likewise. In summary, intratumoural RP1 injection leads to early, systemic but not intratumoural PD-L1 upregulation, as detected via anti-PD-L1 immunoPET. Forthcoming studies will assess the potential of 89Zr-DFO- $\alpha$ PD-L110F.9G2 to guide the onset of anti-PD-(L)1 ICI treatment postOV.
\end{abstract}

References: 1 Ribas, A., et Wolchok J.D., Cancer Immunotherapy Using Checkpoint Blockade. Science, 2018. 359: 1350-1355 2 Ferris, R.L., et al., Nivolumab for Recurrent Squamous-Cell Carcinoma of the Head and Neck. N Engl J Med, 2016. 375:1856-1867 3 Samson, A., et al., Intravenous Delivery of Oncolytic Reovirus to Brain Tumor Patients Immunologically Primes for Subsequent Checkpoint Blockade. Sci Transl Med, 2018. 10(422): eaam7577. 4 Ribas, A., et 
al., Oncolytic Virotherapy Promotes Intratumoral T Cell Infiltration and Improves Anti-PD-1 Immunotherapy. Cell, 2017. 170(6): 1109-1119

\section{Image/Figure:}

https://www.xcdsystem.com/wmis/abstract/File6959/GA574_ImageFigure_0614072819.png

Image/Figure Caption: Figure 1: Tracking changes of PD-L1 expression via immunoPET A. Illustration of experimental timeline B. Biodistribution data from days 3 and 7 post-RP1 or PBS injection; organ uptake values from tumours, spleens and tumour draining (TD) lymph nodes are expressed as \% injected dose per gram (ID/g); bars indicate mean average of each group C.

Representative PET/CT scans of RP1-treated mice taken at $48 \mathrm{~h}$ post-89Zr-DFO- $\alpha \mathrm{PD}-$

L110F.9G2 injection

Full Name of Abstract's 1st Author : Julia Hoebart

Complete Status: Complete

First Name: Julia

Last Name: Hoebart

Email: Julia.Hoebart@icr.ac.uk

Organization: The Institute of Cancer Research

Country: United Kingdom 


\title{
ID: GA575 \\ A genetic targeted mechanogenetics by noninvasive ultrasound
}

\author{
Xuandi Hou, The Hong Kong Polytechnic University, xuandi.hou@connect.polyu.hk
}

\section{Category: Neuroscience}

\begin{abstract}
Body : Techniques for noninvasive and remote brain stimulation have long been desired for both probing brain functions and treating brain dysfunctions. Potential technologies candidate for brain stimulation include optical, magnetic, electric, and acoustic brain stimulation [1-5]. Of these, ultrasound brain stimulation is considered among the most promising technologies since ultrasound can non-invasively penetrate through the skull into deep brain structures with high spatiotemporal resolution [6]. Recently, ultrasound neuromodulation has gained increasing attention, although the spatial resolution of ultrasound is sufficient for regionspecific brain stimulation, it lacks cellular and molecular specificity. Here, we developed an ultrasonic mechanogenetic tool to manipulate the neuronal activity and signaling with excellent precision by introducing gas-filled nanostructures, gas vesicles (GVs). GVs have unique size which can induce highly localized ultrasonic pressure gradient, unique microstreaming and shear stress in low intensity ultrasound field $[7,8]$. In this study, we customized low intensity low frequency pulsed ultrasound stimulation system and a high-speed fluorescence microscopy was employed for calcium imaging. Neuronal calcium concentration was monitored simultaneously as a readout under ultrasonic stimulation. We hypothesized that the GVs could oscillate and consequently trigger widespread and reversible firing of neurons when exposed to ultrasound field. We showed that GVs mediated ultrasound can directly activate cultured neurons and initiate calcium influx (Figure 1). Further, the induced calcium response could be significantly suppressed by inhibiting the mechanosensitive ion channels with ruthenium red (RR), indicating that these ion channels mediated this kind of neuron activation. To manipulate target cells activities, we further sensitized the neurons by overexpression of MscL, a mechanosensitive ion channel. It showed that MscL-neurons could be activated under much lower ultrasound intensity and c-Fos expression significantly increased in the MscL-transfected cells, compared with the control. Furthermore, the parameters of ultrasound excitation we used do not affect cell viability or cell membrane integrity and temperature change. Altogether, our findings demonstrated that under low intensity ultrasound irradiation, local ultrasound pressure will be significantly intensified where the oscillating GVs are placed and this GVs mediated ultrasound system can activate mechanosensitive ion channels and control calcium release in neurons. Moreover, the activity of mechanosensitive ion channels such as MscL stimulated by ultrasound is an important contributor to its ability to stimulate specific cells in vitro.
\end{abstract}

References: [1]. Chen, R., Romero, G., Christiansen, M. G., Mohr, A., \&Anikeeva, P. (2015). Wireless magnetothermal deep brain stimulation. Science, 347(6229), 1477-1480. [2]. Grossman, N., Bono, D., Dedic, N., Kodandaramaiah, S. B., Rudenko, A., Suk, H. J., ...Boyden, E. S. (2017). Noninvasive Deep Brain Stimulation via Temporally Interfering Electric Fields. Cell, 169(6), 1029-1041.e16. [3]. Gradinaru, V., Mogri, M., Thompson, K. R., Henderson, J. M., \&Deisseroth, K. (2009). Optical deconstruction of parkinsonian neural circuitry. Science, 
324(5925), 354-359. [4]. Hamblin, M. R. (2016). Shining light on the head: Photobiomodulation for brain disorders. BBA Clinical, 6, 113-124. [5]. Dayan, E., Censor, N., Buch, E. R., Sandrini, M., \&Cohen, L. G. (2016). Noninvasive brain stimulation from physiology to network dynamics and back Nature Neuroscience Nature Publishing Group. Nat Neurosci, 16(7), 838-844. [6]. Tyler, W. J., Lani, S. W., \&Hwang, G. M. (2018). Ultrasonic modulation of neural circuit activity. Current Opinion in Neurobiology, 50, 222-231. [7]. Shapiro, M. G., Goodwill, P. W., Neogy, A., Yin, M., Foster, F. S., Schaffer, D.V., \&Conolly, S. M. (2014). Biogenic gas nanostructures as ultrasonic molecular reporters. Nature Nanotechnology, 9(4), 311-316. [8]. Yang, Y., Qiu, Z., Hou, X., \&Sun, L. (2017). Ultrasonic Characteristics and Cellular Properties of Anabaena Gas Vesicles. Ultrasound in Medicine \& Biology, 1-9.

\section{Image/Figure:}

https://www.xcdsystem.com/wmis/abstract/File6959/GA575 ImageFigure_0614073913.png

Image/Figure Caption: Figure 1. Left: scheme of GVs mediated neuron stimulation by interacting with membrane surface mechanosensitive ion channel upon ultrasound illuminating. Right: calcium imaging of neurons with and without GVs before and after ultrasound stimulation.

Full Name of Abstract's 1st Author : Xuandi HOU

Complete Status: Complete

First Name: Xuandi

Last Name: Hou

Email: xuandi.hou@connect.polyu.hk

Organization: The Hong Kong Polytechnic University

Country: China 


\title{
ID: GA576 \\ $\mathrm{N}$-acetylcysteine treatment during pregnancy prevents biochemical, neuroanatomical and behavioral deficits in the offspring of a maternal immune stimulation rat model
}

\author{
Nicolás Lamanna-Rama, Fundación para la Investigación Biomédica Gregorio Marañón, \\ nlamanna@hggm.es
}

Category: Neuroscience

\begin{abstract}
Body : Introduction. Schizophrenia (SCZ) is a chronic debilitating psychiatric disorder that affects $1 \%$ of the population worldwide. Although its etiology remains unknown, oxidative stress (OS) and inflammation have been hypothesized to play key roles in the pathophysiology of this disease (1). This has led the researchers to promote the use of anti-inflammatory/antioxidant drugs as therapeutic strategies in the treatment of SCZ. In this sense, $\mathrm{N}$-acetylcysteine (NAC) has gained special attention, since it increases the biosynthesis of the antioxidant glutathione by regulating the cysteine amino acid availability (2). To date, NAC has been tested in patients with schizophrenia or first-episode psychosis as adjunctive drug to antipsychotics, but the results so far diverge between studies. Aim. To evaluate whether NAC administration during pregnancy may prevent neuroanatomic, biochemical and behavioral deficits in the maternal immune stimulation (MIS) rat model of schizophrenia at adulthood. Methods. Pregnant Wistar rats were administered with Poly I:C (4 mg/kg) or Saline in the gestation day (GD) 15. NAC was administered from the immune insult to the end of the gestation (GD15-21) or during the whole gestation period (GD1-GD21). Male pups were divided in six groups: Saline-naïve, Saline-NAC 7 days (NAC7d), Saline-NAC 21 (NAC21d) days, MIS-naïve, MIS-NAC7d and MIS-NAC21d. At postnatal day (PND) 70 the elevated plus maze (EPM) test was performed for evaluating anxiety. At PND100, single-voxel magnetic resonance spectroscopy (MRS) of hippocampus and prefrontal cortex, and T2-weighted images, were acquired. MRS results were analyzed with LCModel software. Morphometric brain changes were determined with voxel-based morphometry (VBM) analyses with SPM12 (3). Data were analyzed by 2-ways ANOVAs. Results. In the EPM test (figure A), MIS-naïve offspring significantly remained more time in closed arms and less time in open arms than Saline-naïve offspring. These behavioral abnormalities were significantly prevented by NAC treatment in MIS-offspring. MRS results (figure B) showed a significant NAC effect in macromolecules and lipids 9 and 20, and a nearly significant effect for glycerophosphocholine plus phosphocholine in hippocampus. Moreover, N-acetylaspartate showed a slight dependence with NAC dose, both in Saline and in MIS. Furthermore, it seems that NAC corrects for the possible NAA deficiency in MIS. Finally, VBM analysis (figure C) showed an interesting significant enlargement in prefrontal cortex in MIS-NAC7d, but not in MIS-NAC21d. Also, MIS-NAC7d showed an augmentation in gray matter volume in septum and piriform cortex. On the other hand, MIS-NAC21d showed bilateral enlargement in the thalamus. Conclusions. Our study demonstrates that the administration of NAC during pregnancy prevented the deficits in anxiety in the MIS model. Moreover, it could prevent the atrophy in thalamus and prefrontal cortex reported in schizophrenia $(4,5)$, and also slightly prevent some metabolic brain changes, such as the deficit of N-acetyl-aspartate in hippocampus. Together,
\end{abstract}


these results suggest that NAC could be useful as therapeutic strategy to prevent or halt the progression into a schizophrenia-like condition after infection-mediated neurodevelopmental disturbances.

References: 1. Wu JQ, Kosten TR, Zhang XY. Prog Neuro-Psychopharmacology Biol Psychiatry. 2013;46:200-6. 2. Berk M, Malhi GS, Gray LJ, Dean OM. Trends Pharmacol Sci. 2013;34(3):167-77. 3. Casquero-Veiga M, García-García D, MacDowell KS, et al. European Neuropsychopharmacol. 2019;29(7):880-96. 4. Csernansky JG, Schindler MK, Splinter NR, et al. Am J Psychiatry. 2004;161(5):896-902. 5. Ohtani T, Levitt JJ, Nestor PG, et al. Schizophr Res. 2014;152(1):184-90.

\section{Image/Figure:}

https://www.xcdsystem.com/wmis/abstract/File6959/GA576_ImageFigure 0614075203.jpg

Image/Figure Caption: Figure 1. A. Elevated plus maze test: time in opened and closed arms, together with number of head dippings, were represented as indirect measurements of anxiety levels. Each column represents the mean \pm SEM. 2-way-ANOVAs were performed, followed by LSD post-hoc test $\left[{ }^{*} \mathrm{p}<0.05,{ }^{* *} \mathrm{p}<0.01,{ }^{* * *} \mathrm{p}<0.001 ; \$ \mathrm{p}<0.05, \$ \mathrm{p}<0.001\right.$, against saline naïve]. B. Magnetic resonance spectroscopy (MRS): brain metabolite levels relative to total creatine were calculated in prefrontal cortex and hippocampus. 2-way ANOVAs + Bonferroni post-hoc test [*p $<0$.05]. Abbreviations: GPC+PCh (glycerophosphocholine+phosphocholine), NAA+NAAG (N-acetyl aspartate), Glu+Gln (glutamate+glutamine), MM09+Lip09 (macromolecule $09+$ lipid 09), MM20+Lip20 (macromolecule $20+$ lipid 20). C. Voxel-based morphometry: voxel-based SPM results in T2 maps overlaid on a T2-MR template showing NAC effect in both Saline and MIS offspring with a treatment of 7 or 21 days of NAC. The color bars represent the T-values corresponding to higher (warm) and lower (cold) gray matter changes. Right (R) and left (L) hemispheres. Abbreviations: Cerebellum (Cb), Prefrontal Cortex (PFC), Globus Pallidum (GP), Thalamus (Th), Piriform Cortex/Amygdala (Pir/AA).

Full Name of Abstract's 1st Author : Nicolás Lamanna-Rama

Complete Status: Complete

First Name: Nicolás

Last Name: Lamanna-Rama

Email: nlamanna@hggm.es

Organization: Fundación para la Investigación Biomédica Gregorio Marañón

Country: Spain 


\title{
ID: GA577 \\ Cannabidiol treatment during adolescence induces volumetric and metabolic changes in the maternal immune stimulation rat model of schizophrenia: a combined T2 and MRS study
}

\author{
Nicolás Lamanna-Rama, Fundación para la Investigación Biomédica Gregorio Marañón, \\ nlamanna@hggm.es
}

Category: Neuroscience

\begin{abstract}
Body : Introduction: Oxidative stress (OS) and inflammation might play an important role on the pathophysiology of mental diseases, which suggest a potential benefit of antiinflammatory/antioxidant compounds in these pathologies[1]. Cannabidiol (CBD), a major nonpsychotropic constituent of cannabis, shows antiinflammatory/antioxidant properties, generating certain interest as a therapeutic strategy for mental disorders[2]. In this sense, CBD has been used as adjunctive therapy in schizophrenia, reducing positive psychotic symptoms and improving better cognitive performance[3]. However, its efficacy in earlier stages of the disease in patients at high-risk of psychosis has not been evaluated yet. Objective: To evaluate the effects of CBD treatment during adolescence on schizophrenia-related deficits in the maternal immune stimulation (MIS) rat model of schizophrenia at adulthood. Methods: Pregnant Wistar rats were administered with Poly I:C (4 mg/kg) or saline in gestational day 15. Male offspring were treated with CBD $(10 \mathrm{mg} / \mathrm{kg})$ or its vehicle (VH, ethanol: cremophor: saline; 1:1:18) i.p. from post-natal day (PND) 35 to PND50. Four groups were evaluated attending to the study factors: MIS condition (Saline, MIS) and treatment (VH, CBD) (N=12 per group). Prepulse inhibition test (PPI) was performed on PND70 to evaluate sensorimotor gating deficits. Single-voxel magnetic resonance spectroscopy (MRS) of hippocampus (Hipp) and prefrontal cortex (PFC), and T2weigthed brain images were acquired on PND100. MRS results were normalized to total creatine, and analysed by means of LC Model software. Volumetric brain changes were determined by conventional regions of interest (ROI) and voxel-based morphometry (VBM) analysis with SPM12. Data were assessed by means of 2-ways ANOVAs followed by Bonferroni post-hoc tests ( $\mathrm{p}$ Results: MIS-offspring showed PPI deficits compared with saline-offspring and CBD showed a trend in the prevention of these behavioral deficits (Fig.1.A). ANOVA analysis of MRS data showed a treatment effect in glutamine + glutamate (Gln + Glu) and Nacetylaspartate $+\mathrm{N}$-Acetylaspartylglutamate (NAA + NAAG) in the PFC; and in glycerophosphocholine + phosphocholine $(\mathrm{GPC}+\mathrm{PCh})$ and NAA + NAAG in the Hipp. A MIS effect was produced on macromolecule $20+$ lipid 20 (MM20 + Lip20). Bonferroni post-hoc test showed that CBD increased Gln + Glu in the PFC of MIS-offspring, as well as NAA + NAAG and MM20 + Lip20 in the PFC of saline-offspring (Fig.1.B). In addition, we found a reduction in volume in the Hipp and PFC, together with a ventricle enlargement in MIS-offspring compared with saline-offspring (Fig.1.C). CBD effects on volumetric changes are still in progress, but we hypothesize that $\mathrm{CBD}$ will prevent the volumetric abnormalities in our MIS model. Conclusions: Our study demonstrates that the administration of CBD during adolescence almost prevented the sensorimotor gating deficits in the MIS model. Moreover, CBD prevented some brain metabolic abnormalities suggesting an improvement of the neuronal integrity and the glutamatergic status,
\end{abstract}


mainly in the PFC. Together, these results suggest that CBD could be a potential therapeutic preventive strategy for the onset of schizophrenia.

References: [1] Upthegrove R, Khandaker GM. Cytokines, Oxidative Stress and Cellular Markers of Inflammation in Schizophrenia. Curr Top Behav Neurosci. 2020;44:49-66. [2] Osborne AL, Solowij N, Weston-Green K. A systematic review of the effect of cannabidiol on cognitive function: Relevance to schizophrenia. Neurosci Biobehav Rev. 2017;72:310-324. [3] McGuire P, Robson P, Cubala WJ, et al. Cannabidiol (CBD) as an Adjunctive Therapy in Schizophrenia: A Multicenter Randomized Controlled Trial. Am J Psychiatry. 2018 Mar 1;175(3):225-231. [4] Paxinos, G. and C. Watson, The rat brain in stereotaxic coordinates. 4th ed. 2008, San Diego: Academic Press.

\section{Image/Figure:}

https://www.xcdsystem.com/wmis/abstract/File6959/GA577_ImageFigure_0614080344.png

Image/Figure Caption: Figure 1. A) PPI study: Averaged PPI values under three different prepulse intensities $(74 \mathrm{~dB}, 80 \mathrm{~dB}$ and $86 \mathrm{~dB})$ in the saline and MIS-offspring. Repeated measures 3-way ANOVA + Bonferroni post-hoc test [\$p N-Acetylaspartylglutamate). Table shows mean values \pm SEM of brain metabolites in Hipp and PFC. Results are expressed as mean \pm SEM. C) Volumetric study: Manual regions of interest (ROI) were segmented as follows (coordinates from Bregma)[4]: Hipp ( -2.12 to $-6.7 \mathrm{~mm})$, PFC (4.70 to $-1.40 \mathrm{~mm}$ ) and ventricles $(1.60$ to $-8.30 \mathrm{~mm})$. Results are expressed as mean $\pm \mathrm{SEM}$.

Full Name of Abstract's 1st Author : Nicolás Lamanna-Rama

Complete Status: Complete

First Name: Nicolás

Last Name: Lamanna-Rama

Email:nlamanna@hggm.es

Organization: Fundación para la Investigación Biomédica Gregorio Marañón

Country: Spain 


\title{
ID: GA578 \\ Synthesis and initial characterization of Gd(III) complex of salinomycin as a novel theranostic probe for magnetic resonance imaging (MRI)
}

\author{
Irena Pashkunova-Martic, Medical University of Vienna, irena.pashkunova- \\ martic@meduniwien.ac.at
}

\section{Category: Oncology}

\begin{abstract}
Body : Recently toxicity concerns associated with the long-term use of low molecular weight acyclic gadolinium based contrast agents (GBCA) have resulted in the restriction of their administration by the European Medicines Agency (EMA) and triggered risk warnings from the U.S. Food and Drug Administration (FDA). Therefore, the research on novel chelators for paramagnetic centers as part of magnetic resonance imaging (MRI) contrast agents with better stability and safer profile are subject of intense research during the past few years. Furthermore, the development of new molecularly targeted contrast agents or agents that can sense
\end{abstract} pathological changes in the tumor microenvironment is among the key goals in modern oncological research. Main purpose of this work was the assessment of novel Salinomycin (SAL) based theranostic probes for magnetic resonance imaging (MRI). SAL is a natural antibiotic produced by Streptomyces albus. SAL was found to selectively eradicate breast cancer stem cells (CSCs) and to be at least 100-fold more effective than paclitaxel (a commonly used breast-cancer drug). Selective towards tumors, theranostic agents can revolutionize early diagnosis and therapy of cancer. In this study, first paramagnetic complex of SAL with Gd(III) was synthesized and characterized by various spectroscopic methods such as IR, EPR and ESI-MS. The potential of the complex as a theranostic agent for MRI was evaluated by in vitro relaxivity measurements on a 9.4 T MR scanner. The elemental analysis demonstrated that the complex is of a composition [Gd(C42H69O11)3(H2O)3]. The ESI-MS spectrum of the compound corresponded to elemental analysis data. In the IR spectrum of the Gd(III) complex two characteristic bands at $1540 \mathrm{~cm}-1$ and $1400 \mathrm{~cm}-1$ corroborated monodentate coordination mode of carboxylate anion to $\mathrm{Gd}(\mathrm{III})$. The shift of the band for the carbonyl group from $1700 \mathrm{~cm}-1$ to $1690 \mathrm{~cm}-1$ compared to the IR spectrum of salinomycinic acid proved participation of the carbonyl group of the antibiotic in weak hydrogen bonds. The strong broad band at $3400 \mathrm{~cm}-1$ confirmed the presence of hydrogenbonded hydroxyl groups. The EPR spectrum of the Gd(III) complex of SAL consisted of several overlapping signals as three of which with effective g-factors $6,2.8$ and 2 dominated. The recorded EPR spectrum described Gd3+ ions in a crystal field with low symmetry and zero field splitting parameter $\mathrm{D}>0.3 \mathrm{~cm}-1$. Overall the spectral data revealed tricapped trigonal prismatic molecular geometry of the Gd(III) complex of SAL. Three salinomycinate monoanions are coordinated to the metal center via deprotonated carboxyl group and terminal hydroxyl group. Three water molecules, located in the inner coordination sphere of Gd(III) stabilize the structure of the complex by hydrogen bonds with the organic ligand. The relaxivity of Gd(IIII) complex of SAL was similar to the relaxivity of the clinical contrast agent Magnevist. Moreover, Gd(III) trisalinomycinate showed pronounced cytotoxicity against three human tumor cell lines A549, non-small cell lung carcinoma (IC50: $0.15 \pm 0.12 \mu \mathrm{M}$ ); SW480, colon cancer (IC50: $0.36 \pm 0.12$ $\mu \mathrm{M}$ ) and $\mathrm{CH} 1 / \mathrm{PA} 1$, ovarian teratoma-cancer (IC50: $0.09 \pm 0.03 \mu \mathrm{M}$ ). Novel paramagnetic 
complex of SAL with Gd (III) was successfully prepared and characterized. This research is the first assessment, which demonstrates the potential of Gd(III) complex of salinomycin as a theranostic agent for MRI. Acknowledgements: The authors acknowledged the financial support from the National Science Fund of Bulgaria (grant KP-06-Austria-6/6.08.2019), the Austrian Federal Ministry of Education, Science and Research (BMBWF) (project No: BG 07/2019) and the FWF (Austrian Science Fond: T 1145-B).

References: 1) Clough T.J., Jiang L., Wong K.-L., Long N. J. Nat Commun 10, 1420-1434, 2019. 2) Dekkers I. A., Roos R., Van Der Molen, A. J. Eur. Radiol. 28, 1579-1584, 2018. 3) Gupta P.B., Onder T.T., Jiang G., Tao K., Kuperwasser C., Weinberg R.A., Lander E.S. Cell, 138, 645-659, 2009. 4) Huczynski A. Chem. Biol. Drug Des, 79, 235-238, 2012. 5) Ivanova J., Pantcheva I.N., Zhorova R., Momekov G., Simova S., Stoyanova R., Zhecheva E., Ivanova S., Mitewa M. J. Chem. Chem. Eng. 6, 2012, 551.

Full Name of Abstract's 1st Author : Irena Pashkunova-Martic

Complete Status: Complete

First Name: Irena

Last Name: Pashkunova-Martic

Email: irena.pashkunova-martic@meduniwien.ac.at

Organization: Medical University of Vienna

Country: Austria 


\title{
ID: GA580 \\ Sonogenetic stimulation of mouse brain by non-invasive ultrasound
}

\author{
Quanxiang Xian, The Hong Kong Polytechnic University, 18072003r@connect.polyu.hk
}

\section{Category: Neuroscience}

\begin{abstract}
Body : Manipulating specific targeted neural activity by physical intervention is a powerful method to gain causal insight into brain functions and treat brains disorders. Ultrasound stimulation is a promising modality for probing brain function and treating brain diseases. It can be non-invasively steered and focused into $\mathrm{mm}$-scale regions across the human skull, facilitated to produce controlled modulation of neuronal activity. However, it still remains challenging to stimulate well-defined neurons in a desired brain area without significant surgical invasion. Here, we introduce a non-invasive and selective neural excitation strategy using ultrasound, through the specific activation a mutant of the large-conductance mechanosensitive channel. Methods we used included ultrasound stimulation, cell culture, viral transduction, patch clamp, calcium imaging, virus injection, immunocytochemical staining, Electromyography (EMG). In the vitro experiment, we first tested Low-intensity ultrasound can stimulate $\mathrm{Ca} 2+$ influx in 293T cells expressing MscL-G22S. Next, we tested the effect of ultrasound on cortical neurons collected from the embryonic mouse brain (E16) to test the feasibility of selectively stimulating neurons by ultrasound. Our data indicate that the expression of MscL-G22S can significantly reduce the ultrasound intensity required to cause $\mathrm{Ca} 2+$ influx in cortical neurons. Then test our setup in vivo. Ultrasound stimulation (recognized by c-Fos expression) of the neuron-induced cortical area in the mouse brain, the data showed that ultrasound treatment induced significantly higher expression of c-Fos in MscL-expressing cortical neuron group compared to the non-ultrasound and control virus cortex treated with ultrasound groups. A similar comparison of sham-injected mice showed that there was no significant difference in cFos expression in the cortex of mice treated with ultrasound or untreated, and judging by body weight, there was no significant health difference in the groups of these mice. Therefore, we indicated that the application of ultrasound in the mouse cortex can induce neuronal activity, but the induced MscL expression makes this effect many times stronger. We also used EMG methods to test the effect of low-intensity ultrasound stimulation different parameters on motor cortex response characteristics. This experiment illustrated the effect of mechanosensitive channel (CamKII-EYFP mice and CamKII-mscl-EYFP mice) on the twitch response to sonication. The data showed that the successful rate in mscl group was significant higher than control group when the mice were treated with $0.05 \mathrm{MPa}, 0.15 \mathrm{MPa}, 0.30 \mathrm{MPa}, 0.35 \mathrm{MPa}, 0.5$ ultrasound stimulation. The ultrasound stimulation parameters correspond to stimulation success rages in mscl group. We next tested the spatial selectivity of the ultrasound stimulation method by using the MscL-G22S virus to sensitize neurons in deeper regions of the brain, the dorsomedial striatum (DMS). Increased c-Fos expression was also observed in neurons expressing the control virus, however, c-Fos of MscL-EYFP neurons was also significantly higher than this group (MscL-EYFP 72.1 c-Fos+ cells/slices compared to 37.9 Ctrl-EYFP), which shows that MscL can effectively sensitize cells to ultrasound stimulation. Thus, we demonstrate an effective non-invasive approach for activating neurons in the intact brain using
\end{abstract}


ultrasound. This experiment confirmed the ability of ultrasound generated by our setup to penetrate regions of the brain deeper, making it suitable for in vivo use in areas of the brain both relatively superficial and deep. The non-invasiveness and penetrative reach of this approach makes it promise for eventual clinical translation, while the cell-type selectivity could facilitate the elucidation and management of neural circuits involved in specific behaviors or disorders.

\section{Image/Figure:}

https://www.xcdsystem.com/wmis/abstract/File6959/GA580_ImageFigure 0614082629.png

Image/Figure Caption: Ultrasound stimulation evokes greater muscular responses and activates more neurons when applied to mouse cortices expressing MscL-G22S.

Full Name of Abstract's 1st Author : Zhihai QIU

Complete Status: Complete

First Name: Quanxiang

Last Name: Xian

Email: 18072003r@connect.polyu.hk

Organization: The Hong Kong Polytechnic University

Country: China 


\title{
ID: GA582 \\ Non-invasively modeling ischemia in Duchenne Muscular Dystrophy
}

\author{
Sarah Hakim, Western University, shakim4@uwo.ca
}

\section{Category: Cardiovascular \& Pulmonary}

\begin{abstract}
Body : Introduction: Duchenne muscular dystrophy (DMD) is the most commonly inherited pediatric musculoskeletal disorder. It is a progressive neuromuscular degenerative disorder caused by either the absence or dysfunction of dystrophin [1]. This results in a loss of cell membrane integrity in skeletal and cardiac muscle, and the brain of those affected. Within DMD muscle, necrotic fibers are often observed in groups, and it has been previously hypothesized that this may be due to a reduction in regional blood supply. Furthermore, repetitive regeneration and degeneration cycles deplete the muscle stem cell pool leading to adipose tissue accumulation and the onset of ischemia [2]. Current methods of diagnosis are invasive and localized to certain areas of the body despite DMD being a systemic disorder [3]. Here we aim to develop an imaging protocol to non-invasively model ischemia and tissue perfusion parameters in DMD patients. We hypothesize that CT will help model DMD disease progression by quantifying changes in hemodynamic and ischemic factors in the heart, brain, and skeletal muscle. Methods: Two cohorts of DMD mice $(n=6)$ were scanned using CT to collect both longitudinal and acute data at differing time points 4-5 weeks, 8-10 weeks, and 15-20 weeks. These critical periods are associated with disease progression and correspond to prefibrotic, fibrotic, and post fibrotic condition respectively. An age-matched wild type control strain (C57bl/10) was used for comparison. Mice in the acute cohort were euthanized at their respective time points and underwent wire myography to study vascular reactivity and validate CT findings. Immunohistochemistry $(n=3)$ was performed for both validation and quantification of the ischemic outcome. Hypoxia-inducible factor 1-alpha (HIF-1a) and myeloperoxidase, were targeted to assess transcriptional regulation within the hypoxic-ischemic environment. In addition, H\&E staining $(n=1)$ was used to observe cell morphology and characteristics pertaining to pathology, while Mason's trichrome staining $(n=3)$ confirmed collagen deposition. Results: DMD mice have qualitatively shown increased levels of collagen deposition within the brain, cardiac, and skeletal tissue in comparison to the wild type strain. In addition, preliminary data has suggested increased levels of collagen deposition correlate with increased HIF-1a and myeloperoxidase expression. Furthermore, wire myography has shown that DMD mice are less reactive to dilatory responses when compared to contractile responses at the 15-20 week time point. These findings correlate well with past CT data collected by our lab. Discussion: Currently, there is little knowledge of functional tissue perfusion parameters in DMD patients. This research will be essential in developing therapeutics to restore tissue integrity and function when the natural ability of the tissues for repair is exhausted. Further, it will serve as a noninvasive diagnostic measure to assess disease progression prior to the onset of fatal complications.
\end{abstract}

References: [1]Timpani, C. A., Hayes, A. \& Rybalka, E. Therapeutic strategies to address neuronal nitric oxide synthase deficiency and the loss of nitric oxide bioavailability in Duchenne 
Muscular Dystrophy. Orphanet J. Rare Dis. 12, 1-11 (2017). [2]Shimizu-Motohashi, Y. \& Asakura, A. Angiogenesis as a novel therapeutic strategy for Duchenne muscular dystrophy through decreased ischemia and increased satellite cells. Front. Physiol. 5 FEB, 1-8 (2014).

[3]Joyce, N. C., Oskarsson, B. \& Jin, L. Muscle Biopsy Evaluation in Neuromuscular Disorders Nanette. Phys Med Rehabil Clin N Am 23, 609-631 (2015).

Full Name of Abstract's 1st Author : Sarah Hakim

Complete Status: Complete

First Name: Sarah

Last Name: Hakim

Email: shakim4@uwo.ca

Organization: Western University

Country: Canada 


\title{
ID: GA583 \\ Comparison between GlucoCEST MRI and 18F-FDG PET in monitoring therapy response in murine breast cancer 4T1 models.
}

\author{
Martina Capozza, University of Turin, martina.capozza@unito.it
}

\section{Category: Oncology}

\begin{abstract}
Body : Introduction New approaches for the early monitoring of treatment response are needed for the selection of the optimal therapeutic strategy to improve the outcome of cancer patients. Sequential 18F-FDG-PET/CT acquisitions are the gold standard at clinical level for assessing early response as a decrease of tumor metabolic activity in several tumors1. However, this nuclear methodology has several drawbacks mainly associated to radiation exposure and high costs that, in turn, limit its wide applicability. GlucoCEST MRI relies on the use of unlabelled D-glucose and it may be applied on ordinary MRI scanners available at research and clinical settings2. It has been proposed as an alternative to the radiolabelled FDG, but few studies have evaluated the glucoCEST capability to monitor the response to therapy and compared to the FDG-PET technique. This work aims at validating the potential of MRIGlucoCEST methodology in monitoring the response to two different treatment regimens based on doxorubicin (DOXO) and dichloroacetate (DCA) in breast tumor (4T1) bearing mice. Methods Balb/C female mice ( $\mathrm{n}=12$ for each group) were inoculated with 4x104 4T1 murine breast cancer cells at both mice flanks. The two therapeutic treatments were based on doxorubicin (dose $5 \mathrm{mg} / \mathrm{kg}$, four intraperitoneal administrations in one week), a potent anthracycline antibiotic and DNA intercalator that inhibits cancer cell growth3 and on Dichloroacetate (intraperitoneal daily administration $200 \mathrm{mg} / \mathrm{kg} /$ day supplemented with oral administration with power dissolved in water at $100 \mathrm{mg} / \mathrm{kg}$ ad libitum), whose mechanism of action consists of the inhibition of pyruvate dehydrogenase kinases (PDKs), which reverses the Warburg effect reducing lactate production and proton accumulation in the extracellular tumor region4. GlucoCEST MR images of control and treated groups were acquired before and after one week of treatment and compared with the analogous groups undergone to the 18F-FDGPET/CT imaging. CEST protocol was performed on a Bruker 7T MRI scanner. Z-spectra before and after glucose $(3 \mathrm{~g} / \mathrm{kg})$ intravenous injection were acquired and CEST contrast was calculated between POST and PRE images. For PET, animals were fasted overnight before the intravenous 18F-FDG injection (about $5.48 \mathrm{MBq} /$ mouse) PET static acquisition was performed for 30 minutes starting 45 minutes after 18F-FDG administration. Results Doxorubicin treated mice showed a marked decrease in tumor growth, being statistically significant from day 21 (after the last doxorubicin treatment), compared to the control group (Figure 1A). The FDG-PET technique showed no difference in mean \%ID/cc values between the control and the treated groups (Figure 1C). Conversely, the calculated average GlucoCEST values between pre- and post-treatment highlighted a significant decrease of the metabolic activity (Figure 1B and D). When mice were treated with DCA any difference was observed in the tumor growth rates between the two groups. Both glucoCEST MRI and 18F-FDG PET did not observe any difference in glucose uptake between the treated and control groups following DCA treatment Conclusions In conclusion, for assessing the response to doxorubicin treated mice, GlucoCEST MRI appears
\end{abstract}


more sensitive than 18F-FDG-PET in reporting the therapy response as it parallels the observations relative to the tumour size reduction. DCA treatment did not result in a therapeutic effect, with similar observed glucose uptake between the two techniques. These findings support the view that glucoCEST may be a sensitive technique in monitoring therapeutic effects, but more work appears necessary to identify cancer types and specific treatments where the methodology can be effective. Funding: This work was supported by the European Union's Horizon 2020 research and innovation programme (Grant Agreement No. 667510) and by the Associazione Italiana Ricerca Cancro (AIRC MFAG 2017 - ID. 20153 project).

References: 1. Avril S, Muzic RF, Plecha D, Traughber BJ, Vinayak S, Avril N. ${ }^{18} \mathrm{~F}-\mathrm{FDG}$ PET/CT for Monitoring of Treatment Response in Breast Cancer. J Nucl Med. 2016;57 Suppl 1:34S-39S. 2. van Zijl PC, Yadav NN. Chemical exchange saturation transfer (CEST): what is in a name and what isn't? Magn Reson Med. 2011;65:927-948. 3. Thorn CF, Oshiro C, Marsh S, et al. Doxorubicin pathways: pharmacodynamics and adverse effects. Pharmacogenet Genomics. 2011;21:440-446. 4. Kankotia S, Stacpoole PW. Dichloroacetate and cancer: new home for an orphan drug? Biochim Biophys Acta. 2014;1846:617-629.

\section{Image/Figure:}

https://www.xcdsystem.com/wmis/abstract/File6959/GA583 ImageFigure 0614103847.jpg

Image/Figure Caption: Figure 1. A) Bar graph showing tumor volume calculation of 4T1 tumour bearing mice over time for control, dichloroacetate and doxorubicin treated mice groups $\left(\right.$ mean $\pm \mathrm{SD}$ ). Two-way ANOVA Bonferroni post, $\left.{ }^{*} \mathrm{p}=0.0004,{ }^{* *} \mathrm{p}<0.0001 ; \mathrm{B}\right)$ Bar graph showing mean \pm SD GlucoCEST contrast obtained injecting glucose at $3 \mathrm{~g} / \mathrm{Kg}$ dose via intravenous bolus for each 4T1 tumour group. Data are reported as the variation $(\Delta \mathrm{ST} \%)$ between the ST effect percentage post minus pre intravenous injection. Unpaired t-test * $\mathrm{p}=$ 0.0341 ; C) Bar graphs show the average values for the injected dose per cubic centimeter (\%ID/cc, mean $\pm \mathrm{SD}$ ); D) Representative GlucoCEST map overimposed on the anatomical image after i.v. injection of D-glucose $(3 \mathrm{~g} / \mathrm{kg})$ of $4 \mathrm{~T} 1$ tumor bearing mice for control and treated groups before and after treatment

\section{Full Name of Abstract's 1st Author : Martina Capozza}

Complete Status: Complete

First Name: Martina

Last Name: Capozza

Email: martina.capozza@unito.it

Organization: University of Turin

Country: Italy 


\title{
ID: GA584 \\ [125I]IPPI for Tau Imaging in Post-Mortem Human Alzheimer's Disease Brain
}

\author{
Jogeshwar Mukherjee, University of California-Irvine, j.mukherjee@uci.edu
}

\section{Category: Neuroscience}

\begin{abstract}
Body : Objective: Alzheimer's disease (AD) is a neurodegenerative disease characterized by aggregation of Tau protein into paired helical filaments causing neurofibrillary tangles (NFT) in the brain. A second generation NFT PET radiotracer, [18F]MK-6240 shows characteristic changes in the brains of patients with AD (Betthauser 2019). The aim of the present study was to develop and evaluate the effectiveness of a novel radioiodinated tracer, 6[125I]iodo-3-(1H-pyrrolo[2,3-c]pyridine-1-yl)isoquinoline ([125I]IPPI), for binding to Tau protein in postmortem human brain (AD and cognitively normal $(\mathrm{CN})$. Availability of an iodine125 azaindole derivative for in vitro studies of NFT will be valuable to complement studies with [18F]MK-6240. Methods: Radiosynthesis of [125I]IPPI was carried out by radioiododestannylation of 6-tributyltin-3-(1H-pyrrolo[2,3-c]pyridine-1-yl)isoquinoline (prepared from BrPPI, the bromo analog of IPPI), and purified chromatographically. Computational modeling studies of IPPI and MK-6240 binding on Tau fibril were evaluated on the reported cryo-EM structure (Fitpatrick et al, 2017). We used the Chimera software to evaluate the binding energies of IPPI and MK-6240 at four binding sites (Murugan et al., 2018). In vitro autoradiography studies were carried out with [125I]IPPI for Tau in AD and CN brains and evaluate drug effects (IPPI, BrPPI, (R)-deprenyl for MAO B and clorgyline for MAO A). Off-target binding to MAO in vivo may not be a concern (Hostetler et al., 2016) and more recently, [18F]MK-6240 showed some propensity of being displaced by high concentrations of MAO inhibitors in vitro (Aguero et al., 2019). Brain slices with [125I]IPPI in 10\% ethanol PBS buffer $\mathrm{pH} 7.4(60 \mathrm{~mL} ; 0.1 \mu \mathrm{Ci} / \mathrm{mL})$ were incubated at $25 \mathrm{oC}$ for $1.25 \mathrm{hr}$. Nonspecific binding was measured in the presence of $10 \mathrm{mM} \mathrm{MK}-6240$. The slices were then washed with cold PBS buffer, 50\% ethanolic PBS buffer twice, PBS buffer and cold water. The brain sections were air dried, exposed overnight on a phosphor film, and then placed on the Phosphor Autoradiographic Imaging System/ Cyclone Storage Phosphor System and extent of binding of [125I]IPPI was measured in DLU/mm2. Results: Binding affinity of IPPI $((\mathrm{Ki}=0.75 \mathrm{nM})$ was similar to MK$6240(\mathrm{Ki}=0.43 \mathrm{nM})$ (Walji et al., 2015). [125I]IPPI was produced in $>95 \%$ purity. Molecular modeling of IPPI revealed binding energies of IPPI $(-7.8,-8.1,-8.2,-7.5 \mathrm{Kcal} / \mathrm{mol})$ at the four sites were comparable to MK-6240 (-8.7, -8.5, -8.3, -7.5 Kcal $/ \mathrm{mol})$. Ratio of average grey matter (GM) [125I]IPPI in AD versus $\mathrm{CN}$ was found to be 7.31 and $\mathrm{AD}$ GM/ white matter (WM) $=4.35$. Ratio of average GM/WM [125I]IPPI in CN was 1.21. It should be noted that the AD WM exhibited higher levels of [125I]IPPI binding compared to CN subjects. Specifically bound [125I]IPPI to Tau in AD brains was displaced by MK-6240 and IPPI (>90\%). Our studies with [125I]IPPI showed that at higher concentrations of $10 \mathrm{mM}$ of (R)-deprenyl (for MAO B) and clorgyline (for MAO A), binding was reduced $13 \%$ and $20 \%$. At lower concentrations of $1 \mathrm{mM}$ of (R)-deprenyl did not effect [125I]IPPI while $1 \mathrm{mM}$ clorgyline had a Conclusion: [125I]IPPI exhibited high binding in human AD frontal cortex and anterior cingulate and is a suitable radioiodinated ligand for Tau imaging. MK-6240 displaced $>95 \%$ [125I]IPPI from the AD
\end{abstract}


brains, suggesting good correspondence in the binding sites of the two radioligands. Both PET and SPECT imaging agents of IPPI are possible, provided in vivo stability and kinetics are found appropriate. For SPECT, [123I]IPPI may be considered and for PET, [124I]IPPI and $[75 / 76 / 771]$ BrPPI may be considered.

References: Aguero C, Dhaynaut M, Normandin MD, Amaral AC, Guehl NJ, Neelamegam R, Marquie M, Johnson KA, El Fakhri G, Frosch MP, Gomez-Isla T. Autoradiography validation of novel tau PET tracer [F-18]-MK-6240 on human postmortem brain tissue. Acta Neuropathologica Commun. 7: 37, 2019. Betthauser TJ. AD molecular: Imaging tau aggregates with positron emission tomography. Prog Mol Biol Transl Sci. 165: 1877-1173, 2019. Fitzpatrick AWP, Falcon B, He S, Murzi AG, Murshudow G, Garringer HI, Crowther RA, Ghetti B, Goedert B, Scheres SHW. Cryo-EM structures of tau filaments from Alzheimer's disease. Nature, 547: 185-190, 2017. Hostetler ED, Walji AM, Zeng ZP, Miller I, Bennacef C, Salinas B, Connolly L, Gantert H, Haley M, Holahan M, Purcell K, Riffel TG, Lohith P, Coleman A, Soriano A, Ogawa S, Xu X, Zhang E, Joshi JD, Rocca D, Hesk D, Schenk DJ, Evelhoch JL. Preclinical characterization of 18F-MK-6240, a promising PET tracer for in vivo quantification of human neurofibrillary tangles. J Nucl Med, 57(10), 1599-1606, 2016. Murugan NA, Nordberg A, Agren H. Different positron emission tomography tau tracers bind to multiple binding sites on the tau fibril: insight from computational modeling. ACS Chem Neurosci. 9: 1757-1767, 2018. Walji A, Hostetler E, Greshock T, Li J, Moore KP, Bennacef I, Mulhearn J, Selnick H, Wang Y, Yang K, Fu J. (2015) WO 2015/188368 A1.

\section{Image/Figure:}

https://www.xcdsystem.com/wmis/abstract/File6959/GA584_ImageFigure 0614104422.jpg

Image/Figure Caption: Chemical structure of [125I]IPPI and comparison of MK-6240 and IPPI at Tau binding sites using Chimera model showing binding of MK-6240 (pink) and IPPI (purple) at Site 1 .

Complete Status: Complete

First Name: Jogeshwar

Last Name: Mukherjee

Email: j.mukherjee@uci.edu

Organization: University of California-Irvine

Country: United States 


\title{
ID: GA585 \\ Molecular Imaging of Tumor Microvesicles that Enhance Metastasis in a Mouse Breast Cancer Model
}

\author{
Masamitsu Kanada, Michigan State University, kanadama@msu.edu
}

\section{Category: Oncology}

\begin{abstract}
Body : Introduction: Extracellular vesicles (EVs) comprise exosomes and microvesicles (MVs) in the physiological condition. Exosomes (40-120 nm in diameter) are produced as components of multivesicular bodies (MVBs) and are released from cells via fusion of MVBs with the plasma membrane. MVs (50-1000 nm in diameter) are formed by the outward budding of the plasma membrane. We have previously characterized the physical properties and the functional transfer of cargo molecules packaged in exosomes and MVs. To further understand the roles of exosomes and MVs in cancer, here we developed a new EV reporter system that enables comparative analysis of distinct EV classes in vivo. Methods: We developed a new EV imaging probe using a highly sensitive red-shifted bioluminescence resonance energy transfer (BRET) protein called red enhanced Nano-lantern (ReNL) with a palmitoylation signal peptide (Palm). Since palmitoylated reporter proteins have been presumed to label all EV subpopulations, we assessed their labeling efficiency in the fractions of exosomes and MVs derived from murine mammary carcinoma 4T1 cells stably expressing PalmReNL. Cellular uptake of the reporter exosomes and MVs by various cell types was analyzed by fluorescence microscopy and measuring luminescence signals. Furthermore, we used in vivo bioluminescence imaging to determine the biodistributions of the reporter exosomes and MVs in healthy and mammary tumor-bearing mice. Results: (1) Exosomes and MVs carrying PalmReNL exhibited red-shifted emission spectra upon the addition of a luminescent substrate. (2) PalmReNL was more efficiently expressed in exosomes than MVs. However, individual MVs expressed a significantly higher number of reporters due to their larger surface areas compared to exosomes. (3) Culturing the reporter exosomes or MVs with various cell types for $2 \mathrm{~h}$ showed the highest cellular uptake efficiency in the recipient cells and the signals decreased over longer periods in culture, followed by the increased proliferation of the recipient cells. (4) Both reporter exosomes and MVs administered intravenously showed similar biodistributions. Both EV classes preferentially accumulated in the lung, followed by liver, spleen, heart, bone, lymph nodes, and brain. (5) Finally, circulating reporter MVs significantly promoted metastatic tumor cell growth in the lung tissues. Summary/Conclusion: Using PalmReNL reporter proteins, we demonstrated the successful tracking of distinct classes of EVs both in vitro and in vivo. Both reporter exosomes and MVs derived from 4T1 cells rapidly transferred the biological signals after their cellular uptake. Moreover, despite their different sizes, both EV classes showed similar organotropisms in healthy mice. Our data suggest that cancer cell-derived MVs, in addition to exosomes, are a critical regulator of systemic promotion of metastasis.
\end{abstract}

Complete Status: Complete

First Name: Masamitsu 
Last Name: Kanada

Email: kanadama@msu.edu

Organization: Michigan State University

Country: United States 


\title{
ID: GA586 \\ Baseline Subject Characteristics Associated with Short Term Changes in Coronary Calcification Assessed by 18F-Sodium Fluoride PET/CT
}

\author{
Lauren Brodsky, University of Pennsylvania, Ibrod@sas.upenn.edu
}

Category: Cardiovascular \& Pulmonary

\begin{abstract}
Body : Introduction:Current standard-of-care tests for atherosclerosis can detect calcification of arteries only at later stages of the disease. Commuted Tomography (CT) calcium score, for example, is only effective once appreciable amounts of plaque have formed. $18 \mathrm{~F}-$ Sodium Fluoride (NaF) is a radiopharmaceutical with affinity of fluoride to hydroxyapatite. For this reason, it is appealing for bone imaging. Recent studies have shown that $\mathrm{NaF}$ could also be used to detect calcified micro-deposits within coronary plaque. The goal of this study was to test if the baseline subject characteristics are associated with the short term changes in coronary microcalcification assessed by NaF PET/CT. Methods: This retrospective study included healthy female $(\mathrm{N}=8$, age $52 \pm 10$ years, BMI $24 \pm 1.7 \mathrm{~kg} / \mathrm{m} 2)$ and male $(\mathrm{N}=15$, age $50 \pm 10$ years, BMI $27 \pm 2.9 \mathrm{~kg} / \mathrm{m} 2$ ) participants who had NaF PET/CT scans taken two years apart. Imaging was performed 90 minutes after intravenous injection of $2.2 \mathrm{MBq}$ of NaF per kilogram of body weight. The analysis regions were selected on CT images by drawing volumes of interest around the entire heart using a semi-automatic segmentation method (PMOD Technologies LLC, Switzerland).SUVmean and SUVmax were calculated in the same regions of the registered PET images. Percent change in SUV between the two time points were correlated against baseline age, BMI, cardiovascular risk factors, and blood chemistry. Results:In males, SUVmean was positively correlated with baseline BMI $(\mathrm{r}=0.89$, $\mathrm{pDiscussion}$ :High BMI is a known risk factor for atherosclerosis. Our data showed that rate of increase in coronary microcalcification over time measured by NaF PET/CT is associated with baseline BMI and some clinical risk factors in males. Lack of such associations in females could be due to low sample size $(\mathrm{N}=8)$. Further prospective studies are needed to determine if baseline BMI and clinical factors could be used to predict rate of increase in coronary microcalcification which could provide the basis for managing the progression of atherosclerosis in patient-specific manner. Keywords:Coronary, Calcification, atherosclerosis, 18F-Sodium Fluoride, NaF, PET/CT, BMI, blood pressure, pulse rate Figure 1: Figure 2: Figure 3: Figure 4: maximum intensity projections of $18 \mathrm{~F}-\mathrm{NaF}$ PET/CT showing increased coronary $\mathrm{NaF}$ tracer uptake in a male $(\mathrm{BMI}=34 \mathrm{~kg} / \mathrm{m} 2)$ after two years.
\end{abstract}

\section{Image/Figure:}

https://www.xcdsystem.com/wmis/abstract/File6959/ImageFigure_GA586_0616010457.jpg

Image/Figure Caption: Maximum intensity projections of $18 \mathrm{~F}-\mathrm{NaF}$ PET/CT showing increased coronary $\mathrm{NaF}$ tracer uptake in a male $(\mathrm{BMI}=34 \mathrm{~kg} / \mathrm{m} 2)$ after two years

Complete Status: Complete 
First Name: Lauren

Last Name: Brodsky

Email:1brod@sas.upenn.edu

Organization: University of Pennsylvania

Country: United States 


\title{
ID: GA708 \\ Evaluating the Role of 68Ga-PSMA PET/CT Imaging in Predicting Intraprostatic Tumor Extent of Tumor Involvement Prior to Various Surgical Protocols in Patients with Known or Diagnosed Prostatic Cancer.
}

\author{
Sikandar Shaikh, Yashoda Hospitals, idrsikandar@gmail.com
}

\section{Category: Oncology}

\begin{abstract}
Body : PURPOSE:The role of performance of Gallium Ga 68 (68Ga)-labeled prostatespecific membrane antigen (PSMA) ligand positron emission tomography/computed tomography (PET/CT) shows very promising results with known or diagnosed prostatic cancers. The evaluation in relation to Intraprostatic involvement ,Local infiltration and Lymph node detection even at low prostate specific antigen (PSA) levels is highly sensitive.In patients with biochemical recurrence after curative therapy for prostate cancer. In this study we evaluated the usefulness of PSMA-PET/CT for the evaluation of intraprostatic tumor extent prior to surgery METHOD AND MATERIALS:This was the retrospective study with diagnosed prostate cancer. 30 patients were included in this study who underwent a 68Ga-PSMA-PET/CT before surgical treatment. All patients underwent either open or laparoscopic radical prostatectomy. Intraprostatic tumor extent was assessed on PET/CT by the peak standardized uptake value (SUV).This was correlated with final Whole gland histopathology including Gleason grade, Lobe infiltration, TNM stage and Seminal vesicle invasion RESULTS: The mean SUV over all patients was 12.64 \pm 9.19 . Median Gleason score was 7. The distribution of TNM stage 2a, 2b, 2c, 3a, 3b, 4 was 0,2 , 6, 5, 2 and 0 patients. Mean intraprostatic SUV for patients with organ confined vs. extraprostatic tumor on histopathology was 6.3 and 15 respectively $(p=0.043)$. Mean SUV for patients with a Gleason score of $\leq 7 \mathrm{a}$ and $>7 \mathrm{a}$ was 6.2 and $13.5(\mathrm{p}=0.046)$.

CONCLUSION:Thus the PSMA PET-CT has very effective value in evaluation of intra and extra prostatic disease involvement

\section{References: 1}

Full Name of Abstract's 1st Author : Sikandar Shaikh

Complete Status: Complete

First Name: Sikandar

Last Name: Shaikh

Email: idrsikandar@gmail.com

Organization: Yashoda Hospitals

Country: India 


\title{
ID: GA709 \\ In Vitro and In Vivo Evaluation of a Potential Radiotracer Targeting Alpha- Synuclein
}

\author{
Ran Sing Saw, Werner Siemens Imaging Center, Department of Preclinical Imaging and \\ Radiopharmacy, Eberhard Karls University of Tuebingen, ransing.saw@med.uni- \\ tuebingen.de
}

\section{Category: Neuroscience}

\begin{abstract}
Body : Introduction: Positron emission tomography (PET) imaging of alpha-synuclein $(\alpha$-syn) aggregates in Parkinson's disease (PD) would be an invaluable tool for non-invasive diagnosis of the disease as well as facilitating the development of novel treatments. However, no validated $\alpha$-syn radiotracer is currently available. Here we evaluate MODAG-005, a derivative of anle138b, which modulates $\alpha$-syn aggregation1, by using in vitro binding assays and an in vivo fibril inoculation model. Methods: In vitro binding affinity and selectivity of [3H]MODAG-005 were evaluated in saturation binding assays using human recombinant $\alpha$-syn, amyloid-beta (A $\beta 1$ 42) and hTau46 fibrils. To evaluate the pharmacokinetic profile in vivo, [11C]MODAG-005 was intravenously injected into healthy rats, as well as rats which were intrastriatally inoculated with $4 \mu \mathrm{L}$ of $\alpha$-syn fibrils (30 $\mu \mathrm{M})$, with a contralateral buffer injection as internal control. Using the volume of the whole striatum based on the rat brain atlas provided in PMOD (44 mm3), the molarity of inoculated fibrils in the right striatum was calculated to be $\approx 2.8 \mu \mathrm{M}$. 60 -minute dynamic PET scans were performed on healthy rats $(n=3)$ and inoculated rats at 1,4 and 7 days post-inoculation (dpi) ( $\mathrm{n}=6$ per time point). Images were analyzed by drawing volumes of interest (VOIs) both on the whole striata based on the rat brain atlas and on the hot spot based on isocontour automatic detection (IAD) set to $70 \%$ hot threshold. IAD was applied in a $2.5 \mathrm{~mm}$ sphere drawn into the hot spot of the fibril-inoculated striatum, which was subsequently reflected contralaterally. Binding potential (BPND) in the right, fibril-inoculated striatum was calculated from individual time-activity curves from 30 to 60 minutes, using the left, buffer-injected striatum as the reference region (DVR-1). The presence of inoculated fibrils was validated ex vivo using thioflavin S staining. Results: [3H]MODAG-005 in vitro binding experiments revealed Kd values of $0.2 \mathrm{nM}$ towards $\alpha$-syn fibrils, $7 \mathrm{nM}$ towards hTau46 and $>100 \mathrm{nM}$ towards A $\beta 1-42$ fibrils. [11C]MODAG-005 showed an excellent penetration of the blood-brain barrier and a fast clearance from the brain, with peak standardized uptake values of more than 1.5 in all rats, indicating a good brain uptake independent from intracranial surgery. For the inoculation model, atlas-based whole striata VOIs revealed BPND of $0.3 \pm 0.1,0.2 \pm 0.1$ and 0.1 \pm 0.1 , whereas the IAD analysis yielded BPND of $0.7 \pm 0.3,1.4 \pm 0.6$ and $0.7 \pm 0.4$, for 1,4 and $7 \mathrm{dpi}$, respectively. Comparing inoculated rats at each time point to healthy rats, the BPND values based on the whole striata VOIs of inoculated rats were statistically significantly higher than healthy rats at $1 \mathrm{dpi}$ (p Conclusion: MODAG-005 exhibited an excellent brain availability, good washout kinetics, as well as in vivo binding to localized $\alpha$-syn aggregation with sufficient signal-to-noise ratio in rats. The potential of the ligand as $\alpha$-syn radiotracer will be further evaluated at lower fibril concentrations to determine the limit of detection and in human pathological brain tissues.
\end{abstract}


References: 1. Wagner, J., et al. (2013). "Anle138b: a novel oligomer modulator for diseasemodifying therapy of neurodegenerative diseases such as prion and Parkinson's disease." Acta Neuropathol 125(6): 795-813.

Full Name of Abstract's 1st Author : Ran Sing Saw

Complete Status: Complete

First Name: Ran Sing

Last Name: Saw

Email: ransing.saw@med.uni-tuebingen.de

Organization: Werner Siemens Imaging Center, Department of Preclinical Imaging and Radiopharmacy, Eberhard Karls University of Tuebingen

Country: Germany 


\title{
ID: GA710
}

Bifunctional Chelator Conjugate as Potential Amyloid Plaque Scintigraphy Probe : Synthesis and Preclinical Evaluation in Traumatic Brain Injury Model

\author{
Anupama Datta, Institute of Nuclear Medicine and Allied Sciences/DRDO, \\ anupama@inmas.drdo.in
}

\section{Category: Neuroscience}

\begin{abstract}
Body : Aim/Introduction: Earliest pathological changes to occur after the incidence of Traumatic Brain Injury (TBI) are considered to be the formation of A $\beta 42$-containing diffuse amyloid plaques which are one of the most prominent hallmarks of Alzheimer's Disease and is a risk factor for developing associated neurological disorders. Early detection of these plaques consequently remains vital for better regulation and management of these disorders. Considering this, we developed a cost-effective, chalcone scaffold-based SPECT radiotracer which will specifically bind detrimental A $\beta 1-42$ plaques. The bifunctional chelating agent, pentapa-en-NH2 was explored for the development of SPECT agent. We hypothesise that the molecule will display enhanced binding affinity and blood brain barrier penetration. Methods: The bifunctional chelator was synthesised through a series of steps in high purity and further conjugated to the chalcone derivative. The molecule was conjugated to $99 \mathrm{mTc}$ and further used for pre-clinical studies. A repetitive mild TBI mouse model was developed, and uptake of tracer was studied. Result and Conclusions: The molecular docking studies reported multiple binding of the tracer at the recognition sites of $A \beta$ fibrils with a significantly high binding score (-12.64) as compared to known A $\beta$ ligands. Chalcone derivative, 6-(\{[(6-carboxypyridin-2-yl)methyl] $(2-\{4-[(2 \mathrm{E})-3-[4-$ (dimethylamino)phenyl]prop-2-enoyl]phenoxy\}ethyl)amino $\}$ methyl)pyridine-2-carboxylic acid, Ch-(pa) 2 was synthesized in 95\% yield with high purity. Radiolabelling was carried out with $99 \mathrm{mTc}$ under mild conditions with $95.4 \%$ efficiency and $103-124 \mathrm{MBq} \mu \mathrm{mol}-1$ specific activity. In vitro binding assay with $\mathrm{A} \beta 1-42$ aggregates displayed high binding affinity of $\mathrm{Ch}-(\mathrm{pa}) 2$ with an inhibition constant of $3.98 \pm 0.62 \mathrm{nM}$. The fluorescent data displayed peaks of absorption/emission at $410 / 540 \mathrm{~nm}$ and exhibited a blue shift with a 10 -fold increase in emission intensity on binding with $A \beta$ aggregates. Blood kinetics of the radiocomplex performed on normal rabbit displayed fast clearance $(\mathrm{t} 1 / 2(\mathrm{~F})=32 \pm 0.04 \mathrm{~min} ; \mathrm{t} 1 / 2(\mathrm{~S})=3 \mathrm{~h} 55 \mathrm{~min} \pm 0.03 \mathrm{~min})$. A repetitive mild TBI mice model was established and ex vivo staining of TBI mice model brain sections with Ch-(pa)2 showed specific binding of complex to amyloid plaques. In vivo scintigraphy displayed more than the two-fold increase of radioligand concentration in TBI model as compared to sham mice owing to higher amyloid burden in TBI model. Ex vivo biodistribution analysis demonstrated high blood-brain barrier penetration in TBI model mice with brain uptake of $5.24 \pm 0.31 \%$ ID g-1 at 2 min p.i. and 3-fold higher than brain uptake in sham mice. These preliminary studies reveal the enhanced amyloid binding affinity by bivalent approach and offer a new perspective in $99 \mathrm{mTc}$ probes for $\beta$-amyloid imaging.
\end{abstract}

References: 1. K.G Kadiyala, T. Tyagi, D. Kakkar, N. Chadha, K. Chuttani, B. G. Roy, M. Thirumal, A. K. Mishra, A. Datta, RSC Advances, 2015, 5, 33963 2. K. Chauhan, A. Arun, S. 
Singh, M. Manohar, K. Chuttani, R. Konwar, A. Dwivedi, R. Soni, A. K. Singh, A. K. Mishra, A. Datta, Bioconjugate Chem., 2016, 27 (4), 961-972

\section{Image/Figure:}

https://www.xcdsystem.com/wmis/abstract/File6959/GA710_ImageFigure 0614020906.jpg

Image/Figure Caption: In vitro Fluorescence studies with amyloid plaques and Histological studies using amyloid specific dyes

Full Name of Abstract's 1st Author : Garima Mann

Complete Status: Complete

First Name: Anupama

Last Name: Datta

Email: anupama@inmas.drdo.in

Organization: Institute of Nuclear Medicine and Allied Sciences/DRDO

Country: India 


\title{
ID: GA711 \\ Novel Technique for Depth-Selective Raman Imaging for Breast Cancer Margin Assessment
}

\author{
Rishikesh Pandey, CytoVeris Inc., rpandey@cytoveris.com
}

\section{Category: Oncology}

\begin{abstract}
Body : There are over 300,000 new cases of breast cancer per year in the United States and breast conservation surgery (BCS) is a standard of care for the treatment of early-stage breast cancer. Even with advancements in the standard of care, repeat surgery is necessary in $20 \%$ to as high as $40 \%$ of BCS cases due to the histopathological determination of positive margins days or even weeks after surgery. Cytological analysis such as touch preparation and frozen section histopathologic assessment are practiced, but these approaches are inadequate owing to sensitivity and specificity concerns, sample preparation artifacts, and sampling errors. Moreover, these methods are time-consuming and complex which further inhibit their broader applicability. Consequently, there is an urgent need for an intraoperative tool that can quickly, accurately, and non-invasively evaluate margins of the resected breast tissue to allow complete removal of all cancer tissue during primary surgery, reducing and ultimately eliminating the need for secondary procedures. Several spectroscopy-based approaches have been proposed for intraoperative margin detection. Intraoperative tools for margin assessment ideally should detect cancer at the surface as well as at a specified depth of tissue due to the current standard requirement of no residual within $2 \mathrm{~mm}$ of the surface of the excised tissue for ductal carcinoma in situ. Raman spectroscopy, in particular, has emerged as a potential tool owing to its ability to provide real-time depth-resolved biomolecular information in a label-free manner. While spatially offset Raman spectroscopy (SORS) has shown excellent promise in providing depthselective Raman spectral information, it requires suffers from poor signal to noise ratio from deep layers due to diffuse scattering of light from tissue. Here we present a novel technique that we call Angular Depth Resolved Raman Spectroscopy (ADRRS). We use a non-diffracting, selfhealing beam to interrogate the tissue sample at an oblique incident angle. As such, the Raman signals from different depth layers can be detected at different lateral $\mathrm{x}$ positions as illustrated in Fig. 1. The use of Bessel beam that propagates in scattering media with less beam divergence enables sensitive Raman measurements from sub-surface tissue layers. This talk will present our recent imaging results in determining its applicability in the surgical setting.
\end{abstract}

\section{Image/Figure:}

https://www.xcdsystem.com/wmis/abstract/File6959/GA711_ImageFigure_0614021717.png

Image/Figure Caption: Figure 1: Schematic of ADRRS concept. Raman signals from different depths will be detected at different lateral $\mathrm{x}$ positions.

Complete Status: Complete 
First Name: Rishikesh

Last Name: Pandey

Email: rpandey@cytoveris.com

Organization: CytoVeris Inc.

Country: United States 


\title{
ID: GA712 \\ Multimodal PET/MRI imaging probe for precise inflammatory macrophage detection
}

\author{
Chao Wang, UC SAN DIEGO, CHW124@UCSD.EDU
}

Category: Immunology: Inflammation \& Infection

\begin{abstract}
Body : Inflammation is associated with a range of diseases including autoimmune and cardiovascular diseases and cancer. The ability to image active inflammatory processes greatly enhances our ability to diagnose and treat these diseases at an early stage. An in vivo multimodal PET/19F MRI imaging probe was designed based on a perfluorocarbon nanoemulsion (NE), which labels monocytes and macrophages in situ and can be used to visualize inflammatory macrophages with high sensitivity, specificity and versatility. The NE has $89 \mathrm{Zr}$ encapsulated within its fluorous oil by a novel fluorous chelator, producing a highly stable PET/MRI tracer for in vivo inflammation imaging. Pharmacokinetic analysis of blood samples using both dosimetry and 19F NMR measurements confirmed a high degree of agent stability in vivo. Following a single injection of $89 \mathrm{Zr}$ NE, probe utility was demonstrated in murine diseases models of acute inflammation, inflammatory bowel disease (IBD) and solid tumor using PET/CT and $19 \mathrm{~F} / 1 \mathrm{H}$ MRI in the same animal. Good correlation was observed between hotspot signals from PET and 19F MRI in each of the models tested, with notable differences suggesting that these techniques are highly complementary. In all mouse models studied, over 10-fold greater agent uptake was observed at the lesion site compared to controls. Histology studies confirmed the presence of NE in macrophages in inflammatory lesions. The high sensitivity of PET enables unambiguous whole-body imaging of macrophage burden at clinically relevant dosage, while 19F MRI yields higher resolution views of the underlying lesion localization. Overall, the $89 \mathrm{Zr}$ NE probe is valuable as a multimodal preclinical biomarker, particularly for imaging tissues where 18F-FDG normally has a high intrinsic uptake.
\end{abstract}

\section{Image/Figure:}

https://www.xcdsystem.com/wmis/abstract/File6959/GA712_ImageFigure_0614022842.jpeg

Image/Figure Caption: Figure 1. Visualization of macrophage burden with PET/CT and 19F/1H MRI in three mouse models: Carrageenan-induced acute inflammation (CD 1), DSSinduced IBD (C57BL/6) and 4T1 solid tumor (Balb/c). Mice received a single dose of $89 \mathrm{Zr}$ NE $(100 \mu \mathrm{Ci})$ via tail vein, and 24 hours thereafter PET/CT (10 min, Inveon, Siemens) and 19F/1H MRI scans (15 min, RARE, 11.7 T Bruker) were acquired.

Full Name of Abstract's 1st Author : Chao Wang

Complete Status: Complete

First Name: Chao 
Last Name: Wang

Email: CHW124@UCSD.EDU

Organization: UC SAN DIEGO

Country: United States 


\title{
ID: GA713 \\ Improved localization of optical absorbing molecules with quantitative photoacoustic imaging
}

\author{
Kenneth Hoyt, University of Texas at Dallas, kenneth.hoyt@utdallas.edu
}

\section{Category: Instrumentation}

\begin{abstract}
Body : Introduction: Recent developments in photoacoustics (PA) have demonstrated that structural information can be obtained from the broadband nature of PA signals. In this regard, we introduced a pseudo quantitative PA (QPA) imaging approach for depicting different sized optical absorbing molecules. In this work, we performed proof-of-concept simulations along theoretical considerations and validated QPA imaging using phantom materials. Methods: QPA images were simulated using an acoustics toolbox for MATLAB (k-Wave; Mathworks, Natick, MA). An advanced time-domain model of ultrasound (US) wave propagation was implemented given a center frequency of $10 \mathrm{MHz}$, sampling frequency 100 $\mathrm{MHz}$, and transducer aperture size of $14.8 \mathrm{~mm}$. The overall dimension of the computational grid was set to $14.8 \times 14.8 \mathrm{~mm}$ with a grid size (spacing) of $11 \mu \mathrm{m}$. PA data was reconstructed using time reversal reconstruction. After, the PA data fields were processed using a novel processing approach that links the local US signal to the size of optical absorbing molecules [1]. More specifically, the spectrum of the PA signal was decomposed using a pair of nth-order Gaussianweighted Hermite polynomial $(\mathrm{GWn})$ functions before taking the signal envelope from a Hilbert transform. The envelope of the original unfiltered data is assigned to the green channel, low frequency information (GH2) to the red channel, and the high-frequency information (GH8) to the blue channel, which completes the RGB colormap and QPA image display. A series of phantom materials were constructed from gelatin embedded with a small volume of optical absorbing molecules with known size. Raw radiofrequency (RF) PA data was collected after laser excitation using the Vevo 3100 + LAZR-X system (FUIJIFILM VisualSonics Inc, Toronto, Canada) equipped with a linear array transducer. PA signal analysis was the same as detailed for the simulation study. All results obtained using QPA processing were compared to traditional PA images. Results and Discussion: Simulated images obtained from the two different phantom types suggest that the QPA images can accurately depict the relative size of different locally spaced optical absorbing molecules. QPA images exhibit a change in hue from bluer to red when the scatterer size increases, which is consistent with theoretical considerations. Phantom materials embedded with different-sized optical absorbers revealed the same trend. A comparison of QPA to traditional PA imaging results revealed a considerably narrower point spread function (PSF) for the former, which suggests QPA improves localization of optical absorbing molecules and yields images of higher spatial resolution. Collectively, these preliminary findings help validate this novel QPA method and more results are forthcoming.
\end{abstract}

References: [1] Basavarajappa L, Hoyt K. High-frequency quantitative photoacoustic imaging and pixel-level tissue classification. IEEE International Symposium on Biomedical Imaging, 308-311, 2020. 


\section{Image/Figure:}

https://www.xcdsystem.com/wmis/abstract/File6959/GA713_ImageFigure 0614025341.jpg

Image/Figure Caption: Figure: Simulated phantoms containing (A) different sized optical absorbing molecules and (C) mini-Derenzo type in addition to the matched QPA image reconstructions from each, (B) and (D), respectively.

Complete Status: Complete

First Name: Kenneth

Last Name: Hoyt

Email: kenneth.hoyt@utdallas.edu

Organization: University of Texas at Dallas

Country: United States 


\title{
ID: GA714 \\ Fluorescent Probe Design: Challenges and Future Perspectives
}

\author{
Adam Sedgwick, The University of Texas at Austin, a.c.sedgwick@utexas.edu
}

\section{Category: New Chemistry, Biology \& Bioengineering}

\begin{abstract}
Body : Fluorescence imaging offers a powerful non-invasive approach for the visualisation of key cellular components/ processes with high spatial and temporal resolution.1 In conjunction with recent advancements in fluorescence imaging technologies, intelligent synthetic probe design has enabled the detection of various biological species in vitro and in vivo.2, 3 Inspired by these examples, a newly reported synthetic method was implemented to achieve the 'first of' fluorescent probe for the rapid detection of hydroxylamine in aqueous solution.4, 5 Unfortunately, when designing this fluorescent probe and other probe examples, a number of issues were encountered, including poor solubility and selectivity. Motivated to overcome these limitations, biocompatible fluorescent probe-protein complexes were developed to allow the detection of a disease-specific biomarkers. The first demonstration resulted in the enhanced in vitro and in vivo detection of the key reactive oxygen species/reactive nitrogen species (RNS/ROS) signalling agent peroxynitrite (ONOO-) - Scheme 1a.6 Recently, this strategy was extended to the detection of an inflammatory-based enzymatic biomarker, human neutrophil elastase (HNE) in vitro.7 This newly reported protein-based hybridization (host-guest assembly) is believed to represent a simple and general strategy for researchers to use to overcome the issues of low solubility and poor cell permeability of newly designed fluorescent probes. Despite the success shown using fluorescent probes, biological systems are complex with more than one species being involved in a given pathway. As a result, current fluorescent imaging platforms can provide a limited picture to a particular biological study. Exploiting various photophysical probes of specific fluorophore scaffolds, so called dual/multi-responsive fluorescent probes or AND-logic gates have been developed (Scheme 1b).8 A novel AND logicbased fluorescence probe for the simultaneous detection of ONOO- and GSH was developed. Cellular imaging experiments using this probe demonstrated the ability to monitor the coexistence of metabolically produced $\mathrm{ONOO}^{-}$and $\mathrm{GSH}$ and evaluate a therapeutic effect.9 This was recently extended to the detection of ONOO-, however, only when the fluorescent probe was bound to amyloid beta peptide, a key biomarker found in Alzheimer's disease.10 Overall, a new strategy has been developed for the detection of a biologically important species through applying newly developed synthetic methodology. Host-guest chemistry has been employed to overcome solubility issues of fluorescent probes and multi-responsive fluorescent probes have been developed that are suitable for complex biological systems.
\end{abstract}

References: 1. E. Haustein and P. Schwille, Hfsp Journal, 2007, 1, 169-180. 2. D. Wu, A. C. Sedgwick, T. Gunnlaugsson, E. U. Akkaya, J. Yoon and T. D. James, Chem. Soc. Rev., 2017, 46, 7105-7123. 3. A. C. Sedgwick, L. L. Wu, H. H. Han, S. D. Bull, X. P. He, T. D. James, J. L. Sessler, B. Z. Tang, H. Tian and J. Yoon, Chem. Soc. Rev. 2018, 47, 8842-8880. 4. L. R. Peacock, R. S. L. Chapman, A. C. Sedgwick, M. F. Mahon, D. Amans and S. D. Bull, Org. Lett. 2015, 17, 994-997. 5. A. C. Sedgwick, R. S. L. Chapman, J. E. Gardiner, L. R. Peacock, G. Kim, 
J. Yoon, S. D. Bull and T. D. James, Chem. Commun. 2017, 53, 10441-10443. 6. H. H. Han, A. C. Sedgwick, Y. Shang, N. Li, T. T. Liu, B. H. Li, K. Q. Yu, Y. Zang, J. T. Brewster, M. L. Odyniec, M. Weber, S. D. Bull, J. Li, J. L. Sessler, T. D. James, X. P. He and H. Tian, Chem. Sci. 2020, 11, 1107-1113. 7. Z. Jia, H-H. Han, A. C. Sedgwick, G. T. Williams, L. Gwynne, J. T. Brewster II, S. D. Bull, A. T. A. Jenkins, X-P. He, H. Schönherr, J. L. Sessler and T. D. James, Front. Chem. 2020, DOI:10.3389/fchem.2020.00389 8. J. L. Kolanowski, F. Liu and E. J. New, Chemical Society Reviews, 2018, 47, 195-208. 9. A. C. Sedgwick, H. H. Han, J. E. Gardiner, S. D. Bull, X. P. He and T. D. James, Chem. Sci. 2018, 9, 3672-3676. 10. A. C. Sedgwick, W. T. Dou, J. B. Jiao, L. L. Wu, G. T. Williams, A. T. A. Jenkins, S. D. Bull, J. L. Sessler, X. P. He and T. D. James, J. Am. Chem. Soc. 2018, 140, 14267-14271.

\section{Image/Figure:}

https://www.xcdsystem.com/wmis/abstract/File6959/GA714_ImageFigure_0614025345.png

Image/Figure Caption: Scheme 1 - (a) Basic schematic of a probe-protein complex for the detection of ONOO- (b) Basic schematic to the design of a multiresponsive fluorescent probe

Complete Status: Complete

First Name: Adam

Last Name: Sedgwick

Email: a.c.sedgwick@utexas.edu

Organization: The University of Texas at Austin

Country: United States 


\title{
ID: GA716 \\ In vitro Dynamic Molecular Imaging of Nanoparticle Permeation Across Membranes Using a Novel Method Based on Nuclear Imaging (PET)
}

\author{
Mahmoud Omar, Université Laval, mahmoud.omar.1@ulaval.ca
}

\section{Category: New Chemistry, Biology \& Bioengineering}

\begin{abstract}
Body : Introduction: In pharmacology, permeation tests are often performed in vitro by using dedicated equipment called Franz diffusion cells. Such tests are used to measure the permeation and interaction of molecules (and nanoparticles) across biological and synthetic membranes (e.g. skin, gastrointestinal tissues, polymer membranes). 1 The device is made of two compartments, one donor (DC) and one acceptor (AC), separated by the tested membrane. Molecules (or nanoparticles) are carried by the fluid of the DC; They diffuse through the tested material, and they are collected by the AC where they are sampled and analysed.2 Unfortunately, in the case of certain highly potent compounds such as nanoparticles carrying anti-cancer drugs ("nanocarriers"), the detection technologies currently used in diffusion cells (as UV-vis, FTIR) are not sensitive enough.3 The sensitivity of the detection could be dramatically enhanced by relying on a nuclear molecular imaging modality. In this work, a novel diffusion cell operating in a positron emission tomography scanner (PET) was designed (Figure 1a-b) as a new tool to improve the sensitivity of permeation studies and to allow real-time monitoring of the permeation process. We report on radioactive nanoparticles (ultrasmall $89 \mathrm{Zr}$ (IV)-gold nanoparticles-GNP), as a model drug nanocarrier for testing with PET, the permeation of nanoparticles and interaction across biological and polymeric membranes. Materials and Methods: GNPs (4nm diam.-TEM; hydrodynamic diam. 19nm diam.-DLS) were grafted with deferoxamine, DFO (chelator for $\mathrm{Zr}(\mathrm{IV})$; Figure 1c), and their physicochemical properties were characterized by Fourier-transform infrared spectroscopy (FTIR), X-ray photoelectron spectroscopy (XPS), thermogravimetric analysis (TGA), and elemental analysis. Radiolabeling was performed with $89 \mathrm{Zr}$ (IV) (half-life: 3.3 days; 95\% radiochemical yield; Au:Zr molar ratio: 1:1.7). FTIR revealed the presence of DFO at the surface of GNPs (hydroxamate peaks: $1629.0 \mathrm{~cm}-1,1569.0 \mathrm{~cm}-1$; amine peak: $3312.0 \mathrm{~cm}-1$ ). XPS revealed the $\mathrm{O}=\mathrm{C}-\mathrm{N}$ C1s peak of DFO at $287.49 \mathrm{eV}$, and upon $\mathrm{Zr}(\mathrm{IV})$ chelation, chelated $\mathrm{Zr} 3 \mathrm{~d} 5 / 2$ peak at $182.4 \mathrm{eV}$ with no ionic $\mathrm{Zr} 3 \mathrm{~d} 5 / 2(183.38 \mathrm{eV})$. TGA showed a two-step degradation at 189 and $320^{\circ} \mathrm{C}$ corresponding to DFO and PEG, respectively. Overall, the physicochemical characterisation techniques confirmed the synthesis of ultrasmall GNP functionalized with DFO capable of chelating 89Zr(IV) for PET imaging. The permeation of GNPs through biological (porcine skin) and polymer (latex and nitrile) membranes was studied with PET (Figure 1d-f). These membranes were placed in the designed diffusion cell, and $5 \mathrm{~mL}$ of a phosphate-buffered aqueous solution containing $89 \mathrm{Zr}$ GNP ( $\sim 5 \mathrm{MBq} ; 0.15 \mathrm{pmol} / \mathrm{mL} \mathrm{Zr}$; and 0.22 or $0.1 \mathrm{mM}$ of $\mathrm{Au}$ for skin and polymeric membranes, respectively) was added in the DC. DC and AC were imaged continuously for a duration of up to 42 hours. Results: Imaging the permeation of GNP revealed kinetic profiles with unprecedented time resolution. Particles kinetic profiles showed three permeation phases. Radiolabeled GNPs were detected at a concentration as low as $6.8 \times 10-6 \mathrm{nM}$ of $89 \mathrm{Zr}-\mathrm{DFO}$ and $1 \mathrm{nM}$ of $\mathrm{Au}$, which is in general 1000 time more sensitive compared with conventional spectroscopic methods. 4
\end{abstract}


Permeation parameters were extracted from these curves: lag time, influx, and diffusion coefficients. Real-time monitoring revealed GNP penetrated in the skin and out with an influx of 7.8 and $4.9 \mu \mathrm{M} \mathrm{mm}-2 \mathrm{hr}-1$, respectively. Alternatively, the GNP permeation profiles through polymeric membranes showed an influx of 12.8 and $22.5 \mathrm{nM} \mathrm{mm}-2 \mathrm{hr}-1$ for latex and nitrile membranes, respectively. Conclusion: This study confirms the strong potential of PET as a highly-sensitive, real-time imaging instrument to monitor the permeation process of nanoparticles (and other substances) across membranes. The study demonstrated quantitatively the permeation profile of GNPs through the skin and polymeric membranes. In the future, this technique will allow the performance of diffusion tests at an unrivalled sensitivity, which is needed for measuring the diffusion of highly potent or toxic molecules across membranes.

References: 1. Bronaugh, L., et al. J. Pharm. Sci., 2004,74. 2. Heylings, J.R. Topical Drug Bioavailability, Bioequivalence, and Penetration, 2014. 3. Vinches, L., et al. J. Phys., 2015, 617. 4. Lippmann-Pipke, J., et al. Comput. Geosci., 2017, 101.

\section{Image/Figure:}

https://www.xcdsystem.com/wmis/abstract/File6959/GA716 ImageFigure 0614044246.jpg

Image/Figure Caption: Figure $1 \mathrm{~A}$ schematic representation of the in vitro permeation test operated in the PET: (a) The designed PET diffusion cell, (b) A cross-section of the diffusion cell inside the PET, (c) The synthesis of gold nanoparticles, (d) PET imaging of the diffusion cell, (e) Analysis of the regions of interests, (f) Nanoparticles permeation profile.

Complete Status: Complete

First Name: Mahmoud

Last Name: Omar

Email:mahmoud.omar.1@ulaval.ca

Organization: Université Laval

Country: Canada 


\title{
ID: GA717 \\ Tuning Photophysical Properties of Nerve-Binding Near Infrared Fluorophores for Fluorescence-Guided Surgery
}

\author{
Antonio Montaño, Oregon Health \& Science University, montao@ohsu.edu
}

\section{Category: New Chemistry, Biology \& Bioengineering}

\begin{abstract}
Body : Iatrogenic nerve injury presents one of the most feared surgical complications and a major source of morbidity across all surgical specialties. Recent statistics have estimated the current volume of surgical procedures annually exceeds 300 million worldwide. Notably, some procedures, such as radical prostatectomy, are known to carry a greater risk of unintended injury than others. Accidental nerve damage during surgery frequently leads to chronic postsurgical pain, loss of sensation, and/or paralysis. While nerve-sparing techniques have been practiced for decades, their success depends on a surgeon's first-hand experience that can only be acquired by performing numerous procedures, each with a variable success rate.[1] Furthermore, because nerves are often translucent and evasive structures, they can be difficult, if not impossible, to visualize adequately during surgery. A surgeon's primary means of navigation remain limited to white light visualization and neuroanatomical knowledge. While some procedures can be supplemented with electromyographical monitoring, this nevertheless amounts to little more than one's hands and eyes, leaving ample room for new methodologies that may enhance detection and visualization of nerves, providing improved surgical outcomes for both patients and surgeons. Fluorescence-guided surgery (FGS) has the potential to revolutionize surgical outcomes by selectively enhancing tissue visibility. Fluorescent molecules with the greatest potential for clinical translation emit in the near-infrared (NIR) region between $650-900 \mathrm{~nm}$, where tissue absorbance, light-scattering and autofluorescence are all at their local minima.[2] However, a limited number of fluorescent molecules have been developed to image nerve tissue, and the vast majority of these are plagued with high non-specific uptake in surrounding tissues and only emit at visible wavelengths. Development of a NIR nerve-specific fluorophore is challenged by the fact that nerve-specific fluorophores must be of sufficiently low molecular weight to cross the blood-nerve-barrier (BNB), however achieving the degree of conjugation (e.g., number of unsaturated hydrocarbons) requisite of long-wave fluorescent emission unavoidably increases molecular weight.[3] Herein, we report our efforts to synthesize libraries of systematically-modified far-red to NIR fluorophores. Nerve-specificity was verified using both murine and swine models following direct and systemic administrations.[4]Modification of lead candidates from our $700 \mathrm{~nm}$ library of nerve dyes, facilitated the design of our first-in-class NIR nerve-binding fluorophores, fully compatible with the $800 \mathrm{~nm}$ channels already present in clinical imaging systems. Comprehensive molecular engineering of these lead compounds have also resulted in new, stable compounds that are water soluble and demonstrate improved photophysical properties compared to parent compounds, without the need to compromise inherent brightness or nerve-specificity Finally, through fluorescence-guided laparoscopic surgery we have successfully identified the first NIR nervebinding fluorophore candidates for clinical translation. This exciting technology is poised for
\end{abstract}


integration into the day-to-day clinical workflow and will enable direct, high-contrast nerve visualization in real-time.

References: 1. Summer, L. G.-S.; Khaled, A. N.; Kenneth, M. F.; Onkar, K.; Yoshitomo, A.; Tiberiu, M. S.; Bruce, F. J.; Nicole, E. B.; Cristina, A. T. H.; John, V. F., Nerve-Highlighting Fluorescent Contrast Agents for Image-Guided Surgery. Molecular imaging 2011, 10 (2), 7290.2010.00026. 2. Gibbs, S. L.; Xie, Y.; Goodwill, H. L.; Nasr, K. A.; Ashitate, Y.; Madigan, V. J.; Siclovan, T. M.; Zavodszky, M.; Tan Hehir, C. A.; Frangioni, J. V., Structure-Activity Relationship of Nerve-Highlighting Fluorophores. PLOS ONE 2013, 8 (9), e73493. 3. Park, M. H.; Hyun, H.; Ashitate, Y.; Wada, H.; Park, G.; Lee, J. H.; Njiojob, C.; Henary, M.; Frangioni, J. V.; Choi, H. S., Prototype Nerve-Specific Near-Infrared Fluorophores. Theranostics 2014, 4 (8), 823-833. 4. Barth, C. W.; Gibbs, S. L., Direct Administration of Nerve-Specific Contrast to Improve Nerve Sparing Radical Prostatectomy. Theranostics 2017, 7 (3), 573-593.

Complete Status: Complete

First Name: Antonio

Last Name: Montaño

Email: montao@ohsu.edu

Organization: Oregon Health \& Science University

Country: United States 


\title{
ID: GA719 \\ Biomarker targeted manganese-based MRI contrast agents and multi-color mapping of cancer molecular signatures by pMRI
}

\author{
zongxiang gui, Georgia State University, ZGUI1@STUDENT.GSU.EDU
}

\section{Category: Oncology}

\begin{abstract}
Body : Liver metastases from different primary sites (i.e., colon, lung, and uveal melanoma (UM)) have differential expressions of multiple key molecular biomarkers, reflecting tumor microenvironment (MTE), and tumor-stroma interactions difference, respond to different types of therapy. Researches about human liver metastases have provided evidence that chemokine receptor type 4 (CXCR4), collagen type 1related to metastatic potential, angiogenesis, and the micro-environment during cancer initiation and progression in several metastatic mouse models. There is an unmet need to develop non-invasive molecular imaging, with the additional capability to quantitatively detect multiple biomarkers at different stages of diseases at the cell, tissue, and body levels. Our team developed a protein MRI contrast agent's platform. We have developed several Gd3 + based contrast agents against various biomarkers, with high relaxivities (e.g. Gd3 + based CXCR4 targeted MRI contrast agent $\mathrm{r} 1=28.3 \mathrm{mM}-1$. s$1, \mathrm{r} 2=40.2 \mathrm{mM}-1 . \mathrm{s}-1$ at $1.4 \mathrm{~T} . \mathrm{r} 1=23.5 \mathrm{mM}-1 . \mathrm{s}-1$ at $1.4 \mathrm{~T}, \mathrm{r} 2=98.6 \mathrm{mM}-1$. s- 1 at $7.0 \mathrm{~T})$. Here, we report first $\mathrm{Mn} 2+$ based protein contrast agents with the capability of targeting collagen type 1 , and advantages such as differential relativity without using Gd3+ as well as PET/MRI imaging capability. It exhibits high relaxivities $(\mathrm{r} 1=30.6 \mathrm{mM}-1$. $\mathrm{s}-1$ at $1.4 \mathrm{~T}, \mathrm{r} 2=61.7 \mathrm{mM}-1$. s- 1 at 1.4T). They also show strong stability and high metal selectivity for $\mathrm{Gd} 3+$ and $\mathrm{Mn} 2+$ to Other physiological metals such as $\mathrm{Ca} 2+$ and $\mathrm{Zn} 2+$, strong targeting capability to overexpressed biomarkers in cancer cells compare with clinical contrast agents. Preliminary results have demonstrated them in vivo imaging capability using animal models. With developed $\mathrm{Gd} 3+$ and $\mathrm{Mn} 2+$ agents targeting different biomarkers, we are developing multi-color MRI methodology, expected to enable precision molecular MR Imaging (pMRI), which has a wide range of applications in tumor diagnosis and treatment stratification.
\end{abstract}

References: Pellicoro, A., Ramachandran, P., Iredale, J. P. \& Fallowfield, J. A. Liver fibrosis and repair: immune regulation of wound healing in a solid organ. Nature Reviews Immunology 14, 181-194 (2014). Erez, N. Fibroblasts form a hospitable metastatic niche in the liver. Nature cell biology 18, 465-466 (2016). Hernandez-Gea, V. \& Friedman, S. L. Pathogenesis of liver fibrosis. Annual review of pathology: mechanisms of disease 6, 425-456 (2011). Salarian, M. et al. Early detection and staging of chronic liver diseases with a protein MRI contrast agent. Nature communications 10, 1-14 (2019). Tan, S. et al. Chemokine receptor 4 targeted protein MRI contrast agent for early detection of liver metastases. Science Advances 6, eaav7504 (2020). Xue, S. et al. Protein MRI contrast agent with unprecedented metal selectivity and sensitivity for liver cancer imaging. Proceedings of the National Academy of Sciences 112, 6607-6612 (2015).

\section{Image/Figure:}


Image/Figure Caption: Working model of multi-color MR imaging. Mn2+ based protein contrast agents with the capability of targeting collagen type 1 and $\mathrm{Gd} 3+$ based protein contrast agents with the capability of targeting CXCR4 were simultaneously administered to tumorbearing mice. By acquiring T1 and T2 mapping at 1.4 T with MRF, concentration distributions of both were extrapolated from tissue relaxivities before and after the administration of contrast agents.

Full Name of Abstract's 1st Author : Zongxiang Gui

Complete Status: Complete

First Name: zongxiang

Last Name: gui

Email: ZGUI1@STUDENT.GSU.EDU

Organization: Georgia State University

Country: United States 


\title{
ID: GA720 \\ Development of 18F-labeled gabapentin for imaging $\alpha 2 \delta$-1 receptors
}

\author{
Yu-Peng Zhou, Massachusetts general hospital, yzhou40@mgh.harvard.edu
}

\section{Category: New Chemistry, Biology \& Bioengineering}

\begin{abstract}
Body : Noninvasive imaging of biochemical processes underlying pain signaling can greatly benefit the diagnosis and treatment of neuropathic pain. Current approaches for imaging neuropathic pain (e.g. CT, MRI, PET, and SPECT) have limited sensitivity or specificity, 1 therefore, novel PET radiotracers are needed. Gabapentin, which targets $\alpha 2 \delta-1$ protein, has been successfully used to treat neuropathic pain. 2 The increased expression of $\alpha 2 \sigma-1$ subunit of voltage-dependent calcium channels (VDCCs) in the spinal cord and dorsal roots during neuropathic pain has been observed in different animal models.3 Based on these results, radiolabeled gabapentin is proposed as a potential radiotracer for imaging pain. Previously reported structure-activity relationship (SAR) studies on gabapentin have shown that 4-methyl and 4-ethyl derivatives of gabapentin are active whereas modification in the 2 position reduce the binding affinity of the molecules. 4 Based on these results, the gabapentin derivative with $18 \mathrm{~F}$ in the 4 position is proposed. The synthesis starts from the commercially available compound 2azaspiro[4.5]decane-3,8-dione, which was protected, reduced, and mesylated subsequently gave the labeling precursor mesylate compound. The $18 \mathrm{~F}$ labeled Boc protected gabapentin lactam was obtained by the reaction $[18 \mathrm{~F}] \mathrm{KF}$ and the labeling precursor in the presence of kryptofix2.2.2.(K222)/K2CO3 at $110 \mathrm{Co}$ for $30 \mathrm{~min}$. $18 \mathrm{~F}$-labeled gabapentin is obtained by the hydrolysis and deprotection of $18 \mathrm{~F}$ labeled Boc protected gabapentin lactam using base and acid, respectively. Reversed-phase HPLC is used for the purification of 18F-labeled gabapentin using XBridge $5 \mu \mathrm{m} \mathrm{C} 18$ semiprep-column $(10 \times 250 \mathrm{~mm})$. The mobile phase is NaH2PO4 EtOH-H2O $(10 \mathrm{mM}, \mathrm{v} / \mathrm{v}=5: 95)$ solution at flow rate of $4 \mathrm{~mL} / \mathrm{min}$. For the autoradiography experiment, mouse spinal cords sections are fixed in formalin for $10 \mathrm{~min}$ and washed with PBS. Sections are incubated with $10 \mu \mathrm{Ci}$ of $18 \mathrm{~F}$-labeled gabapentin with pregabalin $(0-5,000 \mathrm{nM})$ in PBS for 30 min and then washed with ice cold PBS ( $3 \times 0.5 \mathrm{~min})$. After washing, the tissues are dried, exposed to phosphor screen and imaged 24 hours later. 18F-labeled gabapentin was synthesized in $\sim 20 \%$ decay corrected radiochemical yield based on radio-HPLC. The identity and purity was confirmed by analytical HPLC. Autoradiography in mouse spinal cord sections shows binding of $18 \mathrm{~F}-$ labeled gabapentin in dorsal horn region consistent with the reported expression of $\alpha 2 \delta-1$ receptors. 5 Over $85 \%$ of the binding could be displaced by $5 \mu \mathrm{M}$ of the selective $\alpha 2 \delta-1$ drug pregabalin demonstrating high specificity.
\end{abstract}

References: [1] Mol. Imaging. Biol. 20, 188-193 (2018); [2] Nat. Rev. Neurosci. 13, 542-555 (2012); [3] J. Neurosci. 21, 1868-1875 (2001); [4] J. Med. Chem. 41, 1838-1845, (1998); [5] Neuroscience 155, 510-521 (2008)

Complete Status: Complete

First Name: Yu-Peng 
Last Name: Zhou

Email: yzhou40@mgh.harvard.edu

Organization: Massachusetts general hospital

Country: United States 


\title{
ID: GA721 \\ Minicircles for a Two-Step Blood Biomarker and PET Imaging Early Detection Strategy
}

\author{
Elise Robinson, Stanford University, erobin@stanford.edu
}

\section{Category: Oncology}

\begin{abstract}
Body : Introduction: Early cancer detection can dramatically increase treatment options and survival rates for patients1. However, detection of early stage solid tumors remains difficult; blood-based clinical biomarker strategies suffer from high variability due to background expression produced by noncancerous cells, while highly sensitive imaging techniques such as positron emission tomography (PET) remain too expensive for routine screening of large populations2,3. Here, we demonstrate a two-step strategy to detect and locate early cancer growths by delivering tumor-activatable minicircle plasmids (MCs) encoding both blood-based and imaging reporter genes to tumor cells. In long-term clinical strategies, we propose PET imaging to be performed only in patients with secreted biomarker detected in blood, i.e. only those in which a simple and cost-effective reporter gene blood test has indicated the presence of cancer. Methods: We genetically engineered tumor-activatable MCs under the control of the pan-tumor-specific Survivin promoter (MC-pSur) to encode: (1) Gaussia Luciferase (GLuc), a secreted biomarker that is not produced by any other tissues in the body and can easily be assayed in blood samples4; and (2) Herpes Simplex Virus Type 1 Thymidine Kinase mutant (HSV-1 sr39TK), a PET reporter gene that can be used for highly sensitive, deep tissue imaging to determine tumor location(s)5. HSV-1 sr39TK expression can be assessed via retention of two PET reporter probes analogs: tritiated Penciclovir $([3 \mathrm{H}] \mathrm{PCV})$ in cell culture and 9-(4-[18F]fluoro-3-(hydroxymethyl)butyl]guanin ([18F]FHBG) in vivo5. In cell culture, we delivered 500ng of MC-pSur to HeLa cells and measured GLuc secretion and [3H]PCV retention on day 3. In mice bearing subcutaneous HeLa tumors, we delivered three 40ug doses of MCpSur intratumorally on days 0,6 and 7, and assessed tumor-specific expression by measuring levels of GLuc secreted in blood and retention of [18F]FHBG in tumors on day 9. Results: In cell culture, HeLa cells treated with MC-pSur secreted 1.252 x 106-fold higher GLuc levels in media relative to HeLa cells treated with MC-pSur and no transfection agent, or a mock treatment $(\mathrm{n}=3$, day $3, \mathrm{p}=0.0005)$. [3H]PCV levels were 508.2-fold higher with respect to the same treatment groups ( $\mathrm{n}=3$, day 3 , $\mathrm{p}$ Conclusion: We present a minimally invasive two-step strategy for early cancer detection. Our results suggest that tumor-activatable MCs delivered to cancer cells express a secretable biomarker and PET reporter gene to potentially overcome the specificity limitations of endogenous blood-based biomarkers and high costs of routine PET screening. Ongoing work includes detecting metastatic tumors with systemic MC delivery in animal models and determining the smallest tumor detectable by tumor-activatable MCs. This work contributes significantly to early cancer detection strategies by developing novel screening tools and methods.
\end{abstract}

References: 1. Etzioni, R. et al. The case for early detection. Nat. Rev. Cancer 3, 243-252 (2003). 2. Diamandis, E. P. Cancer biomarkers: Can we turn recent failures into success? J. Natl. 
Cancer Inst. 102, 1462-1467 (2010). 3. Yasuda, S. et al. Application of positron emission tomography imaging to cancer screening. Br. J. Cancer 83, 1607-1611 (2000). 4. Tannous, B. Gaussia luciferase reporter assay for monitoring of biological processes in culture and in vivo. Nat. Protoc. 4, 582-591 (2009). 5. Herschman, H. R. PET reporter genes for noninvasive imaging of gene therapy, cell tracking and transgenic analysis. Crit. Rev. Oncol. Hematol. 51, 191-204 (2004).

\section{Image/Figure:}

https://www.xcdsystem.com/wmis/abstract/File6959/GA721 ImageFigure 0614072627.jpg

Image/Figure Caption: Figure 1. (a) HeLa cells treated with tumor-activatable minicircle MCpSur secrete $1.252 \times 106$-fold higher levels of GLuc relative to HeLa cells treated with MC-pSur and no transfection agent, or a mock treatment $(n=3$, day $3, p=0.0005)(b)$ HeLa cells treated with MC-pSur result in 508.2-fold retention of the PET reporter probe [3H]PCV relative to HeLa cells treated with MC-pSur and no transfection agent, or a mock treatment $(\mathrm{n}=3$, day $3, \mathrm{p}$ in vivo produce GLuc that is secreted and measured in whole blood (n=6 for "MC-pSur + jetPEI," $n=7$ for "Mock," day 9, $\mathrm{p}=0.2378$ ). (d) Representative PET/CT images of mice that received either MC-pSur with jetPEI or a mock treatment. [18F]FHBG was injected intravenously and $10 \mathrm{~min}$ static PET scans were acquired 30 min later. Red arrows point to tumor location. (e) Quantification of [18F]FHBG retention within PET ROIs at tumor site $(\mathrm{n}=6$, day $9, \mathrm{p}=0.0058)$. (f) Quantification of [18F]FHBG retention in excised tumors as measured on a gamma counter ( $\mathrm{n}=6$, day 9, $\mathrm{p}=0.0196)$. MC-pSur, minicircle with Survivin promoter; GLuc, Gaussia Luciferase; [3H]PCV, tritiated Penciclovir; [18F]FHBG, 9-(4-[18F]fluoro-3-(hydroxymethyl)butyl)guanine; $\% \mathrm{ID} / \mathrm{g}$, percent injected dose per gram.

Full Name of Abstract's 1st Author : Elise Robinson

Complete Status: Complete

First Name: Elise

Last Name: Robinson

Email: erobin@stanford.edu

Organization: Stanford University

Country: United States 


\title{
ID: GA722 \\ Development of a Novel Small Molecule Photosensitizer with Integrated Photoacoustic Readout
}

\author{
Catharine Brady, University of Illinois at Urbana-Champaign, cjbrady2@illinois.edu
}

Category: New Chemistry, Biology \& Bioengineering

\begin{abstract}
Body : Reactive oxygen species (ROS), such as singlet oxygen (1O2) and superoxide $(\mathrm{O} 2 \bullet-)$, are transient and chemically reactive molecules often generated in the mitochondria. At endogenous concentrations, ROS have been shown to play a role in cellular signaling and redox homeostasis, but increased levels are associated with oxidative stress, can cause downstream damage to biomolecules, and can result in cellular apoptosis and necrosis. Photodynamic therapy takes advantage of this reactivity to kill diseased cells through the use of photosensitizers that generate ROS from molecular oxygen upon light activation. While this therapeutic technique has shown potential, there are few clinically approved photosensitizers which demonstrate little structural diversity because it is difficult to predict photosensitizing ability based on chemical features. This highlights the need for new photosensitizer platforms with improved properties for in vivo applications. To address this, a novel near-infrared (NIR) small molecule photosensitizer with integrated photoacoustic imaging readout was developed, optimized, and evaluated. Various modifications to the hemicyanine dye (HD) scaffold were synthesized and assessed for their photosensitizing, photoacoustic, and general photochemical properties. It was demonstrated that a selenium-containing HD dye showed increased photosensitization capability, and red shifted absorbance and emission maxima. The same wavelength light for singlet oxygen generation was shown to be able to be used for photoacoustic imaging which can allow for in vivo tracking of the photosensitizer at centimeter depths. Further work will include the incorporation of specific disease targeting groups for to help eliminate off-target effects.
\end{abstract}

Full Name of Abstract's 1st Author : Catharine J. Brady

Complete Status: Complete

First Name: Catharine

Last Name: Brady

Email: cjbrady2@illinois.edu

Organization: University of Illinois at Urbana-Champaign

Country: United States 


\title{
ID: GA723 \\ Investigating differential PARP-1 expression for molecular diagnosis of melanoma using a PARP1 targeted fluorescent probe
}

\author{
Aditi Sahu, MSKCC, aditisahu@gmail.com
}

\section{Category: Oncology}

\begin{abstract}
Body : Background: Melanoma is the most aggressive skin cancer with the highest associated mortality[1]. The 5-year survival rates range from $98.3 \%$ (stage I) to $16 \%$ (stage IV) emphasizing the importance of diagnosis at an early stage[2]. Non-invasive morphological imaging approaches such as dermoscopy and high-resolution, morphological reflectance confocal microscopy demonstrate high sensitivity but moderate and variable specificity[3]. Noninvasive gene assays based on PRAME (PReferentially expressed Antigen in MElanoma) expression show similarly high sensitivity but moderate specificity[4]; neither of these approaches have shown potential in stratifying lesions based on malignant potential. Noninvasive molecular imaging using targeted fluorophores such as PARPi-FL (targets PARP1, which is overexpressed in cancers[5]), can enable functional evaluation of PARP1 expression to stratify lesions which are malignant and/or with higher malignant potential[6], thereby improving diagnostic specificity for melanoma detection and prognosis. As a first step towards PARPi-FL imaging in vivo, we investigated the differences in PARP1 expression in the spectrum of melanocytic lesions including melanoma and benign nevi, using histology and PRAME expression as gold standards. Methods: We investigated PARP1 and PRAME expression using immunohistochemistry (IHC) in 25 lesions (14-melanoma, 11-benign nevi) in $5 \mathrm{~mm}$-thin formalin-fixed paraffin-embedded serial sections. IHC was optimized on an automated LeicaBond stainer platform using optimized protocols. IHC and hematoxylin-and-eosin (H\&E) tissue sections were digitized using a MIRAX slide scanner. Manual scoring (by a board-certified dermatopathologist) and IHC quantification (software-based) were performed to assess the differences in PARP1 expression in 14 specimens. Since PARP1 can localize to the nucleolus in addition to the nuclear volume, differential PARP1 staining patterns can be observed in nucleus and nucleolus. Thus, the nuclear and nucleolar positivity were separately evaluated for manual scoring. Manual dermatopathologist scoring was based on number of positive nuclei and nucleoli in the lesion area over the range 0-4, with 0 (absent), 1 ( Results: PARP1 expression was found in all benign and melanoma tissues at different levels. Prominent nucleolar staining was observed in all melanoma tissues, an important feature for detection of malignancy and aggressiveness in pathology[7]. Based on dermatopathologist evaluation, melanoma samples had higher scores for both nucleus, nucleolus area positivity and overall score (range 3-4) as compared to benign nevi (range 1-4). Benign nevi showed high nuclear positivity in some nevus (3 out of 8 specimens were scored 4), however they showed low positivity for nucleolus and overall score. In quantification, melanoma showed significantly higher percentage area and intensity for PARP1 expression as compared to benign nevi ( $\mathrm{p}$ Conclusions: Melanoma shows higher PARP1 expression in terms of intensity and area positivity in direct comparison with benign melanocytic lesions, especially in nucleolus relative to nucleus. These findings will be confirmed on a larger data set to build statistical classifier for benign versus melanoma using both nuclear and
\end{abstract}


nucleolar positivity. Given the IND status of PARPi-FL for use in humans, the results will establish the basis for subsequent in vivo clinical imaging studies for molecular melanoma diagnosis in patients.

References: 1. Karimkhani, C., et al., The global burden of melanoma: results from the Global Burden of Disease Study 2015. British Journal of Dermatology, 2017. 177(1): p. 134-140. 2. Nikolaou, V. and A. Stratigos, Emerging trends in the epidemiology of melanoma. British journal of dermatology, 2014. 170(1): p. 11-19. 3. Guitera, P., et al., In vivo confocal microscopy for diagnosis of melanoma and basal cell carcinoma using a two-step method: analysis of 710 consecutive clinically equivocal cases. Journal of investigative dermatology, 2012. 132(10): $\mathrm{p}$. 2386-2394. 4. Gerami, P., et al., Development and validation of a noninvasive 2-gene molecular assay for cutaneous melanoma. Journal of the American Academy of Dermatology, 2017. 76(1): p. 114-120. e2. 5. Ossovskaya, V., et al., Upregulation of poly (ADP-ribose) polymerase-1 (PARP1) in triple-negative breast cancer and other primary human tumor types. Genes \& cancer, 2010. 1(8): p. 812-821. 6. Kossatz, S., et al., Validation of the use of a fluorescent PARP1 inhibitor for the detection of oral, oropharyngeal and oesophageal epithelial cancers. Nature Biomedical Engineering, 2020. 4(3): p. 272-285. 7. Derenzini, M., L. Montanaro, and D. Treré, What the nucleolus says to a tumour pathologist. Histopathology, 2009. 54(6): p. 753-762.

\section{Image/Figure:}

https://www.xcdsystem.com/wmis/abstract/File6959/GA723_ImageFigure_0614080306.PNG

Image/Figure Caption: PARP1- expression and quantification in melanoma and benign nevi. Representative examples of H\&E and PARP1-IHC stained sections show high and low PARP1 expression in melanoma and nevus, respectively. The dermatopathologist scoring shows higher values for nuclear and nucleolar positivity and overall score for melanoma (range 3-4 versus 1-4 for benign nevi). Prominent nucleolar PARP1 positivity was observed in melanoma, potentially helping differentiate melanoma from nevi. IHC quantification of area positivity and intensity over area was higher for melanoma $(\mathrm{p}<0.05)$ in direct comparison to nevi, except an outlier nevus with congenital pattern shown in the figure. Epidermal PARP1 expression was used as an internal control.

Full Name of Abstract's 1st Author : Aditi Sahu

Complete Status: Complete

First Name: Aditi

Last Name: Sahu

Email: aditisahu@gmail.com

Organization: $\mathrm{MSKCC}$

Country: United States 


\title{
ID: GA724 \\ Visualizing Tumor Microenvironment and subtyping Cancer by pMRI
}

\author{
Jingjuan Qiao, Georgia State University, jqiao2@gsu.edu
}

\section{Category: New Chemistry, Biology \& Bioengineering}

\begin{abstract}
Body : The preferred imaging modality for the detection of tumor extent and treatment response to neo-adjuvant and adjuvant medical therapies involves generation of high-resolution 3D anatomic and dynamic MRI images without exposing patients to ionizing radiation. We have established a series of robust, sensitive protein MRI contrast agents to longitudinally quantify and multi-dimensionally map the expression and distribution of diagnostic tumor biomarkers and tumor microenvironment biomarkers, including collagen1, CXCR4 and PDL-1. Here we report the optimization of production and manufacturing of multiple biomarker targeted contrast agents for collagen1, CXCR4, PDL-1, integrin, HER2, and EGFR, exhibiting high stability, reproducibility and scale up productivity. These developed biomarker targeted protein contrast agents exhibit 10 - to 50-fold increases in both $\mathrm{r} 1$ and $\mathrm{r} 2$ relaxivities, compared to clinicallyapproved $\mathrm{Gd} 3+$ contrast agents, resulting in exceptional imaging capability that can discern heterogeneous tissue signals via a dual MR imaging methodology. These contrast agents have also demonstrated strong metal binding stability for $\mathrm{Gd} 3+$ and significant metal selectivity over physiological ions. The ability of these agents to bind biomarkers CXCR4, collagen, HER2, and PDL1, has also been optimized, and includes the capability to detect overexpressed biomarkers in TME of primary cancers and liver metastasis from pancreatic cancer PDAC, breast cancer, and uveal melanoma. We have further demonstrated their in vivo capability to visualize heterogeneous expression of biomarkers in TME using several mouse models. Our studies in precision imaging with unprecedented sensitivity and accuracy address major medical gaps, including early detection of small lesions and heterogeneity of multiple biomarker expression, especially for high risk patients, and offer means to monitor dynamic changes of biomarkers and the tumor environment, both during disease progression and following treatment with applications in image-guided interventions.
\end{abstract}

References: 1. Pu F, Qiao J, Xue S, Yang H, Patel A, Wei L, Hekmatyar K, Salarian M, Grossniklaus HE, Liu ZR, Yang JJ. GRPR-targeted Protein Contrast Agents for Molecular Imaging of Receptor Expression in Cancers by MRI. Scientific reports 2015;5:16214:PMC4649707. 2. Qiao J, Li S, Wei L, Jiang J, Long R, Mao H, Wang L, Yang H, Grossniklaus HE, Liu ZR, Yang JJ. HER2 Targeted Molecular MR Imaging Using a De Novo Designed Protein Contrast Agent. PLoS One 2011;6(3):e18103:3063795. 3. Salarian M, Turaga RC, Xue S, Nezafati M, Hekmatyar K, Qiao J, Zhang Y, Tan S, Ibhagui OY, Hai Y, Li J, Mukkavilli R, Sharma M, Mittal P, Min X, Keilholz S, Yu L, Qin G, Farris AB, Liu ZR, Yang JJ. Early detection and staging of chronic liver diseases with a protein MRI contrast agent. Nature communications 2019;10(1):4777:PMC6820552. 4. Salarian M, Yang H, Turaga RC, Tan S, Qiao J, Xue S, Gui Z, Peng G, Han H, Mittal P, Grossniklaus HE, Yang JJ. Precision detection of liver metastasis by collagen-targeted protein MRI contrast agent. Biomaterials 2019;224:119478. 5. Tan S, Yang H, Xue S, Qiao J, Salarian M, Hekmatyar K, Meng Y, 
Mukkavilli R, Pu F, Odubade OY, Harris W, Hai Y, Yushak ML, Morales-Tirado VM, Mittal P, Sun PZ, Lawson D, Grossniklaus HE, Yang JJ. Chemokine receptor 4 targeted protein MRI contrast agent for early detection of liver metastases. Sci Adv 2020;6(6):eaav7504:PMC7007242. 6. Xue S, Yang H, Qiao J, Pu F, Jiang J, Hubbard K, Hekmatyar K, Langley J, Salarian M, Long RC, Bryant RG, Hu XP, Grossniklaus HE, Liu ZR, Yang JJ. Protein MRI contrast agent with unprecedented metal selectivity and sensitivity for liver cancer imaging. Proc Natl Acad Sci U S A 2015.

Complete Status: Complete

First Name: Jingjuan

Last Name: Qiao

Email: jqiao2@gsu.edu

Organization: Georgia State University 


\title{
ID: GA725 \\ A miniature 'plug-and-play' microscope for lifetime imaging in preclinical models of brain disease
}

\author{
Janaka Senarathna, Johns Hopkins University School of Medicine, \\ dmmj.senarathna@gmail.com
}

\section{Category: Instrumentation}

\begin{abstract}
Body : Neuroimaging in preclinical models of brain disease is a critical tool enabling both a rigorous assessment of disease pathology and the efficacious development of new drugs. However, brain diseases are complex: i.e. they present densely convoluted neuronal, microcirculatory and immunological dysfunctionalities. Moreover, brain diseases often shift their disease phenotype with progression, therapy, and the extent of therapeutic resistance. State-ofthe-art neuroimaging systems today (e.g. MRI, PET, Multiphoton or confocal microscopy) are often bulky, available only through imaging centers with limited access, expensive, timeconstrained, and typically require the animal to be anesthetized. Consequentially, neuroimaging can only be performed for short durations at discrete time points in the disease lifecycle, critically under-sampling the disease progression. Moreover, independent of the effects of the disease model being investigated, anesthesia alters brain function and may result in erroneous conclusions about disease progression and response to therapy. While miniaturized microscopes that enable imaging in unanesthetized mice have been designed to circumvent this problem1-6, they often lack the capacity to interrogate multiple neurobiological variables, confining the assessment of neurophysiological function. To address these limitations, we developed an affordable, miniature multi-contrast microscope7. It permits interrogating multiple neurophysiological variables in freely behaving mice via multichannel imaging with fluorescence (FL)1, intrinsic optical signals (IOS) 8 and laser speckle contrast (LSC)9, incorporated within a footprint comparable to that of a US quarter (Fig. 1a,b). One can use the FL channel to image neuronal activity with a voltage sensitive dye or a genetically encoded calcium indicator, as well as monitor fluorescently tagged cells. The IOS channel can be used to image microvascular structures, vasodilation and vasoconstriction, while the LSC channel permits imaging blood flow in the brain. A 3D printed disposable plastic head-mount is implanted atop a surgically prepared cranial window that provides optical access to the brain (Fig. 1c). Weighing $3 \mathrm{~g}$ after support from a strain relief, the microscope can be affixed to the head-mount to conduct neuroimaging at a resolution of $5 \mu \mathrm{m}$ over a $3 \times 3 \mathrm{~mm} 2$ field of view $(\mathrm{FoV})$ at 10 frames per second. We also created an easy-to-use graphical user interface (Fig. 1d) to control all parameters related to image acquisition and illumination, thereby making it a 'plugand-play' device with minimal technical knowhow needed for operation. Moreover, one can pack the microscope onto a briefcase for convenient transportation. After validation against a non-miniaturized imaging system, we demonstrate the utility of our microscope by conducting lifetime imaging of vascular changes induced by a brain tumor. We imaged a 9L-GFP tumorbearing mouse daily with our multichannel microscope (Fig. 1e-k). We monitored tumor progression in vivo with the FL channel (Fig. 1e) wherein by day 14 (D14) the entire FoV was occupied by tumor. We successfully tracked the stages of angiogenesis in vivo with the IOS
\end{abstract}


channel (Fig. 1f, and red arrows): microvascular sprouting (D3-D6, Fig. 1j,k), anastomoses and flow establishment (D7-D9), and development of sinusoidal vessels (D7-D15). Fig. 1g shows concomitant, but delayed increase in microvascular perfusion as tumor vessels anastomose with brain microvessels (D7-D9), with mature vessels eventually becoming well perfused (D9-D15). Microvascular connectivity (MC) maps (Fig. 1h) illustrate early stage coherent blood volume fluctuations (i.e. vasodilations and vasoconstrictions) in areas with sprouting tumor vessels (D3D6), which become more correlated (D7-D9), and finally show a decrease of MC due to formation of abnormal blood vessels (D10-D15). Trends of these metrics are plotted in Fig. $1 \mathrm{i}$. We believe that our miniature microscope will herald a new era in preclinical brain disease research by enabling novel insights into the dysfunction of multiple neurobiological variables over the disease lifetime.

References: 1 Ghosh, K. K. et al. Miniaturized integration of a fluorescence microscope. Nat Methods 8, 871-878, doi:10.1038/nmeth.1694 (2011). 2 Sigal, I. et al. Imaging brain activity during seizures in freely behaving rats using a miniature multi-modal imaging system. Biomed Opt Express 7, 3596-3609, doi:10.1364/BOE.7.003596 (2016). 3 Liberti, W. A., Perkins, L. N., Leman, D. P. \& Gardner, T. J. An open source, wireless capable miniature microscope system. J Neural Eng 14, 045001, doi:10.1088/1741-2552/aa6806 (2017). 4 Miao, P. et al. Chronic widefield imaging of brain hemodynamics in behaving animals. Biomed Opt Express 8, 436-445, doi:10.1364/BOE.8.000436 (2017). 5 Jacob, A. D. et al. A Compact Head-Mounted Endoscope for In Vivo Calcium Imaging in Freely Behaving Mice. Curr Protoc Neurosci 84, e51, doi:10.1002/cpns.51 (2018). 6 Barbera, G., Liang, B., Zhang, L., Li, Y. \& Lin, D. T. A wireless miniScope for deep brain imaging in freely moving mice. J Neurosci Methods 323, 56-60, doi:10.1016/j.jneumeth.2019.05.008 (2019). 7 Senarathna, J. et al. A miniature multi-contrast microscope for functional imaging in freely behaving animals. Nat Commun 10, 99, doi:10.1038/s41467-018-07926-z (2019). 8 Hillman, E. M. Optical brain imaging in vivo: techniques and applications from animal to man. J Biomed Opt 12, 051402, doi:10.1117/1.2789693 (2007). 9 Senarathna, J., Rege, A., Li, N. \& Thakor, N. V. Laser Speckle Contrast Imaging: theory, instrumentation and applications. IEEE Rev Biomed Eng 6, 99-110, doi:10.1109/RBME.2013.2243140 (2013).

\section{Image/Figure:}

https://www.xcdsystem.com/wmis/abstract/File6959/GA725 ImageFigure 0614091950.png

Image/Figure Caption: Imaging with the miniature microscope reveals angiogenesis-induced changes over the lifetime of a brain tumor. (a) Schematic of the microscope architecture and (b) the microscope kept next to a US Quarter coin for size comparison. (c) The cranial window preparation and head mount attachment for imaging with the microscope. In set on the top right shows a zoomed in view of cortical microvasculature observed through the cranial window. (d) screenshot of the graphical user interface. Panels illustrating (e) brain tumor progression (GFP, green fluorescence protein), and concomitant changes in (f) cerebral blood volume (CBV, i.e. microvascular structure, with red arrows indicating new vessel formation) and (g) cerebral blood flow (CBF) due to tumor angiogenesis. (h) Microvascular connectivity (MC) changes were also mapped in the freely behaving animal over the entire lifetime of the brain tumor. MC was calculated by correlating CBV fluctuations from the entire FoV over 5 min with those from the seed pixel indicated by crosshairs. (i) In addition, changes in mean vascular density (MVD, light 
grey circles), mean vascular perfusion (MVP, dark squares), extent of area with $\mathrm{MC}>0.5$ ( MCA, light blue triangles, normalized by the value on D3) were also computed on a daily basis. Data is shown only from D3-D11 as beyond this the deterioration of the cranial window due to excessive tumor growth (i.e. panel b, D12-D15) prevented accurate measurement of vascular parameters. All values are normalized to D3 levels and shown over a $0-1$ range. The MCA value for D1 1 was -0.70 and hence not shown within this range. $(\mathrm{j}, \mathrm{k})$ The angiogenic tumor microvasculature at D6 is scrutinized by zooming in on a region of interest (black dotted box). Selected vessel diameters are marked. Data presented is from a single animal and scale bars indicate $5 \mathrm{~mm}, 10 \mathrm{~mm}, 500 \mu \mathrm{m}, 500 \mu \mathrm{m}$ and $100 \mu \mathrm{m}$, respectively for a, c, panels e $-\mathrm{h}, \mathrm{j}$, and $\mathrm{k}$. Sub-figures a-h, and $\mathrm{j}$ adapted with permission under a creative commons license (http://creativecommons.org/licenses/by/4.0/) from Senarathna et al, "A miniature multi-contrast microscope for functional imaging in freely behaving animals." Nature communications 10.1 (2019): 1-13.

Complete Status: Complete

First Name: Janaka

Last Name: Senarathna

Email:dmmj.senarathna@gmail.com

Organization: Johns Hopkins University School of Medicine

Country: United States 


\title{
ID: GA726 \\ PARP1- targeted fluorescent molecular probe for non-invasive confocal microscopic detection of basal cell carcinoma
}

\author{
Aditi Sahu, MSKCC, aditisahu@gmail.com
}

\section{Category: Oncology}

\begin{abstract}
Body : Background: Clinical examination followed by dermoscopy and Reflectance confocal microscopy (RCM) provide high sensitivity (80-95\%) and moderate specificity (60$70 \%$ ) for diagnosis of basal cell carcinoma (BCC)- the most common cancer in the world [1, 2]. $\mathrm{RCM}$ is a high-resolution label-free quasi-histopathological imaging modality based on the detection of reflected (singly-backscattered) light from cellular organelles and tissue[3]. RCM imaging has improved the clinical $\mathrm{BCC}$ diagnostic specificity by $2 \mathrm{x}$, beyond which the grayscale contrast has reached a fundamental limit. This is mainly attributed to the weaker back-scatter from chromatin in nucleus: $\sim 100$ photons/pixel relative to the surrounding cytoplasm and dermis ( $\sim 104$ to $\sim 106$ photons/pixel) because of which structures with high nuclear:cytoplasmic ratio such as BCC tumors, lower epidermis layers and hair follicles are hard to visualize and differentiate, limiting BCC diagnosis[4]. The diagnostic accuracy may be dramatically enhanced with the use of nuclear-targeted fluorescent probes that demonstrate transepidermal delivery following topical application. PARPi-FL is one such small-molecule fluorescent reporter that targets nuclear poly-(ADP-ribose) polymerase [PARP] enzymes [5]. PARPi-FL has shown specific nuclear labeling, high contrast (tumor:normal) imaging [6,7], and has an investigational new drug (IND) status for in vivo studies[8]. To investigate the potential clinical utility of PARPi-FL, three important questions were investigated: i) BCC PARP1 expression, ii) PARPiFL BCC labeling and diagnostic accuracy, and iii) modeling of transepidermal PARPi-FL delivery by passive diffusion following topical application. Methods: PARP1 expression was evaluated on thin FFPE tissue sections of BCCs $(n=95)$. PARPi-FL labeling, and diagnostic accuracy on discarded thick surgical specimens of BCCs $(n=85)$ and normal skin $(n=20)$; while permeability was on normal ex vivo tissue from mastectomy procedures $(n=5)$. In vivo permeability and skin toxicity were evaluated in live anesthetized Yorkshire pigs $(n=3)$. PARP1 expression and PARPi-FL was quantified as area positivity and intensity, respectively, in tumor and normal structures. Diagnostic accuracy was evaluated with blinded reading by two expert confocal readers to assess sensitivity, specificity, positive and negative predictive values (PPV, NPV). Positive nuclear staining in epidermis and underlying dermis indicated successful permeability. Results: Higher PARP1 expression was found in BCCs as compared to normal structures such as epidermis, hair follicles and sebaceous glands ( $p$ Conclusion: Higher PARP1 expression and PARPi-FL staining was observed in BCC tumors. PARPi-FL staining was found to be nuclear-specific and highlighted BCCs in the FCM images, thereby improving the sensitivity (by 10-20\%) and specificity (by 4\%) of RCM+FCM over RCM alone. The permeability and passive diffusion of PARPi-FL through intact stratum corneum following topical application was also confirmed in both ex vivo human skin and in vivo in a pig model in a clinically feasible time range (10-30 minutes). Thus, our innovative findings demonstrate the
\end{abstract}


utility and establish the foundation for prospective in vivo PARPi-FL studies for BCC diagnosis in patients.

References: 1 . Nori, S., et al., Sensitivity and specificity of reflectance-mode confocal microscopy for in vivo diagnosis of basal cell carcinoma: a multicenter study. J Am Acad Dermatol, 2004. 51(6): p. 923-30. 2. Guitera, P., et al., In vivo confocal microscopy for diagnosis of melanoma and basal cell carcinoma using a two-step method: analysis of 710 consecutive clinically equivocal cases. Journal of investigative dermatology, 2012. 132(10): p. 2386-2394. 3. Rajadhyaksha, M., R.R. Anderson, and R.H. Webb, Video-rate confocal scanning laser microscope for imaging human tissues in vivo. Applied optics, 1999. 38(10): p. 2105-2115. 4. Rajadhyaksha, M.M., S. Gonzalez, and J.M. Zavislan, Detectability of contrast agents for confocal reflectance imaging of skin and microcirculation. Journal of biomedical optics, 2004. 9(2): p. 323-332. 5. Reiner, T., et al., Imaging therapeutic PARP inhibition in vivo through bioorthogonally developed companion imaging agents. Neoplasia, 2012. 14(3): p. 169IN1177IN3. 6. Kossatz, S., et al., Detection and delineation of oral cancer with a PARP1 targeted optical imaging agent. Scientific reports, 2016. 6: p. 21371. 7. Irwin, C.P., et al., PARPi-FL-a fluorescent PARP1 inhibitor for glioblastoma imaging. Neoplasia, 2014. 16(5): p. 432-440. 8. Kossatz, S., et al., Validation of the use of a fluorescent PARP1 inhibitor for the detection of oral, oropharyngeal and oesophageal epithelial cancers. Nature Biomedical Engineering, 2020. 4(3): p. 272-285.

\section{Image/Figure:}

https://www.xcdsystem.com/wmis/abstract/File6959/GA726 ImageFigure 0614085727.PNG

Image/Figure Caption: Investigating PARPi-FL for nuclear labeling and improving diagnosis of basal cell carcinoma (BCC) (A) Higher PARP expression was observed in BCCs as compared to normal structures such as epidermis, hair follicles and sebaceous glands $(p<0.05)$ in representative IHC images; higher area positivity (\%) was observed in IHC quantification. (B) PARPi-FL labels nucleus in both epidermis and BCC tumor in skin tissue, higher staining observed in most tumors. The added contrast due to PARPi-FL combined with optical sectioning and resolution during FCM imaging will enable superior visualization and differentiation of tumor and normal structures. (C) To evaluate effect of RCM + complementary PARPi-FL contrast fluorescence confocal microscopy (FCM) over reflectance confocal microscopy (RCM) alone, blinded evaluation by two expert readers was performed. Higher sensitivity ( $97 \%$ from $87 \%$ for reader $1,100 \%$ from $79 \%$ for reader 2 ) and NPV (95\% from $79 \%$ for reader $1,100 \%$ from $81 \%$ for reader 2 ) while almost similar specificity ( $64 \%$ from $60 \%$ for reader $1,81 \%$ unchanged for reader 2 ), and PPV ( $74 \%$ from $73 \%$ for reader $1,83 \%$ from $79 \%$ for reader 2) was found in RCM+FCM images as compared to RCM alone, highlighting the added value of PARPi-FL contrast in BCC detection. (D) After successful BCC labeling and improvements in diagnostic accuracy, permeability of PARPi-FL through intact stratum corneum to mimic non-invasive topical application in humans was shown in human ex vivo tissue and in vivo in pig. Positive nuclear staining in epidermis and underlying dermis within 10-30 minutes indicates successful passive diffusion of PARPi-FL through intact skin. Insets show the methods of topical application- PARPi-FL saturated gauze with occlusion was employed in ex vivo tissue, while plastic wells were tested to store PARPi-FL for the incubation times in vivo in pig. 
Full Name of Abstract's 1st Author : Aditi Sahu

Complete Status: Complete

First Name: Aditi

Last Name: Sahu

Email: aditisahu@gmail.com

Organization: $\mathrm{MSKCC}$

Country: United States 


\title{
ID: GA727 \\ Targeting Cancer Stem Cells for Diagnostic Imaging and Ablation
}

\author{
Kevin Wyszatko, McMaster University, wyszatkt@mcmaster.ca
}

\section{Category: Oncology}

\begin{abstract}
Body : Cancer Stem Cells (CSCs) constitute a malignant subpopulation of tumor cells that drive tumor growth while resisting conventional chemo- and radiation therapy strategies (Singh 2003, Eyler 2008). These refractory tumor cells also drive metastasis and on surviving treatment lead to cancer recurrence (Hermann 2007, Lagadec 2010). Consequently, the CSC population represents an attractive therapeutic target for hard-to-treat tumors. Selectively eliminating CSCs by targeting CSC biomarkers could have broad clinical impact as CSCs have been isolated in virtually every type of cancer (Singh 2003, Al-Hajj 2003, Matsui 2004, Taylor 2005, Han 2014, Suetsugu 2006). In addition to therapeutics, companion diagnostic imaging probes for CSC markers offer to not only stratify patients amenable to CSC targeted therapy, but to also provide valuable information regarding tumor staging and prognosis (Daywood 2014, Chang 2016). Biomarkers suited towards targeting the CSC population for treatment and diagnostic imaging are ideally surface-expressed, sparingly found on regular tissues and lost upon differentiation. Cancer Stem Cell marker CD133 well-embodies these criteria and is exceptionally useful for studying CSCs (Glumac 2018). Previously developed murine and humanized antibodies targeting CD133 for therapy and diagnostic imaging are rare or underperforming. Cancer stem cell resilience to chemotherapy has stifled some CD133 targeted treatment efforts (Dean 2005). Other preclinical therapy studies have shown promising antitumor efficacy especially when multiple doses of the therapy were administered (Swaminithan 2013). Unfortunately these preclinical therapy studies which utilize humanized or murine antibodies would be unsuited for clinical translation due to the human anti-mouse antibody response (HAMA) (Sgro 1995). The long term goal of this project is to leverage highly localized, potent radiation from emerging therapeutic radionuclides with novel, fully human monoclonal antibodies to selectively eliminate cancer stem cells in human patients. Here, RW03, a monoclonal antibody with specificity for CD133 is evaluated as the basis of a versatile targeting platform against CSCs. Robust bioconjugation strategies were used to stably radiolabel RW03 with lutetium-177 achieving $>50 \%$ radiochemical yield and $>99 \%$ radiochemical purity (RCP) after a $1 \mathrm{~h}$ reaction in slightly acidic $(\mathrm{pH} 5.5)$ buffer. Similar strategies were used to produce CD133 targeted companion diagnostic PET/SPECT imaging probes. Radiolabelled conjugates were routinely stable ( $>95 \% \mathrm{RCP})$ in saline for over 1 week, and for several days in mouse serum. Seminal studies evaluating the biodistribution of RW03 directly labelled with lutetium177 demonstrated tumor uptake of $65 \% \mathrm{ID} / \mathrm{g}$ and tumor/ blood ratio of 5:1 in HT-29 xenograft mice. In addition, a biodistribution study demonstrated high uptake $(35 \% \mathrm{ID} / \mathrm{g})$ in CD133 expressing xenograft HCT116 tumors. In HT-29 tumors, tumor uptake was blocked by coadministering 200x excess cold RW03 to demonstrate antigen-mediated tumor uptake. Escalating dose therapeutic studies with [177Lu] Lu-DOTA-RW03 were conducted on the HT29 xenograft model. Mice tolerated the treatment well, and the maximum tolerated dose (MTD) was not reached even upon administering $14.8 \mathrm{MBq}$, while for this dose a significant reduction
\end{abstract}


in tumor growth rate was measured. These promising results were followed up with a multiple dose therapy study that demonstrated a greater reduction in tumor growth rate with a small increase in toxicity to the mice. Tumors from treated and untreated mice were resected and assessed by autoradiography and immunohistochemistry and showed the radiation distribution to be localized to sites of high CD133 expression, causing radiobiological damage to diseased tissue. Companion PET preclinical imaging studies utilizing [89Zr] Zr-DFO-RW03 were conducted and biodistribution results were similar to those for [177Lu] Lu-DOTA-RW03. Future work includes preclinical therapy studies using alpha-emitting radionuclides against patient derived GBM orthotopic xenograft tumor models.

References: Singh, S. K., Clarke, I. D. \& Terasaki, M. Identification of a cancer stem cell in human brain tumors. Cancer Res. 63, 5821-5828 (2003). Eyler, C. E. \& Rich, J. N. Survival of the fittest: cancer stem cells in therapeutic resistance and angiogenesis. J. Clin. Oncol. 26, 28392845 (2008). Hermann, P. C. et al. Distinct populations of cancer stem cells determine tumor growth and metastatic activity in human pancreatic cancer. Cell Stem Cell 1, 313-323 (2007). Lagadec, C. et al. Survival and self-renewing capacity of breast cancer initiating cells during fractionated radiation treatment. Breast Cancer Res. 12, R13 (2010). Al-Hajj, M., Wicha, M. S., Benito-Hernandez, A., Morrison, S. J. \& Clarke, M. F. Prospective identification of tumorigenic breast cancer cells. Proc. Natl. Acad. Sci. U. S. A. 100, 3983-3988 (2003). Matsui, W. et al. Characterization of clonogenic multiple myeloma cells. Blood 103, 2332-2336 (2004). Taylor, M. D. et al. Radial glia cells are candidate stem cells of ependymoma. Cancer Cell 8, 323-335 (2005). Han, J., Fujisawa, T., Husain, S. R. \& Puri, R. K. Identification and characterization of cancer stem cells in human head and neck squamous cell carcinoma. BMC Cancer. 14, 173 (2014). Suetsugu, A. et al. Characterization of CD133+ hepatocellular carcinoma cells as cancer stem/progenitor cells. Biochem. Biophys. Res. Commun. 351, 820-824 (2006). Dawood, S., Austin, L., Cristofanilli, M. Cancer stem cells: implications for cancer therapy. Oncology (Williston Park). 28:1101-1107, 1110 (2014). Chang, J. C. Cancer Stem Cells Role in Tumor Growth, Recurrence, Metastasis, and Treatment Resistance. Medicine Open. 95 (1): e4766. (2016). Glumac, P. M. \& LeBeau, A. M. The role of CD133 in cancer: a concise review. Clinical and Translational Medicine. 7 (2018). Dean, M., et al. Tumor Stem Cells and Drug Resistance. Nat Rev Cancer. 5(4):275-84 (2005). doi: 10.1038/nrc1590. Swaminithan, S., et al. CD133targeted paclitaxel delivery inhibits local tumor recurrence in a mouse model of breast cancer.

\section{Image/Figure:}

https://www.xcdsystem.com/wmis/abstract/File6959/GA727_ImageFigure_0614092646.png

Image/Figure Caption: Therapeutic efficacy of [177Lu] Lu-DOTA-RW03 in HT-29 xenograft mouse models. Single treatment dose targeting cancer stem cells in solid tumors with betaemitting radiation. Mice in the high dose group $(14.8 \mathrm{MBq})$ experienced a significant reduction in tumor growth rate with minimum toxicity.

\section{Complete Status: Complete}

First Name: Kevin

Last Name: Wyszatko 
Email:wyszatkt@mcmaster.ca

Organization: McMaster University

Country: Canada 


\title{
ID: GA728 \\ Molecular mechanism of ultrasound neuron stimulation
}

\author{
JIEJUN ZHU, The Hong Kong Polytechnic University, jiejun.zhu@connect.polyu.hk
}

\section{Category: Neuroscience}

\begin{abstract}
Body : Manipulating a specific neuron or neuron circuits in the brain is a typical way understanding the brain function and treating brain disfunctions [1,2]. Ultrasound, especially for low intensity low frequency ultrasound, is an emerging technique that can noninvasively modulate neuron activity in targeted brain regions with fine special temporal resolution and deep brain penetration $[3,4,5]$. Ultrasound is therefore a promising tool for both probing brain function and treating brain diseases with the advantage of non-invasiveness. However, the minimum focal spot of an ultrasound beam is much larger than a single neuron or a specific small set of neurons. These make it difficult for the ultrasound to probe or target the complex neural circuits entangled with interdependent different neurons. The underlying mechanism of ultrasound neuron modulation is thus the key issue for the best usage of ultrasound neuron modulation in both the basic and clinic neurosciences. Due to the nature of mechanical force of ultrasound, the mechano-sensitive ion channel displayed a promising candidate. Piezo family is so far the most sensitive mechanical ion channel, that could respond quickly to forces as low as $10 \mathrm{pN}$ which is consistent with the scale of ultrasound induced forces [6,7]. Base on this, piezo family is likely to be a factor modulates the ultrasonic neural modulation. To test this hypnosis, several experiments studying the role of exogenous and endogenous piezo1 in the ultrasound neuron modulation were performed. Firstly, 293T was used to test whether piezo1 mediates the ultrasound effect on cells. The results showed that overexpressing the piezol in the $293 \mathrm{~T}$ induce the calcium influx whereas the unexpressed one has little response to ultrasound $(\mathrm{n}=9, * * * \mathrm{p}<$ 0.0001). This indicates the piezo1 mediated the ultrasound effect on cells. To confirm whether the ultrasound neuron modulation is also mediated by piezo1, primary neurons were adopted. Firstly, the endogenous expression of piezol in the primary neurons were tested by immunofluorescent staining. And the function of piezo1 was assessed by calcium imaging with a piezo1 agonist Yoda1 and a piezo1 antagonist GsMTx-4 (Fig A). After the confirmation of piezo1 function in primary neurons $(\mathrm{n}=15, * * * \mathrm{p}<0.001$, Fig $\mathrm{A})$, the ultrasound bio-effect was tested with or without the GsMTx-4. The data showed that the ultrasound alone could activate piezo1, initiating calcium influx with a dose depend of ultrasound intensity. And these effects can be blocked by GsMTx-4 ( $\mathrm{n}=9, * \mathrm{P}<0.05, * * \mathrm{P}<0.01$, *** $\mathrm{P}<0.001$, Fig B). Similarly, the ultrasound increased nuclear c-Fos expressions in primary neurons with a dose depend of ultrasound intensity. And these effects can be reduced if the neurons were pre-treated with GsMTx-4 $(\mathrm{n}=3, * \mathrm{P}<0.05, * * \mathrm{P}<0.01$, Fig $\mathrm{C})$. These findings demonstrated that the piezo1 mediates the ultrasound neuron modulation in primary neurons. To further explore the signalling implications of Piezo1-mediated ultrasound neuron modulation, a mouse neuronal cell line, CLU199, was applied. Using western blot, the protein expression of phospho-CaMKII, phosphoCREB and c-Fos were tested. The results showed that the ultrasound increased the expression of phospho-CaMKII, phospho-CREB and c-Fos in a dose dependent manner of ultrasound intensity. But Piezo1 knockdown significantly reduced this effect $(\mathrm{n}=3, * \mathrm{P}<0.05)$. These
\end{abstract}


findings indicate the piezol mediated ultrasound neuron modulation in vitro and may also provide a possible mechanism of ultrasound brain modulation in vivo. By controlling the expression of piezol we may able to target specific neuronal pathways or nuclei in both the basic and clinical neurosciences (Fig D) [8].

References: 1. Zhang F, Aravanis AM, Adamantidis A, de Lecea L, Deisseroth K. Circuitbreakers: optical technologies for probing neural signals and systems. Nature Reviews Neuroscience. 2007;8(8):577-81. 2. Temel Y, Jahanshahi A. Neuroscience. Treating brain disorders with neuromodulation. Science. 2015;347(6229):1418-9 3. Tyler WJ. Noninvasive neuromodulation with ultrasound? A continuum mechanics hypothesis. Neuroscientist. 2011;17(1):25-36 4. Ye PP, Brown JR, Pauly KB. Frequency Dependence of Ultrasound Neurostimulation in the Mouse Brain. Ultrasound Med Biol. 2016;42(7):1512-30 5. Park TY, Pahk KJ, Kim H. A novel numerical approach to stimulation of a specific brain region using transcranial focused ultrasound. Conf Proc IEEE Eng Med Biol Soc. 2018;2018:3697-700 6. Coste B, Mathur J, Schmidt M, Earley TJ, Ranade S, Petrus MJ, et al. Piezo1 and Piezo2 are essential components of distinct mechanically activated cation channels. Science. 2010;330(6000):55-60 7. Liang X, Howard J. Structural Biology: Piezo Senses Tension through Curvature. Current Biology. 2018;28(8):R357-R9. 8. Qiu Z, Guo J, Kala S, Zhu J, Xian Q, Qiu W, et al. The Mechanosensitive Ion Channel Piezo1 Significantly Mediates In Vitro Ultrasonic Stimulation of Neurons. iScience. 2019; 21, 448-457.

Full Name of Abstract's 1st Author : ZHU JIEJUN

Complete Status: Complete

First Name: JIEJUN

Last Name: ZHU

Email: jiejun.zhu@connect.polyu.hk

Organization: The Hong Kong Polytechnic University

Country: China 


\title{
ID: GA729 \\ Amphiphilic voltage-sensitive dyes for cell membrane potential sensing \\ MIRNA EL KHATIB, University of Pennsylvania, elmirna@pennmedicine.upenn.edu
}

\section{Category: Neuroscience}

\begin{abstract}
Body : Fluorescent voltage-sensitive molecular dyes make up a suitable tool for probing changes in local membrane potentials without perturbing underlying cellular physiology. When combined with in vivo imaging, such probes could enable visualization of neuronal activity, making it possible to study communication between neurons in intact brain. Developed by R. Tsien and E. Miller, amphiphilic voltage sensors based on photoinduced electron transfer (PET) offer fast response kinetics and high sensitivity to membrane potential changes; however, all presently existing molecules are limited due to their inherent poor aqueous solubility, precluding studies in vivo in the brain. Here, we report the development of improved amphiphilic probes, in which a branched dendron-like polyanioic structure is attached to the fluorescent rosamine 'head' of the molecule, imparting water-solubility and enforcing the required orientation during partitioning into the plasma membrane. The new probes exhibit excellent aqueous solubility and do not require organic solvents for their use in biological systems.
\end{abstract}

\section{Image/Figure:}

https://www.xcdsystem.com/wmis/abstract/File6959/GA729_ImageFigure_0614101740.png

Full Name of Abstract's 1st Author : Mirna El Khatib

Complete Status: Complete

First Name: MIRNA

Last Name: EL KHATIB

Email: elmirna@pennmedicine.upenn.edu

Organization: University of Pennsylvania

Country: United States 


\title{
ID: GA730 \\ A Combined MRI, Micro-CT and Optical Imaging Pipeline for Image-Based Vascular Systems Biology of Cancer
}

\author{
Akanksha Bhargava, Johns Hopkins University, bhargav.akanksha@gmail.com
}

\section{Category: Oncology}

\begin{abstract}
Body : Image based systems biology is emerging as a powerful new paradigm to better understand the role of the tumor microenvironment (TME) in cancer progression, metastasis and response to therapy. In spite of the recent advances in vascular imaging approaches, imagebased vascular systems biology has remained challenging because currently there are no methods to label blood vessels simultaneously in all three primary imaging modalities, i.e. magnetic resonance imaging $(\mathrm{MRI})$, micro-CT $(\mu \mathrm{CT})$ and optical microscopy. Consequently, this poses a hurdle to the integration of 3D vascular imaging data with complementary image contrasts acquired at multiple spatial scales, e.g. white matter fiber distributions from MRI (for brain tumors), bone contrast from $\mu \mathrm{CT}$ (to study bone metastasis), protein expression from multiphoton (MPM) or light-sheet microscopy (LSM), all of which can provide invaluable inputs to cancer systems biology models. Moreover, since the spatial resolution of these data may span several orders of magnitudes and having common landmarks visible across spatial scales is often not possible, data integration via image co-registration has also remained challenging. Therefore, we developed a "multicontrast" vascular contrast agent combination that makes blood vessels visible across MRI, CT and optical microscopy. This enabled the development of an easy-to-use method called VascuViz that provides a multimodal, multiscale 3D vascular imaging capability in intact, unsectioned tumors. Here, we showcase the utility of VascuViz for multicontrast and multiscale characterization of the TME in an orthotopic MDA-MB-231 breast cancer xenograft using MRI $(40 \mu \mathrm{m}), \mu \mathrm{CT}(9 \mu \mathrm{m})$ and MPM ( VascuViz enabled concurrent imaging and visualization of the tumor vasculature with complementary image contrasts such as $\mathrm{T} 1 \mathrm{w}$ - and Dw-MRI (Fig. 1a, b), euclidian distance map from $\mu \mathrm{CT}$ (Fig. 1c, d), collagen (col) data from second harmonic generation (SHG) imaging and green fluorescent protein expression (GFP) data from cancer cells using multiphoton microscopy (MPM) (Fig. 1e-g). VascuViz facilitated data integration via a "vascular fiducials" based image co-registration method1. This enabled the generation of co-registered 3D maps of the TME at the macroscopic $(40 \mu \mathrm{m})$, mesoscopic $(9 \mu \mathrm{m})$ and microscopic $(200 \mu \mathrm{m})$ in the tumor center (Fig. 1c). Co-registration between EDM and ADC maps revealed that regions with high mean ADC values $\left(>0.001 \mathrm{~mm}^{2} / \mathrm{s}\right)$ matched with those where inter-vessel distances were in the range of $151-300 \mu \mathrm{m}$ (Fig. 1f-j). These results complemented 3D MPM data ( In conclusion, we developed a multimodality, multiscale vascular imaging approach, VascuViz, using MRI, $\mu \mathrm{CT}$ and LSM and showcased its utility in an orthotopic breast cancer xenograft. In addition to enabling high-resolution vascular mapping in intact unsectioned tumors, this method provides for the first time, the ability to integrate vascular data across multiple image contrast mechanisms from the spatial scale of individual endothelial cell to the whole-tumor. This method has direct applications in image based systems biology of cancer2 and other vascular disease models (e.g. stroke). Bhargava, A. et al. The FASEB Journal 34, 11 (2020). Stamatelos, S. K. et al. Sci Rep 9, 5276, (2019).
\end{abstract}


References: Bhargava, A. et al. The FASEB Journal 34, 11 (2020). Stamatelos, S. K. et al. Sci Rep 9, 5276, (2019).

\section{Image/Figure:}

https://www.xcdsystem.com/wmis/abstract/File6959/GA730_ImageFigure_0614105138.png

Image/Figure Caption: Fig. 1: Multicontrast and multiscale characterization of the TME in a human breast cancer xenograft: VascuViz employed an intravascular polymer mixture (GalRh$\mathrm{BVu}$ ) that consisted of the fluorescently labeled MRI contrast agent Galbumin ${ }^{\mathrm{TM}}-\mathrm{Rhodamine}$ $(\mathrm{GalRh})$ and the radio-opaque BriteVu $\mathbb{}{ }^{\circledR}(\mathrm{BVu})$ to make blood vessels visible in MRI, $\mu \mathrm{CT}$ and optical imaging. (a) T1w-MRI $(40 \mu \mathrm{m})$ of an orthotopic MDA-MB-231 breast tumor perfused with GalRh-BVu. The yellow dotted line represents the tumor volume segmented manually using the soft tissue contrast derived from T1w-MRI. (b) Simultaneous visualization of the tumor vasculature and apparent diffusion coefficient (ADC) derived from Dw-MRI at $50 \mu \mathrm{m}$ wherein high values in the center indicated necrosis. This data could be combined with (c) the microvascular network data obtained from $\mu \mathrm{CT}$ at $9 \mu \mathrm{m}$ spatial resolution. Here, the vascular contrast is due to the presence of radio-opaque BVu. Blood vessels are scaled by their mean diameters (9 to $160 \mu \mathrm{m}$ ). The yellow dotted line represents the tumor boundary overlaid from T1w-MRI (a). Co-registration between T1w-MRI and $\mu \mathrm{CT}$ enabled the generation of the (d) tumor euclidian distance map (EDM) at $9 \mu \mathrm{m}$ spatial resolution that revealed the presence of inter-vessel distances as large as $350 \mu \mathrm{m}$ in the tumor center. These regions matched with those that showed high ADC values (i.e. $>0.001 \mathrm{~mm}^{2} / \mathrm{s}$ ) in (b). MPM image of a $20 \mu \mathrm{m}$ thick section through the tumor center revealed (e) capillaries (red), green fluorescent protein (GFP) expression from cancer cells (green) and SHG based collagen (Col) data (blue). Here, the blood vessel contrast is due to the presence of GalRh. Representative regions of the tumor rim (red box) and tumor center (blue box) from (e) are magnified 10X in (f, g), respectively.

Complete Status: Complete

First Name: Akanksha

Last Name: Bhargava

Email: bhargav.akanksha@gmail.com

Organization: Johns Hopkins University

Country: United States 


\title{
ID: GA731 \\ 800-nm Nerve-Binding Fluorophores Facilitate Buried Nerve Visualization Using Clinical Grade Surgical System
}

\author{
Lei Wang, Oregon Health \& Science University, wanglei@ohsu.edu
}

\section{Category: Neuroscience}

\begin{abstract}
Body : Accidental nerve transection or injury is major morbidity associated with many surgical interventions, resulting in persistent postsurgical numbness, chronic pain, and/or paralysis. Nerve-sparing can be a difficult task due to patient-to-patient variability and the difficulty of nerve visualization in the operating room. Fluorescence-guided surgery (FGS) to aid in the precise visualization of vital nerve structures in real-time during surgery could greatly improve patient outcomes. Currently, almost all clinical FGS systems have an "800 nm" channel designed to image the U.S. Food and Drug Administration (FDA) approved indocyanine green (ICG), which is mainly used to assess blood flow or detect sentinel lymph nodes during surgery as a non-specific fluorescent contrast agent. After nearly two decades, only a limited number of fluorescent molecules have been developed to image nerve tissue, where the vast majority of are plagued with high non-specific uptake in surrounding tissues and mostly fluoresce at visible wavelengths. Developing a nerve binding agent that has near infrared (NIR) fluorescent properties has been a complex and challenging task. While nerve-specific fluorophores must be of sufficiently low molecular weight to cross the blood-nerve-barrier (BNB), the degree of conjugation (e.g., number of unsaturated hydrocarbons) requisite for long-wave fluorescence emission necessarily increases molecular weight. To overcome these opposing constraints, we have taken a medicinal chemistry approach that facilitated the design of our first-in-class NIR nerve-binding fluorophore library, which is derived from $700 \mathrm{~nm}$ analogs through purposeful repositioning of the polar groups and effective elongation of the electronic push-pull system. However, such structures cannot be readily attained via traditional oxazine syntheses. Adapting modern organic synthesis secured the production of these ICG spectral analogs while maintaining nerve-specific contrast that are fully compatible with the $800 \mathrm{~nm}$ channels already present in clinical imaging systems. Utilization of these novel NIR nerve binding fluorophores during FGS will significantly improve nerve visualization, providing an immediate benefit to both patients and surgeons. Additionally, our study design and results demonstrate the feasibility of our molecular engineering approach which can be readily adapted to create other tissuespecific fluorophores.
\end{abstract}

Complete Status: Complete

First Name: Lei

Last Name: Wang

Email: wanglei@ohsu.edu 
Organization: Oregon Health \& Science University

Country: United States 


\title{
ID: GA732 \\ Optimization of combinatorial library-derived peptides targeting the integrin alphavbeta6
}

\author{
Yng (Sarah) Tang, University of California, Davis, yngtang@ucdavis.edu
}

\section{Category: Oncology}

\begin{abstract}
Body : Objectives: Combinatorial library approaches such as the one-bead onecompound (OBOC) and phage display offer a useful tool for rapid identification of novel receptor-targeted peptides, however subsequent lead optimization towards successful imaging agents remains an ongoing challenge.1,2 This work aims to optimize the binding affinity and selectivity of short peptides identified from various combinatorial libraries that target the integrin avb6, a cell surface receptor that is overexpressed in numerous aggressive cancers. $3 \mathrm{C}$-terminal extension of a short $\alpha v \beta 6$-binding peptide with amino acids taken from the well-known $\alpha v \beta 6$ selective A20FMDV2 peptide derived from the foot-and-mouth disease virus (NAVPNLRGDLQVLAQKVART) has previously shown to significantly improve the binding affinity and selectivity for integrin $\alpha v \beta 6$ as well as serum stability.4 Here, we further explore the effect of C-terminal extension of peptides on the binding profiles, serum stability and secondary structure of two peptides from our OBOC library (1 and 2),4 an RTD peptide originally discovered via phage display (3) where the avß6-binding DLXXL motif was first identified,5 and two RGD octamers discovered from a sunflower trypsin inhibitor1-based phage display known as SFLAP3 (4)6 and SFITGv6 (5)7, in in vitro assays. The lead candidate was PEGylated with the goal to further improve serum stability prior to in vivo evaluation. Methods: All peptides were synthesized using standard Fmoc chemistry on solid-phase. Binding affinity and selectivity of peptides were evaluated by competitive binding ELISA with purified integrins (avb6, avb3 and avb8). Secondary structure of the peptides was determined by circular dichroism (CD) spectroscopy. Radiolabeling of peptides at the $\mathrm{N}$-terminus was performed on solid-phase with [18F]FBA, followed by HPLC purification. The 18F-peptides were evaluated in vitro (binding and internalization) in DX3purob6 ( $\alpha \mathrm{v} \beta 6+)$ and DX3puro ( $\alpha v \beta 6-)$ cells, and their stability evaluated in mouse serum at $1 \mathrm{~h}$ post-incubation (p.i) at $37^{\circ} \mathrm{C}$. Results: All modified peptides (6-10) exhibited significantly higher affinity for avb6-integrin in both ELISA (0.4-12 $\mathrm{nM})$. They demonstrated a 1.5-5-fold increase in binding to DX3purob6 cells (3.4-38\% binding) with increased selectivity (up to 12:1) relative to the unmodified parent peptides. CD data revealed that all peptides (1-10) exhibited random coil conformation in CD buffer, but in 30\% TFE a-helices were observed only for the modified peptides (6-10). [18F] 7 showed $20 \pm 0.4 \%$ binding to DX3purob6 cells with highest selectivity ratio of $11.8: 1$, but was only $16 \%$ intact in mouse serum at $1 \mathrm{~h} \mathrm{p}$.i. This peptide was PEGylated and both PEGylated peptide variants $[18 \mathrm{~F}] 11$ and $[18 \mathrm{~F}] 12$ showed $\sim 40 \%$ binding to DX3purob6 cells, $>99 \%$ stability in mouse serum at $1 \mathrm{~h}$ p.i. and [18F]12 showed 2-fold higher selectivity for DX3purob6 cells than [18F]11 (Table 1). Conclusions: Five peptides were synthesized and modified by C-terminal extension. All extended peptides showed improved affinity and selectivity for the integrin $\alpha v \beta 6$. CD data suggested that the formation of an $\alpha$-helix is important for selectivity. [18F]12 is currently under further evaluation in vivo.
\end{abstract}


References: [1] Gray, B.P. et al. Chem Rev, 2014, 114,1020-81. [2] Hu, L.Y. et al. Mol Imaging Biol, 2017, 19, 163-82. [3] Bandopadhyay, A. et al. Curr Drug Targets, 2009, 10, 645-52. [4] Tang, Y.C. et al. Molecules, 2019, 24(2), e309. [5] Kraft, S. et al. J Biol Chem, 1999, 274(4), 1979-85. [6] Roesch, S. et al. J Nucl Med, 2018, 59(11), 1679-85. [7] Altmann, A. et al. Clin Cancer Res, 2017, 23(15), 4170-80.

\section{Image/Figure:}

\section{https://www.xcdsystem.com/wmis/abstract/File6959/GA732 ImageFigure 0614110637.png}

Image/Figure Caption: Table 1 - In vitro cell binding data showing the relative binding affinity of all $18 \mathrm{~F}$-radiolabeled peptides as determined by the \% total binding to the DX3purob6 ( $\alpha \mathrm{v} \beta 6$ + ) cell line, their selectivity for the DX3purob6 cell line as determined by the DX3purob6 /DX3puro binding ratios, and the serum stability as determined by the $\%$ of intact peptide remaining after incubating with mouse serum for $1 \mathrm{~h}$.

Complete Status: Complete

First Name: Yng (Sarah)

Last Name: Tang

Email: yngtang@ucdavis.edu

Organization: University of California, Davis

Country: USA 


\title{
ID: GA733 \\ Zwitterionic water-soluble and cell-permeant rhodamine dyes enable quantitative imaging of therapeutic engagement
}

\author{
Lei Wang, Oregon Health \& Science University, wanglei@ohsu.edu
}

\section{Category: New Chemistry, Biology \& Bioengineering}

\begin{abstract}
Body : Patients with advanced cancers die because their cancer develops resistance to all available therapeutic strategies. "Personalized cancer medicine" strives to use biomarkermatched molecularly targeted therapies to overcome known resistance mechanisms. However, there is currently no methodology to measure the highly interdependent levels of available molecular drug targets, amount of drug binding and the resulting cellular response in a single dynamic, living system. To overcome these limitations, we have developed a dynamic, fluorescence-based, three-compartment model termed intracellular paired-agent imaging (iPAI). iPAI is a fluorescence-based approach that utilizes fluorophore labeled small molecule therapeutics as imaging agents to measure drug target engagement. Herein, we report our designed zwitterionic, water-soluble, and cell-permeable fluorophore pairs, based on the classic tetramethylrhodamine (TMR) and Silicon-substituted TMR (SiTMR). Termed Sulfo-Rh and Sulfo-SiRh, both dyes showed substantially improved optical stability against solvent polarity changes compared to the base fluorophores, TMR and SiTMR. In addition to matched structural features, these newly synthesized fluorophores also exhibited matched photophysical properties, including net charge, molecular size, Stokes' shift, full width at half maximum (FWHM), and brightness. Together, these spectrally-distinct fluorophores offer a unique opportunity for further development into fluorescent labels for quantifying intracellular targets in live cells. In proof of concept studies, we have developed targeted and untargeted versions of the epidermal growth factor receptor (EGFR) tyrosine kinase inhibitor Erlotinib using our novel water-soluble and cellpermeant fluorophore pair. The similarities of the iPAI targeted and untargeted agents to the parent drug were characterized using competitive binding and cytotoxicity assays in cancer cell lines with varied epidermal EGFR expression. Further validation studies were completed demonstrating the ability of iPAI to image drug target engagement, providing a chemical tool kit for quantitative small molecule imaging in the living system.
\end{abstract}

Complete Status: Complete

First Name: Lei

Last Name: Wang

Email:wanglei@ohsu.edu

Organization: Oregon Health \& Science University

Country: United States 


\title{
ID: GA735 \\ Visualization of host-pathogen interactions in nonhuman primate models of infectious diseases: from cell to whole body multimodal imaging
}

\author{
Catherine Chapon, CEA/DRF/IBFJ/IMVA/IDMIT/L3i, catherine.chapon@cea.fr
}

Category: Immunology: Inflammation \& Infection

\begin{abstract}
Body : In the infectious disease domain, exploring hots-pathogen interaction using in vivo imaging is a rapidly evolving area. Although non-human primates (NHP) provide a good model for infectious diseases, their exploration for viral transmission and dissemination by in vivo imaging has not been used extensively. This can be explained by the limited access to adequate structures for imaging these large animals with a high resolution while having specific regulations according to the level of confinement for the human pathogens. Our main objectives are to develop minimally invasive technologies for the longitudinal monitoring of infections, host response and treatments in NHP. NHP models for immune related disorders and human infectious diseases (such as CoVid-19, SIV, flu, yellow fever or whooping cough for instance) for studying pathophysiology and for human vaccines, immunotherapies and anti-microbial treatments are developed. In vivo imaging (near infrared fluorescence, probe based confocal endomicroscopy, echography, two-photon microscopy and PET-CT) is performed in BSL2 and BSL3 conditions. The two-photon microscope and the PET-CT camera suites are separated into two sides: a biologically "hot" side (pathogens present) and a biologically "cold" side (pathogens not present). For the PET-CT camera, a transparent biocontainment polycarbonate tube extends the hot side of the imaging suite into the bores of the imaging system machines on the cold side (Figure 1). The animal is placed on a patient table in the hot side of the barrier wall, which then moves it into the biocontainment tube to be imaged. The clear tubes also allow scientists to observe the animals being imaged from the cold side. The imaging machines themselves are located outside of the containment on the cold side of the barrier wall and are thus accessible for adjustments and maintenance without requiring technicians to enter the hot side and also to allow the decontamination of the imaging equipments easily. The behavior of skin antigen presenting cells following intradermal immunization with different vaccine vectors was characterized using probe based confocal endomicroscopy in order to better understand the mechanisms leading to the induction of cellular and humoral immune responses after vaccination1,2. Furthermore, noninvasive in vivo imaging procedures were developed to track bacterial localization and cellular interactions with host cells in the lower respiratory tract of challenged and naturally infected animals in a model of whooping cough in baboons3. Ongoing studies demonstrates the susceptibility of NHP to SARS-CoV-2 infection and the value of CT and PET-CT for characterizing the lesions of respiratory tract and score the disease severity and treatment impact. Development of PET-CT approaches using immuno-PET or PET reporter gene imaging to track pathogens and immune cells at the whole body scale is also ongoing.
\end{abstract}

References: 1. Todorova, B., Adam, L., Culina, S., Boisgard, R., Martinon, F., Cosma, A. et al. Electroporation as a vaccine delivery system and a natural adjuvant to intradermal administration of plasmid DNA in macaques. Sci Rep. 7, 4122 (2017). 2. Todorova, B., Salabert, N., Tricot, S., 
Boisgard, R., Rathaux, M., Le Grand, R. et al. Fibered Confocal Fluorescence Microscopy for the Noninvasive Imaging of Langerhans Cells in Macaques. Contrast Media Mol Imaging. 2017, 3127908 (2017). 3. Naninck, T., Coutte, L., Mayet, C., Contreras, V., Locht, C., Le Grand, R. et al. In vivo imaging of bacterial colonization of the lower respiratory tract in a baboon model of Bordetella pertussis infection and transmission. Sci Rep. 8, 12297 (2018).

Complete Status: Complete

First Name: Catherine

Last Name: Chapon

Email: catherine.chapon@cea.fr

Organization: CEA/DRF/IBFJ/IMVA/IDMIT/L3i

Country: France 


\title{
ID: GA754 \\ To Evaluate the Unknown Primary Site of Tumour with 68Ga-DOTATOC PET/CT in Patients with Known Metastatic Neuroendocrine Tumor
}

\author{
Sikandar Shaikh, Yashoda Hospitals, idrsikandar@gmail.com
}

\section{Category: Oncology}

\begin{abstract}
Body : PURPOSE: It is very difficult to evaluate the Localization of the primary site of the unknown primary tumor by various modalities. This is critical for management of patients presenting with neuroendocrine tumor (NET) with metastases. METHOD AND MATERIALS: This was the retrospective study which was performed to evaluate the efficacy of 68GaDOTATOC PET/CT in patients with diagnosis of or suspected NET with metastases.A subgroup of patients with metastases and unknown primary after initial work-up was analyzed. The $68 \mathrm{GaGa}$-DOTATOC Whole body PET/CT was done. This was evaluated by the SUV max uptake values. The study was considered true positive if the positive primary site has significant uptake and which was confirmed by histology or follow-up imaging. The scan was considered false positive if no primary lesion was found corresponding to $68 \mathrm{aGa}$-DOTATOC positive site. All negative scans for primary tumor were considered false negative. A scan was classified unconfirmed if 68Ga-DOTATOC PET/CT suggested a primary, however, no histology was obtained and imaging follow-up was not confirmatory. RESULTS:Forty patients with known metastatic NET and unknown primary underwent 68Ga-DOTATOC PET/CT.After evaluation and quantification the study was True positive, false positive, false negative and unconfirmed rates for unknown primary tumor were $38 \%, 7 \%, 50 \%$ and $5 \%$ respectively CONCLUSION:The efficacy of 68Ga-DOTATOC PET/CT is an effective modality in localization of unknown primary in patients with metastatic NET as compared to other modalities including F FDG PETCT
\end{abstract}

\section{References: 1}

Full Name of Abstract's 1st Author : Sikandar Shaikh

Complete Status: Complete

First Name: Sikandar

Last Name: Shaikh

Email: idrsikandar@gmail.com

Organization: Yashoda Hospitals

Country: India 


\title{
ID: GA791 \\ Evaluate the Efficacy of 68Ga-DOTA-TOC and 18F-FDG PET/CT in the Follow- up of Patients with Neuroendocrine Tumor Treated with the First Full Peptide Receptor Radionuclide Therapy Cycle
}

\author{
Sikandar Shaikh, Yashoda Hospitals, idrsikandar@gmail.com
}

\section{Category: Oncology}

\begin{abstract}
Body : PURPOSE:To evaluate the value of 68Ga-DOTA-TOC and 18F-FDG PET/CT for initial and follow-up evaluation of patients with neuroendocrine tumour (NET) treated with peptide receptor radionuclide therapy (PRRT). MATERIAL AND METHODS:We evaluated 33 patients who were histologically proven NET.All these patients underwent both PRRT and three combined 68Ga-DOTA-TOC and 18F-FDG PET/CT studies.68Ga-DOTA-TOC PET/CT was performed before PRRT, 3 months after completion of PRRT and after a further $6-9$ months. 18F-FDG PET/CT was done within 2 months of 68Ga-DOTA-TOC PET/CT. Follow-up ranged from 11.8 to 80.0 months (mean 34.5 months). the first full PRRT cycle. RESULTS:Overall 31 of the 99 18F-FDG PET studies (31\%) were true-positive in 19 of the 33 patients (58\%). Of the 33 patients, 14 (3 grade 1, 11 grade 2) were 18F-FDG-negative initially During follow-up (group 1), 12 (3 grade 1, 6 grade 2, 3 grade 3 ) were 18F-FDG-positive initially and during follow-up (group 2), 5 patients (1 grade 1, 3 grade 2, 1 grade 3 ) were $18 \mathrm{~F}-\mathrm{FDG}$-negative initially but $18 \mathrm{~F}$ FDG-positive during follow-up (group 3), and 3 patients (all grade 2) were 18F-FDG-positive initially but 18F-FDG-negative during follow-up (group 4). CONCLUSION: 18F-FDG PET showed more and/or larger metastases than 68Ga-DOTA-TOC PET in three patients of group 2 and two patients of group 3, all with progressive disease. In two patients with progressive disease who died during follow-up tumour SUVmax increased by $41-82 \%$ from the first to the last follow-up investigation
\end{abstract}

\section{References: 1}

Full Name of Abstract's 1st Author : Sikandar Shaikh

Complete Status: Complete

First Name: Sikandar

Last Name: Shaikh

Email: idrsikandar@gmail.com

Organization: Yashoda Hospitals

Country: India 


\title{
ID: GA792 \\ To Evaluate the Role of FDG PET/CT in Assessing Disease Activity in Large Vessel Vasculitis
}

\author{
Sikandar Shaikh, Yashoda Hospitals, idrsikandar@gmail.com
}

Category: Immunology: Inflammation \& Infection

\begin{abstract}
Body : PURPOSE:To evaluate the role of FDG PET-CT in the assessment of disease activity in large vessel vasculitis (LVV). MATERIAL AND METHODS:54 PET/CT scans were performed in 19 pts with suspected and diagnosed LVV (giant cell arteritis, Takayasu arteritis or idiopatic aortitis). The amount of vascular uptake was graded using a 4-point scale $0=$ no uptake $1=$ less than liver $2=$ similar to liver $3=$ higher than liver Grade $0-1$ was negative, 2 was moderately positive and 3 was markedly positive. This PET/CT was correlated with clinical indices of ITAS (Indian Takayasu Activity Score) and Kerr/National Institute of Health (Kerr/NIH) Serum acute-phase reactants ESR ,C-reactive protein [CRP]) levels ,Interleukin-6 (IL-6) and Soluble IL-6 receptor (sIL-6R RESULTS: 43\% of 54 PET-CT were negative, 31\% were moderately positive, and $26 \%$ were markedly positive. A significant correlation between the SUV uptake and both ESR and CRP levels was found and correlated. Significantly higher ESR values were observed in pts with markedly positive PET/CT $(49.4+36.5 \mathrm{~mm} / 1 \mathrm{st} \mathrm{h})$ compared with moderately positive $(27+21 \mathrm{~mm} / 1 \mathrm{st} \mathrm{h}, \mathrm{p}=0.0001)$ and inactive scans $(22.7+$ $15.9 \mathrm{~mm} / 1 \mathrm{st} \mathrm{h}, \mathrm{p}=0.0001$ ). CRP levels were $0.8+1.0 \mathrm{mg} / \mathrm{dL}$ in pts with inactive scans, $1.3+2.2$ $\mathrm{mg} / \mathrm{dL}$ in pts with moderately positive $(\mathrm{p}=0.001)$ and $3.0+3.6$ in patients with markedly positive scans $(\mathrm{p}=0.0001)$. Higher levels of IL-6 resulted in patients with markedly positive scans $(10.0$ $+8.9 \mathrm{pg} / \mathrm{ml})$ compared to those with inactive scans $(8.1+18.5 \mathrm{pg} / \mathrm{ml}, \mathrm{p}=0.013)$. We found no association between sIL-6R levels and vascular FDG uptake. There was a significant association between vascular FDG uptake and both ITAS and Kerr/NIH scores CONCLUSION:The above findings of PET/CT is a very useful tool for evaluating disease activity in patients with LVV.
\end{abstract}

\section{References: 1}

Full Name of Abstract's 1st Author : Sikandar Shaikh

Complete Status: Complete

First Name: Sikandar

Last Name: Shaikh

Email: idrsikandar@gmail.com

Organization: Yashoda Hospitals

Country: India 


\title{
ID: GA793 \\ Presence of glucosaminoglycans as reported by GAG-weighted MRI may report on joint loading and metabolic response of cartilage in populations susceptible to osteoarthritis
}

\author{
Abigail Cember, University of Pennsylvania, cember@pennmedicine.upenn.edu
}

\section{Category: Immunology: Inflammation \& Infection}

\begin{abstract}
Body : Introduction: Glucosaminoglycans (GAG) are a family of polysaccharides which have an extensive role in the structure of the human body. Osteoarthritis (OA), one of the world's most common musculoskeletal disorders and age-dependent conditions, involves breakdown of joint cartilage, whose molecular structure is largely composed of extracellular glucosaminoglycans[1,2]. GAG-weighted chemical exchange saturation transfer (gagCEST) MRI has been demonstrated a number of times in the literature [3-6]. In this study, a previously described gagCEST protocol is applied for imaging knee cartilage in older adults, a subset of whom have been diagnosed with OA. Methods: This protocol was approved by the Institutional Review Board of the Veterans Affairs Medical Center of Philadelphia. Eighteen subjects participated in the first stage of this study; all were male veterans of the United States armed forces over the age of 55, including both patients diagnosed with osteoarthritis of the knee $(\mathrm{n}=$ 8) and healthy controls. Patients underwent scanning of the knee in which OA was present; in controls with no knee pain, the scan was always performed on the right knee. All MR imaging studies were performed in a Siemens 7T whole-body MRI scanner (Siemens Medical Systems, Erlangen, Germany) using a circularly polarized (CP) transmit /28-channel receive array knee coil of inner diameter $15.4 \mathrm{~cm}$ to $18 \mathrm{~cm}$ ( Quality Electrodynamics, Mayfield Village, $\mathrm{OH}$ ). The imaging protocol consisted of a localizer, a T2 weighted structural image with the full field of view of the knee coil, and a series of measurements specific to the CEST experiment with a field of view (slab) restricted to a smaller volume. These scans were all performed with custom sequences which are described in detail in [6]. Post-processing was done in Matlab. Results: Even amongst healthy controls, gagCEST "hot spots" frequently occurred in the medial articular cartilage; other subjects had relatively homogeneous gagCEST maps. The figure shows images from four subjects, the left knee of one OA patient (red box) and three healthy controls. Axial (left) and coronal (right) views are shown of the same location in the knee joint, with T2weighted structural images shown by themselves alongside the gagCEST overlay. No ROI is drawn on the gagCEST maps; the jet colorscale shown is $0-20 \%$ normalized asymmetry, rendering pixels with no signal navy blue. (It can be seen from this representation that gagCEST is largely able to distinguish cartilage from other tissues, with some occasional spurious signal.) In the figure, the OA subject (red box) clearly has low gagCEST signal throughout the joint, which was typical for all patients. Healthy controls whose CEST maps showed notable features frequently presented the pattern visible in the coronal images here, wherein the medial side of the knee (subjects' left side, L, of right knee) had patches of elevated signal. Conclusions: While this data has so far been subjected to only visual qualitative analysis, it was observed that subjects with heterogenous gagCEST tend to have elevated signal in the medial part of the knee cartilage, which is known to be under high amounts of biomechanical stress in many individuals and is
\end{abstract}


frequently the site of joint pain. Patients with OA generally had very sparse gagCEST signal overall, limiting detection of similar patterns without more quantitative analysis; ongoing work includes the integration of our gagCEST maps with automatic segmentation and thickness calculations performed by PyKneeR [7] The possibility that increased GAG is a physiological response to joint stress suggests that longitudinal studies monitoring the strength of the gagCEST signal in aging joints could shed light on the metabolic etiology of osteoarthritis. In addition, gagCEST can be used to monitor the effecticacy of common interventions such as injection of hydroluoronic acid.

References: 1] Lawrence RC, Felson DT, Helmick CG, et al. Estimates of the prevalence of arthritis and other rheumatic conditions in the United States: part II. Arthritis Rheum 2008;58:26-35. 2] Saarakkala S, Julkunen P, Kiviranta P, M€akitalo J, Jurvelin JS, Korhonen RK. Depth-wise progression of osteoarthritis in human articular cartilage: investigation of composition, structure and biomechanics. Osteoarthritis Cartilage 2010;18:73-81. 3] Ling W, Regatte RR, Navon G, Jerschow A. Assessment of glycosaminoglycan concentration in vivo by chemical exchange-dependent saturation transfer (gagCEST). Proc Natl Acad Sci U S A 2008;105:2266-2270 4] Singh A, Haris M, Cai K, Kassey VB, Kogan F, Reddy D, Hariharan H, Reddy R. Chemical exchange saturation transfer magnetic resonance imaging of human knee cartilage at 3T and 7T. Magn Reson Med 2012;68:588-594. 5]Schmitt B, Zb yn S, Stelzeneder D, Jellus V, Paul D, Lauer L, Bachert P, Trattnig S. Cartilage quality assessment by using glycosaminoglycan chemical exchange saturation transfer and (23)Na MR imaging at 7T. Radiology 2011;260:257-264. 6] Krishnamoorthy G, Nanga RPR, Bagga P, Hariharan H, Reddy R. High Quality Three-Dimensional gagCEST Imaging of In Vivo Human Knee Cartilage at 7 Tesla. Magnetic Resonance in Medicine 77:1866-1873 (2017) 7] Bonaretti S, Gold G, Beaupre G (2019). pyKNEEr: An image analysis workflow for open and reproducible research on femoral knee cartilage. bioRxiv: https://doi.org/10.1101/556423 8] Yushkevich PA, Piven J, Hazlett HC, Smith RG, Ho S, Gee JC, Gerig G. User-guided 3D active contour segmentation of anatomical structures: Significantly improved efficiency and reliability. Neuroimage 2006 Jul 1;31(3):111628.

\section{Image/Figure:}

https:/www.xcdsystem.com/wmis/abstract/File6959/GA793_ImageFigure 0615083637.png

Image/Figure Caption: Figure caption: Images from four subjects, the left knee of one OA patient (red box) and three healthy controls, below. Axial (left) and coronal (right) views are shown of the same location in the knee joint, with T2-weighted structural images shown by themselves alongside the gagCEST overlay. No ROI is drawn on the gagCEST maps; the jet colorscale shown is $0-20 \%$ normalized asymmetry, rendering pixels with no signal navy blue. (It can be seen from this representation that gagCEST is largely able to distinguish cartilage from other tissues, with some occasional spurious signal). The OA subject shown clearly has low gagCEST signal throughout the joint, which was typical for all patients. Healthy controls whose CEST maps showed notable features frequently presented the pattern visible in the coronal images here, wherein the medial side of the knee (subjects' left side, L, of right knee) had patches of elevated signal. Images in this figure are as viewed in ITK-SNAP [8].

\section{Complete Status: Complete}


First Name: Abigail

Last Name: Cember

Email: cember@pennmedicine.upenn.edu

Organization: University of Pennsylvania

Country: United States 


\section{ID: GA794}

Changes in functional connectome to predict protein spreading in neurodegenerative diseases

Federica Agosta, , agosta.federica@hsr.it

Complete Status: Incomplete

First Name: Federica

Last Name: Agosta

Email: agosta.federica@hsr.it 
ID: GA795

Canada's Impact on PET Neuroimaging

Neil Vasdev, Centre for Addiction and Mental Health, neil.vasdev@utoronto.ca

Complete Status: Incomplete

First Name: Neil

Last Name: Vasdev

Email: neil.vasdev@utoronto.ca

Organization: Centre for Addiction and Mental Health

Country: Canada 


\section{ID: GA796}

TBD

Kullervo Hynynen, Sunnybrook Research Institute, khynynen@sri.utoronto.ca

Complete Status: Incomplete

First Name: Kullervo

Last Name: Hynynen

Email: khynynen@sri.utoronto.ca

Organization: Sunnybrook Research Institute

Country: Canada 
ID: GA797

Translating Optical Technologies to Human Trials and Commercial Success

Ralph DaCosta, University Health Network, ralph.dacosta@uhnresearch.ca

Complete Status: Complete

First Name: Ralph

Last Name: DaCosta

Email: ralph.dacosta@uhnresearch.ca

Organization: University Health Network

Country: Canada 


\section{ID: GA798}

The importance of Molecular Imaging for Drug Development: From Cancer to COVID

Kimberly Brewer, Dalhousie University, brewerk@dal.ca

Complete Status: Complete

First Name: Kimberly

Last Name: Brewer

Email: brewerk@dal.ca

Organization: Dalhousie University

Country: Canada 
ID: GA799

Tracking antigen-specific regulatory $T$ cells

Megan Levings, BC Children's Hospital Research Institute, mlevings@bcchr.ca

Complete Status: Incomplete

First Name: Megan

Last Name: Levings

Email: mlevings@bcchr.ca

Organization: BC Children's Hospital Research Institute 


\section{ID: GA800}

New directions in Immunotherapy for Early Stage Cancer - Imaging and Blood Biomarkers of Response

Patrick Forde, Johns Hopkins University, pforde1@jhmi.edu

Complete Status: Complete

First Name: Patrick

Last Name: Forde

Email: pforde1@jhmi.edu

Organization: Johns Hopkins University

Country: United States 


\section{ID: GA801}

Transcriptional recording by CRISPR spacer acquisition from RNA

Florian Schmidt, ETH Zuerich, florian.schmidt@bsse.ethz.ch

Complete Status: Incomplete

First Name: Florian

Last Name: Schmidt

Email: florian.schmidt@bsse.ethz.ch

Organization: ETH Zuerich

Country: Switzerland 
ID: GA802

Sense and Respond Recombinase Circuits

Wilson Wong, , wilwong@bu.edu

Complete Status: Complete

First Name: Wilson

Last Name: Wong

Email: wilwong@bu.edu 
ID: GA803

Integrated radiogenomics for virtual biopsy and treatment monitoring in ovarian cancer

Evis Sala, University of Cambridge, es220@cam.ac.uk

Complete Status: Incomplete

First Name: Evis

Last Name: Sala

Email: es220@cam.ac.uk

Organization: University of Cambridge

Country: United Kingdom 


\section{ID: GA804}

"The Need for Speed": The power of measuring in-vivo dynamics with highspeed imaging and microscopy

Elizabeth Hillman, Columbia, eh2245@columbia.edu

Complete Status: Incomplete

First Name: Elizabeth

Last Name: Hillman

Email: eh2245@columbia.edu

Organization: Columbia

Country: United States 


\title{
ID: GA805
}

Immune Cell trafficking in Inflammatory Diseases

\author{
Gwendalyn Randolph, , gjrandolph@wustl.edu
}

\begin{abstract}
Body : This lecture will briefly cover our long-held interest in monocyte trafficking in atherosclerosis and will point out the utility of PET tracers designed for chemokine receptor detection in studying the disease. The lecture will then transition to new findings in our research on IBD, specifically ileal Crohn's disease and mouse models of it. I will show how intravital imaging, photoconversion imaging coupled with flow cytometry, and PET imaging of chemokine receptors can be utilized together to reveal key aspects of the pathobiological basis of disease and its degree of progression, with the PET tracers having translational potential in a disease where they have not yet been applied.
\end{abstract}

Complete Status: Complete

First Name: Gwendalyn

Last Name: Randolph

Email: gjrandolph@wustl.edu 
ID: GA806

Molecular Imaging for the measurement of CNS Drug Penetration and Exposure

Roger Gunn, Invicro, roger.gunn@invicro.co.uk

Complete Status: Complete

First Name: Roger

Last Name: Gunn

Email: roger.gunn@invicro.co.uk

Organization: Invicro

Country: United Kingdom 


\section{ID: GA807}

Often disregarded but critical: The importance of tissue permeability for imaging success and clinical translation

Thomas Reiner, Novartis, thomas.reiner@novartis.com

Complete Status: Complete

First Name: Thomas

Last Name: Reiner

Email: thomas.reiner@novartis.com

Organization: Novartis

Country: Switzerland 
ID: GA809

Integrated Diagnostics: A Test is a Test

R Bryan, Dell Medical School, University of Texas Austin, Nick.bryan@austin.utexas.edu

Complete Status: Incomplete

First Name: R

Last Name: Bryan

Email: Nick.bryan@austin.utexas.edu

Organization: Dell Medical School, University of Texas Austin

Country: United States 


\section{ID: GA810}

Seeing is not always believing: uncovering truth using the tools of radiology and pathology

Katherine Lucot, Stanford University, kllucot@stanford.edu

Complete Status: Incomplete

First Name: Katherine

Last Name: Lucot

Email: kllucot@stanford.edu

Organization: Stanford University

Country: United States 


\section{ID: GA811}

MALDI Imaging for Molecular Histology

Caitlin Tressler, The Johns Hopkins University School of Medicine, ctressl3@jhmi.edu

Complete Status: Incomplete

First Name: Caitlin

Last Name: Tressler

Email: ctress13@jhmi.edu

Organization: The Johns Hopkins University School of Medicine

Country: United States 


\section{ID: GA812}

Session Introduction

Jack Hoppin, Emit Imaging, jack.hoppin@emitimaging.com

Complete Status: Incomplete

First Name: Jack

Last Name: Hoppin

Email: jack.hoppin@emitimaging.com

Organization: Emit Imaging

Country: United States 


\section{ID: GA813}

(Imaging) Biomarker-Driven Strategies for a New Hypoxia-Activated Prodrug CP-506

Philippe Lambin, Maastricht University, Philippe.Lambin@gmail.com

Complete Status: Incomplete

First Name: Philippe

Last Name: Lambin

Email: Philippe.Lambin@gmail.com

Organization: Maastricht University 


\section{ID: GA814}

Harnessing Radioluminescence and Sound to Reveal Molecular Pathology of Atherosclerotic Plaques

Raiyan Zaman, Harvard Medical School, rzaman@mgh.harvard.edu

Complete Status: Incomplete

First Name: Raiyan

Last Name: Zaman

Email: rzaman@mgh.harvard.edu

Organization: Harvard Medical School

Country: United States 
ID: GA815

Imaging a Pathway to Treatment of Preeclampsia

Carolyn Bayer, Tulane University, carolynb@tulane.edu

Complete Status: Incomplete

First Name: Carolyn

Last Name: Bayer

Email: carolynb@tulane.edu

Organization: Tulane University

Country: United States 


\section{ID: GA816}

Workhorses in the clinic; NET/PSMA

Neeta Pandit-Taskar, Memorial Sloan Kettering Cancer Center, pandit-n@mskcc.org

Complete Status: Incomplete

First Name: Neeta

Last Name: Pandit-Taskar

Email: pandit-n@mskcc.org

Organization: Memorial Sloan Kettering Cancer Center

Country: USA 


\section{ID: GA817}

Amyloid imaging in the time of an AD disease modifying therapeutic

Susan De Santi, Life Molecular Imaging, s.desanti@life-mi.com

Complete Status: Complete

First Name: Susan

Last Name: De Santi

Email: s.desanti@life-mi.com

Organization: Life Molecular Imaging

Country: United States 


\section{ID: GA819}

Terbium 'Sisters' for RadioTheragnostics: from Bench-to-Bedside

Cristina Mueller, Paul Scherrer Institute, cristina.mueller@psi.ch

Complete Status: Complete

First Name: Cristina

Last Name: Mueller

Email: cristina.mueller@psi.ch

Organization: Paul Scherrer Institute

Country: Switzerland 


\title{
ID: GA820 \\ Molecular Imaging of PARP
}

Thomas Reiner, Novartis, thomas.reiner@novartis.com

\begin{abstract}
Body : The poly(adenosine diphosphate-ribose)polymerase (PARP) enzyme familymore specifically, PARP1-is an important participant in the DNA damage response. It is upregulated in many malignancies and has been identified as a critical component for cellular survival. PARP inhibitors have shown therapeutic potential, either in conjunction with DNAdamaging agents or as monotherapies in tumors with defects in other DNA repair pathways. The upregulation of PARP imaging agents opens up a new realm of possibilities in the clinic, with optical imaging agents being used for both diagnostic purposes and improvement of surgical procedures through intraoperative imaging. Radiolabeled probes allow direct measurement of PARP expression in patients, with the goal of improving stratification of patients, quantification of target engagement by therapeutic inhibitors, and monitoring of noninvasive treatment. This presentation will look back at the development of our small molecule imaging platform for PARP1, yielding several translated imaging agents that have originated in our lab. We trace these agents from their beginning in the Lab to their current use as investigational new drugs in oral cancer, oropharyngeal cancer and brain cancer.
\end{abstract}

Complete Status: Complete

First Name: Thomas

Last Name: Reiner

Email: thomas.reiner@novartis.com

Organization: Novartis

Country: Switzerland 


\section{ID: GA821}

Opening Address: Bridging Biology and Medicine with Molecular Imaging

Julie Sutcliffe, University of California at Davis, jlsutcliffe@ucdavis.edu

Complete Status: Complete

First Name: Julie

Last Name: Sutcliffe

Email: jlsutcliffe@ucdavis.edu

Organization: University of California at Davis

Country: United States 


\section{ID: GA822}

Opening Address

Anthony Fauci, NIH, lisa.baird@netzero.com

Complete Status: Incomplete

First Name: Anthony

Last Name: Fauci

Email: lisa.baird@netzero.com

Organization: $\mathrm{NIH}$ 


\section{ID: GA825}

MPI: From the Small to the BIG World

Patrick Vogel, University of Würzburg, patrick.vogel@physik.uni-wuerzburg.de

Complete Status: Complete

First Name: Patrick

Last Name: Vogel

Email: patrick.vogel@physik.uni-wuerzburg.de

Organization: University of Würzburg

Country: Germany 
ID: GA826

A One-Year Perspective on MPI for Cell Tracking

Paula Foster, Robarts Research Institute, pfoster@robarts.ca

Complete Status: Complete

First Name: Paula

Last Name: Foster

Email: pfoster@robarts.ca

Organization: Robarts Research Institute

Country: Canada 


\section{ID: GA834}

Shailendra (Shil) Patel V.P., Codiak Biosciences, Cambridge, MA

Shil Patel, Codiak Biosciences, shil.patel@codiakbio.com

Complete Status: Incomplete

First Name: Shil

Last Name: Patel

Email: shil.patel@codiakbio.com

Organization: Codiak Biosciences

Country: United States 


\section{ID: GA835}

Mohammed Farhoud VP, Sales \& Marketing Emitt

Mohammed Farhoud, Sofie, mfarhoud@gmail.com

Complete Status: Incomplete

First Name: Mohammed

Last Name: Farhoud

Email:mfarhoud@gmail.com

Organization: Sofie

Country: United States 
ID: GA841

Ashley Knight PhD

Ashley Knight, Takeda, ashley.knight@mail.utoronto.ca

Complete Status: Complete

First Name: Ashley

Last Name: Knight

Email: ashley.knight@mail.utoronto.ca

Organization: Takeda

Country: United States 


\section{ID: GA844}

Social justice, decolonization, and equity

To be Announced, , wmis@wmis.org

Complete Status: Incomplete

First Name: To be

Last Name: Announced

Email:wmis@wmis.org 
ID: GA846

Test Test Test

Sylvia Anderson, World Molecular Imaging Society, sanderson@wmis.org

Category: Neuroscience

Abstract Body : Test

References: None

Complete Status: Complete

First Name: Sylvia

Last Name: Anderson

Email: sanderson@wmis.org

Organization: World Molecular Imaging Society

Country: United States 


\section{ID: GA847}

Introduction

Dima Hammoud, National Institutes of health, hammoudd@cc.nih.gov

Complete Status: Incomplete

First Name: Dima

Last Name: Hammoud

Email: hammoudd@cc.nih.gov

Organization: National Institutes of health

Country: United States 


\section{ID: GA850}

Bioluminescent Probes for Bacteria

Matthew Bogyo, Stanford University, mbogyo@stanford.edu

Complete Status: Incomplete

First Name: Matthew

Last Name: Bogyo

Email: mbogyo@stanford.edu

Organization: Stanford University

Country: United States 


\section{ID: GA851}

18F-avb6-targeting peptide PET for COVID fibrosis

Julie Sutcliffe, University of California at Davis, jlsutcliffe@ucdavis.edu

Complete Status: Incomplete

First Name: Julie

Last Name: Sutcliffe

Email: jlsutcliffe@ucdavis.edu

Organization: University of California at Davis

Country: United States 


\section{ID: GA852}

FDS Clinical Study

Alvaro Ordonez, Johns Hopkins University School of Medicine, aordone2@jhmi.edu

Complete Status: Incomplete

First Name: Alvaro

Last Name: Ordonez

Email: aordone2@jhmi.edu

Organization: Johns Hopkins University School of Medicine

Country: United States 


\section{ID: GA853}

PET Imaging of Pseudomonas

Clemens Decristoforo, Medical University Innsbruck, clemens.decristoforo@i-med.ac.at

Complete Status: Incomplete

First Name: Clemens

Last Name: Decristoforo

Email: clemens.decristoforo@i-med.ac.at

Organization: Medical University Innsbruck

Country: Austria 


\section{ID: GA854}

Image-guided in situ detection of bacterial biofilms in a human prosthetic knee infection model

Marleen van Oosten, , m.van.oosten01@umcg.nl

Complete Status: Incomplete

First Name: Marleen

Last Name: van Oosten

Email: m.van.oosten01@umcg.nl

Country: Netherlands 


\section{ID: GA855}

Drug biodistribution PET imaging

Filipa Mota, Johns Hopkins University School of Medicine, fdamota2@jhmi.edu

Complete Status: Incomplete

First Name: Filipa

Last Name: Mota

Email: fdamota2@jhmi.edu

Organization: Johns Hopkins University School of Medicine

Country: United States 


\section{ID: GA856}

CNS infections and intravital two-photon microscopy

Dorian McGavern McGavern, NIH, mcgavernd@ninds.nih.gov

Complete Status: Incomplete

First Name: Dorian McGavern

Last Name: McGavern

Email:mcgavernd@ninds.nih.gov

Organization: $\mathrm{NIH}$ 


\section{ID: GA857}

Alanine, etc. for bacteria

David Wilson, University of California, San Francisco, David.M.Wilson@ucsf.edu

Complete Status: Incomplete

First Name: David

Last Name: Wilson

Email: David.M.Wilson@ucsf.edu

Organization: University of California, San Francisco

Country: United States 


\section{ID: GA858}

Pathogen-specific imaging in theranostics

Jung-Joon Min, Chonnam National University, jjmin@jnu.ac.kr

Complete Status: Incomplete

First Name: Jung-Joon

Last Name: Min

Email: jjmin@jnu.ac.kr

Organization: Chonnam National University 


\section{ID: GA859}

Closing Remarks: What we are looking for in the field for the upcoming year

Sanjay Jain, Johns Hopkins University, sjain5@jhmi.edu

Complete Status: Incomplete

First Name: Sanjay

Last Name: Jain

Email: sjain5@jhmi.edu

Organization: Johns Hopkins University

Country: United States 
ID: GA860

Shripad Bangale, FujiFilm VisualSonics Application Specialist

Shripad Bangale, FujiFilm VisualSonics, shripad.bangale@fujifilm.com

Complete Status: Complete

First Name: Shripad

Last Name: Bangale

Email: shripad.bangale@fujifilm.com

Organization: FujiFilm VisualSonics 